


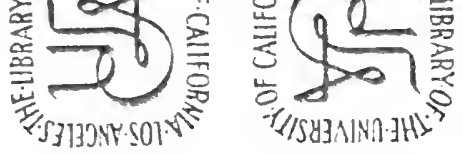
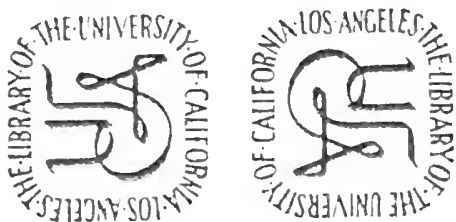

NE.LIBRARYOF

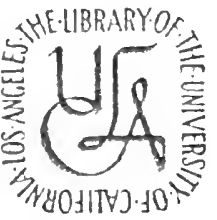

告
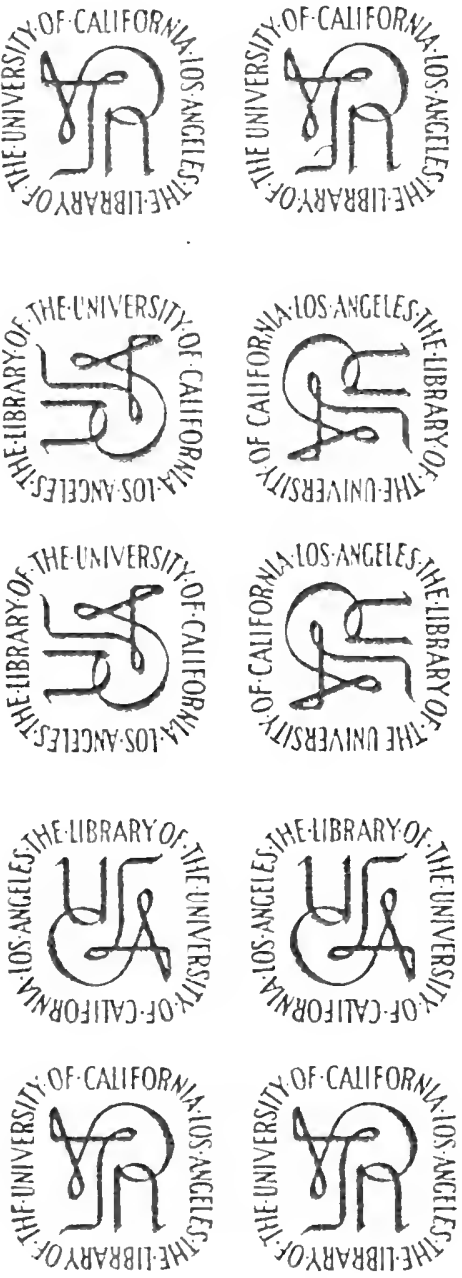

美 d d d
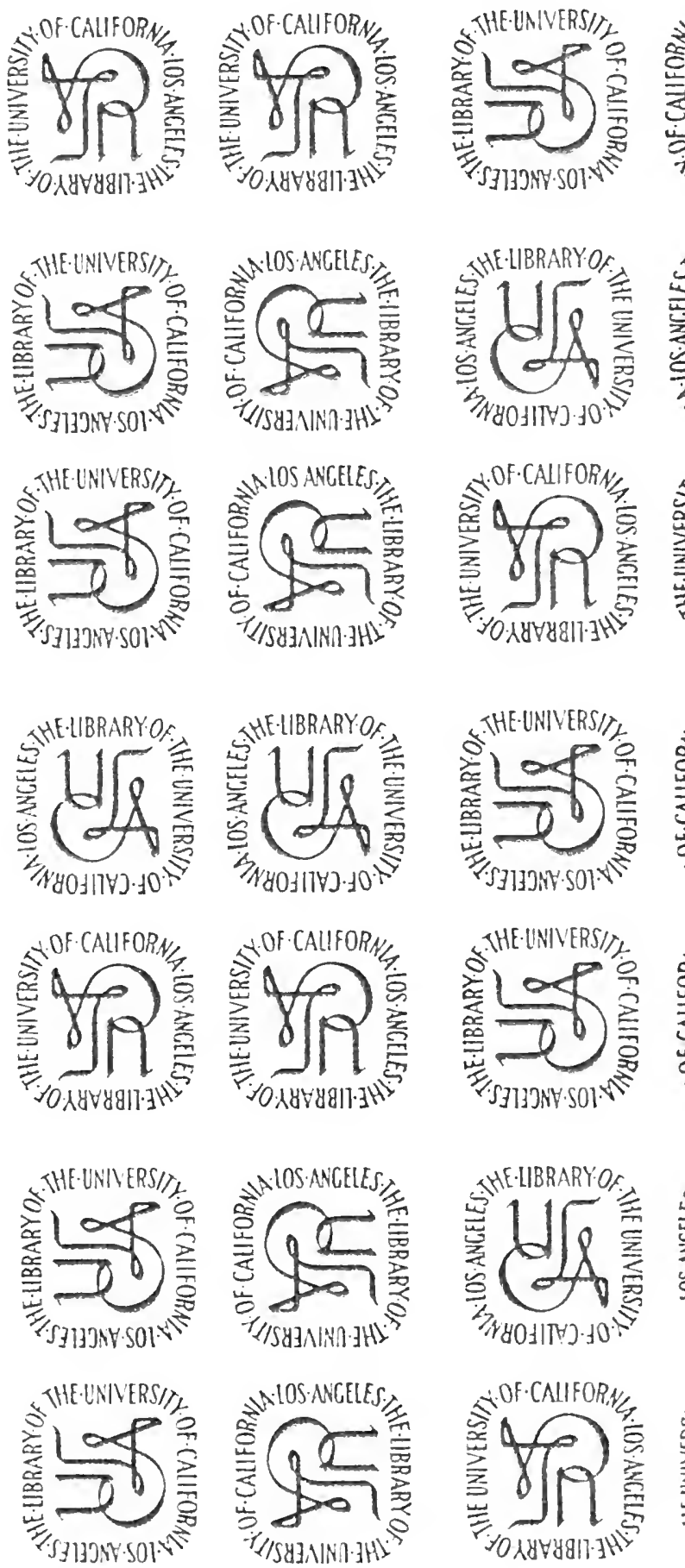
Digitized by the Internet Archive in 2007 with funding from Microsoft Corporation 




\section{THE CITIES AND TOWNS}

\section{OF CHINA}

A GEOGRAPHICAL DICTIONARY

B Y

G. M. H. PLAYFAIR

HIS BRITANNIC MAJESTY'S CONSUI, AT FOOCHOW

SECOND EDITION

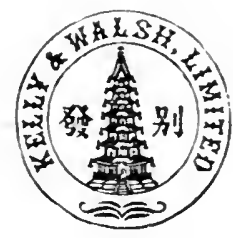

S HA NG HA I

KELLY \& WALSh, LIMITED

HONGKONG-YOKOHAMA-SINGAPORE

1910 



\section{Preface to the Second Edition.}

That over a quarter of a century has elapsed since the "Cities and Towns of China" was published would, of itself, be a sufficient excuse for issuing a second edition. There are, however, other reasons which call for it. The catacly'sm of 1900 provoked an increased interest in this country, one result of which was a run on books of reference connected with China. As a consequence of this the "Cities and Towns of Clina" has for many months been out of print, and the demand for it still contirues. In the preface to the first edition I alluded to a rmmour that "the Chinese Govermment has it in contemplation to establish gradually an Inperial postal system throughout the Empire." Twenty years were needed for the realisation of that intention, but the postal system is with us now and is extending its network over the provinces. Moreover, all the sigus of the times point in the direction of the opening of China to the world.

This second edition will be found to be increased in bulk. The appendices have been enlarged by the acldition of lists of rivers and lakes, of places mentioned by Marco Polo, of towns in Japan and of a table indicating the distribution of Mongolian Tribes. Thongh Formosa is no longer a part of the Empire of China, I have retained the enumeration of its cities as they existed under Chinese rule. The chief alteration, which it has been my aim to effect by the very considerable revision the book has undergone. is the correction of the plentiful inaccuracies with which my earlier pages were sprinkled. These, I hope, I have succeeded in elininating for the most part in the present edition.

G. II. H. PIAYYFAIR.

Foochow, September 1910. 



\section{Preface to the First Edition.}

The following work is in the main a Revisal of Bıor's "Dictionnaire des Villes Chinoises," supplemented by a catalogue of the more important ninor towns of the Enpire, inserted in their proper alphabetical places.

B1or published his work as far back as in 18 12 , and, even in conservative China, many changes have taken place during these thirty-six years necessitating not a few additions, corrections, and, occasionally, eliminations. Moreover the systen of orthography employed is not such as to make it a book of easy reference to most Europeans, and no longer finds the same favour as when the French Roman Catholic Missionaries were the chief authorities on watters relating to China. The revision I have undertaken has therefore resulted in an entire rearrangement of Bro'r"s materials.

'The utility of the list of ninor towns of the Empire, which constitutes my independent contribution to the work, may not be readily apparent. It slonld be considered, howerer, that the general nuarch of events in China tends more and more to an opening up of the country to foreign knowledge. The last few years have scen travellers penctrate, botli in an official and a private capacity, into the little known regions of Yünnan and Ssŭch'nan; the Baron von Richthofen has publisled the first instalment of his great work on China, the fruit of journeys and observations in the interior. Tlie fanine, which for so many months has been raging in Shansi and Honan, has been the cause of philanthropic missions to that part of the country in the endearour to relieve, however partially, the terrible distress there prevalent. The Chefoo Convention has opened new ports on the coast and on the Great River, has stationed a Consular Official in the middle of Ssŭch 'uan, has provided for tle residence of a representative of the British Government in Yünnan, and has prepared the way for a mission to Thibet. The rumour that the Chinese Government has it in contemplation to establish gradually an Imperial postal system thronghout the Empire, is the possible foreshadowing of a step in the same direction, and its realisation would of itself necessitate the compilation of some such list as that to which I am now alluding. The opening of the countryto Europeans must inevitably bring into prominence placis which, not baing marked on European naps, it would be a matter of great diffculty to identify. without sone such aid as this book will give. Incomplete as this list necessarily is, it is offered as a contribution towards a more thorough knowledge of the geography of China than is yet possessed by Europeans. 
In the dearth of other sources of information within my reach, the degrees of Latitude and Longitude have, with few exceptions, been taken from Bror's work and have little claim to be considered scientifically accurate. In the few instances where it was possible, they have been corrected from the British Adniralty Charts and from calculations placed at my disposal by the courtesy of Dr. FrITsche of the Russian Observatory at Peking, and in these instances the errors have been found to be of considerable moment. The Latitudes and Longitudes of localities in Manchuria, Mongolia, 'Turkestan and Thibet have been taken from Dr. Willidus' map of the Chinese Fimpire and from Johnsros's General Gazeteer. Elsewhere, in cases in which the above authorities have supplied no information, they have been calculated as carefully as possible from the Chinese Atlas. Though scientifically imperfect, they will enable the towns to be found readily on any foreign or native map of China, and this is all I claim on their account.

The orthography I have chosen is that of the $T z \ddot{u}$-êrh-chi and, on the ground that "good wine needs no bush," the choice should perhaps require no justification. Sir THOM.dS WADE's system of romanisation has, however, so often been declared unsuitable for general purposes, however well adapted to the requirements of Pekinese Mandarin, that I think it advisable to state briefly my reasons for considering it peculiarly fitted for the purposes of this book.

'This orthography', as developed in the $T z \breve{u}-\hat{c} r-h-c h i$, is beyond denial purely and simply that of the Pekinese dialect; it must be also admitted that only a sinall proportion of the Chinese people use this Northern Court dialect as their native tongue at all, and that the class which lias acquired it by education is not very numerous. Still the variety of Chinese spoken in and about the Capital is the language of polite society and bears much the same relation to other dialects as the French of Paris does to that of the provinces. Pekinese is the Langue d'Oil of China; Nankinese its Langue d'Oc. He would be a bold innovator who should propose to the world in general to pronounce all French proper names according to some provincial dialect, say Provençal or Gasconnais, for no other reason than that "ParisianFrench is too peculiarly the dialect of Paris." Yet those who would reject Pekinese as the standard dialect for China are virtually assuming this position. They persist in advocating the claims of the Iangue d'Oc though the nation itself has declared for the Langue d'Oil. Pekinese I believe to be the most suitable dialect in which to romanise Chinese proper names, (with a few exceptions to be noted presently) and to say that Sir THomas WADE's Pekinese orthography is the best, as being the most scientific, is to make a statement as nearly as possible axiomatic.

The exceptions to which I refer above are inevitable and are to be met with iu every language. In Europe an Englishman speaks of Brussells, Florence, Vienna and Copenhageu, not of Bruxelles, Firenze, Wien or Kjöbenhavn; the 
former method of spelling has become so engrafted on the language as to make a change equally impossible and undesirable. Similarly in China we must continue under ordinary circumstances to speak or write of Foochow, Amoy, Canton and Peking; no one will advosate the expediency of clanging to Fu-chou, Hsia-mên, Kuang-chou or Pei-ching. The sane rule applies to the names of the provinces, which are all inore or less familiar in their Southern form to European ears. In referring to the provinces I have therefore employed the Nankinese form, romanised in accordance with Sir TIrom.tS WADE's principles. To avoid confusion between the characters 金 and 画 I have applied the same rule to the names of the Chinese dynasties. To facilitate reference to Dr. Wri,riars' map of China, which I have assumed to be that most commonly used by foreigners in this country, I have in the case of all administrative cities added Dr. 'Virliams' orthography after the Chinese characters.*

The Chinese works which I have consulted are the Chin-shin 指紳 or "Chinese Red Book," which affords a complete list of the cities of China; the great Chinese Atlas published at Wu-ch'ang $F u$ and called the Ta-ch'ing Yi-t'ung Y $\ddot{u}-t^{\prime} u$ 大清壱統舆圖; ny edition is dated 1863. The two works just mentioned have chiefly to do with the molern part of my materials. For the ancient, or historical portion, my authority has been the Li-tai Ti-li-chih 㑛代地理志, an acconnt of the political geography of China since the Han Dynasty, published in the winter of 1870-71. I have only made use of about one-fifth of the matter contained in the Seven Historical Volumes of this Chinese work, and am not therefore in a position to claim anything like completeness for the historical portions of the following pages. Had the entire contents of these Seven Volumes been utilised, the present work would have been more than doubled.

Among other works consulted, I may mention the Cyclopædia called the $Y \ddot{u}$-hai 王海, which supplies many details about the geography of the ancient dynasties.

For the identifications of varions cities in Turkestan and elsewhere to the West of China, I have to acknowledge my obligations to Dr. BRETSCHNEIDER's able articles in the Chinese Recorder and in the Transactions of the North China Branch of the Royal Asiatic Society.

Finally to Dr. E. J. Erres, Inspector of Schools, Hongkong, my best thanks are due for his care and attention in superintending the passage of this book through the press.

G. M. H. PIAYYFAIR.

H.B.M. Consulate, Foochow, 22nd August, 1879.

* Even in one or two cases retaining obvious printers' errors, as in the instance of Fan-chih District city Shansi, which on the map has been written Fän-chi, by error for Fan-chi. 


\section{EXPLANATION}

OF

\section{'TERMS AND ABBREVIA'TIONS}

C. I. C..

C.

Chou, 州, department: the division of a province ranking above a district and below a subprefecture; usually called San Chou, 费州, in contradistinction to the next. Under the $T$ ' $a$ ng dynasty a first-class Chou comprised 30,000 fanilies or more; a second-class Chou, over 20,000 but under 30,000 families.

Chih-li Chou, ifi 潇州, independent department; i.e., independent of a prefecture. This territorial division seems to be of modern institution; as also the following.

C.I. 'T.

Chih-li T'ing, 值绿触, independent subprefecture; a division of a province ranking innediately after a prefecture and dependent directly on a circuit.

C. Wars Civil Wars; the historical period comprised between 481 and $255 \mathrm{~B}$. C.

Chên

鎬, now generally a market-town, thongh the name is also applied to the district under the connunand of a Brigade General or Chên-tai. Under the kin dyriasty the Chên seens to have been of greater inportance than at the present clay.

cistor

州, the designation of the great divisions of the Empire nswally ascribed to the Emperor Yü, and which are said to have been instituted after the Clinese Deluge. The Chou existed as a division nutil the T'ang dynasty, and represented the modern province.

Chün 那, As a territorial division the Chün varied under various dynasties from almost a province to a prefecture. As a general rule it may be taken to correspond pretty nearly to the prefecture of the present day. The word Chün is still nsed in the literary language as a synonym for Fu.

Circ. Circuit, 湴 or 路. These two Clinese characters were in use under various dynasties to denote essentially the same thing. Like most of the territorial divisions the circuit has varied greatly. Under the T'ang, it replaced the Cinot", thus corresponding to a province, the character 椺 being then in use. Under the Yüan the name 路 seens to have been given botl to what represents the morlern circuit and to the chief prefecture, or 尚府, of that circuit. The modern circuit, called 椺, consists of one or 
more prefectures, independent subprefectures or independent departments under the administrative control of an Intendant or Taotai. See also Tao infra.

Distr. District, see H.

Di'n. Dŕnasty.

E. East.

Fu Prefecture, 拊. The largest subdivision of the modern province, corresponding to the Chün of carlier dynasties.

H. District 牃. The lowest subdivision of a province, dependent on a prefecture or independent department, and sometimes, though rarely, on an independent subprefecture.

Is. Islands.

K. Kingdon 國.

Ken Adninistrative division in Japan, corresponding, in most respects, to a prefecture in China.

Lat. Iatitude. In the following pages, North is always to be understood.

Li 里, the Chinese mile, about three of which go to the mile English.

Long. Longitude. In the following pages East is always to be understood.

Marq. Marquisate, 伤國, nane given to some of the petty fendal Kingdonis in ancient times.

Mil.

or Milit.

Mil. Post Chai, 基 or 紫; the two characters are synonymons, but were used under different dyuasties.

Mil.

Station

Mil. Circ. Military circuit, 钲路, corresponding, mutatis mutandis, to the civil circuit.

Mil. Distr. Military district, 軍, 䚘 or 監.

Prefecture See Fu.

Prov. Province 少, the largest division of the Empire, of which there are eighteen in China and three in Manchuria. They correspond to the ancient Chou and also partly to the circuit 道 of the T"ang dynasty and to the Chün.

N. Nortl1.

Obs. Obsolete.

Orig. Originally.

P. Posterior.

Fao 㷛, an outpost in the less civilised parts of the Enpire; also a subdivision of a Sš̆ or township.

Post-town 馹, stations, varying in size and inportance, on the roads travelled - by Government couriers. On Clinese maps they are marked by a triangle.

R. River.

S. South. 
S. A. Spring and Autumn, 春秋, the historical period comprised between 722 and 481 B.C.

$S h \hat{e}$ 社, in Formosa, the head-quarters of a native tribe.

So 所, a guard-house, or mil. station, of less importance than a 䞑 Wei.

Ss̆̌ 司, a township.

T. T'ing 嗄, subprefecture, a provincial division subordinate to a prefecture; some having territorial jurisdiction, are marked "terr. jur."

T. C. 士 州, a native department.

T. F. 土府, a native prefecture.

T. S. 土司, a native township.

T. T. 土 屯, a native village.

Tao 道, in Corea, seems to correspond to the 路 of the Yüan dynasty, in that it apparently designates the head prefecture 首 府 in a circuit. See circ. supra.

Three Ks. Three Kingdonss; the historical period comprised between 220 and 277 A.D.

W. West.

Wei 衛, See mil. station and district.

Y. Ying 營, a camp.

Figures between square brackets refer to the rivers on which the towns are situated. See list of rivers in Appendix.

The Appendix also contains a list of the places mentioned by Marco Polo, the Chinese names of cities in Japan and the distribution of Mongol tribes. 


\section{THE DYNASTIES OF CHINA.}

NAME OF DYNASTY.

DA'I'E.

ENGIISH.

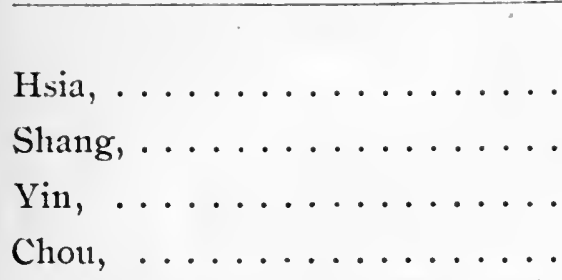

Period embraced by the " Spring and Autumn of Confucius,"...

Period knou'n as the "Civil Wars," Ts'in,

Western Han,..........

Wang Mang, (nsurper),......

Eastern Han, ..........

Three Kingdoms, ........

Minor Han, .............

Wei,

Wu

Tsin

W. Tsin,
E. Tsin,

1st Sung

$T s^{\prime} i, \ldots \ldots \ldots \ldots$

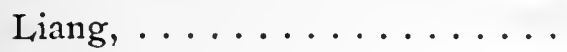

Ch'ền,

$\mathrm{N}$

\section{W}

E. Wei, . . . . . . . . .

N. Ts $^{\prime} \mathrm{i}, \ldots \ldots \ldots \ldots \ldots$

N. Chou, $\ldots \ldots \ldots \ldots \ldots$
Chinfse. Bfgan. Ended.

13.c.

1766

1401

1122

255

481

255

206

A. 1 .

9

25

221

277

263

265

277

420

317

$+20$

479

502

557

589

535

557

550

577

589 
NAME OF DYNASTY.

DA'T:

ENGir.sh.

ENTHSH.

\begin{tabular}{|c|c|c|c|}
\hline Sni, . . . . . . . . . . & 陵 & $\begin{array}{l}\text { A.D. } \\
589\end{array}$ & $\begin{array}{l}\text { A.D. } \\
618\end{array}$ \\
\hline $\mathrm{T}^{\prime}$ ang,$\ldots \ldots \ldots \ldots$ & 荅 & 619 & 907 \\
\hline Five Dynasties, . . . . . . . . & 五代 & 907 & 960 \\
\hline Posterior Liang, . . . . . . . . & 後梁 & 907 & 92.3 \\
\hline Posterior T"ang.$\ldots \ldots \ldots \ldots$ & 後颐 & 923 & 936 \\
\hline Posterior $T \sin , \ldots \ldots \ldots \ldots \ldots$ & 後丽 & 936 & 947 \\
\hline Posterior $\operatorname{Han}, \ldots \ldots \ldots \ldots \ldots$ & 後漢 & 947 & 951 \\
\hline Posterior Chou, ........... & 後周 & 951 & 960 \\
\hline Sing, $\ldots \ldots \ldots \ldots \ldots$ & 本 & 960 & 1127 \\
\hline S. Sung, $\ldots \ldots \ldots \ldots \ldots$ & 南㷊 & 1127 & 1278 \\
\hline Yüan, $\ldots \ldots \ldots \ldots$ & $\overrightarrow{\mathrm{U}}$ & 1260 & 1368 \\
\hline $\operatorname{Ming}, \ldots \ldots \ldots \ldots \ldots \ldots \ldots \ldots \ldots$ & 明 & 1368 & 1644 \\
\hline 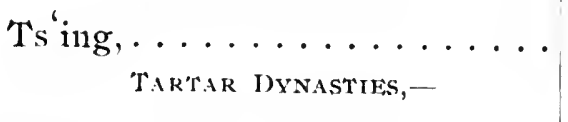 & 清 & 1644 & - \\
\hline Liao, . . . . . . . & 逊 & 907 & 1125 \\
\hline W. Liao, $\ldots \ldots \ldots \ldots \ldots$ & 西迩 & 1125 & 1163 \\
\hline Kin, $\ldots \ldots \ldots \ldots \ldots$ & 金 & 1115 & 1234 \\
\hline
\end{tabular}




\section{THE CITIES AND TOWNS OF CHINA.}

\section{A \\ 阿}

1 A-an 阿安, T-S., near Sungp'an 'T., Ssŭch nan.

2 A-chï 埧, Y., near P'ingfan H., Kansul.

3 A-chï | 埧, T-S., near Sungp'an 'T., Ssŭch' 'uan.

4 A-ên-t'ung | 恩, 峝, T-S., near Sung-p an T., Ssurch' nan

5 A-êrl1-kê-ling-t' u| 尔噶礐 圖 Araalinton, town in Dsassaktu Klianate; Iat. $47^{\circ} 30^{\prime}$, I.ong. $92^{\circ} 20^{\prime}$.

6 A-êrl1-shan| 尔飠 Arasan, town in Tarbagatai; Lat. $45^{\circ} 30^{\prime}$, Long. $83^{\circ} 15^{\prime}$.

7 A-êrh-t u-shih | 尔 圖 什 Aratuchi, town in Yarkand; Lat. $39^{\circ} 30^{\prime}$, Long. $69^{\circ} 10^{\prime}$.

8 A-lia-li | 哈里 Agari-kaul, town in Ulianghai; Lat. $50^{\circ}$ Long. $98^{\circ}$.

9 A-hsi 「細, T-S., near Sungp'au 'T., Ssŭch' uan.

\section{阿}

10 A-kê | 苹, 'T-S., near Sungp'an T., Ssŭcli nan.

11 A-k' $\mathrm{e}-\mathrm{su}$ | 克蘇 Aksu, or $1^{\circ}$ Oksn, town in Yarkand; Lat. $41^{\circ} 09^{\prime}$, Itong. $79^{\circ}$ $13^{\prime}$. See 7030 .

$2^{\circ}$ Circuit in the New Donninion; ; it comprises Harashar C.I.'T, Kuché C.L.T, Ush C.I.'T, Wênsu C.L.C., and Kurkurausu C.L.'T.

12 A-la-11111-t ê $\mid$ 刺模武 Alamut, town in Persia, 45 miles from the Caspian.

$13 \mathrm{~A}-\mathrm{la}-\mathrm{k}^{\prime} \hat{\mathrm{e}} \mid$ 拉克 Alakabo, town in Cobclo; Lat. $49^{\circ}$ $20^{\prime}$, Long. $93^{\circ} 40^{\prime}$.

$14 \mathrm{~A}-\mathrm{la}-\mathrm{k}$ 'ê-shr1o | 拉克碩, TS., in Kokonor.

15 A-lê-ch' u-k ê chucu, town in Kirin; Lat. $45^{\circ} 55^{\prime}$, Long. $126^{\circ} 40^{\prime}$. $16 \mathrm{~A}-1 \mathrm{i}-111 \mathrm{a}$ | 里馬, or A-li-mali | 力麻里, Almalik, an ancient town identified with the modern Ili. 


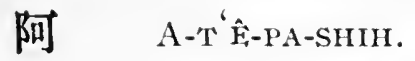

17 A-li-ma-li | 力麻里, see No. 16.

18 A-lu-huan | 鱟歡, Yüan, a region in Uliasutai.

19 A-ln-k'ê-êrh-ch'in |嚕科爾 沈 Arn-khorchin, Mongol Tribe.

20 A-mi | 迷 O-ni, C., in Lin96] an Fu, Yünnan; Lat. $23^{\circ} 43^{\prime}$, Long. $103^{\circ} 27^{\prime}$; Yüan, A-ning | 戛; Ming, A-1ni | 迷, C. Anin (Polo).

21 A-mu-li | 模里 Amol, town in Persia, prov. Mazanderan.

22 A-11ing | 察, see No. 20.

23 A-pa-ha-êrh|八哈耳 $\mathrm{Ab}$ har, town in Persia, prov. Irak-ajemi.

24 A-pa-ha-11a|巴哈納 Abaganar, Mongol Tribe.

25 A-pa-kê | 巴葛 Abaga, Mongol Tribe.

26 A-p’o 策, see No. 3838.

27 A-sa-k'ê|薩克, 'T-S., i1 Kokonor.

28 A-shn | 樹, T-S., near Sung-p'an T., Ssŭch 'nan. 29 A-ssŭ $\mathrm{t}^{6} \mathrm{a}-\mathrm{k}^{\prime} \mathrm{e}$ | 斯 塔克 Astak, town in Onter Kansuh ; Lat. $42^{\circ} 50^{\prime}$, Long. $93^{\circ} 25^{\prime}$.

30 A-tê-ch 'iao | 得 橋, T-S., near, Mien-11ing H., Ssŭch 'uan.

31 A-t'ê-pa-shih| 武八失, or A-t i-pa-shih | 骨䨐八什
要

AI-CHIH.

Atbasl, town in Independent Tartary; Lat. $40^{\circ}$, I,ong. $75^{\circ} 40^{\prime}$.

32 A-t i-pa-shili | 體入什, see No. 31.

33 A-tu| 都, T-S., near Hsich'ang H., Ssŭch' uan.

34 A-tuan | 端, Aduan, Ming, military district in Kokonor.

35 A-tung | 東, Shê in $\mathrm{N}$. Forniosa, near Changhura $\mathrm{H}$.

36 A-yi |邑, see No. 6742 . 37 A-yütan / 原, (obs.) $100 \mathrm{li}$ N.E. Huan H., Kansuh;

Sung, pao in Huan C. 38 A-yung $\mid$ 永, T-S., in Kokonor.

Abhar, see No. 23.

Abaga, see No. 25.

Abaganar, see No. 24.

Acbaluc, see No. 487.

Aduan, see No. 34.

Agari-Haul, see No. 8.

\section{AI \\ 艾}

39 Ai 艾, see No. 7458 .

40 Ai-hui | 輝 or 琿 Aihom, town in Heh-lung-kiang; Lat. $50^{\circ} 20^{\prime}$, Long. $127^{\circ}$.

\section{惑}

41 Ai-chih / 知, Aichi ken in Japan, chief town $\mathrm{Na}$ goya. 
愛

42 Ai-min | 民, (obs.) N.W. of the Aru Khorchin tribe of Mongols; Lico, $\mathrm{H}$., in $\mathrm{Wu}$ 岛 $\mathrm{C}$.

43 Ai-ÿüan | 媛, Ehimé, ken in Japan, chief town Matsuyama.

\section{霍}

44 Ai-la-k'ê town in Dsassaktu Klinnate, Lat. $49^{\circ}$, Lonng. $96^{\circ}$.

Aichi, see No. 41. Aihom, see No. 40. Airak, see No. 44. Akita, see No. 1307. Aksı, see No. 11. Alakabo, see No. 13. Alamut, see No. 12. Alimalik, see No. 16. Altai, see No. 1041. Altchucu, see No. 15. Amol, see No. 21. Amoy, see No. 2571.

\section{AN}

\section{安}

45 An 安 Ngan.

$1^{\circ} \mathrm{C}$. in Pao-ting $\mathrm{Fu}$, Chihli; Lat. $38^{\circ} 53^{\prime}$, Long. $115^{\circ} 53^{\prime} ; T^{\prime}$ ang, Wu-hsing 武 興; Kin, Yüan, Ming, An $\mid \mathrm{C}$.

$2^{\circ} \mathrm{H}$. in Mien C., Ssŭch 'uan; Lat. $31^{\circ} 35^{\prime}$, Long. $104^{\circ} 31^{\prime}$; Han Min-chiang 汶江; Yüan An $\mid \mathrm{H}$.
安

\section{AN-CHI}

$3^{\circ}$ (Obs.) $25 l i \mathrm{~N} . \mathrm{W} . \mathrm{Wu}$ ch'iao H., Chihli; $W$. Han, Marq. in $\mathrm{P}^{\prime}$ ing. yüan 平原Chïn。

$4^{\circ}$ (Obs.) in Chehkiang; E. Han, H. in $\mathrm{Wu}$ 峚 Chün.

$5^{\circ}$ (Obs.) in Shingking;

Itiao, C. in Tung-ching 東京 circ.

$6^{\circ}$ (Obs.) 90 li N.E. An

H., Ssŭch' 'uan; Yüan C. in Ch'êng-tı1 战都 circ. $7^{\circ}$ See Nos. 83, 652, 880, 1098, 1404, 2724, 5767, 5893, 6306.

46 An-ch'ang | 昌Ngan-cliang. $1^{\circ}$ Chên near Lu-ch' êng H., Shansi.

$2^{\circ}$ (Obs.) Sui, H., S. E. of Ho-nei Fu, Honan.

$3^{\circ}$ See Nos. 435, 1504.

47 An-ch' êng | 战, see No. 584. 48 An-cl'êng 城。

$1^{\circ}$ Chên in Pin C.,

Kuangsi.

$2^{\circ} \mathrm{Fu}$ in Ching-ch'i 京畿 circ. Corea.

$3^{\circ}$ See Nos. 64, 1384, 7743.

49 An-clii | 基, (obs.) $120 / i \mathrm{~S}$. of 'T'êng H., Knangsi; Sui, H., in Yung-p ing 永平Chün。

50 An-chi | 吉 Ngan-kih, H., in Huchou Fin, Chehkiang; Lat. $30^{\circ} 40^{\prime}$, I.ong. $119^{\circ} 36^{\prime} ; \mathrm{W}$. Han, 
努 $\mathrm{AN}-\mathrm{CH}$ iNG.

Kn-chang故彰; E. Han, An-chi | ; Sung, C., in Chê-hsi 淅西 circ.

51 An-ch'i | 溪 Ngan-ki.

[261] $1^{\circ} \mathrm{H}$. in Ch iian-chon $\mathrm{F} u$, Fuhkien; Lat. $25^{\circ} 12^{\prime}$, Long. $118^{\circ} 10^{\prime}$; Five dy'n. Ch'ing-ch' i 清 溪; Sung, An-ch ‘ $\mid, \mathrm{H}$.

$2^{\circ}$ Chên néar Yüi-hang H., Chehkiang.

52 An-chia | 家, Y., near Yung-shon H., Shensi.

53 An-chiang | 漭, Chên near Tan-yang H., Kiangsu.

54 An-chiang | 汇.

$1^{\circ}$ (Obs.) near Yüanchou F11, Honan; Sung, military station in Yüan 沅 C.

$2^{\circ}$ See No. 3456.

55 An-ch'ing | 磨 Ngan-king, [528] $\mathrm{Fu}$ in the An-Lï-Ch $11-$ Ho circ. of Anhuii; Lat. $30^{\circ} 32^{\prime}$, Long. $117^{\circ}$ $0 \tau^{\prime}$; this city is the capital of the province, and forms also the District city of Huai-ning; E. Han, Wan 皖 $\mathrm{H}$.

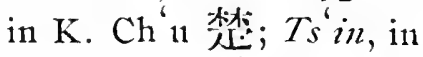
Chin-chiang 九江 chïn; Han, in Lü-chiang 盧江 chïn; $W_{e i}$ and $W_{u}$, Chung-chên 重 鎭; Liang, Chin 晋 C.; Sui,
发 $\mathrm{A} \times-\mathrm{CH} \ddot{\mathrm{U}}$.

T'ung-an 同努; T'ang，

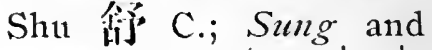
Yïan, An-ch'ing | Nanghin (Polo). Port of call.

56 An-ch'in | 邱 Ngan-ki11, [503] H., in Ching-chou Fu, Shantung; Lat. $36^{\circ} 23^{\prime}$, Long. $119^{\circ} 42^{\prime}$; orig. $\mathrm{Ch}^{\prime}{ }^{\mathrm{ii}}$-Ch'in 渠 邱; $W$. Han, Ch'ang-an 星 H. in K. Kao-mi 高密; E. Han, Ch'ang-an 昌 | Marq. in K. Pei-hai 北海; Tsin, Ch'ang-an 昌| H. in Ch'êng-yang 城陽 chün; Sui, Mou1shan 牟 III; S. Sung, Ch'ang-an 昌| H. in Kao-mi 高密 chïn; $N$. $W_{e i} \mathrm{Ch}^{\prime}$ ang-an 昌 $\mathrm{H}$. in P'ing-ch'ang 平昌 chiin.

57 An-chon | 州 Fun in P'ingan 平妄 Circnit, Corea. 58 An-chï| 居.

$1^{\circ}$ (Obs.) S. W. Suining H., Ssŭch' nau; Sui, H. in Tzŭ-yang 資荡 chün; $T^{\prime}$ ang and Sung, H. in $\mathrm{P}^{\prime}$ u 望 $\mathrm{C}$. $2^{\circ}$ (Obs.) near Wênch'uan H., Ssŭch'uan; T'ang, H. in Pao 保 C.

$3^{\circ}$ (Obs.) in T'ung-liang H., Ssŭch'uan; Ming, $\mathrm{H}$. in Chung-ch'ing 重度 Fu。 
安

ANi-F̂े̃G.

59 An-chnang | 荘, post-town near Chên-ning $\mathrm{H}$, Kueichou.

60 An-clnung | 䈨, see No. 435. 61 An-fêngl風, (obs.) $130 \mathrm{li}$ S.IV. of Ho-chin H., Anhui ; $V V$. Han, H. in K. Lint-an 六 $\mid$;E.Han, Marq. in I, ï-chiang 盧江 chïn; $7 \sin , \mathrm{H}$. in Anfêngr |

62 An-fêng 豐.

$1^{\circ}$ Chên near Hsi-an Fin, Shensi.

$2^{\circ}$ Chên near Fun-ning H., Kiangstu.

$3^{\circ}$ Sung, military district in Huai-hsi 淮 陹 circ. $4^{\circ}$ (Obs.) E. of Ku-shint H., Honan; W. Han, H. in K. I,in-an 六|; E. Han, H. in I,iichiang 盧江.chün; Tsin, H. in An-fêng | chün. $5^{\circ}$ (Obs.) 130 li S.W. Ho-ch iu H., Anlıui ; $T \sin , S$. Sung and $S$. Ts'i, chün and H. in Yii 預 Curo:

$6^{\circ}$ (Obs.) in Tê-hura H., Kiangsi ; S. Sung, H. in Hsün-yang 潯陽 chün. $7^{\circ}$ (Obs.) 60 li S.W. of Shou C. Anhui ; N. Wei, chün and H. in Ho 篗 CHOU and Yang 掦 Cuor ; Sui, H. in Huainan 淮南 chün; T"ang,
安

AX-HUA.

H. in Shou 㗲 C.; Sung,

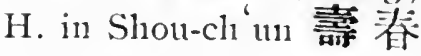
Fu ; Yüan, H. in An-fêng circ.

$S^{\circ}$ (Obs.) in Shingking; Kin, H. in Ch'ïan 솟․ $9^{\circ}$ (Obs.) in Shon C.,

Anluni; Yïan, circ. in Honan. $10^{\circ}$ See No. 6179.

63 An-fu| 卢, see No. 1184.

64 An-fu| 鬸 Ngan-ful.

[450] $1^{\circ}$ H. in I.i C., Hunan;

Iat. $29^{\circ} 22^{\prime}$, I ong. $111^{\circ}$ $25^{\prime}$.

$[6+4] 2^{\circ}$ H. in Clii-an Fin, Kiangsi; I at. $27^{\circ} 70^{\prime}$, Long. $114^{\circ} 34^{\prime}$; W. Han, An-p ing $7^{3}$ and Anch'êng 城; E. Han, P'ing-tu年都; T'ang An-fu | $\mathrm{H}$.

$3^{\circ}$ (Obs.) Sui H., IV. of

Chï̈n C., Hupeh.

65 An-han | 漢, see No. 5815. 66 An-hua | 论, Ngan-hwa.

$1^{\circ} \mathrm{H}$., forming the prefectural city of Chingyang, Kansul1; Lat. $36^{\circ}$ 03', Long. $107^{\circ} 43^{\prime}$; Han, Ÿ̈̈-chil 椎郅; Sui, Ho-shui 合 水; Sung. An-hua | | $\mathrm{H}$.

[389] $2^{\circ} \mathrm{H}$. in $\mathrm{Ch}^{\prime}$ ang-slia $\mathrm{Fu}$, Hunan, orig. Mei-shan 梅山 H.; J.at. $25^{\circ} 24^{\prime}$, Long. $111^{\circ} 25^{\prime}$. 
$3^{\circ} \mathrm{H}$., forming the prefectural city of Ssŭ-nan, Kuei-chon; Lat. $27^{\circ} 56^{\prime}$, Long. $108^{\circ} 26^{\prime}$.

$4^{\circ}$ Chên 35 li S.W. of

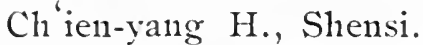
Kin, | chên.

$5^{\circ}$ (Obs.) $W . W_{c i}, \mathrm{H}$. near Sui C., Honan.

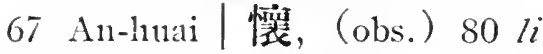
S.E. Kuei-shan H., Kuangtung; S. Sung | H.; S. Tsi, TungKuan 東官 chün.

68 An-hnang | 兴; see No. 6306.

69 An-hui | 徽 Ngan-hwui, a province of Clina ; literary name Wan 皖; also called $T^{6}$ ung-an 同安 and Wan-cliiang 皖江; capital, An-cl'ing Fil; it contains 3 circ., $8 \mathrm{Fu}$, 1. T., 5 C.I.C., 4 C., and $52 \mathrm{H}$.

70 An-hsi | 陌 Ngan-si.

$1^{\circ}$ C.L.C. in Kansuh; Lat. $40^{\circ}$, Long. $95^{\circ} 45^{\prime}$; Sung, An-hrsi | H. Ÿ̈an Kua 瓜 C.

$2^{\circ}$ (Obs.) near Turfan; $T^{\prime}$ ang, Fin in Lung-yu 㗥右 circ.

$3^{\circ}$ See Nos. 101, 2438, 2620.

71 An-hsi /息, ancient name of Parthia.

\section{努 AN-JUNG。}

72 An-Hsiang-Yün-Ching | 裹 珼荆, circ. in Hupeh, comprising An-lu Fu, Hsiang-yang Fu, Yünyang Ful, and Chingmên C.

73. An-hsiang | 郷 Ngan-hiang. $1^{\circ}$ H. in Li C., Hunan ; Lat. $29^{\circ} 22^{\prime}$, I ong. $112^{\circ}$ $02^{\circ}$; Han, $\mathrm{Ch}^{\prime}$ uan-ling 孱陵; Tsin, Nan-p'ing 南本; $C h h^{6} e n$, An-hsiang $\mathrm{H}$.

$2^{\circ}$ See No. 6889.

74 An-hsing / 興, (obs.) near Ching-chou Fin, Hupeh. $T$ 'ang, $\mathrm{H}$.

75 An-jên|仁 Ngan-jin.

$1^{\circ} \mathrm{H}$. in Hêng-chon $\mathrm{F} n$, Hunani ; Lat. $26^{\circ} 43^{\prime}$, Long. $113^{\circ} 04^{\prime}$; orig. a chên; Sung, Yüan, Ming, H. in Hêng-chon.

[58] $2^{\circ}$ H. in Jao-chou Fin, Kiangsi ; I at. $28^{\circ} 25^{\prime}$, Long. $117^{\circ} 02^{\prime}$.

$3^{\circ}$ Chên near Chao-yi H., Shensi.

$4^{\circ}$ (Obs.) in A nnanin; Sui, H. in Chiao-chih 交趾:chün. $5^{\circ}$ (Obj.) 30 li S.E. Tayi H., Ssŭch' uan ; $T$ 'ang and Sung, H. in Chiung 邛 C.

76 An-jung | 我, (obs.) E. of Ch'in-an H., Kansuh ; $N$. $W_{e i}, \mathrm{H}$. in Lüehyaug 略陽 chïn。 
安

77 An-k'ang | 康 Ngan-kang.

$1^{\circ} \mathrm{H}$. forming the prefectural city of Hsing-an, Shensi ; Iat. $32^{\circ} 31^{\prime}$, Long. $109^{\circ} 22^{\prime}$.

$2^{\circ}$ See Nos. 1969, 2815.

78 An-kou | 跟.

$1^{\circ}$ Chên near Yent-clíang H., Sliensi.

$2^{\circ}$ See No. 3388.

79 An-kn| 固.

$1^{\circ}$ Chên near I,ung-tê H., Kansuh.

$2^{\circ}$ (Obs.) Liang, H. near $\mathrm{P}^{\prime}$ êng C., Ssŭch' nan.

$3^{\circ}$ See Nos. 3024, 5200.

80 An-kuei | 貴, (obs.) $I V$. $W_{e i}$, H. near Sui C., Honan.

81 An-lê | 樂, (obs.) T'ang, town S. of Y'u H., Hunan.

82 An-ling | 陵, chên, 25 li N.W. of Wu-ch'iao H., Cliihlil.

83 An-lu| 陸 Ngan-luh.

$1^{\circ}$ Fu in An-Hsiang-YünChing circ., Hupel, forming also the District city of Chung-hsiang; Lat. $31^{\circ} 07^{\prime}$, Long. $112^{\circ}$ $39^{\prime}$; S. A. and C. Wars, Ying-chung 鹃中 in $\mathrm{K}$. $\mathrm{Cl}_{1}{ }^{\prime}$ 焚; $T s^{6}$ in, in Nan 南 Chün；Han, Yün-tı 雲杜; Ching-ling 竟 陵 S. Sung and Sici, chuint; Wei, Ying 趴 C.; Liang,
安 Ax-MIX.

Nan-siŭ 南司 and Pei$h_{1} \sin$ 北新; $W . H_{c i}, \mathrm{An}$ | C., and Wên, 泪 C.; P. Chou, Shili-cli'êng 石 城; T'ang, Ching-shan 京山 and Fu-sluni 富 水; Yïan, An-lu! Fin ; Ming, Ch' êng-t ien 张天 Fin.

$2^{\circ} \mathrm{H}$. forming the prefectural city of Tê-an Fin, Hupelı; Iat. $31^{\circ} 20^{\prime}$, Long. $113^{\circ} 37^{\prime} ; \mathrm{Han}$, An-lu | H.; Sui, Chirang 吉陽; T'ang, C. in Huai-nan 淮南 circ.; Five dyn. An-lu| |C. $3^{\circ}$ See Nos. 1949, 196\%, 6306.

84 An-L ï-Ch' ${ }^{\prime}$-Ho | 盧滁利, circ. in Anluni, comprising An-ching Fin, I, ïChou Fu, Ch' 11 C. and Ho C.

85 Anl-ling | 隆, see No. 2480.

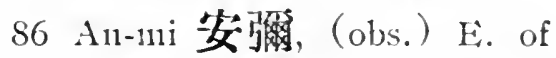
Su C., Kansul, E. Han, Tsin, H. in Clint-ch '̈̈an 酒泉chiin。

ST An-min| 尼.

$1^{\circ}$ (Obs.) in the Ongniod country, N. of Jehol; Liao, H. in Jao 鲜 C. $2^{\circ}$ (Obs.) in Shing-king; Liao, H. in Iien 連 C. $3^{\circ}$ (Obs.) near Hsï-chon Fin, Ssurch 'uan; Kin, Chên in 徐 $\mathrm{C}$. 
安

AN-P'ING.

88 An-11an| 南 Ngan-11an.

$1^{\circ} \mathrm{H}$. in Hsing-yi $\mathrm{Fu}$, Knei-chon; Lat. $25^{\circ} 48^{\prime}$, Long. $105^{\circ} 13^{\prime}$.

$2^{\circ}$ (Obs.) T"ang, Fu in

Ling-11an, 領南 circ.

$3^{\circ}$ Tributary Kingdon

(Annan).

89 An-ning | 杼 Ngan-ning; C.

[371] in Yün-11an $F$ n, Yünnan;

Lat. $25^{\circ}$, Long. $102^{\circ} 38^{\prime}$;

Orig. 'T"ang-lang-ch" "wan

螳蛝川 in K. Tien 滇;

Han, Lien-jan 連然;

Tang, An-ning | $\mathrm{H}$.

90 An-pei | 北(obs.) T"ang, C.

91 An-pien / 罌 Ngan-pien.

$1^{\circ}$ '. in Yentan Fin,

Shensi; Lat. $37^{\circ} 40^{\prime}$,

I.ong. $107^{\circ} 42^{\prime}$.

$2^{\circ}$ Chên near Iing C.,

Shansi.

$3^{\circ}$ Fin in Hsien-p ing 战车。 circ., Corea.

$4^{\circ}$ See No. 3407.

92 An-ping 4 \% Nant-ping.

[351] $1^{\circ}$ T. in $\mathrm{K}^{\prime}$ ai-hna $\mathrm{F} u$,

Yünnan; I at. $23^{\circ} 02^{\prime}$,

Long. $104^{\circ} 50^{\prime}$.

[225] $2^{\circ}$ 'T-C. in 'T'ai-p'ing Fu,

Kuangsi; I at. $22^{\circ} 43^{\prime}$,

Long. 106 49'; Han, Po

波 C.; Sung, An-p ing

$3^{\circ}$ H. in Shên C., Chihli;

Lat. $38^{\circ} 16^{\prime}$, Long. $115^{\circ}$

$38^{\circ}$; Han, An-p'ing |

| : \%sin, Po-ling 博陵;

Sui, Shên 深 C.
然 AN-SHUN

$4^{\circ} \mathrm{H}$. in An-shun $\mathrm{Fu}$, Kueichou; Iat. $26^{\circ} 23^{\prime}$, Long. $106^{\circ} 12^{\prime}$.

$5^{\circ}$ Chên near 'T'ai-wan Fu Fulikien.

$6^{\circ}$ Ss̆̆ near An-jên H., Hunan.

$7^{\circ}$ Post-town near Paoying H., Kiangsu.

$S^{\circ}$ Post-town near $K^{6}$ neichou Fu, Ssŭch' "ran.

$9^{\circ}$ See Nos. 64, 4945, 7252. 93 An-p $n$ | 浦, (obs.) $W u, \mathrm{H}$. 30 li W. of L,ê-an H., Kiangsi.

94 . In-sai | 藇 Ngan-sai, H. in [536] Yen-an Fn. Shensi; Lat. $36^{\circ} .48^{\prime}$, Long. $109^{\circ} 13^{\prime}$; Han, Kao-nu 高奴; P. llici, Kuang-lê 厝樂; Suri, Chin-ming 金明; Yüan, An-sai $\mid \mathrm{H}$.

95 An-sha 妾沙, Ssŭt near Yung-an H., Fulukien.

96 An-shan | Ilchün in Chingcli’i 京畿 circ., Corea.

97 An-shan | 傸 H. in Chiangyüan 江原 circ., Corea. 98 An-shun | 順 Ngan-shun. [326] $1^{\circ} \mathrm{Fu}$ in Kuei-lisi circ., Kueichou, forming also the District city of $\mathrm{P}^{\prime} \mathrm{u}$ ting; Lat. $26^{\circ} 13^{\prime}$, Long. $105^{\circ} 53^{\prime}$; Yüan, Hsi-an 習努 C.; Ming, An-shun C.

$2^{\circ}$ T-C. in Au-sluun Fu, Kueichou. 
安 Aヘー゙。

99 An-su | 藏 Ngan-sulı.

$1^{\circ}$ Circ. in Kansuh, con1prising A $11-h_{1}$ siC., and SuC. [356] $2^{\circ} \mathrm{H}$. in Pao-ting F ${ }^{\prime}$, Chihli; Iat. $39^{\circ} 02^{\prime}$, Long. $115^{\circ} 47^{\prime}$; Han, Sui-ch'êng 遂城; Fice dy'n. An-su $\mid$.

100 An-tê | 德, see Nos. 3949. 4951, 6305.

101 An-ting | 定 Ngan-ting. [78] $1^{\circ} \mathrm{H}$. in Yen1-an $\mathrm{Fu}$, Sliensi ; Lat. $37^{\circ} 15^{\prime}$, Long. $109^{\circ} 29^{\prime} ; W^{\prime}$. I'ci Fên | 汾 C. ; Sung, Yich'uan 宜川; Ÿ̈an, An-ting $\mid$.

[221] $2^{\circ}$ H. in Kung-ch 'ang Fu, Kansulı; Lat. $35^{\circ} 38^{\prime}$, I.ong. $104^{\circ} 38^{\prime}$; Sung, An-hsi | 西, walled city in Hui 會 C.; Kin, Anlısi | 西 H., in Ku1ng 琴 C. ; Yüan, Ting-lnsi 定西 C., in Sllensi.

$3^{\circ}$ T-S., near Ssŭ-ên Fu, Kuangsi.

$4^{\circ}$ Post-town near ling C., Kansuh.

$5^{\circ}$ (Obs.) military station 1,500 li S.W. Kan-chon Fu, Kansuh ; also known as Sarihuiur.

$6^{\circ}$ See Nos. 3288, +250, 4672,5171 .

102 An-tsê $\mid$ 涬, see No. $761 \mathrm{~s}$. 103 An-t 11 圖, T-S., in Kokonor.

\section{发 AN-YH.}

140 An-tung | 東 Ngan-tung. $1^{\circ} \mathrm{Fu}$ in Ch'ing-Slang 磨 们 circ. Corea; Lat. $36^{\circ}$ $40^{\prime}$, Long. $128^{\circ} 35^{\prime}$.

[148] $2^{\circ}$ H. in Huai-an Fu, Kiangsu; Lat. $33^{\circ} 47^{\prime}$, Long. $119^{\circ} 27^{\prime}$; $\mathrm{H}^{\circ}$. W' $\mathrm{W}^{\prime}$, Hai-an 海 | ; Sui, Lien shui 速水; Sinng, Antuing $\mid$.

$5^{\circ} \mathrm{Y}$. near Y'ung-1ning $\mathrm{H}$., Hunan.

$4^{\circ} \mathrm{H}$. in Fêng-hnang C.L.'T., Shing-king.

$5^{\circ}$ (Obs.) 7'ang, Fu; Sung C. in Hnai-nan 淮南 circ.; Yüan, C. in Huaian 淮 | circ. ; lling, H. in Huai-an 淮发 Fu.

105 An-tz'u 管 次, see No. 6698.

106 An-wu | 奖, (obs.) 50 li S.lW. of Ching H., Anhui ; Tsin. S. Sung, and s. Tsi, H. in Hsüancliêng 宣城 chün.

107 An-yang | 院 Ngan-yang.

$1^{\circ} \mathrm{H}$., forming the prefectural city of Chang-tê, Honan; Lat. $36^{\circ} 0 \bar{r}^{\prime}$, Long. $114^{\circ} 30^{\prime}$; oris. . Hsiang 相; Tin, Anyang | ; Han, Tangvin 港陵; Sui, Hsiang 相 C.

$2^{\circ}$ An-yang | 陽, se No. 6242.

108 An-yeh | 菜, see No. 416 . 
109 An-yi | 宜, (obs.) S.W. Pao-ying H., Kiangsu; S. Ts ${ }^{\prime} i, \mathrm{H}$. in Yang-p ing 陽平 chïn; Sui, H., in Chiang-yang 江陽 chiin.

110 An-yi | 義 Ngan-i, H., in [124] Nan-k'ang Fin, Kiangsi ; Lat. $28^{\circ} 46^{\prime}$, Long. $115^{\circ}$ $30^{\prime}$.

111 An-yi | 沂, (obs.) 120 li S. of 'T' êng H., Kuangsi ; $S$. Sung and S. Ts' $i, \mathrm{H}$. in Yung-p'ing 永平 chiun.

112 An-yi|费.

$1^{\circ}$ (Obs.) E. of Hsi-ning H., Kansuh; W. Han, E. Han, H. in Chin-ch êng 金城 chïn; $T \sin , \mathrm{H}$. in Hsi-p ing 西平 chiin.

$2^{\circ}$ (Obs.) S. of Wu-ch' uan H., Kneichou; Sung, H. in Ssŭ 思 C.

$3^{\circ}$ (Obs.) near Hsï-chou Fu, Ssŭch' nan; Sung, nililitary station in Ch' angning 長漂 chün.

113 An-yi | 邑 Ngan-yih, H., [537] in Chieh C., Shansi ; Lat. $35^{\circ} 05^{\prime}$, Long. $110^{\circ} 58^{\prime}$; orig. An-yi | |, the residence of the Emperor Yü 禹; Sui, Yü 虞 C.

114 An-yin | 陰 Ngan-yin, H., in Ch ing-Shang 慶份 circ., Corea ; Lat. $35^{\circ} 12^{\prime}$, Long. $127^{\circ} 10^{\prime}$.
安

AN-FA.

115 An-yin-tzŭ | 銀子, posttown near Huai-jên H., Shansi.

116 An-yo | 岳 Ngan-yoh.

[553] $1^{\circ} \mathrm{H}$. in T'ung-ch'uan Fu, Ssŭch' 'uan; Lat. $30^{\circ} 07^{\prime}$, Long. $105^{\circ} 23^{\prime}$; Liang, $\mathrm{P}^{\prime} \mathrm{u}-\mathrm{tz}$ ' ù 前慈; $P$. Chou, An-yo | ; T'ang, $\mathrm{P}^{\prime} \mathrm{u}$ 音 C.

$2^{\circ} \mathrm{H}$. in Corea; Lat. $38^{\circ}$ $50^{\prime}$, Long. $125^{\circ} 34^{\prime}$.

117 An-yüan | 遠 Ngan-yuen. [299] $1^{\circ}$ H. in Kan-chou Fu, Kiangsi ; Lat. $25^{\circ} 17^{\prime}$, Long. $115^{\circ} 13^{\prime}$; Han, Yï-tu雱都; Liang, Anyüan

$2^{\circ}$ Chên near Fu-ch'iang H., Kansuh.

$3^{\circ}$ Ss̆̌ near Ninghua H., Fuhkieil.

$4^{\circ}$ (Obs.) Sung H. near

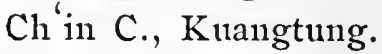

$5^{\circ}$ See No. 6306.

鞍

118 An-chiang-t'un 鞍匠驰， Ss ̌̆ near I tuan-p ing H., Chihli.

\section{俺}

119 An-tu-huai 俺都淮 Andkhui, town W. of Balkh, Bokhara.

\section{潒}

$120 \mathrm{An}$-fa 菼盐 $\operatorname{chên}$ in Haiyang $H$., Kuangtung. 
奥 AO-SIIAN-WEI.

Andkluui, see No. 119.

Anin, see No. 20.

Annam, see No. 88.

\section{AO}

\section{奥}

121 Ao-lung 奥竜, chên near Wu-hu H., Anlui.

\section{澳}

122 Ao-mên 澳 門 Macao, Portuguese Colony situated in Hsiang-slian $\mathrm{H}$., Kuangtung ; Lat. $22^{\circ} 12^{\prime}$, Long. $113^{\circ} 30^{\prime}$.

\section{瞥}

122a Ao-chin 敬禁, see No. 5822 .

\section{媪}

123 Ao-wei 媼圍, see No. 7032.

\section{敖}

124 Ao-ch' êng 敖城, ssü near T'ai-110 H., Kiangsi.

125 Ao-han 敖哭 (or 漢) Aoklian, Mongol Tribe. 126 Ao-han | 漢, see No. 125. 鰲

127 Ao-shan-wei 謷山衛, $S$ s near Chi-mo H., Shantung.

Aokhan, see No. 125. Araalintou, see No. 5. Arasañ; see No. 6. Aratuchi, see No. 7. Aru-Khorchin, see No. 19.
奥 AO-SHAN-WEI.

Astak, see No. 29.

Atbash, see No. 31.

Awomori, see No. 1218.

Babu, see No. 4775.

Bactria, see No. 6035.

Badakshan, see No. 4779.

Bagdad, see No. 4799.

Baharain, see No. 4806.

Bai, see No. 4817.

Bakamanitou, see No. 4316.

Balkl, see No. 4770.

Bamian, see No. 4774.

Banka, see No. 4402.

Barchin, see No. 4759.

Barchuk, see No. 4762.

Bardaa, see No. 4760.

Barin, see No. 4772 .

Barkhalighend, see No. 4759.

Barkoul, see No. 425.

Barluk, see No. 4764.

Barsis, see No. 4797.

Barskoto, see No. 4769.

Bashi, see No. 4777.

Bathang, see No. 4781.

Bavurd, see No. 4785.

Berim, see No. 2106.

Bishbalik, see No. 5099.

Bocca Tigris, see No. 2167.

Bodognikdai, See No. 5255.

Bokhara, See No. 5270.

Bokokar, See No. 5226.

Bolo, See No. 5227.

Boinbay, See No. 4392.

Bost, See No. 5284.

Bukukhan, See No. 5226.

Bulkur, See No. 5272.

Burgultai, See No. 5268. 
扎 CHA-MU-TA.

Burkat olgurta, See No. 5269.

Buroul, See No. 5273.

Cacan fu, See No. 2064.

Caohan fu, See No. 5318.

Caicli11, See No. 829.

Caiju, See No. 3334.

Cain, See No. 1621.

Caindu, See No. 171.

California, See No. 1041.

Cambaluc, See No. 5822.

Cannal, See No. 1907.

Canton, See No. 3393.

Caracoran, See No. 1901.

Carajan, See No. 6049, 7813.

Casa Branca, See No. 938.

Cascar, See No. 3224.

Cashmere, See No. 658.

Cangigu, See No. 786.

Canly, See No. 363.

Cayı, See No. 3171.

\section{CHA}

扎

128 Cha-ha-ch'in 扎哈 沁, Djakchin, Mongol Tribe.

129 Cha-kê-êrh | 噶忽 Yikar, town in Thibet; Lat. $29^{\circ}$ 10', Lo11g. $91^{\circ} 50^{\prime}$.

130 Clia-lai-t'ê | 賃特 Djalaid, Mongol Tribe.

131 Cha-lu-t'ê魯特 Djarnd, Mongol Tribc.

132 Cha-mu-ta | 穆達 Ghianda, town in Thibet; Iat. $30^{\circ}$, Inong. $93^{\circ} 40^{\prime}$.
札 ChA-PU.

133 Cha-shih-lu-mu-pu | 什贈 穆布 Teshihlumbu, town in Thibet, also written Cha-shih-lu11-pu | 什倫布; capital of Ulterior Thibet; Lat. $29^{\circ}$, Long. $89^{\circ}$. Also called Zhikatse.

134 Cha-shili-lun-pu| 什倫布, sec No. 173.

135 Clia-ya / 雅 Djaya, town in 'Thibet; Lat. $31^{\circ} 45^{\prime}$, Long. $98^{\circ}$.

\section{札}

136 Cha-hsi-kang 札 䤼 剛 Teshigang, town in Thibet; Lat. $32^{\circ}$, Long. $79^{\circ} 45^{\prime}$.

137 Cha-hnang | 愰 Sapporo, name of a ken and its chief town, Japan.

138 Cha-1nu | 木 Jann, town in Yarkand; Lat. $41^{\circ} 15^{\prime}$, Long. $79^{\circ} 50^{\prime}$.

139 Cha-sa-ku-t’u Han | 薩 古圆汗 Dsassaktı Khanate, in Mongolia, extending from Outer Kansuh on the $S$. to Ulianghai on the $\mathrm{N}$; bounded on the E. by Sainnoin and on the W: by Cobdo.

140 Clia-wu | 武, T-S., in Kokonor.

141 Cha-ling, see No. 147.

142 Cha-pu, see No. 152. 
后 CH'A-P'U.

\section{CH'A}

\section{补}

143 Ch'⿳亠口冋-ho 歪河, T-S., near Sh1nn-111ng Fu, Yünnan. 144 Ch'a-k'ou1-pao | 口堡, posttown near P'ing-fan $\mathrm{H}$., Kansulh.

$145 \mathrm{Ch}^{6}$ a-tao-k'oul | 道口, posttown near Yen-ch'ing C., Chihli.

\section{莱}

$146 \mathrm{Cl}^{\prime}$ a 菻, chên near Shihch iuan H., Shensi.

147 Cl'a-ling | 陵 Clia-ling, C. [302] in Ch'ang-sha Fu, Hunan ; Lat. $26^{\circ} 54^{\prime}$, Long $113^{\circ} 23^{\prime} ; \quad T^{\prime}$ ang Ch'a-ling | 陵H.W. Har. Yeh-ling 䒩陵.

148 Ch'a-yang | 陽, post-town near Yen-p'ing Fu, Finhkien.

149 Ch'a-yiian | [国, chên near Shun-an H., Chehkiang.

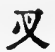

$150 \mathrm{Cl}^{\prime}$ a-t ang 紧, chên near Chieh-hsin H., Shansi.

151 Ch'a-chien 没澗, chên near T'ien-ch'ang H., Anhui.

\section{乍}

152 Ch'a-p' u 乍浦 Cha-pu, town near $\mathrm{P}^{6}$ ing-hu H., Chelnkiang, the residence of a high military official and of 2 Subprefect.
祭

СH'A-MU-'TO

查

153 Ch'a-ch'êng 查城, posttown near Yung-ning C., Kueichou.

\section{祭}

$154 \mathrm{Ch}$ 'a-ch'ih 察赤, also written Shih 石, Chê-shih 柘石, Chê-chih 柘支, Chê-chê 柘 折, Shash, ancient name of 'T'ashih-yiian 塔失元, otherwise Ta-shih-han 達失 干, or 'T'a-shih-han 塔 什干, Taskkend, a town of Independent Turkestan, 90 miles N.W. Khokand.

$155 \mathrm{Ch}$ 'a-han-sont-êrh | 漢搜尔 Chagen-suol, town in Sainnoin ; Lat. $46^{\circ} 50^{\prime}$, Long $97^{\circ} 30^{\prime}$.

156 Ch'a-han-t'o-lo-hai | 漢托 羅海, post-town near Wan-cl' '̈̈an H., Chihli.

$157 \mathrm{Ch}$ 'a-ha-êrlı | 哈 Chahar, region $\mathrm{N}$. of Shansi ; partly in Kuei-shi T., Shansi, partly in Hsüan-hua Fu, Clihli. The Mil. Lientenant Governor resides at Kalgan.

$158 \mathrm{Ch}$ 'a-mu-to | 稳采Chamdo, town in Thibet; Lat. $32^{\circ}$ $05^{\prime}$, Long. $97^{\circ} 30^{\prime}$.

Chagen-snol, see No. 194. 
毛

Chan-'T.

\section{CHAl}

\section{笔}

159 Chai-lung 宅龍, T-T., near Lü̈-slian H., Ssŭch' uan.

\section{CH'Al}

\section{柴}

$160 \mathrm{Ch}^{\prime}$ ai柴, (obs.) S. T'ai-a11

H., Shantung; W. Han,

H. in T'ai-slian 泰山 chiin.

$161 \mathrm{Cl}^{\prime}$ ai-sang | 桑, see No. 7146.

$162 \mathrm{Ch}^{\prime}$ ai-ts'un | 村, (obs.) in Shansi; Kin, chên in Chiang 綘 C.

163 Chama, see No. 1318.

164 Chamdo, see No. 196.

\section{CHAN}

165 Chan-ta 琶迋 T.S. South of T'êng-yüeh T., Yünnan.

\section{占}

166 Chan-pei-k'ou 占北口, Y., near Mi-yün H., Chihli.

167 Chan-t'i | 蛼, (obs.) S.W.

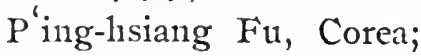
E. Han, H. in Lê-lang 樂浱 chiun。

\section{站}

毡

168 Chan-ti 毡的 Djend, tow11 near mouth of R. Silion.
㳣

Chan-wU.

\section{浔}

169 Chan-hua 露化 Chen-hwa, [284] H. in Wu-ting Fu, Shantung; Lat. $37^{\circ} 40^{\prime}$, Long. $118^{\circ} 04^{\prime}$; orig. Po-hrai 渤 海; Sung, Chao-an 招安; Kin，Clran-hua 化.

170 Chan-yi | 昷 Chen1-yil, C. [335] in Ch'üi-ching Fu, Yünnan; Lat. $25^{\circ} 38^{\prime}$, Long. $103^{\circ} 4^{\prime}$; Han, Yüan-wên 苑溫; $T^{\prime}$ ang, Hsi-p ing 西平; Yïan, Chan-yi 盆睢。

171 Chan-tui 睢 對, the local name of the Chien-cli ang region in Ssŭch'uan [see No. 848]. From the sound of these characters is supposed to be derived Marco Polo's "Cain-du" [Baber]. Also known as 敏你雅克 Miniak or Menia.

172 Chan-tui-ju 曕對茹, T-S., near Ta-chien-lu $T$.; Ssŭch 'uan.

173 Chan-tui-sa-tun | 對撒墩, T-S., near Ta-chien-lu T., Ssŭch'uan.

\section{湛}

174 Chan-wu 湛梧, (obs.) in Annam; $T \sin , H$. in Chiur-chên 九真 chün. 
張 Chang-Chia-K'ou.

\section{CH'AN}

\section{糏}

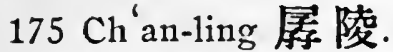

$1^{\circ}$ Ssŭ near Kung-an H., Hupeh.

$2^{\circ}$ See Nos. 73, 2214, 3502.

\section{滻}

$176 \mathrm{Cl}^{\prime}$ 'an-ts'un 㶐村, (obs.) near Ching C., Hunan ; Sung, proo in Cling 靖 C.

Chan-du, See No. 553.5

\section{CHANG}

\section{長}

177 Chang-tzŭ $\mid$ 子 Chang-tsz; [104] H. in Lu-an Fu, Shansi; Lat. $36^{\circ} 05^{\prime}$, Long $112^{\circ}$ 50'; Han, Chang-tzŭ 子; 2nd Wei, Lê-yang樂 陌; sui, Chi-shil 黛氏.

\section{張}

178 Chang-ch' êng 張城, (obs.) formerly town near Chieh C., Shansi.

179 Chang-chi | 吉, chên near Jung-ho H., Shansi.

180 Chang-ch' $\mathrm{i}$ | 溪, chên near Tung-liu H., Anhui.

181 Chang-chia-chi | 家集, sš̆ near Kuang-hua H., Hupetr.

182 Chang-chia-k'ou | 家口 Chang-kia, or Kalgan, T.
張 ChaNG-TENG.

in Hsüan-hua Fu, Chihli, and included in the $\mathrm{K}$ 'onpei circ.; Lat. $40^{\circ} 51^{\prime}$, Long $114^{\circ} 53^{\prime}$..

183 Chang-chia-p ing | 家坪, ssŭ near Hsin-ning $\mathrm{H}$., Hunan.

184 Chang-chia-wan | 家灣, walled town near ' $\mathrm{T}$ 'ung C., Chihli.

185 Chang-ch iao / 橋.

$1^{\circ}$ Chên near Fu-p'ing H., Shensi.

$2^{\circ}$ Post-town near Sui-ning H., Kiangsu.

186 Chang-chin | 金, chên near Mêng H., Honan.

187 Chang-ch'iu 張秋, chên near Tung-ch ang $F u$, Shantung.

188 Chang-chu | 濐, chên in

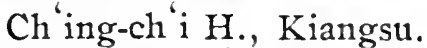
189 Chang-chuang | 庄, chên near Huo-chia H., Honan. 190 Chang-hung | 洪, chên near San-shui H., Shensi.

191 Chang-hsia | 夏, chên in $\mathrm{Cl}^{\prime}$ ang-ch'ing $\mathrm{H}$., Shantung.

192 Chang-ku | 谷, chên near P'ing-lu H., Shansi [No. $5117]$.

193 Chang-lan | 蘭, chên near Hsiao-yi H., Shansi.

194 Chang-san | 三, Y., near Fêng-ning H., Chihli.

195 Chang-têng | 登, ssü in

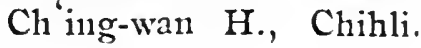




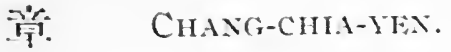

196 Chang-ts'un | 村.

$1^{\circ}$ Post-town near Fu C., Shensi.

$2^{\circ}$ [Obs.] 60 li N.W. Têng C., Honan ; Kin, chên in Têng 鄧 C.

197 Chang-yeli | 掖 Chang-yé.

(The second character has also the sound $y i$.)

$1^{\circ} \mathrm{H}$. forming the prefectural city of Kan-chon, Kansuhn; Lat. $39^{\circ} 01^{\prime}$, - Long. $100^{\circ} 56^{\prime}$; Ming, Shanhsi 傸西 ss̆̌ in Shensi.

$2^{\circ}$ (Obs.) Han, chün occupying part of Kansull.

198 Chang-yen | 医, chên near

Chin-shat1 H., Kiangsu. 199 Chang-yi-ch'êng | 儀城, (obs.) formerly town $\mathrm{E}$. of Pao-ning Fin, Ss̆̆. chinan.

200 Chang-ỹo | 岳, chên near Yi-shih H., Shansi.

\section{章}

201 Chang-an 章 妍.

$1^{\circ}$ (Obs.) 115 li S.E. Lillhai H., Chehkiang ; E. Han, H. in Kneichi 會稽 chün; Tsin, $S$. Sung, and S. Ts' $i, H$. in Lin-hai 臨海 chün.

$2^{\circ}$ See Nos. 3024, 3738, $3860,6185,6421$.

202 Cliang-chia-yen | 家 医, chên near $\mathrm{Cl}_{1}^{\text {'ing'p }}$ ' $n \mathrm{H}$., Kiangsu.
漳 $\mathrm{CHANG-CHOC}$.

203 Chang-chia-tı | 家渡, chên near Ching H., Anluii.

204 Chang-ch'in | 邱 Changkiu, H. in Chi-nan Fu, Shantung; Lat. $36^{\circ} 49^{\prime}$, Long. $117^{\circ} 36^{\prime} ;$ S. $A$. Kao-t'ang 高 唐; Han, Yang-ch iu 晹 邱; Sui, I,in-chi 臨沙and Changch'in | 邱.

205 Cliang-ku| 谷, T-S., near Ta-chien-lı 'T., Ssŭch-llan.

206 Chang-kung | 貢, see No. 3099.

207 Cliang-shan | Il, (obs.) Sui town near Chingmên C., Hupel.

\section{漳}

208 Chang 漳 Chang.

$1^{\circ}$ (Obs.) H. in Kungcli 'ang Fin, Kansulı; Lat. $34^{\circ} 40^{\prime}$, Long. $104^{\circ} 38^{\prime}$. $2^{\circ}$ See Nos. 210, 214.

209 Chang-chiang | 江.

$1^{\circ}$ Ss̆̈ in $\mathrm{P}^{\prime}$ ing-ho H., Fuhkien.

$2^{\circ}$ See No. 6236 .

210 Chang-clion | 州 Chang[272] chatl, Ful in T'ingChang-Lung circ., Fuhkien, forming also the district city of Lung-ch' $\mathrm{i}$; Iat. $24^{\circ} 31^{\prime}$, Long. $117^{\circ}$ $43^{\circ}$; Chou, $\mathrm{Ch}^{6} \mathrm{i}-\mathrm{min}$ 七 閩; Han in Kuei-chi 會 嵇 $c h u ̈ n ; \quad T^{\prime} a n g$, 
樟 ChANG-SHU。

Chang | C., and Changp'u | 浦; Ning, Changchon | 州 Fin. Zayton [Polo] teste Phillips.

211 Chang-hsien | 繋, posttown in Irung-hsi, H., Kansul.

212 Chang-la ! 臟, Y., near Sung-p an T., Ssŭch'nan.

213 Chang-p'ing | 平 Chang[95] ping, H. in Funing $\mathrm{Fu}$, Fuhkieu ; Lat. $25^{\circ} 16^{\prime}$, I ong. $117^{\circ} 38^{\prime}$.

214 Chang-p' $u$ | 浦 Chang-pu. $1^{\circ} \mathrm{H}$. in Chang-chou $\mathrm{Fu}$, Fuhkien; Lat. $24^{\circ} 07^{\prime}$, I,ong. $117^{\circ} 49^{\prime} ; T^{\prime}$ ang, Chang-p' $\mathrm{u}$ | 浦; Ÿ̈̈an, Cliang | C.

$2^{\circ}$ See No. 210.

215 Chang-t'ing | 汀, ssŭ near P'ing-ho H., Fulhkien.

216 Chang-tsê / 澤, chên near I,11-ch'êng H., Shansi.

217 Cliang-yin | 隐, See No. 6990.

\section{毫}

218 Chang-lin 樟林, sš̆ near Cl' ao-chou Fu, Kwangtunig.

219 Chang-num | 木 chên in Cl' êng-hai H., Kuangtung.

220 Chang-shu | 樹, chên in Ch'ing-chiang H., Kiangsi.
彰 Chang-Tt̂.

221 Chang-ts'un | 村, chên near Hsin-ch'êng H., Kiangsi.

\section{彰}

222 Chang-huna 彰化 Changhwa, H., in 'T'ai-wan Fu, Fulıkien; Lat. $24^{\circ}$ $30^{\prime}$, I ong. $120^{\circ} 53^{\prime}$.

223 Chang-ming | 明 Chang[125] ming, H., in Lung-an Fu, Ssŭch'nan; I at. $31^{\circ} 37^{\prime}$, Long. $104^{\circ}$ 48'; Han, Fou 涪 C.; Tsin, Han-ch'ang 漢昌; Swi, Ch'ang-lung 昌隆。 in Chin-shan 金川 chün; $T^{\prime}$ 'ang, Ch'ang-ming 昌 明; $P$. Chou, Chang-ming 明.

224 Chang-pa-li 彰八里, or Cli ang-pa-la 昌八刺, or Ch'ang-chi 昌吉, Djambalik, town E. Manas, Outer Kansulh.

225 Chang-tê | 德 Chang-telı, [562]Fu in Ho-pei circ., Honan, forming also the district city of An-yang. Lat. $36^{\circ}$ $07^{\prime}$, Long. $114^{\circ} 30^{\prime} ;$.S.A., Tung-yang 東隄 in $\mathrm{K}$. Chin 㠫; C. Wars, Weiyeh 魏㸁; Ts'in, Shangtang 上黨 and Han-tan 期貚; Han, Wei 魏 chün; Wei, Yelı-tu 業都; 2nd Wci, Hsiang 相 C. ; Five dy'n., Chang-tê | 德. 
丈 Chang-JÊN.

226 Chang-wu | 武, see Nos. 2995, 6799, 8417.

\section{彰}

227 Chang部, (obs.) Ts'in, chün, comprising part of Anhui, see No. 2358.

\section{丈}

228 Chang-jên 丈人, chển near T'un-lin H., Shansi.

Chang, see No. 309.

Chang-chai, see No. 230.

Chang-chau, see Nos. 210, $242,312$.

Chang-chi, see No. 238.

Chang-chun, see No. 244.

Chang-ling, see No. 250.

Chang-hwa, see Nos. 222, 291.

Chang-kia, see No. 182.

Chang-kih, see No. 288.

Chang-kiu, see No. 204.

Chang-koh, see No. 253.

Chang-li, see No. 293.

Cliang-lol,, see Nos. 256, 292, 297.

Chang-111, see No. 6521.

Chang-ngan, see No. 229.

Chang-ning, see Nos.

263, 297, 317.

Chang-o, see No. 2102.

Chang-ping, see Nos. 213, 299.

Chang-pu, see No. 214.

Chang-sha, see No. 267.

Chang-shan, see Nos. 268, 319.
長 CH'ANG-AN.

Chang-shau, see No. 271.

Chang-shuh, see No. 320. Chang-tai, see No. 274.

Chang-teh, see Nos. 225. 321.

Chang-ting, see No. 276.

Chang-tsing, see No. 241. Chang-tsz', see No. 177.

Chang-tu, see No. 302.

Chang-twan, see No. 245. Chang-w11, see No. 279. Chang-yang, see No. 280. Chang-yé, see No. 197.

Chang-yih, see No. 306.

Chang-yuen, see Nos. 283, 284, 307.

\section{CH'ANG}

\section{長}

229Ch'ang-an 長安Chang-ngan.

$1^{\circ}$ T. in Pao-ch'ing $\mathrm{Fu}$, Hunan.

$2^{\circ} \mathrm{H}$., forming with Hsienning $\mathrm{H}$., the prefectural city of Hsi-an, Shensi ; Lat. $34^{\circ} 17^{\prime}$, Long. $108^{\circ} 58^{\prime}$; Chou, Haoching 鎬京; $T s^{6}$ in, Han, Sui, T'ang, Sung, and $K$ in, H. in Chingchao 京兆 $\mathrm{Fu} ; Y$ ïan, H. in Fêng-yüan 奉元 circ. ; Ming H. in Hsian 西努 $\mathrm{Fu}$, Shensi.

$3^{\circ}$ Chên near Hai-ning C., Chehkiang. 
長 $\mathrm{CH}^{\prime} \mathrm{ANG}^{-\mathrm{CH}}{ }^{\prime} \mathrm{I}$.

$4^{\circ} S s \breve{u}$ near Huai-yüan $H$., Kuangsi.

$5^{\circ} \mathrm{Y}$. near T'ung-tao H., Hunan.

$6^{\circ}$ Post-town near Linhsiang H., Hunan.

$7^{\circ}$ Post-town near Yench'ing C., Chihli ; Ming 11 pao.

230 Ch'ang-chai | 寨 Changchai, T., in Kuei-yang Fu, Kueichou; Lat. $25^{\circ}$ $30^{\prime}$, Long. $107^{\circ} 10^{\prime}$.

$231 \mathrm{Ch}^{\prime}$ 'ang-ch' êng | 城.

$1^{\circ}$ Chên near Ho-shun H., Shansi.

$2^{\circ}$ Post-town near $T^{\prime}$ ai-an $\mathrm{Fu}$, Shantung.

$3^{\circ} \mathrm{Fu}$ in $\mathrm{Ch}^{\prime}$ üan-lo 全 羅 circ., Corea.

$232 \mathrm{Ch}$ 'ang-chi $\mid$ 吉, sš̌ near Ch'ang-ning H., Kuangtung.

$233 \mathrm{Ch}^{\prime}$ ang-ch' $\mathrm{i}$ | 溪.

$1^{\circ}$ (Obs.) S. of Fu-ning Fu, Fulikien ; $T^{\prime}$ ang and Sung, H. in Fu 福 C. $2^{\circ}$ See Nos. 1866, 1876, 4707.

$234 \mathrm{Ch}^{6}$ ang-ch'i | 歖 H. in Ch'ing-shang 庻们 circ., Corea.

235 Ch âng-ch' $i$ | 崎 Nagasaki, ken and chief town, Japan.
長 $\mathrm{CH}^{\prime} \mathrm{ANG}-\mathrm{CHOU}$.

$236 \mathrm{Ch}^{6}$ ang-chiang | 江.

$1^{\circ}$ (Obs.) 60 li W. of P'êng-ch 'i H., Ssŭch'uan; Sui, H. in Sui-ning 遂 俩 chün.

$2^{\circ}$ (Obs.) W. of P'êngcli'i H., Ssŭch'uan. $T^{\prime}$ ang, H. in Sui 遂 C.

$237 \mathrm{Cl}$ 'ang-chiang-pu | 江垆, ssŭ near Ying-ch' êng H., Hupeh.

238 Ch'ang-chil | 诒 Changchi, H., forming the prefectural city of $\mathrm{I}_{11-a 11}$, Shansi; Lat. $36^{\circ} 07^{\prime}$, Long. $113^{\circ} 13^{\prime}$.

$239 \mathrm{Ch}^{\prime}$ ang-ch'ih | 池, (obs.) S. of Nan-chiang H., Ssŭch'nan; Sui, H. in Ch'ing-pei 清北 chiin.

$240 \mathrm{Ch}$ 'ang-ching | 津 Fu in Hsien-ching 咸鏡 circ., Corea.

$241 \mathrm{Ch}^{\prime}$ ang-ch'ing | 清 Changtsing, H. in Chi-nan Fu, Shantung ; Lat. $36^{\circ} 45^{\prime}$, Long. $116^{\circ} 4^{\prime}$; Han, In 盧; Sui, Ch'angch'ing | 清; T'ang, Shan-jên 山荏.

242 Ch'ang-chon | 洲 Changchau.

$1^{\circ} \mathrm{H}$., forming with $\mathrm{Wu}$ H., and Yüan-ho H., the prefectural city of Su-chou, Kiangsu ; Lat. $31^{\circ} 28^{\prime}$, Long. $120^{\circ} 44^{\prime}$; 
長 Ch'ANG-HSING.

orig. Ch'ang-chon-yüan 洲苑; $T^{6} \mathrm{ang}, \mathrm{Ch}$ 'angchon1 洲.

$2^{\circ}$ see No. 5904.

$2+3 \mathrm{Cl}^{\prime}$ ang-chon-y ïan 長洲苑, see No. 242.

244 Ch'ang-clunan |湍 Fu in Ching-ch'i 京畿 circ., Corea. Lat, $38^{\circ} 18^{\prime}$, Long. $126^{\circ} 50^{\prime}$.

245 Clı'ang-cl'‘un | 春 Changchunn, C.L.'T'. in Chi-lin circ., Kirin. Lat. $43^{\circ} 40^{\prime}$, Long. $125^{\circ} 05^{\prime}$; Liao, C. and H. in Shang-ching 上京 circ.; Kin, H. in $\mathrm{T}^{6} \mathrm{ai}$, 瑃 $\mathrm{C}$.

246 Ch'ang-fêng | 豐, (obs.) 60 li N.E. Jên-ch 'iu H., Chilli; T'ang, H. in Mo 莫 C.; Kin, chên in Mo 莫 $\mathrm{C}$.

247 Ch'ang-fêng | 楓, chên in Huai-ning H., An-lutii.

248 Ch'ang-fu | 甫 Ss̆̌ in Huang-yen H., Chehkiang.

249 Ch'ang-huai | 淮, military station near Chin-shan H., Kiangsu.

250 Ch'ang-hsing | 舆 Changhing, $H$. in Hu-chou Fu, Chehkiang; Lat. $31^{\circ} 01^{\prime}$, Long. $119^{\circ} 43^{\prime}$; Han, Ku-chang 故鄣; Tsin, Ch' ang-ch'êng
長 CH'ANG-L Ê.

城; T'ang, Chih 雉 C.; Five dyn., Clin ang-hsing 興.

$251 \mathrm{Ch}$ 'ang-hising | 行, sš̆ near T'êng H., Kuangsi.

$252 \mathrm{Ch}^{\prime}$ 'ang-jun | 閏, chen near Ch' êng-ch' êng $\mathrm{H}$., Shensi.

253 Ch'ang-kê | 党 Chang-koh.

[405] H. in Hsü C., Honan; Lat. $34^{\circ} 15^{\prime}$, Long. $113^{\circ}$ $03^{\prime} ;$ S.A., Ch' ang-kê 营; Han, Cl' ang-shê 社.

254 Ch' 'ang-kung | 蓊, (obs.) $T^{\prime}$ ang, H., W. of 'T'êng H., Kunangsi.

$255 \mathrm{Ch}^{\prime}$ ang-lan | 蘭, chên near Chu1-chi H., Chehkiang.

256 Ch' 'ang-lê | 樂 Chang-lol. $[554] 1^{\circ} \mathrm{H}$. in Yi-ch'ang $\mathrm{Fu}$, Hupeh ; Lat. $30^{\circ} 10^{\prime}$, Long. $111^{\circ} 15^{\prime}$.

$2^{\circ} \mathrm{H}$. in Fu-chon $\mathrm{Fu}$, Fuhkien; Lat. $25^{\circ} 55^{\prime}$, Long. $119^{\circ} 20^{\prime}$; orig., Min 閩 H.; T⿱ ang, Hsin- $^{\prime}$

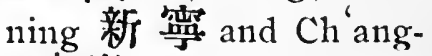
1e | 樂.

$3^{\circ} \mathrm{H}$. in Chia-ying C.,

Kuang-tung; Lat. $24^{\circ}$ $02^{\prime}$, Long. $115^{\circ} 36^{\prime}$; $T s^{\prime}$ in, Hsing-ning 興富; Ming, Ch' ang-1ê $\mid$ 樂 $\mathrm{H}$. $4^{\circ}$ See Nos. 576, 1870, $1871,3174,5704$. 
長 Ch'ANG-Ning.

257 Ch'ang-li | 利, (obs.) Han, town W. of Yünyang $H$. Ssŭch'nan; see also No. 5170.

$258 \mathrm{Cl}^{\prime}$ ang-lien | 連, H. in Huang-hai 哄海 circ., Corea.

$259 \mathrm{Cl}^{\prime}$ ang-lin | 林, (obs.) $T^{6}$ ang, H. near Chingmên C., Hupeh.

$260 \mathrm{Ch}$ 'ang-lu | 蘆, see No. 6521.

261 Ch'ang-lung | 龍.

$1^{\circ}$ Chên near Hsiang-y üran H., Shansi.

$2^{\circ}$ Ss s̆̈ in Clı̀nng-yi H., Kiangsi.

$262 \mathrm{Ch}$ 'ang-1ning | 明, (obs.), rang, H. near Yao C., $\mathrm{Y}$ ünnan.

263 Ch'ang-ning | 耍 Changning.

$[197] 1^{\circ} \mathrm{H}$. in Nan-ch' ang Fu, Kiangsi; Lat. $28^{\circ} 53^{\prime}$, Long. $115^{\circ} 37^{\prime}$.

$2^{\circ} \mathrm{H}$. in Hsü-chou $\mathrm{Fu}$, Ssŭch 'uan; Lat. $28^{\circ} 15^{\prime}$, Long. $104^{\circ} 50^{\prime} ; \mathrm{Han}$, Chien-wei 腱 焉; Sui, Lü-ch' '1an 瀘川; 'T"ang $\mathrm{Ch}^{\prime}$ ang-ning | 耍.

$3^{\circ} \mathrm{H}$. in Hui-chon Fu, Kuang-tung; Lat. $24^{\circ}$ $07^{\prime}$, Long. $113^{\circ} 51^{\prime}$.

$4^{\circ}$ Chên near $\mathrm{Ch}^{\prime} \mathrm{ien} C$. . Shensi.
長 CH'ANG-SHA.

$5^{\circ}$ Chên near Hant-chung Fu, Shensi.

$6^{\circ}$ Chên near Shun-hua H., Shensi.

$7^{\circ}$ Post-town near Hsünhua T., Kansuh.

$8^{\circ}$ Post-town near Ninglisia Fu, Kansull.

$9^{\circ}$ (Obs.) S. Sung, military district in $\mathrm{T}^{\prime}$ 'ungchun 潼川 circ.

$10^{\circ}$ (Obs.) T"ang, H. near Ching-chou Fit, Hupeh.

$11^{\circ}$ See Nos. 539, 957.

264 Clinang-Pao 長質, circ. in Hunan, conrprising Ch'ang-sha Fu and Paoch ing Fu.

$265 \mathrm{Ch}$ 'ang-pin | 筫, (obs.) in Annam; Tang, H. in T'i-shang 提上.

206 Ch'ang-p'ing | 平.

$1^{\circ}$ Post-town near Kaoping H., Shansi.

$2^{\circ}$ Post-town near C $h_{1}^{\prime}$ unrran H., Fulhkien.

$3^{\circ}$ See Nos. 3156, 6546.

267 Ch'ang-sha | 沙 Changsha.

$[17 \%] 1^{\circ} \mathrm{F} 11$ in Cli ang-Pao circ., Hunan, forming the two District cities of Cl' angsha and Shan-lina; capital of the province; Lat. $28^{\circ} 12^{\prime}$, Long. $112^{\circ}$ $47^{\prime}$; Chom, Hsing-sha 星. 
長

沙; S.A. and C. Wars, Ch'ien-chung 默中 and Hsinng-hsiang 熊湘 in K. Cl' 11 楚; $T s^{\prime}$ in and Han, Ch'ang-sha | 沙; Three $K ' s$, 1st nnder Minor Han, 2nd under Wu; Tsin, Hsiang 湘 C., Sui, and $T^{6}$ ang, T'an 潭 C.; Sung, Wu-an 武安; Ming: Ch'ang-sha|沙 Fit.

$2^{\circ} \mathrm{H}$. forming witl Shanhua $H$. the prefectural city of $\mathrm{Ch}^{\text {'ang-sha, }}$ Hunan; Iat. $28^{\circ} 12^{\prime}$, Long. $112^{\circ} 47^{\prime} ; T s^{\prime} i n$, Hsiang 湘 H.; Han, Lin-hsiang 臨湘; Sui, Ch'ang-sha |沙; Five $d y^{\prime \prime}$., Lung-hsi 龍喜.

$3^{\circ}$ Y. near Lê-hui H.,

Kuangtung.

$4^{\circ}$ (Obs.) Ts'in and Han, chün comprising Hunan. $5^{\circ}$ See No. 2031.

268 Ch'ang-shan | U Changshan.

[186] $1^{\circ} \mathrm{H}$. in Chi-nan $\mathrm{Fu}$, Shantung; Iat. $36^{\circ} 56^{\prime}$, Long. $118^{\circ} 04^{\prime}$; Han, Yü-ling 於 陵; 1st Sung, Wu-ch'iang 武强; Sui, $\mathrm{Cl}^{6}$ 'ang-shan | 山.

$2^{\circ}$ See No. 1023.

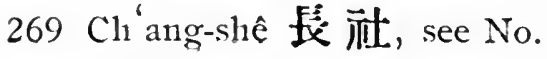
253.
長

CH'ANG-T'ING

$270 \mathrm{Ch}^{\prime}$ 'ang-shou | 收, chên near Wei-11an H., Shensi.

$271 \mathrm{Cl}^{\text {' }}$ ang-shou | 訾 Changshau.

$1^{\circ} \mathrm{H}$. in Chung-ch' ing Fu, Ssŭch 'nan; Lat. $29^{\circ} 58^{\prime}$, Long. $107^{\circ} 09^{\prime}$; orig., Ch'ien-chung 黔 中; Tang, Lê-wên 樂 溫.

$2^{\circ}$ Ss̆̆ near $\mathrm{P}^{\prime}$ ing-chiang H., Hunan.

$3^{\circ}$ See No. 2515.

$272 \mathrm{Ch}^{\prime}$ ang-shui | 水.

$1^{\circ}$ (obs.) $T s^{\prime}$ in, $H$. near

Chia-hsing Fu, Chehkiang. $2^{\circ}$ See No. 699.

273 Ch'ang-sung | 松, (obs.) S.W. Wên H., Kansuh ; Sui, H. in Wutu 武都 chiïn.

274 Ch'ang-t'ai | 泰Chang-tai, [95] H. in Chang-chou $\mathrm{Fu}$, Fulukien; Lat. $24^{\circ} 3 \tau^{\prime}$, Long. $117^{\circ}$.

$275 \mathrm{Ch}^{\prime}$ ang-tao | 道.

$1^{\circ}$ Post-town near Li H., Kansuh.

$2^{\circ}$ See Nos. 2458, 3729.

$276 \mathrm{Ch}$ 'ang-t'ing | i丁 Changting.

$1^{\circ} \mathrm{H}$. forming the prefectural city of $T$ 'ingchou, Fuhkien; Lat. $25^{\circ}$ $45^{\prime}$, Long. $116^{\circ} 30^{\prime} ;$ Tsin, Hsin-1o 新 羅; $T^{\prime}$ ang, Ch'ang-t'ing | i丁. $2^{\circ}$ See No. 1249. 
長 CH'ANG-YÜAN.

$277 \mathrm{Ch}^{\prime}$ ang-tun | 敦, ssŭ near $\mathrm{Cl}_{1}{ }^{\text {in }}$; C., Kuangtung.

278 Cli'ang-wu | 武 Chang-wu, [67] H. in Pin C., Shensi; Lat. $35^{\circ} 12^{\prime}$, Long. $107^{\circ}$ $46^{\prime}$.

$279 \mathrm{Ch}^{\prime}$ ang-yang | 洯 Chang[74] yang, H. in Yi-ch'ang Fu, Hupeh; Lat. $30^{\circ} 32^{\prime}$, Long. $111^{\circ} 07^{\prime}$; Han, Lang-shan 狠 U; Sui, Ch'ang-yang | 陟; T'ang, Mn 睦 C. $N$. Wei Cl'ang-yang | 楊 $\mathrm{H}$.

280 Ch'ang-yang | 楊,-See No. 279.

281 Ch'ang-yelı | 野, Nagano, Ken in Japan; chief town Yenkoji.

282 Ch'ang-yi 長 宜, (obs.) near Fêng-t'ien Fu, Shingking; Kin, chên in Liao-yang 迩陽 Fu.

283 Ch'ang-yüan | 垣 Changyuen.

$1^{\circ} \mathrm{H}$. in Ta-ming Fin, Chihli ; Lat. $35^{\circ} 18^{\prime}$, Long. $114^{\circ} 56^{\prime}$; orig. K' vang-yi 匡邑; Han, Cli'ang-ÿïan| 垣; Sui, K'uang-ch'êng 匡 城; Ming, Ch'ang-yüan | 垣 $\mathrm{H}:$ in $\mathrm{K}^{6}$ ai 開 $\mathrm{C}$. $2^{\circ}$ (obs.) N. E. Ch'angyüan H., Chihli ; $W$.
昌 $\mathrm{CH}^{\prime} \mathrm{ANG}-\mathrm{CH}-\hat{\mathrm{H} N G}$.

Han, Cli'ang-yüan | 垣 H. in Ch'ên-lin 陳留 chün; E. Han, Marq. in Ch'ên-lin 陳留; chün; $7 \mathrm{sin}, \mathrm{Ch}$ 'ang-yüan | 玨 H. in K. Ch'ên-lin 陳 留; N. Wei, Ch' ang-yüan 垣 H. in Tung 東 chiin.

$3^{\circ}$ (obs.) E. P'o C. Anhui; S. Sung, Ch' ang-yüan $\mathrm{H}$. in $\mathrm{Ch}^{\prime}$ iao 譙 chün.

$4^{\circ}$ (obs.) S.W. Ch' angyüan H., Chihli; Sung, Ch'ang-yïan | 坦 H. in K'ai-fêng 開封 Ftr.

$5^{\circ}$ (Obs) in Honan; Kin, $\mathrm{Cl}^{\prime}$ 'ang-yüan | 坦, H. in K'ai-fëng 開 封 Fu.

$6^{\circ}$ (Obs) 40 li N. E. Ch'ang-yïan H., Chihli ; Kin and Yüan, H. in $\mathrm{K}^{6}$ ai 開 C.

284 Clíang-yüan | 淵 Changyuen, H. in Hnang-hai 黄 海 circ., Corea; Lat. $39^{\circ} 23^{\prime}$, Long. $125^{\circ}$.

\section{昌}

$285 \mathrm{Ch}$ 'ang 昌.

$1^{\circ}$ (Obs) S. Sung, C. in $T^{\prime}$ 'ung-ch'nan 澧川 circ.

$2^{\circ}$ See Nos. 3044, 6531.

286 Ch'ang-an | 努, see No.56. 287 Ch'ang-ch'êng | 城 Fu in P'ing-an斥娄city Corea. 
昌

Ch' ANG-hua.

288 Ch'ang-chi | 吉Chang-kih. $1^{\circ} \mathrm{H}$. in Ti-hula $\mathrm{F} n$ Kansulı; Lat. $44^{\circ}$, I.ong. $88^{\circ}$. $2^{\circ}$ See No. 224.

$289 \mathrm{Cl}^{\prime}{ }^{\circ}$ ang-chiang $\mid$ 江.

$1^{\circ}$ (Obs) 60 li W. Pêngclı̀i H., Ssŭclı nan; Sui, H.in Sui-ning 遂密 chïn. $2^{\circ}$ (Obs) W. P'êng-cli ${ }^{\prime} \mathrm{i}$ H., Ssŭch uan ; Tang, H. in Sui 透 C. Sung, H. in Sni-ning 遂管 Fu.

290 Ch'ang-clion | 州, 'T-S. near Ning-ÿ̈an $F u$, Ssŭch' "1an.

291 Ch'ang-litua 昌化 Changhwa.

$1^{\circ} \mathrm{H}$. in Hang-chon $\mathrm{F} n$, Chelnkiang; Lat. $30^{\circ} 10^{\prime}$, Long. $119^{\circ} 13^{\prime} ; T^{\prime}$ ang, Tzŭ-ch'i 紫溪, Wulung 武隆, and T'ang-slian 㲥川; Five dyn., Wucl'ang 吳 $\mid$; Sung, Ch ang-huna | 化.

$2^{\circ} \mathrm{H}$. in $\mathrm{Cl}^{\circ}$ inng-chou Fu, Knangtung; Lat. $19^{\circ}$ $12^{\prime}$, Long. $108^{\circ} 44^{\prime}$; Han, Clih-lai 至來, sui, Cl' ang-hura 化. $3^{\circ}$ (obs.) Sung, military district in Kuang-hsi 雷 西 circ.

292 Clı ang-lê | 樂 Chang-loh. $1^{\circ} \mathrm{H}$. in Clìng-chou Fu, Slianl-tu11g; Lat. $36^{\circ} 42^{\prime}$, Long. $118^{\circ} 58^{\prime}$; Han,
昌

Chi ANG-I,CNG

Ying-ling 營陵, Swi, Ying-ch'iu 營邱; Sung, Ch'ang-lê | 樂.

$2^{\circ}$ See No. 4956.

293 Ch'ang-li | 黎 Chang-li.

$1^{\circ}$ H. in Yung-p ing Fin, Chilhli ; Lat. $39^{\circ} 43^{\prime}$, Long. $119^{\circ} 10^{\prime}$; orig. in Ying 管 CHOU; Kin, H. in Ln1-ping 路 平 C.; Yiuan, H. in Yung-p ing 永平 circ, Ming, H. in Yung-p ing 永平 Fu。 $2^{\circ}$ (Obs.) in Slingking; $T \sin$, chün and $\mathrm{H}$. in P'ing 平 $\mathrm{C}$.

$3^{\circ}$ (Obs.) 25 li W. An-su H., Chihli; N. Wei, chiü in Southern Ying 營 chot:

$4^{\circ}$ (Obs.) near Chin-chon Fu, Shingking; N. $W_{c i}$, chün in Ying 營 chOv. $5^{\circ}$ (Obs.) E. T'ung C., Chihli ; Tang, H. in $\mathrm{Cl}^{\prime}$ ung 祘 $\mathrm{C}$.

294 Clíang-lï | 慮, (obs.) 60 li S.E. 'T'êng H., Shantung; W. Han, Marq. in Tunghai 軗海 chün; E. Han, and $T \sin , H$. in 'Tunglai 東海 chün; S. Sung, and $N$. Wei, H. in Lanling 蘭陵 chïn

$295 \mathrm{Cl}^{\prime}{ }^{\prime}$ an1g-lung | 隆, see No. 223. 
昌 $\mathrm{CH}^{\prime}$ ANG-SUNG.

296 Ch'ang-1ning | 明, see No. 223.

297 Ch'ang-ning | 覃 Changning, $\mathrm{H}$. in Ch'ing-Shang 度台 circ. Corea, Lat. $35^{\circ} 16^{\prime}$, Long. $128^{\circ} 54^{\prime}$.

$298 \mathrm{Ch}^{\prime}$ ang-pa-la / 剌, see No. 224.

299 Ch'ang-p'ing | 本 Changping.

$1^{\circ} \mathrm{C}$. in Shun-t ien Fu, [380] and in the Pa-Ch'ang circ., Chihli; Lat. $40^{\circ} 12^{\prime}$, Long. $116^{\circ} 10^{\prime}$; Han, Chün-tu 軍 都; Wei, Ch'ang-p'ing |本; Five dyn., Yen-p'ing 䜩平.

$2^{\circ}$ Post-town near Yenchou $\mathrm{Fu}$, Shantung.

$3^{\circ}$ (Obs.) $W u$, H., E. of

Hêng C., Kuangsi.

$4^{\circ}$ (Obs.) town near Ssŭshui H., Shantung; birthplace of Confucius.

$5^{\circ} \mathrm{H}$. in $\mathrm{Ch}^{\prime}$ üan-lo 全羅 circ., Corea.

300 Ch'ang-p' u 昌蒲, (obs.) near Tun-huang $H$, Kansuh ; $T \sin , \quad H$. in Tun-huang 敦煌 chün. 301 Ch'ang-sung | 松, (obs.) W. of Ku-lang H., Kansuh; $N$. Wei, chün in Liang 梁 $\mathrm{CHOU} ;$ Sui, H. in -Wu-wei 武威 chün; T'ang, H. in Liang 凉 c.

\section{常 $\mathrm{CH}^{6}$ ANG.}

$302 \mathrm{Ch}^{\prime}$ ang-t' ${ }^{\prime}$ | 圆, or Ch'ang[483] t'u-ê-1ê-kê |圖額勒克, Chang-tu, Fu in Tung-pien circ., Shing-king; Lat. $42^{\circ} 55^{\prime}$, Long. $123^{\circ} 05^{\prime}$.

$303 \mathrm{Ch}^{\prime}$ ang-wei | 魏, (obs.)

Three Ks., S. W. Fang H., Hupeh.

304 Clíang-yang | 陽, see No. 3574.

305 Ch' ang-yel | 掖; Changyeh 張掖 chün (No. 197,20 ) is sonnetimes erroneously so written.

306 Ch'ang-yi | 邑 Chang-yih; $1^{\circ} \mathrm{H}$. in Lai-chon $\mathrm{Fu}$, Shantung; Lat. $36^{\circ} 52^{\prime}$, Long. $119^{\circ} 43^{\prime}$; Han, 'Tu-ch' 'ang 都 |; Sung, Clíang-yi 邑.

$2^{\circ}$ (obs.) formerly, town

$N$. of Nan-ch'ang Fu, Kiangsi.

307 Ch'ang-yüan | 原 Changyuen, $\mathrm{Fu}$ in $\mathrm{Ch}$ 'ingShang 度而 circ., Corea; Lat. $34^{\circ} 52^{\prime}$, Long. $128^{\circ}$ $30^{\prime}$.

$308 \mathrm{Ch}^{\prime}$ ang-yüan $\mid \overrightarrow{\mathrm{U}}$.

$1^{\circ}$ (Obs.) N. W. Jungch ang H., Ssŭch uan; T'ang and Sung, H. in Ch'ang 昌 C. $2^{\circ}$ Sce No. 3044.

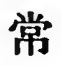

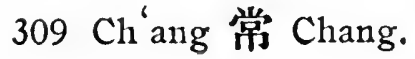


常

$1^{\circ}$ C. in Corea, Lat. $40^{\circ}$, Long. $125^{\circ} 40^{\prime}$.

$2^{\circ}$ (Obs.) S. Sung, C. in Chê-hsi 浙西 circ.

$3^{\circ}$ See No. 312.

310 Ch' ang-an | 努, chên near Lo-jung H., Kuangsi.

311 Ch'ang - Chên - T'ung - Hai 常鎮通海，circ. in Kiangsu, comprising Ch' ang-chou Fu, Chênchiang $\mathrm{Fu}$, T'ung $\mathrm{C}$. and Hai-mên T.

312 Ch'ang-chow / 州 Cliangchau, $\mathrm{Fu}$ in the $\mathrm{Cl}^{\text {'ang- }}$ Chên-T'ung-Hai circ., Kiangsu, forming also the two district cities of Wu-chin and Yang-hu; Lat. $31^{\circ} 47^{\prime}$, Long. $119^{\circ}$ $56^{\prime}$; Chou, 1st in K. Wu 员, $2 n d$ in $\mathrm{K}$. Yüeh 越, $3 r d$ in $\mathrm{K}$. Ch' $\mathrm{u}$ 楚; Tsin, Yen-ling 延陵 in Kuei-chi 會稽 chiun; $T \sin , \mathrm{P}^{6} \mathrm{i}-\mathrm{ling}$ 忽陵; $E$. Tsin, Tsin-ling 晋陵; T'ang and Sung, Ch'ang |c. Chinginju (Polo). 313 Ch'ang-fên | 分, (obs.) S. W. T'ao-chou T., Kansuls; Sui, H. in 'T'ungch'ang 同昌chün; T'ang H. in Tieh 觛 C.

$314 \mathrm{Ch}^{\prime}$ ang-fêng | 豈.

$1^{\circ}$ (Obs.) 5 li S. W. T'ung-jên Fu, Kueichou; Tang, H. in Chin 錦 C.
常 CH'ANG-TÊ.

$2^{\circ}$ (Obs.) T'ang, H., W. of Ma-yang H., Hunan.

$315 \mathrm{Ch}^{6}$ ang-k'ang | 康, see No. 4573.

316 Ch'ang-lê | 贸, chên near $P^{\prime}$ u-ch'êng H., Shensi.

$317 \mathrm{Cl}^{6}$ ang-ning | 管 Changning, $H$. in Hêng-chou $\mathrm{Fu}$, Hunan ; Lat. $26^{\circ} 26^{\prime}$, Long. $112^{\circ} 11^{\prime}$; Han, Lei-yang 未晹; $W u$, Hsin-ning 新 䆓; 1st Sung, Ch'ang-ning | 箩. 318 Ch'ang-p'ing | 平; (obs.) Wei, H., S. W. Hsiangyang $\mathrm{Fu}, \mathrm{Hupeh}$.

319 Ch'ang-shan |山 Changshan.

[56] $1^{\circ} \mathrm{H}$. in $\mathrm{Ch}^{\prime}$ ï-chou $\mathrm{Fu}$, Chehkiang ; Lat. $28^{\circ} 55$, Long. $118^{\circ} 40^{\prime} ; S u^{\prime} i$ Ting-yang 定陽; T'ang, Ch'ang-shan | II. Chanshan (Polo).

$2^{\circ}$ (obs.) chiun under the Han dyn.

$3^{\circ}$ See No. 487.

$320 \mathrm{Cl}^{\prime}$ ang-shu | 熟 Changshuh, H. in Su-chou Fu, Kiangsu; Lat. $31^{\circ} 45^{\prime}$, Long. $12^{\circ} 36^{\prime}$. This district city serves also for Chao-wên H.; orig., E. part of Hai-yü 海虞; Tsin, Nan-sha 南沙; Liang, Ch'ang-shu | 熟. $321 \mathrm{Ch}$ 'ang-tê | 德 Chang-teh. 
常

Chao.

$1^{\circ}$ Fu in the Yo-Cl' angLi circ., Hunan ; forming also the district city of Wu-ling; Lat. $29^{\circ} 01^{\prime}$, Long. $111^{\circ} 27^{\prime} ;$ S.A. and C. Wars, in K. Ch" $\mathrm{u}$ 楚; Ts'in, $\mathrm{Cl}^{\prime}$ ien-chung 默 中; Han, Wu-ling 武 陵, Han-chou 漢菷 and Yi-ling 義陵; Three Ks., 1st under Minor Han, 2nd under $\mathrm{Wu} ;$ Liang, Wu 武 C. and Yüan 沅 C.; Sui and $T^{6}$ ang, Lang 期 C.; Sung, Ting 鼎 C. and $\mathrm{Ch}^{\prime}$ ang-tê | 德; Ming, Ch'ang-tê | 德 $\mathrm{Fu}$.

$2^{\circ}$ (Obs.) S. Sung, Fu in Hu-pei 湖北 circ.

322 Cl'ang-ts'un 常村, chenn near Hsiang-yŭan $H$., Shansi.

$323 \mathrm{Cl}^{\prime}$ ang-yi | 邑.

$1^{\circ}$ Post-town near Yichêng H., Kiangsu. $2^{\circ}$ See No. 7693.

Chan-shan, See No. 319.

\section{CHAO}

\section{趙}

324 Chao 趙 Chau.

[34] $1^{\circ}$ C. L. C. in the Ch'ing-ho circ., Chihli; Lat. $37^{\circ} 48^{\prime}$, Long. $112^{\circ}$
趙 ChaO-TE.

$56^{\prime}$; orig., centre of $\mathrm{K}$. Chao |; N. Ts ${ }^{\prime} i$, Chao C.; Sung, Ch'ing-yüan 慶源 $\mathrm{Fu}$, in Ho-pei 河 北 Western circ.

[121] $2^{\circ}$ C. . in Ta-li $\mathrm{Fu}$, Yünnan; Lat. $25^{\circ} 35^{\prime}$, Long. $100^{\circ} 31^{\prime}$; $\operatorname{Han}$, Yung-ch' ${ }^{\prime}$ ang 永昌; under the Mêng 蒙 barbarians, Chao | C.; under the Chia 㷉 barbarians, 'T'ien-shui 天水. $3^{\circ}$ (Obs.) Ts ${ }^{\prime}$ in, Han-tan 邯鄲 chün; A.D. 4001300 , K. Chao |, comprising parts of Shansi and Chihli.

325 Chao-ch' êng | 城 Chau[123] ching, H. in Ho C., Shansi; Lat. $36^{\circ} 23^{\prime}$, Long. $111^{\circ} 42^{\prime}$; orig., principality conferred by Mu Wang 穆王 of the Chou dyn. on Tsao-fu 造 父; Sui, Chao-ch êng 城. 326 Chao-chia-wan | 家 灣, T-S., near Nien-po H., Kansuh.

327 Chao-ch' ü | 曲, chên near T'ai-p'ing H., Sliansi.

328 Chao-ch' uan-pao | 川堡, post-town near Lung-inên H., Chihli.

329 Chao-k'ang | 康, chen near Chiang H., Shansi,

330 Chao-tê | 德. 
趙

$1^{\circ}$ Y. near Shê H., Honan.

$2^{\circ}$ Y. near 'T'ai-ho H.,

Anhui.

331 Chao-tien / 克, chên near Li-ch' êng H., Shansi.

332 Chao-ts'un | 村.

$1^{\circ}$ Chên near Chao-ch' êng H., Shansi.

$2^{\circ}$ Chên near Li-ch'üan H., Shensi.

333 Chao-tı | 渡, chên near Chao-yi H., Shensi.

334 Chao-wang | 王, chên near Hu H., Shensi.

\section{笔}

335 Chao-ch'ing 肇度 Chan-

[165] king, Fu in the Ling-hsi circ., Kuangtung, forming also the district city of Kao-yao; Lat. $23^{\circ}$ $05^{\prime}$, Long. $112^{\circ} 30^{\prime}$; $T s^{\prime}$ in, in Nan-hai 南海 chïn; Han, in Ts'angw11 蒼梧 chün; 1st Sung, Sui-chien 䌅建; Liang, Kao-yao 高要; Sizi, Hsin-an 信安; T'ang, Tuan 端 C., and Chêng 湞 C.; S. Sung, Chao-ch'ing | 鹿 Fu in Kuang-tung 庴東 circ.

$$
\text { 召 }
$$

336 Chao-kung 吕公, chên near Fu-fêng H., Shensi.

\section{昭}

337 Chao 昭。
昭 CHAO-WÊN.

$1^{\circ}$ (Obs.) S. Sung, C. in Kuang-hsi 廣西 circ. $2^{\circ}$ See No. 5169.

338 Chao-ch'ing | 慶, see No. 2154.

339 Chao-ha | 哈, post-town near Ta-t ${ }^{\prime}$ nng $F u$, Shansi.

340 Chao-hua | 化 Chau-hwa, $\mathrm{H}$. in Pao-ning $\mathrm{Fu}_{\mathrm{u}}$ Ssŭch 'nan ; Lat. $32^{\circ} 16^{\prime}$, Long. $105^{\circ} 50^{\prime}$; Han, Chia-1nêng 殿萌; 1st Sung, Yi-ch'ang 昷昌; Sung, Chao-hua | 化.

341 Chao-ling | 陵, see No. 5570 .

342 Chao-nan | 南 (obs.) $W$. Han, H. in Ch'ing-chin Fi1, Shantung.

343 Chao-p'ing | 平 Chat1-

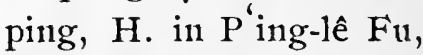
Kuangsi ; Lat. $23^{\circ} 45^{\prime}$, Long. $110^{\circ} 32^{\prime}$.

344 Chao-tê | 德.

$1^{\circ}$ (Obs.) formerly $\mathrm{H}$., W. of Hsin-ch'êng H., Chehkiang.

$2^{\circ}$ Sce No. 4098.

345 Chao-t'ung, | 通 Chautung, $F u$ in the Yi-tung circ., Yünnan ; forming also the district city of Ên-an; Lat. $27^{\circ} 20^{\prime}$, Long. $103^{\circ} 50^{\prime}$.

346 Chao-wên | 文 Chau-wăn, $\mathrm{H}$. in Su-chou $\mathrm{Fu}$, Kiangsu ; this district 
昭

ChAO-NING.

city serves also for

Ch ang-shn H.; Lat. $31^{\circ}$ $45^{\prime}$, Long. $120^{\circ} 36^{\prime}$.

347 Chao-wu 炤武, see No. 5569.

348 Chao-wu-ta|島達 Chao

Uda, Mongol League.

349 Chao-yang | 缡, see No. 5570 .

350 Chao-yi | 義, see No. 4098.

照

351 Chao-ling 照陵, post-town near Fêng-t ien Fu, Shingking.

\section{招}

352 Chao-an 招努, see No. 169.

353 Chao-huai | 懷, (obs.) in Kuangtung; $S$. Sung and $S$. Ts' $i, H$. in Haich'ang 海昌chiin.

354 Chao-hsien | 賢.

$1^{\circ}$ Chên near Yung-ning C., Shansi.

$2^{\circ}$ Chên near Lung-tê $\mathrm{H}$, Kansuh.

355 Chao-hsin | 信, (obs.) $S$. Sung, military district in Huai-tung 准東 circ.

356 Chao-lun | 論, (obs.) Tang, H., W. of $\mathrm{Ch}^{6} \mathrm{ên}^{6}$ chou $\mathrm{Fu}, \mathrm{Hunan.}$

357 Chao-ning | 耍, ss̆̌ in Ch'ao-yang H., Kuangtung.
朝

Chiao-hsien.

358 Chao-nung | 農, (obs.) in Such'ien H., Kiangsu ; $N$. Wei, H., in Chinning 晋察 chün.

359 Chao-yi|练, (obs.) T'ang H., $70 l i \mathrm{~W}$. of Shihch'êng H., Kuangtung.

360 Chao-yüan | 遠 Chat1yuen, H. in Têng-chout Fu, Shantung; Lat. $37^{\circ}$ $22^{\prime}$, Long. $120^{\circ} 49^{\prime}$; Han, Yeh 掖 H. ; Kin, Chao-yüan|遠.

\section{詔}

361 Chao-an 詔发 Chan-ngan, $H$. in Chang-chon Fin, Fuhkien; Lat. $23^{\circ} 43^{\prime}$, Long. $117^{\circ} 18^{\prime}$.

\section{朝}

362 Chao-cls'êng 朝城 Chanching, H. in Ts ao-chou Fu, Shantung; Lat. $36^{\circ}$ $08^{\prime}$, Long. $115^{\circ} 45^{\prime}$; Shang, Imperial domain; Han, Tung-wu-yang 東 武陽; T'ang, Wu-shêng 武聖 and Chao-ch'êng |城.

363 Chao-hsien | 鮮 Chosen, or Chatı-sien.

$1^{\circ} \mathrm{K}$. of Corea, also called Kao-li 高高䴡 and

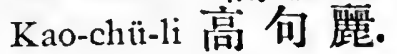
$2^{\circ}$ The capital city of Corea, also called Ching- 
chi Tao 京畿道 Kingkitan; Lat. $37^{\circ} 36^{\prime}$, Long. $127^{\circ} 08^{\prime}$. Called Söul or Seoul. [Cauly] (Polo.) Also known as Hancl' êng 漢城 from being sitnated on the Han River.

$3^{\circ}$ See Nos. 1968, 3805.

364 Cliao-kê | 歌, see Nos.616, 6971.

365 Chao-t'ien | 天, post-town near Chung-ch'ing $\mathrm{Fu}$, Ssŭch' uan.

366 Chao-yang | 陽 Chau[430] yang, Fu in K' ou-wai-Jêho circ., Chihli ; Lat. $41^{\circ}$ $30^{\prime}$, Long. $120^{\circ} 25^{\prime}$. Local name-Tso-t'a 座塔.

367 Chao-yi | 邑 Chau-yih, H. [148] in Hsi-an Fu, Shensi ; Lat. $34^{\circ} 48^{\prime}$, Long. $110^{\circ}$ $02^{\prime} ; S . A$., $\mathrm{P}^{\prime} \mathrm{u}$-kuan 蒲 關; Han, Lin-chin 臨 晋; $W . W e i$, Chao-yi

邑; T'ang, Ho-hsi 河西.

Chao Uda, see No. 348.

\section{CH'AO}

\section{潮}

$368 \mathrm{Ch}^{\circ}$ ao 潮.

$1^{\circ}$ (Obs.) S. Sung, C. in Kuang-tung 廣東 circ. $2^{\circ}$ See No. 369.

$369 \mathrm{Ch}^{\prime}$ 'ao-chou, | 州 Chat1[134] chan, $\mathrm{Fu}$ in the HuiCh'ao-Chia circ., Kuangtung; forming also the
巢 $\mathrm{CH}^{\prime}{ }^{\prime} \mathrm{O}$.

district city of Hai-yang ; Lat. $23^{\circ} 34,{ }^{\prime}$ Long. $116^{\circ}$ $36^{\prime} ; T^{\prime} s^{\prime}$ in and Han, in Nan-hai 南海 chïn and Chieh-yang 揭㖴 chïn; $T$ sin, Yi-an 義安; Liang, Ying 滋 C.; Sui, Ch' ao C.; T'ang, Ch'ao-yang 陽; Ming, Ch' ao-chou 州 Fu.

$370 \mathrm{Ch}$ ao-ho | 河, Y. near Mi-yün H., Chihli.

$371 \mathrm{Ch}^{\prime}$ ao-lien | 連, ssŭ in Hsin-hui H., Kuangtung. 372 Ch'ao-yang | 陽, see No. 369.

373 Ch'ao-yang | 陽, Chauyang, $\mathrm{H}$. in $\mathrm{Ch}^{\prime}$ ao-chou $\mathrm{Fu}$, Kuangtung; Lat. $23^{\circ} 22^{\prime}$, Long. $116^{\circ} 41^{\prime}$; Ts'in, Ch'ao-yang | 陽.

\section{巣}

$374 \mathrm{Ch}^{\prime}$ ao 巢 Tsau.

[150]1 ${ }^{\circ} \mathrm{H}$. in Lü-chou $\mathbf{F u}$, Anhui ; Lat. $31^{\circ} 35^{\prime}$, Long. $117^{\circ} 46^{\prime}$; orig., in K. Ch' ao |; Ts in, Chü-ch'ao 居 ; Liang, $\mathrm{Ch}^{\prime} \mathrm{i}$ 䩶 H.; S. Sung, $S$. $T s^{\prime} i$ and $N$. Wei, $\mathrm{Ch}^{\prime} \mathrm{i}$ 鞓 $\mathrm{H}$. in Nanch'iao 南譙 chün; Sui, Hsiang-an 襄安 H. in Lü-chiang 盧江 chün. $2^{\circ}$ (Obs.) formerly a $\mathrm{K}$. comprising part of Anhui. $3^{\circ}$ See No. 7158. 
Charchan, see No. 401.

Cliau, see No. 324.

Chau-chau, see No. 369.

Chau-chi, see No. 1346.

Chau-ching, see Nos. 325, 362.

Chau-hwa, see No. 340 .

Chau-king, see No. 335.

Chau-ngan, see No. 361.

Chau-ping, see No. 343.

Cliau-sien, see No. 363.

Chatu-tung, see No. 345.

Chaut-wăı, see No. 346.

Chau-yang, see Nos. 366, 373.

Chan-yih, see No. 367.

Chau-yuen, see No. 360.

Che-ching, see No. 377.

Che-foo, see No. 977.

\section{CHÊ}

\section{柘}

375 Chê 柘, see No. 377.

376 Chê-chê | 折, see No. 154.

377 Chê-chêng | 城 Che-ching,

$\mathrm{H}$. in Kuei-tê $\mathrm{Fu}_{\text {u, }}$

Honan; Lat. $34^{\circ} 08^{\prime}$,

Long. $115^{\circ} 38^{\prime}$; orig.,

Chu-hsiang-shih-yi 朱衰

氐邑; Han, Chê | H.;

Sui, Chê-ch' êng | 城.

378 Chê-chi 柘 磯, chên near Hu-k'ou H., Kiangsi.

379 Chê:ch'i | 溪, T-S., near

Yin-chiang H., Kueichou.

380 Chê-chih | 支, see No. 154 .

哲 CHê-SHUi.

381 Chê-chung | 鍾, (obs.) in Ssŭch'uan; T'ang, C. in Lung-yu 隌右 circ.

382 Chê-kao | 棉, chên near Lii-chou Fu, Anhui.

383 Chê-lin | 林 sš̌ in Jaop'ing H., Kuangtung.

384 Chê-lin-pao | 林堡, posttown near Fêng-hsien H., Kiangsu.

385 Chê-nan | 南, see No. 1138.

386 Chê-shih | 石, see No. 154.

387 Chê-t'ang | 塘, chển near

Li-shui H., Kiangsu.

388 Chê-yang | 洋 ssŭ, near Fu-ting H., Fuhkiell.

\section{浙}

389 Chê-chiang 浙江 Chehkiang.

$1^{\circ}$ A province of China; literary names Chê 淛, Yiieh 越 Wu-shan 员 川 and Chê-shui 浙水; capital Hang-chou $\mathrm{Fu}$; it contains 4 circ., 11 Fu, 2 C. L. T., 1. T., $1 \mathrm{C}$. and $75 \mathrm{H}$.

$2^{\circ}$ Chên near Hang-chou $\mathrm{F} \mathfrak{}$, Chehkiang.

哲

390 Chê-li-1nu 哲里木 Cherin, Mongol League.

391 Chên-hsi | 西, (obs.) $S$. Sung, circ.

392 Chê-shui | 水, see No. 389. 
草

Cheh-Kiang.

\section{草}

393 Chê-pao 者 保, T-S., near Ning-yüan Fu, Ssŭch' uan. 394 Chê-hai | 海, ssŭ near Tung-ch' wan $\mathrm{Fu}$, Yünnan.

\section{赫}

395 Chê-yang 赫陽, post-town near Yü C., Honan.

396 Chê 淛, see No. 389.

\section{$\mathrm{CH}^{\prime} \hat{\mathrm{E}}$ \\ 車}

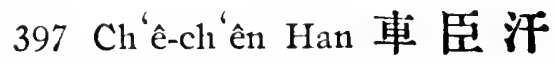
Tsetsen Klianate in Mongolia; bounded on N. by Siberia, on E. by Hehlung-kiang, on S. by Inner Mongolia and on W. by 'Tuchetu.

398 Ch' ê-li | 里, ssŭ near Chênyüan T., Yünnan.

399 Ch'ê-p' an 盤, post-town near Ch'ien-shan H., Kiangsi.

$400 \mathrm{Ch}^{\prime} \hat{e}$-tu | 度, Chên near P'n-ch' êng, H., Shensi.

\section{扯}

$401 \mathrm{Ch}^{\prime} \hat{\mathrm{e}}-1 \mathrm{i}-\mathrm{ch}{ }^{\prime}$ ang 扯力昌 Charchan, a town in Harashar, mentioned by Marco Polo.

Cheh-chuen, see No. 2427. Cheh-kiang, see No. 389.
䔬 - Cht̂N-Lung.

- Chemulpo, see No. 605. Chen-hwa, see No. 169. Chen-peh, see No. 4671. Chen-yih, see No. 170.

\section{CHÊN}

点

402 Chên 畺

$1^{\circ}$ (Obs.) N. W. Mou C., Ssŭch' uan; T'ang, C. in Chien-nan 劍南 circ. $2^{\circ}$ (Obs.) in Annam; T'ang, C. in Ling-nan 領 南 circ.

$1^{\circ}$ See No. 7468.

403 Chên-ch'i | 溪, post-town near Chien-wei H., Ssŭch'uan.

404 Chên-cliün | 君, (obs.) in Hsiao-yi H., Shansi ; $N$. $W e i$, chün in Hsien 顯 chov.

405 Chên-fu | 符.

$1^{\circ}$ (Obs.) N. of Yang H., Shensi ; $T^{6}$ ang, H. in Yang 洋 C.

$2^{\circ}$ (Obs.) N. E. Yang H., Shensi ; Sung, H. in Yang 洋 C.

$3^{\circ}$ (Obs.) in Mou C., Ssŭch'uan; T'ang, H. in Chên $\mid \mathrm{C}$.

406 Chên-ho-chih 㯖 和志, town in Liuch iu Islands. 407 Chên-lung | 龍, (obs.) in Annam; Sui, H. in Haiyin 海陰 chün. 
萁 CHÊN-AN.

408 Chên-ning | 徎, see No. 485.

409 Chên-pao | 望, H. in Ch 'ing-shang 度份了 circ., Corea.

410 Chên-pi | 壁, town in Li11ch'iu Is.

411 Chên-ting | 定, see No. 487.

412 Chên-yang | 陽, see No. 488.

413 Chên-yi | 義, chên near Su-chou Fu, Kiangsu.

414 Chên-yūan | 源, see No. 4124.

\section{錞}

415 Chên 鎮

$1^{\circ}$ (Obs.) Sung, C., N.E. of Kan-ên H., Kuangtung. $2^{\circ}$ see No. 487.

416 Chên-an | 安 Chin-ngan. $[164] 1^{\circ} \mathrm{Fu}$ in Kuangsi, Tsochiang circ.; forming also the district city of ' $T$ ' ienpao; Lat. $23^{\circ} 20^{\prime}$, Long. $106^{\circ} 19^{\prime}$; orig., land of the Yüeh 䊀 tribes; Han, in Chiao-chih 交阯 chün; Sung, Chên-an|安.

$2^{\circ} \mathrm{H}$. in Sliang C., Shensi ; Lat. $33^{\circ} 16^{\prime}$, Long. $109^{\circ}$ 14'; orig., Fêng-yang 豐 陽; Tang, An-yeh 安 業; Five dyn., Ch'ien-yu 乾形右.
鑥 CHÊN-CH' IEN.

$3^{\circ} \mathrm{H}$. in $\mathrm{Ch}^{6}$ ïan-lo 全羅 circ. Corea ; Lat. $35^{\circ} 30^{\prime}$, Long. $126^{\circ} 50^{\prime}$.

$4^{\circ}$ Ss ̌̆ near Kuei-yang $H$, Hunan.

417 Chên-ch'i | 溪.

$1^{\circ}$ (Obs.) near Clı ên-chon Fin, Hunan ; Sung military station in $\mathrm{Ch}^{\prime}$ ên 辰 C.

$2^{\circ}$ (Obs.) near Lü C., Ssŭcliuan; Snng, pao in Lï 瀘 $\mathrm{C}$.

418 Chên-chiang | 江 Chin[528] kiang, Fu in the Ch angChên-'T'nng-Hai circ., Kiangsu, forming also the district city of Tan-t ${ }^{6} 1$; Lat. $32^{\circ} 10^{\prime}$, Long. $119^{\circ}$ $29^{\prime}$; one of the ports open to foreign trade; $S . A$., $1 s t$ in $\mathrm{K}$. Wu 吳, $2 n d$ in K. Yäeh 越; C. Wars, in K. Ch ' ${ }^{\prime}$ 楚; $T s^{\prime} i n$, in Kuei-chi會稽 chün; $W u$, Mo-ling 秣陵; 1st Sung, Nan-1sï 南徐; Sui, Jun 润 C.; T ang, 'Tan-yang 丹陽; Sung, Chênchiang | 江. Fu, Chinghian fu (Polo).

419 Chên-ch'iang 鎮看, posttown near $\mathrm{P}$ ing-fan $\mathrm{H}$., Kansuh.

420 Chên-ch'ien | 前 ssŭ in Wêr1-ch'ang H., Kuangtung. 
鍡

421 Chên-ching | 靖, Y. near

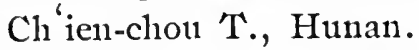

422 Chên-ch'uan | 川 H. in Chung-clin'ing 忠清 circ., Corea.

423 Chên-fan | 番 Chin-fan, $\mathrm{H}$. in Liang-chou $\mathrm{Fu}$, Kansuh ; Lat. $38^{\circ} 35^{\prime}$, Long. $103^{\circ} 08^{\prime}$; E. Han, Wu-wei 武威; Yïan, Hsiao-ho-ch'êng 小河 城; Ming, military district in Shensi.

424 Chên-hai | 海 Chin-hai.

$1^{\circ} \mathrm{H}$. in Ning-po $\mathrm{Fu}$, Chehkiang; Lat. $30^{\circ} 01^{\prime}$, Long. $120^{\circ} 40^{\prime}$.

$2^{\circ} \mathrm{Y}$. near Hsi-ning $\mathrm{Fu}$, Kansuh.

$3^{\circ} \mathrm{H}$. in $\mathrm{Cl}^{6}$ ing-shang 慶

份 circ., Corea.

$4^{\circ}$ See No. 1204.

425 Chên-hsi | 西 Chin-si.

$1^{\circ}$ C. L. T. in the Chên-

Ti circ., New Dominion ;

Lat. $43^{\circ} 40^{\prime}$, Long. $94^{\circ}$; called also Barkonl 巴 尔庫.

$2^{\circ}$ (Obs.) near Turfan; T'ang, C. in ' T'iao-chih 條支 Fu。

$3^{\circ}$ (Obs.) near $\mathrm{P}^{6}$ ing-liang Fu, Kansuh ; Sung, poo in Chên-jung 鑥我 military district.

$4^{\circ}$ (Obs.) near Ning-yüan Fu, Ssŭch' 'uan; Ming, So
鉏 ChÊN-NAN.

in Yüeh-sui 越㑺 military district.

$5^{\circ}$ (Obs.) near Yünnan;

Yüan, circ. in Yünnan. $6^{\circ}$ (Obs.) N. W. Ch'ingchên H.,Kueichou; Ming, military district in Kueichou.

426 Chên-lısia | 俠, sš̆ near Kung-ch 'ên'g H., Kuangsi.

427 Chên-hsiung | 雄 Chinhiung.

$[471] 1^{\circ}$ C. in Chao-t 'ung Fu, Yünnan; Lat. $27^{\circ} 18^{\prime}$, Long. $103^{\circ} 50^{\prime}$.

$2^{\circ}$ (Obs.) 7 li S. W. Chên-

hsiung, Yünnan; Ming $\mathrm{Fu}$ in Ssŭch'uan.

428 Chên-jung | 求, (obs.) in

Ku-yüan C., Kansuh ;

Sung, military district in Ch'in-fêng 秦 鳳circ., Shensi ; Kin, C. in Fênghsiang 鳳翔 circ.

429 Chên-k'ang | 康, T-C. [171] near Mien-ning T., Yünnan; Lat. $24^{\circ} 12^{\prime}$, Long. $99^{\circ} 36^{\prime}$.

430 Chên-lo | 羅, Y. near P'ing-ku H., Chihli.

431 Chên-nan 鎮南 Chin-nan. [277] $1^{\circ} \mathrm{C}$. in Ch' $\mathrm{u}$-hsiung $\mathrm{Fu}$, Yünnan; Lat. $25^{\circ} 16^{\prime}$, Long. $101^{\circ} 24^{\prime}$; orig., Shih-ku 石踣; r'ang， Tsung 宗 $\mathrm{C}$. in Chiennan 劍南 circ.; Yüan, Chên-nan | 南 C. 
鎮

CHÊN-TI.

$2^{\circ} S s \breve{u}$ near Tan C., Kuangtung.

$3^{\circ}$ (Obs.) $T^{\prime}$ ang, H. near Ling-shan H., Kuangtung.

432 Chên-11an-ch'ang | 南長, (obs.) Ming, H. in S. W. of Hunan.

433 Chên-ning | 隔 Chin-ning. [346] $1^{\circ}$ C. in An-shun Fu, Kueichou ; Lat. $26^{\circ} 02^{\prime}$, Long. $105^{\circ} 42^{\prime}$.

$2^{\circ}$ (Obs.) Sung. C., W. of Ssŭ-ên H., Kuangsi.

$3^{\circ} \mathrm{Y}$. near $\mathrm{Ch}^{\prime}$ ien-clou T., Hunan.

$4^{\circ}$ Post-town near Hno-lu H., Chihli.

434 Chên-pien | 邊.

$1^{\circ}$ C. I. T. in Yi-11an circ., Yünnan.

$2^{\circ} \mathrm{H}$. in Kuei-shan C. L. C., Kuangsi. Formerly, Chên-an 安 Assistant sub-prefecture.

435 Chên-p'ing | 平 Chin-ping. [245] $1^{\circ} \mathrm{H}$. in Nan-yang Fu, Honan; Lat. $33^{\circ} 10^{\prime}$, Long. $112^{\circ} 18^{\prime} ; \operatorname{Han}$, An-chung 安䍃; Tsin， An-ch'ang 安昌; Yïan, Chên-p'ing $\lceil 4$.

[134] $2^{\circ} \mathrm{H}$. in Chia-ying C., Kuangtung ; Lat. $24^{\circ}$ $40^{\prime}$, Long. $116^{\circ} 30^{\prime}$.

436 Chên-Ti $\mid$ 建, circ. in the New Dominion, compris-
鋪 CHÊN-YỬAN.

ing Chên-hısi T., Ti-hua Fu, Ha-mi T., and the towns Pidjan, Urumtsi and Murui.

437. Chên-tsên | 岑 H. in Chung-ch'ing 忠声 circ., Corea.

438 Chên-yang | 洋 Chin-yang, $\mathrm{H}$. forming also the city of 'T'ai-ts'ang C., Kiangsu ; Lat. $31^{\circ} 30^{\prime}$, Long. $120^{\circ} 52^{\prime}$.

439 Chên-yi / 荑, Y. near Su C., Kansuh ; Ming, So in Shensi.

440 Chên-yüan | 嗃 Chin-yuen. [20] $1^{\circ} \mathrm{Fu}$ in Kuei-tung circ. Kuei-chou, forming also district city of same name; Lat. $27^{\circ} 01^{\prime}$, Long. $108^{\circ} 18^{\prime}$; orig., Ta-tien 大田 and Ch'i-tung 溪洞; Yüan, Chên-yüan |壹 Fur

$2^{\circ} \mathrm{H}$. forming the prefectural city of the same name in Kueichou; Lat. $27^{\circ} 01^{\prime}$, Long. $108^{\circ} 18^{\prime}$; Ming Chên-yïan | 遠 $\mathrm{H}$. $3^{\circ} Y$. near Chiang-hua H., Hunan.

$4^{\circ}$ T-C. near Yang-li C., Kuangsi.

$5^{\circ}$ T-S. near Tai-kung T., Kueichou.

$6^{\circ}$ Ss̆̌ in $\mathrm{T}^{\prime}$ ien-chu H., Kueichour. 
鎭 CHỀN-SHAN.

441 Chên-yüan | 原 Chin-yuen, [523] H. in Ching C., Kansuh; Lat. $36^{\circ} 02^{\prime}$, Long. $107^{\circ}$ $03^{\prime}$; Han, Kao-p'ing 高 平; $T^{6}$ ang, Yüan 原 $\mathrm{C}$.; Yïan, Chên-yüan | 原 C. in Shensi.

442 Chên-yïian | 沅 Chin-yıen. [362] $1^{\circ}$ C. L. T. in Yi-nan circ., Yünnan ; Lat. $23^{\circ}$ $47^{\prime}$, Long. $101^{\circ} 06^{\prime}$; orig., Land of S. W. barbarians; $T^{\prime}$ ang, Yinshêng 銀生 $\mathrm{Fu} ; Y \ddot{i} a n$, in Wei-yüan 威遠 Fu; Ming, 1st, Chên-yïan | 沅 Fu, 2nd, Chên-yuian | 沅 C.

$2^{\circ} \mathrm{Fu}$ and $\mathrm{C}$., see above.

\section{針}

443 Chên-t'ang 針塘 Chên in Chia-shan $\mathrm{H}$., Chehkiang.

㽎

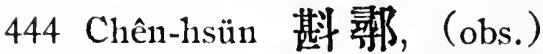
formerly town near Wei H., Shantung.

445 Chên-kuan | 灌, see No. 5753.

\section{珍}

446 Chên 珍, (obs.) E. of T'unğ-tzŭ H., Kueichou ; Sung, C. in K' vei-chon 配州 circ.

447 Chên-shan | I chün in Ch'üan-lo 全羅 circ., Corea.
溱 CHỀN.

448 Chên-tao | 島 chün in Ch' üan-lo 全羅 circ., Corea.

449 Chên-yüan | 原 H. in Ch'üan-lo 全羅 circ., Corea.

\section{振}

450 Chên 振, see No. 7224.

451 Chên-wei | 威 H. in Chingch'i 京畿 circ., Corea.

452 Chên-wı| 武.

$1^{\circ} \mathrm{Y}$. near $\mathrm{Ch}^{\prime}$ ien-chon

T., Hunan.

$2^{\circ} \mathrm{Y}$. near Yin-chiang H., Kuei-chou.

\section{震}

453 Chên-tsê 震澤 Chin-tseh. $1^{\circ} \mathrm{H}$. in Su-chou $\mathrm{Fu}$, Kiangrsu, serving also as the district city of Wuchiang; Lat. $31^{\circ} 12^{\prime}$, Long. $120^{\circ} 20^{\prime}$.

$2^{\circ}$ Chên near the above.

454 Chên-wei / 威, (obs.) near Yü-lin Fu, Shensi; Sung, walled town in $\mathrm{Fu}$ 你 $\mathrm{C}$.

\section{淎}

455 Chên 溱.

$1^{\circ}$ (Obs.) Tang, C. comprising Chêng-an $\mathrm{C}$. and T'ung-tzŭ H., Kueichou. $2^{\circ}$ (Obs.) S. of Ch'i-chiang H., Ssŭch'uan; T'ang, C. in Chiang-nan 江南 
溙

CH.̂ेN.

circ.; Sung, C. in Chungch'ing 重庱 Fu.

$3^{\circ}$ See No. 3010.

456 Chên-ch'i | 溪, (obs.) S. of Cl' i-chiang H., Ssŭch'uan; Sung, military station in Nan-p'ing 南 本 military district.

457 Chên-t'ung | 潼, chên near Hsing-hua H., Kiangsu.

\section{溙}

458 Chên-tzŭ 榛㝋, chên $90 \mathrm{li}$ W. of Luan C., Chihli.

\section{搼}

459 Chên-tung 臻洞, ssŭ near

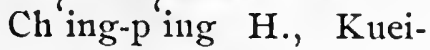
choul.

\section{貞}

460 Chên-fêng 貞豐 Chingfung, C. in Hsing-yi $F^{\prime} u$, Kueichou ; Lat. $25^{\circ} 44^{\prime}$, Long. $105^{\circ} 40^{\prime}$.

461 Chên-hsiang | 拫, see No. 715.

\section{粗}

462 Chên 緽, see No. 2365 .

\section{CH'ẾN}

陳

$463 \mathrm{Ch}^{\prime}$ ên 陳.

$1^{\circ}$ (Obs.) Chou and S.A., $\mathrm{K}$. in Honan.
陳 $\mathrm{CH}^{\prime} \hat{\mathrm{E}}_{\mathrm{N}-\mathrm{CHOU}}$.

$2^{\circ}$ (Obs.) N.E. Hsiangch'êng H., Honan; $S$. Sung, and $S$. TS' $i$, chion in $\mathrm{Yü}$ 豫 $\mathrm{cHOU}$.

$3^{\circ}$ (Obs.) in Anhui; $S$.

Sung, H. in Nan-liang 南梁 chiin.

$4^{\circ}$ (Obs.) 44 li E. T'ai-h11 H., Anhui; S. Ts'i, H. in Hsi-ju-yin 西汝陰 chün.

$5^{\circ}$ (Obs.) near Ju-ning Fu, Honan; N. Wei, chün in Ho 霍 C. $6^{\circ}$ See Nos. 467, 2248.

$464 \mathrm{Ch}^{\prime}$ ên-chia | 家, Y. near Kuang-hua, H., Hupeh. 465 Ch'ên-chia-ku 陳家谷, $\mathrm{Y}$. near Mi-yün H., Chihli.

$466 \mathrm{Ch}^{\prime} \mathrm{e}$ en-ch'iao / 橋.

$1^{\circ}$ Chên near Fêng-ch'in H., Honan.

$2^{\circ}$ Chên near Jung-tsê H., Honan.

467 Ch'ên-chou | 州 Chin-chan, [470] $\mathrm{Fu}$ in the Ho-nan-K'aiKuei-Ch' ên-Hsü circ., Ho-nan : forming also the district city of Huai-ning; Lat. $33^{\circ} 46^{\prime}$, Long. $115^{\circ}$ $03^{\prime}$; Chou and $S . A ., \mathrm{K}$. $\mathrm{Ch}^{\prime}$ ên I $\quad T_{\text {sin, }}$ Yingch'nan 穎川; Han, Ch' ${ }^{\prime}$ ên $\mid$ H.; P. Chou, $\begin{array}{lll}\mathrm{Ch}^{\prime}{ }^{\prime} \mathrm{e}_{1} & \text { C.; Sui, Wan- }\end{array}$ ch'iu 宛邱; Ming, $\mathrm{Ch}^{\prime}$ ên-chou | 州 Fu. 


\section{陳 $\mathrm{CH}^{\circ} \mathrm{E} N-\mathrm{CH}^{\prime} \mathrm{I}$.}

$468 \mathrm{Cl}^{\prime}$ 'ên-ling-wu | 陵矿, ssŭ near Yo-chou Fu, Hunan.

469 Ch'ên-liu | 留 Chin-liu.

[157] $1^{\circ} \mathrm{H}$. in $\mathrm{K}^{\prime}$ ai-fêng $\mathrm{Fu}$, Honan; Lat. $34^{\circ} 45^{\prime}$, Long. $114^{\circ} 40^{\prime}$; orig., Yu-shên 有萃; $T s^{\prime}$ in, Ch'ên-liu | 留; Ming, $\mathrm{Ch}^{\prime}$ ên-liu | 留 Fu.

$2^{\circ}$ (Obs.) Han, chün in Honan.

$3^{\circ}$ See No. 3061.

470 Ch'ên-mêng | 蒙, (obs.) Yïan, H., S. E. of Tuyün $\mathrm{F}$, Kueichon.

$471 \mathrm{Ch}^{\prime}$ ên-mu | 墓, chên near Cl' 'ing-p'u H., Kiangst. $472 \mathrm{Ch}^{\prime}$ ên-ts'ang-ch'êng | 倉 城, (obs.) formerly town near Fêng-hsiang Fu, Shensi.

$473 \mathrm{Ch}^{\prime}$ ên-ts'un | 村.

$1^{\circ}$ Chên near Fêng-hsiang $\mathrm{Fu}$, Shensi.

$2^{\circ}$ (Obs.) in Chiao C.,

Shantung; Kin, chên in $\mathrm{Mi}$ 密 $\mathrm{C}$.

\section{展}

474 Ch'ên 辰, see Nos. 476, 4949 and 7765.

475 Ch'ên-ch'i | 溪 Shin-ki.

[561] $1^{\circ} \mathrm{H}$. in $\mathrm{Ch}^{\prime}$ ên-chou $\mathrm{Fu}$, Hunan ; Lat. $27^{\circ} 56^{\prime}$, Long. $109^{\circ} 53^{\prime}$; Han, Ch'ên-yang | 晹; Sui,

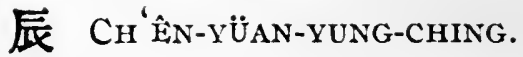

H. in Yüan-ling 沅陵 chiun; T'ang and Sung, H. in Ch' ên $\mid$ C.; Yiüan. $\mathrm{H}$. in Ch'ên-chou | 州 circ.; Ming, $\mathrm{H}$. in $\mathrm{Ch}^{\prime}$ ênchou | 州 Fu.

$2^{\circ}$ See No. 4269.

$476 \mathrm{Ch}^{\prime}$ ên-chon | 州 Shin-chau, [561] $\mathrm{F} n$ in the $\mathrm{Cl}_{1}$ ên-YüanYung Ching circ., Hunan; forming also the district city of Yüan-ling; Lat. $28^{\circ} 22^{\prime}$, Long. $110^{\circ} 09^{\prime}$; orig. land of the Man 筑 barbarians; S. A., in $\mathrm{K}$. $\mathrm{Wu}$ 吳; $T s^{\prime}$ in in $\mathrm{Ch}^{\prime}$ ienchung 駖中 chiin; Han, Ching 荆 C.; Chêen, Yüanl-ling 沅 陵; Sui, $\mathrm{Ch}^{\prime} \hat{e ̂}^{\mathrm{n}} \mid \mathrm{C}$.; T'ang, Luch'i i 慮溪; Ming, Ch'ênclou |'州 Fu.

477 Cli ên-hran 辰 韓, (obs.) Han K. in S. E. Corea. $478 \mathrm{Cl}^{\prime}$ 'ên-yang | 陽.

$1^{\circ}$ Post-town near Ch'ênchou Fu, Hunan.

$2^{\circ}$ See No. 475.

479 Ch' ên-Yüan-Yung-Ching | 元 永靖, circ. in Hunan, comprising Ch'ênchou $\mathrm{Fu}$, Yüan-chou $\mathrm{Fu}$, Yung-shun $\mathrm{Fu}$, Ching C.; Fêng-huang C. L. T., Yung-sui C.L.T., Huangchou C.L.T. and $\mathrm{Ch}^{\text {'ien- }}$ chou C. L. T. 
椰

CHÊNG-NING.

\section{椰}

$480 \mathrm{Cl}^{\prime}$ ên 林 Chin.

[17] $1^{\circ}$ C.L.T. in the HêngYung-Ch' ên-Kuei circ., Hunan; Lat. $25^{\circ} 47^{\prime}$, Long. $112^{\circ} 38^{\prime}$; S. A. and C. Wars, in K. Ch' $\mathrm{u}$ 楚; $T^{\text {' in }}$, in Ch'angsha 長沙 chïn; Han, Kuei-yang 桂陽; Three $K s$., subordinate to $\mathrm{Wu}$; Sui and T'ang, Ch'ên C.; Five dyn., Tun 敦 C.; Ming, $\mathrm{Ch}^{\text {ên }}$ | C. $2^{\circ}$ See Nos. 3453, 7419.

481 Cl' ên-yang | 陽, (obs.) $T s^{\prime}$ in, H. near Cli ề C.

\section{CHÊNG}

\section{正}

482 Chêng-an 正 安 Ching[185] ngan, C. in Tsun-yi Fu, Kueichou; Lat. $28^{\circ} 40^{\prime}$, Long. $107^{\circ} 55^{\prime}$.

483 Chêng-chieh | 階, see No. 5655.

484 Chêng-hsi | 西 (obs.) 55 li S. W. of Wên H., Kansuh ; Sui, H. in Wutu 武都 chün.

485 Chêng-ning | 塪 Chingning, $\mathrm{H}$. in $\mathrm{Ch}^{\text {'ing-yang }}$ $\mathrm{Fu}$, Kansuh ; Lat. $35^{\circ}$ $22^{\prime}$, Long. $108^{\circ} 10^{\prime}$; Han, Yang-chou陽周; Swi, Lo-ch'uan 䍜川; T'ang,
正 ChêEg-YANG.

Chên-ning 真 管。

486 Chêng-ta 正大, $Y$. near 'T'ung-jên Fitu, Kueichou.

487 Chêng-ting | 起 Chingting.

[143] $1^{\circ} \mathrm{Fu}$ in the $\mathrm{Cl}^{\prime}$ ing-ho circ., Chihli ; forming also the district city of the same name; Lat. $38^{\bullet} 20^{\prime}$, Long. $114^{\circ} 40^{\prime}$; Chou, Ping \# C.; C. Wars, in K. Chao 趙; $T s^{\prime}$ in, in Chü-lu 鉅 麇 chün; Han, Hêng-shan 恒 山 and Chên-ting 鼻定; Trang, Hêng 恒 C. and Chên 鎮 C.; Ming, Chên-ting 真定 Fur

$2^{\circ} \mathrm{H}$. forming the prefectural city of the same name; Lat. $38^{\circ} 20^{\prime}$, Long. $114^{\circ} 40^{\prime}$, orig. Tung-yüan 東垣; Han, Chêng-ting 真定; Sui, Ch'ang-shan 常 山; T'ang, Chung-shan 中 II, Acbaluc (Polo).

488 Chêng-yang | 晹 Chingyang, $\mathrm{H}$. in Ju-ning $\mathrm{Fu}$, Honan ; Lat. $32^{\circ} 35^{\prime}$, Long. $114^{\circ} 20^{\prime}$; Han, Shên-yang 慣陽; Sung, Chên-yang 蒖陽.

489 Chêng-yang Kuan | 隄 關 Ching-yang Kwan, $T$. in Fêng-yang $\mathrm{Fu}$, Anhui ; Lat. $32^{\circ} 28^{\prime}$, Long. $116^{\circ}$ $30^{\prime}$. 
政

CHÊNG.

\section{政}

490 Chêng-'ho 政和 Ching-ho, [420] H. in Chien-1ning $\mathrm{Fu}$, Fuhkien ; Lat. $27^{\circ} 27^{\prime}$, Long. $119^{\circ}$; Five dyn., Kuan-li 關埭.

491 Chêng-pin | 䆩.

$1^{\circ}$ (Obs.) N. W of Ch'ingyüan H., Knangtung ; Sui, H. in Nanhai 南 海 chiin.

$2^{\circ}$ See No. 1261 .

492 Chêng-p'ing | 平, chên near Ch'ang-wu H., Shensi.

\section{征}

493 Chêng-li 征里, chên near K' un-shan H., Kiangsu.

\section{徵}

494 Chêng 徵, see No. 539.

\section{鄭}

495 Chêng 鄭 Ching.

[32] $1^{\circ} \mathrm{C}$. in $\mathrm{K}^{\prime}$ ai-fêng $\mathrm{Fu}$, Honan ; Eat. $34^{\circ} 46^{\prime}$, Long. $113^{\circ} 56^{\prime}$; Chou, 1st principality of Kuanshu 管叔; 2 nd K. Chêng |; Ts'in, Kuan 管 H.; E. Wei, Kuang-wu 厝 武; $T^{\prime \prime a n g, ~ C h e ̂ n g ~ \mid ~ C . ~}$ $2^{\circ}$ (Obs.) B. C. 827, K. in Hsi-an Fu, Shensi; B. C. 770 removed to $\mathrm{K}$ ai-fêng $\mathrm{Fu}$, Họnan.
成 $\mathrm{CH}_{\mathrm{E}}$ ÊNG.

$3^{\circ}$ (Obs.) Ts' in, H., N. of Hua C., Shensi.

$4^{\circ}$.See Nos. 2984, 3005, 4938.

496 Chêng-chia | 家.

$1^{\circ}$ Sš̆ near Yüi-shan $\mathrm{H}$., Kiangsi.

$2^{\circ}$ Y. near Ning-ch'iang C., Shensi.

$3^{\circ}$ Post-town near T'aoyüan H., Hunan.

497 Chêng-chia-k'ou, | 蒙 口, chên near Ku-ch'êng H., Chihli.

498 Chêng-lu | 陸, chên near $\mathrm{Ch}$ ang-chou $\mathrm{Fu}$, Kiangsu.

499 Chêng-yi | 邑, see Nos. 1546, 4109. 7388.

\section{滇}

500 Chêng 滇 see Nos. 335, 5886.

501 Chêng-ch'ang | 昌, see No. 4948.

502 Chêng-yang | 陽.

$1^{\circ}$ Post-town near Ying-tê H., Kuangtung.

$2^{\circ}$ See Nos. 7041, 7610.

\section{CH'ÊNG}

成

$503 C^{\prime}$ 'êng 成 Ching.

[30] $1^{\circ}$ H. in Chieh C., Kansuh; Lat. $33^{\circ} 48^{\prime}$, Long. $105^{\circ}$ $45^{\prime}$; orig., Land of W. 
成

Сh' ÊNG Ching.

barbarians 西戎; $N$ Wei, Cl'oul-ch'ih 仇池 chün; Liang, Nan-ch'in 南秦; $W . W e i, \mathrm{Cl}^{\prime} \hat{e ̂}_{\mathrm{ng}}$ | C.; Sui , Han-yang 谨 陟; 'T'ang, T' 'ung-ku同 谷.

$2^{\circ}$ See No. 1701.

$504 \mathrm{Cl}^{\prime}$ êng-an 成努 Chingngan.

[10] $1^{\circ} \mathrm{H}$. in Kuang-p ing Fu, Chihli ; Lat. $36^{\circ} 29^{\prime}$, Long. $113^{\circ} 53^{\prime}$; orig., $\mathrm{Cl}^{\prime}$ ien 乾, Sui and $T^{\prime}$ ang, H. in Wei 魏 chün; Sung, H. in Ta-ming 大 名 Fu; Kin, H. in Lo 洛 C.; Yüan, H. in 'Tz'ù 磁 C.; Ming, H. in Kuang-p'ing 廣平 Fu。 $2^{\circ}$ (Obs.) in $\mathrm{K}^{\prime}$ ao-ch'êng H., Honan; W. Han, $\mathrm{H}$. in $\mathrm{Ch}^{6}$ en-lin 陳留 chiün.

$3^{\circ}$ (Obs.) 30 li S. E. Ju C., Honan: W. Han, Marq. in Ying-ch' 'uan 穎 川 chïn.

505 Ch' êng-chi | 紀, see No. 1105.

$506 \mathrm{Ch}^{6}$ êng-chin | 晉, posttown near Hsin C., Sliansi.

$507 \mathrm{Ch}^{\prime}$ êng-ch' uan | 川 Chingchiuen, $\mathrm{F}_{n}$ in $\mathrm{P}^{\prime}$ ing-an 平 少 circ. Corea ; I.at. $39^{\circ}$ $18^{\prime}$, Long. $127^{\circ}$.
成 CH' ${ }^{\prime} \hat{E}$ NG-TU.

$508 \mathrm{Cl}^{\text {' }}$ êng-kao | 泉, see No. 5881.

509 Ch êng - Lung - T' 'ung-MienMou, | 龍潼綿茂, circ. in Ssŭch 'nan, consisting

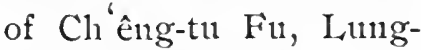
an-Fin, 'T'ung-ch' nan $F$ in, Mien C., Mou C., Li-fan C.L.T., Mon-kung C.L.T. and Sung-p an C.L.T.

$510 \mathrm{Ch}^{6}$ êng-p ing | 评, (obs.) formerly H., N. of Fuch' êng H., Chihli.

$511 \mathrm{Ch}^{\prime}$ êng-tu | 都 Ching-tu. $1^{\circ} \mathrm{Fu}$ in the $\mathrm{Cl}^{\prime}$ êng-LungT'ung-Mient-Mon circ., Ssŭ-ch' 'ran ; forming also the district cities of

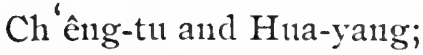
the capital of the province; Lat. $29^{\circ} 34^{\prime}$, Long. $103^{\circ}$ $11^{\prime}$; orig., in K. Shu 蜀; Han, Tsin, S. Sung and $S . T s^{\prime} i$, in Shu 蜀 chïn; Han, Kuang-han 廣漢 and Ning 帘 C.; Minor Han, Inperial residence; Chên, Chêngt11 都 and Chin-ch' êng 錦城; T'ang, Clien-nan 劍南, Nan-ching 南京 and Hsi-ch' uan 西川; Ming, Ch'êng-tı | 都 Fu.

$2^{\circ} \mathrm{H}$., forming together with Hua-yang $\mathrm{H}$. the prefectural city of Ch' êng-tu, Ssứclı́ uan ; 
成

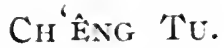

Lat. $30^{\circ} 41^{\prime}$, Long. $103^{\circ} 11^{\prime} ; \mathrm{Ts}^{\prime} \mathrm{in}, \mathrm{Cl}^{\prime} \mathrm{e}$ eng$\mathrm{t}$ 11 $\mid$ 都.

$3^{\circ}$ (Obs.) in Honan ; $E$. Han, H. in Nan-yang 南䍀chün。

512 Ch' êng-wu | 武 see No. 521. 513 Cl1'êng-yi | 宜, (obs.) W. of the old Han H. of Chin-yïan 九原, in the Elenth conntry, W. end of Inner Mongolia; $W$. Han, and E. Han, H. in Wu-yiian 五. 原 chïn。

\section{城}

514 Ch'êng-anl 城安, Y. near P'ei C., Kiangsu.

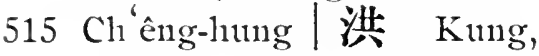
town in Outer Kansuh; Lat. $42^{\circ} 10^{\prime}$, Long. $91^{\circ}$ $30^{\prime}$.

$516 \mathrm{Cl}^{6}$ êng-k'ou $/$ 口 Chingkan.

[427] C.L.T. in Ch'nan-ting Circ., Ssŭch nan, I at. $30^{\circ}$ $20^{\prime}$, Long. $108^{\circ} 30^{\prime}$.

$517 \mathrm{Cl}^{\prime}$ êng-kn | 固 Ching-ku. [133] $1^{\circ} \mathrm{H}$. in Han-chung Fu, Shensi ; Lat. $33^{\circ}$, Long. $107^{\circ} 37^{\prime} ; T^{\prime}{ }^{\prime} i n, \mathrm{Cl}^{\prime}$ êngku | 固; Minor Han, Lê-ch'êng 樂 城.

$2^{\circ}$ See No. 7234

518 Ch'êng-pu | 热 Ching-pu, [153] H. in Tao-ch'ing Fu, Hunan ; Lat. $26^{\circ} 18^{\prime}$, Long. $110^{\circ} 13^{\prime}$.
遈 CH' ${ }^{\prime} \hat{\mathrm{E} N G}$-SU.

$519 \mathrm{Cl}^{\text {' }}$ êng-tu | 都, (obs.) S. E. $P^{6}{ }^{\prime}$ C., Sliantung; $U$. Han, Marq. in Shan-yang 山防 chiin。

$520 \mathrm{Ch}$ 'êng-tzŭ $\mid$ 子 chên in Têhua H., Kiangsi.

$521 \mathrm{Ch}^{\prime}$ êng-wu| 武 Ching-wu, [340] H. in 'Ts'ao-chou Fu, Shantung; Lat. $35^{\circ} 05^{\prime}$, Long. $110^{\circ} 13^{\prime}$; Han, Cl'êng-wi 成 武; $N$. $T s i$, Yung-ch' ang 永昌; Sui, Tai 戴 C.

522 Ch' êng-yang | 陽, see No. 3674.

\section{誠}

523 Ch'êng 誠, see No. 1157.

\section{称}

524 Cl'êng-kou 科 鈎, posttown near An-ting $\mathrm{H}$., Kansul.

\section{呈}

$525 \mathrm{Ch}^{\prime} e^{\mathrm{e}} \mathrm{g}$-kinng 呈 貢 Ching[461] kung, H. in Yün-nan Fin, Yünnan; Lat. $24^{\circ}$ $56^{\prime}$, Long. $102^{\circ} 56^{\prime}$; Han, chên; Yüan, Shêngkung 晟 貢; Hing, $\mathrm{Cl}^{6}$ êng-kung | 貢.

\section{这}

$526 \mathrm{Cl}^{\prime}$ êng-su 逞宿, chên near Hua-t ing H., Kansull. 
程 CH't̂NG-FAN.

\section{程}

527 Ch'êng-fan 程番.

$1^{\circ}$ T-S. near Ting-fan C., Kuei-chon.

$2^{\circ}$ See No. 3477.

$528 \mathrm{Cl}^{\prime}$ êng-11siang | 既, see No. 708.

\section{乘}

$529 \mathrm{Ch}^{\prime}$ êng 乘, see No. 3643 .

$530 \mathrm{Cl}^{\prime}$ êng-lisi, | 西 T-S. in K'ai C., Kneichou.

\section{㮃}

531 Cli êng 嶵 Ching, H. in Shao-11sing Fin, Chehkiang; Lat. $29^{\circ} 36^{\prime}$, Lonng. $120^{\circ} 43^{\prime} ; H a n$, Yen 敞; $T^{k}$ ang, Yen-ch êng 䜪城, Siung, Ch' êng $\mid \mathrm{H}$.

\section{氶}

$532 \mathrm{Cl}^{6}{ }^{\mathrm{e}} \mathrm{ng}$ 永, sec Nos. 59+4, 7518.

$533 \mathrm{Ch}^{\prime}$ êng-cll ênn $\mid$ 城, see No. 7518.

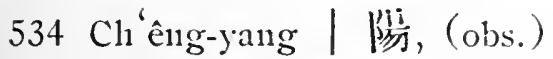
Han, town W. of Hêngchou Fin, Hunan.

\section{承}

$535 \mathrm{Ch}$ 'êng-ên 承恩， (olss.) $35 l i$ W. of Hsin-wu H., Honan: Kin, chân in Huai 栾 C.
澄 CI ${ }^{\prime} \hat{\mathrm{N} G}-\mathrm{K}^{\prime} \mathrm{UNG}$.

$536 \mathrm{Ch}^{6}$ êng-k' on | 口, chên near Hui-ch'ang $\mathrm{H}$., Kiangsi.

$537 C^{\prime} h^{6}$ êng-tê | 德 Ching-teh. [199] $1^{\circ} \mathrm{F} n$ in the $\mathrm{K}^{\prime}$ on1-waiJê-ho circ., Chillıi ; also know11 as Jê-ho 熱河 Jehol; I at. $40^{\circ} 59^{\prime}$, Long. $117^{\circ} 59^{\prime}$.

$2^{\circ} \mathrm{H}$. forming the prefectural city of Fêngt'ien, Shingking; Lat. $41^{\circ} 51^{\prime}$, Long. $123^{\circ}$ $05^{\prime}$.

\section{澄}

$538 \mathrm{Ch}^{\prime} \mathrm{e}$ eng 澄, (obs.) T'ang, C., comprising part of Lin-chon Fin, Kuangsi.

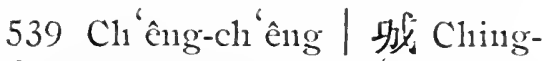
[207] ching, H. in T'tung-chon Fur, Shensi; I at. $35^{\circ} 12^{\prime}$, Long. $109^{\circ} 51^{\prime}$; Han, Chêng 徽 H.; 2nd $W_{e} i$, $\mathrm{Cl}^{\prime}$ 'êng-ch' êng | 城; Tang Ch'ang-ning 变 䁇.

$540 \mathrm{Cl}^{\prime}{ }^{\text {êng-hai }}$ 澄 海 Chinghai, H. in Ch' ao-chon Fin, Knangtung; Lat. $23^{\circ} 22^{\prime}$, Long. $116^{\circ} 41^{\prime}$.

$541 C^{\prime}{ }^{\prime}$ êng-k'ung | 空, (obs.) near T'ai-an Ful, Shantung ; Kin, chên in Tungp ing 染本 Fin, Shantung, Vestern circ. 
澄 $\mathrm{CH}^{\prime} \hat{\mathrm{E} N G}-\mathrm{MAI}$.

542 Ch'êng-mai | 邁 Chingmai, $\mathrm{H}$. in $\mathrm{Ch}^{6}$ inng-chou Fu, Kuangtung; Lat. $19^{\circ}$ 56', Long. $109^{\circ} 10^{\prime}$; Sui, Cli'êng-mai | 邁.

\section{激}

543 Ch'êng 澂, see No. 544.

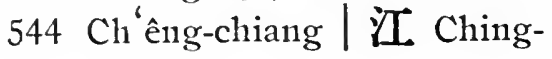
kiang.

$1^{\circ} \mathrm{Fu}$ in the Yi-tung circ., Yünnan; forming also the district city of Hoyang; I at. $24^{\circ} 42^{\prime}$, Long. $103^{\circ} 04^{\prime}$; orig., Land of S. W. barbarians ; C. Wars, Clinêng I, in K. Tien 滇; Han, Yü-yüan 兪 元 in Yi 谷 CHOU; Sui, K'un 昆 C.; under the Mêng 蒙 barbarians, Ho-yang 河晹; Yïan, Ch'êng-chiang | 江; Ming, Ch' êng-chiang 江 Fu.

$2^{\circ}$ (Obs.) E. of Ho-yang H., Yünnan; Yiüan, circ. in Yünnan.

Cherim, See No. 390.

\section{CHI}

\section{䩪}

545 Chi 蕇芹 (Obs.) S. of Su C., Anhui ; W. Han, H. in P'ei 浦 chün; E. Han,
Chi-wu.

H. in $\mathrm{P}^{6}$ ei 沛 K.; $T$ sin and S. Sung, H. in Ch'iao 譙 chün; S. Ts'i, H. in Pei-ch'iao 北譙 chïn; Sui, H. in P'êngch'êng 彭城 chiun.

\section{羈}

546 Chi 羈, properly 霸 $\mathrm{Pa}$; Sung, C. in Ssŭch uan.

547 Chi-mi | 縻.

$1^{\circ}$ (Obs.) Sung, C. in S. W. Hunan and N. Kueichon.

$2^{\circ}$ See Nos. 3398, 6169.

\section{皘}

548 Chi-ch'êng 積城 H. in Ching-ch'i 京幾 circ., Corea.

\section{㖟}

549 Chi 稳, See No. 8108.

550 Chi-shan | 山 Tsih-shan, [123] $1^{\circ} \mathrm{H}$. in Cliang C., Shansi ; Lat. $35^{\circ} 35^{\prime}$, Long. $111^{\circ} 01^{\prime} ;$ 2nd $\mathrm{Wei}$, Kao-liang 裔凉; N.Ts'i Hsün 輜 C., Sui, Chishan | II.

$2^{\circ} \mathrm{H}$. in Chung-ch'ing 忠清 circ., Corea.

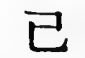

551 Clii-wu已䎊.

$1^{\circ}$ (Obs.) 40 li $\mathrm{S} . \mathrm{W}$. Ning-ling H., Honan; 
己

ChI-IVU.

E. Han, H. in Ch' êll-liu 陳留 chiin；N.Wei， H. in Ma-t'ou 馬頱 chiin.

$2^{\circ}$ (Obs.) 30 li N. Huaiyüan H., Anhui ; $S$. $T^{6} s i, \mathrm{H}$. in Ma-t'on 熙 頙 chiin；N.Wei，H. in $\mathrm{P}^{6}$ ei 浦 chün.

$3^{\circ}$ (Obs.) S. E. Ling-pi H., Anhui ; N. Wei, H. in Lin-huai 臨淮 chün.

\section{紀}

552 Chi 紀

$1^{\circ}$ (Obs.) Chou, a K. which included Yi-shui H., Shantung.

$2^{\circ}$ (Obs.) Sui, C., N. of Ch'in-an H., Kansuh.

\section{劇}

553 Chi 劇, (obs.) formerly $\mathrm{H}$. near Yü-ch' êng, H., Shantung.

554 Chi-k uei | 魁, (obs.) N. W. Cl' 'ang-lê H., Shantung ; W. Han, Marq. in Pei-hai 北海 chün.

\section{嵇}

555 Chi-hsü 稽 徐, (obs.) in Annam; Han and $T s^{\prime} i n$, H. in Chiao-chih 交 趾: chiün.

\section{計}

556 Chi-chin 辣 斤, (obs.) S.
䌖 CHI-CH'UAN.

W. Chiao C., Shantung; $W$. Han, H. in Lang-yeh 斑 躯 chün.

557 Chi-tu | 都, chên near Huat'ing H., Kansuh.

\section{欶}

558 Chi-ch'i 棘深, post-town near Chiang-ching $\mathrm{H}$., Ssŭch' 'uan.

\section{極}

559 Chi-kao 極 高, Ssŭ in H. Sin-ch êng H., Kuangtung.

\section{基}

$560 \mathrm{Chi}$ 基, (obs.) T'ang, C. comprising part of Chingmên C., Hupeh.

\section{箕}

$561 \mathrm{Chi}$ 筫.

$1^{\circ}$ (Obs.) $80 l i \mathrm{~N}$. of Chü C., Shantung; W. Han, Marq. in Lang-yeh 瑯 㑚 chün.

$2^{\circ}$ See No. 3801.

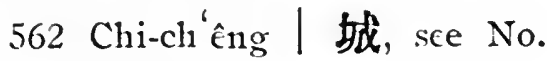
2460.

\section{垁隹}

563 Chi 雞, Y. near Tao C., Hinan.

564 Chi-ch' wan | Ji, (obs.) Sung, H., N. W. of Ch' inan H., Kansul. 
維

565 Chi-lung | 籠 Kelung (or, Keelung, ) formerly one of the open ports in the North of For-mosa ; Lat $25^{\circ} 11^{\prime}$, Loing. $121^{\circ} 44^{\prime}$. T. in T'ai-pei Fu. (Also written 监榢.) In the Ming Shih the nane is given to the whole of Formosa.

566 Chi-t'on|碩, Y. near I,inwu H., Hunan.

567 Chi-tsê | 漴 Ki-tseh, H. in [128] Knang-p ing Fu, Chilnli ; Lat. $37^{\circ}$, Long. $115^{\circ}$; Han, Knang-p'ing 穞平; Sui, Clii-tsê | 澤.

568 Chi-tsui | 嘴, Y. near Tao C., H111:11.

569 Chi-tsung | 宗. (obs.) 40 li S. of Mon C., Ssŭcl' 'uan; Sung, Customs barrier in Mon1 荗 C.

570 Chi-wei | 惟. (obs.) near Hsüchon Fì, Ssŭch' nan ; T'ang, H. in Clih 誌 C.

\section{筸}

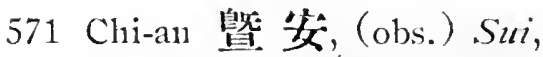
town N. of $\mathrm{Cl}_{1}$ ang-lina $\mathrm{H}$., Kuangtung.

572 Chi-yang| 榢, sce No. 760.

\section{機}

573 Chi-chang 機 張 H. in Cl'ingr-shang 愛份 circ., Corea.
CHI.

\section{波}

574 Clii 汲 $\mathrm{Ki}$.

[496] $1^{\circ} \mathrm{H}$. forming the prefectural city of Weilini, Honan; Lat. $35^{\circ}$ $28^{\prime}$, Long. $114^{\circ} 16$; Shang, Mn-yeli 牧野; Han, Cli |; N. Wei, 1st Chin-mên 金閒 H., in Chin-mên 金門 chün; 2nd Hsi-an 新安 $\mathrm{H}$., Hsi-ÿian 西垣 H., and Tung-yï «n H. in Hsinan 新安 chïn; 3rd Pein1ien-ch'ih 北㴡池 $\mathrm{H}$. in Mien-cls'ih 范池 chiun; 4th Nan-mien-cli il 南溜 池 H., in Yi-yang 宜陽 chïn; Yïan, Wei-hui 衛 輝 circ. in Chung-shu 中 書 province; Ming, Wei-hui 衛 輝 Fu, Honan. $2^{\circ}$ See No. 6971.

\section{极}

575 Chi-ch'iao 极欈 chên near Ning C., Kansul.

\section{器}

$576 \mathrm{Chi}$ 冀 $\mathrm{Ki}$.

[325] $1^{\circ}$ C.L.C. in the Ch 'ing-110 circ., Clinhli; Lat. $37^{\circ}$ $38^{\prime}$, Long. $115^{\circ} 42^{\prime}$; orig., Tung-yang 東妵; E. Han, Chi $\mid \mathrm{C} ., \quad I V$. Han, Hsin-tu信都 $\mathrm{K}$. 
冀

CHi.

and $\mathrm{H}$.; from $\mathrm{Han}$ to Yïan, Hsin-tu 信都 $\mathrm{H}$. $2^{\circ}$ See No. 1887.

577 Chi-ch'êng | 城, see No. 1887.

578 Chi chou / 州, one of the nine great divisions of China made by Yü 禹; it comprised Chilili, Shansi and parts of Honan and Manchuria.

579 Chi-11ing | 家, circ. in Shansi, comprising T'aiyüan Fu, Fên-chon Fu, I, 11-an Fin, Tsê-chon Fin, Liao C., $\mathrm{Cl}^{\text {' }}$ in C. and $\mathrm{P}^{\prime}$ ing-ting $\mathrm{C}$.

\section{鮌}

580 Chi 副 $\mathrm{Ki}$.

[217] $1^{\circ}$ C. in Slunn-t ien Fu, Chillili; Lat. $40^{\circ} 05^{\prime}$, I.ong. 117 22'; Chou, centre of K. Yen 郝; $T s^{\prime} i n$ and Han, Yü-yang 漁防； Trang, Chi |C. $2^{\circ}$ See No. 6037.

581 Chi-ch'iu | 邱, formerly a town near Shun-t ien Fin, Chilıli.

582 Chi-pei | 北, see No. 950

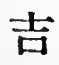

\section{Chi 吉 Kih.}

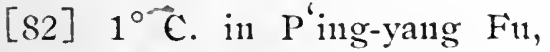
Shansi ; Lat. $36^{\circ} 06^{\prime}$, Long. $110^{\circ} 35^{\prime}$; Han,
Chi-t,IN.

Pei-ch'ii 北 愿; 2nd $\mathrm{WCi}$, Ting-yang 定璌; P. Chou, Fên 汾 C.; Sui, Kêng 耿 C.; T"ang,

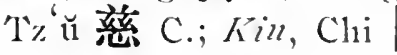
c.

$2^{\circ}$ (Obs.) S. Sung, C. in Chiang-hsi 江. 西 circ. $3^{\circ}$ See Nos. 584, 3847, 4141, 7862.

584 Chi-an | 管 Kih-ngan.

[208] $1^{\circ}$ Fn in the Chi-NanKan-Ning circ., Kiangsi; forming also the district city Lï-ling; Lat. $27^{\circ}$ $02^{\prime}$, Long. $115^{\circ} 05^{\prime}$; $S . A$., in K. Wu 紧; $C$. Wars, in K. Cln' 1 楚; Ts'in, in Chin-chiang 儿江 chïll and Ch'angsha 長沙 chïln; Han, I,ii-ling 盧陵; Threc K's., An-ch'êng 发城; Sui and $T^{\prime}$ ang, Chi $\mid \mathrm{C}$; Ming, Chi-an| 安 Fu. $2^{\circ}$ S šr in Chis ao-yang H.,

Kuangtung.

585 Chi-chia | 家 Y. near $\mathrm{Cl}_{1}^{6} \mathrm{i}$ H., Honan (No. 616).

586 Chi-lin | 林 Kirin.

$1^{\circ}$ Province of Manchuria, East of Hel1-l1111g-kiar.s. $2^{\circ}$ Prefecture in the Chilin circ., Kirin ; Capital of the province; also called Kirin-wn-la | 林 烏 拉 Lat. $43^{\circ} 45^{\prime}$, Long. $126^{\circ} 25^{\prime}$. 
$3^{\circ}$ Circnit in Kirin, comprising Chi-lin $\mathrm{Ft}$, Pedne C.L.T., Ch'angch' 11 C.L.T., Pin-chon C.L.T. and Wu-ch' ang C.L.T.

587 Chi-lin-wu-1a | 林 鳥拉, see preceding.

588 Chi-Nan-Kan-Ning | 南 赎察, circ. in Kiangsi, comprising Chi-an Fu, Nan-an 'Fu, Kan-chou Fu and Ning-tur $C$.

589 Chi-p'ang / 龎, (obs.) in Annam ; $S . T s^{6} i, H$. in Chin-chên 九 真 chün.

590 Chi-shui | 水 Kih-shwui. [208] $1^{\circ} \mathrm{H}$. in Clii-an $\mathrm{Fu}$, Kiangsi ; Lat. $27^{\circ} 16^{\prime}$, Long. $115^{\circ} 14^{\prime}$; Han, Chi-yang | 陽; $S$. $T^{6}$ ang, Chi-sluti | 水. $2^{\circ}$ Ss̆̌ near Wang-chiang H., Anluti.

591 Chi-ssŭ-t'a-ni | 思塔 你 Constantinople.

592 Chi-yang | 陽.

$1^{\circ}$ Chên near Tung-liu H., Anhui.

$2^{\circ}$ (Obs.) Sui, H., S. of Ying-shan H., Hupelı. $3^{\circ}$ (Obs.) E. Tsin, town W. of Chu1-ch'i H., Hupeh.

$4^{\circ}$ (Obs.) T'ang, $100 \mathrm{li}$ N.W. of Yai C., Knangtung.

$5^{\circ}$ See Nos. $83,590,5170$.
源 Chr.

\section{徏}

593 Chi-lun | 佶 倫 Kih-lun, T-C. in T'ai-p'ing $F u$, Knangsi ; Lat. $23^{\circ} 20^{\prime}$, Long. $105^{\circ} 53^{\prime}$; Ming, Chieh-hun 結 倫 C. in T'ai-p'ing Fu, Kuangsi.

\section{集}

594 Chi 集, (obs.) Sui, C. comprising parts of Nan-chiang H. and $\mathrm{Pa}$ C., Ssŭch' uan. 595 Chi-cling | 津, (obs.) N. E. of Shan C., Honan; Kin, chên in Shan 陝 C. 596 Chi-hsien | 賢, post-town near Wan H., Ssŭch' 'uan. 597 Chi-11nu-êrh-yi-k'ê | 謨尔 邑克 Zimeriek, town in Tarbagatai ; Lat. $46^{\circ} 55^{\prime}$, Long. $83^{\circ}$.

\section{結}

598 Chi-ch'i 績 溪 or 績 笑人 [480] Tsih-ki, H. in Hui-chon $\mathrm{Fu}$, Anhui ; Lat. $30^{\circ} 09^{\prime}$, Long. $118^{\circ} 38^{\prime} ; T^{\prime}$ ang, H. in Hsi 䣄 C. ; Sung, H. in Hui 徽 C. ; Yüan, H. in Hui-chon 徽 州 circ. ; Ming, H. in Huichou 徽州 Fu.

$599 \mathrm{Ch}_{11}-\mathrm{cl}_{1}$ ' $\mathrm{i}$ / 筃谷, see preceding.

\section{濟}

600 Chi 濟 Tsi. 
$1^{\circ}$ C. in Chi' üan-lo 全 羅 circ., Corea ; Lat. $34^{\circ}$ $40^{\prime}$, Long. $125^{\circ} 12^{\prime}$. Situated on Qualpaert Island.

$2^{\circ}$ (Obs.) Chou, a feudal state; S.A., a K. in $\mathrm{N}$. of Shantung.

$3^{\circ}$ See Nos. 601, 602.

601 Chi-nan | 南 Tsi-nan, Fu in the Chi-Tung-T'ai-WuLin circ., Shantung ; forming also the district city Li-ch' êng ; capital of the prov.; Lat. $36^{\circ} 40^{\prime}$, Long. $117^{\circ} 01^{\prime} ;$ S.A. and $C$. Wars, in K. Chi $\mid$; $s^{\prime}$ in, in $\mathrm{Ch}^{\prime} \mathrm{i}$ 湾 chïn; Han, $\mathrm{P}^{\prime}$ ing-yüan 平原, Cl'iench'êng 干乘 and Po-hai 渤 海; 2nd $W e i, \mathrm{Chi}$ C.; T'ang, Lin1-tzŭ 臨 淄; Ming, Chi-nan | 南 Fu. Chinang-li (Polo).

602 Chi-ning | 耍 Tsi-ning C. [322] C. L.C. in the Yen-YiTs'ao-Chi circ., Shantung; Lat. $36^{\circ} 50^{\prime}$, Long. $116^{\circ}$ $58^{\prime}$; orig., K. Jên 任; Han, Jên-ch'êng 任城; 2nd Wei, Chi | C.; Sui, Chi-pei | 北; Yüan, Chining | 嘎. Sinju1natı (Polo).

603 Chi-pei | 北, sce preceding.

604 Chi-Tung-T'ai-Wu - Lin |

東泰武臨， circ。 in
Shantung, comprising Chinan Fu, Tung-ch'ang Fu, T'ai-an F $\mathrm{w}$, Wu-ting Fu, and Lin-ch' ing C.L.C.

605 Chi-wu-p' $u$ | 物浦 Chemulpo, sec No. 2986.

606 Chi-yang | 䛷 Tsi-yang.

[148] $1^{\circ} \mathrm{H}$. in Chi-nan Fu, Shantung; Lat. $37^{\circ}$ $03^{\prime}$, Long. $117^{\circ} 20^{\prime}$; Chou, Shih 郝; Sui, Kao-yüan 高范; $T^{6}$ ang, Chi-yang | 陽.

$2^{\circ}$ See No. 7417.

607 Chi-yin | 陰, see Nos. 6537, 6141, 6701.

608 Chi-yüan | 源, Tsi-yuen, $H$. in Huai-ch'ing Fu, Honan; Lat. $35^{\circ} 07^{\prime}$, Long. $112^{\circ} 39^{\prime}$; Chou, Imperial domain; $T s^{\prime}$ in, Chih 軹 H.; Sui, H. in Ho-nei 河內 chün; T"ang, Sung, Kin and Yüan, H. in Mêng 孟. C.; Ming, H. in Huai-ch'ing 懷慶 Fin.

\section{郎}

609 Chi-fei 的裴, (obs.) W. of Fei-hsiang H., Clihhli; $W$. Han, Marq. in Wei 魏 chiin。

610 Chi-lai | 来, (obs.) near Yi-clon F11, Shantung; $W$. Han, Marq. in Langyeh 撕 chün. 
郎

611 Chi-mo | 墨 Tsih-moh, H. in Lai-clion Fu, Shantıng; Lat. $34^{\circ} 15^{\prime}$, Long. $120^{\circ}$ 44'; Han, Chi-mo| 墨.

\section{祭}

612 Chi-ch'êng 祭城, (obs.) formerly town N. E. of $\mathrm{Ch}^{\prime}$ ang-yüan $\mathrm{H}$., Chihli.

Chi-chan, see No. 986.

Chi-chwang, see No. 6976.

Chi-fau, see No. 977.

Clii-hing, see No. 5655.

Chi-kiang, see Nos. 957, 961.

\section{$\mathrm{CH}^{\prime} \mathrm{I}$}

旗

613 Ch'i-han 旗後 see No. 6126.

\section{棋}

$614 \mathrm{Cl}^{\text {‘ } \mathrm{i}}$ 棋, (obs.) 40 li S. W. $\mathrm{K}$ 'ai-yiian $\cdot \mathrm{H}$., Shingking; Liao, C. in I,iao 楒 C.

\section{期}

$615 \mathrm{Ch}^{6} \mathrm{i}$-ssŭ 期思.

$1^{\circ}$ (Obs.) N. W. of K11sliih H., Honan; $W$. and $E$. Han, H. in J11-11an 漖南 chün; $T$ sin, S. Sung and $S$. $T s^{\prime} i$, H. in Yi-yang 防 chïl；N. Wei, H.
基

CH'I-TS'UN.

in 1st, Ju-yin 汶㓌 chiü, 2nd Yi-yang 陽 chün, 3rd Pie11ch' êng 邊城 chiin.

$2^{\circ}$ (Obs.) E. of Shangcl'êng H., Honan; Sui, H. in Yi-yang $\mathbb{C}$ 陽 chiun.

\section{淇}

$616 \mathrm{Cl}_{1}{ }^{\circ} \mathrm{i}$ 淇 $\mathrm{Ki}, \mathrm{H}$. in Wei-hui Ful, Honan1; Lat. $35^{\circ} 38^{\prime}$, Long. $114^{\circ} 21^{\prime}$; orig., Chao-kê 朝歌; Chou, Mei-yi 沫畠; Yüan, C., Wei-hui 衛 輝 circ.; Ming, H. in Wei-hu1 衛 敞 Fin.

$617 \mathrm{Cl}^{\text {' }} \mathrm{i}$-ao | 澳, ss̆̈ near Hsiang-shan H., Knanytimg.

618 Ch'i-mêt1 |师, (obs.) $50 \mathrm{li}$ N. E. of Clii H., Honan; Kin, chên in Hsïn 溶 C.

\section{蔡}

$619 \mathrm{Cl}^{\prime}$ i-chiang 棊江 Ki-kiang, [25] H. in Cl' 'ung-ch' ing Fu, Ssŭch 'van; Iat. $28^{\circ} 56^{\prime}$, I.ong. $106^{\circ} 49^{\prime}$; orig., $\mathrm{Cl}^{1}$ i-shih, | 市; Ming, H. in $\mathrm{Cl}^{\prime}$ 'ung-ch 'ing 重序 Fu. $620 \mathrm{Cl}^{6} \mathrm{i}$-slinili $\mid$ 市, see preceding.

$621 \mathrm{Ch}^{\circ} \mathrm{i}$-ts'u11 | 村, (obs.) 40 li W. of Sha-ho H., Clilili; Kin, chên in Hsing 邲 C. 
騏 $\mathrm{CH}^{6} \mathrm{I}$.

\section{騏}

$622 \mathrm{Ch}$ 'i 䮲, (obs.) S. E. of Hsiang-ning H., Shansi ; $\mathrm{W}$. Han, Marq. in Hotung 河 東 chïn.

\section{杷}

$623 \mathrm{Ch}^{\prime} \mathrm{i}$ 杞 $\mathrm{Ki}$.

[157] $1^{\circ} \mathrm{H}$. in $\mathrm{K}$ ai-fêng $\mathrm{F}_{11}$, Honan ; Lat. $34^{\circ} 42^{\prime}$, Lolig. $114^{\circ} 55^{\prime}$; Shang and Chou, a feudal state; Han, Yung-ch' 'in 䔨邱; Sui, Chi ${ }^{\circ}$ C.; Five dyn., $\mathrm{Ch}^{\prime} \mathrm{i} \mid \mathrm{H}$.

$2^{\circ}$ See No. 2611.

$624 \mathrm{Ch}^{\prime}$ 'i-wei | 惟, (obs.) near Turfan; T"ang, C. in Yüeh-chih 月支 Fin.

\section{郎}

$625 \mathrm{Ch}^{\prime} \mathrm{i}$ 䢞, (obs.) Han, town N. of Yi-ch'êng H., Hupeh.

\section{起}

$626 \mathrm{Ch}^{\prime} \mathrm{i}$ 起, see No 1414.

$627 \mathrm{Ch}^{\text {' }}$ i-êrh-man 兒 漫 Kermané, also called H11-ssŭ 虎 司; a town between Samarcand and Bokhara. $628 \mathrm{Ch}^{\prime} \mathrm{i}-\mathrm{t}$ 'a | 塔, T-S. near Hsi-ning Fu, Kansul.

\section{庴}

$629 \mathrm{Ch}^{\prime} \mathrm{i}$ 庴 See Nos. 1245, 3277.
埼

CH'I-Ÿ̈.

\section{溪}

[N. B. This character is frequently read "hsi" in Peking.]

$630 \mathrm{Ch}^{6} \mathrm{i}$ 㳂.

$1^{\circ}$ (Obs.) S.E. Yung-shun H., Hunan; T"ang, H. in Cliang-nan 汭南 circ.

$2^{\circ}$ (Obs.) N. Ssŭ-ên H., Kuangsi ; Sung, C. in Kuang-nan 厤南 Western circ.

$3^{\circ}$ See Nos. 4949, 7970.

631 Cli'i-k'ou | 口, post-town near Yung-ting H., Ful kien.

$632 \mathrm{Cl}_{1}{ }^{\mathrm{i}}$-tung $\mid$ 洞, see No. 440 .

\section{棌倠}

$633 \mathrm{Cl}^{\text {'i-t }}$ 'on 溪待頭, chên near Wan-an H., Kiangsi.

\section{奇}

634 Ch'i-t'ai 奇臺 Ki-tai, H. in Ti-hua C., Kansulı; Lat. $43^{\circ} 40^{\prime}$, Long. $91^{\circ}$.

$635 \mathrm{Ch}$ 'i-yen-ts'ai-kon | 才 漂, 'T-S. near Nie11-po H., Kansuh.

\section{埼}

636 Ch'i-yï 埼王, Saitana, Ken in Japan. Chief town Urawa. Also written 琦玉: 
崎

CH'I-SHAN.

\section{崎}

$637 \mathrm{Ch}^{\text {'i }}$-shan 崎 山, ss̆̌ near Ying-chêng H., Hupeh.

$638 \mathrm{Ch}^{\dagger} \mathrm{i}-\mathrm{y} \ddot{\mathrm{i}} \mid \mathrm{E}$, see No. 636.

\section{岐}

$639 \mathrm{Ch}^{\prime} \mathrm{i}$ 岐.

$1^{\circ}$ (Obs.) Five dyn., $\mathrm{Ch}^{\prime} \mathrm{i}$ c.

$2^{\circ}$ See No. 1724 .

$640 \mathrm{Ch}^{\prime} \mathrm{i}$-fu |阜 Gifu, ken and chief town, Japan.

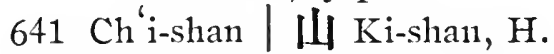
[469] in Fêng-hisiang $\mathrm{Fu}$, Shensi ; Lat. $34^{\circ} 20^{\prime}$, Long. $107^{\circ} 40^{\prime}$; orig., land of Chou 周; P. chou, Sanlung 三龍; Sui, Ch'ishan III.

642 Ch'i-t'ing | 䂛 Ki-ting.

[27] $1^{\circ} \mathrm{T}$. in Huang-chou $\mathrm{Fu}$,

Hupeh ; Lat. $31^{\circ} 05^{\prime}$,

Long. $114^{\circ} 38^{\prime}$.

$2^{\circ}$ (Obs.) Ts' $i$ and Liang,

H., W. of Ma-ch 'êng

H., Hupeh.

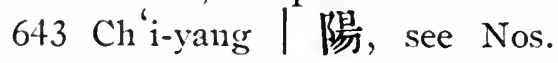
1724, 1817.

\section{砥}

644 Ch'i-p'ing | 砥 雨 chän in Ching-ch"i 京 綏 circ., Corea.

\section{龩}

$645 \mathrm{Ch}^{\prime} \mathrm{i}$ 踭 $\mathrm{Ki}$.
蔪 Ch' I-CH'Un.

[528] $1^{\circ} \mathrm{C}$. in Huang-chou $\mathrm{Fu}$, Hupeh ; Lat. $30^{\circ}$ 03', Long. $115^{\circ} 25^{\prime} ; T^{\prime}$ ang, $\mathrm{Ch}^{6}{ }^{\mathrm{i}-\mathrm{ch}}{ }^{\mathrm{i}} \mathrm{un}$ | 春; $T \sin$,

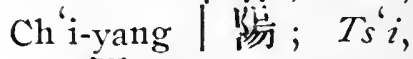
Lo 羅 C.; P. Chou, $\mathrm{Ch}^{\prime}{ }^{\mathrm{i}} \mid \mathrm{C}$.; Sung, $\mathrm{Ch}^{\prime} \mathrm{i}$ |C. and $\mathrm{Ch}^{\prime} \mathrm{i}$-ch'un | 春 H. in Huai-nan 淮 南 circ.; Yüan, Ch' $\mathrm{i}$ ch'un | 春 H.; Ming, $\mathrm{Ch}^{\prime} \mathrm{i} \mid \mathrm{C}$. Port of call. $2^{\circ}$ (Obs.) near Kuei-tê Fu, Honan; N.Wei, H. in $1 s t \mathrm{Ch}$ iao 譙 chiin, 2nd Ma-t'ou 馬頭 chün, Nan1-yen 南兗 cHou.

$3^{\circ} N$. Wei, H. in Ma-t on 馬頭 chiun, $\mathrm{Ch}^{\mathrm{u} \mathrm{u}}$ 楚 CHOU; now (obs.) near Fêng-yang Fu, Anhuni. $4^{\circ}$ (Obs.) N. W. of $\mathrm{Ch}^{\prime} \mathrm{i}$ C., Hupeh ; T'ang, C. in Huai-nan 淮南 circ.; Five dyn., $\mathrm{Ch}^{\prime} \mathrm{i} \mid \mathrm{C}$. $5^{\circ}$ (Obs.) $36 l i \mathrm{~S}$. of $\mathrm{Su}$ C., Anhui ; T Tang, Sung and $K i n, H$. in $\mathrm{Su}$ 宿 C.

$6^{\circ}$ See No. 2282.

$646 \mathrm{Ch}^{\text {'i-ch' }}$ êng $\mid$ 城, see No. 2242.

$647 \mathrm{Ch}^{\mathrm{i}} \mathrm{i}$-ch'un | 春.

$1^{\circ}$ (Obs.) N. W. of $\mathrm{Ch}^{6} \mathrm{i}$ C., Hupeh; W. Han, H. in Chiang-hsia 江 夏 chün ; E. Han, Marq. in Chiang-11sia 江 夏 
䂋 CH'I-CH'Uก.

chün; Tsin, H. in Yiyang 一陽 chün; Sui, chiin and H. in Yang 揚 CHOU; $T^{6}$ ang, H. in $\mathrm{Ch}^{\prime} \mathrm{i} \mid \mathrm{C}$.

$2^{\circ}$ See Nos. 645, 648, 3391, 4038.

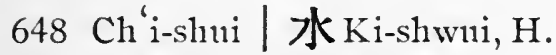
in Huang-chon $\mathrm{Fu}$, Hupeh; Lat. $30^{\circ} 27^{\prime}$, Long. $115^{\circ}$ 22'; Han, $\mathrm{Cl}^{\prime}{ }^{\prime} \mathrm{i}-\mathrm{ch}$ 'un | 春; Liang, Cli'i-shui | 水; T'ang, Lan-ch'i 蘭 |.

649 Ch'i-yang | 陽, see No. 645.

\section{所}

$650 \mathrm{Cl}^{\prime} \mathrm{i}$-li 行黎, (obs.) near Shun-t ien Fu, Chihli; T'ang. C. in Fêng-cl' êng 清誠 $\mathrm{Fu}$.

$651 \mathrm{Cl}^{\prime}$ 'i-ming | 命, T-S. near Sung-p an T., Ssŭch' uan.

\section{竝}

$652 \mathrm{Cl}^{\prime} \mathrm{i}$ 阽 $\mathrm{Ki}$.

[448] $1^{\circ} \mathrm{C}$. in Pao-ting $\mathrm{Fu}$, Chiltli ; Lat. $38^{\circ} 27^{\prime}$, I.ong. $115^{\circ} 26^{\prime}$; Han, K. An 安; Sui, Yi-fêng 哦豐; T'ang, Ch'i | C.; Sung, P'u-yin 蒲 陰 $\mathrm{H}$.

[385] $2^{\circ}$ H. in T'ai-ÿ̈an Fu, Shansi ; Lat. $37^{\circ} 23^{\prime}$, Long. $112^{\circ} 18^{\prime}$; orig., $\mathrm{Ch}^{\prime} \mathrm{i}-\mathrm{cl}{ }^{\prime} \mathrm{i} \mid$ 溪; Han, Ch'i | Sui, H. in

\section{乞 CH $^{\prime}$ I-I,I-MANG-SHA-HANG.}

T'ai-yüan 太原 chün; T"ang, Sung and Kin, H. in T'ai-yüan 太原 $\mathrm{Fu}$; Yüan H. in Chining 蕉蒚 circ.; Ming, H. in T'ai-yian 太原 Fur.

$3^{\circ}$ (Obs.) S.E. of Ch'i $\mathrm{H}$., Shansi ; Han, H. in T'ai-yüan 太原 chün; $T \sin , \mathrm{H}$. in K. T'aiyïan 太原; N. Wei, H. in T'ai-yizan 太原 chiin.

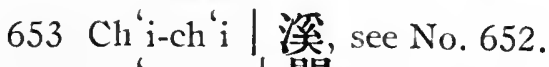
654 Ch'i-mên |閒 Ki-mun, H. [314] in Hui-chon Fu, Anhui ; Lat. $29^{\circ} 55^{\prime}$, Long. $118^{\circ}$ $18^{\prime}$; orig., $\mathrm{Yi}$ 黟 $\mathrm{H}$.;

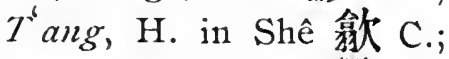
Sung, H. in Hui 徽 C.; Yïan, H. in Hui-chon 徽 州 circ.; $\operatorname{Ming}, \mathrm{H}$. in Hui-clou 徽 州 Fu。 655 Ch'i-yang | 陽 Ki-yang, $\mathrm{H}$. [177] in Yung-chou Fin, Hunau ; Lat. $26^{\circ} 30^{\prime}$, Long. $111^{\circ}$ 44'; Han, Ch'üan-ling 泉 陵; Wu, Ch'i-yang | 陽.

\section{气}

$656 \mathrm{Ch}^{\prime}$ i-li-man 乞里蠻 Kerman, town in Persia; Lat. $29^{\circ} 48^{\prime}$, Long. $56^{\circ} 30^{\prime}$.

$657 \mathrm{Ch}^{\prime} \mathrm{i}-\mathrm{li}$-1nang-sha-hang | 里 芒沙杭 Kirmanshahan, town in Persia; Lat. $34^{\circ}$ $18^{\prime}$, Long. $47^{\circ} 12^{\prime}$. 
乞 $\mathrm{CH}^{\mathrm{I}} \mathrm{I}-\mathrm{SH} H \mathrm{H}-\mathrm{MI}$.

658 Ch'i-shih-111 | 石 洣, or Kê-shil1-mi 簓烦密, or Ch'ieh-shih-mi-êrh 怯 失 迷兒, or Chia-shih-1ni-lo 迦濕张羅, Caslmere; Lat. $24^{\circ} 05^{\prime}$, Long. $74^{\circ}$ $57^{\prime}$.

\section{椿}

$659 \mathrm{Cl}^{\prime}$ i-hsia 椿 霞 Tsi-hia, H. in Têng-chou Fin, Shantung; Lat. $37^{\circ} 17^{\prime}$, I.ong. $121^{\circ} 18^{\prime}$; Han, Shui 腄 H.; Kin, Ch'i-hsia | 霞 $\mathrm{H}$.

$660 \mathrm{Ch}^{6}$ i-yü| 豫, (obs.) T'ang, C. near Ta-yao H., Yünnan.

\section{t}

661 Ch'i-cliia 七家, post-town near Linan C., Chihli.

$662 \mathrm{Ch}^{\prime} \mathrm{i}$-êrl1-pao | 兒堡, T-S. near Mien-ning H., Sš̆ch' wan.

663 Clik-kung | 公, (obs.) in Nankung H., Chillit; Kin, chên in Chi 黛 C.

$664 \mathrm{Cl}^{\prime}$ 'i-kung | 琽 ssü in Yangshan H., Knangtung.

$665 \mathrm{Ch}^{6} \mathrm{i}$-1nin | 淈.

$1^{\circ}$ (Obs.) Chou, Division in Fuhkien.

$2^{\circ}$ See No. 1871.

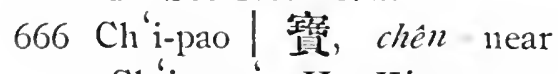
$\mathrm{Cl}^{\text {'ing-p' }}$ " $\mathrm{H}$., Kiangsu.

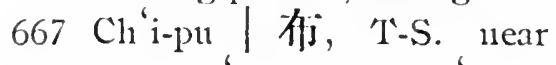
Sung-p'an T., Ssŭcl' 'nan.
驾 $\mathrm{CH}^{6} \mathrm{I}-\mathrm{CH} \mathrm{I}^{\prime} \mathrm{I}-\mathrm{HA}-\hat{\mathrm{E}} \mathrm{RH}$.

$668 \mathrm{Ch}^{\prime}{ }^{\mathrm{i}}-\mathrm{p}^{6} \mathrm{u}$ | 浦, ss̆̌ in 'T'aits'sang C., Kiangsu.

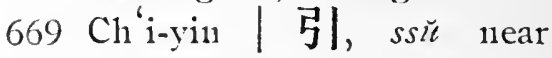
Ying-shan H., Anluni.

670 Ch'i-yiian | 源, (obs.) near T'ai-p'ing Fin, Kuangsi ; T"ang, C. in Ling-nan 欲 南 circ.; Stung, H. in Yung 慧 C.

\section{㔖然}

$671 \mathrm{Cl}^{6} \mathrm{i}$ 呍.

$1^{\circ}$ (Obs.) Tsin, chiun in Shantung.

$2^{\circ}$ See No. 601.

$672 \mathrm{Cl}^{1}$ i-an $\mid$ 管.

$1^{\circ}$ Post-town near Huangchou Fu, Hupeh.

$2^{\circ}$ See Nos. 2252, 7252.

673 Ch'i-cl'ang 昌, (obs.) $T^{6}$ si and $T^{6}$ ang, H., N. of Hsing-ning $\mathrm{H}$., Kuangtung.

674 Ch'i-ch'êng | 城, see No. 3160.

$675 \mathrm{Cl}^{\prime} \mathrm{i}$-chi | 基, (obs.) $40 \mathrm{li}$ W. of Kuan H., Ssŭchinan; S. Ts $i, H$. in Min-shan 汶川 chïn.

$676 \mathrm{Ch}^{6} \mathrm{i}$-ch ${ }^{6} \mathrm{i}-\mathrm{e} r \mathrm{rh}-\mathrm{kê}-\mathrm{na}$ | 基分 噶納 Tsitsirkana, town in Cobdo; Lat. $50^{\circ}$, Long. $91^{\circ} 50^{\prime}$.

$677 \mathrm{Ch}^{\prime} \mathrm{i}$-ch'i-ha-êrh|㔖哈尔 Tsitsihar.

$1^{\circ}$ Town, capital of Hehlung-kiang; Lat. $47^{\circ}$ $20^{\prime}$, Long. $123^{\circ} 30^{\prime}$. 
啒 $\mathrm{CH}^{6} \mathrm{I}-\mathrm{CH}{ }^{6} \mathrm{I}-\mathrm{HA}-\hat{\mathrm{E}} \mathrm{RH}$.

$2^{\circ}$ See No. 2007.

$678 \mathrm{Ch}^{6} \mathrm{i}$-chia-clunang | 家生, post-town near Lê-t'ing H., Chihli.

$679 \mathrm{Ch}^{6} \mathrm{i}-\mathrm{ho}$ | 河 Tsi-ho, H. in [148] Chi-nan Fin, Shantung ; Lat. $36^{\circ} 42^{\prime}$, Long. $116^{\circ}$ $50^{\prime} ;$ Han, Chu-a 祝阿; Sung, Ch"i-ho 河.

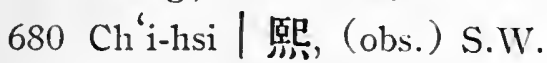
of Yung H., Kuangsi ; $S$. $T s^{\prime} i$ chion in 1st Kuang 廣 cHOU, 2nd Hsiang 湘 CHOU.

$681 \mathrm{Ch}^{6} \mathrm{i}-\mathrm{h}$ sing | 興, (obs.) $T s^{\prime} i$, town E. of Yün-nan Fù, Yünnan.

$682 \mathrm{Ch}^{6} \mathrm{i}-\mathrm{k}$ 'ai | 開, (obs.) in Ssŭch'nan; S. Ts'i, Tso 左 chiun in $\mathrm{Yi}$ 益 chov.

$683 \mathrm{Ch}^{6} \mathrm{i}-1$ ê | 樂, see No. 6206. $684 \mathrm{Cl}^{\prime} \mathrm{i}-\mathrm{l}$ 1111g | 隆, (obs.) in Kuangtung; S. Ts'i, chiin in Yüeh 越 chot.

$685 \mathrm{Ch}^{6} \mathrm{i}$-tung | 東 Tsi-tung. [148] $1^{\circ} \mathrm{H}$. in Chi-nan Fu, Sliantung; Lat. $37^{\circ}$ $17^{\prime}$, Long. $117^{\circ} 37^{\prime}$; Han, 'Tson-p'ing 娜压; Yïan, Ch i-tung | 東. $2^{\circ}$ (Obs.) near the above; Yizan, H. in Ho-chien 河 間 circ.; Ning, H. in Clii-nan Fu, Shantung. 686 Ch'i-t'ung | 通, see No. 4365.
嘉

\section{涂}

$687 \mathrm{Ch}^{\mathrm{i} i-k 11}$ 漆 谷 Fu in Ch'ing-shang 慶何 circ., Corea.

$688 \mathrm{Ch}^{\prime} \mathrm{i}$-yüan | 原 $\mathrm{H}$. in Ch'ing-shang 鹿䀺 circ., Corea.

689 Ch i-yiian 漆垣, (obs.) in Shensi; Han, H. in Shang 上 chïn.

\section{CHIA}

\section{加}

690 Clia-111011-shan 加参蛅， post-town near Fêng-t icn Fu, Shing-king.

691 Clia-p'ing | 4 Fin in Clining-ch'i 京畿 circ., corea.

\section{架}

692 Chia-chon 架州, T-S. near Mien-ning H., Ssŭch' nan.

\section{迦}

693 Chia-shih 迦師, see No. 3224.

694 Chia-shih-1ni-lo | 濕彌維, see No. 658.

\section{嘉}

695 Chia 嘉, see No. 755.

696 Chia-cli êng | 誠 see No. 5977. 
壱

CHIA-HO.

697 Chia-ho | 瓜 Kia-ho, H. in Kueiyang C., Hunan ; Lat. $25^{\circ} 31^{\prime}$, Long. $112^{\circ}$; see also No. 699.

698 Chia-hsiang | 祥Kia-siang,

[15] H. in Chi-ning C., Shantung; Lat. $25^{\circ} 32^{\prime}$, Long. $116^{\circ} 30^{\prime}$; orig., Chï-yeh 鉅野, Kin, Chia-hsiang 祥.

699 Chia-hsing | 與 Kia-hing. $1^{\circ} \mathrm{Fu}$ in the Hang-Chia$\mathrm{Hu}$ circ., Chehkiang; forming also the two district cities Clia-hising and Hsiu-shui; Lat. $30^{\circ} 48^{\prime}$, Long. $120^{\circ}$ 43'；S.A., Tsui-li 售 李 and $\mathrm{Ch}^{6}$ ang-shui 長 水, $1 s t$ in $\mathrm{Ks}$. Wu 奌 and Yüeh 越, $2 n d$ in K. Yïeh 越; $T s^{\prime}$ in, in Kuei-chi 會秏 chiin; $W u$, Chia-ho|禾 and Chia-hsing | 與; Sui, in Sil 䔡 C.; Trang, in Hang 杭 C.; Five dyn., Hsin 秀 C.; Ming, Chia-hsing | 舆 Fu. Ciangan (Polo).

$2^{\circ} \mathrm{H}$. forming with Hsinshui $H$. , the prefectural city of Chia-hsing, Chehkiang; Lat. $30^{\circ}$ $48^{\prime}$, Long. $120^{\circ} 43^{\prime}$; S.A., Yii-êrh 禦耳; Ts'in, Yu-ch'üan H 拳; $W u$, Chia-ho|禾
咟

ChiA-TING.

and Chia-hsing | 興; Five dyn., Hsiu 秀 C. 700 Chia-lin $\lceil$ 鹿粦, (obs.) in Wu-wei H., Kansuh; T'ang, C. in Liang 凉 CHOU.

701 Chia-ming | 明, chên near Lung-ch'ang H., Ssŭch' uan.

702 Chia-shan | III Kia-shan, chiun in P'ing-an 平安 circ., Corea ; Lat. $40^{\circ} 04^{\prime}$, Isong. $125^{\circ} 40^{\prime}$.

703 Chia-shan | 善 Kia-shen, H. in Chia-hsing Fu, Chehkiang; Lat. $30^{\circ} 53^{\prime}$, Long; $120^{\circ} 40^{\prime}$; orig., Wei-t'ang 魏 塘 chên.

704 Chia-shum |㮌, post-town near Nien-fo H., Kansul. 705 Chia-ting | 定 Kia-ting.

[307] $1^{\circ} \mathrm{Fu}$ in the Chiench'ang-shang-nan circ., Ssŭch uan ; forming also the district city Lêshan; Lat. $29^{\circ} 34^{\prime}$, Long. $104^{\circ} 00^{\prime} ; T_{s}^{\prime} i n$, in Shu 蜀 chün; Han, Han-chia漢 | inChienwei 㯬第 chiun; Sui, Mei-shan 首山; T ang and Sung, Chia $\mid \mathrm{C}$.; Ming, Chia-ting | 定 Fu.

$2^{\circ}$ H. in T'ai-ts'ang C., Kiangsu ; Lat. $31^{\circ} 22^{\prime}$, Iong. $121^{\circ} 03^{\prime}$; Sui, K'un-shan 峎山; Ming Chia-ting 定 $\mathrm{H}$. 
嘉 ChIA-YANG-Cil ÊNG.

706 Chia-yang-ch' êng 嘉陽城, (obs.) Han, town near P'ing-hsiang H., Clihli.

707 Chia-yi 笔 Kia-yi, H. in T'ai-wan Fu, Fuhkien; Lat. $23^{\circ} 20^{\prime}$, Long. $120^{\circ}$ 30'; formerly Chin-lo 諸 羅 $\mathrm{H}$.

708 Clia-ying | 應 Kia-ying, [134] C.L.C. in the Hui-Cli aoChia circ., Kuangtung; Lat. $24^{\circ} 10^{\prime}$, Long. $106^{\circ}$ $03^{\prime}$; Han, Cliiel-yang 揭 陽; Tsin, Chia-ying | 應; Sung, Mei 梅 C. in Kuang-11an 鹿南 E. circ.; Yüan, Mei 梅. C., Kuangsi.

709 Chia-yü | 魚 Kia-yü, H. in [528] Wu-cli ang Fu, Hupeh; Lat. $30^{\circ}$, Long. $113^{\circ} 47^{\prime}$; $T \sin$, Sha-yang 沙陽; $S$. Tang, Chia-yü 魚; Sung, H. in O 鄂 C.; Yüan, $\mathrm{H}$. in Wu-cl' ang 武昌 circ.; Ming, H. in Wu-ch' ang Fu.

710 Chia-yü Kuan | 峪關, Kia-yu Kwan, ssü in Su C., Kansul.

\section{珼}

711 Chia-chia 貿家, X. near Ning-hsia T., Shensi.

712 Chia-ch'ü|曲, chên near $\mathrm{P}^{\mathrm{a}} \mathrm{u}$-ch ềng H., Shensi.
賈 Chin-shui.

713 Chia-tș inn | 村, chên near Pao-chi H., Shensi.

\section{段}

714 Chia-shih 段氏， see No. 7002 .

搌

715 Chia 㜊 Kia, C. in Yü-lin [148] Fu, Shensi ; Lat. $38^{\circ} 08^{\prime}$, Long. $110^{\circ} 20^{\prime} ; \mathrm{Han}$, Yin-yin 直陰; P. Chou, Clung-lisiang 中 鄉; Sui, Chên-hsiang 貞鄉; Kin, Chia | C.

716 Chia-mêng | 萌, see Nos. $340,3429$.

\section{恰}

717 Chia-k ê-t un 恰克圖, Kiachta, or Mai-m1nai Chên 買 賣 鍢, a town on the frontiers of Russia and Mongolia; Lat. $50^{\circ} 16^{\prime}$, Long. $106^{\circ} 44^{\prime}$.

\section{甲}

718 Chia-fu 甲 府 Kôfu, chief town of Yamanashi Ken, Japan.

719 Chia-ma | 馬 Y. near Wuch'êng H., Shantung.

720 Chia-shan | I4 Fu in Hsien-ching 㭜 鏡 circ., Corea.

721 Chia-shui | 水 (obs.) Sui, $\mathrm{N}$. of $\mathrm{Cl}^{\prime}$ in $\mathrm{C}$., Shansi. 
甲

722 Clia-tzŭ $\mid$ 子, ssü near Hui-lai H., Kuangtung.

723 Chia-wa | 世, T.S. near Sung-p'an T., Ssŭch' nan.

\section{夾}

724 Clia-chiang 夾汇: Kiahkiang.

[72] $1^{\circ} \mathrm{H}$. in Chia-ting Fu, Ssŭch 'uan ; Lat. $29^{\circ}$ $38^{\prime}$, Long. $103^{\circ} 44^{\prime}$; Han, Nan-an 南岀; Sui, Chia-chiang | 江; $T^{\prime} a n g$, H. in Clia 嘉 C.; Sung and Yiumn, H. in Cliia-ting Frn.

$2^{\circ}$ (obs.) $80 l i \mathrm{~N}$. of Chiachiang H., Ssŭclh' uan ; Sui, H. in Mei-shan 眉山chün.

725 Chia-ku | 谷, see No. 3573. 726 Chia-p'u|浦, chent near $\mathrm{Ch}^{\prime}$ ang-lising H., Chehkiang.

\section{郊}

727 Chia 郟 Kiah, H. in Ju C., Honan ; Lat. $34^{\circ} 04^{\prime}$, Long. $113^{\circ} 10^{\prime} ;$ S.A., in K. Chêng 鄭; Han, Clia | H.; 2nd Wei, Lunngshan 龍 山; Sui, Ju-nan 汝南 and Clia-cl' êng | 城

728 Chia-ch'êng | 城, see No. 727.
嘜 Chiang-CH ÊNG.

唊

729 Chia-k'on 脥口, chên near Cli' ang-hina H., Chehkiang.

\section{CHIANG}

\section{港}

730 Chiang-k'ou 滛 口, ssŭ near $\mathrm{P}^{i}$ 11-chl'i $\mathrm{i}$., Hupeh.

\section{it}

731 Chiang 江 Kiang.

[309] $1^{\circ}$ T.C. in T'ai-p'ing

Fin, Kuangsi ; Lat. $22^{\circ}$ $21^{\prime}$, Long. $107^{\circ} 05^{\prime}$;

Sung Chiang / C.

$2^{\circ}$ See Nos. 735, 1281; $1583,1949,4562,4752$, 5049.

$3^{\circ}$ (obs.) S. Sung, C. in Chiang-hsi | 西 circ. $4^{\circ}$ (obs.) S. Sung, C. in Fu-chien 福 建 circ. $5^{\circ}$ (obs.) chou formed A. D. 280 from parts of Ching 制 chov and Yang 揚 CHOU.

732 Chiang-an | 努 Kiang[528] ngan, H. in1 Lü C., Ssŭch 'nan; Lat. $28^{\circ} 42^{\prime}$, Long. $105^{\circ} 38^{\prime}$; Han, Chiang-yang | 陽; Tsin, Han1-an 漢安; Siui, Chiang-an| 安.

733 Cliang-ch' êng | 乘, see No. 5548. 
江

Chiang-chieh.

734 Chiang-clieh | 界, Fin in P'ing-an 平资 circ, Corea.

735 Chiang-ching | 津 Kiangtsin.

[528] $1^{\circ}$ H. in Chring-cle'ing Fu, Ssŭch uan; Lat. $29^{\circ} 15^{\prime}$, Long. $106^{\circ}$ $20^{\prime}$; Han, Chiang | C.; $P$. Chou, Chiang-yang | 陽; Sung, Yiuan and Ming, H. in Chungch'ing Fu.

$2^{\circ}$ (obs.) S. W. Chiangching H., Ssŭch' 'uan; Sui, H. in Pa 巴chiin; T’ang, H. in Yü 满 C.

736 Chiang-ch naan | 川 Kiangchnen.

[190] $1^{\circ} \mathrm{H}$. in Ch'êng-chiang $\mathrm{Fu}$, Yünnan; Lat. $24^{\circ}$ $32^{\prime}$, Long. $102^{\circ} 58^{\prime}$; Han, Lu-yün 碌雲 and Yi-ch êng 舆城;

Yüan, Cliiang-ch uan | 川.

$2^{\circ}$ See No. 7460.

737 Chiang-hu | 滻, ssǐ near Fêng-ch' êng H., Kiangsi.

738 Chiang-hu|户 Yedo, old name of Tokio, Japan.

739 Chiang-hua | 華 Kianghwa.

$1^{\circ} \mathrm{H}$. in Yung-chon $\mathrm{F} n$, Hunan; Lat. $25^{\circ} 19^{\prime}$, Long. $111^{\circ} 32^{\prime}$; Han, Fêng-ch' êng 傌 漛; Tang, Chiang-hina 華.
汇 Ching-k'ou.

$2^{\circ}$ See No. 6275.

740 Chiang-hisi | 西 Kiang-si.

$1^{\circ}$ Province of China;

literary nanles Yü-chang

豫章: and Hung-tu

洪都; capital, Na11-

clı ang $\mathrm{Fu}$; it contains

4 circ., $13 \mathrm{Fu}, 3$ T,

1 C.L.C., 1 C., and 75 $\mathrm{H}$.

$2^{\circ}$ H. in P'ing-an 本安 circ., Corea; Lat. $39^{\circ}$ $15^{\prime}$, Long. $125^{\circ} 35^{\prime}$. $3^{\circ}$ (obs.) S. Sung, circ.

741 Chiang-11sia | 夏 Kianghia.

$1^{\circ} \mathrm{H}$. forming the prefectural city of Wu-cl' ang, Hupel1; Lat. $30^{\circ} 33^{\prime}$, Long. $114^{\circ} 27^{\prime}$; Han, Sha-yi 沙羡; Tsin, Ju1-11an 汝南; Sui, Chiang-hsia | 夏.

$2^{\circ}$ (obs.) Han, chiun in Hupel.

$3^{\circ}$ See Nos. 1967, 6306, 7052.

742 Chiang-k'on | 口.

$1^{\circ}$ Chên near P'eng-shni H., Ssŭcl' nan.

$2^{\circ}$ Chền near T'ung-chiang H., Ssŭch nan.

$3^{\circ}$ Ss ̌̆ near Sung-tzŭ H., Hnpel.

$4^{\circ}$ Ss $\breve{u}$ near San-shui H., K nangtung.

$5^{\circ}$ Chên in Lo-jung H., Kuangsi. 
江 Chiang-K'ov.

$6^{\circ} \mathrm{Y}$. near Hsiao-yi 'T., Shensi.

743 Chiang-lan | 㯺, Kianglan, T. in Yung-cliou Fu, Human.

744 Chiang-ling | 陵 Kiangling.

$1^{\circ} F_{11}$ in Chiang-yüan 江 原 circ., Corea; Lat. $38^{\circ}$, Long. $128^{\circ} 50^{\prime}$.

$2^{\circ} \mathrm{H}$. forming the prefectural city of Ching-chou, Hupeh ; Lat. $30^{\circ} 27^{\prime}$, Long. $112^{\circ} 05^{\prime} ; T_{s}^{\prime}$ in, Ying 趴; Han, Chiangling | 陵.

$3^{\circ}$ (Obs.) S. Sung, Fu in Hu-pei 湖北 circ.

$4^{\circ}$ See No. 1142.

745 Chiang-mên | m. .

$1^{\circ}$ Post-town near $\mathrm{Na}$-ch $\mathrm{i}$ H., Ssŭcli'uan.

$2^{\circ}$ Post-town near Hsiangshan $\mathrm{H}$., Kuangtung.

746 Chiang-nan | 南 Kiangnan, a province comprising Anluti and Kiangsu; Sung, Chiang-nan | 南 circ.

747 Chiang-nan-hsi 南 函, (obs.) T"ang, circ.

748 Chiang-nan-tung | 南棸, (obs.) T"ang, circ.

749 Chiang-ning | 俩 Kiangning.

$1^{\circ}$ Circ. in Kiangsu, consisting of Chiang-ning Fu.
江 Chiang-no.

$2^{\circ} \mathrm{F} n$ in the Chiang-ning [528] circ., Kiangsu ; forming also the two district cities of Chiang-ning and Shang-yüan ; formerly an Imperial residence and thercfore called Nan-ching 南京 Nanking, the Southern capital; capital of the province; Lat. $32^{\circ} 05^{\prime}$, Long. $118^{\circ} 47^{\prime}$; orig., Chin-ling 金陵, 1 st in $\mathrm{K}$. Wa 紧, 2nd in $\mathrm{K}$. Yüch 越, 3rdin K. Ch' 1 楚; Ts'in, Mo-ling 秝陵 in Ch'ang 長 chün; Han, Tanl-yang 丹陸; Chêen, Chiang 蔣 C.; T'ang, Chiangning | 南 and Shêng 景 C., Ming, Yingt'ien 應 天 Fu.

$3^{\circ} \mathrm{H}$. forming with Shang[528] yüan $H$. the prefectural city of Chiang-ning, Kiangsu ; Lat. $32^{\circ}$ $05^{\prime}$, Long. $118^{\circ} 47^{\circ}$; $T s^{\prime}$ in, Mo-ling 秣 陵; Tsin, Chien-yeh 建業; $T^{6}$ ang, Chin-ling 金陵 and Pai-hsia 白下; Five dyn., Chiang-ning | 察.

$4^{\circ}$ Ssŭ near Li-shui H., Kiangsı.

750 Chiang-no | 那 post-town 
江

CHANG-No.

near K'ai-hua Fu, Yüinnan.

751 C̣liang-pei 江 北 Kiangpel, T. in Clinng-cli'ing Fin, Ssŭch'uan ; Lat. $29^{\circ}$ 50', Long. $107^{\circ} 05^{\prime}$.

752 Chiang-p'o | 防, (obs.) 40 li N. W. of Vingshang H., Anhuni Kin, chên in Ying 穎 C.

753 Chiang-p' 11 | 涌 Kiang-pu, [528] H. in Cliang-ning Fu, Kiangsu ; Lat. $32^{\circ} 05^{\prime}$, Long. 118. 39'; orig., 'T'ang-yi 棠 邑 in K. $\mathrm{Cl}^{6} \mathrm{u}$ 楚.

754 Chiang-shan| |⿱㇒日勺 Kiang[112] shan, H. in Ch'̈̈̈-chou Fit, Chelıkiang; Lat. $28^{\circ}$ $47^{\prime}$, Long. $118^{\circ} 51^{\prime}$; $T^{6}$ ang, Hui-chiang 䫉 !; Five dy'n., Chiang-shan | U.

755 Chiang-su | 䰻 Kiang-sı, province of China; literary names $\mathrm{Wu}$ 谷; Chin-ling 金陵 and Chien-k'ang 建 廊; capital, Chiangning $F u ;$ it contains 5 circ., 8 Fu, 1 C.I.'T., 3 T., 3 C.L.C., 3 C. and $62 \mathrm{H}$.

756 Chiang-tsui | 㧘, posttown near Lang-ch'inng H. $\rightarrow$ Yünnan.

757 Chiaing-tu | 都 Kiang-tu. $1^{\circ} \mathrm{H}$. forming witl Kanch'üan $H$. the pre-
iL CHIANG-Yin.

fectural city of Yangclou, Kiangsu; Iat. $32^{\circ} 21^{\prime}$, Long. $119^{\circ}$ 15'; Ts'in, Kuang-ling 復陵; Han, Clinangtı | 奇i'; T'ang, Chiangyang |核.

$2^{\circ}$ (Obs.) 46 li S.W. Chiang-tu H., Kiangsu; W. Han, H. in K. Kuang-ling 廣 陵; $E$. Han, Tsin, S. Sung and $S . T s i, H$. in Kuang-ling 廣陵chün; Sui, H. in Chiang-tu | 都chün.

$3^{\circ}$ See No. 7468.

758 Chiang-tung | 東 Kiangtunig.

$1^{\circ}$ Fin in P'ing-an 平努 circ., Corea ; Lat. $39^{\circ}$ $25^{\prime}$, I.ong. $126^{\circ} 35^{\prime}$.

$2^{\circ}$ Sš̆ near Chang-chou Fu, Fulkien.

$3^{\circ}$ (Obs.) S. Sung, circ. $758^{\wedge}$ Chiang-ts'un | 村 $S s \breve{u}$ in Shun-tê Fu, Kuangtung.

759 Chiang-yang | 灌, see Nos. $732,757,785,1858,4133$, 4549.

760 Chiang-yin | 院 Kiang-yin. $1^{\circ} \mathrm{H}$. in Chang-chou $\mathrm{Fu}$, Kiangsu ; Lat. $31^{\circ} 54^{\prime}$, Long. $120^{\circ} 18^{\prime} ; \mathrm{Han}$, Pi-ling 牦陵; Tsin, Chi-yang 䜿览; Liang Chiang-yin | 陸. Port of Call. 
$2^{\circ}$ (Obs.) S. Sung, military district in Chê-hsi 浙西 circ.

$3^{\circ}$ (Obs.) P. Chont, H., S. of Wu1-shan H., Ssŭch'nan.

761 Chiang-yn | 湔 Kiang-yu, [125] H. in I,ung-an Fun, Ssŭcl' 'van; Lat. $31^{\circ} 46^{\prime}$, Long. $104^{\circ} 52^{\prime} ; \quad T s^{\prime}$ in, land of the Ti-cll'iang EE 花; $W$. Wei, L 111ng 龍 C.; P. Chou, Chiang-yn | 油.

762 Chiang-yüan | 原.

$1^{\circ}$ (Obs.) 10 li E. of Clı' 'ung-ch' 'ing C., Ssŭclinan; Han and Tsin, H. in Shu 蜀 chïn; S. Sung, H. in Chin-yüan 㕷愿 chïn；S. Ts'i, H. in Chin-k'ang 亚 亦象 chiin.

$2^{\circ}$ (Obs.) 30 li S. E. of Ch'nung-ch'ing C., Ssŭchnan; Sung, H. in $\mathrm{Ch}^{6}$ ing-cling 装度 Fir.

$3^{\circ}$ See No. 1589, 6118.

763 Chiang-yüan | 源.

$1^{\circ}$ (Obs.) W. of Sung-p'an T., Ssŭch 'nan; Sui, H. in Min-shan 汶 II chiin.

$2^{\circ}$ (Obs.) in Mon C., Ssŭch'uan; Tang, H. in Chï 崌 C.
美

ChIANG.

\section{㚇}

764 Chiang-niang 羔艮, chên near San-shui H., Shensi.

765 Chiang-yen | 堰, chên near T'ai C., Kiangsu.

766 Chiang-yi | 邑, (obs.) Chon, town near Fênghsiang Fu, Shensi.

\section{紅}

767 Chiang 紅虹, see No. 2380. 768 Chiang 些 Hung.

$1^{\circ} \mathrm{H}$. in Fêng-yang $\mathrm{Fu}$, Anhui; Lat. $33^{\circ} 26^{\prime}$, Long. $117^{\circ} 55^{\prime}$; orig., Hsia-po-yi 夏伯邑; $H a n, H s i a-c h$ in 夏邱; $P$. Chou, Chinsling 晢 陵; T'ang, Chiang | $\mathrm{H}$.

$2^{\circ}$ See No. 3966.

\section{綘}

769 Chiang 綘 Kiang.

$[123] 1^{\circ}$ C.L.C. in the Ho-tung circ., Shansi ; Lat. $35^{\circ}$ $37^{\prime}$, Long. $111^{\circ} 29^{\prime}$; Han, Liir-fên 臨汾; 2nd $W_{c i}$, Tung-yung 東 䔨; $P$. Chou, Chiang $\mid \mathrm{C}$.

$2^{\circ}$ H. in Chiang C., [415] Sliansi; Lat. $35^{\circ} 29^{\prime}$, I.ong. $111^{\circ} 40^{\prime} ;$ S. $A$., Hsin-t'ien 新田; Han, Chiang $\mathrm{H}$; 2nd Wei, Nan-chiang 南 | 
姜 Chiang.YI.

770 Chiang-yi | 邑, see No. 7514.

将

771 Chiang-chün 将軍, Y. near P'ing-ku H., Chihli.

772 Chiang-lê $\mid$ 樂 'Tsiang-lol,

[55] H. in Yen-p ing $F$ n, Fulukien; I at. $26^{\circ} 43^{\prime}$, Long. $117^{\circ} 20^{\prime} ; \quad W u$, Chiang-lê | 樂; Five dy'n., Yung 鏞 C.

773 Chiang-ling | 陵, see Nos. $4385,8164$.

774 Chiang-t'ai | 台, posttown near Wu-ch' ang $\mathrm{H}$., Hupel.

\section{蔣}

775 Chiang 蔣.

$1^{\circ}$ (Obs.) S.A., K. in

Honan.

$2^{\circ}$ See Nos. 749, 3430.

776 Chiaung-chuang | 生, chên near Chin-shan H., Kiangsu.

777 Chiang-tsê | 潞 Jansujung or Gyangtse, town in Thibet; Lat. $28^{\circ} 50^{\prime}$, Long. $89^{\circ} 45^{\prime}$.

\section{漿}

778 Chiang-shui-cli'ang 漿水 算, see No. 6501 .

\section{- 講}

779 Chiang-chu 朱, (obs.) 100 li S. W. Ho C.,
德 China-chiang.

Kansulı; Sung, fort in Ho 河 C.

\section{CH'IANG}

\section{羌}

$780 \mathrm{Ch}^{\prime}$ iang-pai 侇白, chèn near T'ung-cliou $\mathrm{Fu}$, Shensi.

781 Chi'ang-yüan | 原 chên in Futping H., Shensi.

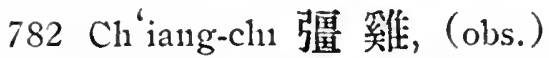
near Ya-chou $\mathrm{Fu}_{11}$, Ssŭch'uan; T'ang, C. in Chien1-11an 劍南 circ.

\section{CHIAO}

\section{交}

783 Chiao 交.

$1^{\circ}$ (Obs.) W. Han and $T$ sin, chov.

$2^{\circ}$ (Obs.) T’ang, C., W. of $\mathrm{Ch}^{\prime}$ in-an H., Kansull. $3^{\circ}$ See No. 7199.

784 Chiao-clı'êng | 城 Kiauching, H. in 'T" ai-yüan Fu, Slansis ; Lat. $37^{\circ} 36^{\prime}$, Long. $112^{\circ} 06^{\prime}$; Han, Chin-yang 玨陽; Sui, Chiao-cl' êng; T'ang, Ling-clin uan 靈川.

785 Chiao-chiang | it, (obs.) in Annam; Sui, H. in Lin-yi 林邑 chiin. 
786 Cliao-chih | 趾 [or, 阯], (obs.) Han, Chün, now Cochin-Clina, Cangigu (Polo).

787 Chiao-11o | 河 Kiat1-ho.

[10] $1^{\circ} \mathrm{H}$. in Ho-clien $\mathrm{Fu}$, Chillili Lat. $38^{\circ} 06^{\prime}$, Long. $116^{\circ} 20^{\prime}$.

$2^{\circ} \mathrm{F} 1$ in Ching-ch'i 京䰹 circ., Corea ; Lat. $37^{\circ}$ $57^{\prime}$, Long. $125^{\circ} 35^{\prime}$.

788 Chiao-lisieh | 斜, chên near T'ung-chou Fu, Shensi.

789 Chiao-k'ou | A.

$1^{\circ}$ Chên 11ear Yü-shê H., Shansi.

$2^{\circ}$ Chên near Kao-ling H., Shensi.

$3^{\circ}$ Chèn near Yen-ch' uan H., Shensi.

790 Chiao-li | 黎, (obs.) near $\mathrm{Ch}^{\prime}$ 'ang-li H., Chihli; $W$. Han, H. in Liao-hsi 邀 䍀 chün.

791 Chiao-tao | 道, chenn near Fu C., Shensi.

\section{榄}

792 Chiao 巢, formerly K. in Anhui.

\section{教}

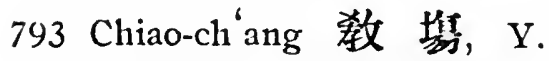
near Chiang-hua H., Hunan.
蹘

794 Chiao 袋 Kiau, C. L. C. in Teng-Lai-Gh'ing-Chiao circ., Shantung; Lat. $36^{\circ} 14^{\prime}$, Long. $110^{\circ} 24^{\prime}$, $\mathrm{Han}, \mathrm{Cl}_{1}$ 'ien-tsou 騟 陬; 2nd Wei, Chiao C.; Sui, Chiao-11si | 阿.

795 Chiao-hisi 膠西.

$1^{\circ}$ (Obs.) S. W. Kao-mi H., Sha11tung; Sui, H. in Kao-mi 高密 chün. $2^{\circ}$ See No. 794.

796 Chiao-sliui | 水, see No. 5196.

797 Chiao-tung | 枯.

$1^{\circ}$ (Obs.) S. E. P'ing-tu C., Shantu11g; W. Han, Clino-tung | 東 $\mathrm{K}$.

$2^{\circ}$ (Obs.) in P'ing-tu C., Shantung; E. Han, Pei-hai 北海 Marq. in

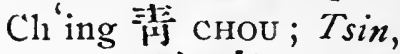
Clii-11an 濟南 chün in $\mathrm{Cl}^{\prime}$ 'ing 青 cHou; $S$. Sung, II. in Pei-hai 北 海 chïn.

$3^{\circ}$ (Obs.) in Hai C., Kiangsı, $S . T s^{\prime} i, H$. in Pei-hai 北海 chïn.

$4^{\circ}$ (Obs.) 50 li E. of Wei H., Shantung; Han, H. in Pei-hai 北海 chün.

$5^{\circ}$ See Nos. 5196, 7003.

\section{焦}

798 Chiao 焦, (obs.) formerly 
焦 CHIAO.

a feudal state in Honan, comprising Hsia-yi $\mathrm{H}$.

\section{蕉}

799 Chiao-ts' un 蕉村, chen near Ning C., Kansul.

\section{雀}

800 Chiao-ch'i 雀溪, T-S. near T'ung-jên Fu, Kueichous.

\section{椒}

801 Chiao-ch'iu-ch'êng 㑐邱 城, (obs.) formerly town $\mathrm{N}$. of Nan-ch'ang Fu, Kiangsi.

802 Chiao-p'o | 陂, (obs.) 60 li S. of Fu-yang H., Anhuii ; Kin, chên in Ying 顆 C.

\section{CH'IAO}

\section{喬}

$803 \mathrm{Ch}$ 'iao-cluu 喬 珠, (obs.) W. of 'Tielh-ch' $\mathrm{i}$ Y., Ssŭch'uan; T'ang, H. in Chê 柘 C.

$804 \mathrm{Cl}^{\prime}$ iao-11siao | 孝, chen, near Hung-tung H., Shansi.

$805 \mathrm{Ch}^{\prime}$ iao-t'ung | 桐 H. in Ching-ch'i ${ }^{\prime}$ 京畿 circ., Corea.

\section{橋}

$806 \mathrm{Ch}^{\prime}$ iao 橋, (obs.) T'ang $\mathrm{C}$, in $\mathrm{Cl}^{\prime}$ 'ing-yang $\mathrm{Fu}$,
橋 Сн'

Kansuli.

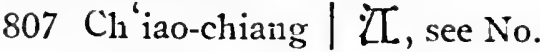
7763.

$808 \mathrm{Cl}^{\text {'iao-ssŭ }}$ 司, chên near Hai-ning C., Chehkiang.

$809 \mathrm{Cl}^{\prime}$ iau-t ou | 頭, post-town near Hsiang-yin H., Hunan.

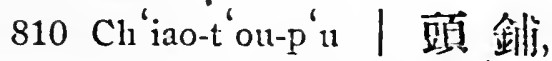
post-town near Hai-ch' êng H., Slingking.

\section{IIj}

811 Ch'iao-clia-ying 巧家營 Kiau-kia-ying, $T$. in Tungch'vail $\mathrm{Fu}$, Yünnan; Lat. $26^{\circ} 51^{\prime}$, Long. $103^{\circ} 05^{\prime}$.

焦

$812 \mathrm{Ch}^{\prime}$ iao 焦 formerly K. in Anluiv.

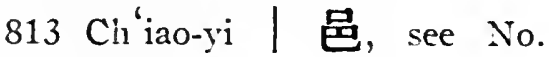
5229.

\section{嶣}

814 Ch'iao-1nu 嶣木, chên near Lung-tê H., Kansuh.

\section{樵}

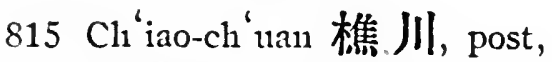
town near Shao-wu FuFullkien.

\section{陗}

$816 \mathrm{Ch}^{\text {' }}$ iao-shih 陗石, chên near Chien-ch'ang $\mathrm{Fu}$, Kiangsi.

Chiba, see No. 945. 
介

ChIFH.

\section{CHIEH}

\section{介}

817 Chielı 介, see No. 1688.

818 Chieh-lısin | 休 Kiai-linu, H. in Fên-chon Fu, Shensi; Lat. $37^{\circ} 05^{\prime}$; Long. $111^{\circ}$ $51^{\prime} ; T^{\prime}$ in, Chieln-hsiu

休; 2nd Wei, P'ing-ch' ang 平昌.

819 Chieh-tung $\mid$ 本, (obs.) in Ch'i-tung H., Shantung; Kin, chên in Tzŭ 畄 C.

\section{价}

820 Chieh 价 Yïan C. in P'ingjang Fu, Corea.

821 Chieh-ch' uan 价 川 Kiaichnen, chïn in P'ing-an 不安 circ., Corea; Lat. $39^{\circ} 50^{\prime}$, Long. $126^{\circ} 50^{\prime}$.

\section{界}

822 Chieh-ho 界河, post-town near T'êng H., Sliantung.

823 Chieh-ho-k ou | 河 口, chên near Ian H., Shansi.

824 Chieh-hisiu|休 Han, H. in Shansi.

825 Chiels-p'ai一牌 ssŭ in Iaipin H., Kuangsi.

826 Chieh-shou| 首, chên in Kao-yu C., Kiangsi.

827 Chieh-t 'ing | 亨, post-town near $\mathrm{Ch}^{6}$ ên-chou Fin, Hunan.
界

CHIEH.

\section{堺}

828 Chich 堺 Sakai, ken in Japan. Chief town Sendai.

\section{解}

829 Chich 解: Kiai, C. L. C. in [533] the Hotung circ., Shansi ; Lat. $34^{\circ} 59^{\prime}$, Long. $110^{\circ} 50^{\prime}$; orig., domains of the En11peror Shun 舜; S. A., Chieh-liang | 梁; Sui, Yü-lisiang 盧 拫; Five dy'n., Chieh | C. Caichu1 (Polo).

830 Chieln-liang | 梁, see Nos. $829,3848$.

\section{湝}

831 Chiel1-k'ou 湝 口, chên near Ch'i-mên H., Anhui.

\section{階}

832 Chieh 階 Kiai.

$1^{\circ}$ C. I. C. in the KungCh'in-Chieh circ., Kan1sull ; Lat. $33^{\circ} 19^{\prime}$, Long. $105^{\circ} 04^{\prime}$; Han, Wu-tu 武都; $W$. Wei, Wu-chieh武 |; T'ang, Wir 武 C. and Chieh C.; Five dy'n., Chielı C.; Sung, Chieh | C. and Fu-ching 酔津 H.; Yüan, Chieh | H. in Shensi; Ming, C. in Kung-cli'ang 粱 昌 Fu. 
界

С HIEн.

$2^{\circ}$ (Obs.) N. W. of Chieh C., Kansul1; $T^{\prime}$ ang, C. in Lung-yu䈍右 circ.

\section{街}

833 Chieh-k'ou 街 口, ssŭ in Hsi H., Anhui.

\section{蝢}

834 Chieh-ch'iu 蝢 邱, see No. 3557.

\section{結}

835 Chiel-an 結婪 Kiai-1ngan, T-C. in T'ai-p'ing Fu, Knangsi ; Lat. $23^{\circ} 18^{\prime}$, Long. $106^{\circ} 55^{\prime}$.

836 Chieh-ch'êng | 城 H. in Chung-ch'ing 忠清 circ., Corea.

837 Chieh-lun | 倫, see No. 593.

\section{揭}

838 Chieh-yang 揭 陽 Kiehyang.

[315] $1^{\circ} \mathrm{H}$. in Ch'ao-chou $\mathrm{Fu}$, Kuangtung ; Lat. $23^{\circ}$ $32^{\prime}$, Long. $116^{\circ} 20^{\prime}$. $2^{\circ}$ See Nos. 369, 708, 1937, 2975, 4708.

\section{鿼}

839 Chieh-slih 礍 石 Kiehshih.

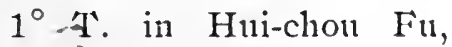
Kuangtung; Lat. $22^{\circ}$ $50^{\prime}$, Long. $116^{\circ}$.

$2^{\circ}$ See No. 3193.
且. CHIEN-AN.

CH'IEH

怯

840 Ch'ieh-shih 怯失 Kish, an island in the Persian Gulf.

841 Ch'ieh-shih-mi-êrh|失迷 胃, see No. 658.

且.

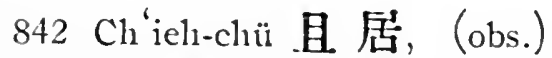
$60 l i$ E. of Hsüan-hua Fu, Chihli ; $W$. Han, H. in Shang-ku上谷 chün.

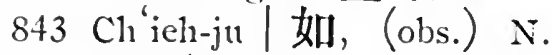
of 'T'ien-chên H., Shansis; W. Han, H. in Tai 代 C. 844 Clı'iell-lï | 慮, (obs.) E. of Lell-lung H., Chihli; $W$. Han, H. in Liao-hsi 遼 西 chiin.

\section{CHIEN}

\section{建}

845 Chien 建, see Nos. 859, 1871, 4937.

846 Chien-an | 安 Kien-ngan.

$1^{\circ} \mathrm{H}$. forming with Ouning $H$. the prefectural city of Chien-ning, Fullkien ; Lat. $27^{\circ} 04^{\prime}$, Long. $118^{\circ} 25^{\prime}$; Han, Hou-kuan 侯官; Wu, Chien-an | 努.

$2^{\circ}$ Ss̆̆ near Hsing-an H., Kuangsi.

$3^{\circ}$ See No. 859. 
且

CHITE-CH ANG.

847 Chien-ch'ang | 昌 Kienchang.

[52] $1^{\circ} \mathrm{Fu}$ in the Nan-FuChien circ., Kiangsi; forming also the district city of Nan-ch' êng; Lat. $27^{\circ} 34^{\prime}$, Long. $118^{\circ} 28^{\prime} ;$ S. A., 1 st in $\mathrm{K}$. Wu 吳, $2 n d$ in $\mathrm{K}$. $\mathrm{Cl}^{\prime}{ }^{\prime}$ 楚; $T s^{\prime}$ in, in Chiuchiang 九 II chïn; Han. Kan-chiang 旰 江 in Yü-chang 预 意: chün; $W_{b l}, \mathrm{~L}$,in-ch' uan 臨 川; S. T'ang, Chien-wu 建武; Sung, Chien-ch'ang | 昌.

[193] $2^{\circ} \mathrm{H}$. in Nan-k'ang Fu, Kiangsi ; Lat. $29^{\circ} 05^{\prime}$, Long. $115^{\circ} 43^{\prime} ; W$. Han, Hai-hun 海昏; E. Han, Chien-ch'ang $\mid$ 昌.

$3^{\circ} \mathrm{H}$. in $\mathrm{Cl}_{1}$ ao-yang $\mathrm{Fu}$, Clihli; Lat. $41^{\circ} 15^{\prime}$, Long. $118^{\circ} 50^{\prime}$. L Local name: 'Ta-t $z \breve{r} \mathrm{k}$ 'ou 塔 子 口.

$4^{\circ} \mathrm{Y}$. in $\mathrm{Ch}^{\prime}$ ien-an H., Chihli. Kin, 11 chên.

$5^{\circ}$ See Nos. 1158, 6069, 7615.

848 Chien-ch' ang-shang-nan 昌上南, circ. in Ssŭch' 'uan, comprising Ya-chou Fu, Ning-yüan Fu, Cliating Fu, Mei C. and Chil1ng C. See No. 171.
建 CHIEN-LING.

849 Chien-ch' êng | 城, see Nos. 1236, 2727, 3124, 5512.

850 Chien-ch'i | 溪, post-town near Chien-yang H., Fulnkien.

S51 Chien-ch' 11 | 初.

$1^{\circ}$ (Obs.) in Annann; Tsin, S. Sung and $S$. $T s^{\prime} i$, H. in Chiu-chên 九 董 chün.

$2^{\circ}$ (Obs.) in Kuangsi ; $S$. Sung, H. in Y'ii-lin 朢 林 chün; S. Ts'i, H. in

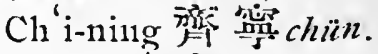

852 Chien-chung | 忠.

$1^{\circ}$ Post-town near $\mathrm{Li}$ ch'üan H., Shensi.

$2^{\circ}$ Post-town near Sanyüan $\mathrm{H}$., Shensi.

853 Chien-fu | 裀, (obs.) Sing, H., 50 li E. of Wêng-yüan H., Kuangtimng.

854 Chien-hising | 興, (obs.) $W$. Wei, H. near Mienyang C., Hupeh.

855 Chien-hsinng | 雄.

$1^{\circ}$ (Obs.) 35 li E. of Fukou H., Honan ; Kin, chên in K'ai-fêng 開 封 $\mathrm{Fu}$. $2^{\circ}$ See No. 5200.

856 Chien-k'ang | 康.

$1^{\circ}$ (Obs.) S. Sung, Fu in Chiang-tung 江. 年 circ. $2^{\circ}$ See No. 755 .

857 Chien-ling | 陵, see Nos. $2870,3446$. 
建

CHIEN-NaN.

858 Chien-nan | 南, chên near Li-ch' uan H., Hupeh.

859 Chien-ning | 俩 Kienning.

[306] $1^{\circ}$ Fin in the Yen-ChicnShao circ., Fuhkien; forming also the two (iistrict cities of Chicnan and Ou-ning; Lat. $27^{\circ} 04^{\prime}$, Long. $118^{\circ}$ 25'; Chou, Cli'i-min 閩; Wu Chien-an | 些; T'ang, Chien |C.; Sung, Chien-ning | 寡 Oueiln Fu (Polo).

[324] $2^{\circ} \mathrm{H}$. in Shao-wu Fu, Fuhkien; Lat. $26^{\circ} 49^{\prime}$, Long. $116^{\circ} 59^{\prime}$; Sung, Sui-ch'êng 綏 城; S. Tang, Chien-ning | 流.

$3^{\circ}$ Chền near Ling-ch' 'uan II., Shansi.

$4^{\circ}$ (Obs.) H. Wu, W. of Hsiang-t'an H., Hunan. $5^{\circ}$ (Obs.) Sung, H. near Ma-ch' êng H., Hupeh. $6^{\circ}$ (Obs.) T'ang, H., S. E. of Po-pai H., Kuangsi.

$7^{\circ}$ See No. 7813.

860 Chien-ping | 4 S Kicnping.

[417] $1^{\circ} \mathrm{H}$. in Kuang-tê C., Anliui ; Lat. $31^{\circ} 12^{\prime}$, Long. $119^{\circ} 05^{\prime}$.

$2^{\circ}$ Post-town near Kuei C., Hupeh.
建 CIIEXT-TU.

$3^{\circ} \mathrm{H}$. in Ch'ao-yas.g Fu, Chihli ; $240 \mathrm{Li}$ N. F. of Chien-ch' ang $\mathrm{H}$.

$4^{\circ}$ See No. 7150.

861 Chien-shih | 始 Kien-chi ; [181] $H$. in Shih-nan Fu, Hupeh ; Lat. $30^{\circ} 42^{\prime}$, Long. $109^{\circ} 45^{\prime}$; Han, Wu 巫. H.; $T^{\prime} i$, Chienshih|始; P. Chou, Yeh 莱 C.

862 Chien-shui | 水 Kienshwui.

$1^{\circ} \mathrm{H}$. forming the prefectural city of Lin-an, Yünnan; I at. 23' $37^{\prime}$, Long. $103^{\circ} 05^{\prime}$; Yüan, Chien-shui | 水 $\mathrm{C}$.

$2^{\circ}$ (Obs.) $T^{\prime}$ ang, H., W. of Ting-an H., Kuangtung.

863 Chien-tê | 緦 Kicn1-tel1.

$1^{\circ} \mathrm{H}$. in Clin'ih-chou Fu, Anliui ; Lat. $30^{\circ} 08^{\prime}$, Long. $117^{\circ} 12^{\prime}$; Han, Shih-chêng 石城; Five dyn., Cliien-tê $\mid$ 德.

$2^{\circ} \mathrm{H}$. forming the prefectural city of Yenchou, Chehkiang; Lat. $29^{\circ} 27^{\prime}$, Long. $119^{\circ}$ $35^{\prime} ;$ Han, Fin-ch' 'un 富春; $W u$, Chien-tê |德.

$3^{\circ}$ See No. 0759 .

864 Clıien-tu | 都!, (obs.) in Yünnan; S. Sung, chün 
in Ning 密 chov.

865 Chien-wei | 威, (obs.) S. E. Chielı C., Kansuh; Sui, H. in Wu-tı 武都 chïn. 866 Chien1-wu | 武, Y. near Ch'ang-11ing H., Ssŭcl' '11an; see also No. 847.

867 Chien-yang | 渮 Kienyang.

$1^{\circ} \mathrm{H}$. in Chien-11ing Fu, Futhkien ; Lat. $27^{\circ} 23^{\prime}$, Long. $118^{\circ} 13^{\prime}$; orig., Chien-anl 安; Ts in, Chien-yang | 晹; Stung, Chia-ho 棠禾. $2^{\circ}$ (Obs.) formerly town E. of $\mathrm{Ch}^{6} \mathrm{u} \mathrm{C}$., Anhui. $3^{\circ}$ (Obs.) $W u$, town E. of Shilı-shou H., Hupeh. $5^{\circ}$ Chên in Ching-mêên C., Hupel.

$6^{\circ}$ See No. 355\%.

868 Chien-yeh | 業, see No. 749.

869 Chien-yiuan | 元, (obs.) near Ning-kuo Fu, Anhui; $S . T s^{\prime} i, H$. in Hsüanch'êng 宣城 chün.

\section{㯬}

870 Chien犍, (obs.) near Ssŭnan Fu, Kueichon; T $T^{6}$ ang, C. in Chiang-nan 江. 南 circ.; Sung, C. in Shaoch'ing 紹磨 Fu.

871 Chien1-wei | 䨌 Kien-wei.
[307] $1^{\circ} \mathrm{H}$. in Chia-ting Fu, Ssŭch wa11; Lat. $29^{\circ}$ $09^{\prime}$, Long. $104^{\circ} 08^{\prime}$; $H a n$, Nan-an 南努; $P$. Chou, Wu-yang 武陽; Sui, Clinen-wei | 篇.

$2^{\circ}$ (Obs.) S.W. Yi-pin H., Ssŭch' 11an; Han, chïn in $\mathrm{Yi}$ 䀂 $\mathrm{cHov;}$ S. Ts' $i$, chiun in Ning 寉 chov; Sui, chïn in Liang 梁 chOU.

$3^{\circ}$ (Obs.) 15 li N. E. P'êng-shan H., Ssŭch'uan; E. Han, Tsin and $S$. Sung, chïn in $\mathrm{Yi}$ 会 chou.

$4^{\circ}$ (Obs.) near Hsï-chon Fı, Ssŭcl'uan; $E$. Han, tributary $\mathrm{K}$. in $\mathrm{Yi}$ 谷 chov.

$5^{\circ}$ (Obs.) in Yünnıan; $S$. $T^{\prime} i$, H. in Sung-ch' ang 来昌chïn。

$6^{\circ}$ (Obs.) S. E. of Chienwei H., Ssŭch' 'uan ; Sui, H. Chien-wei | 爲 chïn; $T^{i}$ ang, H. in Chia 嘉 C.

$7^{\circ}$ See Nos. 263, 705, 2895.

\section{澗}

872 Chien1-ch“i 澗溪, chên near Lai-an H., Anluni.

873 Chien-hisia-chiang | 洽江., sš̌ near Shunl-11ing $F$ u, Yün11an. 
簡

Chines.

\section{簡}

874 Cliien 簡 Kien.

[118] $1^{\circ} \mathrm{C}$. in Cl' êng-tu Fin, Ssŭch' 'uan; Lat. $30^{\circ}$ 25', Long. $104^{\circ} 38^{\prime}$; $W$. Wei, Yang-an1 隄 安; Yüan, Chien|C. $2^{\circ}$ (Obs.) $C h^{\prime} e ̂ n$ and $T^{\prime}$ ang, C. near Nan-1ning Fu, Kuangsi.

875 Chien-ch'ih-chü | 池垬, ssü near 'Ting-yüạn ' $\mathrm{T}$., Shensi.

876 Chien-ts'un | 村, chên near Wu-chiang H., Kiangsu.

877 Chien-yang | 榢, see No. 2018.

\section{兼}

878 Chien-chon 啸州, or 徒建 州, or Chien-chien-chou 儉儉州, Kemkemdjoute or Kemkentchik, a town in Siberia on the upper Yenisei.

\section{儉}

879 Chien-chien-chou 儉儉州, see No. 878.

\section{䜪}

880 Chien 劍 Kien.

[30] $1^{\circ}$ C. in Pao-ning Fu, Sșŭch' 'uan; Lat. $32^{\circ}$, Long. $105^{\circ} 38^{\prime}$; Han, 'Tzŭ-t' $1111 \mathrm{~g}$ 梓滰; Liang, An 妍 C.; S. Ts $i$,
簡 CHIEN-P'U.

Nan-an 南努, chün and H., in Yi 征 C.; T ang, Chien | C.

$2^{\circ}$ (Obs.) S. Sung, C. in Fu-chien 的畐建 circ.

$4^{\circ}$ See No. 7368.

881 Chien-ch'ang $\mid$ 長, (obs.) Ming, H. near Shih-nan Fu, Hupeh.

882 Chien-ch'iang | 卼, see No. 7964.

883 Chien-ch' uan | M Kienchiten.

[42] $1^{\circ}$ C. in I i-chiang Fin, Yünnan; I at. $26^{\circ} 35^{\prime}$, Long. $100^{\circ} 06^{\prime}$.

$2^{\circ}$ See No. 4181.

884 Chien-fu | 甫, see No. 4615.

885 Chien-kuan | 關, post-town near Chao-hua H., Ssŭch' uan.

886 Chien-mên|肳, (obs.) 60 li N.E. Chien C., Ssŭch'uan; Tang, H. in Chien | C.; Sung, H. in Lrung-ch'ing 隆磨 Fu.

887 Chien-11an | 南, (obs.) $T^{6}$ ang, circ. in ancient Liang 梁 CrIOU; see also No. 572 .

888 Chien-nieh | 聶, (obs.) $T \sin , \mathrm{H}$. near Chien C., Ssŭch' ıan.

889 Chien-p'u| 浦; Sung, H. in Fuhkien. 


\section{堅}

890 Chien 堅, Yüana, C. in Shansi.

891 Clien-k'un 堅昆, (obs.) T"ang, Fu in the country of the Khalkas, Kuan-nei 關肉 circ.

\section{監}

892 Chien-hai 監 海, chên near Chia C., Shensi.

893 Chien-li | 利 Kien-li， H. [528] in Ching-chou $\mathrm{Fu}, \mathrm{Hu}-$ peh; Lat. $29^{\circ} 49^{\prime}$, Long. $112^{\circ} 43^{\prime}$.

894 Chien-tn | 茶, post-town near Ku-yüan C., Kansul.

\section{洊}

895 Chicn-shui 洊水, (obs.) Sui, H., E. of Huai-chi H., Knuangsi.

\section{湔}

896 Chient-ti 湔 氏, (obs.) N. W. of Sung-p'an 'T., Ssŭcli ${ }^{\prime}$ un ; $I V$. Han and $E$. Han, circ. in Shu 蜀 chiiln.

\section{箭}

897 Chien-chu 箭 你, posttown near Yung-ting H., Fulkien.

898 Chien-kan | 桿, T-S. near Yün-lung $C$., Yünnan.

\section{CHIEN}

\section{汧}

$899 \mathrm{Ch}^{\text {'ien }}$ 湃, see No. 4230.

$900 \mathrm{Ch}^{\prime}$ ien-yang | 陽 Kien[48] yang, H. in Fêng-lisiang Fu, Sliensi ; Lat. $34^{\circ} 35^{\prime}$, Long. $107^{\circ} 11^{\prime}$; Han, Yümi 喻 㧘; P. Chou, $\mathrm{Cl}^{\prime}$ ien1-yang | 陽.

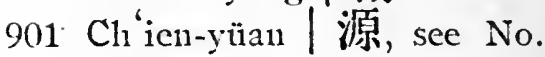
4230.

\section{默今}

$902 \mathrm{Cl}_{1}$ ' ien 點今.

$1^{\circ}$ (Obs.) T ang, C. con1prising parts of Kueichon and Ssŭclı' uan.

$2^{\circ}$ (Obs.) S. Sung, C. in Huai-tung 淮東 circ.

$3^{\circ}$ See No. 3476.

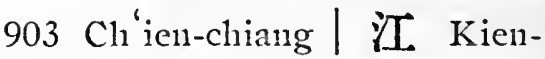
[449] kiang, II. in Yu-yang C., Ssŭch naan; Lat. $29^{\circ}$ $21^{\prime}$, Loing. $106^{\circ} 23^{\prime}$; Sui, Shilh-chên!r 石城; T'ang,

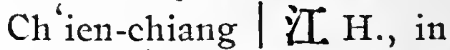
$\mathrm{Cl}^{\prime}$ ien $\mid \mathrm{C} . ;$ Sung and Yiüan, H. in Shao-ch'ing 紹禾 $\mathrm{Fu} ;$ Ming, H. in $\mathrm{Cl}^{\prime}$ 'ung-ch' ing 重嚴 $\mathrm{Fu}$.

$904 \mathrm{Cl}^{1}$ ieil-chung | 中.

$1^{\circ}$ (Obs.) Ts'in, Cln'ienclung | if or Wu-ling 武陵 chüll; comprising parts of Hunan, Kueichon, Hupeh and Ssŭch' '11an. 
汧

$2^{\circ} T^{\prime} a n g$, circ.

$3^{\circ}$ See Nos. 271, 321, $3727,5858,5868,5955$.

$905 \mathrm{Ch}^{\prime}$ ien-l1si | 西 Kien-si, C. [381] in Ta-ting Fu, Kueichou;

Lat. $27^{\circ} 10^{\prime}$, Long. $106^{\circ}$ $10^{\prime}$.

906 Cli ien-tsou | 陬, see No. 794.

907 Ch'ien-yang | 隄 Kien[561] yang, H. in Yüan-clion Fin, Hunan ; Lat. $27^{\circ} 09^{\prime}$, Long. $109^{\circ} 30^{\prime}$; Han, Hsün-ch' êng鐔城; Liang,

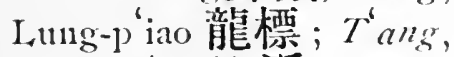
Lang-ch'i 朗溪; Sung, $\mathrm{Cl}_{1}$ ien-yang | 陽.

\section{虔}

908 Ch'ien 宏, see Nos. 3098, $3099,4558$.

$909 \mathrm{Ch}^{\prime}$ ien-nan | 南, see No. 4198.

\section{要}

910 Ch 'ien 瞹, sce Nos. 1661, 3901.

$911 \mathrm{Cl}^{6}{ }^{\prime}$ ien-an | 安'Tsien-ngan, [271] H. in Yung-p'ing Fu, Chihli; Lat. $40^{\circ} 05^{\prime}$, Long. $118^{\circ} 44^{\prime}$; Han, Ling-chil 令支; Kin, Ch'ien-an | 㚣.

912 Ch'ien-chiang | 江 Tsienkiang.

[416] $1^{\circ} \mathrm{H}$. in Ssŭ-ên Fu, Kuangsi ; Lat. $23^{\circ} 40^{\prime}$, Long. $118^{\circ} 40^{\prime}$.
CHIHN-CHHH.

$2^{\circ}$ (Obs.) N. of preceding; Sung, H. in P'in 篟 C.; Yïan, H. in Pin-chon 賽州 circ.; Ming, H. in I,in-choil 棑州 Iㄴ.

913 Ch'ien-l1119-chai | 龍 檈, ss̆̆ near Nan1-11ing Fin, Knangsi.

$91+C h{ }^{2} i e 11-l u n g-t$ ' u11 | 隆崖 'T-S. near 'T'ali-p ing 'T-C., Knangsi.

915 Ch ic11-111in| |民.

$1^{\circ}$ (Obs.) ncar Fêng-t ien Fu, Shingking; Lico, H. in L,1110 龍 C.

$2^{\circ}$ (Obs.) E. of Fin-11ing H., Clilili ; Liao, H. in $\mathrm{Ch}^{\text {'ien }} \mathrm{C}$.

$3^{\circ}$ (Obs.) 100 li $\dot{\mathrm{H}}_{\mathrm{s}}$ of Fin-11ing H., Chilnli ; Kin, chên in Jui 瑞 C’.

\section{釿}

916 Ch'ien-kung 錐眯, (obs.) near Ya-chon Fin, Ssŭch'nan; Tang, C. in Chien1-11an 劍南 circ.

$917 \mathrm{Cl}^{\prime}$ ien-tun | 敦, (obs.) near Turfan; Tang, C. in Hsieh-fêng 竄鳳 Fu。

\section{酸}

918 Ch'ien-chieh 潛 街 (obs.) near Ning-yüan Fu, Ssŭch'uan; $W$. Han, H, in Yüeh-sui 越鹪 chün. 
CH'IEN-SHAN.

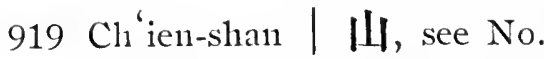
933.

\section{乾}

$920 \mathrm{Ch}^{\text {'ien }}$ 乾 Kien.

[312] $1^{\circ}$ C. L. C. in the Hsi$\mathrm{Ch}$ 'ien-Fu circ., Shensi; Lat. $34^{\circ} 37^{\prime}$, Long. $108^{\circ} 20^{\prime}$; Han, Ch' ihyang 池陽; $T^{6}$ ang, Fêng-t'ien 奉 天 and $\mathrm{Cl}_{1}$ 'ién $\mathrm{C}$.

$2^{\circ}$ Chên near 'T'ien-111ên H., Hupeh.

$3^{\circ}$ See No. 504.

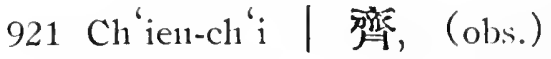
$110 l i \mathrm{~S}$. W. of Yü-mên H., Kansulı ; Han, H. in Chin1-ch'ïan 酒良 chïn; Tsin, H. in Tu11-huang 敦 煌 chiun.

922 Ch'ien-chon | 州 Kien[561] chan, C. L. 'T. in Hunan ; Lat. $28^{\circ} 10^{\prime}$, Long. $109^{\circ}$ $30^{\prime}$.

923 Ch'ien-chü|埧, ssŭ near Iai-fêng H., Hupelı.

$924 \mathrm{Ch}^{\prime}$ ien1-fêng | 封.

$1^{\circ}$ (Obs.) S. E. of T'ai-an H., Shantung; $T^{6}$ ang, H. in Yen 亮 C.

$2^{\circ}$ (Obs.) in Kansulı; T'ang, C. in Lung-yu 隴右 circ.

$925 \mathrm{Ch}^{\prime}$ ien1-fu| 符.

$1^{\circ}$ (Obs.) N. E. of 'T's'ang C., Chihli ; T"ang, $\mathrm{H}$. in Ts'ang 滄 $\mathrm{C}$.
硎 CH'IEN-SHAN.

$2^{\circ}$ (Obs.) $100 l i \mathrm{~N} . \mathrm{E}$. of 'Ts'ang C., Chihli ; Kin, chên in 'Ts'ang 渝 $\mathrm{C}$.

$926 \mathrm{Ch}$ 'ien-ning | 耍, see No. 1197.

927 Ch'ien-yu | 形右, see No. 416.

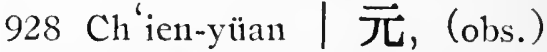
80 li N. of Chên-an H., Shensi; Trang, H. in Shang 率 C.

\section{鉛}

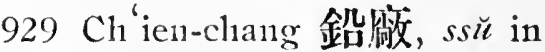
Ch' '1119-yi H., Kiangsi.

930 Ch'ien-shan | II Yuen[211] shan, H. in Kuang-hisin Fin, Kiangsi ; Lat. $28^{\circ} 15^{\prime}$, Long. $117^{\circ} 50^{\prime} ; T^{6}$ ang, $\mathrm{Ch}^{\text {'ien-shan }}$ |ll.

\section{潛}

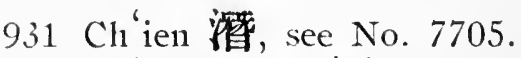

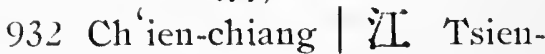
kiang.

$1^{\circ} \mathrm{H}$. in An-lu $\mathrm{F} u$, Hupeh ; Lat. $30^{\circ} 28^{\prime}$, Long. $112^{\circ} 40^{\prime}$; Han, Chiang-ling 江 陵; Sung, Ch'ien-chiang | 江.

$2^{\circ}$ (Obs.) N. W. of preceding; Sung, $H$. in Chiang-ling 江 陵 Fit 933 Ch'ien-shan | 山 (also

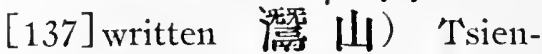
shan $\mathrm{H}$. in An-cl' ing Fu, 
型

Ch' IEN-SHAN.

Anhui ; Lat. $30^{\circ} 43^{\prime}$, Long. $116^{\circ} 38^{\prime} ; S$. Sung and $S$. TS $i$, Chin-hsi 育 熙 chiün in Yï 豫 CHOU; Yüan Clı'ien-shan | 山.

\section{錢}

$934 \mathrm{Ch}^{\prime}$ ient-chiang 錢江, see No. 2988.

935 Cln'ien-ch'ing | 清, chên near Sliao-hsing $\mathrm{F} t$, Cliehkiang.

$936 \mathrm{Cl}^{\prime}$ ien-t'ang | 塘 Tsientang.

$1^{\circ} \mathrm{H}$. forming with Jên1 1o $\mathrm{H}$. the prefectural city of Hang-chou, Chehkiang; Lat. $30^{\circ}$ 。 $12^{\prime}$, I rong. $120^{\circ} 12^{\prime}$; Ts'in, Ch'ien1-t'ang | 塘; Sung, Lin1-an 臨 岀 Fin.

$2^{\circ}$ See No. 1993.

937 Ch'ien-ts'ang | 倉, chên near P'ing-yang H., Cliehkiang.

\section{前}

$938 \mathrm{Cl}^{\prime}$ ien-slan-clai 前山钭 Tsien-shan-chai, 'T. in Kuang-chou $\mathrm{F} u$, Kuangtung; Lat $22^{\circ} 16^{\prime}$, Iong. $110^{\circ} 50^{\prime}$. Called by the Portuguese Casa Branca.

$939 \mathrm{Ch}^{\prime}$ iell-so | 所, ssŭ near T'ai-chou $\mathrm{Fu}$, Chehkiang. $940 \mathrm{Ch}^{\prime}$ ien-tsang | 藏.

\section{櫭 CHHH-CH ${ }^{6} \hat{\mathrm{NG}}$.}

$1^{\circ}$ Anterior Thibet, laving for capital H'lassa.

$2^{\circ}$ See No. 3555.

\section{千}

941 Ch'ien1-ch' êng F. 乘, see Nos. 601, 5125.

$942 \mathrm{Ch}^{\prime}{ }^{\mathrm{i}} \mathrm{ent}-\mathrm{ku}$ | 谷, post-town near Yen-ch' ang $\mathrm{H}$., Shensi.

$943 \mathrm{Ch}^{\prime}$ ien-t'ung | 童, (obs.) 80 li S. E. of Nan-p i H., Chihli; W. Han, H. in Po-hai 勃海 chiun.

944 Ch ienl-wan-kuan / 萬 貫, T-S. near I,ci-po 'T., Sscrucli' vian.

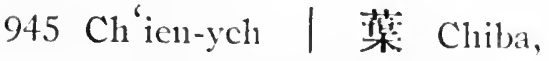
Ken and chief town, Japan.

\section{茜苜}

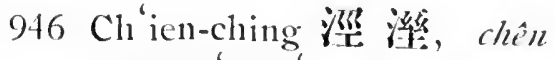
near 'T'ai-ts' ang C., Kiangsu.

\section{CHIH}

\section{氏}

947 Chilh-ch'ilh 氏 池 (obs.) S. IV. of Shan-tan H.; Kansulh. Han, H. in Chang-yeh 張掖 chiün.

\section{雉}

948 Cliih 雉, see No. 250.

949 Chill-ch' êng | 城. see No. 4563. 
道

ChIH-I,I.

\section{直}

950 Chih-li 直疑 Chilnli, a province of China, containing the capital of the Empire, Peking; literary nanies, Yen-yün 燕竝 and Ching-chi 京 畿; called also Chi-pei 魝 北 and Yu1-yen幽䜩; capital, Pao-ting Fin; it contains 7 circ., 11 Fil, 3 'T., 6 C. I. C., $17 \mathrm{C}, 124 \mathrm{H}$.

\section{多直}

951 Chilh-ch'ien 殖錢, chên near Li-shui H., Kiangsu.

治

952 Chilh-p'ing 治平, (obs.) Tang, H. 80 li $\mathrm{N}$. of Hsin-yü H., Kiangsi.

\section{陟}

953 Chih 陟, see No. 7058.

紙

954 Chilh-fang 紙序, chên near Ch'êng H., Kansull.

\section{厥}

955 Chil 䋝, see No. 1691.

\section{支}

956 Chilh-chiang 支江: (obs.) S. W. of Kung H., Ssŭch'nan; T'ang, H. in Ting 定 $\mathrm{C}$.
枝

Chin-chiang.

\section{枝}

957 Chilh-chiang 枝 江 Chikiang.

[528] $1^{\circ} \mathrm{H}$. in Clining-chou $\mathrm{Fu}$, Hupeh; Lat. $30^{\circ} 24^{\prime}$, Long. $111^{\circ} 22^{\prime}$; orig.,

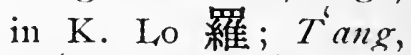
Clinang-ning 長 察; Sung, H. in Cliangling 汭. 陵 $\mathrm{F}_{11}$; Yü̈an, H. in Chung-hsing 中 興 circ.; Ming, H. in Ching-chou 荆州 Fu. $2^{\circ}$ (Obs.) E. of preceding; $W$. Han, H. in Nan 南 chiin; E. Han, Marq. in Nan 南 chion; Tsin, S. Sung, S. Ts'i and $S u i$, H. in Nan 南 chün; Tang, H. in Chiang-ling 江 陵 Fu. 958 Cliilh-yang | 陽, see No. 2342.

\section{拓}

959 Chih 拓, (obs.) Wei, C. near Yi-ch' ang Fu, Hupeh.

\section{秩}

960 Chih-ling-kuan 秩陵關, sš̆ near Li-shui $\mathrm{H}$., Kiangsu.

\section{芷}

961 Chih-chiang 芷 江 Chikiang, $H$. forming the prefectural city of Yüan- 
枝 CHIH-CHIANG.

chou, Hunan; Lat. $27^{\circ}$ $41^{\prime}$, Long. $109^{\circ} 25^{\prime}$; Sung, Yïan and Ming, Yüan 沅 C.

962 Chill-yang | 晹, see No. 2691.

\section{至}

963 Chih-lai 至 來, see No. 291.

964 Chih-tê | 德 Trang, H. in Anhui.

\section{銍}

965 Chih 銍, see No. 5914.

\section{織}

966 Chih-pu 織布, chên near Ho-yang H., Shensi.

\section{職}

967 Chih-t'ien 職 田, chên near San-shui H., Shensi.

\section{知}

968 Chilı 知, (obs.) near Ssŭnan Fu, Kueichou; Sung, C. in Shao-ch'ing 紹 殿 Fu.

969 Chilh-li | 禮 H. in Ch'ingshang 慶䀺 Circ., Corea. 970 Chih-nien 念, town in the Litl-ch'in Islands.$$
\text { - 智 }
$$

971 Chilı 智, see Nos. 2065, 6941.
智 CHIH-T'TIEN.

\section{指}

972 Chih-hui 指揮, Y. near Ch' ên-cliou F't1, Hunan.

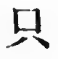

973 Chih-ch'êng 只城, chên near Chi-yüan H., Honan.

\section{枳}

974 Chil 枳, see Nos. 1753, $4568^{2}, 7084$.

\section{軹}

975 Chih 軹, see No. 608.

$$
\text { 之 }
$$

976 Chih-chiang 之 江, see No. 1993.

977 Chih-fou | 啉 Chi-fan, or Chefoo, known to the Chinese as Yen-t'ai 烟喜, one of the ports open to foreign trade, near 'Têngchou Fru, Shantung; Lat. $37^{\circ} 33^{\prime}$, Long. $121^{\circ} 25^{\prime}$; the name is also written 芝罧。

\section{芝}

978 Chih 芝, see No. 2772.

979 Chih-ch' uan | 川, chên near Ho-yang H., Shensi.

980 Chili-fou, | 學, see No. 977.

981 Chih-t'ien / 田, chên near Hung H., Honan. 
制

Chin-jung.

制

982 Chill-ju11g 制 我, (obs.) N. of Anting H., Shensi; Sung, fort in Yen-an 延 发 Fin, Shensi.

\section{質}

983 Chih-h11 質狐, (obs.) 50 li F. of Kao-lan H., Kansuh; Kin, pao in Lan1 蘭 C.

Cliil1-chi11g, see No. 991. Chih-fung, see No. 996. Chih-ki, see No. 992.

\section{$\mathrm{CH}$ 'IH}

\section{银}

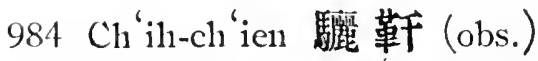
S. of Yung-ch'ang $\mathrm{H}$., Kansul1 ; Han, H. in Chang-yel 張 掖 chïn; $T \sin , \mathrm{H}$. in Wn-wei 武威 chiin.

\section{池}

$98.5 \mathrm{Ch}^{\prime}$ ih 池, see Nos. 986,3475.

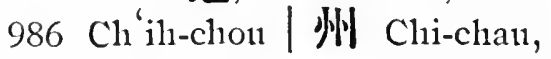
[528] Fin in the Hui-NingCh' ih - T' ai-Knang-tê-chon circ., Anhui; forming also the district city of Kueich'ih; Lat. $30^{\circ} 40^{\prime}$, Long. $117^{\circ} 30^{\prime}$; S. A., 1 st in K. $\mathrm{Wu}$ 奚, $2 n d$ in $\mathrm{K}$. $\mathrm{Ch}^{\prime}$ ' 楚; $T s^{\prime}$ in, in Chang 部
池 CH'

chün; Han, in Tan-yang 丹

陽 chïn; $W u$, Shih-cleêng 石城; $T \sin$, in Yü-chang 豫章 chiin, Liang, in Nan1-ling 南陵 chiun; Sui, Ch' in1-p' 11 秋浦 and Ch' ${ }^{2}$ ihyang 池陽; T゙ang and Sung, Ch' ih $\mid \mathrm{C}$.

$987 \mathrm{Ch}$ 'ih-ho | 河, ss̆̈ near Lai-an H., Anhui.

988 Ch' 'ih-p'êng | 蓬, (obs.) near Ch'ên-chon Fu, Hunan ; Sung, military station in Ch' ên 辰 C.

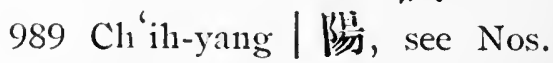
920, 986, 1114, 3734, 5404.

\section{赤}

990 Ch'ih-an 赤岸, chên near $T^{\prime}$ 'an-ch ềng H., Shantung. $991 \mathrm{Cl}^{6}{ }^{\prime} \mathrm{ih}-\mathrm{ch}$ 'êng | 城 Chil1ching.

[337] $1^{\circ} \mathrm{H}$. in Hsiian-hua $\mathrm{F} u$, Chihli; Lat. $40^{\circ} 58^{\prime}$, Long. $115^{\circ} 40^{\prime}$.

$2^{\circ}$ Chên, near Ning C., Kansul.

$3^{\circ}$ Post-town near Lin-an H., Chellkiang.

$4^{\circ}$ See No. 6185.

$992 \mathrm{Ch}^{\prime}$ ihl-ch'i | 溪 Chilh-ki, C. L. T. in Shao-Lo circ., Kuangtung.

993 Ch'ill-chin-hu-t'ai | 金湖 台, post-town near Yümên H., Kansuh. 
赤 CH'IH-CHIN-HSIA-T'AI.

$994 \mathrm{Ch}^{\prime} \mathrm{ih}_{\text {-chin-hsia-t’ai }}$ 金峽 台, post-town near Yümên H., Kansulı.

995 Ch'ih-chin | 斤, (obs.)

Yüan, military district,

Outer Kansulh.

996 Ch'ilı-fêng | 笔 Chih-fung, [239] also called Ha-ta 哈達 or Wu-lan-ha-ta 鳥蘭哈 達, H. in Cl' êng-tê $\mathrm{Fu}$, Clihli; Lat. $42^{\circ} 30^{\prime}$, Long. $119^{\circ}$.

997 Cli'ih-hn| 湖, (obs.) 10 li W. of Ch'u C., Anluni; N. $\mathrm{We} i, \mathrm{H}$. in Hsin-ch ang 新昌 chïn。

998 Ch'ilh-mu-êrl | 木 兒, a city S. of Alimalik (Ili).

999 Clìih-niao | 息, see No. 3025.

$1000 \mathrm{Ch}^{\circ}{ }^{\mathrm{ih}}$-sha | 沙, chên near $\mathrm{Cl}^{\prime}$ ing-shuti H., Kansul.

1001 Ch'ih-shih | 石.

$1^{\circ} S s \breve{u}$ near $\mathrm{Cl}^{\prime}$ ên $\mathrm{C}$., Hunan.

$2^{\circ}$ Ss ̌̆ in Ta-yü H., Kiangsi.

1002 Ch'ih-shui-hsün | 水沨, post-town near Hsü-yung T., Ssŭch'uan.

1003 Ch'ih-yen | 岩, ssü near Chêng-ho H., Fuhkien.

\section{斥}

1004 Ch'ih-chang 压漳, see No: 6950.

$1005 \mathrm{Ch}^{\text {i }}$ ih-ch'iu | 邱, see No. 1300.
金

Chin-CHI.

昛

$1006 \mathrm{Ch}$ 'ih-tiel1-kon 叫迭淨, 'T-S. near Hsi-ning $\mathrm{Fu}$, Kansuh.

\section{CHIN}

\section{金}

1007 Chin 金 Kin.

$1^{\circ} \mathrm{H}$. in I an-cliou $\mathrm{Fu}$, Kansuh ; Lat. $35^{\circ} 55^{\prime}$, Long. $104^{\circ} 08^{\prime}$; Han, Chin-ch' êng | 城; Kin, Chin | C.

$2^{\circ}$ (Obs.) S. Sung, C. in

Li-chon 利 州 circ.

$3^{\circ}$ (Obs.) formerly C. near

Lei-yang H., Hunan.

$4^{\circ}$ See Nos. 1025, 2815.

$1007^{\wedge}$ Chin-ao | 鎞, see No. 5822.

1008 Chin-ch'ai | 鍃, Y. near Kuang-chou Fu, Kuangtung.

1009 Chin-ch' ang | 昌, see No。 2079.

1010 Chin-ch' êng | 城.

$1^{\circ}$ Chên near Huai-ch'ing Fu, Honan.

$2^{\circ}$ Ss̆̆ near Nan-ning Fu, Kuangsi.

$3^{\circ} \mathrm{H}$. in Chiang-yüan il: 原 circ., Corea.

$4^{\circ}$ See Nos. 1007, 2829, 2905, 3583, 6358.

1011 Chin-chi | 雞, Y. in Polo H., Kuangtung. 
金

1012 Chin-chi | 積, see No. 4698.

1013 Chin1-ch'i | 溪, post-town near Ju11g H., Kuangsi.

1014 Chin-ch'i | 谿 Kin-ki, H. [218] in Fu-chon Fin, Kiangsi ; Lat. $27^{\circ} 52^{\prime}$, Lo11g. $116^{\circ}$ $53^{\prime} ; T^{6}$ ang, Shang-1nu 上 莫 chên; S. T"ang, Sung, I'üan and Ming, Chin-ch"i 笑谷 $\mathrm{H}$.

1015 Chin-ch ih | 池, (obs.) in Ssŭch' 11 an; T Tang, H. in Yen 㬴 C.

1016 Chin-chon | 州 Kin-chan, 'I. in Fêng-t'ien Fin, Shingking; Iat. $39^{\circ} 07^{\prime}$, Long. $121^{\circ} 43^{\prime}$.

1017 Chin-chn-ling | 竹嶺, ssŭ near Sni-ch'ang H., Chelikiang.

1018 Chin-clı'ü | 渠, chên near Mei H., Shensi.

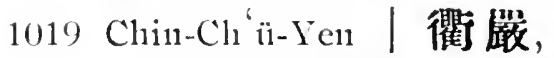
circ. in Chelnkiang, con1prising Chin-huna Fin, Cl' ï-clion Fu and Yenchou Fin.

1020 Chin1-ch'nan | 川, chün in Huang-liai 黃海 circ., Corea.

1020 Chin-ch'ïeh | 閶, see No. 5822.

1021 Chin-hai | 海 Kin-hai, Fu in Ch'ing-shang 慶份 circ., Corea; Lat. $34^{\circ} 45^{\prime}$, Lo11g. $129^{\circ} 10^{\prime}$.
金 Chin-hsiang.

1022 Chin-hang | 杭, ss̆̌ in $\mathrm{Cl}^{\prime}$ 'ung-yi H., Kiangsi.

1023 Chin-hua | 華 Kin-hwa.

[520] $1^{\circ} \mathrm{F} u$ in the Chin-Ch' îYen circ., Chehkiang, forming also the district city of the same name; Lat. $29^{\circ} 11^{\prime}$, Long. $119^{\circ} 51^{\prime}$; S.A., W. frontier of $\mathrm{K}$. Yüeh 越; $T s^{6} i n$, in Kuei-chi 會 稽 chün; Wu, Tung-yang 東陽; Liang, Chin-lina | 華; $C h^{6} \hat{e} n$, Chin 繣 C.; Sui, Wu 愁 C.; Sung, Pao-w1 雃菂; Ming, Clin-hua | 華 Fin. Vuju (Polo).

$2^{\circ} \mathrm{H}$. forming the prefectural city of the same name; I at. $29^{\circ} 11^{\prime}$, Long. $119^{\circ}$ 51; Han, Ch'ang-shan 長 山; $W u$, 'Tung-yang 東陽; Liang, Clin-hua | 華.

1024 Chin-hua | 化, H. in Cliang-yüan 江 原 circ., Corea.

1025 Chin-hsiang | 组 Kin[189] hiang, H. in Chi-ning C., Shantung; Lat. $35^{\circ} 11^{\prime}$, Long. $116^{\circ} 35^{\prime} ; S$. A., Sung-yi 柇邑; Han, 1st Tung-min 東 悢，2nd Chin-lisiang | 细; T'ang, Chin |C. 
金

ChIN-HSIEN.

1026 Chin-hsien | 仙 (obs.) formerly town near 'la-li Fu, Yünnan.

1027 Chin-kang | 崗, ssŭ near Hsüan-ên H., Hupel.

1028 Chin-k'ou | 口, ssü in Chiang-hsia H., Hupeh.

1029 Clin-kuei | 庴 Kin-kwei, $\mathrm{H}$. in Chang-chou Fit, Kiangsu; this city serves also for Wu-hisi H.; Lat. $31^{\circ} 38^{\prime}$, Long. $120^{\circ} 08^{\prime}$.

1030 Chin-li $\mid$ 利, ssŭ in Nanhai H., Kuangtung.

1031 Clin-lin | 鄰, (obs.) in Annann; T'ang, C. in Ling-11an 領南 circ.

1032 Chin-ling | 陵, post-town near Clinang-ning $\mathrm{Fu}$, Kiangsu; see also No. 749. 1033 Chin-ling | 嶺, chên in Yi-tu H., Shantung, Kin | chên.

1034 Chin-lung | 龍, (obs.) in Annam; Tang, C. in Ling-nan 嶺南 circ.

1035 Chin-111ên 阴.

$1^{\circ}$ Chên in T'ung-an, Fuhkien.

$2^{\circ}$ (Obs.) S. of Yung-11ing H., Honan ; N. Wei, chiin and $\mathrm{H}$. in Yang 陽 cHov.

$3^{\circ}$ See Nos. 574, 5822.

1036 Chin-ming | 明, see Nos. $94,7358$.

1037 Chin-niu| 牛, chên near Lü-chiang H., Anluii.
金 ChIN-T'ANG.

1038 Chin-p' $u$ | 浦, H. in Ching-chi 京畿 circ., Corea.

1039 Chin-sha | 杪, chên near Kuei-ch'i H., Kiangsi.

1040 Clinin-sha-chiang | 沙 江, ssŭ in Wu-ting $\mathrm{C}$., Y ünnan.

1041 Chin-shan | Ill Kin-shan. $1^{\circ} \mathrm{H}$. in Sung-cliang F $\mathrm{k}$, Kiangsu; Lat. $30^{\circ} 45^{\prime}$, Long. $121^{\circ} 30^{\prime}$.

$2^{\circ}$ Name given to Chinese Altai, and popularly to California and Australia. $3^{\circ}$ Ss ̌̈ in Hua-t'ing H., Kiangsu.

$4^{\circ}$ Chïn in Cl' 'ing-sliang 鹿佔 circ., Corea.

$5^{\circ}$ See No. 1045.

1042 Chin-shil-fan | 石 番, T-S. near 'Ting-fan C., Kueichon; Ming, ss̆̌ in Kuei-yang 筫陽 Fu.

1043 Chin-shu| 墅, chên near Su-chou Fu, Kiangsu.

1044 Clini-t'ai | 臺, see No. 5822.

1045 Chin-t'an | 壇 Kin-tan, $\mathrm{H}$. in Chên-chiang $\mathrm{Fu}$, Kiangsu ; Lat. $31^{\circ} 50^{\prime}$, Long. $119^{\circ} 33^{\prime} ; T_{s}^{\prime}$ in, Ch'ü-a 曲阿; Sui, Clininshan |山; T゙ang, Chint'an | 壇.

1046 Chin-t'ang | 堂 Kin-tang, $\mathrm{H}$. in Ch'êng-tu $\mathrm{Fu}$, Ssŭch'uan; Lat. $30^{\circ} 52^{\prime}$, 
金 ChIN-T'ANG.

Long. $104^{\circ} 22^{\prime}$; Han, Hsin-tı 新 都; $T^{6}$ ang, Chin-t'ang | 堂.

1047 Chin-ti 堤, chïn in $\mathrm{Cl}^{\prime}{ }^{\prime i} \mathrm{ian}-\mathrm{lo}$ 全 羅 circ., Corea.

1048 Chin-tou|斗.

$1^{\circ}$ Post-town near Lï-chou Fu, Anhui.

$2^{\circ}$ (Obs.) formerly town W. of Ling-pi H., Anliui.

1049 Chin-tsê / 㵏 Kanazawa, cliief town of Islikawa ken, Japan.

1050 Chin-ts' 111 | 村, (obs.)

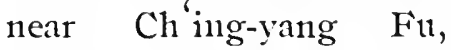
Kansul1; Stung, pao in Ch'ing-yang 慶 陽 Fu.

1051 Chin-tui | 堆, chên near Hua-yin H., Shensi.

1052 Chin-tzŭ-chi | 子 磯, ssü in Wu-ch'ang H., Hupeh.

1053 Chin-wei | 微, (obs.) in the Khalka country; $T^{6}$ ang; Fu in Kuan-nei 關 內 circ.

1054 Chin-wu ! 武, town in the Liu-ch'iu Islands.

1055 Chin-yai | 崖, (obs.) in Kansuh; Sui, H. in T'ungch'ang同昌chün.

1056 Chin-yang | 陽, (obs.) Sung, town near Pao-an H., Shensi.

1057 Chin-yüan | 原, (obs.) in Ssŭch' uan ; T'ang, H. in Kuei-chou 軌 州 Fu.
金 CHIN-SHAN.

1058 Chin-yüan | 源, (obs.) in the Khorchin comitry; Liao and Kin, H. in Ta-ting 大䇥 Fu；Yüan， H. in 'Ta-ning 大要 circ.

1059 Chin-yüan | 淵, (obs.) $W$. Wei, $\mathrm{H}$. near Chint'ang H., Ssŭch' uan.

\section{錦}

1060 Chin 錦 Kin, H. forming the prefectural city of Chin-chou, Shingking ; Lat. $41^{\circ} 06^{\prime}$, Long. $121^{\circ}$ $18^{\prime}$.

1061 Chin-cl'êng | 城, sce No. 511.

1062 Clin-chou | 州,Kin-chan, Fut in the Fêng-ChinShan-hai circ., Shingking; forming also the district city of Chin; Lat. $41^{\circ} 06^{\prime}$, Long. $121^{\circ} 18^{\prime}$.

1063 Chin-kang | 崗, sš̆ in Chiang-hua H., Hunan.

1064 Clin-kuan | 官, post-

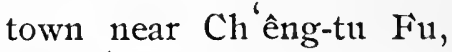
Ssŭch 'nan.

1065 Chin-p'ing | 屏 Kin-ping. $1^{\circ} \mathrm{H}$. in Li-p'ing Fu, Kueichon ; Lat. $26^{\circ}$ $30^{\prime}$, Long. $109^{\circ}$.

$2^{\circ}$ Post-town near Paoning Fu, Ssŭch' uan.

$1065^{\mathrm{A}}$ Chin-shan | 山, chün in Ch'üan-lo 全 羅 circ., Corea. 
近

ChIN-T'ifin.

1066 Chin-t'ien | 田, ssü near Chiang-hua H., Hunan.

\section{近}

1067 Chin-chi-li-k' ê 近吉里克 Dzindzilik, town in Cobdo; I at. $49^{\circ} 20^{\prime}$, Long. $95^{\circ} 40^{\prime}$.

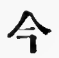

1068 Chin-kuei-jên 今踥仁, town in the Lin-ch 'in Islands.

\section{福}

1069 Chin-ch' uan 袊川, H. in Ching-chi 京 畿 circ., Corea.

\section{禁}

1070 Chin-ch'ên 禁宸, see No. 5822.

$1070^{A} C^{\prime}$ hini-ch 'êng | 城, see No. 5822.

\section{進}

1071 Chin-hao 進浩, chên near Lo-ch' nan H., Shensi.

1072 Chin-hsien | 賢, Tsin[375] hien, H. in Nan-ch' ang Fu, Kiangsi ; Lat. $28^{\circ}$ $29^{\prime}$, Long. $116^{\circ} 17^{\prime} ; T_{\sin }$, Chung-ling 鍾陵; T'ang, Chin-hsien 賢 H.

\section{晉}

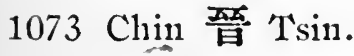

$1^{\circ} \mathrm{C}$. in Chêng-ting $\mathrm{Fu}$, Chihli; Lat. $38^{\circ} 06^{\prime}$, Long. $115^{\circ} 12^{\prime} ; S . A$.,

\section{前 Chin-Chiang.}

K. Ku 鼓; Sui, Hsiyang 暮陽 and Kucliêng 鼓城; Yïan, Chin $\mid \mathrm{C}$.

$2^{\circ}$ C. in Corea ; Lat. $35^{\circ}$, I,ong. $128^{\circ} 05^{\prime}$.

$3^{\circ}$ (Obs.) Chou and $S$. $A$, a $\mathrm{K}$. comprising most of Shansi.

$4^{\circ}$ See Nos. 55, 1023, 1098, 3858, 5200, 5462.

1074 Chin-an | 发.

$1^{\circ}$ Post-town near $\mathrm{Ch}^{6}$ ïanchou Fu, Fuhkien.

$2^{\circ}$ See Nos. 1077, 1871, 5311.

1075 Chin-ch'ang 昌, see No. 6451.

1076 Chin-ch'êng | 城.

$1^{\circ}$ Chên near P'u-ch'êng H., Shensi.

$2^{\circ}$ (Obs.) formerly town E. of Tzŭ C., Ssŭch'uan.

$3^{\circ}$ See No. 2453.

1077 Chin-chiang | 江 Tsinkiang.

$1^{\circ} \mathrm{H}$. forming the prefectural city of $\mathrm{Ch}^{\text {'üan- }}$ chou, Fuhkien; Lat. $24^{\circ} 56^{\prime}$, Long. $118^{\circ}$ $51^{\prime} ; T \sin$, Chin-an 安; Sui, Nan-an 南 安; Trang, Sung, Yüan and Ming, Chin-chiang 江 $\mathrm{H}$.

$2^{\circ}$ See No. 2361. 
1078 Clin-chou | 州 $\mathrm{Fu}$, in Ch'ing-shang 慶攸 circ., Corea.

1079 Chin-fêng | 豐, (obs.) near Ch'êng-tı Fir, Ssŭch'uan; S. Sung, H. in Shih-k'ang 始康 chïn。 1080 Chin-hsi | 熙.

$1^{\circ}$ (Obs.) $T \sin , H$. near Tê-yang H., Ssŭchnan.

$2^{\circ}$ See Nos. 933, 4435.

1081 Chin-hsi | 西, see No. 4435.

1082 Chin-hsing | 與, see Nos. $75,2827$.

1083 Chin-k'ang | 康.

$1^{\circ}$ Ss̆̆ near Hsi-ning H.,

Knangtung.

$2^{\circ}$ (Obs.) Sung, H., N. of Ling-shan $\mathrm{H}$, Kuangtung.

$3^{\circ}$ See No. 1589.

1084 Chin-ling | 陵, see Nos. 312, 768, 7154 .

1085 Clin-ning | 耍 Tsinning.

[461] $1^{\circ}$ C. in Yün-11an $\mathrm{F} u$, Yïnnan; Lat. $24^{\circ} 47^{\prime}$, I.ong. $102^{\circ} 51^{\prime}$; Han, Tien-ch'in 滇池; $T$ sin, Chin-ning 富。

$2^{\circ}$ See No. 5200 .

1086 Chin-sui | 綏, (obs.) near Ch'ü-ching Fu, Yünnan; $S$. Sang and $S$. $T s^{6} i, H$. in Hsi-p'ing 西 本 chïn.

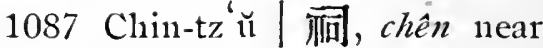
T'ai-yüan H., Shansi.

1088 Chin-yang | 陽.

$1^{\circ}$ (Obs.) formerly town E. of P'êng-tsê H., Kiangsi.

$2^{\circ}$ See Nos. 784, 6174.

1089 Chin-yüan | 原.

$1^{\circ}$ (Obs.) $10 \quad l i$ E. of Ch'ung-ch'ing C., Ssŭch' wan; S. Sung and S. Ts'i, chün in Yi 谷 CHOI.

$2^{\circ}$ See Nos. 1589, 6118.

\section{䌐}

1090 Chin 繢, see No. 1023.

1091 Chin-shan | Ll Kin, H. in Chihli.

1092 Chin-yün | 堅 T'sin-yun, [135]H. in Ch'11-chou Fu, Chehkiang; Lat. $28^{\circ} 42^{\prime}$, Long. $119^{\circ} 53^{\prime} ; T^{*}$ ang, Chin $\mathrm{H}$.

Chin, see No. 480.

Chin-chau, see No. 467.

Clinin-fan, see No. 423.

Chin-hai, see No. 429.

Chin-hiung, see No. 427.

Chin-kiang, see No. 418.

Chin-liu, see No. 469.

Chin-nan, see No. 431.

Chin-ngan, see No. 416.

Chin-ning, see No. 433.

Chin-ping, see No. 435.

Chin-si, see No. 425. 
浓 CH' ${ }^{\prime N}-\mathrm{CH}^{\prime} \mathrm{ANG}$.

Clinin-tseh, see No. 453.

Chin-yang, see No. 438.

Clinilywen, see No. 442.

Clini-wang-tao, see No. 1108.

\section{CH'IN}

\section{湓}

1093 Ch'in-ch'ang 摛昌, see No. 2613.

\section{芹}

1094 Ch'in-ch'ïan 芹泉.

$1^{\circ}$ Chên near Liao C., Shansi.

$2^{\circ}$ Post-town near $\mathrm{P}^{\prime} i n g$ ting C., Shansi.

\section{銫}

$1095 \mathrm{Ch}$ 'in 䣄 Kin, C.I.C. in

[63] I,ien-cl in circ., Kuangtung; Lat. $21^{\circ} 58^{\prime}$, Long. $109^{\circ} 07^{\prime} ; \mathrm{Ts}^{\prime} \mathrm{in}$, in Hsiang 象 chün; Liang, An 安 C.; Sui, Ch'in| ; S. Sung, $\mathrm{Cl}_{1}$ in C. in Knang-hisi 展西 circ.

1096 Chin-cliang | 汇, (obs.) $50 l i \mathrm{~N}$. E. of Ch' in C., Kuangtung; Sui, H. in Nan-yïeh 南越 chün; T'ang, H. in Ch'in $\mid \mathrm{C}$.

1097 Chờin-fêng | 風, (obs.) [104] 40 li S. of Hsin-yang H., Shantung; Kin, Chên in Ti 棣 C., Shantung.
沈 $\mathrm{CH}^{\prime}$ IN-YÜAN.

\section{沙}

1098 Ch'in 沈 Tsin, C. L. C. in the Chi-hing circ., Shansi ; Lat. $36^{\circ} 40^{\prime}$, Long. $112^{\circ} 46^{\prime} ; S . A$., ' $\mathrm{T}^{\prime}$ ung-ti 銅 鞮 in $\mathrm{K}$. 'Tsin, 至; C. Wars, 1 st in $\mathrm{K}$. Han 韓, 2nd in $\mathrm{K}$. Chao 趡; $T s^{\text {' }}$ in and Han, in Shang-tang 上. 黨 chiun: 2nd $W_{e i}$, Yi-11ing 青察; Sui, Ch'in C.; T“ang, Yang-ch'êng 晹城 and 'T'ung-ti 銅 鞮; Sung, Wei-shêng 威 勝 and 'T'ung-ti 銅 鞮; lling, 1st Ch' in | C., 2nd Ch'in-chou | 州 Fu. See No. 1103.

1099 Ch'in-chon |'州, see preceding.

$1100 \mathrm{Ch}^{\prime} \mathrm{int}-\mathrm{sh}$ | | 水 Tsin[294] shwui, H. in 'Tsê-chon Ful, Shansi ; Lat. $35^{\circ} 40^{\prime}$, Long. $112^{\circ} 15^{\prime} ; \mathrm{Han}$, Ch'in-shui | 水 $H$.; $P$. Chou, Knang-ning 展察。

1101 Ch'in-ta-lan | 達 蘭 Chintalan, town in Tarbagatai; Lat. $45^{\circ}$, Long. $82^{\circ} 25^{\prime}$.

1102 Ch'in-yang | 陽, posttown near $\mathrm{Cl}^{\prime}$ in-yüan $\mathrm{H}$., Shansi.

1103 Ch'in-yüan | 源 Tsin[64] ytuen, H. in Ch'in C., Shansi ; Lat. $36^{\circ} 35^{\prime}$, 
秦 $\mathrm{CH}^{\prime}$ IN-YÜAN.

Long. $112^{\circ} 23^{\prime}$. Han, Kul-yiian 嵘 遠; $N$. Wei, Yi-ning 義 耍 chiin; Sui, H. in Shangtang上黨 chïn; T'ang, $\mathrm{Cl}^{\prime}$ in | C.; Sung, H. in Wei-slêng 威 勝 military district; Kin, Yüan and Ming, H. in $\mathrm{Ch}^{\text {'in }}$ | C.

\section{留}

$1104 \mathrm{Ch}^{\prime}$ in 椄, see No. 3287.

\section{秦}

$1105 \mathrm{Ch}{ }^{\prime} i n$ 秦 $\mathrm{T} \sin$.

[498] $1^{\circ}$ C. I. C. in the Kung-Ch' in-Chieh circ., Kansuh ; Lat. $34^{\circ} 36^{\prime}$, Long. $105^{\circ} 40^{-\prime}$; Sung, Cl'êng-chi 成 紀; Han, T'ien-shui 天 水; Wei, Ch'in $\mathrm{C}$.; Sung, C. in Ch'in-fêng | 鳳 circ.; Kin, C. in Fêng-hsiang 鳳 翔 circ.; Yüan, C. in

Shensi ; Ming, C. in Kung-ch'ang 瑟 昌 Fin.

$2^{\circ}$ (Obs.) Chou, feudal state, con1prising Kungcli' ang $F u$, Kansuh, and I, ung C., Shensi.

$3^{\circ}$ See Nos. 2066, 5487.

$1106 \mathrm{Cl}^{\prime}$ in-an | 㚣 Tsin-ngan, [219]H. in Ch in C., Kansuh ; Lat. $34^{\circ} 52^{\prime}$, Long. $105^{\circ}$
秦 Ching.

$50^{\prime} ;$ Wei, Lung-ch 'êng 隴 城; Kin, Cl'in-an | 努. $1107 \mathrm{Cl}^{\prime}$ in-ch' ang | 昌, see No. 6069.

$1108 \mathrm{Ch}$ 'in-hwang tao | 皇島 Chinwangtao or Tsin-wang Tao; port near Fu-ning $\mathrm{Fu}$, Chihli, opened to trade by the Chinese Government.

$1109 \mathrm{Cl}^{\prime i n-p}$ ing | 平, see No. 1724.

1110 Clin'in-yu | 郵, see No. 3171.

\section{䅈}

1111 Ch'in-hsï 岀䅈岨, ssŭ near Fu-ting H., Fuhkien.

Chinangli, see No. 601. Chinese Altai, see No. 1041.

\section{CHING}

\section{泾}

1112 Ching 涇 King.

[67] $1^{\circ}$ C. L. C. in the P'ing-

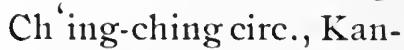
suh; Lat. $35^{\circ} 22^{\prime}$, Long. $107^{\circ} 20^{\prime} ;$ Han, Shun-ku 敦解; 2nd Wei, Ching C.; Sui, An-ting 安 这.

[73] $2^{\circ} \mathrm{H}$. in Ning-kuo Fu, Anluii ; Lat. $30^{\circ} 46^{\prime}$, Long. $108^{\circ} 22^{\prime}$; Han, Cling | H.; T'ang, Yu 觔 C. 
涇

Ching.

$3^{\circ}$ See Nos. 1227, 6169, 6406.

1113 Ching-1no | 陌, chên near Cling-ning C., Kansuh.

1114 Ching-yang | 陽, H. in [67] Hsi-an Fu, Shensi; Lat. $34^{\circ} 30^{\prime}$, Long. $108^{\circ} 45^{\prime}$; Ts'in, Ching-yang | 陽; Han, Cl'ih-yang 池陽; T'ang, Ting 鼎 $\mathrm{C}$.

1115 Ching-yüan | 原, see No. 5171.

\section{經}

1116 Ching-lüeh 絕略, (obs.) $T^{6}$ ang, military district in Ling-nan 嶺南 circ.

1117 Ching-yün | 雲, (obs.) in Yünnan ; S. Sung, H. in Chien-tu 建都 chïn.

\section{竟}

1118 Ching-ling 党 陵, see Nos. 83, 1134, 4445.

\section{鏡}

1119 Ching 鏡, (obs.) T゙ang, C. near Ta-li Fu, Yünnan.

1120 Ching-ch'êng | 城 Fu in Hsien-ching 咸鏡 circ., Corea.

\section{京}

1121 Chirig-ch'êng 菒城, see No. 5288.

1122 Ching-ch'i | 畿.
景

Ching.

$1^{\circ}$ (Obs.) $T^{6}$ ang, circ.

$2^{\circ}$ Circuit in Corea, con1prising Li 驪 C., Yang 楊 C. and P'o 坡 C. $3^{\circ}$ See Nos. 363, 950, 5822 . 1123 Ching-hsi | 西, (obs.) Sung and S. Sung, circ.

1124 Ching-pei | 北, post-town near Hsi-an Fu, Shensi.

1125 Ching-shan | H Kingshan.

$1^{\circ}$ H. in An-lu Fu, Hupelı; Lat. $31^{\circ} 05^{\prime}$, Long. $113^{\circ} 03^{\prime}$; Han, Yün-tu 雲杜; Liang, Hsinyang 新陽; 2nd $\mathrm{Wci}$, IVên 溫, C.; Sui Chingslian | Ill.

$2^{\circ}$ Ss ̈̆ near 'Tung-kuan H., Kuangtung.

1126 Ching-shil | 陁 Kinsay, see Nos. 1993, 5822.

1127 Ching-tu | 都 Kioto, Fu in Japan; see also No. 5288 .

1128 Ching-tung | 東, (obs.) Sung, circ.

1129 Ching-yüan | 原, posttown near Ching-slian $\mathrm{H}$., Hupel.

\section{景}

1130 Ching 景 King, C. in Hochien Fu, Chihli ; Lat. $37^{\circ}$ $54^{\prime}$, Long. $116^{\circ} 20^{\prime} ; S$. A., in K. 'Tsin 晉; Han, Kuang-ch'wan 廣川; Sui, Hsiu 修 H.; T'ang, Ching | C. 
景 CHING-CH'

1131 Ching-ch'êng | 城, see No. 2697.

1132 Cling-chih | 芝, chên near An-ch'iu H., Shantung.

1133 Ching-lıo|和, chên near Ch'ing H., Chillil.

1134 Ching-ling | 陵.

$1^{\circ}$ (Obs.) Five dyn., H. in Lat. $30^{\circ} 42^{\prime}$, Long. $113^{\circ}$ $05^{\prime}$; Han, Ching-ling 竟陵; Sui, Fu 復 C.

$2^{\circ}$ (Obs.) formerly $\mathrm{H}$. near P'ing-yao H., Shansi.

1135 Ching-11ing | 察, King[493] ning, H. in Ch' 11-chon Fin, Chellkiang; Lat. $27^{\circ}$ 56', Long. $119^{\circ} 40^{\prime}$; orig., Clin'ing-tien 苚田.

1136 Ching-tê | 德, chên near Fu-liang H., Kiangsi; commonly known as King-tel Chên, the great centre of porcelain manufacture.

1137 Ching-tu | 渡, chên near Fu-p ing H., Shensi.

1138 Ching-tung | 東 Kingtung.

[333] $1^{\circ}$ C. L. T. in the Yi-nan circ., Yünnlan; ; Lat. $24^{\circ}$ $31^{\prime}$, Long. $101^{\circ} 04^{\prime}$; orig., Cliê-nan 柘南; Tong, K. Nan-chao 南詔, land of the Mêng 蒙 barbarians, and Yin-shêng 銀 生 Fu；Ÿïan, K'ai-nan 開 南 C.; Ming, Chingtung | 東 Fu.
荆 ChIng-Chou.

$2^{\circ}$ T. F. near the preceding.

$3^{\circ}$ (Obs.) Fu; see above.

\section{䠲}

1139 Ching-jung 歌找, (obs.) near An-hua H., Kanstulı; Sung, pao in Ch'ing-yang 慶陽 $\mathrm{Fu}$, Shensi.

\section{荆}

1140 Ching 制.

$1^{\circ}$ One of the nine chou, or divisions of the En11pire, 111ade by Yii 禹, comprising Hunan, Hupeh, Kuangsi and parts of Ssŭch '1an, Kueiclıou and Kuangtung.

$2^{\circ}$ (Obs.) T"ang, Fin.

$3^{\circ}$ See Nos. 1142, 6333.

1141 Ching-cli $i \mid$ 溪 King-ki. $1^{\circ} \mathrm{H}$. in $\mathrm{Cl}$ ang-chon $\mathrm{F} 11$, Kiangsu; this city serves also for Yi-hsing $\mathrm{H}$; Lat. $31^{\circ} 28^{\prime}$, Long. $119^{\circ} 29^{\prime}$.

$2^{\circ}$ See No. 7629.

1142 Ching-chon | 州 King[528] chan, Fin in the Shangching-nan circ., Hupeh ; forming also the district city of Chiang-ling; Lat. $30^{\circ} 27^{\prime}$, Long. $112^{\circ} 05^{\prime}$; S. A., chief department of $\mathrm{K} . \mathrm{Ch}^{6} \mathrm{u}$ 楚; Han, 
荆

Ching-chou.

Lin-chiang 臨 江 and Ching | C.; Three Ks., under 1st Minor Han, 2nd Wu; Tsin, Hsin 新 chïn; Sui and $T^{6}$ ang, Chiangling 江. 陵 H., and Nan 南 chün; Sung, Chingnan | 南; Ming, Chingchon | 州 Fu.

1143 Cling-ho 荆河 in Chienli H., Hupelı. Port of call.

1144 Cling-hul-pei | 湖 北, (obs.) Sung, circ.

1145 Ching-111ên | 門 Kinginun. C. L. C. in the AnHsiang-Yün-Cling circ, Hupeh; Lat. $31^{\circ} 05^{\prime}$, Long. $112^{\circ} 05^{\prime} ; \mathrm{Han}$, in Nan 南 chiin; Tsin, Wu-ning 武 耍; Tang, H. in Chiang-ling 江 陵 Fu ; Yïan, C. in Honan ; Ming, C. in $\mathrm{Ch}^{\prime}$ êng-t'ien 承天社。

1146 Ching-nan | 南.

$1^{\circ}$ Post-town near Chienli H., Hupeh.

$2^{\circ}$ See No. 1142.

1147 Ching-shan |山, (obs.) Liang, town N. of Huaiyüan H., Anhui.

1148 Ching-t'ai | 臺, (obs.) Sui, town W. of Chingmên_.C., Hupel.

1149 Clining-tzŭ-kuan | 紫關, post-town near Chê-ch' naan T., Honan. 垟i Ching.

1150 Cling-Yi-Shil | 宜. 施, another name for the Shang-Ching-Nan circ., Hupeh (5496.)

1151 Ching-ÿüan | 原, (obs.) formerly town near 'Tuyïn Fu, Kueichou.

\section{津}

1152 Ching 津, (obs.) in Ssŭch'tian ; T'ang, C. in Inng-yu隴石 circ.

1153 Ching-hsinng | 雄, posttown near P'ing-yang Fu, Shansi.

1154 Ching-shil 市, chên in Li C., Hunan.

1155 Ching-wu| 梧, (obs.) in Annanin; S. Sung, H. in Chin-chên 儿 眞 chün.

\section{蛙}

1156 Ching-ling or Cly'ing-ling 蜻蛉, see No. 6109.

\section{靖}

1157 Ching 靖 Tsing.

$[110] 1^{\circ}$ C. L. C'. in the ShênYüan-Yung-Ching circ., Hunan; Lat. $26^{\circ} 35^{\prime}$, Long. $109^{\circ} 28^{\prime}$; orig., Yung-p'ing 永平; $T s^{\prime}$ in, in Ch'ien-clnung 騟中 chïn; Han, in 'T'sang-kê 羘河 chün; T'ang, Ch'êng 誠 C.; Sung, Ch'ï-yang 渠 晹 and Cling | C. 
站言

Ching.

$2^{\circ}$ Sce Nos. 1452 and 3027.

1158 Ching-an | 努 Tsing-

[8] ngan, H. in Nan-ch'ang $\mathrm{Fu}$, Kiangsi ; Lat. $28^{\circ} 48^{\prime}$, Long. $115^{\circ} 21^{\prime} ; \quad T$ sin, Chien-ch'ang 建 昌; Tang, Cling-an | 安.

1159 Ching-chiang | 江. Tsing[528] kiang, H. in Ch ang-clon: Fu, Kiangsu ; Lat. $32^{\circ} 05^{\prime}$, Long. $120^{\circ} 14 .^{\prime}$

1.160 Ching-hai-wei | 海 衞, ssŭ near Wên-têng H., Shantung.

$1160^{\mathrm{A}}$ Ching-hs, Kuan 站西熨 'I-H Ya Cloou Fiu, Ssŭch' van.

1161 Ching-pien | 邊 T'singpien.

$1^{\circ} \mathrm{H}$. in Yen-an Fin, Shensi ; Lat. $37^{\circ} 20^{\prime}$, Long. $108^{\circ} 02^{\prime}$.

$2^{\circ}$ Post-town near Liangchou Fin, Kansul.

1162 Cling-yiuan | 遠 Tsing[148] ynen, H. in Lan-clion Fin, Kansul1 ; Lat. $36^{\circ} 38^{\prime}$, Long. $104^{\circ} 08^{\prime}$.

\section{墔}

1163 Ching 青争, (obs.) T'ang, C. comprising Chao-p'ing H., Kuangsi.

1164 Cling-clinang | 江. $1^{\circ}$ (obs.) S. Sung, Fu in Kuang-hsi 触西 circ. $2^{\circ}$ Sce Nos. 3446, 3876.

1165 Ching-chou | 州, T-S. near Mon C., Ssŭch' 'nan.
青爭 CHING-L, E.

1166 Ching-chü | 店, (obs.) S. W. Sung-p an T., Ssŭcli'uan; Trang, H. in Ching | C.

1167 Cling-fan | 蕃, (obs.) near Slun11-t'ien Fu, Chihli; Tang, H. in Hsüan 主 C.

1168 Cling-fêng | 封.

$1^{\circ}$ (obs.) in the Khorchin country; Kin, H. in Ta-ting 大定 Fit.

$2^{\circ}$ (obs.) 40 li E. of 'T'ai-an H., Shantung; Kin, chên in T'ai-an 泰㚣 C., Sliantung.

1169 Ching-hai 静 海 'Tsing[322] hai, H. in T'ien-ching Fu, Chilhli; Lat. $38^{\circ} 55^{\prime}$, Long. $116^{\circ} 45$; Han, Ching-hai | 海.

1170 Ching-jung | 戎, (obs.) N. W. Cling-sluii H., Kansuh ; Sung, military station in $\mathrm{Ch}^{\dagger}$ in 秦 $\mathrm{C}$., Shensi; Kin, chên, in $\mathrm{Cl}^{\prime}$ in 泰 C.

1171 Cling-kang | 阙 Slidzuoka, ken and clief town, Japan.

1172 Ching-lê | 樂 Tsing-lol,, [123] H. in Hsin C., Shansi; Iat. $38^{\circ} 31^{\prime}$, Long. $111^{\circ}$ $57^{\prime} ;$ Han, Fên-yang 汾 晹; Sui, Lon-fan 樓煩 chiün, K'ê-lan 㟧風 H., and Ching-lê | 樂 H.; T'ang, Kuan 管 C. 
静 CHING-NING.

1173 Ching-ning 窂 Tsing-ning, [219] C. in P'ing-liang Fin, Kansul1; Lat. $35^{\circ} 35^{\prime}$, Isong. $105^{\circ} 45^{\prime}$; orig., Lung-cll'ien 瀧千; $T^{\prime}$ ang, Wei 渭 C.; Yiuan, Cliningning | 荤.

\section{粘}

1174 Ching 精 Dzing, town in Ili ; Lat. $44^{\circ} 30^{\prime}$, Long. $84^{\circ}$; also called An-fu 安 卓。

$1174^{A}$ Ching-ho | 河 C.L.T. in Tarbagatai.

\section{旌}

1175 Ching-shan 㫋宩, chï̈n in Chiang-yüan 江 原 circ., Corea.

1176 Ching-tê $\mid$ 得 Tsing-tell, [296] H. in Ning-kuo $F u$, Anhui,; Lat. $30^{\circ} 35^{\prime}$, Long. $118^{\circ} 34^{\prime}$.

1177 Ching-yang | 陽, (obs.) $W_{u}, \mathrm{H}$., S. of Chih-chiang H., Hupel.

\section{井}

1178 Ching 井, see No. 1179 . 1179 Ching-l1sing | 䣎 Tsing[453] hing, H. in Clhêng-ting Fu, Chilhli; Lat. $38^{\circ} 02^{\prime}$, Long: $113^{\circ} 10^{\prime}$; Kin, Wei 威 C. in Ho-pei 河北 Western circ.; Sung, T'ien-wei 天威 military
井 Ching-yi.

district in Ho-pei 河 北 Western circ.

1180 Ching-p'ing | 坪, posttown near P'ing-lu $\mathrm{H}$., Shansi [5176].

1181 Ching-yen | 研 Tsing[323] yen, H. in Tzŭ C., Ssŭcl' 'uan; Lat. $29^{\circ} 32^{\prime}$, Long. $104^{\circ} 08^{\prime}$.

1182 Chinig-yi | 邑 H. in Ch'üanl-lo 全籊 circ. Corea.

Clining, see Nos. 495, 503, 531.

Ching-ching, sẻe No. 539. Ching-chnen, see No. 507. Ching-fung, see No. 460. Ching-hai, see No. 540. Ching-lio, see No. 490. Ching-kau, see No. 516. Ching-kiang, see No. 544. Ching-ku, see No. 517. Ching-kung, see No. 525 . Ching-111ai, see No. 542 . Ching-11gan, see Nos. 482, 504.

Cling-ning, see No. 485. Ching-pu, see No. 518 . Ching-teh, see No. 537. Ching-ting, see No. 487. Ching-tu, see No. 511. Ching-wul, see No. 521. Cining-yang, see No. 488. Ching-yang-kwan, see No. 489.

Clinglian fu, see No. 418 . Chinginju, see No. 312 . 
慶

CH'iNG.

\section{CH'ING}

\section{度}

1183 Ch'ing 慶, see No. 1191. $1184 \mathrm{Ch}$ 'ing-an | 安, (obs.) formerly town near Pai-sê T., Kuangsi.

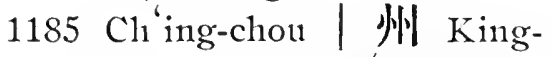
chau, Fu in Cling-sliang 慶们 circ., Corea; Lat. $35^{\circ} 52^{\prime}$, Long. $129^{\circ} 10^{\prime}$.

$1186 \mathrm{Cl}^{\prime}$ ing-fu | 符 King-fu,

[421] H. in Hsü-chou Fu, Ssŭch'nan; Lat. $28^{\circ} 21^{\prime}$, Long. $104^{\circ} 33^{\prime}$; Han, Nan-kuang 南廣; Sung, Cll'ing-fu| 符.

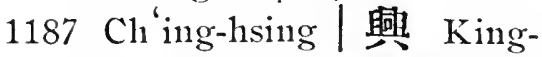
ling, Fu in Hsien-ching 战鏡 circ., Corea; Lat. $42^{\circ} 30^{\prime}$, Long. $130^{\circ} 30^{\prime}$.

$1188 \mathrm{Ch}^{\prime}$ ing-shan |山.

$1^{\circ}$ H. in Cl' ing-shang 度 攸 circ., Corea.

$2^{\circ}$ See No. 3894.

1189 Ch'ing-shang | 份 circ., in Corea.

$1190 \mathrm{Ch}^{\prime}$ ing-tı | 都', see No. 6947.

$1191 \mathrm{Ch}^{\prime}$ 'ing-yang | 陽 King[285] yang, Fu in the P'ingCh'ing-Ching circ., Kansulı; forming also the district city of An-huna; Lat. $36^{\circ} 03^{\prime}$, Long. $107^{\circ}$ $43^{\prime}$; S.A., Yi-ch 'ii 義渠; $T s^{\prime}$ in, Pei-ti 北地; 2 nd Wei, So 朔 C.; Sui and
慶 CH' ${ }^{6}$ ING-YÜN.

Tang, Cll'ing | C.; Sung, Cl'ing-yang|陽; Ming, $\mathrm{Cl}^{\prime}$ ing-yang | 陽 Fu.

$1192 \mathrm{Cl}^{\prime}$ ing-yüan | 遠 King[274] yuen, Fu in Yu-chiang circ., Kuangsi ; forming also the district city of Yi-shan; Lat. $24^{\circ} 26^{\prime}$, Long. $108^{\circ} 25^{\prime}$; Han, in Chiao-chili 交趾 chiun and Jih-nan 日南 chün; Tang, Yüel 宩 C., Yi 目. C. and Lung-shui 龍 水; Sung, Ch'ing-yüan | 遠.

$1193 \mathrm{Cl}^{6}$ ing-yüan | 源 Kingyuen.

$1^{\circ}$ Fu in Hsien-ching 咸 鏡 circ., Corea; I, at. $42^{\circ} 30^{\prime}$, I.ong. $130^{\circ} 25^{\prime}$. $2^{\circ}$ See No. 324 .

1194 Ching-yüan | 元 Kingyuen.

[420] $1^{\circ} \mathrm{H}$. in $\mathrm{Cl}^{-}{ }^{\prime} \mathrm{u}$-chon $\mathrm{Fu}$, Chehkiang; Lat. $27^{\circ}$ $42^{\prime}$, Long. $119^{\circ} 04^{\prime}$; Sung, Ch'ing-yüan | $\overrightarrow{\mathrm{J}} \mathrm{H}$.

$2^{\circ}$ See Nos. 4702.

1195 Cling-yïn 一 雲 Kingyun.

$1^{\circ} \mathrm{H}$. in $\mathrm{T}^{\prime}$ ien-cling $\mathrm{Fu}$, Chilili; Lat. $37^{\circ} 55^{\prime}$, Long. $117^{\circ} 30^{\prime}$; Han, Yang-hsin 隄信; Sui, Wu-ti 無 㴍; Ming, H. in 'Ts'ang 滄 C. 


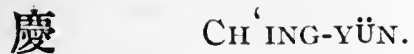

$2^{\circ}$ (obs.) $40 l i \mathrm{~s} . \mathrm{W}$. of K'ai-yüan H., Shingking; Liao, H. in Cli $\mathrm{i}$ 棋 C.; Kin, H. in Hsien-p'ing 威平 Fu。

\section{馨}

$1196 \mathrm{Cl}^{\prime}$ ing-slih 磬石, ssü in I,ê-ch 'ing H., Chelikiang.

\section{青}

$1197 \mathrm{Cl}^{6} \mathrm{ing}$ 青 $\Gamma$ sing.

[322] $1^{\circ} \mathrm{H}$. in $\mathrm{T}^{\circ}$ icn-ching $\mathrm{F} u$, Chillili; Lat. $38^{\circ} 37^{\prime}$, Long. $116^{\circ} 54^{\prime} ; T^{6}$ ang; Ch'ien-ning 乾 耍; Five dyn., Yu11g-an 永 安; Sung, Ch'ing 清 C.; Kin, Hui-ch nan 會川.

$2^{\circ}$ See Nos. 1204, 4365.

1198 Ch'ing-chên | 銃, ss̆̈ in T'ung-hsiang H., Chel1kiang.

1199 Ch'ing-ch' êng | 城 T'singching.

[148] $1^{\circ} \mathrm{H}$. in Wu-ting Fin, Shantung; Lat. $37^{\circ} 13^{\prime}$, Long. $117^{\circ} 51^{\prime}$; Han, 'Tsou 哪; Yüan, Ch' ingcl'êng|城.

$2^{\circ}$ (obs.) P. Chou, H., S. of Kuan H., Ssŭchrónan.

$1200 \mathrm{Ch}^{\prime}$ ing-ch'i | 溪, see No. 5830.

1201 Ch'ing-chia | 䓠, post-
荓 $\mathrm{CH}$ ING-KANG.

town near Ching-ning $\mathrm{C}$., Kansuh.

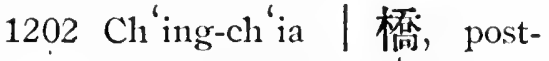
town near Pao-ch'êng H., Shensi.

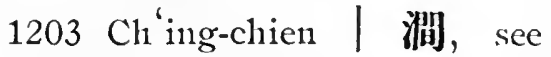
No. 1237.

$1204 \mathrm{Ch}^{\prime}$ ing-chon | 州 Tsing[304] chan, Fu in the Têng-LaiCh'ing-Chiao circ., Shantung; forming also the district city of Yi-tu; Lat. $36^{\circ} 44^{\prime}$, Long. $118^{\circ} 44^{\prime}$, Chou, 1st in Ch ing | CHOU, 2nd in K. Chi, 㴒; T'in, Lang-yel 瑯躯 in Ch'i 唯 chion; Han, Pei-hai 北 海; Wei, Yi-tu 谷都; Sui and T'ang, Ch'ing | C.; Sung, Chê̂n-hai 䥓 海; Ming, Cling-chou 州 Fu.

1205 Ch'ing-ch' uan | 川, so, near Lung-an Fu, Ssŭch'nan.

$1206 \mathrm{Cl}^{\prime}$ 'ing-hai | 海 Tsinghai, or Kokonor, province of Mongolia, bounded on the $\mathrm{N}$. by Kansuh, the E. by Kansul and Ssŭch' 'nan, the S. by 'Thibet, and the $W$. by Thibet and Harashar.

1207 Ch'ing-hua | 化, chênnear Chou-chih H., Shensi.

$1207^{\mathrm{A}} \mathrm{Ch}$ 'ing-kang 毒剧 H. in Hai-Luh C.L.T., Tsitihac. $1208 \mathrm{Ch}^{\prime}$ ing-kang | 崗, post- 
青 CH'iNG-KANG.

town near Yo-chou Fu, Hunan.

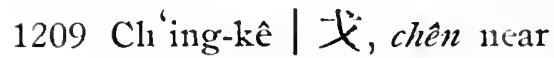
Ning-kuo Fu, Anhui.

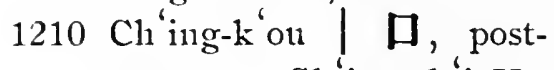
town near $\mathrm{Ch}^{\text {'ing-ch }}{ }^{\prime} \mathrm{i} \mathrm{H}$., Ssŭch 'uan.

$1211 \mathrm{Ch}$ 'ing-ling | 蛉, see No. 6109.

1212 Ch'ing-lı | 盧, (obs.) near Ch'ang-ning 長 富 H., Ssŭch' nan; T'ang, H. in $\mathrm{Cl}_{1}$ ang-ning 長哥 $\mathrm{C}$.

1213 Cly ing-lung | 龍.

$1^{\circ}$ Chên near Futp'ing H., Shensi.

$2^{\circ}$ Post-town near Yungning C., Shansi.

1214 Ch'ing-lung | 童, chên near Ch'i H., Honan (No. 616.)

1215 Ch'ing-pin | 算, (obs.) S. of Hsing-wên $H$., Ssŭch'nan; T'ang, II. in Yïelı 誢 C.

1216 Ch'ing-p'o | 坡（or 清 坡), ssŭ near Wu-kang C., Hunan.

1217 Ch'ing-p'u | 淮 Tsing-pu, $H$. in Sung-chiang F'tu, Kiangsu ; Lat. $31^{\circ} 10^{\prime}$, Long. $120^{\circ} 53^{\prime}$; orig., Yu-ch'üan 由 手.

$1218 \mathrm{Ch}^{\prime}$ ing-sên | 森 Awomori, Ken and chief town, Japan.

1219 Ch'ing-shan | 山, chên near Yü-hang H., Chehkiang.
青 CH'ING-T'IFN.

1220 Ch'ing-shên | 袖 Tsingshin.

[307] $1^{\circ}$ H. in Mei C., Ssŭch'tuan; Lat. $29^{\circ} 48^{\prime}$, Long. $103^{\circ} 51^{\prime}$; Han, Nan-an 南安; $W$. Wei, Ch'ing-yi | 衣; P. Chou, Tang, Sung, Yïan and Ming, Ch'ing-shên | 耐 $\mathrm{H}$. in Mei 肖 C.

$2^{\circ}$ (obs.) $20 \quad l i \mathrm{~S}$. of above; Sui, H. in Meishan 眉山 chïn.

$1221 \mathrm{Ch}^{\prime}$ ing-shui | 水, posttown near Chin H., Kansuli.

$1222 \mathrm{Cl}^{\prime}$ ing-sung | 松 Fu, in

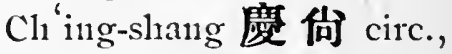
Corea.

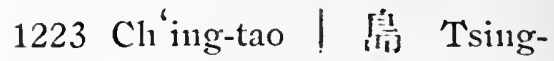
tao Island, at the nouth of Chiao-chon bay, scized by the Germans in 1897 and now held by them on a 99 ycars' lease from China. Lat. $36^{\circ} 10^{\prime}$, Long. $120^{\circ}$ $15 .^{\prime}$

$1224 \mathrm{Ch}^{\prime}$ ing-t'ien | 田 Tsingtien.

[370] $1^{\circ} \mathrm{H}$. in $\mathrm{Ch}^{\prime} \mathrm{u}$-chou $\mathrm{Fu}$, Chehkiang; Lat. 28 , Long. $120^{\circ} 21^{\prime}$; Sui, Kua-ts'ang 括、蒼; T'ang, Cling-t'ien | 田.

$2^{\circ}$ See No. 1135. 
青 CH'ing-wU.

$1225 \mathrm{Cl}^{\prime}$ ing-wu | 烏, (obs.) $40 l i \mathrm{~W}$. of Ning-ch'iang C., Shensi ; Sung, chên in 'ra-an 大，努 wilitary district.

1226 Ch'ing-yang-shan | 羊 IU, see No. 5184 .

1227 Ch'ing-yang | 晹 Tsingyang.

[510] $1^{\circ} \mathrm{H}$. in $\mathrm{Cl}_{1}{ }^{\mathrm{ih}}$-chour $\mathrm{Fu}_{1}$, Anhui ; Lat. $30^{\circ} 45^{\prime}$, I,ong. $116^{\circ} 46^{\prime}$; Han, Ching 鳐 $\mathrm{H}$; $W u$, Linclìêng 臨 城; T'ang, Cl'ing-yang | 陽.

$2^{\circ}$ Chên near Ying-shan H., Anhui.

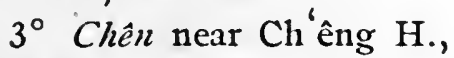
Kansuh (also written | 羊).

$4^{\circ}$ Post-town near Linch'ing C., Shantung.

$5^{\circ}$ Post-town near Mien H., Shensi.

$6^{\circ}$ Post-town near Sui-tê C., Shensi.

$1228 \mathrm{Ch}$ 'ing-yang | 暘, chen near Chiang-yin $\mathrm{H}$., Kiangsu.

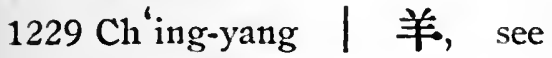
$1227,3^{\circ}$.

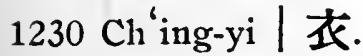

$1^{\circ}$ (obs.) N. of Ya-chou Fut, Ssŭch'uan; $W$. Han, H. in Shu 虽 chün.

$2^{\circ}$ See Nos. $1220,4737$.
清 Ch'ING-CHIANG.

\section{蚌}

1231 Cly'ing-ling or Ching-ling 蜻 蛉, see No. 6109 .

\section{溚}

1232 Ch'ing 声, see No. 1197.

$1233 \mathrm{Cl}^{\prime}$ ing-an | 安 H. in Chung-ch'ing 忠清 circ., Corea.

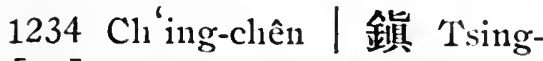
[24] chin, H. in An1-shun F., Kueichou. Lat. $26^{\circ} 35^{\prime}$, Long. $106^{\circ} 25^{\prime}$. Ming, Wei-ch'ing 威清 Wei.

$1235 \mathrm{Cl}^{\prime}{ }^{\mathrm{ing}} \mathrm{g}-\mathrm{ch}$ 'i | 溪 Tsing-ki. [254] $1^{\circ}$ H. in Ya-chou Fu, Ssŭcl' 'uan; Lat. $29^{\circ} 45^{\prime}$, Long. $102^{\circ} 50^{\prime} ; T^{\prime}$ ang, Sung and Yüan, Li 黎 C. and Han1-yüan1 漢源 $\mathrm{H}$.

[20] $2^{\circ}$ H. in Ssŭ-chou Fu, Kueichou; Lat. $27^{\circ} 06^{\prime}$, Long. $108^{\circ} 25^{\prime}$.

$3^{\circ}$ Chên near Ho-shan H., Anhui.

$4^{\circ}$ (obs.) 7"ang, H. in Tzŭ 資 C., N. E. of Nei-chiang H., Ssŭch'uan.

$5^{\circ}$ See No. 51.

$1236 \mathrm{Cl}^{\prime}$ ing-chiang | 江 Tsingkiang.

[81] $1^{\circ} \mathrm{T}$. in Chên-yüan $\mathrm{F} u$, Kueichou; Lat. $26^{\circ} 30^{\prime}$, I song. $108^{\circ} 50^{\prime}$. 
清 CH'ing-CHIANG.

$2^{\circ} \mathrm{H}$. forming the prefectural city of Lin-chiang, Kiangsi ; Lat. $28^{\circ}$ ()6 $6^{\prime}$, Long. $115^{\circ} 27^{\prime}$; Han, Chien-ch'êng 建 城; Tang, Kao-an 高㚣; Sung, Lin-chiang 臨 江 military district and $\mathrm{Cl}^{\prime}$ 'ing-chiang | 江. $\mathrm{H}$.; Yüan, L,in-chiang 臨 江 circ. and $\mathrm{Ch}^{\text {'ing- }}$ chiang | 江 H.; Ming, Lin-chiang 臨江. Fu and $\mathrm{Cl}^{\prime}$ 'ing-chiang | 江 $\mathrm{H}$.

$3^{\circ}$ (obs.) 40 li E. of Ên-shih H., Hupeh; Sui, chiin in Ching 荆 cHOU.

$4^{\circ}$ See Nos. 1616, 5673.

$1237 \mathrm{Cl}^{\prime}$ 'ing-chien|減, or 毒 [78] 澗,'Tsing-kien, H. in Suitê C., Shensi; Lat. $37^{\circ}$ 12', Long. $110^{\circ} 03^{\prime}$; Han, Pai-t'u 白土; Sui, Hsinp'ing 新平; T'ang, K' nan 望 C.; Sung, Ch ing-chien 广滥.

$1238 \mathrm{Ch}$ 'ing-chien-so | 迸所, ssŭ near Wên-ch ang $\mathrm{H}$, Knangtung.

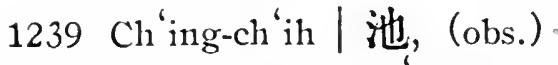
$40 l i$ S. E. of 'Ts'ang C., Chihli ; Sui, H. in Po-hai 渤海 chün; T'ang, Sung, Kin and Yïan, H. in 'Ts'ang C., Chihli.
清 $\mathrm{CH}^{\text {'ING-HO. }}$

$1240 \mathrm{Ch}$ 'ing-ch'üan | 泉 Tsingtsiuen, $H$. forming with Hêng-yang H. the prefectural city of Hêng-chou, Hunan ; Lat. $26^{\circ} 55^{\prime}$, Long. $112^{\circ} 23^{\prime}$.

1241 Ch'ing-fêng | 封, chên near Ho-ching H., Shansi.

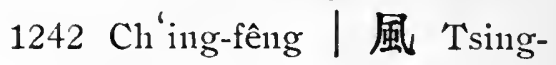
fung.

$1^{\circ} \mathrm{H}$. in Chu11ng-ch'ing 忠 清 circ., Corea; Lat. $37^{\circ} 38^{\prime}$, Long. $128^{\circ} 13^{\prime}$.

$2^{\circ}$ Post-town near Yenp'ing Fu, Fuhkien.

1243 Ch'ing-fêng | 豐 Tsingfung.

[289] $1^{\circ} \mathrm{H}$. in Ta-ming $\mathrm{Fu}$, Chihli; Lat. $35^{\circ} 58^{\prime}$, Long. $115^{\circ} 21^{\prime}$; Han,

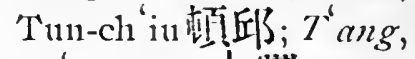
Ch'ing-fêng | 㟽

$2^{\circ}$ (obs.) IV. of preceding; T $T^{6} \mathrm{ng}, \mathrm{H}$. in Shan 滂 $\mathrm{C}$.

$3^{\circ}$ (obs.) N. W. of $1^{\circ}$ above; Sung, H. in K'ai-tê 開 德 Fu; Kin and Yïan, H. in $\mathrm{K}^{\prime}$ ai 開 $\mathrm{C}$.

$1244 \mathrm{Cl}^{\text {'ing-hai }}$ 海.

$1^{\circ}$ (obs.) $T^{\prime}$ ang, military district in Ling-11an 領 南 circ.

$2^{\circ}$ See No. 3393.

$1245 \mathrm{Ch}$ 'ing-ho | 河 'Tsing-ho. 
清

CH'ING-HO.

$1^{\circ}$ Circ. in Chihli comprising Pao-ting Fu, Chêngting Fu, Chi C. (No. 576), Chao C., Shên C. and Ting $\mathrm{C}$.

[10] $2^{\circ}$ H. in Kuang-p'ing Fu, Chihli; Lat. $37^{\circ} 09^{\prime}$, I.ong. $115^{\circ} 46^{\prime}$; orig., Kan-ch'üan1-shih 甘家 节; $T s^{\prime}$ in, 'Ts'o 庴 $\mathrm{H}$. Han, Hsin-ch'êng 信 成 and Kan-ling 甘 陵; Tsin, $\mathrm{Ch}^{\text {'ing-ho- }}$ 河; $T^{6}$ ang, Pei 具 C.

[322] $3^{\circ} \mathrm{H}$. in Huai-an $\mathrm{Fu}$, Kiangsu; Lat. $33^{\circ} 35^{\prime}$, Long. $119^{\circ} 01^{\prime}$; orig., Ssŭ 泗 C.; Sung, Ch'ing-ho | 河.

$4^{\circ}$ H. in Corea; Lat. $36^{\circ}$ $51^{\prime}$, Long. $129^{\circ} 27^{\prime}$.

$5^{\circ}$ See Nos. 1606, 3254, 7054.

$1246 \mathrm{Ch}^{\prime}$ ing-hu | 湖, chên near Chiang-shan H., Chehkiang.

1247 Ch'ing-hua | 化.

$1^{\circ}$ Chên near Fu-fêng H., Shensi.

$2^{\circ}$ Chên near Huai-ch'ing $\mathrm{Fu}$, Honan.

$3^{\circ}$ Sš̆ near An-fu H., Hunan.

$4^{\circ}$ See No. 5673.

1248 Ctr'ing-hsiang | 湘, see No. 1491.

$1249 \mathrm{Ch}$ 'ing-liu |·流, Tsingliu.

\section{清 CH'ING-T'AI.}

[388] $1^{\circ}$ H. in $T^{\prime}$ ing-chon $F u$, Fulukien ; Lat. $26^{\circ} 07^{\prime}$, Long. $116^{\circ} 59^{\prime}$; Han, Ch'ang-t'ing 長 汀; Sung, Ch'ing-liu | 流. $2^{\circ}$ See No. 3557 .

$1250 \mathrm{Ch}^{\prime}$ ing-p'ing | 布 Tsingping.

[286] $1^{\circ} \mathrm{H}$. in 'Tung-ch'ang Fu, Shantung; Lat. $36^{\circ} 58^{\prime}$, I,ong. $116^{\circ}$ $12^{\prime}$; Han, Pei-ch'in 具邱; Sui, Ch'ing$\mathrm{p}$ ing.

$2^{\circ}$ H. in Tu-yün Fu, Kueichou; Lat. $26^{\circ}$ $38^{\prime}$, Long. $107^{\circ} 38^{\prime}$.

$3^{\circ}$ Post-town near Huan H., Kansuh.

1251 Ch'ing-p'o | 坡, see No. 1216.

$1252 \mathrm{Ch}^{\prime}$ ing-shui | 水 Tsingshwui.

[328] $1^{\circ} \mathrm{H}$. in $\mathrm{Ch}^{\prime}$ in C., Kansulı; Lat. $34^{\circ} 42^{\prime}$, Long. $106^{\circ} 12^{\prime}$.

$2^{\circ}$ Chên near Ch'ienchiang H., Kuangsi.

$3^{\circ}$ Y. near T'ai-kı H., Shansi.

$4^{\circ}$ Post-town near Ling C., Kansuh.

$1253 \mathrm{Ch}^{\prime}$ ing-shui-ho | 水 河 [516] Tsing-shwui-ho, T. in So-p'ing Fu, Shansi; Lat. $40^{\circ}$, Long. $111^{\circ} 50^{\prime}$.

$1254 \mathrm{Ch}^{\prime}{ }^{\prime}$ ing-t ${ }^{\circ} \mathrm{ai} \mid$ 秦, see No. 3152. 
清 CII'ING-T'AN.

1255 Ch'ing-t'an | 潭, (obs.) $W$. Wei, town E. of Tsaoyang $H .$, Hupeh.

$1256 \mathrm{Ch}^{\prime}$ ing-tao | 道 T'singtaul.

$1^{\circ} \mathrm{Fut}$, in $\mathrm{Cl}_{1}{ }^{\text {ing-shang }}$ 慶 份 circ., Corea. Lat. $35^{\circ} 34^{\prime}$, Long. $128^{\circ} 50^{\prime}$.

$2^{\circ}$ Ss ̌̆ in Hai-k'ang H., Kuangtung.

1257 Ch'ing-tsê | 澤, sce No. 7829.

1258 Ch'ing-yi | 邑, see No. 5616.

1259 Ch'ing-yi | 易, chên near Kung H., Honan.

1260 Clin'ing-ying | 澦, posttown near Yii C., Honan (No. 7687.)

$1261 \mathrm{Cl}^{6}$ ing-yian | 遠 Tsing[22] ynen, H. in Kuang-chon Fu, Kuangtung; Lat. $23^{\circ}$ $49^{\prime}$, Long. $112^{\circ} 46^{\prime}$; Han, Chung-su 中宿; Liang,

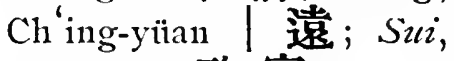
Cliêng-pin 政實.

$1262 \mathrm{Ch}$ 'ing-yüan | 源.

$1^{\circ}$ Post-town near Linch'ing C., Shantung.

$2^{\circ}$ (obs.) T'ang, Sung, Kin, Yü̈an and Ming, H. in T'ai-yüan $\mathrm{Fu}$, Shansi ; Lat. $37^{\circ} 37^{\prime}$, Long. $112^{\circ} 16^{\prime} ;$ S. A., Kêng-yang 梗陽.

$3^{\circ}$ See Nos. 1491, 2905.
清 ChIU-hSIEN.

1263 Ch'ing-ytian-hsiang | 源 悱, ssŭ near Hsü-kou $H$, Shansi.

1264 Ch'ing-yüan | 輀, see No. 3850.

$126.5 \mathrm{Ch}$ 'ing-yüan | 苑 Tsingyuen.

$1^{\circ} \mathrm{H}$. forming the prefectural city of Pao-ting, Clihli ; Lat. $38^{\circ}$ 53', Long. $115^{\circ} 36^{\prime}$. Han, Fan-hsing 禁 興; Sui, Ch'ing-yüan | 苑.

$2^{\circ}$ Post-town near Yi C., Chihli.

$3^{\circ}$ See Nos. 2714, 4955.

Chinju, see No. 6768.

Chintalan, see No. 1101.

\section{CHIU}

\section{舊}

1266 Chin 䳡, chên near Pohsing H., Shantung.

1267 Chin-ch' êng | 城, T-S. near Ssŭ-ên Fu, Kuangsi.

1268 Chiu-chon | 州.

$1^{\circ}$ Chên near Mien H., Shensi.

$2^{\circ} \mathrm{Y}$. near $\mathrm{Kul}$-an $\mathrm{H}$, Chilhli.

1269 Chin-hsien | 縣.

$1^{\circ}$ Chên in Sung H., Honan.

$2^{\circ}$ Chên near Fan-ch'ang H., Anlui. 
售

Chiu-pien.

1270 Chiu-pien | 邊, post-town near Fêng-t ien Fu, Shingking.

1271 Chin-p'u| 鋪, chên near Hsii-yi H., Anhui.

1272 Chintien | 店, Y. near Fei-lisiang H., Chilhli.

1273 Chin-wei | 徨, chên near P'ing-hu H., Chehkiang.

1274 Chiu-wei-p'ing | 㣮扞, post-town near Yung-pei T., Yünnan.

\section{久}

1275 Chin-clı'ang 久長, chên near Liching H., Shantung.

12.76 Chin-chih | 志, town in the Lin-ch'in Islands.

1277 Chiu-mi | 米, town in the Lit1-ch iu Islands.

\section{九}

1278 Chin1-chên 九真.

$1^{\circ}$ (obs.) in Annani; $W$. Han, chün; E. Han, $T \sin , S$. Sung and $S$. $T s^{\prime} i$, chïn in Chiao 交 CHOU.

$2^{\circ}$ (obs.) in Annam; Sui, chion and $H$. in Yang 揚 cHOU; $T^{6}$ ang, H. in $\mathrm{Ai}$ 零 $\mathrm{C}$.

1279 Chin-chì | 無谷, ssŭ in 'Tzŭ-li H., Hunan.

1280 Chill-chia-chiang | 管港, T-S. near Hsi-ning Fin, Kansuh.
九 ChIU-ChiANG-Ch' ${ }^{6} \hat{x g}$.

1281 Chin-cliiang | 江 Kinkiang.

[528] $1^{\circ} \mathrm{Fu}$ in the Kuang-JaoChin-Nan circ., Kiangsi; forming also the district city of Tê-l1ua; Lat. $29^{\circ} 42^{\prime}$, Long. $116^{\circ} 08^{\prime}$; one of the ports on the Yangtsze open to forcign trade; $S$. A., in $\mathrm{K}$. Wu 哭 and K.Ch'u楚; Ts'in, in Clin-cliang | 江 chiin; Han, in Yiichang 豫章 chiin; $E$. Han, in P'êng-tsê 彭 澤 chiinn; Wei, in Wucli'ang 武昌 chïn; Tsin, Hsün-yang 潯 晹; Liang, Clining 江 C.; Sui, Chiu-chiang I 江; Sung, Tingcliang 起江; Ming, chin-chining | 江 Fur.

$2^{\circ}$ (obs.) W. Han and E. Han, chïn in Anluui; $T s^{\prime}$ in, chiun in Kiangsi. $3^{\circ}$ (obs.) Sui, chün in Clining 㓞 chou.

$4^{\circ}$ (obs.) 65 li N. W. of Ting-yüan H., Anhui; E. Han, chiön in Yang 揚 chou.

$5^{\circ}$ See Nos. 5749, 6312.

1282 Chin-chiang-cli'êng | 江. 城, (obs.) formerly town near Huang-mei H., Hupel. 
九 ChIU-ChOU.

1283 Chiu-chon | 洲, see No. 2336.

1284 Chiu-chung | 重, see No. 5822.

1285 Chin-fêng | 姫, ssŭ near Lê-ch' ang H., Kuangtung.

$1285^{\mathrm{A}}$ Chiu-hua 九華, see No. 5822.

1286 Chiu-lising | 姓, T-S. near Hsing-wên H., Ssŭch'uan.

1287 Chiu-hsing-hsiang | 姓 拫, post-town near Hsingwên H., Ssŭch' uan.

1288 Chiu-lung | 龍. $1^{\circ} S s \breve{u}$ in Hsin-an H., Kuangtung, Kowloon. $2^{\circ}$ See No. 3095.

1289 Chin-mên $\mid$ 哃, (obs.) 25 li N. W. of Kao-ch'êng H., Chihli ; Han, Tsin and $N$. Wei, $\mathrm{H}$. in Ch'ang-shan 常山 chün; Sui, H. in Hêng-shan 恒 II chïn; T'ang, H. in Chên 鎭 $\mathrm{C}$.

1290 Chiu-tao-liang ! 道梁, ss̆̌ near Pao-k ang $\mathrm{H}$., Hupeh.

1291 Chint-yi | 疑, ssŭ near Chiang-hua H., Hunan.

1292 Chin-yüan | 原.

$1^{\circ}$ Post-town near Hsin C., Shansi.

$2^{\circ}$ (Obs.) $T s^{\prime}$ in, chün.

\section{鳩}

1293 Chiu-tzŭ-yi 鳩获邑, see No. 7163.
邱 $\mathrm{CH}^{\prime} \mathrm{IU}-\mathrm{L}$.

\section{酒}

1294 Chiu-ch 'üan 酒泉.

$1^{\circ}$ Post-town near Su C., Kansuh.

$2^{\circ}$ See No. 5920.

1295 Chiu-tien / 古, post-town near Kung-ch'ang $F \mathfrak{w}$, Kansuh.

\section{遒}

1296 Chin 澊, see No. 3568.

\section{就}

1297 Chiu-tien 就 舫, chên near Fên-hsi H., Shansi.

\section{CH'IU}

\section{怒}

1298 Ch'iu-ts'un 各村, chên near Han-ch'êng H., Shensi.

\section{厹}

1299 Ch'in-yu 㕕唒, see Nos. 5893, 5915.

\section{邱}

1300 Ch'in 邱 Kitt, H. in Lin[10] ch'ing C., Shantung; Iat. $36^{\circ} 47^{\prime}$, Long. $115^{\circ} 20^{\prime}$; Han, Ch'ih-ch'iu 厉|; Yüan, Ch'in | .

1301 Ch'iu-lu | 盧, (obs.) near Ning-yüan Fu, Ssŭch'uan; Tang, C. in Chien-nan 劍南 circ. 
邱

Ch'iU-ma.

1302 Ch'in-1na | 馬 Cha-ma, Y. in Inner Mongolia; Lat. $41^{\circ} 40^{\prime}$, Long. $100^{\circ}$ $03^{\prime}$.

1303 Cl'in1-pei | 北 Kin1-peh, [89] H. in Kuang-hsi C., $Y$ innunan ; Lat. $24^{\circ} 10^{\prime}$; Long. $104^{\circ} 15^{\prime}$.

\section{求}

$1304 \mathrm{Cl}^{\prime}$ int-li | 求 禮 H. in Chïan-10|全 羅 circ., Corea.

\section{秋}

1305 Ch'iu-p " 1 秋浦, see Nos. $986,3475$.

$1306 \mathrm{Cl}^{\prime}{ }^{\prime} \mathrm{in}-\mathrm{t}^{\prime} \mathrm{ien}$ |田 Akita, ken and chief town, Japan.

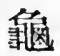

$1307 \mathrm{Ch}^{\text {'i }} 11$-tzŭ 鼠获 (or 滋). $1^{\circ}$ (obs.) N. of Yü̈-lin H., Shensi ; Han, H. in Shang 上 chïn. $2^{\circ}$ (obs.) near Turfan; T'ang, Fin in Lung-yu 隴右 circ.

取

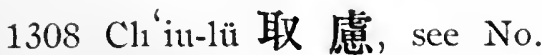
1466.

\section{CHIUNG}

\section{扔}

1309 Clriung IS Kiung, C. L. [307] C. in the Chien-ch'angsliang-nan circ., Ssŭch' 'nan; Lat. $30^{\circ} 28^{\prime}$, Long.
Is ChiUng-Kuo.

$103^{\circ} 34^{\prime}$; Chou, in Jung 榮 C.; Ts'in, Han, Tsin, S. Sung, S. Ti'i, Sui, T'ang and Sung, Lin-

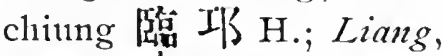

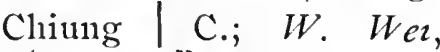
P'u-yüan 蒲原; S. Sung, Chiung | C. in $\mathrm{Cl}^{\prime}$ êng-tı 成 都 circ.

1310 Chinung-ch' ên | 陳, (obs.) near Ya-chon Fu, Ssŭch'van; Sung, C. in Li 黎 cHOU.

$1311 \mathrm{Cl}^{\prime}$ 'iung-chiang | 江. 'T-S. in Lu-cl' 'nan H., Kueichou.

1312 Chiting-pu| 部, T-S. 20 li N. of Yïel1-sui T., Ssŭch 'uan.

1313 Chinng-slu1i | 水, posttown 80 li S. E. of Chênyüan H., Kneichon.

1314 Clinung-tı | 都, (obs.) S. E. of Hsi-cliang $\mathrm{H}$., Ssŭch"nan; Han, Tsin, $S$. Sung and Sui, H. in Yüeh-sui 越鹪 chiün.

简

1315 Chiung-clion 筇洲, (obs.) Yiuan, N. E. of Hsi-o H., Yiinnan.

\section{迴}

1316 Chinung-ch'i 廹岐, ssü in $\mathrm{Cl}^{\prime}$ ing-yüan H., Knangtung.

1317 Chiung-kno | 部, chên near Kung H., Honan. 
廻

Chiung-LiNG.

1318 Chiung-ling | 陵, chên near Clinin-t an H., Kiangstt.

1319 Chiung-lung | 龍, chên near Ho-yüan H., Kuangtu11g.

\section{CHIUNG}

嗄

$1320 \mathrm{Cl}^{\prime}$ 'inng 顀, sce No. 1321.

1321 Ch'iung - chon | 州 Kinng-chan, Fu in the Cli iung-Yai circ., Kuangtung; forming also the district city of $\mathrm{Ch}^{\prime}$ iungslian ; Lat. $19^{\circ} 56^{\prime}$, Long. $110^{\circ} 15^{\prime}$; the prefecture includes the whole island of Hai-nan 海 南, and the city, or practically the village of Hai-k'ou 海 口, Hoi-llow, in its vicinity, is one of the ports open to foreign trade; Han, Chulyai 珠 崖 and Tanêrin 碓耳; Liang, Yai 崖 C.; T'ang, Clin'iungshan | I and Ch'iung |

C.; Ming, Ch'iung-chou 州 Fit.

$1321^{A}$ Cl'iung-lin | 林, see No. 5822.

1322 Ch'iung-shan | Ill Kiungslian.

$1^{\circ} \mathrm{H}$. forming the prefectural city of Cl' iungchou, Kuangtung; Lat.
卓 CHO-K't̂.

$19^{\circ} 56^{\prime}$, Long. $110^{\circ}$ 15'; Han, Tai-mei 瑇 琾; T'ang, Ch'iungshan | II.

$2^{\circ}$ See Nos. 1321, 6441.

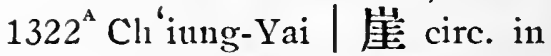
Kwang tung; comprising Ch'iung chon Fu and Yai C.L.C.

骂

$1323 \mathrm{Cl}^{\prime}$ 'iung 弅, (obs.) near Mou C., Ssŭch'uan; T"ang, C. in Chien-nan 劍 南 circ.

\section{CHO}

洮

1324 Cho 浭 Chol, C. in Shun[341] t'ien F11, Chihli ; Lat. $32^{\circ} 15^{\prime}$, Long. $118^{\circ} 20^{\prime}$; orig., Cho-yi | 邑; Wei, Fan-yang 范陽; Tang, Cho C. Juju (Polo).

1325 Cho , (obs.) Han, chïn in Chihli; see No. 4955. 1326 Clio-lu | 鹿.

$1^{\circ}$ Post-town near Cho C., Chihli.

$2^{\circ}$ (obs.) town in Chihli; capital of the Emperor Huang Ti 畵帝.

$3^{\circ}$ See No. 4947.

1327 Cho-yi | 邑, see No. 1324. 卓

1328 Cho-k'ê 卓科, T-S. near 'Ta-chien-1ı T., Ssŭch' uan. 


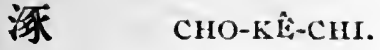

1329 Clio-kê-chi | 克 基, T-S near Sung-p an T., Ssŭch' uan.

1330 Cho-ni | 泥, T-S. near T'ao-chou T., Kansuh.

1331 Cho-so-t'u | 索 圆 Chosotu, Mongol League.

\section{綿}

1332 Cho-êrh 綽 雨 Cholin; town in Inner Mongolia; Lat. $46^{\circ} 30^{\prime}$, Long. $122^{\circ}$ $45^{\prime}$.

1333 Cho-liuo-erl | 水爾. T-S, in Kokonor.

1334 Cho-lo-k'ê | 䕴克, or | 洛克, Chulouk, town in Yarkand; Lat. $38^{\circ}$, Long. $77^{\circ} 15^{\prime}$

1335 Cho-lo-ssŭ | 羅斯 Choros, Mongol Tribe.

1336 Cho-shu-êrh | 樹 Tsushoul, town in Thibet; Lat. $29^{\circ} 20^{\prime}$, Long. $91^{\circ} 25^{\prime}$.

1337 Cho-ssǔ-chia-pu | 斯甲布, 'I-S. N. of Ta-chien-lu. In Thibetan comma called Tchro-shiop. [Baber].

Choh, see No. 1324.

Chơlin, see No. 1332.

Choros, see No. 1335.

Chosotu, see No. 1331.
Chov-chin.

CHOU

周

1338 Chou-an 周 安, chên in Shang-liu H., Kuangsi.

1339 Cliou-cl' $\mathrm{i}$ 溪, post-town near Wan H., Ssŭch 'uan.

1340 Chou-chiang | 港, chèn near Tzŭ-ch'i H., Chehkiang.

$13+1$ Cliou-n.n | 南, see Nos. 1346, 4063.

1342 Chou-tsuan | 纂, chên near Kaop'ing H., Shansi.

1343 Cloun-ts'un | 村.

$1^{\circ}$ Chên near $\mathrm{Ch}^{\prime}$ 'ung-shan H., Shantung.

$2^{\circ}$ (obs.) near T'sê-chon Fu, Shansi ; Kin, chên in Huai 懹 C.

\section{緘}

1344 Chou 絧，(obs.) formerly C., N. of Yi-wu H., Chehkiang.

州

1345 Chou-ling 州 陵, (obs.) Han, H., S. E. of Mienyang $H$. , Hupeli.

\section{䀅}

1346 Chou-chih 致厓 Chat1-chi, $\mathrm{H}$., in Hsi-an $\mathrm{Fu}$, Shensi ; Lat. $34^{\circ} 10^{\prime}$, Long $108^{\circ}$ 16'; Han, Chou-chih | 屋; P. Chou, Chou-nan 周南; Trang, Yi-shon 宜 壽 and Choul-chih | 厔. 
仇 CH'OU-CH'

\section{CH'OU}

仇

$1347 \mathrm{Cl}^{\prime}$ ol1-ch'ih 仇池.

$1^{\circ}$ (Obs.) in Nan-chêng H., Shensi ; $S$. $7 s^{\prime} i$, chïn in $\mathrm{Ch}^{\prime}$ in 秦 crrou. $2^{\circ}$ See No. 503.

1348 Ch'ou-yu | 唒, see No. 7080 .

\section{$\mathrm{CHU}$}

沮

$1348^{\wedge} \mathrm{Cl}_{111}$ 沮, see Nos. 4157, 4675.

\section{本}

1349 Chul-chia-chiao 朱家角, chèn near Ch'ing-p' $u$ H., Kiangsu.

1350 Chu-chia-pao | 管 堡, T-S. near Ta-t'ung $H$., Kansul.

1351 Chn-clı'iao | 橋, chên in Yeh H., Shantung.

1352 Chul-fêng | 叟, (obs.) S. of Ho-p $u$ H., Kuangtung; S. $T s^{\prime} i, \mathrm{H}$. in Ho-p' $\mathrm{u}$ 合 浦 chïn.

1353 Chu-hsiang | 襄, see No. 377.

1354 Chu-hsien | 仙, chên in Hsiang-fu H., Honan.

1355 Ch11-hsü| 虛, (obs.) 60 li E. of Lin-ch' ${ }^{\prime} \mathrm{i}$ H., Shantung; $W$. Han, H. in Lang-yeh 珇躯 chün;
朱 ChU-yai.

E. Han, Marq. in $\mathrm{K}$. Pei-hai 北海; Tsin, H. in Tung-kuan 東莞 chün; S. Sung and N. Wei, H.

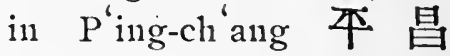
chïn.

1356 Chu1-kang |風, sš̆ in Lien C., Kuangtung.

1357 Chn-li-chang | 里章 Djurdjan, a town of Persia, Province of Khorassan.

1358 Chu-liu | 柳, chên near Ch' ang-lina H., Chehkiang.

1359 Chu1-1u| 盧, (obs.) 30 li

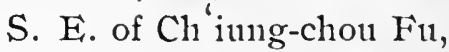
Kuangtung; $W$. Han, $S$. Sung and $S . T s^{\prime} i, H$. in Ho-p'u 合浦 chïn.

1360 Chu-lü | 盧; sometimes the preceding is so written.

1361 Chu-lung | 龍, chên near Chung-pu H., Shensi.

1362 Chu-t ${ }^{\prime} \mathrm{ai} \mid$ 喜. (obs.) in Huan H., Kansuh; Sung, pao in Huan 環 C.

1363 Chu-wu | 吾, (obs.) in Annaun; Han, $T \sin , S$. Sung and $S$. Ts' $i, \mathrm{H}$. in Jih-nan 日南 chün; Sui, H. in Pi-ching 比景 chïn.

1364 Chu-yai | 崖, (obs.) 30 li

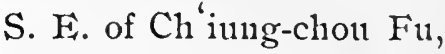
Kuangtung; $E$. Han, $\mathrm{H}$. in Ho-p' u 合浦 chïn; $S$. Sung, military district in Kuang-hsi 簧西 circ. 
珠 ChU-Ch'ANG.

\section{珠}

1365 Chu1-ch'ang 珠㙋, ssü in Ho-p' $u$ H., Kuangtung. The open port, Pakhoi, is situated in this ssü.

1366 Chut-kuan | 官, see No. 3815.

1367 Clin-lung-ch'iao | 龍橋, chên near Lai-an H., Anluni.

1368 Chil-yai | 崖, see Nos. 1321, 6215, 6889, 7224.

\section{制}

1369 Chu 制.

$1^{\circ}$ (obs.) Chou, K. in Shantung, comprising T'êng H., Tson H., etc.

$2^{\circ}$ See Nos. 2289, 6309, $6601^{*}$.

1370 Chu-ch'êng | 城, see No. 2282.

\section{渚}

1371 Chu-ch'i 渚溪, ssŭ in Hsing-tzŭ H., Kiangsi.

1372 Chll-yang-ch'êng | 城陽, (obs.) formerly town near Jên H., Chilhli.

\section{諸}

1373 Chu 諸.

$1^{\circ}$ (obs.) $30 l i \mathrm{~S}$. W. of Chu-ch êng H., Shan1tung; Han, H. in Langyeh 郎 哪 chiun; Tsin; $\mathrm{H}$. in $\mathrm{Ch}^{\prime}$ êng-yang 陽 城 chüu; S. Sung, $N$.
竹 Chu-chas.

Hei, H. in 'lung-kuan 東莞 chün.

$2^{\circ}$ (obs.) in Kiangsu; .S. Sung, H. in Tung-kuan 東莞 chün.

$3^{\circ}$ See No. 1374.

1374 Chutclı'êng | 城 Chn1ching, $\mathrm{H}$. in Ch'ing-chou Fu, Shantung; Lat. $36^{\circ}$, Long. $119^{\circ} 58^{\prime} ; \mathrm{Han}$, Chin | H.; Sui, Chucli êng | 城; T゙'ang, Mi 密 $\mathrm{C}$.

1375 Chn1-chi | 朢 Chut-ki, H. [369] in Shao-hsing Fu, Chehkiang; I alt. $30^{\circ}$, Long. $119^{\circ} 58^{\prime}$ :

1376 Chun-chü | 翟, chèn in Chia-ting H., Kiangsu.

1377 Chu-liang-yi | 梁邑, see No. 5582 .

1378 Chul-lo | 羅, see No. 707. 主

1379 Chu-yüel1-k'on 主月口, post-town near $\mathrm{Ch}$ ien-shan H., Anhui.

\section{竹}

1380 Chn-chan 竹塹, shê near Tannsui, N. Formosa; Lat. $24^{\circ} 49^{\prime}$, Long. $121^{\circ} 15^{\prime}$; this place, locally known as Tukcham, is the residence of the Tamsui Subprefect. Now Hsin-chu $\mathrm{H}$. See No. 2745 , 
竹 CHU-CH'I.

1381 Chu1-ch'i | 䈶 Chulh-ki, H. [469] in Yün-yang $\mathrm{F}^{*} u$, Hupelı; Lat. $32^{\circ} 10^{\prime}$, Long. $109^{\circ}$ $50^{\prime}$.

1382 Clun-cli' i-so | 崎所, ssŭ near Min-ch'ing H., Fuhkien.

1383 Chn1-k'ou | 口, chên near Ch'ing-yüan H., Chel!kiaug.

$1383^{A}$ Ch11-1111-k'an | 木坎; T-S. near Mon C., Ssŭch'nan.

1384 Chu-shan | U Chuh-shan. [469] $1^{\circ} \mathrm{H}$. in Yün-yang $\mathrm{F} u$, Hupeli; Lat. $32^{\circ} 08^{\prime}$, I,ong. $110^{\circ} 21^{\prime} ; T^{\prime}$ in, Shang-yung 上庸; Liang, An-ch'êng 安 城; W. Wei, Chu-shan 山.

$2^{\circ}$ Sš̆ in Hiang-shan H., Chelikiang.

1385 Chn-yüan-ts'un | 園村, ssŭ near Mi-lê H., Yünnan.

\section{筑}

1386 Chu1-yang 筑陽.

$1^{\circ}$ (obs.) Han, H., 4 li E. of Ku-ch'êng H., Hupeh.

$2^{\circ}$ See No. 3317 .

\section{朝}

1387 Chu1-10 朝那, see No. 5171.

\section{燭}

1388 Chut-lung 燭 龍，（obs.）
柷 CHU-CH' $\hat{\mathrm{E}}^{\mathrm{N}}$.

near Ning-hsia Fu, Kansuln; T'ang, C. in Kuannei 閣內 circ.

祝

1389 Chu 形兄, (obs.) Chou, feuclal state near Y ï-ch'êng H., Shantung; see No. 7688.

1390 Chn1-a | 阿, see No. 679. 1391 Chr1-clii | 其.

$1^{\circ}$ (obs.) $50 l i \mathrm{~S}$. W. of Kan-yü H., Kiangsu; Han and Tsin, H. in Tung-lai 東海 chün. $2^{\circ}$ (obs.) in Tan-yang H., Kiangsu；S. Tsi $i, \mathrm{H}$. in Nau Tung-hai 南東 海 chiün.

1392 Cln-lin-tsung | 林總, post-town near Tsao-yang H., Hupeh.

1393 Chnt'tang | 塘, chên near Chiang-yin H., Kiangsu.

\section{柷}

1394 Cl111-cl' êng 柷城, (obs.) Han, town N. of Chungch'ing Fin, Ssŭch' uan.

Cluı, see No. 1397.

Chit-chatı, see No. 1396.

Chu-cling, see No. 1374. Chn-ki, see No. 1375.

Chı1-kui, see No, 1455. 
處

Crí

\section{$\mathrm{CH} \cdot \mathrm{U}$}

\section{處}

$1395 \mathrm{Cl}^{\prime} \mathrm{u}$ 處.

$1^{\circ}$ (obs.) S. Sung, C. in Chê-tung 浙東 circ.

$2^{\circ}$ See No. 1396.

1396 Ch'u-chou / 州 Chu-chan, [330] Fu in the Wên-Ch' $u$ circ., Chelikiang; forming also the district city of Li-shuti ; Lat. $28^{\circ} 26^{\prime}$, Long. $119^{\circ}$ $57^{\prime} ; S$. A. and C. Wars, land of Yiieh 越; $T s^{\prime} \mathrm{in}$, in Kuei-chi 會嵇 chün; Han, K. Tung-ou 東甌; $T \sin$, Y ung-chia 永嘉; Sui, $\mathrm{Cl}^{\prime} \mathrm{u} \mid \mathrm{C}$. and Kuats'ang 括蒼; Tang, Kua 括 C. and Clinn-yün 䌁 雲; Ming, $\mathrm{Ch}^{6} \mathrm{u}$-chou | 洲 Fu.

\section{滁}

1397. Ch' 1 滁 Chu, C. L. C. in the An-Lii-Ch'u-Ho circ., Anhui; Lat. $32^{\circ} 15^{\prime}$, Long. $118^{\circ} 20^{\prime} ; S . A$., frontiers of $\mathrm{K}$. Wu 吳 and $\mathrm{K} . \mathrm{Ch}^{\prime} \mathrm{n}$ 楚; $T \sin$, in Chint-chiang 九 江 chiin, Han, in Huai-nan 淮南 chïn; E. Tsin, Nan=ch'iao 南譙; $N$. $T$ s $i$, Pei-ch"iao 北譙; T'ang, C. in Huai-nan 淮 南 circ.; Sung, H. in
楚 Cir U-HSIUNG.

Tung Huai-11an 東淮南 circ.; Yüan, C. in Yangchon 揚州 circ.; Ming, C. in Nan-ching 南京 province.

$1398 C^{\prime} h^{\prime} u-k$ ou | 口, ssŭ in Hsing-ning H., Hunan.

\section{初}

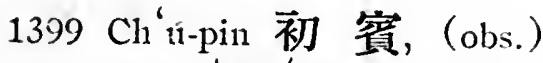
near K'ai-p'ing H., Knangtung; $S$. Sung and $S$. $T s^{\circ} i, \mathrm{Cl}^{\prime}$ 11-pin |㯵 H., in Hsin-1nui 新 會 chiun.

\section{楚}

$1400 \mathrm{Ch}^{\prime} \mathrm{u}$ 楚.

$1^{\circ}$ (obs.) S. $A_{\text {., }, \mathrm{K} \text {. with }}$ centre near Chih-chiang H., Hupeh ; N. Frontier between the Yangtsze and the Yellow River; S. Frontier to S. of the Yangtsze.

$2^{\circ}$ See Nos. 957, 1583, 2159, 2244.

1401 Ch' $^{\prime}$ 11-ch' êng | 城, (obs.) Tang, H. 90 li $\mathrm{s}$. of Chitt-chiang Fu, Kiangsi.

$1402 \mathrm{Ch}^{\prime}$ u-ch'iu | 邱, (obs.) formerly H., S. of 'Ts'aochou Fu: Shantung.

$1403 \mathrm{Ch}^{\prime} 11-1111 \mathrm{sh}{ }^{\prime}$ | 呼楚. See No. 5933.

$1404 \mathrm{Ch}^{\prime}$ n-hsiung | 雄 Tsuhiung. 
[277] $1^{\circ} \mathrm{Fu}$ in the Yi-lisi circ., Yünnan ; forning also a district city of the salne nanle; Lat. $25^{\circ} 02^{\prime}$, Long. $101^{\circ} 43^{\prime}$; orig., in K. Tien 滇; $H a n$, in Yi 賓 $\mathrm{CHOU} ; T s^{\prime} \mathrm{in}$, An 安 C.; T*ang, Weich" 11 威 |; Ming, Ch'n-hsiung | 雄 Fin.

$2^{\circ} \mathrm{H}$. forming the prefectural city of the same name; Lat. $25^{\circ}$ $02^{\prime}$, Long. $101^{\circ} 43^{\prime}$.

$1405 \mathrm{Cl}^{\prime \prime 1-c h}$ 'nan | J Fu in P'ing-an 本 安 circ. Corea.

1406 Clinn-wang | 早, chén near Nei-hnang H., Honan.

$1407 \mathrm{Cl}^{\prime}$ n-wang-cli êng | 王 城, (obs.) S. 4., capital of K. Clin|, near Yünnnêng H., Hupeh.

\section{$\mathrm{CHUi}$}

们

1408 Chïi-chiang | 㴀, chên near Ching-chiang H., Kiangsu.

$1+09$ Chü-jung | 容 Kü-yung, [65] H. in Chiang-ning Fin, Kiangsu ; Lat. $31^{\circ} 58^{\prime}$, Long. $119^{\circ} 09^{\prime}$; Han, Chujung | 容; T⿱ang, Mao 㣢 C.; Sung, the second of the Imperial domains.
拒 Ch ̈̈-NAN.

\section{E}

1410 Chü-cli 巨㴒 Kiu-tsi, H. in Corea, Lat. 34。 24', Long. $128^{\circ} 10^{\prime}$.

1411 Chü-ch'iao | 橋, see No. 3498.

1412 Chü-ching | 津, (obs.) 300 li $\mathrm{N}$. of Li-chiang Fu, Yünnan; Yüan and Ming, C. in Li-chiang F'n.

\section{釬}

1413 Chü 鈣, (obs.) Trang C., $N$. of Ssŭ-nan Fin, Kueichou.

1414 Chï-lı | 鹿 Kin-luh. [432] $1^{\circ}$ H. in Shun1-tê Fin, Chilhli; Lat. $37^{\circ} 26^{\prime}$, I.ong. $116^{\circ} 33^{\prime}$; Han, Na11-luan 南絲; Sui, Chii-lı| 鹿 $T^{6}$ ang, 起 C.

$2^{\circ}$ (obs.) Tsin, chün counprising $N$. Slantung and part of Chilhli.

$3^{\circ}$ See Nos. 3175, 5821, 7015.

1415 Chïi-yeh | 野 Kin-yé.

[102] $1^{\circ} \mathrm{H}$. in 'Ts'ao-chou $\mathrm{Fu}$, Shantung; Lat. $35^{\circ}$ 29', Long. $1161^{\prime}$; orig., Ta-yel1 大野; $T^{\prime}$ ang, Lin 麟 C.

$2^{\circ}$ See No. 698 .

\section{拒}

1416 Chü-nan 拒 南, see No. 4068. 
菊 $\mathrm{CH} \ddot{U}_{-}-\mathrm{CH}^{6} \mathrm{IH}$.

\section{菊}

1417 Chü-ch'ih 菊池, (obs.) in $\mathrm{I}, \mathrm{il}$ C., Ssŭch 'ran ; T'ang, H. in Nêng 能 C.

\section{属}

1418 Chü-cl'an 屈產. See No. 5718 .

\section{具}

1419 Chï-chih-ch'nan 具志川, town in Liu-ch in Islands. 1420 Chï-chilt-t'on | 志顛, town in Lin-ch in Islands.

\section{埧}

1421 Chü-t'on-ts'un 埧頭村, ss̆̈ near $\mathrm{P}^{i}$ ing-rïian $\mathrm{H}$., Kuangtung.

\section{葛}

1422 Clıü 营 Kü, C. in Yi-chou [404] Fu, Shantung; Lat. 35 $35^{\prime}$, Long. $119^{\circ} 20^{\prime}$; Chour, Clı̈̈-tzŭ ج; Han, H.; P. Chou, C.

1423 Chü-tzŭ | 子, see No. 1422.

\section{居}

1424 Chü 居.

$1^{\circ}$ (obs.) in Mon C., Ssŭch'uan; T'ang, C. in Chien-nan 劍 南 circ.; Sung, C. in Mon 茂 $\mathrm{C}$.

$2^{\circ}$-(obs.) near Hsü-chon Fu, Ssŭch'uan; $T^{*}$ ang; C. in Chien-nan 劍南 circ.
苴 С

1425 Chü-ch'ang | 昌 H. in Ch'ing-shang 度少 circ., Corea.

1426 Chï̈-ch'a | 集, see No. 374.

1427 Chïi-fêng | 䖝, (obs.) $\mathrm{N}$. W. of Ch ing-luna 清化 Fu, Annam; Han, H. in Chin-chên 九真 chïn.

1428 Chü-yung | 庸, (obs.) E. of Yen-ch ing C., Chiluli; Han, $T \sin$ and $N$. Wei, H. in Shang-ku 上谷 chiin.

1429 Chü-yung-kuan | 庸關, post-town near Changping C., in the Nankow Pass.

\section{崌}

1430 Chü 崌, (obs.) in Mon C. Ssŭch'nan; T'ang, C. in I,ung-yu 隴右 circ.

\section{曲}

1431 Chï-yiü 曲逆, see No. 6909.

沮

1432 Chü-yang-ch'êng 沮 陽 城, (obs.) near Pao-an C., Chilıli; Han, capital of Shang-ku上谷 chiin.

直.

1433 Chï-ch'üeh 草, 却, ssur near Yung-pei $T$., Yünnan. 
聚 CHÜ-I,ANG-PAO.

\section{聚}

1434 Chü-liang-pao 聚梁堡, post-town near Yang-kao H., Shansi.

\section{車}

1435 Chü-sliilı 車 師, see No. 7184.

\section{CH'U}

\section{句}

$1436 \mathrm{Ch}^{\prime}$ ï-t'ing 句町, see No. 3838.

\section{渠}

$1437 \mathrm{Ch}^{\prime} \ddot{\mathrm{u}}$ 渠 Kü, H. in Sui[111] ting Fu, Ssŭcli' nan; Lat. $30^{\circ} 53^{\prime}$, Long. $106^{\circ} 53$; Han, Tang-ch 'ì 宕|; $W$. Wei, Liu-chiang 流 江; S. Sung, C. in 'T'un1'-ch'nan 潼川 circ.

$1438 \mathrm{Ch}^{\prime}$ ï-cliêng | 城, sš̆ near Li-ling H., Hunan.

$1439 \mathrm{Cl}^{\prime}$ ïi-chiang | 江. $1^{\circ}$ (obs.) 12 li N. of Kuang-an C., Ssŭch tran; Trang, H. in Clı' ï | C.; Sung, H. in Ning-lisi 耍西 nilitary district.

$2^{\circ}$ See No. 3388.

$1440 \mathrm{Ch}^{\prime}$ ü-clı'iu | 邱, see No. 56.

$1441 \mathrm{Ch}$ 'ü-li | 黎, (obs.) near Turfan; T*ang, $\mathrm{F}_{11}$ in Lung-yu 隴右 circ.

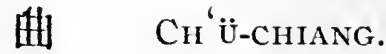

1442 Ch'ï-yang | 㧛, see No. 1157.

\section{翟}

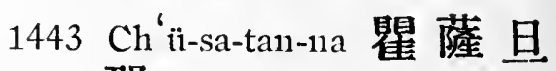
那, see No. 2057.

\section{踭}

$1444 \mathrm{Cl}^{\prime}$ i⿺ 雚.

$1^{\circ}$ (obs.) S. Sung, C. in Chê-tung 浙東 circ.

$2^{\circ}$ See Nos. 1465, 2438.

$1445 \mathrm{Ch}^{\prime}$ ü-chou | 州 Kü-chaut, [112] Fu in the Chin-Ch' ü-Yeir circ., Chehkiang; forming also the district city of Hsi-all ; Lat. $27^{\circ} 55^{\prime}$, Long. $119^{\circ} 12^{\prime} ; \quad T^{\prime} \mathrm{in}$, T'a-mo 太条 H.; $W$. $H a n, H$ sin-an 新安; Sui, San-ch'ü 三 T; $T^{\prime}$ ang, Ch $\mathrm{Ch}^{6} \mid \mathrm{C}$. Chint-ju (Polo).

\section{曲}

$1446 \mathrm{Ch}^{\prime}$ ü 曲, see No. 1452.

$1447 C^{6}{ }^{6} \ddot{i}-a$ | 阿, see Nos. 1045, 6211, 7060 .

$1448 \mathrm{Cl}^{\prime} \mathrm{i}$-an | 安, see No. 1415.

$1449 \mathrm{Cl}^{\prime}$ ì -ch ${ }^{\prime} \hat{e} 1 \mathrm{~g}$ 曲城, (obs.) $\operatorname{Han}, \mathrm{H} .60 l i \mathrm{~N}$. E. of Lai-chou Fu, Shantung.

$1450 \mathrm{Ch}^{\prime}$ ü-chiang | 江 Kiullkiang. 
曲

$1^{\circ} \mathrm{H}$. forming the prefectural city of Shaochou, Kuangtung; Lat. $25^{\circ} 07^{\prime}$, Long. $113^{\circ} 09^{\prime}$; Han, Ch' ï-chiang | 江; Wu, Shih-hising 始 與; T $T^{6}$ ang, P'an 番 $\mathrm{C}$.

$2^{\circ}$ Ss̆̌ in Chien-shui H., Yünnan.

$3^{\circ}$ See No. 3644.

$1451 \mathrm{Ch}^{\prime}$ ü-ch'ien | 千, chên near Huan H., Kansull.

$1452 \mathrm{Ch}^{\prime}$ ü-ching | 靖 Kiuh[335] tsing, Fu in the Yi-tung circ., Yünnan; forming also the district city of Nan-ning ; Lat. $25^{\circ} 32^{\prime}$, Long. $103^{\circ} 50^{\prime}$; Han, Yeh-lang 夜朗 and Mei 味 H.; Minor Han, Hsing-kn 興古; Liang, Hsi-ts'uan 西䁷; 7'ang, $\mathrm{Cl}^{6}$ ï $\mid$ C., Ching 靖 C. and Shilı-ch'êng 石i 城; Yïan, Ch'ü-ching | 靖; Ming, Cl' ${ }^{\prime}$ ï-ching | 靖 Fu.

1453 Ch'ü-chov | 周 Kiuh[128] chau, H. in Kuang-p 'ing Fiv, Clihli ; Lat. $36^{\circ} 52^{\prime}$, Long. $115^{\circ} 06^{\prime}$; Han, $\mathrm{Cl}^{6}$ üi-chou | 周; Wei,

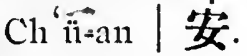

$1454 \mathrm{Cl}^{\prime}$ ü-fu | 阜 Kiulı-fau, $[130] \mathrm{H}$. in Yen-chon Fin, Shantung; Lat. $35^{\circ} 36^{\prime}$,
曲 CII ${ }^{\prime}$-YANG.

Long. $117^{\circ}$; Chou, centre

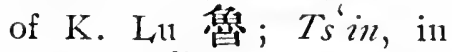
Hsüeh 薛 chiun; Sui, Wên-yang 汶陽; Sung, Hsien-yian 仙源.

$1455 \mathrm{Cl}^{6}$ ü-hui | 惠 Cluıkıi, tow11 in Harashar; Lat. $42^{\circ} 10^{\prime}$, Long. $88^{\circ} 40^{\prime}$.

$1456 \mathrm{Ch}^{\prime} \mathrm{i}$-hisien | 先, (obs.) S. W. of Su C., Kansulı ; Ming, military district.

$1457 \mathrm{Ch}$ 'ü-hsï| 胥, (obs.) in Annan ; Tsin, H. in Chin-tê 九 德 chïn.

$1458 \mathrm{Ch}^{\prime} \ddot{\text { ü-liang }}$ | 梁, see No. 7861.

$1459 \mathrm{Cl}^{\prime}$ ï̈-shui | 水, see No. 7007.

$1460 \mathrm{Cl}^{\prime}$ 'ï-t'ai | 臺, (obs.) Tang, H., S. E. of Laichou $\mathrm{Fu}$, Shantung.

1461 Ch' ï - $\mathrm{ti}$ |堤, (obs.) $30 \mathrm{li}$ N. E. of Chi-yang H., Shantung; Kin, chên in Chi-nan Fu, Shantung.

$1462 \mathrm{Cl}^{6}$ ü-wei | 洧, see No. 6983.

$1463 \mathrm{Cl}^{\prime}$ ï-wu | 沃 Kiuh-yu,

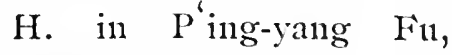
Shansi ; Lat. $35^{\circ} 42^{\prime}$, Long. $111^{\circ} 41^{\prime}$; Ts in, fendal state; Han, Cliiang 綘 H., 2nd $W e i, \mathrm{Cl}^{6}$ ï-wu | 沃.

1464 Ch'ï-yang | 陽 Kinhyang. 
抖

Ch' ${ }^{\mathrm{U}}$-YANG.

$1^{\circ} \mathrm{H}$. in Ting C., Chihli; Lat. $38^{\circ} 39^{\prime}$, Long. $114^{\circ} 38^{\prime}$; Han, Ch ïyang | 陽; Sui, Hêngyang 恒陽.

$2^{\circ}$ Post-town near Yingclı' êng H., Hupelı.

$3^{\circ}$ See No. 6462.

\section{属}

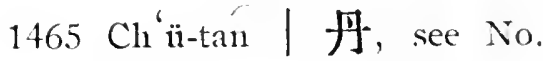
2057.

\section{取}

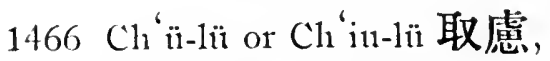
(obs.) S. W. of Huai-11ing H., Kiangsu ; $W$. Han, H. in Lin-hn1ai 臨 淮 chïn; E. Han and Tsin, H. in Hsia-p'ei 下䂙 K.; N. Wei, H. in Lin1-t' $11 n \mathrm{~g}$ 臨漌 chiin.

\section{CHUAN}

\section{磷}

1467 Chnan-ho 礵河, chîn near 'T's'ang C., Chillit.

\section{顓}

1468 Chutan-yii 顓乎.

$1^{\circ}$ (obs.) $70 l i \mathrm{~N} . \mathrm{W}$. of Fei H., Shantung; Sut, H. in I ang-yel 班 㑚 chiin.

$2^{\circ}$ See No. 4381.
川 Ch'UaN-Sha.

\section{CH'UAN}

\section{沌}

$1469 \mathrm{Cl}^{\prime}$ 'uan-k'on 沌口, sš̌ in Han-yang H., Hupeh.

$1470 \mathrm{Ch}^{\prime}$ wan-yang | 陽, see No. 1967.

\section{船}

$1471 \mathrm{Cl}^{\prime}$ 'nan-kao 船高, chên near Ho-ching H., Shansi.

1472 Ch'nan-shê | 舍, chên near 'T'ung-chon $F_{11}$, Shensi.

$1473 \mathrm{Cl}^{\prime}$ '11an-ssŭ-k'11119 |司空, (obs.) N. E. of Hua-yin H., Shensi; E. Han, H. in Ching-chao 京兆 circ.

\section{川}

1474 Ch' nan-chê 川柘, T-S. near Sung-p'an 'T., Ssŭch'uan.

1475 Ch' nan-nan-Yung-ning, | 南永脢, circ. in Ssŭch uan comprising Hsïchou $F$ n, Lü C., Tzŭ C. and Yung-ning T-F., Hsiiyung $T$. and Lei-po ' $T$.

1476 Ch'nan-pei | 北, circ. in Ssŭch 'uan, comprising Paoning $F u$ and Slun-ch ing Fu.

1477 Ch'uan-sha | 沙 Clunensha, T. in Sung-chiang Fut, Kiangst ; Lat. $31^{\circ} 08^{\prime}$, I.ong. $121^{\circ} 20^{\prime}$. Ter. jur. 
川

Ch'Uan-tung.

$1477^{\wedge} \mathrm{Ch}^{\prime}$ uan-tung | 東, circ. in Ssŭch' uan, comprising Chung-ch'ing Fu, K'neichou Fu, Sui-ting Fu, Chung C., Yu-yang C., Shih-chu 'T. and Ch'engkon 'T.

\section{漝}

1478 Ch' uan-t'ing 溇亭, see No. 4014.

\section{第}

1479 Clı 'uan-shan 第山, ss̆̈ in Ch' ên-hai H., Chelıkiang.

\section{CHUIAN}

\section{管}

1480 Chüan 焱, (obs.) formerly town near $\mathrm{K}$ ai-fêng $\mathrm{F}$, Honan.

\section{鄄}

1481 Chüan1-ch'êng 㖶城, see No. 5267.

\section{CH'UAN}

\section{勸}

1482 Ch '̌̈an-numg 勸装, (obs.) in the Khorchin country; Liao, H. in Ta-ting 大 定 Fin.

\section{全}

$1483 \mathrm{Cl}^{\prime}$ ïan 全 'Tsinen.
泉 $\mathrm{C}_{\mathrm{H}} \dddot{U}_{\mathrm{U}} \mathrm{AN}$.

[177] $1^{\circ}$ C. in Kuei-lin $F_{11}$, Kuangsi ; Lat. $25^{\circ} 42^{\prime}$, Long. $111^{\circ} 06^{\prime} ; \mathrm{Ts}^{\circ} \mathrm{in}$, in Ch'ang-sha 長沙 chün; Suri, Hsiang-yüau 湘源； Five dyn. Ch iian $\mathrm{C}$.

$2^{\circ}$ C. in Chiun-1o 全羅 circ., Corea; Lat. $35^{\circ}$ $48^{\prime}$, Long. $126^{\circ} 45^{\prime}$.

$1484 \mathrm{Cl}^{\prime}$ üan1-chiao | 椒 Tsiuen[105] tsian, H. in Cln' ${ }^{\prime}$., Anluni ; Lat. $31^{\circ} 05^{\prime}$, I. ong. $118^{\circ} 10^{\prime} ;$ Tsin, Ch' üan-chiao | 椒; Liang, Pei-clı'iao 北譙.

$1485 \mathrm{Ch}^{6}$ iian-lo 全羅 circ., in Corea.

$1486 \mathrm{Cl}^{6}$ üan1-shêng | 勝, $\mathrm{Y}$. near Ma-yang H., Hunan.

$1487 \mathrm{Ch}^{\text {' }}$ üan-wang | 日., ss̆̈ in Hsi-an H., Chellkiang.

$1488 \mathrm{Cl}^{\prime}$ ïan1-yi | 義 H. in Chunng-ch'ing 忠清 circ., Corea.

\section{泉}

$1489 \mathrm{Cl}^{\text {‘ }}$ üan 泉.

$1^{\circ}$ Chên near Fan-chil H., Shansi.

$2^{\circ}$ (obs.) S. Sung, C. in Hul-11an 湖 南 circ.

$3^{\circ}$ (obs.) S. Sung, C. in Fu-chien 福建 circ. $4^{\circ}$ (obs.) Thang, C., N. of Ching-yang $\mathrm{H}$, Shensi. 
泉

CH' $\mathfrak{U}_{A N}$.

$5^{\circ}$ See Nos. 1491, 1871, 3545, 4936.

1490 Clinana-chinng | 江, see No. 4181.

1491 Ch'ïan-chou | 州 Tsinen-

[61] chan, Fu in the HsingCly iuan-Yung circ., Ful1kien; forming also the district city of Chinchiang; Lat. $24^{\circ} 56^{\prime}$, I ong. $118^{\circ} 51^{\prime}$; orig., Ch'i-min 七閩; Han, in Knei-chi 會穕 chün; Liang, Nan-an 南安; Sui, Cling-lisiang 清湘 and Wên-ling 瀿陵; Tang, Clı üan | C., and Ch'ing-yiuan 清源; Sung, P'ing-hai 平海; Ming, Ch üan-chon | 州 Fu Zayton, (Polo) according to Yule.

1492 Ch'üan-hsia | 下, chên near Hsing-p'ing H., Shensi.

$1493 \mathrm{Cl}^{\prime}{ }^{\prime}$ ïant-ling | 陵, see Nos. $655,3934$.

\section{CHUANG}

\section{莊}

1494 Clinang 莊, (obs.) T ang, C. near Ssŭ-11a11 Fu, Kueichou.

1495 Chnang-hsing | 行, chên near Chin-shan H., Kiangsu.
揽 CHÜFH-HSI.

1496 Chnang-lang | 浱 Chwanglang.

[117] $1^{\circ} \mathrm{T}$. in Liang-clou $\mathrm{F} 11$, Kansuh ; Lat. $36^{\circ} 47^{\prime}$, Long. $103^{\circ} 18^{\prime}$.

$2^{\circ}$ (obs.) Yüan, H. in Lat. $35^{\circ} 27^{\prime}$, Long. $105^{\circ} 56^{\prime}$; Ts in, in Pei-ti 北 地 chïn; now called Chuang-langhsiang | 泿 楖.

1497 Chuang-lang-hisiang | 源 鄉, see preceding.

1498 Chnang-li | 裏, chên near Fin-p'ing H., Shensi.

\section{CH'UANG}

\section{春}

$1499 \mathrm{Cl}^{\text {' }}$ nang-ling 春陵, see No. 6531.

\section{CHÜEH}

\section{覺}

1500 Chüeh-pa-la 覺 巴拉, T-S. in Kokonor.

\section{橛}

1501 Chüeh-hsi 厥西.

$1^{\circ}$ (obs.) N.W. of Sui C., Hupeh; Tsin, H. in Yi-yang 義陽 chïn. $2^{\circ}$ (obs.) S. W. of Anhsiang H., Hunan; $S$. Sung and $S . T^{6} s i, \mathrm{H}$. in Yi-yang 義晹 chün. 
缺 $\mathrm{CH}^{\prime} \dddot{\mathrm{U}} \mathrm{FH}-\mathrm{K}^{\prime} \mathrm{OU}$.

\section{CH'ÜEH}

缺

$1502 \mathrm{Ch}^{\prime}$ üel1-k'ou 缺 口, ssŭ in Tung-kuan H., Knangtung.

\section{散}

$1503 \mathrm{Cl}_{1}$ 'üeh-kê 鵲 個, T-S. near Sung-p'an T., 'Ssŭch' uan.

\section{確}

1504 Ch'üeh-shan 確 山 Kiohshan, H. in Ju-ning $F u$, Honan; Lat. $32^{\circ} 51^{\prime}$, Long. $114^{\circ} 01^{\prime}$; Han, An-ch'ang 㚣昌; E. Han, Lang-shan 朗山; Sung, Ch`üeh-shan | 山.

\section{閣}

$1505 \mathrm{Cl}^{\prime}$ üeh-1ısi 閶 西, (obs.) N. W. of Sui C., Hupei; S. Sung, H. in Sui-yang 㯾陽 chiin; S. Ts $i, \mathrm{H}$. in Sui 随 chiün.

Chuen-sha, see No. 1477. Chuh-ki, see No. 1381. Cliuh-shan, see No. 1384.

\section{CHUI}

樌

1506 Chati-cli'i 鳌其 (obs.) N. of Hsï-yi H., Âuhui; $W$. Han and $T \sin , H$. in Linhuai 臨淮 chün.
春 CHUN-KU。

\section{CH'UI}

\section{所}

$1507 \mathrm{Ch}$ 'ui-lêng-to-êrh-to 吹冷 㴽尔含，T-S. in Kokonor.

\section{椎}

$1508 \mathrm{Ch}^{\prime}$ ui-mei 椎梅, (obs.) in Ya-chou Fu, Ssŭch' 'uan; Tang, C. in Chien-nan 劍南 circ.; Sung, C. in $\mathrm{Ya}$ 雅 cHOU.

Chulouk, see No. 1334.

\section{CHUN}

準

1509 Chun1-chiel1-êrl 準硶尔 Dsungaria, a region comprising Ili, Tarbagatai, Kurkara-usu, \&c.

\section{CH'UN}

春

$1510 \mathrm{Ch}^{\prime}$ 'u 春, see No. 7256.

$1511 \mathrm{Cl}^{\prime}$ 'un-chi | 䅨 chundzi, town in Ili ; Lat. $43^{\circ} 30^{\prime}$, Long. $81^{\circ}$.

$1512 \mathrm{Ch}^{\prime}$ un-cl' uan | 川 Fu in Chiang-yuan 江原 circ., Corea.

$1513 \mathrm{Cl}^{\prime}$ 'un-k'ê | 科, 'T-S. near Sung-p'an 'T., Ssŭch' 'uan. $1514 \mathrm{Cl}^{\prime}$ un-ku|嵘, see Nos. 1630, 4601. 
春 Ch'UN-LING.

1515 Chr 'nn-ling | 陵, see Nos. $4640,6531$.

$1515^{\mathrm{A}} \mathrm{Ch}^{6}$ inl-1ning | 明, see No. 5822.

\section{朐}

$1516 \mathrm{Ch}$ 'n1 胸.

$1^{\circ}$ (obs.) S. of Hai C., Kiangsn ; Han and $T \sin , \mathrm{H}$. in 'Tung-hai 東海 chïn.

$2^{\circ}$ (obs.) W. of Cliên1chiang Fin, Kiangsu; S. Stung, H. in Nantu11g-hai 南東海 chïn.

$3^{\circ}$ See No. 1913.

1517 Ch' $1 n-j$ ên or $\mathrm{Ch}^{\prime}{ }^{\prime}$ nn-jun 䏰，(or｜忍 or 洵䏰). See Nos. 3790, 7818.

\section{純}

$1518 C h ' 11 n$ 純, (obs.) Sung, C. comprising L, ii C., Ssiúcli 'tan.

$1519 \mathrm{Cl}^{\prime}$ 'n11-yi | 戔, (obs.) $T^{6}$ ang, H. 40 li S. of Yung-an C., Knangsi.

\section{CHUN}

淁

1520 Chün-chi 淩稭 (obs.) Tang, C. in Kuan-nei 關内 circ。

\section{均}

1521 Chün 均 Kinus.
軍 CHÜN-WEI.

$1^{\circ} \mathrm{C}$. in Hsiang-yang Fu, Hupeli; Lat. $32^{\circ} 42^{\prime}$, Long. $111^{\circ} 08^{\prime}$; Liang, Hsing 舆 C.; Sui, Yüan and Ming, Chün $\mathrm{C}$.

$2^{\circ}$ (obs.) N. of preceding; $T^{6}$ ang, C. in Shan-nan 山南 circ.; S. Sung, C. in Ching-hsi 京西 circ.

1522 Chün-yang | 䍀, (obs.) Liang, town N. IV. of Chïn C., Hupeh.

\section{鈞}

1523 Chiü11 鈞, see No. T687.

\section{群}

1524 Chiin-111a 群 馬 Gumma， ken in Japan, chief town Takasaki.

\section{軎}

1525 Chrün-clı'êng 軍城, chên near Futp ing H., Chillli. 1.526 Chün-tı| 都.

$1^{\circ}$ (obs.) $17 l i \mathrm{~W}$. of Ch'ang-p'ing C., Chihli; W. Han, H. in Shang$\mathrm{ku}$ 上谷 chïn; $E$. Han, H. in Ch'ingyang 度陽 chïn; Tsin and $N$. Wci, H. in K. Yen 燕.

$2^{\circ}$ See No. 299.

1527 Chün-wei | 成 H. in Clı'ing-shang 殿份 circ., Corea. 
軍

Chusc:

Chundzi, see No. 1512.

\section{CHUNG}

\section{中}

1528 Chung 中.

$1^{\circ}$ Chên near Hali C., Kiangsu.

$2^{\circ}$ (obs.) 400 li E. of Hsi-ch'ang H., Ssŭclinan; Yïan, H. in Chien-ch'ang 建 昌 circ, l'ünnan.

1529 Chung-ch'a | 盆, '-S. near Sung-p an T., Ssŭch' nan.

1530 Chung-ch' êng | 城, town in the Lin-ch in Islands.

1531 Chung-chiang | 港, she near Tan-shui H., Fuhkien.

1532 Chung-chiang|江 Chung[259] kiang, H. in 'T’ungch 'uan Fu, Ssŭchiuan; Lat. $31^{\circ} 04^{\prime}$, Itong. $104^{\circ}$ $49^{\prime}$; llinor Han, Wuch'êng 伍 城; P. Chou, Hsüan-wu 妾. 武; Sui, K'ai 凱 C.; Sung, Chungchiang | 江.

1533 Chung-ch ing | 腐, sce No. 7813.

1534 Chung-ch 'in | 邱, see No. 4651.

1535 Chung-chü| 埧, ssŭ near Chang-ming H., Ssŭch'uan.
中 Chusg-Li:

1536 Chung-chiuan | 川, chề near I, H., Kansuh.

1537 Chnng-ho | 和.

$1^{\circ}$ Ss ̌̆ in Huang-an H., Hupeh.

$2^{\circ} F_{n}$ in P’ing-an 平安 circ., Corea.

1538 Chung-hou So | 後 所, ss $\breve{~ i n ~ N i n g-y i u ̈ a n ~} \mathrm{H}$, Shingking.

1539 Chung-lisiang | 拫, see Nos. 715, 4654.

1540 Chung-hisin | 信, ss̆̈ near Lien-p ing C., Knangtung.

1541 Chung-kung | 公, chên near Chi-yïan H., Honan.

1542 Clunug-kung | 宫, (obs.) IV. of I.i-clienty H., Shantung; Kin, thên in Chi-nan Fin, Shantung.

$15+3$ Chnng-lin | 林, T-S. nean Hsia-chiang T., Kneichon.

1544 Chung-lu | 盧.

$1^{\circ}$ (obs.) S.IV. of Hsiangyang $H .$, Hupelı; $I I$. Han, H. in Xan 南 chïn; E. Han, Marq. in Nan 南 chïn; Tsin, S. Sung and S. Ts'i, H. in Hsiang-yang 衰 陽 chün.

$2^{\circ}$ (obs.) 60 li N. E. of Nan-chang H., Hupelı; Sung, H. in Hsiangyang 哄陽 Fu。

1545 Cliung-lï | 盧, (obs.) Han, town E. of Nanchang H., Hupel. 
中

1546 Chung-n110 | 牟 Chung-

[32] man, H. in $\mathrm{K}^{\prime}$ ai-fêng $\mathrm{F} u$, Honan; Lat. $34^{\circ} 43^{\prime}$, I ong. $114^{\circ} 11^{\prime}$; orig., Pultien 固田; S. A., Chêng-yi 酣邑; Han, Chunng-inou| 牵; Trang, Mou 车 $\mathrm{C}$.

1547 Chung-pu | 部 Clinng-pu, [264] H. in Fu C., Shensi; Lat. $35^{\circ} 38^{\prime}$, Long. $109^{\circ}$ $12^{\prime}$; orig., Hsi-tao 習道; 2nd Wei, Chung-pu | 部; Sui, Nei-pu 內部.

1548 Chnng-shan |山, see Nos. 487, 3969, 6440.

1549 Chung-shon-chiang | 受 降,' (obs.) T'ang, fort in Fêng 豐 C.

1550 Chung-so | 所, T-S. near Yen-yüan H., Ssŭch' uan.

1551 Chung-su | 䈹, see No. 1261.

1552 Chung-t'ang | 堂, ssŭ in 'Tung-kuan H., Kuangtu1ng.

1553 Chung-tien | 佃 Chung[409] tien, T. in Li-chiang Fu, Yünnan; Lat: $27^{\circ} 32^{\prime}$, Long. $100^{\circ} 08^{\prime}$; Thibetan name Djiedan [Baber].

1554 Chung-tsang | 藏 Central Thibet; see No. 2502.

1555 Chung-ts' ao | 曹, T-S. near Ch'ing-chên H., Kueichon.

1556 Chung-tso So / 左所, see No. 2571,
Chung.

1557 Chung-ts'un | 村.

$1^{\circ}$ Chên near T'un-liu H., Sliansi.

$2^{\circ}$ Chên near Yi-ch' êng H., Shansi.

$3^{\circ}$ T-S. near Mien-ning H., Ssŭch tuan.

1558 Chung-tı | 都.

$1^{\circ}$ (obs.) N.W. of $\mathrm{P}^{\mathrm{i}}$ ingyao H., Sliansi; Han and $N$. Wei, H. in T'ai-yüan 太原 chiun. $2^{\circ}$ See No. 7016.

1559 Chnung-wei | 衛 Chungwei.

$1^{\circ} \mathrm{H}$. in Ning-hsia $\mathrm{F} u$, Kansulh ; Lat. $37^{\circ} 35^{\prime}$, Long. $100^{\circ} 05^{\prime}$.

$2^{\circ}$ Chên near Yi-ch'êng H., Shansi.

\section{忠}

1560 Chung 忠 Chung.

[528] $1^{\circ}$ C.I.C. in Ch 'nan-tung circ., Ssĭch'uan; Lalt. $30^{\circ} 28^{\prime}$, Long. $108^{\circ}$; orig., in K. Pa 巴; Han, Lin-chiang 監江; Sui, Lin 臨 C.; T ang, Clining $\mid \mathrm{C}$.

$2^{\circ}$ T-C. in Nan-ning Fin, Kuangsi ; Lat. $22^{\circ} 26^{\prime}$, Long. $107^{\circ} 27^{\prime}$.

$3^{\circ}$ (obs.) formerly C., W. of Ting-an H., Kuangtung. 
中 Chung-ch' ing.

1561 Chung-ch'ing | 清 circ., in Corea.

1562 Chung-hsiao | 孝, sš̌ near Li-ch' uan H., Hupeh.

1563 Chung-lu | 路, post-town near Li-ch nan $H$. ., Hupelı.

1564 Chung-miao | 廟, chên near Ying-shan H., Anluii.

\section{畩}

1565 Chung-tê-li-k'ê 婊得里 克 Gundelik, town in Yarkand; Lat. $37^{\circ} 40^{\prime}$, Long. $79^{\circ}$.

\section{重}

1566 Chunng-chên | 鎭, see Nos. 55, 2282, 7515.

1567 Chung-ch'iu | 邱, see No. 5679.

1568 Chung-ch'üan | 泉, see No. 5314.

\section{鍾}

1569 Chnnng-ch'i 鍾溪, chên near Chin-kuei H., Kinngsu.

1570 Chung-hsiang | 硉Clın1gsiang, $H$. forming the prefectural city of An-1u, Hupelı; Lat. $31^{\circ} 0 \bar{\iota}^{\prime}$, Long. $112^{\circ} 39^{\prime}$.

1571 Chung-li | 離. $1^{\circ}$ (obs.) $20 l i \mathrm{~N}$. E. of Fêng-yang Fu, Anhui; Han, H. in Chinchiạng 北 江. chïn.
絡 Chung.

$2^{\circ} 20$ li N.N.E. of Fêngyang Fu, Anluui ; Tsin, H. in Huai-nan 淮諵 chïn; S. Sung and $S$. $T s^{\prime} i$, chïn in Hsii 徐 crov; Sui, chion and H. in Yang 揚 curou; T'ang, Sunng and Yüan, H. in Hao 濠 C.

$3^{\circ}$ (obs.) S. E. of T'ang H., Honnil ; N. IV ci, H. in Hsi-hniai 西淮 chïn.

$4^{\circ}$ (obs.) $T_{s}^{\prime}$ in and $H_{a n}$, feudal state in Anhui.

$5^{\circ}$ See No. 1731.

1572 Chnnng-ling! 陵, sec No. 1072 .

1573 Chning-shan | II, Chungslian, $T$. in P'ing-lè Firu, Kunngsi, near $\mathrm{Ho} \mathrm{H}$.

1574 Chnng-tai | 帶, chênn near P'ing-hu H., Chehkiang.

1575 Chung-wu|吾.

$1^{\circ}$ Post-town near Silch'ien H., Kianirsus.

$2^{\circ}$ (obs.) formerly feudal state in Kiangsu.

$3^{\circ}$ See No. 5915.

1576 Clunng-wu 鍾武, (obs.) Han, town W. of Hêngyang $\mathrm{H}$., Hunan.

\section{終}

1577 Chung 終, (obs.) Five $d y^{\prime \prime}$., C. 


\section{終 ChUNG-NAN.}

1578 Chung-nan | 南.

$1^{\circ}$ Chên near Hsing-p ing H., Shensi.

$2^{\circ}$ (obs.) $W$. Wei, H., E. of Chou-chilı .H., Shensi.

\section{鐘}

1579 Chung-cl' êng 鐘城, Fu in Hsien-ching 咸 鏡 circ, Corea.

1580 Clnung-ch'i | 溪 ssŭ, in Yi-hnsing H., Kiangsu.

1581 Chung-ming | 鳴, chên near Mêng-ch' êngr $H$., Anluni.

Chn11ng-kiang, see No.1532. Chung-king, see No. 1583. Chung-nuan, see No. 1546. Chung-siang, see No. 1570.

\section{CHUNG}

\section{重}

1582 Cl'nung-an 重发.

$1^{\circ}$ Ss ̌̆ near Huang-p ing C., Kueichon.

$2^{\circ}$ (obs.) $80 l i \mathrm{~S} . \mathrm{W}$. of Hêng-yang H., Hunan; E. Han, Marq. in Lingling 零陵 chïn; Tsin and $S$. Sung, H. in Hêng-yang 衡陽 chün; S. $\mathcal{T} s^{6} i, \mathrm{H}$. in Hsiangtung 湘東 chün。

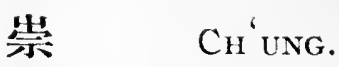

$1583 \mathrm{Ch}$ ' ung-ch'ing/展 Chung[528] king, $\mathrm{Fu}$ in the $\mathrm{Ch}^{\text {'uan- }}$ tung circ., Ssŭch'uan; forming also the district city of $\mathrm{Pa}$; Lat. $29^{\circ} 34^{\prime}$, Long. $106^{\circ} 50^{\prime}$; Chou, in K. $\mathrm{Pa} 巴 ; s^{\prime} i n$ in $\mathrm{Pa}$ 巴 chion; Han, 1st Chiang 江 C.; 2nd Yung-ning $\overrightarrow{\text { 水 }}$ 軍; Liang, Ch 1 楚 C.; Sui, Ÿ̈ 渝 C.; T'ang, Nan1-p'ing 南㝋; Sung, Ch' ing-ch'ing | 愿.

$1583^{\star} \mathrm{Ch}^{\prime}$ 'ung-ch ‘ïan | 泉, see No. 5314.

\section{充}

$1584 \mathrm{Ch}$ 'ing 充.

$1^{\circ}$ (obs.) Han, fendal state in Ssŭch uan.

$2^{\circ}$ (obs.) W. of An-fu H., Hunan; Han, H. in Wu-ling 武 陵 chiön; Tsin, H. in 'T'ien-mên 天門 chiun.

$3^{\circ}$ (obs.) near An-hula H., Kuei-clon ; $T^{6} a n g$, C. in Cliang-nan 江 南 circ.

$4^{\circ}$ See Nos. $4568,4617$.

$$
\text { 览 }
$$

$1585 \mathrm{Cl}^{\prime} \mathrm{ung}$ 崇.

$1^{\circ}$ (obs.) Yin, K. in Shensi.

$2^{\circ}$ (obs.) Liang, C. near Yao C., Shensi.

$3^{\circ}$ See Nos. 2178, 5597. 
崇 $\mathrm{CH}^{6}$ UNG-AN.

1586 Ch'ung-an | 安 Tsung[306] ngan, H. in Clicn-ning Fur, Fullkien ; Lat. $27^{\circ}$ 46 , Long. $118^{\circ} 08^{\prime}$; Five dyn.,; Wên-ling 溫 領 chên Sung, Ch'ung-an | 发:

1587 Ch'nung-chêng | 正:, chên near Fu-fêng H., Shensi.

$1588 \mathrm{Cl}^{\prime}{ }^{\prime}$ nng-ch'i | 潆, chên near Cli'ien-shan H., Kiangsi.

$1589 \mathrm{Cl}^{\prime}$ ungr-ch'ing / 慶 Tsungking.

[307] $1^{\circ} \mathrm{C}$. in Ch'êng-tu Fiv, Ssŭch'nan1; Lat. $30^{\circ}$ $36^{\prime}$, Long. $103^{\circ} 43^{\prime}$; Han, Chiang-yïan 江 原; S. Ts $i$, Chin$\mathrm{k}^{\prime}$ ang 晋康； Sui， Chin - yüan 晋 原; Tang, Shu1 置 C.; Sung, Ch'nng-ch'ing | 度。

$2^{\circ}$ (obs. S. Sung, Fu in Cl'êng-tı 成都 circ.

$1590 \mathrm{Ch}^{\prime}$ 'ung-hsin | 信 Tsungsin, H. in Cling C., Kansulı ; Lat. $35^{\circ} 19^{\prime}$, Long. $107^{\circ} 02^{\prime}$.

$1591 \mathrm{Ch}^{\prime}$ 'ung-jên | 仁 Tsungji11.

$1^{\circ} \mathrm{H}$. in Fi1-chou $\mathrm{F} u$, Kiangsi; Lat. $27^{\circ} 44^{\prime}$, Long. $116^{\circ}$; E. Han, Lin-ju 臨 汝; Wu, Hsin-chien 新 建;
崇 CH'UNG-SHUN.

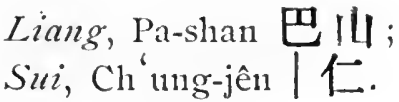
$2^{\circ}$ Chên near Ch'êng H., Chelikiang.
$3^{\circ}$ (obs.) Tang, H., S. W. of I.i-p 11 H. Kuangsi.

1592 Ch'nng-1u | 路, T-S. near Sung-p'an 'T., Ssŭch'nan.

$1593 \mathrm{Ch}^{6}$ inng-ming | 明 Tsungming, H. in T'ai-ts'ang C., Kiangsi ; I,at. $31^{\circ} 36^{\prime}$, Long. $121^{\circ} 19^{\prime}$; orig., Hai-mên 海 阴; Yüan, $\mathrm{Cl}^{\prime}$ 'ung-ning | 明.

$1594 \mathrm{Cl}^{6}{ }^{\prime} 1111 \mathrm{~g}-11 \mathrm{ing}$ | 察 l'sungning.

$1^{\circ} \mathrm{H}$. in Cln êng-tu Fu, Ssŭch'uan; Iat. $30^{\circ}$ $55^{\prime}$, Long. $103^{\circ} 53^{\prime}$; T'ang, 'T'ang - ch' ang 府昌; Five dyn., Kuei-luna 站化; Sung, Cl' 'ung-ning | 窎。

$2^{\circ}$ Chên near San-yüan H., Shensi.

$3^{\circ}$ Chên near Wei-yüan H., Kansul1.

$4^{\circ}$ Post-town near Huochia H., Honan.

$1595 \mathrm{Cl}^{6}$ ung-shan | 美: Tsungshen, $H$. forming the prefectural city of 'T'ai-p'ing, Kuangsi ; Lat. $22^{\circ} 25^{\prime}$, Long. $107^{\circ} 07^{\prime}$.

$1596 \mathrm{Ch}$ ung-shun | 順, ssü near Lai-pin H., Kuangsi 
崇

Ch'ung-wu.

1597 Ch'ung-wu | 武, see No. 3160 .

$1598 \mathrm{Cl}^{\prime}$ 'ung-yang | 陽 Tsungyang, H. in Wu-ch'ang Fu, Hupeh; Lat. $29^{\circ} 34^{\prime}$, Long. $113^{\circ}$; $\operatorname{Han}$, Hsiatsun 下售; T' Rng, 'Tsun 焦 C.; Five dyn., Cli' ungyang | 陽.

$1599 \mathrm{Ch}^{\prime}$ 'ung-yi | 載, Tsung-i, $\mathrm{H}$. in Nan-an Fu, Kiangsi; Lat. $25^{\circ} 46^{\prime}$, Long. $113^{\circ}$ $02^{\prime}$.

\section{沖}

1600 Ch'ung-shêng 蛙絙 ken in Japan.

Chusan, see No. 6447.

Chwang-lang, see No. 1496.

Ciangan, see No. 699.

Cobdo, see No. 3231.

Cobdo Khanate, see No. 3231.

Cochin China, see No. 786.

Coigan-ju, see No. 2244.

Constantinople, see No. 591.

Corea, see No. 363.

Cotan, see No. 2057.

Cui-ju, see No. 3476.

Cuncun, see No. 1950.

Dalny, see No. 6054.

Damascus, see No. 6366.

Damegan, see No. 6149.

Damietta, see No. 6212.

Daran, see No. 6365.
Demavend, see No. 6052.

Derbend, see No. 6613.

Detsin, see No. 6308.

Dilem, see No. 6349.

Djakchin, see No. 128.

Djalaid, see No. 130.

Djambalik, see No. 224.

Djarud, see No. 131.

Djaya, see No. 135 .

Djend, see No. 168.

Djiedam, see No. 1553.

Djumba, see No. 4529.

Djuzdjan, see No. 1357 .

Dodon, see No. 6480.

Dolonnor, see No. 6475.

Dsassaktu, see No. 139.

Dsungaria, see No. 1509.

Durban Keuked, see No. 5892.

Durgen, see No. 6614.

Dzacharkang, see No. $6503^{\mathrm{A}}$.

Dzangamrin, see No. 5556.

Dzindzilik, see No. 1067.

Dzing, see No. 1174.

\section{$\hat{E}$ \\ 額}

1601 Ê-ku-tê 額古德 Egudenkalka, town in Tsetsen ; Lat. $47^{\circ} 30^{\prime}$, Long. $111^{\circ}$.

$1602 \hat{\mathrm{E}}-1 \mathrm{lu}-\mathrm{t}^{\prime} \mathrm{e}$ | 魯特 Oelots or Elenths, Mongol Tribe.

\section{訛}

1603 Ê-ta-la 訛打刺, see next. 
訛

$\hat{\mathrm{E}}-\mathrm{T}^{\prime} \mathrm{A}-\mathrm{L}, \mathrm{A}$.

1604 Ê-t a-la | 塔刺 (or Ê-ta-la 打剌. or Wu-t'i-la-êrh 儿提刺耳, or Wo-t'o-loêrh 幹脱纙兒), Otrar, a tow11 in Khokand, New Dominion.

1605 E-yi-han | 跡邗 Uzgend, a town in Kliokand, New Dominion.

Eguden-kalka, see No. 1607.

Egypt, see No. 4415.

Ehime, see No. 43.

Eleuths, see No. 1602.

Emil, see No. 7339.

\section{$\hat{E} \mathbf{N}$}

\section{恩}

1606 Ên 恩 Ngăn.

$1^{\circ} \mathrm{H}$. in Tung-ch ang $\mathrm{Fu}$, Shantung; Lat. $37^{\circ}$ $23^{\prime}$, Long. $116^{\circ} 20^{\prime}$; Han, Cl ing-lio 清河; $P$. Chou, Pei 具 C.; Sung, Kin and Yüan, Ên | C.; Ming, H. in Kao-t'ang 高唐 C. $2^{\circ}$ (obs.) N. of Ên-p ing H., Kuangtung; Tang, C. in Ling-nan 嶺南 circ.

$3^{\circ}$ - (obs.) in Shingking; Liao, C. in Ta-ting 大定 $\mathrm{Fu}$.

$4^{\circ}$ See Nos. $1245,7252$.

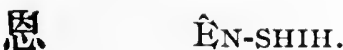

1607 Ên-an | 安 Ngăn-11gan, $\mathrm{H}$. forming the prefectural city of Clrao-t'ung, Yünnan; Lat. $27^{\circ} 20^{\prime}$, Long. $103^{\circ} 50^{\prime}$.

1608 Ên-clı' êng | 城, (obs.) T'ang, C. 250 li N. of T'ien T-C., Yünnlan.

1609 Ên-chin | 勤, (obs.) T"ang, H., E. of Chaop'ing H., Kuangsi.

1610 Êt1-ching | 津 H. in Chung-ch'ing 忠清 circ., Corea.

1611 Ên-fêng | 封, (obs.) in Kuangsi ; T'ang, H. in Yen 硕 $\mathrm{C}$.

1612 Ền-1ê | 樂 Ngăn-loh, (obs.) formerly $\mathrm{H}$. in Chên-yüan Fu, Yünnan; Lat. $24^{\circ} 10^{\prime}$, Long. $101^{\circ}$ $05^{\prime}$.

1613 Ên-lung | 隆 Ngăn1-lung, $\mathrm{H}$. in Ssŭ-ên Fù, Kuangsi. Lat. $23^{\circ} 42^{\prime}$, Long. $106^{\circ}$ $38^{\prime}$; formerly T'ien 田 C. Reduced in rank to a District in 1873. It lies a short way to the N.W. of Fêng-Y C., Kuangsi.

1614 Ên-na 納, town in the Lit1-ch iu Islands.

1615 Ền-p'ing | 平 Ngăn-ping, [444] H. in Chao-ch'ing $\mathrm{Fu}$, Kuangtung; Lat. $22^{\circ} 06^{\prime}$, Long. $112^{\circ} 14^{\prime}$.

1616 Ên-shih | 施 Ngăn-shi, $\mathrm{H}$. forming the prefectural 
因 ÊN-SHIH.

city of Shih-11an, Hupeh ; Lat. $30^{\circ} 16^{\prime}$, Long. $109^{\circ}$ $26^{\prime}$; Tsin, and S. Sung, Sha-ch' it 沙渠 H.; Sui, Ch'ing-chiang 清江 H.; T'ang, and Sung, Shih 施 C. and Ch'ing-chiang 清江 H.; Ming, Shih 施 C. and Wei, in Hukuang. 1617 Ên-yen | 晏, (obs.) formerly H. near Hsing-wên H., Ssŭch'nan.

\section{ÊRH}

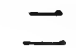

1618 Êrrh-lung-tu 二龍渡 ssü

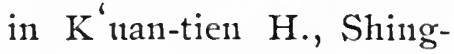
king.

1619 Êrh-p' $\mathrm{t}$ |舖.

$1^{\circ} \mathrm{Y}$. near Yüan-wu $\mathrm{H}$, Honan.

$2^{\circ}$ Post-town near Weining C., Kneichou.

\section{玶}

1620 Êrrli-ling 珥陵, chên near Chin-t'an H., Kiangst.

\section{婼}

$1620^{A}$ Êrh-ch' iang 婼 党, H. in Yen-ch'i Fu, New Dominion.

Esmok, See No. 5866.
繁

FAN.

\section{FA}

\section{法}

1621 Fa-kuo 法 國, France; also Fa-lan-hsi | 監西, or 蘭西, or Fa-lang-hssi |浱西。

$1621^{\mathrm{A}} \mathrm{Fa}-y 1_{11}$ |因, or 發因, or 没銀、Cain, a city S. W. of Herat in Afghanistan.

\section{"翟}

1622 Fa-yin 留 因, see No. $1621^{\mathrm{A}}$.

1623 Fa-yin | 銀, see No. $1621^{\mathrm{A}}$.

$162+$ Fa-ying-ch'êng | 英城, (obs.) formerly town in Kuangsi.

1625 Fa-yı | 油, chên near Tzŭ C., Ssŭch'uan.

\section{FAN}

凡

1626 Fa11-ch'a 凡蚠, (obs.) Han, town near Ling C., Kansulı.

1627 Fan-ch'êng | 城, see No. 2373.

\section{繁}

1628 Fan 繁.

$1^{\circ}$ (obs.) N. E. of Hsinfan H., Ssŭch' '1an; Han, Ts in, S. Sung, and $S . T^{*} s i, \mathrm{H}$. in Shu 罢 chün.

$2^{\circ}$ See No. 2749. 
慗

FAN-AN.

1629 Fanl-an 安, (obs.) near $\mathrm{Cl}^{\prime}$ ing-chou. Fin, Shantung; $W$. Han, Marq. in Ch'ien-ch'êng 千乘 chiün.

1630 Fan-ch'ang | 昌 Fanchang, H. in "' "ai-p ing Fu, Anlhui; Lat. $31^{\circ} 08^{\prime}$, Long. $118^{\circ} 26^{\prime}$; Han, $\mathrm{Ch}^{\prime} \mathrm{un}^{\mathrm{k}} \mathrm{ku}$ 春踒; Tsin, Fan-ch'ang |昌; Liang, Nan-ling 南陵.

1631 Fan-chih | 诗, Făn-chi, H. in Tai C., Shansi; Lat. $39^{\circ} 12^{\prime}$, Long. $113^{\circ}$ $16^{\prime}$; Han, Fan-chil | 時; E. Wei, Wu 武 C.

1632 Fanl-sluni | 水, see No. 4596.

\section{訤}

1633 Fan 焚.

$1^{\circ}$ (obs.) $60 l i \mathrm{~S} . \mathrm{W}$. of Tzŭ-yang H., Shan-. tung; $W$. Han, H. in K. Tung-p'ing 東平; E. Han, H. in Jênclı'êng 任城 chïn.

$2^{\circ}$ (obs.) in Kiangsu; $S$. $T s^{\prime} i$, H. in Nan-lu 南 惫 chïn.

$3^{\circ}$ (obs.) in Lïi-chou $\mathrm{Fu}$, Anhui ; S. Tis $i$, H. in Nan Jn-yin 南湤陰 chiin.

$4^{\circ}$ (obs.) in Ssŭch'uan; $T^{\prime}$ ang, H. in Chiangnan 江南 circ.
FAN-SHUI.

1634 Fan1-cli'a | 汉, chen near Hsing-hua H., Kiangsu.

1635 Fan-ch'êng | 城.

$1^{\circ}$ Post-town near Hsingyang Fu, Hupeh.

$2^{\circ}$ (obs.) formerly town

near Hsiang-yang $\mathrm{F} u$, Hupeh.

1636 Fan-ch'iao | 橋, chênnear Lin-chin H., Shansi.

1637 Fan-ch' uan | J, see No. 2691.

1638 Fan-11sing | 與, see No. 1265.

1639 Fan-yï| 舆, (obs.) S. E. of Cl' ing-yüan H., Chihli ; $W$. Han, Marq. in Cho 溷 C.

\section{范}

1640 Fan 范 Fan, H. in Ts'aochou Fu, Shantung; Lat. $36^{\circ}$, Long. $115^{\circ} 45^{\prime}$; Han, Fan.

1641 Fan-ts' 11 | 村 chen in T'ai-ku H., Shansi.

1642 Fan-yang | 陽, (obs.) A.D. 221-907, chün in Chihli; see also Nos. $1324,1665,5822,5825$, 6452 .

\section{蕃}

1643 Fan 蕃, see No. 6336.

\section{畨}

1644 Fan-shui 水, see No. 4926. 
方 FAN-CHANG.

Fan-chang, see No. 1630.

Fan1-yü, see No. 4927.

Făn-chau, see No. 1688.

Făı11-chi, see No. 1631.

Fă11-i, see No. 1686.

Făn-shwui, see No. 1684.

Făn-si, see No. 1691.

Făn-yang, see No. 1693.

\section{FANG}

\section{方}

1645 Fang 方, see No. 7453.

1646 Fang-ch" êng | 城, see Nos. 3281, 7698.

1647 Fang-chia-yen | 家堐, sš̆ near Yi-ch' êng H., Hupeh.

1648 Fang-ch'iaol憍, chèn near Yii-yao H., Chehkiang.

1649 Fang-ch'ü|渠, see No. 2266.

1650 Fang-fan | 番, T-S. in Ting-fan C., Kueichou; Ming, ssü.

1651 Fang-hsiang | 相, chen near Lo-ch' nan H., Shensi.

1652 Fang-shan | Il, chên near Lan H., Shansi.

1653 Fang-ts'un | 村.

$1^{\circ}$ Chên near Wu-hu H., Anhui.

$2^{\circ}$ Post-town near Li-po H., Kueichou.

1654 Fang-wei | 維, (obs.) 220 li N. E. of $\mathrm{P}^{\text {ing-wu H., }}$ Ssŭch'uan; Sui, H. in P'ing-wu平武 chün.

1655 Fang-yi | 義, see No. 5059.
房

FANG-LI.

\section{坊}

1656 Fang 坊, chèn near Hoyang H., Shensi.

1657 Fang-hu| 湖 chen in $\mathrm{Cl}^{\prime}$ ien-shan H., Kiangsi.

1658 Fang-t'ou | 䫓, chèn near $T$ 'ung-chou $F u$, Shensi.

\section{芳}

1659 Fang-ch'ih 芳池.

$1^{\circ}$ (obs.) near Ning-hsia Fu, Kansuh; $T^{b}$ ang, $\mathrm{Fu}$ in Kuan-11ei 關 內 circ.

$2^{\circ}$ (obs.) ne $\mathrm{Cl}^{\text {Ie }} \mathrm{Ch}^{\text {ing- }}$ yang. Fiı, Kansuh; $T^{6}$ ang, Fu in Kuan-nei 礔 队 circ.

1660 Fang-lan | 蘭, chên near Ting-hsiang H., Shansi.

\section{房}

1661 Fang 序 Fang.

[313] $1^{\circ} \mathrm{H}$. in Yün-yang $\mathrm{Fu}$, Hupeh; Lat. $32^{\circ} 01^{\prime}$, Long. $110^{\circ} 42^{\prime}$; orig., K. Tan-chu f 朱; Han, Fang-ling | 陵; P. Chou, $\mathrm{Cl}^{\prime}$ ien 遥 $\mathrm{C}$.; $T$ ang, Fang | C. $2^{\circ}$ (obs.) S. Sung, C. in Ching-hsi 京西 circ.

1662 Fang |, chên near $\mathrm{Ch}^{\prime}$ angshan H., Shantung.

1663 Fang-li | 里, shê near Tan-shui H., Fuhkien. 
房 FANG-LING.

1664 Irang-ling | 陵, see No. 1661.

1665 Fang-shan | II Fangshan.

[341] $1^{\circ}$ H. in Shun-t ien Fu, Chillili Lat. $39^{\circ} 33^{\prime}$, Long. $116^{\circ} 03^{\prime}$; orig., Fan-yang 范陽; Kin, Fêng-hsien 奉 先; Yïan, Fang-shan |山. $2^{\circ}$ See No. 5179.

1666 Fang-tzŭ $\mid$ 子.

$1^{\circ}$ (obs.) S. A., K. i11cluding Sui-p'ing $H$. , Honan.

$2^{\circ}$ See No. 5964.

1667 Fang-tzŭ-yi |子邑, see No. 3842.

$1667^{\mathrm{A}}$ Fang-ch'êng 防城, H. in Ch'in C.L.C., Kuangtung. Formerly Fang-kung 方 可 $\mathrm{Y}$.

\section{防}

1668 Fang-fêng-shilı 防風氏, see No. 7073.

1669 Fang-kung | 功, see No. $1667^{\mathrm{A}}$.

1670 Fang-t'ing | 亭, see No. 5689.

1671 Fang-tung | 東, (obs.) S. W. of Chin-hsiang H., Shantung; E. Han, H. in Shan-yang 山陽 chiin.

Fâtshau, see No. 1779.

Fau, see No. 1780.

Fau-ching, see No. 1783.

Fant-kang, see No. 1784.
子 FEI-TZÜ.

Fau-liang, see No. 1793. Fau-ning, see No. 1786. Fan-ping, see No. 1787. Fall-shan, see No. 1794. Fall-yang, see No. 1789.

\section{FEI}

\section{腢}

1672 Fei-ch'êng 肥城 Fi-ching, H. in 'T'ai-an Fu, Shantung; Lat. $36^{\circ} 20^{\prime}$, Long. $116^{\circ} 53^{\prime}$; orig., K. Feitzŭ |子; Han, Fei-ch'êng 城.

1673 Fei-hsiang | 拫 Fi-hiang, $\mathrm{H}$. in Kuang-p'ing $\mathrm{F} u$, Chihli ; Lat. $36^{\circ} 40^{\prime}$, Long. $115^{\circ} 02^{\prime} ; \operatorname{Han}, \mathrm{P}^{\prime} \mathrm{u}$ 蒲; Wei, Fei-hsiang | 拫. 1674 Fei-ju | 如.

$1^{\circ}$ (obs.) $30 l i \mathrm{~N}$. of Lulung H., Chihli ; Han, H. in Liao-hsi 逰西 chün.

$2^{\circ}$ (obs.) N. of Lil-lung H., Chihli ; $T \sin$, and $N$. Wei, H. in Liao-hsi 遼西chün。

1675 Fei-lieh | 罚 or 累, (obs.) 7 li S. W. of Kao-ch'êng H., Chihli ; W. Han, H. in K. Chên-ting 兵定.

\section{Fei-tzŭ|子.}

$1^{\circ}$ (obs.) formerly $\mathrm{K}$. comprising Fei-ch'êng H., Shantung.

$2^{\circ}$ See No. 1672. 
淝

FEI-Shui.

淝

1677 Fei-shui 淝水, see No. 4374.

\section{飛}

1678 Fei 飛, (obs.) in Ssŭch' nan; T ang and Sung, Fei $\mid C$.

1679 Fei-1111-koul 狐口, see No. 3389.

1680 Fei-niao | 鳥, (obs.) Sui, H., S. E. of Chnng-chiang H., Ssŭch 'ran.

1681 Fei-wu | 鳥, another version of the preceding, and more correct.

\section{廢}

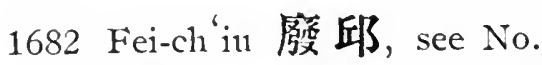
2829.

\section{FÊN}

\section{分}

1683 Fên-ning 分管, see No. 7458.

1684 Fên-shui | 水 Făn-shwui, $H$. in Yen-chon Fu, Chelnkiang; Lat. $29^{\circ} 59^{\prime}$, Long. $119^{\circ} 20^{\prime}$.

1685 Fên-yen | 尶, ssŭ in Chien-li H., Hupeh.

1686 Fên-yi | 宜 Făn-i, H. in [552] Yüan-chon Fin, Kiangsi; Lat. $27^{\circ} 46^{\prime}$, Long. $114^{\circ}$ 43'; Han, Yi-ch' 111 春 宜; Sung, Yüan and Ming, Fên-yi | 宜 H.
汾 FÊN-YANG.

\section{汾}

1687 Fên 汾, see Nos. 583, 1688, 2098, 7255.

1688 Fên-chou | 州 Făn-chau, [543] Fu in the Chi-ning circ., Shansi; forming also the district city of Fên-yang; Lat. $37^{\circ} 19^{\prime}$, Long. $111^{\circ}$ $41^{\prime}$; S.A., $1 s t$ in $\mathrm{K}$. Tsin 晋, $2 u d$ in $\mathrm{K}$. Wei 魏; C. Wars, in K. Chao 趙; $T s^{\prime}$ in and Han, T' $\mathrm{T}^{\prime}$ ai-yüan 太原; Three Ks., Hsi-ho 西河; 2nd $\mathrm{Wei}$, Fên

C.; N. Ts'i, Nan-so 南 䍭; P. Chou, Chieh 介 C.; 7'ang, Hao 浩 C.; Sung, Fên-yang | 陽; Ming, Fên-chou ? 州 Fu.

1689 Fên-ch' P. Chou, H., E. of Yich' uan H., Shensi.

1690 Fên-hu| 湖, chên near Wu-chiang H., Kiangsu.

1691 Fên-hıi | 西 Făn-si, H. [123] in P'ing-yang $\mathrm{Fu}$, Shansi ; Lat. $36^{\circ} 40^{\prime}$, Long. $111^{\circ}$ $32^{\prime}$; Han, Chil 猒 H.; 2nd Wei, Lin-fên 臨汾; Sui, Fên-hsi | 西.

1692 Fên-shni | 水.

$1^{\circ}$ Chên near Yen-ch'ang H., Shensi.

$2^{\circ}$ Post-town near Hsinch êng H., Chihli.

1693 Fên-yang | 陽 Făn-yang. 
汾

$1^{\circ} \mathrm{H}$. forming the prefectural city of Fênchou, Shansi ; Lat. $37^{\circ}$ $19^{\prime}$, Long. $111^{\circ} 41^{\prime}$.

$2^{\circ}$ Post-town near the preceding.

$3^{\circ}$ See Nos. 2814, 3602.

1694 Fên-yin | 陰, see Nos. 3048, 6894.

\section{焚}

1695 Fên 焚, (obs.) in Ssŭch'uan; Sung, C. in Shaohsing 紹興 Fu.

1696 Fên-lon 艏, chên near

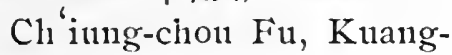
tung.

\section{坟}

1697 Fên-t'ai 坆台, chên near Yeh H., Honan.

\section{FÊNG}

\section{封}

1698 Fêng 封.

$1^{\circ}$ (obs.) Sui, C. comprising Fêng-ch' uan H., Knangtung.

$2^{\circ}$ See Nos. 1701, 5159.

1699 Fêng-ch“i 谿, (obs.) in Annam; E. Han, H. in Chiao-chih 交趾: chïn; $T s i n$, and $S$. Ts $i, H$. in Wu-p ing 武平 chün。

1700 Fêng-ch iu | 邱 Fungkiu, H. in Wei-hui $F u$, Honan; Lat. $35^{\circ} 05^{\prime}$, Long. $114^{\circ} 37^{\prime}$; orig., $\mathrm{K}$.
風 FÊNG-CHING。

Fêng-fu | 㕮; Han, Fêngch'iu | 邱, and $\mathrm{P}$ 'ingchin 平邱。

1701 Fêng-ch' 11an | Jí Fêng[416] chuen, H. in Chao-ch 'in

Fu, Kuangtung; Lat. 23 25', Long. $111^{\circ} 03^{\prime}$; Han, Kuang-11sin 算信; Liang,

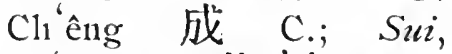
'T's'ang-wu 蒼梧 chün; T'ang, Fêng | C.

1702 Fêng-fu| 父, see No. 1700.

1703 Fêng-hsiang | 拫, (obs.) Han, H. 100 li E. of Ho H., Knangsi.

1704 Fêng-lising | 與, (obs.) Tsin, town $\mathrm{N}$. of Fêngch' uan H., Kuangtung.

1705 Fêng-lê | 樂, (obs.) $T \sin , \mathrm{H} .$, W. of Hsin-hui H., Knangtung.

1706 Fêng-p'ing | 平, (obs.) Sung, H., W. of Hsin-hui H., Kuangtung.

1707 Fêng-shan | II, (obs.) $T s^{6} i$, H., S. W. of Lienchon Fin, Kuangtung.

1708 Fêng-ssŭ $\mid$ 斯, (obs.) 20 li N. W. of Chao C., Chilıli; $W$. Han, Marq. in Ch'ang-shan 常 山 chïn.

1709 Fêng-yang | 陽, see No. 3060 .

\section{風}

1710 Fêng-ching 屈經, chên in Chia-shan H., Chehkiang. 
風 FÊNG-K'ANG.

1711 Fêng-k'ang 風 方, posttown near Ching $\mathrm{H}$., Auhui.

$1711^{A}$ Fêng-ch' ên 祳, see No. 5822.

\section{楓}

1712 Fêng-ch'iao 楀 橋, chên near Chu-chi H. Chehkiang.

1713 Fêng-ching/淡, chên near

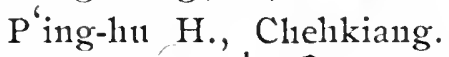

1714 Fêng-hisiang | 香, posttown near T'ai-hn H., Anlini.

$1714^{\wedge}$ Fêng-pi | 陛, see No. 5822.

171.5 Fêng-t'ing | 家, ssŭ near Hui-an H., Fuhkien.

\section{鳳}

1716 Fêng | 碱 Fung.

[330] $1^{\circ} \mathrm{H}$. in Han-chung Fu, Shensi ; Lat. $33^{\circ} 55^{\prime}$, I,ong. $106^{\circ} 42^{\prime} ; T \sin$, Lung-hsi 隌西; 2nd $W e i$, Liang-ch' üan 梁 泉; E. Wei, Fêng | C., Sui, Ho-ch'ih 河 池 chïn.

$2^{\circ}$ (obs.) S. Sung, C. in I,i-chon 利 州 circ.

1717 Fêng-ch'êng ! 城, posttown near $\mathrm{Ch}$ ao-chou $\mathrm{Fu}$, Kuangtung. See also No. 5822.

1718 Fêng-ch'i|棲, post-town near $\mathrm{Yi}$-ch' ang $\mathrm{Fu}$, $\mathrm{Hupeh}$; see also No. 3920 .
鳳 FÊNG-HSIANG.

1719 Fêng-ch'ih | 池, see No. 5822.

1720 Fêng-ch' uan | 川, chên near Ho-shui H., Kansuh.

1721 Fêng-ch' üeh | 閶, see No. 5822.

1722 Fêng-huang | 凰 Funghwang.

[514] $1^{\circ}$ C.I.T. in Ch 'ên-YüanYung-Ching circ., Hunan; I,at. $28^{\circ}$, Long. $109^{\circ} 30^{\prime}$.

$2^{\circ} \mathrm{Y}$. near the preceding. $3^{\circ}$ C. L. T. in Tungpien circ., Shingking. Formerly Fênghuang, Ch'êng| 城 ssü.

1723 Fêng-huang Ch'êng | 圈 城, See No. $1722,3^{\circ}$.

1724 Fêng-lisiang | 翔 Fungtsiang.

[497] $1^{\circ} \quad F u$ in the Fêng-Pin circ., Shensi ; forming also the district city of the same name; Lat. $34^{\circ} 35^{\prime}$, Long. $107^{\circ}$ $50^{\prime} ;$ Chou, land of $\mathrm{Ch}^{\prime} \mathrm{i}$ 岐; S.A., land of $\mathrm{Ch}^{\text {' in }}$ 秦; $T s^{\prime}$ in, in Nei-shih 內史 chün; Han, Yufu-fêng 右扶風; $T \sin$, K. Ch'in 秦; E. Wei, Ch'i-yang 岐晹; Sui, $\mathrm{Ch}^{\prime} \mathrm{i}$ 歧 C.; 7 'ang, Fêng-hsiang | 㓞 and Hsi-ching 㛉京; Five dyn., Kuan-hsi 關西; Ming, Fêng-hsiang ! 翔 Fu. 
鳳 FÊNG-HSIANG.

$2^{\circ} \mathrm{H}$. forming the prefectural city of the sane name, Shensi ; Lat. $34^{\circ}$ $35^{\prime}$, Long. $107^{\circ} 50^{\prime}$; S.A., Ku-yung 古䧹; Ts'in, Yung 㠍; 2nd Wei, Ch'in-p'ing 泰 平; Sui, Fu-fêng 扶 風; $\tau^{\prime} a n g$, Fênghsiang | 翔.

1725 Fêng-kang | 阔 sš̆, in Cl’ 'ung-jên H., Kiangsi.

1726 Fêng-lin | 林, chên near Chiang-shan H., Chehkiang.

1727 Fêng-Pin | 邠, circ. in Shensi, comprising Fênghsiang $F u$ and Pin $C$.

1728 Fêng-shan | U Fungshan.

$1^{\circ} \mathrm{H}$. in $\mathrm{T}^{\prime}$ ai-wan $\mathrm{Fu}$ Fuhkien; Lat. $22^{\circ} 34^{\prime}$, Long. $120^{\circ} 20^{\prime}$; also locally known as $\mathrm{P}^{\prime} \mathrm{i}$ t'ou 埤頭 Petow.

$2^{\circ}$ Post-town near $\mathrm{P}^{\prime} \mathrm{u}$-ch ' $\mathrm{i}$ H., Hupeh.

1729 Fêng-t'ai | 荳 Funng-tai.

$1^{\circ} \mathrm{H}$. forming the prefectural city of Tsêchou, Shansi ; Lat. $35^{\circ}$ $30^{\prime}$, Long. $112^{\circ} 50^{\prime}$.

$2^{\circ} \mathrm{H}$. in Fêng-yang $\mathrm{Fu}$, Anhui; this city serves -also for Shou C.; Lat. $32^{\circ} 34^{\prime}$, Long. $116^{\circ} 43^{\prime}$. 1730 Fêng-t'on | 韻, chên near Fền-hsi H., Shansi.
F̂̀̃G.

1731 Fêng-yang | 晹 Fungyang.

$1^{\circ} \mathrm{F} u$ in the Fêng-Ying circ., Anhui ; forming also the district city of the same name; Lat. $32^{\circ} 54^{\prime}$, Long. $117^{\circ}$ 35'; orig., Tu-shan 罡 山; C. Wars, in $\mathrm{K}$. $\mathrm{Ch}^{\prime}$ ' 楚 and Huai-nan 淮南 chïn; Ts'm, in Chin-chiang 九 江 chion; Han, K. Chungli 鍾離; $N$. T s i $i$, Hsi-cli"n 西楚; Sui, $T^{\prime} a n g$, and Sung, Hao 嫁 C.; Ming, Fêngyang | 隄 Fu.

$2^{\circ} \mathrm{H}$. forming the prefectural city of the same name; Lat. $32^{\circ} 54^{\prime}$, Long. $117^{\circ} 35^{\prime}$.

1732 Fêng-Ying | 穎, circ. in Anhui, comprising Fêngyang Fu, Ying-chou Fu, Lin-an C. and Ssŭ C.

1733 Fêng-yü-hsiang | 牙、拫, T-S. near Lang-ch'iung H., Yünn11n.

\section{蘛}

1734 Fêng 嵦 Fung.

[407] $1^{\circ} \mathrm{H}$. in Su-chou $F u$, Kiangsu ; Lat. $33^{\circ} 46^{\prime}$, Long. $116^{\circ} 50^{\prime} ; T^{\prime}$ in, Fêng-yi | 邑.

$2^{\circ}$ (obs.) Sung, C. near Yü-lin Fu, Shensi. 
$3^{\circ}$ (obs.) Chou, a town near Hsi-an Fn, Sliensi ; a royal residence.

$4^{\circ}$ See No. 4558.

1735 Fêng-an | 安, (obs.) Han, H., S. W. of P' $11-$ chiang H., Chehkiang.

1736 Fêng-chên | 鎮 Finngchin, ' $T$. in 'Ta-t' ${ }^{\prime}$ ng $F 11$, Shansi; Lat. $40^{\circ} 30^{\prime}$, Long. $113^{\circ} 15^{\prime}$.

1737 Fêng-ch'êng | 城 Fungching.

[40] $1^{\circ} \mathrm{H}$. in Nan-ch'ang $\mathrm{F}_{11}$, Kiangsi ; Lat. $28^{\circ} 10^{\prime}$, Long. $115^{\circ} 50^{\prime}$; $W u$, Fin-ch'êng 富城; Tsin, Fêng-ch'êng | 城; Sui, Knang-fêng 展|; Five $d y n$. Wn-kao 無暃.

$2^{\circ}$ (obs.) $\mathrm{Wei}$, town near Ch'ing-chou Fu, Shantung.

1738 Fêng-ch'i | 溪, chên near Pao-k'ang H., Hupelı.

1739 Fêng-ch'uan | 川 Fu, in Huang-hai 黃海 circ., Corea.

1740 Fêng-fu| 阜, chên near Wei-nan H., Shensi.

1741 Fêng-hnan-ch'êng | 莧 城, town in the Lin-ch "iu Islands.

1742 Fêng-hsiang | 拫, (obs.) $T s^{\prime} i$, H. near Ching-mên C., Hupeh.

1743 Fêng-jun | 潤 Fung-jun, H. in 'Tsun-hua C., Chihli ;
Lat. $37^{\circ} 53^{\prime}$, Long. $118^{\circ}$ $05^{\prime}$.

1744 Fêng-ku | 固, chên near Li-ching $\mathrm{H}_{\text {., Shantung. }}$

1745 Fêng-kuo | 國, chên in Li-ching H., Shantung.

1746 Fêng-ning | 富 Fungning.

$1^{\circ}$ H. in Ch'êng-tê Fu, Chihli; Lat. $41^{\circ} 22^{\prime}$, Long. $117^{\circ} 05^{\prime}$.

$2^{\circ}$ T-S. near T11-shan C., Kueichou.

1747 Fêng-shun | 順 Fungshun, $\mathrm{H}$. in $\mathrm{Ch}^{\prime}$ ao-chou Fur, Kuangtung; Lat. $23^{\circ}$ $41^{\prime}$, Long. $116^{\circ} 40^{\prime}$.

1748 Fêng-tê | 德 Fung-teh, Fu in Corea; Lat. $37^{\circ} 15^{\prime}$, Long. $125^{\circ} 48^{\prime}$.

1749 Fêng-tı | 都, see No. 1753.

1750 Fêng-yang | 陽, see Nos. $416,5469^{\mathrm{P}}$.

1751 Fêng-yi | 邑, see No. 1734.

1752 Fêng-yiian | 原, posttown near Wei-nan $H$, Shensi.

\section{辢}

1753 Fêng-tu 哑都 Fung-tu. [528] $1^{\circ}$ H. in Cliung C., Ssŭch'uan; Lat. $30^{\circ} 10^{\prime}$, Long. $107^{\circ} 48^{\prime}$; orig., Chih 枳 H.; Han, $P^{\prime}$ ing-tu 4 都; Sui, Fêng-tu 豊都; $T^{6}$ ang， 
Sunng, and Yïan, Fêngtu豐栺; Ming; Fêngtu䧣都。

\section{傌}

1754 Fêng-ch'êng 罵乘.

$1^{\circ}$ (obs.) Han, H., W. part of Fu-ch' uan $H$., Kuangsi.

$2^{\circ}$ See No. 739.

1755 Fêng-lei | 雷, chên near Pai-shui H., Shensi.

1756 Fêng-yüan | 原, chên near Ch' êng-ch' êng H., Shensi.

\section{綘}

17.57 Fêng-chang 綘 帳, chên near Wu-kung H., Shensi.

\section{篻}

1758 Fêng 等, (obs.) in Annan; T゙ang, C. in Ling-nan 額 南 circ.

1759 Fêng-ch'an-wu-p'ing | 产 武坪, T-S. near Min C., Kansuh.

1760 Fêng-mên | 門, post-town near Ching-yein H., Ssŭch' nan.

1761 Fêng-shih | 市, posttown near I'ung-ting $\mathrm{H}$. Fulıkien.

\section{逢}

1762 Fêng-lung 逢龍, (obs.) N. W. of Cho C., Chihli ; T'ang, H. in Shên 㨧 C.

\section{奉}

1763 Fêng-chieh 奉筒 Funngtsiel, $H$. forming the prefectural city of $\mathrm{K}^{\prime}$ ueichou, Ssŭch' uan; Lat. $31^{\circ}$ $10^{\prime}$, Long. $109^{\circ} 35^{\prime}$; $T s^{\prime}$ in, Yü-fu 俥復; linor Han, Yung-an $\overline{\text { 水 }}$ 安; T'ang, Fêng-chieh 䈟。

1764 Fêng-Chin-Shan-hai | 錦 山海, circ. in Shingking, comprising Fêng-t ien Fu, Chin-chou $F$ and the city of Shan-hai Kuan.

176.5 Fêng-fu | 符, see No. 6176.

1766 Fêng-hua | 化 Fung-hwa, [426] H. in Ning-po Fu, Chehkiang; Lat. $29^{\circ} 43^{\prime}$, Long. $121^{\circ} 26^{\prime} ; T s^{\prime} i n$ and Han, Yin 鄞; T゙ang, Fêng-hua | 化.

1767 Fêng-hsien | 先 see Nos. $1665,5314$.

1768 Fêng-lisien | 賢 Funghien, H. in Sung-chiang Fu, Kiangsu ; I at. $29^{\circ}$ $50^{\prime}$, Long. $121^{\circ} 19^{\prime}$.

1769 Fêng-hsin | 新 Fung-sin. [124] $1^{\circ} \mathrm{H}$. in Nan-ch'ang Fu, Kiangsi ; Lat. $28^{\circ} 41^{\prime}$, Long. $115^{\circ} 19^{\prime}$; Han, Hai-hun 海 昏; E. $H a n, H \sin -w u$ 新吳; S. T'ang, Fêng-hsin | 新. 
$2^{\circ}$ Post-town near Chinghai H., Chinli.

1770 Fêng-kn1o | 國, (obs.) Liang, H., E. of 'Ts'angch'i H., Ssŭcl' "uan.

1771 Fêng-1ning | 明, (obs.) Han, H., N. of Hsi-an $\mathrm{Fu}$, Shensi.

1772 Fêng-shêng | 仼, see No. 4947.

1773 Fêng-t'ien | 天 Fungtien, or Moukden, or Shingking.

[158] $1^{\circ} \quad F_{11}$ in the Fêng-ChinShan-hai circ., Shingking; also known as Shêng-ching 盛 京; forming the district city of Ch' êng-tê; Lat. $41^{\circ}$ $51^{\prime}$, Long. $123^{\circ} 38^{\prime}$; capital of the province; orig., Shên 澢 C. and Shên-yang 潘隄; also known as Shêng-yang 盛陽。

$2^{\circ}$ See No. 920 .

1774 Fêng-yi | 議 Fung-i, C. in Chên-an Fur, Kuangsi ; Lat. $23^{\circ} 42^{\prime}$, Long. $106^{\circ}$ $39^{\prime}$.

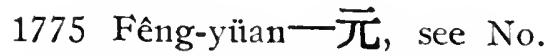
229.

\section{捧}

1776 Fêng-cha-hsün 捧䰻洲, sš̆ near Hsing-yi $\mathrm{Fu}$, Kueichou.
Ferghana, see No. 6103. Fi-ching, see No. 1672. Fi-liang, see No. 1673.

\section{FO}

\section{佦}

1777 Fo-kang 俳岡 Foh-kang, [22] C.I.T. in Hui-Chao-Chia circ., Kuangtung; Lat. $24^{\circ} 53^{\prime}$, Long. $112^{\circ} 43^{\prime}$.

1778 Fo-p'ing | 本 Foh-ping, [433] $\mathrm{T}$. in Han-chung $\mathrm{Fu}$, Shensi ; Lat. $33^{\circ} 35^{\prime}$, Long. $107^{\circ} 53^{\prime}$; Terr. jur. 1779 Fo-shan | W Foh-shan [416] or Fat-shan, T. in Kuangchou $\mathrm{Fu}$, Kuangtung; Lat. $23^{\circ} 08^{\prime}$, Long. $112^{\circ}$ $55^{\prime}$.

Foh-kang, see No. 1777. Foh-ping, see No. 1778. Folh-shan, see No. 1779. Foochow, see No. 1871.

Formosa, see Nos. 565, 6194.

\section{FOU}

\section{涪}

\section{Fou 涪 Fau.}

[528] $1^{\circ} \mathrm{C}$. in Chung-ch'ing $\mathrm{Fu}$, Ssŭch' uan ; Lat. $29^{\circ}$ $45^{\prime}$, long. $107^{\circ} 36^{\prime}$; orig., in K. Pa 巴; Han, Foul-ling | 陵; $P$. Chou, Han-p'ing 漢平; $T^{\prime}$ ang, Fou | C. 
涪

Fou.

$2^{\circ}$ See Nos. 223, 4433.

1781 Four-ch'êng | 城, (obs.) Sui, H., N. W. of 'T'ungch' 'uan Fu, Ssŭch'

1782 Font-ling | 陵.

$1^{\circ}$ Post-town near Fon C., Ssŭch' ian.

$2^{\circ}$ See Nos. 1780, 7084.

\section{FU}

宾

1783 Fin-ch'êng 阜 城 Fauching.

[10] $1^{\circ} \mathrm{H}$. in Ho-chien F 11 , Chihli; Lat. $37^{\circ} 55^{\prime}$, Long. $116^{\circ} 13^{\prime}$; Han, Ful-ch'êng|城; T'ang, Han-fu 漢 |.

$2^{\circ}$ Chên near $\mathrm{Cl}^{\prime}$ ing-yang Fin, Kansul.

$1783^{\wedge} \mathrm{F} u-h \sin \mid$ 新 H. in Ch ${ }^{\prime}$ aoyang $\mathrm{Fu}$, Clihlii ; $180 \mathrm{Li}$ N. of No. 366.

1784 Fil-k'ang | 㞗 Fan1-kang, H. in Ti-hua C., New Dominion; Lat. $44^{\circ}$, Long. $89^{\circ} 05^{\prime}$.

1785 Fu-ling | 陵, (obs.) Han, H., S. W. of Ch' 11 C., Anhui.

1786 Funing | 察 Fan-ning, [148] $\mathrm{H}$. in Huai-an $\mathrm{F}_{11}$, Kiangsu ; Lat. $33^{\circ} 43^{\prime}$, Long. $119^{\circ} 51^{\prime}$.

1787 Fu-p ing | 平 Fall-ping, [379] $\mathrm{H}$. in Chêng-ting $\mathrm{F}_{11}$, Chihli; Lat. $38^{\circ} 52^{\prime}$,
浮 FU-SHAN.

Long. $114^{\circ} 17^{\prime} ;$ Sui, Hsing-t'ang 行唐; Kin, Fu-p ing | 平.

1788 Futs'ai 剘, (obs.) S.E. of Mêng-ching H., Honan; Sung, fort in Ho-nan 河 南 $\mathrm{Fu}$.

1789 Fi1-yang | 隄 Faut-yang, $H$. forming the prefectural city of Ying-chou, Anhui ; Lat. $32^{\circ} 58^{\prime}$, Long. $115^{\circ}$ $57^{\prime}$.

$1790 \mathrm{~F}_{11-\mathrm{y}} \mid$ 由, $s s \breve{u}$ in Chishui H., Kiangsi.

\section{孚}

$1791 \mathrm{~F}_{11}$ 孚.

$1^{\circ}$ (obs.) E. of Nd-ti T.C., Kuangsi ; Sung, C. in Kuang-hsi 廣 西 S. circ.

$2^{\circ}$ See No. 4546.

\section{浮}

1792 Fin-cl1'êng 浮城, (obs.) $W_{e i}, \mathrm{H}$. near Ying-ch' êng H., Hupeh.

1793 Fulliang | 梁 Fantliang, [14] $\mathrm{H}$. in Jao-chou $F u$, Kiangsi ; Lat. $29^{\circ} 20^{\prime}$, Long. $117^{\circ} 19^{\prime}$; Han, $\mathrm{P}^{\prime}$ o-yang 翻晹; $T^{\prime}$ ang, Hsin-ch'ang 新昌, and Fulliang | 梁.

1794 Fi1-shan | If Fan-shan, $\mathrm{H}$. in $\mathrm{P}^{6}$ ing-yang $\mathrm{Fu}$ Stansi ; Lat. $35^{\circ} 59^{\prime}$ Long. $111^{\circ} 53^{\prime}$; orig., 
Tan1-chnt-ch 'êng 丹朱城, i.e., city of Tan chn, son of Emperor Yao: Han, Hsiang-ling 襄陵; $P$. Chou, Fit-shan | ; T'ang, Shên-shan 神 山.

1795 Fu-shui, see No. 7352 .

$1796 F_{11-t}$ u | 圖, (obs.) near Yen-an Fu, Shensi; Sui, pao in Sui-tê 総 德 military district.

$1797 \mathrm{~F}_{\mathrm{u}-\mathrm{t}} \mathrm{t} \mathrm{u}-\mathrm{y}$ üi | 圖 峪, posttown near Knang-ch' ang H., Chilili.

1798 Fu1-yang | 陽, (obs.) formerly H., N. of Nan-p $i$ H., Chihli.

1799 Futyang | 洋, ss $\breve{u}$ in Haiyang $\mathrm{H}$., Knangtung.

\section{府}

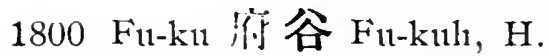
[148] in Yü-lin Fu, Shensi; Lat. $39^{\circ} 08^{\prime}$, Long. $110^{\circ}$ $43^{\prime} ; T s^{\prime}$ in, 'T'ai-yuian 太 原; T"ang, Fu-ku | 谷; Sung, Yung-an 永安 nilitary station.

\section{符}

$1801 \mathrm{~F}$ 符.

$1^{\circ}$ (obs.) $6 l i \mathrm{~W}$. of Hochiang H., Ssŭch' tuan; W. Han, H. in Chienwei 腱鹰 chän; Tsin, $\mathrm{H}$. in Cliang-yang 江. 陽 chün.

$2^{\circ}$ See No. 2122. \\ 滏 FU-YANG.}

$1802 \mathrm{Fu}$-1i | 離.

$1^{\circ}$ (obs.) in Su C., Anhui; Han, Tsin, Sui and Kin, Fil-li。 離 $\mathrm{H}$.

$2^{\circ}$ See No. 5914.

附

$1803 F_{\mathfrak{u}-c h}$ 'êng 附 城, chên near Tsê-chon Fu, Shansi.

1804 Fu-ên | 恩, (obs.) N.W. of $\mathrm{P}$ ing-yao $\mathrm{H}$., Shansi; N. Wei, chün in Yü 蔚 cHov.

\section{鄜}

$1805 F_{1}$ 鄜 $F_{11}$, C. L. C. in [264] the Hsi-Ch' ien-Fu circ., Shensi; Lat. $36^{\circ} 05^{\prime}$, Long. $109^{\circ} 18^{\prime} ; \operatorname{Han}, \mathrm{Fn}$ $\mid \mathrm{H} . ;$ Wei, Fu $\mid \mathrm{C}$.

$1806 \mathrm{~F}^{\prime}$ 11-ch êng | 城, post-town near the preceding.

\section{父}

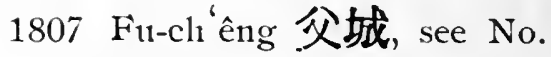
4938.

\section{釜}

1808 Fu-shan 釜 U Fusan, port south of Tung-lai 東萊 $\mathrm{F} t$, Corea, opened by treaty to Japanese trade.

\section{㵚}

$1809 \mathrm{Fu}$-yang 滏陽, see No. 6868. 
夫 FU-JÊEN.

\section{夫}

1810 Fu-jên 夫 人, (obs.) $T \sin$, Fort $\mathrm{N}$. W. of Hsiang-yang $\mathrm{Fu}$, Hupeh.

1811 Fu-tsu | 种, (obs.) in Corea ; Han, H. in Lêlang 樂浱 chün.

1812 Fil-yi | 夷.

$1^{\circ}$ (obs.) N.E. of Hsinning H., Hunan; Han, H. in Ling-ling 零陵 chün; Tsin, H. in Ling 陵 chïn.

$2^{\circ}$ See No. 4937.

\section{扶}

$1813 \mathrm{Fu}$ 扶.

$1^{\circ}$ (obs.) N. E. of Hsinning H., Hunan; $S$. Sung and $S . T s^{\prime} i, \mathrm{H}$. in. Shao-ling 郡 陵 chün.

$2^{\circ}$ (obs.) in Wên H., Kansuh ; $T^{6}$ ang, C. in Shan-nan 山南 circ.

$3^{\circ}$ (obs.) near Hsü-chou Fu, Ssŭch'uan; T'ang, H. in Wei 稨 C.

1814 Fu-an | 㚣 Fu-ngan, H.

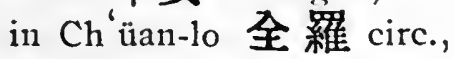
Corea ; Lat. $35^{\circ} 37^{\prime}$, Long. $127^{\circ} 07^{\prime}$.

1815 Fu-cl'i | 其, (obs.) N. W. of Chu-ch'êng H., Shantung; $N$. Wei, $\mathrm{H}$. in Tung-wu 東武 chün。

1816 Fu-ch'i | 溪, ssŭ in Jênhua $\mathrm{H}$., Kuangtung.
扶 FU-KOU.

1817 Fu-fêng | 風 Fu-fung.

[4977] $1^{\circ}$ H. in Fêng-hsiang F $\mathrm{u}$, Shensi ; Lat. $34^{\circ} 20^{\prime}$, Long. 107 56 ; Chou, Ch'i-yang 岐陽; Han, Mei-yang 美陽; $P$. Chou, Yen 燕 C.; $\tau^{6}$ ang, Wei-ch'uan 湋 川, and Fin-fêng | 風.

$2^{\circ}$ (obs.) N.W. of Chingyang H., Shensi ; T sin, chiin in Yung 䔨 CHOr.

$3^{\circ}$ (obs.) 4 li $\mathrm{E}$. of $\mathrm{K} 11-$ cl'êng H., Hupeh; $S$. Sing and $S . T s^{\prime} i$, chiin in Yung 䔨 CHOU. $4^{\circ}$ (obs.) in Ssŭcl' 'uan; $S$. Ts' $i$, chïn in $\mathrm{Yi}$ 䀂 CHOU.

$5^{\circ}$ (obs.) near Mien C., Ssŭch' 'uan; S. Ts'i, H. in Yi $\mathrm{Yint}$ chou.

$6^{\circ}$ (obs.) in Anhui; $N$. Wei, chïn in Ho 霍 cHOU.

$7^{\circ}$ (obs.) $10 l i \mathrm{~N} . \mathrm{E}$. of $\mathrm{Cl}^{\prime}$ ien C., Shensi; $N$. Wei, chïn in Yung 倠 cHOU.

$8^{\circ}$ (obs.) S. of Fêninglusiang Fu, Shensi ; Sui, chün in Yung 淮 CHOU. $9^{\circ}$ See No. 1724.

1818 Fu-kou | 清 Fulkau, H. [32] in Ch 'ên-chou Fu, Honan; Lat. $34^{\circ} 12^{\prime}$, Long. $114^{\circ}$ $34^{\prime}$. 
扶 FU-LAI.

1819 Fu-lai | 來.

$1^{\circ}$ (obs.) $T^{\prime}$ ang, H., E. of Pei-lin H., Kuangsi.

$2^{\circ}$ (obs.) IV. of Hsingwên H., Ssŭch' $T^{6}$ ang, H. in Yen 晏 C.

1820 Fulai | 萊, another way of writing No. $18191^{\circ}$.

1821 Fur-lung | 隆, Y. near

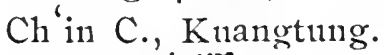

1822 Fin-yang | 陽, (obs.) Sui, H. near Ssŭ-nan Fun, Kueichou.

1823 Fut-yü | 餘 Fu-yu.

$1^{\circ} \mathrm{H}$. in Chung-ch'ing 忠清 circ., Corea; Lat. $36^{\circ} 54^{\prime}$, Long. $126^{\circ} 32^{\prime}$.

$2^{\circ}$ See No. 3074 .

1824 Fut-yü|重, (obs.) 25 li S. E. of Ch'ing-yüan H., Chihli ; N. Wei, H. in Kao-yang 高㜔 chïn.

\section{笑}

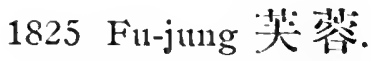

$1^{\circ}$ Ss ̌̆ near Shao-clion Fu, Kuangtung.

$2^{\circ}$ (obs.) W. of Tsuu1-yi H., Kuei-chou; $T^{6} a n g$, H. in Po 播 C.

\section{夫乃}

1826 Fin 夫邞, (obs.) S. W. of Chiao C., Shantung; $W$. Han, H. in Lang-yeh 郎 躯 chïn。
FU-YI.

䇥

1827 Fu 撫, (obs.) S. Sung, c. in Chiang-hsi 江 西 circ.; see also No. 1829.

1828 Fin-an | 安; post-town near Kan-ch' ïan $H$., Shensi.

1829 Fut-chou | 州 Fu-chau, [509] Fu in the Nan-Fu-Clien circ., Kiangsi ; forming also the district city of Lin-ch' tuan; Lat. $27^{\circ} 56^{\prime}$, Long. $116^{\circ} 18^{\prime}$; S.A., in $\mathrm{K}$. Wu 㕦; C. Wars, in K. Ch' ${ }^{\prime}$ 楚; $T s^{\prime} i n$; in Chin-chiang 阽汇 chïn; Han, in Y ïi-chang 预管 chïn; Three Ks., Lincli'tran 臨 川; Sui and $\tau^{\prime}$ ang, Fil $\mid \mathrm{C}$.

1830 Futk'ang | 康, ssŭ in Yï-lin C., Kuangsi.

1831 Fu-ning | 耍 Fu-ning, H. in Yung-p'ing Fu, Chilili; Lat. $39^{\circ} 56^{\prime}$, Long. $119^{\circ} 20^{\prime}$; Han, Li-ch'êng 蕄城; Kin, F11-ning | 富.

1832 Fu1-shun | 順, post-tow11 near Fêng-t'ien Fu, Shingking.

1833 Fu1-yi | 粰 Fin-i， T. in Kan-cliou Fu, Kaisulı; Lat. $39^{\circ} 10^{\prime}$, Long. $100^{\circ}$ $25^{\prime} ; W$. Han and N.Wei, Furyi | 夷 $\mathrm{H}$.

1834 Fulyi | 夷, see No. 1833. 
㭪

FU-CH' 1 .

\section{㭪}

1835 Fu-ch'i 㭪溪, post-town near L,ê-ch'ing H., Chehkiang.

\section{敷}

$1836 \mathrm{Fu}$ 敷

$1^{\circ}$ (obs.) in Ssŭclíuan; Sung, C. in Shao-ch'ing 紹磨F11.

$2^{\circ}$ (obs.) formerly C. near Lin-pa T., Shensi.

1837 Fu-clı' êng | 城, (obs.) formerly $H$. near Liu-pa T., Shensi.

1838 Fu-hisi | 西, (obs.) S. W. of Hua-yin H., Shensi; $N$. Wei, H. in Hua-shan 華山chïn。

1839 Fu-shui | 水, chên near Hua-yin H., Shensi.

1840 Fu-wên|文, (obs.) Han, $\mathrm{H}$. near Lan-chou $\mathrm{Fu}$, Kansul.

1841 Fulyung | 行, see No. 2873.

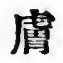

1842 Ful-kung 膚 公, (obs.) S. W. of Hsi-11ing H., Kansuh ; Sung, fort in K' 10 C., Shensi.

1843 Fi1-shih | 施 Fut-shi.

$1^{\circ} \mathrm{H}$. forming the prefectural city of Yen1-an, Shensi; Lat. $36^{\circ} 42^{\prime}$, Long. $109^{\circ} 28^{\prime}$; Han, Fu-shih | 施 $\mathrm{H}$.
窝 FU-JÊN.

$2^{\circ}$ Post-town near An-sai H., Shensi.

$3^{\circ}$ (obs.) 50 li S. E. of Sui-tê C., Shensi ; Han, $\mathrm{H}$. in Shang 上 chïn. $4^{\circ}$ (obs.) near Ch'ing-yang Fu, Kausuh ; $N . W_{c i}$, H. in Hsiang-lê 襄樂 chïn.

$5^{\circ}$ (obs.) E. of Fu-shih H., Shensi; Sui, H. in Yen-an 延安 chün.

\section{富}

1844 Ful 富.

$1^{\circ}$ T. C. in Knang-nan F., Yuinnan.

$2^{\circ}$ See No. 2824.

1845 Fin-ch'êng | 站, see No. 1737.

1846 Fu-ch' uan | 川Fu-chuen, [416] H. in P'ing-lê Fu, Knangsi; Lat. $24^{\circ} 33^{\prime}$, Long. $111^{\circ}$ $03^{\prime} ;$ Han, Fil-cli uan | 川; T'ang, Fu-shui | 水. 1847 Fil-chuang | 蒋, posttown near Chiao-11o H., Chilili.

1848 Fu-ch 111 | 春, see Nos. 1862, 7085.

1849 Fu-jên $\mid$ 仁.

$1^{\circ}$ (obs.) near $\mathrm{Ch}{ }^{\prime} \mathrm{inng-y}$ üan Fu, Kuangsi ; Sung, fort in Ch'ing-yiian 度 遥 Fu.

$2^{\circ}$ (obs.) near Ya-chon Fu, Ssŭch'uan; Sung, C. in Ya 雅 C. 
富富保IN.

1850 Fu-lin | 林, (obs.) T ang, H. $100 \mathrm{li} \mathrm{N}$. of Yangch'un H., Kuangtung.

1851 Fin-lo | 羅, (obs.) T'ang, H., S. W. of Tan C., Kuangtung.

185.2 Futlu | 醁, chên near Yung-ning C., Kuangsi.

1853 Fu-min |民 Fu-min.

[371] $1^{\circ} \mathrm{H}$. in Yün-nan Fu, Yünnan; Lat. $25^{\circ} 20^{\prime}$, Long. $101^{\circ} 55^{\prime}$; Han, Hsi-11ing-li 西耍利 C.; Yïan, Fu-nin | 民 $\mathrm{H}$.

$2^{\circ}$ (obs.) N. of Yu-yü 右玉 H., Shansi; Liao and Kin, H. in Fêng 豐 C.

1854 Fu-ning | 察 Fu, in Hsien-cling 諴鏡 circ., Corea.

1855 Futping | 4 Futping, H. in Hsi-an Fu, Shensi; Lat. $34^{\circ} 42^{\prime}$, Long. $108^{\circ}$ $47^{\prime} ; T s^{\prime}$ in, P'in-yang 頻 血; Sui, Fu-p ing 本; T ang, Mei-yüan 美原.

1856 Fu-sha11 | Il, ken in Japan.

1857 Fu-shui | 水, see No. 1846.

1858 Fu-shun | 㮌, Fu-shun, [265] H, in Hsü-chon Fu, Ssŭch'nan; Lat. $31^{\circ}$, Long. $104^{\circ} 22^{\prime}$; Ts in, in Pa 巴 chïn; Han, Chiang-yang 江榢; $P$. Chou, Lo-yüan
福 FU-AN.

洛原; Sung, Fu-shun | 䐜。

1859 Fut'tien | 田, ssŭ, in Lï̈ling H., Kiangsi.

1860 Fu-ts'un | 村, post-town near Hsi-ch' ung H., Ssŭch' tran.

1861 Fu-t'un | 屯, post-tow1 near Shinn-ch'ang $H$, Fulkien.

1862 Fr1-yang | 晹 Fin-yang, [49] H. in Hang-chou Fu, Chehkiang; Lat. $29^{\circ} 58^{\prime}$, Long. $119^{\circ} 59^{\prime}$; Han, Fi1ch' 1 in | 春 $\mathrm{H}$.; Wu, Tungan 東安; T sin，Fun-yang | 隊.

1863 Fi1-yün | 雲, (obs.) W. of Wan 萠 C., Kuang-

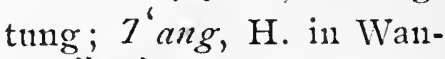
an 萬发 $\mathrm{C}$.

\section{偪}

1864 Fu-yang 偪晹, see No. 5037.

\section{㧫}

$1865 \mathrm{Fu}$ 福, (obs.) S. Sung, C. in Fu-chien | 建 circ.; see also No. 1871.

1866 Ful-an | 安 Fuh-11gan.

[33] $1^{\circ} \mathrm{H}$. in Fu-ning $\mathrm{Fu}$, Fullkien; Lat. $27^{\circ} 05^{\prime}$, Long. $117^{\circ} 47^{\prime}$; Han, $\mathrm{Cl}^{\prime}$ 'ang-ch'i 長 沵; Sung, Fu-an|安.

$2^{\circ}$ See No. 1876. 
融

FU-CHIF,

1867 Fut-chien | 建 Fuh-kien.

$1^{\circ}$ A province of Clinia; literary names Min 閩, Min-chung 閩 : 1 ' and Chin-an 晉安; capital, Fu-chour Fi1; it con1tains 5 circ., $11 \mathrm{Fu}, 5$ T., 2 C.L.C. and $66 \mathrm{H}$. $2^{\circ}$ (obs.) S. Sung, circ.

1868 Fit-ching | 津.

$1^{\circ}$ (obs.) S. E. of Chieh C., Kansuln; $T^{6}$ ang, $\mathrm{H}$. in Chieh C.

$2^{\circ}$ See No. 832 .

1869 Fu-ching | 井 Funai, clief town of Oita Kell, Japan.

1870 Fut-ch' ing | 清 Ful11-tsing, H. in Fu-chou Fu, Ful1kien; I at. $25^{\circ} 41^{\prime}$, I,ong. $119^{\circ} 25^{\prime}$; orig., $\mathrm{Ch}^{\prime}$ ang-lê 長樂; Trang. Wan-an 茧安；Ming，Fu1-ch'ing | 清.

1871 Fu-chon | 州 Fuh-chan [306] or Foochow; $\mathrm{F}_{11}$ in the Fu-chou-Fu-ning circ., Fuhkien ; forming also the two district cities of Min and Hout-kuan; Lat. $26^{\circ}$ $03^{\prime}$, Long. $119^{\circ} 25^{\prime}$; capital of the province; one of the ports open to

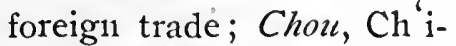
min- 七閩; $T s^{\prime}$ in, Min-

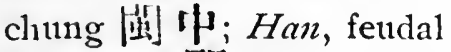
state Min 閨 in K. Yüeh 越; $T \sin$, Chin-an 互㚣;
福 Fu-SHAN。

Sui, Min 閩 C. and $\mathrm{Cl}^{\prime}$ ïan 良 C.; T'ang， Chien 建 C. and Ch'ang-lê 長樂; Sung, $\mid$ C. and Wei-wu 威武; Ming, Fi1-clion | 州 Fu. Fuju (Polo).

1872 Fu1-chou-Fun-ning | 州福 穿, circ. in Fuhkien, conlprising Fu-clion $F$ Fu-11ing Fin.

1873 Fur-kang | 佸 Fukuoka, ken and chief tow 11, Japan.

1874 Fut-ling | 陵, post-town near Fêng- $t^{\prime}$ ien Fin, Shingking.

1875 Fur-lu | 醁.

$1^{\circ}$ (obs.) Han, H., E. of

Su C., Kansulı.

$2^{\circ}$ (obs.) $T^{*}$ ang, H., E. of

Kuei-lin Fu, Kuangsi.

1876 Fun-11ing | 察 Fuh-1ning, Fu in the Fu-chon-Fin-ning circ., Fuhkien ; forming also the district city of Hsia-p' 1 ; Lat. $26^{\circ} 54^{\prime}$, Itong. $120^{\circ}$ 05'; Chou, $\mathrm{Ch}^{6} \mathrm{i}$-min $七$ 閩; Ts'in, Min-chung 閩中; $T \sin$, Wên-111a 溫麻; Sui and T'ang, Cli'ang-chli 严溪; Sung, Fin-an1 玹; Ming, F11-11ing | 窗 F11.

1877 Fur-shan | III Fuh-shan, $H$. in 'lêng-chou F'u, Shautung; I.nt. $37^{\circ} 33^{\prime}$, Long. $121^{\circ} 35^{\prime}$; Han, Shni 看 H.; Kin, Fin-shan | 山. 
तin

Fu-sHur.

1878 Fu-shui | 水, sš̆ near 'Tung-kuan H., Kuangtiung.

1879 Futrao |島 Fukttshina, ke11 and chief town, Japan. 1880 Fin-ting | 鼎 Full-ting, [488] H. in Fu-ning Fut, Finhkien; Lat. $27^{\circ} 30^{\prime}$, Long. $120^{\circ} 20^{\prime}$.

1881 F 11-yiu | 餘, (obs.) Ming, nililitary district in Mongolia.

\section{復}

1882 In 1 復 Fin1.

$1^{\circ}$ C. in Fên Sliningking; Lat. $39^{\circ}$ $40^{\prime}$, Long. $121^{\circ} 43^{\prime}$.

$2^{\circ}$ (obs. S) S. Sung, C. in Hi1-pei 湖 北 circ.

$3^{\circ}$ See No. 1134.

1883 Fr11-lei 棌, (obs.) near P'ing-liang Fin, Kansuh; W. Han, H. in An-ting 学定 chïn.

188t Fin-yang | 陽, see No. 6817.

\section{踓}

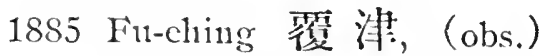
S.E. of Chieln C., Kansul, ; Sui, H. in Wr1-tu 武都 cliinn.

\section{伏}

1886 Fu-cl1'êng 伏 城, (obs.) formerly town W. of 'T'ang H., Chillli.

\section{伏 FU-KUH.}

1887 Fu-ch'iang | 庵 Fuhkiang, H. in Kung-ch' ang Fu, Kansuh; Lat. $34^{\circ} 38^{\prime}$, Isong. $105^{\circ} 24^{\prime}$; orig., Chi 冀; Han, Hanlyang，漠 陽; 2nd $W e i$, Tang-t'ing 當寄; P. Chou, Chicli êng 蕒 城; T'ang, Fu-ch'iang | 范.

1888 Fut-ju11g | 我, (obs.) on frontiers of Kansulı; Sui, H. in Ch'ieh-mo 且末 chïn.

1889 Fur-li | 荔, ssŭ near Yangso H., Kuangsi.

1890 Furlu | 慮, (obs.) near Turfan; Tang, C. in Yüeh-chil 月支 Fu.

1891 Fu-1u | 洼, see No. 3080. 1892 Fn-p'i | 皮, chên near Fin-fêng H., Sliensi.

1893 Fut-yü | 虞.

$1^{\circ}$ (obs.) $80 l i \mathrm{E}$. of $\mathrm{Yi}-$ linng H., Ssŭch'uan; Sui, H. in Cl'ing-hua 清化 chïn.

$2^{\circ}$ (obs.) E. of Yi-lung H., Ssŭch' nan; T'ang and Sung, H. in P'êng 蓬 C.

Fu-cliau, see No. 1829. Fut-chuen, see No. 1846. Fit-fung, see No. 1817. Fu1-i, see No. 1833. Fuju, see No. 1871. Fu-kau, see No. 1818. Fu-kuh, see No. 1800. 
Fukuoka, see No. 1873:

Fukushima, see No. 1879.

Funai, see No. 1869.

Fu-ngan, see No. 1814.

Fu-ping, see No. 1855.

Fu-shi, see No. 1843.

Fu-yt1, see No. 1823.

Fuh, see No. 1882.

Ful1-cliau, see No. 1871.

Fuh-kiang, see No. 1887.

Fuh-kien, see No. 1867.

Fuh-11gan, see No. 1866.

Full-11ing, see No. 1876.

Fuh-shan, see No. 1877.

Ful1-ting, see No. 1880.

Fulh-tsing, see No. 1870.

Fung, see Nos. 1716, 1734.

Fung-chin, see No. 1736.

Fung-ching, see No. 1737.

Fung-chuen, see No. 1701.

Fung-hien, see No. 1768.

Fung-hwa, see No. 1766.

Fung-hwang, see No. 172.2.

Fu11g-i, see No. 1774.

Fung-jun, see No. 1744.

Fung-kiu, see No. 1700.

Fung-ning, see No. 1746.

Fung-shan, see No. 1728.

Fung-shun, see No. 1747.

Fung-sin, see No. 1769.

Fung-tai, see No. 17'29.

Fung-teh, see No. 1748.

Fung-tien, see No. 1773.

Fung-tsiang, see No. 1724.

Fung-tsieh, see No. 1763.

Fung-tu, see No. 1753.

Fungul, see No. 2895.
Fung-yang, see No. 1731.

Gachoun, see No. 3194.

Ganft1, see No. 3101.

Gensa11, see No. 7760.

Ghazna, see No. 3205.

Ghianda, see No. 132.

Ghitiju, see No. 1445.

Ghizning, see No. 3205.

Ghorlos, see No. 3540.

Gumboslioka, see No. 3505 .

Gu11111, see No. 1524 .

Gundelik, see No. 1565 .

Gutchen, see No. 3254.

Gyangtse see No. 777.

\section{HA}

㭘

$1893^{\mathrm{A}} \mathrm{Ha}$-êrh-pin 哈 源 Harbin. City close to Hulan, Tsitsiluar. It is the residence of the Intendant of custonns and railways.

1894 Ha-êrli-shou 哈 尔 受, T-S. in Kokonor.

$1895 \mathrm{Ha}$-êrh-t' ' -po | 尔圆博 Kartube, town in the New Donninion; I at. $42^{\circ}$ $30^{\prime}$, Long. $93^{\circ}$.

$1896 \mathrm{Ha}^{\prime} \mathrm{k}^{\prime} \hat{\mathrm{e}}-\mathrm{t}^{\prime} \mathrm{u}$ | 克圆, see No. 4321.

1897 Ha-la | 拉 Kara-koto, town in the New Dominion; Lat. $43^{\circ} 40^{\prime}$, Long. $94^{\circ}$.

$1898 \mathrm{Ha} \mathrm{la}-\mathrm{ch}^{\prime}$ in | 喇汇 (or k'ê-êrh-la-ch'in 喀爾喇 沈）Kharachin， Mongol Tribe. 
哈 HA-LA-HA-SHIH.

1899 Ha-la-ha-shilı | 喇哈什, Karakash, town in Yarkand ; Lat. $37^{\circ} 25^{\prime}$, Long. $80^{\circ}$.

1900 Ha-la-lio-cho | 拉和卓, or Ha-la-huo-chê | 刺 火 者, or Ha-la-ho-chou 拉霍州，Karakhodjo; also called Ho-chou 合州 or 和州, or Huo-chou 火 州, and anciently Kao-ch'ang 婯昌; (obs.) formerly a town 30 li E. of Turfan.

1901 Ha-la-ho-lin | 刺 和林 Karakorum, also called Ho-lin 和林, a city between Urga and Uliasutai Caracoran (Polo).

1902 Ha-la-l1o-chon | 刺墔州, see No. 1900.

1903 Ha-la-huo-chon | 刺火州, see No. 1900.

1904 Ha-la-sha-êrl | 喇沙竹, [205] or K'ê-la-sha-êrh 喀拉 沙尔, Harashar.

$1^{\circ}$ Formerly Province in Mongolia. Bounded on the N. by Ili and Kansul1; on the E. by Kansull and Kokonor; on the S. by Thibet; and on the W. by Yarkand.

$2^{\circ}$ C.L.T. in the Aksu circ., New Dominion. Lat. $42^{\circ} 15^{\prime}$, Long. $87^{\circ}$ 05. See No. 7386 .
HAI.

1905 Ha-lê-kê-a-man | 勒噶 阿满. See No. 3254.

1906 Ha-lieh 哈 列, or Yeh-li 也里, or Yi-li 沙里, or 哜離, Herat or Heri, in Afglianistan.

1907 Ha-mi | 密 Hami or Khamil (also written Kêmu-li 柯模里, Ha-mi-li 哈迷里, and Kan-mu-lu 感木惫), T. in Chên-11si C.L.T. and in the ChênTi circ., New Dominion ; Lat. $42^{\circ} 45^{\prime}$, Long. $93^{\circ}$ 35'. Camul (Polo).

1908 Ha-mi-li | 迷里, see preceding.

1909 Ha-shili-ha-êrh / 保哈胃, see No. 3224.

1910 Ha-ta | 達, see No. 996. 1911 Ha-t'a-pu-ch'i | 塔布㤃 Kadabzi, town in Sain11oin ; Lat. $44^{\circ} 10^{\prime}$, Long. $100^{\circ} 50^{\prime}$.

1912 Ha-t ê-1111-êrh | 呼持尔 Hatur, towl1 in Ulianghai ; Lat. $50^{\circ} 20^{\prime}$, Lo1ng. $100^{\circ}$ $10^{\prime}$.

\section{HAI}

\section{海}

\section{Hai 海 Hai.}

$1^{\circ}$ C.L.C. in the Hsü-Hai circ., Kiangsu; Lat. $34^{\circ} 29^{\prime}$, Long. $119^{\circ}$ $27^{\prime}$; orig., K. 'T'an 炎队 $T s^{\prime}$ in, Ch'ü 朐 
H.; Han, Tung-hai 東 海; $W e i$, '小'an 炎 $\mathrm{H}$.; T'ang, Hai | C.

$2^{\circ}$ C. in Hnang-liai 黄海 circ., Corea.

$3^{\circ}$ See No. 6185.

1914 Hai-an | 安.

$1^{\circ}$ Chên near Ju-kao H., Kiangsu.

$2^{\circ}$ (obs.) $T^{6}$ ang, H. near

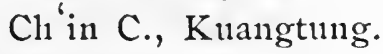

$3^{\circ}$ See Nos. 10t, 7152.

1915 Hai-ch'êng | 城 Hai[84] ching.

$1^{\circ} \mathrm{H}$. in Ku-yüan C., Kansul, formerly Yench'a 鹽荼 T. Lait. $41^{\circ}$ $50^{\prime}$, Long. $105^{\circ} 25^{\prime}$.

$2^{\circ}$ H. in Fêng-t'ien Fu, Shingking ; Lat. $41^{\circ}$ $45^{\prime}$, Long. $121^{\circ} 55^{\prime}$.

1916 Hai-cl' êng | 颈 Hai[95] cling, H. in Chang-chon Fu, Fulukien; Lat. $24^{\circ}$ $25^{\prime}$, Long. $118^{\circ} 03^{\prime}$.

1917 Hai-ch' ${ }^{\prime} \mathrm{i} \mid$ 曲, see No. 2995.

1918 Hai-fêng | 嵦 Hai-fung.

$1^{\circ} \mathrm{H}$. in Wu-ting $\mathrm{Fu}$, Shantung; Lat. $37^{\circ}$ $50^{\prime}$, Long. $117^{\circ} 46^{\prime}$; Han, Yang-hsin 陽信; Sui, Wu-ti 無 様; Ming, Hai-fêng|豐 H. $2^{\circ-}$ H. in Hui-chon $F u$, Kuangtung ; Lat. $22^{\circ}$ $54^{\prime}$, I,ong. $115^{\circ} 19^{\prime}$; Han, in Tung-kuan 東
官 chün；Tsin，Haifêng | H. H. Ln1-an 陸安.

$3^{\circ}$ (obs.) $80 l i \mathrm{~N}$. E. of Yen-shan H., Chihli; Kin, chên in Ts'ang 洽 $\mathrm{C}$.

1919 Hai-hun | 昏, or | 居, see Nos. 847, 1769, 7035. 1920 Hai-hsi | 西.

$1^{\circ}$ (obs.) 120 li S. of $\mathrm{Hai}$ C., Kiangsu; Han, Sung, S. Ts' $i$ and $\mathrm{IVe}$, Hai-hsi 西 $\mathrm{H}$.

$2^{\circ}$ (obs.) N. of An-tung H., Kiangsu ; N. Wei, chïn in $\mathrm{Hai}$ 海 cHov.

1921 Hai-k'ang | 氺 Hai-kang, $\mathrm{H}$. forming the prefectural city of Lei-cliou, Kuangfung ; Lat. $20^{\circ} 52^{\prime}$, Long. $112^{\circ} 40^{\prime} ; \mathrm{Han}, \mathrm{H}_{\text {süi-wên }}$ 徐牂；Sui, Hai-k'ang | 康。

1922 Hai-k'ou | 口, Hai-kau. $1^{\circ} \mathrm{H}$. in Corea; Lat. $38^{\circ}$ $34^{\prime}$, Long. $126^{\circ} 05^{\prime}$. $2^{\circ}$ See No. 1321.

1923 Hai-la-tı | 喇都, posttown near Hsi-ho H., Kansul.

1924 Hai-lin | 粼), (obs.) near Chao-ch ing $\mathrm{Fu}$, Kuangtung; $S$. Sung and $S$. $T^{\prime} s i$, H. in Sung-k'ang 宋康 chiin.

1925 Hai-ling | 陵, see Nos. 1928, 2821, 6175, 6768 . 


\section{海 HAI-LING-SIIAN.}

1926 Hai-ling-slian | 陵 山, ssĭ near Tien-pai H., Kuangtung.

1927 Hai-lu1 海 倫, C.I.T. in Sui-Lan-Hai circ., Tsitsihar.

1928 Hai-lu1ng 海龍, Fu, in Fêng-Chin-Slian-Hai circ., Shingking. Lat. $42^{\circ} 30^{\prime}$, Long. $125^{\circ} 20^{\prime}$.

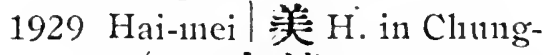
ch'ing 忠清 circ., Corea.

1930 Hai-11ên|門 Hai-1nun.

$1^{\circ}$ C.L.T. in the $\mathrm{Ch}^{\prime}$ angCh' ên-T'tung-Hai circ., Kiangsu ; Lat. $32^{\circ} 12^{\prime}$, Long. $121^{\circ} 06^{\prime}$; Han, Hai-ling | 陵; Five dyn., Hai-nên | 門 $\mathrm{H}$. $2^{\circ}$ (obs.) 40 li E. of $\mathrm{T}^{\prime} \mathrm{ung}$ C., Kiangsu ; Sung, Yïan and Ming, H. in 'T'ung C.

$3^{\circ}$ Y. in Haii-ch êng H., Fulıkien.

1931 Hai-mên-shan | 門山, ssŭ near Hai-ch' êng H., Fulhkien.

1932 Hai-n1ing | 冥 Han, H., in Corea.

1933 Hai-11an | 南 Hai-11an.

$1^{\circ}$ A large island off the coast of Knangtung, constituting the prefecture of $\mathrm{Cl}^{\prime}$ 'inung-chou. $2^{\circ} \mathrm{H}$. in Ch'iüan-lo circ., Corea, Lat. $34^{\circ} 55^{\prime}$, Long. $125^{\circ} 20^{\prime}$.
HAI-YANG.

1934 Hai-ning | 字 Hai-ning. $1^{\circ} \mathrm{C}$. in Hang-chou $\mathrm{Fu}$ Chehkiang; Lat. $30^{\circ}$ $24^{\prime}$, Long. $120^{\circ} 35^{\prime}$; Wu, Yen-kuan 監官; Yüan, Hai-ning | 漂 $\mathrm{H}$.

$2^{\circ}$ (obs.) Ming, H., W. of Hai-yen H., Chelıkiang.

1935 Hai-pin | 演, (obs.) $60 \mathrm{li}$ E. of Fin-ning H., Chihli ; Liao, H. in Jun 潤 C.; Kin, H. in Jui 瑞 C.

1936 Hai-p'ing | 平, (obs.) Liang, H., N. E. of Tienpai H., Kuangtung.

1937 Hai-cliên-Wei | 參 衛, see No. 2376.

1938 Hai-t'ang | 塘, see No. 4715.

1939 Hai-yang | 陽 Hai-yang. $1^{\circ} \mathrm{H}$. in Têng-chon $\mathrm{Fu}$, Shantung; Lat. $36^{\circ}$ $42^{\prime}$, Long. $121^{\circ} 13^{\prime}$.

$2^{\circ} \mathrm{H}$. forming the prefectural city of Ch'aoclou, Kuangtung; Lat. $23^{\circ} 34^{\prime}$, Long. $116^{\circ}$ 36'; Han, Chiel1-yang 揭隊； Tsin, Hai-yang |陪.

$3^{\circ}$ (obs.) Han, H., S. W. of Linan C., Chihli. $4^{\circ}$ (obs.) $T s^{\prime} i, H .$, N. of $\mathrm{Cl}^{\prime}$ 'ang-shu H., Kiangsu. $5^{\circ}$ See Nos. 2858, 4154, 6175. 
海

HAI-IMN.

1940 Hai-yen | 覧 Hai-yen, H. in Chia-hsing Fu, Chel1kiang; Lat. $30^{\circ} 35^{\prime}$, Long. $120^{\circ} 40^{\prime} ; T \sin$, Hai-yen I 鹽; T"ans, Wu-yüian 武原.

1941 Hai-ÿ̈ | 虞.

$1^{\circ}$ (obs.) E. of Ch'ang-shu H., Kiangsu ; $T \sin , S$. Sung and $S . T s^{\prime} i, \mathrm{H}$. in $\mathrm{Wu}$ 受 chün.

$2^{\circ}$ See No. 320 .

Hai-cling, see Nos. 1915, 1916.

Hai-fung, see No. 1918.

Hai-kang, see No. 1921.

Hai-kall, see No. 1922.

Hai-1nun, see No. 1928.

Hakodate, see No. 2625.

Hakone, see No. 2624.

Hanil, see No. 1907.

\section{HAN}

\section{漢}

1942 Han 漠 $\mathrm{Han}, \mathrm{C}$. in Ch' êng-tu Fur, Ssŭch'uan; Lat. $31^{\circ}$. Long. $104^{\circ} 22^{\prime}$; Han, Lo 雒 H.; Tsin, Hsin-tu 新都; Liang, Kuang-lian 廣漢; T"ang and $S$. Sung, Han $\mid C$.

1943 Hanlan | 安.

$1^{\circ}$ (obs.) 60 li E. of Chiang-an H., Ssŭch' nan ; E. Han, H. in Chien-wei 揵篇 chïn;
漢 HAN-CH'UAN.

$T \sin , S$. Sung and $S$. $T s^{\prime} i, H$. in Chiangyang 江跑 chïn.

$2^{\circ}$ (obs.) N. of Nanch' 'ung H., Ssŭch' nanı;

$S$. Sung and S. Ts' $i$,

H. in Tang-ch'ü 宕渠 chïn.

$3^{\circ}$ (obs.) near Mei C., Ssŭch' nanı ; S. Sung and $S .7 s^{\prime} i, H$. in Cliang-yang 江 階 chïn.

$4^{\circ}$ See No. 4133.

1944 Han-ch' ang | 嗩, see Nos. 223, 6524 .

1945 Han-ch'êng | 城, Fu in Cling-ch'i 京嫘 circ., Corea. See No. 363.

1946 Han-chia | 嘉, see Nos. $705,4462$.

1947 Han-chiang | 江, posttown near Hsiang-yang Fu, Hupeh.

1948 Han-ch' ' | 初.

$1^{\circ}$ (obs.) in Ssŭch'uan; S: $T s^{\prime} i, H$. in Tangcl'ì 宕渠 chün.

$2^{\circ}$ (obs.) S. of Nan-ch'ung H., Ssŭch 'uan; S. Ts' $i$, H. in $\mathrm{Cl}^{\prime}$ ï-liao 渠釈 chïn.

1949 Han-ch'uan | 川 Hanchuen.

$1^{\circ} \mathrm{H}$. in Hanlyang $\mathrm{Fu}$, Hupeh ; I at. $30^{\circ} 43^{\prime}$, I long. $113^{\circ} 42^{\prime}$; Han, 
漢

HAN-CH'UAN.

A11-11 努 陸; Wei, Chiang 江. C.; P. Chou, Tsêng-shan 解 II; Sui, Han-ch'nan | 川. $2^{\circ}$ See No. 1950.

1950 Han-chung | 中 Han[133] chung, Fu in the Shan-an circ., Shensi ; forming also the district city of Nanchêng; Lat. $32^{\circ} 56^{\prime}$, Long. $107^{\circ} 12^{\prime} ;$ S.A. and $C$. Wars, in K. Ch'in 柇 and K. $\mathrm{Cl}^{\prime} \mathrm{u}$ 楚; $T s^{\prime}$ in, Hanchung | 中 chïn; Han, Han-wang-tu | 王都, imperial residence; $W e i$, Liang 梁 C.; P. Chou, Han-ch'nan \{川; T'ang. and Sung, Hsing-yüan 興 $\overrightarrow{\mathrm{U}} ;$ Ming, Han-chung | 中 Fn. Cun-cnn (Polo).

1951 Han-fêng | 岦, see No. 3057.

1952 Han-fu | 阜, see No. 1783.

1953 Han-Huang-Tê | 弪: 德, circ. in Hupeh, comprising Han-yang Fu, Hnang-chon Fu and Tê-an Fu.

1954 Han-lısi | 西, (obs.) 55 li S.W. of Ch' ien C., Shensi ; $N$. Wei, H. in Wu-kung 此功 chïn.

1955 Han-hsing | 興, see No. 5314.

1956 Han-kao | 香, see No. 1957.
漢 HAN-YANG.

1957 Han-k'ou | 口 Han-kow ; 「528] in Hsia-k'ou 夏口 T., a subprefecture of Hanyang F., Hupel. Literary name Hant-kao | 臬. A port open to foreign trade. Lat. $30^{\circ} 33^{\prime}$, Long. $114^{\circ}$ $20^{\prime}$.

1958 Han-kung | 攻, (obs.) near Cling-chou $\mathrm{Fu}$, Hupei ; $S . T s^{6} i$, H. in Hsin-hsing 新興 chïn.

1959 Han-nan | 南, (obs.) formerly $\mathrm{H}$., $\mathrm{N}$. of Yicl'êng H., Hupeh.

1960 Han-11ing | 耍, see No. 2827.

1961 Han-nin | 牛, T-S. near Lü-shan H., Ssŭch'uan.

1962 Han-p'ing | 本, see No. 1780.

1963 Han-shou | 䩧, see Nos. $321,3429$.

1964 Han-tu | 都, see No. 2079.

1965 Han-tung | 東.

$1^{\circ}$ (obs.) in Sui C., Hupeh; Sui, chïn in Ching 荆 chou.

$2^{\circ}$ (obs.) N. of Chunglisiang H., Hupeh; Sui, H. in Ching-ling 竟陵 chïn.

$3^{\circ}$ See No. 5955.

1966 Han-wang-tı | 王 都, see No. 1950.

1967 Han-yang | 榢 Han-yang. 
漢

HAN-YANG.

[528] $1^{\circ}$ F'u in the Han-Huang'Tê circ., Hupelı ; forming also the district city of the same name; Lat. $32^{\circ} 32^{\prime}$, Long. $114^{\circ} 14^{\prime}$; orig., in K. Yün 鄖; C. Wars, in K. Clin 楚; Ts'in, in Nan 南 chïn; Han, in Chiang-hsia 江夏 chün; T'ang, Mien 洏 C. and Han-yang | 陽; Ming, Han-yang | 院 Fu.

$2^{\circ} \mathrm{H}$. forming the prefectural city of the same name, Hupeh; Lat. $32^{\circ} 32^{\prime}$, Long. $114^{\circ} 14^{\prime} ;$ Han, An-lu 首陸; $T \sin$, Clnwanyang 沛陽; Sui, Hanyang 隄; Tang， Mien 唡 $\mathrm{C}$.

$3^{\circ}$ Post-town near Hanchung Fu, Shensi.

$4^{\circ}$ (obs.) Han, H., S. of

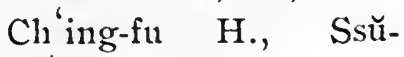
ch'tuan.

$5^{\circ}$ See Nos. 1887, 3521, 3729.

1968 Han-yang-ch'êng | 陽城, a name for the capital of Corea; see No. 363.

1969 Han-yin | 䇣 Han-yin. [222] $\mathrm{I}^{\circ} \mathrm{T}$. in Hsing-an $\mathrm{F} u$, Shensi; Lat. $32^{\circ} 40^{\prime}$, Long. $108^{\circ} 28^{\prime} ;$ Han, Han-yang | 隄; Tsin,
韓 HAN-CII ÊNG.

An-k'ang 努亦; T'ang， Han-yin | 陰 H. Terr. jur.

$2^{\circ}$ See No. 6843.

1970 Han-yüan | 源.

$1^{\circ}$ (obs.) 30 li S. of $\mathrm{Ch}^{\text {'ing-ch' }}{ }^{\mathrm{i}} \mathrm{H}$., . Ssŭchnan; Sui, H. in I,in-clining 臨工 chïn. $2^{\circ}$ (obs.) N. of Hsi-ho H., Kansuh; Tang, H. in $\mathrm{Cl}^{6}{ }^{6} \mathrm{e} n \mathrm{~g}$ 成 C.

$3^{\circ}$ See No. 1235.

\section{涵}

1971 Han-clniang 消江 ssü in $P^{\prime}$ u-t'ien H., Fullkien.

\section{韓}

1972 Han 韓.

$1^{\circ}$ At first a feudal state in Slensi; during 5 th, th and $3 r d$ centuries B. C. it was a kingdom comprising parts of Shansi and Honan.

$2^{\circ}$ (obs.) near Moukden; Liao and Kin, Han | C. $3^{\circ}$ See No. 2618.

1973 Han-ch'êng | 城 Hanching.

[148] $1^{\circ}$ H. in ' $T^{\prime}$ ung-chou Fu, Shensi ; Lat. $55^{\circ} 32^{\prime}$, Long. $110^{\circ} 24^{\prime}$;. Ts $s^{6}$ in. Hsia-yang 夏 Han-ch'êng | 塯.

$2^{\circ}$ Chên in Yi-yang H., Honan. [No. 7441]. 
溒 HAN-CHIA-CHI.

1974 Han-chia-chi | 冢 集, T-S. near Ho C., Kansuh.

1975 Han-hung | 洪, chên near Ch'in-yüan H., Shansi.

1976 Han-shan | Ill, chün in Chung-ch'ing 忠清 circ., Corea.

1977 Han-yang | 晹, chên near P'u-cliou Fu, Shansi.

\section{阰}

1978 Han-kou | 浘, (obs.) Han, H. near Fei-hsiang H., Chihli.

1979 Han-tan | 鄲 Han-tan.

$1^{\circ} \mathrm{H}$. in Kuang-p'ing Fu, Chihli ; Lat. $36^{\circ} 40^{\prime}$, I,ong. $114^{\circ} 40^{\prime}$; Han, capital of K. Chao 趙; $T$ s'in, Hanl-tan | 鄲 chïn.

$2^{\circ}$ See No. 225.

\section{喿}

1980 Han-tıng品東, (obs.) in Tun-huang H., Kansulı; Yüan, military district; also written 捍東.

\section{捍}

1981 Han-shu捍浆, (obs.) N. of Shoul-yang H., Shansi; $N$. Wei, H. in Ta-an 大 发 chïn.

1982 Han-tung | 東, see No. 1980.
含 HAN-YANG.

\section{函}

1983 Han-ku 函 谷, see No. 3965.

1984 Han-kuan | 關, post-town near Hsin-an H., Honan.

1985 Han-kuan | 館 ken in Japan.

\section{舆}

1986 Han-hu 寒湖, chên near Ho-shun H., Shansi.

1987 Han-shui | 水, Y. near Tung-an H., Hunan.

1988 Han-t'ao | 桃, chên near Liao C., Shansi.

\section{含}

1989 Han-shan 含 [l Hanshan, H. in Ho C., Anliui ; Lat. $31^{\circ} 47^{\prime}$, Long. $118^{\circ}$ 03'; Han, Li-yang 歴陆; E. Tsin, Lung-k'ang 龍 元; T*ang, Han-slian | 山.

1990 Han1-tzŭ | 㓌, (obs.) S. of Ching-chi 'Tao, Corea ; Han, H. in Lê-lang 藥 浪 chün; Tsin, H. in Tai-fang 带方 cliün.

Hanl-ching, see No. 1973. Han-chuen, see No. 1949. Han-fung, see No. Ha11-hing, see No. 2689. Han-kow, see No. 1957. Han-ning, see No. 2691. Han-tsung, see No. 2694. Han-yang, see Nos. 1967, 2695. 
杭

HANG.

\section{HANG}

\section{杭}

1991 Hang 杭, see No. 1993.

1992 Hang-Chia-Hu | 嘉湖, circ. in Chehkiang connprising Hang-clion $\mathrm{F} t 1$, Clina-lising Fin and Huchou Fu.

1993 Hang-chon | 州 Hang[49] chan, Fin in the HangChia-Hu circ., Chelıkiang ; forming also the two district cities of $\mathrm{Ch}^{\text {'ien- }}$ t'ang and Jên-ho; Lat. $30^{\circ} 12^{\prime}$, I,ong. $120^{\circ} 12^{\prime}$; is capital of the province; literary name Chilı-chiang 之江. S.A., in K. Wu 尖 and K. Yiiel 越; C. Wars, in K. Ch' 11 楚; Ts'in, in Kuei-chi 會嵇 chiin ; E. Han, in Wu 吴 chiun; Tsin, Ch' ien-t'ang 錢磨; Sui, 'Hang | C.; T'ang, Yii-hang 餘 1 ; Sung, Lin-an 臨安; Ming, Hang-chon | 州 Fu. Kinsay (Polo).

1994 Hang-ts'un | 村, sš̌ in Kuang-tê C., Anluui.

Hang-chau, see No. 1993. Hăng-cliau, see No. 2031. Hăng-chun, see No. 2014. Hăng-slıa11, see No. 2032. Hăng-slıwui, see No. 2033. Hăng-yang, see No. 2035.
浩 HAO-CH'I-T⿱⺈.

\section{HAO}

\section{滈}

1995 Hao 滈, (obs.) T"ang, C., S. of P'ing-slian H., Ssŭclı' uan.

\section{高}

1996 Hao 鄗, see Nos. 3170, 4892.

1997 Hao-cli'êng | b佬, posttown near Chno C., Chilnli.

\section{鎬}

1998 Hao-ching 鎘京, see No. 229.

\section{第}

1999 Hao-ching 䧛䇦, (obs.) Liang, H., S. of Chiop'ing H., Knangsi.

2000 Hao-shil | 石, (obs.) $T^{\prime}$ ang, H., E. of Pei-liu H., Kuangsi.

\section{濠}

2001 Hao 嫁, see Nos. 1731, 3865.

2002 Hao-yi 费, (obs.) near Hsü-choı F11, Kiangsu; N. Wei, H. in Sn-yü 宿 豫 chïn.

\section{浩}

2003 Hao 浩, see Nos. 168s, 5052.

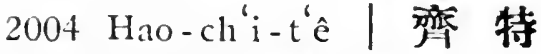
Khaochid, Mongol 'Tribe. 
浩 HARASHAR.

Harashar, see No. 1904.

Harbin, see No. 2174.

Hatur, see No. 1912.

Hal1-kwan, see No. 2139.

Hel1-lung-kianır, see No. 2007.

Heh-yen-tsing, see No. 2011 .

\section{HEI}

\section{紫}

2005 Hei-ch'ih' 黑池, chên near Ho-yang H., Shensi.

2006 Hei-ch'üan | 泉, posttown near Kao-t'ai H., Kansulı.

2007 Hei-lung-chiang | 龍 江 Heh-lung-kiang, a province of Manchuria, also called

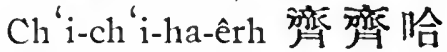
尔 Tsitsihar; bounded on the N. by Siberia; on the E. by Kirin; on the S. by Kirin and Inner Mongolia; and on the IV. by Siberia and Tsetsen. Local naine Pu-k'uei 个奎.

2008 Hei-shan-h11-t'ail 湖台. $1^{\circ}$ Post-town near Kao-t' ai H., Kansulh.

$2^{\circ}$ Post-town near Yü̈-mên H., Kansulh.

2009 Hei-shil-tı | 石渡, chên near Kung H., Honan.

2010 Hei-sung | 松, post-town near Ku-lang H., Kansuh.

2011 Hei-yen-ching | 監井, Hieh-yen-tsing, important
HÂNG-NUNG.

salt wells in the Cli ' 11 hsiung Prefecture, Yünnan; Lat. $21^{\circ} 20^{\prime}$, Long. $101^{\circ} 55^{\prime}$.

2012 Hei-yü | 峪, chên near Sung H., Honan.

\section{HÊNG} 恒

2013 Hêng 恒, (obs.) E. Wei, $\mathrm{H}$. near $\mathrm{Cl}_{1}$ 'ing-yang $\mathrm{Fu}$, Kansuh; see also No. 2031.

2014 Hêng-ch' ${ }^{6}$ | | 春 Hăngchun, H. in T'ai-wan $F u$, Fuhkien; Lat. $22^{\circ} 02^{\prime}$, I ong. $120^{\circ} 43^{\prime}$; this city, near the $S$. Cape of Formosa, was established in 1874 , on the site of a village called Hou-tung 猴洞.

2015 Hêng-nung | 宸.

$1^{\circ}$ (obs.) near Nan-yang Fu, Honan; N. Wei, chiin and H. in Ching 荆 cHOU.

$2^{\circ}$ (obs.) near Ying-chou Fu, Honan; $N$. Wei, H. in Hsi-hêng-nung 西 | 险 chiin and in Cl'ên-liu 陳留 chün. $3^{\circ}$ (obs.) $40 l i \mathrm{~S}$. of Lingpao H., Honan; $N$. $W e i, \mathrm{H}$. in Hêng-nung | 屒 chün, Shan 傸 CHOU.

$4^{\circ}$ See No. 5483. 
恒

HÊNG-SHAN.

2016 Hêng-shan | IU, see No. 487.

2017 Hêng-yang | 陽, see No. 487.

\section{横}

2018 Hêng 橫 Hwang, C. in [198] Nan-ning Fu, Kuangsi ; Lat. $22^{\circ} 37^{\prime}$, Long. $108^{\circ}$ $57^{\prime}$; Han, Kao-liang 高 梁; Liang, Chien-yang 簡陽; Sui, Yüan 緣 C.; Tang, Hêng | C.

2019 Hêng-ch' a †查, sš̆ in Kao-yao H., Kuangtung.

2020 Hêng-ch' êng | 城, H. in Chiang-yüan 江 原 circ., Corea.

2021 Hêng-cl' êng-k'oul城口, post-town near Ling C., Kansuh.

2022 Hêng-ch' ü | 渠, chên near Chou-chilh H., Shensi.

2023 Hêng-ch' 'nan | 川, chên near Cli'êng H., Kansulı.

2024 Hêng-lin | 林, chên near Ch' ang-chou Fu, Kiangsu.

2025 Hêng-ling | 嶺, chên near Ho-shum H., Sliansi.

2026 Hêng-pin | 演, Yokohama, open port; chief town of Kanagawa Ken, Japan.

2027 Hêng-p'u | 浦, see No. 4558:

2028 Hêng-slian | IL, (obs.) Tang, H., W. of Nanning $\mathrm{Fu}$, Knangsi.

\section{衡 HÊNG-SHAN.}

2029 Hêng-shui | 水.

10 Chên near Wên-lisi H., Shansi.

20 Chên near Fêng-hsiang Fu, Shensi.

\section{衡}

2030 Hêng 衡.

$1^{\circ}$ S. Sung, C. in Hu-nan 湖南 circ.

$2^{\circ}$ See Nos. 1829, 2031.

2031 Hêng-chou | 州 Hăng[177] chan, Fu in the HêngYung-Ch 'ên-Kuei circ. of Hunan ; forming also the two district cities of $\mathrm{Cl}^{\text {' }} \mathrm{ing}$ clı 'üan and Hêng-yang ; Lat. $26^{\circ} 55^{\prime}$., Long. $112^{\circ}$ $23^{\prime} ;$ S. A., in K. Cl' u楚; $T s^{\prime}$ in, in Ch' ang-sha 長沙 chiin; Han, in Kuei-yang 桂陽 chün; Wu, Hsiangtung 湘東 and Hêng-yang | 陽; Liang, Hêng | C.; Sui, Hêng-shan |山; $T^{\prime}$ ang and S. Sing, Hêng

| C. in Hul11an 湖南 circ. ; Ming, Hêng-chon | 州 Fin.

2032 Hêng-shan | U4 Hăıg[177] shan.

$1^{\circ}$ H. in Hêngr-cliou Fu, Hunan ; Lat. $27^{\circ} 14^{\prime}$, Isong. $112^{\circ} 38^{\prime} ; \mathrm{Han}$, Hsiang-nan洣南; $T$ sin, Hêng-shan | III.

$2^{\circ}$ See No. 2031. 
2033 Hêng-shui | 水 Hăng[143] shwui, H. in Chi C. L. C., Chihli; Lat. $37^{\circ} 45^{\prime}$, Long. $115^{\circ} 43^{\prime} ;$ Han, 'T'ao桃 H.; sici, Hêng-shui | 水.

2034 Hêng-ts'ai | 材, (obs.) 120 li S. of Lai-yang H., Shantunng; Kin, chên in Lai 荻 C.

2035 Hêng-yang | 晹 Hăngyang.

$1^{\circ} \mathrm{H}$. forming with $\mathrm{Cl}_{1}$ ingcl' ïan H.the prefectural city of Hêng-chon Fin, Hunan ; Lat. $26^{\circ} 55^{\prime}$, Irong. $112^{\circ} 23^{\prime} ; \mathrm{Han}$, Ling 靈 $\mathrm{H} . ; \quad W u$, Lin1ch'êng 臨丞; T'ang, Hêng-yang |陽.

$2^{\circ}$ See No. 2031.

2036 Hêng-Yung-Clı ề11-Kuei | 丞湘桂, circ. in Hn11an connprising Hêng-chon F'n, Ynung-chon Fu, Chên C. and Knei-yang C.

Herat, see No. 1906.

Heri, see No. 1906.

Hia, see No. 2560.

Hia-kiang, see No. 2534.

Hia-lui, see No. 2544.

Hia-pu, see No. 2574.

Hia-tsin, see No. 2561.

Hia-yih, see No. 2570.

Hiang-ching, see No. 2596. Hiang-ho, see No. 2090. Hiang-ning, see No. 2585. Hiang-shan, see No. 2591.
Hiang-wu, see No. 2595. Hian-fung, see No. 2639. Hian-kan, see No. 2641. Hian-i, see No. 2642. Hien, see No. 2697. Hil, see No. 5599. Hilı-kuh, see No. 5600 . Hin, see No. 2721.

Hin-cling, see No. 2722. Hing, see No. 2814. Hing-hwa, see No. 2821. Hing-i, see No. 2840. Hing-kwoh, see No. 2824. Hing-ngan, see No. 2815. Hing-nieh, see No. 2839. Hing-1ling, see No. 2807. Hing-ping; see No. 2829. Hing-shan, see No. 2830. Hing-tai, see No. 2850. Hing-tang, see No. 2845. Hing-wăn, see No. 2836. Hiogo, see No. 5133. Hiroshima, see No. $3416^{\mathrm{a}}$. Hi11-11ing, see No. 2858. Hinng, see No. 2877. Hiung-chne11, see No. 2880. H'lari, see No. 3553. $\mathrm{H}^{\prime}$ lassa, see No. 3555. H'lunchub, see No. 3842. $\mathrm{H}^{\prime}$ lurungjung, see No.4027.

\section{$\mathrm{HO}$} 不

2037 Ho-yüan 乔源, ss̆̆ near Lien-hua 'T., Kiangsi.

\section{和}

2038 Ho 和 Ho. 
和

Ho.

$1^{\circ}$ C. I. C. in the An-LüCh $\mathrm{u}-\mathrm{Ho}_{0}$ circ., Anhui; Lat. $31^{\circ} 42^{\prime}$, Long. $118^{\circ}$ $25^{\prime}$, C. Wars, in K. Ch' ' 楚; $T s^{\prime}$ in, Li-yang 歴陽 H. in Chin-chiang 九汇. chiin; Han, in Hnai-nan 淮南 chün; Three Ks., under IVu; $N, T s^{\prime} i$ and S. Sung, Ho |C.

$2^{\circ}$ See Nos. 3005, 4575.

2039 Ho-chêng | IE, post-town near 'Ti-tao C., Kansuh.

2040 Ho-ch'êng | 城, (obs.) Liang, town $220 l i \mathrm{E}$. of P'êng-tsê H., Kiangsi.

2041 Ho-cl' $\mathrm{i}$ | 溪.

$1^{\circ}$ Ss ̌̆ near Nan-cling H., Fulukien.

$2^{\circ}$ (obs.) $20 \quad l i \quad \mathrm{~W}$. of

Yo-ch 'ilı H., Ssŭch' uan;

Sung, H. in Hsi-ning 西察 military district.

2042 Ho-chou | 州, see No. 1900.

2043 Ho-ch' ${ }^{6}$ i | 曲 Ho-kinh, (obs.) Yüan, C. near W11ting C., Yünnnan.

2044 Ho-han | 含, sš̆ near Lin-kao H., Kuangtung.

2045 Ho-ho | 合, post-town near Hsiang-ho H., Chilıli. 2046 Ho-lisiao | 孝., Y. near Hsin-yang C., Honan.

2047 Ho-jung | 我, (obs.) near Kung-ch'ang Fil, Kansuh; Sui, H. in 'Tang-ch' ang 䇹昌 chiun.
和 Ho-shco-r't

2048 Ho-kê-shan | 歌山, Wakayama, ken and chief town, Japan.

2049 Ho-lin | 林, see No. 1901. 2050 Ho-lin-kê-êrh | 林格爾 [518] Horinkar, 'T. in So-p'ing Fit, Shansi ; Lat. $40^{\circ} 15^{\prime}$, Long. $112^{\circ} 35^{\prime}$.

2051 Ho-lin $\mid$ 琳 = | 林, see No. 2049.

2052 Ho-p'ing | 平, Ho-ping.

[243] $1^{\circ}$ H. in Hui-chon Fu, Kuangtung; Lat. $24^{\circ}$ $30^{\prime}$, Itong. $114^{\circ} 55^{\prime}$; orig., Lung-ch'uan 龍 川I.

$2^{\circ}$ Chên near An-chi H., Chehkiang.

$3^{\circ}$ Ss ̌̌ n near Ch' ao-yang $\mathrm{H}$., Kuangtung.

2053 Ho-shin | 閐, see No. 2058.

2054 Ho-shang | 份, ss̆̌ in Iiu-an C., Anhui.

2055 Ho-shun | 順, H. in Liao [75] C., Shansi ; I at. $37^{\circ} 30^{\prime}$, I ong. $113^{\circ} 36^{\prime}$; S. A., Yüi-ch' êng 榆城; $N$. Ts'i, Liang-yii 梁榆; Sui, Hoshu11 顺.

2056 Ho-sln110-mang-k'ê | 碩 茶㖟 Hocho-mancan, town in Inner Mongolia; Lat. $43^{\circ} 40^{\prime}$, I,ong. $121^{\circ} 30^{\prime}$.

2057 Ho-sln110-t'e | 碩 特

Khoshoit, Mongol Tribe. 
和 HO-'`'

2058 Ho-t'ien |闐, (also called Chï-sa-tan-11a 㲘薩旦那; Hul-t'an 忽崖; Huan-11a 渙那; $\mathrm{Cl}^{\prime} \mathrm{\prime} \mathrm{u}-\tan$ 屈丹; Y ï-tun 于遁; Huo-tan 豁旦; Wn-tuan五端; Wo-tuan 斡端; and $\mathrm{Ho}$ shan 和閭) Khoten or Yi-li-ch'i 伊里㧝 Ilchi, C. L. C. in Kashgar circ., New Dominion, I at. $37^{\circ}$, Long. $80^{\circ} 30^{\prime}$. Coten (Polo). 2059 Ho-tu | 都, (obs.) in Ya-chou Fu, Ssŭch' 'uan; T'ang, C. in Chien-nan 劍南 circ.; Sung, C. in $\mathrm{Li}$ 黎 chou.

\section{啝}

2060 Ho-tzŭ 啝子, chên near Ho C., Shansi.

2061 Ho-yüan | 源, (obs.) $T^{*} a n g$, town near Ling-shih H., Shanıs.

\section{河}

2062 Ho 河 Ho, C. in Lan[428] chou Fu, Kangsuh; Lat. $35^{\circ} 44^{\prime}$, Long. $103^{\circ}$; Han, 'Ta-hsia 大夏; $T s^{6}$ in, Ho

2063 Ho-ch'i | 溪, ssŭ near Ch'ien-chon 'T., Hunan.

[064 Ho-chien |閒 Ho-kien. 2119] $1^{\circ} \mathrm{F} u$ in the $\mathrm{T}^{\prime}$ ien-chingHo-chien circ., Chiluli ; forming also the district sity of the same name;
河 Ho-ching.

Lat. $38^{\circ} 33^{\prime}$, Long. $116^{\circ}$; S. A., in Tung-yaug 東 陽; C. Wars, in 1st $\mathrm{K}$. Yen 菬, 2nd K. Chao 拈，3rd K. Chi 淬; $T^{\prime}$ 'in, in Chü-lu 鎹麇 chïn and Shang-ku上. 谷 chïn; Han, Po-hai 渤海 and Ho-chien | 間; Sui and T'ang, Ying 瀛 C.; Sung, Ying-hai 海海; Ming, Ho-chien | 間 Fu. Cacanfu (Polo).

$2^{\circ} \mathrm{H}$., forming the prefectural city of the same name, Chihli; Lat. $38^{\circ}$ $33^{\prime}$, Long. $116^{\circ}$.

2065 Ho-ch $^{6} \mathrm{ih}$ / 池 Ho-chi.

$1^{\circ} \mathrm{C}$. in $\mathrm{Cl}^{\prime}$ ing-yüan $\mathrm{Fu}$, Kuangsi; I at. $24^{\circ} 42^{\prime}$, Long. $107^{\circ} 43^{\prime} ; T^{\prime}$ ang, Chih 智 C.; Sung, Hocl'ih 池 C.

$2^{\circ}$ (obs.) $50 l i \mathrm{~W}$. of $\mathrm{Hui}$ H., Kansuh; Han and Tsin, H. in Wu-tu 武 都 chïn; Sni, H. in Ho-ch'il | 池 chün; T゙ang, H. in Fêng 鳳 C.

$3^{\circ}$ See Nos. 1716, 2357. 2066 Ho-ching | 津 $\mathrm{H}_{\mathrm{O}-\mathrm{t} \text { sin. }}$. [123] $1^{\circ} \mathrm{H}$. in Chiang C., Shansi ; Lat. $35^{\circ} 38^{\prime}$, Long. $110^{\circ} 46^{\prime}$; orig., K. Kêng 聎; $\mathcal{T} s^{\prime} i n$, P'i-slih 支 E; 2nd 
河

Ho-ching.

$W e i$, Lung-mên 龍門; T'ang, Ch'in 秦 $\mathrm{C}$.; Sung, Ho-ching | 津. $2^{\circ}$ (obs.) IV. of Ho C., Kansuh ; Sui, H. in Chiao-ho 澆河 chün. $3^{\circ}$ (obs.) $2 l i \mathrm{~W}$. of Hoching H., Shansi ; Kin, H. in Ho-chung 河中 Fu.

2067 Ho-ch'ing | 清, see Nos. 4388, 6271.

2068 Ho-cliou-wei | 州衞, T-S. near Ho C., Kansul.

2069 Ho-ch' $u$ | 除, (obs.) N. E. of Yï-lin H., Shensi; E. Han, H. in Wn-yüan 五原 chïn.

2070 Ho-ch' ii | 曲 Ho-kiuh, [257] H. in Pao-tê C., Shansi; Lat. $39^{\circ} 15^{\prime}$, Long. $111^{\circ}$ $02^{\prime}$; orig., Hsiung-yung. 雍勇; Sung, Huo-shan 水仙; Kin, Ho-ch' ï | 曲. $2070^{\mathrm{a}}$ Ho-chuang | 藞, sš̆ in Hsiao-shan H., Chehkiang. 2071 Ho-chung | 中.

$1^{\circ}$ (obs.) in Vung-chi H., Shansi; T"ang. Fu in the Ho-tung 河東 circ.; Sung, Fu in Xung-lising

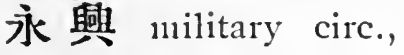
Shensi; Kin, Fu in Na11 Ho-tung 南河東 circ.; Yüan, Iin in Clin11ning 登掌 circ.

$2^{\circ}$ See Nos. 5318, 5342.
河 Ho-k"ou".

2072 Ho-hui 河 會, Y. near Ho-ch ï H., Shansi.

2073 Ho-11si | 西 Ho-si.

[26] $1^{\circ} \mathrm{H}$. in Lin-an Fu, Yünnani Lat. $24^{\circ} 15^{\prime}$, Long. $102^{\circ} 43^{\prime} ; T^{\prime}$ ang, T'sung 宗 C.; Yiuan, Ho-hsi 西 $\mathrm{H}$.

$2^{\circ}$ Post-town near Mi-chih H., Shensi.

$3^{\circ}$ T-S. near Mien-ning H., Ssŭch '11an.

$4^{\circ}$ 'T. T'. near Wên-ch' uan H., Ssŭch' 'uan.

$5^{\circ}$ (obs.) in Lin-fên H., Shansi ; N. Wei, chün in Chin 晋 CHоU.

$6^{\circ}$ (obs.) E. of Chao-yi H., Shensi ; I'ang, H. in Ho-chung $\mid$ 中 Fu. $7^{\circ}$ (obs.) N. E. of Ho-hsi H., Yünnan; T Tang, H. in 'sung 崇 C.

$8^{\circ}$ (obs.) near Hsü-chou $\mathrm{Fu}$, Ssŭch' 'uan; T' $T^{\prime}$ ang, H. in Chih 志 C. $9^{\circ}$ See Nos. 367, 5610.

2074 Ho-hsi-wu | 西務, ssŭ in Wu-ch' ing $\mathrm{H}_{\text {., Clihli. }}$

2075 Ho-hsün | 沉, T-S. near

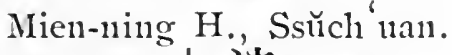
2076 Ho-jung | 溶. ssü near Chien-li $\mathrm{H}_{\text {., Hupeh. }}$

2077 Ho-k'ou | 口.

$1^{\circ}$ Sš̆ near Chiang-ning Fu, Kiangsu.

$2^{\circ}$ Ssŭ near $\mathrm{Ch}^{\prime}$ ien-shan H., Kiangsi. 
河

2078 Ho-li-ch'i | 擵溪, chên near Ning-kno Fin, Anhni.

2079 Ho-11an | 南 Ho-nan.

$1^{\circ}$ A province of China; literary name, Yü 㐨; capital, K'ai-fêng F'u; it contains 3 circ., 9 Fi1, 3 T., 4 C. L. C., $6 \mathrm{C}$, and $96 \mathrm{H}$.

$2^{\circ} \mathrm{Fu}$ in the Ho-nan-ShanJu circ, Honan ; forming also the district city of Lo-yang; Lat. $34^{\circ}$ $43^{\prime}$, Long. $112^{\circ} 28^{\prime}$; Ts'in, San-ch'nan 三 川; Han, Han-tu 漢 都 H.; $W_{e i}$, Ssŭ 司 C.; 2nd $W e i$, I, 洛 C.; P. Chou, 'Tung-ching 束京; Sui, Yï 㐨; 7'ang, Tung-tu 東都; Sung, Hsi-ching 芫京; Kin, Chin-ch'ang 金. 昌; Ming, Honan | 南 Fin。

$3^{\circ}$ Chên near $\mathrm{H}_{u} \mathrm{H}$., Shensi.

$4^{\circ} T^{6}$ ang, circ. and Fu.

$5^{\circ} T^{\prime}$ in and Han, chïn.

$6^{\circ}$ See No. 3005.

2080 Ho-nan-K'ai-Kuei-Ch' ên-

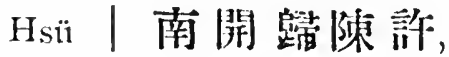
circ. in Honan, comprising $K^{\prime}$ ai-fêng Fu, Kuei-tê Fu, Ch 'ên-chon Fu and Hsü C.

2081 Ho-nan-Shan-Ju | 南傸 汝, circ. in Ho-11an, con1-
河 Ho-SHANG.

prising Ho-nan Fu, Shan C. and $\mathrm{Ju} C$.

2082 Ho-nei | 內 Ho-nui.

$1^{\circ} \mathrm{H}$. forming the prefectural city of Huai-ch'ing, Honan; Lat. $35^{\circ} 07^{\prime}$, I,ong. $113^{\circ}$; Han, Yel1wang 野王; Sui, Honei 內 ; 7'ang, Huai 懐 C.

$2^{\circ}$ (obs.) Han, chïn comprising parts of Shansi and Honan.

$3^{\circ}$ See Nos. 2228, 2759.

2083 Ho-pei | 北.

$1^{\circ}$ Circ. in Honan, connprising Chang-tê Fu, Wei-hui $F u$, and Huaicli'ing Fin.

$2^{\circ}$ (obs.) $T^{6}$ ang, circ. comprising parts of Chi 懻 $\mathrm{CHOU}$ and $\mathrm{Y}$ и 幽 CHOU; Sung, circ.

$3^{\circ}$ (obs.) E. Han, chïn comprising part of Shansi.

$4^{\circ}$ See No. 5177.

2084 Ho-pin | 溜, (obs.) in Inner Mongolia, N. of Shensi (the Ordos country); T'ang, H. in Shêng 勝 C.; Liao, H. in 'Tung-shêng 東勝 C.

$2084^{\mathrm{a}}$ Ho-po | 婆, ssŭ in Chiehyang $H .$, Knangtung.

2085 Ho-shang | 上, (obs.) Ts'in and Han, chïn. 
河

Ho-T'ou.

2086 Ho-t'ou | 䫓, chên near 'Тz'ŭ-ch'i $\mathrm{H}$., Chelıkiang.

2087 Ho-tu-t'ien | 都田, posttown near An-nan $\mathrm{H}$, Kueichou.

2088 Ho-tung | 東.

$1^{\circ}$ Circ. in Shansi, com1prising $\mathrm{P}^{\prime} \mathrm{u}$-clion $\mathrm{Fu}$, P'ing-ting $\mathrm{C}$., Chiang C., Ho C. and Chieh C. $2^{\circ}$ Post-town near $\mathrm{P}^{\prime}$ uchou Fu, Shansi.

$3^{\circ}$ 'T-S. near Lei-po 'T., Ssŭcli'uan.

$4^{\circ}$ T. T. near Min-ch' nan H., Ssŭch' tuan.

$5^{\circ}$ (obs.) N. of Hsia H., Shansi ; $I V$. Han, chün. $6^{\circ}$ (obs.) W. of Sung-tzŭ H., Hupelı; S. Sung, chiin in Ching 荆 CHOU. $7^{\circ}$ (obs.) S. E. of Yungchi H., Shansi; $N$. Wei, chiun in $\mathrm{Cl}^{\prime}$ in 奉 CHOU.

$8^{\circ}$ (obs.) in Yung-chi H., Shansi; Sui, chïn and $\mathrm{H}$. in $\mathrm{Chi}$ 蕉 cHou; $T^{6}$ ang, H. in Ho-chung | 中 $\mathrm{Fu} ;$ Sung, H. in Ho-chung | 中 Fu, in Yung-hsing 永圔 military" circ.; Kin, H. in Ho-chung | 中 $\mathrm{Fu}$, Ho-tung | 東 circ.; Yüan, H. in Ho-chung 中 $F u$, Clin-ning 留戛 circ.
河 HO-YÜAN.

$9^{\circ}$ (obs.) in Ya-cloou $\mathrm{Fu}$, Ssŭch uan; T'ang; C. in Li 黎 CHOU; Sung, H. in Li 黎 chou.

$10^{\circ}$ (obs.) Ts' $i n$ and $H a n$, chïn.

11 ${ }^{\circ}$ (obs.) T"ang, circ. comprising part of Chi 䓔 chov.

$12^{\circ} \mathrm{H}$. in $\mathrm{Cl}_{1}$ 'ing-shang 磨份 circ., Corea.

$13^{\circ}$ See Nos. 5200, 6546.

2089 Ho-yang | 陽 Ho-yang.

$1^{\circ} \mathrm{H}$. forming the prefectural city of $\mathrm{Ch}^{\prime}$ êngchiang, Yünnan; Lat. $24^{\circ} 42^{\prime}$, Long. $103^{\circ} 04^{\prime}$; $T^{6}$ ang, land of S. barbarians; Yïan, Ho-yang H. ; Ming, Ch'êngchiang 徽江 Fu。

$2^{\circ}$ Ss̆̈ near Ting-hsing H., Chihli.

$3^{\circ} \mathrm{H}$. in $\mathrm{Ch}^{\prime}$ ing-shang 慶 份 circ., Corea.

$4^{\circ}$ See Nos. 544, 4385, 4388.

2090 Ho-yin | 陵, (obs.) Han, H. in Lat. $34^{\circ} 58^{\prime}$, Long. $113^{\circ} 42^{\prime}$; Ts'in, $\mathrm{P}^{\prime}$ ing-yin 平陰. Now the following.

2091 Ho-yin-hsiang | 陰鸟, ssŭ near Jung-yang $\mathrm{H}$., Honan. See Preceding.

2092 Ho-yü | 裕, chên in Tangyang H., Hupeh.

2093 Ho-yüan | 源 Ho-yuen. 
河

HO-YÜAN.

[478] $1^{\circ}$ H. in Hui-chon Fu, Knangtung; Lat. $23^{\circ}$ $42^{\prime}$, Long. $114^{\circ} 34^{\prime}$; Han, Lung-ch'nan 龍 川; S. $T s^{i} i$, Ho-yiuan | 源 $\mathrm{H}$.

$2^{\circ}$ (obs.) S. of Kokonor; Sui, chiin in Yung 䔨 cHou.

\section{何}

2094 Ho-pao 何 保, Y. near Ho-ch' '̈i H., Shansi.

2095 Ho-t'ang | 塘, post-town near Li-ling $\mathrm{H}$., Hunan.

\section{賀}

2096 Ho 賀 Ho, H. in P'ing-lê [416] Fu, Kuangsi; Lat. $24^{\circ} 08^{\prime}$, Iong. $111^{\circ} 17^{\prime} ;$ Han, Linho 臨賀; T'ang, Ho $\mid \mathrm{C}$.

\section{款}

2097 Ho 呩, see No. 4220.

\section{霍}

2098 Ho 霍 Holl.

[123] $1^{\circ}$ C. L. C. in the Ho-tung circ., Shansi; Lat. $36^{\circ}$ $34^{\prime}$, Long. $111^{\circ} 43^{\prime}$; orig., Ch' 'in-yang 邱陽; E. Han, Yung-an 永 安; Sui, Fên 汾 C. and Ho-yi | 邑; Tang, Lii 吕 C.

$2^{\circ}$ See No. 4005.

2099 Ho-chao | 趙, chên near Yi-shilh H., Shansi.
霍

HO-SHAN.

2100 Ho-ch'iu | 邱 Hohkiu, H. in Ying-chou Fu, Auhui; Lat. $32^{\circ} 23^{\prime}$, Long. $116^{\circ}$ $13^{\prime}$; Chou, Ho-shu-fêng-yi | 叔封邑, appanage of Ho-shu; S.A., K. Liu-liao Tsin, 六㢣; Ho-ch'iu | 邱.

2102 Ho-êrlı-chang-kı | 耳부 谷, T-S. north of Ta-chien1u, Ssǔch' uan. Thibetan name Tclirango (Baber); Chango (Rockhill).

2103 Ho-êrh-cho-k'ê / 耳卓科, T-S. nortliwest of 'T'a-chienlu, Ssŭch'uan. Thibetan name Djego (Baber); Chuwo (Rockhill).

2104 Ho-êrh-kan1-tzŭ-ma-shı | 耳甘孜麻書, T-S. northwest of Ta-chien-1ı, Ssǔcl' 'uan. Thibetan name Mazur (Rockhill). See No. 3094.

2105 Ho-êrh-k'ung-sa|耳孔撒, T-S north-west of 'Ta-chienlı, Ssŭclı' nan. Tliibetan name Kungsar (Baber); Kangsar (Rockhill).

2106 Ho-êrl1-kno-ssŭ | 耳果 斯; sš̆ in Chên-hsi T., in the New Dominion.

2107 Ho-êrlı-pai-li | 耳百利, T-S. north-west of 'Tachien-lu, Ssụ̆ch' 'ıan. Thibetan name Pere (Baber); Berim (Rockhill).

2108 Ho-shan | 山L Ho-shan. 
霍

HO-SHAN.

$1^{\circ} \mathrm{H}$. in Liu-an C., Anhui ; Lat. $31^{\circ} 30^{\prime}$, Long. $116^{\circ} 20^{\prime}$; orig., Yo-an 岳安, in Lilt-an 六多.

$2^{\circ}$ Post-town near Ho C., Shansi.

$3^{\circ}$ See No. 4005.

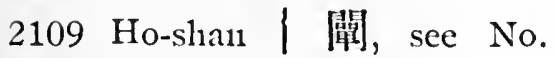
2414.

2110 Ho-shu-fêng-yi | 叔封邑, see No. 2100.

2111 Ho-yi | 邑, see No. 2098.

\section{寉}

2112 Ho-yu-p'ing 崔遊坪, post-town near fion C., Ssŭch '11an.

\section{寉鳥}

2113 Ho 鶴, see No. 2115.

2114 Ho-ch' êng | 城, (obs.) $K$. $W e i$, town near $\mathrm{Ch}^{\prime}$ angyüaıı $\mathrm{H}$., Chihli.

2115 Ho-ch' ing | 慶 Hol-king, [398] C. in I,i-cliang Fu, Yünnan ; Lat. $26^{\circ} 32^{\prime}$, Long. $100^{\circ} 22^{\prime}$; orig., Ho | C.; Yïan, Ho-ch ing | 度.

2116 Ho-êrlh-tsa 菆咱, 'T-S. near Sung-p an T., Ssŭch'uan.

2117 Ho-fêng | 䎑 Hoh-fung, [266] C.L.T. in Shil1-Ho circ., Hupeh ; Lat. $30^{\circ} 10^{\prime}$, Long. $110^{\circ}$.

2118 Ho-mao-ho|毛 河, chên near T'ung-ling $H$., Anluni.
合 Ho-crü.

2119 Ho-shan | II Hoh-shan, [444] H. in Chao-ch'ing Fu, Knangtıng; Lat. $23^{\circ} 08^{\prime}$, Long. $112^{\circ} 28^{\prime}$.

\section{合}

2120 Ho 合 Hoh.

[30] $1^{\circ}$ C. in Chung-ch'ing Fu, Ssŭch ' $11 a 11$; Lat. $30^{\circ} 08^{\prime}$, Long. $106^{\circ} 24^{\prime}$; orig., in K. P'u 鉴; $T s^{\prime}$ in, Tien-chiang 熱江. H.; E. Wei and S. Sung, Ho | C.; S. Ts' $i$, Tungtang-ch'ii 束宕渠 chiin in Liang 梁 CHOU ; Sui, Fon 楛 $\mathrm{C}$.; Trang, $\mathrm{Pa}$ ch'van 巴川.

$2^{\circ}$ See Nos. 3668, 3815, 4140.

2121 Ho-ch'i | 溪, chên near Ch'ang-hsing H., Chehkiang.

2122 Ho-chiang | 江 Holl-kiang. [528] $1^{\circ}$ H. in I,ü C., Ssŭch ' 'wan; Lat. $28^{\circ} 48^{\prime}$, Long. $105^{\circ}$ $56^{\prime} ;$ Han, Fu 符 $\mathrm{H}_{\text {. }}$; P. Chou, Ho-chiang | 江.

$2^{\circ}$ (obs.) formerly H., S. E. of 'Tu-yün Fu, Kueichon.

2123 Ho-cliao / 角, chên near Yang-wu H., Honan.

2124 Ho-chon | 州, see No. 1900.

2125 Ho-chü | 埧, 'T-S. near Sung-p'an T., Ssŭch' uan. 
合

2126 Ho-fei | 肥 Holl-fi, H. forming the prefectural city of Lï-chon, Anluni; Lat. $31^{\circ} 50^{\prime}$, Long. $117^{\circ}$ 15'; Han, Ho-fei | 肥; Sung; Ju-yin 沙陰; Sui, Liî-chiang 㢝江 chün; T'ang, Lï 盧 C.

2127 Ho-11o | 河.

$1^{\circ}$ Chên near Hno-clia H., Honlan.

$2^{\circ}$ See No. 2814.

2128 Ho-ho-ching | 河 津, (obs.) near 'T'ai-yüan Fin, Shansi; Kin, chên in Lan 颳 $\mathrm{C}$.

2129 Ho-hui | 會, Y. near Lan H., Shansi.

2130 Ho-la-huo-chê | 刺火者, see No. 1900.

2131 Ho-lai | 懶, see No. 2132.

2132 Ho-lan | 蘭, C. L. T. in Ning - Hsing - Hu11 circ. Kirin. Locally called Ninguta. See No. 4694. Kin, Ho-lai | 懶 circ.; Yïan, Ho-lan || Fu.

2133 Ho-p $u$ | 浦 Hoh-pu, H. forming the prefectural city of Lien-chou, Knangtung; Lat. $21^{\circ} 39^{\prime}$, Long. $108^{\circ} 59^{\prime}$.

2134 Ho-shui | 水 Hoh-shwui. [38] $1^{\circ} \mathrm{H}$. in $\mathrm{Ch}^{\prime}$ ing-yang $\mathrm{F} 11$, Kausuh ; Lat. $36^{\circ} 03^{\prime}$, Long. $107^{\circ} 58^{\prime}$; Han, Kuei-tê 蹼德; E. IVei, Yü 蔚 C. and Hua-ch 'ilı
合 HOH-Y $\mathrm{YNG}^{x}$.

華池; Sui，Lê-fan 樂 蟠; $T^{6}$ ang, Ho-shui | 水.

$2^{\circ}$ Chên near Wu-yang H., Honan. $3^{\circ}$ See No. 66.

\section{郃}

2135 Ho-yang 命陽 Hol1-yang, [54] H. in T'nung-chon Fu, Shensi ; Lat. $35^{\circ} 18^{\prime}$, Long. $110^{\circ} 05^{\prime}$; orig., $\mathrm{K}$. Hua 華; Han, Ho-yang 隰。

Ho-bé, see No. 6217. Ho-chi, see No. 2065. Ho-kie11, see No. 2064. Ho-kiul, see Nos. 2043, 2070.

Ho-1111, see No. 2082. Ho-ping, see No. 2052. Ho-si, see No. 2073. Ho-tsin, see No. 2066. Ho-yuen, see No. 2093. Hochomancan, see No. 2056.

Hoh, see Nos. 2098, 2120. Hoh-fi, see No. 2126. Hoh-fung, see No. 2117. Hoh-kiang, see No. 212.2. Holl-king, see No. 2115. Hoh-kiu, see No. 2100. Hol1-pu, see No. 2133. Hoh-slia11, see No. 2108, 2119.

Hoh-shwui, see No. 2134. Holl-yang, see No. 2135. 
合 Hornow.

Hoilow, see No. 1321.

Hongkong, see No. 2588.

Horinkar, see No. 2050.

Hormulu, see No. 2192.

\section{HOU}

\section{媵}

2136 Houl-lung 啳壠, shê near 'Tanshui H., N. Formosa, (Fuhkien).

2137 Houı-pu | 部, ssŭ in T'aihu H., Anluni.

2138 Ho-tsang | 藏, the name given to Ulterior 'Thibet and its capital, 'Teslinh'lumbı; see No. 133.

\section{榐}

2139 Houl-kuan 候官 Hankwan.

$1^{\circ} \mathrm{H}$. forming witl $\mathrm{Min}$ H. the prefectural city of Fu-chou, Fulukien; Lat. $26^{\circ} 03^{\prime}$, Long. $119^{\circ} 25^{\prime}$.

$2^{\circ}$ See Nos. 3268, 4558, 4615, 6799.

2140 Hon-1na | 馬, chên in Ch' ï-wu H., Shansi.

\section{猴}

2141 Hont-tung | 猴洞, see No. 2015.

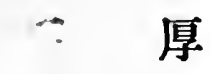

2142 Hol1-clı'iu 厚邱, see No. 5773.
Hu.

2143 Hon-chon | 州 Fin in Hsien-ching 威鏡 circ., Corea.

2144 Houl-tzŭ | 子, chên near Wei-yüan H., Kansılı.

\section{HU \\ 胡}

2145 Hu-ching 胡津, (obs.) S. W. of Kung H., Ssurclı' uan; T"ang, H. in Hsiel 協 C.

2146 Hii-chnang-t'un | E屯, post-town near Hai-ch 'êng H., Shingking.

2147 Hu-fan \璠, (obs.) S. of Hsing-wêt1 H., Ssŭcl' 'nan; T゙'ang, H. in Xüeh 悅 $\mathrm{C}$.

2148 Hun-lê | 樂, ssŭ in Ningkno H., Anluni.

2149 Hu1-su | 蘇, (obs.) 20 li S. W. of Ning-ching $H$., Chillili; Sui, H. in P'ingyiian 平原 chïn.

2150 Hu-wa-êrl | 互耳, Klıavar, a town in the Persian province Mazanderan, S. of Sari.

$2151 \mathrm{Hu}$ 湖.

\section{湖}

$1^{\circ}$ Chên in Lung-yu, Chel1kiang.

$2^{\circ}$ (obs.) E. of Wênlısiang H., Honan; $W$. Han, E. Han and Tsin, $\mathrm{H} 11$ H.

$3^{\circ}$ See Nos. 2154, 7033, 7198. 
湖

$\mathrm{HU}-\mathrm{CH}^{\prime} \mathrm{A}$.

2152 Hu-ch'a | 良, chên near Chin-t'an H., Kiangsu.

2153 Hut-ch'i | 溪, chên near Chin-t'an H., Kiangsu.

2154 Hu-chou | 州 Hu-chan, $\mathrm{Fu}$ in the Hang-Chia- $\mathrm{H}_{\mathrm{u}}$ circ., Chehkiang; forming also the two district cities of Kuei-an and Wu-ch' êng; Lat. $30^{\circ} 48^{\prime}$, Long. $120^{\circ}$ $03^{\prime} ; S . A$., in K. Wu 吳 and K. Yüeh 越; $C$. Wars, Kul-ch'êng 㼋城; Ts'in, Wu-ch'êng 岛程; Han, in Knei-chi 會稽 chïn and Tan-yang 丹陽 chiin; Three Ks., Wu-lising 吴興; Sui and $T^{6}$ ang, $\mathrm{Hu}_{\mathrm{C}}$ C.; Sung; Chaoch'ing 昭㢁; Ming, Huchou 州 Fu. Vuju (Polo).

2155 Hu-jun-chai| 潤些, ssŭ near Yang-li C., Kuangsi.

2156 Hu-kou |口 Hu-kan, H. [528] in Chin-chiang F'n, Kiangsi ; Lat. $29^{\circ} 54^{\prime}$, Long. $116^{\circ} 18^{\prime}$.

2157 Hu-kuang | 廣 Hn-kwang, (obs.) Ming, a province comprising Hunan and Hupeh; this name, or that of Liang-11u 兩 !, is still applied to these two provinces spoken of collectively, especially as a Governor-Generalship.

2158 Hu-11an | 南 Hul-11an.
虎

HU-M̂̂N.

$1^{\circ}$ A province of China; literary names Hsiang 湘, Hsiung-hsiang 熊 湘 and T'an-chon 潭 州; capital, Ch' ang-sha $\mathrm{Fu}$; it contains 4 circ., $9 \mathrm{~F} 11,4$ C. L. T., 4 T., 4 C. L. C., 3 C. and $64 \mathrm{H}$.

$2^{\circ}$ Sung and S. Sung, circ. 2159 Hu-pei | 北 Hu-pel.

$1^{\circ}$ A province of China; literary names $O$ 鄂, $\mathrm{Ch}^{\prime} \mathrm{u}$ 楚, O-chu鄂渚 and Chiang-hsia 江夏; capital, Wu-ch'ang Fin; it contains 4 circ., 10 Fu, 3 T., 1 C. L. C., 7 C., and $60 \mathrm{H}$.

$2^{\circ}$ Sung and S. Sung, circ.

2160 Hul-p'o | 防, ss̆̌ in Juichin H., Kiangsi.

2161 Hu-pu| 团 Hu-pu, T. in Hsü-chon Fu, Kiangsu.

2162 Hu-shu | 執, chên near Chï-jung H., Kiangsu.

2163 Hn-tai 埭, chên near Clin-kuei H., Kiangsu.

\section{虎}

2164 Hu-chui 虎陮, T-S. near Kuei-yang Fu, Kueichou. 2165 Hu-lang | 郎, T-S. near Mien-ning H., Ssŭch' nan. 2166 Hu-mên|門 Hu-111un or Bocca Tigris, 'T. in Kuangchou Fu, Kuangtung; Lat. $22^{\circ} 40^{\prime}$, Long. $110^{\circ} 52^{\prime}$. 
虎 Hu-ssü.

2167 Hut-ssŭ $\mid \overline{0}$, see No. 627. 2168 Hu-t'ou-chêng | 頙城, (obs.) formerly town near Fı-shuı H., Ssŭcli' 11an.

胡

2169 Hul-lu-tun 胡监墩 site of 'T'ai-chung Fu, in MidFormosa.

2170 Hut t’out-kuan | 預關, ssü near Ma-ch' êng H., Hupeh.

2171 Hul-tu-k'ou 渡口, ss in Chiang-ling H., Hupeh.

2172 Hu-wei 虎威, Y. near Chiang-hua H., Hunan.

2173 Hul-yii | 峪, chên near Ho-shun H., Shansi.

\section{呼}

2174 呼菊 Hu-lan, Fu in SuiLan-Hai circ., 'Tsitsiluar; Lat. $46^{\circ} 30^{\prime}$, Long. $126^{\circ}$ $05^{\prime}$.

2175 Hu-lun-pei-êrlı | 倫具爾 Hurnupir, town in Hel1lung-kiang; I at. $49^{\circ}$, Long. $119^{\circ}$.

2176 H11-t $11-p i$ |圖必, or Kut'a-pa 古塔巴，Kntopi， also called Klintukbai and Khutaiyai ; in Cli'ang-chi H., New Dominion. Lat. $44^{\circ} 10^{\prime}$, Long. $87^{\circ} 50^{\prime}$.

\section{焉}

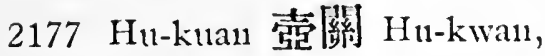
[402] H. in I I1-an Fu, Sliansi; I at. $36^{\circ} 02^{\prime}$, Long. $113^{\circ}$ $05^{\prime}$; orig., in K. Li 黎; $\mathcal{T} \sin$, Hul-kuan | 關.
HU-CHIUNG.

\section{紫及}

2178 Hu 檽 Hu, H. in Hsi-an Fin, Shensi ; Lat. $34^{\circ} 08^{\prime}$, Long. $108^{\circ} 38^{\prime}$; Hsia, in in $\mathrm{K}$. $\mathrm{H}_{11}$ 愿; Yin, in $\mathrm{K}$. Ch'ung 紫; Ts'in, Kan. ting 甘亭; Han, Hu $\mid \mathrm{H}$.

$2179 \mathrm{H} n$ 愿.

$$
\text { 愿 }
$$

$1^{\circ}$ (obs.) Hsia, K. in Shensi.

$2^{\circ}$ See No. 2178.

\section{渥}

2180 Hu 㵿, see No. 5503.

2181 Hul-wei | 尾, see No. 6217.

\section{的斗.}

2182 Hutien 獬苦, chên near Wan-clı 'ïan II., Shansi.

狐

2183 Hu-chê 狐 諿, see No. 7849.

2184 Hu-11u 奴, (obs.) 30 li N. E. of Shun-yi H., Chiluli; Han and Tsin, Hi1-n11 $\mathrm{H}$.

2185 Hu-su | 襙 (obs.) in Chi11 H., Shingking; $W$. Han, H. Liao-lisi 潼西 chün.

\section{護}

2186 Hu-cl' êng 護 城, posttown near Sui-ning H., Kiangsu.

2187 Hu-chiung | IB, (obs.) near Ya-chou Fu, Ssŭcl'uan; Sung, C. in Li 黎 CHOU, 
花 HU-HUANG.

\section{潡}

2188 Hn-huang 湔䔮, chên in Chiang-hsia H., Hupeh.

\section{忽}

2189 Hu-chan 忽暟, see No. 2414.

2190 Hu-ch'an | 䌊, see No. 2414.

2191 Hu-ch'ien | 染, see No. 2414.

2192 Hu-li-1nu-tźn | 里模子 Hormuz, an island in the Persian Gulf; Lat. $27^{\circ}$ $05^{\prime}$, Long. $56^{\circ} 29^{\prime}$.

2193 Hu-t'an | 崖, see No. 2058.

Hu-chau, see No. 2154.

Hu-kau, see No. 2156.

Hu-kwan, see No. 2177.

Hu-kwang, see No. 2157.

H11-111111, see No. 2166.

Hu-pel, see No. 2159.

In-wei, see No. 6217.

Hii, see No. 2885.

Hï-i, see No. 2892.

\section{化}

2194 Hua 化 Hwa, C. in Kaochou Fu, Kuangtung; I.at. $21^{\circ} 37^{\prime}$, Long. $110^{\circ} 48^{\prime}$; Ts'in, in Hsiang 集 chün; Liang, Lo 矅 C.; Sui, Shil-1ung 石龍; T'ang, Pan 辦 C., and Iingshui 陵水; Sung, Hua | C. in Kuang-hsi 擴西 circ.
花 HUA-SHAO.

2195 Hua-cl' êng | 城, see No. 4752.

2196 Hua-chu | 洼, (obs.) Sung, H. near Kuangning $H_{\text {., Knangtung. }}$

2197 Hua-lê | 櫟, chên near P'u H., Shansi.

2198 Hua-lung | 隆.

$1^{\circ}$ (obs.) near Chao

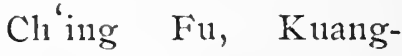
tung; $S$. Sung and S. $T s^{\prime} i, H$. in Sung-k'ang 本康 chïn。

$2^{\circ}$ See No. 2483.

2199 Hua-1nêng | 蒙.

$1^{\circ}$ (obs.) S. of Yung-an

C., Kuangsi ; S. Sung and $S$. Ts $i, H$. in Suichien 綏 建 chün; Sui, H. 引n Nan-liai 南 海 chïn; $T^{\prime}$ ang, H. in Kuang 黁 C.

$2^{\circ}$ (obs.) Ist Sung, H. near Kuang-ning H., Kuangtung.

2200 Hua-11u穆, (obs.) 1st Sung, H. near Kuang-ning H., Kuangtung.

2201 Hua-p'ing | 4 Hwa-ping, chên near Hua-t'ing H., Kansuh.

2202 Hua-p'ing-ch'nan | 平川

[67] Hwa-ping-cluen,C.I.T. in the P'ing-Ch'ing-Ching circ., Kansuh ; Lat. $35^{\circ}$ $35^{\prime}$, Long. $106^{\circ} 30^{\prime}$.

2203 Hua-shao | 稍, Y. in Hs ï̈an-hua H., Chihli. 


\section{花}

HUA-SHIH.

2204 Hua-sliih | 石, chên near Clnung-pu H., Sliensi.

\section{花}

2205 Hna 花 Hwa, H. in Kwang-chon Fit, Kuangtung; Lat. $23^{\circ} 24^{\prime}$, I.ong. $113^{\circ} 10^{\prime}$.

2206 Hua-J1ua | 花, chên near Ling-t'ai H., Kansull.

2207 Hua-ma-ch'il | 監池, post-town near I.ing C., Kansulı.

\section{華}

\section{Hua 華 Hwa.}

[498.] $1^{\circ}$ C. in $\mathrm{T}^{\prime}$ unng-chon Fu, Shensi ; Lat. $34^{\circ} 30^{\prime}$, Long. $109^{\circ} 51^{\prime} ; T^{\prime}{ }^{\prime} \mathrm{in}$, Nei-sliih 队史; Han, Ching-chao 京兆 and Hung-11ung 弘農； $P$. Chou, Hua-shan | 山 ; W. Wei, Hua $\mid \mathrm{C}$.

$2^{\circ}$ (obs.) Liang, C. wear Knang-ÿ̈аı H., Ssŭcli'nan.

2209 Hua-chia 管, Y. near Fo-p ing T., Shensi.

2210 Hua-ch' ${ }^{\prime} h_{1}$ | 池.

$1^{\circ}$ Chên near Ho-shui H., Kansuh.

$2^{\circ}$ (obs.) $\mathrm{N}$. E. of $\mathrm{Ho}-$ shui H., Kansulı; Sui, 'T'ang and Kin, Huacl' ${ }^{\mathrm{ih}}$ | 池 $\mathrm{H}$.

$3^{\circ}$ See Nos. 2134, 5404.
華 HUA-T'ING.

2211 Hua-ch'ing | 清, (obs.)・ T'ang, H., S.IV. of $\mathrm{Ch}^{\prime}$ in C., Knangtung.

2212 Hua-ch' ${ }^{\prime}$ 1an | 川, see No. 7463.

2213 Hna-fêng | 崶, sš̆ near Chang-chon Fitr, Fullkien.

2214 Hua-jung | 容 Hwa-yung. $1^{\circ} \mathrm{H}$. in Yo-chon Fu, Hunan; Lat. $29^{\circ} 30^{\prime}$, Long. $112^{\circ} 22^{\prime}$; $W u$, $\mathrm{Cl}^{\prime}$ an-ling 屡 陵; Ts'in, Nan-an 南安; Sui, Hua-jung | 点; $\tau^{\prime}$ ang, Jung-ch êng 容 城.

$2^{\circ}$ Post-town near IV11cli'ang H., Hupelı.

$3^{\circ}$ See No. 5734.

2215 Hua-pu|哇, chên near $\mathrm{K}^{6}$ ai-luna H., Clielikiang.

2216 Hua-shan |山, see No. 2208.

2217 Hua-shih | 石, chên near Chiang-yin $\mathrm{H}$., Kiangsu.

2218 Hua-t'ing | 竞 Hwa-ting. [202] $1^{\circ}$ H. in P'ing-liang Fil, Kansul ; I at. $35^{\circ} 18^{\prime}$, Long. $106^{\circ} 35^{\prime}$; Ts 'in, Lung-11si 㜔然; Sui, Hua-t'ing | 党; Five $d y^{\prime \prime},, \mathrm{Yi}$ 義 $\mathrm{C}$.

$2^{\circ} \mathrm{H}$. forming with I.ou H. the prefectural city of Sung-chiang, Kiangsu ; Lat. $31^{\circ} 03^{\prime}$, I.ong. $121^{\circ} 15^{\prime}$; Han, Lon 蒌 
H.; $\quad W u$, Hua-t'ing 亭; Tsin, Yü11-chien 雲 間.

2219 Hua-yang | 陽 Hwa-yang. $1^{\circ}$ H. forming with Ch'êng-tı H. the prefectural city of $\mathrm{Ch}^{\text {'êng- }}$ tı, Ssŭch 'uan; Lat. $30^{\circ}$ $41^{\prime}$, Long. $103^{\circ} 11^{\prime}$; Ts'in, Ch'êng-tı 成都; $\tau^{\prime}$ ang, Hua-yang | 陽. $2^{\circ}$ Chên, in Wang-ching H., Anhui.

$3^{\circ}$ (obs.) 1st Sung, tow1 near Kuang-yïan H., Ssŭch' uan.

$4^{\circ}$ (obs.) $T^{6}$ ang, H. 40 li $\mathrm{S}$. of Jui-chou $\mathrm{Fu}$, Kiangsi.

$5^{\circ}$ (obs.) 2nd Wei, H., $\mathrm{S}$. E. of Mien H., Shensi.

2220 Hua-yin | 陸 Hwa-yin. [498] $1^{\circ}$ H. in T'ung-chou Fin, Shensi ; Lat. $34^{\circ}$ $35^{\prime}$, Long. $109^{\circ} 57^{\prime} ; C$. Wars, Yin-clini 陰䑒; Han, Hua-yin | 陰; Sung, T'ung-ching 潼 津。

$2^{\circ}$ (obs.) 1st Sung, H. near Hsiang-yang Fu, Hupeh.

2221 Hua-yo|岳, chên near Hua-yin H., Shensi.

2222 Hua-yïan | 原, see No, 7322 .

\section{滑}

2223 Hua 滑 Hwal, H. in Weihui Fu, Honan; Lat. $35^{\circ}$ $38^{\prime}$, Long $114^{\circ} 48^{\prime}$; orig., Wei 衛; $T s^{\prime}$ in, in Tung 東 chiin; Han, Pu-yang 熦陽; Sui, Hua |C.

\section{HUAI}

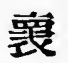

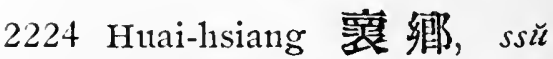
near Hsin-yi H., Kuangtung.

\section{襄}

2225 Huai 懷.

$1^{\circ}$ (obs.) S. W. of Wuchith H., Honan; Han and $T \sin , \mathrm{H}$. in Ho-nei 河內 chïn; N.Wei, H. in Wuttê 武德 chïn.

$2^{\circ}$ (obs.) in Hupeh; $S$. $T s^{\prime} i$, H. in An-1nan-tso 努綮庄 chïn。

$3^{\circ}$ (obs.) in Shingking; Lico, C. in Shang-ching 上京 circ.

$4^{\circ}$ See Nos. 2082, 2228.

2226 Huai-an | 安 Hwai-ngan.

$1^{\circ} \mathrm{H}$. in Hsüan-hua $\mathrm{F} u$, Chihli ; Lat. $40^{\circ} 27^{\prime}$, Long. $114^{\circ} 32^{\prime}$.

$2^{\circ} \mathrm{Y}$. near Liang-chon $\mathrm{Fu}$, Kansul.

$3^{\circ}$ (obs.) S. Sung, military district in $\mathrm{T}^{6}$ 'ning. cl'nan 潼川 circ. 
裂 HUAI-AN.

$4^{\circ}$ (obs.) Sung, town W. of Fin-chon Fu, Fuhkien.

$5^{\circ}$ (obs.) Sung, H., S. E. of Chin-t'ang H., Ssuŕch'van.

2227 Huai-chi | 集 Hwai-tsil,, [416] H. in Wu-chou Fu, Kuangsi ; Lat $23^{\circ} 55^{\prime}$, Lrong. $111^{\circ} 47^{\prime} ; T_{s i n}$, Huai-yïan | 咏; 1 st Sung, Huai-clii | 集; Tang, Wei 威 C.

2228 Huai-ch'ing | 度 Hwai[64] king $F 11$ in the Ho-pei circ., Honan; forming also the district city of Honei; Lat. $35^{\circ} 07^{\prime}$, Long. $113^{\circ}$; Chou, San-chien 三 監; C. Wars, in $\mathrm{K}$. Wei 魏 and K. Chêng确; Ts'in, San-ch'tuan 三川 chün; Han, Ho-nei 河成; 2nd Wei and T'ang, Huai C.; Ming, Huai-ch'ing 度 $\mathrm{Fu}$ 。

2229 Huai-ên | 恩

$1^{\circ}$ (obs.) S.E. of $\mathrm{Ch}^{\prime} \mathrm{ing}$ ho H., Kiangsu; $N$. Wei, H. in Huai-yin 淮 陰 chün.

$2^{\circ}$ (obs.) $T^{6}$ ang, H., S. IV. of Chang-p' 1 H., Fulikien.

2230 Huai-hua | 化.

$1^{\circ}$ - (cbs.) Previous to

Sung; C., W. of Nal1-

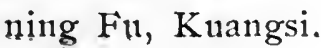

滚 HUAI-JUNG.

$2^{\circ}$ See No. 4927 .

2231 Huai-1ısi | 熙,(obs.) near Wu-chon Fin, Kuangsi ; S. Sung and . Ts $i, \mathrm{H}$. in 'l's'ang-wu蒼梧 chün.

2232 Huai-jên| 仁 Hwai-jin.

[376] $1^{\circ}$ H. in Ta-t'ung Fin, Shausi ; Lat. $39^{\circ} 54^{\prime}$, Long. $113^{\circ} 07^{\prime} ; T_{s}^{\prime}$ in, Yü11-chu1ng 雲 中; Han, Sha-11an 沙南; Sui, Ta-li 大利; Liao, Huai-jên $\mid$ 化.

$2^{\circ}$ (obs.) 23 li W. of Kan-yü H., Kiangsu ; $N$. Wei, H. in Yit'ang 義塘 chïn.

$3^{\circ}$ (obs.) 20 li S. E. of Tê-p'ing H., Shantung; Sui, H. in 'Tung-hai 東海 chiun; T'ang, H. in Hai 海 C.; Kin, H. in $\mathrm{T} \hat{\mathrm{e}}$ 德 $\mathrm{C}$.

$4^{\circ} \mathrm{H}$. in Hsing-ching C.I.T., Shingking.

$5^{\circ}$ H. in Chung-ch'ing 清 忠 circ., Corea.

$6^{\prime}$ See No. 3100.

2233 Huai-jon | 柔 Hwai-jan, [337] H. in Shun-t'ien Fu, Chihli; Lat $40^{\circ} 19^{\prime}$, Long. $116^{\circ} 39^{\prime}$.

2234 Hnai-jung | 戎.

$1^{\circ}$ (obs.) $70 l i \mathrm{~S}$. W. of Pao-an C., Chihli; Sui, H. in Cho 溷 chiün. 
$2^{\circ}$ (obs.) in Huai-lai H., Chillili T ang H. in Kuei 媱 C.

$3^{\circ}$ (obs.) E. of Chingyüan H., Kansuln; Sung, pao in Hui會 C.

2235 Huai-kuei | 踏.

$1^{\circ}$ (obs.) in Ssŭch'nan ;

S. Sung, H. in Pa-hisi

巴西 chïn。

$2^{\circ}$ (obs,) 20 li N. W. of Chnng-chiang H., Ssŭchnan; S. Sung, H. in Hsin-cli êng 新城。 chiin.

2236 Huai-lai | 來 Hwai-lai, [227] H. in Hsuian-hua Fu, Chillili I at. $40^{\circ} 23^{\prime}$, Long. $115^{\circ} 48^{\prime} ; T^{\prime}$ ang and Sung, Kuei 娞 C.; Liao and Yïan, Huai-lai 來 H.; Ming, Huai-lai 來 wei.

2237 Huai-ning | 㑑 Hwaining, $H$. forming the prefectural city of Anch'ing, Anhui ; Lat. $30^{\circ}$ $32^{\prime}$, Long. $117^{\circ} 07^{\prime} ;$ Han, Wan 皖 $\mathrm{H}$; $W u$, Wanch'êng 皖城; E. T S sin, Hnai-ning | 焉; Sui, Hsi 熙 C.; T $T^{6}$ ang. Shu 舒 C.

2238 Huai-tê | 德.

$1^{\circ} \mathrm{H}$. in Ch'ang-tu F., Shingking.

$2^{\circ}$ H. in Chnne-ch'ing 忠清 circ., Corea.
淮

HuAI.

$3^{\circ}$ (obs.) T'ang, H. 50 li N.E. of Hsin-yi H., Kuangtung.

2239 Huai-wei | 威, (obs.) 200 li N. E. of An-hua H., Kansul1, Sung, pao in $\mathrm{Cl}^{\prime}$ ing-yang 慶陽 Fu.

2240 Huai-wên | 文, see No. 5773.

2241 Huai-yi 邑, see No. 7058.

2242 Huai-yüan | 遠 Hwaiyuen.

$1^{\circ} \mathrm{H}$. in Yüi-lin $\mathrm{Fu}$, Shensi ; Lat. $37^{\circ} 54^{\prime}$, I ong. $108^{\circ} 50^{\prime}$.

$2^{\circ} \mathrm{H}$. in Fêng-yang $\mathrm{Fu}$, Anhui ; Lat. $33^{\circ}$. Long. $117^{\circ} 17^{\prime} ;$ S.A., $\mathrm{P}^{\prime}$ ei 泫; Wei, Cli' i-ch'êng 㖕 城; Sung, Huaiyüan|遠.

[255] $3^{\circ} \mathrm{H}$. in Liu-chou $\mathrm{Fu}$, Kuangsi ; Lat. $25^{\circ} 16^{\prime}$, Long $109^{\circ} 18^{\prime}$; orig., in Tsang-kê 㸼抲 chïn; Sung, Huai-yïan | 遠. $4^{\circ}$ Chên near Ch'ing-yiuan I't1, Kuangsi.

$5^{\circ}$ See No. 2227.

\section{准}

2243 Huai 淮.

$1^{\circ}$ Chên near Hsien H., Chibli.

$2^{\circ}$ (obs.) S. E. of Chêngyang H., Honan; $N$. Wei, Huai-chou 淮州 
淮

Huar.

chïn in Hsi-hnai 近 cHov.

$3^{\circ}$ See No. 6246.

2244 Huai-an | 娄 Hwai-ngan, [322] Fu in the Huai-yang circ., Kiangsu; forming also the district city of Shan-yang; Lat. $33^{\circ} 25^{\prime}$, Long. $119^{\circ}$ $22^{\prime} ;$ S.A., in 1 st K. Wu 㕦, 2 d $\mathrm{K}$. Yüeh 越; $C$. Wars, in K. Ch"u楚; $T_{s}{ }^{\prime}$ in, Huai-yin | 隐 in Chin-chiang 九江 chinn; Han, Lin-huai 臨 | and Kuang-ling 贋陵; Tsin, Shan-yang II 第; Sui, and 'ang, Ch'u 楚 C.; S. Sung, Huai-an 努 C. in Huai-tung | 東 circ. Coiganju (Polo).

2245 Huai-hsi | 西, (obs.) $S$. Sung, circ.

2246 Huai-k'ang | 康, see No. 3010.

2247 Huai-nan | 南, (obs.) Han, chïn in Anluni; see No. 2038.

2248 Huai-ning | 嘎 Hwaining, $H$. forming the prefectural city of Ch' ênchou Fu, Honan ; Lat. $33^{\circ} 46^{\prime}$, Lonig. $115^{\circ} 03^{\prime}$; Han and $T \sin , \mathrm{Ch}^{\prime} \mathrm{ên}^{\circ}$ 棵 H.; S. $7 s^{\prime} i$, Nan-clı'ên 南陳 H.; T'ang, Kin and Yüan, Cl'ên 陳 C.

2249 Huai-pin | 演, see No. 6291.
HUAI-YIN.

2250 Huai-tung | 東, (obs.) S. Sung, circ.

2251 Huai-yang | 榢 Hwai-yang, Fin in Chiang-yïan 江源 circ., Corea; Lat. $38^{\circ}$, Jong. $128^{\circ} 40^{\prime}$.

2252 Huai-Yang | 掦 circuit in Kiangsu, comprising Huaian Fu and Yang-chou Fur.

2253 Huai-yin |陰.

$1^{\circ}$ Post-town near Huai-an Fu, Kiangsu. $2^{\circ}$ See No. 2244.

2254 Hnai-yüan | 源, (obs.) N. IV. of Hsin-yang C., Honan; Sui, H. in Yiyang 载防 chïn.

\section{槐}

2255 Huai-li 槐里, see No. 2829.

2256 Huai-pai | 柏, chên near Lo-ch nan H., Shensi.

2257 Huai-shan | II Hwaishan, chïn in Chung-ch'ing 忠澅 circ., Corea; Lat. $37^{\circ} 03^{\prime}$, Long. $127^{\circ} 55^{\prime}$.

2258 Huai-shui | 水, post-town near Pai-hsiang H., Chihli.

2259 Huai-tien-chi | 店集, post-town near Shê $H$, Honan.

2260 Huai-ya | 芽, chên near Mei H., Shensi.

2261 Huai-vin | 陰, chên near Wu-t'ai H., Shansi. 
猚

HuaI-Lu.

\section{獲}

2262 Huai-1u 獲鹿, see No. 2423.

\section{HUAN}

筫

2263 Huan 䌙, see No. 4312.

\section{澴}

2264 Huan 澴, see No. 2641.

2265 Huan-ch uan | 川, posttown near Hsiao-kan H., Hupel.

\section{環}

$2266 \mathrm{H}_{\text {uan }}$ 環 Hwan.

[147] $1^{\circ}$ H. in Ch'ing-yang Fin, Kansuh; Lat. 36? $39^{\prime}$, Long. $107^{\circ} 07^{\prime}$; orig., ' T'ung-yüan 通 遠; Chou, So-fang 朔 方; Han, Fang-ch' ï 方渠; P. Chou, Hui 會 C.; Sui, Huan | C.; Five dyn., Wei 威 C. $2^{\circ}$ See No. 7760 .

\section{皖}

2267 Huan 皖, see Nos. 55, 2237, 6164.

2268 Huan-ch' êng | 城, see No. 2237.

\section{换}

2269 Huan-ma 換馬, chên near Kao-p'ing H., Shansi.
㬈:

Huang-Chia.

\section{渙}

2270 Hnan1-110 渙那, see No. 2058.

\section{莧}

2271 Huan-ch'iao 䓍橋, chên near Hai-ning C., Chehkiang.

\section{HUANG}

\section{黃:}

2272 Huang 黃 Hwang.

$1^{\circ} \mathrm{H}$. in Têng-choi $\mathrm{Fu}$, Shantung; Lat. $37^{\circ}$ $37^{\prime}$, Long. $120^{\circ} 47^{\prime}$; orig., capital of K. Lai 萊; Han, $\mathrm{H}_{\text {uang }}$ | $\mathrm{H}$. $2^{\circ}$ (obs.) S. A., K. comprising parts of Honan and Hupeh.

$3^{\circ}$ See Nos. 2282, 2301, 3430 .

2273 Huang-an | 安 Hwang[276] ngan, H. in Huang-chou Fu, Hupeh; Lat. $31^{\circ} 24^{\prime}$, Long. $114^{\circ} 25^{\prime}$.

2274 Huang-ant-ch'êng | 安城, (obs.) E. Wei, town S. of Chien C., Ssŭch' uan (No. 880).

2275 Huangr-chang | 漳, chên near Liao C., Shansi.

2276 Huang-chia 家, Y. near Sui-ting Fu, Ssŭch' uau.

2277 Huang-chia | 甲, Y. near Chiang-hua H., Hunan. 
黃

HUANG-Chiao.

2278 Huang-chiao | 角, Hwangkiol, $\mathrm{T}$. in Chia-ting $\mathrm{F} \mathrm{u}$, Sstuch 'uan; Lat. $29^{\circ} 08^{\prime}$, Long. $104^{\circ} 08^{\prime}$.

2279 Huang-chien | 㵎 H. in Chung-ch ing 忠清 circ., Corea.

2280 Huang-cls'ih | 池.

$1^{\circ}$ Chên near Wu-lıu H., Anluti.

$2^{\circ}$ (obs.) near Kung H., Ssŭch'uan; I'ang, H. in Lung 隆 C.

2281 Huang-ch in | 沈 ' $\mathrm{l}$. in Huai-ch'ing Fin, Honan.

2282 Huang-chou | 州 Hwangchat.

$1^{\circ} \mathrm{F} \mathfrak{u}$ in Huang-hai 翼 海 circ., Corea; Lat. $39^{\circ}$, Long. $126^{\circ} 05^{\prime}$.

[528] $2^{\mathrm{C}} \mathrm{Fu}$ in the Han-Huang'lê circ., Hupeh; forming also the district city of Huang-kang ; Lat. $30^{\circ} 26^{\prime}$, Long. $114^{\circ} 54^{\prime}$; S.A., 1 st K. Huang |, 2nd Chuch'êng 制 城; $T s^{\prime} i n$, in Nan 南 chïn; Han, Hsi-ling 函陵 in Chiang-hsia 江夏 chün; Three Ks., Chung-chên 重鎮; Tsin, Hsi-yang 酶䍀; S. Ts'i, Ch'i-an

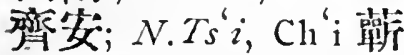
C.; Sui, Yung-an 永 发; Tang, Huang | C. Port of call.
翼: HUANG-KANG.

2283 Huang-chung-pao | 忠 堡, ssŭ near 'Tung-lisiang H., Ssŭch 'tıan.

2284 Huang-fu | 甫.

$1^{\circ}$ Chên near Fu C., Shensi.

$2^{\circ}$ Y. near T'ai-ku H., Shansi.

$2284^{\mathrm{a}}$ Huang-hai | 海, Circuit in Corea.

2285 Huang-lut-tien | 湖店, post-town near Hsin-t'a H., Honan.

2286 Huang-hu| 游, chên near Mêng-ch'êng H., Anhui.

2287 Huang-hsing | 與.

$1^{\circ}$ Chền near Ch'ing-chên H., Kueichon.

$2^{\circ}$ (obs.) Tsin, H., $80 l i$ N.W. of Yung-hsin H., Kiangsi.

2288 Huang-jut-t'un | 如屯, sš̌ in Fêng-ning H., Chihli.

2289 Huang-kang | 阙 Hwangkang.

$1^{\circ} \mathrm{T}$. in $\mathrm{Ch}$ 'ao-chou $\mathrm{Fu}$, Kuangtung; Lat. $23^{\circ}$ $42^{\prime}$, Long. $117^{\circ} 10^{\prime}$.

$2^{\circ} \mathrm{H}$. forming the prefectural city of Huangchou, Hupeh ; Lat. $30^{\circ}$ $26^{\prime}$, Long. $114^{\circ} 54^{\prime}$; Han, Chu制 H. and Hsi-ling 西陵; Tsin, Chu 制 H.; N. Ts'i, $\mathrm{Pa} 巴 \mathrm{C} . ;$ Sui, Huangkang | 阔; Trang, Mu-lan 木蘭. 
翼 HUANG-KANG.

$3^{\circ}$ Chên near Chao-an H., Fuhkien.

$4^{\circ}$ Ssžt in Yi-ch' 'un H., Kiangsi.

2290 Huang-lang | 蝍, ssì near L.ei-po T., Ssŭch'nan; also T-S. in the same neighbonrhood.

2291 Huang-liang | 良, chên near Hsi-an Fin, Shensi.

2292 Huang-lien | 連, see No. 4685.

2293 Hnang-ling-ch' ${ }^{\prime} \mathrm{n} g$ | 陵 城, (obs.) formerly tow1 near Ma-yang H., Hunan.

2294 Huang-lo-ho | 各隹河, chên in Wu-wei C., Anhui.

2295 Huang-lung | 龍.

$1^{\circ}$ Chên in Fu C., Shensi. $2^{\circ}$ (obs.) near Slinn-t ien $\mathrm{F}_{11}$, Chillil ; $T^{6}$ ang, $\mathrm{H}$. in $\mathrm{H} \sin$ 信 $\mathrm{C}$.

$3^{\circ}$ (obs.) in $\mathrm{K}$ 'ai-yüan

H., Shing-king ; Liao,

H. in Lunng 龍 C.

$4^{\circ}$ Chên in Yün H., Hupeh.

2296 Huang-mao | 伢.

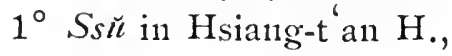
Hu11an.

$2^{\circ}$ Ss̆̌ near Yung-lisin H., Kiangsi.

2297 Huang-mei | 悔 Hwang111ei.

$1^{\circ} \mathrm{H}$. in Huang-chou $\mathrm{Fu}$, Hupeh; Lat. $30^{\circ} 12^{\prime}$, Long. $116^{\circ} 03^{\prime}$; Ts in, Hsin-ts'ai 剩蔡; Sui,
翼 HUANG-P'ING.

T'ang and Sung, Huang-mei | 梅 $\mathrm{H}$. $2^{\circ}$ (obs.) N.W. of Huangmei H., Hupel1; Sui, H. in Ch'i-ch' 'un 䉓春 chiin.

2298 Huang-ni-ho | 泥河, chên near Lü-chiang $\mathrm{H}$., Anluni.

2299 Huang-ni-wan | 泥灣, sš̆ near Yang-ch' 'un H., Kuangtung.

2300 Huang-pa | 㶚, posttown near Ning-ch iang C., Shensi.

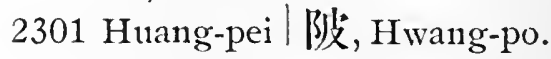
$1^{\circ} \mathrm{H}$. in Han-yang $\mathrm{Fu}$, Hupelı; Lat. $30^{\circ} 56^{\prime}$, Long. $113^{\circ} 15^{\prime}$; Han, Hsi-ling 证陵; Sui, Huang-pei | 陵; $P$. Chou, Huang |; Sung, Yïan and Ming, - Huang-pei | 陂 $\mathrm{H}$.

$2^{\circ}$ Ss ̌̆ in Lien-hua T., Kiangsi.

2302 Huang-pei-clian | 媩站, (or, Huang-p'o-chan), ssü near Huang-an H., Hupeh.

2303 Huang-p'ên | 湓, chên near Ch'ili-chou Fu, Anhui.

2304 Huang-p'ing | 平, Hwangping, C. in Cliên-yuian $\mathrm{Fu}$, Kueichon ; Lat. $26^{\circ} 30^{\prime}$, Long. $106^{\circ} 48^{\prime}$; Ming, Hsing-1n1ng 與隆; Wei, in P'ing-yüeh 平越 $\mathrm{Fu}$, Kueichou. 
黃 HUang-P ${ }^{6}$ ING-ChIU-Chov.

2305 Huang-p ing-chiu-chou | 平舊州, ssŭ near the preceding.

2306 Huang-p'o-chan | 济站, see No. 2302.

2307 Huang-p'o|坡, Y. near

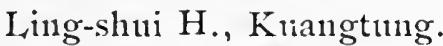

2308 Huang-pu | 戋, sš̌ in Hsiang-shan H., Kuangtu1ng.

2309 Huang-pu | 埔 Whanpoa, one of the ports open to foreign trade; Lat. $23^{\circ}$ $05^{\prime}$, Long. $113^{\circ} 27^{\prime}$; it is close to Canton.

2310 Huang-p'u | 浦, ssŭ in Shang-hai H., Kiangsu.

2311 Huang-san | 徐, Y. near Sui-ning H., $\mathrm{H}_{\text {unan. }}$

2312 Huang-sha | 沙, posttown near Pao-ch'êng H., Shensi.

2313 Huang-shan | 山, sš̆ in Shê H., Anhuii.

2314 Huang-shan-kuan | 山館, sš̌ near Huang $\mathrm{H}$, Shautung.

2315 Huang-shih-hsiang | 石 港, chên in Ta-yeh H., Hupeh. Port of call.

2316 Huang-ssŭ | 絲, posttown near Kuei-ting $\mathrm{H}$, Kueichon.

2317 Huang-t'ang | 塘, sš̌ near 'T'êng $H$. , Kuangsi.

2318 Huang-tao | 道, T-S. in Ssŭ-chou Fu, Kueichou.
相 HUANG-CH'

2319 Huang-tao-ch'i | 道溪, (obs.) near Ssŭ-chou Fu, Kueichon, Ming, ssŭ in Ssŭ-chon Fu.

2320 Huang-t'ien | 田, posttown near Ku-t ien $H$, Fulkien.

2321 Huang-ting | 鼎, sš̆ in Nan-hai H., Kuangtung.

2322 Huang-tu| 渡, chên near

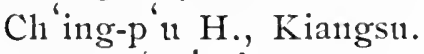

2323 Huang-t' 1 土, (obs.) $P$. Chou, H., E. of Yün-hsi H., Hupeh.

2324 Huang-tun-pao | 墩堡, post-town near An-hsi C., Kansul.

2325 Huang-yai | 崖, Y. near Chi C., Shun-t'ien Fu, Chihli.

2326 Huang-yen | 蕌, Hwang[571] yeu, H. in 'T'ai chou Fu, Chehkiang; Lat. $28^{\circ} 42^{\prime}$, Long. $121^{\circ}$ 18'; Han, Yung-11ing 永䡐; Sui,

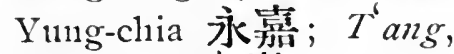
Huang-yen | 㒈

2327 Huang-shan 㗂 U; Fu in Ch'iian-lo 全羅 circ., Corea.

\section{䓍}

2328 Huang-ching 璜溜, chên near Ch' ang-chou Fin, Kiangsu.

\section{皇}

$2328^{\mathrm{a}}$ Huang-ch i 皇畿, see No. 5822 . 
兴: HUANG-CHOU.

$2328^{\mathrm{b}}$ Huang-chou | 洲, see No. 5822.

2329 Huang-hua | 華.

$1^{\circ}$ Post-town near Tê-an Fu, Hupel.

$2^{\circ}$ Post-town near Kueiyang Fin, Kneichon.

$2329^{\mathrm{a}}$ Huang-tu| 都, see No. 5822.

\section{湟}

2330 Huang 涅, see No. 2483. 2331 Huang-chung | 中, see No. 2483.

\section{晃}

2332 Huang-chou 晃州 Kwang[561] chan, C. I. T. in Ch' ênYiuan-Iung-Ching circ., Hnnan ; Lat. $27^{\circ} 20^{\prime}$, Long. $109^{\circ} 15^{\prime}$.

\section{荒}

2333 Huang-fu 芜服, see No. 7862.

\section{況}

2334 Huang-ch'i 況其, an erroneons way of writing Chu-ch'i 㒭其.

\section{HUI}

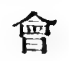

2335 Hui 會, see Nos. 2266, 2342, 4498.

2336 Hui-ch'ang | 昌 Hwuichang.
會 HUI-T'ING.

[228] $1^{\circ}$ II. in Kan-clou Fu, Kiangsi ; Iat. $25^{\circ} 22^{\prime}$, Long. $115^{\circ} 42^{\prime}$; orig., Chiu-chon 九州 chên; Sung, Hui-ch'ang | 昌. $2^{\circ}$ See No. 3894.

2337 Hui-ch'êng | 城, see No. 3174.

2338 Hui-cl'i | 溪, (obs.) near

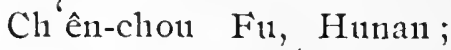
Sung, fort in $\mathrm{Cl}^{\prime}$ ên 辰 C'.

2339 Hui-ching | 津, (obs.) in Ning-ching H., Chihli ; Kin, chên in Ching 景 C. 2340 Hui-ch' uan | 川, see No. 1197.

2341 Hui-li | 理 Hwui-li, C. [429] iı Ning-yüan Fu, Ssŭch'nan; Lat. $26^{\circ} 34$, Long. $102^{\circ} 56^{\prime}$; Han, $T$ sin, S. Sung and $S$. $T s^{\prime} i, \mathrm{Hui}$-wu | 無 $\mathrm{H}$.; I'ïan, Wn1-a11 武努 C. and Hui-li | 理 C.

2342 Hui-ning | 宊 Hwni-ning. $1^{\circ} \mathrm{F} n$ in Hsien-ching 成 鏡 circ., Corta; Lat. $42^{\circ} 40^{\prime}$, Long. $129^{\circ} 20^{\prime}$. $2^{\circ} \mathrm{H}$. in Kung-cl ang $\mathrm{F} u$, Kansuh ; Lat. $35^{\circ}$ 45/, Long. $105^{\circ} 07^{\prime}$; Han, Chih-yang 枝陽; 2nd Wei, Hui-ning | 宿; E. Wei, Hui | C.; Kin, Hsi-ning 西家.

2343 Hui-t'ing |亭, post-town near Yung-ch' êng H., Honan. 
算

HUI-TSÊ.

2344 Hui-tsê | 澤 Hwui-tseh, $\mathrm{H}$. forming the prefectural city of Tung-ch'nan, Ssŭch' 'uan; Lat. $26^{\circ} 21^{\prime}$, Long. $103^{\circ} 26^{\prime}$.

2345 Hui-t'ung / 同 Hwui-tung. [110] $1^{\circ} \mathrm{H}$. in Ching C., Hunan; Iat. $26^{\circ} 50^{\prime}$, Long. $109^{\circ} 27^{\prime}$; orig., Lang-chiang 狠 讧; Sung, Hui-t ung | 同H. $2^{\circ} \mathrm{H}$. in $\mathrm{Cl}^{\prime}$ inng-chon $\mathrm{Fu}$, Knangtung ; Lat. $19^{\circ}$ $10^{\prime}$, Long. $111^{\circ} 09^{\prime}$; 7'ang, Lê-luni 㴡 |; Yüan, Hui-t'ung|同. $3^{\circ}$ (obs.) in An H., Ssŭch nan; Sung, pao in Shih-ch'üan 石泉 military district.

2346 Hui-wu | 热, see No. 2341.

2347 Hui-yeh | 野, (obs.) Trang Sung, C., N. IV. of Ya-chou Fu, Ssŭch'uan.

\section{懀}

2348 Hui 懀, (obs.) Chou, feudal state in $E$. of Shensi.

\section{回}

2349 Huil-110 回紀, see No. 7005.

2350 Hurillu | 鶻, see No. 7005.

2351 Hui-lung | 龍, chên near Shih-lou H., Shansi.
徽 HuI-chov.

2352 Hui-p u | 浦, see No. 4682.

\section{䧃}

2353 Hui 䧃, (obs.) in Kuangsi; Sung, a district in Y'ung 邑 C.

\section{灰}

2354 Hui-ho 灰河, chín near T'ung-ling H., Anluni.

2355 Hui-pu | 垚, post-town in $P^{\natural}$ ing-tn C., Shantung.

\section{滇}

2356 Hui-chiang 澒江, see No. 754.

徽

$2357 \mathrm{H}_{\text {ui }}$ 徽 Hwui.

$1^{\circ} \mathrm{H}$. in $\mathrm{Cl}^{\circ}$ in $\mathrm{C}$., Kansul, L Lat. $33^{\circ} 46^{\prime}$, Long. $106^{\circ} 07^{\prime}$; $\mathrm{Han}$, Ho-ch' ilh 河池; 2nd Wei, Kuang-lina 腐化; Yüan, Hui | C. $2^{\circ}$ (obs.) T"ang, C., W. of Yao C., Yünnan. $3^{\circ}$ (obs.) S. Sung, C. in Cliang-tung 江串 circ. $4^{\circ}$ See No. 2358.

2358 Hui-chou / 州Hwui-chan, [377] $\mathrm{Fu}$ in the Hui-Ning-Ch 'ih'T'ai-Knang-tê-chou circ, Anhui ; forming also the district city of Shê ; Lat. $29^{\circ} 59^{\prime}$, Long. $118^{\circ} 28^{\prime}$; $S . A$., in $1 s t \mathrm{~K}$. Wu 吳, 
2nd $\mathrm{K}$. Yueh 越; C. Wars, in K. Ch' 11 楚; Ts'in, in Chang 鄣 chïn; Han, in Tan-yang 丹陽 chün; Wu， $\mathrm{H} \sin 1 \mathrm{t}$ 11 新都; $T$ sin, Hsin-an 新安; Sui and T'ang, Shê 歌 C.; Sung, Hui $\mathrm{C}$.

2359 Hui - Ning - Cl' ih - T'ai Kuang-tê-chou | 篮池太 愿德州, circ. in Anhui, comprising Hui-chou Fu, Ning-kno $\mathrm{F}$ n, Ch'ih-chon Fu, T'ai-p'ing Fu and Kuang-tê C.

\section{㭧}

2360 Hui 惠, see No, 2365.

2361 Hui-an | 安 Hwui-ngan, H. in Clı 'üan-clion $\mathrm{Fu}$, Fuhkien ; Lat. $25^{\circ} 02^{\prime}$, Long. $119^{\circ} 02^{\prime}$; orig., Chin-chiang 晉江; Sung, Hui-an 安:

2362 Hui-an1-pao | 安:堡, posttown near Ling C., Kansuh.

2363 Hui-Ch' ao-Chia | 潮嘉, circ. in Kuangtung comprising Hui-chou Fu,

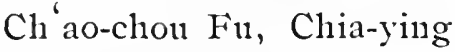
C. and Fo-kang $\mathrm{T}$.

2364 Hui-chia|佳, (obs.) in T'ien T-C., Kuangsi; 7'ang, H. in T'ien 田 $\mathrm{C}$. 2365 Hui-chou | 州Hwui-chan, [478] Fu in the Hui-Ch' ao-Chia circ., Kuangtung; forming also the prefectural city of
Kuei-shan ; Lat. $23^{\circ} 02^{\prime}$, Long. $114^{\circ} 13^{\prime}$; Han, in Nan-hai 南海 chün; Liang, Liang-lina 梁化; $S_{u i i}$, Hsün 循 C. and Lung-cl' 'uan 龍川; Sung, Hui | C.; and Po-lo 情羅; $T^{6}$ ang, Hai-fêng 海豐 and Chêng 䅡 C.; Hing, H.uichou 州 Fu.

\section{Hui-huai | 㗒.}

$1^{\circ}$ (obs.) W. of Mienyang C., Hupelı; $S$. Sung and $S$. Ts'i, H. in Chiang-hsia 江 夏 chïn.

$2^{\circ}$ (obs.) in Honan; $S$. $T s^{6} i, H$. in Hsin-yeh 新䟥 chün.

2367 Hui-hui-pao |回堡; posttown near Yü̈-mên $H$., Kansuh.

2368 Hui-lai | 來 Hwui-lai, H. in Ch'ao-clou Fu, Kuangtung; Lat. $23^{\circ} 10^{\prime}$, Long. $116^{\circ} 20^{\prime}$; Ming, Hui-lai | 來 $\mathrm{H}$.

2369 Hui-1nin | 民 Hwui-min.

$1^{\circ} \mathrm{H}$. forming the prefectural city of Wu-ting, Shantung ; Lat. $37^{\circ}$ 33', Long. $117^{\circ} 41^{\prime}$. $2^{\circ}$ (obs.) in Ku1-yüan C., Kansuh ; Sung, pao in Huai-tê 襄德, military district. 
㦛 HUI-MIN.

$3^{\circ}$ (obs.) $60 l i \mathrm{~s}$. of Chiung C., Ssŭch'uan; Sung, fort in Clining I I $\mathrm{C}$.

2370 Hui-ning | 耍.

$1^{\circ}$ Town near 'lurfan in the New Dominion; also known as Pa-lik'un巴里坤。

$2^{\circ}$ Town near Ili.

2371 Hui-yüan / 遠; see No. 7469.

\section{暉}

2372 Hui 腪, (obs.) in Ssŭch'nan; $T^{6}$ ang, C. in Chiang-nan 江. 南 circ.; Sung, C. in Shao-ch'ing 紹慶 Fu。

\section{輝}

2373 Hui 輝 Hwui, H. in Wei-lui Fu, Honan; Lat. $35^{\circ} 30^{\prime}$, Long. $114^{\circ}$; orig., $\mathrm{K}$. Kung 共; Han, Kung 共 H. ; Sui, Kung-cln'êng 共 城; Tang; Fanch' êng 凡 城; Kin, Sumên蘇偬 and Hui $\mid \mathrm{C}$; Yïan and Ming, Hui | $\mathrm{H}$.

2374 Hui-t'ê | 特 Khoit; Mongol tribe.

\section{HUN}

\section{- 渾}

2375 Hun 渾, (obs.) near Yen-an Fu, Shensi; $T^{*}$ ang, C. in Kuan-nei 關內 circ.
紅

HUNG-CH' I.

2376 Hu11-ch' '111 | Hwăn-chn11.

1。 I't1 in the Ning-HsingHur circ., Kirin ; Lat. $42^{\circ} 45^{\prime}$, Long. $126^{\circ}$ $25^{\prime}$.

$2^{\circ}$ The Russian town of Wladiwostok, lying some distance E. of the preceding. Also called Hai-shên Wei 海參徫.

2377 Hun-mi|彌, (obs.) in Corea; Han and Tsin, H. in Lê-lang 樂浱 chïn. 2378 Hun1-yüan | 源 Hwănyuen.

$[160] 1^{\circ}$ C. in 'Ta-t'nug Fu, Shansi ; Iat. $39^{\circ} 41^{\prime}$, Long. $113^{\circ} 45^{\prime} ; T^{\prime} \mathrm{in}^{\prime}$, Yen-mên 鴈楻; T'ang, Hu11-yüan | 源 C.

$2^{\circ}$ (obs.) W. of preceding; Liao and Kin, H. in Ying 應 $\mathrm{C}$.

\section{混}

2379 Hun1-t'ung 混同, (obs.) N. E. of the city of Kirin, Liao, H. in Ning-chiang 富 江 C.

\section{HUNG}

\section{紅.}

2380 Hung 紅, (obs.) E. Han, H. in K. P'ei 浦 in Yü 豫 CHOU.

2381 Hung-ch'i | 旗, Y. near Luan-p'ing H., Chihli. 
紅 HUNG-CHI'

2382 Hung-ch'in | 沈, posttown near Sui-ning H., Kiangsu.

2383 Hung-luna-pu | 花㸃, post-town near 'l"an-ch' èng H., Shantung.

238t Hung-mei | 梅, ssü in in Nan-hsiung C., Kuangtung.

2385 Hung-pu-clı̈̈|卜苜, TS. near Hui-li C., Ssŭ. ch' uan.

2386 Hung-shan | I4, posttown near I,ing C., Kansuh.

2387 Hung-shui - chün-t'ang | 水軍塔, post-town near P'ing-fan H., Ssŭch'uan.

\section{虹.}

2388 Hung-cliang | 江. Hungkiang, $\mathrm{T}$. in $\mathrm{Cl}^{\prime}$ üan-chou Fu, Fulkien.

\section{広}

2389 Hung-lina 答化, posttown $20 l i \mathrm{~N}$. of An-lina H., Kansuh.

2390 Hung-lu | 路, post-town near Fu-clı'ing H., Fuhkicn.

2391 Hung-nung | 農, （obs.） $T s^{\prime}$ in, H. near Clin-chiang Fu, Kiangsi.

2392 Hung-nung 弘睑.

$1^{\circ}$ (obs.) $40 \quad l i \mathrm{~S}$. of I.ing-pao H., Honan; Han, Tsin and Sui, chïn and H.; $T^{6}$ ang, H. in Kuo 貌 C.
泓

HUNG-CHOU.

$2^{\circ}$ (obs.) near Físiangyang $H$. , Hupeh; $S$. Sung and S. Ts'i, chiün in Yung 雃 chou. $3^{\circ}$ (obs.) in Ssŭch' ${ }^{6}$ tan ; S. Ts' $i$, chïn in Liang 梁 chou.

$4^{\circ}$ See Nos. 2208, 3965.

2393 Hung-wên | 聞, (obs.) WV. of Ping-jang Fin, Corea; Lico, H. in Lu 湶 C.

\section{泓}

2394 Hung-chil 泓芝, chên near Yi-sliih H., Sliansi.

\section{胫}

2395 Hung 洪 Hung.

$1^{\circ}$ C. in Chung-ch'ing 忠 清 circ., Corea; Lat. $37^{\circ} 51^{\prime}$, Long. $125^{\circ} 30^{\prime}$. $2^{\circ}$ See Nos. 3847, 4562.

2396 Hung-ao | 澳, ssŭ in Hsiang-shan H., Kuangtung.

2397 Hung-ch' êng | 城, posttown near Ku-lang $\mathrm{H}$, Kansul.

2398 Hung-chiang | II., ssŭ in Hui-t'ing H., Hunan. Sung, military post in Yüan $\overrightarrow{\text { J } ~ C . ~}$

2399 Hung-cl'ih | 池, chên, near P'ing-lu H., Shansi [No. 5177.]

2400 Hung-chou | 州, ssĭ near Yung-ts' ung H., Kueichou. 
獲

HuNG-Ch'UAN.

2401 Hung-ch' wan | M, H. in Chiang-yïan 江. 原 circ., Corea.

2402 Hung-fan | 番, ssŭ ncar 'Ting-fan C., Kueichou; Ming, ssü in Kuei-yang. Fin, Kueichon.

2403 Hung-lu | 盧, (obs.) near Hsü-chou Fu, Ssŭch' 'uan; $\tau^{\prime}$ ang, H. in Nan 南 C.

2404 Hung-shan | 善, posttown near P'ing-yao H., Shansi.

2405 Hung-shui | 水, chên near I,iao C., Shansi.

2406 Hung-tu | 都, see No. 4562.

2407 Hung-tung | 洞 Hung[123]tung, H. in P'ing-yang Fin, Shansi ; I at. $36^{\circ} 17^{\prime}$, Long. $111^{\circ} 42^{\prime}$; S.A., K. Yang 楊; Han, Yang 揚 H.; Sui, Hung-tung | 洞. 2408 Hung-ya |. 雅 Hung-ya, [72] H. in Clina-ting Fir, Ssŭch' 'uan; Lat. $29^{\circ} 55^{\prime}$, Long. $103^{\circ} 50^{\prime}$.

2409 Hung-yüan | 源 Hungyuen, H. in Hsien-cling 咸鏡 circ., Corea; Lat. $40^{\circ}$, Long. $128^{\circ} 41^{\prime}$.

\section{滺}

2410 Hung 渄, (obs.) in Ssŭch'nan; Trang, C. in Chiang-nan 江南 circ.; Sung, C. in Shao-ch'ing 紹磨 Fu.

\section{淿 HuO-SHAO-KOU.}

2411 Hung-kon | 濰, see No. 2460 .

2412 Hung-ıê11 | P', (obs.) S. IV. of Shên-1111 H., Sliensi; Han, H. in Hsiho 西河 chün.

2413 Hung-shan| 山 $H$. in Clnung-cli ing 忠清 circ., Corea.

Hung-kiang, see No. 2388. Hung-yuen, see No. 2409.

\section{HUO}

\section{火}

2414 Huo-chan 火 占 (also written Hu-chan 尔部, Hu-clı'ien 忽雤, Hu-ch'an 忽緸，Ho-slian 霍间 and K'11-chan 苦盖), Khodjend, town in Kokand, Independent Turkestan.

2415 Huo-cling $\mid$ 护, (obs.) P. Chou, H., S. W. of Chiung C., Ssŭch' 'uan.

2416 H110-chon | 州, see No. 1900.

$24 i 7$ Huo-fêng-t ang | 箱 㙋, post-town near Kuei C., Hupel.

2418 Hno-shan| II, see No. 2070.

2419 Huo-shao | 畓, Y. near Fo-p'ing T., Shensi.

2420 Huo-shao-kon | 㷥 等, post-town near Yü-nnên H., Kansuh. 
水 HuO-'TS'UAN.

HWUI-CHAU.

2421 Huo-ts'nan | 䥊, chên near Liang-tang H., Kansuli.

\section{獲}

2422 Huo-clia 獲嘉 Hwolkiah, H. in Wei-hui Fu, Honan; Lat. $35^{\circ} 20^{\prime}$, Long. $113^{\circ} 47^{\prime} ; \mathrm{Han}$, Hno-chia | 嘉; Tsin, Yin 殷 C.; E. Wei，Hsin-wu 修武.

2423 Huo-lu (or, Huai-11) | 鹿 Hwoh-luh, H. in Chêngting F11, Chihli; Lat. $38^{\circ}$ $08^{\prime}$, Long. $114^{\circ} 26^{\prime}$; orig., Shih-yi 石邑; Sui, Lu1ch'üan 鹿泉; T'ang， Huro-lu | 歴.

\section{楅}

2424 Huo-nei 榾眉, (obs.) near Ya-chou Fu, Ssŭch'nan; Trang, C. in Chien-11an 劍南 circ.

\section{沿问}

2425 Houl-mei 哈 筫, (obs.) near Ya-chou $F u$, Ssŭch'uan; Sung, C. in Ya 雅 CHOU.

\section{蔹}

2426 Hulo-tan 製旦, see No. 2058.

Hurunpir, see No. 2075.

Hwa, see Nos. 2194, 2205, 2208.

Hwa-ping-clnten, see No. 2202.

Hwa-ting, see No. 2218.

Hwa-yang, see No. 2219.

Hwa-yin, see No. 2220.

Hwa-yung, see No. 2214.

Hwah, see No. 2223.

Hwai-jau, see No. 2233.

Hwai-ji1, see No. 2232.

Hwai-king, see No. 2228.

Hwai-lai, see No. 2236.

Hwai-ngan, see Nos. 2226, 2244.

Hwai-ning, see Nos. 2237, 2248.

Hwai-shan, see No. 2257. Hwai-tsih, see No. 2227.

Hwai-yang, see No. 2251. Hwai-yue11, see No. 2242. Hwan, see No. 2266.

Hwăn-chuın, see No. 2376. Hwăn-yıen, see No. 2.378. Hwang, see Nos. 2018, 2272.

Hwang-chan, see No. 2282. Hwang-kang, see No. 2289. Hwang-kiol, see No. 2278. Hwang-11ei, see No. 2297. Hwang-ngan, see No. 2273. Hwang-ping, see No. 2304. Hwang-po, see No. 2301. Hwang-ye11, see No. 2326. Hwoll-kia, see No. 2422. Hwoh-1111, see No. 2423. Hwui, see No. 2373.

Hwui-chang, see No. 2336. Hwui-chan, see Nos. 2358, 2365. 
HsI.

Hwui-ki, see No. 3469.

Hwui-lai, see No. 2368.

Hwui-li, see No. 2341.

Hwni-nin, see No. 2369.

Hwui-11gan, see No. 2361.

Hwui-ning, see No. 2342.

Hwui-tseh, see No. 2344.

Hwui-tu1ng, see No. 2345.

\section{HSI}

\section{淅}

$2426^{\text {a }}$ Hsi 淅, see Nos. 2427, 7823.

2427 Hsi-ch' nan | 川 Cliehchuen, 'T. in Nan-yang Fu, Honan ; Lat. $33^{\circ} 05^{\prime}$, l.ong. $111^{\circ} 27^{\prime}$; Han, Hsi C. | Ming, Hsich'uan| 川.

$2427^{\mathrm{a}}$ Hsi-yüan | 原 (obs.) in Liü C., Ssŭclı' 'uan; T'ang, H. in $\mathrm{H}_{\mathrm{si}}$ 淅 C.

\section{浠}

2428 Hsi-ch'nan 浠川, posttown near $\mathrm{Cl}_{1}$ i-sliui $\mathrm{H}$, Hupeh.

\section{点}

2429 Hsi-wu-wu 賞屋武, town in the I,in-ch"in Islands.

\section{㚜}

$2430 \mathrm{Hsi}$ 淺, (obs.) in Ssŭ-nan Fu, Kueichour ; T'ang, C. in Chiang-nan 江南 circ.

\section{冥腎}

2431 Hsi 熙.

$1^{\circ}$ (obs.) N. W. of $\mathrm{P}^{6} \mathrm{ing}$ jang Fn, Corea; Yüan, C. in Tinng-ning 帮 家i circ.

$2^{\circ}$ See Nos. 2237, 6358.

2432 Hsi-an | 安, see No. 4927. 2433 Hsi-ch nan 川 chün in P'ing-an 平安 circ., Corea.

2434 Hsi-p ing 本, (obs.) $W u$, H., W. of Lien-slan T.. Kuangtung.

\section{戲}

2435 Hsi-yang-ch' êng 践呦城, (obs.) S.A., town E. of Chang-tê Fu, Honan.

\section{細}

2436 Hsi-yang 細烫, see No. 6163.

\section{西}

2437 Hsi 西.

$1^{\circ}$ (obs.) 120 li S. W. of $\mathrm{Ch}^{\prime}$ in C., Kansuh ; $W$. Han, H. in Lung-hnsi 隴 I chiin; E. Han, H. in Han-yang 谟陽 chïn. $2^{\circ}$ (obs.) S. E. of Shan$\tan$ H., Kansul ; Tsin, chïn in Liang 凉 chou.

$3^{\circ}$ (obs.) in Yi-ch' êng H., Hupeh ; S. Sung and $S . T s^{\prime} i, H$. in Nan T'ien-shui 南天水 chiill. 
$4^{\circ}$ (obs.) in Ssŭclı' uan ; S. Sung and S. Tis $i$,

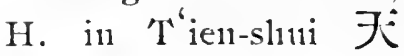
水 chïn

$5^{\circ}$ (obs.) $40 \mathrm{li} \mathrm{W}$. of Mien 活 H., Shensi; Sui, H. in Han-ch 'uan 漢 川 chïn; $T^{\prime}$ ang and Sung, H. in Hsing-

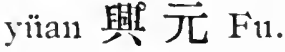

$6^{\circ}$ (obs.) near 'Turfan; $T^{*}$ ang, C. in Lung-yu 隴右 circ。

$7^{\circ}$ (obs.) formerly C., N. IV. of Chiang-ning Fu, Kiangsu.

$8^{\circ}$ See No. 4442.

2438 Hsi-an | 努 Si-ngan.

$1^{\circ} F_{11}$ in the HsiCh' ien-Fu circ., Shensi ; forming also the two district cities of $\mathrm{Cl}^{6}$ angan and Hsien-ning; Lat. $34^{\circ} 17^{\prime}$, Isong. $108^{\circ} 58^{\prime}$; capital of the province; Ts' in, Kuanchung 關中; Han, Wei-nan 消南 and Nei-slinh 內史; E. Han, Yung 䔨 C.; T'ang, Kuan-nei 關內; Sung, Yung-lising 水 與; Yïan, An-lisi 姲|; Ming, A11-11si 岁|Fin; capital of China under the 'Ts'in Han and T'ang dynasties. $2^{\circ} \mathrm{H}$. forming the prefectural city of $\mathrm{Cl}_{1}^{\text {' }}$ iิclou, Clielskiang; Lat. $28^{\circ} 55^{\prime}$, I Long. $119^{\circ}$ $12^{\prime} ; T s^{\prime} i n, \mathrm{~T}^{\prime}$ a-mo 太 末; Han, Hsin-an 新㚣; $\mathcal{T} \sin$, Hsin-an 信安； T'ang，Hsi-an 发.

$3^{\circ}$ Post-town near Huaijên H., Sliansi.

$4^{\circ}$ (obs.) $30 l i \mathrm{~W}$. of Lintzŭ H., Shantung; Han, S. Sung and $N$, Wei, H. in Ch'i 㧝 chün.

$5^{\circ}$ (obs.) in Kiangsu; $S$. Sung and $S$. Ts $i, \mathrm{H}$. in Nan-1ı 南畕 chün.

$6^{\circ}$ (obs.) near Hsing-yi Fu, Kueichou; S. Sunr, H. in Hsing-ku 與 古 chïn.

$7^{\circ}$ (obs.) in Aunam; $S$. Sung and $S . T s^{\prime} i, \mathrm{H}$. in Chin-tê 九德 chün.

$8^{\circ}$ (obs.) near Chiangning Fu, Kiangsu; $S$.

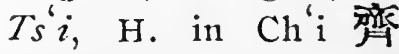
chiun.

$9^{\circ}$ (obs.) in Nan1-chêng H., Shensi ; S. Ts' $i$, H. in Tung-ning 東蕣 chün.

$10^{\circ}$ (obs.) S.W. of Kung H., Ssŭch'uan; T'ang, H. in Hsiel 恊 C. 
焝

HSI-AN.

$11^{\circ}$ (obs.) N.W. of Kuyüan C., Kansulı; Sung, C. in Ch'in-fêng 秦愿 circ.

$12^{\circ}$ H. in Hai-lunn Fu, Shingking.

$13^{\circ}$ See No. 229.

2439 Hsi-cli' ang | 昌 Si-cliang. $1^{\circ} \mathrm{H}$. forming the prefectural city of Ningyüan, Ssŭch' uan; Lat. $27^{\circ} 50^{\prime}$, Long. $102^{\circ} 12^{\prime}$. $2^{\circ}$ See Nos. 2738, 6179.

2440 Hsi-chêng-yang | 佂陽, ssü near Ho-ch'in H., Anhui.

2441 Hsi-ch'êng | 城, see No. 2815.

2442 Hsi-ch'i | 溪, chên in Yï-hang $H$. , Chehkiang.

2443 Hsi-ch'iang | 菟, see Nos. 2483, 6358.

2444 Hsi-chiao | 交, chển near P'ing-ting C., Shansi.

2445 Hsi-chiel1-t o | 界沱, sš̆ near Clıung C., Ssŭcli nan.

2446 Hsi-Cl'ien-Fin | 乾塵, circ. in Shensi comprising Hsi-an Fu, Ch' ien C., Fu $C$., and ' $\mathrm{T}$ 'ung-chon $\mathrm{Fu}$.

2447 Hsi-cling | 京, see Nos. 1724, 2079, 6174.

2448 Hsi-cling | 津, post-town near Ch'ien C., Shensi.

2449 Hsi-chon | 周, see No. 2724.

2450 Hsi-ch “ 1 l楚, see No. 1731. 2451 Hsi-ch' uan | 川.
西 JSI-FC-FÊNG。

$1^{\circ}$ 'T-S. near Hsi-ningr Fin, Kansulı.

$2^{\circ} T \sin , H$. in Ku-yiuan C., Kansul.

$3^{\circ}$ See No. 511.

2452 Hsi-chung | 中, (obs.) in Hsing-yi Fin, Kueichou ; S. $T s^{6} i, \mathrm{H}$. in Hsing-ku 典南古 chion.

2453 Hsi-ch' nng | 充 Si-chnng, [291] H. in Shlun-cll'ing Fu, Ssŭch' uan; I Iat. $31^{\circ} 02^{\prime}$, Long. $105^{\circ} 52^{\prime}$; Han, in $\mathrm{Pa}$ 巴 chiön; Tsin, Hsicling | 充; $W . W_{e i}$, Chin-ch' êng 至城; T'ang, Sung, Yüan and Ming, Hsi-cl' 'ung | 忘 H.

2454 Hsi-fêng | 封, (obs.) T"ang, H. near Mou C., Ssŭ́ch 'nan.

2455 Hsi-fêng | 豐.

$1^{\circ}$ (obs.) 50 li S. W of Lin-ch 'van H., Kiangsi;

$T$ sin, S. Sung and $S$. $T s^{6} i$, H. in Lin-ch 'van 臨川 chün。

$2^{\circ}$ (obs.) IV. of Shanyang H., Shensi; $S$. Sung, H. in Pei-shanglo 北上洛 chiin.

$3^{\circ}$ H. in Hai-lung Fn, Shingking.

$4^{\circ}$ See No. 3856.

2456 Hsi-fu-fêng | 扶風, (obs.) in Nan-cliêng H., Shensi ; S. Sung and $S$. Ts'i, chiin in $\mathrm{Cl}_{1}^{\prime}$ in 泰 chot. 
西 HSI-HÊNG-NUNG.

2457 Hsi-hêng-nung | 恒農, (obs.) 40 li $\mathrm{S}$. of Lingpao H., Honan; $N$. Wei, chïn in Shan 㻖 cHou.

2458 Hsi-ho | 和 Si-ho.

$1^{\circ}$ H. in Kung-ch' ang Fu, Kansulı; Lat. $34^{\circ}$, Long. $107^{\circ} 53^{\prime} ; T s^{\prime} \mathrm{in}$, Lin-t'ao 臨洮; Han, Shang-lu 上. 酻; $W$. $W e i, \mathrm{Ch}^{\prime}$ 'ang-tao 長道; $2^{\circ}$ See No. 4457.

2459 Hsi-ho | 河, see Nos. 1688, 3858.

$2460 \mathrm{Hsi}-\mathrm{hua}$ | 華 Si-hwa.

[32] $1^{\circ}$ H. in $\mathrm{Cl}_{1}^{\prime}$ ên-chou Fu, Honan; Lat. $33^{\circ} 53^{\prime}$, Long. $114^{\circ} 38^{\prime} ; \mathrm{Han}$, Hsi-hua | 華; Sui, Hung-kou溤灌; $T^{6}$ ang, Chii-ch'êng 筫城.

$2^{\circ}$ See No. 6802.

2461 Hsi-hua-ch'ih | 華池, chên near Ning C., Kansuh.

2462 Hsi-huai | 淮, (obs.) S. E. of 'T'ang H.; Honan; N. Wei, chiull in Nanhsiang 南鸾 crou.

2463 Hsi-hung-1111ng | 弘訾, (obs.) in Honan; S. Ts $i$, chün in Ning-111an 宿㝈 Fin.

2464 Hsi-hsia-clii | 快集, ss̆̌ near Nei-lisiang $H$, Honall.

2465 Hsi-hsiang | 拫 Si-hiang.
西 HSI-LIANG.

[514] $1^{\circ} \mathrm{H}$. in Han-chung $\mathrm{Fu}$, Shensi ; Lat. $32^{\circ} 42^{\prime}$, Long. $107^{\circ} 53^{\prime}$; Minor Han, Nan-hsiang 南 坬; T Tsin, Hsi-hsiang | 悱.

$2^{\circ}$ Ss ̌̆ near Ling-shan H., Kuangtung.

2466 Hsi-hsin | 新; sometimes written for $N$. Wei, Hsihsin-yang | 新莏 $\mathrm{H}$.

2467 Hsi-hising | 舆, post-town near Cling-t ien H., Chehkiang.

2468 Hsi-jung | 我.

$1^{\circ}$ (obs.) near Ch'ingyang $\mathrm{Fu}$, Kansuli ; T'ang, C. in Yi-ling 宜定 Fu。

$2^{\circ}$ (obs.) near Turfan ; $T^{6}$ ang, C. in Yüehchil 月支 Fu.

2469 Hsi-ku | 固 so near

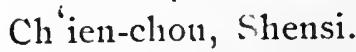

2470 Hsi-kuei | 音, (obs.) near Ning-lisia Fu, Kansulı; Tang, C. in Kuan-nei 關 內 circ.

2471 Hsi-kung | 頁 Saigon, the capital of French Cambodia.

2472 Hsi-kung | 琹, post-town near Hui-ning H., Kansuh.

2473 Hsi-lê | 樂, post-town near Hsin-lê H., Chihli.

2474 Hsi-liang | 倞, see No. 7836. 
西 HSI-I.IANG-SHAN.

2475 Hsi-liang-shan | 梁 仙, chên near Wu-lıu H., Anluni.

2476 Hsi-liao | 透, (obs.) Ts in, chïn.

2477 Hsi-lin | 林 Si-lin, H. in [416] Ssŭch êng Fu, Kuangsi ; Lat. $24^{\circ} 15^{\prime}$, Long. $105^{\circ}$ $55^{\prime}$.

2478 Hsi-ling | 陵, see Nos. $2282,2284,4257,7420$, 7812 .

2479 Hsi-lï | 瀘, (obs.) 25 li S.IV. of Hsi-ch'ang $\mathrm{H}$., Ssǔcl' 'uan; 7'ang, H. in Sui 崔 C.

2480 Hsi-lung | 隆 Si-lung, [96] C.I.C. in the Yn-chiang circ., Knungsi ; Lat. $24^{\circ}$ 32', Long. $105^{\circ} 35^{\prime}$; Ming, An-lung 妿隆 ssü in Kuangsi.

2481 Hsi-mu1-ni:nng | 模娘 Semnan, town in Persia, S.E. of Teheran.

2482 Hsi-nan | 南, sš̆ near San-shui H., Kuangtung. 2483 Hsi-ning | 稿 Si-ning.

[155] $1^{\circ} \mathrm{F} u$ in the Hsi-ning circ., Kansull ; forming the district city of the same name; Iat. $36^{\circ}$ $39^{\prime}$, Long. $101^{\circ} 48^{\prime}$; orig., Huang-chung 湟 中, land of the Hsich 'iang | 羔, W. barbarians; Han, $\mathrm{P}^{\prime} \mathrm{o}-$ ch 'iang 破着; E. Han,
西 Hsi-P'ing.

Hsi-p'ing | 平; $T$ sin, Hsi-tu | 都; Sui, Shan

盖 C.; Sung, Huang 滛 C. and Hsi-ning |

$2^{\circ} \mathrm{H}$. forming the prefectural city of the same name; Lat. $36^{\circ} 39^{\prime}$, Long. $101^{\circ} 48^{\prime}$.

[376] $3^{\circ} \mathrm{H}$. in Hsüan1-11й $\mathrm{Fu}$, Chilhli; Lat. $40^{\circ} 06^{\prime}$, Long. $114^{\circ} 13^{\prime}$.

[227] $4^{\circ} \mathrm{H}$. in Lo-ting C., Kuangtung; Lat. $23^{\circ}$ $10^{\prime}$, I, ong. $110^{\circ} 36^{\prime}$.

$5^{\circ}$ (obs.) Liang, town 63 li $\mathrm{S}$. of Ch' 'ung-jên H., Kiangsi.

$6^{\circ}$ See No. 2342.

2484 Hsi-ning-li | 穿利, see No. 1853.

2485 Hsi-nung | 農, (obs.) near T'ai-p'ing Fu, Kuangsi; Sung, C. in Vung 魯 cHOU.

2486 Hsi-pao | 堡, sš̆ near Chên-ning C., Kueic!ıun.

2487 Hsi-p ing | 平 Si-ping.

$1^{\circ} \mathrm{H}$. in Julning $\mathrm{F}_{11}$, Honlan; I at. $33^{\circ} 27^{\prime}$, Lonig. $114^{\circ} 08^{\prime}$; orig., Pai 柏 feudal state; Han, Hsi-p'ing | 平. $2^{\circ}$ (obs.) T'ang. C. near Ln1-liang C., Yünnan.

$3^{\circ}$ See Nos. 170, 2483, $3856,5291,7152$. 
西 HSI-P'ING-CH'ANG.

2488 Hsi-p'ing-ch'ang | 平昌, see No. 6314.

2489 Hsi-p'ing-ti | 平 E; erroneous way of writing $T \sin , \mathrm{P}^{\prime}$ ing-shih 平氏 $\mathrm{H}$.

2490 Hisi-p'o | 坡, post-town near An-11an H., Kneichon.

2491 Hsi-shan | 山.

$1^{\circ}$ Ss $\breve{\imath t}$ near Hsin-hsing H., Kunangtung.

$2^{\circ}$ Y. near Kn-chon T., Kneichon.

$3^{\circ} \mathrm{Y}$. near Huai-yüanı H., Knangsi.

2492 Hsi-shang-yung |上唐, (obs.) near Han-chung Fu, Shensi; $S . T s^{\prime} i, \mathrm{H}$. in Han-chung 漢中 chïn.

2493 Hsi-shih-mên 石 門, (obs.) in Shensi; N. Wei, H. in Ch'oul-ch'ih 仇池 chiin.

2494 Hsi-shont-chiang | 受降, (obs.) on N. bank of the Yellow River; $T^{6}$ ang, fort in Fêng 装. C.

2495 Hsi-shui | 水.

$1^{\circ}$ (obs.) Liang, H., E. of P'êng-tsê H., Kiangsi. $2^{\circ}$ (obs.) P. Chou, H., W. of Pao-11ing Fin, Ssŭch' nan.

2496 Hsi-sung | 杂, (obs.) T"ang, C. near Ho-cli ${ }^{\natural} \ddot{\mathrm{i}}$ C., Yünnan.

2497 Hsi-ta-cl' ï | 大渠, T-S. near P'ing-fan H., Kansuh.
牙

HsI-wU.

2498 Hsi-ta-t'ung | 大通, post.town near Kn-lang $\mathrm{H}$, Kansuh.

2499 Hsi-tang-ch' ì | 岩渠, (obs.) N.W. of Yent-t'ing H., Ssŭch'uan; S. Ts $i$, chïn in $\mathrm{Yi}$ 雀 cHOU.

2500 Hsi-tien | 后, chên near Fêng-hua H., Chehkiang.

2501 Hsi-t'ing | 亭, chên near Ling-t'ai H., Kansuh.

2502 Hsi-tsang | 藏, Thibet; also called 'T'u-po-t'ê 伯持, 'Ti1-po-t' ê 整字特, T"11-po 土波 and ' T"11-fan 吐蕃, or 土舀; the conntry is divided into Ch'ien-tsang 前趾 Anterior Thibet, Chnung-tsang 中藏 Central 'Tlibet, and Houl-tsang 後藏 Ulterior Thibet; the capital of the

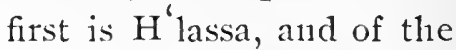
last 'Teshuh'lumbu.

2503 Hsi-ts' uan | 鼠蛋, see Nos. 1452, 2761.

2504 Hsi-tu | 都.

$1^{\circ}$ (ohs.) in Hsiao-yi H., Shansi; $W$. Han, H. in Hsi-ho | 河 chïn. $2^{\circ}$ See No. 2483.

2505 Hsi-tn-êrih-han | 都尔漭, post-town near Ta-t ung H., Kansuh.

2506 11si-t'un| 屯, chên near I.ing-t'ai H., Kansuh.

2507 Hsi-wu | 点, chên near Fêng-lıua H., Chehkiang. 
西 HSI-IVU-CH'

2508 Hsi-wu-ch' $\ddot{i}$ |无渠, T-S. near Ku-lang H., Kansul.

2509 Hsi-yang | 防荡.

$1^{\circ}$ Chên near San-yüan H., Shensi.

$2^{\circ}$ See Nos. 2282, 3433.

2510 Hsi-yen | 烟, chên near Hsin C., Shansi.

2511 Hsi-ying | 營, chên near Ch'in-yüan H., Shansi.

2512 Hsi-yü|于, (obs.) in Annam; Han, $T \sin , S$. Sung and $S$. $T s^{6} i, H$. in Chiao-chih 交趾: chïn.

2513 Hsi-yüan | 原 Si-yuen.

$1^{\circ}$ 'Town in Lin-ch'iu Islands.

$2^{\circ}$ (obs.) S. W. of Hsinning C., Kuangsi ; $7^{\prime}$ ang, H. in Ling-nan 領南 circ。

$3^{\circ}$ (obs.) in Ho C., Kansuh; Sung, pao in Ho 河 C.

2514 Hsi-yuian | 垣, see No. 574.

\section{㪘}

2515 Hsi 隰 Siel,, C.L.C. in [174] Shansi ; Lat. $36^{\circ} 40^{\prime}$, Long. $110^{\circ} 56^{\prime} ; C$. Wars, Hsi-l1o 西河; Han, $\mathrm{P}^{6} \mathrm{ul}$ tzŭ 莆子; P. Chou, Ch'ang-shou 長䩧; Sui, $\mathrm{Hsi} \mid \mathrm{C}$.

2516 Hsi-ch' êng | 域, see No. 2642.
鍟

HSI-HSÜEH.

2517 Hsi-wu | 沃, see Nos. $5323,5553$.

\section{習}

2518 Hsi-an 習努, see No. 98. 嶀

2519 Hsi-o 崤峩 Sih-ngo, H. [113] in Lin-an Fin, Yünnan; Lat. $24^{\circ} 24^{\prime}$, Long. $102^{\circ}$ $36^{\prime}$.

\section{压}

2520 Hsi-chih 急 直, (obs.) formerly $\mathrm{K}$. in $\mathrm{E}$. of Corea.

$2521 \mathrm{H}$ si-lin | 鄰, (obs.) in Mou C., Ssŭch' 'uan; $T^{\prime}$ ang, H. in $T u$ 染 C.

\section{息}

$2522 \mathrm{Hsi}$ 息 Sih.

[144] $1^{\circ}$ H. in Kuang C., Honan ; Lat. $32^{\circ} 25^{\prime}$, Long. $114^{\circ} 45^{\prime}$; S.A., feudal state Hsi ; Han, $\mathrm{H}_{\mathrm{si}} \mid \mathrm{H}$.

$2^{\circ}$ (obs.) S.A., feudal state in Honan.

2523 Hsi-fêng| 烽, (obs.) 110 li N.E. of Kuei-yang Fu, Kueichou, Ming, so in Fulyung 敷勇 military district, Kneichon.

\section{鍟}

$2524 \mathrm{H}_{\text {si }}$ 錫, see No. 7823.

2525 Hsi-lisüel |穴. see No. 7820 . 
錫 HSI-LIN-KUO-L $\hat{\mathrm{E}}$.

2526 Hsi-lin-kuo-lê | 林郭勒 Silinghol, Mongol League.

2527 Hsi-po-t' 11 伯圖 Silato, town in Tarbagatai; Lat. $46^{\circ}$, Long. $83^{\circ} 15^{\prime}$.

\section{析}

2528 Hsi-ching 析 津.

$1^{\circ}$ (obs.) S.W. of Shunt'ien Fu, Chilnli; Sung, H. in Yen-slian 燕 山 $\mathrm{F}_{11}$; Liao, I'11 and H. in Nan-ching 南京 circ. $2^{\circ}$ See No. 6037.

2529 Hsi-yang | 陽 (obs.) $S$. $T s^{\prime} i$, chïn in Honan.

\section{淅}

$2529^{\wedge}{ }^{A}$ Hsi-ch'uan 浙川 C.L.T. in Nan-jn-kuang circ., Hona11. Lat. $33^{\circ} 05^{\prime}$, Long, $111^{\circ} 27^{\prime}$, Han and Ming, Hsi-chuan $\mathrm{H}$.

2530 Hsi-yüan 淅源, (obs.) in Lü C., Ssŭch'uan ; T'ang, H. in Hsi 淅 C.

$$
\text { 晅 }
$$

2531 Hsi-la-ssŭ 萌 刺思, Serakhs, town in Independent Turkestan, S. W. of Merv.

2532 Hsi-yang | 陽, see No. 1073.

\section{犀}

2533 Hsi 犀, (obs.) in Ssŭcli'uan; T'ang, C. in Lung-yu 隴右 circ.
下 HSIA-KUEI.

\section{HSIA}

\section{下}

2534 Hsia-chiang $下$ 江 Hiakiang, T. in Li-p'ing Fu, Kueichou ; Lat. $27^{\circ} 32^{\prime}$, Long. $108^{\circ} 50^{\prime}$.

2535 Hsia-chieh , chêu in Wei-nan $\mathrm{H} \cdot \mathrm{S}_{\text {hensi. }}$

2536 Hsia-chu | 制, sš̆ in Yi-hsing H., Kiangsu.

2537 Hsia-ch" ï-yang | 曲陽, [515] (obs.) Han, H., N. of Chin C., Chillli.

2538 Hsia-clnuang | 获, sš̆ in Chêng-ho H., Fuhkien.

2539 Hsia-hsiang | 相, see No. 5915.

2540 Hsia-jên | 任, chên near Yi-shilı H., Shansi.

2541 Hsia-kê | 閣, chên near Ch' ao H., Anliui.

2542 Hsia-k'ou| | 口, chên near Nan-p'i H., Chilıli.

2543 Hsia-kuei | 邽.

$1^{\circ}$ (obs.) $50 l i$ N.E. of Wei-nan H., Shensi; W. Han, H. in Chingchao 兆京 district; $T \sin$ and Sui, H. in Fêng-yi 傌䇉 chün; $T^{6}$ ang, Sung and Kin, H. in Hua 華 C.

$2^{\circ}$ (obs.) in Ssŭch'uan; S. Sung, H. in An-ku 发固 chïn。 
下

$3^{\circ}$ (obs.) in Nan1-chêng H., Shensi; S. Ts $i$, H. in Fêng-yi 证呀 chïn.

$4^{\circ}$ See No. 6988.

2544 Hsia-lei | 霓 Hia-lui, [225] T-C. in Chên-an Fu, Kuangsi ; Lat. $22^{\circ} 55^{\prime}$, Long. $106^{\circ} 34^{\prime}$.

2545 Hsia-li | 歴, ssü near Ting-nan T., Kiangsi.

2546 Hsia-liang | 良, chên near Ch'in-yüan H., Shansi.

2547 Hsia-ma-kuan | 馬 關, see No. 5208.

2548 Hsia-ni | 泥, T-S. near Sung-p'an T., Ssŭch' uan.

2549 Hsia-p'ei | 䂙.

$1^{\circ}$ (obs.) 3 li $\mathrm{E}$. of $\mathrm{P}^{\prime} \mathrm{ei}$ C., Kiangsu; Han, Hsia-p'ei | 䂙 H.; $E$. Han, K. Hsia-p'ei | 䂙; S. Sung and $N$. Wei, chün.

$2^{\circ}$ (obs.) in Kiangsu; $S$. Sung and $S . T s^{6} i$, Hsia-p'ei | 不 H. $3^{\circ}$ (obs.) S.E. of Su-ch'ien H., Kiangsu; Sui, chïn in Hsï 徐 cHOU. $4^{\circ}$ See No. 5035.

2550 Hsia-po | 博, see No. 5628.

2551 Hsia-shê / 攝, ssŭ near Hsiang-t an H., Hnnan.

2552 Hsia-shih-hsi 石 西, T.C. in 'T'ai-ping Fu,
夏 HSIA.

Kuangsi ; Lat. $22^{\circ} 10^{\prime}$, Long. $106^{\circ} 36^{\prime}$; Ming, C. in Ssŭ-ming 思明 Fu.

2553 Hsia-ti | 廸, chên near Chi-shan H., Shansi.

2554 Hsia-ts'ai | 蔡.

$1^{\circ}$ Chên near Fêng-t'ai H., Anhui.

$2^{\circ}$ See No. 8657.

2555 Hsia-tsun | 售.

$1^{\circ}$ (obs.) Han, town E. of Yüan-chiang $\mathrm{H}$., Hunan.

$2^{\circ}$ See Nos. 1598, 4773, 6771, 7765.

2556 Hsia-tung | 東, chên near Wên-hsi H., Shansi.

2557 Hsia-yi|邑, see No. 2570.

2558 Hsia-chih | 雉, (obs.) Han, H. near Hsing-kuo H., Kiangsi.

\section{治}

2559 Hsia-ho 治 峆, chên in Ching-yang H., Shensi.

\section{夏}

$2560 \mathrm{Hsia}$ 夏 Hia.

$1^{\circ} \mathrm{H}$. in Chieh C., Shansi ; Lat. $35^{\circ} 10^{\prime}$, Long. $111^{\circ} 12^{\prime}$; orig., residence of the Einperor Yï 禹; 2nd $\mathrm{Wei}$, Hsia $\mathrm{H}$.

$2^{\circ}$ Chên near $\mathrm{P}^{\prime} \mathrm{ei} \mathrm{H}$, Kiangsu.

$3^{\circ}$ See No. 4687. 
夏 HSIA-CHING.

2561 Hsia-ching | 津 Hia-tsin, H. in Lin1-ch'ing C., Shantung; Lat. $37^{\circ} 03^{\prime}$, Long. $116^{\circ} 10^{\prime} ; C$. Wars, Yaoching 要津; Han, Shu 俞 H.; T"ang, Hsia-ching | 㴖 $\mathrm{H}$.

2562 Hsia-ch'in | 邱, see No. 768.

2563 Hsia-jui | 汭, see No. 7052.

2564 Hsia-k'ou | 口 T. in Han-yang Fin, Hupeh. See No. 1957.

2565 Hsia-po-yi | 伯是, see No. 768.

2566 Hsia-tien | 尔容, chên near Hsiang-yüan H., Shansi.

2567 Hsia-wu | 屋, (obs.) town N. of 'T'ang H., Clihli.

2568 Hsia-yang | 陽.

$1^{\circ}$ Ss̆̌ in P'ei H., Kiangsu.

$2^{\circ}$ See No. 1973.

2569 Hsia-yang-shih | 荡市, sš̆ near Knei-hula H., Fuhkien.

2570 Hsia-yi 邑 Hia-yih, H. in Kuei-tê $\mathrm{F} u$, Honan; Lat. $34^{\circ} 20^{\prime}$, Long. $116^{\circ}$ 19'; Han, Hsia-yi 下邑; 2nd Wei, H. in Tang 碭 chïn.

\section{厦}

2571 Hsia-111ên 厦胣 Annoy, T. in Chang-chou Fu, Fuhkien ; Lat. $24^{\circ} 31^{\prime}$,
硤 HSIA-SHIH.

Long. $117^{\circ} 04^{\prime}$; one of the ports open to foreign trade. Literary name Lntao 踏岛; was occupiea by Koxinga. He gave it the name of Su-min C. 蘇 民 in 1648. Under the Ming dynasty it was called Clinug-tso So 中 左闹。

\section{㞋}

2572 Hsia 琲, (obs.) formerly town S.W. of Chieln C., Shansi.

2573 Hsia-ch'iu | 邱, see No. 6863.

\section{昙需}

2574 Hsia-p' $u$ 霞浦 Hia-pu, $\mathrm{H}$. forming the prefectural city of Fu-ning, Fuhkien ; Lat. $26^{\circ} 54^{\prime}$, Long. $120^{\circ}$ $05^{\prime}$.

\section{䧿}

2575 Hsia-111an 轄 漫, T-S. near Sung-p'an T., Ssŭch' uan.

\section{阧}

2576 Hsia-ch' nan 堘川 Kiahchnen, Fin in Cling-shang 磨何 circ., Corea; Lat. $35^{\circ} 08^{\prime}$, Long. $128^{\circ}$.

\section{陜}

2577 Hsia-shih 磱石, T-S. near $P^{6}$ ing-fan H., Kansul. 
阦

HsIA.

性。

$2578 \mathrm{H}$ sia 快.

$1^{\circ}$ (obs.) S. Sung, C. in

Hu-pei 测北 circ.

$2^{\circ}$ See No. 7420.

2579 Hsia-chiang | 江 Kiall[208] kiang, H. in Lin-chiang Fu, Kiangsi ; Lat. $27^{\circ}$ $34^{\prime}$, Long. $115^{\circ} 15^{\prime}$; Ming, Hsia-chiang | 江. $\mathrm{H}$.

2580 Hsia-lısi | 西, (obs.) Sung, circ.

2581 Hsia-k'ou |口.

$1^{\circ}$ Chên near Yü-shan H., Kiangsi.

$2^{\circ}$ Post-town near Mien H., Shensi.

$3^{\circ}$ Post-town near Yungclíang H., Kansul.

2582 Hsia-shan | IL, chên near Wu-wei C., Anluni.

2583 Hsia-shil | 石.

$1^{\circ}$ Chên near Hai-yen H., Chehkiang.

$2^{\circ}$ Post-town near Shan C., Honan .

\section{㶲}

2584 Hsia-chu 师竹, T-S. near Sung-p an T., Ssŭch'nan.

\section{HSIANG}

\section{拫}

2585 Hsiang-ning 拫家 Hiang-

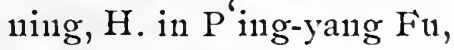
Shansi; Lat. $36^{\circ}$, Long.
香 HSIANG-TS'AO.

$110^{\circ} 46^{\prime} ;$ Han, Lin1-fên 臨汾; 2nd Wei, Cli'angning 昌俩; Five dyn., Hsiang -11ing | 窂.

2586 Hsiang-t'on | 解, chên near Chieh C., Shansi.

\section{香}

2587 Hsiang-clı'i 香溪, chên near Lall-ch'i $\mathrm{H}$., Chehkiang.

2588 Hsiang-chiang | 港 Hongkong, an island, formerly in Hsin-an H., Kuangtung, but now a British Colony ; Lat. $22^{\circ} 16^{\prime}$, Long. $114^{\circ} 11^{\prime}$.

2589 Hsiang-ch'ïan | 泉, chên near Pao-chi H., Shensi.

2590 Hsiang-ho | 河 Hiang-lıo, [191] H. in Shun-t ien Fu, Chihli ; Lat. $39^{\circ} 50^{\prime}$, Long. $117^{\circ} 02^{\prime}$; orig., Wu-ch'ing 武清; Liao, Hsiang-lio | 咑.

2591 Hsiang-shan | III Hiangshan.

$1^{\circ} \mathrm{H}$. in Kuang-chou $\mathrm{Fu}$,

Kuangtung; Lat. $22^{\circ}$ $32^{\prime}$, Long. $113^{\circ} 15^{\prime}$.

$2^{\circ}$ Post-town near Kuangcli ang H., Chihli.

2592 Hsiang-tsa | 㕷, T-S. near Sung-p’an T., Ssŭch'tran.

2593 Hsiang-ts، ao | 草, chên near Wang-chiang $\mathrm{H}$., Anhui. 
向

Hsiang.

\section{向}

2594 Hsiang 向.

$1^{0}$ (obs.) Tkang, C., comprising part of Mou C., Ssŭch "uan.

$2^{\circ}$ (obs.) Han, H. near Han-shan H., Anhui.

2595 Hsiang-wu | 武 Hiangwu, T.C. in Chên-an Fut, Kuangsi ; I at. $23^{\circ} 12^{\prime}$, Long. $106^{\circ} 45^{\prime}$.

\section{項}

2596 Hsiang-cli êng 項城Hiang[212] ching, H. in Hsiu C., Honlan; Lat. $33^{\circ} 20^{\prime}$, Lonng. $114^{\circ} 53^{\prime}$; orig., K. Hsiang-tzŭ | 子; Han, Hsiang-ch êng | 城; $E$. $W_{e} i$, Mo-ling 秝陵; Sui, Hsiang-ch' êng | 战.

2597 Hsiang-ts'tun | 村, ssü near Wu-yüan H., Anhui.

2598 Hsiang-tž̆ $\mid$ 子

$1^{\circ}$ (obs.) formerly K. in Honan.

$2^{\circ}$ See No. 2596.

\section{象}

2599 Hsiang 豖 Siang.

[569] $1^{\circ}$ C. in Liu-chou $\mathrm{Fu}$, Kuangsi ; Lat. $23^{\circ} 59^{\prime}$, Long. $109^{\circ} 26^{\prime} ; C_{l}{ }^{\prime} \hat{e} n$, Hsiang |chün; Sui and $T^{*}$ aug, Hsiang $\mid \mathrm{C}$. $2^{\circ}$ (obs.) $T \sin$ and $C h^{\prime} e ̂ n$, chïn comprising parts
群

HSIANG-FU.

of Kuangtung and Kuangsi.

$3^{\circ}$ See Nos. 2194, 3668, 3815, 3981.

2600 Hsiang-lit | 胡, sš̆ in Ying-tê $\mathrm{H}$., Kuangtung.

2601 Hsiang-kang / 岡, ssŭ near Wêng-yüan H., Kuangtuing.

2602 Hsiang-lei | 霄, (obs.) in the Tumed country near Kuei-hua 'T., Shansi; Liao, H. in Hsing-chung 興需 Fu。

2603 Hsiang-shan | II Siangshau, H. in Ning-po Fu, Chehkiang; Lat. $29^{\circ} 35^{\prime}$, Long. $121^{\circ} 42^{\prime} ; T^{\prime}$ in, Ning-hai 富 海; T $T^{6}$ ang, Hsiang-shan |山.

2604 Hsiang-tung-chai | 洞些, ssü near Shang-hang $\mathrm{H}$., Fullkien.

\section{橡}

2605 Hsiang-1nu 橡木 Tochigi, ken in Japan.

\section{翔}

2606 Hsiang-lung 翔龍, (obs.) 1st Sung, H. on an island S. of Wi1-cl' 'uan H., Knangtung.

\section{洋}

2607 Hsiang-fu 祥符 Siang-fu, $\mathrm{H}$. forming the prefectural city of $\mathrm{K}^{\text {'ai-fêng }} \mathrm{Fu}$, Honan; Lat. $34^{\circ} 52^{\prime}$, 
祥

Long. $114^{\circ} 33^{\prime} ;$ Han, Hsün-yi 淩儀; $E$. Wei, Liang 梁 C.; P. Chou, Pien 汒 C.; Sung, Hsiung$\mathrm{fu}$ | 符.

2608 Hsiang-yüan | 原, chün in P'ing-an 4 安 circ.,

Corea.

\section{Hsiang 衰}

\section{襄}

$1^{\circ}$ (obs.) Han and Wei, K. in Chihli.

$2^{\circ}$ See Nos. 2616, 2850, $5582,5821$.

2610 Hsiang-an | 岀.

$1^{\circ}$ (obs.) S. of Wu-wei C., Anhuii; Han and $T \sin , \mathrm{H}$. in Lü-chiang 盧江 chiin.

$2^{\circ}$ See No. 374 .

2611 Hsiang-clı' êng | 城, Siang[200] ching, H. in Cl' ên-chon Fu, Honan; Lat. $33^{\circ} 52^{\prime}$, Long. $113^{\circ} 36^{\prime} ; S$. A., $\mathrm{Ch}^{\prime} \mathrm{i}$ 杞 place of retirement of the Prince of Hsiang | ; Han, Hsiang-clı' êng | 城; $T^{\prime}$ ang, Ju 汶 C.

2612 Hsiang-lê | 樂, chên near Ning C., Kansult.

2613 Hsiang-ling | 陵.

[123] $1^{\circ} \mathrm{H}$. in $\mathrm{P}$ ing-yang $\mathrm{Fu}$, Shansi; Lat. $36^{\circ} 02^{\prime}$, Long. $111^{\circ} 26^{\prime}$; Han, Hsiang-ling | 陵; 2nd Wei, Ch'in-ch'ang 摛 昌.

$2^{\circ}$ See No. 1794.
HSIANG-YANG.

2614 Hsiang-pei 营.

$1^{\circ}$ (obs.) $120 l i \mathrm{~S} . \mathrm{W}$. of Lan-shan H., Shantung; Han and $T \sin$, H. in Tung-hai 草海 chiin.

$2^{\circ}$ (obs.) in Tan-yang H., Kiangsu；S. Ts $i, \mathrm{H}$. in Nan Tung-hai 南 乘海落 chïn.

$3^{\circ}$ (obs.) N. of An-tung H., Kiangsu ; S. Sung, H. in Tung-hai 身i海 chion, S. Ts'i, H. in Pei 'Tung-hai 北東海 chïn; N. Wei, H. in Hai-lısi 海西 chïn.

2615 Hsiang-wu | 武, see No. 4332.

2616 Hsiang-yang | 榢 Siangyang.

$1^{\circ}$ Fu in Cliang-ÿüan iT 原 circ., Corea; Lat. $37^{\circ} 45^{\prime}$, Long. $129^{\circ}$.

[133] $2^{\circ} \mathrm{F} 11$ in the An-HsiangYün-Ching circ., Hupel,; torming the district city of the same name; Lat. $32^{\circ} 06^{\prime}$, Long. $113^{\circ} 05^{\prime}$; S.A., in K. Ch'u 楚; $7 s^{\prime}$ in and Han, in Nan 南 chün; Wei, Hsiang-vang / 陽; $T \sin$, Ching 汧 C.; $E$. Tin, Yung 䔨 C.; $\mathrm{W}$. $W_{e i}$, Hsiang | C.; Sui, Shan-11an 山南; Ming, Hsiang-yang | 防 $\mathrm{Fu}$. 
偯 HSIANG-YANG.

$3^{\circ} \mathrm{H}$. forming the prefectural city of the same name, Hupeh; Lat. $32^{\circ} 06^{\prime}$, Long. $113^{\circ}$ $05^{\prime} ;$ Han, Hsiang-yang １陽； T'ang and Sung, Hsiang |C.

$4^{\circ}$ (obs.) S. Sung, Fin in Ching-hsi 京西 circ.

2617 Hsiang-yi| 邑, see No. 5951.

2618 Hsiang-yüan | 坦 Siangyuen.

[104] $1^{\circ} \mathrm{H}$. in Itu-an $\mathrm{F} u$, Shansi ; Lat. $36^{\circ} 27^{\prime}$, Long. $113^{\circ} 06^{\prime}$; Ts ${ }^{\prime} i n$, Hsiang-yüan | 垣; $P$. Chou, Han 韓 C.

$2^{\circ}$ (obs.) N. of preceding; $H a n$ and $T \sin , H$. in Shang-tang 上黨 chïn; N. Wei, 1st chün and H. in Ping 扭 CHOU, 2nd $\mathrm{H}$. in $\mathrm{H}$ siang 鄉 chiin; Sui, H. in Shang-tang 上镗 chïn. $3^{\circ}$ (obs.) S.W. of Wu-hu H., Anhui ; S. Sung and $S . \quad T^{s} s$, H. in Huai-nan 淮南 chün. $4^{\circ}$ See No. 7163 .

\section{相}

2619 Hsiang 相, see Nos. 107, 225.

.2620 Hsiang-an | 岀, ssü in Nan-k'ang H., Kiangsi.

\section{湘 HsIANG-HSIANG.}

2621 Hsiang-chi | 篗, (obs.) near Ning-11sia Fu, Kansulı; T'ang, Hsiang-chi | 彩 $\mathrm{H}$.

2622 Hsiang-hsiu | 胥, chên near Yi-ch' uan H., Shensi.

2623 Hsiang-ju | 邚.

$1^{\circ}$ (obs.) in $P^{\prime}$ êng C., Ssŭch' uau; S Sui, H. in Pa-hsi 巴西 chiun; T'ang, H. in Kuo 果 C.; Sung and Yïan, H. in P'êng 逢 C. $2^{\circ}$ See No. 7593.

\section{箱}

2624 Hsiang-kên 箱根 Hakone, town in Japan.

2625 Hsiang-kuan | 館 Hakodate, ken and chief town, Japan. An open port.

\section{厢}

2626 Hsiang-hsi 相 西, chên near Lo-ch' nan H., Shensi.

\section{湘}

2627 Hsiang 湘, see Nos. 267, 2158.

2628 Hsiang-lisi | 西, (obs.) S. of Hisiang-t an H., Hunan ; $T s i n, S$. Sung and S. Ts'i, H. in Hêng-yang 衡陽 chiin.

2629 Hsiang-hsiang / 拫 Siang[247] hiang, $\mathrm{H}$. in Cli 'ang-sha Fu, Hunan; Lat. $27^{\circ} 47^{\prime}$, Long. $112^{\circ} 16^{\prime} ; T^{\prime}$ in, 
湘

HSIANG-HSIANG.

Hsiang-nan | 南; Han, Hsiang-hsiang /拫; $T^{6}$ ang, Lung-ch'êng 龍 城.

2630 Hsiang-k'ou |口, posttown, near $\mathrm{Yung}$-chon $\mathrm{Fu}$, Hunan.

2631 Hsiang-nan | 南, see Nos. 2032, 2629, 2633.

2632 Hsiang-pin | 筫, (obs.) Liang, H., N.E. of Hsiangyin $\mathrm{H}$., Hunan.

2633 Hsiang-t’an | 潭 Siangtall.

[177] $1^{\circ} \mathrm{H}$. in Ch'ang-sha $\mathrm{Fu}$ Hullan; Lat. $27^{\circ} 53^{\prime}$, Long. $112^{\circ} 42^{\prime} ; \mathcal{T s}^{\prime} \mathrm{in}$, Hsiang-nan / 南; Lians, Hsiang-t'an | 潭.

$2^{\circ}$ See No. 5475.

2634 Hsiang-tung | 東.

$1^{\circ}$ (obs.) in Hêng-yang H., Hunan ; Tsin, chion in Ching 形 chou; $S$. Sung and $S$. $T s^{\prime} i$, chïn in Hsiang I chou.

$2^{\circ}$ See No. 2031.

2635 Hsiang-yin | 陵, Siang[177] yin, H. in Ch'ang-slia Fu, Hunan; Lat. $28^{\circ} 42^{\prime}$, Long. $112^{\circ} 38^{\prime}$; orig., L.o 維; Ts'in, Lo 羅 H.; 1 st Sung, Hsiang-yin | 院.

2636 Hsiang-yüan! 源.

$1^{\circ}$ (obs.) $7 \quad l i \mathrm{~W}$. of $\mathrm{Cl}^{\prime}$ üan C., Kuangsi ; Sui, H. in Ling-ling
HSIAO-YI.

零陵 chiin; T6ang, H. in Yung 永 C.

$2^{\circ}$ See No. 1483.

\section{HSIAO}

\section{教}

2637 Hsiao-cli ang 䒨昌, see No. 2641.

2638 Hsiao-chao | 叉, chên near IVên-shui H., Shansi.

2639 Hsiao-fêng | 豐 Hianfung, $\mathrm{H}$. in $\mathrm{Hu}$-chou $\mathrm{Fu}$, Chehkiang; Lat. $30^{\circ} 30^{\prime}$, Long. $119^{\circ} 36^{\prime}$.

2640 Hsiao-huai | 懐, (obs.) in Hupelı $_{1} S . T s^{\prime} i$, Hsiaohuai | 懁 $\mathrm{H}$.

2641 Hsiao-kan | 感 Hian-kan, [146] H. in Han-yang Fu, Hupelı ; Lat. $30^{\circ} 56^{\prime}$, L,ong. $113^{\circ} 50^{\prime}$; 1st Sung, Hsiao-ch'ang | 昌; T'ang, Huan 澴 C.; P. T ang, Hsiao-kan | 感.

2642 Hsian-yi | 義 Hiaul-i.

[45] $1^{\circ}$ T. in Hsi-an Fu, Shensi ; Lat. $33^{\circ} 25^{\prime}$, Long. $109^{\circ} 45^{\prime}$.

[543] $2^{\circ}$ H. in Fên-chou $\mathrm{Fu}$, Shansi; Lat. $37^{\circ} 10^{\prime}$, Long. $111^{\circ} 45^{\prime}$; orig., T'ai-yüan 太原; Wei, Chung-yang 中 陽; Tsin, Hsi-ch' êng 筸 城; T'ang, Hsiao-yi | 義. 
小 HstaO-CH '̂NNG.

$$
\text { 小 }
$$

2643 Hsiao-ch'êng 小徵, ss̆̌ in Lou H., Kiangsu.

2644 Hsiao-cl' êng-fan | 程番, (obs.) near Kuei-yang Fu, Kneichou； Ming, ssŭ in Kuei-yang Fu.

2645 Hsiao-ch`i 溪, see No. 5963.

2646 Hsiao-clı'i-p' $u$ | 浫 鋪, post-tow11 near Cli angning H., Kiangsi.

2647 Hsiao-ch 'ih | 池, posttown near $\mathrm{Cl}_{1}$ ien-shan $\mathrm{H}$., Anhui.

2648 Hsiao-ch'ih-k'ou | 池口, sš̆ near Huang-mei $\mathrm{H}$., Hupeh.

2649 Hsiao-clini-cli'nan | 金 川, ss̆̆ near 'T'ien-ch' "üan C., Ssŭch' '1an.

2650 Hsiao-chu | 制.

$1^{\circ}$ (obs.) formerly K. in Yïnn11an.

$2^{\circ}$ See No. 294.

2651 Hsino-ch' uan | 川, chền near Ch' êng H., Kansulı.

2652 Hsiao-hei-shan | 哭 ll, post-town near Hsin-yen T., Shingking.

2653 Hsiao-lio | 河.

$1^{\circ}$ Chên near Wan-ch üan H., Sliansi.

$2^{\circ}$ Y. near Lung-an $\mathrm{Ft}$, Ssŭcl' uan.

2654 Hsiao-lio-ch'êng | 河 城, see No. 423.
小 HsiaO-Ning.

2655 Hsiao-ho-ch'i | 河 溪, post-town near Tê-an $\mathrm{Fu}$, Hupel.

2656 Hsiao-hsing | 姓, T-S. near Sung-p an 'T., Ssŭch' van.

2657 Hsiao-ku-shan | 姑 川, ssŭ near Wang-chiang $\mathrm{H}$., Anhui.

2658 Hsiao-kuan | 關.

$1^{\circ}$ Chên near Têng-fêng $\mathrm{H}$., Honan.

$2^{\circ}$ Post-town near $\mathrm{P}^{\prime} \mathrm{u}$ ch'êng H., Fullkien.

2659 Hsiao-lao-mên | 落門, (obs.) 30 li E. of Ningyiian H., Kansulı; Sung, military post in $\mathrm{Ch}^{\prime}$ in 秦 $\mathrm{C}$.

2660 Hsiao-li-t'an | 里潭, ssŭ near T'ien-nên H., Hupel.

2661 Hsiao-ling | 淩, chên near 'Tê-ch'ing H., Chelikiang.

2662 Hsiao-lu| 酰, town in the Lin-cl' in Islands.

2663 Hsiao-lung | 龍, (obs.) near Kuei-yang Fu, Kueichou; Ming, ssü in Kueiyang $\mathrm{Ft1}$.

2664 Hsiao-nan | 難, chên in Yüan-ch' êng H., Chilıli.

2665 Hsiao-ning | 富.

$1^{\circ}$ (obs.) Han, H. near Yell-ch' ing C., Chihli.

$2^{\circ}$ (obs.) Yüan, H. near Pao-ning Fu, Ssŭclíuan. 
小 HsiaO-Shin.

2666 Hsiao-shih | 市, chên near An-chi H., Chehkiang.

2667 Hsiao-tien | 店.

$1^{\circ}$ Chên near Clining-lê H., Sliansi.

$2^{\circ}$ Chên near T'ai-yüüan H., Shansi.

\section{藏}

2668 Hsiao 藏 Siau, H. in Hsü-chou Fu, Kiangsu; Lat. $34^{\circ} 12^{\prime}$, Isong. $117^{\circ}$ $13^{\prime}$; orig., feudal state Hsiao | Han, Hsiao | H.; Sui, Lung-clı' êng 龍 城.

2669 Hsiao-kuan | 館, posttown near An-sai H., Shensi.

2670 Hsiao-shan | !L Sianshan, H. in Hang-chon Fu, Chelnkiang; Lat. $30^{\circ}$ 05', Long. $120^{\circ} 20^{\prime}$; Han, Yü-chi 餘毁; $W u$, Yunghising 永 與; 7 ' ang, Hsiao-shan III.

\section{HSIEH}

\section{歇。}

2671 Hsieh-111 晶馬, Y. near T'ung-hsï H., Honan.

\section{協}

2672 Hsielı 協, (obs.) Sui， C., F. of Cl' ${ }^{\prime}$ ü-cling $\mathrm{Fu}$, Yünnan, and comprising part of Hsii-chou Fu, Ssŭch' tran.

\section{HSIEH.}

\section{謝}

2673 Hsieh-1111 謝 沭, (obs.) $H a n, \quad \mathrm{H}$, N. of $\mathrm{K}^{\prime}$ aichien H., Kuangtung.

2674 Hsieh-shui-pao | 水堡, post-town near An- i C., Kansuh.

2675 Hsielı-ts' un | 村, chênn near Ch' êng-ku H., Shensi.

\section{邪}

2676 Hsieh-lung 邪龍.

$1^{\circ}$ (obs.) in Mêng-liua T., Yünnan; $H a n, H$. in Yi 䇥 $\mathrm{CHOU} ; T \sin , \mathrm{H}$. in Yün11-11an 空南 chün. $2^{\circ}$ See No. 4941.

2677 Hsiel1-mi-ssŭ-kan | 迷思 干, see No. 5342 .

2678 Hsiel1-t'on1-11ei | 頭昧; IV. Han, H. in Corea.

\section{斜}

2679 Hsiel1-chieh 斜階, see No. 5655.

2680 Hsieh-k'on | 口, chên near Lint' 'ung H., Shensi.

2681 Hsielı-kung | 鿗, (obs.) in Ya-chon Fu, Ssŭch' 'uan; 7'ang, C. in Chien-nan 劍南 circ.

2682 Hsiel-t’ang | 楛, chên near Chia-hsing Fu, Chehkiang.

\section{薛}

$2683 \mathrm{Hsieh}$ 薛。 
$1^{\circ}$ (obs.) Ts'in, chïn in Shantung; S.A., K. L, 鲁; Tsin，Ln鲁 chïn. $2^{\circ}$ See Nos. 1454, 7373.

2684 Hsieh-cli'êng | 城.

$1^{\circ}$ (obs.) formerly town near T'êng H., Yenchou, Shantung.

$2^{\circ}$ See No. 4946.

\section{泄}

2685 Hsieh-la-shih 泄刺失, see No. 5677.

\section{HSIEN}

\section{成}

2686 Hsien-an 咸安.

$1^{\circ}$ chiun in $\mathrm{Ch}^{\text {ing-shang }}$ 慶少 circ., Corea.

$2^{\circ}$ See No. 5058 .

2687 Hsien-ch' ang | 昌 H. in $\mathrm{Cl}^{\prime}$ 'ing-shang 慶仯 circ, Corea.

2688 Hsien-ching | 鏡 circ., in Corea.

2689 Hsien1-fêng | 豉 Han1-fung, 449] H. in Shilinnan Fu, Hupeh ; Lat. $29^{\circ} 54^{\prime}$, Long. $109^{\circ} 08^{\prime}$.

2690 Hsien-hsing | 興 Hanhing, Tao in Hsien-ching 咸鏡 circ., Corea； Iat. $39^{\circ} 51^{\prime}$, Long. $128^{\circ} 28^{\prime}$.

2691 Hsien-ning | 傢 Hanning.

$1^{\circ} \quad \mathrm{H}$. forming with $\mathrm{Cl}^{\text {' }}$ ang-an $\mathrm{H}$. the pre- fectural city of Hsi-an, Shensi; Lat. $34^{\circ} 17^{\prime}$, Long. $108^{\circ} 58^{\prime}$; $T s^{\prime} i n$, Chih-yang 芷陽; Han, Pa-ling 露陵 and Tuling 杜陵; P. Chou, Wan-nien 萬年; Sui， Ta-hsing 大興; Five dy'n., Ta-1:ie:1 大作; Sung, Fan-ch' uan 焚 川.

[129] $2^{\circ} \mathrm{H}$. in Wu-ch' ang Fu, Hupeh; Lat. $29^{\circ} 55^{\prime}$, Long. $114^{\circ} 06^{\prime}$; Sui, Chiang-hsia 江 夏; Tang, Yung-an 永安; Sung, Hsien-11ing | 察. $3^{\circ}$ See No. 4573.

2692 Hsien-ping | 平.

$1^{\circ} \mathrm{H}$. in Ch' üan-lo 全羅 circ., Corea.

$2^{\circ}$ See No. 6782.

2693 Hsien-shun | 浣, (obs.) near Chung C., Ssŭch' uan; Sung, Fu in K'uei-chon 窪州 circ.

2694 Hsien-ts'ung | 從 Han1tsung, Fu in P'ing-an 平 努 circ., Corea; Lat. $39^{\circ}$ $16^{\prime}$, Long. $125^{\circ} 30^{\prime}$; Yiäan, $\mathrm{H}$. in Huang 盐 C.

269.5 Hsien1-yang | 陽 Hanyang.

$1^{\circ}$ chiin in $\mathrm{Ch}^{\prime}$ ingr-shang 慶 眮 circ., Corea; Lat. $35^{\circ} 03^{\prime}$, Long. $127^{\circ} 15^{\prime}$. 
战

HSIEN-YANG.

[498] $2^{\circ}$ H. in Hsi-an Fu, Shensi; I at. $34^{\circ} 20^{\prime}$, Long. $108^{\circ} 39^{\prime} ; \mathrm{Ts}^{\prime} \mathrm{in}$, Hsien-yang | 陽; Han, Wei-ch'êng 渭 城; Tsin, Shil1-an 石安.

2696 Hsien-yüch | 悅 H. in $\mathrm{Ch}^{\prime}$ 'üan-lo 全. 羅 circ., Corea.

\section{獻}

2697 Hsien 獻 Hien, H. in [143] Ho-chien Fu, Chihli; I at. $38^{\circ} 20^{\prime}$, I ong. $116^{\circ} 05^{\prime}$; Han, I,ê-ch' êng 樂 城 and Ching-ch'êng 澩城; Suir, Kuang-ch'êng 廣城; Kin, Hsien | H.

\section{弦}

2698 Hsien 綮.

$1^{\circ}$ (obs.) S.A., K. in Honan.

$2^{\circ}$ See Nos. $3430,3433$.

\section{縣}

2699 Hsien1-t’ou 縣頭, chên near Ch' ien-yang H., Shensi.

\section{顯}

2700 Hsien 顯, see Nos. 4935, 6246.

$2701 \mathrm{Hsien-ch}$ in | 親, (obs.) near $\mathrm{Ch}^{\prime}$ in-an H., Kansuh; E. Han, H. in Han-yang 漢陽 chiin.
仙 HSIFN-T'AI.

2702 H:ien-ch' wan | 川; T゙ang, H. in Kuangsi.

2703 Hsien-11sin | 新, (obs.) N.W. of Clintin H., Kansuln; $T$ sin and $N$. Wei, H. in ' ' "ien-shni 天 水 chiün.

\section{鮮}

2704 Hsien-yü 鮮虞, see Nos. 6246, 6440 .

\section{仙}

2705 Hsien 仙, (ols.) T'ang, C. near Yeh H., Honan.

2706 Hsien-chên | 銭, Y'. near Ch' ien-chon 'T., Hn11ran.

2707 Hsien-chü / 居 Sien-kü. [252] $1^{\circ} \mathrm{H}$. in T"ai-chon Fu, Chehkiang; 1.at. $28^{\circ}$ $52^{\prime}$, Long. $120^{\circ} 46^{\prime}$; $T \sin$, Lê-an 樂 安; Sung, Hsien-chï | 居 $\mathrm{H}$.

$2^{\circ}$ (obs.) 50 li W. of Knang-shan H., Honan; $T^{\prime}$ ang and Sung, $\mathrm{H}$. in Kuang 光 C.

2708 Hsien-chü-k'ou | 居口, ssŭ near Yi-ch' êng H., Hupeh.

2709 Hsien-fu | 島, see No. 5582 .

2710 Hsien-k'on 口, Y. near Win-hsüan H., Kuangsi.

2711 Hsien-t'ai | 臺 Sendai, chief town of Niyagi ken, Japan. 
仙 HSIEN-T'AO.

2712 Hsien-t'ao | 桃, chên near 'T'ien-mên H., Hupeh.

2713 Hsien-t' $i \mid$ 提, (obs.) 10 li E. of Shan-tan H., $\mathrm{Kansuh} ; T \sin , \mathrm{H}$. in $\mathrm{Hsi}$ 砳 chïn.

2714 Hsien-yu | 遊, Sien-yu, [537] $\mathrm{H}$. in Hsing-hua $\mathrm{Fu}$, Fuhkien; Lat. $25^{\circ} 18^{\prime}$, Long. $118^{\circ} 58^{\prime} ; T^{\prime}$ ang, 1st Ch'ing-yiuan 清源, 2nd Hsien-yu | 遊.

2715 Hsien-yüan | 原, see No. 1454.

2716 Hsien-yüan 源, the same as the preceding.

\section{HSIN}

\section{所}

2717 Hsin-k'an-ch'êng 欣坎 城, post-town near An-lisi C., Kansull.

2718 Hsin-k'an-pu| 坎布, post-town near An-hsi C., Kansull.

2719 Hsin-lê | 樂, ssŭ near Hui-chou Fu, Kuangtung. 2720 Hsin - tao | 道, (obs.) $T^{\prime}$ ang, H. $80 l i \mathrm{~N} . \mathrm{W}$. of Yung H., Knangsi.

\section{忻}

2721 Hsin 忻 Hin, C.L.C. in [565] the Ying-P'ing-Ta-So Ning - Hsin - 'Tai-Pao circ., Shansi ; Lat. $38^{\circ} 26^{\prime}$, Long. $112^{\circ} 43^{\prime}$; Han,
新

Hsin.

Yang-ch'ü 陽曲; $E$. $H a n, H \sin -c h$ ' $\ddot{~}$ 新曲; $T \sin$, Chiu-yüan 九原 H.; 2nd Wei, Ssŭ 肆 C.; Sui, Hsiu-jung.秀容 $\mathrm{H}$.; $T^{\prime}$ ang, Hsin $\mid \mathrm{C}$.

2722 Hsin-ch'êng | 城 Hinching.

$1^{\circ} \mathrm{H}$. in $\mathrm{Ch}$ ing-yüan $\mathrm{F} u$, Kuangsi ; Lat. $24^{\circ}$, Long. $108^{\circ} 25^{\prime} ; T^{\prime} a n g$, Hsin-ch'êng | 城.

$2^{\circ}$ T. H. near the preceding; $T^{6}$ ang, Chih 艺 C.

\section{新}

$2723 \mathrm{Hsin}$ 新.

$1^{\circ}$ Chên near Hua H., Honan.

$2^{\circ}$ Y. near Chin H., Kansuh.

$3^{\circ}$ (obs.) in Nan-cliêng H., Shensi ; S. Sung, H. in 'T'ien-shni 天水 chïn.

$4^{\circ}$ (obs.) 80 li N.E. of Yen-ch'ang H., Shensi;

Sung, military post in Yen-an 延安 Fu.

$5^{\circ}$ (obs.) near Ch' ing-chou $\mathrm{Fu}$, Shantung; Kin, chên in Yi-tu商都 Fu. $6^{\circ}$ (obs.) in Lin-yi H.; Shantung; Kin, chên in Chi-nan Fu.

$7^{\circ}$ (obs.) Tsin, chün comprising part of Hupeh. 
$8^{\circ}$ See Nos. 1142, 2761, 4947, 6795.

2724 Hsin-an | 努 Sin-11gan.

$1^{\circ}$ (obs.) H. in Pao-ting Fu, Chihli; I,at. $38^{\circ}$ $56^{\prime}$, Long. $116^{\circ} 02^{\prime}$; Han, Jung-ch'êng 容 城; Kin, An 安 C.; Yüan, Hsin-an 安 $\mathrm{H}$. $2^{\circ} \mathrm{H}$. in Ho-nan $\mathrm{F} u$, Honan; Lat. $34^{\circ} 45^{\prime}$, Long. $112^{\circ} 06^{\prime}$; S..4., Hsi-chon 西周; $T s^{i} i n$, Hsin-an | 穻; P. C hou, 'Tung-yuian 東垣.

$3^{\circ} \mathrm{H}$. in Knang-clion Fin, Kuangtung; Lat. $22^{\circ}$ $36^{\prime}$, Long. $114^{\circ} 04^{\prime}$.

$4^{\circ}$ (obs.) E. of Mien-ch 'ilı H., Honan; Han and $T \sin , \mathrm{H} \operatorname{sin-an}$ |发 $\mathrm{H}$.; N. Wei, Hsin-an 发 chïn and H., in Lo 洛 chov.

$5^{\circ}$ (obs.) W. of Slin11-an H., Cliellkiang; $T \sin$, S. Sung and S. Ts'i, chïn in Yany 揚 chou. $6^{\circ}$ (obs.) in Hupelı; $S$.

Sung, H. in Ho-nan 湖南 chül。

$7^{\circ}$ (obs.) near Yüun-vangr Fin, Hupelı; S. Sung and $S . T s^{\prime} i, H$. in Shang-yung 上唐 chün. $8^{\circ}$ (obs.) in Ssŭch '11an; S. Sung, H. in Hsin-pa 巴 chïn. $9^{\circ}$ (obs.) in Yünnan; S $S$. Sung, H. in Chien-tu 建都 chion; S. $T s^{\prime} i$, H. in Chien-ning 建 家 chiin.

$10^{\circ}$ (obs.) in $\mathrm{Ho-p} \mathbf{1}^{\prime} \mathrm{H}$., Knuangtung ; S. Sung, and s. $T s i$, H. in Ho-p" "n 合浦 chïn. $11^{\circ}$ (obs.) near Hsiangyang Fir, Hupel1; S. $T s^{\prime} i$, H. in Ts'ai-yang 蔡陽 chül。

$12^{\circ}$ (obs.) N.W. of Nancliang H., Hupeln; S. Ts' $i, \mathrm{H}$. in An-ting 安 定 chiü.

$13^{\circ}$ (obs.) W. of Tai C.,

Shansi ; N. Wei, chï̈ in $\mathrm{Wn}$ 武 chov.

$14^{\circ}$ (obs.) in Yri-cli êng

H., Shansi ; N. $W_{c i}$,

H. in Pei-chiang 北綘 chün.

$15^{\circ}$ (cbs.) near 'Ts'ao H.,

Sllantung; N. Wei, H. in P'ei 浦 chiun.

$16^{\circ}$ (obs.) E. of Hsin-an

H., Honan ; Suli, H. in

Honan 河南 chïn.

$17^{\circ}$ (obs.) in Annan1;

T*ang, C. in Ling-nan 領南 circ.

$18^{\circ}$ (obs.) near Ssŭ-nan

$I^{*} 11$, Kneichon; $T^{*} a n g$,

H. in Chuang 莊 C.

$19^{\circ}$ (obs.) in Shingking; Liao, H. in Ning 耍 C. 
$20^{\circ}$ (obs.) near Kuangp'ing Fu, Chihli ; Kin, H. in Lo 洛 C.

$21^{\circ}$ (obs.) 20 li $\mathrm{W}$. of Fei-hsiang H., Chihli; Kin, chên in Lo 洛 C. $22^{\circ}$ (obs.) N. of Yüi-ch êng H., Shantung; Kin, chên in Chi-nan 溜 南 Fu.

$23^{\circ}$ Chên near Fei-hsiang H., Chinli.

$24^{\circ}$ Chên near Hai C.,

Kiangsu.

$25^{\circ}$ See Nos. 256, 267, $574,2858,4561$.

2725 Hsin-an-pien 发邊, Y. near Ting-pien H., Shensi.

2726 Hsin-chai | 憲, chên near Fei-hsiang H., Chihli.

2727 Hsin-ch'ang 昌 Sinchang.

$1^{\circ} \mathrm{H}$. in Shao-hsing $\mathrm{Fu}$, Chehkiang; Lat. $29^{\circ}$ $32^{\prime}$, L ong. $12^{\circ} 50^{\prime}$; Han, E. part of Shan 剡; Liang, Hsin-ch'ang 昌.

[13] $2^{\circ} \mathrm{H}$. in Jui-chou $\mathrm{Fu}$, Kiangsi ; Lat. $28^{\circ} 18^{\prime}$, I tong. $114^{\circ} 38^{\prime}$; $\operatorname{Han}$, Chien-ch'êng 建 城; $W u$, Yi-fêng 宜 豐; Sui, Hsin-ch ang | 昌. $3^{\circ} \mathrm{H}$. in Chung-ch ing 忠清 circ., Corea.

$4^{\circ}$ See Nos. 1793, 2730, 5843.
2728 Hsin-chao | 招, (obs.) Sung, S. of Kulang-ning H., Kuangtung.

2729 Hsin-chêng | 觙 Sin[405] ching, H. in $\mathrm{K}^{\prime}$ ai-fêng $\mathrm{Fu}$, Honan; Lat. $34^{\circ} 26^{\prime}$, Long. $113^{\circ} 56^{\prime}$.

2730 Hsin-ch'êng | 城 Sinching.

[341] $1^{\circ} \mathrm{H}$. in Pao-ting $\mathrm{Fu}$, Chihli ; Lat. $39^{\circ} 21^{\prime}$, Long. $115^{\circ} 54^{\prime}$; Han, Hsin-ch'ang 昌; $T^{\prime}$ ang, Hsin-ch' êng | 城.

[186] $2^{\circ}$ H. in Chi-nan Fin, Shantung; Lat. $37^{\circ}$ $02^{\prime}$, Long. $118^{\circ} 08^{\prime}$; orig., Ma-t'ai 馬臺 in K. Chi 裂；Yüan, Hsin-ch'êng | 城.

$3^{\circ} \mathrm{H}$. in Hang-chou $\mathrm{Fu}$, Chehkiang; Lat. $30^{\circ}$ $05^{\prime}$, Long. $119^{\circ} 43^{\prime}$.

[52.] $4^{\circ} \mathrm{H}$. in Chien-ch' ang Fu, Kiangsi ; Lat. $27^{\circ}$ $13^{\prime}$, Long. $116^{\circ} 54^{\prime}$ : orig., Nan-ch'êng 南 城; Sung, Hsin-ch' êng 城。

$5^{\circ} \mathrm{F} 11$ in Chi-lin circ., Kirin, Lat. $45^{\circ} 10^{\prime}$, Long. $125^{\circ} 40^{\prime}$; formerly Po-tu-no 伯都訥 Pedhe, C.L.T. See No. 5236. 
$6^{\circ}$ Chên near Yüan-wu H., Honan.

$7^{\circ}$ Chên near Ch'ienchiang H., Hupeh.

$8^{\circ}$ Chên near Ch'ing-t'ien $\mathrm{H}$., Chehkiang.

$9^{\circ}$ Ssŭ near Lei-yang H., Hunan.

$10^{\circ}$ Post-town near Hsing-

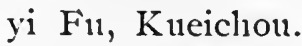

$11^{\circ}$ (obs.) Chên, town E. of Hêng-chou Fin, Hunan.

$12^{\circ}$ See Nos. 1661, 7495. $2730^{\mathrm{A}}$ Hsin-chi | 汲, see No. 6983.

2731 Hsi11-chi|集, chên near Ch'ing H., Cliihli.

2732 Hsin-ch'i | 溪 $\mathrm{H}$. in Huang-hai 黃海 circ., Corea.

2733 Hsin-ch 'i-k'ou 雞问, ss near Han-yang Fu, Hupeh.

2734 Hsin-ch' $i \mid$ 郪.

$1^{\circ}$ (obs.) 70 li N. of T'ai-ho H., Anhui ; $W$. Han, H. in Ju-nan 汝 南 chïn.

$2^{\circ}$ See No. 5551.

2735 Hsin-chiang | 港, ssü in Ching-chiang H., Kiangsu.

2736 Hsin-cliiang | 疆 New Dominion (formerly called Outer Kansuh 外甘肅)

Province. Capital Ti-hua Fu; contains 4 circuits, 3 Fu, 7 C.L.'T., 4 C.L.C., $2 \mathrm{~T}$. and $14 \mathrm{H}$.
2737 Hsin-chieh | 街.

$1^{\circ}$ Chên near Lin-t ung H., Shensi.

$2^{\circ}$ Chên near Pao-chi H., Shensi.

$3^{\circ}$ See No. $6774^{\mathrm{A}}$.

2738 Hsin-chien | 建 Sin-kien. $1^{\circ} \mathrm{H}$. forming with Nanch'ang $H$. the prefectural city of Nan-cl' 'ang, Kiangsi ; Lat. $28^{\circ} 33^{\prime}$, Long. $116^{\circ} 01^{\prime}$; Tsin, Yi-fêng 宜. 豐; $C h^{6} e ̂ n$, $\mathrm{Hsi}-\mathrm{ch}$ any 西昌; Sung, Hsin-chien | 建. $2^{\circ}$ See No. 1591.

2739 Hsin-ch'in | 奏.

$1^{\circ}$ (obs.) 40 li N. of Shên-1nu H., Shensi; $T^{\prime}$ ang and Sung, H. in Lin 鹿粦 C.

$2^{\circ}$ See No. 5610 .

2740 Hsin-ching | 津 Sin-tsin, [307] H. in Ch êng-tu Fu, Ssŭch'nan; Lat. $30^{\circ} 25^{\prime}$, Long. $103^{\circ} 50^{\prime}$; Han, Wu-yang 武 陽; S'su, Hsin-ching | 津 $\mathrm{H}$.

2741 Hsin-ching | 井, (obs.) $T \sin , \mathrm{H} .$, N.E. of Nan-pu H., Ssŭch uan.

2742 Hsin-cling | 經, ssü in $\mathrm{Cl}^{\prime}$ 'ing-p' $\mathrm{u} \mathrm{H}$., Kiangsi1.

2743 Hsin-chon-ch'êng | 州城, (obs.) formerly town N.W. of Hui-chou Fu, Anhui.

2744.Hsin-chu | 住, chên near Lin-t'ung H., Shensi. 
新

2745 Hsin-chu $/$ 竹 Sin-cluul, H. in T'ai-pei Fu, Fulnkien, recently founded in N. Formosa. See No. 1380 .

2746 Hsin-chï|埧, chên near Hai C., Kiangsu.

2747 Hsin-ch' üan | 泉, ssŭ near I.ung-yen C., Fulnkien.

2748 Hsin-chnang | 庄, shêe near Tan-shni H., Fulhkien.

2749 Hsin-fan | 繁 Sin-fan, H. in Cli êng-tı Fin, Ssŭ. ch' tran; Lat. $30^{\circ} 52^{\prime}$, Long. $104^{\circ} 06^{\prime} ;$ Han, Fan 繁 H.; T'ang, Hsin-fan 掣 $\mathrm{H}$.

2750 IIsin-fáng | 坊, chên near Yüan-chon Fu, Kiangsi.

2751 Hsin-fêng | 豐.

$1^{\circ}$ Chên near Linl-t'nug H., Shensi.

$2^{\circ}$ Chên near P'ing-lin H., Chehkiang.

$3^{\circ}$ Chên near Tan-yang H., Kiangsu.

$4^{\circ}$ Sš̆ in Nan-ch' êng H., Kiangsi.

$5^{\circ}$ (obs.) N.E. of Lint'ung H., Shensi; Han, Hsin. fêng | 豈 $\mathrm{H}$.

$6^{\circ}$ (obs.) in Chiang-ling H., Hupelı; S. Sung and $S . T s^{\prime} i, H$. in Hsin-hsing | 與 chün.
新 HSin-Fu,

$7^{\circ}$ (obs.) near Hsiangyang Fu, Hupelı; $S$. Sung and $S$. Ts'i, H. in Cling-chao 京兆 chimn.

$8^{\circ}$ (obs.) in Yüunnan; $S$. Sung and S. Ts'i, H. in Liang-slnni 梁 水 chïln.

$9^{\circ}$ (obs.) S.E. of Ch'angning $\mathrm{H} .$, Knangtung; S. Ts' $i, H$. in Nan-hai 南海 chïn.

$10^{\circ}$ (obs.) S.E. of Clit1ch'i H., Hupeh; $S$. $T s^{6} i, H$. in Shang-yung 上庸 chïn。

$11^{\circ}$ (cbs.) near Ta-li Fu, Yünnan; S. Ts'i, H. in Hsi-a 西阿 chïn.

$12^{\circ}$ (obs.) in Anluii; $N$. Wei, H. in An-ting 安 定 chün.

$13^{\circ}$ (obs.) 70 li N. of Su C., Auluni ; N. Wei, H. in Sui-nan 脽南 chïn.

$14^{\circ}$ (obs.) near Ch' ï-ching Fu, Yünnan; $T^{\prime}$ ang, H. in Nan-ning 青䡐 C.

$15^{\circ}$ See Nos. 3894, 6988. 2752 Hsin-fêng | 封, (obs.) $S$. Sung, C. in Kuang-tung 鹰東 circ.

$2753 \mathrm{Hsin}-\mathrm{fu}$ | 挴, ss ̌̆ near $P^{\prime}$ u-êrh Fu, Yünnan. 
新

Hsin-HO.

2754 Hsin-ho | 河 Sin-ho.

[325] $1^{\circ}$ H. in Chi C. L. C., Chillli ; Lat. $37^{\circ} 36^{\prime}$, Long. $115^{\circ} 16^{\prime}$; Han, 'T'ang-yang 堂晹.

$2^{\circ}$ Post-town near Shan$\tan \mathrm{H}$., Kansuh.

2755 Hsin-hua | 化 Sin-hwa. $1^{\circ} \mathrm{H}$. in Pao-ch 'ing Fu,

Hunan; I at. $27^{\circ} 32^{\prime}$, Long. $111^{\circ} 10^{\prime}$; Han, Yi-yang 感陽; Sung， Hsin-hua | 代.

$2^{\circ}$ T-S. near Hsia-chiang T., Kneichou.

$3^{\circ}$ See No. 6137.

2756 Hsin-huai | 懹, (obs.) in Ch'üel1-shan H., Honan; $N$. Wei, H. in Yü 豫 C.

2757 Hsin-hui | 會 Sin-hwui. $1^{\circ} \mathrm{H}$. in Kuang-chon $\mathrm{F} u$, Kuangtung; Lat. $22^{\circ}$ $30^{\prime}$, Long. $113^{\circ} ; T$ sin, Hsin-1uii 會; $T^{\prime}$ ang, Kang 閑 C.

$2^{\circ}$ See No. 2774.

2758 Hsin-hsi | 熙, (obs.) near Kuang-chon Fu, Kuangtung; $S$ Sung and $S$. $T s^{i} i, H$. in Hsin-hui | 會 chïn.

2759 Hsin-hsiang | 拫 Sin[496] hiang, H. in Wei-hui Fu, Honan; Lat. $35^{\circ} 22^{\prime}$, Long. $114^{\circ} 04^{\prime}$; Han, Honei 河內; Sui, Hsinhsiang | 拫.
新 HSIN-K'AI-CH' $\hat{\text { F̂NG. }}$

2760 Hsin-hisieh | 瀉 Nii-gata, Prefecture and chief town in Japan. It is an open port.

2761 Hsin-hsing | 興 Sin-ling. $1^{\circ} \mathrm{C}$. in $\mathrm{Cl}^{\prime}$ êng-chiang Fut, Yünnan; Lat. $24^{\circ}$ $30^{\prime}$, Long. $102^{\circ} 40^{\prime}$; orig., in $\mathrm{K}$. 'Tien 滇; Liang, Hsi-ts nan 近 薮; Yïan, Hsin-hsing 舆直.

$2^{\circ} \mathrm{H}$. in Chao-ch ing Fu, Kuangtung ; Lat. $22^{\circ}$ 52', I,ong. $112^{\circ}$; Han, Lin1-yün 臨尤; Tsin, Hsin-hsing | 與; Liang, Trang, Sung and Yüan, Hsin $\mid \mathrm{C}$.

$3^{\circ}$ Chên near Shon-yang H., Shansi.

$4^{\circ}$ Chên near P'ing-hı H., Chehkiang.

$5^{\circ}$ Post-town near Chiench'ang H., Kiangsi.

$6^{\circ}$ Post-town near $P^{\prime}$ in-an T., Kneichon.

$7^{\circ}$ See Nos. 4181, 5843, 6890.

2762 Hsin-hsü | 虛, Y. near Sui-ch'i H., Kuangtung. 2763 Hsin-jung | 容, (obs.) in Annan; Sui, H. in Haiyin 海陰 chün.

2764 Hsin-k'ai-cl'êng | 閶城, ss̆̆ in Huang-wei $\mathrm{H}$, Hupeh. 
新

HSIN-KAN.

2765 Hsin-kan | 涂 Sin-kan, [208] H. in Jin-chiang $\mathrm{Fu}$, Kiangsi ; Lat. $27^{\circ} 42^{\prime}$, Long. $115^{\circ} 24$; Ts'in, Hsin-kan | 洤; $W_{u} u$, Pach'in 巴邱.

2766 Hsin-k'ang | 沗, see No. 4689.

2767 Hsin-kuan | 管, post-town near Wn-ch'ang H. Hupeh.

2768 Hsin-lê | 樂 Sin-lol, H. [379] in Clêng-ting Fu, Chilıli; Iat. $38^{\circ} 25^{\prime}$, Long. $114^{\circ}$ $56^{\prime} ;$ Han, Hsin-shih | 市; Sui, Hsin-lê | 樂.

$2769 \mathrm{Hsin-1i} \mid$ 黎.

$1^{\circ}$ (obs.) in Elenth conntry, W: end of Inner Mongolia; T ang, C. in Kuan-nei 關內 circ.

$2^{\circ}$ (obs.) N.W. of Cho C., Chill1i ; T'ang, $\mathrm{H}$. in $\mathrm{I}, \mathrm{i}$ 黎 $\mathrm{C}$.

$2770 \mathrm{H}$ sin-1o | 羅.

$1^{\circ}$ (obs.) T'ang, K. in S.W. of Corea.

$2^{\circ}$ (obs.) Tsin, H., S.W. of T'ing-chon Fu, Fuhkien.

$3^{\circ}$ See Nos. 276, 4217, 6470.

2771 Hsin-11111 | 民 Sin-1nin, 'T. in Fêng-t ien Fu, Shingking; Lat. $41^{\circ}$ $52^{\prime}$, Long. $121^{\circ} 55^{\prime}$. 'Terr. jur.
新

HSIN-P'ING.

2772 Hsin-11an | 難, ss̆ Han-yang $\mathrm{H}$. , Hupeh.

2773 Hsin-nien|年 $K i n$, chên in Chêng-ting Fu, Chihlli.

2774 Hsin-ning | 寝 Sin-ning. [198] $1^{\circ}$ C. in Nan1-ning Fu, Knangsi ; Lat. $22^{\circ} 35^{\prime}$, I.ong. $107^{\circ} 35^{\prime}$; Sung, $\mathrm{W}_{11-1 i}$ 武黎 H. in Yung 邑 C.

[131 $\left.{ }^{\mathrm{A}}\right] 2^{\circ}$ H. in Pao-ch'ing Fu, Hunan ; Iat. $26^{\circ} 25^{\prime}$, I Iong. $111^{\circ} 44^{\prime}$; Han, feudal state Fu-yi 夫 费; Sung, Hsin-ning 门察.

[382] $3^{\circ} \mathrm{H}$. in Sui-ting $\mathrm{Fun}$, Ssurch 'uan; Lat. $31^{\circ}$ $12^{\prime}$, Long. $107^{\circ} 55^{\prime}$; Han, Tang-ch 'i '宕渠; $W . W e i$, Hsin-ning | 富。

[280] $4^{\circ}$ H. in Kuang-chon Fu, Knangtung; Lat. $22^{\circ}$ $14^{\prime}$, Long, $112^{\circ} 34^{\prime}$; Sui, Hsin-hni | 會.

$5^{\circ} \mathrm{H}$. in $\mathrm{Cl}^{\prime}$ ing-shang 慶 少 circ, Corea.

$2774^{A}$ Hsin-pin | 筫, (obs.) V. of Hsing-wên H., Ssŭch $^{6}$ nan1 ; $T^{6}$ ang, H. in Yen 晏 $\mathrm{C}$.

2775 Hsin-ping | 本 Sin-ping. [363] $1^{\circ} \mathrm{H}$. in Li-chiang $\mathrm{Fu}$, Yünnan; Lat. $24^{\circ} 12^{\prime}$, Long. $102^{\circ} 08^{\prime}$. 
新 HSIN-P'ING.

$2^{\circ}$ Sš̌ in Ch'ang-ning. H., Kiangsi.

$3^{\circ}$ Post-town near Pin C., Shensi.

$4^{\circ} \mathrm{H}$. in Yen-Ch ${ }^{\circ} \mathrm{F}_{11}$,

New Dominion.

$5^{\circ}$ See Nos. 1237, 4828.

2776 Hsin-shih | 市.

$1^{\circ}$ Chên near Ning-yüan

H., Kansul.

$2^{\circ}$ Ss ̌̆ near Hsiang-yin H., Hunan.

$3^{\circ}$ Ssŭ near Tê-ch'ing H., Chehkiang.

$4^{\circ}$ See No. 2768.

2777 Hsin-ssŭ| 寺, chên near Ning-yüan H., Kanstuh.

2778 Hsin-tai | 帶, chên near P'ing-hu H., Chehkiang.

2779 Hsin-t'ai | 泰 Sin-tai.

[502] $1^{\circ}$ H. in T'ai-an Fu, Shantung; Lat. $36^{\circ} 07^{\prime}$, Long. $117^{\circ} 56^{\prime}$; Han, 'Tung-P'ing-yany 東压 嚚; $T \sin , \mathrm{H} \sin -t^{\prime} \mathrm{ai} \mid$ 橈.

$2^{\circ}$ See No. 7482 .

2780 Hsin-ti | 堤, chên near Chien-li H., Hupeh. Port of call.

2781 Hsin-tien | 店.

$1^{\circ}$ Chên near Hua-t'ing H., Kansuh.

$2^{\circ}$ Post-town near $T^{6}$ aoyüan H., Hunan.

2782 Hsin-tien-tzŭ 店子, post-town near $\mathrm{Yi} C$., Shingking.

\section{新 HSIN-TU.}

2783 Hsin-tien | 䍒, T-S. near Kuei-ting H., Kueichou.

2784 Hsin-t'ien | 田 Sin-tien. $1^{\circ} \mathrm{H}$. in Yung-chon $\mathrm{F} 11$, Hunan ; Lat. $25^{\circ} 45^{\prime}$, Long. $112^{\circ} 01^{\prime}$.

$2^{\circ}$ Ssŭ near An-yüan H., Kiangsi.

$3^{\circ}$ See No. 769.

2785 Hsint-ting | 䇥, see Nos. $5751,5958$.

2786 Hsin-ts'ai | 蔡 Sin-tsai. [201] $1^{\circ}$ H. in J11-ning $F_{11}$, Honan; Lat. $32^{\circ} 46^{\prime}$, I.ong. $114^{\circ} 58^{\prime}$; Han, Hsin-ts'ai 蔡; $E$. $W_{e i,}$ 'Ts'ai 蔡 C.; Sui, Shu 卸 C.

$2^{\circ}$ (obs.) Tsin, H. near Chin-chiang Fu, Kiangsi.

$3^{\circ}$ See No. 2297.

2787 Hsin-tsao | 造, Y. near Chiang-hna H., Hnnan.

2788 Hsin-ts'un | 村, ssü near 'Tê-ch'ing C., Knangtıng.

2789 Hsin-tu | 都 Sin-tu.

$1^{\circ}$ H. in Ch êng-tı Fu, Ssŭch' 'nan; Lat. $30^{\circ}$ $50^{\prime}$, Long. $104^{\circ} 15^{\prime}$; Han, Hsin-tu | 都? H.; Liang, Shilh-k'ang 始 㞗; Swi, Hsing-lê罾樂. $2^{\circ}$ (obs.) E. of Hsin-yeh H., Honan; $W$. Han, Marq. in Nan-yang 南 陽 chïn. 
新 Hsin-TU.

$3^{\circ}$ See Nos. 1046, 194?, 2358, 2850, 4708, 5830, 6795, 7394.

2790 Hsin-t11 | 瀆, chên near K'un-shan H., Kiangsu.

2791 Hsin-w11 吳.

$1^{\circ}$ (obs.) W. of Fênng-11sin H., Kiangsi ; $T \sin , S$. Sung and $S . T s^{\prime} i, \mathrm{H}$. in Y'ï-chang 豫 章 chïn.

$2^{\circ}$ See No. 1769.

2792 Hsin-yang | 陽 Sin-yang.

$1^{\circ} \mathrm{H}$. in Su-clion Fu, Kiangsu; Lat. $31^{\circ} 2 S^{\prime}$, Long. $120^{\circ} 48^{\prime}$; the same city serves also for $\mathrm{K}$ ' 111 -shan $\mathrm{H}$.

$2^{\circ}$ See Nos. 1125, 4689.

2793 Hsin-yeh | 野 Sin-yé, H. [338] in Nan-yang Fu, Honan; Lat. $32^{\circ} 40^{\prime}$, Long. $112^{\circ}$ $05^{\prime} ;$ Han, Hsin-yeh | 野; $T \sin , Y$ Y-yang 義陽.

2794 Hsin-yeh | 治, see No. 6941.

2795 Hsin-yen | 堰, post-town near An-hsiang H., Hunan.

2796 Hsin-yi | 夷, (obs.) Wu and $T$ sin, H., $40 l i \mathrm{~W}$. of Hsin-hui H., Kuangtung.

2797 Hsin-yii | 喻 (also written 諭) Sin-yu, H. in Linchiang Fu, Kiangsi; Lat. $27^{\circ} 50^{\prime}$, Long. $114^{\circ} 52^{\prime}$; Han, Yi-ch un 宜春;

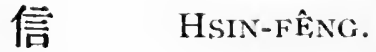

Tsin, S. Sung, Sui and $T^{\prime}$ ang, Hsin-yü | 喻 $\mathrm{H}$.; Yïan, Hsin-yü|噏 C..; Ming, Hsin-yii | 咏 $\mathrm{H}$.

2798 Hsin-ÿ̈ | 諭, see preceding.

\section{辛}

2799 Hsin-chih 辛置, chên near Chao-ch'êng H., Shansi.

\section{信}

$2800 \mathrm{H} \sin$ 信.

$1^{\circ}$ (obs) S. Sung, C. in Chiang-tung 江東 circ.

2 See Nos. 3400, 3491.

2801 Hsin-an | 努.

$1^{\circ}$ Chên near $\mathrm{Pa}$ C., Chibli.

$2^{\circ}$ (obs.) Sung, H., E. of $\mathrm{Pa} \mathrm{C}$., Chillil.

$3^{\circ}$ See Nos. 38, 124, 335.

2802 Hsin-chao | 叉, chên near Fu1-p ing H., Shensi.

2803 Hsin-ch êng | 城, see No. 1245.

2804 Hsin-ch'wan | J, chün in Huang-hai 黃 海 circ.. Corea.

2805 Hsin-fêng | 䇺 Sin-fung, [452] $\mathrm{H}$. in Kan-chon $\mathrm{Fu}$, Kiangsi; Lat. $25^{\circ} 25^{\prime}$, Long. $114^{\circ} 24^{\prime} ; E$. Han Nanan 南努; $T \sin$, Nank'ang 南康; $T^{\prime}$ an. 5 Hsin-fêng | 豈. 
信 HSIN-t,ING-CH t̂A.

2806 Hsin-ling-cl'êng | 陵城, (obs.) $W_{u}$ and $T \sin$, town W. of Pa-tung H., Hupeh. 2807 Hsin-ln11 | 倫, 'I-C., near Yang-li C., Kuangsi.

2808 Hsin-tê | 德, see No. 5821. 2809 Hsin-tı | 都.

$1^{\circ}$ Ss̆̌ in Ho H., Kuangsi.

$2^{\circ}$ See Nos. 576, 4955, 5821.

2810 Hsin-yang | 陽 Sin-yang. [386] $1^{\circ}$ C. in Ju-ning $F t 1$, Honan; Isat. $32^{\circ} 12^{\prime}$, Long. $114^{\circ}$; Han, P'ingshih 平氏; $W_{c i}, Y_{i-}$ yang 義晹; 2nd $\mathrm{Wci}$, Ying 郢 C.; P. Chou, Shên 申 C.; Sung, Hsin-yang | 陽.

$2^{\circ}$ (obs.) S. Sung, military district in Hu-pei 湖北 circ.

2811 Hsi11-yi | 義, see No. 2812.

$2812 H \operatorname{sin-y}$ | 宜 Sin-i。

[468] $1^{\circ}$ H. in Kao-chon Fu, Knangtung ; Lat. $22^{\circ}$ $06^{\prime}$, Long. $111^{\circ} 54^{\prime}$; Han, in 'T's'ang-wu 蒼 梧 chïn; T'ang, Hsinyi 義; Sung, Hsinyi 宜.

$2 \circ$ (obs.) 2 li S. of preceding; Sung, Yiuan and Ming, Hsin-yi 宜 $\mathrm{H}$.
想

Hinga-chi.

\section{HSING}

\section{县}

2813 Hsing 舆 Hing.

[555] $1^{\circ} \mathrm{H}$. in 'T'ai-ÿïan F’n, Shansi ; Lat. $38^{\circ} 38^{\prime}$, Long. $111^{\circ} 02$; Han, Fên-yang 汾陽; P. Chon, Yüi-fên 尉汾; Sui, I,in-ch1 iian 臨泉; T'ang, Lin-ching 臨 津 and Ho-ho 合河; Kin, Hsingr |C.

$2^{\circ}$ See Nos. 1521, $4+42$.

2814 Hsing-an | 安 Hing-11gan. [133] $1^{\circ}$ Fu in the Shan-an circ., Shensi; forming also the district city of An-k'ang; Lat. $32^{\circ} 31^{\prime}$, Long. $109^{\circ} 22^{\prime}$; Han, Hsi-ch'êng 西城; $W$. $W_{c i}$, Chin 金 C.; T'ang, An-k'ang 安 康.

[211] $2^{\circ} \mathrm{H}$. in Kuang-hısin Fun, Kiangsi ; Lat. $28^{\circ} 25^{\prime}$, Long. $117^{\circ} 43^{\prime}$.

[177] $3^{\circ} \mathrm{H}$. in Kuei-lin Fu, Kuangsi ; Lat. $25^{\circ} 32^{\prime}$, L.ong. $110^{\circ} 36^{\prime}$; Han, Shih-an 始安; T’ang, I,i11-yüan 臨源; Sung, Hsing-an | 安.

$4^{\circ}$ See Nos. 2821, 3429.

2815 Hsing-chi | 溛.

$1^{\circ}$ Chên S.E. of Ch'ing H., Chihli. 
與

Hsing-Cht.

$2^{\circ}$ (obs.) Sung, H., N. of Ts'ang C., Chihli.

2816 Hsing-ching | 京, C.L.T. in 'Tung-pien circ., Shingking; site of the Imperial Tombs.

2817 Hsing-Ch' ïan-Yung | 興 永, circ. in Fuhkien, comprising Hsing-hua Fu, $\mathrm{Ch}^{\prime}$ ïan-chou $\mathrm{Fu}$ and Yungch' $111 \mathrm{C}$.

2818 Hsing-chung | 中, (obs.) in the Tumed country (the N. of Shansi); Liao and $K i n, F u$ and $\mathrm{H}$.; Yïan, C. in Ta-ning 太 察 circ.

2819 Hsing-hai | 海 H. in Ch ing-shang 麀份 circ., Corea.

2820 Hsing-hua | 化 Hing-hwa. $1^{\circ} \mathrm{Fu}$ in the HsingCh 'ïan-Yung circ., Fuhkien; forming also the district city of $\mathrm{P}^{\prime} \mathrm{n} 1 \mathrm{-t}$ ' ien ; Lat. $25^{\circ} 25^{\prime}$, Long. $119^{\circ} 17^{\prime}$; Chou, Chimin 七閩; Han, $\mathrm{P}^{6}{ }_{11}$ chung 俈 中; Sung, Hsing-hua 化 and Hsing-an | 娄; Ming, Hsing-hua Fu.

$2^{\circ}$ H. in Yang-cliou Fu, Kiangsu; Lat. $32^{\circ} 51^{\prime}$, Long. $119^{\circ} 48^{\prime} ; T^{\prime}$ ang, Hai-ling 海: 陵; Five dyn., Hsing-hua |. 化.
興

HSING-LUNG.

$3^{\circ}$ Ss ̌̆ near Yung-ting H., Fuhkien.

$4^{\circ}$ (obs.) S. Sung, military district in Fu-chien 福建 circ.

2821 Hsing-jên | 仁.

$1^{\circ}$ (obs.) N. W. of Ts'ao

H., Shantung; Sung,

$F_{11}$ in Ching-tung 京

東 Western circ.

$2^{\circ}$ (obs.) N. of Jehol ;

Liao, Hsing-jên | 仁 $\mathrm{H}$.

$3^{\circ}$ Ss̆̌ in Yü-tı H., Kiangsi,

$4^{\circ}$ See No. 6541.

2822 Hsing-ku | 古, see Nos. 1452, 5291.

2823 Hsing-kuo | 國 Hingkwoh.

[532] $1^{\circ}$ C. in Wu-ch'ang $\mathrm{Fu}$, Hupeh ; Lat. $29^{\circ} 46^{\prime}$, Long. $115^{\circ} 11^{\prime} ; T s^{\prime}$ in, in Nan 南 chün; Sui, $\mathrm{Fu}$ 富 C.; Sung, Hsing-kno | 國.

$2^{\circ} \mathrm{H}$. in Kan-chon $\mathrm{Fu}$, Kiangsi ; Lat. $26^{\circ} 22^{\prime}$, Long. $115^{\circ} 12^{\prime} ; S$. Sung, military district in Chiang-hsi 江西 circ.

2824 Hsing-lê | 樂, see No. 2789.

2825 Hsing-lung | 隆.

$1^{\circ}$ Chên ncar Ch'ienchiang H., Hupeh. 
興

HSING-LUNG .

$2^{\circ}$ T-S. near Ssŭ-ên Fin, Kuangsi.

$3^{\circ}$ See No. 2304.

2826 Hsing-ning | 安 Hingning.

[17] $1^{\circ}$ H. in Ch'ên C., Hunan ;

Lat. $25^{\circ} 55^{\prime}$, Long. $111^{\circ} 59^{\prime}:$ Han, Hanning 漢富; Sui, Chinlising 習 |; Sung, Hsing-11ing | 富.

$2^{\circ} \mathrm{H}$. in Clija-ying C., Kuangtung; Lat. $24^{\circ}$ $12^{\prime}$, Long. $115^{\circ} 48^{\prime}$; Han, Lrung-cl' nan 龍 川 ; E. Tsin, Hsing11ing | 寉.

2827 Hsing-pa | 巴, T-S. in Kokonor.

2828 Hsing-p'ing | 本 Hingping.

[498] $1^{\circ}$ H. in Hsi-an Fu, Shensi; Lat. $34^{\circ} 18^{\prime}$, Long. $108^{\circ} 25^{\prime}$; Chou, 'T'ai-ch'iu 太邱; $T s^{\prime} i n$, Fei-cl' ${ }^{\prime} i 11$ 廢印; $H_{a n}$, Huai-li 槐里, Monling 茂陵 and P'ingling 尔陵; $T_{s i n}$, Shil - p'ing 石 尔; T'ang, Chin-ch'êng 金 城 and Hsing-p'ing | 平.

2\% (obs.) 1'ang, H. near Huai-chi H., Kuangsi. $3^{\circ}$ (obs.) $W u, \mathrm{H} .$, W. of Lê-an H., Kiangsi.
典青 HSING-Y I.

2829 Hsing-shan | 山 Hing[39] slian, H. in Vi-cll 'ang Fin, Hupeh1; Lat. $31^{\circ} 11^{\prime}$, I.ong. $110^{\circ} 46^{\prime}$.

2830 Hsing-shêng $\mid$ 聖, (obs.) T"ang, H. near Ch'angning H., Ssŭch' '11an.

2S31 Hsing-shil | 市, chên near $P^{\prime}$ u-ch' êng H., Sliensi.

2832 Hsing-tao | 道.

$1^{\circ}$ (obs.) Tsin, H., S. E. of Hêng C., Kuangsi.

$2^{\circ}$ (obs.) Tsin, H. near Han-chung Fin, Shensi. 2833 Hsing-tê | 德 H. in $\mathrm{Cl}^{\prime}$ 'üan-lo 全 羅 circ., Corea.

2834 Hsing-t'ien | 田, posttown near Chien-yang $\mathrm{H}$., Fulukien.

2935 Hsing-wên | 文 Hing-wăn, H. in Hsü-chou Fi1, Ssŭclı' '1an; Lat. $28^{\circ} 10^{\prime}$, Long. $105^{\circ} 02^{\prime}$; orig., Y'elh-lang 夜郎; T'ang, Yen 晏 C. 2S36 Hsing-wn | 武, post-town near Ling C., Kansull.

2837 Hsing-yang | 陽 H. in Ch'üan-lo 全羅 circ., Corea.

2838 Hsing-yel1 | 業 Hing-nielı, [176] H. in Yüt-lin Fin, Kuangsi; Iat. $22^{\circ}+5^{\prime}$, Long. $109^{\circ}$ $30^{\prime}$; Chiên, Shilh-11an 石 南; $T$ 'ang, Hsing-yeh | 紧.

2839 Hsing-yi | 義 Hing-i. 
與

HSING-YI.

[358] $1^{\circ} \mathrm{Fu}$ in the Kuei-hsi circ., Kneichon; forming also the district city of the same name; Lat. $25^{\circ} 15^{\prime}$, Long. $106^{\circ}$.

$2^{\circ} \mathrm{H}$, forming the prefectural city of the same name, Kneichon; Iat. $25^{\circ} 15^{\prime}$, I, ong. $106^{\circ}$.

$28+0$ Hsing-yïan | 元.

$1^{\circ}$ (obs.) 2 li E. of Nanchêng H., Shensi ; T'ang, Fin in Slran11an 山南 circ.

$2^{\circ}$ (obs.) S. Sung, Fu in Li-choul 利州 circ.

$3^{\circ}$ See Nos. 1950, 4567.

\section{监}

2841 Hsing-shan 隍 山, posttown near Ching-hising H., Clihlili.

2842 Hsing-yang | 陽, posttown near Wan H., Chilnli.

\section{行}

$28+3$ Hsing-t'ai 行臺.

$1^{\circ}$ (obs.) in Chêng-ting Fu, Chinli; Kin, chên in Chên-ting 真定 Fin. $2^{\circ}$ (obs.) near Cliiang C., Shansi; Kin, barrier in Chiang 綘 C.

2844 Hsing-t'ang | 垫 Hing[379] tangr, H. in Chêng-ting Fin, Chihli; 'Lat. $38^{\circ} 27$, long. $114^{\circ} 42^{\prime}$; Ts' in, Nan-hsing 南行; T⿱㇒ang,
星 Hsing-ts'un.

Hsïan 泣. C.; Five dyn., Chang-w11 彰武 and Yung-ch'ang 永昌.

2845 Hsing-ts'un-chai | 村寨, ss̆̌ near Hai-fêng H., Slantung.

\section{杏}

$2846 \mathrm{Hsing}-c c^{\prime}$ 'üan 杏泉, chên near Ch'ing-shui H., Kansull.

2847 Hsing-t'ou | 頭, chên near Yi-chün H., Shensi.

2848 Hsing-yüan | 苑 see No. 5822 .

\section{邢}

$2849 \mathrm{H}$ sing 郍, see No. 5821.

$2850 \mathrm{H}_{\text {sing-t'ai }}$ 臺 Hing-tai, $\mathrm{H}$. forming the prefectural city of Sluun-tê, Chihli ; Lat. $37^{\circ} 07^{\prime}$, Long. $114^{\circ}$ $39^{\prime} ; \mathrm{Ts}^{\prime} \mathrm{in}, \mathrm{H}$ sin-tu 新都; $W^{2}$,, K. Hsiang 襄; Sui, Lunng-kang 龍阔; Sung, Hsingr-t'ai | 臺.

\section{星.}

2851 Hsing 星 C. in Cln 'ingshimg 慶份 circ., Corea.

2852 Hsing-chu | 渚, see No. 4590.

2853 Hsing-sha | 沙, see No. 267.

2854 Hsing-ts'un | 村, posttown near $\mathrm{Cl}^{\text {' }}$ ung-an $\mathrm{H}$., Fullkien. 
星

HSING-TZÜ.

2855 Hsing-tzŭ |子 Sing-ts's. $1^{\circ} \mathrm{H}$. forming the prefectural city of Nank'ang, Kiangsi ; Lat. $29^{\circ} 23^{\prime}$, Long. $116^{\circ}$ $10^{\prime}$; Han, P'êng-tsê 彭 澤; Five dy'n., Hsingtzŭ $\mid$ 子.

$2^{\circ}$ Ss̆̌̆ near Lienl C., Kuangtung.

\section{HSIU}

\section{休}

2856 Hsilz-chi 休 吉, (obs.) Sui, H., E. of Ho-yüan H., Kuangtung.

2857 Hsiu-na | 納, (obs.) Yüan, H. near Hsin-hsing C., Yünnan.

2858 Hsilt-ning | 舞 Hill-ning. $1^{\circ} \mathrm{H}$. in Hui-chou Fu, Anluti ; Lat. $29^{\circ} 53^{\prime}$, Long. $11 \mathrm{~S}^{\circ} 17^{\prime} ; \mathrm{W} u$, Hai-yang 海陽; Sui, Hsin-11ing | 察.

$2^{\circ}$ See No. 7205 .

2859 Hsint-t $и \mid$ 屠, (obs.) 60 li N. of Wu-wei H., Kansuln; Han and $N$. Wei, Hsiu-t'u| 屠 $\mathrm{H}$.

\section{秀}

2860 Hsiu. 秀, see Nos. 699, 5970.

2861 Hsin-chou | 州, ssŭ in Lung-ch 'üan H., Kiangsi.
HSIU-JÊx.

2862 Hsin-ju11g | 蔉, see No. 6451 .

$2863 \mathrm{H}$ sin-ju11g | 溶.

$1^{\circ}$ (obs.) N. IV. of Hsin

C., Shensi; N. Hei, chïn and H., in Sisu 肆 CHOU.

$2^{\circ}$ See No. 2721.

2864 Hsiu-lao-p'o| 老婆, posttown near Yi C., Shingking.

2865 Hsin-shan | III Sin-shan. $1^{\circ} \mathrm{H}$. in Yn-yang C., Ssŭclınan; Lat. $28^{\circ}$ 28', Long. $109^{\circ} 98^{\prime}$. $2^{\circ}$ See No. 3838 .

2866 Hsin-shni | 水 Sin-shwui, $H$. forming with Chialising $\mathrm{H}$. the prefectural city of Chia-hsing, Chehkiang; Lat. $30^{\circ} 48^{\prime}$, I,ong. $120^{\circ} 43^{\prime} ; W u$, Chia-hsing 嘉興.

\section{岫}

2867 Hsin-yen 岄骹 Sin-yen, C. in Fêng-lnuang C.I.'T., Shingking; Lat. $40^{\circ}$, Long. $123^{\circ}$.

\section{編}

2868 Hsin 緖, (obs.) T $T^{6}$ ang, C. comprising part of Hsinnchou Fu, Knangsi.

\section{修}

2869 Hsin 修, see No. 1130. 2870 Hsin-jên | 仁 Sin-jin. 
修 Hsiu-jêt.

$1^{\circ}$ H. in $\mathrm{P}^{\prime}$ ing-lêe $\mathrm{Fu}$, Knangsi ; Lat. $24^{\circ} 11^{\prime}$, Long. $110^{\circ} 05^{\prime}$; Wu, Chien-ling 建 陵; Trang, Jen 晏 C. and Hsin-jên | 仁.

$2^{\circ}$ (obs.) S. of preceding; T'ang, H. in Knei 桂 C.

2871 Hsin-ling | 領, ss̆u near Kuang-ch' ang H., Kiangsi. 2872 Hsiu-tu |都, (obs.) in the Ordos country, N. of Shensi ; $W$. Han, H. in So-fang 䍭方 chïn.

2873 Hsiu-wên | 文 Siu-wăn, [24] H. in Kuei-yang Fu, Kneichou; Lat. $26^{\circ} 45^{\prime}$, Long. $106^{\circ} 30^{\prime}$; Ming, so in Fil-yu11g 敷勇 military district.

2874 Hsin-wu | 武 Sin-wu.

$1^{\circ}$ H. in Huai-ch'ing Fu, Honan; I at. $35^{\circ} 16^{\prime}$, L Hsin-wu | 武; $T s^{\prime} i n$, Nan-yang 南陽; $E$. Wei, Kuang-ning 廣 富; T゙ang, Ning-yi 耍 邑.

$2^{\circ}$ See No. 2442.

\section{脩}

2875 Hsint-yün 脩雲, (obs.) in Yünnlan; Tang, H. in Chien-ning 建富 chün.

2876 Hsiu-pei 畺北, chên in Ta-li H., Shensi.
許

Hsữ.

\section{HSIUNG}

\section{雄}

2877 Hsinng 雄 Hiung.

[341] $1^{\circ}$ H. in Pao-ting Fin, Chillil; Lat. $39^{\circ} 06^{\prime}$, I.ong. $116^{\circ}$; $\operatorname{Han}, \mathrm{Yi}$ 易 H.; Tsin, Yi-ch 'êng 易战; T゙ang，Kuei-yi 踫易； Five dyn., Hsiung | C.

$2^{\circ}$ See Nos. 4007, 4585.

2878 Hsiung-ch ${ }^{\circ}$ in / 邱, posttown near $\mathrm{Cl}^{\prime} \mathrm{i} \mathrm{H}$., Honan (No. 623).

2879 Hsiung-yung | 勇, see No. 2070.

\section{熊}

2880 Hsiung-ch'tuan 熊川, Hinng-chuen, in $\mathrm{Ch}^{\text {'ing- }}$ shang 慶尚 circ., Corea ; Lat. $34^{\circ} 43^{\prime}$, Long. $128^{\circ}$ $55^{\prime}$.

2881 Hsiun1g-êrh | 耳, see No. 7862.

2882 Hsiung-11siang | 湘, see No. 267.

2883 Hsiung-pên | 本 Kunnanoto, chief town and ken, Japan.

2884 Hsiung-yo-ch' êng | 岳城, post-town near Kai-p ing $\mathrm{H}$., Shingking.

\section{HSÜ}

許

$2885 \mathrm{Hsü}$ 望 $\mathrm{Hü}$. 
許

Hsü.

[550] $1^{\circ}$ C.I.C. in the Ho-nanK'ai - Kuei - Ch' ên - Hsï circ., Hnnan; Lat $34^{\circ}$ $06^{\prime}$, Long. $114^{\circ}$; Chou, K. Hsü $\mid$; Wei, Hsïch'ang | 昌; $N . T s^{6} i$, Nan-chliêng 南鄭; $P$. Chou, Hsü| C.; Sung, Ying-ch'ang 頴昌.

$2^{\circ}$ (obs.) Chou, K. in Honan.

2886 Hsï-ch' ang | 昌, see No. 2885.

2887 Hsï-fêng | 封, (obs.) in Yü C., Honan (No. 7698); Kin, chên in T'ang 庥 C. 2888 Hsü-liang | 底, chên near Huai-ch'ing Fu, Honan.

2889. Hsü-wan | 浿, chên near Fu-chou Fu, Kiangsi.

\section{虚}

2890 Hsü 虛, see No. 4741 .

2891 Hsü-jên | 仁, post-town near Wu-ting C... Yünnan.

\section{时}

2892 Hsü-yi 旰眙 Hü-i.

[162] $1^{\circ}$ H. in Ssŭ C., Anhui; Lat. $33^{\circ} 02^{\prime}$, Long. $118^{\circ} 20^{\prime} ; T^{\prime}$ in, Hsiiyi | 眙; $T \sin$, Lin-huai 臨淮; S. Sung and S. Ts $i$, chün in Nanyen 南兗 CHOU; Yïan and Ming, Hsü-yi | 胎 H. Unwalled city.
棦 Hsit-p' $\mathrm{Y}$.

$2^{\circ}$ (obs.) N. E. of preceding; Han, Tsin, Sui and $T^{6} \operatorname{ang} g$, Hsü-yi | 眙 $\mathrm{H}$.

\section{㟪}

2893 Hsii-ch'êng 㟪城, chên near Hai-yen H., Chehkiang.

\section{叙}

2894 Hsii 叙, see No. 2895.

2895 Hsï-chou | 州 Sü-chan, [528] Fin in the Ch' uan-nanI'ung-ning circ., Ssŭcl' "1wan; forming also the district city of Yi-pin ; I at. $28^{\circ} 47^{\prime}$, Long. $104^{\circ}$ $51^{\prime}$; orig., K. $\mathrm{P}^{6} \mathrm{o}$ 欶; Han, Chien-wei 捉 萹 chün; Liang and Sui, Jung 我 C.; T'ang, Nunch`i 南溪; Sung, Hsü| C.; Ming, Hsü-chou | 州 Fu. Sinigul or Fungul (Polo).

2896 Hsü-yung | 永 Sü-yung, [572] C. L. T. in Ssŭch 'uan; forming also the district city of Yung-ning; Lat. $28^{\circ} 08^{\prime}$, Isong. $105^{\circ} 18^{\prime}$.

\section{漵}

2897 Hsü-p' u 漵浦 Süi-pu, H. [195] in Ch 'ên-chou Fu, Hunan; Lat. $27^{\circ} 50^{\prime}$, Long. $110^{\circ}$ $20^{\prime}$; Han, Yi-ling 義陵; T ang, Hsì-p ${ }^{6}$ | 浦. 
徐 Hsi⿺.

\section{徐}

2898 Hsü 徐.

$1^{\circ}$ One of the chou or territorial divisions established by the Einperor Yii 禹; it comprised parts of Kiangsu, Anhui and Shantung.

$2^{\circ}$ (obs.) Chou, K. comprising parts of Kiangsu and Anhui.

\section{$3^{\circ}$ See No. 2902.}

2899 Hsii-ch'êng | 城, (obs.) Chou, town S. of Shih-tai $\mathrm{H}$, , Anhui; capital of K. Hsü | ; Han, Hsü-ch' êng 城 $\mathrm{H}$.

2900 Hsii-ch'i-k'o11 | 溪 口, post-town near Yungch'êng H., Honan.

2901 Hsü-chia | 家, Y. near 'T'êng H., Shantung.

2902 Hsü-chon | 州 Sü-chau, F11 in the Hsii-Hai circ., Kiangs11; forming also the district city of ' $T$ " ung-shan; Lat. $34^{\circ} 11^{\prime}$, Long. $117^{\circ}$ $32^{\prime}$; S.A., land of Sung 宋; C. Wars, K. Ch' 11 楚; $T s^{\prime}$ in, P'êng-ch'êng 彭城; Han, T ang and Sung, Hsü|C.

2903 Hsü-ch'ï | 渠, (obs.) near Ya-chou Fu, Ssŭch'uan; Trang, C. in Chien-nan 劍南 circ.
揟 HsÜ-TZU .

2904 Hsü-Hai | 海, circ. in Kiangsu comprising Hsüchon $\mathrm{Fu}$ and Hai $\mathrm{C}$.

2905 Hsï-kou | 拲 Sü-kau, H. [485] in 'T'ai-yüan Fu, Shansi; Lat. $37^{\circ} 35^{\prime}$, Long. $112^{\circ}$ $40^{\prime}$; orig., $\mathrm{Cl}^{\prime}$ ing-yüan 清源; Kin, Hsii-kou | 拲.

2906 Hsü-kung-tien | 公 店, post-town near Yi-chou Fu, Shantung.

2907 Hsü-pao|保, chên near Huai-ch'ing Fu, Honaw.

2908 Hsï-wên | 聞 Süi-wăn.

$1^{\circ} \mathrm{H}$. in Lei-chou $\mathrm{Fu}$, Kuangtung; Lat. $20^{\circ}$ $19^{\prime}$, Long. $110^{\circ} 18^{\prime}$.

$2^{\circ}$ (obs.) S. of preceding ; T'ang, Sung and Yüan, Hsü-wên | 聞 $\mathrm{H}$.

$3^{\circ}$ See Nos. 1921, 3668, 5961.

2909 Hsï-w1 | 無, (obs.) W. of 'Tsun-hus C., Chihli; Han and $T \sin , H$. in Pei-p ing 北平 chïn; $N$. $W e i$, H. in Yü-yang 魚 陽 chïn。

\section{趈}

2910 Hsü-chiang 㔻 江, ssŭ near San-shui H., Kuangtung.

\section{揟}

2911 Hsü-tzŭ 揟次, (obs.) N of Ku-lang H., Kansuh; 
揟

Hsí̛-'TzÜ.

Han, H. in Wu-wei 武 威 chïn; N. Wei, H. in Ch'ang-sung 昌菘 chün.

\section{須}

2912 Hsï-chiang 須江, (obs.) 35 li S. W. of Chiangshan H., Cliehkiang;

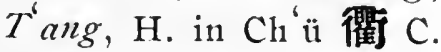

2913 Hsü-chü| 句, see No. 6745.

\section{旭}

2914 Hsü-ch' uan 旭川, see No. 3043.

\section{HSÜAN}

\section{立}

2915 Hsüan 立., see No. 6768. 2916 Hsüan-ch'ih | 池.

$1^{\circ}$ (obs.) in Ching-lê H., Shansi ; T'ang, H. in Hsien 畫 C.

$2^{\circ}$ (obs.) near Turfan; $T^{\prime}$ ang, Fu in Lung-yu 隴右 circ.

2917 Hsüan-fêng | 風 H. in Ch'ing-shang 慶佮 circ., Corea.

2918 Hsüan-t'u | 蒬.

$1^{\circ}$ (obs.) N. of Hsingching in Shingking; W. Han, chün; E. Han, C. in Yu 幽 CHOU.
宣 HSÜAN-FU.

$2^{\circ}$ (obs.) N.E. of Hsienp'ing Fun, Corea; Tsin, chün in P'ing $4 \overline{1}$ CHOU.

2919 Hsüan-wu | 武, see No. 6772.

\section{泣}

2920 Hsïan 泣, see No. 2845.

2921 Hsüan-11an-pu | 南㸃, post-town near Hsin-ts ai H., Honan.

2922 Hsüan-shih | 氏, see Nos. $3156,3951$.

\section{宣}

2923 Hsüan 宣, see Nos. 2924, 3417, 4696.

2924 Hsüan-ch' êng | 城 Sinenching.

$1^{\circ} \mathrm{H}$. forming the prefectural city of Ning-kuo, Anluni; Lat. $30^{\circ} 50^{\prime}$, Long. $118^{\circ} 41^{\prime}$; orig., Wan-ling 宛陵; Han, Hsüan-ch' êng | 城; Sui, Hsüan |C.

$2^{\circ}$ See No. 4696.

2925 Hsüan-ch'üan | 川 Siuenchnen, Fu in $\mathrm{P}^{\text {'ing-an }}$ 本 安 circ., Corea; Lat. $39^{\circ}$ $45^{\prime}$, Long. $125^{\circ} 02^{\prime}$.

2926 Hsüan-ên | 恩 Sinenngăn, H. in Shih-nan Fu, Hupeh ; Lat. $30^{\circ}$, Long. $109^{\circ} 30^{\prime}$.

2927 Hsüan1-fu|撫, ssü near Yüeh-sui T., Ssŭch'uan. 
宣 HSÜAN-HAN.

2928 Hsüan-han | 漢, see No. 6127.

2929 Hsïan-ho|河, chên near Chêng-ning $H$., Kansuh. 2930 Hsian-hua | 化 Sinen-hwa. [522] $1^{\circ} \mathrm{Fu}$ in the $\mathrm{K}^{\prime}$ ou-pei circ., Chihli; forming also the district city of the same name; Lat. $40^{\circ} 37^{\prime}$, Isong. $115^{\circ}$ $08^{\prime} ; T^{\prime}$ ang, Wu 武 C. ; Liao, Kuei-hua 跡化; Kin, Hsïan-tê | 德. Sindachu (Polo).

$2^{\circ} \mathrm{H}$. forming the prefectural city of the same name, Chihli; Lat. $40^{\circ}$ $37^{\prime}$, Long. $115^{\circ} 08^{\prime}$.

$3^{\circ} \mathrm{H}$. forming the prefectural city of Nan-ning, Kuangsi ; Lat. $22^{\circ} 43^{\prime}$, Long. $108^{\circ} 03^{\prime}$; Han, Ling-fang 領方; Sui, Hstian-hua | 化; T'ang and Sung, Yung 舀 C. $4^{\circ}$ Post-town near Tinghsing H., Chillit.

2931 Hsüan-lê | 樂, (obs.) Sui, H., S. of Yang-shan H., Knangtung.

2932 Hsüan-p'ing | 平 Sinen[35] ping, $\mathrm{H}$. in $\mathrm{Ch}^{6} \mathrm{u}$-chou $\mathrm{Fu}$, Chehkiang; Lat. $28^{\circ} 45^{\prime}$, Long. $119^{\circ} 20^{\prime}$; orig., Lishui 霹水.

2933 Hsüan-tê | 德, sẹe No. 2930.
勳

HsƯN.

2934 Hsüan-wei | 威 Sinenwei.

[358] $1^{\circ} \mathrm{C}$. in $\mathrm{Ch}^{\prime}$ ì-ching $\mathrm{Fu}$, Yünnan ; Lat. $26^{\circ} 25^{\prime}$, Long. $104^{\circ}$.

$2^{2}$ See No. 6306.

\section{HSÜEH}

\section{薛}

[See also under the syllable HSIEH.]

2935 Hsüieh 薛, chên near T'ai. p'ing H., Anluni.

2936 Hsüel1-ch'êng | 城, see No. 2684.

2937 Hsüeh-chi | 穫, chên near Wan-ch' ìan H., Shansi.

2938 Hsüeh-chia | 家, chên near $P^{\prime}$ u-cli'êng $H$., Shensi.

2939 Hsïeh-fêng | 齐, chên near Han-ch'êng H., Shensi.

2940 Hsüeh-lu|祿, chên E.

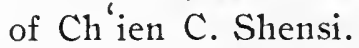

2941 Hsüeh-pu | 垚, chên near Li-slı1i H., Kiangsu.

\section{島}

2942 Hsïeh-li 雪裏, posttown near Fêng-t'ien $F u$, Shingking.

\section{HSÜN}

\section{動}

2943 Hsün 勳. 
勳

HsüN.

$1^{c}$ (obs.) in Ssŭch 'uan; $T^{\prime}$ ang, C. in Cliangnan 江南 circ.; Sung, C. in Shao-hsing 紹興 Fu.

$2^{\circ}$ See No. 550 .

\section{鐔}

2944 Hsün 鐔, see No. 7368. 2945 Hsün-ch' êng | 城, see No. 907.

2946 Hsün-ching | 津, (obs.) T'ang, H., N. of 'T'êng H., Kuangsi.

2947 Hsün-fêng|封, (obs.) S. E. of 'Tsun-yi H., Kueichou; Han, H. in 'Tsangkê 年柯 chün; $T \sin , \mathrm{H}$. in Hsing-ku 興古 chïn; S. Sung, H. in Liangshui 梁水 chün.

\section{语}

2948 Hsün-ssŭ-kan 䙓思干, see No. 5342.

2949 Hsün-tien | 甸 Sinn-tien, [1] C. in Ch ü̈-ching $\mathrm{Fu}$, Yünnan; Lat. $25^{\circ} 34^{\prime}$ Long. $103^{\circ} 19^{\prime}$; Ming, Hsün-tien 甸 Fu.

2950 Hsün-tien | 甸, see No. 2949.

2951 Hsün-yüan | 源, (obs.) in Yün-lien H., Ssŭch 'uan; $\tau^{\prime}$ ang, H. in Yiin 筷 C.

\section{棂}

2952 Hsün-ssŭ-kan 沀思干, see No. 5342.
䛻

HsüN.

\section{浔}

2953 Hsün 輩, see No. 2955.

2954 Hsün-chiang | 江, see No. 2955.

2955 Hstin-clıon | 州 Sin-chan, [198]Fu in Yu-chiang circ., Knangsi, forming also the district city of Kuei-p 'ing; Lat. $23^{\circ} 26^{\prime}$, Long. $199^{\circ}$ $51^{\prime} ; T^{\prime}{ }^{2}$ in, in Kuei-lin 桂 林 chün; Han, in Yü-lin 數林 chiön and 'Ts'ang-wu 蒼梧 chün; Liang, Kueip'ing 社平; T ang, Hsünchiang | 江 and Hsün I C.

2956 Hsün-yang | 陽, see Nos. 1281, 6312.

\section{溶}

2957 Hsiun 澹 (or 淩) Siün, H. in Wei-hui Fu, Honan; Lat. $35^{\circ} 45^{\prime}$, Long. $114^{\circ} 50^{\prime}$; orig., Wei-yi 衞邑; Han, Li-yang 黎 骝; Five dyn., Hsün | H.

浚

2958 Hsün 浚, see No. 2957.

2959 Hsün-yi | 儀, (obs.) N.W. of Hsiang-fu H., Honan ; Han and $W_{e} i, \mathrm{H}$. in $\mathrm{Cl}^{\prime}$ 'ên-liu 陳留 chün.

毁川

2960 Hsün 馴, see No. 5216.

\section{循}

2961 Hsün 话. 
$1^{\circ}$ (obs.) S. Sung, C. in Knang-tn11g 廣東 circ. $2^{\circ}$ See No. 2365.

2962 Hsün-hıa | 化 Siun-hwa, $\mathrm{T}$. in Hsi-ning Fu, Kansuh; Lat. $36^{\circ}$, Long. $102^{\circ} 30^{\prime}$.

\section{巡}

2963 Hsïn-chie11 巡 檢, ssü near Lï-shan H., Ssŭ. ch'van.

\section{葋}

2964 Hsün-ch' êng | 节城, see No. $2966^{\mathrm{A}}$.

\section{洵}

2965 Hsün 洵, see No. 2966.

2966 Hsün - yang | 陽 Siunyang, $\mathrm{H}$. in $\mathrm{H}$ sing-an $\mathrm{Fu}$, Shensi ; Lat. $32^{\circ} 40^{\prime}$, Long. $109^{\circ} 36^{\prime}$; Han, Hsün-yang | 陽; T'ang, Hsün $\mid \mathrm{C}$.

\section{㿟}

$2966^{A}$ Hsün 创!

$1^{\circ}$ (obs.) formerly town near Yi-shih H., Shansi; also called Hsü11-ch 'èng 荷城。

$2^{\circ}$ See No. 4369.

I, see Nos. 7443, 7508. Ibaraga, see No. 6860.

I-chang, see Nos. 7419, 7420 .
I-chan, see No. 7461.

I-ching, see Nos. 7421, 7468, 7514.

I-chuen, see No. 7422 .

I-chun, see No. 7423.

I-fung, see Nos. 7426, 7450.

I-hing, see Nos. 7429 . 7456.

I-ho, see No. 7427.

I-hwang, see No. 7428.

I-kao, see No. $7466^{\mathrm{A}}$.

I-kh Char, sce No. $7466^{\mathrm{B}}$.

I-kiun, see No. 7424.

I-lan, see No. 7430 .

I-lanhala, see No. 5377.

I-liang, sce No. 7431.

I-lung, see No. 7453.

I-ning, see No. 7458.

I-pin, see No. 7436.

I-shan, see Nos. 7437, 7460 .

I-shi, see No. 7510.

I-shwui, see No. 7462.

I-tu, see No. 7440.

I-wu, see No. 7463.

I-yang, see Nos. 7441, 7495, 7517.

Ilbalik, see No. 7519 .

Ilchi, see No. 2057.

Ili, see No. 7469.

Ilkoun, see No. 7466.

Imperial tombs, see No. 2817.

Ingearik, see No. 7235 .

Inner Mongolia, see No. 465.5 .

Ishikawa, see No. 5705. 
的

Ispahan, see No. 7522 .

Iwate, see No. 7397.

\section{J}

Jam, see No. 138.

Jan-su-jung, see No. 777.

\section{JANG}

\section{壤}

2967 Jang-tu 壤渡, post-town near Wan H., Ssŭch' 'uan.

\section{瀼}

2968 Jang 浧, (obs.) T'ang, C., 280 li S. of Nan-ning $\mathrm{Fu}$, Kuanorsi.

2969 Jang-chiang | Z[, (obs.) in Shang-ssŭ C., Kuangsi; $T^{\prime}$ ang, H. in Jang | C.

\section{穰}

2970 Jang-tung 醙東, (obs.) $60 l i \mathrm{~N}$. E. of 'T'êng C., Honan; Kin, chên in Têng 鄧 C.

2971 Jang-yi | 邑, see No. 6333.

\section{JAO}

\section{餻}

2972 Jao 琵, (obs.) S. Sung, C. in Chiang-tung 江東 circ.

2973 Jäo-an | 努, (obs.) T $\sin$ and T"ang, H., E. of Ts'ang C., Chihli.
JÊ-WU.

2974 Jao-chou | 州 Jao-chau, [14] $\mathrm{F}_{11}$ in the Kuang-JaoChin-Nan circ., Kiangsi ; forming also the district city of $\mathrm{P}^{\prime}$ o-yang; Lat. $28^{\circ}$ $59^{\prime}$, Long. $116^{\circ} 46$; S.A. and $C$. Wars, in K. Ch "u 楚; $T s^{\prime}$ in, $\mathrm{P}^{\prime}$ o-yang鄱陽 in Chiu-chiang 九 江 chion; Han, in Yü-chang 豫章; Liang, Wu 呂 C.; Sui, I'ang and Sung, Jao $\mid \mathrm{C}$.

2975 Jao-p'ing | 本 Jatt-ping, [149] $\mathrm{H}$. in $\mathrm{Ch}^{\text {' ao-chou }} \mathrm{Fu}$, Kuangtung; Lat. $23^{\circ} 56^{\prime}$, Long. $116^{\circ} 51^{\prime}$; Han, Chieh-yang 揭陽.

2976 Jao-yang | 陽 Jall-yang, $\mathrm{H}$. in Shên C., Chihli ; Lat. $38^{\circ} 15^{\prime}$, Long. $115^{\circ} 51^{\prime}$.

Jall-chan, see No. 2974. Jall-ping, see No. 2975. Jau-yang, see No. 2976.

\section{J $\hat{E}$}

染

2977 Jê-ho 熱河, see No. 537. 2978 Jê-lang-wa | 郎晆, T-S, near Yüeh-sui T., Ssŭch' nan.

2979 Jê-tang | 當, T-S. near Sung-p' an T., Ssŭch' uan. 2980 Jê-wu ! 霧, T-S. near Sung-p'an T., Ssŭch' uan. 
㦚

JEGHEN.

Jeghen, see No. 3217.

Jehol, see No. 537.

\section{JÊN}

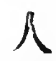

2981 Jên-110 人和, post-town near Ch'ung-an H., Fuhkien.

\section{任}

2982 Jên 任 Jin.

[432] $1^{\circ} \mathrm{H}$. in Shunn-tê $\mathrm{Fut}$, Chihli; Lat. $37^{\circ} 12^{\prime}$, Long. $114^{\circ} 48^{\prime}$.

$2^{\circ}$ See No. 602.

2983 Jên-ch'êng | 城, see Nos. 602, 7373.

2984 Jên-ch'in | 邱 Jin-kiu, [558] H. in Ho-chien Fin, Chihli ; Lat. $38^{\circ} 43^{\prime}$, Long. $116^{\circ} 15^{\prime}$; orig., Kao-yang 高陽; Han, Jên-ch'in | 邱; Sung, Chêng 唒 $\mathrm{C}$.

2985 Jén-shih | 䔈 H. in Ch'ïan1-10 全 羅 circ., Corea.

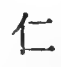

2986 Jên-ch 'uan 仁川 Fin in Ching-ch'i 京畿 circ., Corea. The port nearest the capital; open to foreign trade. Local name Clii-wup' 1 洉物浦, Chemulpo.

2987 Jên-fêng | 豈; (obs.) 60 li N.E. of Chi-yang H.,
化 J⿸厃㔾N-SHOU.

Shantung; Kin, chên in Chi-nan Fu, Shantung.

2988 Jên-ho | 和 Jin-ho, $H$. forming with Ch' ien-t'ang $\mathrm{H}$. the prefectural city of Hang-chou, Chehkiang; Lat. $30^{\circ} 12^{\prime}$, Long. $120^{\circ}$ $12^{\prime}$; Han, Yen-knan 監葛; Five dy'n., Ch'ien-chiang 錢江; Sung, Jên-ho.

2989 Jên-hua | 化 Jin-hwa, H. [60] in Shao-chou Fu, Kuangtung ; Lat. $25^{\circ} 15^{\prime}$, Long. $113^{\circ} 13^{\prime}$; orig., Chüchiang 曲 江; T T ang, Jên-hura | 化.

2990 Jên-huai | 覆, (obs.) W. of Jên-huai H., Kueichou; Ming, H. in Chên-an 且 安 $\mathrm{C}$.

2991 Jên-huai | 懷 Jin-hwai.

[51] $1^{\circ}$ C. L.T. in the Kuei-

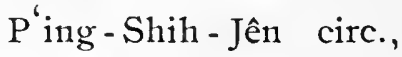
Kueichou ; Lat. $28^{\circ}$ $15^{\prime}$, Long. $106^{\circ} 55^{\prime}$.

[51] $2^{\circ} \mathrm{H}$. in Tsun-yi-Fu, Kueichou; Lat. $28^{\circ}$ $20^{\prime}$, Long. $105^{\circ} 40^{\prime}$.

2992 Jê11-shou | 壽 Jin-shau.

$1^{\circ}$ H. in Tzŭ C., Ssŭch' 11an; Lat. $30^{\circ}$, Long. $104^{\circ} 20^{\prime}$; Han, Wuyang 武榢; $W$. Wei, $\mathrm{P}^{\text {' }}$ 1-ning 普富; Sui, Jên-shou | 䩧.

$2^{\circ}$ Post-town near Shunch'ang H., Fuhkien. 
仁 JF̂n-T'Ung.

2993 Jên-t'ung | 同 Fu in Ch'ing-shang 度尚 circ., Corea.

2994 Jên-yi | 義, ssŭ near Lingshih H., Shansi.

\section{JIH}

H

2995 Jih-chao 日照 Jih-chau, [126] H. in Yi-chou Fu, Shantung ; Lat. $35^{\circ} 27^{\prime}$, Long. $119^{\circ} 53^{\prime} ;$ Han, Hai-ch ' ï 海曲; Kin, Jih-chao | 照. 2996 Jih-hsia 日下, see No. 5822.

2997 Jih-kê-tsê | 噶則 Zhikatsé, town in Thibet; Lat. $29^{\circ} 15^{\prime}$, Long. $89^{\circ}$.

2998 Jih-kuang 日光 Nikko, town in Japan.

2999 Jih-nan |南, name given to Annam or Tonquin ; now also called Yüeh-nan 越南, see No. 1192 .

Jil-chan, see No. 2995.

Jin, see No. 2982.

Jin-ho, see No. 2988.

Jin-hwa, see No. 2989.

Jin-hwai, see No. 2991.

Jin-kiu, see No. 2984.

Jin-shan, see No. 2992.

\section{JO}

? 若

3000 Jo-clı'i 落溪, (obs.) near Yüan-choı $\mathrm{F}$, Hu1nan;
汝

Ju.

Sung, military post in Yüian 沅 C.

\section{都}

3001 Jo 都, (obs.) Ts in, H., W. of Yi-ch'eng $\mathrm{H}$., Hupeh; S. A., Jo $\mid \mathrm{K}$.

\section{JOU}

\section{柔}

3002 Jol1-yüan 柔遠, post-town near - Liang-chou $\mathrm{F} u$, Kansuh.

\section{JU \\ 儒}

$3003 \mathrm{Ju}$ 儒.

$1^{\circ}$ (obs.) near $\mathrm{Ch}^{\prime}$ ing-yang Fu, Kansul, T Tang, C. in An-huna 安化 $\mathrm{Fu}$.

$2^{\circ}$ (obs.) in Kueichou ;

Sung, C. in Shaoch'ing 紹慶 Fu.

$3^{\circ}$ See No. 7363.

\section{濡}

$3004 \mathrm{Ju}$ 濡, (obs.) in Kueichour; T'ang, C. in Chiang-nan 江南 circ.

\section{汝}

$3005 \mathrm{Ji1}$ 汝, Ju.

[200] $1^{\circ}$ C.L.C. in the Ho-nanShan-Ju circ., Honan; Lat. $34^{\circ} 14^{\prime}$, Long. $112^{\circ} 54^{\prime}$; S.A., in $\mathrm{K}$. 
Chêng 郳 and K. Ch' $\mathrm{u}$ 楚; C. Wars, in $\mathrm{K}$. Han 韓 and $K$. Wei 魏; Ts'in, in Sanch'uan 三川 chïn; Han, in Honan 河南 chiun; P. Chou, Ho 和 C.; Sui, Ju | C.; $T^{6}$ ang, Ju $\mid \mathrm{C}$.

$2^{\circ}$ See No. 2611.

3006 Ju-ch'êng | 城, see No. 3453.

3007 J11-fên | 垻, see No. 5582 . 3008 Ju-ho | 河, chên near Sung H., Honan.

3009 Ju-nan | 南.

$1^{\circ}$ Chên near Hsin-ts'ai

H., Honan.

$2^{\circ}$ See Nos. 727, 3010.

3010 Ju-ning | 家 Ju-ning, Fu [201] in the Nan-Ju-Kuang circ., Honan ; forming also the district city of Ju-yang; Lat. $33^{\circ} 01^{\prime}$, Long. $114^{\circ}$ $21^{\prime} ; S . A$., in K. Shên 沈 K. Ts'ai 蔡; $T s^{\prime} i n$, in Ying-ch' 'uan 穎川 chïn; Han, Ju1nan | 南; $P$. Chou, Yü 豫 C.; Sui, Shu 舒 C. and Chên 溱 C.; T'ang, Ts'ai 蔡 C.; Sung, Huai-k'ang 淮康; Yüan, Ju-ning | 富.

3011 Ju-yang | 陽 Ju-yang, $\mathrm{H}$. forming the prefectural city of Ju1ning, Honan; Lat. $33^{\circ} 01^{\prime}$, Long. $114^{\circ}$
乳 JU-YÜAN.

21; Han，Ju-yang | 陽; Sui, Yin-shui 溵水。

3012 Ju-yin | 㓌, see Nos. 2126, 7582.

3013 Ju-yüan | 源, (obs.) in Honan; Sui, H. in Hsiangch'êng 襄战 chiin.

\section{如}

$3014 \mathrm{Ju}$ 如, (obs.) in Ssŭch'uan; T'ang, C. in Lung-yu 隴右 circ.

3015 Ju-hsi-t'ung | 晋峝, sš̆ near $\mathrm{Ch}^{\text {'in } \mathrm{C} ., \text { Knangtung. }}$

3016 Ju-kao | 泉 Ju-kau, H. in T'ung C., Kiangsu ; Lat. $32^{\circ} 22^{\prime}$, Long. $120^{\circ} 36^{\prime}$; Han, Kuang-ling 廣陵; Tsin, Jul-kao | 泉.

\section{茹}

3017 Ju 茹, (obs.) S. of Hsüan-hua H., Chihli; $W$. $H a n, \mathrm{H}$. in Shang-ku 上 谷 chïn.

3018 Ju-na | 麻, sš̆ near Ching H., Anhui.

3019 Ju-p ing | 平, chên near Yi-ch' uan H., Shensi.

\section{乳}

3020 J11-yüan 乳源 Ju-yuen, [310] $\mathrm{H}$. in Shao-chou $\mathrm{Fu}$, Kuangtung; Lat. $24^{\circ} 52^{\prime}$, Long. $112^{\circ} 50^{\prime}$; orig., $\mathrm{Ch}^{\prime} \ddot{\mathrm{u}}$-chiang 曲江.; Sung, Ju1-yüan | 源. 
㳄

JU-KAU.

J11-kau, see No. 3016.

Ju-yuen, see No. 3020.

\section{JUAN}

軟

3021 Juan-ch'iao 軟 橋, posttown near $\mathrm{P}^{\prime}$ un-an $\mathrm{T}$., Kueichou.

3022 Juan-hung 軟紅, see No. 5822 .

\section{JUI \\ 瑞}

3023 Jui 唡, (obs.) S. Sung, C. in Chiang-hsi 江西 circ.

3024 Jui-an | 岀 Sui-ngan.

$1^{\circ} \mathrm{H}$. in Wên-chon Fin, Chehkiang; Lat. $27^{\circ}$ $47^{\prime}$, Long. $120^{\circ} 45^{\prime}$; Han, Chang-an 章安; $W u ，$ Lo-yang 羅陽; Tsin, An-ku 安固; T'ang, Jui-an 安.

$2^{\circ}$ See No. 7022.

3025 Jui-ch'ang | 昌 Sui-cliang, H. in Clint-chiang Fu, Kiangsi ; Lat. $29^{\circ} 49^{\prime}$, Long. $115^{\circ} 44^{\prime}$; Three $K s ., \mathrm{Ch}_{1}$ ih-11iao 赤鳥; $S$. T'ang, Jui-ch'ang 昌. 3026 Jui-chin | 金 Sui-kin, H. [228] in Ning-tı C., Kiangsi ; Lat. $25^{\circ} 49^{\prime}$, Long. $116^{\circ}$ $01^{\prime}$; Han, Yüi-tu 雱都; $T^{*}$ ang, Jui-chin | 金.
容 JUI-YING。

3027 Jui-chou | 州 Sui-chan, [403] Fu in the Jui-Yian-Lin circ., Kiangsi ; forming also the district city of Kao-an; Lat. $28^{\circ} 25^{\prime}$, Long. $115^{\circ} 14^{\prime} ; S . A$., in $\mathrm{K}$. W11 吳; $C$. Wars, in K. Ch' 1 楚; $T s^{\prime} i n$, in Chiu-chiang 九江 chün; $H a n$, in Yü-chang 豫章 chiun; $7^{6}$ ang, Ching 垟 C., $\mathrm{Mi}$ 米 C. and $\mathrm{Yün}$ 㐨 C.; Sung, Jui | C.

3028 Jui-hsing | 興 Sui-hing, Fu in Huang-hai 翼: 海 circ., Corea ; Lat. $38^{\circ} 46^{\prime}$, Long. $126^{\circ} 20^{\prime}$.

3029 Jui-lin | 林, ssĭ in Juichin H., Kiangsi.

3030 Jui-shih | 石, post-town near Ling-shih H., Shansi.

3031 Jui-Yüan-Lin | 袁林, circ. in Kiangsi comprising Jui-chou F11, Yüan-chon $F u$ and Lin-chiang Fı.

\section{苻}

3032 Jui-ch'êng 苪城 Jui[148] ching, H. in Chieh C.., Shansi; Lat. $34^{\circ} 50^{\prime}$, Long. $110^{\circ} 22^{\prime}$; orig., K. Jui |; P. Chou, Jui-ch' êng 城.

3033 Jui-clia | 家, Y. near Chung-wei H., Kansuh.

\section{容}

3034 Jui-ying 旁營, chên near Mei H., Shensi. 
潤

JUI-CHING.

Jui-ching, see No. 3032 .

J11-j11, see No. 1324 .

\section{JUN}

润

3035 Ju1 㵎, see No. 418.

\section{JUNG}

\section{容}

3036 Jung 容 Yung, H. in [457] Wu-chou Fu, Kuangsi ; Lat. $22^{\circ} 53^{\prime}$, Long. $110^{\circ}$ $03^{\prime}$; Han, Ho-p " 1 合浦; Sui, P'u-ning 晢窗; T'ang, Jung | C.

3037 Jung-ch'êng | 城 Yungching.

$1^{\circ} \mathrm{H}$. in Pao-ting $\mathrm{F}_{11}$, Chihli; Lat. $39^{\circ} 04^{\prime}$, Long. $116^{\circ} 58^{\prime}$.

$2^{\circ}$ See Nos. 2214, 2724.

3038 Jung-shan | 山, (obs.) Sung, H., N.W. of Yiilin C., Kuangsi.

\section{榕}

3039 Jung-ts' un 榕村, Y. near Yai C., Kuangtung.

\section{茸}

3040 Jung-ch'êng 咠. 城, see No. 5970.

\section{戈}

3041 Jung 戎.
榮 JUNG-CHING.

$1^{\circ}$ (obs.) in Yi-pin H., Ssŭch' uan; $T^{\prime}$ ang, C. in Chien-nan 劍南 circ.

$2^{\circ}$ (obs.) in Hsing-wên H., Ssǔch'uan ; Yïan,

C. in Ma-hu 馬湖 circ. $3^{\circ}$ See No. 2895.

3042 Jung-ch' êng / 城, (obs.) Sui, $20 l i \mathrm{~S}$. of Wu-chou Fu, Kuangsi.

\section{笨}

3043 Jung 笨 Yung.

[204] $1^{\circ} \mathrm{H}$. in Chia-ting $\mathrm{Fu}$, Ssŭchŭ 'nan1; Lat. $29^{\circ}$ $30^{\prime}$, Long. $104^{\circ} 30^{\prime}$; Sui, T'ai-lao 太年; T'ang, Hsii-ch 'uan 旭 川 and Jung $\mid \mathrm{C}$.

$2^{\circ}$ Chên near Lii-chiang H., Anhui.

3044 Jung-ch'ang | 昌 Yung[563] chang, H. in Chungch'ing Fu, Ssǔch'uan; Lat. $29^{\circ} 34^{\prime}$, Long. $105^{\circ}$ $38^{\prime}$; orig., Nei-chiang 內 江; T'ang, Ch'ang-yïan 昌元 and Ch'ang 昌 C.

3045 Jung-ch'êng | 城 Yungching, H. in Têng-chou Fu, Shantung; Lat. $37^{\circ}$ $30^{\prime}$, Long. $122^{\circ} 30^{\prime}$.

3046 Jung-ching | 經 Yungking, $\mathrm{H}$. in Ya-chou $\mathrm{Fu}$, Ssŭch'nan; Lat. $29^{\circ} 52^{\prime}$, Long. $102^{\circ} 55^{\prime}$. 
策 JUNG-CH'UAN.

3047 Jung-ch' uan | 川, chün in

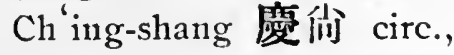
Corea.

3048 Jung-ho | 河 Yung-ho, H. in $\mathrm{P}^{\prime} \mathrm{u}$-chou $\mathrm{Fu}$, Shansi; Lat. $35^{\circ} 23^{\prime}$, Long. $110^{\circ}$ $25^{\prime}$; Hsia, Shao-k'ang-yi 少康邑; Tsin, Fên-yin 汾陰; Tang, Pao-ting 珼鼎; Sung, Jung-ho | 河.

3049 Jung-yin | 因, (obs.) "'ang, H., N. of Huaichi H., Kuangsi.

\section{㮡}

$3050 \mathrm{Jung} \cdot$ tsê 榮澤 Yung-tseh, [32] $\mathrm{H}$. in $\mathrm{K}$ ai-fêrı $\mathrm{Fu}$, Honan; Lat. $34^{\circ} 57^{\prime}$, Long. $113^{\circ} 44^{\prime} ; T s^{\prime} i n$, in San-ch'11an 三川 chïn; Sui, Jung-tsê | 裟.

3051 Jung-yang | 陽 Yung[32] yang, H. in $K^{\prime}$ 'ai-fêng $F ı$, Honan; Lat. $34^{\circ} 53^{\prime}$, Long. $113^{\circ} 35^{\prime}$; orig., E. part of K. Ku1 絾; Han, Jung-yang | 陽; T'ang, $\mathrm{Wu}-\mathrm{t}^{\prime} \mathrm{ai}$ 武泰.

\section{KA}

\section{蓋}

$3052 \mathrm{Ka}$ 荒.

$1^{\circ}$ (obs.) Chou, town in K. Chi 沙 near Chinan $\mathrm{F} u$, Shantung.
鬥

K'AI.

Kabaran, see No. 3191.

Kabatah, see No. 3191.

Kabul, see No. 3233.

Kadabzi, see No. 1911.

Kagoshima, see No. 4659.

\section{KAI}

\section{䀉}

$3052^{\mathrm{A}}$ Kai 莣, see Nos. 3156, 6546.

3053 Kai-hsün | 遜, T-S. near Ho C., Kansuh.

3054 Kai-p'ing | 平 Kai-ping, $\mathrm{H}$. in Fêng-t'ien $\mathrm{Fu}$, Shingking; Lat. $40^{\circ} 10^{\prime}$, Long. $121^{\circ} 40^{\prime}$; Liao, Ch'ên 辰 C.

Kai, see No. 3057.

Kai-cbing, see No. 3058.

Kai-fung, see No. 3061.

Kai-hwa, see No. 3062.

Kai-kien, see No. 3060.

Kai-ping, see No. 3068.

Kai-tai, see No. 3070 .

Kai-ylten, see No. 3074 .

\section{K'Al}

\section{開}

3057 K'ai 開 Kai.

[289] $1^{\circ} \mathrm{C}$. in Ta-ming Fu, Chihli; Lat. $35^{\circ} 46^{\prime}$, Long. $115^{\circ} 16^{\prime}$; orig., Shan-y üan 溳淵; Han, T un-ch'iu 頓邱; $T^{\prime}$ ang, Shan 演 C.; 
型

$\mathrm{K}^{\prime} \mathrm{AI}$.

Kin, Yïan and Ming, $\mathrm{K}^{\prime} \mathrm{ai} \mid \mathrm{C}$.

$2^{\circ} \mathrm{C}$. in Kuei-yang $\mathrm{Fu}$, Kueichun ; Lat. $27^{\circ}$ 15', Long. $107^{\circ} 05^{\prime}$; Ming, K'nai-hsi 戗西 ssü and $\mathrm{K}^{\prime}$ ai $\mid \mathrm{C}$.

$3^{\circ} \mathrm{H}$. in $\mathrm{K}^{\prime}$ nei-chou $\mathrm{Fu}$, Ssŭch 'uan; Lat. $31^{\circ}$ $18^{\prime}$, Long. $108^{\circ} 30^{\prime}$; Han, Han-fêng 漢豐; IV. Wei, Yung-ning 永耍; Sui, K' ai $\mid \mathrm{C}$. Tang and Sung, $\mathrm{K}^{\prime}$ ai-chiang $\mid$ 江 $\mathrm{H}$.

$4^{\circ}$ (obs.) near Ju-ning Fu, Honan ; N.Wei, H. in $\mathrm{Ch}^{\prime} \mathrm{ên}^{\mathrm{n}}$ 像 chien.

$5^{\circ}$ (obs.) S.E. of Fênghuang-ch' êng, Shingking; Liao, C. in Tung-ching 東京 circ. $6^{\circ}$ See No. 3058.

3058 K'ai-ch'êng | 城 Kaiching.

$1^{\circ} \mathrm{F} 11$ in Cling-ch ‘ $\mathrm{i}$ 宗 畿 circ., Corea; Lat. $37^{\circ} 55^{\prime}$, L Long. $126^{\circ}$ 48'; Sung and Ming, $\mathrm{K}^{\prime}$ ai $\mid \mathrm{C}$.

$2^{\circ}$ Chên near Yi-ch' nan H., Shensi.

$3^{\circ}$ See No. 3288.

3059 K'ai-chiang | 江, see No. 3057.

3060 K'ai-chien | 建 Kai-kien, [416] $\mathrm{H}$. in Chao-ch'ing $\mathrm{Fu}$,
開 K'AI-HUA.

Knangtung; Lat. $23^{\circ} 45^{\prime}$, Long. $111^{\circ} 27^{\prime}$; Han, Fêng-yang 封隄; 1st Sung, K'ai-chien 建; Liang, Nan-ching 南静. 3061 K'ai-fêng / 封 Kai-fung. [148] $1^{\circ}$ I'u in the Ho-nanK'ai - Knei - Ch' ên - Hsü circ., Honan; forming also the district city of Hsiang-fu ; Lat. $34^{\circ} 52^{\prime}$, Long. $114^{\circ} 33^{\prime}$; capital of the province; S.A., in K. Chêng 觙, K. Wei 魏 and K. Ch' ên 陳; C. Wars, Ta-liang 大梁 in K. Wei 魏; $T s^{\prime}$ in, in San-ch' uan 三川 chïn; Han, in $\mathrm{Ch}^{\prime}$ ên-liu 棵留 chïn; E. Wei, Liang 梁 C. and $\mathrm{K}^{\prime}$ ai-fêng | 封; $T^{\prime}$ ang, P'ien 沛 C.; P. Liang, 'Tung-ching 東京; Sung, Pienching 汒京; Yïan, Pien-liang 沐梁; Ming, $\mathrm{K}^{\prime}$ ai-fêng | 封 Fin.

$2^{\circ}$ (obs.) 50 li $\mathrm{S}$. of preceding ; Han, Tsin, $W c i$ and Sui, K'aifêng | 封 $\mathrm{H}$.

3062 K'ai-hua | 化 Kai-hwa. [351] $1^{\circ} \mathrm{Fu}$ in the Lin-K'aiKuang circ., Yünnan ; forming also the district city of Wên-shan; Lat. $23^{\circ} 10^{\prime}$, Long. $104^{\circ} 50^{\prime}$. 
開 K'AI-HUA.

[56] $2^{\circ} \mathrm{H}$. in $\mathrm{Ch}^{6} \mathrm{ui}$-chou $\mathrm{Fu}$, Chehkiang; I at. $29^{\circ}$ $09^{\prime}$, Long. $118^{\circ} 36^{\prime}$.

$3063 \mathrm{~K}^{\prime}$ ai-hnua-ch' êng | 化城, (obs.) W. Wei, town W. of Yiin-yang Fu, Hupeh.

3064 K'ai-nan | 南, see No. 1138.

3065 K'ai-ning | 夏 H. in Ch'ing-shang 慶少 circ., Corea.

3066 K'ai-pao | 珼, (obs.) Sung, H., E. of Ku-yïan C., Kansuh.

3067 K'ai-pien | 邊, chên near Chên-yüan H., Kansuh.

3068 K'ai-p'ing | 平 Kai-ping. [406] $1^{\circ} \mathrm{H}$. in Chao-ch'ing Fu, Kuangtung; Lat. $22^{\circ} 30^{\prime}$, Long. $111^{\circ}$ $54^{\prime}$.

$2^{\circ} \mathrm{Y}$. near Fêng-jun H., Chihli.

3069 K'ai-shun | 順, chên near Liu-an C., Anhui.

3070 K'ai-t'ai | 秦 Kai-tai, H. in Li-p'ing Fu, Kueichou; I at. $26^{\circ} 10^{\prime}$, Long. $109^{\circ}$. 3071 K'ai-tu | 都, chên near Wên-shui H., Shansi.

3072 K'ai-yang | 晹, see No. 4039.

3073 K'ai-yi | 夷, (obs.) 60 li N. of Ề-shih H., Hupeh; Sui, H. in Ching-chiang 清江 chiun.
甘 $\mathrm{KaN-ChOU.}$

3074 K'ai-yüan | 原 Kai-yuen, H. in Fêng-t'ien Fu, Shingking; Lat. $42^{\circ} 40^{\prime}$, Long. $124^{\circ} 15^{\prime}$; Liao, Fuyï 扶餘 H. in Huai 懷 C. and L lung 龍 C. in Tung-ching 東京 circ.; Yïan, $\mathrm{K}^{\prime}$ ai-ÿian | 元 circ. in Liao-yang 遥陽 province.

3075 K'ai-yüan $\mid \overrightarrow{\text { 兀 }}$.

$1^{\circ}$ Chên near Ch'êng H., Chehkiang.

$2^{\circ}$ (obs.) near Ning-hsia Fu, Kansuh ; T'ang, C. in Ching-pien 靜邊 $\mathrm{Fu}$.

$3^{\circ}$ See No. 3074.

凱

3076 K'ai-li 凱里, Y. near Ch'ing-p'ing H., Kueichous.

Kalgan1an, see No. 3254. Kalgan, see No. 182 .

Kampicliu, see No. 3079. Ka11111, see No. 1907.

\section{KAN}

甘

3078 Kan1-ching 甘井, chên near Ho-yang H., Shensi. 3079 Kan-chou | 州 Kan-chat, [390] Fu in the Kan-Liang circ., Kansul1; forming also the district city $0^{\circ}$ Chang-yeh; Lat. $39^{\circ} 01^{\prime} \mathrm{f}$ 
甘

Long. $100^{\circ} 56^{\prime}$; Han, in Chang-yeh 㲀 夜 chïn; W. Wei, Kan $\mid$ C. Kampichu (Polo).

3080 Kan-ch'ïan | 泉 Kantsituen.

[264] $1^{\circ}$ H. in Yentan Fin, Shensi ; Lat. $36^{\circ} 24^{\prime}$, Long. $109^{\circ} 13^{\prime}$; Han. Tiao-yin 雕陰; Tang, Fn-111 伏陸 and Kanch'üan广泉.

$2^{\circ} \mathrm{H}$. forming the prefectural city of Yang-chou, Kiangsu; Lat. $32^{\circ} 21^{\prime}$, Loing. $119^{\circ} 15^{\prime}$.

$3^{\circ}$ Yost-town near Kanchou Fu, Kansuh.

$4^{\circ}$ Post-town near $\mathrm{Ch}^{\prime}$ êng H., Kansuh.

3081 Kan-ch'ïan-shih | 泉市, see No. 1245.

3082 Kan-ho / 河, chên near Hu H., Shensi.

3083 Kan-ku | 谷, (obs.) Kin, H. 70 li E. of 'T'ung-wei H., Kansul.

j084 Kan-kung-ch'êng | 公城, (obs.) formerly town $\mathrm{N}$. of Nan-lê H., Chihli.

3085 Kan-Liang | 凉, circ. in Kansul comprising Kanchou $\mathrm{Fu}$ and Liang-chou Fu.

3086 Kan-ling | 陵, see Nos. 1245, 3277.

3087 Kan-lo-ch'êng | 羅 城, (obs.) formerly town
KAN-ÊN.

N.W. of Huai-an Fu, Kiangsu.

3088 Kan-pei | 北, chên near Shun-hua H., Shensi.

3089 Kan-s1 | 淵 Kan-suh, a province of China ; capital, Lan-chou Fu; literary names Lung-hsi 隌西 and Hsi-chiang 西羌; it contains 7 circ., $8 \mathrm{Fu}, 1$ C.L.T., 9 T.. 6 C.I.C., $1 \mathrm{C}$. and $61 \mathrm{H}$.

3090 Kan-sung | 松, (obs.) in Ssŭch'uan; S. Ts'i, chün in $\mathrm{Yi}$ 盆 $\mathrm{CHOU} ; T^{\mathrm{l}}$ ang, H. in Yen 崖 C.

3091 Kan-t'ang | 棠.

$1^{\circ}$ Post-town near Shan C., Honall.

$2^{\circ}$ Post-town near Changp'u H., Fuhkien.

$3^{\circ}$ See No. 7441.

3092 Kan-tu1ng | 東, (obs.) N.W. of Yang-ch' 'un H., Kuangtung ; S. Sung, H. in Hsin-ning 新察 chün.

3093 Kan-ts'ao | 草, ssŭ in T'ai-ts'ang C., Kiangsu.

3094 Kan-tzŭ-ma-shu | 孜麻 펼, T-S. near Ta-chien-lu T., Ssŭch 'van.

\section{感}

3095 Kan-ên 笼恩 Kan-ngăn, H. in Ch'iung-chou $\mathrm{Fu}$, Kuangtung; Lat. $18^{\circ} 50^{\prime}$; Long. $108^{\circ} 20^{\prime}$; Han, Chiu-lung 九 龍; Sui, Kan-ên |恩 $\mathrm{H}$, 
感

KAN-MU'-I.U.

3096 Kan-1mu-lu | 木嶨, see No. 1907.

3097 Kan-yi | 義 (obs.) T゙ang H., W. of T'êng H., Knangsi.

\section{贑}

3098 Kan 贑 Kan。

$1^{\circ} \mathrm{H}$. forming the prefectural city of Kanchou, Kiangsi ; Lat. $26^{\circ} 02^{\prime}$, Long. $115^{\circ}$ $02^{\prime} ;$ Han, Kan | H.; $T \sin$, Nan-k'ang 南氺 and $\mathrm{Ch}^{\prime}$ ien 虔 $\mathrm{C}$.

$2^{\circ}$ (obs.) S. Sung, C. in Chiang-hsi 江. 西 circ.

$3^{\circ}$ See Nos. 3095, 4708.

3099 Kan-chou | 州 Kan-chau, [22S] Fu in the Chi-Nan-KanNing circ., Kiangsi ; forming also the district city of Kan ; Lat. $26^{\circ}$ $02^{\prime}$, I Long. $115^{\circ} 02^{\prime} ;$ S.A., in $K$. $W_{11}$ 岁 and $K$. Yüelı 越; C. Wars, in K. $\mathrm{Ch}^{\prime} \mathrm{u}$ 楚; $T s^{\prime}$ in, in Chiuchiang 九江 chïn; Han, Chang-kn1ng 章貢 in Y $\mathrm{Y}$ chang 娥算 chion; $T$ sin, Nan-kang 南康; Sui and T'ang, Ch'ien 虔 C.; Sung, Kan | C.

3100 Kan-yü | 榆 Kan-yu.

$1^{\circ} \mathrm{H}$. in Hai C., Kiangsu; Lat. $34^{\circ} 52^{\prime}$, Long.
抨

KAN-CH $\hat{\text { HNG }}$.

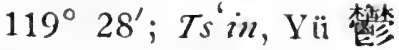
C.; Han, Tung-hai 東海; T"ang, Kan-y ï 榆; Five d’'n., Huaijên 懁仁.

$2^{\circ}$ (obs.) N. E. of preceding; Han and Tsin, Kan-yiu | 榆 H.

\section{澉}

3101 Kan-p'u 澉浦, chên near Hai-yen H., Chelikiang; the "Ganfu" of Marco Polo.

\section{干}

3102 Kan1-yao 干窑, chên near Chia-hsing Fu, Chehkiang.

3103 Kan-tun |墩, chên near $\mathrm{Ch}^{6}$ in-p' $1 \mathrm{H}$., Kiangsu.

幹

3104 Kan-lu-hn-11u | 魯忽奴, (obs.) in Clihli; Ming, so in Tung-shêng 東勝 military district, Slınnsi.

\section{邗}

$3105 \mathrm{Kan}$ 干阝, see No. 7308.

3106 Kan-kon | 溝, see No. 3171.

扞

3107 Kan1-111 扞彌, see No. 3227.

\section{扞}

3108 Kan-ch'êng 扞城, chïn in Chiang-yiian 江 原 circ.; Corea. 
扞

K.x-chue.

Kan-chan, see Nos. 3079, 3099.

Kan-ngăı, see No. 3095.

Kan-suh, see No. 3089.

Kan-tsiuen, see No. 3080.

Kan-yu, see No. 3100 .

Kanagawa, see No. 5611.

Kanazawa, see No. 1049.

\section{K'AN}

堪

3109 K'an-ts'un 堪村, chên near Ch' ang-hua H., Chelnkiang.

\section{閔}

3110 K'an-t uan-chi 臂傳集, ss $\breve{k}$ near Chien-p ing $\mathrm{H}$., Anhui.

\section{KANG}

\section{岡}

3111 Kang 䎟, see No. 2757.

3112 Kang-shan | LL Okayama, ken and chief town, Japan.

\section{風}

3113 Kang-ti 呞氐, (obs.) E. of Ping-wu H., Ssŭch' 'nan; Han, circ. in Kuang-han 廣漢 chün.

Kang-tsin, see No. 3117. Kang-sar, see No. 2105.
高

K.10.

\section{K'ANG}

康

3114 K'ang 康, see Nos. 4039, 6309.

$3115 \mathrm{~K}^{\prime}$ ang-ch' êng | 城, chèn near Shih-lon H.. Shansi.

3116 K'ang-ch'iao | 橋, chên near Fu-ping H., Slicnsi.

3117 K'ang-ching / 津 Kangtsin, H. in Ch' üant-lo 全 羅 circ., Corea; Lat. $34^{\circ}$ $54^{\prime}$, Long. $125^{\circ} 30^{\prime}$.

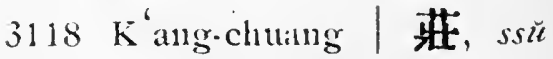
near Chênu-fềng C., Kneichon.

$3119 \mathrm{~K}^{\prime}$ aning-lê | 樂.

$1^{\circ}$ (obs.) Tsin, H. $20 l i$

F. of Wants :ii H., Kiangsi.

$2^{\circ}$ See No. 6905.

$3119^{\prime} \mathrm{K}$ 'ingrping | 平 H. in $\mathrm{Ch}^{\prime}$ ang-t" $" \mathrm{~F} n$, Shingkingr. $3120 \mathrm{~K}$ 'ang-sung | 碩 $\mathrm{H}$. in Huany-hai 黃海 circ. Corea.

$3121 \mathrm{~K}^{\prime}$ anş-tien / 店, sšr in Nin1-an H., Fuhbien.

$3122 \mathrm{~K}^{\prime}$ 'ang-tso | 佐, ssĭ near Chên-fêng C., Knueichon.

Kanibarlan, see No. 4791.

\section{KAO}

高

3123 Kao 高 Kau. 
$1^{\circ}$ H. in Hsü-chon Fin, Ssŭch uan; I at. $28^{\circ}$ $18^{\prime}$, Isong. $104^{\circ} 33^{\prime}$; orig., Yeh-lang 液郎. $2^{\circ}$ (obs.) S. Sung, C. in Kuang-lisi 風西 circ. $3^{\circ}$ (obs.) Liang, C. near I,ê-an H., Kiangsi.

$4^{\circ}$ See No. 3135.

3124 Kao-an 努 Kan-ngan, H. forming the prefectural city of Jui-chon, . Kiangsi ; Lat. $28^{\circ} 25^{\prime}$, Long. $115^{\circ}$ 18'; Han, Chien-ch 'êng 建城: E. Han, Shangts ai 上蔡; $T^{\prime}$ ang; Kaoal 安.

3125 Kao-ch'ai | 柴.

$\mathrm{l}^{\circ} \mathrm{Y}$, near Chiang-hua $\mathrm{H}$., Human.

$2^{\circ}$ (obs.) in Kiangsin: $W$. Han, Marq. in P'ei 沛 chün.

3126 Kao-ch'ang | 昌.

$1^{\circ}$ (obs.) Wu, town 50 li W. of Chi-an $\mathrm{F}$, Kiangsi.

$2^{\circ}$ See No. 1900.

3127 Kao-ch'ê | 車。

$1^{\circ}$ (obs.) near Fêng-hsiang

Fin, Shensi; $N$. $W_{e i}$,

H. in Wu-tu 武都 chïn.

$2^{\circ}$. See No. 7005.

3128 Kao-ch'êng | 成, see No. $-5982$.

3129 Kao-ch'êng | 城. $1^{\circ}$ Chün in Chiang-yüan 江原 circ., Corea.

$2^{\circ}$.Ss̆̈ in Sui C., Hupeh.

$3^{\circ}$ see Nos. 3175, 7352.

3130 Kalo-ch'i | 㟢 Takasaki. ken in Japan; chief town Gumuna.

3131 Kao-chia | 蒙, ihên in Tan-tu H., Kiangsu.

3132 Kau-chia-clíang | 家第: ssü near $\mathrm{Cl}^{\prime}$ ien-chiang $\mathrm{H}$., Hupelı.

3133 Kao-ch'iao | 橋.

$1^{\circ}$ Chên near Lini-t'ung H., Shensi.

$2^{\circ} S_{s}$ ŭ in Pilo-shan H., Kiangsur.

$3^{\circ}$ Ss̆̆ in IVu-lısi H., Kiangsu.

$4^{\circ} S$ s̆̆ in Hai C., Kiangsu.

.3134 Kao-ch'iao-ho | 橋 河, ss ̌̆ near Hai C., Kiangsu.

$313 t^{\mathrm{A}} \mathrm{Kao}-\mathrm{chih}$ | 知 Kòchi, ken and chief town, Japan.

3135 Kao-chou | 州 Kan-chau, [468] Fu in the Kao-Lei-Yang circ., Kuangtung ; forming also the district city of Ion-ming; Lat. $21^{\circ} 48^{\prime}$, long. $110^{\circ} 26^{\prime} ; T^{\prime}{ }^{\prime} i n$, in Nan-hai 南海 chiun ; Han, Kao-liang | 凉; Three Ks., Kao-lising | 興; Lians, Kao | C.

3136 Kao-chü-li | 句 麗, see Nos. 363, 3805. 3137 Kao-chü-li | 句騕. 
䯩

$1^{\circ}$ (obs.) N.E. of Hsienlising Fin, Corea; $T$ sin, H. in Yüan-t $n$ 元 营 chiin.

$2^{\circ}$ (obs.) N. of Hsingching, Shingking; Han, H. in Y Yian-t $n$ 元 菟 chiin .

3138 Kao-ch' $\ddot{\text { ì }}$ |渠.

$1^{\circ}$ Chên near Pin C'., Shensi.

$2^{\circ}$ (obs.) $W$. Wei, H. near Yen-t'ing $H$, Ssŭch' 'uan.

3139 Kao-fêng | 紫, (obs.) in Kansuh ; Sung, pao in Hsi-an 西安 C., Shensi.

3140 Kao-ho|河, chên near Ch'ang-tzŭ H., Shansi.

3141 Kao-hsien | 縣, chên near $\mathrm{Ch}^{\prime}$ ii-wu H., Shansi.

3142 Kao-hsing | 興, see No. 3135.

$3142^{\mathrm{A}}$ Kao-kê | 柯, ssĭ in Liyang $H .$, Hunan.

3143 Kao-lei-yang | 雷陽,circ. in Kuangtung comprising Kao-chou Fu: Lei-chou $\mathrm{Fu}$, and Ching-yang C.L.T.

3144 Kao-li | 麗, see Nos. $363,3805$.

3145 Kao-liang | 凉, see Nos. 3135, 5696, 7257.

3146 Kao-liang-ch'êng | 梁城. $1^{\circ}$ (obs.) Han, H. near Hêng C., Kuangsi.
高 KAO-PI.

$2^{\circ}$ (obs.) 2nd Wei, $\mathrm{H}$. near Chi-shan $H$. , Shansi.

3147 Kao-ling | 陵 Kau-ling. [79] $1^{\circ}$ H. in Hsi-an Fn, Shensi; Lat. $34^{\circ} 30^{\prime}$, Long. $109^{\circ} 04^{\prime}$; Ts'in, Kaoling | 陵; Han, L,11yiian 鹿苑。

$2^{\circ}$ Town in the Lin-ch'in Islands.

3148 Kao-ling | 靈 $H$. in Ch'ing-shang 慶攸 circ., Corea.

3149 Kao-liu | 流 chên, in Muyang $\mathrm{H}$., Kiangsu,

3150 Kao-lung | 陪, sš̆ near $\mathrm{Cl}^{6}$ a-ling C., Hunan.

3151 Kao-mi | 密 Kan-mih, [349] H. in Chia C. L. C. Shantung; Lat. $36^{\circ} 23^{\prime}$, Iong. $120^{\circ} 11^{\prime}$; Han and S. Sung, Yi-an 英安 H.; N. Wei, Kao-mi | 密rhün. 3152 Kao-ning / 明 Kan-ming, [556] H. in Chao-ch'ing $\mathrm{F} u$, Kuangtung; Lat. $22^{\circ} 51^{\prime}$, Jong. $112^{\circ} 18^{\prime} ;$ Sui, Ch'ing-t'ai 清泰.

\section{Kao-nu | 奴.}

$1^{\circ}$ (obs.) E. of Fu-shil H., Shensi ; Han, H. in Shang 上 chün. $2^{\circ}$ See No. 94.

3154 Kao-pai | 白, chên near Hsü-kou H., Shansi.

3155 Kao-pi | 敞 H. in Ch' 'ïanlo 全羅 circ., Corea. 
高

KAO-P'ING.

3156 Kao-p'ing | 平 Katl-ping. '[443] $1^{\circ} \mathrm{H}$. in 'Tsê-chou Fu, Shansi ; Lat. $35^{\circ} 45^{\prime}$, Long. $112^{\circ} 43^{\prime} ; C$. Wars, Cli'ang-p'ing 長 尔; Han, Hsüan-slil 泣氏; $N$. Ts' $i$, Kaopng | 平; $T^{6}$ ang, $\mathrm{Kai}$ 蓋 C!

$2^{\circ}$ Post-town near P'ingliang Fu, Kansuh.

$3^{\circ}$ Post-town near Kuangning $\mathrm{H}$., Shingking.

4: (obs.) $W u$, town S. of Hsin-hna H., Hunan.

$5^{\circ}$ See Nos. 441, 3288, 6.546 .

3157 Kito-shan | |山.

$1^{\circ}$ Post-town near Tat'ung H., Shansi.

$2^{\circ}$ H. in Ch'üan-lo 全羅 circ., Corea.

3158 Kao-shun | 湆 Kau-shun, [445] H. in Chiang-ning Fu, Kiangsu ; Lat. $31^{\circ} 28^{\prime}$, Long. $118^{\circ} 58^{\prime}$; Ming, $\mathrm{H}$. in Ying-t'ien 應天 $\mathrm{F} n$.

3159 Kao-t'ai | 壷 Kau-tai, H. [334] in Su C., Kansuli; Iat. $39^{\circ} 25^{\prime}$, Long. $100^{\circ} 17^{\prime}$; Ming, so in Shensi.

3160 Kao-t'ang | 唐 Kantang. [284] $1^{\circ} \mathrm{C}$. in Tung-ch 'ang Fiu, 'Shantung; Lat. $37^{\circ} 05^{\prime}$, Long. $116^{\circ} 19^{\prime} ;$ S.A. Kao-t'ang | 唐; Han, Kao-t'ang | 唐 $\mathrm{H}$.;
宫 KAO-YANG.

T゙ang, Ch'ung-wu 䕀 武; Five dyn., Yüich'iu 魚邱 and $\mathrm{Cl}^{\circ} \mathrm{i}$ ch'êng 呍城.

$2^{\circ}$ See No. 204.

3161 Kao-t'ang | 塘, see No. 5916.

3162 Kao-ti | 隄.

$1^{\circ}$ Chên near Nei-huang H., Honan.

$2^{\circ}$ (obs.) E. of 'Tsaoch'iang H., Chilhli;

Han, H. in K. Hsin$\mathrm{tu}$ 信都.

3163 Kao-tien |古, chên near Mei H., Shensi.

3164 Kao-ts'ai |才, ssü near Ta-tien H., Fuhkien.

3165 Kao-ts'un | 村, ss̆̌ near Fêng-hnang T., Hunan.

$3166 \mathrm{Kao}$-tu | 都.

$1^{\circ}$ Chên near Kao-p ing H., Shansi.

$2^{\circ}$ (obs.) 30 li N.E. of Fêng-tai H., Shansi ; Han and $T \sin , H$. in Shang-tang 上黨 chion;

N. $W_{i} i$, chiin and $\mathrm{H}$. in Chien 建 CHOU.

$3^{\circ}$ See No. 7666.

3167 Kao-tzŭ|資, chên near Chên-chiang $\mathrm{Fu}$, Kiangsu. 3168 Kao-yang | 陽 Kaulyang. $1^{\circ} \mathrm{H}$. in Pao-ting $\mathrm{Fu}$, Chihli; Lat. $38^{\circ} 44^{\prime}$, I.vilg. $115^{\circ}$ 56'; Han, Kilo-yang | 陽; Sui, Man 滿 C. 
$2^{\circ}$ H. in Ching-ch i 京畿 circ., Corea.

$3^{\circ}$ Post-town near Wushan H., Ssŭch 'uan.

$4^{\circ}$ (obs.) Han, town near Kao-111 H., Shantung. $5^{\circ}$ (obs.) Sung, town near Ho-clien Fu, Chihli.

$6^{\circ}$ (obs.) formerly tow near Ch'i $\mathrm{H}_{\text {. }}$ Honan (No. 623).

$7^{\circ}$ See No. 2984.

3169 Kao-yao | 要 Kall-yau.

$1^{\circ} \mathrm{H}$. forming the prefectural city of Chaocl 'ing Fu, Kuangtung; Lat. $23^{\circ} 05^{\prime}$, I,ong. $112^{\circ} 30^{\prime} ;$ Han, Kao-yao 要; S Sti, Tuan 端 C. $2^{\circ}$ See Nos. 335, 5.392 .

3170 Kao-yi | 邑 Kat1-yih.

[145] $1^{\circ}$ H. in Chao C., Chihli ; I,at. $37^{\circ} 40^{\prime}$, I,ong. $114^{\circ} 47^{\prime} ; H a n$, Han 鄗 H.; E. Han, Kaoyi 邑.

$2^{\circ}$ See No. 6514 .

3171 Kao-yu / 䑶 Kan-yu, C. in [322] Yang-chon Fu, Kiangsu; Lat. $32^{\circ} 46^{\prime}$, Long. $119^{\circ}$ 22'; Wu, Han-kon 干乃溝; $T \sin , \mathrm{Ch}$ 'ing-yu 秦郵; Han, Kao-ru | 郵. Cavu (Polo).

3172 Kao-yï | 魚, (obs.) formerly town near Yüuch êng H., Shantung. \\ 郜}

3173 Kao-yüan | 原.

$1^{\circ}$ (obs.) Yüan, H. in。 Hsing-ho 與利 circ.

$2^{c}$ Chïn in Hsien-ching 咸鏡 circ., Corea。

3174 Kao-yüa11 | 苑 Kat1-yuen [183] H. in Ch'ing-chou Fu, Shrontung; Lat. $37^{\circ} 10^{\prime}$, Lone. $118^{\circ} 12^{\prime}$; orig.. l'ian-ch'iang 苑墙; Hon, Kao-yian! 苑; 1st. Sung, Cha ang-lê 長樂; Sui, Hui-cli êng 偣城.

\section{韾}

3175 Kao-cliêng 宫城 Kan[143] ching. H. in Chêng-ting Fu, Chillit; Lat. $38^{\circ} 05^{\prime}$, Long. $114^{\circ} 59^{\prime}$; Han, Kao-clı'êng | 城; $N . T s^{\prime} i$, Kao-ch'êng 高城; Sini,

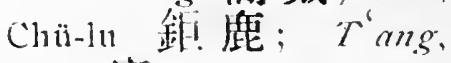
I ien 筆 $\mathrm{C}$.

\section{泉}

3176 Kao-lan 翡闌 Kau-lan, $\mathrm{H}$. forming the prefectural city of Lan-cliou, Kansulu ; Lat. $36^{\circ} 08^{\prime}$, Long. $113^{\circ}$ $55^{\prime}$.

317 Kao-yii | 虞, (obs.) 50 li N. E. of Clii-mo H., Shantung; W. Han, Marq. in Lang-yel 郎躯 chiin.

\section{郜}

3178 Kao-ch'êng 郜城, chên near' Têng-fêng H., Honan. 


\section{K'AO}

\section{考}

3179 K'ao-ch'êng 考城 Kan[148] ching, H. in Wei-hni Fu, Honan; I.at. $34^{\circ} 47^{\prime}$, Long. $110^{\circ} 26^{\prime} ;$ S.A., Kn-ch'êng 踒城; T's ' Tzŭ 淄 C.; E. Han, $\mathrm{K}^{\prime}$ : 10 -ch' êng / 城.

$3180 \mathrm{~K}$ ao-yang $/$ 陽, see No. 7450 .

\section{㛈}

3181 K'ao-1mu㛈姥, chinn near P'"l-chou Fu, Shansi.

\section{KAR}

Karakash, see No. 1899.

Karakliodjo, see No. 1900.

Karakormm, see No. 1901.

Karakoto, see No. 1897.

Kartubé, see No. 1895.

Kash, see No. 3193.

Kashan, see No. 3202.

Kashgar, see No. 3221.

Kassan, see No. 3201.

Kasvin, see No. 3232.

Kath, see No. 3203.

Kau, see No. 3123.

Kall-chat1, see No. 3135.

Kall-ching, see Nos. 3175 , 3179.

Kall-lan, see No. 3176.

Kall-ling, see No. 3147.

Kảl1-milı, see No. 3151 .

Kall-ming, see No. 3152.

Kall-ngan, sce No. 3124.
Kau-ping, see No. 3156. Kau-shun, see No. 3158. Kall-tai, see No. 3159.

Kan-tang, see No. 3160. Kan-yang, see No. 3168. Kan-yan, see No 3169. Kall-yih, see No. 3170. Kan-yu, see Xo.. 3171. Kall-yueu, see No. 3174. Kazerun, see No. 3238. Kelung see No. 565.

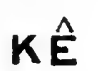

\section{浩}

3182 Kê-n1ên 浩䡒 (obs.) E. of Nien-po H., Kansuh; Han and $T \sin , \mathrm{H}$. in Clin-ch'êng 金城 chïn.

\section{渴}

3183 Kê-shih 渴西, see No. 3193.

\section{葛}

3184 Kê-chao 葛趙, chen near P'ing-lu H., Shansi. [5177.]

3185 Kê-ku|. 沽 Koku, Y. near ' $T$ 'ien-ch'ing Fu, Chihli.

3186 Kê-kung | 公, chên near Chien-tê H., Anhui.

3187 Kê-lu | 盧, (obs.) near Lai-chou Fu, Shantung; E. Han, H. in Tung-lai 東萊 chün。 


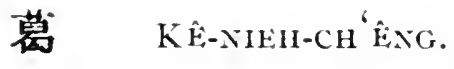

3188 Kê-nieh-ch'êng | 熞城, (obs.) formerly town near Kuang-p'ing Fu, Chihli.

3189 Kê-po | 伯.

$1^{\circ}$ (obs.) formerly $\mathrm{K}$. in Honan.

$2^{\circ}$ See No. 4697 .

3190 Kê-yang | 陽, see No. 7517.

\section{葛}

3191 Kê-ma-lan 噶瑪蘭 Kabaran (written Kabatah in Williams' Map of China), $T$. in ' $T$ 'ai-wan $F n$, ForInosa ; Lat. $24^{\circ} 37^{\prime}$, Lonn. $122^{\circ}$. Now Yi-lan 宜蘭 I-lan, H., in T'ai-pei Fu, Formosa. See No. 7430.

3192 Kê-pu-la | 有i喇, posttown near Fêng-t'ien Fu, Shingking.

3193 Kê-shih | 石, or 渴石, or Chieh-shil 磶石, Kash, town in Independent Turkestan, S. of Samarcand.

3194 Kê-slıun | 順 Gachom ; there are several towns of this name in Mongolia; one is in Outer Kansulı; I at. $41^{\circ} 30^{\prime}$, Long. $94^{\circ}$ $15^{\prime}$.

\section{滑}

3195 Kê-ho 渦河, chên near Fu-ning H., Kiangsu.

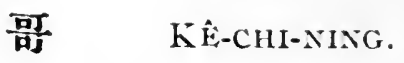

3196 Kê-yang | 晹 Ko-yang, $\mathrm{H}$. in Ying-chon $\mathrm{Fu}$, Anhui ; Lat. $33^{\circ} 35^{\prime}$, Long. $116^{\circ} 13^{\prime}$; see No. 4374 .

柯

3197 Kê-ch' êng 柯 城, (obs.) $S$. $A$., town near Neihsiang H., Honan.

3198 Kêeclı'iao | 憍, ss̆̈ in Shang-yin H., Chehkiang.

3199 Kê-11111-1i | 模里, see No. 1907.

3200 Kê-san | 三, see No. 3201.

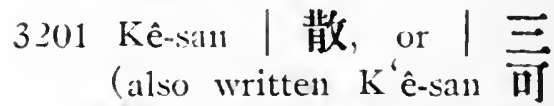
散 or 可冞) Kassan, town in Independent Turkestan; 1at. $41^{\circ}$, Long. $71^{\circ} 30^{\prime}$.

3202 Kê-shang | 傷 Kashan, town of Persia, $N$. of Ispalian.

3203 Kê-t ${ }^{\prime} \mathrm{i} \mid$ 提 Kath, a town in Independent Turkestan.

\section{渮}

$320+$ Kê-tsê 渮澤 Ko-tsell, $\mathrm{H}$., forming the prefectural city of Ts ao-chou, Shantung; Lat. $35^{\circ} 20^{\prime}$, Long. $115^{\circ} 36^{\prime}$.

\section{哥}

3205 Kê-chi-ning 哥疾察 Ghizning or Ghazna, town in Afghanistan. 


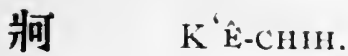

\section{牊}

3206 Kê-chih 师可 支, (obs.) S.W. of Lı̈ C., Ssŭclı' uan ; $T^{6}$ ang, H. in Sung 尔 c.

3207 Kê-lung/龍, (obs.) S.IV. of İ̈̈ C., Ssŭch'uan; $T^{\prime}$ ang, H. in Sung 柇 C.

3208 Kê-wei | 違, (obs.) in Ssŭch' 'uan; Sung, Kê-wei | 逗 C.

\section{蓝}

3209 Kè-shilh-tsa 草什咱, 'T-S. near Ta-chien-lu T. Ssŭch uan.

3210 Kê-wa| 所, 'T-S. near Sunng-p an T., Ssŭch "nan.

3211 Kê-yung | 融, (obs.) near Yüi-lin Fu, Shensi; $N$. Wei, H. in Hual-ch' êng 化城 chiin.

\section{蒚}

3212 Kê 南, (obs.) formcrly $\mathrm{K}$. near P'ing-ÿuan $\mathrm{H}$., Shantung.

3213 Kê-ching | 津, see Nos. $3652,7352$.

\section{格}

$321^{\circ} 4$ Kê 格, (obs.) Sung, C., near Huai-yüan H., Knangsi.

3215 Kê-chieh | 結, T-S. near Mieni-ning H., Ssŭch uan.

3216 Kê-êrh-chi | 尔 吉, T-S. in Kokonor.

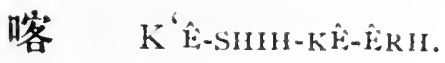

3217 Kê-kên | 根 Jeghen, town in Ili; Lat. $43^{\circ}$, Long. $80^{\circ} 40^{\prime}$.

\section{閣}

3218 Kê-mên 閣壃, (obs.) in Kansuln; T'ang, C. in LA111y-y' 隴右 circ.

\section{箅}

3219 Kê-sinilh-111i 䇢失密, see No. 658.

\section{$K \cdot \hat{E}$}

\section{喀}

$3220 \mathrm{~K}$ 'ê-êrlh-ch' ${ }^{2}$ in 喀尔沉, or 科尔沈, the Khorchin tribes of Mongols near Heh-lung-kiang province.

$3221 \mathrm{~K}^{\prime} \hat{\mathrm{c}}$-êrh-k'ê爾喀Khollkil IIongol 'Tribe.

$3222 \mathrm{~K}$ "ê-êrhl-lit-ch"ius | 爾喇 沈 Khalrachin, IIongol Tribe. See No. 1898.

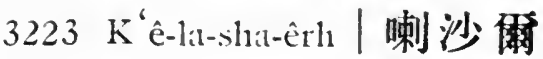
Harashar, C.J.'T. in atk $\div u$ Circuit, New Dominion. See No. 1904.

3224 k'ê-shih-kê-êrh | 什噶 尔, (or K'ê-shih-ha-êrh 可 失哈耳, or $\mathrm{K}^{\prime} \mathrm{e}-\mathrm{s}$-shil1-hatli 可失哈里, or Ha-shihho-êrh "哈實哈兒, or Chia-shil1 迦師,) Kashgar, also called Lai-ning 徠 泶。 


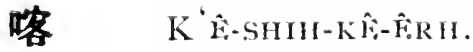

$1^{\circ}$ Town in Yarkand; Lat. $39^{\circ} 25^{\prime}$, long. $73^{\circ}$ $5 \%^{\prime}$. Cascar (Polo). Now Su-lê C.I.C. (No. 5924).

2 Circnit in the New Dominion; comprising Yingeshar C. I. 'l., Inapash C.L.'T.. Sullê C.I.C... So-ch ê C.I.C. and Khoten C.I.C.

\section{克}

$3225 \mathrm{~K}$ ê-êrh-lin-hsi-lao 克尔 林尾老 Kirlentsiloa, tow11 in Dsassaktu; I Iat. $48^{\circ}$, Long. $95^{\circ} 50^{\prime}$.

$3226 \mathrm{~K}^{*} \hat{\mathrm{e}}$-jung $\mid$ 我, (obs.) 60 Ii W. of Sui-tê C., Shensi ; Sung, walled town in Suitê 綏德 military district, Shensi.

$3227 \mathrm{~K}^{\prime} \mathrm{e}-\mathrm{li}-\mathrm{y} \mathrm{a}$ |里雅.

$1^{\circ}$ Keldia, town in Yarkand; Lat. $36^{\circ} 05^{\prime}$. Long. $83^{\circ} 30^{\prime}$.

$2^{\circ}$ Kirrea, towu in Yarkand; Lat $36^{\circ} 15^{\prime}$; Long. $83^{\circ} 20^{\prime}$. Also written Kan-mi 扞彌.

$3228 \mathrm{~K}^{6} \hat{\mathrm{e}}-\mathrm{shih}-\mathrm{k}$ " | 什 庫, post-town near Chiench'ang H., Chihli.

$3229 \mathrm{~K}^{\prime} \hat{\mathrm{e}}$-shih-k' $\mathrm{e}-\mathrm{t}^{\prime} \mathrm{e}$.11 $\mid$ 什克 鶽 Keshikteng, Mongol Tribe.

\section{科}

3230 K'ê-erh-ch'in 科尔沈, see No. 3220 .
岢 $\mathrm{K}^{\prime} \hat{\mathrm{E}}-\mathrm{LAN}$.

3231 K'ê-pu-to | 布悉 Cobdo. $1^{\circ}$ A Khanate in Mongolia; bounded on the $\mathrm{N}$. by Siberia, on the E. by Dsassaktu, on the S. by 'Tarbagatai, and on the II. by Siberia and Tarbagatai.

$2^{\circ}$ The chief town of the preceding; Isit. $47^{\circ}$ $40^{\prime}$, I ong. $89^{\circ} 30^{\prime}$.

\section{可}

$3232 \mathrm{~K}^{\prime} \mathrm{e}-\mathrm{cli}-\mathrm{y}$ in 可疾云 Kasrin, or Khezovin, town of Persia, between Teheran and 'Tabriz.

$3233 K^{\prime}$ ê-pu-li | 不里, Kabul, town in Afghanistan.

3234 K'ê-san | 散, see No. 3201.

3235 K'ê-san | 茶, see No. 3201.

3236 K'ê-shih-ha-êrh |. 失哈 耳, see No. 3224 .

$3237 \mathrm{~K}^{\prime} \hat{e}$-shih-ha-li | 失哈里, see No. 3224.

3238 K'ê-tsa-lung | 咱隆 $\mathrm{Ka}$ zerun, town of Persia, in province Fars.

\section{荘}

3239 K'ê-lan 㟧颳 Ko-lan, C. [236] in T'ai-yüan Fu, Shansi ; I at. $38^{\circ} 52^{\prime}$, Long. $111^{\circ}$ $23^{\prime}$; Han, Fên-yang 汾陽; Wei, Lan 嵐 C.; $T^{\prime} a n g$, Lan1-ku 嵐谷. 
Keldia, see No. 3227.

Kemkenjonte, see No. Si8. Kemkentchik, see No. 878.

\section{KÊN}

根

3240 Kên-shih 根窒 Nenuro, ken and chief town, Japan.

\section{KÊEG}

邢

3241 Kêng-cli $\hat{e n}^{\prime}$ 那城, see No. $32+3$.

\section{耿}

3242 Kêng 耿.

$1^{\circ}$ (obs.) Shang, K. in Shansi.

$2^{\circ}$ See Nos. 583, $206,6$.

3243 Kêng-ch'êng | 城（ancientl! also written 邢 城), (obs.) s'hang, the capital of the Empire; in Shansi.

\section{梗}

3244 Kêng-yang, 梗陽, see No. 1262.

\section{K'ÊNG}

\section{坑}

3245 K'êng-pu 坑埠, chên near Sing-shan H., Anlui.

Kenjanfu, see No. 2438. Kerman, see No. 656.
Kermané, see No. 627.

Keshikteng, see No. 3229.

Khalkha, see No. 3221.

Khamil, see No. 1907.

Khar, see No. $32 j 0$.

Kharachin, see Nos. 1898. 3222 .

Khavar, see No. 2150.

Khezovin, see No. 32.32.

Klochid, see No. 2004.

Khodjend, sce No. 2.14.

Khoit, see No. 2374.

Khorchi1, see No. 3220.

Khoten, see No. 2057.

Khuar, see No. 3270.

Khutaiyai, see No. 2176.

Khutukbai, see No. 2176.

Ki, see Nos. 57t, 576 , $580,616,623,645,652$.

Ki-kiang, see No. 619.

Ki-mun, see No. 654.

$\mathrm{Ki}$-shan, see No. 641.

Ki-shwui, see No. $6+8$.

Ki-tai, see No. 634.

Ki-ting, see No. 642.

Ki-tseh, see No. 567.

Ki-yang, see No. 655.

Kia, see No. 715 .

Kia-hing, see No. 699.

Kia-ho, see No. 697.

Kia-i, see No. 707 .

Kia-slran, see No. 702.

Kia-shen, see No. 703.

Kia-siang, see No. 698.

Kia-ting, see No. 705.

Kia-ying, see No. 708.

Kia-yu, see No. 709.

Kia-yu Kwan, see No. 710. 
KIACH'TA.

Kiachta, see No. 717 .

Kiah, see No. 727.

Kial1-chuen, see No. 2576.

Kiah-kiıng, see Nos. 724, 2579.

Kiai, see Nos. 829, 832. Kiai-chuen, see No. 821 . Kiai-hi11, see No. 818.

Kiang, see Nos. 731, 769. Kiang-chuen, see No. 736. Kiang-hia, see No. 741. Kiang-hwa, see No. 739. Kiany-lan, see No. 743 . Kiang-ling, see No. 744. Kiang-nan, see No. 746. Kiang-11gan, see No. 732 . Kiang-11ing, see No. 750. Kiang-peh, see No. 750. Kiang-pu, see No. 753. Kiang-slian, see No. 754. Kiang-si, see No. 740. Kiang-su, see No. 755. Kiang-tsin, see No. 735. Kiang-tu, see No. 757. Kiang-tung, see No. 758. Kiang-yin, see.No. 760. Kiang-yu, see No. 761. Kiau, see No. 794. Kiall-ching, see No. 784. Kiau-ho, see No. 787. Kiau-kia-ying, see No. 811 . Kie-ngan, see No. 835. Kieh-shih, see No. 839. Kieh-yang, see No. 838 . Kien, see Nos. 874, 880, 920.

Kien-chang, see No. 847. Kien-chau, see No. 922.
KING-MUN.

Kien-chi, see No. 861. Kien-chuen, see No. 883. Kien-kiang, see No. 903. Kien-li, see No. 893. Kien-ngan, see No. 846. Kien-ning, see No. 859 . Kien-ping, see No. 860 . Kien-shwui, see No. 862. Kien-si, see No. 905. Kien-tel, see No. 863. Kien-wei, see No. 871. Kien-yang, see Nos. 867, 900, 90\%.

Kih, see No. 583.

Kih-lu1, see No. 593.

Kih-ngan, see No. 584.

Kih-shwui, see No. 590.

Kin, see Nos. 1007, 1060, 1095.

Kin-chau, see Nos. 1016, 1062.

Kin-hai, see No. 1021. Kin-hiang, see No. 1025. Kin-hwa, see No. 1023. Kin-ki, see No. 1014. Kin-kwei, see No. 1029. Kin-ping, see No. 1065. Kin-shan, see No. 1041 . Kin-tan, see No. 1045. Kin-tang, see No. 1046. King, see Nos. 1112, 1130. King-chau, see Nos. 1142, 1185.

King-fu, see No. 1186. King-hing, see No. 1187. King-ki, see No. 1141. King-ki-tau, see No. 363 King-mu11, see No. 1145 . 
King-Ning.

King-ning, see No. 1135 . King-shan, see No. 1125. King-tel1-chên, see No. 1136.

King-tung, see No. 1138. King-yang, see Nos. 1114, 1191.

King-yuen, see Nos. 1192, 1193, 1194.

King-yun, see No. 1195. Kinsay, see No. 1993.

Kiol1-shan, see No. 1504.

Kioto, see No. 1127.

Kiri11, see No. 586.

Kirlentsiloa, see No. 3225. Kirmanshalıan, see No. $65 \%$.

Kirrea, see No. 3227.

Kish, see No. 840.

Kiu, see No. 1300.

Kill-kiang, see No. 1281.

Kin-luh, see No. 1414.

Kill-peh, see No. 1303.

Kint-tsi, see No. 1410.

Kil1-yé, see No. 1415.

Kiuh-chau see No. 1453.

Kiuh-fau, see No. 1454.

Kiuh-kiang, see No. 1450. Kiuh-tsing, see No. 1452. Kiuh-yang, see No. 1464. Kiuh-yu, see No. 1463.

Kiun, see No. 1521.

Kiung, see No. 1309.

Kiung-chau, see No. 1321.

Kitıng-shan, see No. 1322.

Ko-ku, see No. 3185.

Ko-lan, see No. 3239.

Ko-tseh, see No. 3204.
口 K'OU-WAI-J角-HO.

Ko-yang, see No. 3196. Kobé, see No. $5607^{\wedge}$. Kôchi, see No. $3134^{A}$. Kôfu, see No. 718. Koh, see No. 3538. Kokand, sec No. 6103. Kokonor, see No. 1206.

\section{KOU}

分

3246 Kon1-chang 句章, see Nos. $6447,6863,7560$.

\section{狗}

3247 Konl-cl' ang 狗 場, Y. near Hsing-ri Fu, Kueichous.

\section{K'OU}

\section{䌊}

3248 K'ou1-shih 緱 氐, (obs.) C. Wars, $\mathrm{H}$. near Honan Fin, Honan.

\section{口}

3249 K'ou-pei 口北, circ. in Chihli comprising Hsüanhua Fu, Chang-chia-k'on T., Tu-shilh-k ou T. and To-lun-no-êrh $T$.

3250 K'oul-wai-Jê-ho | 外熱河, circ. in Chihli comprising Ch' êng-tê Fu (Jehol), Chaoyang $F u$ and the Imperial cattle and horse-farms in the North of the province. 
辟寊

Ku-cu' Uax.

\section{KU}

\section{顀}

3251 Ku-ch'nan | 顧川, chên in Chiang-yin $\mathrm{H}$., Kiangsu.

\section{古}

$3252 \mathrm{k} u$ 古, (obs) sung, C., N. W. of Li-p ing Fu, Kueichon.

3253 Kli-cliang-ping | 文坪 (or | 仗坪) Kn-cliangping, 'T. in Yung-shun Fu, Hunan; Lat. $28^{\circ} 35^{\prime}$, Long. $107^{\circ} 30^{\prime}$.

3254 Kn1-ch' êng | 城.

1 Erutchen (or Ha-lê-kèa-111an 哈勒噶阿瑞 Kalyanan), town in Harashar ; I att. $41^{\circ} 30^{\prime}$. Long. $86^{\circ} 50^{\prime}$.

2" Chèn ne:ir 'T"ali-p'ing H., Shansi.

. Ssü near 'l'ing-chon I'u, Finhkien.

$1{ }^{\circ}$ Post-town near 'l’: yüan H., Kiangsu.

$5^{\circ}$ Ss $\breve{u}$ in Chi-t ai H., Kansuh.

3255 Kul-ch'êng-ch'ü| 城渠, 'T-S. near P'ing-fan H., Kansuh.

3256 Ku-chou | 州 Ku-chau.

[473] $1^{\circ}$ T. in Li-p'ing Fu, Kueichou; Lat. $25^{\circ} 50^{\prime}$, Long. $108^{\circ} 45^{\prime}$.

$2^{\circ}$ T-S. riear Li-p ing F 11 , Kueichou.
顧

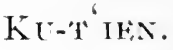

3257 Ku-fu| 卓. Ku-fau, chün in Ch'üan-lo 至羅 circ., Corea ; i.at. $35^{\circ} 42^{\prime}$, Long. $125^{\circ} 45^{\prime}$.

$3258 \mathrm{Ku1-fu|}$ 符, (obs.) in Yüilin C., Kuangsi; Tang, H. in 'Tang 黨 C.

3259 Kn-lunng | 洪, town in Inner Mongolia; I at $41^{\circ}$ $36^{\prime}$, Long. $99^{\circ} 05^{\prime}$.

$3260 \mathrm{Kn}-\mathrm{lang}$ | 浱 Kuslang, $\mathrm{H}$. in Liang-chou $\mathrm{Fu}$, Kaulsul, Lat. $37^{\circ} 30^{\prime}$, Long. $103^{\circ} 05^{\prime}$.

3261 Ku-lang-fan | 浪番, T-S. near the preceding.

3262 Krt-lin-chon/闎州, ssu in Hsŭ-yung 'T., Ssŭch' 'nan.

$3263 \mathrm{Kn}$-ling | 寒, T-S. near Ssŭ-ên Fu, Kuangsi.

3264 K11-pai-shn | 柏樹, T-S. near Yen-yïan H., Ssŭch' tian.

3265 K11-shu|書, (obs.) in Krangsi ; Trang, H. in $\mathrm{Ku} \mid \mathrm{C}$.

$3266 \mathrm{Ku}-\mathrm{su}$ | 蘇, post-town near Su-chon Fu, Kianysu.

3267 Ku1-t'a-pa | 塔巴, see No. 2176.

3268 Ku-t'ien | 田 Kn-tien.

[41] $1^{\circ}$. H. in Fin-chou Fin, Fulkien ; Lat. $26^{\circ} 41^{\prime}$, Long. $118^{\circ} 54^{\prime}$; orig., Hou-kuan 侯 官; T'ang, Ku-t'ien | 田. $2^{\circ}$ See No. 4707. 
倠我

Ke-Tu.

3269 Kul-tu | 都, (obs.) in Annam; T゙ang, Ku-tu | 都 $\mathrm{H}$.

3270 Ku-wa-êrh | 酉耳, kh,u, or Khurar. a city of Persia, S.E. of 'Teheran.

3271 Kil-yeh | 冾, (obs.) Han, H., N. of Eitu-chou Fu, Fuhkien.

3272 Ku1-yu-p ing | 敒坪, posttown near $\mathrm{Cl}^{\prime}$ ên-chou $\mathrm{Fu}$, Hunan.

3273 Ku-yung 雃, see No. 1724

\section{㚲}

327+ Ku-slun 姑 新, see No. 6169.

\section{故}

3275 K11-in 故安, (obs.) S.E. of Yi C., Chibli; Han, H. in Cho 涾 chän; Tsin, H. in k. Fan-yang 范陽。

3276 Ku-chang | p章, see Nos. $50,250$.

3277 Ku-ch'êng | 城 Kn-ching, H. in Ho-chien Fu, Chillli; Lat. $37^{\circ} 29^{\prime}$; Long. $116^{\circ}$ 16'; orig., $\mathrm{Cl}_{1}{ }^{\prime} \mathrm{ing}$-ho 清 河; $\mathrm{Han}, \mathrm{Cl}_{1}{ }^{\prime} \mathrm{i}$ 厝 $\mathrm{H}$. and Kan-ling 甘陵; Sui, Li-t'ing 歷亭; Kin, Kul ch'êng | 城.

3278 Ku-shang-shil |上石, T-C. near Ning-ming C., Kuangsi.
閑 Кレ゙-sแแ1.

\section{沽}

$3279 \mathrm{~K} 11$ 沾; sometimes the $N$. $W_{e i}$, Clan 活 H., is erroncously so written.

\section{沽}

$3280 \mathrm{Ku}$ 诂, chên near Yiclı êng H., Sliansi.

3281 Ki1-an | 努 K11-11gan, H. [549] in Shlun-t ien I'u, Chihli: I at. $39^{\circ} 25^{\prime}$. I ong. $116^{\circ}$ $22^{\prime} ;$ Han, Fang-ch'êng 方城; Sui, Ku-an.

3282 Kn-chên | 鐄, ssü in Ling-pi H., Anhui.

3283 Ku-ch ĉngr | 城.

$1^{\circ}$ Chên near Ning-süin H., kiangatu.

$2^{\circ}$ Chèn newr kilo-shnn H. Kiallgsu.

3 (abs.) formenly town near 'Ting C., Chihli.

$328+$ kn-cliang | 江. chen in I. ü-ling H., Kiangi.

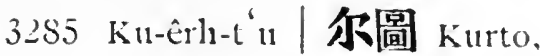
town in 'Aarbagatai: I,alt. $44^{\circ} 15^{\prime}$, Iong. $54^{\circ} 40^{\prime}$.

3286 Ku-ling | 陵, see $\mathrm{No}$. $3+91$.

3287 Ku-shil| 始 Ku-chi. H in Kuang C., Honan: Lat. $32^{\circ} 18^{\prime}$, Long. $115^{\circ}$ $37^{\prime} ;$ S.A., K. I,iao 葓: $H a n, \mathrm{Ch}^{i}$ in 將 $\mathrm{H}$; $E$. IIan, K11-shih|始. 
固

KU-YÜAN.

3288 Ku-yüan | 原 Ku-yuen, C.I.C. in P'ing-Cln'ingChing circ, Kansuh ; 'Lat. $36^{\circ} 04^{\prime}$, Itong. $106^{\circ} 21^{\prime}$; Han, Kao-ping 高平; $T^{6}$ ang, Yrüan 原 C.; Yüan, K"ai-ch'êng 開城; $\operatorname{Ming}$; Kn-ÿian | 原 $\mathrm{C}$.

\section{崮}

3289 Ku-shan 臭!山, post-town near Chang-ch'ing H., Shantung.

\section{賈}

3290 Ku-chia 貿 家, see No. 711.

3291 Ku-ch'ï | 曲, sce No. 712 .

$3292 \mathrm{~K} n-\mathrm{fu}-\mathrm{ch}{ }^{6} \hat{\mathrm{e}} \mathrm{ng}$ | 復城. (obs.) formerly town $\mathrm{N}$. of $\mathrm{T}$ 'ang $\mathrm{H}$. , Cliblili.

3293 Kn-ling | 令, chên near Chi H., Shansi.

3294 Knts in | 村, see No. 713.

\section{鼓}

3295 Ku-cl' êng 鼓城, see No. 1073.

$32.96 \mathrm{Kultzŭ} \mid$ 子, (obs.) S.A., $\mathrm{K}$. comprising Chin $\mathrm{C}$., Chihli.

\section{㙰}

3297 Ku-chung 韾塚, chên near Y'ïan-ch 'ii H., Shansi.
谷 $\mathrm{KU}-\hat{\mathrm{E}} \mathrm{RH}-\mathrm{PA}$.

\section{折}

3298 Ku 㧓, (obs.) T'ang, C. 40 li S.E. of P'ing-lê Fu, Kuangsi.

3299 Ku-chu| 竹, sce No. 4154.

3300 Kn-shan ||山, ssŭ in Fênghuang T., Shingking.

3301 Ku-shu|抒, (obs.) near Turfan; T"ang; Fu in L rung-yul 隴右 circ.

3302 Ku-yiu-shu| 榆樹, ssü in Pedué T., Kirin.

3303 Kn-yiun | 星, (obs.) in Ssŭclı' nan; T'ang, H. in '111 影孤 $\mathrm{C}$.

\section{孤}

3305 K11-ch'êng 孤城, (obs.) formerly town $\mathrm{S}$. of $\mathrm{Hu}^{-}$ chon Fu, Chehkiang.

\section{骨}

3306 Ku-lung 滑龍, (obs.) Yiüan, N.' of Kuei-yang Fin, Kueichou.

\section{合}

3307 Ku-ai 谷 隘, post-town near Ch'ung-an H., Fuhkien.

3308 Ku-ch'êng | 城, H. in Ch'iian-lo 全羅 circ., Corea.

3309 Ku-êrh-pa | 尔埧, T-S. near Sung-p'an T., Ssŭch'van. 
谷 $\mathrm{Ku}-\mathrm{K}$ 'ov.

3310 K11-k'on | 口, see No. 3734 .

3311 Ku-lung | 龍, T-S. near Kuei-ting H., Kueichon.

3312 Kn-shan | I Fin in Huang-hai 黃海 circ., Corea.

3313 Kn-tan | 旦, chên near Mêng H., Honan.

3314 K 11-yang | 陽, see No. 6209.

\section{㓽}

$3315 \mathrm{Ku}$ 稁.

$1^{\circ}$ (obs.) Kin, C. near Ch' in C., Shansi.

$2^{\circ}$ See Nos. 3317, 3432.

3316 Ku-ch'ang | 昌, (obs.) Han, H. $10 l i \mathrm{~N}$. of Yïnnan Fin, Yünnan.

3317 Ku-cli êng | 城 Ku-ching, $\mathrm{H}$. in Hsiangryang $\mathrm{Fu}$, Hupeh; Lat. $32^{\circ} 18^{\prime}$, Long. $111^{\circ} 40^{\prime} ;$ orig., land of $\mathrm{K}_{11} \mid$; Han, Chuyang 筑陽; Sui， $\mathrm{K}_{\mathrm{u} \text { - }}$ cli êng | 城; $T$ 'ang, Tso 贊 $\mathrm{C}$.

3318 Kn-shui | 水, post-town near Lan-ch'i H., Chehkiang.

3319 K11-yiian | 遠, see Nos. 1103, 7618.

Kul-chang-ping, see No. 3253.

Ku-cha11, see No. 3256.

Ku-chi, see No. 3287 .
庫 $\mathrm{K}^{\prime} \mathrm{C}-\hat{\mathrm{E}} \mathrm{R} H-\mathrm{K}^{\prime} \hat{\mathrm{K}}-\mathrm{L}-\mathrm{L}$

K11-ching, see Nos, j277, 3317.

Ku-fun, see No. 3257.

Kungan, see No. 3281.

$\mathrm{K}_{11-t i e n,}$, see No. 3268.

Ku-yuen, see No. 3288.

\section{$\mathbf{K} \cdot \mathbf{U}$}

\section{苦}

$3320 \mathrm{~K}^{\prime} \mathrm{u}-\mathrm{ch}^{\prime} \mathrm{a}$ 苦叉, see No. 3328.

$3321 \mathrm{~K}^{6}$ 11-clian | 蓝, see No. $2+14$.

$3322 \mathrm{~K}^{-11}$-chn | 竹, (obs.) Wu, town S. of Shaolising Fu, Cliehkiang.

$3323 \mathrm{~K}^{6}$ 11-chut-pa | 竹埧, T-S. near Hui-li C., Ssŭch uan.

$332+\mathrm{K}^{6} \mathrm{n}-\mathrm{fa}$ | 法 Kufa, town 25 miles $\mathrm{S}$. of the ruins of Pabylon.

$3325 \mathrm{~K}^{\prime} 11-$ shui | 水, post-town near Lan-clron Fu, Kansuh.

$3326 \mathrm{~K}^{6} \mathrm{n}$-tzŭ $\mid$ 子, Y. near Yung-ming H., Hunan.

$3327 \mathrm{~K}^{\prime}$ ur-yang | 骝, (obs.) Tsin, town near Mêng H., Honan.

\section{㮺}

$3328 \mathrm{~K}^{\prime} \mathrm{u}$-ch'ê車車, or K'uch'a 苦叉, Kuchê, C.L.C. in Aksu circuit, New Dominion. Lat. $41^{\circ} 37^{\prime}$, Long. $82^{\circ} 55^{\prime}$.

$3329 \mathrm{~K}^{\prime} \mathrm{n}$-êrh-k' $\mathrm{e}-\mathrm{la}$ | 尔喀喇 烏蘇, Kurkara-usu, also 
庫 $K^{\prime} \mathrm{U}-\hat{\mathrm{H}} \mathrm{RH}-\mathrm{K}^{\prime} \hat{\mathrm{k}}-\mathrm{L} \mathrm{A}$.

called Ching-sui 慶綏; $T$. in Sui-ching Fn, 'Tarbagatai. Lat. $44^{\circ} 15^{\prime}$, Long. $85^{\circ} 10^{\prime}$.

3330 K' $11-e ̂ r l 1-l e ̂ \mid$ 尔勒; Kurli, [205] town in Harashar; Lat. $41^{\circ} 20^{\prime}$, long. $86^{\circ} 10^{\prime}$.

$3331 K^{\prime} 11-k^{\prime} \hat{e}-k u-y e h \mid$ 克古 野, tow 11 in Yarkand; Lat. $41^{\circ} 20^{\prime}$, Isong. $82^{\circ}$ $10^{\prime}$.

$3332 \mathrm{~K}^{\prime}{ }^{11-1 u n}$ | 倫 Kurun, also called Urga, town in Tuchetu: Lat. $47^{\circ} 55^{\prime}$, Jong. $106^{\circ} 50^{\prime}$.

\section{Kü}

Kü, see Nos. 1422, 1437. Kü-cha11, see No. 1445. Kü-yung, see No. 1409.

\section{KUA}

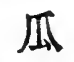

3333 Kua 瓜, see No. 70.

3334 Kua-chon 州.

$1^{\circ}$ Chên near Yang-chou $\mathrm{F} u$, Kiangsu. Caiju (Polo).

$2^{\circ}$ Y. near An-hisi C., Kansuh.

3335 Kua-ning | 临, (obs.) formerly town near Paoclii H., Shensi.

3.336 Kua-pieh | 刷, T-S. near Yen-yüan H., Ssŭch' nan.

3337 Kua-pu | 倌, chên in Liu-ho H., Kiangsu.
官

KUAN-SHUI.

\section{括}

3338 Kua 括, see No. 1396.

3339 Kua-ts'ang, | 蒗.

$1^{\circ}$ Post-town near $\mathrm{Ch}^{\prime} \mathrm{n}$ chou $\mathrm{Fu}$, Chehkiang.

$2^{\circ}$ See Nos. 1224, 1396, 3738 .

\section{K'UAI}

\section{四}

3340 K' nai-hsi 非西.

$1^{\circ}$ T-S. near Li-po H., Kueichou.

$2^{\circ}$ (obs.) Yüan, H. near Kuei-yang Fu, Kueichou.

$3^{\circ}$ See No. 3057.

\section{KUAN}

\section{官}

3341 Kuan-ch'ang 官鹪, chên near Yüan-ıw H., Honan.

3342 Kuan-chou | 州, Y. near T'ung-jên Fu, Kueichou.

3343 Kuan-chuang | 庄, chên near Wu-ping H., Ful1kien.

3344 Kuan-chün | 軍, chên near Ch'in-yüan H., Shansi.

3345 Kuan-fı | 富, sš̆ near Hsin-an $\mathrm{H}$, Kuangtung.

3346 Kuan-nu-ch êng | 奴域, (obs.) Han, town E. of Ning-po Fu, Chehkiang.

3347 Kuan-shui | 水, chên near T'ai-ping H., Shansi. 
官 KLAN-T'Axg.

3348 Knan-t'ang | 塘, posttown near $\mathrm{P}^{\prime} \mathrm{u}$-chl ${ }^{\prime} \mathrm{i} \mathrm{H}$. Hupeh.

3349 Kuan-tao | 道, chên near Fu-p'ing H., Shensi.

$3350 \mathrm{~K}_{\text {uan-ti }}$ 底, chên near Fr1-p ing H., Shensi.

3351 Kuan-tien | 店, chên near Mêng H., Honan.

3352 Kuan-t'ing | 亭, chên in Ho-fei H., Anhui.

3353 Kuan-tu-pao | 渡堡, sš̆ near Pao-k'ang H., Hupeh.

\section{館}

3354 Kuan-cli ‘ien 館前, posttown near $\mathrm{T}^{6}$ ing-chou $\mathrm{Fu}$, Fulkien.

3355 Kuan-t'ao | 陶 Kwan-tau. [496] $1^{\circ}$ H. in Tung-ch' ang Fu, Shantung; Lat. $36^{\circ} 42^{\prime}$, Long. $115^{\circ} 39^{\prime} ;$ S. A., Kuan-shih-yi 冠氏邑; Han, Kuan-t'ao | 陶; P. Chou, Mao 毛 C. $2^{\circ}$ See No. 3367 .

\section{管}

3356 Kuan 管, see Nos. 495, 1172.

3.357 Knan-ch' êng | 城, posttown thear Cliêng C., Honan; Sui to Yiüan, H.

3358. Kuan-chieh | 界, ssŭ in Chên-hai H., Chehkiang.
KUAN.

\section{灌}

3359 Knan 灌 Kwan, H. in [307] Chia-ting Fu, Ssŭch' 'uan; Lat. $30^{\circ} 59^{\prime}$, Long. $103^{\circ}$ $4.2^{\prime}$; Winor Han, Tulan, 都努; T“ang, P'an-lung 盤龍, Tao-cliang 導 and $\mathrm{K}_{\text {uan }}$ / C.

3360 Knan-ching | 津 or 觀津. $1^{\circ}$ (obs.) S.E. of Wu-r.i H., Chihli; IV. Han, H. in K. Hsint-tu 信 都; E. Han and Tsin, H. in K. An-p'ing 安 本; N. Hei, H. in Wu-yi 武邑 chün. $2^{\circ}$ (obs.) 25 li S. E. of Wri-yi H., Chilhli; Kin, chên in Chi 冀 C.

3361 Knan-k ou-chai | 口砦, post-town near Cli'ang-t'ai H., Fuhkien.

3362 Kuan-yang | 陽 Kwan[223] yang, H. in Kuei-lin Fu, Kuangsi ; Lat. $25^{\circ} 22^{\prime}$, Long. $110^{\circ} 59^{\prime}$; Han, Ling-ling 零陵; $W u$, Kuan-yang | 隄.

3363 Kuan-ying-ch 'êng | 嬰城, (obs.) formerly town $\mathrm{E}$. of Nan-ch'ang Fu, Kiangsi.

\section{觀}

3364 Kuan 觀, (obs.) Sung, C., E. of Nan-tan T. C., Kuangsi ; see ạ̣so No. 6727. 
觀

KUAN-CH'

3365 Kuan-ch'êng | 城, Kwancling, H. in T's ao-chon Fin, Shantung; I at. $36^{\circ}$, Long. $115^{\circ} 37^{\prime}$; Hsia, K. Shao-k'ang 少康; $H a n$, Pan-k11an 㫠|;E. Han, K. Wei 衛; Sui, Kua11ch'êng | 城.

3366 Knan-cling | 津, see No. 3360 .

\section{它}

3367 K11an 赾, Kwan, H. in Tung-ch ang Fu, Shantung; Lat. $36^{\circ} 33^{\prime}$, Long. $115^{\circ} 39^{\prime}$; S.A.,' Kuanshih-yi 氐岳; Han, Krian-t'ao 館陶; Yüan, Kuan | C.

3368 Kuan-clıüı | 軍, (obs.) 40 i N. W. of 'Têng C., Howan; Han, Tsin, $S$. Sung, S. Ts $i$ and Sui, H. in Nan-yang 南陽 chün; N. Wei, H. in 1st Nan-yang 南陽 chïn, 2nd Ln1-yang 鼻晹 chün.

3369 Knan-sliih-yi | 氏邑, see No. 3367 .

\section{關}

3370 Knan-cli êng 關城.

$1^{\circ}$ Chên near Chên-yiian H., Kansul.

$2^{\circ}$ Post-town near Lnanclı'êng H., Clinili.

3371 Knan-chung |中, see Nos. 2438, 5487.
寬 K'UAN.

3372 Kuan-hsi | 西. $1^{\circ}$ (obs.) Han, chiïn W. of Hsi-an Fu, Shensi. $2^{\circ}$ (obs.) E. of Hua-yin H., Shensi ; Kin, chên in Hua 華 C. $3^{\circ}$ See No. 1724.

3373 Knan-k'on | 口, Y. near Kuei-hua T., Kueichou.

3374 Knan-li | 䍵, see No. 490. 3375 Kuan-ling-lisïn | 嶺汎, post-town near Y111g-1ning c., Kneichon.

3376 Kran-nei | 內.

$1^{\circ}$ (obs.) Trang, circ., formerly Yung 䔨 CHOU.

$2^{\circ}$ See No. 2438.

3377 Kuan-shan| IU, chên in Lin-t'ing H., Shensi.

3378 Knan-t 'ou | 頙, chên near Yung-shon H., Shensi.

3379 Knan-t11ng | 東.

$1^{\circ}$ A general name for the Manchurian provinces. $2^{\circ}$ (obs.) $50 \quad l i \mathrm{~W}$. of Wên-lisiang H., Honan; Kin, chên in Shan 傸 C.

3380 Knan1-tzŭ|子, chên near Fu-cl' iang H., Kausuh.

3381 Kuan-yang | 陽, chên in Pi H., Sliantung.

\section{K'UAN}

\section{寬}

3382 K' 1an 蜜, see No. 1237 . 


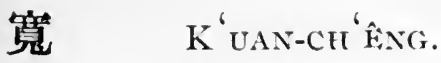

3383 K' 11an1-clı'êng | 城, post-

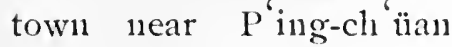
C., Chinli.

3384 K'uan-kon | 尠, posttown near $\mathrm{P}$ ing-fan II., Kansuh.

3385 K' 'nan-tien | 旬, H., in Fêng-huaig C. L. T., Shingking.

\section{KUANG}

\section{鹿}

3386 Knang 廣 Kwang.

$1^{\circ}$ C. in Corea ; Lat. $37^{\circ}$ $50^{\prime}$, Long. $127^{\circ} 23^{\prime}$.

$2^{\circ}$ (obs.) S. Sung, C. in Kuang-tung cire.

$3^{\circ}$ See Nos. 3393, 4109.

3387 Kuang-a | 阿], see No. 4247.

3388 Knang-an | 桇 Kwangan.

$1^{\circ} \mathrm{C}$. in Shun-ch ing Fin, Ssŭch'nan; Iat. $30^{\circ}$ $31^{\prime}$, Long. $106^{\circ} 39^{\prime}$; Han, 'Yang-ch'ii 岩渠 and An-kon 安拲; Sung, Kllang-an| 安; military district in T'ung-ch'nan 潼川 circ.; Yüan, Clï̈chiang 渠 江 $\mathrm{H}$.

$2^{\circ}$ Chên near 'la-ch' êng H., Chihli.

$3^{\circ}$ Ss $\breve{k}$ in Kuang-tê C., Anluni.

$4^{\circ}$ See No. 6670.
贋 Kuang-chou。

3389 Knang-ch'ang / 昌 Kwangchang.

[107] $1^{\circ}$ H. in Yi C., Chihli; Lat. $39^{\circ} 34^{\prime}$, Long. $114^{\circ} 20$; orig., Feihu-k'ou飛狐口; Han， Kuang-ch'ang | 昌; P. Chou, Wn-lung 五龍.

[509] $2^{\circ} \mathrm{H}$. in Chien-ch ang Fn, Kiangsi ; Lat. $26^{\circ} 45^{\prime}$, Long. $116^{\circ} 12^{\prime}$; orig., Nan-fêng 南豐; Sung, Knang-ch'ang | 昌.

3390 Knang-Chao-I, 庴倠羅 circ., in Kuang-tung, comprising Knang-chon Fu, Chao-cl' ing $F_{12}$ and Loting C.I.C.

3391 Kuang-ch'êng | the

$1^{\circ}$ Chên near Ning C., Kansul.

$2^{\circ}$ See No. 2697.

3392 Knang-chi | 湾 Kwangtsii, H. in Huang-chon Fu, Hupeh; Lat. $30^{\circ} 10^{\prime}$, Long. 115 38 ; Han, $\mathrm{Ch}^{6} \mathrm{i}-\mathrm{ch}^{6} \mathrm{nu}$ 暲春; $T^{6}$ ang Knang-chi | 䛚.

3393 Knang-chon 州 Kwangchan.

$1^{\circ}$ Canton; Fin in the Kuang-Chao-Lo circ., Kuangtung ; forming also the two district cities of Nan-hai and $\mathrm{P}^{6}$ an-yï ; the capital of the province; Lat. $23^{\circ}$ $08^{\prime}$, L ong. $111^{\circ} 17^{\prime}$; 
Literary names Yangcl'êng 羊城 and Suiyiuan 㯖 坦; S.A., Yang-ch êns 羊 城; Ts'in, in Nan-hai 南 海 chïn; Wu, P'an 畨 C.; T'ang and Sung, Ch'ing-hai 清 海; Ming, Kuang-chou | 州 Fu。

$2^{\circ} \mathrm{Fu}$ in Ching-ch i 京 䋨 circ., Corea.

$3393^{\mathrm{A}}$ Knang-chon Wan | 州 灣, bay on the north side of Lei-chon Peninsula, Kuangtung; annexed by France in 1897.

3394 Kuang-ch'nan | 川, see Nos. 1130, 6130.

3395 Kuanı̀ofêng | 噩 Kwangfung.

[211] $1^{\circ}$ H. in Kuang-hsin Fin, Kiangsi ; I at $28^{\circ} 28^{\prime}$, Long. $118^{\circ} 06^{\prime}$; orig.

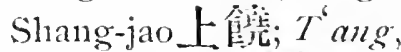
Yung-fêng 永豐.

$2^{\circ}$ See No. 1737.

3396 Kuang-lian | 漢, see Nos. $511,19+2,5059,6795$, 7354.

3397 Knang-lina $\mid$ 化. $1^{\circ}$ (obs.) Hu, H., N. W. of Yang-chiang 'l', Kuangtung.

$2^{\circ}$ See No. 2357.

3398 Knang-hisi | 西 Kwang-si.

$1^{\circ}$ A province of China; literary nanles I,ing-y u
領右, Clien-ling 建 西 ; capital，Kuei-lin Fu; it contains 3 circ., 11 Fin, 1 C.L.T., 6 T., 2 C.L.C., 16 C., 29 T. C., 49 H., and 3 T.H.

[355] $2^{\circ}$ C. I. C. in Yi-tung circ., Yümnan; Lat. $24^{\circ}$ $40^{\prime}$, Long. $103^{\circ} 50^{\prime}$; orig., K. Tien 滇; Han, Yi 䀂. C. and 'T'sang-kê 梌柕 chiun ; T゙ang, Chi-1111 墕麼 C.; Yüan, Kuang-hsi 西 Fu.

3399 Kunng-hsi | 熙, (obs.) $100 l i \mathrm{~S}$. of Lo-ting C., Knangtung; S. Ts ${ }^{\prime} i$, chiün in Kntang | chov.

3400 Knang-hisin | 信 Kwangsiil.

[211] $1^{\circ} \mathrm{Fu}$ in the Kuang-JaoChin-Nan circ., Kiangsi; forming also the district city of Shangjao ; Lat. $28^{\circ} 28^{\prime}$, Long. $118^{\circ} 06^{\prime} ;$ S.A. and $C$. Wars, in $\mathrm{K}$. $\mathrm{W}_{\mathrm{u}}$ 吳 and $\mathrm{K} . \mathrm{Cl}^{\prime} \mathrm{u}$ 楚; Ts' ${ }^{\prime} \mathrm{n}$. in Knei-chi 會稽 chunn; Han, Yï1-kan 餘汗 and T'a-mo 太龙 H.; T"ang and Sung, Hsin 信 C.; Ming, Kuanglisin | 信 Fu.

$2^{\circ}$ Ses Nos. 1701, 6526. 
腐 KUANG-HSING.

3401 Kuang-hsing | 興, see No. 5576.

3402 Knang-Jao-Cluin-Nan | 鲜 九南, circ. in Kiangsi, comprising Kuang-lisin Fu, Jao-chou Fu, Chiucliang Fu and Nan-k'ang Fur.

3403 Kuang-jên | 仁 Kwangjin, town in Ili; Lat. $44^{\circ}$ $05^{\prime}$, Long. $82^{\circ} \quad 10^{\prime}$.

3404 Kuang-jon | 柔, see No. 5704.

3405 Kuang.lê | 樂, see No. 94. 3406 Kuang-ling | 陵.

$1^{\circ}$ Post-town near Yangchou $\mathrm{Fu}$, Kiangsu.

$2^{\circ}$ See Nos. 757, 1593, 2244, 3016, 6406.

3407 Kuang-ling | 霝 Kwangling, H. in 'Ta-t 'nung Fin, Shansi; Lat. $38^{\circ} \cdot 46^{\prime}$, Long. $114^{\circ} 21^{\prime}$; Han, Ven-ling 延陵; T゙ang, Au-pien 安邊; Five dyn. Kwang-ling | 靈.

3408 Knang-ming | 明, (obs.) Tang, H., E. of Vi-ning H., Knangsi.

3409 Kiang-na | 納, (obs.) T'ang, H., S. of 'T'ungchiang H., Ssŭch'uan.

3410 Knang-nan | 南 Kwang[416] natr, $F t$ in the Lin-K'aiKuany circ, l'ünnan; forming also the district city of l'ao-ning; Iat. $24^{\circ}$
庶 Kuang-P'ING.

10', Long. $105^{\circ} 06^{\prime}$; Sung， T'ê-mo 特磨; Yiian, Kuang-nan | 南 Fi1.

3411 Kuang - Nan-Shao-Lien |

南韶連, formerly circ. in Kuang-tung, comprising Kuang-chou Fu, Nanhsiung C., Shao-chou Fu and Iien $C$.

3412 Kuang-ning | 察 Kwangning.

[416] $1^{\circ} \mathrm{H}$. in Chao-cli ing Fu, Kuangtung; Lat. $23^{\circ}$ $39^{\prime}$, Long. $111^{\circ} 59^{\prime}$.

$2^{\circ}$ H. in Chin-chou I'u, Shingking; Lat. $41^{\circ}$ $40^{\prime}$, Long. 122 ; Han, Wu-lü 無慮 $\mathrm{H}$.

3413 Kuang-p’ing | 本 Kwangping.

[128] $1^{\circ}$ Fin in the 'ra-Shut1Kuang circ., Chiluli; forming also the district city of Yuny-nien; Iat. $36^{\circ} 46^{\prime}$, Long. $114^{\circ}$ $55^{\prime} ;$ S.A., in K. Clin 晉; C. Wars, in $\mathrm{K}$. Cliao 趙; Ts'in in Han-tan 林 鄲 chün; Han, Kuang - p ing | 平; Sui, IVu-an 武 安; Trang, Ming 洛 C.; Ming, Kuang-p'ing 平 $\mathrm{Ft}$.

[10] $2^{\circ}$ H. in Knang-p ing Fin, Chihli; Lat. 36 34'. Long. $115^{\circ} 06^{\prime}$. 
3414 Kuang-shou | 㗲, see No. 7969.

3415 Kuang-shui-tien | 水古, post-town near Ying-sli:ın H., Hupeh.

3416 Knang-shun | 順 Kwang[24] shun, C. in Kuei-yang Fu, Kueichon; I at. $26^{\circ} 0 S^{\prime}$, Long. $106^{\circ} 14^{\prime}$.

$3416^{\mathrm{A}}$ Kuang-tao $\mid$ 耑 Hiroslinu, ken and chief town, Japan. 3417 Knang-tê / 德 Kwang-teh. $[417] 1^{\circ}$ C.L.C. in the Hui-NingCh'ilı - T'ai - Knang - têchou circ., Anlu1i; Lat. $31^{\circ}$, Long. $119^{\circ} 23^{\prime}$; S.A., 1st, 'T'ung-jui 相 㑂 in $\mathrm{K}$. Wn 叁; 2nd, in K. Yiieln 越 and K. $\mathrm{Cl}^{\prime}{ }^{\prime} \mathrm{n}$ 楚; Ts'in, in Chang 鄣 chïn; Han, Kuang-tê | 德; Liang, Sliil-fèng 石 封 and Ta-liang 大粱; Sui,

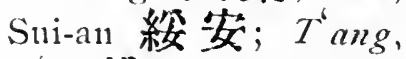
'T'ao 桃 C. and Hsïan 宣 C.; Ming, Kuang-tê 鲉。

$2^{\circ}$ (obs.) S. Sung, military district in Chiangtung 江 東 circ.

$3^{\circ}$ See No. 3828.

3418 Knang-ting | 定, see No. 5316.

3419 Knang-tsung | 佘Kwangtsung.

$1^{\circ}$ H. in Shun-tê $\mathrm{Fu}$, Chihli ; Lat. $37^{\circ} 10^{\prime}$,
Long. $115^{\circ} 18^{\prime}$; Han, T'ang-yang 堂陽; Minor Han, 'TsungCh'êng 宗城; T'ang, 'sn11ng 宗 C.; Yüan, Kuang-tsung | 宗 $\mathrm{H}$. $2^{\circ}$ (obs.) $20 l i \mathrm{E}$. of Wei H., Chihli; E. Han, H. in Cliü-lu 鉋豦 chïn; $T \sin$, Marq. in K. An-ping 安本; N. Wei, Kulang-tsung | 宗 chïn and $\mathrm{H}$.

$3^{\circ}$ (obs.) near Chi-nan $\mathrm{Fu}$, Shantung; S. Sung and $N$. Wei, H. P'ingyüan 平原 chün。

3420 Kuang-tu | 都.

$1^{\circ}$ (obs.) S.E. of Ch 'êngtı H., Ssŭch' 'uan; Han, Tsin, S. Sung and $S . T s^{\circ} i, H$. in Shu 蜀 chïn。

$2^{\circ}$ (obs.) WV. of An-su H., Clinhli; $N$. IVei, H. in Chien-tê 建 德 chün. $3^{\circ}$ (obs.) in Knang-ning H., Shingking; $N$. Wei, H. in Chien-tê 建德 chïn.

$4^{\circ}$ (obs.) 7 li S. E. of Slunang-liı H., Ssŭch'nan; T'ang and Sung, H. in Ch' êng-tı 成都 Fu。

3421 Kuang-t11ng | 東 Kwangtu1ng. 
廣

KUANG-TUNG.

$1^{\circ}$ A province of China; literary names Pai-yüeh 百然，Ling-nan 嶺南 and Yüeln-tung 宩東; capital, Kuang-chou Fu; it contains 5 circ., 9 Fu, 2 C.L.'T., 9 T., 4 C.I.C., 7 C. and $78 \mathrm{H}$. $2^{\circ}$ Sung and S. Sung, circ.

3422 Kuang-t' ung | 通 Kwangtung.

[27 ] $1^{\circ} \mathrm{H}$. in $\mathrm{Cl}_{1}{ }^{\prime} \mathrm{u}-1, \operatorname{sinumg} \mathrm{Fu}$, Yünnan; I at. $25^{\circ} 10^{\prime}$, Long. $101^{\circ} 55^{\prime}$; orig., Ln1-t'an 路賧.

$2^{\circ}$ Chên near Kao-shun H., Kiangsu.

3423 Kuang-wei | 威; (obs.) S. E. of Hsi-ning $\mathrm{H}$., Kansuh; Tang, H. in $\mathrm{K}$ "no 部 $\mathrm{C}$.

3424 Kuang-wu | 武.

$1^{\circ}$ Y. near Chung-wei H., Kansul.

$2^{\circ}$ Military post near Laian H., Anluti.

$3^{\circ}$ Post-town near Jung-tsê H., Honan.

$4^{\circ}$ See Nos. 495, 6157, 7754 .

3425 Kuang-wu-ch'êng | 武城. $1^{\circ}$ Ss̆̆ near Ning-wu Fu, Shansi.

$2^{\circ}$ (obs.) Sui, town near

Lan-chou Fu, Kansul.

3426 Kuang-yang | 陽.
光

KUANG.

$1^{\circ}$ (obs.) Han and $T^{\circ}$ ang, chïn comprising parts of Chihli and Shansi.

$2^{\circ}$ (obs.) 2nd $\mathrm{Wei}, \mathrm{H}$. near Lin-t'ung $H$., Shensi.

$3^{\circ}$ See Nos. 5792, 5822.

3427 Kuang-yü| 框, see No. 3473.

3428 Kuang-yüan | 源, (obs.) in Knangsi; Sung, C. in Yung 画 $\mathrm{CHOU}$.

3429 Kuang-yüan | 元 Kwangyuen, H. in Pao-ning Fu, Ssŭch' 'uan; Lat. $32^{\circ} 20^{\prime}$, Long. $105^{\circ} .57^{\prime} ; T^{6}{ }^{\circ} \mathrm{in}$, Chia-mêng 碾萌; Minor Han, Han-shon 漢䩧; 2nd $W e i, H \operatorname{sing-an}$ 興安; Liang, Li 黎 $\mathrm{C}$; $W$. Wei， Li 利 C.; Sui, Mien-ku 緜谷; Yüan, Kuang-ÿüan | $\overrightarrow{\text { 兀 }}$ circ.; Ming, Knang-yüan | H.

\section{光}

3430 Kuang 光 Kwang.

[154] $1^{\circ}$ C.L.C. in the Nan-JuKuang circ., Honan; Lat. $32^{\circ} 13^{\prime}$, Long. $115^{\circ}$; S.A., in the feudal states Hsien 弦. Huang 黃 and Chiang 蔣; Han, Yi-yang 晹; Liang, Kuang C.; S. Sung, C. in Huai-11si 淮西 circ. 
光

$2^{\circ} \mathrm{C}$. in Ch'üan-lo 全羅 circ., Corea; Lat. $35^{\circ}$ $25^{\prime}$, Long. $126^{\circ} 04^{\prime}$.

3+31 Kuang-fu|丽畐, chên in Wu H., Kiangsu.

3432 Knang-hna | 化 Kwanghwa.

[133] $1^{\circ}$ H. in Hsiang-yang Fu, Hupeh; Iat. $32^{\circ}$ $27^{\prime}$, Long. $111^{\circ} 45^{\prime}$; orig., Ku 教; Han, Ts'uan 贊 $\mathrm{H}$; $\mathrm{W} i$, Yin-ch'êng 陰 城; Sung, Kuang-luua | 化. 2" (obs.) S. Sung, nilitary district in Clninghsi 京西.

3433 Kuang-slian | 山, H. in [154] Knang C., Honan; Lat. $32^{\circ} 08^{\prime}$, Long. $114^{\circ} 51^{\prime}$; S.A., Fendal state Hsien 弦; Han, Hsi-yang 西 陽；Tsin，Knang-shan| 山.

343+ Kuang-tsê | 涬 Kwangtseh, H. in Shao-wu Fu, Fuhkien; Lat. $27^{\circ} 32^{\prime}$, Long. $117^{\circ} 28^{\prime}$; orig. Shao-wu 別 武; Sung, Kuang-tsê | 湍.

3435 Kuang-wu-ch êng | 武城, (obs.) Han, town S. of Ho-11an Fu, Honan.

3436 Kuang-yang | 陽 H. in Ch'iianl-lo 全 羅 circ., Corea.

3437 Kuang-yi | 義, see No. 4567.
桂

KUEI-LIN.

\section{洸}

3438 Kuang-chiang, 洮江, ssü in Ying-tê H., Kuangtung.

\section{K'UANG}

\section{匡}

$3439 \mathrm{~K}$ 'uang 匡, (obs.) formerly C. near $Y u ̈-l i n ~ C$. , Kuangsi ; see also Nos. $7+31,7813$.

3440 K' 'wang - ch'êng | 城, see Nos. 283, 6165.

$344 \mathrm{i}$ K'uang-k'ou| | near Hsin-an H., Honan.

3442 K'uang-yi| 邑, see No. 279.

Kuché, see No. 3328.

\section{KUEI}

\section{桂}

3443 Kuei 桂.

$1^{\circ}$ (obs.) $T^{6}$ ang; Fu.

$2^{\circ}$ See No. 3446 .

3444 Kuei-ch i | 溪, (obs.) 30 li N. E. of Tien-chiang H., Ssŭch 'uan; T'ang, H. in Chung 忠 C.

3445 Kuei-k'ou | 口.

$1^{\circ}$ Ss̆̈́ near Ch'ang-ỹang H., Hupeh.

$2^{\circ}$ Ss̆̈ in T'ung-an H., Fuhkien.

34+6 Kuei-lin | 林 Kwei-lin. 
桂

KUEI-LIN .

[242] $1^{\circ}$ Fun in the Knei-P'ingWu-Yü circ., Kuangsi ; forming also the district city of Lin-knei ; Lat. $25^{\circ} 13^{\prime}$, Itong. $110^{\circ}$ $14^{\prime}$; capital of the province; orig., land of the Yüiel 䁠 tribes; $C$. Wars, forming the frontiers of K. Yüel

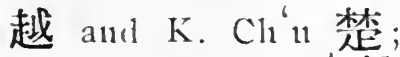
Ts'in, in Kuei-lin 林 chiun; E. Han and $W_{u}$, Shil1-an 始安; Liang, Knei|C.; Trang, Chienling 建 陵; Sung, Ching-chiang 静 江; Ning, Knei-lin | 林 Fin.

$2^{\circ}$ (obs.) Ts'in, chiin comprising part of the preceding.

$3^{\circ}$ See No. 3561.

344 i Kuei-ling | 嶺, (obs.) Sui, H., E. of Ho H., Knangsi.

3448 Knei-ping | 平 Kweiping, $H$. forming the prefectural city of Hsünchou, Kiangsi; Lat. $23^{\circ}$ 26', Long. 109 51': Han, Kuei-shan | 山: Sui, Kuei-p ing | 平.

3449 Kuei-P'ing-Wn-Y Yü | 本 梧穮, circ. in Kungsi, comprising Knei-lin fin. P'ing-lê Fu, Wu-chon Fu and Yü-lin $C$.
桂 Kuniraxg.

3450 Kuei-slan | 山.

$1^{\circ}$ Ss̆̌ in Wêng-y̆üan

H., Kuangtung.

$2^{\circ}$ See No. 3448.

3451 Kuei-t' un | 屯 Knni-tun, town in 'Tarbagatai; Lat. $44^{\circ} 10^{\prime}$, Long. $85^{\circ} 35^{\prime}$.

3452 Kuei-tung | 東 Kwei-tung. $1^{\circ} \mathrm{H}$. in $\mathrm{Ch}^{\prime} \hat{e ̂}_{11} \mathrm{C}$., Hunan; Lat. $26^{\circ} 0 t^{\prime}$, Isong. $113^{\circ} 3 t^{\prime}$; Tang, Yicliang 義且; Sung. Knei-tung | 東.

$2^{\circ}$ (obs.) near the preceding; Yüan and Ming, H. in C C ${ }^{\prime} \hat{e}_{11}$ 湘 C.

3453 Kuei-yang | 陽 Kweiyang.

$1^{\circ}$ C. L. C. in the HêngY'ung-Ch ên-Kuei circ., Hunan; Lat. $25^{\circ} 48^{\prime}$, Long. $112^{\circ} 23^{\prime}$; Han, Knei-yang | 陽: Tsin, P'ing-yang 平陽。

$2^{\circ}$ H. in Ch ên C., Honan; Lat. $25^{\circ} 35^{\prime}$, Long. $113^{\circ} 16^{\prime} ;$ Han, Ch' ${ }^{\prime} \hat{e ̂}_{11}$ 梆; Tsin, Ju-cli êng 汝 城; T゙ 義昌; Sung, Fueiyang | 陽

$3^{\circ}$ (obs.) Han, chïn in Knangtung.

$4^{\circ}$ (obs.) S. Sung, militars district in Hn-11an 湖 南 circ. $5^{\circ}$ See Nos. 3924, 5576. 


\section{歸}

3454 Kuei 菷 Kwei.

[528] $1^{\circ}$ C. in Yi-ch'ang Fit, Hupel1; Iat. $30^{\circ} 58^{\prime}$, I,ong. $110^{\circ} 58^{\prime}$; orig., in K. K' nei 櫒; $P$. Chou, Tzŭ-kuei 䄰 |; T'ang, C. in Shan-11an III南 circ.; Ming, C. in Ching-chou 荆州 Fu.

$2^{\circ}$ (obs.) near Ning-hsia Fu, Kansuh; T"ang, C. and H. in Chingpien 静邊 Fr1.

$3^{\circ}$ (obs.) S. of Knei C., Hupelı; Sung and Yüan, Knei | C.

$4^{\circ}$ (obs.) 90 li S. W. of Kai-p'ing H., Shingking ; Liao, C. in Tung-cling 東索 circ.

3455 Knei-an | 努 Kwei-ngan, $\mathrm{H}$. forming with Wuch'êng H. the prefectural city of Hu-chou, Chehkiang; Sung, Kuei-an | 安.

3456 Kuei-hua | 化 Kwei-hwa. [475] $1^{\circ}$ T. in So-p'ing Fu, Shansi; generally known as Kuei-huach'êng | 化 城; Lat. $41^{\circ}$, Long. $112^{\circ} ; T^{6}$ ang, Tung-shou-chiang 東 受降, walled town in Fêng 塭 C.
[326] $2^{\circ}$ T. in An-shun Fu, Kueichou ; Lat. $26^{\circ}$, Long. $105^{\circ} 25^{\prime}$.

[388] $3^{\circ}$ H. in ' $\mathrm{T}^{\prime}$ ing-chou $\mathrm{Fu}$, Fuhkien; Lat. $26^{\circ} 20^{\prime}$, Long. $117^{\circ} 19^{\prime}$.

$4^{\circ}$ (obs.) Yïan, H. in Yünnan ; Iat. $24^{\circ} 52^{\prime}$, Long. $102^{\circ} 56^{\prime}$; Han, An-chiang 岀江.

$5^{\circ}$ See Nos. 1594, 2930, 6181.

3457 Knei-hua-ch"êng | 化城, see preceding.

3458 Kuei-hua-ku | 化 故, post-town near Ch'êngkung H., Yünnan.

3459 Kuei-jên|仁, (obs.) Sui, H. near Pa C., Ssŭch' 'uan. 3460 Kuei-shan | 善 Kweishen, H. in Hui-chou Fu, Kuangtung ; Lat. $23^{\circ} 02^{\prime}$, Long. $114^{\circ} 13^{\prime}$; Han, Polo 博羅; $C h^{6} e ̂ n, K u e i-$ shan | 盖; Sui, Hsïn 循 C.

3461 Knei-shun | 順 Kweishun.

[225. $11^{\circ}$ C. L. C. in 'T'ai-p'pingSsŭ-Shun circ., Kuangsi ; Lat. $23^{\circ} 10^{\prime}$, Long. $106^{\circ} 04^{\prime}$.

$2^{\circ}$ Y. near Hsing-yi Fu, Kueichon.

3462 Kuei-ssŭ | 思, (obs.) E. of Hsin-ch' êng T. H., Ssŭch'uan; T'ang, C. in Ling-nan 領南 circ. 
歸

KUEi-SuI.

3463 Kuei-Sui | 綏, circ. in Shansi, conprising Kueihua T. and Sui-yianan T.

3464 Knei-tê | 德 Kwei-teh.

[359] $1^{\circ}$ Ful in the Ho-nanK'ai - Kuei - Cli'ên - Hsï circ., Honan ; forming also the district city of Shang-cin'iu; Lat. $34^{\circ}$. $28^{\prime}$, Long. $115^{\circ} 51^{\prime}$; S. A., land of Sung 来; Ts'in, Shang-ch'iu 衙邱 and Po-yi 毭邑; Han, Sui-yang 脽陽; Sui, Sung 笨 C.; Five dyn., Kuei-tê | 德; Sung, Ying-t'ien 應 天; Ming, Knei-tê | 德 Fu。

$2^{\circ}$ T. C. in Nan1-ning Fin, Ktlangsi ; Lat. $23^{\circ} 20^{\prime}$, Long. $107^{\circ} 20^{\prime}$.

$3^{\circ}$ See No. 2134.

3465 Kuei-yang / 陽, ssŭ near Ch' ang-ning H., Hunan.

3466 Kuei-yi | 義.

$1^{\circ}$ Post-town near Hsiung H., Chilili.

$2^{\circ}$ Post-town near Hsiangyin $\mathrm{H}$., Hunan.

$3^{\circ}$ (obs.) $T^{6}$ ang, H. near I.iang-hisiang H., Chihli. $4^{\circ}$ See No. 7277.

3467 Knei-yi | 易, see No. 7877.
貴

KUFI.

軌

3468 Kuei 軌, (obs.) T'ang, C., W. of Shinng-p'an T., Ssŭchi itan.

\section{會}

[See also under the syllable Hur.]

3469 Kuei-chi 會稽 Hwni-ki.

$1^{\circ} \mathrm{H}$. forming the prefectural city of Shaolnsing, Chelnkiang; Iat. $29^{\circ} 56^{\prime}$, I,ong. $120^{\circ}$ $39^{\prime} ; 7 \sin$, Shan1-yin III 陰; $C h^{6} e ̂ n, K_{n e i-c l i \mid}$ | 嵇.

$2^{\circ}$ Ts'in tc T"ang, chiun conprising Chehkiang, the S. of Anhui and the N. of Fulnkien.

$3^{\circ}$ See Nos. 2154, 7130.

\section{檜}

3470 Knei-wu 檜無, sometimes written for $T \sin , \mathrm{H}$ ui-wu 會無 $\mathrm{H}$.

\section{辞}

3471 Kuei 涂, see No. 7514.

\section{愴}

3472 Knei 郃, (obs.) formerly feudal state near Jung-yany H., Honan.

\section{貴}

3473 Kuei 贵 Kwei。 
县

[198] $1^{\circ} \mathrm{H}$. in Hsün-chon $\mathrm{F}^{\prime} 1$, Kuangsi ; Lat. $23^{\circ} 07^{\prime}$, Long. $101^{\circ} 89^{\prime}$; Han,

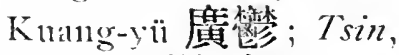
Yii-p ing 㭸雬平; Liang, Ting 定 C.; T T a lng. Knei | C.

$2^{\circ}$ (obs.) S. S smug, C. in Kuang-11si 愿西 circ.

3474 Knei-ch'i | 溪 Kwei-ki, [211] H. in Kuang-hisin Fu, Kiangsi ; Lat. $28^{\circ} 17^{\prime}$, Long. $117^{\circ} 17^{\prime} ;$ Han, Yü-kan 餘汗; $T^{6}$ ang, Knei-cl1'i | 溪.

3475 Knei-ch'ih | 池 Kwei-chi, $\mathrm{H}$. forming the prefectural city of Ch'ih-chou, Anluni; Lat. $30^{\circ} 40^{\prime}$, Long. $117^{\circ}$ 30'; Han, Shih-ch' "êng 不 城; Sui, $\mathrm{Cl}^{\prime}{ }^{\prime i 11-p} \mathrm{p}$ 秋浦; Five dyn., Knei-ch'ih | 池.

3476 Knei-chon | 州 Kwei-chan, a province of Clina; literary names Cl'ien-chung 騟中, 'Tsang-kê 牷将可 and Ch'ien 黔; capital Kueiyang Fin; contains 3 circ, $12 \mathrm{Fu}, 4$ C. L. T., 10 T., 1 C.L.C., 13 C., 34 H. Cui-ju (Polo).

3477 Kuei-chu | 筑 Kwei-clunh, $\mathrm{H}$. forming the prefectural city of Kuei-yang, Kueichon; Lat. $26^{\circ} 30^{\prime}$, Long. $106^{\circ} 36^{\prime}$; Ming, Ch 'êngfan 程番 ssü.
貴 KUEI-YANG.

3478 Knei-hsi | 西, circ. in K ueichou comprising $\mathrm{P}^{\prime}{ }_{1}$ an T., An-shun F'u, Hsingvi $F u$, Ta-ting $F u$ and T'su11-yi Fin.

3479 Knei-hsiang | 㴙, see No. 7756.

3+80 Knei-p ing | 本, (obs.) Wei, H., E. of Shunang-liu H., Ssŭch' nan.

3481 Knei-P'ing-Shilh-Jên | 平 石仁, circ. in Kneichon comprising Kuei-yang Fu, P'ing-yüeh C., Shilh-ch 'ien Fit and Jên-huai $T$.

3482 Kuei-tê | 德 Kwei-teh, T. [148] in Hsi-11ing Fu, Kansuh; Lat. $35^{\circ} 50^{\prime}$, Long. $101^{\circ}$ $15^{\prime}$.

3483 Knei-ting | 定 Kwei-ting, H. in Kuei-yang Fu, Kueichou; Lat. $26^{\circ} 30^{\prime}$, Jonig. $107^{\circ} 06^{\prime}$.

3484 Knei-tung | 東, circ. in Kueichou comprising Sungt'ao T., Li-p'ing Fu, Tuyün $F u$, Chên-yüan $F u$, Ssŭ-chon Fu, 'T'ung-jên Fun and Ssŭ-nan Fun.

3485 Kuei-yang | 荡 Kwei[327] yang, Fin in the KueiP'ing-Shih-Jên circ., Kueiclour; forming also the district city of Kuei-chu; Lat. $26^{\circ} 30^{\prime}$, Long. $106^{\circ}$ $36^{\prime}$; capital of the province; orig., land of S.W. barbarians; Han and 
$T^{\prime}$ ang, included in T'sangkê 踤物 chiun but unsubdued; Sung, Ta-wan-kulao 大萬谷落; Yüan, Shun-yuan 順元 circ.; Ming, Knei-yang | 隄 Fu.

$$
\text { 黛: }
$$

3486 Kuei 媱, see Nos. 2236, 7363.

\section{刍鼠}

3487 Kuei-ch'êng 龜城 Fin in P'ing-an 平安 circ., Corea.

\section{K'UEI}

\section{葵}

3488 K'uei-ch'in 葵邱, posttown near Sui C., Honan. 3489 K'uei-t'an | 潭, ssü in Hui-lai H., Kuangtung.

\section{蒥}

$3490 \mathrm{~K}$ 'tei 蹈.

$1^{\circ}$ (obs.) S.A., K. in Ssŭch' uan; $S$. Sung, circ.; and C. T'ang, C. $2^{\circ}$ See No. 3536.

3491 K'uei-clon | 州 Kwei[528] chau, Fu in the Ch' uantung circ., Ssŭch' 'nan ; forming also the district city of Fêng-chielı; Lat. $31^{\circ} 10^{\prime}$, Long. $109^{\circ} 35^{\prime}$; S.A., K. K'nei |; Ts'in, in $\mathrm{Pa} 巴$ chiin; Han,
Yung-ning 永寉 and $\mathrm{Pa}$ turge 巴東; Ninor Han, Kun-ling 固陵; Liang, $\mathrm{H} \div$ in 信 C.; T⿱ $T^{\prime}$ ng, Yünan 雲安; Sung, K"nei C.; lling, K'nei-chon 州 Fu.

3492 K'nei-lung | 龍, (obs.) near Ya-chon Fin, Sšnch'nan; T'ang and Sung, Knei-lung | 龍 C.

Kufa, sce No. 3324.

Kui-tun, see No. 3451 .

Kukukoti, see No. $59+5$.

Kumamoto, see No. 2883.

\section{K'UN}

\section{昆}

$3493 K^{6}$ แ1 昆.

$1^{\circ}$ (obs.) W. of $\mathrm{K}^{\prime} \mathrm{mn-ming}$ H., Yünnan; T゙ang, C. in Chien-nan 劍南 circ.

$2^{\circ}$ See Nos. 544, 3496, 4095, 7813.

$3494 \mathrm{~K}^{\prime}$ un-ch'ih | 池, (obs.) near Hsï-chou Fu, Ssŭclinan; $T^{\prime}$ ang, H. in Ts'ung 從 C.

$3495 \mathrm{~K}^{\text {'un-ling }}$ | 陵, post-town near $\mathrm{Ch}$ ang-chou $\mathrm{Fu}$, Kiangsu.

3496 K'un-ming | 明 Kwănming. 
昆

K'UN-MING.

$1^{\circ} \mathrm{H}$. forming the prefectural city of $Y$ ünnan, Yünnan; Lat. $25^{\circ} 06^{\prime}$, Long. $102^{\circ}$ $52^{\prime} ;$ Han, in K. Tien 潩, Sui, K'un | C.; Yïan, $\mathrm{K}^{\prime}$ in1-111ing.

$2^{\circ}$ See No. 3737 .

3497 K'nn-sha | 沙, chên near Kao-ling H., Shensi.

3498 K'un-yang | 際 Kwănyang.

[461] $1^{\circ}$ C. in Yün-nan $\mathrm{Fu}$, Yünnan; Lat. $24^{\circ} 45^{\prime}$, Long. $102^{\circ} 45^{\prime}$; orig., . in K. Tien 潩; Sung, Chü-ch'iao E湓; Yüan, K'un-yang |晹; Ming, $\mathrm{K}$ 'un-yang | 陽 C.

$2^{\circ}$ Chïn in $\mathrm{Ch}^{\prime}$ ing-shang 慶少 circ., Corea.

\section{崑}

3499 K'un-hsï 茎墟, (obs.) near Turfan; Tang, Fu in Lung-yu 隴右 circ.

3500 K'un-shan | | Kwănshan.

$1^{\circ} \mathrm{H}$. in Su-chou $\mathrm{F} u$, Kiangsu; this city serves also for Hsinyang $\mathrm{H}_{\text {; }}$ L Lat. $31^{\circ} 28^{\prime}$, Long. $120^{\circ} 48^{\prime} ; T^{\circ}$ sin, $^{\circ}$ Lon 婁 H.; Liang, K'un-shan | 山.

$2^{\circ}$ See No. 705 .
貢 KUNG-ChING.

\section{KUNG}

\section{公}

3501 Kung 公 Kn111g, C. in Chnng-ch'ing 忠清 circ., Corea ; Lat. $37^{\circ}$, Long. $126^{\circ} 57^{\prime}$.

3502 Kung-an | 安 Kung[142] ngan, H. in Ching-chou Fin, Hupeli; Lat. $30^{\circ} 01^{\prime}$, I.ong. $111^{\circ} 57^{\prime}$; Han, $\mathrm{Cl}^{\prime}$ 'nan-ling 㩐 陵; 1st Sung, Nan-p'ing 南平; Chền, Ching 荆 C.

3503 Ku11g-kuan | 官, posttown near Mêng-ch êng H., Anhui.

3504 Kung-lang | 郎, T-S. near Shun-11ing Fu, Yünnan.

3505 Kung-pu-shno-kê | 布碩 噶 Gumboshoka, town in Thibet; Lat. $29^{\circ} 30^{\prime}$, Long. $94^{\circ} 15^{\prime}$.

\section{功}

3506 Kung 功, (obs.) in Ssŭch"van; T'ang, C. in Cliang-11an 南汇 circ.

\section{䝯}

3507 Kung-chên 貢珍, (obs.) in Shing-king; Liao, $\mathrm{H}$. in Po 渤 C.

3508 Kung-ching | 井, posttown near Shê-hung H., Ssŭch' 'uan. 
共

KUNG.

\section{共}

3509 K $11 n g$ 共.

$1^{\circ}$ (obs.) formerly K. in Honan.

$2^{\circ}$ (obs.) in Hui H., Honan ; Han, Tsin and $N . W e i$, Kung | $\mathrm{H}$.

$3^{\circ}$ (obs.) formerly $\mathrm{H}$. near Mi-yüı H., Chihli. $t^{\circ}$ See No. 1373.

3510 Kung-ch'êng | 城, see No. 1373.

\section{宮}

3511 Knng-ch èng 宮城 Miyagi, ken in Japan; chief town, Sendai.

\section{拱}

3512 Kung-ch 'ên 拱辰, chên near Wang-tı H., Chilıli.

\section{珙}

3513 Kung 珙 Kung, H. in [229] Hsï-chon Fu, Ssŭch' uan; Lat. $28^{\circ} 15^{\prime}$, Long. $104^{\circ}$ +2'; orig., land of S. IV. barbarians; $T^{\prime}$ ang, Kung Ic.

\section{恭}

3514 Kung 菾 (obs.) Sui, C. comprising Chia-chiaing H. and $\mathrm{Pa} \mathrm{H}$., Ssŭch' '11an. 3515 Kung-ch' êng | 城 Kung[273] ching, H. in P'ing-lê Fu, Kuangsi ; Lat. $24^{\circ} 33^{\prime}$,
垶 KUNG-CH ANG.

Long. $110^{\circ} 46^{\prime} ; H a n$, in Ts'ang-wn 蒼梧 chiun; Siui, Kung-ch êng | 城.

3516 Kung-hua | 化, (obs.) $T^{\prime}$ ang, H., E. of Yungning $C_{\text {. }}$, Knangsi.

\section{韶}

3517 Kung 熊, (obs.) T'ang, C. comprising $\mathrm{P}^{\text {ing-11nan }}$ H., and part of Hsün-chon Fu, Kuangsi; see also Nos. $4711,5178$.

3518 Kung-ch iu | 邱, see No. +711 .

3519 Knnurgt'an / 灘, chên in Yu-yang C., Ssŭch 'uan.

\section{㛙}

3520 Kung 琗 Kung.

[263] $1^{\circ}$ H. in Ho-nan Fin, Honan; Lat. $34^{\circ} 53^{\prime}$, Long. $113^{\circ} 06^{\prime}$; Chon, Kung-po-yi | 伯邑; Han, Kung | H.

$2^{\circ}$ See No. 3521.

3521 Kung-ch' ang | 昌 Kung[498] chang, Fu in the Knng-

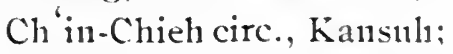
forming also the district city of I ung-hsi; Lat. $34^{\circ}$ $56^{\prime}$, Loug. $104^{\circ} 4 t^{\prime} ; S$. $A$, land of the Ch'iangjung 羌戊 barbarians; $T s^{\prime}$ in, in Lung-hsi 隌覀; Han, T'ien-shui 天水; E. Han, Han-yang 漢陽 and Nan-an 南安; Tsin, 
簕

KUNG-CH'ANG.

in K. Chin 晋; $T^{\prime}$ ang, Wei 㴘 C.; Sung, Kung C.; Ming, Ku11-clíang 昌 Fu.

3522 Kung-Ch'in-Chieh | 泰 階, circ. in Kansul con1prising Kung-ch'ang Fu, $\mathrm{Cin}^{\prime}{ }^{\mathrm{in}} \mathrm{C}$. and Cliveh $\mathrm{C}$.

3523 Knng-11ing | 耍, see No. 6356.

3524 Kung-po-yi | 伯邑, see No. 3520 .

\section{宮}

3525 Kung-lısia oshita, town in Japan.

3526 K 11110 -slun- ving-shêng | 湖器彗 see No. 5822 .

Ku11g, see No. 515 .

Kung-cliang, see No. 3521. Kung-cling, see No. 3515 . Kung-ngan, see No. 3502 . Ku11gsar, see Nos. 2105, 3529.

\section{K'UNG}

\section{孔}

3527 K'nug-cli'êng 孔垛, chên near 'T'n1ng-clı'êng $H$., Anluni.

3528 K'ung-lnng | 龍 chển in Huang-mei H., Hupeh.

$3529 \mathrm{~K}^{\prime}$ 'ung-sa | 撒, T-S. near Tacliien-lı T., Ssŭclı' 'uan. See No. 2105.
絖皆 KUo.

$3530 \mathrm{~K}^{\prime}$ ung-t'i | 提, (obs.) 200 li N. E. of Chieh C., Kansuh ; $N$. Wei, H. in Wu-tu 武都 chïn.

3531 K'ung-tson | 走, chên near Ch'êng-ch' êng H., Shensi.

\section{KUO}

罙

3532 Kno 怆 Kwo, see Nos. $4568^{\mathrm{s}}, 5615$.

3533 Kno-ch" nan |川 H. in

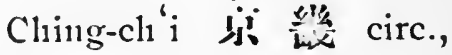
Corea.

3E3 K Kun-liua | 化 Kwo-luwa, 'I. C. in Nan-ning Fu, Kuangsi ; Lat. $23^{\circ} 21^{\prime}$, Long. $107^{\circ} 07^{\prime}$.

\section{鉛问}

3:35 Kuo-ti-wan 銆底灣，ssü near Mien-yang C., Hupeh.

\section{舵}

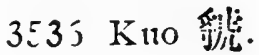

$1^{\circ}$ (obs.) Chon, K. in Honan ; divided into WV. Kno (now Lu-shih H.), E. Kno (now $\mathrm{K}^{\prime}$ ai-fêng $\mathrm{F}$ ù and $\mathrm{Ch}^{\prime} \mathrm{i}$ H. [No. 6:3] ), and N. Kuo (now Îê:ı H.) $2^{\circ}$ (obs.) IIan, town near Chi C. L. C., Chihli. $3^{3}$ See No. 4084. 
舵

KUO-YANG.

3537 Kuo-yang | 陽, (obs.) Han, town N. E. of Jaochou $\mathrm{Fu}$, Kiangsi.

\section{逗}

3538 Kuo 崞 Koh, H. in 'Tai [143] C., Shansi; Lat. $38^{\circ} 55^{\prime}$, Long. $112^{\circ} 48^{\prime} ;$ Han, Kno | H.; P. Chou, Shihch'êng 石城; Five dyn. Pai-lu白鹿.

\section{郭}

3539 Kuo-ch'êng 郭城, posttown near Ching-ÿ̈an H., Kausuh.

3540 Kuo-chia | 家.

$1^{\circ}$ Chen near Ti-tao C., Kansuh.

$2^{\circ}$ Chên in Fêng-ning H., Chihli.

$3540^{A}$ Kun-êrh-lo-ss u | 爾羅斯 Ghorlos, Mongol Tribe.

3541 Kuo-pi | 壁, chên near Ch'in-shui H., Shansi.

3542 Kuo-shan | III Kwohshan, chün in P'ing-an 4. $37^{\circ} 43^{\prime}$, Long. $125^{\circ} 15$.

3543 Kuo-tien | 店.

$1^{\circ}$ Chên near Shili-mên H., Chehkiang.

$2^{\circ}$ Post-tuwn N. of Hsinchêng $\mathrm{H}$., Honan.

3544 Kno-ts'un | 村, chên near Hsi-an Fu, Shensi.
䦞 KWĂN-YANG.

\section{淤}

$3545 \mathrm{~K}$ uo 漷, (obs.) Ning, H. 45 li S. of ' $T$ ' ung C., Chihli; Han, Ch'üan 沓 C.; Liao, Kuo-yin | 陰.

3546 Kno-yin | 院, see preceding.

\section{或}

$35+7$ Kun-t on 國頙, town in the Linch'in Islands; probably meant to indicate the capital.

\section{K'UO}

\section{闊}

3548 K' 11o-tuan 闊辞住; one way of writing Khoten; see No. 2057.

$3549 \mathrm{~K}^{\prime}$ 110-yüan | 源, (obs.) in Ssŭch'uan; T゙ang, H. in $\mathrm{K}^{\text {'no }} \mid \mathrm{C}$.

Kurkura-1tsu, see No. 3329 .

Kurli, see No. 3-30.

Kurto, see No. 3285.

Kurun, see No. 3332.

Kusatsu, see No. 6532.

Kutopi, see No. 2176.

Kwan, see Nos. 3359, 3367.

Kwan-ching, see No. 3365. Kwan-tau, see No. 3355.

Kwan-yang, see. No. 3362 . Kwăn-ming, see No. 3196. Kwăn-shan, see No. 3500 . Kwăn-yang, see No. 3498 . 
KWANG.

Kwang, see Nos. 3386, 3430 .

Kwang-chang, see No. 3389.

K wang-chau, see No. 3393. Kwang-fung, see No. 3395. Kwang-liwa, see No. 3432 . Kwang-jin, see No. 3403. Kwang-ling, see No. 3407. K wang-11an, see No. 3410. Kwang-11gan, see No. 3388 . Kwang-ning, see No. 3412. Kwang-ping; see No. 3413. K wang-shan, see No. 3133 . Kwang-shun, see No. 3416. Kwang-si, see No. 3398. Kwang-sin, see No. 3100 . Kwang-teh, see No. 3417. Kwang-tseh, see No. 3434 . Kwang-tsi, see No. 3391. Kwang-tsung, see No.3419. Kwang-tung, see Nos. $3421,3422$.

K wang-ynen, see No. 3429. Kwei, see Nos. $3454,3473$. Kwei-cha11, see Nos. 3476 , 3491.

Kwei-clii, see No. 3475.

Kwei-clutl, see No. 3477 . Kwei-hwa, see No. 3456. Kwei-ki, see No. 3174. Kwei-lin, see No. 3446. Kwei-ngan, see No. 3454 . Kwei-ping, see. No. 3448. Kwei-shen, see No. 3460 . Kwei-shun, see No. 3461 . Kwei-teh, see Nos. 3464, 3482.
來

LAI.

Kwei-ting, see No. 3483. Kwei-tung, see No. 3452 . Kwei-yang, see Nos. 3453, 3485.

Kwo, see No. 3532.

K.wo-hwa, see No. 3534.

Kwoll-shan, see No. 3542 .

\section{LA \\ 喇}

3550 La-kun，喇渷， T-S near Ta-chien-1u 'T., Ssŭch 'uan.

3551 La-ya | 嚚, ssŭ near Kuei-ting H., Kueichou.

\section{拉}

3552 La-êrh-chi 拉尔吉, T-S. in Kokonor.

3553 La-li | 里 H'lari, tow1 in Thibet; Lat. $31^{\circ}$, Long. $94^{\circ} 20^{\prime}$.

3554 La-lin | 林 Larin, ss̆̌ in Shunang-clı' êng T., Kirin ; Lat. $45^{\circ} 25^{\prime}$, Long. $125^{\circ} 40^{\prime}$.

$3555 \mathrm{La}$-sa | 薩 H'lassa (also called Pu-ta-la 布達 and $\mathrm{Ch}^{\prime}$ 'jen-tsang 前藏), the capital of Anterior Thibet; Lat. $30^{\circ} 48^{\prime}$, Long. $91^{\circ} 25^{\prime}$.

\section{LAI \\ 來}

3556 I ai 來, (obs.) $130 l i \mathrm{~S} . \mathrm{W}$. of Ning-yüan C., Shingking; Liao, C. in Chungching 中京 circ. 
來

LAI-AN.

3557 Lai-an | 㚣 Lai-ngan, H. in $\mathrm{Ch}^{\prime}$ " C., Anluni; Lat. $32^{\circ} 25^{\prime}$, Long. $118^{\circ} 26^{\prime}$; Han, Chien-yang 建陽; 1st Sung, Chieh-ch 'iu 蝢 邱; Sui, Ch'ing-liu 清 流; S. T'ang, Lai-an | 努:

$3557^{\mathrm{A}}$ Lai-fêng | 鳳 Lai-fung.

[21] $1^{\circ} \mathrm{H}$. in Shih-nan Fu, Hupeh ; Lat. $29^{\circ} 50^{\prime}$, Long. $109^{\circ} 40^{\prime}$.

$2^{\circ}$ Post-town near Chungch'ing Fu, Ssŭch' iran. 3558 Lai-fêng|录, (obs.) in Ya-chou Fu, Ssŭch'nan; Sung, C. in Ya 雅 chou. 3559 Lai-hua | 化, chên near T'ung-chou Fu, Shensi.

3560 Lai-kon | 满, post-town near P'ei C., Kiangsu.

3561 Lai-pin | 寅 Lai-pin.

[416] $1^{\circ} \mathrm{H}$. in Lil1-chou Fil, Kuangsi; Lat $23^{\circ} 38^{\prime}$, Long. $109^{\circ} 06^{\prime} ; T s^{\prime}$ in, Kuei-lin 柱林; $T^{6}$ ang, Lai-pin | 濒.

$2^{\circ}$ (obs.) near Hstij-chou Fu, Ssŭch' 'ıan; T'ang, H. in Wn-ch'ang 武 昌 C.

$3^{\circ}$ (obs.) 110 li S.W. of Nien-po H., Kansuh; Sung, town in Lê 樂 C. $4^{\circ}$ (obs.) 130 li S.IV. of Ning-yüan C., Shingking ; Liao, H. in I ai C.
萊 LAI.

3562 Lai-su | 蘇. $1^{\circ}$ (obs.) N.E. of Lianghsiang, Chihli ; $T^{\prime}$ ang, H. in Yi-pin 类賓 C. $2^{\circ}$ (obs.) in Ning-hai 宣 海 H., Shingking; Lico, H. in Su 蘇 C.

3.563 Lai-t'n11ng | 同, (obs.) IV. of Ho C., Kansuh ; Sung and $K i n$, Lai-t'ung | [⿵冂⿱一口] pao.

3564 I,ai-wei | 唯, (obs.) W. of Wên-shan H., Yünnan; $W$. Han, H. in Yi-clion 盛州 chün。

3565 Lai-yin | 銀, (obs.) in I,ü C., Ssŭch' 'uan; T'ang, H. in Nêng 能 C.

3566 Lai-yuian | 猿, (obs.) in Lï C., Ssŭch 'nan; $T^{\prime}$ ang H. in Sh111 㮌 $C$.

\section{徠}

3567 Lai-ning 徠耍, see No. 3224.

\section{淶}

3568 Lai-shui 淶水 Lai-shwui, [107] H. in Yi C., Chihli; Iat. $39^{\circ} 25^{\prime}$, Long. $115^{\circ} 47^{\prime}$; Han, Chin 遒 H.; $P$. Chou, Lai-shnii | 水.

\section{萊}

3569 I ai 萊, (obs.) T゙ang, Sung, Kin, Yüan and Ming, C. in Shantung; see No. 3570 . 
3570 Lai-chou | 州 Lai-chau, $\mathrm{Fu}$ in the Têng-Lai-Ch 'ingChiao circ., Shantung; forming also the district city of Yeh; Lat. $37^{\circ} 10^{\prime}$, Long. $120^{\circ} 10^{\prime}$; orig., land of the Lai | tribes; $S . A$., K. Lai-tzŭ $\mid$ 子; $T s^{\prime}$ in, in Ch'i 驾 chïn; Han, Tung-lai 東萊; 2nd Wei, Kuang 光 C.; Sui and $T^{6}$ ang, Lai | C.

3571 Lai-tzŭ |子, (obs.) S.A., $\mathrm{K}$. in Shantung; see preceding.

3572 Lai-tzŭ-clìng | 子城, (obs.) S.A., capital of preceding, near Huang $\mathrm{H}$. . Shantung.

3573 Lai-wu 無 Lai-wu.

$1^{\circ} \mathrm{H}$. in ' $\Gamma^{\circ}$ ai-an $\mathrm{Fu}$, Slantung; Lat. $36^{\circ}$ 16', Long. $117^{\circ} 51^{\prime}$; S.A., Chia-ku 夾谷; Han, Lai-wu | 燕.

$2^{\circ}$ (obs.) S.E. of Tzŭclinan H., Shantung; Han and $T \sin , \mathrm{H}$. in T'ai-shan 泰山 chïn. $3^{\circ}$ (obs.) 8 li S.E. of Lai-wu H., Sliantung;

Sung, fort.

3574 Lai-yang | 晹 Lai-yang, [811] H. in Têng-chou $F u$, Shantung; Lat. $37^{\circ}$, Loung. $120^{\circ} 59^{\prime} ;$ Han, Ch'angyang 昌陽; T'ang, Laiyang 防.
蘭 $\mathrm{LAN}-\mathrm{CH}^{\prime} \mathrm{IH}$.

Lai-chau, see No. 3570 . Lai-fung, see No. $3557^{\mathrm{A}}$. Lai-ngan, see No. 3557 . Lai-shwui, see No. 3568.

\section{LAN}

\section{橍}

3575 Lan-ch'ê 欄席, chên near Tsê-chon Fu, Shansi.

\section{爛}

3576 Lan-t’ $\mathrm{u}$ 爛土, T-S. near Li-po H., Kueichou.

\section{蘭}

3577 Lan 闌.

$1^{\circ}$ (obs.) Yiüan, C. in Yünnan; Lat. $26^{\circ} 33^{\prime}$, Long. $99^{\circ} 46^{\prime}$; Han, Po-nan 博南.

$2^{\circ}$ See No. 3583.

3578 Lau-ch'i | 祺, (obs.) in Sliantung; W. Han, Marq. in Tung-hai 其海 chün.

3579 Lan-ch'i | 溪.

$1^{\circ} S \operatorname{să}$ in Ch'i-shni H., Hupel1.

$2^{\circ}$ See No. 648 .

3580 Lan-cl1 i | 䈶 Lan-ki, H. [231] in Chin-hua Fu, Chehkiang; Lat. $29^{\circ} 05^{\prime}$, Long. $119^{\circ} 45^{\prime}$; T'ang, Lan-ch ${ }^{\prime}$ i 綵 $\mathrm{H}$.

3581 Lal1-chia | 家, Y. near Ch'ing-ch'i $\mathrm{H}$., Ssŭch '

3582 Lan-ch'il | 池 
蘭

LAN-CH' III.

$1^{\circ}$ (obs.) in Slıan-tan H., Kansuln ; Tsin, H. in Hs: 唒 chiïn.

$2^{\circ}$ (obs.) near Ning-hsia Fu, Kansuh; T"ang, Fu in Kuan-nei 昡新 circ.

3583 Lan-chou | Hil Lan-chan. $1^{\circ}$ Circ. in Kansul comprising Lan-chon Ftt.

[148] $2^{\circ}$ Fu in Lan-chon circ.,

Kansulh; forming also district city of Kao-lan; Lat. $36^{\circ} 08^{\prime}$, Lonig. $103^{\circ} 55^{\prime}$ : capital of t! $1 \mathrm{e}$ province; orig., I anch'üan | 期; IIrn, Chin-cliêng 金城; Sui, Lan | C.

$3^{\circ}$ 'T-S. near Wei-hsi 'T',

Yütnlın.

3584 Lan-clïian | 泉.

$1^{\circ}$. Post-town near Lanchou $\mathrm{Fu}$, Kansulı.

$2^{\circ}$ See No. 3583.

$3584^{\mathrm{A}}$ Lan-hsi | 西, H. in Hulan $\mathrm{Fu}$, Tsitsilar.

3585 Lan-kan | F, (obs.) Han, H. comprising part of Kung-ch'ang Fu, Kansult.

3586 I all-ling | 陵, see Nos. 7060, 7518.

3587 Lanl-pa-sa-êrl | 巴撒耳 Lembesser, a town of Persia.

3588 Lan-shan | I4 Lan-shan, $H$. forming the prefectural

\section{LAN-SHUI.}

city of Yi-chou, Shantung; Lat. $35^{\circ} 15^{\prime}$, Long. $118^{\circ}$ 35'; Sui, T'ang, Sung, Kin and Yiian, Lin1-yi 臨励 $\mathrm{H}$.

3589 Lan-shui | 水, (obs.) Liang, H., S. of Changchou Fu, Fulukien.

3590 Lan-tê 华, chên near Wên-lısi H.. Shansi.

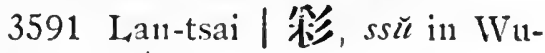
cliang, T., Kirin.

3592 Lan-ts'ang|你, N. IVei, H. in $\mathrm{K}$ insull.

3593 Lan-yang | Fon, see No. $3 ; 9 t$.

359+ Lan-yi | 倍 L,an-i, H. in [148] K'ai-fêng Fu, Honan; Lat. $34^{\circ} 54^{\prime}$, Long. $114^{\circ}$ $59^{\prime} ; \quad T s^{\prime}$ in, Tung-ming 楫明; Han, Tung-hun 将昏; Kin, Lan-yang | 涴.

\section{蓝}

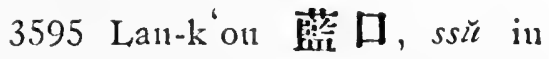
Ho-yiian H., Knangtung.

3596 Lan-p'n | 浦 H. in Chungcli 'ing 忠清 circ., Corea.

3597 Lan-shan | II Lan-shan, $\mathrm{H}$. in Knei-yang C., Hunan; Lat. $25^{\circ} 48^{\prime}$, Isong. $112^{\circ} 23^{\prime}$; Han, Nan-p'ing 南 $4^{*} ; T^{*}$ ang, Lan-shan | 4 .

3598 Lan-shni | 水, (obs.) $I V$. Wei, town S.W. of An-'u Fu, Hupeh. 


\section{藍}

LAN-T' IFN.

3599 Lan-t ien | 田 Lan-tien, [235] H. in Hsi-an Fu, Shensi ; Lat. $34^{\circ} 05^{\prime}$, Long. $109^{\circ}$ $20^{\prime} ; T^{\prime}$ in, Lan1-t'ien | 田; P. Chou, Yü-shan 玉 IL and Pai-1u 白鹿.

3600 I an1-t ai-ch'ing-hua | 臺 清話, see No. 5822 .

3601 I Aan-w1 | 屋, post-town near Wu-p'ing $H_{\text {., Ful }}$ kien.

$3601^{*}$ Lan-reli | 掖, see No. 5822 .

\section{風}

3602 Lan 葻 Lan, H. in T"aiyiian $\mathrm{F} u$, Shansi; Lat. $38^{\circ} 25^{\prime}$, Long. $111^{\circ} 35^{\prime}$; Han, Fên-yang 汾陽; Tang, Ian | C.: ser No. 3239.

3603 Lank-ku | 谷, see No. 3239.

Lan-chan, see No. 3583. Lan-i, see No. 3594. Lan-ki, see No. 3580 . Ian-tien, see No. 3599.

\section{LANG}

\section{朗}

3604 Lang 朗, see No. 321 .

3605 Lang-ch'i | 溪.

$1^{\circ}$ (obs.) in Ch'ien-yang $\mathrm{H}$., Hunan; T'ang, $\mathrm{H}$. in Hsü 叙 C.
浱 LANG-MI.

$2^{\circ}$ (obs.) in Ssŭ-nan Fu, Kueichon; Ming; ss̆̆ in Ssŭ-nan Fir.

$3^{\circ}$ See No. $90 \%$.

3606 Iang-ch'il | 池, see No. 7593.

3607 Lang-hua | 化, (obs.) T'ang, H., W. of Shaochou Fu, Kuangtung.

3608 Lang-ning | 蒠, see No. 4609.

3609 Lang-shan | 山, see No. 1504.

3610 I ang-tai | 岱 Lang-tai, T. in An-sliun Fu, Kneichour; Lat. $26^{\circ} 15^{\prime}$, Long. $105^{\circ} 45^{\prime}$. Terr. jur.

3611 Lang-tê | 德, (obs.) Chêen, H., N.W. of Tienpai H., Kuangtung.

\section{浪}

3612 Lang-cli inng 泿穹 Lang[303] kiung, H. in Ta-li Fu, Yünnan; I at. $26^{\circ} 08^{\prime}$, Isong. $100^{\circ} 08^{\prime}$.

3613 Lang-ch' 'uan | 川, (obs.) $T^{\prime}$ ang, C., S.W. of L,ei-po T., Ssŭch uan.

3614 Lang-mi | 彌.

$1^{\circ}$ (obs.) near Ning-yüan $\mathrm{Fu}$, Ssŭch'uan; $T^{\prime}$ ang, C. in Chien-nan 劍南 circ.

$2^{\circ}$ (obs.) in Ya-chou $\mathrm{Fu}$, Ssŭch'uan; T'ang, C. in Chien-nan 劍南 circ. 
泿

LANG-MI.

3615 Lang-mi | 满, (obs.) in Ssŭch uan ; Sung, C. in $\mathrm{Li}$ 黎 CHOU.

3616 Lang-tzŭ-shan | 子 IL, post-town near Fêng-t 'ien Fin, Shingking.

\section{莨}

361 广 Lang-ch' ii 港薄, (obs.) 80 li S. of Yung-pei T., Yünnan ; Yïan, C. in Lichiang 麗江 circ.

\section{狼}

3618 I rang-chiang 狠江.

$1^{\circ}$ (obs.) near Cling $C$.. Hunan; Sung, military post in Ching 端 C.

$2^{\circ}$ See No. 2345.

3619 Lang-ch'uan | 川, H. in Chiang-rüan 汇.原 circ. Corea.

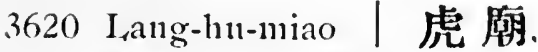
chên near Wên-an H., Chibli.

3621 Lang-nêng | 丞. see No. 7255 .

3622 Lang-shan | IL, see No. 279.

\section{珢}

$3622^{A}$ Lang-chung 珢中, see No. 7473.

3623 Lang-kuei | 瑰 (or huai 槐) (obs.) 110 li N. E. of Lê-an H., Shantung; $W$. Han, H. in Ch'iench'êng F·乘 C.
閔 LANG-YHN-TSING。

362+ Lang-nei |队, see No. 3631.

3625 I Aang-yeh | 㑚 (or｜邪, the second clatracter being read yeh), (obs.) $T s^{\prime} i n$, chiün comprising F. part of Shanting; see Nos. 1204, 7461 .

3626 Lang-yen-ching | 監井 Lang-yen-tsing, important salt-wells in ch' 'n-lisiung Fu, Yünnan; Lat. $25^{\circ} 20^{\prime}$, Long. $101^{\circ} 50^{\prime}$.

\section{郎}

3627 I ang 郎, (obs) T'ang, C. comprising part of $C h^{\prime}$ ï-ching $F u$, Yümman.

3628 Lang-cliêng | 城, ssh̆ near Huang-p'ing C.. K neichou.

3629 Lang-to | 惰, 'I'-S. near Sung-p an 'Г., Ssŭch'nan.

閏

3630 I ang-chn1119閶中 I angchung, $H$. forming the prefectural city of Paoning, Ssŭclı nan ; Lat. $31^{\circ}$ $32^{\prime}$, Lonng. $105^{\circ} 59^{\prime} ; T s^{\prime}$ in, I ang-chung | 中; Tsin, Pa-hisi 巴西; Sui, rangnei 珢內。

3631 Lang-riian | 苑, see No. 5822 .

Lang-kiung, see No. 3612 . Lang-yent-tsing, see No. 3626. 


\section{LAO}

\section{老}

3632 Lao-an 老岸, chển near Hua H., Honan.

3633 Lao-clı'in | 邱, (obs.) formerly town near Cll ênliin H., Honan.

3634 Lao-t'ang | 䛸, Y. near Cliang-luna H., Hunan.

3635 Lao-ya-pan | 雅堡, T-S. near Nien-po H., Kansuh.

\section{落}

3636 Lao-chi 落不渠, (obs.) in Mou C., Ssŭclin 'uan; T"ang, H. in Chï 崌 C.

3637 Lao-hui | 厄, (obs.) S.E. of Yi-shan H., Knangsi.

3638 Lao-t'u11 | 屯, (obs.) 50 li N.E. of Yai C., Knangtung; $T^{\prime}$ ang, H. in Chên 振 C.

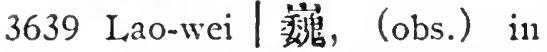
Yi-shan H., Kuangsi : $T^{\prime}$ ang, $\mathrm{H}$ in Knei-hua 部化 $\mathrm{C}$.

3640 Lao-wu | 吴, (obs.) in Ssŭch' 'uan; T'ang, H. in $\mathrm{K}$ 'no 闊 $\mathrm{C}$.

\section{澇}

3641 Lao-tien 澇店, chênn near Hn H., Shensi.

Larin, see No. 3554. Lau, see No. 4071.
樂

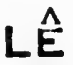

樂

3642 Lê 樂. see No. 5169. ¿643 Lê-an | 'L̀ Lolingan. [489] $1^{\circ} \mathrm{H}$. in Ch' ing-chon $\mathrm{Fu}$, Shautung; Lat. $37^{\circ}$ 05', Long. $118^{\circ} 38^{\prime}$; Hlin, Lê -an | K女: T ang, Ch'êning 乘 C.; S. Sung. Lề-an | 文: cliein.

$2^{\circ}$ II. in Fu-chon $\mathrm{Fu}$, Kiangsi ; Lat. $27^{\circ} 20^{\prime}$, I.ong. $115^{\circ} 51^{\prime}$.

$3^{\circ} \mathrm{Ch} u ̈ n$ in $\mathrm{Cl}_{1}$ 'ïan-lo 全

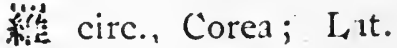
$35^{\circ} 03^{\prime}$, Long. $126^{\circ}$.

$4^{\circ}$ Sš̆ near Yai C., Kuangtung.

$5^{\circ}$ (obs.) N. of Po-hsing H., Shantung; Han, H. in Cl' ien-ch' êng 7.乘 chain.

$6^{\circ}$ (obs.) N. of Kao-yüan H., Shantung ; E. Han, $\mathrm{K}$. in Ch'ing 悲 chou. $7^{\circ}$ (obs.) 12 li E. of Hsin-ch ềng H., Shantunng; $r$ sin, $\mathrm{K}$. in $\mathrm{Cl}_{1}$ ing 青 chou.

$8^{\circ}$ (obs.) E. of Tê-lising 德與 H., Kiangsi; Tsin, S. Sung and $S$. $T s^{\prime} i, \mathrm{H}$. in $\mathrm{P}^{\prime}$ o-yang 鄱㧛 chiin. 
$9^{\circ}$ (obs.) W. of Hsienchii H., Chehkiang; $S$. Sung and $S$. T's $i$, H. in Lin-hai 臨 滩 chün.

$10^{\circ}$ (obs.) $50 l i \mathrm{~W}$. of Kuang-sl:an H., Honail; .S. Sung and Sui, H. in Yi-yang 代防 chür.

$11^{\circ}$ (obs.) near Hsiangyang Fu, Hupeh; $S$. $T s^{\prime} i, H$. in Ts'ai-yang 蔡览 chiun.

$12^{\circ}$ (obs.) in Ssŭch 'uan; S. $T s^{6} i, H$. in Nan-an 南安 chün.

$13^{\circ}$ (obs.) E. of Ho-shan H., Anhui ; N. Wri, chïn and $\mathrm{H}$. in $\mathrm{Ho}_{\mathrm{O}}$ 霍 cHOU.

$14^{\circ}$ (cbs.) in Ssŭ-uan Fu,

Kueichou； T'ang. H. in Chrlang 莊 C.

$15^{\circ}$ (obs.) in Aunam ; T'ang, H. in Chün 郡 C.

$16^{\circ}$ (obs.) $30 l i \mathrm{~N}$. E. of

Yang-ku H., Shantung;

Kin, chên in Tungp'ing 東平 Fu。

$17^{\circ}$ See Nos. 2707, 3656. 3644 I,ê-ch'ang|昌 Loh-chang. [507] $1^{\circ} \mathrm{H}$. in Shao-chou $\mathrm{Fu}$, Kuangtung; Lat. $25^{\circ}$ $15^{\prime}$, Long. $112^{\circ} 48^{\prime}$; Han, Ch'ï-chiang 此 江; Liang, Liang-liua
治

梁化 and P'ing-shih 4.石; Sui, I,ê-ch'ang 昌。

$2^{\circ}$ See No. 4596.

3645 Lê-ch' êng | 成, see No. 3648.

3646 Lê-ch' êng | 城.

$1^{\circ}$ Post-town near Hsien H., Chihli.

$2^{\circ}$ (obs.) Sui, tow11 $50 \mathrm{li}$ E. of P'êng-tsê H., Kiangsi. Formerly a town called $T^{\prime}$ ien-shui 涾.

$3^{\circ}$ See Nos. 517, 2697.

3647 Lê-chieh | 待, (obs.) in Shansi ; Han, H. in Hsiho 西测 chiin.

3648 L,ê-chih | 至 Loh-chi, H. in 'T'ung-ch' uan Fu, Ssŭch'nan; Lat. $31^{\circ} 09^{\prime}$, Long. $105^{\circ} 1 \mathrm{i}^{\prime} ;$ orig., $\mathrm{P}^{\prime} \mathrm{u}$ 酱 $\mathrm{C}$; $T^{6}$ ang, Lêchih 至.

3649 Lê-ch 'ing | 声 Loh-tsing, $H$. in Wên-chou Fur. Chellkiang; Lat. $28^{\circ} 10^{\prime}$, Long. $121^{\circ} 11^{\prime}$; Han, Yung-ning 永富; $T$ sin, Lê-clì êng | 成; Liang, Lê-ch'ing | 欂.

3650 Lê-hui | 會 L,oh-hwui.

$1^{\circ} \mathrm{H}$. in Ch'iung-chou $\mathrm{Fu}$, Kuangtung; Lat. $19^{\circ} 40^{\prime}$, Long. $110^{\circ}$ $33^{\prime}$.

$2^{\circ}$ See No. 2345. 


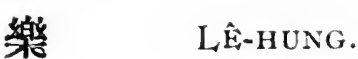

3651 Lê-hung | 鸿, (obs.) in Ssŭch'nan; $T^{*}$ ang, H. in Hung 鴻 C.

$36.51^{\wedge}$ Lê-jung | 容, (obs.) near Ning-hsia Fu, Kansun; T'ang, Fu in Kuan-nei 關 內 circ.

3652 Lê-ling | 陵 Loh-ling.

$1^{\circ} \mathrm{H}$. in IVu-ting $\mathrm{Fu}$, Shantung; Lat. $37^{\mathrm{C}}$ 48 , Long. $117^{\circ} 18^{\prime}$; Han, Lê-ling | 陵; Sui, Kê-cling 高津.

$2^{\circ}$ See No. 7003.

36.53 Lê-lo| 箔. (obs.) Han, town $110 \mathrm{li} \mathrm{W}$. of Yai C., Knangtung.

3654 Lê-fan | 蟠, see No. 2134. 36.55 Lê-pang | 榜, ssŭ near Ch'ing-p ing H., Kneichoul.

3656 Lê-p'ing | 4i Loh-ping. $1^{\circ}$ (obs.) H. S. of P'ingting C., Shansi; I,at. $37^{\circ} 38^{\prime}$, I,ong. $113^{\circ}$ 45'; Han, Tien 沾 H.; $T \sin , \mathrm{I}, \hat{\mathrm{e}}-\mathrm{p}$ 'ing | 尔.

[240] $2^{\circ} \mathrm{H}$. in Jao-chon F11, Kiangsi; Lat. $28^{\circ} 5 \tau^{\prime}$, I.ong. $117^{\circ} 16^{\prime}$; Han, $\mathrm{Y}$ üi-kan 餘干; E. Han, I,ê-an |安; Trang, Lề-p'ing $4 \mathbf{s}$.

$3^{\circ}$ Y. near Yai C., Kuangtung.

$4^{\circ}$ Post-town in P'ingting C., Shansi.
絈 LÂ-YÜAN.

$5^{\circ}$ T-S. near Kuei-ting H., Kueichou.

$6^{\circ}$ See Nos. 6313, 7016.

36.57 Lê-shan | |l| Loh-shan.

$1^{\circ} \mathrm{H}$. forming the prefectural city of Chiating, Ssŭch' 1 an; Lat. $29^{\circ} 28^{\prime}$, Long. $103^{\circ}$ $55^{\prime}$.

$2^{\circ}$ See No. 7763.

3658 I.ê-ting | 亭 Loh-ting, $\mathrm{H}$. [271] in Yung-p'ing Fu, Chihli; Lat. $39^{\circ} 29^{\prime}$, Lo11g. $118^{\circ}$ 55'; orig., I,11-lung 盧龍; $T^{*}$ ang, Ma-ch'êng 齿坆; Kin, Lế-t'ing | 察.

3659 I.ê-tı | 都.

$1^{\circ}$ (obs.) in Shantung; W. Han, Marq. in Pei-hai 北海 chün.

$2^{\circ}$ (obs.) in Corea; $E$. Han, H. in Lêe-lang | 源 chïn.

3650 Lê-wên | 泳.

$1^{\circ}$ (obs.) N.W. of $\mathrm{Ch}^{\prime}$ angshou H., Ssŭch' "uan; $T^{6}$ ang and Sung, $\mathrm{H}$. in Foll 涪 $\mathrm{C}$.

$2^{\circ}$ See No. 271.

3661 Lê-yang | 陽, see No. 177. 3662 Lê-yüan | 源, (obs.) 70 li W. of Chêng-an H., Kueichou ; $T^{6}$ ang, H. in Chên 淎 C.; Sung, H. in Po 播 $\mathrm{C}$. 
勒

LÊE-MU-CHIN.

\section{勒}

3663 I.ê-111u-chin 勒穆金 I.en1tsin, (obs.) formerly town in Mongolia.

\section{LEI}

\section{露}

3664 Lei 雷, see No. 3668.

3665 Lei-chiang | 港, chên near Wang-chiang $\mathrm{H}$., Anhui.

3666 I,ei-chiang | İL, see No. 4180.

3667 Lei-Ch'iung | 隻, (obs.) formerly circ. in Kuangtung comprising Lei-chou $\mathrm{Fu}$ and $\mathrm{Cl}_{1}$ 'iung-chon $\mathrm{F}$ 11.

3668 Lei-chou | 州 Lui-chau, Fu in the Kao-I,ei-Yang circ., Kuangtung; forming also the district city of Hai-k'ang; Lat. $20^{\circ} 52^{\prime}$, Long. $109^{\circ} 40^{\prime}$; orig., land of the Yüelı 舆 tribes; $T s^{\prime}$ in, in Hsiang 象 chïn; Han, Hsü-wên 徐聞; Liang and Sui, Ho 合 C. and Hai-k'ang 海康; $T^{6}$ ang, Lei | C.

3669 Lei-ch'uan | J, (obs.) Liang, H., W. of the preceding.

3670 Lei-hsiang | 拫, see No. 4180.

3671 Lei-pol波 Lui-po, C.L.T. in Ch' 'uan-nan-Yung-ning circ, Ssích uan1; I att. $28^{\circ}$ $30^{\prime}$, I. ong. 102 $55^{\prime} ;$. Wing, l,ei-p

36 广2 I ei-po| 坡, see prececling. 3673 I,ei-t ang | 噇, post-town near J,in-chon I'n, Kuangsi.

36ìt Lei-tsê | 沙, (obs.) Sui, 90 li S.E. of P'" C., Shantung; Han, Ch'êngyang 城陽.

3675 Lei-wei | 衛, chén near Par-shui H., Shensi.

3676 I,ei-yang | 荡.

$1^{\circ}$ Chên near Ching-ning C., Kansuh.

$2^{\circ}$ Post-town near L teichou Fu, Kuangtung.

\section{来}

3677 Lei-yang 未晹 Lui-yang. [23] $1^{\circ} \mathrm{H}$. in Hêng-chou $\mathrm{Fu}$, Hunan; Lat. $26^{\circ} 33^{\prime}$. Long. $112^{\circ} 41^{\prime} ; T s^{\circ}$ in, I,ei | H.; Han, Leiyang | 晹. $2^{\circ}$ See No. 317 .

\section{䋈}

3678 I,ei 抙. (obs.) S. of Ch'ang-li H., Chihli; $W$. Han, H. in Liao-l1si 泳 西 chün.

Lembesser, see No. 3587. Lemtsin, see No. 3663. 
冷 LÊNG-PIEN.

\section{LENG}

冷

3679 Lêngr-pien 冷邊 T-S. near Jung-ching H., Ssŭch nan.

3680 Lêng-shui | 水, Y. near Lai-pin H., Kuangsi.

\section{楞}

3681 Lêng-ts'un 楞村, posttown near Yi-slian $\mathrm{H}$, Knangsi.

Lew-cliew, see No. 4004.

\section{LI \\ 戟}

3682 Li 䍮:, (obs.) formerly C. near Yung-pei T., Yünnan. 3683 Li-kung|茶, (obs.) in Ya-chou Fu, S: ̌̆ch'uan; Sung, C. in Ya 雅 CHOU. 3684 Li-po | 播, (obs.) Yüan, $\mathrm{H}$. near Huang-p'ing $\mathrm{C}$., Kueichou.

\section{李}

3685 Li-kuan-t'un 李官屯, post-town near Kai-p'ing H., Shingking.

3686. Li-wang | 看., post-town near Ku-yüan C., Kansuh.

3687 Li-wên | 文, (obs.) 60 li S. of An-ch 'in H., Shantung; Kin, chên in Mi 密 C.
LI-HU.

\section{里}

3688 Li-ho 里河, chên near Li-ching H., Sliantung.

3689 Li-hsing | 舆, sš̆ near T'ai-wan Fu, Fulhkien.

3690 Li-t. ien I田 Y. near Ch ên C., Hunan.

\section{理}

3691 Li-fan 理番 Li-fan, also [307] called Tsa-ku 雑谷 Tsah-kuh, C. L. T. in $\mathrm{Cl}^{\prime}$ êng-Lung- 'T' ung-MienMon circ., Ssŭcl' 'uan; Lat. $31^{\circ} 50^{\prime}$, I ong. $103^{\circ} 32^{\prime}$.

3692 Li-ting $\mid$ 定, (obs.) T'ang, $60 \mathrm{li}$ W. of Yai C., Kuangtung.

3693 Li-yao | 猺 Li-yau, (obs.) formerly C.L.T. in KuangNan - Shao - Lien circ., Kungtung; Lat. $25^{\circ} 10^{\prime}$, Long. $112^{\circ} 40^{\prime}$.

\section{裹}

3694 Li-t'ang 裹塘 Lithang, T-S. near 'T a-chien-lu $T$., Ssŭch 'uan.

\section{蒩}

$3595 \mathrm{Li}$ 整, (obs.) formerly town near $\mathrm{K}^{\prime}$ ai-fêng $\mathrm{Fu}$, Honan.

\section{離}

3696 Li-hu 離狐.

$1^{\circ}$ (obs.) S. E. of Tungming H., Chihli; $W$. Han, H. in Tung 東 
離 L.I-HU.

chän; E. Han and Tsin, H. in Chi-yin 泖 降 chïn; Sui, H. in 'Tung 東 chïn.

$2^{\circ}$ (Also written | 解) see No. 5467.

3697 Li-hu | 解, see preceding. 3598 Li-shilı / $\mathbf{i}$, see Nos. $3837,4689$.

3699 Ii-shih-yi | 不邑, see No. 7862.

\section{利}

3700 Li 不",.

$1^{\circ}$ (obs.) S. Sung, C. in Huai-hsi 顶 唒 circ.

$2^{\circ}$ (ubs.) IV. H'ei, C. comprising Kuang-บüan II. and T's ang-ch' $\mathrm{i} \mathrm{H}$., Ssǔclı' 'uaiı.

$3^{\circ}$ See No. $3+29$.

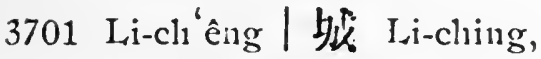
H. in Corea; Lat. $40^{\circ}$ $25^{\prime}$, Long. $128^{\circ} 55^{\prime}$.

3702 Li-ch'iao | f f fir, Y. near Liang-tang H.. Kansuh.

3703 Li-ching | 津 Ii-tsin, [148] H. in Wu-ting Fu, Sliantuing; Lat. $37^{\circ} 43^{\prime}$, Long. $118^{\circ} 26^{\prime}$;-orig., Po-hai 渤 海 chün; Han, Li-ching | 津.

3704 Ii-chou | 州, (obs.) S. Sung, circ. comprising parts of Ssŭch'uan and Shensi.

3705 Li-cli' 'uan | M Li-chuen.
黎 LI.

[74] $1^{\circ}$ H. in shih-nan Hu, Hupelı; I at. $30^{\circ} 15^{\prime}$, I.ong. $109^{\circ} 06^{\prime}$.

$2^{\circ}$ Fu in Ching-ch i 京 the circ. Coreat.

3706 Li-fêng | 䇺.

$1^{\circ}$ (obs.) 3 li S. of 'T'ung C., Kiangsu; Sung. fort in 'T'ung 通 C. $2^{\circ}$ See No. 7352 .

$3707 \mathrm{Li}-\mathrm{kung} \mid$ 菜, (obs.) N.W. of Sung-p'an T., Ssŭch'11an ; $T^{6}$ ang, H. in Li11 䗲 C.

3708 Li-kn1o | 紫, ssĭ near T'ung-shan H., Kiangsu.

3709 Li-min | let, (obs.) S. of Ch'êng-tê F11, Chilnli; Liao, H. in Pei-an 北 这 $\mathrm{C}$.

3710 Li-yüan | 原 H. in Hsienching 故鏡 circ., Corea.

\section{睝}

$3711 \mathrm{Li}$ 稆.

$1^{\circ}$ (obs.) near Ning C., Yünnan; $T^{\prime}$ ang, C. in Cliien-nan 劍南 circ. $2^{\circ}$ See No. 4672 .

3712 Li-clíiu | 邱, see No. 3899.

\section{黎}

3713 Li 醔。

$1^{\circ}$ (obs.) 29 li W. of Yün-ch'êng H., Shantung; $W$. Han, H. in

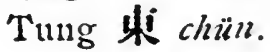


$2^{\circ}$. Sui, Li-yang $\mid$ 曜 $\mathrm{H}$.

$3^{\circ}$ (obs.) N. IV. of Cho C.. Clihhli; I"ang, C. in Ho-pei 河北 circ.

$t^{\circ}$ (obs.) S. Sung, C. in Ch'êng-tu 成都 circ.

$5^{\circ}$ (obs.) formerly K. in Shansi.

$6^{\circ}$ See Nos. 1235, 3429. 3714.

$371+$ Li-ch'êng | 城 Li-ching, [104] H. in Lu-an Fu, Shansi; Lat. $36^{\circ} 25^{\prime}$, Long. $113^{\circ}$ $27^{\prime}$; orig., K. Li |; Sui. Li-ch' êng | 城.

3715 Li-clı' $\mathrm{i}$ |溪, (obs.) $100 \mathrm{li}$ S.W. of Huil-li C., Ssŭch'uan; Yïan, C. in Huich'nan 會川 circ.

3716 Li-ch'i-chou | 溪州, T-S. near Hui-li C., Ssŭch' 'uan.

3717 Li-chon | 州, T-S. in Ch'ing-ch'i H., Ssŭch' wan.

3718 Li-li | 里, chên near Wuchiang H., Kiangsu.

3719 Li-min | 民, chên near Jên-hnai 'T., Kueichon.

3720 Li-p'ing | 平 Li-ping, Fu [394] in the Kuei-tung circ., Kneichou; forming also the district city of $\mathrm{K}^{\prime}$ ait'ai; Lat. $26^{\circ} 10^{\prime}$, Long. $109^{\circ}$; Han, in Tsang-kê 辉牁 chiün; Five dyn., in Ssŭ 思 CHOU; Yüan, 'T'an-ch“i 潭溪; Ming, Li-p'ing | 平 Fu.
禮 LI-Ax。

3721 Li-yang | 陽.

$1^{\circ}$ (obs.) Sui, H. in Honan.

$2^{\circ}$ See Nos. 295\%, 3713.

\section{螭}

3722 Li-ao 螭坳, see No. 5822 .

3723 I,i-pi | 陛, see No. 5822.

\section{豊}

3724 Li-chi 豊基, chün in

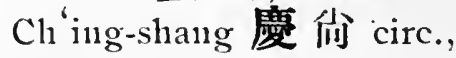
Corea.

3725 Li-shun | 順, ss̆̌ near Chia-ying C., Kuangtung.

3726 Li-tê | 德 $F_{11}$ in Chingch'i 京畿 circ., Corea.

\section{澧}

3727 Li 澧 Li, C.L.C. in the [244] Yo-Ch'ang- $\mathrm{L}_{\mathrm{i}}$ circ., Hu11 an ; Lat. $29^{\circ} 37^{\prime}$, Long. $111^{\circ} 43^{\prime} ; T s^{\prime} i n, C h{ }^{\prime}$ ienchung 默中; Tsin, Nanp'ing 南 4 ; Sui and Ming, Li $\mid \mathrm{C}_{\text {. }} \quad T^{\prime}$ ang, Li-yang | 陽.

$3728 \mathrm{I}$ i-vang $\mid$ 陽, see preceding.

\section{朊典}

$3729 \mathrm{Li}$ 澧 $\mathrm{Li}, \mathrm{H}$. in $\mathrm{Ch}^{\prime}$ in [175]C., Kausulh; Lat. $34^{\circ} 10^{\prime}$, l,ong. $105^{\circ} 10^{\prime} ; T s^{\prime}$ in. T'ien-chia 态嘉; Han, Han-yang 漢陽; Sung, Ch' ang-tao 長道.

3730 Li-an | 安 H. in Ch'ingshang 㾍份 circ., Corea. 


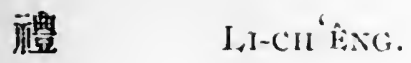

3731 Li-ch'êng | 城, (obs.) native place of Eimperor Shun 棌: near P'ing-yang Fu, Shansi.

3732 Li-shan / II H. in Chungclining 出清 circ, Corea.

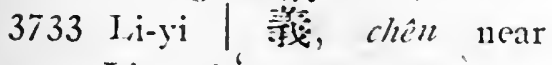
Ling-ch nan H., Shansi.

$$
\text { 触 }
$$

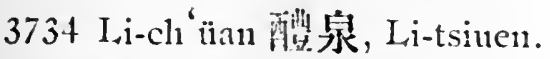
[206] $1^{\circ}$ H. in Hsi-an 'ìu, Shensi ; Lat. $34^{\circ} 30^{\prime}$, Longr: $108^{\circ} 20^{\prime}$; Han, $\mathrm{Ku}$-k'on 谷口; $E$. Han, Cl'ih-yang 池 陽; Kin, Li-cl' üan 泉 H.; T'ang, Wên1 sin 㴓秀.

$2^{\circ} \mathrm{Chün}$ in Ch'ing-sheng 慶偷 circ., Corea.

3735 Li-ling | 陵 Li-ling, II. [79] in Cli ang-sha Fu, Hunan; Inat. $27^{\circ} 41^{\prime}$, Iong. $113^{\circ}$ $16^{\prime} ;$ Han, I in1-lisiang 監 洲; E. Han, I, i-ling | 陵 H.

\section{幽:}

3736 Li 鹿, see No. 7855 .

3737 Li-chiang | 汀. Li-kiang.

[557] $1^{\circ} \mathrm{Fu}$ in the Yi-lisi circ., Yünnan ; fornining also the district city of the same nane; Lat. $26^{\circ}$ 52', Long. $100^{\circ} 27^{\prime}$ : Yüan, I,i-chiang | 汀. circ.; Ming, Li-chiang 江 $\mathrm{Fu}$.

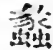
I.I-WL.

$2^{\circ} \mathrm{H}$. forming the prefectural city of the sane name, Yünnan; Iat. $26^{\circ} 52^{\prime}$, Long. $100^{\circ} 27^{\prime}$; Han, Tingtso 定管; Tang, Kunning 昆明; Ming, 'T'ung-an 通娄 C.

$3^{\circ}$ See No. 6169.

3738 Li-shui | 水 Li-shwui, $H$. forming the prefectural city of Ch' "n-chou, Chelnkiang; Lat. $28^{\circ} 26^{\prime}$, Long. $119^{\circ} 57^{\prime}$; Han, Chang-an 章: 安; Sui, Kua-ts'ang 括蒼; Yiuan Li-shui | 水 $\mathrm{H}$.

3739 Li-yang | 陽, ss̆̈ in Chung-lisiang H., Hupeh.

\section{露}

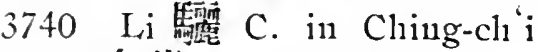
京畿 circ., Corea.

$37+1$ Ii-ch'êng 艇, see No. 1890.

$37+2$ Li-yi | 邑, (obs.) Tsin, II., E. of Lint-t ${ }^{6}$ ing H., Sliensi.

\section{盖}

$3743 \mathrm{Li}$, 蟹 $\mathrm{Li}, \mathrm{H}$. in Palo-ting Fu, Cliillii; Lat. $38^{\circ} 32^{\prime}$. Long. $115^{\circ} 40^{\prime}$; Han,

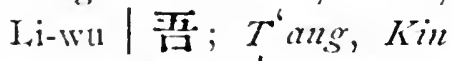
and Ÿ̈an, Li | C.; Ming, $\mathrm{L}_{\mathrm{i}}$ H. Sung Yung-hing 永然 Mil. District.

3744 Li-wu | 菭, see preceding and No. 5277 . 
荔莎 I.

\section{菬}

3745 Li 菬, see No. 3747 .

$37+6 \mathrm{~L}, \mathrm{j}-\mathrm{po}$ 波 Li-po, H. in [474]'Tu-yün I'n, Kneichon; I at. $25^{\circ} 32^{\prime}$, Long. $107^{\circ}$

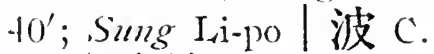

$37+7$ I i-p 11 |浦 L,i-pu, H. in [192]P'ing-lê Fu, Kuangsi; Lat. $24^{\circ} 20^{\prime}$, Itong. $110^{\circ}$

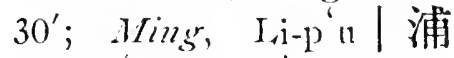
H.; Thlag, Li | C.

\section{力}

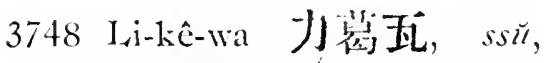
near Wên-ch nan H., Sš̆ ch' nan.

\section{螭}

$37+9$ I,i-ao 螭坟放, see No. 5822 . $3749^{*}$ I,i-pi | 陛, sce No. 5822.

\section{殐}

3750 I i i-ts'm 粱村.

$1^{\circ}$ Chîn near WWên-1ısi II., Shansi.

$2^{\circ}$, is̆̌t near Inn1wg-hisin H., Kiangsi.

\section{溧}

3751 I,i-shui 溧水 I, ih-shwui,

[65] H. in Chianng-ning Fu, Kiangsu; Iat. $31^{\circ} 43^{\prime}$, I.ong. $119^{\circ} 07^{\prime} ;$ Han I, yang | 陽; Sui, I, i-shni 水 $\mathrm{H}$.

3752 I,i-yang | 陽 Lilh-yang. [19t] $1^{\circ}$ H. in Cliên-chiang Fì, Kiangsu; I at. $31^{\circ} 32^{\prime}$, I.ong. $119^{\circ} 32^{\prime} ; T^{*}$ ang,

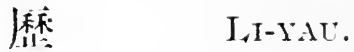

Li-yang | 陽 $\mathrm{H} . ; W_{u}$, Yu11\%-an 永笠; $T \sin$, Yung-shili 永愔.

$2^{\circ}$ See No. 3751 .

\section{if.}

3753 I i-chiang | 将 S'š̆ in Hsin-lising H., Kuangtuly.

3754 Li-shan III, see No. 78.35 .

糜勧

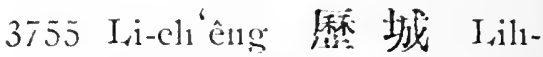
ching, II. forming the prefectural city of Clinan, Shantung; Iat. $36^{\circ} 40^{\prime}$, long. $117^{\circ} 01^{\prime} ;$ S.A. I. ilisia $\mid$ 下in K. Clii 濟; T'ang, P'ing-ling 平陵;

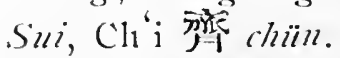

3756 I i-11sial TF, sce preceding. 3757 Ii-ling | 陵, see No. 6306. 3758 I i-t'irg | ist, see No. $327 \%$.

3759 Li-rang | 榢, see Nos. 1989, 2038.

I,i-cling, see Nos. 3701, 3714.

I.i-clne11, see No. 3705. Ii-kiang, see No. 3737. I,i-ping, see No. 3720. I i-pu, sce No. 3747. Ii-sliwui, see No. 3738. Iithang, see No. 3694. Li-tsin, see No. 3703. Ii-tsinen, sce No. 3734. Ii-yau, see No. 3693. 
兩

I.IANG.

\section{LIANG}

\section{兩}

3760 Liang-an 兩娄, chên, near Ch'êng-ch'êng H., Shensi.

3761 Liang-chê | 浙, (obs.) sung, circ. (This term is now used to designate the eastern and western divisions of Chelikiang, in connection witl the salt administration.)

3762 Liang-cliang | 江. Liangkiang, collective name given formerly to the provinces of Fiangnan and Kiangsi, but now connprising Kiangsi, Kiangsu and Anhui.

3763 Liang-h1n | 湖, or Huknang 湖黁, collective name for the provinces of Hunan and Hnpeh.

3764 I,iang-1nnil | 淮; a term used to designate the northern and sonthern divisions of the Hnai-yang salt administrative district.

3765 Liang-kuang | 栱 Liangkwang, or Liang-yüel | 畗, collective name for the provinces of Kuangtu11s and Knangsi.

3766 Liang-tang | 當 Liang[336]tang, H. in Cl' in C., Kansuls ; I at. $33^{\circ} 58^{\prime}$,
長 IIANG-T'TFN.

Long. $106^{\circ} 22^{\prime} \mathrm{Han}$, Wutu武都; Sung, Liang-tang 嘗 $\mathrm{H}$.

3767 Liang-t'on-mên | 領旧, chên near Ch'êng H., Chehkiang.

3768 Liang-tı | 渡, chên near Ling-shih H., Shansi.

3769 Liang-yiich | 椄, see No. 3765.

\section{鼠}

3770 Liang-chai 主寨, T-S. near Sung-p an T., Ssŭcli'nan.

3771 Liang-chang | 章, Y. near Yung-sui 'T., Hunan.

3772 I,iang-ch $\mathrm{i} \mid$ 溪, 'T-S. near Yin-cliang H. Kneichou.

3773 Liang-ho|不, sš̆ near Ch'i-mên H., Anhui.

3774 I iang-hsiang | 细 Liang[549]hiang, H. in Shnn-t ien Fu, Chihli; Lat. $39^{\circ} 44^{\prime}$, Long. $116^{\circ} 05^{\prime}$; orig., in K. Yen 燕; Sung, Lianglısiang | 经 $\mathrm{H}$.

3775 I, iang-hsing | 與, ss $\check{6}$ in Hui-an H., Fnhkien.

3776 Liang-kung | 茶, (obs.) 130 li S. E. of Min H., Kansuh ; Sui, . H. in Tang-clı'ang 宕昌 chün; T'ang, H. in 'Tang 宕 C.

3777 Liang-shê | 舍, chên near Lunng-tê H., Kansulh.

3778 Liang-t'ien|田, ssü in Ch' ên C., Hunan. 
宠

LIANG-I'I.

3779 Liang-yi | 邑, see No. 5750 .

3780 Liang-vïan | 原, (obs.) 90 li N. W. of I,ing-t ai H., Kansulı; Sui, H. in An-ting 学定 chïn; T'ang, Sung, and Kin, H. in Cling 涩 C.

\section{梁}

3781 Liang 梁.

$1^{\circ}$ One of the nine chou or great divisons of the Empire instituted by Yii 需; comprising Ssŭch 'ran and parts of Shensi, Kansuh, and Hupeli. The mane is sometimes also written 淙。

$2^{\circ}$ (obs.) Han, K. in Kiangsn.

$3^{\circ}$ (obs.) E. Mci, C. near K'ai-fêng I'n, Honan.

$4^{\circ}$ See Nos. 1950, 2607, 3005,3061 , and 4675 .

37S2 I,iang-ch'i | 期, (obs.) I幺.

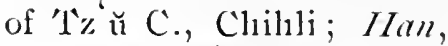
H. in Wei 魏 chän.

3783 Liang-chia | 家 Y. near Han-chung Fin, Shensi.

3784 I iang-chiang $\mid$ 江, (obs.) formerly town near I,iangshan H., Ssŭch'nan.

3785 Liang-ch'ïan | 泉, see No. 1716.
凉 LIANG.

3786 Liang-chuang | 莊, posttown near Kn-cli êng H., Chihlit.

3787 Liang-fêng | 豐, (obs.) near Kuang-chou Fin, Kuangtung; $N$. $\mathrm{V}_{e} e, \mathrm{H}$.

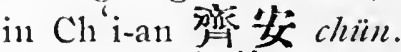

3788 Liang-111 | 湖, ssü in Shang-y ï H., Clielikiang.

3789 Liang-hua | 化, see Nos. $2365,3644$.

3790 Liang-shan | Ifl Liangslian.

$1^{\circ}$ Chïn in Cl'ing-shang 慶 份 circ., Corea; Iat. $35^{\circ} 16^{\prime}$, Long. $129^{\circ} 35^{\prime}$.

[295] $2^{\circ}$ H. in Chung C., Ssŭclinan; I at. $30^{\circ} 46^{\prime}$, Long. $107^{\circ} 5 \mathrm{i}^{\prime}$; Han. Ch'nn-jên 胸䏰; Sui liang-shan | iil.

$3^{\circ}$ Post-town near fiêng H., Shensi.

3791 I,iang-shan | 舎, chên near Ningr-y ï:un H., Kansul.

3792 L iang-! in $\mid$ 榆, see No. 2055 .

3793 I.iang-yiuan | 園, chển in Ho-fei H., Anluui.

379+ I,iang-youan | 原, chên near Ling-t'ai H., Kansul.

3795 Liang-yiian | 源, chên near Chnng-pu H., Shensi.

\section{涪}

3796 I iang 谅, sec Nos. 3781, 3797. 
凉

IAANG-CHOV.

3797 Liang-chon | 州 Liang[230] chan, Fu in the KanLiang circ, Kansuin; forming also the district city of Wu-wei; Lat. $37^{\circ}$

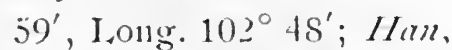
in Wu-wei 武威 chïn; T'ang, Liang|C. Yüan Hsi-liang 西淙 C.

3798 Liang-clnang | 菲 I,iangclwang, 'I. in I iang-chon Fin, Kansuls.

3799 Liang-shan-yi |山 菼, T-S. near Yüieli-sui ' 1 ., Ssŭch' nan Ming, I,iangchai | 寨 ssŭ.

\section{亮}

3800 Liang-ch'ai 亮柴, T-S. near Hsia-chiang ' $T$. Kneichon.

Ijang-chan, sec No. 3797. I,iang-clıwang, sec No. 3798.

Liang-hiang, see No. 3774. I iang-kiang, see No. 3762 . I iang-kwang, see No. 3765 .

\section{LIAO}

恣

3801 I iao 拈 I,ian, C.L.C. in

[75] the Chining circ., Shansi; I at. $37^{\circ} 03^{\prime}$, Irong. $113^{\circ}$ 28; S.A., in K. Clin 晋; in C. Il tors, 1st, K. Han 㴔, 2nd, K. Chao 趋; Tsin and Han,
涾 I,IAO-PN.

Shang-tang 上䉪；2nd Wir, Iino-yang | 陽; Sui, Liao-slan | III T'ang, Liao | C.., Clii 筫: C. and $\mathrm{Yi}$ 儀 $\mathrm{C}$; . Ming, Liao $\mid \mathrm{C}$.

\section{J.iao-11si| 西.}

$1^{\circ}$ (obs.) E. of Ist-lung H., Chihli; $I V$. Han, I iaco-hsi |西 chiin.

$2^{\circ}$ (obs.) W. of Fu-11ing H., Chihli ; E. Han, and Tsin, chion in Y'n 幽 crov.

$3^{\circ}$ (obs.) $30 l i \mathrm{~N}$. of Itllung H., Chilhli; $N$. WCi, chün in P'ing 平 choc. Han Fei-ju 肥 如 $\mathrm{H}$.

$4^{\circ}$ (obs.) $\mathrm{W}$. of the right wing of the 'Tumed tribe (near Knei-luna 䟺化 T., Shansi); Sui, chiin in Clii 犦 chov.

$5^{\circ}$ (obs.) to $/ i \mathrm{I}$. of $\mathrm{Yi}$ c., Shingking; Lino, C. in Hsien 顯 CHOr'; Kin, chên in Knangning 鹰 藏. Fu. Lian Ch`ang-ch 'ing 長磨 H. $6^{\circ}$ See No. 3805.

3803 Iiao-pin | 寶, (obs.) 180

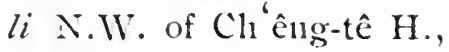
Slningking; Lino, H. in Liao C.; $/$ Kin, H. in Shên 潘 $\mathrm{C}$. 
3804 Liao-shan | IL, see No. 3801 .

3805 Liao-tung | 東.

$1^{\circ}$ Literary name of the province Slingking; orig., in K. Yen 澡; Ts'in, Yu 陷 CHOU or Liao-lisi | 西, and Ying 管 CHOU or Liaotung |東; Han, in Chao-lisien 朝鮮; Sui, Kao-cliü-li 高何 屚; Five dyn., Tw11g-ching 東宗; Kin, Liao-yang 隄.

$2^{\circ}$ (obs.) in Chin-clion Fu, Shingking; E. Han, K. in Yu幽 C. $3^{\circ}$ (obs.) 25 li $\mathrm{W}$. of An-su H., Chilliti $N$. $W_{c i}$, chïin in S. Ying 營 CHOC.

$4^{\circ}$ (obs.) in Liao-yang C., Shingking; Ming, ss $\breve{t}$ in Shantung.

3806 Liao-yang | 晹 Lian-yang. [439] $1^{\circ}$ C. in Fênng-t'ien Fu, Shingking; Lat. $41^{\circ}$ $10^{\prime}$, Long. $123^{\circ} 27^{\prime}$; Ming Tzŭ-tsai 自存 C. I1 1905 port open to trade.

$2^{\circ}$ See Nos. 3801, 3805.

\section{侧}

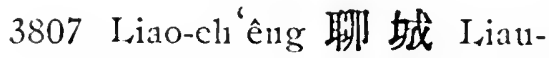
ching, $H$. forming the prefectural city of 'Tung- cliang, Shantung; Lat. $36^{\circ} 37^{\prime}$, Lo11g. $116^{\circ} 12^{\prime}$; S.A., I I iao-sliê | 攝; Sung, Jiao-ch'êng | 城。 $\mathrm{H}$. and $\mathrm{Po}$ 博 $\mathrm{C}$.

3808 I,iao-shê $\mid$ 攝, see preceding. 琴

3809 Iiao 曑, (ols.) S.A., K. in Honan; see No. 3287.

Liat, see No. 3801.

Iian-ching, see No. 3807. Lianl-yang, see No. 3806.

\section{LIEH}

多)

3810 Lieh-jên 列 人: (obs.) formerly H., N. of Feihsiang If., Chihli.

3811 Lieh-yï $\mid$ 路, T'S. in Kokonor.

\section{LIEN}

排实

3812 Lien 麻, (obs.) S. Sung, C. in Knang-lisi 廣 皈 cire.; see Nos. 3175, 3815. 3813 Lien-chiang $\mid \mathrm{KT}^{\circ}$, (obs.) N. of Shili-ch êng H., Knangtung; $T^{\prime}$ ang, H. in Lo 羅 C.

3814 Lien-ch'in | 鍁, circ. in Kuangtung, comprising I,ien-chon Fin, and Ch'in C.

3815 I.icn-chon | 恻 Sicn-chan, 
蓼 I, IFN-CHOU.

[248] Fin in the Kalo-I,ien citc., Knangtung; forming also the district city of $\mathrm{Ho-p} \mathrm{p}^{\prime} \mathrm{u}$; I at. $21^{\circ} 39^{\prime}$, Long. $108^{\circ}$ $59^{\prime} ; T^{\prime}$ in, in Hsiang 集 chän; E. Han, Ho-p $u$ 合 浦; Wu, Cluu-kuan 珠管; 1st Sung, Yïlel 越 $C$.; Sui, Ho 含 C.; T'ans, Lien | C.; Sung, 'T"aip'ing 太平; lling, I,ienclon 州 Fir.

\section{瀲}

3816 Iien-ch'êng 瀕城, ss near Futung H., Fulıkien.

\section{練}

3817 I ienlt'an 練 潭, chên in 'I'ung-ch' êng H., Anlıui.

\section{栔}

3818 Lien-hsiang-knan 煤袋 關, 'T-S. near I,n-fêng H., Yünnan.

3819 Iien-fan | 攀, (olss.) near P'ing-yang Fu, Shansi; Sung, military post in P'ing-yang Fin.

\section{連}

3820 Iien 連 I.ien, C.I.C. in [150] the Kuang-Nan-Shao-Lien circ., Kuangtung; I at. $24^{\circ} 50^{\prime}$, Long. $112^{\circ} 14^{\prime}$; IV:Han, Knei-yang 桂 陽; 7"ang, Lien | C.

3821 Jien-ch' êng | 城 I,ienching.
速 IAFN-SIIX.

$1^{\circ}$ H. in 'in'ing-chon Fin, Fulıkien ; I at. $25^{\circ} 37^{\prime}$, Ifolg. $116^{\circ} 50^{\prime}$.

$2^{\circ}$ 'T'S. near Ku-lang H., Kausulı.

$3^{\circ}$ (obs.) Trang, H. 50 li E. 'T's'ên-ch' i H., Kunangi.

3822 I ien-chiang | 江. I,ienkiang.

$1^{\circ} \mathrm{H}$. in Fin-clion Fu, [on sea] Fulukien; I at. $26^{\circ} 08^{\prime}$, I.ong. $119^{\circ} 20^{\prime}$; Tsin, Irên-11na 溫 麻; T"ans, I,ien-chiang | 泣.

$2^{\circ}$ (obs.) İ. of 'lien-pai H., Kuangtung; Sui, II. in Kao-liang 䓪凉 chiinl.

3823 I,ien-cln'ih | 池, (obs.) near Hsü-chou Fu, Ssŭcli'uan; Y゙ang, H. in Cling 鏡 $\mathrm{C}$.

$382+$ Iien-ch' nan | M. in Cling-chii 京畿 circ., Corea.

3825 Iien-jan | 然, see No. 89. 3826 Lien-p'êng 朋, post-town near Yao C., Yümnan.

3827 Iien-p ing | 平 I,ien[311] ping, C. in Hui-chon Fu, Knangtung; I at. $24^{\circ} 19$, I,ong. $114^{\circ} 18^{\prime}$.

3828 Iien-shan | U Lien-shan. [138] $1^{\circ}$ C.I..'T. in Kuang-NanSlrao-I,ien circ., Kuangtung; I at. $2 t^{\circ} 45^{\prime}$, 
Long. $112^{\circ}$; orig., Knei-yang 桂 防; Liang, Knang-tê 黁 嘼; Ming I,ien-shan |

Ill H. Terr. jur.

$2^{\circ}$ H. in Chnno-ch'ing 出清 circ., Corea; Lat. $36^{\circ} 35^{\prime}$, Long. $127^{\circ} 04^{\prime}$.

$3^{\circ}$ Post-town neat Fêngt'ien-I'n, Shingking.

3829 Lien-shui|水, see No. 104.

3830 Lien-tao | 滇, (obs.) Han, II., IV. of Yi-yang H., Itnnan.

3831 Lien-wo / 㩦, chên near 'Tung-kuang H., Clinhli.

3832 Lien-yü11 | Ssŭclìuan; Sung, pao iin Shih-cl üan 石泉 nilitary district.

\section{瓘}

3833 Licn-shih 琶速柿, ss̆̆ in Knei-an H., Chelnkiang.

\section{蓮}

383+ Lien-lua 蓮花 Lien-luwa. $1^{\circ}$ 'T. in Clii-an Ent, Kiangsi; I iat. $26^{\circ} 50^{\prime}$, I.ong. $114^{\circ}$. Terr. jur. $2^{\circ}$ Chên near Clh ing-hui H., Kansult.

$3^{\circ}$ Y. near Yang-chiang '., Kuangtung.

3835 Lien-t'ang | 塘, posttown near Yang-chiang 'le, Kunngtung.

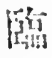

\section{命}

3836 Lien-cliti 命 愿, (obs.) N.II. of P'ing-fan $\mathrm{H}$., Kansun; Han, H. in Chini-clìng 金城 chün.

Lien-clian, see No. 3815.

Lien-ching, see No. 3821. I.ien-hwa, see No. 3834.

Lien-kiang, see No. 3822 . I icn-ping, se No. 3827.

Lili-ching, sce No. 375.5.

I,ih-shwui, see No. 3751 .

Lihlyang, see No. 3752.

\section{LIN}

\section{臨}

\section{Lin 臨 Iin.}

[98] $1^{\circ} \mathrm{H}$. in Fên-chon Fu, Shansi; Lat. $38^{\circ} 05^{\prime}$, Long. $110^{\circ} 58^{\prime}$; Han, I.i-shil 離盾; $P$. Chou, Mn-t n 息笑; Sui, 'l'ai-ho太和; T $T^{*}$ ang, Lincch ïin|沓.

$2^{\circ}$ Chên near Hsing-p'ing H., Shensı. Yïan|H. $3^{\circ}$ See Nos. 1560, 6358. 3838 Lin-an | 姲 Lin11ngan. [96] $1^{\circ}$ Fin in the Yi-nan circ., Yiimuan; forming also the district city of Chien-slui; Lat. $23^{\circ}$ $37^{\prime}$, I.ong. $103^{\circ} 05^{\prime}$; orig., Cly ï̈-t'ing 们町; I Ian, in Tsang-kê 㸼 嗬 chïn; $T^{6} a n g$, 
'T"ung-laai 通海 chiun; Sing, Hsill-shan 秀 山; Yйаn, A-p'o 阿整 in I in1-an | 安 circ.; Ming, Jin-an| 安 Fu. $2^{\circ}$ H. in Hang-chon $\mathrm{Fu}$, Chelıkiang; Lat. $30^{\circ}$ $16^{\prime}$, Long. $119^{\circ} 42^{\prime}$; Han, Lin-shnii | 水; Tsin, Lin1-i11 | 努.

$3^{\circ}$ (obs.) in Shantung; $\mathrm{W}$. Han, Marq. in Lang-yel 瑯㑚 chiin. $4^{\circ}$ (obs.) 5 li N. E. of 'T'ung-laai H., Yünnnan; Yüan, circ. in Yünnnan. $5^{\circ}$ See No. 936.

3839 Lin-clang | 漳 Lin-cliang. [10] $1^{\circ} \mathrm{H}$. in Chang-tê $\mathrm{F} 1$, Honan; Lat. $36^{\circ} 30^{\prime}$, I.ong. $114^{\circ} 46^{\prime}$; Han, Yell 嶫, Ming L,inchang | 漳 H.; $W$. Wei, Yeh 牟 $\mathrm{H}$.

$2^{\circ}$ Post-town near Clinngp'u H., Fuhkien.

3840 Lin1-chên | 德, (obs.) 170 li E. of Kan-cli iian H., Shensi; N. Wei, H. in Tinng-yang 定陽 chiun; Sui, H. in Yel1-an 延岀 chün; T"ang, H. in Yen 延 C.; Sung and Kin, H. in Yen-an 延岁 Fin.

$38+1$ Æin-cll ên | 鹿, (obs.) WV. of Hsüau-hua H., Kuangsi;

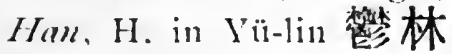
chïn.
$38+2$ Lin-ch' êng | 城 Lin-ching. [459] $1^{\circ} \mathrm{H} .{ }^{\circ}$ in Chao C., Clithli ; I.at. $37^{\circ} 27^{\prime} ;$ I,ong. $114^{\circ} 46^{\prime}$; orig., Fangtzŭ-vi 房子邑; $T^{\prime}$ ang L.inl-ch' êng | 城 H. Ling-iu (Polo.)

$2^{\circ} C^{\circ} \hat{e}^{\prime \prime}$ in 'T êng H., Sliantu11g.

$3^{\circ}$ See No. $122 \%$.

3843 Lin-cll ềng | 烝, see No. 2035.

3844 Lin-chi | 濟, see No. 20.t. 3845 I,in-cli ${ }^{\circ}$ | 淇, (obs.) 70 li S. E. of I,in H., IIonan; N. Woi, H. in I, inllï 林 虑 chïn.

$3846 \mathrm{I}_{\mathrm{in1}}-\mathrm{ch}_{1}{ }^{\mathrm{i}} \mathrm{i} \mid$ 深.

$1^{\circ}$ (obs.) $50 \mathrm{li} \mathrm{N}$. of $\mathrm{P}^{\prime \prime} \mathrm{n}-$ chianng H., Ssŭcli' "1an; T"ang, H. in Clining 巧 C. S S r i , Pn-che 蒲 溪 $\mathrm{H}$.

$2^{\circ}$ See No. 6316.

3847 Lin-chiang i. I. In-kiang. [552] $1^{\circ} \mathrm{F}_{11}$ in the Jui-YüanLin circ., Kiangsi; forming also the district city of Cll' 'ingchiang; Lat. $28^{\circ} 06^{\prime}$. I.ong. $115^{\circ} 27^{\prime}$; S.A., $1 s / \mathrm{in} \mathrm{K}$. Win 哭, 2 d in K. Yiueh 越; C. llars, in K. Chin 楚 $7 s^{\circ} \mathrm{m}$, in Chitu-chiang 九江. chiinn; Hon, Yüi-cliang

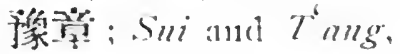




\section{䟢}

IAN-CHIANG.

Hung 洪 and Chi 吉; S. Sung, Lin-chiang |

江., military district in Chiang-1ssi 江. 西 circ.

$2^{\circ}$ Clion in Yi-lan Int, Kirin.

$3^{\circ}$ Ss s̆ near Yo-chon Fu Inu11an.

$4^{\circ}$ Post-town near Shanyang $H$. , Shensi.

$5^{\circ}$ (cbs.) S. of Ju-kao H., Kiang-su ; S. Sung and S. Ts $i, \mathrm{H}$. in Hai-ling 海陵 chiinl

$6^{\circ}$ (obs.) in Yü̈n1 Sung, H. in Chien-tu 建都 chïn.

$7^{\circ}$ (obs.) N. F. of Ho C., Anhui ; S. Ts'i, chün

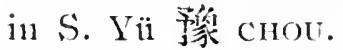
$8^{\circ}$ (obs.) 140 li $\mathrm{S}$. of Min C., Kansuh, Sung, military post in Min 距 C.

$9^{\circ}$ See Nos. 1142, 1236, 1560, 6381.

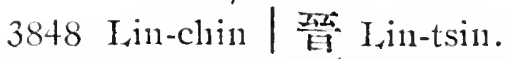
$1^{\circ} \mathrm{H}$. in $\mathrm{P}^{\prime}$ t1-clion Fu, Shansi; I at. $35^{\circ} 10^{\prime}$, Jong. $110^{\circ} 36^{\prime} ; T^{\prime} \mathrm{ang}^{\prime}$ I.in-chin 登 H.; Han; Clich-liang 解梁; Sni, Sang-ch'ïan 汖. $2^{\circ}$ Sce No. 367, 6051. 3849 Lin ching | 津. $i^{\circ}$ (obs.) 130 lis. E. of Cliven C., Ssŭclı' "uan; (No. 910); Sui, H. in
IAN-CH $\mathrm{U}$.

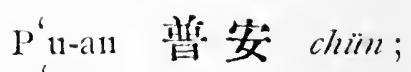
T'ang, IH. in Chien 劍 $\mathrm{C}$.

$2^{\circ}$ (obs.) $50 l i \mathrm{~N} . \mathrm{W}$. of $\mathrm{Yi}$ lising H., Kiangsu; ; S. Sing, and $S .7 s^{6} i \mathrm{H}$. in Yi-lnsing 義與 clün. $3^{\circ}$ (obs.) $20 l i$ S. W. of Ning-cling 俩津 H., Clinhli ; $T^{6}$ ang, H. in Ching 景 C.

$4^{\circ}$ (obs.) in Ssŭcl 'uan; T゙ ang, H. in Pêng 彭 C. $5^{\circ}$ (obs.) near Hsii-chon Fin, Ssŭch' nan; T゙ang, H. in I $\mathrm{I}_{\mathrm{O}}$ 洛 C.

$6^{\circ}$ (obs.) in Shingking; Kin, H. in Han 故 C. $7^{\circ}$ See No. 2814.

3850 Lin-ch' ing | 清 Liu-tsing, [322]C. L. C. in the Chi-'tung'T"ai-Wu-Lin circ., Shantung; Lat. $37^{\circ} 03^{\prime}$, Long. $115^{\circ} 50^{\prime}$; Han, Cly ingyüan 清淵; Wing Linch'ing | 清 C.

3851 Lin-chinng | I

$1^{\circ}$ (obs.) W. of Ya-an H., Ssŭclı nan; Sui, chiiln in Liang 梁 chov. $2^{\circ}$ See Nos. 1309, 5316, 7213.

3852 I in1-ch'n-chêng | 涂城, (obs.) N. Tsi town E. of $\mathrm{Cl}^{\prime}{ }^{\prime} \mathrm{C}$., Anhui.

3853 Lin-chü | 店, (obs.) in Yü1-lien H., Ssŭclı́nan; T*ang, H. in Yün 筠 C. 
臨 $\mathrm{I}$ NN-CḦ̈.

3854 Lin-chü | 沮.

$1^{\circ}$ (obs.) N. W. of Tangyang $\mathrm{H}$. Hupels ; Han, H. in Nan 南 chün; Ysin, H. in Hsiangyang 裂榢j chïn; $S$. Sung and S. Ts'i, H. in Nanl 㑲 chün.

$2^{\circ}$ See Nos. 4561, 7746.

3855 Lin-cln'ï| 朐 Lin-kï.

[304] $\mathrm{l}^{\circ} \mathrm{H}$. in Clı'ing-chou $\mathrm{Fu}$, Shantung; Lat. $36^{\circ}$ 35', I.o11g. $118^{\circ} 50^{\prime}$; S.A., $\mathrm{P}^{6}$ ien 鹖, town in K. Clii 湾; Han, I,in-clïi | 居.

$2^{\circ}$ (obs.) N. of Yeli $\mathrm{H}$., Shantung; $W$. Han, H. in 'lung-lai 束倠 chiin.

$3855^{\mathrm{A}}$ L Lin-cli $^{6} \ddot{i} \mid$ 沟 See No. 5373.

3856 Lin-ch'nan | 川 Linchnen.

$1^{\circ} \mathrm{Fu}$ in Corea; Lat. $36^{\circ}$ $50^{\prime}$, I.ong. $125^{\circ} 55^{\prime}$.

$2^{\circ} \mathrm{H}$. forming the prefectural city of Fu-cliou, Kiangsi ; Iat. $27^{\circ} 57^{\prime}$, I.o11g. $116^{\circ} 18^{\prime}$; Han, Nan1-ch'êng 南少成; $E$. Han, I,in-ju 江; Wu, Hsi-p'ing 䣲 惊; -Tsin, Hsi-fêng 西䓙; Liang, 'ling-cl' "nan 缹 川 ; T'ang I,iu-clin 'nan | 川.
潐 I.NヘーHU.

$3^{\circ}$ (obs.) T"ang, H., S. E. of Yai C., Kuangting. $4^{\circ}$ See No. 4568.

3857 I in1-cli 'üan | 泉, see Nos. 2814, 3837.

3858 Lin1-fên | 汾 Lin-făn.

$1^{\circ} \mathrm{H}$. forming the prefectural city of $\mathrm{P}^{\text {'ing- }}$ yang, Shansi; Iat. $36^{\circ}$ $06^{\prime}$, Itong. $111^{\circ} 33^{\prime}$; Han, Lin-fên | 汾; T'ang Chin 丝 C.; T"ang, Hsi-110 西河.

$2^{\circ}$ (obs.) $25 l i \mathrm{~N}$. E. of Chiang C., Shansi ; $I V$. Han, H. in Hotung 湖東 chïn; $T s^{\prime} i m$, and N. IVci, H. in P'ingyang 快晹 chïn.

$3^{\circ}$ See Nos. 769, 1691, 2585.

3859 I in-fên|顀, see No. 5582. 3860 Lin-hai | 海 I,in-liai, H. forming the prefectural city of ' $\mathrm{T}$ 'ai-chon, Cliehkiang; Lat. $28^{\circ} 54^{\prime}$, Long. $121^{\circ} 10^{\prime} ;$ Han, Cliang-an 章发; Tsin, Lin-hai 「海 H.; T'ang, I.in |C.

3861 Lin-han $/$ 嫁, see No. 6462. 3862 Lin-110|河, see No. 7364. 3863 Lin-lio | 賀, see No. 2096. 3864 Lin-hu | 湖 (obs.) s0 li S. W. of Wu-wei C., Anhui; Han and $T$ sin, H. in Lï-chiang 瀘江. chïn. 
臨

$3 \$ 65$ I in-huai | 潐.

$1^{\circ}$ (obs.) $80 \mathrm{li} \mathrm{N}$. W. of Hsü-yi H., Anhui ; II. Han, chïn.

$2^{\circ}$ (obs.) in Kiangsu; $S$. Sung and S. Ts' $i$, chiin in S. Hsii 徐 cHOt.

$3^{\circ}$ (obs.) S. E. of Lingpo H., Anluni; $N$. Hei, 1st, chïn in Jên 仁 chov; 2nd, chün and H. in Pien 汒 CHOr.

$4^{\circ}$ (obs.) in Anhui; $N$. Wii, H. in 1st, Hsiatśai 下蔡 chiin, 2nd in Ju1ng-yang 笑 陟 chïn, 3rol in Pei-t' $u n g$ 北通 chiinn.

$5^{\circ}$ (obs.) 1 li $\mathrm{N}$. of Hsïi H., Anluni; $T^{6}$ ang, Sung, Kin and Yüan, II. in s.sŭ 沉 C. $\dddot{T}^{6} a n g$ Ss

$6^{\circ}$ (obs.) 20 li N. E. of

I'êng-ỹang Fu, Aului; Ming, H. in Fêng-yang Fin; Lat. $32^{\circ} 55^{\prime}$, Long. $117^{\circ} 30^{\prime}$; Han, Chung$1 i$ 鉬離; Sui, Hao 嫁 C.; now a village, Lin1ln1ai-1siang | 淮楖. See No. 3866.

$7^{\circ}$ See Nos. 2244, 2892.

3866 I.in-1nnai-11siang | 淮 细, $C h^{6} \mathrm{cn}$ in Fêng-yang Fin, Kiang-su; sce also preceding.

\section{臨 IAN-K'AI-KUANG。}

3867 Lin-hung | 洪, (obs.) 60 li S. of Kan-yü H., Kiangsu; Kinl, chên in Hai 海 C.

3968 I,in-hsi | 西.

$1^{\circ}$ (obs.) in Annan; T Tsin, and $S . T s^{\prime} i, H$. in Hsin-ch' ang 新昌chïn. $2^{\circ}$ (obs.) in Li-cliang II., Yünnan; Yüan, H. in Chü-ching 巨津 C.

3869 Lin-lnsiang | 洲 Lin-siang. [528] $1^{\circ}$ H. in Yo-chon F'n, Hunan; Jat. $29^{\circ} 34^{\prime}$, Long. $113^{\circ} 03^{\prime}$; Han, Pa-ling 巴陵; Sung, I,in-lisiang | 湘.

$2^{\circ}$ See Nos. 267, 3735 .

3870 Iin-lisü $\mid$ 徐, (obs.) in Annam; $N$. HCi, H. in Clin iao 譙 C.

3871 Lin-ju 汶, see Nos. 3856, 7428.

3872 I in-jung | 我.

$1^{\circ}$ (obs.) W. of ancient So-fang 朔少 H.; Han, H. in So-fang 桄 chün.

$2^{\circ}$ (obs.) N. W. of Yich' nan H., Sliensi ; $N$. Hei, H. in Tung-lisia 東夏 $\mathrm{C}$.

3873 Lin-K'ai-Kuang | 開 廣, circuit in Yünnan, con1prising Lin-an Fu, K'ailuna Fu and Knang-nan Fn. 
䂑

L,IN-KANG.

3874 Lin-kang · 鼡, (obs.) Sung, H., $120 \mathrm{Li}$ S. IV. of Suining H., Hunan.

3875 Lin-kao | 高 Lin1-kau, H. [on sea] in Clining-clon $\mathrm{Fu}$, Kuangtung; Lat. $19^{\circ}$ $47^{\prime}$, Long. $109^{\circ} 54^{\prime}$.

3876 Lin-kuei 桂 Lin-kwei, H. forming the prefectural city of Knei-lin, Kuangsi ; Lat. $25^{\circ} 13^{\prime}$, Long. $110^{\circ} 14^{\prime}$ : Han，Shill-an 始发; T'ang, I,in-knei | 桂 $\mathrm{H}$.

3877 Lin-k'un | 鼠, (obs.) in Yün-lien H., Ssŭch nan; $T^{6} a n g, \mathrm{H}$. in Yün 笏 C.

3Sis Lin-lê | 樂, (obs.) $I V$. Han, Marq. N. of Ningchin H., Cliihli.

3879 Lin-li | 澧, see No. 6865. 3580 Lin-p'ing | 平.

$1^{\circ}$ Chên near Ch'ien C., Shensi.

$2^{\circ}$ Chîn near 'lê-ch'ing H., Chelikiang.

3981 Lin1-po | 波 Lin1-po, H. in Ch'inan - lo 全. 羅 circ., Corea ; I at. $35^{\circ} 58^{\prime}$, I.ong. $125^{\circ} 30^{\prime}$.

$3 \% 82$ Liu-p' 1 | 浦.

$1^{\circ}$ Chên near Hsiao-shan

H., Chelikiang.

$2^{\circ}$ See No. 5117.

3883 I,in-shan | Ml, militar! post near Yiï-yo H., Cliehkiang.

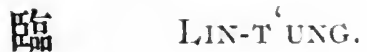

$388+$ Lin-shui | 水, sce Nos. 3838, 6868.

3885 I i11-sh111-pao 水堡, poittown near Su C., Kansuh. 3886 Lin-sui | 睢( (obs.) 3 li $\mathrm{x}$. E. of Yung-ch êng H., Honan; E. Han, H. in P'ei 淽 K.

3887 Lin-su1ng | 泾 (obs.) W. of Ho C., Kansuln; Sung, pao in Clii-slih 積不 military district.

3888 Lin-t'an | 漝 (obs.) T'ang, Sui, and H. near ' 1 'aochon 'T., Kansul.

3889 Lin-t'ao | 㯇, Y. near Kinyüan C., Kansuh.

3890 Lin-tao | 洮.

$1^{\circ}$ 'T-S. $70 \mathrm{Li}$ l. of 'l'i-talo C., Kansul.

$2^{\circ}$ See Nos. 2458, 4457 . $6273,6358$.

3991 I,int ting | ij, post-town near 'T'ing-chon Fin, I'ulnkien.

3992 Lin-tsung | 宗, (obs.) in Nien-po H., Kanssh ; Sung, military post in Lê 樂 C., Shensi.

3893 Lin-tu | 都, (obs.) near Fêng-yang Fin. Anlnii; IV. Han, II. in P'ei 声 cliïl.

$389+$ I in-t ung | 潼 Lin-tung. [498] $1^{\circ}$ H. in Hoi-an I'in, Shensi; Lat. $34^{\circ} 20^{\prime}$, 
臨

LIN-T'บับั

Long. $109^{\circ} ; T_{s}^{\prime}$ in, Li-yi 蹗邑; Han, Hsin-fêng 新豐; T"ang, Ch'ingshan 滰 以 and Hui-ch' ang 會昌; Sung $\| \mathrm{H}$.

3895 Lin1-tzŭ | 淄 Lin1-tsz.

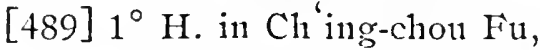
Shantung; Lat. $36^{\circ}$ $55^{\prime}$, Long. $118^{\circ} 32^{\prime}$; orig., Ying-cli'in 管邱; W. Han, Ch'i 驾穿 chïn and Lin-tzŭ | 淄 $\mathrm{H}$. in Cling 毒 chou; Sui, Tang, and $S$. Sung; Lin-tzŭ | 淄 H. $2^{\circ}$ (obs.) S. E. of Liu-ho H., Kiangsu; $S . T s^{6} i$,

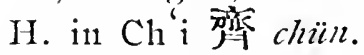
$3^{\circ}$ See No. 601.

3896 Iin-wu | 武 Lin-wu.

[507] $1^{\circ} \mathrm{H}$. in Knei-yang C., Hunan ; Lat. $25^{\circ} 22^{\prime}$, Long. $112^{\circ} 14^{\prime}$; Han, Iin-wn 武 H.; T'ang, I,111\%-wn 隆武.

$2^{\circ} \mathrm{Y}$. in Janl-shan II., H111:a11.

3897 Lin-wu| 舞, see No. 5073. 3898 L.i11-yi | 源.

$1^{\circ}$ (obs.) 50 li N. of Ian-sha11 H., Sliantung; $I V$. Han, H. in Tu11g-lıai 東海 chün; E. IIan and $T \sin , \mathrm{H}$. in Lang-yel 郎躯 $\mathrm{K}$.; N. $W \mathrm{ei}, \mathrm{H}$. in 'T'an 炎队 chïn.
監 LIN-I $\ddot{U} A N$.

$2^{\circ}$ (obs.) $30 l i$ N. E. of Shang-yüan H., Kiangsu; S. Sung and $S$. $T s i$, H. in S. Langyeh 䶂躯 chïn.

$3^{\circ}$ (obs.) in Anluni; $N$. IVei, H. in Jung-yang 冢曜 chün and Peit'n11\% 北道 chün。

$4^{\circ}$ Sce Nos. 3588, 7461.

3899 I.in-yi | 芑 Iin-yih, H. in Cli-nan Fu, Sliantung; Lat. $37^{\circ} 19^{\prime}$, Long. $117^{\circ}$ $0 t^{\prime}$; orig., Li-ch'in 剓邱; Sung, Iin-yi| 呈.

3900 Lin-ying | 頴 Lin-ying, H. in Hsï C., Honan; Lat. $33^{\circ} 51^{\prime}$, Long. $114^{\circ}$ $03^{\prime} ;$ Sui, | 穎 H.

3901 Lin1-yü| 楡 Lin1-yu.

$1^{\circ}$ H. in Inng-p ing Fir, [on sea] Cliilnli; Iat. $40^{\circ} 08^{\prime}$, Iong. $129^{\circ}$ $10^{\prime}$; Lian, Cll ien 酸 C.; Sin, Shanhai ll范. $2^{\circ}$ See No. 3902.

3902 I in-yü | 渝 (or | 榆, ) (obs.) W. of Clinêng-tê H., Shingking; Han, H. in Ijao-1ssi 㟫西 chün.

3903 Lin-yïan | 原, (obs.) E. of Lill-ch' ï JH., Shantung; W. Han, Marq. in Iangyeh 瑯哪 chïn.

3904 Ji11-ÿian | $\vec{\pi}$, see No. 3906. 
臨

LIN-YÜAN.

3905 Lin-yian| 沅 (obs.) IV. of Wu-ling H., Hunan; Han, T'sin, S. Sung and S. $T^{\prime} s i, H$. in Wuling 武陵 chiin.

3906 I,in-rün | 尤 (sometimes wrongly written I,in-yüan | 元), see No. 2761 .

\section{林}

3907 I,in 林 Lin H. in Clan@-tê [562] Fu, Honan; Lat. $36^{\circ} 08^{\prime}$, Iong. $113^{\circ} 55^{\prime} ;$ Han, I nung-lï 隆㑕; 2nd $W^{\prime \prime} c i$, I.in-lü| 慮; Sini, Yc11

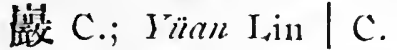

3908 I.in-ch'nan | 川 chün in Clunng-ch'ing 出清 circ., Corea.

3909 Lin1-clun11 | 中, (obs.) in Wn-wei H., Kansulı; $N$. $W c i, \mathrm{H}$. in Wu-wei 武 威 chïn。

3910 Li11-lisi | ju․, (obs.) in Annan1 ; T"ang, C. and H. in ling-nan 献倩 circ.

3911 I.in-l1sii | 雀 sš̆ i: I,ingrshan H., Kuangtung.

3912 Li11-k'on-p1o 田堡, T-S. near Min C'., Kansult.

3913 Li11-lï | 剫, see No. 3907. 3914 Lin-ts' ung | 牭, 'í-S. near Sunı-p'pan 'T., Ssŭch' nan.

3915 Iin1-tz11-k'ou | 子 1 , post-town near ILining-yin H., Hunan.
I,IN-SHUI.

3916 Lin1-tzŭ | 样, chên near Ju-kao H., Kiangsu.

\section{淋}

3917 I,in-ts'ung 淋琣, (obs.) near Issï-chon Fu, Ssŭch'nan; T'ang, H. in Cliing 鏡 C.

\section{麟}

3918 I,i11 蚍数.

$1^{\circ}$ (obs.) formerly C., W. of Ya-chou Fin, Ssŭchinan.

$2^{\circ}$ See No. $1+15$.

3919 I.in-ti | 战 H. in Cliangyüan 汭. 原 circ., Corea. Same as No. 3925.

3920 Iin-yu| 遊 I,in-yu, H. [508]in Fêng-hısiang Fin, Shensi; I at. $34^{\circ} 40^{\prime}$, I,ong. $107^{\circ} 45^{\prime} ;$ Han, 'l'n-yang 杜陽；Sui；. I'êng-chi 鳳模 and I,in-yil 遊 $\mathrm{H}$.

\section{粼}

3921 lin 粼, sce No. 3923.

3922 Lin-shan| Ill, see No. 3923.

3923 Iin-sluni | 水 I,in-shwui, [295]H. in Shnu1-cl ing I'u, Ssŭclı' 1101 ; I at. $30^{\circ} 23^{\prime}$, Itong. $106^{\circ} 5 t^{\prime} ; \mathrm{Han}$, 'Jang-ch'ii 宕渠; Liang Lin1-shui | 水 and Lin | C.; T*ang, Lin-shan | IL and $\operatorname{Iin} \mid \mathrm{C}$. Sung $\mid$ 水 H. 
隣 LIN-CH'

\section{隣}

3924 Iin-cli i 隣溪, (obs.) in Ssŭcli'van; S. Ts'i, chün in I,iang 梁 chov.

\section{獜}

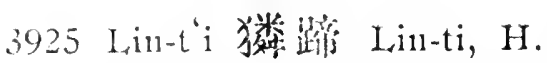
in Corea; Lat. $37^{\circ} 52^{\prime}$, I.o11g. $128^{\circ} 25^{\prime}$. See No. 3919.

Lin-ching, sce No. 3842 . I.in-cluen, see No. 3856. Li11-f $\breve{a ̆}_{11}$, see No. 3858 . Jin-kau, see No. 3875. I,in-kiang, sce No. 3847 . I.in-kü, see No. 3855 . Jink-kwei, see No. 3375. I.in-11gan, see No. 38.38 . I.in-sliwui, see No. 3923. Lin-siang, see No. 3869. Iin-ti, see No. 3925. I.in-tsin, see No. 3848. Lin-tsing, see No. 385 (). I.in1-ts\%', see No. 3895. I,intlung, see No. 3894. I.in-yih, see No. 3899. I in1-yu, see No. 3901.

\section{LING}

$$
\text { 命 }
$$

[see also under IJIEN.]

3926 I ing-cli'i命支.

$1^{\circ}$ (obs.) W. of $\mathrm{Cl}^{\prime} \mathrm{ien}$ an İ., Chillit ; Man, II. in 1,iao-lisi 漼阿 chiin.

$2^{\circ}$ See No. 911.

\section{零 LING-LÜ.}

3927 Ling-chieh | 階, (obs.) 7 li W. of Shilh-hsing $\mathrm{H}$, Kuangtung; $S . T s^{\prime} i, \mathrm{H}$. in Shih-hsing 始與 chün.

3928 Ling-hu | 狐, (obs.) Chou, town in K. Cli in 奉, Shansi.

3929 I,ing-lo | 羅, (obs.) Tang, H., 120 li $\mathrm{N}$. of Hua C., Kuangsi.

\section{椧}

3930 Iing-lun 椧淪, (obs.) near Turfan; $T$ 'ang, C. in Hsiel1-fêng 竄鳳 Fin.

3931 Iing-tao | 道, see No. 4716.

\section{卧}

3932 Ling-ch'i 褰溪 ssŭ in Ching C., Hunan.

3933 Ling-k'ou | 口, chênn near Lin-t ung H., Shensi.

5934 Ling-ling | 陵 Iing-ling. $1^{\circ} \mathrm{H}$. forming the prefectural city of Yungchou, Hunan; I at. $26^{\circ}$ $08^{\prime}$, Long. $111^{\circ} 35^{\prime}$; Han, Ch'üan-ling 泉 陵; Sull, I,ing-ling | 淩 Chïn and $\mathrm{H}$.

$2^{\circ}$ See No. 3362 .

3935 Ling-lii | 曜.

$1^{\circ}$ Ss̆̈ near Sui-ch'i H., Kuangtung.

$2^{\circ}$ (obs.) T"ang H., W. of Shih-cliêng H., Kuangtung. 
領

IING-YANG.

3936 Ling-yang | 陽, see No. 5721.

\section{領}

3937 Ling-fang 領方, see Nos. $2630,5117,5517$.

\section{嶺}

3938 Ling-hsi | 西, (obs.) formerly circ. in Kuangtung comprising Chao-ch'ing Fu, Yang-chiang $T$. and Lo-ting $\mathrm{C}$.

3939 Ling-nan | 南, (obs.) T'ang, circ. corresponding to the S. part of Yang 揚 cHov. See No. 3421.

3940 Ling-tung | 東, Y. near Chiang-hnta H., Hunan.

3941 Ling-yu | 右, see No. 3398.

\section{綾}

3942 L.ing 綾 C. in Ch'iuan-lo 全羅 circ., Corea.

凌

3943 Ling-chiang 凌江, posttown near Nan-hsiung C., Kuangtung.

3944 Ling-ho 河, post-town near Yi C., Shingking.

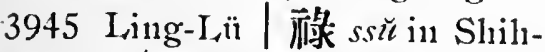
ch êng H., Knaungtung.

3946 Ling-t' ang | 塘, chên near Chia-ting H., Kiangsu.

3947 Ling-yün | 雲 Ling-yun, H. in Ssŭch'êng Fin, Kuangsi ; Lat. $22^{\circ} 46^{\prime}$, Long. $106^{\circ} 52^{\prime}$.
IANG-YÜAN.

\section{菱}

3948 Ling-t'ang 菱塘, ssŭ in P'an-yii H., Knangtung.

\section{陵}

3949 Ling 陵 Ling.

[284] $1^{\circ}$ H. in Chi-nan Fu, Shaintung; I at. $37^{\circ}$ $27^{\prime}$, Long. $116^{\circ} 40^{\prime}$; orig, Chung-ch'in 重 邱; $T \sin , A n-$ ling 安 陵; Sui, P'ing-yüan 本原 chüu and Chiangling 將陵 H.; Yïan, Ling | C.

$2^{\circ}$ (obs.) T'ang, H., S.E. of Pei-liu H., Knangsi.

3950 Ling-ch' ien | 前, chên, near Fu-p ing H., Shensi.

3951 Ling-ch' nan | 川 Lingchuen, H. in 'Tsê-chou $\mathrm{Fu}$, Shansi ; Lat. $35^{\circ} 42^{\prime}$, Long. $113^{\circ} 17^{\prime}$; Han, Hsüan-shih 泣氏; Sui, Ling-ch'nan | 川.

3952 Ling-sluii $\mid$ 水 Ling-shwui. $1^{\circ} \mathrm{H}$.in $\mathrm{Ch}^{\prime}$ inng-chon $\mathrm{Fu}$,

Kuangtung; Lat. $18^{\circ}$ $30^{\prime}$, Long. $109^{\circ} 33^{\prime}$; Ling-sluni | 水. Ming $\mathrm{H}$. $2^{\circ}$ See No. 2194.

3953 Ling-t'ing | 亭, chên near Hsing-hua H., Kiangstr.

3954 Ling-yang | 陽, chên near Shih-tai H., Anhui.

3955 Ling-yüan1 沅, see No. 7080. 
虞 LING-CH'IU.

\section{溚}

3956 Ling-ch“iu 毇邱, see No. 7829.

\section{㒘}

3957 Ling 霝 Ling.

[84] $1^{\circ}$ C. in Ning-hsia Fu, Kansulı; Lat. $37^{\circ} 40^{\prime}$, I fong. $105^{\circ} 11^{\prime} ;$ Ming Ling $\mid \mathrm{C}$.

$2^{\circ}$ (obs.) $T^{\prime}$ ang, H., E.

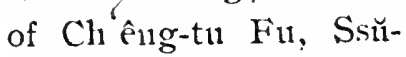
clinan.

$3^{\circ}$ (obs.) Han, Tsin, H., S. W. of Kao-t'ang C., Shantung.

3958 Ling-ch'i | 溪.

$1^{\circ}$ (obs.) in Knangtung; S. Ts'i, H. in Shillhsing 始興 chün.

$2^{\circ}$ See No. 7970.

3959 Ling-ch'ih | 池, (obs.) N. W. of Chien C., Ssŭch'nan (No. 904); T'ang, H. in Cliêng-tu 成都 Fi1. 3960 Ling-ch'iu | 邱 Ling-kiu. [448] $1^{\circ}$ H. in Ta-t' $1111 \mathrm{~g}$ F 11 , Shansi; Iat. $39^{\circ} 28^{\prime}$, Long. $114^{\circ} 15^{\prime}$.

$2^{\circ}$ See No. 7738.

3961 Ling-clinan | 川 Lingchuen.

$1^{\circ} \mathrm{H}$. in Knei-lin $\mathrm{Fu}$, Kuangsi ; Lat. $25^{\circ} 25^{\prime}$, Long. $110^{\circ} 20^{\prime}$. $2^{\circ}$ See Nos. 784, 7458.

3962 Ling-cli' ïan | 䍝.
LING-SHAN .

$1^{\circ}$ (obs.) Sung, H., E. of Ch' êng-tii Fin, Ssŭch'nan.

$2^{\circ}$ (obs.) P. Chou, town W. of Chang-tê Fin, Honan.

3963 Lrng-kwang | 光 chïn in Cli 'üan-lo 全 羅 circ., Corea.

3964 I.ing-mên | PJ, (obs.) 120 li from Chï C., Shantu1ng; $W$. Han, H. in Lang-yeh 郳躯 chïn.

3965 Ling-pao | 算 Ling-pa1, [148]H. in Slian C., Honan; Lat. $34^{\circ} 42^{\prime}$, Long. $110^{\circ}$ $50^{\prime}$; Chou, capital of $\mathrm{K}$.

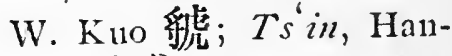
kn 函谷; Han, Hungn11119 弘娄; Sui, T'ao-lin 桃㷊; T'ang, Ling-pao |珼.

3966 Ling-pi | 璧 Ling-pih, H. in Fêng-yang Fu, Anhui; I at. $33^{\circ} 33^{\prime}$, Long. $117^{\circ}$ $33^{\prime} ;$ Sui, Chiang 虹 C.; Sung, Ling-pi | 璧.

3967 Ling-shan / /4 Ling-shan. [63] $1^{\circ} \mathrm{H}$. in Jien-chou Fu, Knangt111g; Lat. $22^{\circ}$ $24^{\prime}$, long. $109^{\circ}$; Han, Ho-p' 11 命浦; Sui, Nan-piu 南賓; T'ang, Ling-shan |l.

$2^{\circ}$ Ssŭ in Chiao C., Shantung.

$3^{\circ}$ H. in Ch'ing-shang 慶 少 circ, Corea. 
3968 Ling-sliilı | 不 Ling-shih, [123]H. in Ho C., Shansi; Lat. $36^{\circ} 53^{\prime}$, Long. $110^{\circ}$ 46'; Han, Chiel-11siu 介 休; Sui, I,ing-shin | 石.

3969 Iing-shou | 裉 Ling-shan, [491] H. in Chêng-ting Fu, Chilıli; I at. $38^{\circ} 18^{\prime}$, Long. $114^{\circ} 31^{\prime}$; orig., Clunng-shan 中 II Sui, Yen 㔨 C.

3970 Ling-t'ai | 臺 I,ing-tai, H. in Ching C., Kansulı; Lat. $34^{\circ} 59^{\prime}$, Long. $107^{\circ}$ 23'; Chou, K. Mi-hsï 密 須; N. Wei and Sin, Shun-ku 熟解 $\mathrm{I}$.

3971 I ing-tien | 质, chên near Ch'ang-wu H., Shensi.

3972 Ling-wu | 武, (obs.) Ts'in, H., E. of Hsienyang $H$. , Sliensi.

3973 Ling-wu-cl' êng | 武 城, (obs.) formerly town near Ning-lısia Fu, Kansuh.

3974 Ling-yen | 岩 Ling-yen, chün in Ch' ïan-lo 全羅 circ., Corea ; Lat. $35^{\circ} 11^{\prime}$, Long. $125^{\circ} 26^{\prime}$.

3975 L.ing-yüan | 源.

$1^{\circ}$ (obs.) in $\mathrm{Cl}_{1}$ 'êng-tê H., Shingking; Liao, $\mathrm{H}$. : Shên 潘 C.

$2^{\circ}$ (obs.) Wei, H. nestr Wei-nan H., Shensi.
柳

\section{㢦}

3976 Ling 歌 Ling.

$1^{\circ} \mathrm{H}$. in Hêng-chon Fin, Hunan; I at. $26^{\circ} 32$, Long. $113^{\circ} .35^{\prime} ; \mathrm{Han}$, Ch'a-ling 茶陵; Sung, Ling $\mid \mathrm{H}$.

$2^{\circ}$ (obs.) Han, H. $12 \mathrm{li}$

E. of Hêng-chou Fu, Hunan.

I,ing-chuen, see Nos. 3951 3961.

Ling-kin, see No. 3960. Ling-pan, see No. 3965. Ling-pil, see No. 3966. Ling-shan, see No. 3969. Ling shwui, see No. 3952. Ling-tai, see No. 3970. I,ing-yu11, see No. 3947. Lin-gin, see No. 3842. Lioh-yang, see No. 4157.

\section{LIU 柳}

3977 Liu 柳, see No. 3981.

3978 Iilu-clìêng | 城 Liuclining.

$[274] 1^{\circ} \mathrm{H}$. in Liti-chou Fu, Kuangsi ; Lat. $24^{\circ} 25^{\prime}$, Long. $108^{\circ} 58^{\prime}$; Liang, Lrung-ch'êng 龍 城; $T^{6}$ ang, Iung | C.; Sung, Liul-ch' êng | 城. $2^{\circ}$ See No. 4107.

3979 Lin-ch'ilı | 池 (obs.) near Hsï-chou Fu, Ssŭclı' 1 an ; $T^{\prime}$ ang, I,ill-ch'ih | 池 $\mathrm{H}$. 
柳 LIU-CH'iH-KOU.

3980 Liu-ch'il-kou | 池 㴖, post-town near Chien C., Ssŭch' tuan. (No. 910.)

3981 Liu-chou | 州 Lit1-chan [274] Fu in the Yu-chiang circ., Knangsi, forming also the district city of Ma-p'ing; Lat. $24^{\circ} 14^{\prime}$, Long. $109^{\circ}$ $09^{\prime} ; T s^{\prime}$ in, in Knei-lin 桂 林 chïn; Han, in Yü-lin 槐紧林 chün; Chên, in Hsiang 象 chün; T'ang, Lungr-clı'êng 龍城 and Liu $\mid \mathrm{C}$.

3982 Li11-chung |

$3982^{\text {A }}$ Lint-ho | 测, H. in HaiLung Fu, Shingking. See No. 4107.

3983 I,in-kon | 淮, Y. near Yen-ch'ing C., Chihli.

3984 Lini-lin | 林, chên near Yung-ning C., Shansi.

3985 Liu-ts'un | 村, chên near Ch'ien-yang H., Shensi.

3986 Lin-ying | 焪 ss Ch'ang-t'ai H., Fulhkien.

\section{漗}

3987 Liu-chi 留集, chên near Fin-p'ing H., Shensi.

3988 Liu-huang | 隍 ssŭ in Fêng-shum H., Kuangtung.

3989 Liul-ku | 古, chên near Fu-p'ing H., Shensi.
流 LIU-CH'

3990 Lin-li | 利, chên near Lin-t 'nug H., Shensi.

3991 Lin-ling-chi | 陵集, posttown near Ying-shan $\mathrm{H}$., Anliui.

3992 Liu-pa | 䩗 Lin-pa, T. in Han-chung Fu, Shensi; Lat. $33^{\circ} 30^{\prime}$, Long. $107^{\circ}$ 25.' 'Terr. iur.

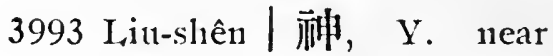
Sui-clı'i H., Knangtung.

\section{埕}

3994 Iin-chia 㔀家, ssŭ near Ho-fêng C., Hupelı.

399.5 Lit1-chia-kê | 家隔, ssŭ near Hsiao-kan H., Hupelı.

3996 Lin-p'n | 音 post-town near Yï-ch'êng H., Shantung.

\section{瀏}

3997 Jiu-yang 瀏陽 Lil1-yang, [256] H. in Ch'ang-sha $\mathrm{F} 11$, Hunan; Lat. $28^{\circ} 10^{\prime}$, Long. $113^{\circ} 27^{\prime}$; Han, Lin-11siang 臨湘; $W u$, Liulyang | 晹.

\section{流}

3998 Li11-ch'i 流溪.

$1^{\circ}$ (obs.)S. IV. of Na11cl'ung H., Kno 果 C.; Ssŭch 'uan; $T^{6}$ ang $\mathrm{H}$. in Sung, H. in Shunch'ing順度 Fu.

$2^{\circ}$ Ssü in Ts'ung-hua H., Kuangtung. 
流

I,IU-CHIANG.

3999 Liu-chiang | 江, see No. 1437.

4000 Liu-fêng | 風, (obs.) S. of Pai-ho H., Shensi; $S$. $T s^{\prime} i, H$. in Nan Shang-lo 南上洛 chün.

4001 Liu-ho | 河, chên near Ch'ing H., Chilili.

4002 Liu-min | 比.

$1^{\circ}$ (obs.) in Pai-ho H., Shensi ; S. Sung and $S$. $T s^{\prime} i$. H. in Nan Shanglo 南上洛 chïn.

$2^{\circ}$ (obs.) near Shang C., Shensi; S. Sung and $S . T s^{\natural} i$, H. in Pei Shang-lo 北上洛 chiin. 4003 Lin-nan | 南, (obs.) Liang, H., S.W. of Yangch' nan H., Kuangtung.

\section{琉}

4004 Iin-ch 'iu琉球 Lew-chew, a group of Islands in the N. Pacific Ocean, between Formosa and Japan, extending from Lat. $26^{\circ}$ to $27^{\circ}$, in I.ong. $128^{\circ}$. Chief town Na-pa 那將, or Na-pa-kiang.

\section{朳}

4005 Liulan 六发 L,uh-ngan. [361] $1^{\circ}$ C.I.C. in the Fêng- Ying circ., Anhui ; Lat. $31^{\circ} 49^{\prime}$, Long. $116^{\circ} 31^{\prime}$; orig., $\mathrm{K}$. Liu-liao | 萎; Han,
$\frac{1}{N}$ Lo.

Lill-an | 努; Sui, Ho. shan 霍山; $T^{6}$ ang, Ho 霍 C.

$2^{\circ}$ (obs.) $13 l i \mathrm{~N}$. of preceding; $W$. Han, K. Lin-an | 安; E. Han, H. in I,ï-chiang 盧江 chïn.

4006 Lin1-ch' ên | 陳, Y. near Jung H., Kuangsi.

4007 Liu-ho | 合 Luh-hoh, H. [105] in Chiang-ning F $11, \mathrm{~K}$ iangsu ; Lat. $32^{\circ} 20^{\prime}$, Long. $118^{\circ} 50^{\prime} ;$ orig., T'ang-yi 栄邑 in K. Ch'in 楚; Tsin, in $\mathrm{Ch}^{\prime} \mathrm{i}$ in 秦 chïn; P. Chou, Lin-ho|合; Five dy'n., Hsinng 雄 C.

4008 Iju-hsien | 蔣, (obs.) formerly town $\mathrm{N}$. of Liuan C., Anluni.

4009 Lit1-liao | 壮, see Nos. 2100, 4005, 5749.

4010) Liu-tzŭ |子, ss̆̌ near Ts'êng-ch'i $\mathrm{H}$., Knangsi.

Lit1-ching, see No. 3978.

\section{LO \\ 稆保}

4011 Lo 羅.

$1^{\circ}$ (obs.) formerly K. in Hunan.

$2^{\circ}$ (obs.) 1st Sung, C. 110 li N. W. of Wuch'wan H., Kuangtung. 
羅 LO-CH'-ANG-HO.

$3^{\circ} \mathrm{C}$ in $\mathrm{Ch}^{\prime}$ ïan1-lo 全羅 circ., Corea.

$4^{\circ}$ See Nos. 645, 2635, $5140,5696$.

4012 Lo-ch' ang-ho| 昌河, chên near T'ung-ch êng $H$., Anlini.

4013 Lo-ch" êng | 城 Lo-ching, [481] H. in Iin-chou $\mathrm{Fu}$, Kuangsi ; Lat. $24^{\circ} 44^{\prime}$, Long. $108^{\circ} 38^{\prime}$.

4014 Lo-chiang | İ Lo-kiang. $1^{\circ} \mathrm{H}$. in Mien C., Ssŭchnan; I at. $31^{\circ} 18^{\prime}$, Long. $104^{\circ} 36^{\prime} ; T$ sin, Wan-an 萬 䘫 and Ch'van-t'ing 㴟 亭; T"ang, Lo-chiang | 江. $\mathrm{H}$.

$2^{\circ}$ (obs.) near Fin-chou Fin, Full-kien ; $T \sin$, S. Sung and S. Ts' $i, H$. in Clinu-an 留妾 chion.

4015 Lo-ching | 鏡, so near I.o-ting C., Kuangtung.

4016 Lo-ch'nan | 川, see No. 485.

4017 Lo-fan | 畓, 'T-S. near Knei-yang Fu, Kneichon; Ming, Lo-fan | 番 ssŭ.

4018 I,o-hung | 虹, (obs.) near Hsü-clon Fu, Ssŭcl' uan; $T^{\prime}$ ang, H. in W11-cl' ang 武昌 C.

4019 Lo-hsin | 新, (obs.) near Hsï-clıı Fì, Ssŭch 'uan ; $T^{\prime}$ ang, H. in Wu-ch'ang 武昌 C.
維

LO-PAI.

4020 Lo-hsing | 星, Y. nea1 Ch'ang-ning H., Ssŭch'nan.

4021 Lo-hsing-t'a | 星塔Pagoda Island near Foochow, the name Ma-wei 馬尾 is also applied to the locality.

4022 Lo-hsiu | 秀, Y. near Jung H., Knangsi.

4023 Lo-lisinng | 雄, see No. 4032.

4024 Lo-kang | 鄌 ssü in $\mathrm{Cl}^{*}$ enp'ing $\mathrm{H}$. Knangtung.

4025 L。o-kung | 菾, (obs.) near Ssŭ-11an Fı, Kueichou; T'ang, H. in Ying 應 C. 4026 Io-ling | 龍

$1^{\circ}$ (obs.) in Kuangsi; $T^{\prime}$ ang, H. in Lung 籠 c.

$2^{\circ}$ (obs.) in Yün-lien H., Ssŭch' 'ıan; $T^{6}$ ang, $\mathrm{H}$. in Lien 連 $\mathrm{C}$.

4027 Lo-lung-tsung | 隆宗 H'lurungjung, town in 'Thibet; Lat. $30^{\circ} 20^{\prime}$, L,ong. $96^{\circ}$.

4028 Lo-111êng | 蒙, (obs.) in T'ung-tao H., Hunan; Sung, military post in Ching 靖 C.

4029 Lo-pai | 白, T. H. near 'T'ai-p'ing 'T. C., Kuangsi; Lat. $22^{\circ} 22^{\prime}$, Long. $107^{\circ} 14^{\prime}$. 
羅

LO-P' ÊNG.

4030 Lo-p'êng | 蓬, (cbs.) in Ya-chou F., Ssŭch' uan ; T'ang and Sung, Lo-p'êng | 蓬 C.

4031 Lo-pien | 辨 (obs.) T'ang, H., S. E. of Pei-liu H., Kuangsi.

4032 Lo-p'ing | 平 Lo-ping, C.

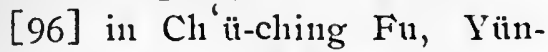
nan; Lat. $24^{\circ} 58^{\prime}$, Long. $104^{\circ} 18^{\prime}$; Minor Han, Hsing-ku 與古; Yüan, I.o-lisiung 雄 C. ; Ming, Iso-p'ing $45^{\circ}$.

4033 Lo-shan | !L Lo-shan, H. [144]in Ju-ning Fu, Honan; Lat. $32^{\circ} 15^{\prime}$, Long. 114 2.4'; Han, Mêng 䢻 $H$.; N. Ts'i, Kao-an 高发; Sui, Lo-shan | 山.

4034 Lo-shui / 水, (obs.) formerly H., W. of Yangch'un H., Knangtung.

4035 Lo-t'ang (or 蘿) 塘, ss̆̈ in An-fu H., Kiangsi.

4036 Lo-tien | 甸, (obs.) T'ang and Sumg, K. in Kueichon.

4037 Lo-tien / 店, chên near Chia-ting H., Kiangsu.

4038 Lo-t'ien| 日] Lo-tien.

$1^{\circ} \mathrm{H}$. in Huang-chon $\mathrm{Fu}$, Hupeh ; Lat. $30^{\circ} 53^{\prime}$, Long. $115^{\circ} 22^{\prime}$; Han, $\mathrm{Ch}^{\prime} \mathrm{i}-\mathrm{ch}^{\prime} \mathrm{n}$ 龩 替; Liang, $\mathrm{Yi}$ 燨 $\mathrm{C}$; S Sui, Lo-t'ien | 日日.
LO-YANG.

$2^{\circ}$ See No. 7609.

4039 Lo-ting | 定 Lo-ting, C. L. C. in Chao-I,O circ., Kuangtung; Lat. $22^{\circ} 55^{\prime}$, Long. $111^{\circ} 20^{\prime}$; $S . A$., land of the Yiieh 宩 tribes; Han, Tuan-ch' $\mathrm{i}$ 端溪; Ts' in, Lung-hsiang 龍拫 and P'ing-yüan 本 原; Liang， I,ung 瀧 C.; Sui, Lung-shui 潵水; T'ang, K'ai-yang 開陽; Sung, K'ang 康 $\mathrm{C}$.; Ming, 1st, I, ung-slui 瀧 水, $2 n d$, Lo-ting $\mid$ 定 $\mathrm{C}$.

4040 Lo-tsun | 邆, (obs.) in Yi-shan 宜山H., Kuangsi ; $r^{\prime}$ ang, H. in Ssŭshun 思䐓 C.

4041 Lo-tu | 渡, ssü near L,êch'ang H., Kuangtung.

4042 Io-tz' $\breve{u} \mid$ 次 I,o-tsz'，H. [463] in Yün-nan Fu, Yünnan; Lat. $25^{\circ} 23^{\prime}$, Long. $102^{\circ}$ 23'; Yüan, Lo-tz ${ }^{\circ}$ 品 次 $\mathrm{H}$.

4043 Lo-wei | 圍, (obs.) in Lü C., Ssňch' 'tan; T'ang, H. in $\mathrm{Na}$ 納 $\mathrm{C}$.

4044 Lo-yang | 榢 Lo-yang.

$1^{\circ}$ T. H. in T'ai-p'ing

Fu, Kuangsi; Iat. $22^{\circ}$

$53^{\prime}$, I ong. $107^{\circ} 31^{\prime}$.

$2^{\circ}$ (obs.) $7 s^{\circ} i, \mathrm{H} ., \mathrm{W}$. of

Po-lo H. Kuangtung.

3• See No. 3024. 
羅 LO-YEN.

4045 Lo-yen | 岩, (obs.) formerly C. 481 li W. of Ya-chon Fin, Ssŭ-ch' 'uan.

4046 I,o-yü | 余, (obs.) in Yün-lien H., Ssŭch'uan; $T^{6}$ ang H. in Yün 鈞 $\mathrm{C}$.

4047 Lo-yïan | 源 Lo-yïen, H. in Fu-chou $\mathrm{F} u$, Fuhkien; Lat. $26^{\circ} 26^{\prime}$, Long $119^{\circ}$ 45'; T'ang, Yung-chên 永具; Sung, Lo-yüan | 源 $\mathrm{H}$.

4048 Lo-yün | 雲, (obs.) in Kansul; $T^{\prime}$ ang, C. in Lnng-yu站右 circ.

\section{逬}

4049 Lo-p'êng 暒蓬, (obs.) in Lü C., Ssuch' uan; $T^{6} \mathrm{ang}$, H. in Fêng 奉 C.

\section{鹳}

4050 Lo-t'ang 雉䛸 see No. 4035.

\section{洛}

4051 Lo 洛, see Nos. 2079, 5550 .

4052 Lo-an 发, see No. 4084.

4053 Lo-ch' êng | 城, (obs.) Liang, town near Chinch'i H., Kiangsi.

4054 Lo-ch'i | 溪, (obs.) E. of Kung H., Ssŭch' 'nan; T'ang, H., in Ssŭ-o 思 峩 C.
I,O-YANG.

4055 L,o-chiao | 交, ancient name of Fin C.., Shensi.

4056 Lo-ch'uan 川 Lohchuen, $H$. in $F u$ C., Shensi ; Lat. $35^{\circ} 58^{\prime}$, Long. $109^{\circ} 31^{\prime}$; Han, Fu 鄜 $\mathrm{H}$. and I,o-ch 'nan | 川; 2nd $W c i$; Fu-ch'êng 整城; P. Chou， Iroch'nan | 川.

4057 L Lo-jung | 容, see No. 4067 .

4058 Lo-kang | 旤, chên near Yeh H., Honan.

4059 Lo-k'ou| 口, chên near Kung H., Honan.

4060 J,o-kuei | 襄, (obs.) near Fêng-t'ien Fu, Shingking; $T^{\prime}$ ang, C. in Fêng-ch' êng 奉誠 $\mathrm{F}$.1.

4061 Lo-m1nan | 涔, chên near Liul-ch 'êng H., Kuangsi.

4062 Lo-tı | 都.

$1^{\circ}$ (obs.) in Shensi; $W$. Han, H. in Shang 上. chïn.

$2^{\circ}$ (obs.) in Yi-shan H., Knangsi ; $T$ 'ang, H. in Kuei-hua 蹛化 C.

4063 Lo-yang | (or 雒) 陽 Loh-yang.

$1^{\circ} \mathrm{H}$. forming the prefectural city of Ho-nan, Honan ; Lat. $34^{\circ} 43^{\prime}$, Long. $112^{\circ} 28^{\prime}$; orig., Chou-11an 周南; Han, Lo-yang | 韵. 
倠

Lo-yítAN.

$2^{\circ}$ Ss̆̈ near Hui-an, H., Fuhkien.

4064 Lo-yüan | 原, see No. 1858.

4065 Lo-yüan | 源, (obs.) N. E. of An-hua H., Kansul, Sui and $T^{\prime}$ ang, Lo-yüan | 源 $\mathrm{H}$.

\section{雒}

4066 Lo 雒, see No. 1942.

4067 Lo-jung | 容 I,oh-yung, [569] H. in Liu-chou $F u$, Kuangsi ; Lat. $24^{\circ} 24^{\prime}$, Long. $109^{\circ} 21^{\prime} ; T^{\prime}$ ang, Lo-jung 洛容.

4068 Lo-nan | 南 Lolı-nan, H. [263]in Shang C., Sheusi ; Lat. $34^{\circ} 06^{\prime}$ Long. $110^{\circ} 06^{\prime}$; Han, Shang-lo 上! Sui, Lo-nan | 南.

4069 Lo-yang | 陽; see No. 4063.

\section{㫫各}

4070 Lo-t'o-tien 頤駝店, ssiu near Hsin-yang, C., Honan.

I,o-ching, see No. 4013. Lo-kiang, see No. 4014. Lo-ping, see No. 4032. Loztien, see No. 4038. Lo-tsz, see No. 4042. Lo-yen, see No. 4047. Loh-chang, see No. 3644.
婁 LOU-FAN.

Loh-chi, see No. 3648.

Loh-chnen, see No. 4056.

Loh-11wui, see No. 3649.

Loh-ling, see No. 3652.

Lol1-nan, see No. 4068.

Loh-11gan, see No. 3643.

Loh-ping, see No. 3656.

Loh-shan, see No. 3657.

Loh-ting, see No. 3658.

l,oh-tsing, see No. 3648.

Loh-yang, see No. 4063.

Isoh-yung, see No. 4067.

L tok-kong, see No. 4119.

\section{LOU}

\section{䔎}

4071 Loul 装 Lau.

$1^{\circ} \mathrm{H}$. forming with Hua$t$ 'ing $H$. the prefectural city of Sung-chiang, Kiangsu; Lat. $31^{\circ} 03^{\prime}$, Long. $121^{\circ} 15^{\prime}$

$2^{\circ}$ See Nos. 2218, 3500, 5970.

4072 Lou-chung | 中, see No. 6865.

4073 I,oun-fan | 煩, chên near Cling-lê H., Shansi.

\section{樓}

4074 Lou-fan 樓煩.

$1^{\circ}$ (obs.) N.F. of Kuo H., Shansi ; Han, H. in Ying-mên 雁睯 chiin. 
婁 I,OU-HISÜ。

$2^{\circ}$ (obs.) near Ying-chou Fu, Anhui; S. Sung, H. in Ju-yin 汝陰 chiin.

$3^{\circ}$ (obs.) $70 \quad l i \mathrm{~s}$. of Ching-lê H., Shansi; Trang, H. in Hsien 慧 C.

$4^{\circ}$ See No. 1172.

4075 Lot1-hsü | 虛, (obs.) N. of P'ing-yiian H., Shantung; $W$. Han, Marq. in $\mathrm{P}^{\prime}$ ing-yüan 隹 原chïn.

4076 Lon-tzu |子, Y. near Ho-ch' ii H., Shansi.

\section{漏}

4077 I,ou-chiang 漏江, (obs.) in Ho-yang H., Yünnan; Han, H. in Tsang-kê 佯 㱒 chün; S. Sung, H. in Chien-ning 建富 chiun; S. $T^{6} s i, \mathrm{H}$. in Clien-p'ing 建平 chün.

\section{LU}

\section{盧}

4078 L11 覽.

$1^{\circ}$ (obs.) 30 li $\mathrm{W}$. of Ch'ang-ch'ing H., Shantung; $H a n, \mathrm{~L}, \mathbf{H}$.

$2^{\circ}$ (obs.! S. W. of Sliillp'ing, H., Shantung;

N. Wei, Sui and T'ang, L11 $\mathrm{H}$.

$3^{\circ}$ (obs.) 60 li s. W. of

- Kai-p'ing H., Shingking; lino, C. in
LU-IVU.

Tung-ching 東京 circ.

Now a post-town called Issinng yo-ch 'êng 能岳 城; see No. 2884.

$4^{\circ}$ See Nos. 241, 4079, 5205.

4079 Lr11-ch'i | 溪.

$1^{\circ}$ (obs.) S. W. of J,ü-chi H., Honan; Tang, $\mathrm{H}$. in $\mathrm{Cl}^{\prime}$ ên 辰 $\mathrm{C}$.

$2^{\circ}$ See No. 4134.

4080 Lu1-fan | 番, ssŭ near Ting-fan C., Kueichou; Ming, ss̆̈.

4081 Lt1-jung | 容, (obs.) in Annam; Han; H. in Jil1nan 日南chün.

4082 I n1-lung | 龍 I, 11-lung.

$1^{\circ} \mathrm{H}$. forming the prefectural city of Yungp'ing, Clinlili; Isat. 36; $50^{\prime}$, Long. $118^{\circ} 50^{\circ}$ orig., K. Fei 肥; Sui, L11-ling | 龍; T'ang, P'ing 震 $\mathrm{C}$.

$2^{\circ}$ See No. 7965.

4083 L11-nu | 奴, see No. 6440. 4084 I,11-shih | 氏 L,u-shi, H. in Shan C., Honan; Lat. $34^{\circ} 01^{\prime}$, Long. $110^{\circ} 56^{\prime}$; Chou, W. part of K. Ku1O 彩; Han, I,11-shih | 氏; 2nd Wei, Lo-an 洛安; W. Wei, Yi-ch' 'nan 義川; Sui, Kuo 毮 C.

4085 Lu-wu | 콤, (obs.) S. W. of L,ï C., Ssŭch ' 1 an; $T^{\prime}$ 'ang, H. in Sung 笨 C. 
路

L,U-YANG.

4086 Lu-yang | 荡, (obs.) Sung town W. of Na-yang H., Hunan.

\section{蘆}

4087 I 1 覽, chên near 'T'unghsiang H., Chehkiang.

4088 Ju1-ch'i | 溪, ssü near $\mathrm{P}^{\prime}$ ing-1ısiang $\mathrm{H}$., Kiangsi.

4089 Ln-lnung | 洪 ssŭ in Tung-an H., Hunan.

4090 I,11-kou1-ch'iao | 䩶橋, post-town near Shun-t ien Fin, Chihli.

4091 Lun-shan | 山 Lï-shan,

[72] $\mathrm{H}$. in Ya-clion $\mathrm{Fu}$, Ssŭch 'uan; I at. $30^{\circ} 28^{\prime}$, Long. $102^{\circ} 48$.

4092 Ln1-t’ang|塔, chén near Chin-ÿïn H., Chelikiang. 4093 Lu1-yi | 移, (obs.) N. of An-ting H., Shensi ; Sung, pao in Yïnnan 延努 Fin.

\section{路}

4094 Lu1-cli'iao 路橋, chên near Huang-yen H., Chehkiang. 4095 Ln1-nan | 南 L,n-nan, C. [335] in Ch'êng-chiang F'u, Yünnan; I at. $24^{\circ} 48^{\prime}$, l,ong. $103^{\circ} 21^{\prime} ; T^{\prime}$ ang, K'un 昆 C.; Ÿ̈an, I,11-11an 南.

4096 I,11-t'an | 賧, see No. 3422.

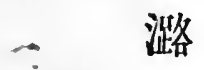

4097 Lu潞, see No. 4098, 6768. 4098 Lu-an | 安 Lu-ngan, Fu [402] in the Chi-ning circ.,
LU-CH' ${ }^{\mathrm{E}} \mathrm{NG}$.

Shansi; forming also the district city of $\mathrm{Ch}^{\prime}$ angcliih ; Lat. $36^{\circ} 07^{\prime}$, Long. $113^{\circ} 13^{\prime} ;$ Slang, 1st, K. $\mathrm{L}, \mathrm{i}$ 黎, 2nd, land of the $\mathrm{Ch}_{1}$ ih-ti 浾狄 tribes, in K. I,11 ; C. Wars, in 1st, K. Han 嘹, 2nd, K. Chao 赫; Tsin, Shang-tang 上. 黨; P. Chou, I, I1 C.; T"ang，Chao-yi 昭義; Sung, Chao-tê 昭 德 and Ln111g-tê 隆德: MIing, I, 11an 努 Fin.

4099 L,n-ch' êng | 城 Lt1-ching, H. in L I1-an Fu, Shansi : Lat. $36^{\circ} 15^{\prime}$, Long. $113^{\circ}$ $13^{\prime} ; H_{a n}, \mathrm{I}+\mathrm{t}-\mathrm{ch}^{\prime}$ êng | 城; 2nd Wei, Yi-ling X $X_{1}$ 陵.

4100 Ln-chiang | 讬, (obs.) near Yung-chíang Fin, Y'ünnan; Ming, I,11-chiang | 江 ssŭ.

4101 Ln-110 | 润, chên 11ear I,nch'êng H., Shansi.

\section{急}

4102 Lu 舅, Chou and S. A., K. comprising $S$. part of Shantung; Tsin, Hsüch 薛 chün; $T s^{i}$ in, Ln l l chün; see Nos. 2683, 4109, 6745, 7373.

4103 Ln1-ch'ên | 際, see No. 4107.

4104 In-cl'êng | 城 (obs.) Chou, capital of K. Lu near Ch' ${ }^{\prime i}-f u$ H., Shantung. 
惫 I,U-CHIANG.

4105 Lu-chiaug | 港, chêu near IVuhu H., Anhui.

4106 Itu-ch'iao / 橋, chên near San-yüan H., Shensi.

4107 Lon-k'ê-cin'in | 克沈（or, Lıl-kul-ch'ên | 古厑, or, Lu-ch'ên | 䢴) Lukchak, a town $60 \mathrm{li}$ S. E. of Turfan; formerly I,iuchung 柳中 H. and Iinch'êng 柳城.

4108 I, 11-ku-ch'ên | 古鹿, see preceding.

4109 Lu-shan | II I,u-shan. [384] H. in Ju C., Honan; Lat. $33^{\circ} 50^{\prime}$, Isong. $112^{\circ} 51^{\prime}$, S.A., Chêng-yi 伯院; Han, In-rang | 陽; Wei, Kuang 廣 C.; 2nd $W e i$, I, $\mid$ C.; T"ang, I,n-shan | II.

4110 Ln-tien | 旬 Lu-tien, T. [389]in Chao-t'ung Fu, Yünnan ; Lat. $27^{\circ} 25^{\prime}$, Itong. $103^{\circ} 50 .^{\prime}$

4111 L Ll1-yang | 晹, see No. 4109.

\section{碌}

4112 I,11-yïn 碌堅, see No. 736.

\section{形尔}

4113 I 11-ch'ïan 海花勸, Lu[352] kinen, H. in Wu-ting C., Yünnan; Lat. $25^{\circ} 35^{\prime}$, Long. $102^{\circ} 34^{\prime}$.
鹿 LU-YI.

4114 Iı-fêng | 豈 I,uh-fung, H. in Yün-naı Fu, Yünnan; I at. $25^{\circ} 09^{\prime}$, Long. $102^{\circ} 14^{\prime} ;$ Yüan, I,n-fêng |H.

4115 Iı-pı 㨝 ssŭ, in Kaoyao H., Kuangtung.

\section{淥}

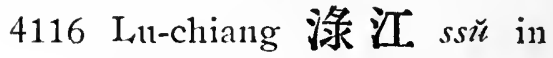
Li-ling H., Honan.

\section{角}

4117 In-chih 解直, chên near K'un-shan H., Kiangsı.

\section{鹿}

4118 Lu-ch'êng 鹿战, see No. 5767.

4119 Lu1-cliang | 江 Lokkong, port on the west coast of Formosa, north of Anping.

4120 Ln1-chiao | 角, ss̆̆ near Yo-chou Fin, Hunan.

4121 Li1-ch 'ïan | 泉, see No. 2423.

4122 Lu-niu| 4 ssŭ in $\mathrm{P}^{\prime}$ anyï H., Kuangtu11g.

4123 Lı1-pu| 步, sš̆ near Kuang-chou Fu, Kuangtung.

4124 In-yi | 邑 Luh-yih, H. [172] in Kuei-tê Fu, Honan; Lat. $33^{\circ} 57^{\prime}$, Long. $115^{\circ}$ $35^{\prime} ;$ S. A., Ming-lı 鳴 |; Han, To 鄲 H.; E. Han, 


\section{陸}

L,U-YÜAN.

Wu-ping 武 本; Sui, In-yi 邑; T"ang; Chênyüan 蒙源.

4125 Lu1-yüan | 苑, see No. 3147.

\section{魔}

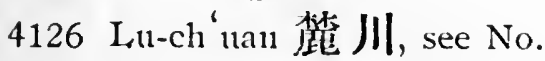
6030.

\section{陸}

4127 L 11-an 陸安, (obs.) formerly H., S. W. of Haifêng H., Kuangtung.

4128 Lu-ch'van | 川 Luh[93] chnen, H. in Yü-lin C., Kuangsi; Lat. $22^{\circ} 25^{\prime}$, Long. $109^{\circ} 49^{\prime}$; Sui, L11ch'nan | 川.

4129 L,11-fêng, | 豐 Ltull-fung, $\mathrm{H}$. in Hui-chou $\mathrm{Fu}$, Kuangtung; Lat. $22^{\circ} 57^{\prime}$, Long. $115^{\circ} 55^{\prime}$.

4130 L L11-11un | 渾.

$1^{\circ}$ (obs.) 50 li N. li. of Sung H., Honan; Han, $\mathrm{H}$. in Hung-11ung 弘 晨 chiin; Tsin, H. in Honan 河南 chün. $2^{\circ}$ (obs.) $30 l i \mathrm{~N}$. E. of Sung H., Honan; Sui, H. in Ho-nan 河 南 chün; T"ang, H. in Ho-nan 河南 Ful

$3^{\circ}$ See No. 5990.

4131 Lu-liang / 凉 Luh-liang [335] C. in Ch'ü-ching $\mathrm{F}_{\mathfrak{u}}$, Yünnan; Lat. $25^{\circ} 08^{\prime}$,
旅 Lï-CH' I.

I.ong. $103^{\circ} 44^{\prime}$; Han, P'ing-yi 平夷; Minor Han, 1st, Hsing-ku 興 古, 2nd, Lu-liang | 凉.

$L_{11}$, see No. 4133

Lu-ki, see No. 4134.

Lul-ngan, see No. 4098.

I.11-shi, see No. 4084.

\section{LU \\ 旅}

4132 Liì-shun-k'ou 旅顺口 Port Arthur. Port at the southern extremity of Mianchuria, once held by Russia from China on lease of indefinite duration.

\section{瀘}

4133 L.̈̈ 瀘 Li1, C. L. C. in [528] the Ch' nan-nan-Yung-ning circ., Ssŭch 'uan ; Lat. $28^{\circ}$ $56^{\prime}$, Long. $105^{\circ} 33^{\prime}$; orig., in K. Pa 巴; E. Han, Cliang-yang 江陽; Liang, I,ii | C.; Sung, I, iï-chnan | 川; Ming, I in | C.

\section{Lii-ch'i | 溪 Lii-ki.}

[561] $1^{\circ} \mathrm{H}$. in Clı'ên-chou $\mathrm{F}_{11}$, Hunan; I at. $28^{\circ} 15^{\prime}$, Long. $107^{\circ} 33^{\prime}$; Han, Yüan-ling | 元 陵; Liang, L11 盧C.; T'ang, Lu1-ch'i 盧溪.

[269] $2^{\circ} \mathrm{H}$ in Chien-ch' ang $\mathrm{Fu}$,

Kiangsi ; Lat. $27^{\circ} 33$, Long, $117^{\circ} 07^{\prime}$. 


\section{慮 I,Ü-CH'vaN.}

4135 Lïi-ch' 'uan | 川, see Nos. 263, 4133.

4136 Lï-ku| 活, (obs.) 120 li N. of Hsi-ch' ang H., Ssŭch' $112 n ; Y$ Yüan, H. in $\mathrm{Li}$ 禮 $\mathrm{C}$.

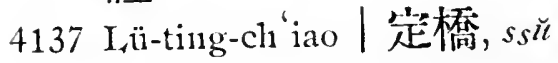
near Jung-cling H., Ss̆̆clínan.

4138 J $\ddot{1}$ 感.

\section{虔:}

$1^{\circ}$ (obs.) Chou, K. in Anluni.

$2^{\circ}$ See Nos. 2126, 4140.

4139 I $[167] 1^{c}$ H. in Iii-chon Fu, Anhui ; Lat. $31^{\circ} 17^{\prime}$, I.ong. $117^{\circ} 17^{\prime}$; Han, Lung-shu 龍舒; Liang, Lii-chiang | 江; Tsin and S. Sung, Shu 抒 H.; $S . T s^{\prime} i$, Lü-chiang | 江 chün; Sui, Lncliang | 江 $\mathrm{H}$.

$2^{\circ}$ (obs.) 120 li W. of İ̈̈-chiang H., Anhıi; $W$. Han and E. Han. Lï-chiang | 江 chüin. $3^{\circ}$ (obs.) $55 \mathrm{li} \mathrm{W}$. of Hoch'iu H., Anhuii; $T$ sin. chiin in Yang 揚 chov. $4^{\circ}$ (obs.) $30 l i \mathrm{~N}$. E. of Ho-shan H., Anhni; $S$. Sung, chiun in S. Yï 豯 cHOr.

$5^{\circ}$ See Nos. 2126, 4140.

\section{Lï.}

4140 Lü-clion | 州 İ̈i-chau, Fu [317] in the An- $\mathrm{L} \ddot{\mathrm{u}}-\mathrm{Ch}^{6}{ }^{6} \mathrm{n}-\mathrm{Ho}$ circ., Anhuii ; forming also the district city of Ho-fei ; Lat. $31^{\circ} 50^{\prime}$, Long. $117^{\circ}$ 15'; Chou, K. Lü $\mid$; S. A., in K. Slin 得; $C$. IVars, in K. Clin 楚; $r s^{\prime}$ in, in Chin-chiang 九 江 chü ; Han, Lü-chiang IIL; E. Han, Ho-fei 合 肥; Lia $g$. Ho 合 C.; Sui, I üu C.

4141 Lï-ling | 陵 Lü-ling.

$1^{\circ} \mathrm{H}$. forming the prefectural city of Chi-an, Kiangsi ; Lat. $27^{\circ} 02^{\prime}$, Lolig. $115^{\circ} 05$; Han, Liï-ling | 陵; Ts'in, Shih-yang 石陽; Sui, Clii 吉 C.

$2^{\circ}$ (obs.) formerly town E. of Fang $\mathrm{H}$., Hupeh. $3^{\circ}$ See Nos. 584, 4181, 7847,7852 .

\section{虑。}

4142 I ü-ssŭ 慮㐆.

$1^{\circ}$ (obs.) N. of Wu-t'ai H., Shansi; Ha, $\mathrm{H}$. in T'ai-yian太原chïn. $2^{\circ}$ See No. 7121.

\section{毁盧}

4143 Lï-yi 驉夷, see No. 7121. 品

4144 Lï 号

$1^{\circ}$ (obs.) formerly town in K. Ch' u 楚. 
槊

L.ن̈-ru.

$2^{\circ}$ (obs.) Chou, town in N. of K. Chi 㴒.

$3^{\circ}$ See No. 2098.

4145 Liü-tu | 都, (obs.) 30 li S. W. of Kê-tsê H., Shantung; $W$. Han, H. in Chi-yin 濟院 chün.

4146 Lï̈-tung | 来sš̆ ini T'ung C., Kiangsin.

4147 Lï-yen | 医, sš̆ near Tsao-yang H., Hupeh.

\section{問}

4148 Lï 誾, (obs.) N. E. of Knang-ning $H$., Shingking; Liao, C. in Shangcling 上京 circ.

4149 I,ü-yang 陽, post-towi near Yi C., Slingking.

\section{湺}

4150 Lü-chung 漊中, (obs.) N. W. of $\Lambda \mathrm{n}-\mathrm{fu}$ H., Hunan. $T$ sin, $S$. Sung and $S$. $T s^{\prime} i, H$. in 'T'ienl-mên 天 門 chïn.

Lï-chat, see No. 4140.

Lï-kiang, see No. 4139.

\section{LUAN}

稖米

4151 I,11an 耪, (obs.) T'ang, C., N. of Yung-shun H., Kuangsi.

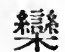

4152 Luan-ch'êng 誉篹城 Lwan[34] ching, H. in Chêng-ting
畧 I Ü̈HH-YANG.

Fu, Chihli ; Lat. $37^{\circ} 50^{\prime}$, I,ong. $114^{\circ} 47^{\prime}$; orig., Luan-wu | 武; E. Han, Luan-chêng | 城.

4153 Luan-wu | 武, see preceding.

4154 Inan 㜻 Lwan, C. in [271] Yung-p'ing Fin, Chihli; I at. $39^{\circ} 48^{\prime}$, Long. $118^{\circ}$ $50^{\prime}$; Shang, Ku-chu孤竹; Han, Hai-yang 海陽; Five dyn., Luan | C.; Liao, Kin and Yüan, Yi-fêng 義 豈 $\mathrm{H}$.

4155 Lnan-ching | 宗, see No. 6206.

4156 I,11an-p'ing | 位 L,nanping, H. in Chêng-tê Fu, Cliilili; Lat. $40^{\circ} 5 \tau^{\prime}$, Long. $11747^{\prime}$.

\section{LÜEH}

\section{晏}

4157 Lüelı-yang 畧陽 Liol1[30] yang, $H$. in Han-chung Fu, Sliensi; Lat. $33^{\circ} 22^{\prime}$, Long. $106^{\circ} 08^{\prime}$; Han, Chï 沮 H.; Tsin, Lîel-yang |院; $W^{\prime}$. Wei, Shn11chêng 順政.

Luhchuen, see No. 4128 . I,ulh-fung, see Nos. 4114, 4129.

Luh-lioh, see No. 4007.

Luh-kinen, see No. 4113. 
倫

I,UN.

I,uh-liang, see No. 4131.

Luh-ngan, see No. 4005.

Luh-yih, see No. 4124.

Lui-chu, see No. 3668.

Liu-po, see No. 3671.

Lui-yang, see No. 3677 .

Lukchak see No. 4107 .

\section{LUN}

4158 Lun 倫.

$1^{\circ}$ (obs.) near Nan-ning 南富 Fu， Kuangsi; $T^{\prime} a n g, C$. in Ling-nan 領南 circ.

$2^{\circ}$ (obs.) in Kuangsi ; Sung, C. in Yung 峞

cHOU.

4159 Lun1-chu-pu| 珠布 Hlunchub; town in Thibet; Lat. $30^{\circ}$, Long. $92^{\circ} 10^{\prime}$.

\section{綸}

4160 I 1111 綸, see No. 7653.

輸

4161 Ld111-t'ai 㫻荎.

$1^{\circ} \mathrm{H}$. in Yen-ch'i Fin.

New Dominion.

$2^{\circ}$ (obs.) near 'Turfan; T'ang, Fu in Lung-yu 隴右 circ.

\section{LUNG}

\section{竜}

4162 Lung-ch'ih 竜池, sš̆ near Hsin-chêng H., Kiangsi

4163 Lu11y-chü-chai | 駒些, post-town near Shang C., Shensi.
龍 LUNG-AN.

4164 Lung-fan | 沓, T-S. near 'Ting-fan C., Kueichon.

4165 Lung-kang / 岡, post-town near Shun-tê Fu, Chihli.

4166 Lung-shan | 山, chên near Chang-ch'iu $\mathrm{H}$., Shantung.

4167 I.ung-têng | 䲲, post-town near Nan-ch'i H., Ssŭch'nan.

\section{龍}

4168 Lung 龍.

$1^{\circ}$ (obs.) N. W. of Chiangyu H., Ssŭch'uan; Yüan, C. in Kuang-yüan 廣 $\overrightarrow{\mathrm{J}}$ circ.

$2^{\circ}$ See Nos. 761, 3074, 3978, 4169, 4177, 5199 .

4169 Lung-an | 努 Lung-ngau. [125] $1^{\circ}$ Fin in the Ch'êngLung-'T'ung-Mien-Mon circ., Ssŭch'uan, forming also the district city of $\mathrm{P}^{\prime}$ ing-wu; Lat. $32^{\circ}$ $22^{\prime}$, I,ong. $104^{\circ} 39^{\prime}$; $7 s^{\prime} \mathrm{in}$, land of the Tich'iang 氏善 tribes; Han, Yin-p'ing 陰平; 2nd Wri, Chiang-yu 江 油; Sui, P'ing-wu 45 武; $T^{\prime}$ ang, Lung-mên | 門; Ming, 1st, Lung C., 2nd, Lung-an | 娄 Fu.

$2^{\circ}$ Chên near Hsin-ch'êng H., Kiangsi. 
龍 LUNG-CH'ANG.

$3^{\circ}$ (obs.) T'ang, H., 30 li N. E. of A11-yi H., Kiangsi.

$4^{\circ}$ (obs.) Tang; H., E. of An H., Ssŭchìnan.

$5^{\circ}$ H. in Chüan-lo 全 羅 circ. Corea.

4170 Lung-ch' ang / 㙫, Y. near Jênlıuai 'T., Kueichon.

4171 Lung-ch' êng | 城; see Nos. 2629, 2668, 3978, 3981, 5762 .

4172 Lung-chi | 集, (obs.) Sui, H., E. of Huang-an H., Hupel.

4173 Lung-ch'i | 溪 Lung-ki, $\mathrm{H}$., forming the prefectural city of Cliang-chou, Fuhkien ; Lat. $24^{\circ} 31^{\prime}$, Long. $117^{\circ} 53^{\prime}$.

4174 Lung-chih | 支, (obs.) S. of Nien-po H., Kansulh; Sui and $T^{\prime}$ ang, Lung-chil | 支 H.; Sung, walled town, in Hsi-ning 西䡐 c.

4175 L,ung-ch'ih | 池.

$1^{\circ}$ Ss̆̆ in Nan-fêng $\mathrm{H}$, Kiangsi.

$2^{\circ}$ (obs.) S. W. of Po-pai H., Knangsi ; $T^{6} a n g, \mathrm{H}$. jn Yin 演 C.

$2^{\circ}$ (obs.) near Turfan; Tang, C. in Hsin-chon 脩州 Fu。
龍允 LUNG-CH'UAN.

$3^{\circ}$ (obs.) in Lï C., Ssŭclituan; T'ang, H. in Shun 順 $\mathrm{C}$.

$4^{\circ}$ See No. 5822 .

4176 Lung-ch'in | 邱, see No. 4220.

4177 I.ung-chon | 州 Lung[198]chatn, T. in T'ai-p'ing Fu, Kuangsi ; Lat. $22^{\circ}$ $22^{\prime}$, Long. $107^{\circ} 39^{\prime}$; Sung, Lung | C.

4178 Lung-chü | 沮, (obs.) 60 li S. of Hai 海 C., Kiangsu ; $N . W_{e i}, \mathrm{H}$. in P'êng-cli'êng 彭城 chün.

4179 Lung-ch' $\ddot{i} \mid$ 渠, (obs.) 180 li S. E. of Chung C., Ssŭch'uan; Sung, H. in Hsien-shun 咸湆 Fin。

4180 Lung-clı'nan | 川 I,ungchuen.

$1^{\circ} \mathrm{F} 11$ in P'ing-an 平努 circ., Corea ; Lat. $36^{\circ}$ $50^{\prime}$, I.ong. $125^{\circ} 55^{\prime}$.

$2^{\circ} \mathrm{H}$. in Hui-chon Fin, [478] Kuangtung; Iat. $24^{\circ}$ I,ong. $115^{\circ} 08^{\prime} ; T \sin$. I,ung-ch' tran | 川; T'ang, Lei-l1siang 零 拫; Sung, I, ei-chiang 霜江 and $\mathrm{H}_{\text {sinn }}$ 䞄 $\mathrm{C}$.; Yüan, Hsün 循 C.

$3^{\circ}$ Post-town near 'T'êng-

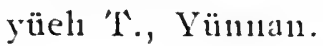

$4^{\circ}$ See Nos. 2052, 2365. 
龍 LUNG-CH'

4181 Lnng-clı'üan | 泉 Lungtsinen.

$1^{\circ} \mathrm{H}$. in $\mathrm{Cl}_{1}{ }^{\prime} 11-\mathrm{chlon} \mathrm{F}^{\mathrm{u}} \mathrm{u}$,

[424] Chellkiang; Lat. $28^{\circ}$ 08', Long. $119^{\circ} 09^{\prime}$; $T^{\prime}$ ang, Lung-tsung |

祭; Sung, Chien-ch "nan 劍川 and Ln1ng-cli' 'ïan 川.

$2^{\circ}$ H. in Chi-an Fin, Kiang-

[249] si ; Lat. $26^{\circ} 18^{\prime}$, Long. $114^{\circ} 25^{\prime} ;$ Han, Lü̈-ling 盧 陵; $W$, Hsinlnsing 新 興; $T \sin$, Sui-hsing 乿 與; $S$. $T^{\prime}$ ang, Lning-ch' uan | 川; - Sung, Cl1'üanchiang 家江.

$3^{\circ}$ H. in Shil1-ch ien Fu,

[77] Kueichou; Lat. $28^{\circ} 20^{\prime}$, Long. $108^{\circ} 30^{\prime}$.

$4^{\circ}$ Sš̆ near Chien C., Ssŭcli 'wan.

$5^{\circ}$ Y. near Hêng C., Knangsi.

4182 Lung-fêng | 逢, (obs.) in Ya-chon Fu, Ssŭch 'nan; $T$ 'ang, C. in Chien-nan 劍读 circ.

4183 Ling-11o | 榈 see No. 5977.

4184 Lung-hu | 湖, see No. 5216.

4185 Lung-hua | 萑.

$i^{\circ}$ Chên near Sung-chiang $\mathrm{Fu}$, Kiangsu.
LUNG-LI.

$2^{\circ}$ Chên in Ching C., Chilili.

4186 Lung-hua | 化, (obs.) T"ang, H., S. E. of Luch' uau H., Kuangsi.

4187 Irung-hsi | 喜, see No. 267.

4188 Lung-hisiang | 拫, see No. 4039.

4189 Lung-hsing | 與, see No: 4938.

4190 Lrung-jên | 仁 H. in Ching-ch'i 京畿, circ., Corea.

4191 Lung-kang | 阔 Lungkang.

$1^{\circ}$ H. in P'ing-an 雨妿 circ., Corea; Lat. $39^{\circ}$ $12^{\prime}$, I Long. $125^{\circ} 32^{\prime}$.

$2^{\circ}$ See No. 2850.

4192 Lung-k'ang | 六.

$1^{\circ}$ (obs.) Liang, town near Hnai-yüan $\mathrm{H}$, Anhui.

$2^{\circ}$ See No. 1989.

4193 Lu111g-k11n | 溒 ssüin Wan C., Kuangtung.

4194 Lung-kung | 宫 chün in Cl1'ing-sliang 愛佾 circ., Corea.

4195 Lung-li | 里 I rung-li.

[85] $1^{\circ} \mathrm{H}$. in Kitei-yang $\mathrm{Fu}$ Kueichon ; I at. 26 $28^{\prime}$, Long. $106^{\circ} 48^{\prime}$. 
龍

$2^{\circ}$ T-S. near Hsia-chiang T., Kueichou.

4196 I rung-ling | 陵 L,ung-ling, $T$. in Yung-ch' ang Fu, Yinnan; Lat. $24^{\circ} 45^{\prime}$, Long. $99^{\circ}$.

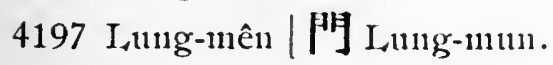

$1^{\circ} \mathrm{H}$. in Hsüan-hua $F_{n 1}$, Chihli; Lat. $40^{\circ} 40^{\prime}$, Long. $115^{\circ} 48^{\prime} ; T^{\circ}$ ang, H. in $\mathrm{H}$ sin 新 $\mathrm{C}$.; Ming, mil. district; Yüan, Wang-「ün 望 雲 $\mathrm{H}$.

[472] $2^{\circ}$ H. in Kuang-chon I’u, Kuangtung; Lat $23^{\circ}$ $44^{\prime}$, Iong. $114^{\circ} 04^{\prime}$; Han, Po-lo 博 羅; Wing, I, nng-mên|'P $\mathrm{H}$.

$3^{\circ}$ Ss̆̈ in Yi-shan H., Kuangsi.

$4^{\circ}$ Y. near Ch'in C., Knangtung.

$5^{\circ}$ (obs.) 2 li W. of $\mathrm{Ho}$ ching H., Shensi ; N. Wei, chïn in Nan-fên 南汾 CHOL ; Sui, H. in Ho-tung 河東 chïn. $6^{\circ}$ (obs.) in Ch' ang-ning H., Ssŭch'uan; T'ang, $\mathrm{H}$. in $\mathrm{Ch}^{\prime}$ ang-ning $\mathrm{C}$. $7^{\circ}$ (abs.) $20 \mathrm{li}$ E. of $\mathrm{L}_{\mathrm{O}}-$ yaing H., Honan; Kin, chên in Ho-nan 河南 Fu.
龍 LUNG-SHIH.

$8^{\circ}$ (obs.) $30 l i \mathrm{E}$. of $\mathrm{Cl}^{\prime} \mathrm{ih}_{1-}$ ch'êng H., Chihli ; Ming, so.

$9^{\circ}$ See Nos. 2066, 4169.

4198 I.ung-nan | 南 I,ung-nan, [452]H., in Kan-chon Fu, Kiangsi; Lat. $24^{\circ} 52^{\prime}$; Long. $114^{\circ} 37^{\prime}$; Han, Nanyeh 南埜, $T^{*}$ ang, Ch'iennan 虔南; Sung, I,ungn:111南.

4199 L.11119-p ‘iao / 標, see No. 907 .

4200 I, 1nng-p ing | t伻, ss $\check{\text { n near }}$ Kuang-chi H., Hupeh.

4201 I, nng-shan | !l!.

$1^{\circ}$ If. in l'ung-shum Fin, [21] Hunan.

$2^{\circ}$ Ċhên in I.i-ch'êngr H., Shantung.

$3^{\circ}$ See No. 727.

4202 I t11n-shao † 沙 ss̆̈ in Cliang-ling H., Hupelı.

4203 I,ung-shêng | 勝 I,ungshing.

$1^{\circ}$ T. in Kuei-lin Fin, Kuangsi ; Iat. $25^{\circ} 30^{\prime}$, I.ong. $109^{\circ} 40^{\prime}$.

$2^{\circ}$ Post-town in Yi-ning H., Knangsi.

4204 Lung-slih | tebe (obs.) near Ning-yüun Fu, Ssŭch'nan; T'ang, C. in Chien-nan 劍南 circ. 
能 LUnG-SHU.

4205 Lun1ng-shu | 抒, see Nos. 4139, 5762, 6808.

4206 Lung-shui | 水, see Nos. 1192, 7437.

4207 Lung-su | 蕉等.

$1^{\circ}$ (obs.) Sung, H., S. E. of Ho-p' u H., Kuangtung.

$2^{\circ}$ (obs.) N. E. of Ho-p' 11 H., Knangtung; $S$. $T s^{\prime} i$, chith and H. in Yiiell 越 chOU; Sui H. in Ho-p' $n$ 合浦 chïn. 4208 Lung-t'an | 潭.

$1^{\circ}$ Ss $\check{\iota}$ in Chï-jung H., Kiangsu.

$2^{\circ}$ Post-town near Cliaohua H., Ssŭch' '11an.

$3^{\circ}$ Post-town near Yu-yang C., Ssŭch' tıan.

$4^{\circ}$ Ss s̆̋ in Hsü-p'ı H., Hunan.

4209 Lung-t'an-ch'iao | 潭橋, ssü. in Lung-yang $\mathrm{H}$., Hunan.

4210 Lung-tien | 甸, see No. 7810 .

7211 Lunng-t'on | 頙.

$1^{\circ}$ Y. near Knei-ting $\mathrm{H}$, Kueichou.

$2^{\circ}$ Y. near Sung-t'ao T., Kueichon.

$3^{\circ}$ Y. near Tan C., Kuangtung.
龍 IUNG-YU.

4212 Lunng-tsê | 濢 H. in Cln'üan1o 全羅 circ., Corea.

4213 Lung-tsung | 宗, see No. 4181 .

4214 Lung-tu | 都, chün near Chï-jung H., Kiangsu.

4215 Lung-wan | 灣, ssŭ near Cliven-li H., Hupeh.

4216 Lung-yang | 陽 Lungyang.

[561] $1^{\circ}$ H. in Clíang-tê Fiu, Hunan; Lat. $28^{\circ} 52^{\prime}$, Long. $111^{\circ} 38^{\prime}$; Han, So 䒺 H.; Wu, Lungyang | 陽; Sung, Ch'ênyang 辰晹.

$2^{\circ}$ Chên near T'ung-chou Fu, Shensi.

4217 Lung-yen | 袮 Lung-yen. [95] $1^{\circ}$ C. L. C. in the T'ingChang-Lung circ., Fulnkien ; Lat. $25^{\circ} 08^{\prime}$, Long. $117^{\circ} 08^{\prime}, T$ sin, Hsin-lo 新 羅; Tang, I, tung-yen

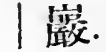

$2^{\circ}$ See No. 5504.

4218 Lung-yi | 義, ssü near Hsin-kan, H., Kiangsi.

4219 Lung-ying | 英 Lungying, 'T. C. in 'T'ai-p'ing Fu, Knangsi ; Lat. $22^{\circ}$ $56^{\prime}$, Long. $106^{\circ} 48^{\prime}$; Yüan, Lung-ying | 莫 $\mathrm{C}$.

4220 Lung-yu | 游 Lung-yu. 
龍

LUNG-YÜAN.

[112] $1^{\circ} \mathrm{H}$. in $\mathrm{Cl}^{\prime}$ ii-chou $\mathrm{Fu}$, Cheh-kiang; Lat. $29^{\circ}$ $04^{\prime}$, Long. $119^{\circ} 22^{\prime}$; Long. $119^{\circ} 22^{\prime} ; T_{s}^{\prime}$ in, T'a-110 太 末; $W u$, Lung-ch iu|邱; $T^{\prime}$ ang, Hu 敹 C.; Fize dy'n., Lung-yu|游.

$2^{\circ}$ (obs.) formerly H. near Chia-ting Fiı, Ssŭch'nan.

4221 Lung-yüan | 源.

$1^{\circ}$ (obs.) near Ssŭ-ên H.,

Kuangsi ; $T^{6}$ ang, H. in Huan 環 C.

$2^{\circ}$ (obs.) near Nan-ning Fu, Knangsi; $T^{\prime}$ ang, H. in Wei 椳 C.

4222 Lung-yüan | 原, (obs.) N.W. of Yi-pin H., Ssŭch'uan: T'ang, H. in Yin 殷 C.

4223 Lung-su 湇蘇, (obs.) N. E. of Ho-p'u H., Kuangtung; S. Sung, chün in Yüeh 越 C.

\section{㯙}

4224 I unng 櫳, (obs.) Sung, C. near 'Tung-lan C., Kuangsi.

4225 Lung-clı'ing | 傜, see No. 7373.

\section{瀧}

4226 Iting or, Shuang 㴰.

$1^{\circ}$ (obs.) 100 li E. of Lo-ting C., Kuangtung,

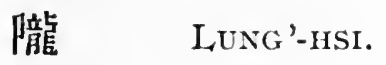

T'ang, C. in Ling-nan 領南 circ.

$2^{\circ}$ See No. 4481.

4227 Itung-ch'ien or, Shuangch'ien 千; see No. 1173.

4228 Lung-shui or, Shuang-shui | 水, see No. 4039 .

\section{等管}

4229 Lung 錇, (obs.) in Kuangsi; $T$ 'ang, C. in Ling-nan 領南 circ.

\section{噰}

4230 Irung 龖 Lung.

[48] $1^{\circ} \mathrm{C}$. in Fêng-lisiang Fu, Sliensi ; Lat. $34^{\circ} 48^{\prime}$, I.ong. $106^{\circ} 58^{\prime}$; Han,

- Clı'ien 汗 H.; 2nd Wei, Lung-tung | 東; W. Wei, I,ung |C.

$2^{\circ}$ See No. 5487.

4231 Itung-ch' êng | 城, see No. 1106.

4232 Lung'-1ısi | 건 Lung-si.

$1^{\circ} \mathrm{H}$. forming the prefectural city of Kungcli'ang, Kansuh; Lat. $34^{\circ} .56^{\prime}$, Long. $104^{\circ}$ 44'; Han, Hsiang-wu 留武; Sui, Lung-hsi |西.

$2^{\circ}$ (obs.) S. W. of the above; Tsin, chiin in $\mathrm{Cl}^{\prime}$ in 秦 chou; $N$. Wei, chïn in Wei 渭 


\section{䧇}

LUNG-NU.

CHOU; Sui, chün in Y111ng 雃 cHou.

$3^{\circ}$ (obs.) in Nan-chêng H., Shensi; S. Sung and $S . T s^{\prime} i$, chizh in $\mathrm{Cl}^{\prime}$ in 柇 CHOU.

$4^{\circ}$ (obs.) N.E. of L I1111ghisi H., Kansull; Sui and $T^{\prime}$ ang, $\mathrm{H}$.

$5^{\circ}$ (obs.) near Fêng-lisiang Fu, Shlensi ; K’in, chên in Linng $\mid C$.

$6^{\circ}$ See Nos. 2218, 63.58, 6789.

4233 L tung-1111 | 木, 'P-S. in Mon C., Ssŭcl nan1.

4234 L_1111g-ti | 隄, (obs.) i11 $\mathrm{Cl}_{1}{ }^{\prime}$ ï-ching Fin, Yünnan; Tang, H. in Nan-ning 南嶰 $\mathrm{C}$.

4235 Ji11ng-tung | 束.

$1^{\circ}$ (obs.) $40 \mathrm{li} \mathrm{W}$. of

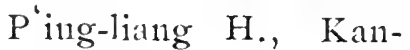
st1h; N. Wei, chïn in Ching 汾 CHOE.

$2^{\circ}$ See No. 4230.

4236 Lung-yu | 右, (obs.) T'ang circ. representing parts of Yung 䔨 $\mathrm{CHOU}$ and Liang 梁 cHOU.

\section{隆}

4237 Lung 隆.

$1^{\circ}$ (obs.) S. Sung. C. in $C^{\prime}{ }^{6} e_{11 g}-t u$ 成都 circ. $2^{\circ}$. (obs.) W. Wei, C.
隆 LUNG-PING.

near Pao-ning Fu, Ssŭch' '1an.

3。 See No. 4951.

4238 Inng-an | 安 Lung-ngan, [416] H. in Nan-11ing Fu Knangsi; Lat. $23^{\circ} 15^{\prime}$, Long. $107^{\circ} 20^{\prime}$.

4239 Lung-clíang | 昌 Lung[281] cliang, H. in Hsï-chon Fit, Ssŭch' '1an1; Lat. $29^{\circ}$ 28', Long. $105^{\circ} 19^{\prime}$.

$42+0$ J nnng-ch'ing | 磨, see No. 7363.

42+1 L.111ng-pa | 埧, T-S. in Kokonor.

4242 I unng-fang | 坊, chên near Clunng-pı H., Shensi.

4243 Linng-hua | 化, (obs.) $T^{6}$ ang, H. near Nanclı 'uan H., Ssŭch'

4244 Lung-hrsing | 與.

$1^{\circ}$ (obs.) S. Sung, Fu in Cliang-hisi 江西 circ. $2^{\circ}$ See No. 4562.

4245 Lung-lii | 虑, see No. 3907.

4246 Lung-ping | 蕜, post-town near Fu C., Shensi.

4247 Lung-ping | 本 Lung[459] ping, H. in Chao C., Cliiluli; Lat. $37^{\circ} 25^{\prime}$. Long. $114^{\circ} 54^{\prime}$; Han Kuang-o 庴阿; Sui, Hsiangch' êng 象城 and Ta-ling 大陵; Sung, Lung-p'ing |本. 


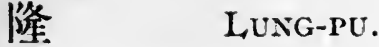

4248 Lung-pu | 布, T-S in Kokonor.

4249 Lung-shan, | 山, see No. 5049.

4250 Lung-tê | 㘻 Lung-teh. [462] $1^{\circ} \mathrm{H}$. in $\mathrm{P}^{\prime}$ ing-liang $\mathrm{Fu}$, Kansuh; Lat. $35^{\circ} 40^{\prime}$. Long. $106^{\circ} 10^{\prime}$; Han, An-ting 安定; Sung, Lung-tê | 德.

$2^{\circ}$ See No. 4098.

4251 Lung-t’un|頙ss $\breve{c}$ in L,uugshan H., Hupel.

4252 Lung-tung | 東, T-S. in Kokonor.

4253 Lung-t'ung | 同 ssü in Shao-yang H., Hunan.

4254 Lung-wu | 武, see No. 3896.

Lung-chang, see No. 4239. I.ung-chau, see No. 4177. Lung-chuen, see No. 4180. Lung-ki, see No. 4173. Lung-mun, see No. 4197. Lung-ngan, see Nos. 4700, $4169,4238$.

Lung-ping, see No. 4267. Lung-shing, see No. 4203. Lung-si, see No. 4232. Lung-tel, see No. 4250. Lung-tsiuen, see No. 4181. Iwan, see No. 4154. Lwan-ching, see No. 4152. Lwan-ping, see No. 4156.
MA-LI-WU.

\section{MA}

称

4255 Ma-a 麻阿, contraction for Mavarannahar, the regions $\mathrm{E}$. of the Oxus; Transoxiana.

4256 Ma-chang | 章, T-S. near Min C., Kansuh.

4257 Ma-ch'ing | 城 Ma-ching, [348 ${ }^{\mathrm{A}}$ ] H. in Huang-chou $\mathrm{Fu}$, Hupeh; Lat. $31^{\circ} 14^{\prime}$, Long. $114^{\circ} 52^{\prime} ;$ Han, Hsi-ling 西陵; Ch'en, Ting 定 C., P. Chou, T'ing 亭 C.; Sui, Ma-chêng | 城; $T^{\prime}$ ang, Yang-ch'êng陽城.

4258 Ma-êrh-yi-nang | 耳亦捿, see No. 4280.

4259 Ma-fu | 夫, chên near Ling-t ai $\mathrm{H}$., Kansuh.

4260 Ma-lıa | 哈 Ma-o.

[282] $1^{\circ}$ C. in Tu-yün Fu, Kueichou; Iat. $26^{\circ} 26^{\prime}$, I ong. $107^{\circ} 24^{\prime}$.

$2^{\circ}$ Sis $\breve{c}$ near the above.

4261 Ma-hui|| 會, chên near Hsin C., Shansi.

4262 Ma-hsiang | 响, ss̆̈ near Ch'ang-chai T., Kueichou.

4263 Ma-li-wu | 里 $\pi$, or Ma-lu 馬魯, Meru or Merv, town in Independent, Turkestan, 300 miles S. E. of Khiva. 
麻后

MA-LUNG.

$426+$ Ma-lung | 龍, (obs.) 50 li N. of Huti-li C., Ssŭch'tran; Yürn, C., in I: 11 ich'nan 會' II! circ.

4265 Ma-sha-chieh|沙街, posttown near Chien-yang H., Fulhkien.

4266 Ma-t'ien | 田 chïn in Ching-ch'i 等㱍 circ., Corea.

4267 Ma-ts'11n | 村, (obs.) formerly town near Fang $\mathrm{H}$. H.upel.

4268 Ma-wên-jên |文仁, town in the Lituch in Is.

4269 Ma-yang | 骝Ma-yang, H. [283] in Yüan-chon Fn, Hunan; Lat. $27^{\circ} 3 S^{\prime}$, L.ong. $109^{\circ} 22^{\prime} ;$ Sui, Yüan-ling 沅陵 and Cl1 ${ }^{6} \mathrm{e}_{1}-\mathrm{ch}^{6}{ }^{\mathrm{i}}$ 辰 筀; T'ang, Na-yang | 陽.

\section{馬}

4270 Ma 馬, (obs.) T'ang C., $N$. of Ma-lung C., Yünnau.

4271 Ma-ch' êng | 成, ssü near $\mathrm{Cl}_{1}{ }^{\prime}$ ang-wn $\mathrm{H}$., Shensi.

4272 Ma-ch'êng | 城, see No. 3658.

4273 Ma-clii | 績, sš̆ in Ya11ghu H., Kiangsu.

4274 Ma-ch'i-ho | 溪河, ss̆̈ near Hsiao-kan H., Hnpel..

4275 Na-clia | 家.
臨 MA-HU.

$1^{\circ} \mathrm{Y}$. near Han-yin T., Shensi.

$2^{\circ}$ Y. near Hsin-ts'ai H., Honan.

4275 Ma-clia-hsiang/家苍, Ma[95] kia-hsiang, T. in $\mathrm{Cl}_{1}$ üanchon Fu, Ftrhkien; Lat. $24^{\circ} 30^{\prime}$, Long. $118^{\circ} 08^{\prime}$.

4277 Ma-cliang | 江, (obs.) S. E. of Chao-p'ing H., Knangsi ; $T^{\prime}$ angr, H. in $\mathrm{Fu}$ 富.

4278 Ma-clitang | 庄, chên near Hsien-yang H., Shensi.

4279 Ma-ê | 額, chên near Weinan H., Shensi.

4280 Ma-êrll-k'ê-lang | 兒喀朗, or Ma-êrh-yi-nang 麻耳亦 雭, Marglinan, or Margijan, town in Independent 'Turkestan, S. E. of Kokand.

4281 Ma-fang | 坊.

$1^{\circ}$ Chên near Ho-shun H., Sliansi.

$2^{\circ}$ Chên near Hu H., Shensi.

4282 Ma-han | 㛺, (obs.) for1merly K. in IV. of Corea.

4283 Ma-h11 | 湖, (obs.) in P'ing-shan H., Ssŭch' tuan; Lat. $28^{\circ} 31^{\prime}$, Long. $104^{\circ}$ $19^{\prime}$; - orig., land of the Iao 㤿 tribes, in $\mathrm{K}$. P'o 整; Han, in Chien1wei 腱篇 chiun and 


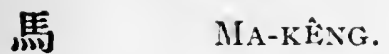

Tsang-kê 㲏 䋍 chün; Yïan, Ma-ln1 | 湖 circ.; Ming, Ma-1un | 湖 Fu.

4284 Ma-kêng | 莖 T'ung-jên Fu, Kueichou.

4285 Ma-k où | П], ssŭ near Hsing-kuo C'., Hupeh.

4286 Ma-k'uai | 塊, chên near Kung-clı'ang Fn, Kansul.

4287 Ma-la | 喇, T.-S. near Hsin-ning H., Ssŭch' 'uan. 4288 Ma-lan|欄, chên near Yi-chün H., Shensi.

4289 Ma-lan-kuan | 蘭 寎, a Customs station in the Great Wall, N.W. of 'Tsunhua C., Chinli, nuder the special jurisdiction of the Brigade General resident at Yung-p'ing Fu.

4290 Ma-li-chil | 力䚳, chên near Ningr-yüan H., Kansuh.

4291 Ma-ling-ch' ii | 勈 曲, chên near Ho-shun H., Shansi.

4292 Ma-lu | 學, see No. 4263. 4293 Ma-lu-ch'a-yelı-k'ê| 售祭 葉 订 Marnclak, a town in Independent Turkestan, 140 miles S. of Merr.

4294 Ma-lu| 鹿, chên near Ninglısia $F \mathrm{~F}$, Kansulh.

4295 Ma-lung | 龍 Ma-lung. [478] $i^{\circ} \mathrm{C}$. in Clı 'ü-cling Fu, Y'ünnan; Lat. $25^{\circ} 29^{\prime}$, I,ong. $103^{\circ} 37^{\prime}$, Minor
馬 MA-T'OU.

Han, Hsing-ku 興古; Yüan, Ma-lung 龍 C.; Ming, Ma-lung / 龍 $\mathrm{H}$. $2^{\circ}$ (obs.) formerly H. near Hui-li C., Ssŭch' naı. $3^{\circ}$ See No. 6137.

4296 Ma-mên | Py, (obs.) in Kuangsi ; S. Ts'i, chïn in Yïelı 越 Crrou.

4297 Ma-pien | 邊 Ma-pien, T. [88] in Hsü-chou Fu, Ssŭch 'uan; Lat. $29^{\circ} 05^{\prime}$, Long. $104^{\circ}$. Terr. jur.

4298 Ma-p'ing | $\mathrm{H}$. forming the prefectural city of Liul-chou, Kuangsi ; Lat. $24^{\circ} 14^{\prime}$, Long. $109^{\circ}$ $09^{\prime} ;$ Han, '个'an-chung 潭 中; $W u$, Ma-ping|本.

$\$ 299$ Ma-shou | 首, post-town near Pao-ch' êng H., Shensi.

4300 Ma-shour-yi | 苗邑, see No. 5753.

4301 Ma-t'a-slih | 踏 不i, sš̆ near An-clı'ing Fu, Anhui. 4302 Ma-t'ai| 臺, see No. 2730. 4303 Na-tangl 當, sš̆ in P'êngtsê H., Kiangsi.

4304 ina-ti | 底, post-town near $\mathrm{Ch}^{6}$ ên-chou Fu, Hunan.

4305 Ma-t'on | 此.

$1^{\circ}$ Chể near 'Tung-kuang H., Chilıli.

$2^{\circ}$ Chên near Ning-kno Fin, Anluni. 
監 MA-T'OU-CHI.

$3^{\circ}$ Chên near Jui-ch' ang H., Kiangsi.

$4^{\circ}$ Y. near J,ê-t'ing H., Chilili.

$5^{\circ}$ Post-town near Hsiangho H., Chihlit.

4306 Ma-t'ou-chi | 箕集, ss in Lin1-an C., Anluni.

4307 Ma-tsu Niao | 祖廟, see No. 4790.

4308 Ma-ts' 111 个村.

$1^{\circ}$ Chên near Kao-p'ing

H., Shansi.

$2^{\circ}$ Chên near Hsing-p'ing H., Shensi.

4309 Ma-wang-ch'êng | 王城, (obs.) formerly fort W. of Cling C., Hunan.

4310 Ma-wei | 尾, see No. 4021. 4311 Ma-yi | 邑.

$1^{\circ}$ (obs.) near So C., Shansi; Lat. $39^{\circ} 28^{\prime}$, long. $112^{\circ} 35^{\prime}$; Ts ${ }^{\prime} \mathrm{in}$, Ma-yi 邑 H., Sui, Shan-yang 鄯陽; Five dy'n., Huan 噮 C.; now Ma-li-hsiang | 邑斑. $2^{\circ}$ See No. 5843 .

\section{嗎}

4312 Ma-ni-t’ $u$ 㜯尼圖 Manito, town in Tarbagitai; Lat. $45^{\circ} 50^{\prime}$, Long. $83^{\circ}$.

\section{理}

4313 Ma-chung-kê 瑪鍾 噶, post-town near Ta-chien-lu T., Ssŭch' nau.
MAI-MAI-CHÊN.

4314 Ma-la-pa-shih | 喇巴什 Marapash, C.L.T. in Kashgar circ., New Dominion.

4315 Ma-11a-ssŭ | 納 斯, see No. 5940.

4316 Ma-ni-t' $u$ | 尼圖, Bakamaniton, town in Outer Kansuh ; Lat. $40^{\circ} 10^{\prime}$, Long. $90^{\circ} 40^{\prime}$.

4317 Ma-yen-ho| 娫河, ssŭ in Pin-chon T., Kirin.

Ma-ching, see No. 4257. Ma-kia-hiang, see No. 4276. Ma-o, see No. 4260.

Ma-ping, see No. 4298.

Macao, see No. 122.

\section{MAI}

憖

4318 Mai-chêng 类 城, (obs.) $S$. $A$., town in K. Ch' $\mathrm{u}$ 楚, near Tang-yang H., Hupeh.

4319 Ma-ti | 地, Y. near Sungt'ao T., Kueichou.

4320 Mai-tsa ! 襍, T. S. near Sung-p'an T., Ssǔch' 'uan.

\section{貲}

4321 Mai-mai-chên 買 賈 鎮, (i. e., Mart, or tradingtown,) town in Tuchetu Khanate, on the Russian frontier, corresponding to the town of Kiachta （Chia-k'ê-t'u 恰 克圖， 
蠻

MaN-haO.

or, Ha-k'ê-t " 1 哈克圖) immediately adjoining, on Siberian territory. See No. 717.

\section{MAN}

\section{䆊}

4322 Man-hao 蠻耗, Man-hao, Yïnnan frontier town on the Songka. It is the highest point to which navigation is practicable.

4323 Man-tung 蠻洞, see No. 4626.

4324 Man1-yi | 隽.

$1^{\circ}$ (obs.) Yüan, H., S. W. of Ssŭ-nan Fu, Kneichou.

$2^{\circ}$ T. S. near Yin-chiang H., Kneichon; Ming, ssü.

$3^{\circ}$ (obs.) in Yünnan; Ming, ssŭ in Ma-hu 馬湖 Fu.

\section{霂}

4325 Man 滿, see No. 3168.

4326 Man-ch'êng | 城, Mwan[92] cling, H. in Pao-ting Fu, Chilli; I at. $39^{\circ}$, Long. $115^{\circ} 20^{\prime} ;$ Han, Pei-p'ing 北斥; $T^{6}$ ang, Man-ch êng 下城.

4327 Man-chia | 家. Y. near Chin-lisiang H., Shantung.
毛 $\mathrm{MAO}-\hat{\mathrm{F}} \mathrm{RH}-\mathrm{K} \hat{\mathrm{E}}$.

4328 Man-yün | 雲 Manwyne, town in Yünnan, near Chan-ta [No. 165].

\section{漫}

4329 Man-ho 漫 矿, chèn near Fei-ch'êng H., Shantung.

Manas; see No. 5940.

\section{MANG}

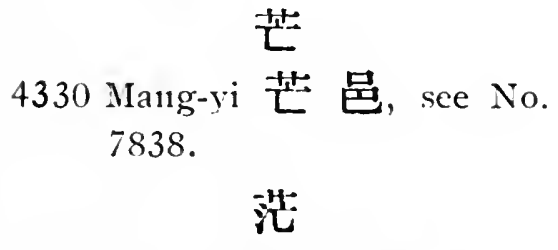

4331 Mang-shih 茫施, (obs.) in Yünnan; Yüan, circ.

Manhao, see No. 4322.

Manito, see No. 4312.

Manwyne, see No. 4328.

\section{MAO}

\section{毛}

4332 Mao 毛.

$1^{\circ}$ (cbs.) Chou, fendal state in Honan.

$2^{\circ}$ See No. 3355.

433.3 Mao-chia | 家, Y. near Yung-ming $H$., Hunan.

4334 Mao-êrll-kê | 胃革, T-S. near Sunng-p an 'T., Ssŭch'uan. 
毛 MAO-HSI-LI.

4335 Mao-h1si-li | 夕里, Moussonl, city in Asiatic Turkey. 4336 Mao-pu-la |卜 喇, posttown near Ling C., Kansi11.

4337 Mao-tsung | 栄, (obs.) 30 li E. of $\mathrm{Ch}^{\prime}$ iieh-shan $\mathrm{H}$., Honan; Kin, chên in 'Ts'ai 蔡 C.

4338 Mao-yang | 晹, chên near Mêng-yin, H., Shantung.

4339 Mao-yü | 峪, chển near Trung-chiang H., Ssücli'nan.

\section{帽}

4340 Mao-êrlı-shan 棺兒山, ssü in 'T'ung-hna H., Shingking.

\section{猄}

4341 Nao-ching 第 津, chên near P'ing-lu H., Shansi [No. 5177].

4342 Mao-shan | 山U, chên in Ch' i C., Hupel.

4343 Mao-tíen | 田, ss̆̌ in Tsêng-ch' êng H., Knangtw11ng.

\section{吅}

4344 Mao-tung 卯 洞, sšr in Lai-fêng H., Hupelı.

\section{愺}

4345 Mao-111êng 貓孟, shê near Tan-shwui H., Fuhkien.

\section{梅 Mri-chu.}

4346 Mao-wu|第, ssü near Chang-hua H., Formosa.

Marapash, see No. 4314. Marghinan, see No. 4280. Margijan, see No. 4280. Maruchak, see No. 4293 Matsu, see No. 4790. Matsuyama, see No. 5980. Matsuyé, see No. 5970. Ma11, see No. 4498. Ma11-chang, see No. 4699. Maut-ku1ng, see No. 4496. Mall-1ning, see No. 4505. Mavarannahar, see No. 4255.

Mazur, see No. 2104.

Medjugung, see No. 4487.

\section{MEI}

\section{梅:}

4347 Mei 梅.

$1^{\circ}$ (obs.) S. Sung, C., in Kuang-tung 奮東 circ. $2^{\circ}$ See No. 708.

4348 Mei-clıai | 寨, ss̆̆ in Huaiyüan H., Knangsi.

4349 Mei-cli'i | 溪.

$1^{\circ}$ Ss $\breve{~ i n ~ A n-c h i ~ H ., ~ C h e l ı-~}$ kiang.

$2^{\circ}$ See No. 4451.

4350 Mei-ch'iu | 邱, Sui C., chên in Hupeh.

4351 Mei-cluu | 渚, ss̆̌ in Clienp'ing H., Anluni. 
梅 MEI-CH'UAN.

4352 Mei-ch' ${ }^{6}$ an | 川, posttown near Knang-cini H., Hupeli.

4353 Mei-hni | 迴, (obs.) in Tai C., Shansi ; Kin, chên, in Tai 代 $\mathrm{C}$.

4354 Mei-11sin | 心, post-tow1 near Slıu-ch' ên H., Anhui.

4355 Mei-lin | 林.

$1^{\circ}$ Chên near Ning-kuo Fu, Anlui.

$2^{\circ}$ Chên near $\mathrm{Ch}^{\prime}$ ang-clion Fu, Kiangsu.

4356 Mei-shan, | U, see No. 66. 4357 Mei-yen | 堤, chên near Wu-chiang J., Kiangsu.

4358 Mei-ying|埂, chên, near Ch'ih-chou Fit, Anlini.

4359 Mei-yün | 园, ssï near Ch'ang-sha Fin, Hunan.

\section{美}

$4360 \mathrm{Mei}-1 \mathrm{i}$ 美 里, town in Lin1-ch 'in Islands.

4361 Mei-tı1-kon | 都湛, T. S. near Ta-t' ung H., Kansuh.

4362 Mei-yang | 陽, see Nos. 1817, 7076.

4363 Mei-yüan | 原.

$1^{\circ}$ Chên near P'u-ch' êng H., Shensi.

$2^{\circ}$ (obs.) N. E. of Fit-p'ing

$\because$ H., Shensi; $T^{\prime}$ ang, Sung and Kin, Meiyüan | 原 $\mathrm{H}$.

$3^{\circ}$ See No. 1855.
留

\section{MHI.}

\section{味}

4364 NIei 味味, See Nos. 1452, 4609.

\section{眉}

4365 Mei 置, Mei, C. I. C. in [307] the Chien-ch ang-Shangnan circ., Ssŭ-cl' 'nan; Iat. $30^{\circ}$ 06' $^{\prime}$, Long. $103^{\circ} 52^{\prime}$; Ts'in， in Shu 蜀 chiün; Han, in Chien-wei 犍篇 chiun; Liang, Ch'ing 青 C.; Sui, Mei-shan|山; T"ang, Clia 嘉 C.; Sung, Mei $\mid \mathrm{C}$.

4366 Nei-1i 筸里, see No. 4412. 4367 Mei-slan / 山, see Nos. 705,4365 .

\section{涺}

4368 Mei-t'an | 㴮潭, Mei-tan, $H$. in P'ing-yüelı C., Kueichon; Lat. $27^{\circ} 40^{\prime}$, Long. $108^{\circ}$.

\section{郡}

4369 Mei 詞 Mei.

[498] $1^{\circ}$ H. in Fêng-hisiang Fin, Shensi; I at. $34^{\circ}$ $13^{\prime}$, Long. $107^{\circ}, 50^{\prime}$; 2nd. Wei, P'ing-yang 平陽: Sui, Mrei-ch' êng 城; T'ang, Hsïn 船 C.; Sung, Mei | H.

$2^{\circ}$ (obs.) N. E. of the above; Han, Tsin and Sui, H. in Fut-fêng 扶 風 chiun. 
眉 MEI-CH' HNG.

$3^{\circ}$ (obs.) near Hsiangyang Fu, Hupelı; $S$. Sung and $S$. TS $i, \mathrm{H}$. in F'n-fêng 扶風 chïn. $4^{\circ}$ (obs.) in Nan-chêng H., Shen-si ; S. Sung and $S . T s i, \mathrm{H}$. in Fufêng 扶風 chïn。

4370 Mei-ch'êng | 城, see No. 4369.

泍

4371 Mei-yi 沫邑, see No. 616.

Mei-tan, see No. 4368.

Meli, see No. 4412.

\section{MÊN}

PI

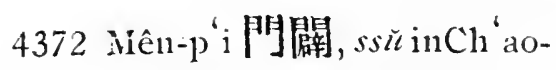
yaug $H_{\text {, }}$ Knangtung.

\section{MÊNG}

觜

4373 Mêng 蒙.

$1^{\circ}$ (obs.) 22 li N. E. of Shang-ch'in H., Honan; Han and Tsin, H. in K. I,iang 梁.

$2^{\circ}$ (obs.) 200 li $\mathrm{s}$. of Shou C., Anluni; $S$. Sung, S. T's $i$, and $N$. IV i, H. in Liang 梁, chiën.

$3^{\circ}$ (obs.) 3 li N. W. of Mêng-ch'êng H. Anhui;
蒙 MÊNG-HUA.

S. Sung, H. in Ch'iao. 譙 chïn; N. Wei, chün and $\mathrm{H}$. in $\mathrm{Ch}^{\text {'iao }}$ 譙 CHOL.

$4^{\circ}$ (obs.) in Kuangtung: $S . T s^{6} i, \mathrm{H}$. in Yungning 永要 chïn.

$5^{\circ}$ (obs.) N. E. of Shang-

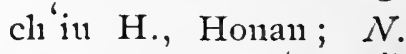
Wei, H. in $\mathrm{Cl}^{6}$ iao 譙 chiin.

$6^{\circ}$ (obs.) near Ch'u C., Anlnui ; N. Wei, H. in Nan-liang 南梁 chün. $7^{\circ}$ (obs.) N. of Yung-an 永 娄 C., Kuangsi ; 7 ang; C. in Ling-nan 領南 circ.

$8^{\circ}$ (obs.) S. A., town in Kiangsu.

4374 Mêng-chêng | 城 Nungching.

$1^{\circ} \mathrm{H}$. in Ying-chon Fit, Anluni ; Lat. $33^{\circ} 23^{\prime}$, Long. $116^{\circ} 38^{\prime}$; Han, Shan-sang U桑; Wei, Kê-yang 渦 晹; Sui, Fei-shui 浿水; $F^{\prime}$ ang, Mêng-ch' êng | 城.

$2^{\circ}$ Post-town near $T^{\prime}$ aip'ing H., Shansi.

4375 Meng-hua / 化 Mung-hwa. $1^{\circ}$ C. I. T. in the Yi-hsi [530] circ., Yünnan; I at $25^{\circ}$ $18^{\prime}$, Long. $100^{\circ} 30^{\prime}$; Han, in $\mathrm{Yi}$ 䀂 $\mathrm{CHOU}$; 
蒙 MÊNG-KÊ-CHIEH.

$T^{\prime}$ ang, in 1st, Yao 姚 C., 2nd, Yang-kua 陽 瓜 chün; Yüan, Mênghua |化; Yüan, Mênghua | 化 Fu.

$2^{\circ}$ T. F. near the above. 4376 Mêng-kê-chieh | 荌結, T-S. near Sung-p'an T., Ssŭch 'uan.

4377 Mêng-lai | 萊, (obs.) in Yünñan ; Yüan, circ.,

$4377^{\mathrm{a}}$ Mêng-li | 里, Y. near Yung-shun H., Kuangsi.

4378 Mêng-shan | 山, see Nos. $4462,7213$.

$4378^{\mathrm{a}}$ Mêng-shih | 氏, see No. 7813.

4379 Mêng-tsê | 澤.

$1^{\circ}$ (obs.) T'an.g, H., W. of Hêng C., Knangsi.

$2^{\circ}$ See No. 6537.

4380 Mêng-tzŭ | 自 Mung-tsz', [11] H. in Lin-an Fu, Yünnan; Lat. $23^{\circ} 34^{\prime}$, Long. $103^{\circ}$ $36^{\prime}$; Yüan, Mêns-tzŭ | 自. 4381 Mêng-yin | 陰 Mung-yin, [484] H. in vi-chou Fu, Shantung; Lat. $35^{\circ} 53^{\prime}$, Long. $118^{\circ} 08^{\prime}$; orig.., Chuan-yü 顓鬼; Han, Mêng-yin | 陰.

\section{㴚}

4382 Mêuy 濛, see No. 50'6.

4383 Mêing-chih| 池 (obs.) near Turfan ; T"ang, $\mathrm{F}_{\mathbf{1 1}}$ in l,ung-yu 龍右 circ.
III. MT̂:NG-MAI.

4384 Mêng-li | 裹, sš̆ in Cli 'üchiang H., Kuangtung.

\section{III.}

4385 Mêng 率 Mung, H. in [148] Huai-ch'ing Fin, Honan; I.at. $34^{\circ} 55^{\prime}$, Long. $112^{\circ}$ $50^{\prime}$; Chou, Mêng-ching | 津; Han, Ho-vang 河隄 ; T'ang, Mêng | C.

4386 Mêng-ch'êng | 城, posttown near Kao-yu C., Kiangsu.

4387 Mêng-chia | 蒙.

$1^{\circ}$ Chên near Chung-pu H., Shensi.

$2^{\circ} \mathrm{Y}$. near Chia-chiang $\mathrm{H}$. Ssŭch' tran.

43Ss Mêng-ching | 津 Mungtsin.

$1^{\circ}$ H. in Ho-nan Fu, Ho11an ; Lat. $34^{\circ} 52^{\prime}$, I.ong. $112^{\circ} 38^{\prime}$; Han, Ho-yang 河陽; T'ang, Ho-ch'ing 河清, Kin, Mêng-ching | 津. $2^{\circ}$ See No. 4385.

4389 IIêng-pa | 埧, chên near Chên-ỹüan H. Kansuh.

4390 Mêng-lun | 倫, (obs.) in Yünnan; Ming, ssŭ.

4.391 Mêng-ma | 麻, post-town near $C_{1}{ }^{\text {iì-ching }} \mathrm{F}_{\mathrm{t}}, \mathrm{Y}$ ïnnan.

4392 Mêns-mai | 筫 Bombay. 
猛 MÂNG-MÂN.

4393 Mêng-nuên | 师, (obs.) $120 \mathrm{li}$ W. of Yung-ning C., Shansi; Kin, H. in Shih 不 $\mathrm{C}$.

4394 Mêng-shan | 山 H. in P'ing-an 平安 circ, Corea.

4395 Mêng-shu | 蜀, see No. 4946.

4396 Mêng-ting | 定, T. F., [321] W. of Chên-yïan T., Yünnan; Lat. $23^{\circ} 37^{\prime}$, Long. $99^{\circ} 14^{\prime}$.

\section{猛.}

4397 Mêng-ling 猛陵.

$1^{\circ}$ (obs.) Han, H., W. of Wu-cliou Fu, Kuangsi.

$2^{\circ}$ See No. 5178.

4398 Mêng-mêng | 猛, T-S. near Wei-yuan T., Yünnan.

4399 Mêng-mien | 緬 Mung[321] mien, or Mien-ning 緬 急, T. in Shun-11ing Fu, Yünnan; Lat. $24^{\circ}$., Long. $100^{\circ} 40^{\prime}$.

4400 Mêng-t'tung | 統, ss̆̈ in Ching-tung 'T., Yünnan.

4401 Mêng-yang | 養, T-S., S. of Chên-yüan 'T., Yünnan.

\section{艋}

4402 Mêng-cliia 艋 舣, ssu in N. Formosa, generally known, from the local pronunciation of the name, as Banka, or Bangka ;
MI.

it is close to ' ' '“ai-pei 臺 北 Fu. [No. 6191].

批

4403 Nêng 邼, see No. 4033.

Menia, see No. 171.

Merguen, see No. 4488.

Meru, or Merv, see No. 4263.

\section{MI \\ 应}

4404 Mi.縻, (obs.) N. W. ef Tayao H., Yünnan; $T^{6}$ ang, C. in Chien-nan 劍南 circ.; also written 犘.

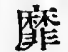

4405 Mi 靡, see No. 4404.

\section{彌}

$4406 \mathrm{Mi}-\mathrm{le}$ 彌勒 Mi-lih, H. in [355] Kuang-lısi C., Yünnan ; I at. $24^{\circ} 40^{\prime}$, Long. $103^{\circ}$ $32^{\prime}$; Yïan Mi-lê | 勒 C. 4407 Mi-niu| 牛, chên near Chin-t ang H. Ssŭch 'uan. 4408 Mi-tu | 渡, post-town near Mêng-hua T. Yünnan.

\section{米}

4409 Mi 米.

$1^{\circ}$ Chén near Kao-p'ing H., Shausi.

$2^{\circ}$ (obs.) Sung, C., N. of

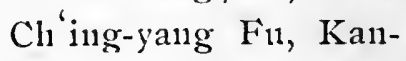
suls. 
密

MI-CHIH.

$3^{\circ}$ See No. 3027.

4410 Mi-chil | 脂 Mi-chi, H. [83] in Sui-tê C., Shensi; I,at. $37^{\circ} 52^{\prime}$, I.ong. $110^{\circ}$; Han, in Shang 上 chün; Sung and Kin, military post in Sui-tê紾德 C.; Yiüan and Ming, H. in Sui-tê C.

4411 Mi-la-kou | 喇渄, T-S. near Nien-po H., Kansuh. $4412 \mathrm{Mi}-1 \mathrm{l}$ |里 (or Mei-li 首 里) Meli, region W. of Mien-ning H., Ssŭch'nan. 4413 Mi-ssŭ-êrlı | 思耳, see No. 4415.

\section{迷}

4414 Mi-k'nn迷昆, see No.5774. 4415 Mi-ssŭ-êrh | 思耳 or 米 思 T羊, or Mi-hsi-êrh 密 苩兒, Misraim, i.e. Egypt. 4416 Mi-yi-so| 易所, T-S. near Hui-li C., Ssŭch' nan.

\section{臂}

$4417 \mathrm{Mi}$ 籍.

$1^{\circ}$ (obs.) S.A., K. comprising parts of Hupeh and Hnnan.

$2^{\circ}$ See Nos. 7615, 7820 .

\section{密}

4418 Mi 密 Mil.

$1^{\circ} \mathrm{H}$. in $\mathrm{K}^{6}$ ai-fêng $\mathrm{Fu}$, [405] Honan; Lat. $34^{\circ} 34^{\prime}$, Long. $113^{\circ} 27^{\prime}$; orig., K. Mi $|; \operatorname{Han}, \mathrm{Mi}| \mathrm{H}$.
㾰 MiaO-SHaN.

$2^{\circ}$ (obs.) formerly K. in Honan.

$3^{\circ}$ See No. 1374.

4419 Mi-hsi-êrh | 羔兒, see No. 4415.

4420 Mi-kung | 茶, (obs.) S. IV. of T'ao-chon T., Kansuh ; $T^{6}$ ang, H. in Lung-y'11 隴石 circ.

4421 Mi-11sü | 須, see No.3970, 4422 Mi-yang | 陽 Mih-yang, Fu in Ch'ing-shang 慶省 circ., Corea; I at. $35^{\circ} 12^{\prime}$ I,ong. $129^{\circ} 25^{\prime}$.

4423 Mi-yün 密雲 Milh-yun.

$1^{\circ} \mathrm{H}$. in Shun-t'ien $\mathrm{Fu}$, [337] Chilhli; Iat. $40^{\circ} 24^{\prime}$, Long. $116^{\circ} 45^{\prime} ; T^{\prime}$ ' in and Han, Yü-yang 漁 陽; Wei, Mì-yün 尝; Sui, T’an 檀 C.

$2^{\circ}$ (obs.) $70 l i \mathrm{~N}$. E. of the above; $N$. $W_{c i}$, chïn in An 安 chov.

Mi-chi, see No. 4410. Mi-lih, see No. 4406.

\section{MIAO}

\section{廟}

4424 Miao-ch'ien 廟前, post town near Jung-ho H., Shansi.

4425 Miao-shan | I ssŭ in Y'üyao H., Clielıkiang. 
苗 MIAO-TZŬ-CHIAO.

4426 Miao-tzŭ-chiao|子角 $s s \breve{t}$ in Lung-nên H., Kuangtung.

4427 Miao-wan | 灣.

$1^{\circ}$ Chên in Ju-yang H., Honan.

$2^{\circ} \mathrm{Y}$. near Fu-ning $\mathrm{H}$. Kiangsu.

\section{获}

4428 Miao-min 苗民, (obs.) in Kueichou；Ming， ssŭ in Shih-ch'ien 石阶 Fu.

4429 Miao-shêng | 勝, Y. near P'ei C., Kiangsu.

\section{MIEN}

\section{緬}

4430 Mien-11ing 緬㛹, see No. 4399.

\section{曻}

4431 Mien-ning 得窎 Mien[6] ning, H. in Ning-yüan Fu, Ssŭch'11an; Lat. $28^{\circ}$ $30^{\prime}$, Long. $102^{\circ} 20^{\prime}$.

4432 Mien-shan | II, Y. near the preceding.

\section{綿}

[N. B. this character and the next, 跞, are frequently interchanged.]

4433 Mien 綿 Mien, C.I.C. in [125] the Ch'êng-Lung-T'ungMien-Mon circ., Ssŭch'uan; Lat. $31^{\circ} 28^{\prime}$, Itong. $104^{\circ} 53^{\prime}$; Han,
綿 MIEN-SSŬ.

Fou 涪 H.; W. Wei, 'T'ung 潼 C.; S. Sung and $S . T s^{\prime} i$, Pa-lisi, 巴 西 chïn and 'Tzŭ-t'ung 梓境 chiun; N. Wei, Tzŭ-t"ung 梓潼 chïn ; Sui, Mien $\mid \mathrm{C}$. and $\mathrm{Pa}$ hsi 巴侮 H.; $T^{\prime}$ ang and Sung, Pa-hsi 巴西 H.

4434 Mien-chu|諳, (obs.) 45 li E. of $\mathrm{Ch}^{\prime}$ in 秦, C., Kansulı; $W$. Han, circ. in $\mathrm{T}^{6}$ ien-shui 天水 chïn; $N$. Wei, H. in Lüelnyang 略㸞chïn.

4435 Mien-chu | 竹 Mien-chuh, H. in Mien C., Ssŭch 'uan; Lat. $31^{\circ} 17^{\prime}$, Long. $103^{\circ}$ $16^{\prime}$; Han, Mien-ch11 | 竹; S. Sung, Chin-hssi 晉西 chiin and $\mathrm{H}$. in $\mathrm{Yi}$ 益 chou; S. Ts'i, Chin-hsi 㕕西 H. in S. Hsin-pa 新巴chiun。

4436 Mien-kn | 谷, see No. 3429.

4437 Mien-shang | 上, chên near Ch' in-yüan H., Shansi.

4438 Mien-shui | 水, (obs.) Wei, H., S.E. of P'êngshan H., Ssŭch' uan.

4439 Mien-ssŭ | 虍, or 繫虎. $1^{\circ}$ (obs.) W. of Min ch' '1an H., Ssŭch' 11an; W. Han, H. in Shu 
罢 chïn; E. Han, circ. in Shu 蜀 chïn.

$2^{\circ}$ See No. $4455 .^{B}$

\section{緜}

[N. B. this character and the last, 綿 are frequently interchanged.]

4440 Mien 㨇, (obs.) S. Sung, C. in Ch'êng-tu 成 都 circ.

4441 Mien-ssŭ | 虎, see No. 4439.

\section{泟}

[N. B. this character and 䥺, are frequently interchanged.]

4442 Mien 活 Mien.

$1^{\circ} \mathrm{H}$. in Han-clunng $\mathrm{Fu}$,

[133] Sliensi; Lat. $33^{\circ} 05^{\prime}$, Long. $106^{\circ} 45^{\prime}$; Han, Mien-yang | 陽; 2nd, $W e i$, Po-chung 嶓冢; $W$. Wci, Hsing 興 C. Sui, Hsi 西 H.; $S$. Sung.Mien $\mathrm{C}$.

$2^{\circ}$ See No. 1967, 4445.

4443 Mien-ch'ih | 池, see No. 4447.

4444 Mien-ch'nan | Chïn in Chung-ch'ing 忠 清 circ, Corea.

Mien-yang|陽 Mien-yang.

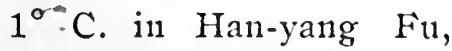
Hupeh ; Lat. $30^{\circ} 12^{\prime}$, Long. $113^{\circ} 12^{\prime}$; Han,
Ching-ling竞陵; Liang; Mien-yang | 陽; Sui, Mien $\mid \mathrm{C}$.

$2^{\circ}$ See No. 4442 .

䏍

4446 Mien-ch'in 把池 see No. 4447.

\section{泟}

[N. B. this character and 涉, are frequently interclianged.]

4447 Mien-ch'in 铚池 Minchi, H. in Honan Fu, Honan; Lat. $34^{\circ} 46^{\prime}$, I, ong. $111^{\circ} 41^{\prime}$; Han, and $T \sin$, Mien-ch'in 苞池 H.; $N$. Wei, Mien-ch' ih I池 chün; Kin, Shao 䍄 C. and Miencl! ih 泟 池 H.; $T^{6}$ ang, Sung, and Ming, Miench'il | 池 H.; Yüan,

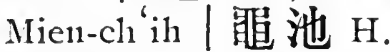
See also No. 590.

Mien-chuh, see No. 4435. Mih, see No. 4418.

Milh-yang, see No. 4422. Mih-yun, see No. 4423.

\section{MIN}

\section{閩}

4448 Min 閩 Min.

$1^{\circ} \mathrm{H}$. forming with Hour[306] kuan $H$. the prefectural 
壁

city of Fin-chou, Fuhkien ; Lat. $26^{\circ} 03^{\prime}$, Long. $119^{\circ} 05^{\prime}$; Han, Tung-yel 東治; Tsin, Yüan-fêng 原䇺; Sung, Yïan and Ming, Min H. $2^{\circ}$ (obs.) N. E. of the above; Sui, H. in Chienan 建努, chiin; $T^{\prime}$ ang, H. in F 11 融 C.

$3^{\circ}$ (obs.) Chou, chiün connprising the greater part of Fuhkien.

$4^{\circ}$ See Nos. 256, 1867, 1871 .

4449 Min-an | 岁, chên near

Fu-chou Fu, Ful1kien.

4450 Min-Chê | 浙 Min-Cheh, collective name for the two provinces of Fuhkien and Chehkiang.

4451 Min-ch 'ing / 清 Min-tsing, [306] H. in Fu-chou Fu, Fuhkien; Lat. $26^{\circ} 13^{\prime}$, Long. $119^{\circ}$ $02^{\prime}$; orig., Hou-kuan 侯 官; T'ang，Mei-ch'i 梅 溪 H., Five dyn., Minch'ing 清 H. Unken (Polo).

4452 Min-chung | | Ts'in, Min-chung | 中 chün; see Nos. 1871, 6185.

毁

4453 Min-1nsing 閔 行, chên near Chin-shan H., Kiangsu.

\section{MiN.}

\section{解}

4454 Min-ni-ya-k'ê 敏 你 雅 克, see No. 171.

\section{汶}

4455 Min 汶, (obs.) Tang and $P$. Chou, C. near Shihch'üan H., Ssŭch' uan.

$4455^{\mathrm{A}}$ Min-chiang | it.

$1^{\circ}$ (obs.) W. Han, H. 50 li N. of Mon C., Ssŭch"nan.

$2^{\circ}$ See No. 45 .

$4455^{\mathrm{B}}$ Min-ch' nan | 川 Wăn[307] chuen, H. in Mon C., Ssŭch 'lian; Lat. $31^{\circ} 22^{\prime}$, Long. $103^{\circ} 36^{\prime}$; Han, Mienssŭ 綿虎; T T sin, Min$\mathrm{cll}^{\prime}$ nanl 川.

$4455^{\mathrm{c}}$ Nin-tung | 東, (obs.) T'ang, C. near Ya-chou $\mathrm{Fu}$, Ssŭch 'uan.

\section{民}

4456 Min 民; S. Ts $s^{6} i$, Lill-min 流| H. is sometimes erroneonsly so written.

$$
\text { 崕 }
$$

4457 Min 即 Min, C. in Kung[455] ch'ang Fu, Kansuh; Lat. $34^{\circ} 24^{\prime}$, Long. $103^{\circ} 58^{\prime}$; orig., Yi 溢, land of the Hsi-ch'iang 西 恙 tribes; $T s^{\prime}$ in, in Lin-t'ao 臨 洮 chïn; Wei, Sui, T'ang and Yïan, Min | C.; Sung, $\operatorname{Min} \mid \mathrm{C}$., and Hsi-ho 酉 和 H.; Ming, Min-chou | 州 inilitary district. 
峎

Min-chov.

4458 Min-chou | 州, see the preceding.

\section{悢}

4459 Min 紙, (obs.) S. A. town in K. Sung 㷊, S. E. of Kao-p'ing H., Shansi.

Min-cheh, see No 4450.

Min-clii, see No. 4447.

Min-tsing, see No. 4451.

\section{MING}

4460 Ming-hu 名護, town in the Liu-ch' in Is.

4461 Ming-ku Wu | 古屋 Nagoya, chief town, Aichi ken, Japan.

4462 Ming-shan | II Ming-shan, [72] H. in Ya-chou Fu, Ssŭclınan; Lat. $30^{\circ} 10^{\prime}$ Long. $103^{\circ} 11^{\prime} ;$ E. Han, Hanchia 漢嘉; Wei, Mêngshan 蒙徜; Sui, Mingshan | II.

\section{若}

4463 Ming 䒚 T. C. in Kuangsi. 4464 Ming-ying | 盈 Ning-ying, [507] T. C. in T'ai-p'ing Fu, Knangsi ; Lat. $23^{\circ} 10^{\prime}$, Long. $107^{\circ} 05^{\prime}$; Sung, Ming-ying | 感 C.

\section{洛}

4465 Ming 洛, see No. 3413. 4466 Ming-shui | 水, see No. 6950.
鳴 MING-T'IAO.

\section{明}

4467 Ming 明, see No. 4702 . 4468 Ming-a-t'ê | 阿 特 (or Ming-kê-t'ê | 噶特) Minigad, Mongol Tribe.

4469 Ming-anl 努: Ming-an, town in Dsassaktu; Lat. $44^{\circ} 20^{\prime}$, Long. $96^{\circ} 55^{\prime}$; there is also a town of the same name in Outer Kansuh, to the E. of Hami.

4470 Ming-chêng | 正, T-S. near Ta-chien-lı ' $\mathrm{T}$., Ssŭch' uan.

4471 Ming-chiang | 港, posttown near Hsin-yang C., Honan.

4472 Ming-chiang | 江 Mingkiang, T. in T'ai-p'ing Fu, Kuangsi; Lat. $23^{\circ} 15^{\prime}$, Long. $106^{\circ} 40^{\prime}$.

4473 Ming-ch' uan | 川 Fu in Hsien-ching 咸 鏡 circ., Corea.

$$
\text { 鳴 }
$$

4474 Ming-chien 鳴 溗, posttown near Ta-ning $\mathrm{H}$, Shansi.

4475 Ming-ho-ch'êng | 鶴城, (obs.) formerly fort, W. of 'Ti-tao C., Kansuh.

4476 Ming-lı| 鹿, see No. 4124. 4477 Ming-sha|沙, (obs.) E. of Ning-hsia Fu, Kansuh.

4478 Ming-t ‘iao | 條, (obs.) Hsia, a royal residence near An-yi H., Shansi. 
鳴

Ming-TU.

4479 Ning-tu | 犢, chén S. E. of Hsien-ning H., Shensi.

\section{冥}

4480 Ming-an 冥安, (obs.) E. of An C., Kansuh; Han and $T \sin , \mathrm{H}$. in Tun-hruang 敦 煌 chiinn.

Milig-kiang, see No. 4472. Minigad, see No. 4468. Miniak, see No. 171. Mito, see No. 5798. Miyag, see No. 3811. Miyanoshita see No. 3525 . Miyé, see Noo. 5368.

\section{MO}

\section{徝}

4481 Mo-hsiang 貃貌 ssŭ in $\mathrm{Cl}^{6}$ ung-ming $\mathrm{H}$., Kiangsu.

\section{摩}

4482 No-ch'u摩舅, see No. 4558.

4483 Mo-11i | 尼, (obs.) in Kneichon; Ming, so in

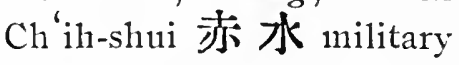
district, Kueichou.

\section{磨}

4484 Mo-hsia 磨下, T-S. near Sung-p'an T., Ssŭch' 'uan. 4485 Mo-p'an | 盤.

$1^{\circ}$ Chên near Sung-tzŭ H., Hupeh.
秝

MO-LI.

$2^{\circ}$ Ss ̌̆ in Yi-t'ung C., Kirin.

\section{沒}

4486 Mo-kou, 沒溝, (or Yingtzŭ 營子), Y. near Haich'êng H., Shingking; Lat. $40^{\circ} 40^{\prime}$, Long. $122^{\circ}$ $14^{\prime}$; residence of the Subprefect of Ying-k'ou 營 口; here is situated the foreign settlement of the open port Newchwang; the town of Niu-chuang-ch êng 牛生城, which gives its name to the port, is situated fartleer inland in Lat. $40^{\circ}$ $53^{\prime}$, Long. $122^{\circ} 07^{\prime}$.

\section{墨}

4487 Mo-chu1-kung 墨竹工 Medjugung, town in Thibet: Lat. $29^{\circ} 50^{\prime}$, Long. $93^{\circ}$.

4788 Mo-êrh-kên | 尔 根 Merguen, town in Heh-lungkiang; Lat. $49^{\circ} 16^{\prime}$, Long. $124^{\circ} 40^{\prime}$.

\section{秝}

4489 Mo-ling 秣 陵.

$1^{\circ}$ Chên in Chiang-ning Fu, Kiangsu.

$2^{\circ}$ See Nos. 749, 2596, 5548.

\section{莫}

4490 Mo-li 離, chên near L.och'êng H., Kuangsi. 
鄚

MO-CH' ÊNG.

\section{鄚}

4491 Mo-ch'êng 鄚 城, posttown near Jên-ch' 'in H., Chilıli.

Momein, see No. 7183.

Morioka, see No. 5645.

\section{MOU}

\section{车}

4492 Moul 卒.

$1^{\circ}$ (obs.) S.A., K. in Shantung.

$2^{\circ}$ See Nos. 1546, 4682, 6330.

4493 Mou-p'ing | 平, see No. 4682.

4494 Mou-shan | 山, see No. 56. 4495 Mou-t'o | 托, 'T-S. near Mou C., Ssŭch' "uan.

\section{歓}

4496 Mou-kung 禁功 Mankung.

$1^{\circ}$ C. I. 'T. in Ssŭch' 'uan; Lat. $31^{\circ} 38^{\prime}$, Long. $103^{\circ} .54^{\prime}$.

$2^{\circ} \mathrm{Y}$. near Ta-yi H., Ssŭch' uan.

\section{䰑。}

4497 Mou 䯹, (obs.) in Ta-yao H.; Yïnnan; T⿱ang C. in Chien-11an 劍南 circ., see also No. 6462.
莫陪 Moussour.

\section{茂}

4498 Mou 茂 Mau，C. L. C. in [31)7] the Ch'êng-Lung-T' 'ungMien-Mon circ., Sstŭch 'tuan; Lat. $31^{\circ} 40^{\prime}$, Long. $104^{\circ}$ 15', Han, Wên-shan 文 山; Liang, Shêng 秥 C.; Sui, Hui 會 C.; $T^{6}$ ang Non $\mid \mathrm{C}$.

4499 Mou-ch'ang | 長 Mauchang, $\mathrm{H}$. in $\mathrm{Cl}^{\text {'üan-lo }}$ 丕羅 circ., Corea; Lat. $34^{\circ} 52^{\prime}$, Long. $127^{\circ} 21^{\prime}$.

4500 Mon-ch'i | 溪 H., in

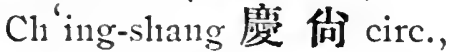
Corea.

4501 Mon1-chu|朱 H. in Ch'üanlo 全羅 circ., Corea.

4502 Mout-lin | 林, chên near Wu-kung H., Shensi.

4503 Mout-ling | 陵, see No. 2829.

4504 Moun-ming, | 名 Nau-ming, $\mathrm{H}$. forming the prefectural city of Kao-chou Fu, Kuangtung; I at. $21^{\circ} 48^{\prime}$, Long. $110^{\circ} 26^{\prime} ; T^{6} \sin$, Mol1-ming | 名; T'ang, P'an 潘 C.; Fine dy'n., Y'üel-shang 越堂。

4505 Mou Mingan | 明安 Mow Mingan, Mongol Tribe.

Monkden, see No. 1773.

Moussoul, see No. 4335. 
田

Mow-MINGAN.

Mow Mingan, See No. 4505.

\section{母}

4506 Mu-hisiang | 享, ss̆̆ near Chên-hsiung C., Yünnan. 4507 Mu-tsu-wa | 足正, T-S. near Mien-ning H., Ssŭch' nan.

\section{茬}

4508 Mu-hsia 姥下, chên near Ho C., Anhui.

\section{慕}

4509 Mu-hua 慕化, see No. $7862,3^{\mathrm{A}}$.

4510 Mu-jung | 谷, (obs.) formerly town $\mathrm{E}$. of $\mathrm{L}$ iichiang H., Anluui.

4511 Mu-tê | 德 ssŭ in P'anyï H., Kuangtung.

4512 Mu-yi | 役 ssŭ in Yungning C., Yünnan.

\section{募}

4513 Mu-fu 募復, ss̆̌ near Yung-ning C., Keichou.

眭

4514 Mu 睦, see No. 7394.

4515 Mun-ts'un | 村, chên near Hsien-yang H., Shensi.

\section{穆}

4516 Mu-hsi 穆喜, post-tow11 near Fêng-t'ien Fin, Shingking.

\section{木 \\ MUU-LEI.}

4517 Mu-ling-kuan | 陵關, ss̆ near Lin-ch 'ü H., Shantung.

4518 Mu-p'ing|坪, see No. 4529.

4519 Mu-to / 委, T-S. in Kokonor.

4520 Mulyang | 洋, chên, near Fu-an H., Fuhkien.

\section{木}

4521 Mu-ch'nan 木川.

$1^{\circ}$ 'T-S. near Chien-wei H., Ssŭch 'uan.

$2^{\circ}$ H. in Chung-ch'ing 忠

清 circ., Corea.

4522 Mu-jên | 仁, (obs.) near Tung-o H., Shantung; Kinn, chên in Tung-p'ing 東平 Fu。

4523 Mu-kua | 瓜.

$1^{\circ} S$ š̆ near Ch'ang-chai T., Knei-chou.

$2^{\circ}$ T-S. in Ting-fan C.

Knei-chou.

4524 Mu-lai | 來, (obs.) in Yün11an ; Yüan, Mu-lai | 來 $\mathrm{H}$.

4525 Mutlan | 闌.

$1^{\circ}$ Post-town near $\mathrm{Ch}^{6}$ êng H., Kansul.

$2^{\circ} \mathrm{H}$. Ho-lan $\mathrm{F} u$, Tsitsihar. $3^{\circ}$ See No. 2289.

4526 Mu-lei | 壘 Murui, town in The New Dominion and included in the Chên-Ti circ.; Lat. $44^{\circ} 35^{\prime}$, Long. $91^{\circ} 50^{\prime}$. 
木

Mu-Pang.

4527 Mu-pang | 邦, (obs.) in Yünnan; Yüan, circ.; Ming, ssŭ.

4528 Mu-p'ing-ch'êng | 本城, ssü near Tien-pai H., Kuangtung.

4529 Mun-p'ing | 坪, or Mu1p'ing 穆坪, 'T-S. near T'ien-ch ‘üan H., Ssŭcl' 'uan, called in Thibetan Djumba [Baber].

4530 Mu-po / 波 (obs.) Kin, chên S. of Huan H., Kansuh.

4531 Min-po | 錸.

$1^{\circ}$ Chên near Huan H., Kansuh.

$2^{\circ}$ Chên near Yunus-ning C., Shansi.

4532 Mu-shu | Jit, T-S. near Mien-ning H., Ssŭch' nan.

4533 Mu-ts'un | 村, T-S. near Yin-chiang H., Kueichon.

4534 Mut-tu | 瀆, chên near Suclon Fu, Kiangsu.

4535 Mu-tung | 洞 chên in Pa H., $\mathrm{Ss}^{\breve{\mathrm{l}}} \mathrm{ch}$ 'uan.

\section{湫}

$4536 \mathrm{Mu}$ 沭, (obs.) T'ang; C. near O-mei H., Ssŭclı nan.

Muang-la, see No. 5866. Muang-shan, see No. 5819. Mung, see No. 4385.
那 NA-PA.

Mung-ching, see No. 4374. Mung-liwa, see No. 4375. Mung-mien, see No. 4399. Mung-tsin, see No. 4388. Mung-tsz', see No. 4380. Nung-yin, see No. 4381. Murui, see No. 4526. Mwan-cling, see No. 6326.

\section{NA}

\section{那}

4537 Na-fu 服扶, Y. near Yangchiang ' $\mathrm{I}$., Kuangtung.

45.38 Na-ha-wan-ti | 哈完的 Nahavand, town in Persian Kurdistan, S. E. of Kermanshah.

4539 Na-hei-sha-pu | 黑沙不 Naksheb, town in Independent Turkestan, S. W. of Samarcand; also written Na-sê-po | 色波.

4540 Na-hno | 水, Y.near Tan C., Kuangtung.

4541 Na-la-t'ê | 喇特 Narat, town in Ili, Lat. $43^{\circ}$, Long, $84^{\circ}$; written Naral in Williams's map.

4542 Na-ma | 馬, T-S. near Ssŭ-ên Fu, Kuangsi.

4543 Na-ni | 尼, chên near Michih H., Shensi.

4544 Na-pa | 霸 Na-pa; this name is written by a misprint Naha in Williams's Inap; see No. 4004. 
那

NA-SÊ-PO.

4545 Na-sê-po|色波, see No. 4539.

4546 Na-ti | 地 Na-ti, T. C. in [518] Ch'ing-yüan Fin, Kuangsi; Lat. $24^{\circ} 44^{\prime}$, I,ong. $107^{\circ}$ $12^{\prime} ; T^{\prime}$ ang, Nan1-yao 辢 獾; Sung; Fu孚 C.

\section{拏}

4547 Na-k'on 㛌 口, ssŭ near Shao-wu Fu, Fukien.

\section{納}

$4548 \mathrm{Na}$ 納, (obs.) T'ang, C. near Kıng H., Ssŭch nan.

4549 Na-ch'i | 溪 (or, | 笑谷) [528] Nahki, H. in Lü C., Ssŭch' 'nan; Lat. $28^{\circ} 48^{\prime}$, Long. $105^{\circ} 23^{\prime}$; Han, Cliiangyang 江骝; Sung, Yüan and Ming, Na-chi i 溪 $\mathrm{H}$.

4550 Na-cli'i 笑谷, see preceding.

4551 Na-kê-êrh-tsê | 噶爾澤 Nagarjung, town in Thibet; Lat. $28^{\circ} 40^{\prime}$, Long. $90^{\circ}$ $20^{\prime}$.

4552 Na-lin-ch'ung | 林冲, T-S. near Ta-chien-lu T., Ssŭch' 'uan.

4553 Na-lon | 樓, T-S. near Lin-an Fu, Yiünan.

\section{蒳}

4554 Na-tzı̆-shan 蒳子山, See No. 5131.
南 NAN.

Na-ha, see No. 4544.

Na-pa, or Na-pa-kiang, see No. 4544.

Na-gano, see No. 281.

Nagarjung, see No. 4551.

Nagasaki, see No. 235.

Nah-ki, see No. 4549.

Nahavand, see No. 4538.

\section{NAl}

乃

4555 Nai-sha-pu-êrh 乃沙不耳 (or, Ni-slaa-pu-êrli 你沙 不兒, or, Ni-sla-pu-li 你沙卜里, or, Ni-ch'awu-êrl 医祭匹兒） Nishapur, a city of Persia, province Khorassan.

\section{奈}

4556 Nai-man 奈曼 Naiman, Mongol tribe.

Naiman, See No. 4556.

Naksheb, see No. 4539

Namoa, see No. 4559.

\section{NAN}

\section{南}

4557 Nan1 南.

$1^{\circ}$ Chên near Fu-ting H., Fulkien.

$2^{\circ}$ (obs.) Han, chïn comprising Hupeh; Ts'in, chïn. 
$3^{\circ}$ (obs.) S. Sinng. C. in Fu-chien 福建 circ.

$4^{\circ}$ See Nos. 1142, 5673.

4558 Nan-an | 努 Nan-11gan.

$1^{\circ} \mathrm{Fu}$ in the Chi-Nan-

[50] Kan-Ning circ., Kiangsi ; forming also the district city of Ta-y ï ; Lat. $25^{\circ} 42^{\prime}$, Long. $114^{\circ} 18^{\prime}$; orig., in K. $\mathrm{Wu}$ 吳 and $\mathrm{K} . \mathrm{Ch}^{\prime}$ " 楚; Tsin, in Chinchiang 九 江 chïn; Han, Hêng-p " in Yï-chang 豫 童 chïn; Sui and T"ang, in Ch'ien 虔 C.; Sung, Nan-an | 努 mil. district in Chiang-nan it. 南 W. circ.; Yüan, circ. in Kiangsi ; Ming, Nantan 姿 Fut.

$2^{\circ} \mathrm{C}$. in Ch ${ }^{\prime}$ ll-hsiung $\mathrm{Fu}$, Yïnnan; Lat. $24^{\circ} 58^{\prime}$, Long. $101^{\circ} 45^{\prime}$; orig., Mo-cli'n 摩留; Yïan, and Ming, Nan-an | 安 C.

$3^{\circ} \mathrm{H}$. in $\mathrm{Ch}^{\prime}$ iuan chou $\mathrm{Fu}$, [61] Fulkien; Iat. $25^{\circ} 07^{\prime}$, Long. $118^{\circ} 20^{\prime}$; Han, Hou-kuan 侯 官; Wu, Tung-an 東技; Liang， Nan-an|岁; $T$ 'an. Fêng 嵦 C., and Nan-an 娄 H. $4^{\circ}$ Y. near 'T'êng H., Kuangsi.

$5^{\circ}$ Post-town near Ho-shun H., Shansi.

$6^{\circ}$ Post-town near Hsiangt'an H., Hunan.

$7^{\circ}$ (obs.) 20 li N. W. of Chia-chiang H., Ssŭch'uan; Han, Tsin, S. Sung, and $S . T s^{\prime} i, \mathrm{H}$. in Chien-wei特偁 chün. $8^{\circ}$ (obs.) N. E. of Lunghsi H., Kansuh ; Tsin, chïn in Ch'in 秦 cHOU. $9^{\circ}$ (obs.) W. of Hua-jung H., Hunan; $T \sin , \mathrm{H}$. in Nan-p'ing 南斥 chün。 $10^{\circ}$ (obs.) in Nan-clêng

H., Shensi; S. Sung and $S$. $T s^{\prime} i$, chïn in $\mathrm{Ch}$ in 秦 chou. $11^{\circ}$ (obs.) $50 \mathrm{li} \mathrm{W}$. of Weiyüan $H$., Ssŭclı' "uan; $S$. $T s^{\prime} i$, chïn in Liang 梁 cHOU.

$12^{\circ}$ (obs.) in Kuangtung; S. $T^{i} i, \mathrm{H}$. in Kao-hsing 高與chïn。

$13^{\circ}$ (obs.) in Hupeh; $S$. $T s^{\prime} i, H$. in 1 st, Yi-yang 義陽 chïn, 2nd, $\mathrm{Ch}^{\prime} \mathrm{i}$ an 㔖安 chinn, 3rd, Hsi-hsin-an 西新安 chïn.

$14^{\circ}$ (obs.) S. of Shê H., Honan; $N$. Wei, chiün in Hsiang 裏 cHou. 
南

NAN-AO.

$15^{\circ}$ See. Nos. 867,871 $880,1077,1220,2214$, 2805, 3521, 4590, 4737, 6206.

4559 Na11-ao | 澳 Namoa, or Nan-11ga11.

$1^{\mathrm{c}}$ T. in $\mathrm{Ch}^{6}$ ao-chou $\mathrm{Fu}$, Kuangtung; Lat. $23^{\circ}$ $40^{\prime}$, Long. $117^{\circ} 20^{\prime}$; situated on the Island of Namoa, part of which belongs to Knangtung and part to Fuhkien.

$2^{\circ}$ Chên near Chao-an H., Futhkien.

4560 Nan-ch' a-ching | 平井, chên near Ning C., Kansuh. 4561 Nan1-changl 漳Nan-chang, [290] H. in Hsiang-yang $\mathrm{Fu}$, Hupeh ; Lat $31^{\circ} 47^{\prime}$, Long. $111^{\circ} 42^{\prime}$; Han, I,in-chü 臨 沮; Tsin, Nan-chang |漳; S. Ts $i, H \sin -\mathrm{a} 11$ 新安 $\mathrm{H}$.

4562 Nan-ch'ang | 昌 Nancliang.

$1^{\circ}$ Fu in the Nan-Fu-Chien [403] circ., Kiangsi; forming also the two district cities of Hsin-chien and Nan-ch'ang; Lat. $28^{\circ}$ $33^{\prime}$, Long. $116^{\circ} 01^{\prime}$; capital of the prov.; orig., in $\mathrm{K}$. Wu 吴 and K. Ch' 11 楚; $T s^{\prime} i n$, in Chin-chiang九iLchün;
南 NAN-ChaO.

Han, in Yï-chang 豫 章 chïn; Ts'in, Chiang 江 C.; Sui and T'ang, Hung 洪 C., S. T'ang, Nan-ch'ang | 昌; Sung, Lung-hsing 隆 與; Ming, 1st, Hung-tu 洪 都 Fu, 2nd, Nan-ch' ang 昌 $\mathrm{Fu}$.

$2^{\circ} \mathrm{H}$. forming with Hsinchien $H$. the prefectural city of Nan-ch'ang, Kiangsi ; Lat. $28^{\circ} 33^{\prime}$, Isong. $116^{\circ} 01^{\prime}$; Han, Nan-ch'ang | 莦; Sui, Yü-cliang 豫章.

$3^{\circ}$ (obs.) $T^{6}$ ang, H., S. E. of Po-pai H, Kuangsi.

4563 Nan-chao | 石 Nan-chau, [368] H. in Nan-yang Fu, Honan; Lat. $33^{\circ} 33^{\prime}$, Long. $112^{\circ} 38^{\prime}$, S. $A$., land of $\mathrm{Ch}^{\prime} \mathrm{u}$ 楚; $T$ sin. Chih-ch 'êng 雉 城; Ming, Nan-chao | 召.

4564 Nan-chao | 照, chên near 'T'ai-ho H., Anhui.

4565 Nan-chao | 詔, see Nos. 438, 6049.

$4565^{\mathrm{A}}$ Nan-ch'ên | 陳.

$1^{\circ}$ (cbs.) in Anhui; $S$. Sung, chïn in Nan-yï | 豫 chou; N. Wei, chün and H. in Ho 霍 cHOU.

$2^{\circ}$ (obs.) near Lüi-chou Fu, Auhui; S. Ts'i, chün 
in Nan1-Ju-yin | 汝院 CHOU.

$3^{\circ}$ (obs.) E. of Shangch'ênng H.,Honan; $N$. $W e i$, chïn and $\mathrm{H}$. in Chien 建 chor.

$4^{\circ}$ See No. 2396.

4566 Nan-chêngl 凨 Nan-ching. $1^{\circ} \mathrm{H}$. forming the prefectural city of Hanchung, Shensi ; Lat. $32^{\circ}$ 56', I, ong. $107^{\circ} 12^{\prime}$, $H a n$, Nan-chêng | 觙; $W . W e i, \mathrm{~K}$ uang-yi 光 義; Sung, Hsing-yüan 興 元 $\mathrm{F} \mathfrak{u} ; S$. Sung, Shang-kuei 上䛾 H.; Yüan, Hsing-yüan1 與! 元 circ.

\section{$2^{\circ}$ See No. 2885.}

4567 Nan-chêng | 城 Nan-cling.

$1^{\circ} \mathrm{H}$. forming the prefectural city of Chiench'ang, Kiangsi ; Lat. $28^{\circ} 34^{\prime}$, Long. $118^{\circ} 28^{\prime}$; Han, Nan-ch'êng | 城;

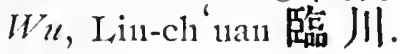

$2^{\circ}$ Post-town near Chi-ning C., Shantung.

$3^{\circ}$ See No. 2730.

$4568^{\mathrm{A}}{ }^{\mathrm{N}}$ Nan1-ch' êng | 呈, chên near Ch' ang-tzŭ H., Shansi. $4568^{\mathrm{B}} \mathrm{Nan1}^{-\mathrm{ch}^{\mathrm{i}} \mathrm{i}}$ | 溪 Nan1-ki.

$1^{\circ 0}$ H. in Hsï-chon $\mathrm{Fu}$,

[528] Ssŭch 'uan; Lat. $28^{\circ} 48^{\prime}$, Long. $105^{\circ} 03^{\prime}$; Han,
南 NAN-CHIN.

Nan-kuang | 廣; Sung, Nan1-ch'i | 溪.

$2^{\circ}$ (obs.) W. of the above;

Sui, H. in Chient-wei

犍篇 chiür.

$3^{\circ}$ See No. 2895.

$4568^{\mathrm{c}}$ Nan-chiang | 江 Nankiang.

$1^{\circ} \mathrm{H}$. in Pao-ning $\mathrm{Ftl}$, [36] Ssŭch 'uan; Lat. $32^{\circ} 20^{\prime}$, Long. $106^{\circ} 55^{\prime}$; orig., K. Pa 巴; T⿱ang, Suti and Sung, Nan-chiang 䧼 江, Ming, Nanchiang | 江. H.

$2^{\circ}$ Y. near Tê-ch'ing C., Knangtung.

4568 Nan1-clı'iao | 橋, chênnear Fêng-lısien H., Kiangsu. $4568^{\mathrm{E}}$ Nan1-ch' iao | 譙, see No. 1397.

$4568^{\mathrm{F}}$ Nan-chien | 润, T.C. near Yung-pei T', Yünnan; Lat. $25^{\circ} 06^{\prime}$, Long. $100^{\circ} 43^{\prime}$. $4568^{\circ}$ Nan1-chien | 簡.

$1^{\circ}$ Ss̆̆ near Mêng-hnua T., Yünnan.

$2^{\circ}$ See No. 6456.

$4568^{\mathrm{H}}$ Nan1-chien | 劍, see No. 7368.

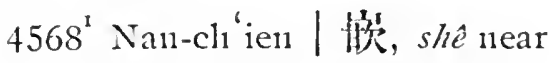
Tan-shui H., Formosa.

4568 Nan-chin | 晉, (obs.) $T^{\prime} a n g$, C. near Nan-ning $\mathrm{Fu}$, Kuangsi. 
南

NAN-CH'IN.

$4568^{\mathrm{K}} \mathrm{Nan1}^{-\mathrm{ch}}{ }^{\mathrm{in}}$ | 秦.

$1^{\circ}$ (obs.) near Hsü-chou Fu, Ssŭch'uan; $T$ sin, S. Sung and S. Ts' $i$, H. in Clii-shil 朱提 chiin.

$2^{\circ}$ See No. 503.

$4568^{\mathrm{L}}$ Nan-cling | 京, see Nos. $511,749$.

$4568^{\mathrm{Mr}}$ Nan-ching 靖 Nant-tsing,

[272] H. in Chang-chon Fu, Fullkien ; Lat. $24^{\circ} 38^{\prime}$, I,ong. $117^{\circ} 25^{\prime}$.

$4568^{N}$ Nan-cling | 靜, see No. 3060.

$4568^{\circ}$ Nan-ching | 津.

$1^{\circ}$ Post-town near Tzŭyang H., Ssǔch'uan.

$2^{\circ}$ Ss̆̆ in Ssŭ-liui H., Kuangtung.

$4568^{\mathrm{P}}$ Nan-chu-shil | 朱 提, (obs.) near Yi-pin H., Ssŭch'nan; S. Ts'i, chïn in Ning 夏 chov.

$4568^{2}$ Nan-ch' 11 an | N Nanchnen.

$1^{\circ} \mathrm{H}$. in Clunng-ch'ing Fu, [364] Sst̆-ch'tan; Lat. $29^{\circ}$ $08^{\prime}$, Long. $107^{\circ} 13^{\prime}$; Han, Chil 枳 $\mathrm{H}_{\text {; }}$; T'ang, Nan-ch nan. 川.

$2^{\circ}$ T-S. near Hsi-ning Fu, Kansul.

$4568^{\mathrm{R}} \mathrm{Nan}^{-c h}{ }^{\prime} \mathrm{u}$ | 婳, chên, near Lu-clı'êng H., Shansi.
南 NAN-FÊENG.

$4568^{\mathrm{s}}$ Nan-ch'ung | 充 Nanchung.

$1^{\circ} \mathrm{H}$. forming the prefectural city of Shunch'ing Fu, Ssüch'uan; Lat. $30^{\circ} 49^{\prime}$, Long. $106^{\circ} 08^{\prime}$; Han, K. Ch" ung 充; $E$. Han, Nanch'ung | 充; 1st Sung, Tang-ch' ï 宕渠chün; T'ang, K110 果 C.

$2^{\circ}$ (obs.) N. of the above; Sui, T'ang, Sung, Yüan and Ming, Nan-ch'ung 充 $\mathrm{H}$.

$3^{\circ}$ See No. 5815.

$4568^{\mathrm{T}}$ Nan-ên | 恩.

$1^{\circ}$ (obs.) S. Sung, C. in Kuang-tung 廣東 circ. $2^{\circ}$ (obs.) E. of $\mathrm{Ch}^{\prime} \mathrm{i} \mathrm{H}$., Shansi; N. Wei, H. in Chên-hsing 畺興 chün. $3^{\circ}$ See No. 7252.

4569 Nan-fang | 方, (obs.) $T^{6}$ ang, C. near Pin C., Kuangsi.

$4570 \mathrm{Nan1}$-fêng-yiian | 風原, town in the Lin-ch" itu Islands. 4571 Nan-fêng | 豈 Nan-fung. $1^{\circ} \mathrm{H}$. in Clien-ch'ang $\mathrm{Fu}$, Kiangsi ; Lat. $27^{\circ} 04^{\prime}$, Long. $116^{\circ} 28^{\prime}$; orig., Nan-ch-êng / 城; $T^{\prime}$ ang, and Sing, Nan-fêng | 豈 H.; Yüan, Nan-fêng | 豐 C.; Ming, Nanfêng 豈 $\mathrm{H}$. 
南

$2^{\circ}$ (obs.) W. of Kuangch'ang H., Kiangsi; $T \sin , S$. Sung and $S$. $T_{s}{ }^{6} i, \mathrm{H}$. in Lin-cli' nan 瀶川 chün。

$3^{\circ}$ See No. 7823.

4572 Nan-Fu-Chien | 撫 建, circ. in Kiangsi comprising Nan-ch'ang Fu, Fu-chou Fu and Chien-ch' ang Fur.

4573 Nan-hai | 海 Nan-hai.

$1^{\circ} \mathrm{H}$. forming with $\mathrm{P}^{\prime}$ anyii $H$. the prefectural city of Kuang-chou, Knangtung ; Lat. $23^{\circ}$ $07^{\prime}$, Lollg. $113^{\circ} 15^{\prime}$; Sui, Nan-hai | 海; T*ang, Hsien-ning 战 應 and $\mathrm{Ch}^{\prime}$ 'ang-k' ang 常康。

$2^{\circ}$ (obs.) Ts'in, chïn comprising part of Kuangtung and having for capital P'an-yï 番禹. $3^{\circ}$ H. in Ch' ing-shang 度 们 circ., Corea.

$4^{\circ}$ See No. 4585.

4574 Nan-han-chung | 漢 中, (obs.) in Ssŭch' 1 an ; $S$. Sung, chün in Yi 益 $\mathrm{CHOU}$.

4575 Nan-ho | 和 Nan-ho, H. in Shun-tê Fu, Chihli; Lat. $37^{\circ} 05^{\prime}$, Long. $114^{\circ}$ $51^{\prime} ; H a n, N a 11-110$ |和 ; $T^{\prime}$ ang, $\mathrm{Ho}$ 和 $\mathrm{C}$.
南 NAN-HSIUNG.

4576 Nan-ho | 河.

$1^{\circ}$ Chên near P'u-chêng H., Shensi.

$2^{\circ}$ Ss̆̌ near Shang C., Shensi.

4577 Nan-ho-tung|泃東, (obs.) IV. of Sung-tzŭ H., Hupeh; S. Sung, chïn in Ching 䰜 chov.

4578 Nan-hni | 匯 Nan-hwui, H. in Sung-chiang Fu, Kiangsu; Lat. $30^{\circ}$, Long. $121^{\circ} 30^{\prime}$.

4579 Nan-hsiang | 河, see No. 2465.

4580 Nanl-lisiang | 翔, chênnear Chia-ting J1., Kiangsu.

4581 Nan-11sin | 新, (obs.) in Corea; $T \sin , \mathrm{H}$. in Taifang 帶方 chiun.

4582 Nan-hsin-shil | 新 市, (obs.) Han, town near Ching-shan H., Hupeh.

4583 Nan-lising | 與, (obs.) $T \sin$, H., N. E. of Hsinlısing H., Kuangtung.

4584 Nan-11sing | 行. see No. 2845.

4585 Nan-hsiung | 雄 Nanhitung.

$1^{\circ}$ C. L. C. in the ShaoNan-Lien circ., Knangtung; Lat. $25^{\circ} 26^{\prime}$, l,ong. $113^{\circ} 52^{\prime} ; C$. Wars, in K. Ch'u楚; 
南

NAN-HSÜ.

Ts'in, in Nan-liai | 海 chün; Han, Hsinng 婎 C. in Kuei-yang 桂陽 chiun, Sung, Nan-lisinng 雄 and Pao-ch' ang 保昌; Ming, Nanhsinng | 雄 Fu.

$2^{\circ}$ (obs.) S. Sung, C. in Kuang-tu1ng 庶東 circ.

4586 Nan-hsü| 徐, see No.6209.

4587 Nan-hsün | 潯, chên in Wu-ch' êng H., Chehkiang.

4588 Nan-Ju-Kuang | 汝 光, circ. in Honan, comprising Nan-yang Fu, Ju-ning Fu and Kuang C.

4589 Nan-ju-ynan | 沙 原, see No. 3005.

4590 Nan-k'ang | 康 Nan-kang.

$1^{\circ} \mathrm{F} 11$ in the Kuang-Jao[366] Chin-Nan circ., Kiangsi; forming also the district city of Hsing-tzü; Iat. $29^{\circ} 23^{\prime}$, Loung. $116^{\circ}$ $10^{\prime} ; S . A$., in K. W $\mathrm{W}_{11}$ 㕦 and K. $\mathrm{Cl}^{\prime} \mathrm{u}$ 楚; $C$. Wars, in K. Clin 11 楚; $T s^{\prime}$ in, Hsing-clnu星渚 in Clint1-cliiang 九 江 chïn, Han, P'êng-tsê 彭裟 in Yii-chang 豫 章 chiun; T'ang, in Chiang 江 chou; $S$. Sung, Nan-k'ang | 康 military district.
南

NAN-LI.

$2^{\circ}$ H. in Nan-an Fin, [50] Kiangsi ; Lat. $25^{\circ} 57^{\prime}$, Long. $114^{\circ} 54^{\prime}$; Han, $\mathrm{Kan}$ 贑 $\mathrm{H}$; $W u$, Nanan 努; $T \sin$, Nan$\mathrm{k}$ 'ang 康.

$3^{\circ}$ See Nos. 2805, 3098, 5546.

4591 Nan-kon | 浶, chên near Sui-tê C., Shensi.

4592 Nan-k'on | П Nankow, name of a pass and of a town near Ch' ang-p'ing C., Cliilili.

4593 Nain-kuan | 關, Y. near Yeh H., Honan.

4594 Nan-knang | 廣, see No. $4568 .^{.}$

4595 Nan-kung | 宮 Nan-kung. $1^{\circ}$ H. in Chi C. L. C., Chihli; Lat. $37^{\circ} 27^{\prime}$, Long. $115^{\circ} 30^{\prime}$; Ming, Nan-kung | 宮 $\mathrm{H}$.

20 (obs.) N. W. of the above; Han, Nan-kung | 窎 $\mathrm{H}$.

4596 Nan-lê | 樂 Nan-loh, H. [289] in Ta-ming Fu, Chillit; Lat. $36^{\circ} 09^{\prime}$, Long. $115^{\circ} 26^{\prime}$; Han, Lê-clı'ang 樂 昌; $W e i, \mathrm{Cl}^{\prime}$ 'ang-lê 昌樂; Sui, Fan-shni 繁水; Five dyn., Nan-lê | 樂.

4597 Nan-li | 里, sš̆ near Nanning Fu, Kuangsi. - 
南

NAN-L,I.

4598 Nan-li | 里 H. in 'T'ai-1tan Fu, Formosa.

4599 Nan-liang | 梁, see No. 4951.

4600 Nan1-lin | 潾, (obs.) Trang, C. near Cli'ang-slion H., Ssǔch' uan.

4601 Nan-ling | 陵 Nan1-ling.

$1^{\circ}$ H. in Ning-kno Fin, [400] Anluni;' Iat. $31^{\circ} 03^{\prime}$, I.ong. $118^{\circ} 18^{\prime} ; \operatorname{Han}$, Ch'nn-ku春媇; Liang, Nan-ling | 陵.

$2^{\circ}$ See Nos. 986, 1630, 6825.

4602 Nan-liu | 罥, chên near Po-p'ing H., Shantung.

4603 Nan-liu | 流, see No. 7713. 4604 Nan-lou-fan | 樓煩, (obs.) near An-clin ing Fn, Anlui; S. Ts' $i$, H. in Chin-hisi $\frac{\text { Xá }}{\mathrm{G}}$ 熙 chïn.

4605 Nan-1u-hun | 陸渾, (obs.) $30 l i \mathrm{~N}$. E. of 'Sung H., Honan ; N. Wei, H. in Yi-yang 伊陽 chün.

4606 Nan1-luan | 綡, sec No. 1414.

4607 Nan-1nien-ch'ih | 淺池.

$1^{\circ}$ (obs.) W. of Yuncr-ning H., Honan ; N. IV $i, \mathrm{H}$. in Chin-mên 金师 ‘̆hün.

$22^{\circ}$ See No. 574 .

4608 Nan-mo | pît, chên ncar I,i-ch'êng H., Shansi.
南 NAN-P'I.

4609 Nan-ning | 峛 Nan-ning. $1^{\circ} \mathrm{F} u$ in the Tso-cliang [198] circ., Kuangsi; forming also the district city of Hsiian-liua; Lat. $22^{\circ}$ 43', I.011\%. $108^{\circ} 03^{\prime}$; Chou, land of N. Yiuel 䊦 tribes; $T s^{\prime}$ in, in Knei-lin 桂 林 chün; Han, in Yii-lin 槐林; r'ang, Yung 䍃 C. and Lang-ning 副 耍; Sung, Yung-ning 永 留:; Ning, Nan1ning | 寉 Fi1。

$2^{\circ} \mathrm{H}$. forming the prefectural city of Clïiching, Yünnan; I at. $25^{\circ} 32^{\prime}$, L,ong. $103^{\circ}$ $50^{\prime}$; Han, Wei 味; T'ang, Nan-ning | 俩. $3^{\circ}$ See Nos. 4672, 7813.

4610 Nan-pa | 巴, (obs.) Liang, H., $\because$ \% of Kao-chou fiu, Kuangtung.

4611 Na11-pao-chung | 苍中, (obs.) in Ssŭclı "lan; $S$. Sung, H. in Nan-lianchung | 漢中 chïn.

4612 Nan-p'i / 皮 Nan-pi.

$1^{\circ}$ H. in ' $\mathrm{T}^{6}$ icn-ching Fu, [322] Chilhli; Iat. $38^{\circ} 08^{\prime}$, I,ong. $116^{\circ} 43^{\prime} ; N$. Wei, Sui, T"ang, Sung, K'in. Yïan and Ming, Nan$\mathrm{p}$ 'i | 皮 $\mathrm{H}$. 
南

NAN-P'I.

$2^{\circ}$ (obs.) 8 li N. E. of the above; Han, Tsin and S. Sung, Nan-p'i | 皮 $\mathrm{H}$.

4613 Nan-pin | 筫.

$1^{\circ}$ (obs.) Sui, H., E. of

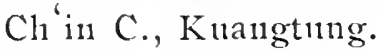

$2^{\circ}$ (obs.) 70 li S. E. of Fêng-tı H., Ssŭch' 'uan; T'ang, Sung and Yïan, Nan-pin | 富 $\mathrm{H}$.

$3^{\circ}$ See No. 3967.

4614 Nan1-pin | 国粨, see No. 5127. 4615 Nan-p'ing | 平 Nan-ping. $1^{\circ} \mathrm{H}$. forming the prefectural city of Yenp'ing, Finlikien; Lat. $26^{\circ} 38^{\prime}$, Long. $118^{\circ}$ 18' Han, Hou-kuan 侯 官; $W_{n,}$ Nan-p ing | 平; $T s^{\prime}$ in, Yen-p'ing 延斗; $S . T^{6}$ ang, Chien$\mathrm{fu}$ 劍甫.

$2^{\circ}$ (obs.) S. Sung, military district in $\mathrm{K}^{\prime}$ uei

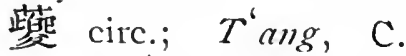
near $\mathrm{Cl}_{1}$ 'ung-ch'ing $\mathrm{Fu}$, Ssŭch 'uan.

$3^{\circ}$ Sec Nos. 73, 1583, 3502, $3597,3727$.

4616 Nanl-p ing | 坪, chên near Li-ch'nan H., Hupeh.

4617 Nau1-pu | 部 Nan-pu, H. [30] in Pao-ning Fu, Ssŭch ' $11 a n$; I at. $31^{\circ} 19^{\prime}$, Long. $106^{\circ}$

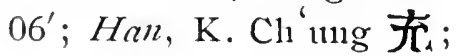

南 NAN-TANG-CH' $\dddot{\mathrm{U}}$.

Liang, Nan-pu | 部; $W$. Wei, Hsin-an 新多.

4618 Nan1-slıa | 沙, see No. 320. 4619 Nan-shan | 山, (obs.) formerly C. near Lung $C$., Shensi.

4620 Nail-shih-hisiu | 始新, (obs.) near Lintan C., Anhui ; N. Wei, H. in Liichiang 盧 汇 chün.

4621 Nan1-so | 朔, see No. 1688. 4622 Nan-ssü | 司, see No. 83. 4623 Nan-su | 蘇, (obs.) near Hsing-cliing 興京, Shingking; $T^{6} a n g, C$. in Ho-pei 河北 circ.

4624 Nan1-ta-t'ung | 大通 T-S. near Ku-lang H., Kansuh.

4625 Nan1-t'ai-yüian | 太 原, (obs.) in Nan-chêng $H$., Shensi; S. Sung and $S$. $T s^{\prime} i$, chiun in $\mathrm{Ch}^{\prime}$ in 秦 chOV.

4626 Nan-tan | 丹 Nan1-tan, $1^{\circ}$ T.C. in Ch'ing-yüan Fu, Kuangsi ; Lat. $25^{\circ}$, L.ong. $107^{\circ} 23^{\prime}$; orig., Man-tung 變润; Ming, Nantan 1 月 $\mathrm{C}$.

$2^{\circ} \mathrm{H}$. in Cl' üan-1o 全羅 circ. ,Corea.

4627 Nan-tang-ch' ü | 宕渠. $1^{\circ}$ (obs.) N. of Nancli'nung H., Ssüch'nan; $S$. Sung and S. Ts'i, chün in Liang 梁 cHov. 
南 NAN-TANG-CHÜ.

$2^{\circ}$ (obs.) in Ssŭch' 'uan; S. Sung, chün in $\mathrm{Yi}$ 盆 $\mathrm{CHOU}$.

4628 Nan-t'ao | 桃, chên near Chi-y ïan H., Honan.

4629 Nan-tien | 们, post-town near 'T'êng-ı̈ïeh T., Yünnan.

4630 Nan-ting | 定, Y. near Lin-kao H., Knangtung.

4631 Nan1-t ou | 投, post-town near Chang-hua H., Fuhkien.

4632 Nan-ts'un | 村, ss ̋̆ near Mien-ch'ih H., Honan.

4633 Nan-tsung | 宗, (obs.) in Hsi-ning H., Kansulı; - Sung, pao in I.ê 樂 C., Shensi.

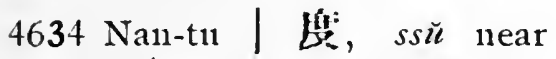
Ts'ên-cl'i H., Kuangsi.

4635 Nan-tı | 渡.

$1^{\circ}$ Chên near Hsin-ning H., Anhui.

$2^{\circ}$ Chên near Ning-kuo Fu, Anhui.

4636 Nau-t'ı11 | 屯, chên near Ching-yang H., Shensi.

4637 Nan-tzun-t'ung | 梓潼, (obs.) in Ssŭch' '11an; $S$. $T s^{\prime} i$, chïn in Liang 梁 CHOU.

4638-Nan-wan-shan | 萬 山, $Y$. near Ling-shui $H$., Kuangtung.
南 NaNg-YaNG.

4639 Nan-wei | 溻, (obs.) Ming, C., IV. of Yung-shun H., Hunan.

4640 Nan-yang | 陽 Nan-yang. [368] $1^{\circ} \mathrm{Fu}$ in the Nan-JuKuang circ., Honan ; forming the district city of the same name; Lat. $33^{\circ} 06^{\prime}$, Long. $112^{\circ} 34^{\prime}$; oriz., in $\mathrm{K}$. $\mathrm{Cl}^{\prime}$ ' 1 楚 and $\mathrm{K}$. Han 韓; Ts'in, Nan-yang | 晹 chün, with capital city IVan 宛; Han, Cln'n1n-ling 春陵; $S_{u i}$, 'Têng 登 C.; T'ang and Sung, Wan 宛 C.; Kin, Shên 申 C.; Ming, Nanyang 陽 Fu.

$2^{\circ} \mathrm{H}$. forming the prefectural city of the same name, Honnan ; Lat. $33^{\circ}$ $05^{\prime}$, Long. $112^{\circ} 34^{\prime}$; orig., K. Shên 申;

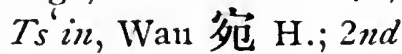
Wei, Shang-mo 上陌; $P$. Chou, Shang-wan 上宛; Sui, Nan-yang 踢。

$3^{\circ} \mathrm{Fu}$ in Ching-ch'i 京 畿 circ., Corea ; Lat. $37^{\circ} 32^{\prime}$, Long. $125^{\circ} 46^{\prime}$. $4^{\circ}$ (obs.) $W$. Wei, town $\mathrm{W}$. of Tsao-yang $\mathrm{H}$, Hupeh.

$5^{\circ}$ See No. 2874. 
南

NAN-YHH.

4641 Nan-yeh | 埜, see No. 4198.

4642 Nan-yen |燕, see No. 6583. 4643 Nan-yi | 移, (obs.) in Annam; S. Ts' $i, H$. in Wu-ping 武平 chïn。

4644 Nan-yiian | 原 Nan-yuen, Fin in Ch'iian-lo 全羅 circ., Corea; Lat. $35^{\circ} 08^{\prime}$, Long. $126^{\circ} 25^{\prime}$.

4645 Nan-yüan | 源, (obs.) in Kuangsi; Sung, C. in Yung 品 CHOU.

\section{䧼}

4646 Nan-chiang 難江, see No. $4568^{\mathrm{c}}$.

\section{郝}

4647 Nan-tsei 郝睋, post-town near $\mathrm{Ch}^{\prime}$ ien C., Shensi.

Nan1-chang, see No. 4562. Nan-chan, see No. 4563. Nail-ching, see Nos. 4567, 4568.

Nan1-chtien, see Nos. $4568^{2}$. Nan-chung, see No. $4568^{\mathrm{s}}$. Nan-fung, see No. 4571. Nanghin, see No. 55.

Nan-hiung, see No. 4585. Nan-hwui, see No. 4578. Nan-kang, see No. 4590. Nan-ki, see No. $4568^{\mathrm{B}}$. Nan-kiang, see No. $4568^{\circ}$. Narl-king, see No. 749.
內 NEII-CH'iU.

Nan1-kow, see No. 4592. Nan-loh, see No. 4596. Nan1-11gan, see No. 4558. Nan-1ngau, see No. 4559. Nan-pi, see No. 4612.

Nan-ping, see No. 4615. Nant-tsing, see No. $4568^{\mathrm{M}}$. Nan-ynen, see No. 4644.

\section{NAO}

\section{砲}

4648 Nao-chun 砲洲, chên in Wu-ch'uan H., Kuangtung. It is an island which produces edible birds' nests.

\section{閙}

4649 Nao-11i 閙泥, post-town near Ning-wu Fu, Shansi.

Narat, see No. 4541.

\section{NEI}

\section{內}

4650 Nei-chiang 內 江 Nui[265] kiang, H. in Tzŭ C., Ssŭcl'uan; Lat. $29^{\circ} 40^{\prime}$, I.ong. $105^{\circ} 08^{\prime}$; Han, Tzŭchung 凟 中; Sui, Neicliang Tे. $\mathrm{H}$.

4651 Nei-ch'iu | 邱 Nui-kiu, H. iu Shun-tê Fu, Chilıli; Lat. $37^{\circ} 15^{\prime}$, Long. $115^{\circ}$ 35'; T'ang, Chung-ch'iu 中邱; Sui, Nei-ch'iu| 邱. 
4652 Nei-fu | P 付, see No. 6137. 4653 Nei-huang|黃 Nui-hwang, [564] H. in Chang-tê F $u$, Honan; Lat. $36^{\circ} 02^{\prime}$, Long. $115^{\circ}$ $09^{\prime}$; Han, Nei-huang | 黃.

4654 Nei-lısiang | 拫 Nui-hiang, [477] $H$. in Nan-yang Fu, Honan; Lat. $33^{\circ} 10^{\prime}$, Long. $111^{\circ} 55^{\prime}$; $T s^{6} i n$, Chunghsiang 中 郷; $W$. Wei, Nei-hsiang | 哏.

4655 Nei-mêng-ku | 蒙古, Inner Mongolia, a province in Mongolia, bounded on the $\mathrm{N}$. by Hellungkiang, Tsetsen, Tuchet $u$ and Dsassaktu; on the E. by Kirin; on the S. by Shingking, Chihli, Shansi, Shensi and Kansuln; and on the IV. by Yarkand.

4656 Nei-pu | 部. see No. 1547. 4657 Nei-shil |史.

$1^{\circ}$ (obs.) $T s^{\prime}$ in, chïn in Shensi.

$2^{\circ}$ See Nos. 1724, 2208, 2438 and 6802.

4658 Nei-t'ing | 掌, (obs.) Sui, $\mathrm{H}$., W. of Ling-shan H., Kuangtung.

Nemuro, see No. 3240.

Nêrtchinsk, see No. 4660. Newcliwang, sec No. 4486. New Dominion, see No. 2736.

\section{內} NIF.

Ngan, see No. 45.

Ngan-ful, see No. 64.

Ngan-hiang, see No. 73.

Ngan-hwa, see No. 66.

Ngan-hwui, see No. 69.

Ngan-i, see No. 110.

Ngan-jin, see No. 75 .

Ngan-kang, see No. 77 .

Ngan-ki, see No. 51.

Ngan-kih, see No. 50.

Ngan-king, see No. 55.

Ngan-kin, see No. 56.

Ngan-luh, see No. 83.

Ngan-nan, see No. 88.

Ngan-ning, see- No. 89.

Ngan-pien, see No. 91.

Ngan-ping, see No. 92.

Ngan-sai, see No. 94.

Ngan-shun, see No. 98.

Ngan-si, see No. 70.

Ngan-suh, see No. 99.

Ngan-ting, see No. 101.

Ngan-tung, see No. 104.

Ngan-yang, see No. 107.

Ngan-yih, see No. 113.

Ngant-yin, see No. 114.

Ngan-yoh, see No. 116.

Ngan-yuen, see No. 117.

Ngăn, see No. 1606.

Ngăı-loh, see No. 1612.

Ngăı-lıng, see No. 1613.

Ngăn-11gan, see No. 1607.

Ngăn-ping, see No. 1615.

Ngăn-slii, see No. 1616.

Ngatl-1ing, see No. 4749.

Ngo-11ei, see No. 4737.

Ngo-pien, see No. 4739. 
泥

Ni-ShaN.

\section{NI}

筧

4659 Ni-shan 筧山 Kagoshima, ken in Japan.

\section{尼}

$4660 \mathrm{Ni}-\mathrm{pu}-\mathrm{cl}$ ' u 尼有楚 Nipchu, or Nertchinsk, town in Hellungkiang on the Russian frontier; Lat. $51^{\circ}$ $49^{\prime}$, Long. $121^{\circ}$.

4661 Ni-shan 尼山, H. in Clunng-ch ‘ing 忠清 circ., Corea.

\section{泥}

$4662 \mathrm{Ni}$-ch'a 泥汉, chênn near Wu-wei C., Anhuii.

4663 Ni-chi $i$ 溪, T-S. near P'ing-slaan H., Ssŭch' '1an. 4664 Ni-yang | 陽.

$1^{\circ}$ Chền near Ch' êng H., Kansuli.

$2^{\circ}$ See No. 4672.

\section{你}

4665 Ni-sha-pu-êrh 你沙 不 尔, see No. 4555.

4666 Ni-sha-pu-li | 沙卜里, see No. 4555.

\section{匿}

4667 Ni-ch'a-w11-êrh 匿察 儿 兒, see No. 4555 .

\section{NIAO}

\section{鳥}

4668 Niao-chü 鳥取, kell in Japan.
踾 NING.

\section{NIEH}

\section{涅}

4669 Nielı 涅, see Nos. 7066, 7669.

\section{NIEN}

\section{鮎}

4670 Nien1-yii 鮎 魚, sš̆ near Wri-ch'ang H., Hupeh.

\section{替}

$4670^{\wedge}$ Nien1-ku畒酦, see No. 5822 .

\section{碳}

4671 Nien-po 碾伯 Clien-peh, H. in Issi-ning Fu, Kansulı; Lat. $36^{\circ} 30^{\prime}$, Long. $102^{\circ} 50^{\prime}$.

Niigata, see No. 2760.

Nikko, see No. 2998.

\section{NING}

\section{察}

4672 Ning 恧 Ning.

[28571 ${ }^{\circ} \mathrm{C}$. in Ch' ing-yang $\mathrm{Fu}$, Kansulh; Lat. $35^{\circ} 35^{\prime}$, Long. $107^{\circ} 51^{\prime} ; s^{\prime} i n$, Yi-ch ü 義渠; Han, Ni-yang 泥榢; Sui, $T^{\prime}$ 'ang and Sung, Tingan 定安 H.; 2nd Wei, Hua 華 C.; W. Wei, Ning | C. 
蒠

NING.

$2^{\circ} \mathrm{C}$. in Lin-an Fu, Yïannan; Lat. $24^{\circ} 18^{\prime}$, Long. $103^{\circ} 05^{\prime} ; \mathrm{Han}$, in $\mathrm{Yi}$ 盛 cHOU; Tsin, Nanning 南|; Tang, $\mathrm{Li}$ 黎 C.; Yüan, Ning | C.

4673 Ning-ch'êng | 城, posttown near Ning-ling $\mathrm{H}$., Honan.

4674 Ning-chiang | 江, (obs.) N. E. of the town of Kirin; Liao, C. in Tung-ching 東京 circ.

4675 Ning-cll iang | 差 Ning[133] kiang, C. in Han-chung Fu, Shensi ; Lat. $32^{\circ} 42^{\prime}$, Long. $106^{\circ} 27^{\prime}$; Han, Chu 沮 H.; Minor Han, Liang 梁 C.; $T \sin , \mathrm{Wu}$-tu 武都.

4676 Ning-clin | 至 Ning-tsin, H. in Chao C., Chillli; Lat. $37^{\circ} 44^{\prime}$, Lo11g. $115^{\circ}$; $T$ sin, Ying-t'ao 浿 陶; T'ang, Ning-chin | 㛩.

4677 Ning-ching | 津 Ning[325] tsin, H. in Ho-chien Fu, Clihli; Lat. $37^{\circ} 48^{\prime}$, Long. $116^{\circ} .32^{\prime} ;$ Sung, Yang-lisin 陽 信; Kin, Ning-ching | 津.

$467 \dot{8}$ Ning-êrh | 洱 Ning-'rh, $\mathrm{H}$, forming the prefectural city of $\mathrm{P}^{\prime} \mathrm{u}$-êrh, Yünnan ; Iat. $23^{\circ} 02^{\prime}$, I,ong. $101^{\circ}$ $10^{\prime}$.

\section{耍 NING-HO.}

4679 Ning-fan | 番, (obs.) near Mien-ning H., Ssŭch'uan ; Ming, Ning-fan | 番 111ilitary district.

4680 Ning-fêng | 風, (obs.) 100 li W. of 'T'êng H., Kuangsi ; T'ang, H. in I,ing-11an1 領南 circ.

4681 Ning-fêng | 豐, (obs.) Sing, H., IV. of Fu-ku H., Shensi.

4682 Ning-hai | 海 Ning-liai.

$1^{\circ} \mathrm{Fu}$ in $\mathrm{Ch}^{\prime}$ 'ing-shang 慶 份 circ., Corea. Lat. $37^{\circ} 15^{\prime}$, Long. $129^{\circ}$ $10^{\prime}$.

$2^{\circ}$ C. in Têng-chon $\mathrm{F} \mathfrak{u}$, Shantung; Lat. $37^{\circ}$ $25^{\prime}$, Long. $121^{\circ} 40^{\prime}$; Han, Mour-p'ing 车斥; T'ang, Mon 牟 C.; Kin, Ning-liai | 海.

$3^{\circ} \mathrm{H}$. in T'ai-clon Fin, Chehkiang; Lat. $29^{\circ}$ $35^{\prime}$, Long. $120^{\circ} 46^{\prime}$; Han, Hui-p'u司浦; Tsin, Ning-hai | 海.

$4^{\circ}$ Ss̆̌ near Hsïi-wên H., Kuangtung.

4683 Ning-ho | 和, chên near Ti-tao C., Kansul.

4684 Ning-ho | 河 Ning-lio, $\mathrm{H}$. in Shun-t' ien Fu, Chilli; Lat. $39^{\circ} 22^{\prime}$, Long. $117^{\circ}$ $43^{\prime}$. 
4685 Ning-huna | 化 Ning-hwa, [388] H. in 'ing-chon Fu, Fulukien; Iat. $26^{\circ} 13^{\prime}$, Long. $116^{\circ} 49^{\prime} ; T^{\prime}$ ang, Huang-lien 黄 連 and Ning-hua | 化.

4686 Ning-hsi | 西.

$1^{\circ}$ (obs.) 12 li N. of Knang-an C., Ssŭch'tran; Szung, military district in 'T' $11 n$ g-c.l 'uan 潼川 circ.

$2^{\circ}$ (olss.) $40 \mathrm{li} \mathrm{VV}$. of Hsining Fu, Kansuln; Sung, walled town in Hsi-ning 西 | C

4687 Ning-hısa | 夏 Ning-hia. $1^{\circ}$ Fu in the Ning-hsiaHo - tung - Ho-hsi circ. Kansul ; forming also the two district cities of Ning-lisia and Ning-so; Lat. $38^{\circ} \hat{\jmath} 3^{\prime}$, Long. $106^{\circ} 08^{\prime}$; orig. land of the Ch'iang 息 tribes; ITan, So-fang 朔 $7 \mathrm{j}$; a non-Chinese K. Hsia 夏; $T \sin , 2$ nd $I V C i$ and T"ang, Hsia夏C.; Sung, Hsing 與 C.; Ming, Ning-hsia | 夏military district.

$2^{\circ} \mathrm{H}$. forming with $\mathrm{Ning}$ so $H$. the prefectural city of Ning-hsia, Kansuls; Lat. $35^{\circ} 33^{\prime}$, Long. $106^{\circ} 08^{\prime}$.
等 NING-JÊN.

4688 Ning-hsia-Ho-tung-Ho-hisi |夏河東河西, circ. in 'Kansulı, comprising Ning-lısia Fu, witl general supervision over the regions on the West and E. of the Yellow River.

4689 Ning-hrsiang | 廓 Ninghiang.

$1^{\circ}$ H. in Fên11-clinu Fur [316] Shansi; Iat. $37^{\circ} 22^{\prime}$, Long. $111^{\circ} 10^{\prime}$; $\operatorname{Han}$, L,i-shih 離 石 chiin; Sui, Tang and Sung, Pingryi 本夷 H.; $P$. Chou, Ning-lısiang | 拫 $\mathrm{H}$.

$2^{\circ} \mathrm{H}$. in Yo-chou Fu, [69] Hunan; Iat. $28^{\circ} 18^{\prime}$, Long. $112^{\circ} 08^{\prime}$; Han, Yi-yang 谷陽; Wu, Hsin-yang 新陽; $T \sin$, Hsin-k'ang 新 㞗; Sung, Ning-lisiang | 手.

$3^{\circ}$ Chên near Hsing-lina H., Kiangsu.

4690 Ning-11sin | 新, (obs.) $S$. Sung and $S . T s^{\prime} i$, H., S. E. of T's'ang-wu H., Knangsi.

4691 Ning-Hsing-Hun | 姓渾, circ. in Kirin, comprising Ho-lan C. L. T., San-lısing C. L. 'T'. and Hun1-ch' 'n11 Fin.

4692 Ning-jên|人. 
察

$1^{\circ}$ (obs.) N. E. of Jung H., Kuangsi ; Sui, H. in Yung-p'ing 永 4 chïn.

$2^{\circ}$ (obs.) in Shansi; Lino, H. in Yiinn-nei 雲內 chiin.

4693 Ning-jên | 仁.

$1^{\circ}$ (obs.) N.of Yiü-lin C., Kuangsi ; $T^{6}$ ang, H. in 'Tang 黨 C.

$2^{\circ}$ (obs.) near So-p 'ing Fu, Shansi; Kin, chên in Yün-nei 昰內 chän.

4694 Ning-kn-t a | 古塔 Ninguta, see No. 2131.

4695 Ning-ku-chai | 谷貿, ssü S. W. of P'u-ting $\mathrm{H}$., Kweichou.

4696 Ning-kno | 國 Ning-kwol. $1^{\circ} \mathrm{Fu}$ in the Hui-Ning[423] Ch'ilı - 'Tai - Knang - tê Chou circ., Anhui; forming also the district city of Hstian-cl' êng; Lat. $30^{\circ} 50^{\prime}$, Long. $118^{\circ} 41^{\prime}$; orig., in 1 st., $\mathrm{K}$. $\mathrm{Wu}$ 只, $2 n d, \mathrm{~K}$. Yüch 越, 3rd, K. Cli'n 楚; Ts'in, in Cliang 鄣 chiin; Han, 'Tanyang 丹晹 and Wanling 宛陵; E. Han, - Hsïan-cln êng 宣城; Sui and $T^{6}$ ang, Hsüan 㫜 C.; Sung, Ning-kno 厂國 Fil.

\section{夏} NiNG-PO.

$2^{\circ}$ H. in Ning-kuo Fu, Anluti; Lat. $30^{\circ} 3^{\prime}$, Long. $118^{\circ} 58^{\prime} ; H_{a^{n}}$,

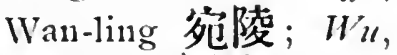
Ning-kno 國 H.

4697 Ning-ling | 陵 Ning-ling, [320] H. in Kuei-tê Fin, Honan; Lat. $34^{\circ} 30^{\prime}$, Long. $115^{\circ}$ $.31^{\prime}$; orig., K. Kê-po 葛 伯; Tsin, Tang 碭 H.; Han, Ning-ling | 陵.

4698 Ning-ling | 霝 Ning-ling, T. in Ning-lisia Fn, Kansuh ; I at. $37^{\circ} 35^{\prime}$, Long. $105^{\circ} 25^{\prime}$; lately established on the site of a village called Chin-chi 金 積 pro.

4700 Ning-1ning | 明 Ning[309] ming, C. in T'ai-p ing Fu, Kuangsi; Lat. $23^{\circ}$ $15^{\prime}$, Long. $106^{\circ} 35^{\prime}$.

4701 Ning-pien | 邊 Ning-pien, Fin jin P'ing-an 作发 circ. Corea ; Lat. $39^{\circ} 57^{\prime}$, I.ong. $126^{\circ} 05^{\prime}$.

4702 Ning-po | 波 Ning-po, Fu [426] in the Ning-Shao-T'ai circ., Chehkiang; forming also the district city of Yin ; Lat. $29^{\circ} 49^{\prime}$, I,ong. $121^{\circ} 35^{\prime}$; literary names, Yang 角 (from the river) and Ssǔ'ming 四明 (from a 1nountain;) onc of the ports open to foreign trade; orig., Yung-tung 角束; 
$T s^{\prime}$ in, in Kuei-chi 曾 嵇 chïn; Sui, Yüeh 越 C.; $T^{6}$ ang, Ming 明 C.; Sung, Ch'ing-yianan 慶元; Ming, Ning-po | 波 Fu.

4703 Ning-shan | 陝 Ning-slien. $1^{\circ}$ T. in Hsi-an Fu, Shensi ; I,at. $33^{\circ}$, I,ong. $109^{\circ} 10^{\prime}$.

$2^{\circ} \mathrm{Y}$. near the above.

$470+$ Ning-Sháo-'l'ai | 紹台, circ. in Chelnkiang comprising Ning-po Fin, Shaohsing Fn and 'T'ai-chon F11.

4705 Ning-so | 朔 Ning-soh, $\mathrm{H}$. forming with Ning-hisia H. the prefectural city of Ning-lısia, Kansuh; L Lat. $38^{\circ} 33^{\prime}$, Long. $106^{\circ} 08^{\prime}$.

4706 Ning-tan |單 (cbs.) in Knangtnng; S. $T s^{i} i, \mathrm{H}$. in Kao-lising 高與 chïn.

4707 Ning-tê | 德 Ning-telı, H. in Fu-11ing Fin, Fuhkien; Lat. $26^{\circ} 37^{\prime}$, Long. $119^{\circ}$ $18^{\prime}$; orig., Ch'ang-ch'i 長 溪 and Ku-t'ien 古田; Fize dyn., Ning-tê | 德.

4708 Ning-tu | 都 Ning-tu.

$1^{\circ}$ C. L. C. in the ChiNan-Kan-Ning circ., Kiangsi; Lat. $26^{\circ} 28^{\prime}$, Long. $115^{\circ}$ 51'; Han, $\mathrm{Kan}$ 贑 $\mathrm{H}$.; $W u$, Hsin- $\mathrm{t} 11$ 新都; $T \sin$, Chiel1yang 揭晹; 1st Sung， Ning-t11 都.

$2^{\circ}$ (obs.) N. of the above; $S$. Sung and $S . T s^{6} i$, H. in Nan1-k'ang 南康 chïn.

$3^{\circ}$ (obs.) $70 l i$ S. E. of Han-yin T., Sliensi; $S$. Sung and $S . T s^{\prime} i, H$. in An1-k'ang 妿康 chïn; $N$. Wei, H. in IVeiming 魏明 chïn.

$4^{\circ}$ (obs.) $24 l i \mathrm{~W}$. of Hanyin T., Shensi; N. Wei., H. in An-k'ang 努康 chïn.

$5^{\circ}$ See No. 5696.

4709 Ning-wei | 喿, (obs.) in Kansuh ; Sinng, pao in Hsi-an 西安 C., Shensi. 4710 Ning-wu | 武 Ning-wn. $1^{\circ} \mathrm{F} u$ in the Yen-P'ing[376] Ta-So-Ning-Hsin-TaiPaocirc., Shansi ; forming also the district city of the sane name; 利l at. $39^{\circ} 08^{\prime}$, I Iong. $112^{\circ} 08^{\prime}$.

$2^{\circ} \mathrm{H}$. forming the prefectural city of the same name, Shansi ; Lat. $39^{\circ}$ $08^{\prime}$, L Long. $112^{\circ} 08^{\prime}$.

4711 Ning-yang / 晹 Ning-yang. $1^{\circ} \mathrm{H}$. in Yen-chou $\mathrm{F} 11$, [262] Shantung; Lat. $35^{\circ} 55^{\prime}$, 


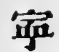

NING-YANG.

Long. $117^{\circ}$; orig., Shanyi 關邑in K. In魯; Han, Ning-yang | 晹; 2nd Wei, P'ing-ÿïan 平 原; Sui, Kungch'iu 驡邱.

$2^{\circ} \mathrm{H}$. in Lung-yen C., [97] Fulukien; Lat. $25^{\circ} 35^{\prime}$, Long. $117^{\circ} 30^{\prime}$.

4712 Ning-yi | 夷.

$1^{\circ}$ (obs.) in Annam; $S$. Sung, H. in Chin-chên 九異 chinn.

$2^{\circ}$ (obs.) N. E. of Licli'nan H., Sliensi; $N$. Wei, H. in Hsien-yang 战陽 chün.

$3^{\circ}$ See No. 5701 .

4713 Ning-yi | 邑 (obs.) Yin., $H$. near Huo-chia H., Fuhkien; see also No. 2874.

4714 Ning-yïan | 遠 Ning[6] ynen.

$1^{\circ} \mathrm{Fu}$ in the Chien-ch' angSliang-nan circ., Ssŭch'nan; forming also the district city of $\mathrm{Hsi}$ clíang; Lat. $\left.27^{\circ} 50\right)^{\prime}$, Long. $102^{\circ} 12^{\prime}$.

$2^{\circ}$ Chün in P'ing-an 4 安 circ., Corea ; Iat. $40^{\circ}$ $05^{\prime}$, I,ong. $126^{\circ} 55^{\prime}$.

$3^{\circ}$ T. in So-p'ing Fu, Shansi; Lat. $40^{\circ} 20^{\prime}$, I,ong. $112^{\circ} 55^{\prime}$.
寉 NING-TEH.

$4^{\circ}$ C. in Chin-chou $\mathrm{Fu}$, Shingking; Lat. $40^{\circ}$ $30^{\prime}$, Long. $120^{\circ} 40^{\prime}$.

$5^{\circ}$ H. in Kung-ch' ang Fu, [498] Kansuh; Lat. $34^{\circ} 38^{\prime}$, Long. $104^{\circ} 58^{\prime} ; T^{\prime}$ ang, I lung-hsi 骝西; Sung; Ning-youan|遠.

$6^{\circ}$ H. in Yung-chon Fu, [132] Hunan; Lat. $25^{\circ} 33^{\prime}$, Long. $111^{\circ} 48^{\prime}$; Han, Lêng-tao 冷, 道; Five $d y n$., Yen-ch' ang 延党; Sung, Ning-yüan |遠.

$7^{\circ} \mathrm{H}$. in Hui-li Fu, Ili. Lat. $43^{\circ} 25^{\prime}$, Long. $82^{\circ} 50^{\prime}$.

$8^{\circ}$ See No. 7224.

4715 Ning-yüeh|越 Ning-yueh.

$1^{\circ}$ Chün in Chiang-yüan 江原 circ., Corea; Lat. $37^{\circ} 45^{\prime}$, I song. $128^{\circ}$ $12^{\prime}$.

$2^{\circ}$ Y. near Ch'ing-ch'i $\mathrm{H}$., Ssŭch 'uan. Also called Hai-t'ang 海塘(Baber).

Ning-hia, see No. 4687.

Ning-hiang, see No. 4689.

Ning-hwa, see No. 4685.

Niug-kiang, see No. 4675.

Ning-kwoh, see No. 4696.

Ning-'rh, see No. 4678.

Ning-shen, see No. 4703.

Ning-sol, see No. 4705.

Ning-teh, see No. 4707. 
女 Nï-CH'

Ning-tsin, see Nos. 4676, 4677.

Ning-yueh, see No. 4715.

Ning-yuen, see No. 4714.

Ninguta, see No. 4694.

Nipch11, see No. 4660.

Nishapur, see No. 4555.

\section{NIU}

\section{4}

$4716 \mathrm{Nin}$ 牛, Y. wear Min C., Kansul.

4717 Nin-chieh | 街, post-tow11 near Chên-hısinng C., Yünnan.

4718 Nin-chuang-ch'eng | 庄 城, sš̆ in Hai-ch êng $\mathrm{H}$., Shingking, in Lat. $40^{\circ}$ $53^{\prime}$, Long. $122^{\circ} \quad 07^{\prime}$; giving its name to the open port of Newchwang, which is, however, several miles nearer the sea; see No. 4486.

4719 Niulfêng | 筆, H, in Huang-hai 黃海 circ., Corea.

4720 Nill-k'oul 口, post-town near Hsü-chon Fu, Ssŭch' uan.

4721 Nit1-nla | 馬, shê near Chang-hun H., Fulhkien.

4722 Nin-p'i | 㨢, chên near L,o-jung H., Kunugsi.

4723 Nitl-po-êrh-tsung | 博尔 宗 Ninburdzung, town in Thibet; Lat. $25^{\circ} 15$, Long. $95^{\circ} 40^{\prime}$.

4724 Niut' ang | 堂, Y. near Yung-ming $\mathrm{H}$., Hunan.

4725 Niu-t un-l1o | 屯河, sšr near Wu-hu H., Anhui.

4726 Nin-wu / 武, chên near Fu C., Shensi.

Niuburdzung, see No. 4723.

\section{NO}

\section{糯}

4727 No-pai-wa 糯白瓦, T-S. near Mien-11ing H., Ssŭch'uan.

\section{訥}

4728 No-110-110 訥訥赫 Pencgne, town in Kirin ; I at. $44^{\circ} 20^{\prime}$, Long. $129^{\circ}$.

\section{蓿}

4729 No-shui 諾水, see No. 6772 .

\section{Nü}

\section{女}

4730 Nï-ch'i 女䄮, (obs.) E. of I nung-mên 龍师 $\mathrm{H}$., Chilli; $W$. Han, H. in Shang-kn 上谷 chïn. 
農

NUAN-CHIANG.

NUAN

暖

4731 Nuan-chiang 暖江, (obs.) near $\mathrm{Cl}_{1}$ ï̈-ching $\mathrm{F} u$, Ssŭch'uan; S. Ts'i, H. in Hsi-p'ing 唒 平 chiin.

4732 Nuan-tai-pa | 帶垻, T-S. near O-pien 'T'., Sstŭch 'uan.

4733 Nuan-tai-111 | 帶密, T-S. near O-pien T'., Ssŭchnıan.

Nui-hiang, see No. 4654. Nui-liwang, see No. 4653. Nui-kiang, see No. 4650. Nui-kiu, see No. 4650.

\section{NUNG}

\section{農}

$4833^{A}$ Nung-an 宸 发 H. in $\mathrm{Cl}^{\prime}$ 'ang-ch' un Fin, Kirin.

4734 Nung.ch' êun | 城, (obs.) $T \sin , \mathrm{H} ., \mathrm{W}$. of IVu-clion Fu, Kuangsi.

\section{o}

我鳥

4735 O-lu1ng 我鳥龍, ss ̌̆ in Mach' êng H., Hupelı.

4736 O-pu|望, ssŭ near Haifêng $H .$, Kuangtung.

\section{峨}

4737 O-m1 峨咸 Ngo-mei, H. [38] in Chia-ting Fin, Ssŭch' '11ani Lat. $29^{\circ} 32^{\prime}$, Itong. $103^{\circ} 38^{\prime} ; \mathrm{Han}^{\prime}$, Nan-an 南然; P. Chou,
峨 O-MI.

O-mei | 扂; Sui, Cli'ingyi 青衣。

4738 O-mei-11si | 箩喜, T-S. near Sung-p'an 'T., Ssŭch'nan.

4739 O-pien / 邊 Ngo-pien, 'T. in [434] Chia-ting I't1, Sstich 'nan. Terr. jur.

4740 O-t'ou | 頭, chên near Clı'ang-hua H., Kuangtung.

\section{砧}

4741 O-chia 碳嘉, (obs.) Yïan, H., near Cling-tung ' $T$., Yünnan; Iat. $24^{\circ} .32^{\prime}$, Long. $101^{\circ} 23^{\prime}$; orig., Hsii 虛.

\section{鄂}

4742 O 鄂, see Nos. 2159, 7052. 4743 O-chn | 渚, see No. 2159. $4743^{\circ}$ O-êrh-chê-yi-t” " | 尔哲 衣圖 Olchitu, town in Sainnoin ; Iat. $46^{\circ} 30^{\prime}$, Long. $99^{\circ}$.

4744 O-êrli-to-ssŭ | 爾冬斯 Ordos, Mongol tribe.

4745 O-k'ê-shih | 克什, T-S. near 'Ta-yi H., Ssŭch' nan.

4746 O-tuan | 端 Khoten, see No. 2057.

4747 O-wang-ch'êng | 王 城, (obs.) formerly town near Wu-ch' ang H., Hupel.

O-mi, see No. 20. 
Oelot, see No. 1602.

Oita, see No. 6023.

Okayama, see No. 3112.

Okbara, see No. 7206.

Oksu, see No. 11.

Olchitu, see No. $4743^{\mathrm{A}}$.

Origniod, see No. 7038.

Ordos, see No. 4744.

Osaka, see No. 6022.

Otrar, see No. 1604.

Ôtsu, see No. 6015.

\section{OU}

旉负

4748 On-yang 謁晹.

$1^{\circ}$ Y. near Chiang-hua H., Hunan.

$2^{\circ}$ T-S. near Hsia-chiang T., Kueichou.

\section{區}

4749 On-ning 唨媱 Ngal1-11ing, H. forming with Cliien-an H. the prefectural city of Chien-1ning, Fulkien; Lat. $27^{\circ} 04^{\prime}$, Long. $118^{\circ} 25^{\prime}$.

\section{藕}

4750 On-ch'il 藕池, chên near Shih-shou H., Hupeh.

4751 On-t'ang | 塘, chên near Lai-an H., Anhui.

Ouigour, see Nos. 7005, 7177.
Ourumtsi, see No. 6356. Outer Kansulu, see No. 6887.

Ozaka, see No. 6022.

\section{PA}

\section{巴}

$4752 \mathrm{~Pa}$ 巴 $\mathrm{Pa}$.

$1^{\circ}$ C. in Pao-ning Fin, Ssŭch' 'uan; Lat. $31^{\circ}$ $51^{\prime}$, Long. $106^{\circ} 45^{\prime}$; $T$ s'in, Shang-ku 上谷; $T^{*}$ ang, Yi-cling 空津;

$P$. Chou, Hua-clı' êng 化城.

$2^{\circ} \mathrm{H}$. forming the prefectural city of Chrungch'ing, Ssŭch' uan; Lat. $29^{\circ} 42^{\prime}$, Long. $106^{\circ}$ $42^{\prime}$; orig., K. Pa | $T s^{\prime} i n$, Chiang 江 C.; Minor Han, $\mathrm{Pa} \mid \mathrm{H}$; $T^{\prime}$ ang, Yü 濔 C.

$3^{\circ}$ (obs.) Chon and $T s^{\prime}$ in, $\mathrm{K}$. comprising part of Ssŭch" wan ; Han, Pa | chiin.

$4^{\circ}$ See Nos. 2301, 6093, 7615.

4753 Pa-ch'êng | 城, chên near Cl' ang-chou Fir, Kiangsu.

4754 Pa-chiang | 江 (obs.) S. of An-hua H., Kueichou; $T^{6}$ ang, H. in Man 戀 C. 
巴

PA-CH'IU.

4755 Pa-clı'iu | 邱, see No. 2765.

4756 Pa-ch'ou | 紬, T-S. near Sung-p'an 'T', Ssuch'uan. $4757 \mathrm{~Pa}^{-c_{1}}{ }^{6} \mathrm{i} \mid$ 渠.

$1^{\circ}$ (obs.) 65 li N.E. of K'ai H., Ssŭclı' ıan ;

S. Sung, H. in Pa-tung

| 東 chïn.

$2^{\circ}$ (obs.) N.E. of 'Tunghsiang H., Ssŭch' nan; S. Sung, H. in Pacli'i潗 chion; $T$ 'ang, H. in 'T'n11ng 通 C.; Sung, H. in Ta 達 C. $3^{\circ}$ See No. 6127.

4758 Pa-ch' uan | 川, see No. 2120.

4759 Pa-êrlı-ch'ih-li-han | 耳赤 利䚮, or Pa-êrh-chên 八 兒䓂, or, 八耳真, Barkhalighend, or Barchin, tow1 in the New Dominion, on the Silion.

4760 Pa-êrlı-ta-a | 耳 打阿 Bardaa, town in Persia.

4761 Pa-êrhl-chu-hai | 尔朱海, town in Inner Mongolia; Lat. $40^{\circ} 40^{\prime}$, Long. $109^{\circ}$ $25^{\prime}$.

4762 Pa-êrh-ch'11-'kê|尔楚克 Barchuk, town in Yarkand ; Lat. $39^{\circ} 10^{\prime}$, Long. $72^{\circ-} 25^{\prime}$.

4763 Pa-êrh-k'u | 尔庫, see No. 425.
巴 PA-LI-HEI.

4764 Pa-êrh-lı-kê | 尔 學 克 Barluk, town in Tarbagatai ; Lat. $45^{\circ} 25^{\prime}$, I.ong. $83^{\circ}$.

4765 Pa-lia-o-lo-su-t 'ai | 哈 鄂 羅蘇台 Urusutai, town in the New Dominion; Lat. $40^{\circ} 10^{\prime}$, Long. $93^{\circ}$.

4766 Pa-ho | 润, chên in Ch'iShui $H$.

4767 Pa-lisi | 西.

$1^{\circ}$ (obs.) IV. of Langclunng H., Ssŭch' nan; Tsin, and Sui, chün in Liang 梁 chOU.

$2^{\circ}$ (obs.) S. Sung, C. in I i-chon 利州 circ.

$3^{\circ}$ (obs.) Han and 7 sin, chün, comprising $\mathrm{W}$. part of $\mathrm{Pa} \mid$ chiül.

$4^{\circ}$ (obs.) formerly H., E. of Mien-chu H., Ssŭch'nan.

$5^{\circ}$ See Nos. 3631, 4433, 4951.

4768 Pa-kı11g | 公, ssŭ near Kao-p'ing H., Shansi; Kin, Pa-kung | 公 chên.

4769 Pa-la-ssŭ | 拉 斯 Barskoto, town in Tsetsen; Lat. $48^{\circ} 10^{\prime}$, Long. $113^{\circ}$ $25^{\prime}$.

$4770 \mathrm{~Pa}-$ li-hei | 里黑 (or Pili-han 必里罕, or Panlê-ho 班勒紀, or Pan-li 班里, or Pan 班, ) Balkh, town in Bokhara. 
世 PA-LI-K'UN.

4771 Pa-li-k'un | 里坤, see No. 2370.

4772 Pa-lin | 林 Barin.

$1^{\circ}$ Town in Inner Mongolia ; Iat. $43^{\circ} 50^{\prime}$, Isong. $118^{\circ} 10^{\prime}$.

$2^{\circ}$ Mongol tribe.

4773 Palling | 陵 Pa-ling.

$1^{\circ} \mathrm{H}$. forning the prefectural city of Yo-chou, Hunan; Lat. $29^{\circ} 18$,

I.ong. $113^{\circ} 03^{\prime}$; orig., 'Tung-ling 東陵; Han, Hsia-tsun 下售; Wu, Pa-ling | 陵.

$2^{\circ}$ Post-town near the above.

$477+$ Pa-11no| 巴莫 Bhan1o in Burmah; also written 巴 無. Popular name Hsin-chien 新街.

4775 Pa-pu | 補, or Pa-p'u 八 前, Babu, or Bapu, town in the New Donininion.

4776 Pa-shan 巴山.

$1^{\circ}$ Post-town near Pa-tung H., Hupeir.

$2^{\circ}$ (obs.) Sui, H., S. of Ch'ang-yang H., Hupel.

$3^{\circ}$ See No. 1591.

tio7 Pa-shih | f Bashi, town in Yarkand; Lat. $41^{\circ}$, I ong. $77^{\circ} 30^{\prime}$.

47 78 Pa-shui | 水, post-town

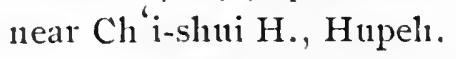

巴 Pa-Tuxg.

4779 Pa-ta-k'ê-shan | 達克山 (or, Pa-ta-ha-shang I. 答 哈 傷, or, Pa-ta-heishang 八 答 黑 商,) Badakshan town near the sources of the Oxus; Lat. $36^{\circ} 28^{\prime}$, Long. $71^{\circ} 23^{\prime}$.

4780 Pa-ta-ha-shang | 答 哈 傷, see preceding.

4781 Pa-t'ang | 咕 Bathang, ' S. near Ta-chien-lı 'T., Ssŭch' nan.

4782 Pa-ti | 底, T-S. near T’ien-cli üan C., Ssŭch'uan.

4783 Pa-tung | 東 Pa-tung:

[528] $1^{\circ} \mathrm{H}$. in Yi-clíang Fu, Hupelı; Lat. $31^{\circ} 02^{\prime}$, Long. $111^{\circ} 15^{\prime}$; Han, IVu 쪼. H.; Liang, Hsin-ling 信陵; Sui, Pa-tung | 東.

$2^{\circ}$ (obs.) N.E. of Fêngchieh H., Ssǔch 'uan; Tsiur and Sui, chün in Liang 梁 chou; $N$. Sung, chïn in Cling 荆 $\mathrm{C}$.

$3^{\circ}$ (obs.) N. W. of Patung H., Hupeh ; Sui, H. in Pa-tung | 東 chiun; T"ang, H. in Kuei 㷌 C.

$4^{\circ}$ (obs.) in Pa-tung H., Hupelı; Yüan and Ming, H.in Kuei 端 C. $5^{\circ}$ See No. 3491. 


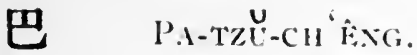

4784 Pa-tzŭ-ch'êng | 子城.

$1^{\circ}$ (obs.) Han, town near Chung C.. Ssŭclinan.

$2^{\circ}$ (obs.) Han, town near Ho C., Ssŭch 'uan.

4785 Pa-wa-êrh-ti |无兒的, or Pal-wa-êrh 八五耳. Bavurd, town of Persia, in the province of Kluorassall.

4786 Pa-wang | 旺, T-S. near 'T"ien-ch"üan C., Ssŭclinan.

4787 Pa-yang | 陽, post-town near Yün-yang H., Ssŭch 'nan.

4785 Pa-yen-jung-ke | 燕我格 [148] Pajenyunk, 'T. in Hsining Fu. Kansith; Lat. $36^{\circ} 10^{\prime}$, Long. $102^{\circ} 50^{\prime}$.

4789 Pa-yen-nang-ch'ien 翼謙, T-S. in Kokonor. 芭

4790 Pa-chiao-shan 芭蕉山 (or, Ma-tsu Miao 馬㓊嘲) Matsu, Island at the month of River Min. Fuhkien.

4791 Pa-lan | Kanibadan, town in Independent Turkestan, between Khodjend and Khokand.

\section{霸}

\section{$4792 \mathrm{~Pa}$ 霸 $\mathrm{Pa}$.}

$1^{\circ} \mathrm{C}$. in Shun-t ien Fu,

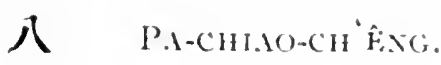

[119] and also included in the Pa-cli ang circ., Chilli, Irat. $39^{\circ} 08^{\prime}$, Jomg. $116^{\circ} 15^{\prime}$; Ts in. Slrang$k 11$ 上谷; $T^{*} a n g, \quad r i-$ ching 额津: : P. C hom, Pal $\mid \mathrm{C}$.

$2^{\circ}$ (obs.) Sung, C. nean Ion C.. Ssunch nan.

$3^{\circ}$ (obs.) Tinng, C. near Chung-ch ing lin, Siñchinan.

4793 Pat-Cliang | 昌, cire, in Chilili, comprising $\mathrm{Pa}$ C. and Ch' ang-p'ing C.

4794 Pa-ch' nan | 川, see No. 2120.

7795 Pa-ling | 陵, see No. 2691.

4796 Pi1-t on | 頭, ssur in P’ingviian $H .$, Kuangtuuy.

\section{拔}

$4797 \mathrm{~Pa}-1$ i-ssŭ 拔里寺 Barsic。 town in Persia.

\section{)}

4798 Pa-chai 八 寨 Pah-chai, 'T. in Tu-yün Fu, Kueichoul ; Lat. $26^{\circ} 05^{\prime}$, I ong. $107^{\circ} 55^{\prime}$.

4799 Pa-chi-ta | 吉打 (or, Paota 報達, ) Bagdad, a cit y of Ariatic 'lurkey; I.at. $33^{\circ} 21^{\prime}$, Long. $44^{\circ} 2 x^{\prime}$.

4800 Par-chiao-cli êng | 角城. post-town near Hsiin-hua T.. Kansulh. 
八 Pa-CHIAO-TIAO.

4801 Pa-chiao-tiao / 角碉, T. $T$. near Wêtr-ch' nan $H$., Ssŭclinan.

+802 Pa-ch'ih-chai | 尺學, ssŭ near Nan-ning Fu, Kuangsi.

4803 Pa-chou | 舟, T-S. near Hsia-chiang 'T., Kueichou. $4804 \mathrm{~Pa}$-êrh-chên |耳 畺, see No. 4759.

4805 Pa-êrh-chên | 兒真, see No. 4759.

4806 Pa-ha-la-yin | 哈刺因 Boharain, an island in the Persian Gulf.

4807 Pa-li-mang | 里范, town in Yarkand, N. of Ush.

4808 Pa-pu | 歩, Y. near Ta-tsu H., Ssŭch'uan.

4809 Pa-p u | 晢, see No. 4775 .

4810 Pa-ta-hei-shang 答黑婮, see No. 4779.

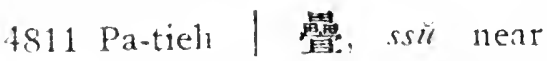
Hsin-ch ang H., Kiangsi.

4812 Pa-tu| 都, chên near Ch'ing-ÿüan H., Chelikiang.

4313 Pa-tu | 渡, chên near Ningr-hisia Fu, Kansuh.

$481+$ ya-t'un| 屯, T-S. near Sung-p'an T., Ssŭch 'nan. 4815 Pa-wa-êrh | 互耳, see No. 4785.
白 $\mathrm{PAI-CH}$ IH.

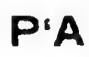

琶

4816 P'a-chiang 琶 江, ssй in Ch'ing-yüan H., Knangtung.

Pagoda Island, see No. 4021.

Pah-chai, see No. 4798.

\section{PAI}

\section{手}

4817 Pai 拜 Bai, see next.

4818 Pai-ch'êng | 城, H. in Wên-su C. L. C., New Dominion; formerly Pai city; Lat. $41^{\circ} 30^{\prime}$, Long. $81^{\circ}$.

4819 Pai-na-mu-ch'êng | 納木 城 Painamjung, town in Thibet; Lat. $29^{\circ}$, Long. $89^{\circ} 30^{\prime}$.

4820 Pai-ti | 底 Pai-ti, town in Thibet; Lat. $29^{\circ}$, I,oung. $90^{\circ} 30^{\prime}$.

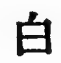

4821 Pai 白, see No. 5252.

4822 Pai-ch'êng | 城, chên near J.o-ch'nan H., Shensi.

4823 Pai-cl!'i | 旗, chên near Ta-p' ‘ H., Kuangtung.

4824 Pai-ch'ih | 池.

$1^{\circ}$ (obs.) E. of Ling 霝 C., Kansuls; T"ang, H. in Yen 鹽 C. 
白 PaI-CH'

$2^{\circ}$ (obs.) near P’ingliang Fu, Kansulh: $V$. Hei, H. in ChingCli êng 長城 chün.

4825 Pai-ch' uan | M, chïn in Huang-hai 黃海 circ. Corea.

4826 Pai-fu| 甫: chên near Mien-ch ih H., Honan.

4827 Pai-ful 仗, post-town near Ch'ien-chiang H., Hupeh. 4828 Pai-ho | 河 Peh-ho, H.in [133] Hsing-an Fu, Shensi; I.at. $32^{\circ} 35^{\prime}$, Long. $110^{\circ} 03^{\prime}$; Ts'in, Han-chung 漢中.

4829 Pai-ho-k'ou | 河 口, posttown near Pao-k'ang $\mathrm{H}$. Hupeh.

4830 Pai-bo | 鶴, post-town near Chiung C., Ssŭch' nan.

4831 Pai-hou | 猴, ssŭ in Tap'u H., Kuangtung.

4832 Pai-hu | 湖.

$1^{\circ}$ Chên near Huang-mei H., Hupeh.

$2^{\circ}$ Sš̆ near Wri-ch ang H., Hupeh.

4833 Pai-hn | 虎, Y. near Hsin-t'ien H., Hunan.

4834 Pai-lnua | 花, l. neat Chiang-hua H., Hunan.

483.5 Pai-hsia $\mid$ F, see No. 749.

4836 Pai-hsia | 霞, ssü near P'ing-lê Fu, Kuangsi.
日 PAI

4837 Pai-ko11 | 溝, post-town near An-su H., Chiluli.

t838 Pai-knng, 忩, (obs.) formerly fort near Yang H. Shensi.

4839 Pai-kung-ch'êng | 公 城. (obs.) formerly fort near Pao-ching Fin, Hunan.

4810 Pai-kno|果, chên near Ira-ch'êng H., Hupeh.

4841 Pai-li| 利, T'S. in Kokonur.

4842 Pai-lien | 蓮, post-toxn neal Knei-hna H., Fuhkien.

4843 Pai-lo 螺, ssü near Chiemli H., Hupel.

4844 Pai-1n | 路, T-S. near Mien-ning H., Ssŭch nan. 4845 Pai-lı | 傂, see Nos. 3538, 3599.

4846 Pai-lung | 龍, see No. 5983.

4847 Pai-m1a-kuan | 熙關, posttown near Ch'èng $H$, Kansuh.

4848 Pai-mang | 芒, Y. near Chiang-hua H., Hunan.

t849 Pai-mao | 荈, ssü in Chaowên H., Kiangsu.

48.50 Pai-mien | 面, ssin near Hsin-t ien H., Hunan.

4851 Pai-na 納, T-S. near Hsin-wên H., Kueichon.

4852 Pai-p'u | 蒲, chên near Ju-kao H., Kiangsu. 


\section{白 PAI-SHA.}

4853 Pai-shal | 沙.

$1^{\circ}$ Chên near Ch'ing-shni H., Kansulh.

$2^{\circ}$ Ss̆̌ in Yi-chang H., Hunan.

$3^{\circ}$ Post-town near Cli angyang $H$. , Hupeh.

$4^{\circ}$ Post-town near Mincling H., Inhkien.

4854 Pai-shan | Ill, T-S. near Ssŭ-ên Fu, Kuangsi.

4855 Pai-shên-miao | 䭪瀚, Y. near Hsiao-yi T., Shensi.

4856 Pai-shih | 市.

$1^{\circ}$ Chên near Chao-yi H., Shensi.

$2^{\circ}$ Post-town near Chungch'ing Fn, Ssüch' uan.

4857 Pai-shib | 不.

$1^{\circ}$ (obs.) $T^{\prime}$ ang, II., S. of Lung-y'u H., Chehkiang.

$2^{\circ}$. Sï̆ in Fin-an II., linhkien.

4858 Pai-shui / 水 Pell-shwui.

$1^{\circ} \mathrm{H}$. in ' $\mathrm{I}^{\prime}$ ung-chon I'u, [348] Shensi; Iat. $35^{\circ} 10^{\prime}$, I.ong. $109^{\circ} 30^{\prime} ; S . A$., P'êng-wei 彭衛; \%' $\mathrm{in}$, Pai-slun | 水; $T^{*}$ ang, Sil-yi 粟邑。

$2^{\circ}$ Chên near P'ing-liang Fu, Kansull.

$3^{\circ}$ Chênnear Kuang-ch' ang $\mathrm{H}$., Kiangsi.
白 PAI-YÜ.

$4^{\circ}$ Ss $\breve{u}$ in Nan-ning $\mathrm{H}$., Yünnan.

$5^{\circ}$ See No. 7769.

4859 Pai-ti-ch'êng|管城, (obs.) formerly fort $\mathrm{E}$. of $\mathrm{K}^{\text {'nei- }}$ chon Fn, Ssŭch' nan.

4860 Pai-t $n$ |. .

$1^{\circ}$ Chên near 'I'ai-ho H., Kiangsi.

$2^{\circ}$ See No. 1237.

4861 Pai-t' $n$ | 息, chèn near Chï-jung H., Kiangsu.

4862 Pai-wên | 文, chên near Hsing H., Shansi.

4863 Pai-yang | 陽, chên near Yi-yang H., Honan (No. 7441).

4864 Pai-yang-clı'êng | 羊城, (obs.) formerly fort W. of Ta-t 'ung Fu, Shansi.

4865 Pai-yang-shan | 羊山, chên near Ch'ung-yang $\mathrm{H}$., Hupeh.

4866 Pai-yang | 洋, chên near 'T'ao-yüan H., Kiangsı.

$4 \$ 67$ Pai-yen-ching| 監井 Pehrent-ting, important saltwells in Yünnan, near $\mathrm{Cl}_{1}{ }^{\prime} 1$-1 cinng Fu; Lat. $26^{\circ}$ $10^{\prime}$, Long. $101^{\circ}$.

4868 Pii-yüan | 元, chên near Yi-yang H., Honan (No. $7695)$.

4869 Pai-ıïn | 雲, post-town near Hsing-an H., Knangsi. 
栢

PaI-ching.

\section{柏}

4870 Pai-ching 柏井, post-town near $P$ 'ing-ting $C$., Sliansi.

4871 Pai-jên |人.

$1^{\circ}$ (obs.) W. of 'T'angshan H., Chihli; Han and $T \sin , \mathrm{H}$. in $\mathrm{K}$. Chao 趋.

$2^{\circ}$ (obs.) in Kiangstt ; S. Sung, H. in Tungknan 東莞 chün.

$3^{\circ}$ See No. 6253.

48 I Pai-jên1仁, see No. 6253. 4873 Pai-lin | 林, post-town near Ningr-chiang C., Shensi.

4874 Pai-lin-kon | 林溝, posttown near Chao-lnua H., Ssŭch' 'uan.

4875 Pai-yang | 陽, ss̆̌ near Kuang-hsin Fu, Kiangsi.

4876 Pai-ฐüan | 源, (obs.) near Ju-ning $\mathrm{Fu}$, Honan; $S$. $T s^{3} i$, Pai-ÿüan | 源 $\mathrm{H}$.

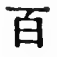

4877 Pai-chang 百文, posttown near Ming-shan $H$, Ssŭch "ran.

4878 Pai-chang-knan | 死關, ss̆̈ near Kuang-ÿüan $\mathrm{H}$, Sxн̆сl' "nan.

4879 Pai-chi | 溜, (obs.) Han and $T$ 'ang, K. in S. W. Corea.
栢

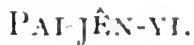

1880 Pai-cliat | 芜, chên near Ch'ing-shui H., Kansulı.

$4 S 81$ Pai-clith | 只, chên near Ven-ch'ang H. Shensi.

4882 Pai-lı11 湖, chên in W11cling H., Ilıpel.

4883 Paj-knan | 笛, chên near Yäi-yas H., Clleiskiang.

4884 Pai-li | 利, T-S. near ' Yachien-lı T., Sis̆̌ch' naı.

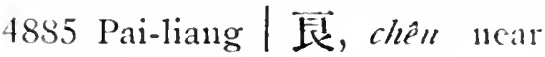
Ho-yany H., Slieusi.

4886 Pai-lon | 樓, (obs.) $T$ 'an.s, fort, S.E. of 'Ting-hsings H., Chihli.

4887 Pai-sê | 出 Pelı-sih, C. I. [416] $\mathrm{T}$. in the Yu-chiang circ., Kuangsi ; I,at. $24^{\circ}$, I,ong. $106^{\circ} 20^{\prime}$.

4888 Pai-slıun | 順, ss̆̌̆in Nanlisinng C.., Kuangtung.

4889 Pai-yi | 径, chên near Chung-pu H., Shensi.

4890 Pai-yüelı | 畧, see No. $3+21$.

\section{栢}

4891 Pai 栢, see No. 2687.

4892 Pai-hsiang/挀 Peln-hiang, [459]H. in Chao C., Chilli; I at. $37^{\circ} 32^{\prime}$, Long. $114^{\circ}$ $50^{\prime}$; Han, Hao 鄗 H.; Sui, Pai-hsiang | 拫.

4893 Pai-jên-yi | 人邑, see No. 6253. 


\section{排}

$\mathrm{P}^{\prime} \mathrm{AI}-\mathrm{PC}$.

\section{P'Al}

\section{排}

4894 P'ai-pu 排拒, ssi near Hsin-ch ang H., Kiangsi.

+895 P'ai-shan / 山、 sš̆ near Hêng-chon Fin, Hunan.

4896 P'ai-shih| 市, chền near Hsing-kuo C.. Hupelı.

+897 P'ai-t'on|頭, chên near Chu-chi H., Chelıkiang.

\section{牌}

4898 P’ai-chou 薭州, ss̆̈ in Chia-yii H., Hupeh.

Painamjung, see No. 4819. Pajenyumk, see No tiss. Pakhoi, see No. 4988.

\section{PAN}

\section{板}

4899 Pan-ch'ino 板橋, chên near L,i-shni H., Kiangsu; also post-town near lüunman H., Y'ünnan.

4900 Panl-li | 栗, Y. near Linwu H., Hunan.

4901 Pan-p u| 浦, chê"n near Hai C., Kiangsu.

1902 Pan-slih | 石, ssin in Anyüan H., Kiangsi.

\section{辦}

1903 P'ill 辦, see N. 2194.

\section{班}

$190+$ I’an 班, see No. 4770.
盤 P'AN-KUAN.

t905 Pan-yu | 侟, 'T-S. near Sung-p'an T., Ssŭch'nan.

1906 Pan-lê-ho| 勒䋁, see No. 4770.

\section{站}

4907 Pan1-iil 站里, see No. 4770. 4908 Pan-ch'in 站渠, (obs.) in Kueichon; $T \sin , H$. in T'sang-kê 羘㟃 chün.

畔

1909 Pan-kuan 使觀, see No. 3365.

\section{P'AN}

\section{般}

4910 P'an-vang 般陽, see No. 6834.

$4911 \mathrm{P}^{\prime}$ an 盤.

$1^{\circ}$ (obs.) T'ang, C. in the present P'n-an 'T., Kueichon, comprising parts of N.E. Yünnan and Kueichon.

$2^{\circ}$ See Nos. 5291, 7524.

4912 P'all-chiang | 江.

$1^{\circ} \mathrm{Y}$. near An-11an H., Kneichon.

$2^{\circ}$ (obs.) formerty $\mathrm{H}$. near Chan-yi C.. Yümman.

1913 P'an-hsiao / 滧, chên near Ch' img-ming H., Kiangsu.

4914 P'an-k $^{\prime}{ }^{\circ}$ | 客, chên near Ning C., Kansul.

t915 P'an-kuan | 關, ssin near Ning-ch" iang C.. Shensi. 


\section{盤}

P'AN-LUNG.

4916 P'an-hung / 龍.

$1^{\circ}$ Chền near Ch'ungming $\mathrm{H}$., Kiangsu.

$2^{\circ}$ See No. 3359.

4917 P'an-p'o / B B, (obs.) $W$. Wei, town near Chingshan H., Hupeh.

4918 P'an-shan | 山, chên near Hsin-yen 'T., Shingking.

4919 P'an-shêng | 勝, Y. near T'ung-jên Fu, Kueichon.

4920 P'an-shih | 石, see No. 6840.

$4921 \mathrm{P}^{\prime}$ an-tao $\mid$ 道, (obs.) $W$. Wei, H., W. of Nanchiang H., Ssŭch'uan.

4922 P'an-ti | 堤, see No. 4923. 4923 P'an-ti | 隄, or | 堤, (obs.) 70 li S. E. of Chieh C., Kansuh; Sui, H. in Wu-tu 武 都 chün; $T^{6}$ ang, H. in Chieh 階 C.

\section{瀊}

4924 P'an-la 瀊拉, 'T-S. near Hsün-hıa T., Kansuh.

\section{番}

$4925 \mathrm{P}^{\prime}$ an 番.

$1^{\circ}$ (obs.) T’ang, principal city in Kao-chou Fu, Knangtung.

$2^{\circ}$ See Nos. $1450,3393$.

4926 P'an-shni | 水 (or, Fanshiui 憣水) (obs.) T'ing, H., N. W. of Kao-chou Fu, Kuangtung.
邦 PANG.

4927 P':m-yัu | 禹 Pwan-yัï.

$1^{\circ} \mathrm{H}$. forming with Nanhai $H$. the prefectural city of Kuang-chou, Knangtung; I I ilt. $23^{\circ}$ $07^{\prime}$, l.ong. $113^{\circ}$ 15';

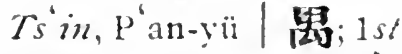
Sung, Hsi-an 熙年 and Huai-hua 䝥化; $T^{6}$ ang, P'an-yü|眮 $\mathrm{H}$.

$2^{\circ}$ (obs.) s. Is. of the preceding; Han and $T \sin , \mathrm{H}$. in Nan-lnai 南海 chün; now, Nanhai H., Kuangtung.

$3^{\circ}$ See No. 4573.

\section{潘}

$4928 \mathrm{P}^{\prime}$ an 潘, see No. 450 t.

$4929 \mathrm{P}^{\prime}$ an-t ien | 由, ssü near Ch'ao-chou Fu, Kuangtung.

4930 P'an-ÿïan | 原, (obs.) 40 li E. of P'ing-liang $\mathrm{Fu}$, Kansuh: $T^{\prime}$ ang, Sung and Kin, P'an-yüan | 源 $\mathrm{H}$.

\section{PANG}

\section{柊}

4931 Pang-lo 杵羅, shên near Ti-tao C., Kansulh.

\section{邦}

4932 Pang 陣, (obs.) near siur nan Fu, Kueichon; $T^{\prime} a n g$. C. in Chiang-nan 江南 
circ.; Sung, C. in Shancing 紹慶 Fin。

l’ăng, see Nos. 5046, 5058. P’ăng-hu, see No. 5057. Păng-ki, see No. 5059. P’ăng-lai, sce No. 5062. Păng-shan, see No. 5049. Păng-shwui, see No. 5051. Păng-tseh, sec No. 5052.

\section{PAO}

\section{算}

1933 Pal1-i11 琵发: see No.6725. 19.3. Pao-cli êng | 城.

$1^{\circ}$ Chül in ( h ' 政 circ., Corea.

$2^{\circ}$ (ohs.) formerly town near Pi H., Ssüch wan.

\$935 Paro-ehi | 雞 Pan-ki, H. [+98] in Fêng-hsiang Fu, Shensi; Lat. $34^{\circ} 20^{\prime}$, I.ong. $107^{\circ}$ 13'; Ts'in, 1s/, Ch' ên-

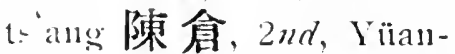
chin 苑 川; P. Chon, Hsien 顯 H.; T゙ang, Paochi | 雞.

1936, Pao-ch'ils | 坻 Pant-ti, H. [191] in Shun-t ien Fu, Chihli; Lat. $39^{\circ} 45^{\prime}$, Long. $117^{\circ}$ $12^{\prime} ; H a n$, Ch 'üan 家 $\mathrm{C}$. fin, Pao-chin|坻 $\mathrm{H}$. in 'Ta-hsing 大興 Fur . Ming, H. in 'T”nng 通 C.
4937 Pao-ch ing | 慶 Pau-king, [490] Fu in the Ch' ang-Pao circ., Hunan; forming also the district city of Shao-yang: Lat. $27^{\circ} 04^{\prime}$, Long. $111^{\circ}$ $21^{\prime}$; S. A. and C. Wars, in K. Ch'n 楚; $T s^{\prime} i m$ and $H / n n$, in Ch'ang-sha 長沙 chiï； " $"$, Shao-ling 邵 陵; Sui, Chien 建 C.

4938 Pao-fêng | 豐 Pau-fung.

$1^{\circ}$ H. in Ju C., Honan; [384] I,it. $33^{\circ} .55^{\prime}$, I.ong. $113^{\circ} 06^{\prime}$; S. A., in $\mathrm{k}$. Chêng 奚; Han, Finch'êng 父城; でang, I.11ng-hsing 龍 興; Simng, Pao-fêng $\mid$ 豐 H. $2^{\circ}$ (obs.) F. of ' $T$ 'ung-shan H., Kiangsu; Sung, fort in Hsü 徐 C.

4939 Pao-jên | 仁, ssŭ near Hoyiiau H., Kuangtung.

4940 Pao-ning | 宇 Pan-ning, H. forming the prefectural city of Kuang-11an, Yünnan; Lat. $24^{\circ} 10^{\prime}$, Long. $105^{\circ} 06^{\prime}$.

1941 Pao-shan / Il Pan-shan.

$1^{\circ}$ H. in 'T'ai-ts'ang C., Kiangsu ; Lat. $31^{\circ} 15^{\prime}$, i.ong. $121^{\circ} 30^{\prime}$.

$2^{\circ}$ (obs.) Yüan, C. near I,i-chiang Fu, Yïnnan; Han, Hsieh-ling 邲龍; Yü̆u, Pao-shan | II C. 
䫍

PAO-IINC:

4942 Pao-ting | 鼎, see No. 3048.

4943 Pao-t ing | 宣, ssh̆ in I,ingshui H., Kuangtung.

4944 Pao-wu| 蔜, see No.1023. 4945 Pao-ying | 應 Pan-ying, [322] H. in Yang-chon Fin, Kiangsu; I at. $33^{\circ} 04^{\prime}$, Long. $119^{\circ} 22^{\prime} ; H_{q u}$, Anp'ing 安平; Liang, Yangping 陽 $4 ;$; $7^{*}$ ang and Sung, Pao-ying | 應 C. Pankin (Polo).

\section{保}

4946 Pao 保 Pan.

$1^{\circ}$ (obs.) Ming, H. in Clı êng-tı Fin, Ssur ch' 'ran; Lat. $31^{\circ} 42^{\prime}$, Long. $103^{\circ} 32^{\prime} ; T^{\prime} a n g$, Hsïeh-ch êng 薛城。 Mêng-shu 孟蜀 and Palo-ning | 耍.

$2^{\circ}$ (obs.) Yüan, C. near Ion C., Ssŭch' nan.

$3^{\circ}$ See No. 4955.

4947 Pao-an | 㚣 Pan-ngan.

$1^{\circ}$ C. in Hsïan-lina Fin, [.376] Chilhli; Lat. $10^{\circ} 22^{\prime}$, l.ong. $115^{\circ} 14^{\prime}$; orig., in K. Ven 灌; \% in Slang-ku上 谷 - achïll Han, Ch10-l11 泳 鹿; T゙ang, Hsin 啋 C.; Licro, Wu-ting 武定 and Fêng-sleêng 奉聖:
保 PAO-NING

Kin, 'Iê-hsing 德 與; Ming, Palo-an 安 C.

$2^{\circ}$ H. in Yen-an Fn, Shensi; Iat. $37^{\circ} 02^{\prime}$, Long. $108^{\circ} 37^{\prime}$; Han, in Shang 上 chïn; T'ang, Yung-k' ang 水 康; Sing, Pao-an| 安.

$3^{\circ}$ Chên near Chung-pn H., Shensi.

4 (obs.) Liang, H., E. of 'lien-pai H., Knangtung.

4948 Palo-chlang|昌 Pall-chang. $1^{\circ}$ (obs.) formerly H. in Nan-lisinug C., Kuangtung; Lat. $25^{\circ} 12^{\prime}$, Long. $113^{\circ} 55^{\prime} ; T s i n$, Shilh-hsing 始 興; T'ang, Chêng-chl'ang 湞昌; Sung, Paoch'ang | 昌.

$2^{\circ}$ See No. 4585.

4949 Pao-ching | 站 Pan-tsing; [21] H. in Vumg-shnn Fin, Hunan ; I at. $28^{\circ} 45^{\prime}$, I,ong. $109^{\circ} 31^{\prime} ;$ Sui, Ch'ên 辰 C.; T"ang, in Cli i 海 C.; Sung, Pao-ching | 靖nililitary district.

4950 Pao-k'ang | 康 Pan-kanng, [313] H. in Yün-yang Fin, Hupelı ; Lat. $31^{\circ} 54^{\prime}$, I ong. $111^{\circ} 15^{\prime}$.

4951 Pao-ning | 寀 Pan-ning. 
保

$I^{\circ}$ Fn in the Ch nan-pei 30] circ., Ssŭch uan; forming also the district city of Lang-chung; Lat. $31^{\circ} 32^{\prime}$, Long. $105^{\circ}$ $59^{\prime}$; S. A., in K. $\mathrm{Pa}$ 巴; Han, Pa-hsi 巴 넌 : Tsin, Lang-chung 閖中; Liang, Nanliang 南梁; W. Wei, Lung 隆 C.; Sung, Antê 安德; Yüan, Рaoning | 管.

$2^{\circ}$ H. in Chung-ch'ing 忠 清 circ., Corea; Lat. $36^{\circ} 55^{\prime}$, Long. $125^{\circ} 30^{\prime}$.

$3^{\circ}$ Post-town near $\mathrm{Hni}$ ning H., Kansuh.

$t^{\circ}$ (obs.) Sung, H., N. of Ilon C., Ssŭch nan.

$5^{\circ}$ (obs.) T'ang, H., E. of Tien-pai H., Knangt1111g.

4952 Pao-shan | Ill Pan-shan, $H$. forming the prefectural city of Yung-ch'ang, Yünnan; I,at. $25^{\circ} 05^{\prime}$, Long. $99^{\circ} 26^{\prime}$.

4953 Pao-tê | 德 P'an-teh, C.l. [148]C. in the Yen-P'nng-Taso - Ning - Hsin - Tai - Pao circ., Shansi; I, at. $39^{\circ}$ $05^{\prime}$, Lon1 . $110^{\circ}+9^{\prime}$; orig. lan 虫 C.: Simng, Pao-té | 德 C.

4954 Pao-tien | 甸, T-S. near Shun-1111g Fu, Yünnan.

\section{遚 PAO-CHUNG.}

4955 Pao-ting | 定 Pau-ting. $1^{\circ} \mathrm{Fu}$ in the Ch ing-ho [92] circ., Chihli; forming also the district city of $\mathrm{Ch}$ 'ing-yüan; Lat. $38^{\circ}$ $53^{\prime}$, Long. $115^{\circ} 36^{\prime}$; capital of the province ; C. Wars, in $\mathrm{K}$. Chao 趙; $T$ s'in in Shang-

- $\mathrm{kn}$ 上谷 chün and Chü-lu 䤧 鹿 chün; Han, Cho 涐 chün and Hsin-tu 新 都; Sui, Ch'ing-yüan 清苑; Sung, Pao | C.; Ming, Pao-ting | 定 Fin.

$2^{\circ} \mathrm{H}$. in Shun-t'ien Fu, [119] Chihli; Lat. $39^{\circ} 02^{\prime}$, Long. $116^{\circ} 25^{\prime}$; Sung, Pao-ting | 定.

$3^{\circ}$ (obs.) Liang and $T^{\prime}$ ang, H., H. of Tienpai H., Kuangtung.

\section{襄}

4956 Pao-ch'êng 鼠城 Pan[391] ching, H, in Han-chung Fu, Shensi ; Lat. $33^{\circ} 04^{\prime}$, Long. $107^{\circ} 07^{\prime} ;$ Han, Pao-chung | 中 or 唩中; Sui, Pao-ch'êng | 城.

t95i Pao-chung | 中, see preceding.

\section{突}

4958 Pao-chung 裹中, (obs.) 10 li E. of Pao-ch'êng H., Shensi: Han and Tsin, 
H. in Han-chung 漢中 Ful ; see No. 4956.

\section{豹}

4959 Pao-t ao 豹慞, Y. near Chiang-hua H., Hunan.

\section{報}

4960 Pao-ên 報 恩 Pan-ngăn, H. in Chung-ch ing 忠清 circ., Corea; L,at. $36^{\circ} 50^{\prime}$ Long. $127^{\circ} 50^{\prime}$.

4961 Pao-ta | 達, see No. 4799.

\section{包}

4962 Pao-chiang 包港、ss̆̈ in Tan-yang H., Kiangsu.

4963 P:10-tso | 坐, I'-S. near Sung-p : :11 T., Ssŭch 'uan.

4964 Pao-tz̆̆-ssŭ| 子寺, '个-S. near sung-p'an 'T'., sisŭchinan.

\section{抱}

4965 Pao-ch'nan 抱川, shïn in Ching-ch i 京畿 circ. Corea.

4966 Pao-han 抱品, see No. 2062.

\section{鮑}

4968 Pao-tien 鮑店, chên near 'T"un-lin H., Shansi.

薄

4969 Pao-chih 薄 知、(ob). near Turfan: T゙rng, C. in Yüeh-chil 月支 Fur.

4970 Pro-sha | 沙, siur in T:an C., Kuangtung.
Parthia, see No. 71.

Pau, see No. 4946.

Pau-chang, see No. 4948.

Pan-ching, see No. 4956.

Pall-fung, see No. 4938.

Pall-kang, see No. 4950.

Pau-ki, see No. 4935.

Pall-kin, see No. 49+5.

Pau-king, see No. 4937.

Pall-11gan, see No. 4947.

Pau-ngăn, see No. 4961.

Pan-ning, see Nos. 4940, 4951.

Pall-shan, see Nos. 1941, 4952.

Pant-teh, see No. 4953.

Pantti, see No. 4936.

Pall-ting, see No. 495.5.

Pall-tsing, see No. 4949.

Pall-ying, see No. 4945.

Pedne, see No. 5236.

Peli-hiang, see No. 4892.

Pehr-ho, see No. 4828.

Peh-lii, see No. 5001.

Peh-shwui, see No. 4858.

Peh-siln, see No. 4887.

Peh-ren-tsing, see No. 4867.

\section{PEI}

焙

49-2 Pei-shui 焙 水, thên near I,in-chon Fu, Kuangsi.

\section{具}

$4 y-3 \mathrm{Pei}$ 具, see Nos. $12+5$. 1606. 
具 Pri-ch'

4974 Pei-ch'in | 邱, see Nos. $1250,6834$.

\section{北}

4975 Pei 北, chên near 'Têngchou Fu, Shantung.

4976 Pei-chai|些, ss̆̈ in Chiehyang $\mathrm{H}$., Kuangtung.

497 7 Pei-ch ên | 除.

$1^{\circ}$ (obs.) in Ch ên-chon Fin, Honan; S. $T s^{6} i$,

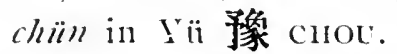

$2^{\circ}$ (obs.) in Honan; $S$. Ts' $i$, H. in Pei-liang

梁 chïn.

$3^{\circ}$ (obs.) in Anluni; $N$. IVei, 1st, chün in $\mathrm{Ho}$ 合 cHOU, 2nd, chün in Jang 掦 croc, 3 rd, H. in Pei-liang | 梁 chïn.

$4^{\circ}$ (obs.) near Ju-ning Fu, Honan ; $N$. IV ei, chïn in Ho 霍 CHOU.

4978 Pei-chi-yin | 㳯㓌, see No. 5467.

4979 Pei-chiao | 菱, ssŭ near I,ien-chiang $H$. , Fulıkien. 4980 Pei-ch'iao / 譙, see Nos. $1397,1484$.

4981 Pei-ching | 京, see Nos. 5822, 6336.

4982 Pei-ch ing | 靑, Fu in Hsien-ching 咸鏡 circ., Corea.
北 Pei-hSIA-P'EI.

4983 Pei-ch ‘ü | 属, see Nos. 583, 6069.

4984 Pei-ch' 'nan | 川, 'T-S. near Hsi-ning Fu, Kansuh.

4985 Pei-ch ‘ueh | 閣, see No. 5822.

4986 Pei-fon | 涪, chên near Fêng-tu H., Ssŭch 'nan.

4987 Pei-ful-fêng|扶風 (obs.) in Nan-chêng H., Shensi; S. Sung, chim in $\mathrm{Ch}^{\text {in }}$ 秦 cHOU.

4988 Pei-lai | 海.

$1^{\circ}$ Pakhoi, ss̆̈̆ near Lienchon Fu, Knangtung; one of the ports open to foreign trade. In Lat. $21^{\circ} 30^{\prime}$, Long. $109^{\circ} 13^{\prime}$. See No. 1365.

$2^{\circ}$ See No. 7003.

4989 Pei-ho-tien / 河店, posttown near Ting-lising $\mathrm{H}$., Chihli.

4990 Pei-huai | 淮, (obs.) in Kiangsu ; S. Sung, chün in S. Yen 党 chov.

4991 Pei-hunng-nung| 弘農, (obs.) in Honan ; S. Ts $i$, chiiln in Ning-man 察蠻 Fin.

4992 Pei-hsia-kuan | 下關, ssŭ near L,ï-chiang H., An1111 .

4993 Pei-hsia-p'ei|下䄧, (obs.) in Kiangsu; $S$. Sung, chiin in S. Yen 兊 chou. 
北

Phi-hisiati.

4994 Pei-lnsiang | 拫, ss $\breve{u}$ in I ang-ch'üan H. near I,ienhua T., Kiangsi.

4995 Pei-lisin | 新.

$1^{\circ}$ (obs.) formerly town near An-su H., Chihli. $2^{\circ}$ See No. 83.

4996 Pei-lising | 杏, see No. 6742 .

4997 Pei-1sii | 徐, see No. $7+61$.

4998 Pei-kê|格, chên near Hsïkon H., Shansi.

4999 Pei-ku | 谷, town in the I,iul-ch'iu Islands.

5000) Pei-li | 黎, Y. near Ch'ang-hua H., Kuangtuing.

5001 Pei-liu | 流 Pel-liu, H. [457]in Yüi-lin C., Kuangsi; Lat $22^{\circ} 45^{\prime}$, Long. $110^{\circ}$ $30^{\prime} ;$ Sui, Pei-liu | 流 H.

5002 Pei-l11-11111 | 陸渾, (obs.) near Yü-lin Fu, Slıensi; T'ang, Fi1 in Knan-nei 關內 circ.

5003 Pei-mêng | 蒙.

$1^{\circ}$ (obs.) in Honan; $S$. $T s^{\prime} i$, H. in Pei-liang | 梁 chün.

$2^{\circ}$ (obs.) in Anl111i; $N$. Wei, H. in Pei-liang |

- 梁 chïn.

5004 Pei-mien-ch'ih | 淹池, see No. 574.
北

PHil-II.

51005 Pei-pa-11.si | 巴西, (obs.) II. of Iang-chming $H$., Siñch ıan ; s'. Sung and S. Ts $i$, chän in I,iang 梁 curot.

5006 Pei-p êng | 蓬, (obs.) in Ya-chon liu, Ssŭch uan: sung, C. in Ch'êng-tn 成都 cire.

5007 Pei-p'ing $\mid$ 庐, see Nos. 4326, 5822, 6909, 7965.

joos Pei-slia | 沙, chên near Fin-11ing H., Kiangsu.

5009 Pei-shan | 山, sš̆ near Kuei H., Kuangsi.

5010 Pei-shêng | 勝, T. C. near Yung-pei 'T., Yünnan; I at. $25^{\circ} 5 t^{\prime}$, I,ong. $101^{\circ}$ $14^{\prime}$; see No. 7964 .

5011 Pei-shih-hsin | 始 新, (obs.) near Liu-an C., Anhui; N. Wei, H. in lïi-chiang 盧江 chün.

5012 Pei-tai-11o|帶河 seaside resort in the neighbourhood of Shan-hai Kuan, frequented by Europeans.

5013 Pei-ti | 地.

$1^{\circ}$ (obs.) Ts'in, chïn in Kansul.

$2^{\circ}$ See Nos. 1191, 1496, 5171.

5014 Pei-ti | 堤, (obs.) $80 \mathrm{li}$ S.Ë. of Ling-pao H., Honan; N.Wei, H. in Shihchêng 石战 chün. 
北

S015 Pei-t ien| 田. chên near Kilo-ling H., Shensi.

$5 n 16$ Pei-ting | 庭, see No. 5099.

5017 Pei-tro| 伎, ss̆n in Kneichn H., Kneichon.

5018 Pei-tu| 杜. chên near Hsien-yang H., Shensi.

5019 Pei-tun| 屯, chên near Ching-yang H., Shensi.

5020 Pei T'nung-chon | 通州, see No. 6768, $1^{\circ}$.

5021 Pei-t'ung-yao | 峝㺕, ssu near Jo-ting C.. Knangtung.

5022 Pei-tzŭ-t'nng | 梓滰、 (obs.) in Ssŭch nan ; S. $T s^{\prime} i$, chïn in I,iang 梁 CHOL.

5023 Pei-wu $\mid$ 巫, (obs.) near Vün-rang Fin, Hupeh: $T s i n$, S. Sung and $S$. $T s^{\prime} i, \quad H$. in Shang-yung 上庸chün。

5024 Pei-yang | 陽, see No. 6246.

5025 Pei-ri-ch'un|宜春, (obs.) 60 li S. W. of Ju-yang H.. Honlan: Han and $T \sin$, H. in Ju-nan 汝南 chion.

5026 Pei-yü 舆, (obs.) W. of Kuei-hna 'T., Shansi; Han, H. in Vün-chung 雲中 chün。
利 P'EI.

\section{扸}

5027 Pei 北, (obs.) Chou. fendal state $\mathrm{N}$. of Weihi1i Fin. Honan.

\section{早}

5028 Pei 卑 (obs.) near Ninghsia Fu, Kansuh; T"ang. C. in Kuan-nei 關內 circ. 5029 Pei-lu| 盧, (obs.) near Ya-chou Fu, Ssŭchinan; Sung, C. in Ya 雅 cног. 5030 Pei-lï / 盧: (obs.) in Yachon Fin, Ssŭch uan; T'ang, C. in Chien-nan 劍南.

5031 Pei, see Nos. 1780, 5035, 50.37 .

\section{P'El}

\section{粦}

[This character may also be read FEI.] $5032 \mathrm{P}^{i}$ ei-ch'êng 裴娍, (obs.) formerly fort $\mathrm{E}$. of Mei C., Ssŭch 'иau.

5033 P'ei-cliia | 芜, Y. near P'ing-fan H., Kansuh.

$503+$ P'ei-shê / ĩt: chên near Hsia H., Shausi.

\section{䄮}

$5035 \mathrm{P}^{\prime}$ ei 䄮 Pei.

$1^{\circ}$ C. in Hsü-choil $\mathrm{Fu}$, Kiangsu; I.at. $34^{\circ} 30^{\prime}$, Long. $118^{\circ} 26^{\prime} ;$ Hsia, 
丕

K. P'ei |: $T$ s'in, Hsiap'ei T|; P. Chou and Yüan, P'ei $\mid \mathrm{C}$.: Ming, P'ei | H. Piju (Polo).

$2^{\circ}$ (obs.) 3 li E. of the above: Kin, C. in Shan-tung 川東 circ.

$3^{\circ}$ (obs.) Hsia, K. in Kiangsu.

\section{懷}

5036 P'ei-hua 栾化, ssü near Ch'ên-ch'i H., Hunan.

\section{沛}

$5037 \mathrm{P}^{\mathrm{e}}$ ei 沛 Pei.

$1^{\circ} \mathrm{H}$. in Hsii-chon $\mathrm{Fu}$, [407] Kiangsu; Lat. $34^{\circ} 49^{\prime}$, Long. $117^{\circ} 10^{\prime}$; orig., feudal state of Pi-yang 偪陽; $T s^{\prime}$ in, $\mathrm{P}^{\prime} \mathrm{ei} \mid \mathrm{H}$. and chün.

$2^{\circ}$ See No. 2242.

\section{徘}

5038 P'ei-hui 徘䧃, chên near Ning-w11 Fu, Shansi.

\section{陪}

5039 P'ei 揢, (obs.) in Ssŭch'uan; $T^{6}$ ang, C. in Lung-yu 践右 circ.

Peking, see No. 5822.
彭 $P^{\prime} \hat{F} \times G$.

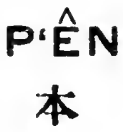

5040 Pên-pn 本部, town in the I.iu-ch'iu Island.

笨.

5041 Pên-chiang 笨港, posttown near Chia-vi $\mathrm{H}$. Fullkien.

\section{奔}

5042 Pên1-niu 㚏牛, chên in Wu-chin H., Kiangsu.

\section{P'EN}

\section{盆}

5043 P'ên-t'ing 盃亭, ssĭ near P'n-ch'êng H., Fuhkien.

$504+$ P'ê11-yïn $\mid$ 无, (obs.) Tsin, H., I: of Hsin-hui H., Kuangtung.

\section{湓}

5045 P'ên-ch'êng 湓城, see No. 6312.

Penang, see No. 5131.

Penegue, see No. 4728.

\section{P'ÊNG}

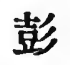

5046 P'ênng 彭 Pång.

$1^{\circ} \mathrm{H}$. in Ch'êng-tu Fu, Ssŭch uan; Lat. $30^{\circ}$ $56^{\prime}$, Long. $104^{\circ} 02^{\prime}$; 
mrig., K. P'êng |; liang, Tung-yi 東监; Sui, \êng 濛 C.; T'ang and s. Sung. P'êng | C.

$2^{\circ}$ (obs.) formerly K. in Ssücli nan.

$3^{\circ}$ (obs.) formerly C. near Chên-yuian H., Kansulı. $50+7$ P'êng-ch'êng | 城.

$1^{\circ}$ Chên, near 'T\%' ŭ C., Chillit.

$2^{\circ}$ Post-town near 'T'iennên H., Hupeh.

$3^{\circ}$ See No. 2902.

$5048 P^{\prime}$ êng-ch'i-ch'êng | 綺城, (obs.) formerly fort near I.ê-p "ing H., Kiangsi.

5049 P'êng-shan | Ul Pangshan, H. in Mei C., Ssŭch' 'na11; Lat. $30^{\circ} 15^{\prime}$, I tong. $103^{\circ} 4^{\prime}$; Ts'in. Wu-yang 武晹; Liang, Ling-shih 靈不; W. Wei, Cliang 江 C.; P. Chou, Lung-shan 隆山; $T^{\prime}$ ang, P'êng-shan | III.

5050 P'êng-shih-ho \& 河, chên nea: 'T'ien-mên H., Hupeh.

5051 P'êng-shui| 水Păng-shwui, $H$. in Yur-yang C., Ssüch 'nan; Lat. $29^{\circ} 14^{\prime}$, Long. $108^{\circ} 14^{\prime}$; Han, Yuyang 酉陽; Sui, P'êngshui | 水
5052 P'êngr-tsê | 澤 Păng-tseh.

$1^{\circ} \mathrm{H}$. in Chint-chiang $\mathrm{F} u$, Kianssi ; Lat. $30^{\circ} 02^{\prime}$, I.ong. $116^{\circ} 35^{\prime}$; Han, P'êng-tsê | 澤; Sui, I.11119-ch 'êng 龍 城; T'ang, Hao 浩 C.

$2^{\circ}$ See Nos. 1281, 2855 , 4590 .

5053 P'êng-tsu | 忨, chên near Ch'ien-yang H., Shensi.

5054 P'êng-wei | 衞, see No. 4858.

5055 P'êngryang | 陽, (obs.) IIan, H., S. W. of Chênyüan H., Kansul.

5056 P'êngr-yüan | 原, (obs.) S. of An-hua H., Kansuh; Sui, H. in Pei-ti 北地 chiun; T'ang, H. in Ning 隺 C.; Sung and Kin, H. in $\mathrm{Cl}^{\prime}$ 'ing-yang 度陽 Fil.

\section{澎}

5057 P'êng-hu 澎湖 Păng-hu, $T$. in the $T$ 'ainan- $P^{\prime}$ enghu circ., Formosa, consisting of the Pescadore Islands, between Formosa and the mainland; Lat. $23^{\circ} 33^{\prime}$, Long. $119^{\circ} 37^{\prime}$.

\section{蓬}

5058 P'êng 蓬 Păng.

$1^{\circ} \mathrm{C}$. in Shun-ch'ing Fu, Ssŭch'uan; Lat. $31^{\circ}$ $05^{\prime}$, Long. $106^{\circ} 20^{\prime}$; 
Han, Tang-chü 宕渠; P. Chou, P'êng $\mid \mathrm{C}$.; $T$ 'ang, Hsien-an 成炎: and P'êng $\mid \mathrm{C}$.

$2^{\circ}$ (obs.) 60 li S. E. of Yi-lung H., Ssŭclı' uan; T'ang, C. in Shan1-11an 山南 circ.; Sung, C. in Li-chon 利州 circ. 5059 P'êng-ch'i | 溪 Păng-ki, [286]H. in T'ung-ch' 'wan Fu, Ssŭch' 'nan; I,at. $30^{\circ} 46^{\prime}$, Long. $105^{\circ} 45^{\prime}$; Han, Kuang-han 廣 漢; $W$. Wei, Fang-yi 方義; T'ang, Wu-fêng 武豐 and P'êng-ch'i $\mathrm{i}$ 溪.

5060 P'êng-lang | 闎, chên near T'ai-ts'ang C., Kiangsu.

5061 P'êng-lai | 萊 Păng-lai.

$1^{\circ} \mathrm{H}$. forming the prefectural city of 'Têngchou, Shantung; Iat. $37^{\circ} 45^{\prime}$, I.ong. $120^{\circ}$ 42'; Han, Tung-lai 東 萊; T'ang, P'êng-lai | 萊.

$2^{\circ}$ Chên near Lê-chih $\mathrm{H}$, Ssŭch 'uan.

$3^{\circ}$ See No. 5822.

5062 P'êng-tao | 島, see No. 5822.

5063 P'êng-ying | 瀛, see No. 5822.

Pere, see No. 2107.

Persia, see No. 5223.
Pescadore Islands, see No. 5057.

Petow, see No. 1728.

Petune, see No. 5236.

\section{PI}

艮

506,4 Pi 貿 $\mathrm{Pi}, \mathrm{H}$. in Yi-chou Fu, Shantung; Lat. $35^{\circ}$ $18^{\prime}$, Long. $118^{\circ} 05^{\prime}$; orig., Pi-pei-ri | 北沂 and K. $\mathrm{Pi} \mid$; Han, $\mathrm{Pi} \mid \mathrm{H}$.

此

5065 Pi-an 比安, H. in Ch ingshang 慶何 circ., Corea.

5066 Pi-p'êng | 蓬, (obs.) in Ya-chon Fu, Ssŭch 'nan; Tang, C. in Chien-nan 㓣南 circ.

5067 Pi-ssŭ | 思, (obs.) in Mou C., Ssŭch uan; T゙ang, Pissŭ | 思 $\mathrm{H}$.

$5068 \mathrm{Pi}$-su | 蘇, (obs.) IV. of Yün-lung C., Yünnan; $W$. Han, H. in Yi-chon 酉州 chün; E. Han and Tsin, H. in Yung-ch'ang 永昌 chün; S. Sung and $S$. $T s^{6} i$, H. in Hsi-ho 西河 chün: also written $\mathrm{P}^{\mathrm{i}} \mathrm{i}$-su 芘 蘇.

\section{萠}

$5068^{\wedge}$ Pi-su 茈蘇, see No. 5068. 
比

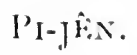

\section{庇}

5069 Pi-jên 庇仁, H. in Clunngcling 忠清 circ, Corea.

\section{鼠}

5070 Pi-sin̆-111ia 瞥思馬, see No. 5099.

\section{必}

50 -1 Pi-li-11a11 必里乫, see No. 4770 .

\section{泌}

5072 Pi 泌, see No. 6246.

5073 li-iang | 陽 l'i-yang, H. in Nan-yang Fin, Honan; I.at. $32^{\circ}+9^{\prime}$, I.ong. $113^{\circ}$ $23^{\prime}$.

\section{畢}

50万t Pi-chich 果笁 Pill-tsieh, [238] H. in lat-ting Fu, Kueichon; I Iatt. $27^{\circ} 12^{\prime}$, Long. $105^{\circ} 13^{\prime}$; Y̌̈̆(1n, in Sh1111-

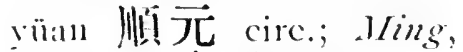

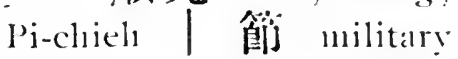
district.

5075 Pi-ti-êrl | 底尔 Pittur, town in Yarkand; Lat. $41^{\circ} 15^{\prime}$, I,ong. $77^{\circ} 35^{\prime}$.

\section{碧}

5076 Pi-chial 碧 甲, ssil in Kuei-shan $\mathrm{H}$., Kuangtung. 5077 Pi-hu | 湖, chên near $\mathrm{Ch}^{\prime}$ u-chon Fu, Chehkiang.
椑 PI.

50 ) \& Pi-t'ung | 潼, chün in P'ing-an 你安 circ., Corea.

5079 Pi-ÿ̈| 表, chên near linch' iang H., Kansull.

5080 Pi-chiany 壁江, see No. 5081.

5081 Pi-shan | Il Pilh-shlin, H. in Chung-ch'ing Fu, Ssüch van; Lat. $29^{\circ} 45^{\prime}$, I.ong. $106^{\circ} 18^{\prime}$; Sung, pi-chiang | 江.

Pi, see No. 5083.

\section{P'I}

\section{㡙}

5082 P'i-ling 㡙陵, see Nos. 312,760 .

\section{軼}

5083 P’i 觙 Pi， H. in Cli êng-

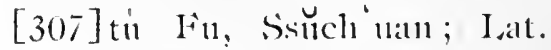
$30^{\circ} 47^{\prime}$, I.ong. $103^{\circ} 56^{\prime}$; $T^{\prime}$ ang, $\mathrm{P}$ i $\mid \mathrm{H}$.

\section{椑}

$508+\mathrm{Pi}$ 椑, (obs.) 73 lis. of Chü C., Shantung; $W$. $H a n$, H. I anng-yel 唒躯 chiin. 
埤

P'I-CH' tิxici.

洠

5085 l'i-ch'êng 粩城, chền near Tan-yang H., Kiangsil.

$5086 \mathrm{P}{ }^{\mathrm{i}} \mathrm{i}-11$ 111 $\mid$ 南, see No. 6193.

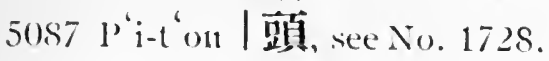

皮

5088 I'i-shil 皮氏, see No. 2066.

\section{披}

$5089 \mathrm{P}$ ‘-sha 披沙, T'-S. near

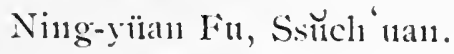

\section{闢}

5090 P'i-chan1 闢展 Pidjan.

$1^{\circ}$ Ss̆̈ in Chên-lisi C. I. 'T., New Donninion.

Iat. $42^{\circ} 45^{\prime}, \mathrm{I}_{\text {ong. }}$. $87^{\circ} 40^{\prime}$.

$2^{\circ}$ Another town of the same name in Varkand, 11:alf way between Ush and Kasligar.

\section{毗}

$5091 \mathrm{P}^{\prime} \mathrm{i}$ 毗, (obs.) in Ssŭch'nan; T'ang, C. in I 1119-y"1 隴右 circ.

\section{茈}

5092 P’i-su 萠蘇, see No. 5068.

\section{正}

5093 P'i.li 正黎, (obs.) near Shun-t ien Fu, Chilli ; Tang, C. in Sung-mo 松漠 $\mathrm{F} 11$.
齽

Pinjax.

\section{番}

5094 P'i 番, see No. 5095.

\section{蕃}

$5095 \mathrm{P}^{\prime} \mathrm{i}$ 蕃, or 番.

$1^{\circ}$ (obs.) in Kiangsu; s.

Sung and s. Ts'i, H.

in Nan P'êng-ch'êngr

南彭城chiin.

$2^{\circ}$ See No. 6336.

Pianfu, see No. 5200.

\section{PIAO}

\section{表}

5096 Pialo-huu 表湖, ssü in V'ung-fêng $H .$, Kiangsí.

发龙

5097 Piao-chiao 彪角, chên near Fêng-hisiang Fin, Shensi.

\section{P'IAO}

\section{驃}

5098 P'iao-fêng 驃封, (obs.) N. E. of Tsun-yi H., Kneichou; S. Ts'i, H. in Lianng-shui 梁水 chiun.

Pidjan, see No. 5090. 
剧

PIFH-SHIH-PA-LI.

\section{PIEH}

別

5099 Pieh-shih-pa-1i 别失入里 (or, Pieh-shilı-pa | 石巴,

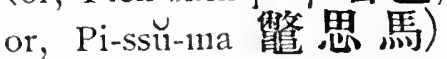
Bislibalik, the ancient name of Urumtsi; $T^{6}$ ang, Pei-t'ing 北庭 Fin.

5100 Pieh-shih-lan |石蘭, a city near-Tashkend.

5101 Pieh-shih-pa | 石巴, see No. 5099.

5102 Piels-ssŭ-man | 思瀮, T. T. near Min-ch' 'uan H., Ssüch' uan.

5103 Pieh-t'ang | 塘, chên near Yün-meng H., Hupeh.

\section{PIEN}

\section{澺}

$510+$ Pien-lnan 邊寒, chên near C'h'ang-tzŭ H., Shansi.

\section{街}

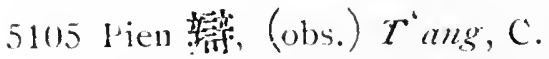
near Wu-chon Fin, Knangsi.

\section{沛}

5106 Pien $\vdots$, see Nos. 2607, 3061.

5107 Pien-ching $\mid$ 京, see No. 3061.

5108 Pien-han | 㛺,(obs.) $\%$ ' in and $H a n$, K. in S. IV. Corea.

5109 Pien-liany | 梁, see No. 3061.
駢・Piju.

\section{便}

5110 Pien 便, see No. 7853 .

5111 Pien-clı‘ | 溪, (obs.) near Yüan-chon Fı, Hunan ; Sung, military post in Yüan 沅 C.

5112 Pien-sluti | 水, ssŭ near Yüan-choı $F u$, Hunan.

Pien-kwan, see No. 5114.

\section{P'IEN}

\section{偏}

5113 P'ien-ch'iao 偏 橋.

$1^{\circ}$ Chên near Yi-chïn $\mathrm{H}$., Shensi.

$2^{\circ}$ T-S. in Chên-yüan Fin, Kueichou.

5114 P'ien-knan | 關 Pien[148] kwan, H. in Ning-wu Fu, Shansi; lat. $39^{\circ} 24^{\prime}$, Long. $111^{\circ} 13$.

\section{騃}

5115 P'ien 駘, see No. 5116.

\section{称}

5116 P ien 政 or 騃, see No. 3855 .

Pilı-shan, see No. 5081. Pil1-tsieh, see No. 5074 . Piju, see No. 5035 . 
賓

$$
\text { PIN. }
$$

\section{PIN}

\section{颁}

5117 Pin 筫 Pin.

$1^{\circ} \mathrm{C}$. in Ssŭ-ên Fin, Kuang[80] si ; Lat. $23^{\circ} 13^{\prime}$, I,ong. $108^{\circ} 36^{\prime}$; Han, Lingfang 領方; $W u, I$, inp'u臨浦; $T^{\prime} a n g$, Pin l C.

$2^{\circ}$ (obs.) S. W. of Liaoyang C., Shingking; Liao, H. in Tung-ching 東京 circ.

5118 Pin-chiang | 江.

$1^{\circ}$ Ss̆̆ in Cl' ingr-yïan H., Kuangtung.

$2^{\circ}$ (obs.) E. of Tê-ch'ing C., Kuangtung; $S$. Sung and $S . T s i, \mathrm{H}$. in Chin-k'ang 琵㞗 chiin.

5119 Pin-cliou | 州, C. L. T. in Chi-lin circ., Kirin.

5120 Pin-ch uan| 川 Pin-chuen, [437] C. in Ta-li Fu, Yünnan; Lat. $25^{\circ} 46^{\prime}$, Long. $100^{\circ}$ $46^{\prime}$; Han, Yeh-yï 葉榆.

5121 Pin-t'ang-ch'i | 唐 溪, (obs.) near Hsü-chou Fu, Ssŭch' 'uan; $T^{\prime}$ ang, H. in Ching 鏡 $\mathrm{C}$. $2^{\circ-}$ (obs.) E. of 'T'ung C.. Chihli ; $T^{\prime}$ ang, H. in Hsien 解 C.
分队 PIN.

5122 Pin-t ${ }^{\prime}$ | 徒, (obs.) near Chin-chon Fu, Shingking; E. Han, H. in K. Liaotung 渴束； Tsin，H. in Chang-li 昌黎 chün.

5123 Pin-yi | 㚕 (obs.) near Hsü-chon Fu, Ssǔch 'uan; I’ang, H. in I, o 洛 C.

5124 Pin-๖iu| 虞, (obs.) formerly town near Yao $C$., Y'innan.

\section{演}

5125 Pin 落 Pin, C. in Wuting Fu, Shantung; Lat. $37^{\circ} 34^{\prime}$, Long. $118^{\circ} 05^{\prime}$; $T^{\prime}$ ang, Ch'ien-ch'êng 千 乘; Five dyn., Pin $\mathrm{C}$.

\section{国祖}

5126 Pin 国团, (obs.) Chou, K. in Shensi; see No. 5127.

\section{邠}

5127 Pin 扮 Pin。

$1^{\circ}$ C. L. C. in the Fêng-

[67] Pin circ., Shensi ; Lat. $35^{\circ} 04^{\prime}$, Long. $108^{\circ}$ $06^{\prime}$ : orig., K. Pin 国国; E. Han, Hsin-p'ing 新 平; 2nd Hei, Nan-pin 南国到; $T^{6}$ ang. Pin | c.

$2^{\circ}$ (obs.) in Mon C., ssŭch'nan; $T^{\prime}$ ang, $C$. in Chien-nan 劍南 circ. 
彬

Pin.

\section{柗}

5128 Pin 涁; Ch' ên 椰 C., Hunan, is sometimes wrongly so written.

Pin-chnen, see No. 5120.

\section{PIIN}

\section{頻}

5129 P'in-yang 頻陽, see Nos. $1855,6806$.

5130 P'in 嬻, (obs.) 120 li N. W. of Hai-ch' êng H., Shing-king; Lino, C. in Hai 海 chor.

\section{檳}

5131 Pin-lang 檳 楖 Penang; also called Na-tzı̆ shan 蒳子14.

\section{PING}

\section{排}

5132 Ping 找, (obs.) Yürn, C. near Chin-t'ang H., Ssŭchinan; see also No. 487.

突

5133 Ping-k' ${ }_{11}$ 兵 庫 Hiogo, ken and chief town, Jay)an.

Ping-fan, see No. 5151. Ping-liai, see No. 5152. Ping-hiang, see Nos. 5159, 5211 .
本 $\mathrm{P}_{\text {ING-AN. }}$

Ping-lio, see No. 5149.

Ping-hu, see No. 5156.

Ping-i, see No. 5203.

Ping-jang, see No. 5162.

Ping-kang, see No. 5165.

l'ing-kiang, see No. 5140.

Ping-kulh, see No. 5167.

Ping-li, see No. 5170.

Ping-liang, see No. 5171.

Ping-lo, see No. 5174.

Ping-lol, see No. 5169.

Ping-lu, see No. 5176.

Ping-lul, see No. 5177.

Ping-11an, see Nos. 5178, 5215.

Ping-shan, see Nos. 5179, 5216.

Ping-siang, see No. 5212. Ping-ting, see No. 5192. Ping-tsinen, see No. 5147. Ping-tu, see No. 5196. P'ing-wu, see No. 5199. Ping-rang, see No. 5200. Ping-yan, see No. 5201. Ping-yin, see No. 5205. Ping-yueh, see No. 5210. Ping-ynen, see Nos. 520s, 5209.

\section{PING}

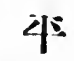

$5134 P^{\prime}$ ing 平.

$1^{\circ}$ (obs.) formerly C. near Pa C., Ssŭch ‘̆

$2^{\circ}$ See No. 7965.

5135 P'ing-anl | 安. 
45

$1^{\circ}$ Chền near Cl ${ }^{\prime}$ nungr-nling H., Kiangstu.

$2^{\circ} 40$ li N. K. of Hsincì'êug H., Sliantung; II: Han, Marce in Ch'ien-ch'êng 干 坓 chïn.

$3^{\circ}$ (obs.) S. W. of Paoying $H_{\text {., }}$ Kiangsu ; $\boldsymbol{H}$. Han, H. in K. Knangling 庴陵; E. Han, H. in Knang-ling 盧 陵 chïl.

$4^{\circ}$ (ols.s.) 80 li $\mathrm{Ir}$. of Chên-yüan H., Kanısulı; Sung anc $k \ddot{i n}$, nilitary post in Yüan 愿 C.

$5^{\circ}$ Circuit in Corea.

5136 P'ing-an-p'n | 㚣鋪, posttown near Hai-fêng H., Kuangtung.

5137 P'ing-ch'ang | 昌.

$1^{\circ}$ Chän in Chiang-yïan 江. 原 cire, Corea.

$2^{\circ}$ See Nos. 818, 5959, 6315.

5138 P'ing-ch'êng | 城.

$1^{\circ}$ Chen near I,ing-ch' nan H., Sllausi.

$2^{\circ}$ Post-town neall P'ingfan H., Kansulı.

$3^{\circ}$ (obs.) $T s^{6}$ in and $M a n$, - town E. of 'la-t'ming Fin, Shansi.

5139 P'ing-clii | 及, (obs.) $T \sin$, one of the chou or

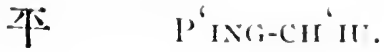

great divisions of the Iimpire, replacing So-fang 朔 方 chov of the Han dynasty.

$5140 \mathrm{P}^{6} \mathrm{ing}$-cliang | 江 Pingkiang.

$1^{\circ}$ H. in Yo-chon Fu, [301] Hunan1; Ialt. $28^{\circ} 42^{\prime}$, Long. $113^{\circ} 24^{\prime}$; oris., 1,0 羅; $W \|$, Ha11ch'ang 漢昌; $T$ ' $\quad r n g$, (;11 ang-chiancr 昌 江 H.; Sung, P'ing-chialng | 江 $\mathrm{H}$.

$2^{\circ}$ Post-town near 'r"unsjên Iin, Kneichon.

$3^{\circ}$ (obs.) S. Simg, lin in (hê-lnsi 浙西 circ.

$4^{\circ}$ See Nos. 5904, 71.30 .

$51+1$ P'ing-cln'iang | 光, posttown $\mathrm{N}$, of I, $\hat{\mathrm{e}}$-slian H., Ssŭch' naı11.

5142 1'ing-ch'il | 沢, H. in Clnnng-elı'ing 忠清 eirc., corea.

5143 P'ing-ch'in | 琴, (obs.) T'ang, H. near Y'ü-lin C., K nangsi.

5114 P'ing-Ch'ing-Ching | 度 㴶, circ. in Kansill, conl1prising P'ingr-liang Fu, Ch ing-yang Fu, Ching C. I.C., Hnil-p ing-ch nan C. I..'T. and Kn-yiian C.I.C. $51+5$ P’ing-chin | 邱, see No 1700. 
平 P'iNG-CH UAN.

5146 P’ing-ch' nan | 川.

$1^{\circ}$.S sü near Tu-shan C., Kneichon.

$2^{\circ}$ See No. 7018.

5147 P'ing-ch'iian | 泉 Pingtsiuen.

$1^{\circ} \mathrm{C}$. in Ch' êng-tê Fu, [354] Chilnli; Lat. $41^{\circ} 05^{\prime}$, I.ong. $118^{\circ} 44^{\prime}$.

$2^{\circ}$ (obs.) Sui, H., S. W. of Chién C., Ssŭch 'nan (No. 874).

5148 P'ing-chin | 春.

$1^{\circ}$ (obs.) N. W. of Hsinyang $C$., Honan; $E$. Han and $T \sin , \mathrm{H}$. in Chiang-hsia 江夏 chän; S. Sung and S. Ts'i, H. in Yi-yang 戥 陽 chïn.

$2^{\circ}$ (obs.) near Ju-ning Fu, Honan ; N. Wci, H. in Ch'êng-yang 城陽 chiin.

$3^{\circ}$ (obs.) in Honan; $N$. Wei, H. in Ch'êngvang 城陽 chiin.

5149 P’ing-ên | 思.

$1^{\circ}$ (obs.) W. of $\mathrm{Ch}_{1} \mathrm{in} \mathrm{H}$., Shantung; W. Han, Narq. in Wei 魏 chän; E. Han, H. in Wei 魏 chiin.

$2^{\circ}$ (obs.) S. E. of $\mathrm{Ch}^{\prime} \mathrm{ii}-$ clion H., Clinhli; Kin, chên in Lo 洛 C.
本 P'ING-HSI.

5150 P'ing-fa | 伐.

$1^{\circ} \mathrm{Y}$. near Lung-li $\mathrm{H}$., Kueichon.

$2^{\circ}$ 'T-S. near Itung-li H., Kueichou.

$3^{\circ}$ (obs.) Yüan, H. 120 li S. E. of Kuei-yang Fu, Kneichon.

5151 P’ing-fan | 番 Ping-fan.

$1^{\circ} \mathrm{H}$. in Liang-chou Fu,

[117] Kansuli; Lat. $37^{\circ}$, Long. $103^{\circ} 20^{\prime}$.

$2^{\circ} \mathrm{Y}$. near Sung-p'an T., Ssŭcli' 'uan.

5152 P'ing-liai | 潗 Ping-liai.

$1^{\circ}$ Chän in Chiang-yüan 江原 circ., Corea; Lat. $37^{\circ} 27^{\prime}$, I.ong. $129^{\circ}$ $02^{\prime}$.

$2^{\circ}$ Post-town near Hsinghua Fu, Fulukien.

$3^{\circ}$ See No. 1491.

$5153 \mathrm{P}^{\text {ing-ho }}$ | 和 Ping-ho, $\mathrm{H}$. in Chang-chon $\mathrm{F} 1 \mathrm{~s}$, Fullkien; I at. $24^{\circ} 18^{\prime}$, Isolng. $117^{\circ} \mathrm{i} 3^{\prime}$.

5154 P'ing-ho'河, see No. 5200.

5155 P'ing-ho| 合, ssŭ near Lo-ting C., Kuangtung.

5156 P'ing-lun | 湖 Ping-lu, $\mathrm{H}$. in Clina-hsing Fin, Chelikiang; Lat. $30^{\circ} 43^{\prime}$, Lonig. $120^{\circ} 46^{\prime}$; orig., 'lang-h11-shih 當湖市; Ming, P'ing-ln1 | 湖 $\mathrm{H}$. 5157 P'ing-hsi | 西. 
平

P'ING-HSI.

$1^{\circ}$ Ssü near Shang-hang H., Fuhkien.

$2^{\circ}$ (obs.) in Hsin-ch ềng T. H., Kuangsi; $T^{6}$ ang, H. in Chill 芝 $C$.

$3^{\circ}$ (obs.) in An-ting H., Kansull; Sung. military post in $\mathrm{H}_{11}$ 會 $\mathrm{C}$.

5158 P'ing-hsia | 夏, (obs.)

Sung, town near Chenyüan $\mathrm{H}$., Kansuiı.

5159 P'ing-hsiang | Ping-hiang, [128]H. in Shun-tê Fun, Chihli; Lat, $37^{\circ} 02^{\prime}$, Long. $115^{\circ}$ 05'; Han,

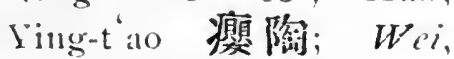
P'ing-hriang | 拫: T゙ang. Fêng 封 C.

$5160 \mathrm{P}^{6} \mathrm{ing}$-lnsing | 與.

$1^{\circ}$ (obs.) 1st Sung, H., S. E. of Chao-ching $\mathrm{Fu}$, Knangtmug.

$2^{\circ}$ (obs.) Han, H., E. of Jung-tsê H., Honan.

5161 P'ing-hsing-knan | 刑關, ssü near Ling-ch'in $\mathrm{H}$., Shansi.

5162 P'ing-jang | 壤 Ping-jang, Fil in P'ing-an 平安 circ., Corea; Lat. $39^{\circ} 16^{\prime}$, Long. $126^{\circ} 03^{\prime}$.

$5163 \mathrm{P}^{\mathrm{i} i n g-j u n g}$ | 戈.

1. Post-town near Hsining Fu, Kansul.

$2^{\circ}$ (obs.) 90 li N.E. of Paoan H., Shensi ; Sung
4 P'INGR.

and Kin, military post in Shensi.

5164 P'ing-kai | 政, ssü in Knei-shan H. Knangtung.

5165 P'ing-kang | 風, ssĭ near Jên-hna H., Knangtung

$5166 \mathrm{P}^{\prime}$ ing-k'ang | 康 Pingkang, H. in Chiang-yiian 江. 原 circ., Corea; Lat. $38^{\circ} 22^{\prime}$, Long. $128^{\circ} 17^{\prime}$.

$5167 \mathrm{P}^{6} \mathrm{ing}$-kn | 谷 Ping-kul, H. in Shun-tien Fu, Chihli; Lat. $40^{\circ} 12^{\prime}$, Long. 117 $07^{\circ}$; Han, P“ing-ku| 谷.

5168 P'ing-lang | 浱, ssü near Tu-yün Fu, Kneichon.

5169 P'ing-lê | 樂 Ping-lolı.

$1^{\circ} \mathrm{F} n$ in the Knei-P'ing-

[224] Wn-Yü circ., Knangsi, forming also the district city of the same name; Lat. $21^{\circ}+2^{\prime}$, Long. $110^{\circ} 29^{\prime}$; Chou, land of the N. Yüeh 䁠 tribes; Ts'in, in Kuei-lin 桂 林chion, Han, in 'Ts'angwu荅梧 chün; $W *$, Shih-an 始安; $T^{6}$ ang, Chao 昭 C.; Yüan, P'ing-lê | 樂.

$2^{\circ} \mathrm{H}$., forming the prefectural city of the same name, Knangsi; Lat. $21^{\circ} 42^{\prime}$, I,ong. $110^{\circ}$ 29'; Wu, P'ing-lê | 樂; T'ang, Lê 樂 C. 
42

$$
\text { P'risti-r.i. }
$$

$3^{\circ}$ Chên in Lo-jung H., Knangsi.

5170 P’ing-li | 利 Ping-li, H. in Hsing-an Fu, Shensi; L.at. $32^{\circ} 18^{\prime}$, I.ong, $109^{\circ}$ 23'; Han、C ' ang-li 長利; $T \sin$, Shang-lien 上. 廉; 1st Sung, Chi-yang 吉陽; T'ang, P'ing-li|利.

5171 P’ing-liang | 凉 Pingliang.

$1^{\circ}$ Fun in the P'ing-Cly ingChing circ, Kansuls; forming also the district city of the same name; l.at. $35^{\circ} 35^{\prime}$, L.ong. $106^{\circ} 41^{\prime} ;$ S.A., Chn1-no 朝那; $T s^{\prime}$ in, in Pei-ti 北地 chïn; Han, Anting 发定; 2nd IVei I11 武 C.; Sni and $T$ ' $\mathrm{ang}$, P'ing-liang | 凉; Sung, Ching-yiian 涇原: lling, P’ing-liang | 凉 ivil.

$2^{\circ} \mathrm{H}$. forming the prefectural city of the same name, Kansulu; Iat. $35^{\circ} 35^{\prime}$, Jong. $106^{\circ}$ $41^{\prime} ; H a n,\left(c_{11-110}\right.$ 朝 那 and Ching-yns 㳸

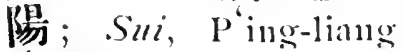
| 凉.

5172 P'ing-liao | 進, (ols.) Tsin, P'ing-liao|遼 chün. 5173 P'ing-ling | P陵, sce Nos. $2829,3755$.
ये P'ixc-shax.

5174 P'ing-lo | 羅 Ping-lo.

$1^{\circ} \mathrm{H}$. in Ning-hisia Iin, Kansuh ; Iat. $38^{\circ} 52^{\prime}$, Long. $106^{\circ} 03^{\prime}$.

$2^{\circ}$ Y. near Fang-shan H. Clinilili.

5175 P'ing-lo | 洛, post-town near Cll'êng H., Kansull.

5176 P’ing-1u | 魯 Ping-ln, H. in So-p ing Fun, Shansi; I ilt. $39^{\circ}+5^{\prime}$, I.011g. $112^{\circ}$ $06^{\prime}$.

5177 P’ing-ln | 陸 Ping-lıll.

$1^{\circ}$ H. in Chieh C., Shan1[148] si ; Iat. $34^{\circ}+\tau^{\prime}$, I,ongr. $111^{\circ} 03^{\prime}$; orig., fendal state Y'ü 虞; E. Han, Ho-pei 河 北; $T^{\prime}$ ang; P'ing-lu| 陸.

$2^{\circ}$ See No. 7016.

5178 P’ing-nan | 南 Ping-nan.

$1^{\circ}$ H. in IIsïn-chon Fin, [16] Knangsi; Lat. $23^{\circ} 32^{\prime}$, I.ong. $110^{\circ} 03^{\prime}$; Han, Nêng-ling 猛. 陵; Tsin, Wu-ch' êng 武 城; T'ang, P'ing-nan | 南; Sung, Kung 熋 $\mathrm{C}$.

$2^{\circ}$ Post-town near $\mathrm{N}$ anching H., Fulnkien.

$3^{\circ}$ See No. 6169.

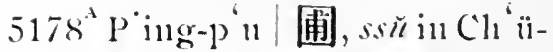
chiang H., Kuingtung.

5179 P'ing-shan | L': P'ing-shatn. 
本

$1^{\circ} \mathrm{H}$. in Chêng-ting $\mathrm{F} \cdot \mathrm{u}$, [143] Chihli; Iat. $38^{\circ} 1 \bar{\gamma}^{\prime}$, Long. $113^{\circ} 10^{\prime}$; orig., P'n-yi 蒲邑; Han, P'n-wu蒲吾; Sui, Fang-shan 房 ll ; T'ang, P'ing-shan | 川.

$2^{\circ}$ Ssü in Mon1-ming H, Knangtung.

$3^{\circ}$ Post-town near Anp'ing H., Kneichon.

$3^{\circ}$ Fu in Huang-1ai 供海 circ., Correa.

5180 P'ing-slih | 氏 see Nos. $2810,3644$.

5181 P'ing-shih | 石, chên near Pi-yang H., Honan.

5182 P'ing-shu|舒.

$1^{\circ}$ (obs.) W. of Ling $\mathrm{H}$., Shansi; Hern and Tsin, H. in Tai 代 chion; N. Hei, H. in Shangkn 上谷 chiin.

$2^{\circ}$ See No. 6006.

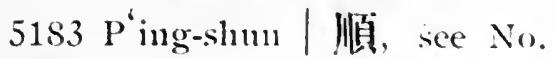
5184.

5184 P’ing-shun1-hsiang | 順良, chôn near I, t1-an Fin, Shansi; orig., Ch ing-yangshan 青羊山; Ming, P'ing-shun | 順 H.

5185 P'ing-so | 索, Y. in Inner Iongolia; Lat. $41^{\circ} 45^{\prime}$, Long. $99^{\circ} 45^{\prime}$.

5186 P'ing-t'ai | 臺.
平

$1^{\circ}$ (obs.) N. F. of P'inghisiang 1I., Chihli; II'. Han, Marq. in Ch angshian 常 l| chïn.

$2^{\circ}($ ols.) S. W. of Yiu C.. Homan (No. 7698); K'in, chîn in l'ii 裕 C.

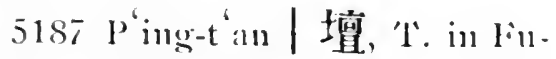
chon Fu, Fullkien on Hait'an 海壇 Island.

5188 P'ing-t'an | 潭, chên near P'ing-ting C., Sli:mini.

5189 P'ing-t'io | 陶, see No. 5201.

5190 P'ing-ti | 隄, (obs.) Is. of 'T'sio-ch' iang H., Chilhli; $W$. Han, Marc. in K. Hsin-tn 信都.

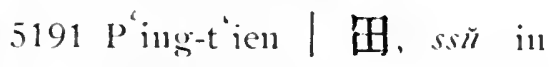
Xi11-l1siung C., K11:111tumg.

5192 P'ing-ting | 筀 Ping-ting. $1^{\circ}$ C. I. C. in the chining circ., Shansis; Latt. $37^{\circ} 52^{\prime}$, l.ong. $11.3^{\circ}$ $35^{\prime} ; / l a m$, Shang-ai 上. 艾; 2nd $\|{ }^{\prime} e$, shih-ai

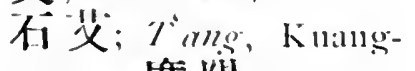

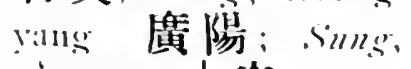
P'ing-ting | 定.

$2^{\circ}$ I'S.S. in Ma-11a C. Kueichon.

$5193 \mathrm{P}^{\prime} \mathrm{ing}$-t' on | 頭.

$1^{\circ}$ S Sin̆ near Sung-t ao T., Kueichon. 
4 P'ING-'T'OU.

$2^{\circ}$ Ss̆̈ near Ning-tu C... Kiangsi.

5194 P'ing-tson | 奏, (obs.) S. of Fêtug-hsiang H., Shensi; N. Wei, H. in $\mathrm{Ch}^{\prime} \mathrm{i}$ 岐 c.

$5195 \mathrm{P}^{\text {ing-tu }}$ | 都.

$1^{\circ}$ (obs.) in An-ting H., Shensi; $W$. Han, H. in Shang | chïn.

2 (obs.) S. E. of An-fu H., Kiangsi; E. Han, Marq. in Yï-chang 瑔 艼. chiin; $T$ sin, $S$. Sung and S'. Ts' $i, \mathrm{H}$. in An-cli êng 妿 城 chiin.

$3^{\circ}$ See Nos. 64, 1753.

5196 P'ing-tu | 度 Ping-tu, C. in Lai-chon Fu, Shantung; Lat. $36^{\circ} 46^{\prime}$, Long. $120^{\circ}$ 20'; Han, P'ing-tu | 度; E. Han, Chiao-tung 東; Sur, Chiao-shui 膠 水.

5197 P'ing-tzŭ|孔, chên near Chêng-ning H., Kansuh.

5198 P'ing-wang|望, ssŭ in Chên-tsê H., Kiangsu.

5199 P'ing-wn | 武 Ping-wn.

$1^{\circ} \mathrm{H}$. forming the prefecturai city of Iungan, Ssŭch '1an ; Lat. $32^{\circ} 22^{\prime}$, Long. $104^{\circ}$ $39^{\prime}$.
平 P'ING-YANG.

$2^{\circ}$ See No. 4169 .

5200 P'ing-yang | 陽 Pingyang.

$1^{\circ} \mathrm{F} n$ in the Yen-P'ing[123] Ta-So-Ning-Hsin-IaiPao circ., Shansi; forming also the district city of I.in-fên ; Lat. $36^{\circ} 06^{\prime}$, Long. $111^{\circ}$ 33'; Hsia, P'ing-yang | 陽, formerly capital of the Eimpire under Yao 龿; S. A., in K. Chin 筸; C. Wars, in K. Chao 趙; Ts'in and Han, Ho-tung 河 冥; 2nd $\mathrm{Wei}$, 'T'ang'唐 C.; Sui, P'ingloo 河 and Lin-fên 臨汾; T゙ang, Chin 䏍 C.; $P$. Liang, 'Tingch'ang 定昌 and Chienhsiung 建雄; Y Y ̈̈an, Chin-ning 㸸寉; Ming, P'ing-yang | 陽, Pianfu (Polo).

$2^{\circ}$ H. in Wên-chon $\mathrm{Fu}$, Chehkiang; Lat. $27^{\circ}$ $42^{\prime}$, I.ong. $120^{\circ} 20^{\prime}$; orig., An-ku 努固 and Shil1-yang 始 陽; rang, Ping-yang | 隄。

$3^{\circ}$ (obs.) C. Wars, town S. of Huai-an Fu, Kiangsu. 
本

$4^{\circ}$ See Nos. 3453, 4369, 6182.

5201 Ping-yao | 遥 P'ing-yau, [139] H. in Fên-chou Fin, Shansi; Lat. $37^{\circ} 12^{\prime}$, Long. $112^{\circ} 08^{\prime}$; orig., 'T'ao 陶; Han, P'ing-t ao | 陶; 2nd Wei, P'ing-yao | 潘.

$5202 \mathrm{P}^{\text {'ing-yi }}$ 荑.

$1^{\circ}$ (obs.) $50 l i \mathrm{~W}$. of $\mathrm{Hsin}$ luii H., Kuangtung; $T \sin , \mathrm{H}$. in Nan-hai 南海 chiin.

$2^{\circ}$ See Nos. 4131, 5203.

5203 P'ing-yi | 萎 Ping-i.

$1^{\circ} \mathrm{H}$. in $\mathrm{Ch}^{\prime} \mathrm{ü}$-ching $\mathrm{Fu}$, [220] Yüınan ; Lat. $25^{\circ} 40^{\prime}$, Long. $104^{\circ} 10^{\prime}$; Han, $T$ sin, $T^{\prime}$ ang and $M i n g$, P'ing-yi | 荑 $\mathrm{H}$.

$2^{\circ}$ T-S. near P'ing-shan H., Ssŭch' 'uan.

5204 P'ing-yi | 邑, (obs.) S. $A$., town 40 li N. E. of Clı'ang-lê H., Shantungr.

5205 P'ing-yin | 陰 Ping-yin.

$1^{\circ} \mathrm{H}$. in Chi-nan $\mathrm{F} n$, [148] Shantung; Lat. $36^{\circ} 23^{\prime}$, Long. $116^{\circ} 35^{\prime}$; Han, I,u 盧 H.; Sui, Yüshan 榆 U and P'ingyin | 除.

$2^{\circ}$ See No. 2090 .

$5206 \mathrm{P}^{\prime}$ ing-yü | 奥.

$1^{\circ}$ (obs.) $60 l i \mathrm{~S}$. E. of Jnyang $\mathrm{H}$., Honan ; Han,
本 P'ING-YÜAN.

$T \sin$ and $S . T s^{i} i, \mathrm{H}$. in Ju1-nan 沙南 chünn. $2^{\circ}$ (obs.) near Han-yang $\mathrm{Fu}$, Hupeh; S. Ts' $i$, H. in Nan Yi-yang 南 義陽 C.

5207 P'ing-yü| 禺, sometimes written by mistake for P'ing-cliang | 昌, chün under the $N$. Wei.

5208 P'ing-yüan | 遠 Pingytuen.

$1^{\circ} \mathrm{C}$. in 'Ta-ting Fin, Kuei[136] chou; Lat. $26^{\circ} 32^{\prime}$, Long. $105^{\circ} 30^{\prime}$.

$2^{\circ} \mathrm{H}$. in Ku-yüan C., Kansuli; original vame of lucality Hsia-mil Kuan下馬關。

$3^{\circ}$ H. in Chia-ying C., Knangtung; I, at. $24^{\circ}$ $47^{\prime}$, Long. $115^{\circ} 54^{\prime}$.

5209 P’ing-yüian | 原 Pingyuen.

$1^{\circ} \mathrm{H}$. in Chi-11:41 Fu, [284] Shantung; Lat. $37^{\circ} 23^{\prime}$, L.ong. $116^{\circ} 34^{\prime}$; orig., K. Kê 鬲; later, capital of K. P'ing-yüan |

原; N. Wei, $\mathrm{P}^{\prime} \mathrm{ing}$-yüan | 原 $\mathrm{H}$.

$2^{\circ}$ Chên near P'ing-liang Fu, Kansulh.

$3^{\circ}$ (obs.) 20 li s. of $P^{\prime}$ ing-yüan H., Shantung; W. Han, chän 
and H.; L. Han, chän and H. in $\mathrm{Ch}^{\circ} \mathrm{ing}$ 奉 chor ; $T$ sin, $\mathrm{K}$. and $\mathrm{H}$. inc (hi 冀 (口m).

$1^{\circ}$ (ols.) 12 li lis of 'Tsom1) ing H., Shantung; S. Simes and $\mathrm{X}$. II H. in P’ing-viin:n1 | 原 chïn.

$5^{\circ}$ (obs.) $15 / i \times 11$. of L,iaro-ch'êng H., Shan1tnung; $x^{*}$. IV ei, chion in Chi 渆 CHOr.

$6^{\circ}$ (obs.) in Anluni ; $\mathrm{Y}$. Il'i, chïn in Ho 霍 cHOE.

$7^{\circ}$ (obs.) near Kungchang lin, Kansuly; $\mathrm{N}$. Il ei, H. in 'l'ien-shni 页水 ‘夰。

$s^{\circ}$ (obs.) in An111111; T'ang, C. in Ling-11an 嶺南 circ.

$9^{\circ}$ See Nos. 601, 3949 , $4039,4711,6305,6578$, 7097 .

5210 P'ing-ÿ̈eh|越 Ping-yneh, [81] C. I. C. in the KneiI'ing-Shlih-Jên cire, Kneichoni; Iat. $26^{\circ} 40^{\prime}$, Long. $167^{\circ} 20^{\prime}$.

\section{洋}

5211 P'ing-lısiang 深榔 Pinghiang, H. in Yüan-chon Fu, Kiangsi; Lat. $27^{\circ}$ $39^{\prime}$, Long. $113^{\circ} 50^{\prime}$; Han,
Yi-chnn 宜奉; Wル, P'ing-hsiang | 拫.

\section{憑}

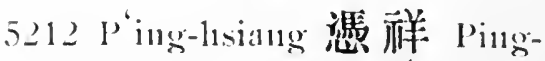
siang, 'T. C. in 'T"ai-p 'ing Fu, Kllangsi ; Lat. $22^{\circ}$ $09^{\prime}$, I.ong. $106^{\circ} 28^{\prime}$, orig., I'ing-shên | 剖 'T'. C.

5213 P'ing-hsin | 信, chên near 'T"ung-chon Fu, Shensi.

$521+$ P'ing-shên | 勈, see No. 5212.

\section{屏}

5215 P'ing-11an 单南 Ping11an, H. in Fin-chou Fu, Fulukien; i at. $26^{\circ} 18^{\prime}$, Long. $118^{\circ} 32^{\prime}$.

5216 P'ing-shan|山 Ping-shan, [528]H. in Hsü-chon Fu, Ssŭcll'nan; I at. $28^{\circ} 39^{\prime}$, Long. $104^{\circ} \quad 05^{\prime}$; orig., I Inng-lun 龍湖; r゙ang and Sung, Hsün 馴 C.

\section{瓶}

5217 P'ing-yao 瓶蜜, chên near Wu-k ang H., Chehkiang.

Pittur, see No. 5075.

\section{PO}

\section{波}

5218 Po 波 Po。 
波

$1^{\circ}$ C. in Coreat I at $40^{\circ}$ $47^{\prime}$, 1.011 $125^{\circ} 57^{\prime}$.

$2^{\circ}$ See No. 92.

5219 Po-clin | 知, (ols.) ne:1r Turfun； T"ang, C. in T:1-han 大汗 1u.

5220 Po-chï|居, (obs.) in Ch' ang-ning IJ., Ssüch'uan; T'ang, H. in

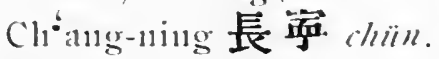

5221 Po-ch'nan | 川, post-town near Hsï-chon Fun, Ssŭclininu.

5222 Po-lo) | 維 Poro, town in Inner Mongolia ; Iat. $-t^{\circ}$ $10^{\prime}$, I 0111 g. $119^{\circ} 10^{\prime}$; also Y. near Yii-lin Fin, Shen$\therefore i$.

5223 P'o-sin | 斯.

$1^{2}$ Persia.

$2^{\circ}$ (ots.s.) ncar 'Turfin ;

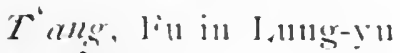
隴不 circ。

\section{嶓}

5221 l'u-chung 嶓家, sec No。 $4+42$.

\section{播}

5225 Po 播, see No. 6593.

$$
\text { 皆 }
$$

5226 Po-kê-han-ch'êny 卓哥品 城 Bokokan, or Buknkhan, eity $\therefore$. E. of Karakorum.
伯 PO-chi-PAO。

5227 l'o-lo | 維 Bolo, anso called P'u-la 诣刺 or Purla 不刺, Pula or Pulat, a city in Turkestan.

\section{渤}

5228 Po-lai 渤海.

$1^{\circ}$ (obs.) Han, chïn and H. in Slanntung, near T'an-ch'êng H.

$2 \circ$ See Nos. 169, 2064 , $3703,6521,7097$.

\section{完}

5229 Po 突 Pols, C. in Ying[213]chou Fin, Anhui; Iatt. $33^{\circ} 58^{\prime}, 1$. ont $115^{\circ} 5 \mathrm{f}^{\prime}$;

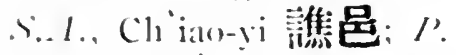
Chon, I'o / C.., anciently the capital of the Emperor

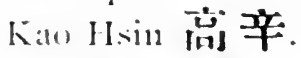

5230 Po-chiêng | Hex se No. 7.699.

5231 Po-ri| 邑, see No. 3t6t. 活

5232 Po 泊, town in the Linch'in Islands.

5233 l'o-kin | 汗, chên in Clunch'êng H., Shantung.

$523+\mathrm{Po}-t^{\prime} \mathrm{ou} \mid$ 䫒, chên ne:ar Nin1-p)'i H., Chihli.

\section{諙}

5235 Po-chi-pao 伯七焦, posttown near An-hsi C., Kansul. 
伯

PO-TU-NO.

5236 Po-tu-no | 都訥 Petune or Pedné, T. in Chi-lin circ., Kirin; Lat. $45^{\circ} 10^{\prime}$. I.ong. $125^{\circ} 40^{\prime}$.

\section{蒲}

5237 Po-ku蒲姑, (obs.) Shang, town near Po-hsing $H$. . Shantung.

\section{傅}

5238 Po 博, see No. $380 \%$.

5239 Po-ch'ang | 昌, see No. 5242.

5240 Po-ch'êng | 城, see No. 6176.

5241 Po-ch'nan | 川, chün in P'ing-an 位安 circ. Corea.

5242 Po-hsing | 與 Poll-hing, H. in Cling-chon Fin, Shantung; I at. $37^{\circ} 15^{\prime}$. Long. $118^{\circ} 20^{\prime}$; Han, Po-cliang 昌.

5243 Po-kung | 基, (obs.) S. W. of Tieh-ch'i Y. (in Nou C.) Ssưch'uan ; T'ang; H. in Knng 茶 c.

5244 Po-lang-sha | 浪少, see No. 7284.

5245 Po-laol勞, (obs.) Liang, H., S. WV. of Chao-p'ing H., Knangsi.

5246 Po-lin | 林, (obs.) Han, town S. IV. of Chaoch'ing Fu, Kuangtung.
博 PO-3: O-I.O-NI-HO-LEI.

5247 Po-ling | 陵, see Nos. $92,5257,6246,6440,7069$.

5248 Po-ling-yi| 陵邑, see No. 5253.

5249 Po-lo | 維 Poli-lo.

[478] $1^{\circ} \mathrm{H}$. in Hui-chou Fu, Kuangtung ; Lat. $23^{\circ}$ $10^{\prime}$, Long. $114^{\circ} 22^{\prime}$.

$2^{\circ}$ See Nos. 2365, 3460, 4197 .

5250 Po-1u | 盧, (obs.) near Ya-chou Fu, Ssŭch'uan; $T^{6}$ ang, C. in Chien-nan 劍南 circ.

5251 Po-nan | 南, see Nos. $3577,7965$.

5252 Po-pai|白 Poh-peh, H. [318] in Yü-lin C., 'Kuangsi ; Lat. $22^{\circ} 23^{\prime}$, Long. $109^{\circ}$ 36'; Han, Ho-p' 11 合浦; $T^{\prime}$ ang, Po-pai | 白.

5253 Po-p'ing | 平 Poh-ping. [476]H. in Tung-cl' ang $F_{11}$, Shantung; I at. $36^{\circ} 43^{\prime}$, Long. $116^{\circ} 18^{\prime}$; orig., Po-ling-yi | 陵邑 in K. Chi 溛; Han, Po-p'ing | 平。

5254 Po-shan | I Po-shan, H. [186] in Ch'ing-chou Fu, Shantung; Lat. $36^{\circ} 35^{\prime}$, Long. $118^{\circ} 04^{\prime}$.

5255 Po-t o-lo-ni-ho-lei | 陀羅 尼霍畺 Bodognikdai, town in Cobdo; Lat. $49^{\circ}$ $25^{\prime}$, Long. $90^{\circ} 49^{\prime}$. 


\section{博}

Po-IVANg.

5256 Po-wang | 望.

$1^{\circ}$ Chén near 'I"ai-p'ing H., Anlini.

$2^{\circ}$ Post-town near Nanyang Fil, Honan.

5257 Po-yeh | 里予 Poll-yé, H. in [448] Pao-ting Fit, Chihli; Lat. $38^{\circ} 30^{\prime}$, Long. $115^{\circ} 34^{\prime}$; $H a n$, Li-wu 簤吾; $E$. Han, Po-ling |陵; $\mathrm{Wei}$, Po-yeh | 野.

5258 Po-yi | 邑, see No. 6176.

Po-yang, see No. 5264.

\section{P'O}

एव

5259 P'o-tao 匹鼠 Port Hamilton; Island off the coast of Corea.

\section{陂}

[N.B. This character is also read PE.I.] 5260 P'o-yang 防 阥, (obs.) $W u$, H., S. of Ning-tu C., Kiangsi.

$$
\text { 坡 }
$$

5261 P'o 坡, C. in Ching-ch $\mathrm{i}$ 京畿 circ., Corea.

\section{破}

5262 P'o-ch'iang 破美, see No. 2483.

$$
\text { 皤 }
$$

5263 P'o-ch'i 皤溪鳥, chên near Hsien-chü H.; Chehkiang.

核䉥

PU.

鄱

5264 P'o-yang 鄱 䛷 Po-yang, $H$. forming the prefectural city of Jao-chon, Kiangsi ; Lat. $28^{\circ} 59^{\prime}$, Long. $116^{\circ}$

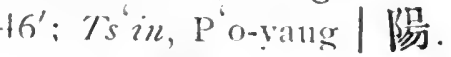

菒束

526.5 P'o-tao 束束道 see No. 7436 .

Pol1, see Nos. 5229, 5266.

Poh-hing, see No. 5242.

Poh-lo, see No. 5249.

Poh-pelh, see No. 5252.

Poh-ping, see No. 5253 .

Poh-shan, see No. 5254.

Poln-yé, see No. 5257.

Poro, see No. 5222.

Port Arthur, see No. 4132.

Port Hamilton, see No. 5259 .

Port Lazareff, see No. $7760^{\wedge}$.

\section{PU}

\section{潂}

$5266 \mathrm{Pu}$ 瀿 Poli.

$1^{\circ} \mathrm{C}$. in 'Ts'ao-chou Fin, [499] Sliantung; I.at. $35^{\circ}$ $48^{\prime}$, Long. $115^{\circ} 33^{\prime} ; S$. A., Wej-ch'êng 得城; Han, Chüan-ch'êng 㖶 城; Sui, Pu|C.

$2^{\circ}$ (obs.) Chou, K. in Ssurch " 11 an. 
淮

Pu-yang.

$3^{\circ}$ See No. 6701.

5267 Pu-yang | 血, see No. 2223.

\section{行}

5268 Pu-êrh-kê-t'ai 杵尔㵧 台 Burgultai, town in Tuchetu; I at. $48^{\circ} 35^{\prime}$, Long. $107^{\circ} 30^{\prime}$.

5269 Pu-êrh-ku-t'ê | 尔古特 Burkut-egurta, town in Tuchetu; Lat. $50^{\circ} 30^{\prime}$, Isong. $101^{\circ} 50^{\prime}$.

5270 Pu-ha-êrh | 哈 尔 or $卜$ 哈兒 (or Pr1-h11a 捕華, or P'u-hna 蒲琵, or Puhua-êrh $卜$ 花思, or $\mathrm{Pu}$ hua-la 不花刺, or Puhuo | 整, or Pn-ho 捕喝) Bokhara, city in the New Dominion Lat. $39^{\circ} 48^{\prime}$, Long. $64^{\circ} 26^{\prime}$.

5271 Pu-huo | 唪, see preceding.

5272 Pu-ku-êrh 有古尔 Bukur, town in Harashar; I,at. $41^{\circ} 28^{\prime}$, Long. $8 t^{\circ} 10^{\prime}$.

5273 Pu-lu-êrh | 翼尔 Buroul, town in the New Dominion Lat. $41^{\circ} 25^{\prime}$, Isong. $97^{\circ}$.

5274 Pu-shan | 山, (obs.) Han, H., 50 li W. of Knei-lin $\mathrm{F} u$, Kuangsi.

5275 Pu-ta-la | 達拉, see No. 3555.

5276 Pu-t'ê-lıa | 特哈 Puteka, town on Heh-lung-kiang;
不 PUTEI.

I.at. $49^{\circ} 05^{\prime}$, Long. $124^{\circ}$ $40^{\prime}$.

\section{闻}

5277 Pn-tien 围田, see No. 1546 .

\section{捕}

5278 Pu-ho 捕晹, see No. 5270. 5279 Pn-hua | 華, see No. 5270. 不

5280 Pu-ch $\mathrm{i}$ 不其, (obs.) S. W. of Chi-mo H., Shantung; $W . H a n$, H. in Lang-yeh 瑯㑚chün; Tsin, S. Sung and $N$. Wei, H. in Ch' angkuang 長 盧 chün.

5281 Pu-ch'i | 期, (obs.) s. W. of Chi-mo H., Shantung; E. Han, Marq. in 'Tunglai 東新 chün。

5282 Pit-êrh | 血, (obs.) N. of Hsien-hsing Tao, Corea; $W$. Han, H. in I,ê-lang 染源 chïn.

5283 Pu-hua-la | 花刺, see No. 5270.

5284 Pu-ssŭ-t’ê | 思武 Bost, town in Afghanistan.

5285 Pu-wei| 军.

$1^{\circ}$ (obs.) $30 l i \mathrm{~N}$. of Paoshan $\mathrm{H}$., Yünnan; $W$. Han, H. in Yi 盆 C.; E. Han and $T \sin , \mathrm{H}$. in Yung-ch'ang 永昌 chiin. 
不

PU-YEH.

5286 P11-yell | 夜, see No.7015.

ト

5287 Pu-ha-êrh 下哈管 or 哈 尔, see No. 5270 .

5288 Pu-lua-êrh | 荅 尔, see preceding.

5289 Pu-hua-êrh | 花 兒, see No. 5270 .

$\mathrm{Pu}$, see No. 5313.

Pu-chau, see No. 5318.

Pu-ching, see Nos. 5303 , 5314.

Pu-ki, see No. 5315.

Pu-kiang, see Nos. 5304, $53 i 6$.

P11-ngan, see No. 5291.

Pu-ning, see No. 5299.

Pu-'rh, see No. 5294.

Pu-shi, see No. 5307.

Pu-tai, see No. 5323.

Pu-tien, see No. 5311.

Pu-ting, see No. 5300.

\section{P'U}

\section{Hife}

$5290 \mathrm{P}^{\prime} \mathrm{u}$ 菖, (obs.) Sui, T'ang and $S$. Sung, C. in Ssǔch'uan, comprising $\mathrm{Ho} \mathrm{C}$. and Lê-chih H.; Liang, $\mathrm{P}^{\prime} \mathrm{u}-\mathrm{tz} z^{\prime} \mathrm{u}$ | 慈.

5291 类P'n-an | Pu-ngan.
前 P'U-NING。

$1^{c}$ C. L. 'T. in Kuei-hsi [567] circ., Kueichou; Lat. $25^{\circ} 45^{\prime}$, Isong. $105^{\circ}$. Terr. jur.

$2^{\circ} \mathrm{H}$. in Hsing-yi $\mathrm{Fu}$, [567] Kueichou; Lat. $25^{\circ} 44^{\prime}$. Long. $104^{\circ} 39^{\prime}$; orig., Yel1-lang 夜郎; $T s^{\prime}$ in, in $\mathrm{Ch}^{\text {'ien-chung 駖 中 }}$ chiun ; Han, in Tsangkê 非妸 chün; Minor Han, Hsing-ku 與古; T'ang, Hsi-p'ing 西坐 and $\mathrm{P}^{\prime}$ an 盘 C.; Yüan, $\mathrm{P}^{6} \mathrm{u}$-an | 发 circ.; Ming, $\mathrm{P}^{\prime} \mathrm{u}$-an 孚 $\mathrm{C}$.

5292 P'u-cli | 紀, (obs.) formerly town near Yungch' uen H., Ssŭch'uan.

5293 P'u-chi-chou | 湇州, T-S. near Hui-li C., Ssŭch' 'uan.

$5294 \mathrm{P}^{\prime}$ u-êrh | 洱 Pu-'rh, Fu in the Yi-nan circ., Yünnan; forming also the district city of Ningr-êrh; Lat. $23^{\circ} 02^{\prime}$, I,ong. $101^{\circ}$ $10^{\prime}$.

5295 P'u-la | 剌, see No. 5227. 5296 P'u-li | 利, post-town near An-slun $\mathrm{Fu}$, Kueichou.

5297 P'u-lung-chou | 隆州, TS. near Hui-li C., Ssŭclinan.

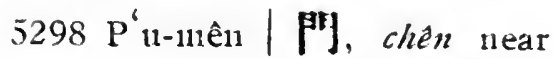
Lan H., Shansi.

$5299 \mathrm{P}^{6}$ u-ning | 耑 $\mathrm{Pu}$-ning. 
䊓

P'U-NING.

$1^{\circ} \mathrm{H}$. in Ch'ao-chon Fu, [315] Knang-tung; Lat. $23^{\circ}$ 32', Iollg. $116^{\circ} 12^{\prime}$.

$2^{\circ}$ See Nos. $2992,3043$.

5300 P'n-ting | 定 Pu-ting, H. [326] forming the prefectural city of An-shun, Kneichon; Iat. $26^{\circ} 13^{\prime} ;$ Long. $105^{\circ}$ 53'; Yïan, P'u-ting | 资. .5301 P’11-t'ung | 通, (obs.) in Kuan H., Shaintung; Kin, rhên in 'Na-1ning 大名 Fu. $5302 P^{6} u-t z z^{\prime} \breve{u} \mid$ 慈, see Nos. $116,5290$.

\section{浦}

5303 P'u-cl' êng浦城 P'u-ching, [319]H. in Chien-ning $\mathrm{Fu}$, Fuhkien; Lat. $28^{\circ} 01^{\prime}$, Long. $118^{\circ} \cdot 37^{\prime} ;:$ Han, Han-11sing 漢與; $W u$, Wn-11sing 贤與; Tang, $P^{\prime \prime 1 i c h}{ }^{\prime} \hat{e n g} \mid$ 战.

5304 P'u-chiang | 江 Pu-kiang, [369] H. in Chin-lina Fu, Cliehkiang; Lat. $29^{\circ}$ $28^{\prime}$, I Iong. $119^{\circ} 49^{\prime}$;

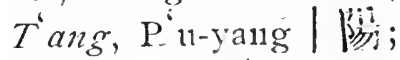
Five dyn., P'n-chiang is..

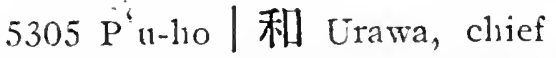
town of Saitama Ken, Japan.

5306, $\mathrm{P}^{\prime} 11-\mathrm{k}^{\prime}$ ou $\mid$ 口, chên near Shang-y ï H., Chelikiang.

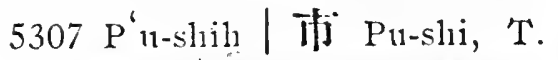
: in Ch ền-chou $\mathrm{F}_{1}$; Hunan;

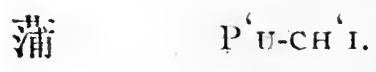

Iat. $28^{\circ} 07^{\prime}$, Long. $107^{\circ}$ $32^{\prime}$.

$5308 \mathrm{P}^{\prime}$ 11-t'ien | 添, town in the Lini-cl' in Islands.

5309 P $^{i}$ 11-yang | 院, see No. 5304 .

\section{莆}

$5310 \mathrm{P}^{6}$ 11-chu11 蔽中, see No. 2821.

$5311 \mathrm{P}^{4}$ u-t ien 田Pu-tien, H. forming the prefectural city of Hsing-lnua, Fullkien; Lat. $25^{\circ} 25^{\prime}$, Long. $119^{\circ}$ $17^{\prime} ; T \sin$, Chin-an 瑟安

$5312 \mathrm{P}^{6}$ 11-yang | 陽, post-town near the preceding.

\section{蒲}

$5313 \mathrm{P}^{6} \mathrm{u}$ 蒲 Pu.

$1^{\circ} \mathrm{H}$. in Hsi C., Shansi ; Lat. $36^{\circ} 18^{\prime}$, Long. $111^{\circ} 06^{\prime}$; S. A., P' ${ }^{\prime 1-}$ ch'êng | 城; 2nd Wei, Slihl1-cls'êng 石 城; $r^{\prime}$ ang, $\mathrm{P}^{\prime} \mathrm{u} \mid \mathrm{H}$.

$2^{\circ}$ See Nos. 1673, 7839.

$5314 \mathrm{P}^{\prime}$ u-cli êng / 城 Pu-ching, [292] H. in T'ung-cliou Fu, Shensi; Lat. $34^{\circ} 58^{\prime}$, Long. $109^{\circ} 28^{\prime} ; T^{\prime}$ in, Chung-ch'üanl重泉; $W$. Wei. P'u-ch'êng | 城; T'ang, Fêng-hsien 泰 先.

5315 P. $^{\prime} 11-$ ch $^{\prime} \mathrm{i} \mid$ 坼 Pu-kí. 
蒲

$1^{\circ}$ H. in Wn-ch'ang Fin, Hupeh; I,at. $29^{\circ} 42^{\prime}$, Long. $113^{\circ} 43^{\prime}$; Han: Slua-yi 沙茨; $W u, \mathrm{P}^{\prime} u$ $\mathrm{ch}^{\circ} \mathrm{i} \mid$ 坼.

$2^{\circ}$ (obs.) S. W. of Chiay'ü H., Hupeh; Tsin, H. in Cli'ang-sha長沙 chiin! $S$. Sung and $S$. $T s^{6} i$. H. in Chiang-hisia 江夏 chiü.

5316 P'u-chiang | 江 Pu-kiang. $1^{\circ} \mathrm{H}$. in Chiung C., Ssŭ[307] ch' 'uan; Lat. $30^{\circ} 12^{\prime}$, I,ong. $103^{\circ} 35^{\prime}$; Han, Lin-chinng 臨 I $; ~ I V$. Wei, Kuang-ting 售定; Ming, P'u-chiang | iI. $\mathrm{H}$.

$2^{\circ}$ (obs.) 1 li $\mathrm{N}$. of the above; Sui, T ang and Sung, P'u-chiang | 江. $\mathrm{H}$.

5317 P'u-ch'il. | 池, (obs.) near Han-chung Fu, Shensi; Tsin, H. in Han-chung 漢中 chiinn.

5318 P'u-chon | 州 Pu-chan, [148] Fu in the Ho-tung circ., Shansi; forming also the district city of yung-chii ; I at. $34^{\circ} 54^{\prime}$, Long. $110^{\circ}$ $15^{\prime} ;$ S. A., $\mathrm{P}^{\prime}$ 11-fan | 坂; P. Chou, $\mathrm{P}^{\prime \prime} \mid \mathrm{C}$.; Tang, Ho-chung 河 中 F 11 ; this was anciently the capital of Emperor Shun. Cachan Hin (Polo).
蒲 P'U-YÜAN。

5319 P'u-fan | 坂, see preceding.

5320 P'u-hna| 萑, see No. 5270.

5321 P'u-kuan | 關, see No. 367.

5322 P'u-mên-so | 關 阔, ss̆ near P'ing-yang H., Cliehkiang.

5323 P'u-t'ai | 墱 Pu-tai.

$1^{\circ} \mathrm{H}$. in Wu-ting $\mathrm{Fu}$, [148] Shantung; Lat. $37^{\circ} 26^{\prime}$, Long. $118^{\circ} 10^{\prime}$; ${ }^{-} \mathrm{Han}$, Hsi-wu 䇣 沃; Sui, $\mathrm{P}^{6} \mathrm{u}-\mathrm{t}$ 'ai | | 整.

$2^{\circ}$ :(obs.) 30 li.S. E. of Pin C., Shantung; Kin, -chên in Pin C.

$5.324 \mathrm{P}^{\prime} \mathrm{u}-\mathrm{t}^{\prime} \mathrm{ing} \mid$ 亭, (obs.) $W$. Wiei, H., S. of Jên-shou H., Ssŭch'nan.

5325 P $11-t^{\prime} 0-k$ ”nig | P它崆, TS. near Iang-ch'iung H., Yünnan.

5326 P'u-tzŭ |子, see No. 2515. 5327 P'u-wu| 需.

$1^{\circ}$ (obs.) S. E. of P'ingshan H., Chihli; Han and $T \sin , \mathrm{H}$. in Cli 'angshan 䈍 山 chïn.

$2^{\circ}$ See No. 5179.

$5328 \mathrm{P}^{\prime}$ u-yi | 源; P'u-cli i | 圻 is sometines wrongly so written.

5329 P" $11-y i \mid$ 邑, see No. 5179. 5330 P'u-yin | 隐, see No. 652. 5331 P'u-yuan | 原, see Nó. 1309. 
鋪

$\mathrm{P}^{\prime} \mathrm{U}$-CH IEN.

\section{鋪}

$5332 \mathrm{P}$ u-ch ien 鋪前, ssü near

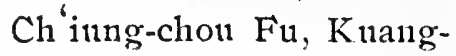
tining.

5333 P'u-1u | 路, chen near 'li. tao C., Kansuh.

\section{楛}

$5334 \mathrm{P}^{\prime} \mathrm{u} \cdot \mathrm{ch}$ ' $\mathrm{i}$ 蒲溪, (obs.) 50 li $\mathrm{N}$. of $\mathrm{P}^{\prime} \mathrm{u}$-chiang $\mathrm{H}$., Ssŭclı' uan; Sui, H. in Lin-chiung 臨 I下 chün.

\section{部:}

$5335 \mathrm{P}^{\prime} \mathrm{u}-\mathrm{t}^{\prime} \mathrm{i}$ 萻䔶, chèn near Yi-ch'uan H., Shensi.

\section{椪}

5336 P'u-ma-luug 椦㢈龍， (obs.) near Kung-ch'ang Iru, Kansuh; Sung, pao in Kung 辞 C.

Pula, see No. 5227.

Pulad, see No. 5227.

Puteka, see No. 5276.

Pwan-yü, see No. 4927 .

Quelin Fu, see No. 859.

Quelpaert Isiand, see No. 6214.

Quinsay, see No. 1993.

Rangoon, see No. 7245.

\section{SA}

撒

5337 Sa-la-hà-hsi 撒刺哈夕, or Hsi-la-ssü 击刺思

\section{SA-LA-CH'I.}

Serakhs, town in Persia, province Khorassan.

5338 Sa-li-mang | 里范 Takhti Soleiman, a ruined city in Persia, province Azerbjan, 125 miles S.S.E. of Tabriz.

5339 Sa-li-wei-wu-êrh | 里畏 匹兒 Sarihuiur, see No. 101.

5340 Sa-li-ya | 里牙 Saria, also called So-li 装里 Sari, town in Persia, capital of the province Mazanderan.

5341 Sa-1n ! 路, T-S. near Sung-p'an T., Ssŭch' 'uan.

5342 Sa-ma-êrlı-kan | 苚身 干 Samarcand, so called under the Yüan dyn.; also known as Hsieh-mi-ssŭkan 邪米思干（or， Hsün1-ssŭ-kan 爵思干, or, 沀思干 Semiscant), city in the New Dominion Lat. $39^{\circ} 50^{\prime}$, Long. $66^{\circ} 50^{\prime} ;$ Han, K' ang-chü 康居; T'ang, $\mathrm{K}^{\prime}$ ang 康 or Sa-1110-ta 薩末韃; Liao, Ho-chung 河 中 $\mathrm{Fu}$.

5343 Sa-wa | 互 Sava, tow11 in Persia, 50 miles S. W. of 'Teheran.

\section{薩}

5344 Sa-la-ch'i 薩拉䂟 Sarar[475] tsi, T. in So-ping Fu, 
薩

SA-LA-CH'I.

Shansi; Lat. $40^{\circ} \quad 45^{\prime}$, Lony. $111^{\circ} 20$.

5345 Sa-mu-kê-na | 䅣啺納 Samkana, town in Cobdo; Lat. $49^{\circ} 20^{\prime}$, J ong. $95^{\circ}$.

5346 Su-mo-ta | 末蓬, see No. 5342 .

Sa-chui, see No. 5424.

\section{SAI}

䑁

5347 Sai-lan 學覽, or 赛藍 or 寉蘭 Sairam, city N. of Tashkend, Russian Turkestan.

5348 Sai-mên | 19 J, (obs.) 150 li N. of An-sai H., Shensi ; Sung, military post in Yen-an 延 放: Fin.

\section{算}

5349 Sai-lan 賽闌, see No. 5347.

5350 Sai-lan|譼, see No. 5347. 5351 Sai-li-mu |里木 Sairim, tow11 in Yarkand; Lat. $41^{\circ}$ 30', I.ong. $82^{\circ}$.

5352 Sai-ma-chi | 馬 集, ssu in Fêng-lunang 'T', Shingrking.

Saianfu, see No. 2616.

Saigon, see No. 2471. $\equiv \quad$ San-chiao.

Sainnoin, see No. 5403.

Sairam, see No. 5347.

Sairim, see No. 5351.

Saitama, see No. 636.

Sakai, see No. 828.

Samarcand, see No. 5342.

Samkana, see No. 5345.

\section{SAN}

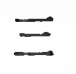

5353 San-cl' ${ }^{\circ}$ 三政.

$1^{\circ}$ Post-town near Fêng H., Shensi.

$2^{\circ}$ Post-town in $\mathrm{Cl}^{\prime}$ in $\mathrm{C}$., Kansul.

5354 San-ch'a-ho|忿河, ssŭ near Hai-clı 'êng H., Shingking.

5355 San-ch'i | 溪.

$1^{\circ}$ Chên in Chings-tê H., Anluui.

$2^{\circ}$ (obs.) S.E. of $\mathrm{Ch}^{\prime} \mathrm{i}$ chiang H., Ssuch' uan; Tang, H. in Nan 南 c.

5.356 San-chia | 礔, H. in Clining-slian 廈侧 circ. Corea.

5357 san-chiang-k ou | 江 口, chên near Lien C., Kuangtung.

5358 San-chiao | 交.

$1^{\circ}$ Chên near Fên-chon $\mathrm{Fu}$, Shansi. 
$2^{\circ}$ Chên near Yung-ning C., Shansi.

5359 San-chiao | 角, chên near Clin H., Kansult.

5360 San-clı'iao | 橋, chên near Hsi-an Fin, Shensi.

5361 San-ch'iao | 槅, chên in Huai-ning $\mathrm{H} .$, Anhui.

5362 San-chieh | 界.

$1^{\circ}$ Chên near Lai-an H., Anluni.

$2^{\circ}$ Chên near Shang-yü H., Chehkiang.

5363 San-cliien | 監, see No. 2228.

5364 San-chih | 缆, Fu in Chiang-yüan1 江原 circ. Corea.

5365 San-chou | 洲.

$1^{\circ}$ Post-town near 'l"ingchon Fu, Fuhkien.

$2^{\circ}$ Ss $\breve{u}$ in Kao-ming $\mathrm{H}$, Kuangtnug.

5366 San-ch “ï $/$ 㨨, see No. 1445.

5.367 San-ch nan | 川.

$1^{\circ}$ Post-town near I,Ocin'nan H., Sliensi.

$2^{\circ}$ (obs.) Tsin and Han, chïn coniprising part of Honan.

$3^{\circ}$ See Nos. 2079, 2228, 2988, 3050, 3061, 3179.

5368 San-ch'üan | 泉.

$1^{\circ}$ Chên near Fên-chon Fu, Shansi. $2^{\circ}$ Chèn near Shan-yin H., Shansi.

5369 San-chung | 重 Miyé, ken in Japan; chief town Yok-。 kaichi.

5370 San-fêng | 封, (obs.) in the Ordos country, N. of Shensi ; on tlie IV. bank of the Yellow R.; Han, H. in So-fang 溯方 chïn.

5371 San-han | 暲.

$1^{\circ}$ (obs.) formerly $\mathrm{K}$. in S. Corea.

$2^{\circ}$ (obs.) S. W. of the country of the Khorchin Mongols ; Liao, H. in Kao 高 C.; Kin, H. in Ta-ting 大定 Fu.

5372 San-ho | 和 San-ho, H. in P'ing-an 平妍 circ., Corea; Lat. $39^{\circ} 08^{\prime}$. Long. $125^{\circ} 30^{\prime}$.

5373 San-ho | 河 Sa11-ho.

$1^{\circ} \mathrm{H}$. in Shun1-t'ien Fin, Clilili ; Lat. $40^{\circ}$, L.ong. $117^{\circ} 01^{\prime} ;$ Han, Linch'ï 陻 沟; T゙ang, San-lio | 润.

$2^{\circ}$ Ss й near Chia-ying C., Kuangtung.

$3^{\circ}$ Ss̆̌ in Nan-hai H., Kuangting.

$537+$ San-hu| $\mid$, (obs.) S.A., town near Ning-ling $H$, Honan. 


\section{$\equiv \quad \mathrm{S}_{\mathrm{AN}-\mathrm{HUA}}$.}

5375 San-hua | 鿊, chên near Wêng-yüan H., Kuangtuing.

5376 San-hui-ch'ang | 淮㙫, post-town near Sui-ting F11, Ssuicli' wan.

5377 San-hsing | 妙 Sansing, C. L. T. in Ning-HsingHun circ., Kirin. Called, in Manchn, Ilan-hala [Ilan $=$ 二; hala $=$ 贺 $]$.

5378 San-kang | 風; Sui, H. in Ssǔch' '11:11 ; Lat. $46^{\circ} 20^{\prime}$, Long. $129^{\circ} 15^{\prime}$.

5379 San-kon | 㸺, post-town near Ying-slian H., Anhui.

5380 San-kung | 菲, (obs.) in Ya-chou Fu, Ssuch' 'uan; Tang, C. in Chien-nan 殮南 circ.

5381 San-lang | 斯, chên near Kn-cli êng H., Chilıli.

5382 San1-li | 里.

$1^{\circ}$ Chên near Cluiang H., Shansi.

$2^{\circ}$ Y. near Shang-lin H., $\mathrm{K}$ tangsi.

5383 San-lin | 林, ssü near Nan-hui H., Kiangsu.

5384 San-lung | 龍, see No. 641. 5385 San-mên | P⿴囗十

$1^{\circ}$ (obs.) 30 li N. IF. _. of $\mathrm{Ch}^{\prime}$ êng-ch' êng H., Shensi; N.Wci, H. in Ch'êng-cli'êng 濯. 找 chün.
三 San-shul.

$2^{\circ}$ (obs.) $40 l i \mathrm{~N} . \mathrm{E}$. of Shan C., Honan; Kin, chên in Shan 陝 C.

5386 San-pan-ch'iao | 板橋, prost-town near $\mathrm{K}$ 'ai-hua $\mathrm{Fu}, \mathrm{Y}$ iunnan.

5387 San-pol泊, (obs.) Yüan, H. in Yünnan, Lat. $24^{\circ}$ 55', I, ong. $102^{\circ} 33^{\prime}$; Sung, Slian-slian 美閏.

5388 San-slia | 沙, ssü near Fu-ning Fu, Fuhkien.

5389 San-shan | II.

$1^{\circ}$ Chên near Wu-hu H., Anhui.

$2^{\circ}$ Post-town near Fuchou Fu, Fulukien.

5390 San-shêng | 聖, post-town near Chia-ting + Fù, Ssŭch'uan.

5391 San-shih-chia-tzŭ |十家 子, post-town near Cliiench'ang H., Chihli.

5392 San-shui | 水 San-shwui. $1^{\circ}$ H. in Pin C., Slensi, Jat. $35^{\circ} 12^{\prime}$, I,ong. $108^{\circ} 18^{\prime}$; orig., Pin

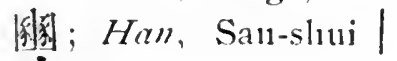
水.

$2^{\circ}$ H. in Kuang-chou $\mathrm{F}_{11}$; [416] Knangtung; Lat. $23^{\circ}$ $12^{\prime}$, I.ong. $112^{\circ} 53^{\prime}$; liang, Nan-hai 南海 and Kao-yao 陑要 Open port.

$3^{\circ} \mathrm{Fu}$ in Hsien-ching 峸 鏡 circ., Corea. 
5393 San-t'ai | 臺 San-tai, H. forming the prefectural city of T'ung-cl' 'uan, Ssŭch 'uan; Lat. $31^{\circ} 09^{\prime}$, Long. $105^{\circ} 11^{\prime}$; Sui, T'ang, Sung and Yiuan, Ch'i 部 $\mathrm{H}$.

5394 San-têng | 登 San-tăng, H. in Corea ; Lat. $39^{\circ} 22^{\prime}$, Long. $126^{\circ} 50^{\prime}$.

5395 San-tiao | 韶, shê near Kelung, N. Formosa.

5396 San-ts'êng-ling | 层領, ssü near Yung-ting $\mathrm{H}$., Fulkien.

5397 San-tsung | 䰚, see No. 6458.

5398 San-tu | 都.

$1^{\circ}$ Ss ̌̆ near I,in-chon Fu, Kuangsi.

$2^{\circ}$ Santu; port in Fu-ning $\mathrm{Fu}$, Fullkien; opened to foreign trade, not by treaty but by the spontaneous action of the Government of China.

5399 San-t'un | 屯, Y. in Ch'ien-an H., Chihli.

5400 San-wang | 望, chên neat Tung-lan C., Kuangsi.

5401 San-yao | 朚, ssŭ near Shang C., Shensi.

5402 San-yen-ching | 眼井, post-town near P'ing-fan H., Kansul.
桑 SANG-CHIANG.

5403 San-yin-110-yen | 音訥顏 Sainnoin, Khanate in Mongolia; bounded on the N. by Dsassaktu; on the L. by Tuchetu; on the S. by Inner Mongolia; and on the IV. by Dsassaktu.

5404-San-yüan | 原 San-yuen. $1^{\circ} \mathrm{H}$. in Hsi-an Fu, [79] Shensi ; Lat. $34^{\circ} 37^{\prime}$, Long. $108^{\circ}$ 53'; Han, Cli'ih-yang 池 晹 ; Tang, Hua-ch il 華 池; Yïan and Ming, San-yïan | 原 $\mathrm{H}$. $2^{\circ}$ (obs). 30 li N. E. of the above; Sui, $T^{6}$ ang, Sung and Kin, Sanyüan 原 $\mathrm{H}$.

San-shwui, see No. 5392. San-sing, see No. 5377. Sant-tai, see No. 5393. San-tang, see No. 5394. San-yuen, see No. 5404. Sanaju, see No. 5408.

\section{SANG}

\section{桑}

5405 Sang 桑, (obs.) Yüan, C. in Kueichon.

5406 Sang-chiang | 江, ssit near Yi-ning $\mathrm{H}$., Kuangsi. 


\section{桑}

SANG-CHIH.

5407 Sang-chih | 植 Sang-chih, [257]H. in Yung-shun $\mathrm{F}_{\mathrm{u}}$, Hunan; I at. $29^{\circ} 25^{\prime}$, Long. $110^{\circ} 03^{\prime}$.

3408 Sang-ch11 | 珠 Sanaju, town in Yarkand; Lat. $37^{\circ}$ $20^{\prime}$, Long. $78^{\circ} 30^{\prime}$.

5409 Sang-ch' üan | 泉, see No. 3848.

5410 Sang-chung | 中, (obs.) S. E. of P'ing-shan H., Chihli; W. Han, Marq. in Chang-shan 常 山 chiin.

5411 Sang-li | 里 Sangri, town in Thibet; Lat. $29^{\circ} 10^{\prime}$, Long. $92^{\circ} 20^{\prime}$.

5412 Sang-pi | 壁. chể near Hsi C., Shansi.

5413 Sang-sê-êrh | 色尔, 'T-S. in Kokonor.

5414 Sang-t'ien | 田, ssĭ near

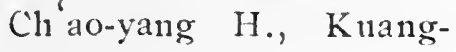
tung.

Sanglii, see No. 5416.

Sangri, see No. 5411.

Santu, see No. 5398.

\section{SAO}

$$
\text { 琭 }
$$

5415 Sao-kung 凌 宮, (obs.) "uệr Hsü-chou Fu, Ssŭch uan; Tang, H. in Kao 高 $\mathrm{C}$.
SHA.

Sapporo, see No. 137.

Sarartsi, see No. 5344.

Sari, see No. 5340.

Saria, see 5340.

Sariluiur, see Nos. 101, 5339.

Savil, see No. 5343.

Sebzivar, see No. 6368.

Semiscant, see No. 5342.

Semnan, see No. 2481.

Sendai, see No. 2711.

\section{SENG}

\section{僧}

5416 sêny-chi-mu 售吉穆 Sanghi, (obs.) Ming, town in Mongolia.

Seonl, see No. 363.

Seraklis, see No. 5337.

\section{SHA}

\section{沙}

5417 Sha 沙 Sha.

$1^{\circ}$ H. in Yen-p ing Fin, [388] Fulhkien; Lat. $26^{\circ} 23^{\prime}$, I.ong. $117^{\circ} 56^{\prime}$; Sung, Sha-ts'un 村1; Sui, Sha $\mathrm{H}$.

$2^{\circ}$ Y. near $\mathrm{Hsing}-\mathrm{yi} \mathrm{Fu}$, Kueichou.

$3^{\circ}$ (obs.) formeriy K. in Honall. 
$4^{\circ}$ See No. 5597.

5418 Sha-chên | 鎮, ssü in Nien-yang C., Hupeh.

5419 Sha-ch'êng | 城.

$1^{\circ}$ (obs.) Tang, H. near Lo-shan H., Honan.

$2^{\circ}$ Ss $\breve{u}$ in Huai-lai H., Chihli; in the 17 th century, H. near Pao-an C., Chilhli, in Lat. $40^{\circ}$ $25^{\prime}$, Long. $115^{\circ} 22^{\prime}$.

5420 Sha-chi $i$ 棇。

$1^{\circ}$ Chển near Lü-cliang H., Anhui.

$2^{\circ}$ Chên near 'T'ai-ts'ang C., Kiangsu.

$3^{\circ}$ Ss̆̌ in Yung-fêng H., Kiangsi.

5421 Sha-chien | 润, post-town near Fan-chih H., Shansi.

$5+22$ Sha-ching | 兰, post-town near Kan-chou Fu, Kansull.

5423 Sha-ch $i$ | 邱, (obs.) S. A., H. near Lai-chon Fun, Shanting.

5424 Sha-chour-clinêng | 州 城 Sha-chou, post-town near An-lisi C., Kansulı ; Lat. $40^{\circ} 15^{\prime}$. L.ong. $94^{\circ} 39^{\prime}$. Sachiu (Polo).

5425 Sha-cliü|埧, 'T-S. near Mou C., Ssŭclı' nan.

5426 Sha-ch'ü | 渠, see Nos. $1616,5673$.
SHA-MA.

5427 Sha-han | 漢 Sha-han, T. in An-1u Fu, Hupeh; Lat. $30^{\circ} 42^{\prime}$, Long. $112^{\circ} 41^{\prime}$.

5428 Sha-ho | 河 Sha-ho.

$1^{\circ} \mathrm{H}$. in Shun-tê Fu, [387] Chihli; Lat. $37^{\circ}$, Long. $114^{\circ} 39^{\prime} ;$ Sui, Sha-ho | 河 ; Tang, Wên 溫 c.

$2^{\circ}$ Chên near Ma-ch êng. H., Hupeh.

$3^{\circ}$ Ssŭ near Lề-t'ing H., Chilili.

$4^{\circ}$ Y. near Han-chung Fu, Shensi.

$5^{\circ}$ Post-town near Kanclion Fu, Kansuh.

$6^{\circ}$ Post-town near Ningyüan C., Shingking.

5429 Sha-kang|閌, sš̆ in K'aip'ing H., Kuangtung.

5430 Slua-kou | 满, Y.near P'ei H., Kiangsu; also posttown near Nien-p; H., Kansuh.

5431 Sha-la-ya-ssü | 喇雅 斯 Sharayas, town in Ili; Lat. $42^{\circ} 50^{\prime}$, Long. $80^{\circ}$.

5432 Sha-lin-p'u|林鋪, posttown near Hai-ch 'êng H., Shingking.

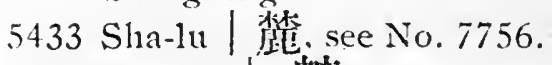

5434 Sha-lu| 輥, shê near Chang-hua H., Formosa.

5435 Slia-ma | 嫼, T-S. near L,ei-po 'T,; Ssĭch' 'nan. 
沙 SHA-MÂN.

5436 Sha-mền | 阴, chên near Wei-hui Fu, Honan.

5437 Sha-mu | \&, sš̆ in Hsinhui H., Kuangtung.

5438 Sha-11an | 南, see No. 2232.

5439 Sha-ni | 泥, post-town near Chin H., Kansuh.

$5+40$ Sha-shih | Cliang-ling $\mathrm{H} ., \mathrm{Hupeh}$.

5441 Sha-shill-ch'êng | 市 城, (obs.) formerly H., S. E. of Cling-chou $\mathrm{Fu}$, Hupeh.

5442 Sha-t'ing | 亭, (obs.) T'ang, H., E. of P'ing-lê $F$ in, Kuangsi.

5443 Sha-ts'un | 村.

$1^{\circ}$ Ss̆̆ near Hsin-hui H., Kuangtung.

$2^{\circ}$ (obs.) 10 li E. of Sha H., Fuhkien; S. Sung and $S . T s^{\prime} i, \mathrm{H}$. in Chienan. 建管 chïn.

$3^{\circ}$ See No. 6084 .

$54+4$ Sha-tu | 圖 Shato, town in Yarkand; Lat. $41^{\circ} 10^{\prime}$, Long. $77^{\circ} 50^{\prime}$.

5445 Sha-wan | 㡉, ssǐ in P'anyii H., Kuangtung.

5446 Sha-ya-êrl | 雅 尔 Shayar, town in Yarkand; Lat. $41^{\circ} 41^{\prime}$, Long. $82^{\circ} 45^{\prime}$.

5447 Sha-yang | 陽, see No. 709. 5448 Slia-yang | 洋, post-town near $\mathrm{Cl}_{1}$ 'ien-chiang $\mathrm{H}$., Hupeh.
山 ShaN-HAt.

5449 Sha-yi | 获, see Nos. 741, 5315.

5450 Slia-ying | 䍃, ssŭ near Chên-ning C., Kueichon.

5451 Sha-yüan | 源, chên near Yung-ning $C$., Shansi.

\section{葆}

$5+52$ Sha-ching 砂 势, posttown near I,an-chou $F$, Kansuh.

\section{殺}

5453 Sha-hu-k ou 晸 点口。 ssŭ in Yu-yü H., Shansi. An important Customs Station, presided over by an Imperial Commissioner.

Sha-chau, see No. 5424.

\section{SHAN}

山

$5+54$ Slian-chai !寨, chên near Hua-t'ing H., Kansulh.

5455 Shan-ch'êng | 城, posttown near Huan $\mathrm{H}$., Kausuh.

5456 Shan-chiao | 角, ssŭ near Clı'üan C., Kuangsi.

5457 Shan-ch' uan-pao | 穿堡, ssŭ in Ma-p’ing H., Kuangsi.

5458 Shan-hai | 海, see No. 3901. 
山 SHAN-HAI-KUAN.

5459 Shan-hai-kuan | 海 關, an inportant frontier town on the borders of Chihli and Shingking near the point at which the Great Wall reaches the sea; this town is included in the jurisdiction of the Intendant of the Fêng-Chin-Shanlai circ., Shingking. The lighest 111litary authority is, however, an official of the province of Chibli.

5460 Shan-ho | 河, chên near Ning C., Kansuh.

5461 Shan-ho-t' $u 11$ |河屯, sš̆ in Wu-ch' ang 'T., Kirin.

5462 Shan-hsi | 䲨 Shan-si.

$1^{\circ}$ A province of China; literary names Chinyang 铂陽, Shan-yu | 朾 and Chin 铂; capital, 'T'ai-yüan Fu; it contains 4 circ., $9 \mathrm{~F} n$, 11 T., 10 C.I..C., 6 C. and $86 \mathrm{H}$.

$2^{\circ}$ (obs.) near P'ing-liang Fu, Kansuh; Sung, pao in Huai-tê 懹德 nnilitary district.

$3^{\circ}$ See No. 6102.

$5462^{\wedge}$ Shan1-k'ou | 口 Yamaguchi, ken and chief town, Japan.

$5462^{\mathrm{B}}$ Shan-1i | 梨 Vannanashi, ken in Japan; chief town, Kôfu.
II SHAN-TAN.

$5462^{\mathrm{c}}$ Shan-hsing | 形 Yamagata, ken and chief town, Japan. $5462^{\mathrm{D}}$ Shan-11an | 南, (obs.) T'ang, circ. in Hupeh, representing the ancient Ching 鼎 CHOU and Liang 梁 chov. It was divided into Eastern and Western divisions.

$5462^{E}$ Shan-pei $\mid$ 北, (obs.) Wei, H., S. E. of Hsi-an Fu, Shensi.

$5462^{\mathrm{F}}$ Shan-p’o | 坡, ssü in Chiang-hsia H., Hupeh.

$5462^{\mathrm{G}}$ Shan-sang | 桑, see No. 4374.

$5462^{\text {B }}$ Shan-shê | 台, Y. near Chiang-luua H., Hunan.

$5462^{\mathrm{I}}$ Shan-shih | 萑.

$1^{\circ}$ (obs.) $40 l i$ N.E. of Ch'ang-ch'ing H., Shantung; Tsin, H. in 'I'aishan 奉 山 chïn; $S$. Sung and N. Wei, H. in 'T'ai-yüan1 太愿 chïn.

$2^{\circ}$ See No. 241.

$5462^{\mathrm{J}}$ Shan-tan |丹 Shan-tan.

$1^{\circ} \mathrm{H}$. in Kan-chou $\mathrm{Fu}$, [390] Kansuh ; Lat. $38^{\circ} 50^{\prime}$, Lung. $101^{\circ} 29^{\prime}$; Han, Tsin, Sui and T'ang, Shan-tan 删丹; Yüan, Shan-tan |丹C.; Ming, Shan-tan ff military district. 
山

SHAN-IT' ANG.

$5462^{\mathrm{K}}$ Shan t'ang | 䛸, posttown near Ch'ên-ch' $\mathrm{i} H$., Hunan.

$5462^{\mathrm{L}}$ Shan1-tsao | 聚, ssŭ in Ch' üan C., Kuangsi.

$5462^{\mathrm{M}}$ Shan-tso | H: see No. $5462^{\circ}$.

$5462^{N}$ Slian-tu| 都, (obs.) Ts ${ }^{6}$ in, H., N. E. of Hsing-yang Fu, Hupeh.

$5462^{\circ}$ Shan-tung | 東 Shan-tung.

$1^{\circ}$ A province of China; literary names 'T'ai-tai 秦岱, Shan-tso | tr or Tung 㸃; capital. Chi-nan $\mathrm{Fu}$; it contains 3 circ., $10 \mathrm{Fu}, 2 \mathrm{C}$. L. C., $9 \mathrm{C}$. and $96 \mathrm{H}$.

$5462^{\mathrm{P}}$ Shan1-yang | 㖴 Shan-yang.

$1^{\circ}$ H. in Shang C., Shensi; [378] Lat. $33^{\circ} 29^{\prime}$, Long. $110^{\circ} 01^{\prime}$; Han, Shangch'êng 阔城; $T$ sin, Fêng-yang 豐晹; $N$. Wei, Shang-ynng 上庸 chiün in I.o 洛 cHOU.

$2^{\circ} \mathrm{H}$. forming the prefectural city of Huai-an, Kiangsu; Lat. $33^{\circ} 25^{\prime}$, I ong. $119^{\circ} 22^{\prime}$; Han, Shê-yang 射隄; $T \sin$, Shan-yang | 陽; Sui, $\mathrm{Cl}_{1}$ ' $\mathrm{u}$ 楚 $\mathrm{C}$.

$3^{\circ}$. See Nos. 2244, 7373.

$5462^{8}$ Shan-yang-ai | 羊险, ssü near Ho-fêng $\mathrm{C}$., Hupeh.
删! Shan-tax.

$5462^{R}$ Shan-yao | 爻, Y., near Chiang-hua H., Hunan.

$5462^{\text {s }}$ Shan-yin | 陰 Shan-yin. $1^{\circ} \mathrm{H}$. in Ta-t'ung F'tu,

[376] Shansi; Lat. $39^{\circ} 33^{\prime}$, Long. $112^{\circ} 58^{\prime}$; $T s^{\prime}$ in, Yin-kuan 隐 领; Sui, Shên-wu 卌武; Kin. Shan-yin | 13 .

$2^{\circ} \mathrm{H}$. forming with Kueichi $H$. the prefectural city of Shao-hsing, Chehkiang; Lat. $29^{\circ}$ 56', Long. $120^{\circ} 39^{\prime}$; Ts'in, Shan-yin | 訟.

$3^{\circ} \mathrm{H}$. in Ch ing-shang 序 份 circ., Corea; Lat. $35^{\circ} 09^{\prime}$, Long. $127^{\circ}$ $30^{\prime}$.

$4^{\circ}$ See No. 3469.

$5462^{\mathrm{r}}$ Shan-yu | 右, see No. 5462.

\section{汕}

5463 Shan-t ou 讪 䫓 Swatow (also called Sha-shan-t'on 沙|敛), a town in Cl' êng-hai IH., Kuangtung; one of the ports open to foreign trade; Lat. $23^{\circ} 20^{\prime}$, Long. $116^{\circ} 39^{\prime}$; it is included in the $\mathrm{T}^{\circ} \mathrm{O}$ p'u䑨浦 ssŭ.

\section{删}

5464 Shan-tan 删丹, see No. $5462^{\mathrm{J}}$. 


\section{杉}

5465 Shan-kuan 杉關, posttown near $\mathrm{K}$ uang-tsê $\mathrm{H}$., Fulukien.

5466 Shan-yang | 隄, chên near Kn-t'ien H., Fuhkien.

\section{單}

5467 Shan 㟕. Shen, H. in [370] Ts'ao-chon Fiu, Shantung; Lat. $34^{\circ} 57^{\prime}$, Long. $116^{\circ}$ $18^{\prime} ;$ S.A., Shan-fu-yi 父邑 in K. I $\mathrm{n}$ 凮; $2 n d$ Wei, Pei-chi-yin 北濟陰; S. Sung, Li-hu 離狐 $\mathrm{H}$.; Five dyn., Shan | H.

5468 Shan-fu-yi | 父邑, see the preceding.

5469 Shan-yü | 于, (cbs.) S. of Kuei-hua T., Shansi ; Tang,.. Fu in Knan-nei

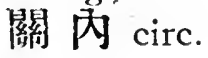

\section{閶}

5470 Shan-hsi 闒 熙, (obs.) E. of Ching-pien H., Shensi ; N. Wei, chün in Hsia 夏 CHOU.

5471 Shan-yi | 邑, see No. 4711.

$$
\text { 司 }
$$

5472 Shan-sha 扇 沙, (obs.) Liang, H., E. of Suicli'i H., Kuangtung.

\section{㷋}

5473 Shan 炎剡, see Nos. 531, 2727.

\section{蚨}

5474 Shan 善, see No. 2483.

5475 Shan-hua | 化 Shen-hwa, $\mathrm{H}$. forming with $\mathrm{Ch}^{\text {' } a n g-}$ sha $H$. the prefectural city of Ch'ang-sha, Hunan; Lat. $28^{\circ} 12^{\prime}$, Long. 112 $47^{\prime}$; orig., Hsiang-t an 湘 潭; Sung, Shan-hua | 化. 5476 Shan-kuang ssŭ | 北寺, Yenkoji, chief town of Nagano Ken, Japan.

5477 Shan-lao | 筞, (obs.) Tang, H., N. of Yü-lin C., Kuangsi.

5478 Shan-shan | Ill Shen-slian, $\mathrm{Fu}$ in Ch 'ing-sliang 敩份 circ., Corea; Lat. $35^{\circ} 51^{\prime}$, Long. $128^{\circ} 10^{\prime}$.

5479 Shan1-shan | 閶, see No. 5387.

5480 Shan-wên | 文, (obs.) N. of Yï-lin C., Kuangsi ; T゙ang, H. in 'lang 䈏 C. 5481 Shan-wu| 無, (obs.) S. of Yu1-yï H., Shansi; Han, H. in 'ling-hsiang 定琵 chiun $; N$. Wei, chïn and $\mathrm{H}$. in Hêng 恒 chov.

\section{部}

5482 Shan-yang, 郘晹, see No. 4312.

傸

5483 Shan 傸 Shen. 
陝

$1^{\circ}$ C. I. C. in the Shan[148] Ju circ., Honan; Iat. $34^{\circ} 45^{\prime}$, Long. $111^{\circ}$ 03'; Han, Slıan|H.; N. Wei, Hêng-1nung 恒 崼 chïn in Shan | CHOU.

$2^{\circ}$ (obs.) N. Wei, chov in Honan.

5484 Shan-an | 发, circ. in Shensi comprising Hanclunng $F u$ and Hsing-an Fu.

5485 Sha11-ch' 11an | 川, see No. 2576.

5486 Shan-clung | 中, (obs.) 110 li S. E. of Shan C., Honan; $N$. Wei, H. in Hêng-nung 恒褽 chün.

5487 Shan-hsi | 西 Shensi.

$1^{\circ}$ A province of China, literary names Wei-nan 渭南, $\mathrm{Cl}_{1}$ in 秦, $\mathrm{K}$ uanclinung 關中, or Lu11ng 散; capital, Hsi-an Fu; it contains 5 circ., $7 \mathrm{Fu}$, 8 T., 5 C. I. C., 5 C., and $73 \mathrm{H}$.

$2^{\circ}$ (obs.) Sung, circ., comprising parts of Shensi and Kansulh. $3^{\circ}$ See No. 197.

5488 Shàn-Kan | 甘 Shen-Kan, collective name of the two provinces, Shensi and Kansulh.
上 Shang-ching.

\section{澶}

5489 Slian 澶, see No. 3057. $5+90$ Slian-yüan | 滩, see No. 3057.

Sha11-si, see No. 5462.

\section{SHANG}

1.

5491 Sliang 上, (obs.) Han and Ts'in, chün in Shensi ; see Nos. 4410, 4947, 5942, 7422 .

5492 Shang-ch' ên | 陳, posttown near $\mathrm{Vi} \mathrm{C}$., Chilnli.

5493 Shang-cl1'i | 溪, (obs.) Yüan and Ming; C., S. E. of Llung-shan H., Hunan.

5494 Shang-chia |甲, (obs.) Liang, H., S. of Hu-k'ou H., Kiangsi.

5495 Shang-clı'iao ho | 橋 河, ss̆̈ in Wu-wei C., Anluui. 5496 Shang-Ching-Nan | 荆南, circ. in Hupel, comprising Ching-chou $\mathrm{Fu}$ and $\mathrm{Yi}$ ch'ang Fu. Also known as Ching-Yi 荆宜 circnit. 5497 Shang-ching | 津, (obs.) 2nd $\mathrm{Wei}, \mathrm{H}$. in Shensi; Lat. $33^{\circ} 02^{\prime}$, Long. $109^{\circ}$ $58^{\prime}$; Han and $T \sin , \mathrm{K}$. Sinang 商; Sui, Tang, Sung and Ming, Shangcling | 津 $\mathrm{H}$. 
上 Shang-Ching-pao.

5498 Shang-cling-pao | 津堡, ss̆̆ near Yün-lisi H., Hupeh.

5499 Shang-ch'ing | 清, chên near Knei-ch i H., Kiangsi.

5500 Shang-ch'nan-k'ou| M 口, 'T-S. near Nien-po H., Kansull.

5501 Shang-ch' nan-ye11 | 川監, ss̆̈ near Yang-cliang 'T., Knangtung.

5502 Shang-fêng | 封, (obs.) S.W. of Cl' in C., Kansul ; N. $\mathrm{We} i, \mathrm{H}$. in 'T'ien-slui 天水 chiin.

5503 Shang-hai | 海 Shanghai, [152] literary nannes Sliên 申, Shang-yang | 洋 and $\mathrm{H}_{11}$ 渥, H. in Sung-chiang Fu, Kiangsi1; Lat. $31^{\circ} 15^{\prime}$, I ong. $121^{\circ} 27^{\prime}$; one of the ports open to foreign trade. 5504 Shang-liang | 杭 Shang[464] hang, H. in 'T'ing-chon F'n, Fulnkien; Lat. $25^{\circ}$, I,ong. $116^{\circ} 32^{\prime} ; T^{\prime}$ ang, I inng-yen 龍 袘; Sung, Shang-hang | 杭.

5505 Shang-linai · 懷, (obs.)

Yüan, H. near L,ung-ying T.C., Kuangsi.

5506 Shang-hnang | 䔡, (obs.) Tsin, H., E. of Nan-chang H., Hupeh.

5507 Shang-liung | 洪, (obs.) E. of Yi-ch' êng H., Hupelı; Sui, H. in Hsiang-yang 垶陽 chiin.
上 Shang-KuFi.

5508 Shang-hisia-l11ng | 一泬, $\mathrm{Y}$. near Fu-ning $\mathrm{H}$, Kiangsi.

5509 Shang-hsia-tu11g |下凍 [198] Shang-hia-tung, 'T. C. in T'ai-p'ing Fu, Knangsi; Lat. $22^{\circ} 25^{\prime}$, I,ong. $106^{\circ}$ $28^{\prime}$.

5510 Shang-jao | 䭗 Shang-jan, H. forming with Kuangfêng $H$. the prefectural city of Kuang-hsin, Kiangsi ; Lat. $28^{\circ} 28^{\prime}$, I.ong. $118^{\circ} 06^{\prime}$; Han, P'o-yang 鄱陽; Wu, Shang-jao| 镬.

5511 Shang-kang | 岡, sš̆ in Yen-ch' êng H., Kiangsu.

5512 Shang-kao | 高 Shang[13] ka11, H. in Jui-chon Fu, Kiangsi ; I,at. $28^{\circ} 11^{\prime}$, I.ong. $114^{\circ} 47^{\prime} ;$ Han, Chien-ch' êng 建城; Tsin, Wang-ts'ai 旺蔡.

5513 Shang-ku | 谷.

$1^{\circ}$ (obs.) Ts'in, chün comprising $\mathrm{N}$. of Chilili.

$2^{\circ}$ (obs.) formerly town N. of Yen-ch'ing C., Chillit.

5514 Shang-knei | 邽.

$1^{\circ}$ (obs.) S.W. of $\mathrm{Ch}^{\prime} \mathrm{in}$ C., Kansuh ; W. Han, H. in Ltung-hsi 龖 西 chiin; E. Han, H. in Han-yang 漢陽 chïn; $T \sin$ and Sui, H. in 
.

Shang-KUhi.

'T‘ien-shni 天水 chün; Tang, H. in Ch'in 秦 $\mathrm{C}$.

$2^{\circ}$ (obs.) in Ssŭch'uan;

S. Sung and S. Ts' $i$,

H. in 'T'ien-shni 天水 chïn.

$3^{\circ}$ See No. 4567.

5515 Shang-liang | 底, chên near Cling-hsin H., Kansul.

5516 Shang-lien | 廉, see No. 5170 .

5517 Shang-lin | 林 Shang-lin.

$1^{\circ} \mathrm{H}$. in Ssŭtề11 Fu,

[151.] Knangsi ; I at. $23^{\circ} 25^{\prime}$, Long. $108^{\circ} 20^{\prime}$; orig., $\mathrm{I}$, ing-fang 領方; T'ang, Shang-lin | 林. $2^{\circ}$ 'T. H. near the above. $3^{\circ}$ See No. 5822.

5518 Shang-lo | 洛, see No. 5550.

5519 Shang-lo 维, see No. 4068.

5520 Shang-lı|䋊, see No. 2458 .

5521 Sliang-lung | 龍 Sluanglung, T. C. in T'ai-p'ing Fu, Knangsi; Lat. $22^{\circ}$ $40^{\prime}$, L Long. $107^{\circ} 42^{\prime}$.

5522 Shang-ma-cl' iao | 馬槅, T-S. near Ting-fan C., Kueichon.

5523 Shang-1110 | 陌.

$1^{\circ}$ Chên near Wn-k'ang H., Chelıkiang.

$2^{\circ}$ See No. 4640.

5524 Shang-1nu | 幕, see No. 1014.
1: Shang-tang.

5525 Sliang-11a-t'o | 納奪, 'T-S. near Sung-p an T., Ssı̆clinall.

5526 Sliang - p'an | 盤, posttown near Hun-yian C., Shansi.

5527 Shang-p'êng | 蓬, (obs.) in Ya-clion Fu, Ssuchluan; Tang, C. in Chienl-11an 劍南 circ.; Sung, C. in Clı êng-tı 成都 Fu, circ.

5528 Shang-p'ing | 坪, sš̆ in I.ien-p ing C., Knangtung.

5529 Shang-slih-hsi | 石 西, 年. c. in 'T"ai-p ing Fu, Knangsi; rang and Sung, Sli1h-11si 石西 C.; lFing, Shang-shih-lisi | 石西 C.; Lat. $22^{\circ} 08^{\prime}$, I,ong. $104^{\circ} 16^{\prime}$.

5530 Shang-ssŭ | 思 Shang-sz’. $1^{\circ}$ C. in '个ai-p ing Fu, [309] Knangsi; Lat. $22^{\circ} 20^{\prime}$,

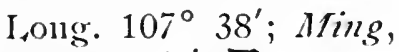
Shang-ssŭ | 思 C.

$2^{\circ} \mathrm{S}$. of the above; Tang and Sung, Shang-ssŭ | 思 C。

5531 Slrang-tang | 嘿.

$1^{\circ}$ (obs.) Ts'in and Han, chiön comprising parts of Cliblili, Shansi and Honan.

$2^{\circ}$ See Nos. 225, 1073, $3801,4098,6840$. 
上 Shang-'T'ANG.

5532 Shang-t'ang | 塘, Y. near Chiang-hua $\mathrm{H}$,,Hunan.

5533 Shang-tê-ch' êng | 得 成, chên near Ch'ing-shui H., Kansull.

5534 Shang-ts'ai | 蔡 Shangtsai.

$1^{\circ} \mathrm{H}$. in Ju-ning Fu, Honan; Iat. $33^{\circ} 19^{\prime}$, Long. 114 $33^{\circ}$; Chou, 'Ts'ai 僖; Han, Shangts'ai 蔡; Sui, W11chien 武建.

$2^{\circ}$ See No. 3124 .

5535 Sliang-tı | 都 Xanadu, also called L11an-ching 灣 京; an Imperial residence under the Yüan dyn.; near Dolonnor 'T., Chilhli. Chandu (Polo).

5536 Shang-t u $\mid$ 士., sš̆ near Ho-shan H., Anluii.

5537 Sliang-tu1ng | 東, chên near Wên-11si H., Shansi.

5538 Shang-wan | 宛, see No. 4640.

5539 Shang-yang | 洋, see No. 5503.

5540 Shang-yang-k'ou | 洋口， post-town near Slin1nch'ang H., Fulıkien.

5541 Shang-yao | 窸, chên near Fêng-t ai H., Anluni.

5542 Shang-yi | 目., (obs.) 35 li N. W. of Ch'ien C., Shensi; Sui, H., in Cling-chao 京兆 chïn.
上 Shang-yüAN.

5543 Shang-yin-t'ai | 㷉 台, (obs.) in Shingking; Han, Shang-yin-t'ai | 殷 台 $\mathrm{H}$.

5544 Shang-yin | 垠, see No. 6667.

5545 Shang-ying | 朠 Shangying, T. C. in T'ai-ping Fu, Kuanosi; Lat. $23^{\circ}$ $03^{\prime}$, Itong. $105^{\circ} 43^{\prime}$.

5546 Shang-yu | 唒 Shang-yu, [395] H. in Nan1-an Fin, Kiangsi ; Lat. $25^{\circ} 50^{\prime}$, Long. $113^{\circ} 18^{\prime}$; Sui, Nan-k'ang 南氺; S. T'ang, Shangy"1 唒.

5547 Shang-yü | 虞 Shang-yut.

$1^{\circ} \mathrm{H}$. in Shao-lising $\mathrm{Fu}$, [539] Chelikiang; Lat. $29^{\circ}$ $52^{\prime}$, Long. $120^{\circ} 58^{\prime}$; T'ang, Shang-yü| 虞 $\mathrm{H}$.

$2^{\circ}$ (obs.) N. W. of the above; Han, Tsin and S. Sung, Shang-yü | 虞 $\mathrm{H}$.

5548 Shang-yüan | 元 Shangyuen, H. forming with Cliang-ning $H$. the prefectural city of Chiangning, Kiangsu; Lat. $32^{\circ}$ $05^{\prime}, \quad$ Long. $118^{\circ} 47^{\prime}$; $T s^{2} i n$, Mo-ling 秝陵 and Chiang-ch'êng 江 乘; T'ang, Shang-yüan | 元. $5548^{\text {A }}$ Shang-yüan | 宛, see No. 5822 . 
上 Shang-yung.

5549 Shang-yung | 庸.

$1^{\circ}$ (obs.) S. E. of Chushan H., Hupeh ; Han, H. in Han-clnnng 漢 中 chïn; Tsin, chïn and H. in Ching 荆 cHOU; S. Sung and $S$. $T s^{6} i$, chïn and H. in Liang 梁 CHOU.

$2^{\circ}$ (obs.) E. of P'ing-li H., Shensi ; S. Sung, H. in Wei-hsing 魏 興 chïn.

$3^{\circ}$ (obs.) S. E. of Chi1ch'i H., Hupeh; Sui, H. in Fang-ling 房陵 chiin; T'ang, H. in Fang 房 C.

$4^{\circ}$ See Nos. 1384, 5704.

\section{商}

5550) Shang 獄 Shang.

$1^{\circ}$ C. I. C.. in the ' 1 'n11g[441] Shang circ., Sliensi ; Lat. $33^{\circ} 51^{\prime}$, Irong. $109^{\circ} 54^{\prime}$; Han, Shanglo 上洛; Wei, Lo 洛 C.; P. T'ang, Shang I C.

$2^{\circ}$ (obs.) Shang, K. in Honan.

$3^{\circ}$ (obs.) Han and Tsin, $\mathrm{K}$. in Hupeh. $4^{\circ}$ - See No. 5497.

5551 Shang-ch' êng | 城 Shangching.
商 SHANG-IA.

$1^{\circ} \mathrm{H}$. in Ju-ning $\mathrm{Fu}$, [114] Honan; Lat. $31^{\circ} 56^{\prime}$, Long. $115^{\circ} 18^{\prime}$; Han, Hsin-ch'i 新都; Sung, Shang-ch'êng | 城. $2^{\circ}$ See No. $5462^{p}$.

5552 Shang-ch'in | 邱 Shangkiı.

$1^{\circ} \mathrm{H}$. forming the prefectural city of Kuei-tê, Honan; Lat. $34^{\circ} 29^{\prime}$, Isong. $115^{\circ} 51^{\prime}$; Han, Sui-yang 㫿陽.

$2^{\circ}$ See No. 3464.

5553 Sliang-ho | 河 Sliang-ho, [476] H. in Wu-ting Fu, Shantung; Lat. $37^{\circ} 23^{\prime}$, Long. $117^{\circ} 18^{\prime} ; H a n, H s i-w u$ 隰沃; Sui, Shang-ho 滴 河.

5554 Shang-lo | 洛, (obs.) $P$. Chou, H., W. of Shang C., Shensi.

5555 Shang-lo | 各隹, chên near Sliang C., Sliensi.

5556 Sluang-11a-1111-lin | 納 穆. 林 Dzangannin; town in Thibet; Lat. $29^{\circ} 40^{\prime}$, Long. $89^{\circ} 10^{\prime}$.

5557 Shang-11an | 南 Shangnan, $H$. in Shang $C$., Shensi; Iat. $33^{\circ} 26^{\prime}$, Long. $110^{\circ} 41^{\prime}$.

5558 Shang-pa | 巴, ’-S. near Sung-p' an T', Ssŭch' 'nan. 
商 Shang-Shli.

5559 Shang-shui | 水 Shang[550] shwui, H. in Ch ên-chon Fu, Honan; I alt. $33^{\circ} 37^{\prime}$, Long. $114^{\circ} 43^{\prime} ; \quad T$ sin, Yin-cliang 溵江.; Sui, Yin-slu1i 澱水; Sung， Shang-slnii | 水.

5560 Shang-yii-cli êng | 於城, (obs.) formerly town near Ning-ling H., Honan.

\section{滴}

5561 Shang-ho 滴河, see No. 5553.

\section{闹}

5562 Shang 何 Shang, C. in Ch' ing-shang 磨 岁 circ., Corea; I at. $36^{\circ}$, Itong. $127^{\circ} 55^{\prime}$.

5563 Shang-chia | 家, Y. near Knang-hua H., Hupelı.

5564 Shang-ssü | 思（olos.）in Annan1 ; T゙ang, C. in I ing-nan 領南 circ.

Shang-ching, see No. 5551. Shang-hia-tnng, see No. 5509.

Shang-jan, see No. 5510. Shang-kant, see No. 5512. Shang-kiu, see No. 5552. Shang-shwui, see No. 5559. Shang-sz', see No. 5530. Shang-tsai, see No. 5534. Shang-yu, see No. 5547 . Shang-yuen, see No. 5548.
邵 ShaO-YANG.

\section{SHAO}

\section{召队}

5565 Shao 邵, see No. 6937.

5566 Shalo-chai | 賽, chên near Ling-t'ai H., Kansuh.

5567 Shao-ling | 陵, see No. 4937.

5568 silıa - po | 諙, chên in Kan-ch' ïan H., Kiangsu. 5569 Shao-wu | 武 Shau-wu.

$1^{\circ} \mathrm{F} u$ in the Yen-Chien[131] Shao circ., Fullkien; forming also the district city of the same; Lat. $27^{\circ} 22^{\prime}$, I,ong. $117^{\circ}$ 33'; Chou, Cli' $\mathrm{i}-\mathrm{min}$ 七閩; Han, in Kueichi 會嵇 chiun; $W u$, Chao-wu 炤 武; $T$ sin, Shao-wul 武; S. Sung, Shao-wu|武 nilitary districț.

$2^{\circ} \mathrm{H}$. forming the prefectural city of the same name, Fulikien; Lat. $27^{\circ} 22^{\prime}$, I,ong. $117^{\circ} 33^{\prime} ; T \sin$, Shaoyang | 晹; Sung, Shaowu|武; T'ang, Suich'êng 綏 城.

$3^{\circ}$ See No. 3268 .

5570 Shao-yang | 陽 Shau-yang.

$1^{\circ} \mathrm{H}$. forming the prefectural city of Paoch'ing, Hunan; Lat $27^{\circ}$ $04^{\prime}$, Irong. $111^{\circ} 21^{\prime}$; 
邵

ShaO-YANG.

Han, Chao-ling 昭陵; E. Han, Chao-yang 船 陽; Tsin, Shao-yang | 瑏

$2^{\circ}$ See Nos. 4937, 5569.

5571 Shao-yüan | 源, chên in Chi-yüan H., Honan.

\section{紹}

5572 Shao-ch ‘ing 紹庱, (obs.) S. Sung, Fu in K' 跸 circ.

5573 Shao-hsi | 熙, (obs.) s. Sung, Fu in 'T 'ung-ch' nan 潼川 circ.

5574 Shao-hising | 興 Shanhing, $F u$ in the NingShao-'T'ai circ., Chehkiang, forming the two district cities of Kuei-chi and Shanyin ; Lat. $29^{\circ} 56^{\prime}$, Long. $120^{\circ} 39^{\prime} ;$ S. A., K. Yüeh 越; $T s^{\prime}$ in and Han, in Kuei-chi 會稽 chün; 1 st Sung, 'Tung-yang 東陽; Sui and T ang, Yüelı 越 C.; S. Sung, Shao-hising I 興 Fu. Tanpiju? (Polo). 韶

5575 Shao 韶, see Nos. 4447, 5576.

5576 Shao-chou | 州 Shan1-cliau, [22] Fu in the Shao-Nan-Lien circ., Kuangtung; forming also the aistrict city of $\mathrm{Ch}^{\prime}$ ü -chiang; Lat. $25^{\circ}$ $07^{\prime}$, Long. $113^{\circ} 09^{\prime}$; orig., in a foreign kingdom;
稍

Shayar.

$T s^{6}$ in, in Nanl-hai 南海 chïn; Han, in Kuei-yang 桂陽 chiün; $W u$, Shihlising 始與; $T^{\prime}$ ang and S. Sung, Shao |C.

5577 Shao-k'ang-yi 少康邑, see No. 7849.

5578 Shao-Nan-Lien 韶南連 circ. in Kuangtung, comprising Lien C., Nanhsiung C., Shao-chou Fu and Lien shan $\mathrm{T}$.

$$
\text { 肇 }
$$

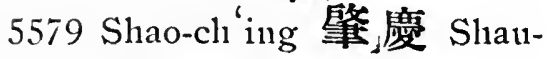
king, see No. 335.

啗

5580 Shao-ma 哨馬, Y. near $P^{6}$ ing-lo H., Kansull. 稍

5581 Shao-yä 稍峪, chên near C'l’ êng H., Kansuh.

Sharayas, see No. 5431. Sliash, see No. 154. Shato, see No. 5444. Shau, see No. 5749. Shat1-chang, see Nos. 5750, 5751.

Shan-chan, see No. 5576. Shath-hing, see No. 5574. Shau-king, see Nos. 335, 5579.

Shat1-kwang, see No. 5753. Shall-ning, see No. 5\%55. Shat1-w11, see No. 5569. Shau-yang, see Nos. 5570, 5756.

Shayar, see No. 5446. 


\section{SHE}

葉

5582 Shê 葉 Sheli.

$1^{\circ}$ H. in Nan1-yang Fut, Honan; I alt. $38^{\circ}$. 43';

Long. $113^{\circ} 22^{\prime}$; orig.,

K. Ying 應; S. A., Chn1-lianly-yi 諸梁邑; Han, Shê | H.; N. Ts'i, Hsiang 襄 C.; T'ang, Hsien1-fu 仙兒; Sui, Ju-fên 汝顀 H.; Kin, Lin-fên 臨填 chênn.

$2^{\circ}$ (obs.) Tang, C. near 'T's'ang-cll'i H., Ssücli itan.

\section{射}

5583 Shê 射, see No. 7347 .

5584 Shê-chiang | 江, see No. 5585.

5585 Shê-lıu11g | 洪 Sliel1-liung, [125] H. in 'I"1111g-cl' 'na11 Fu, Ssŭch'nan; I.at. $31^{\circ}$, Long. $105^{\circ} 20^{\prime}$; Han, 'Tz “ 䣚 H.; $W$. $\mathrm{Hei}$, Shêchiang 江; P. Chou,

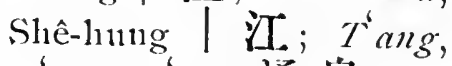
'T'ung-ch"üan 通泉.

5587 Shê-yang | 陽, see No. $5462^{\mathrm{r}}$.

\section{空}

5588 Shê-ch' êng 舍城, (obs.) Sui, H., N. E. of $\mathrm{Cl}_{1}{ }^{\mathrm{i}} \mathrm{iung}$ clion Fil, Knangtung.
5589 Shê-li-ts'u1 | 里 村, post-town Fêng-t ien Fu, Shingking.

\section{捨}

5590 Shê-tzŭ 捨语, (obs.) Yïan, small town near Lin-an Fì, Yünnan.

\section{奢}

5591 Shê-yen 孝 延, post-town near Cling-yang $\mathrm{H}$, , Shensi.

\section{社}

5592 Shê-cl' êng 抏. 城, chên near Yüi-sliê H., Shansi.

5593 Shê-chn! 潜, chên near Chien-p'ing H., Anluni.

\section{賖}

5594 Shê-ch'i 賖旗, chên near Nan-yang Fu, Honan.

\section{攝}

5595 Shê-k'oul 攝 口, see No. 5596.

\section{淪}

5596 Shê-k'ou灄口(or 攝门), ssŭ in Huang-p'ei H., Hupeh.

\section{涉}

5.597 Shê 涉 Slieh, H. in Chang[75] tê Fu, Honan; Lat. $36^{\circ}$ $42^{\prime}$, I,ong. $113^{\circ} 48^{\prime}$; orig., K. Sha 沙; Han, Shê | $\mathrm{H}$; Kin, $\mathrm{Cl}^{\prime}$ ung 崇 C. 


\section{涉}

SHỀ-MO.

5598 Shliê-1110 | 范, (obs.) formerly K. in E. of Corea.

\section{䣄}

5599 Shê 歌 Hilı.

$1^{\circ} \mathrm{H}$. forming the prefectural city of $\mathrm{Hui-}$ chou, Anhui ; Lat. $29^{\circ}$ $59^{\prime}$, L,ong. $118^{\circ} 28^{\prime}$; Ts'in, Hsi IH.; Sui, $\mathrm{H}$ si | C.; Sung, Hui 溦 C.

$2^{\circ}$ See Nos. 598, 2358, 5830.

5600 Shê-ku | 谷 Hiln-kulı, H. in Cliang-yiian 江原 circ., Corea ; Lat. $38^{\circ} 10^{\prime}$, Long. $128^{\circ} 54^{\prime}$.

\section{㩒}

5501 Shê-yüi 搩榆, (obs.) N. E. of 'T'ai-ho H., Yümman; E. Han, H. in Yungclı'ang 永:昌chïn; Tsin, H. in Yün-nan 雲南 chün; S. Sung: H. in Ho-yang 河 陽 chiin; S. Ts'i, H. in 1st 'Tung Ho-yang 東 河陽 chïn, 2ndin Hsi-a 西 阿 chïn.

\section{設}

5602 Shê-la-tzr̆ 設刺子, sec No. 5677 .

5603 Shể-li-wang | 里 壮: (or, Shih-êrl-wan-sha 失兒㡉 沙) Shirvan, formerly a
神

\section{SHÊN-HSIEN.}

province in Transcaucasiana; now a government of Asiatic Russia called Sliemakha.

Sheh, see Nos. 5582, 5597. Shell-hung, see No. 5585. Shemaklia, see No. 5603. Shen, see Nos. 5467, 5483. Shen-hwa, see No. 5475. Shenl-kan, see No. 5488. Shell-shan, see No. 5478. Shen-si, see No. 5487 .

\section{SHÊN}

\section{申}

5604 Shên 中.

$1^{\circ}$ (obs.) formerly K. in Honan.

$2^{\circ}$ See Nos. 2810, 4640.

\section{吩里}

5605 Shlên 触, chên near Hsinkan H., Kiangsi.

5606 Shên-an姼, ssŭ in Nan-hai H., Kuangtung.

$5606^{A}$ Shên-ch'ih | 池 Shin-chi, [376] H. in Ning-wu F'u, Shansi ; Iat. $39^{\circ} 15^{\prime}$, Long. $112^{\circ}$.

5607 Shên-ching | 京, see No. 5822.

$5607^{A}$ Shêln-hı | F Kobé, town and open port in Japan.

5608 Shên-hisien | 仙, Y. near Chiang-hıa H., Hunan. 
部

SHÊN-HSÜAN.

5609 Shên-hı̈̈an | 宣, ss̆̌ near Kuang-yüan H., Ssŭch' 'uan. 5610 Shên-ını | 木 Shin-1nuh, [115] H. in Yii-lin Fu, Shensi; I.at. $38^{\circ} 55^{\prime}$, Lo11g. $110^{\circ}$ 06'; Han, Wu-yüan 五.原 and Ho-l1si 河西; Tang, Hsin-ch'in 新秦; Sung, Shên-1111 | 木.

5611 Shên-11ai-clin ${ }^{\prime}$ |an 矣 川 Kanagawa, ken in Japan; chief town, Yokohanna.

5612 Shên-tao-ling | 道嶺, Y. near Han-ch"êng H., Shensi.

5613 Shên-t'un| 韻, chên near Yen-cl' nan H., Sliensi.

5614 Shên-tıl 都, see No. 5822. 5615 Shliên-w11 | 武.

$1^{\circ}$ Chên near Huai-jên H., Shansi.

$2^{\circ}$ See No. $5462^{\mathrm{s}}$.

\section{莘}

5616 Shên 㴖 Sin.

$1^{\circ}$ H. in 'Tung-ch' ang Fit, Shantung; I at. $36^{\circ}$ $16^{\prime}$, I,ong. $115^{\circ} 53^{\prime}$; Han, Yang-p'ing 陽 庐; P. Chou, Wu-yang 武陽; Sui, Ch'ing-y i 清邑 and Sliê11 H.; T'ang, Shên |C.

$2^{\circ}$ (obs.) formerly K., S. of Ho-yang H., Shensi.

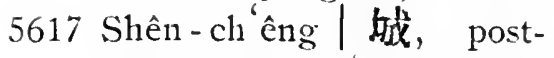
town near Ch'ên-lin H., Hona11.
沈

SHÊEN-LI.

\section{辰}

[See under the syllable $\mathrm{CH}^{\prime} \hat{\mathrm{E}} \mathrm{N}$.]

\section{憤}

5618 Shên 榱, see No. 2584.

5619 Shên-yang | 陽, see No. 488.

\section{瀋}

5620 Shên 瀋, see No. 1773.

5621 Shên-yang | 陽, see No. 1773.

\section{沈}

5622 Shên 沈, (obs.) S.A., K. in Honan; see No. 3010.

5623 Shên-ch'in | 邱 Shin-kin. $1^{c} \mathrm{H}$. in $\mathrm{Ch}$ ' ên-chou $\mathrm{F} n$, [212] Honan; Lat. $33^{\circ} 16^{\prime}$, Long. $115^{\circ} 12^{\prime}$; orig., K. Shên I ; Han, P'ing-11sing 平興; Sui, Shên| C.; T'ang, Shên$\mathrm{ch}^{\prime} \mathrm{in}$ |邱.

$2^{\circ}$ Ss $\breve{u}$ in Fur-yang H., Anhui.

5624 Shên-hisi | 犀, post-town near Chien-wei H., Ssŭch' nan.

5625 Shên-li | 黎, (obs.) 61 li $\mathrm{S}$. E. of Ch'ing-ch' $\mathrm{i} \mathrm{H}$., Ssŭchınan; S. Sung, chün in Yi 㿽 $\mathrm{CHOU} ; S . T s^{\prime} i$, chiin among the Lao 獠 tribes in $\mathrm{Yi}$ 益 $\mathrm{CHOU}$; Sui, H. in Lin-chiung 臨 狝 chün. 
沈 SHÊN-PIEN.

5626 Shên-pien | 邊, 'T-S. near Ch'ing-clı' i H., Ssŭclı 'uan.

5627 Sliên-ts' 111 | 村, post-town near Clı 'ing-ch'i $\mathrm{i}$ H., Ssŭcli' 11:111.

\section{深}

$562 S$ Shên 深 Shin.

$\mathrm{i}^{\circ}$ C.I. . C. in the Cll ${ }^{\prime} \mathrm{ing}$ [119] lo circ, Clibli; Sat. $38^{\circ} 06^{\prime}$, I.ong. $115^{\circ}$ $37^{\prime} ;$ Han, Hsia-po 下 博; Sui, Sliên |c. $2^{\circ}$ See No. 92 .

5629 Shên-ch'ing | 䓫, posttown near Clı ang't ai $\mathrm{H}$., Fullkien.

5630 Shlên-kou | 蒋, post-town near Kao-t ai H., Kansull.

5631 Slıên-tsê | 澤 Shin-tseh, H. in 'Ting C., Chilnli; I at. $38^{\circ} 14^{\prime}$, I ong. $113^{\circ}$ $18^{\prime}$.

\section{模}

5632 Shê̂-ch' 'nan 椹川. $1^{\circ}$ Sš̆ in Sui-ch'i $\mathrm{H}$, Kuangtung.

$2^{\circ}$ See No. 5961.

\section{SHENG}

\section{坐.}

5633 Shêng-mi 生米, chên in Hsin-chien H., Kiangsi.

\section{勝}

5634 Shêng 朌, see No. 7666 .
浅 SHÊNG-CHING.

5635 Shêny-fan1-kou | 番溝, ' 1 -S. near Nien-po H., Kansul.

5636 Shêng-lisiang | 詪, see No. 7860 .

5637 Sliêng-lien | 遇, town in the Lin-ch in Islands.

\section{升}

5638 shêng-hısiang 升 拫, ss̆̆ near L,ien-lua 'I', Kiangsi.

\section{昇}

5639 Shêng 昇, see No. 749.

56 to Sliêng-yüan | 元, (obs.) IV. of Hsiao-yi H., Shansi ; N. Wei, H. in Cliienp’ing 建平 chïn.

\section{彗}

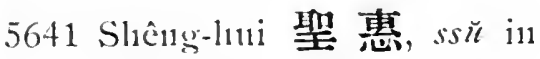
An-yi H., Shansi.

\section{維}

5642 Shêng 綎: see No. 4498.

\section{盛}

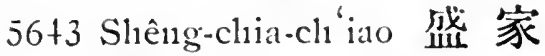
憍, chên near I,ï-chiang H., Anluii.

5644 Sliêng-cling | 京 Slingrking.

$1^{\circ}$ One of the Manclunrian provinces; literary name L,iao-tung 迫真; capital, Fêng-t ien Fur it contains 2 circs., $4 \mathrm{Fu}$, 2 C.L.T., 3 T., 5 C. and $18 \mathrm{H}$.

$2^{\circ}$ See No. 1773. 
盛

SHÊNG-KANG.

5645 Shêng-kang | 開 Morioka, chief town of Iwate ken, Japan.

5646 Shêng-tien | 呄, chên near IVei-yüan H., Kansulı.

5647 Shêng-tsê | 灣, chên near Wu-chiang H., Kiangsu. 5648 Shêng-yang | 陽, see No. 1773.

\section{茂}

5649 Shêtrg-kung 是 頁, see No. 525.

\section{省}

5650 Shêng-ch'i 省溪, 'I'-S. in T'ung-jên Fu, Kneichon;

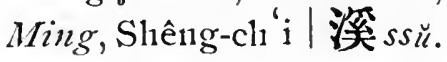

Shi-11an, see No. 5673.

Shi-ping, see Nos. 5.379, 5674.

Shidzloka, see No. 1171.

Shiga, see No. 6816.

Shinline, see No. 6274.

\section{SHIH}

\section{标}

5651 Shih-lisiang 沶㚹, (obs.) $T \sin$, town S. of Fang H., Hupeh.

\section{始}

5652 Shih-an 始 妾:

$1^{\circ}$ (obs.) near Sui-ting Fin, Ssŭch tran ; S. Sung and S. Ts $i, \mathrm{H}$. in $\mathrm{Pa}$ ch'ii 巴渠 chün.
蛞 SHIH-K'ANG.

$2^{\circ}$ (obs.) in Ssŭch'uan; $S$. Ts $i$, chion in Liang 梁 CHOU.

$3^{\circ}$ (obs.) near Huang-chou Fu, Hupelı; S. Ts $i, \mathrm{H}$. in 1st, Ch'i-an 唯安 chiün, 2nd, Hsin-an 新 安 chiin.

$4^{\circ}$ (obs.) in Kueichon; Tang, Shih-an | 努 $\mathrm{H}$.

$5^{\circ}$ See Nos. 3446, 3876, 5169.

5653 Shih-fêng | 䇺, see No. 6421.

5654 Shih-11sin | 新.

$1^{\circ}$ (obs.) W. of Shun-an H., Chehkiang ; Tsin, $S$. Sung and $S$. Ts' $i$, H. in Hsin-an 彩努 chiin.

$2^{\circ}$ (obs.) in Anluni; $S$. Sung and $S .7 s^{\prime} i, \mathrm{H}$. in Lï-chiang 盧 江. chiin.

5655 Shilh-hsing | 與 Shi-hing. $1^{\circ} \mathrm{H}$. in Nan-hsiung C.,

[22] Kuangtu1ng; Lat. $25^{\circ}$, I,ong. $113^{\circ} 36^{\prime}$; $W u$, Hsieh-chieh 斜 階; $T s^{\prime} i$, Chêng-chieh 正 階; Liang, Shih-hsing 與.

$2^{\circ}$ (obs.) P. Han, town S. W. of Shang-yii H., Chehkiang.

$3^{\circ}$ See Nos. 1450, 5576. 5656 Shih-k'ang | 康, see No. 2789. 
始 ShIH-P'ING.

5657 Shilh-p'ing | 平, see No. 2829.

5658 Shil1-yang | 陽.

$1^{\circ}$ Chên near 'T'ien-ch' üan C., Ssŭch' nan.

$2^{\circ}$ See No. 5200.

\section{洔}

5659 Shilı 時, (obs.) in Mon C., Ssŭch' uan; T'ang, C. in Clien1-nan 劍南 circ.; Sung, C. in Mou茂 chov.

5660 Shilı-pao | 堡, sš̌ in Kaoy"u C., Kiangsu.

5661 Shih-p'êng | 蓬, (obs.) in Ya-chon Fu, Ssŭch' 'nan; Tang, C. in Cliien111an 劍 南 circ.; Sung, C. in Ch'êng-tı 成都 circ.

\section{師}

5662 Shih 師, (obs.) in Fangshan H., Chillli; Tang, C. in Ho-pei 河 北 circ.

5663 Shih-tsung! 宗 Sz'-tsung, [96] H. in Kuang-hsi C., Yünnan ; Lat. $24^{\circ} 55^{\prime}$, Long. $104^{\circ} 10^{\prime}$; Yüan and Ming, Shih-tsung | 宗 C.

\section{獅}

5664 Shih-ch'iao 獅留, chên near Ning-kuo Fu, Aului.

5665 Shih-ling | 領; ss in Hua H., Kuangtung.

5666 Shih-tzŭ 子, Y. near Huo-clia H., Honan.
施 SHIH-NAN

\section{柿}

5667 Shih-ch' $i$ 柿深, (obs.) near Li C., Hn11an ; Yüan, Slihl1-ch' $\mathrm{i} \mid$ 深 $\mathrm{C}$.

\section{施}

5668 Shil 施, (obs.) S. Sung, C. in K'nei-chon 淮 州 circ.; see Nos. 1616, 5673.

5669 Shih-ch 'i | 溪, J-S. in Ssŭ-chon Fin, Kneichon; Ming, ssü.

5670 Shih-chor | 州, see No. 5673.

5671 Shih-ho | 鶴 circ. in Hupel, comprising Shithnan Fir and Ho-fêng 'T.

5672 Slihh-jung-ch'i | 溶 深 (obs.) near Yung-shun H., Hunan ; Ming, Shih-jungch'i | 溶溪 ssü.

5673 Shih-nan | 南 Shin-nan,

[74] F11 in the Shilh-Ho circ., Hupeh; forming also the

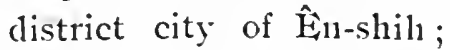
Lat. $30^{\circ} 16^{\prime}$, Long. $109^{\circ}$ $26^{\prime} ;$ S.A., in K. Ch ' 1 楚; $T s^{\prime}$ in, in K. Cli ien-chung 駖中; Han, in Nan 南 chïn; Wu, Sha-clı' $\mathrm{i}$ 沙 渠; Tsin, Yeln-lang 夜 郎; P. Chou, '个ing 家 C. and Ch'ing-chiang 清 江; Sui, Yung 庸 C. and Shih | C.; Trang, Shih | C. and Ch'ing-hura 清化; 
扰

SHIH-NAN.

Sung and Yüan, Shih C.; Ming, Shih-clon 州 military district.

5674 Shih-ping | 秀 Shi-ping, [20] H. in Chên-y ïan Fur, Kueichoul Lat. $27^{\circ}$, Long. $108^{\circ} 02^{\prime}$.

5675 Shih-tien | 佝, sš̆ in PaoSlııı H., Yünınan.

\section{头}

5676 Shih-êrh-wan-sha 失兒䕷 沙, see No. 5603.

5677 Shil1-la-ssŭ | 束川 思 (or Shê-la-tzŭ 設刺子, or Hsieh-la-shih 泄刺失) Shiraz, city of Persia,-in province Farsistan; I at. $29^{\circ} 36^{\prime}$, Long. $52^{\circ} 44^{\prime}$.

\section{蓄}

5678 Shil 荎, (obs.) N. Wei, H. in Chi-11an 沿南 circ.

\section{茌}

5679 Shih (or Tzŭ) 茌 (or 茬) (obs.) Han, H. $40 l i \mathrm{~N}$. $\mathrm{E}$. of $\mathrm{Cl}_{1}$ 'ang-cli'ing $\mathrm{H}$., Shantuing.

5680 Shih-p'ing | 平 Shi-ping, [476] H. in 'I'ung-clı'a11g Fin, Shantung; Lat. $36^{\circ} 42^{\prime}$, Long. $116^{\circ} 25^{\prime}$; orig., Clunug-ch'in 重期; $75^{\prime} \mathrm{in}$, Shih-p'ing | 市.

5681 Shih-shan | II, post-town near the preceding.
什

SHIH-SHÊ.

\section{沶}

5682 Shih-h1siang 沶鄉, see No. 5651.

\section{都}

5683 Shih 郝, see No. 606 .

传:

5684 Shih-lang 侍郎, chên near Cli' ao H., Anluni.

\section{琠}

5685 Shih-ta-kuan 䔰大 關, post-town near Mon C., Ssŭchinan.

\section{$+$}

5686 Shilh-li 十里.

$1^{\circ}$ Y. near Hsin-yang C., Honan.

$2^{\circ}$ Y. near Hsï-y.i H., Anluni.

568т Shilı-tzŭ-110|字河, chên near Wn-wei C., Anhui.

5688 Shih-yi-tı | 一 都, sš̆ near J.ung-cl' nan H., Knangtung.

\section{什}

5689 Shil1-fang 什邡Shih-fang, H. in Ch 'êng-tı Fu, Ssŭch' 'lan1 ; Iat. $31^{\circ} 06^{\prime}$, Long. $103^{\circ} 13^{\prime}$; Han, Shih.fang 邡; P. Chou, Fangting 邡禀。

5690 Shih-shê / 刑, chên near Ning C., Kansul. 
什 Shrh-TZJ.

5691 Shili-tzŭ | 字, chên near Ching C., Kansul.

\section{不}

5692 Shil 石.

$1^{\circ}$ Ssü near Jui-an H., Chehkiang.

$2^{\circ}$ (obs.) T"ang, C. near Tung-hsiang H., Ssŭch' tran.

$3^{\circ}$ See Nos. 154, 7086, 7862 .

5693 Shih-ai | 艾, sce Nos. 5192, 7080.

5694 Sliih-an | 管, see No. 2695 . 5695 Shih-cliê | 哲, chên near Ch'ang-tzun H., Shansi.

5696 Shih-cli êng | b城 Shilıching.

$1^{\circ} \mathrm{H}$. in Ning-tuC., Kiang[62] si ; Lat. $26^{\circ} 18^{\prime}$, I.ong. $116^{\circ} 17^{\prime}$; orig., Ningtu漂 都; S. T'ang, Shih-ch' êng | 城.

$2^{\circ} \mathrm{H}$. in Kao-chou $\mathrm{F} 11$, 193] Kuangtung; I at. $21^{\circ}$ $32^{\prime}$, Long. $109^{\circ} 50^{\prime}$; Han, Kao-liang 宫凉; Liang; Lo 羅C.; T'ang, Shih-cli'êng | 城.

$3^{\circ}$ H. in Chung-ch'ing 忠声 circ., Corea;

- I at. $36^{\circ} 47^{\prime}$, I ong. $126^{\circ} 11^{\prime}$.

$4^{\circ}$ Post-town near An-1n H., Hupeh.
不 SHIH-CH $\dddot{U}$ AN.

$5^{\circ}$ See Nos. 83, 863, 903, 986. $1452,3475,3538$, 5313.

5697 Slih-chia-tien| 家店, chên near Ning C., Kilnsuh.

5698 Shill-chiang | 澕, ss $\ddot{6}$ in Wan-p'ing H., Chilıli.

5699 Shil1-cli iao | 角, Y. in P'ing-shan H., Ssiúch' 'nan.

5700 Shili-ch'iao | 橋, ssī in Ching-mêtı C., Hupelı.

s701 Shih-ch'ien | 阶 Shilh[279] tsien, Fu in the Knei$P^{\prime}$ inıs -Shinh-Jên circ, Kneichon; Lat. $27^{\circ} 30^{\prime}$, Iong. $108^{\circ} 10^{\prime} ; T^{6}$ ang, Yi 美 C., Ning-yi 涪 夷 H., and Yi-clı̈üan 荑泉; Yüan, Shilh-ch'ien| 俐.

5702 Shili-ching-slian | 景山, post-town near I,ianghsiang H., Chihli.

5703 Shih-chu1 | 硅 Shih-chu, C. I.. 'I'., in Ch' 'nan-tung circ., Ssŭch' "ran; Lat. $30^{\circ}$ $30^{\prime}$, I.ong. $108^{\circ} 40^{\prime}$.

5704 Shih-ch'üan | 泉 Shil1tsinen.

$1^{\circ} \mathrm{H}$. in Hoinug-an Fu, [140] Shensi; Lat. $32^{\circ} 51^{\prime}$, Long. $108^{\circ} 30^{\prime}$; Han, Shang-yung 上庸; $W$. Wei, Shih-ch'ïan| 泉; rang, Ni1-an 武管.

$2^{\circ} \mathrm{H}$. in I, ung-an $\mathrm{F}_{11}$, [133] Ssŭch 'nan; I.at. $31^{\circ}$ $47^{\prime}$, I.ong. $104^{\circ} 23^{\prime}$; Han, Knang-jou 腐氶; 
石 SHIH-CH'ÜAN.

T'ang, Shih-ch'ïan |

泉; S. Sung, Shihclïuan | 泉 military district.

5705 Shih-cl' 'uan | 川 Ishikawa, ken in Japan ; chief town Kanazawa.

5706 Shili-chuang | 莊.

$1^{0}$ Chên near Ho C., Sliansi.

$2^{\circ}$ Chên near Kao-p'ing H., Shansi.

$3^{\circ}$ Ss $\breve{\iota}$ in Ju1-kao H.,

Kiangsu.

5707 Shili-fêng | 非, see No. 3415.

5708 Shih-fo | 佛.

$1^{\circ}$ Chên near Ch'in-an H., Kansul.

$2^{\circ}$ Ss̆̈ near Lïi-cl' 'i H., Kiangsi.

$3^{\circ}$ Post-town near Ch 'ingshîn H., Ssŭch' "ıan.

5709 Shih-11si | 西. see No. 5529.

5710 Shih-hsia | 匣, post-town near Mi-yün H., Cliihli.

5711 Shin-k'ang | 康, (obs.) Sung, H., 30 li N. E. of Lien-chon Fn, Knangtung. 5712 Shih-k'ou | 口, chên near Ning-kuo Fu, Anluni.

5713 Shih-ku| 鼓.

$1^{\circ}$ (obs.) formerly H. near Peking.

$2^{\circ}$ (obs.) Liang, H. near Sui-ting Fu, Ssŭch ' $3^{\circ}$ See No. 431.
石

SHIH-M ÊN.

5714 Shih-kuo | 果, sš̆ near Ho-fêng C., Hupel.

5715 Shilh-liang | 粱.

$1^{\circ}$ Chên near' 'T'ien-ch'iang H., Anluni.

$2^{\circ}$ See No. 6406.

5716 Shih-lin-ku | 榴倜, posttown near Hsia-yi H., Honan.

571万 Shih-lo-tzŭ |羅子, see No. 5677.

5718 Shilh-lon | 樓 Shih-lan, [116] H. in Fên-clon F'n, Shansi ; Iat. $37^{\circ} 05^{\prime}$, Long. $110^{\circ} 49^{\prime}$; orig., Cliü-ch' an 屈產; 2nd $\mathrm{Wei}$, T'nching 昍京; Sui, Shihlon | 樓.

5719 Shih-lung | 龍.

$1^{\circ}$ (obs.) 3 li N.E. of Hua

C., Knangtung; Sui, T'ang, Sung and Yüan, Sliih-lung | 龍 H.

$2^{\circ}$ (obs.) near I, nng-an Fu, Ssŭch'nan; T'ang, H. in Hsi-yel 西野 C. 5720 Shilh-ma | 碼, chên in Lung-clı'i H., Inuhkien.

5721 Shilı-ınên| 閏 Slıilı-mun. $1^{\circ}$ H. in Li C., Human; [244] Lat. $29^{\circ} 31^{\prime}$, L,ong. $111^{\circ} 23^{\prime}$; Han, I,ingyang 零陽; $W u, \mathrm{~T}^{i}$ ienmên 天門; Sui, Slrihmên | 門.

$2^{\circ} \mathrm{H}$. in Clia-hsing $\mathrm{Fu}$, Chehkiang; Lat. $30^{\circ}$ $33^{\prime}$, I,ong. $120^{\circ} 25^{\prime}$. 
$3^{\circ}$ Chên near Chiang-shan H., Chehkiang.

$4^{\circ}$ Chên near Chi C., Chih li (No. 580.)

$5^{\circ}$ Ss ̌̆ in Wu-kang C., Hunan.

$6^{\circ}$ Ssŭ in P'o-yang H., Kiangsi.

$7^{\circ}$ (obs.) near Chao-ch'ing $F u, \quad$ Kuangtung; $S$. Sung and $S . T s^{i} i, \mathrm{H}$. in Sung-k'ang 来康 chün.

$8^{\circ}$ (obs.) in Shou-yang H., Shansi ; N. Wei, H. in Kuang-ning 廣察 chün.

$9^{\circ}$ (obs.) N. W. of Chieh C., Kansulp; N. Wei, H. in Wu-tu 武都 chiin.

$10^{\circ}$ (obs.) near Han-chung Fu, Shensi; N. Wei, H. in IV $11-h \operatorname{sing}$ 武興 chïn.

$11^{\circ}$ (obs.) near Kungch'ang Fu, Kansuh ; $N$. Wci, H. in I,in-t ao 監 洮 chïn.

$12^{\circ}$ (obs.) in Kn-yüan C., Kansulı; Sung, pao in Huai-tê 懐德 military. district.

13…(obs.) in Nien-po H.,

Kansulı; Sung, pao in Chên-wu 震武 military district. $14^{\circ}$ (obs.) 50 li W. of 'Tsun-hua C., Chihli; Kin, chên in Su 蘇 C. $15^{\circ}$ See No. 5832.

5722 Shih-mên Chai | 师鼠, Y. in Lin-yö H., Chihli. 5723 Shil-11an | 南, see No. 2839.

5724 Shih-11in| 牛, ss̆̌ in Ninghua H., Fuhkien.

5725 Shih-p'ai | 牌, chên near Ch ien-chiang H., Hupeli.

5726 Shih-pao | 堡, chên near Hsi-lio H., Kansnh.

5727 Shihl-pao-cl'êng | 堡城, post-town near An-hsi C., Kansuh.

5728 Shih-p'ing | 平, see No. 2829.

5729 Shih-p'ing | 坪, see No. 5730.

5730 Shih-p'ing | 屏 Shih-ping, [227] C. in Iin-an Fin, Yünnan; Lat. $23^{\circ} 47^{\prime}$, Long. $102^{\circ}$ $46^{\prime}$; $T^{6}$ ang, land of the Wn-mo 鳥麼 tribes; Ÿ̈an, Shih-p'ing | 坪 C.

5731 Shih-p’o"坡, ss̆̆ near $\mathrm{Cl}_{1}{ }^{\text {ên }}$ C.., Hupelı.

5732 Shih-p "u | 浦 Shih-pn.

$1^{\circ} \mathrm{T}$. in Ning-po Fit, Chelıkiang; I at. $29^{\circ}$ $33^{\prime}$, I,ong. $121^{\circ} 42^{\prime}$. Terr. jur.

$2^{\circ}$ Chên in K' $n$ m-shan H., Kiangsu.

5733 Shih-shan | II, post-town near P'ei C., Kiangsu. 
石

SHIH-SHOU.

5734 Shih-shon | 首 Shih-shan, [528] H. in Ching-chon $\mathrm{Fu}$, Hupeh; Lat. $29^{\circ} 45^{\prime}$, Long. $112^{\circ} 16^{\prime}$; Han, Hua-jung 華容; Tsin, Shilh-shou | 首.

5735 Slihl1-tai | 埭 Shilh-tai, H. [531] in Ch'ih-chou Fu, Anhui ; Lat. $30^{\circ} 27^{\prime}$, Long. $118^{\circ}$ $03^{\prime} ; H a n$, Tan-yang 丹 陽; Liang, Shih-tai |埭. 5736 Shih-t'ai | 臺, (obs.) 40 li S. W. of Ju C., Honan; $N$. Wei, H. in Ju-pei 汝 北 chiin.

5737 Shih-t'ang | 塘, chên near

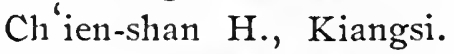
5738 Shih-tao | 道, chên near Têng-fêng H., Honan.

5739 Shih-tao | 島, ssŭ in Jungch'êng H., Shantung.

5740 Shilh-ti $\mid$ 堤, ssü near Yungsui $T$., Hunan.

5741 Shih-t'ou | 頭.

$1^{\circ}$ Post-town near Fêny$t^{\prime}$ ien $F u$, Shingking. $2^{\circ}$ (obs.) formerly town W. of Chiang-ning $\mathrm{F} n$, Kiangsu.

5742 Shih-t'oll-chieh / 頭街, ssŭ in Wan-nien H., Kiangsi.

5743 Shih-t'on-k'on | 頭口, ssŭ in Chia-yü H., Hupeh.

5744 Shih-wan | 灣, sš̈ in Po-lo H., Kuangtung.

5745 Shih-yang | 陽, see No. 4141.
Shou.

5746 Shih-yen | 堰, chển near Tz $\mathrm{ur}^{\mathrm{u}}$-ch ${ }^{6} \mathrm{i} \mathrm{H}$., Chehkiang. 5747 Sliih-yi | 邑, see No. 2423.

\section{適}

5748 Shih-clunng 適 中, posttown near Lung-yen C., Fuhkien.

Shili-ching, see No. 5696. Slili-lau, see No. 5718 . Shih-1nun, see No. 5721 . Shilı-ping, see No. 5730 . Shili-pu, see No. 5732 . Shih-shau, see No. 5734. Shih-tsien, see No. 5701. Shih-tsinen, see No. 5704. Shimané, see No. 6274. Shin, see No. 5628. Shin-chat, see No. 476. Shin-chi, see No. 5606 . Shi11-ki, see No. 475. Shin-kiu, see No. 5623. Shin-1nulh, see No. 5610 . Shin-tseh, see No. 5631. Shing-king, see No. 5644. Shiraz, see No. 5677. Shirvan, see No. 5603. Shobando, see No. 5765 .

\section{SHOU}

\section{壽}

5749 Shou 锋 Shau.

$1^{\circ} \mathrm{C}$. in Fêng-yang Fu, [144] Anlui; this city serves also for Fêng-t'ai H.; Lat. $32^{\circ} 34^{\prime}$, Long. 
SHou.

$116^{\circ} 43^{\prime}$; S. A., I. Iilliao 六蓼 K.; $T s^{\prime}$ in, Shou-ch' un | 春; Han, in Huai-nan 淮南 chün; $W e i$, in Yang 揚 chou; Sui, Shou | C.; W. Han, Chin-chiang 九江 chiin.

$2^{\circ}$ See No. 5750 .

5750 Shou-chang | 張 Shan[148] chang, H. in Yen-chou Fu, Shantung; Lat. $36^{\circ} 07^{\prime}$, Long. $116^{\circ} 05^{\prime}$; S. A., Liang-yi 辰邑; Han, Shou-liang |艮; E. Han, Shon-chang | 㲀; T'ang, Shou | C.

5751 Shoul-ch'ang | 昌 Shancluang.

$1^{\circ} \mathrm{H}$. in Yen-chou $\mathrm{Fu}$,

[2] Chelkkiang; Lat. $29^{\circ}$ $25^{\prime}$, Long. $120^{\circ} 22^{\prime}$; Wu，Hsin-ting 新定; $T \sin$, Shou-ch ang | 昌. $2^{\circ}$ (obs.) S. Sung, military district in Hu-pei 湖北 circ.

5752 Shou-ch' 'un | 春, see No. 5749.

5753 Shon-kuang | 光 Shat1[304] kwang, H. in Ch'ing-clon Fu, Shantung; Lat. $36^{\circ}$ 55', Long. $119^{\circ}$; orig., Chên-kuan 㽎灌; Han, Shou-kuang | 光.

5754 Shour-liang | 豆, see No. 5750 .
舒

Shu.

5755 Shon-ning | 篮 Shan-ning, [466] H. in Fu-ning Fu, Fuhkien ; Lat. $27^{\circ} 32^{\prime}$, Long. $119^{\circ} 30^{\prime}$; orig., Fuh-an 福 努.

5756 Shon1-yang / 陽 Shan-yang, [485] H. in P'ing-ting C., Shansi ; Lat. $37^{\circ} 55^{\prime}$, Long. $113^{\circ} 10^{\prime}$; S $A$., Ma-shol1-yi 馬首邑; Tsin, Shouryang |隄; Tang, Shon 受 C. 收

5757 Slou-ch'i，收溪 (obs.) near Ching C., Hunan; Sung, military post in Ching 竨 C.

\section{受}

5758 Shou 受, see No. 5753 .

\section{首}

5759 Shoun-li-wang-ch' êng 首里 王城 Shridi, town in the Liu-ch' in Islands.

5760 Shon-yang | 陽, see No 6989.

\section{SHU}

舒

5761 Shu 䟥.

$1^{\circ}$ (obs.) formerly K. in Anhui.

$2^{\circ}$ (obs.) 120 li IV. of Lü-chang H., Anhuii; Han, H. in I,ï-chiang 盧江 chïn。

$3^{\circ}$ Sec Nos. 55, 933, 2237, 2786, 3010, 4139, 5762 . 
舒 SHU-CH'

5762 Shu-ch'êng | 城, Shu[46] ching, H. in $\mathrm{I}_{4}$-chou $\mathrm{Fu}$, Anhui; I at. $31^{\circ} 30^{\prime}$, Long. $117^{\circ} 18^{\prime}$; orig., in K. Shu |; Han, Lunngcli'êng 龍 塯; T'ang, Shu1-ch'êng | 城.

5763 Shu-chiu-ch' êng | 鳩城, (obs.) formerly town near the preceding.

5764 Shu1-ch' 'tan | M, chïn in Chung-cling 忠清 circ., Corea.

5765 Shin-pan-to|班多 Shobando, town in Thibet; I at. $30^{\circ} 15^{\prime}$, Long. $95^{\circ} 30^{\prime}$.

5766 Shu 俞.

$$
\text { 命 }
$$

$1^{\circ}$ (obs.) $50 l i \mathrm{~S} . \mathrm{W}$. of P'ing-yüan H., Shantung; Han, S. Sung and $N . W c i, H$. in

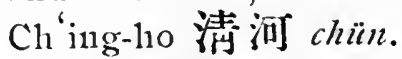
$2^{\circ}$ See No. 2561.

\section{束}

5767 Shu-1u or Sul-1u 束鹿 Shu-lul, H. in Pao-ting Fu, Chihli; Lat. $39^{\circ} 47^{\prime}$, Iong. $115^{\circ} 16^{\prime}$; Han, in Chii-lu 鉅鹿 chion; $N$. $T s^{\prime} i$, K. An 奖; Sui, La1-ch' êng 鹿城; T'ang, Shu1-1u | 鹿.

\section{䄈}

5768 Shu-h111ng 䄈鴻, (obs.) N. of Ta-t'ung H., Shansi; $N$. Wei, H. in Liangch'êng 梁城 chiir.
沭 SHU-YANG.

\section{朱}

5769 Sh11-shih 朱提.

$1^{\circ}$ (obs.) S. W. of Yi-pin H., Ssŭch'uan ; Han, H. in Chien-wei 徒篇 chiin; Tsin, chïn and H. in Yi 监 $\mathrm{cHOU} ; S$. Sung, chïn and $\mathrm{H}$. in Ning 穿 CHOU.

$2^{\circ}$ (obs.) in Ch'ing-fu H., Ssŭch'uau; T"ang, H. in $\mathrm{Cl}^{\prime} \ddot{\text { ï }}$ 曲 $\mathrm{C}$.

\section{殊}

5770 Shu-t 'ui 殊頽, (obs.) N. of Shou-yang H., Shansi; $N$. Wei, H. in Shên-wu 触武 chiin.

\section{蜀}

5771 Shu 蜀.

$1^{\circ}$ (obs.) S.A., K. in Ssŭch nan.

$2^{\circ} T s^{\prime}$ in to T'ang, chiin comprising W. part of Ssŭch' uan.

$3^{\circ}$ See Nos. 511, 1589, 5885.

5772 Sh11-p'o | 陂, (obs.) near Ying-chou $F \mathfrak{n}$, Anlui ; $N$. Wei, H. in Nan-cl' iao 南譙chuin.

\section{沭}

5773 Shu-yang 沭陽 Shuh[404] yang, H. in Hai C., Kiangsu; i,at. $34^{\circ} 08^{\prime}$, Long. $119^{\circ} 08^{\prime} ; H^{\circ}$ an, Honl-ch'in 厚邱; $W_{e} i$, Shu-yang | 陽. 
述

SHU-K' UN.

\section{述}

5774 Sh11-k'11n 述昆, (sometines wrongly written Mik'un 迷昆,) (obs.) S.W. of Yi-shan H., Kuangsi ; T'ang, C. in Ling-nan 領南 circ.; Sung, C. in Ch'ing-y'uan 慶裳 Fin.

Shu1-ching, see No. 5762 .

\section{SHUAI}

\section{率}

5775 Shn1ai-pin 率竇.

$1^{\circ}$ (obs.) in Shingking; Liao, $\mathrm{H}$. in $\mathrm{K}$ 'ang 康 $\mathrm{C}$. $2^{\circ}$ (obs.) S.E. of Ninguta,

Kirin ; Liao, $\mathrm{Fu}$ in Tung-ching 東 京 circ. 5776 Shuai-tao | 道, see No. 7421.

\section{SHUANG}

\section{雙}

5777 Shunang 雙, (obs.) $60 l i \mathrm{~W}$. of T'ieh-ling H., Shingking; Liao, C. in Tungching 東京 circ.

5778 Shuang-ch' êng | 城.

$1^{\circ}$ Post town near Kunangchi H., Hupeh.

$2^{\circ}$ T. in Chi-lin Fu, Kirin.

5779 Shinang-ch' $\mathrm{i} \mid$ 溪, chên near Yü̈-hang $H$. , Chelıkiang.
雙

SinUi-Yaxg.

5780 Shuang-chiang | 江, ssü near 'T'ung-tao H., Hunan.

5781 Shuang-cll iao | 橋, ssŭ in Ho-shan H., Kuangtung.

5782 Shunang-ching | 井, posttown near Su C., Kansul.

5783 Shunang-ch'ing/清, chên in T'ung-shan H., Kiangsu.

5784 Shuang-ch' ïan | 泉, chên near Chao-yi H.., Shensi.

5785 Shnang-fêng | 鳳, Y. near Yin-chiang H., Kneichou.

5786 Shuang-kou | 㩐.

$1^{\circ}$ Chên near 'Tsao-yang H., Hupeh.

$2^{\circ}$ Chên near. Wur-lio H., Anhui.

5787 Shuang-lin | 林, chên near Hu-chon Fu, Chehkiang.

5788 Shuang-liu | 流 Shwangliu, H. in Clı êtug-tı Fu, Ssŭch' 'uan; Lat. $30^{\circ} 37^{\prime}$, I.ong. $104^{\circ}$; Han, Kuangtu腐都; Sui, Shnanglin $\mid$ 流.

5789 Shnang-11niao-chan | 廟 站, post-town near Hsiaokan H., Hupel.

5790 Shunang-tsê | 則, 'T-S. near Sung-p' an T., Ssŭch' 'uan.

5791 Shnang-wei | 威, sš̆ near L u1-ch 'uan H., Kuangsi.

Shuang, see Nos. 4226, $4227,4228$.

Shudi, see No. 5759 .

Shuh-yang, see No. 5773. 
Shui.

\section{SHUI}

腄

5792 Shui 膰, see Nos. 659, 1877.

\section{水}

5793 Shui-an 水安, chên near Wên-shu iH., Shansi.

5794 Shui-ch'êng | 城 Shwniching, T. in Ta-ting $\mathrm{Fu}$, Kueichou ; Lat. $26^{\circ} 35^{\prime}$, Long. $104^{\circ} 15^{\prime}$.

5795 Shui-chih | 治, chên near Chang-tê $\mathrm{F} u$, Honan.

5796 Shui-chin-yüan | 盡源, (obs.) Ming, H. near Shil1nan $\mathrm{Fu}$, Hupeh.

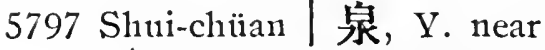
$P$ 'ien-kuan H., Shansi.

5798 Shui-hu | 戸 Mito, chief town of Ibaragi ken, Japan.

5799 Shui-hsiu | 修, see No. 7458.

5800 Shui-jun | 㵎, chên near Chao-ch' êng H., Shansi.

5801 Shui-k'ê | 客, chên near Hua-t'ing H., Kansuh.

5802 Shui-k'ou |口.

$1^{\circ}$ Chên near Lai-an H., Anhui.

$2^{\circ}$ Chên near Ch' ang-hsing H., Chehkiang.

$3^{\circ}$ Ssŭ near Kuang-tsê H., Fuhkien.

$4^{\circ}$ Sš̆ near Yung-an $\mathrm{H}$., Kuangtung.
順

Shun.

$5^{\circ}$ Post-town near $\mathrm{Ku}$ - $\mathrm{t}^{\prime}$ ien H., Fuhkien.

5803 Shui-1i | 豐, ssŭ near Nanching H., Fulhkien.

5804 Shui-tê | 德, T-S. in Ssŭnan Fu, Kueichou.

5805 Shui-t'ou | 頭.

$1^{\circ}$ Chên near Hsia H., Shansi.

$2^{\circ}$ Chên near Yung ho H., Shansi.

$3^{\circ}$ Y. near Lin-wu H., Hunan.

5806 Shui-ts'ao-p'ing | 草 坪, T-S. near Mou C., Ssurch'uan.

5807 Shui-tung | 東.

$1^{\circ}$ Y. near Sui-ch'i H., Kuangtung.

$2^{\circ}$ (obs.) Ming, sš̆ in Kuei-chn H., Kueichou. 5808 Shui-wei | 尾, ssŭ near Lin-kao H., Kuangtung. 5809 Shui-yang | 陽, chên in Hsüan-ch êng H., Anhui. 5810 Shni-yüan | 原 $F u$ in Ching-ch' $\mathrm{i}$ 京畿 circ., Corea.

\section{SHUN}

\section{順}

5811 Shun 順 Shun.

$1^{\circ}$ T. C. near Li-chiang

Fu, Yünnan; Lat. $26^{\circ}$ 39', Long. $100^{\circ} 43^{\prime}$; Yiäan, Shun $\mid \mathrm{C}$. 
順

SHUN.

$2^{\circ}$ (obs.) T'ang and Yïan, C. near Shun-yi H., Chihli.

$3^{\circ}$ (obs.) Trang, C. near Wu-chou Fu, Kuangsi. $4^{\circ}$ See No. 5825.

5812 Shun-an | 安, chên near 'T'ung-ling H., Anluni.

5813 Shun-ch'ang | 昌 Shunchang, H. in Yen-p'ing Fu, Fuhkien ; Lat. $26^{\circ} 48^{\prime}$, Long. $117^{\circ} 58^{\prime} ; T^{6}$ ang, Chiang-shui-ch'ang 漿水 昌; S. T⿱㇒冋厶ang, Shuncli'ang | 昌.

5814 Shun-chêng | 政.

$1^{\circ}$ Y. near Mien H., Shensi. $2^{\circ}$ See No. 4157.

5815 Shun-ch'ing | 度 Shunking, $\mathrm{F} u$ in the $\mathrm{Ch}^{\text {' }}$ wanpei circ., Ssüch'uan; forming also the district city of Nan-ch' ung; Lat. $30^{\circ}$ $49^{\prime}$, Long. $106^{\circ} 08^{\prime}$; Chou, in Yung 倠 CHOU; S.A. and $C$. Wars, in $\mathrm{K} . \mathrm{Pa}$ 巴; $T s^{\prime}$ in, in $\mathrm{Pa}$ 巴 chïn; Han, An-han 安 漢; 1st Sung, Tang-ch ü 宕渠 chün; Sui, Pa-lssi 巴西 chiin; T'ang, Nan1ch'ung 南充 and Kuo 果 C.; Yüan, 'Tung-cl' 'uan 東川; S. Sung and Ming, Shun-ch'ing | 度 Fu.

5816 Shun-ch'uan | M Shunchuen, $F_{n}$ in $P^{\prime}$ ing-an 平
順 SHUN-TÊ.

安 circ., Corea; Lat. $39^{\circ}$ $34^{\prime}$, Long. $126^{\circ} 15^{\prime}$.

5817 Shun-hsing | 與 H. in Ch'ing-shang 产何 circ., Corea.

5818 Shun-lin | 林, ssŭ in $\mathrm{Li}$ C., Hunan.

5819 Shun-ning | 夏 Shu11-ning. $1^{\circ} \mathrm{Fu}$ in the Yi-nan circ., Yünnall; forming the district city of the same name; Lat. $24^{\circ} 38^{\prime}$, Long. $100^{\circ} 08^{\prime}$; Yüan, Shun-ning|莩Fu. Burmese name Shwen-li.

$2^{\circ} \mathrm{H}$. forming the prefectural city of the same name, Yünnan; Lat. $24^{\circ} 38^{\prime}$, Long. $100^{\circ} 08^{\prime}$. 5820 Shun-slih | 石, chên near Shun-hua H., Shensi.

5821 Shun-tê | 德 Shun-teh. $1^{\circ} \mathrm{F} u$ in the Ta-ShunKuang circ., Chinli ; forming also the district city of Hsing-t ai ; Lat. $37^{\circ} 07^{\prime}$, Long. $114^{\circ}$ 39'; Chou, K. Hsing 邢; C. Wars, 1st, in $\mathrm{K}$. Chin 晉, 2nd, in K. Chao 趋; Ts in, Hsintı 信都 in Chï-1u鉅 鹿 chiin; Han, K. Hsiang 桽; Sui, Hsing 邢 C.; T'ang, Chü-lı 鉅 鹿 chïn; Sung, Hsin-tê 信德; Ming, Shun-tê | 德 Fu. 
P'ENG-YING.

$2^{\circ}$ H. in Kuang-chon $\mathrm{F}_{n}$, Kuangtung; Lat. $24^{\circ}$ $49^{\prime}$, Isong. $112^{\circ} 49^{\prime}$; orig., in Nan-hai 南 海 chïn.

5822 Shnn-t'ien $\mid$ 天 Shun-tien, or PEI-Ciring 北京 Peking, an Imperial Prefecture in Chihli; Lat. $39^{\circ} 57^{\prime}$, Long. $116^{\circ} 29^{\prime}$; forming also the two district cities of Ta-11sing and Wan-p'ing; the metropolis of the Empire and residence of the Court; also called Clining-tu 京都, or simply Cling 京; Chou, Yu 幽; Ts'in, Shang-ku .上谷 chïn and Yii-yang 漁晹 chion; Han, Kuang-yang 廣陽 and K. Yen 浾; Tsin, Sui and $T^{6}$ ang, Fan-yang 范陟; Sung, Yen-shan 燕山；Ming，1st，Peipiing 北平 Fu, 2nd, Shun-t'ien|天Fn; Ÿ̈̈an, Ta-tu大都 circ. Cambaluc (Polo).

\section{$2^{\circ} \mathrm{Fu}$ in $\mathrm{Cl}^{6}$ üan1-lo 全躍 circ., Corea.}

N.B.-The following are some of the nanles by which the capital of China is referred to in the native press.]

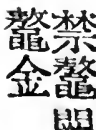

Ao-chin

Chin-ao

,, cli'üich
金闯

, 喜

, 它

禁旅

京城

, 嫘

, 師

, 都

九重

, 華

珼林

䛒

春明

第城

池

閣

楓階

, 宸

泉端

量证

, , 洲

都

杏苑

日下

軟絽。

宮樹鷹聲孫 $\left\{\begin{array}{c}\text { Kung-slun-ying- } \\ \text { shêng }\end{array}\right.$

蘭臺清話 Lan-t'ai-ch'ing-hua

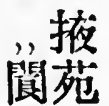

蚊十坐

, 陛

韵池

替噎

北關

落萊

, 息

, 渣
Chin-mên

, t ai

") $\operatorname{cl}^{6} \hat{e}_{11}$

", ch'êng

Ching-ch' êng

, cll' $\mathrm{i}$

"shil

," tu

Chin-chung

," liua

Cly iung-lin

Ch '" thr-1ning Fêng-ch' êng

," cli ${ }^{6} \mathrm{il}$

", cl' ïieh

" pi

" cl'ên

Huang-ch'i

" chou

, tu

Hsing-y ïan

Jilh-hsia

Juan-hung

Lan-yeb

Lang-yuian.

Li-ao

, pi

Lung-cli 'il

Nien-ku

Pei-ch' ïieh

P'êng-lai

,1 tao

", ying 
SHUN-T'IEN.

溘

Shux-AN.

上林

苑

触京

, 都

太掖

丹陛

管城

, 京

, 䦩

天雚

天府

, 階

梁殿

都下

阴

影地

延

紫宸

禁

看池

"

, 量?

掖池

嚥京

市

润洲

御街

苑

王園

, 京

, 階

5823 Shun - t'ung | 通, (obs.)

5824 Shun-yang | 陽.

Shên-ching

T'ai-yeh

'Ta11-pi

'Ti-ch' êng

, cling

"lium

T'ien-ch' ì

'T'ien-f

T'ient-pi

To-tien

Tu-lisia

, mên

'I'ang-ti

T'ing-t'ing

Tzun-ch' ên

" chin

Yao-cls'ih

"pi

, $\mathrm{t}$ ai

Yeli-ch il

Yen-ching

," shih

Ying-cliou

Yï-cliielı

, yïan

" "

", cling

,pi.]

\section{IV. of Ho 河 C., Kansuh ; Stung, pao in Clii-shih 皘 石 nilitary district.}

$1^{\circ}$ Chên near P'u-ch' êng H., Shensi.

$2^{\circ}$ See No. 7698.

5825 Slıu1-yi | 義 Shun-i.

$1^{\circ}$ H. in Shun-t ien Fu, [337] Chillli; Lat. $40^{\circ} 09^{\prime}$, Long. $116^{\circ} 42^{\prime}$; Ts'in, Shang-kn上谷 chün; Han and Wei, Fanyang 范陽; Sni, Shun

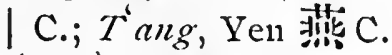
$2^{\circ}$ (obs.) Liang, H. near Sui C., Honan.

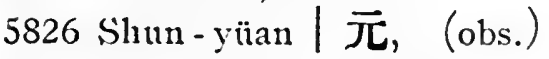
Yüan, circ. in Kueichon; see Nos. 3485, 5073.

\section{鶉}

5827 Shu11-ku 敦孤, see No. 5828.

5828 Shun-ku | 触, or | 孤. $1^{\circ}$ (obs.) N. E. of Lingt ai H., Kansul, ; $W$. Han, H. in Pei-ti 北 地 chiin; E. Han and Tsin, H. in An-ting 安定 chïn.

$2^{\circ}$ See Nos. 1112, 3970.

\section{渲}

5829 Shun 澶.

$1^{\circ}$ (obs.) Tang, C. near

Yuug-slnun I., Kuangsi. $2^{\circ}$ (obs.) T'ang, C. near Ning-lisia Fu, Kansuh. 5830 Shu11-an | 努 Shun1-11gan, [377] H. in Yen-chon Fu, Chehkiang; Lat. $29^{\circ} 34^{\prime}$, Long. 
$119^{\circ} 03^{\prime}$; Han, Hsi 拿 H.; $W u, H \sin -\mathrm{tu}$ 新 都; T'ang, Ch'ing-ch'i 清溪; Sung, Shun1-an | 安.

5831 Shun - ch'ang | 昌, chên

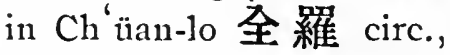
Corea.

5832 Shun-hua | 化 Shun-hwa. $1^{\circ} \mathrm{H}$. in Pin C., Shensi; Lat. $34^{\circ} 55^{\prime}$, Long. $108^{\circ} 30^{\prime}$; Han, Yün-ling 呍陵; 2nd Wei, Yünyang 雲陽; T'ang, Shih-mên 石門; Sung, Shun-hua | 化.

$2^{\circ}$ Chên near Cliang-ning $\mathrm{Fu}, \mathrm{Kiangsu.}$

5833 Shun-jên |人, (obs.) W. of 'T'êng H., Kuangsi ; Sui, Shun-jên |人 $\mathrm{H}$.

5834 Shun-yü|于, (obs.) N.E. of An-ch'iu H., Shantung; Han, Tsin, S. Sung and N. Wei, Shun-yü|于 $\mathrm{H}$.

Shun-chang, see No. 5813. Shun-chuen, see No. 5816. Shun-liwa, see No. 5832. Shun-i, see No. 5825. Shan-king, see No. 5815. Shun-ngan, see No. 5830 . Shun-teh, see No. 5821. Shun-tien, see No. 5822. Shwang-liu, see No. 5788. Shwen-li, see No. 5819. Shwui-ching, see No. 5794. Si-chang, see No. 2439. Si-chung, see No. 2453.
Si-hiang, see No. 2465. Si-ho, see No. 2458. Si-hwa, see No. 2460. Si-lin, see No. $247 \%$. Si-lung, see No. 2480. Si-ngall, see No. 2438. Si-ning, see No. 2483. Si-ping, see No. 2487. Siang, see No. 2599. Siang-ching, see No. 2611. Siang-fu, see No. 2607. Siang-hiang, see No. 2629. Siang-ling, see No. 2613. Siang-shan, see No- 261)3. Siang-tan, see No. 2633. Siang-yang, see No. 2616. Siang-yin, see No. 2635. Siang-yuen, see No. 2618. Sian, see No. 2668. Siau-shan, see No. 2690. Siberia, see No. 7521. Sieh, see No. 2515. Sien-kü, see No. 2707. Sien-yu, see No. 2691. Sih, see No. 2522. Sih-ngo, see No. 2519. Siju, see No. 5915. Silato, see No. 2527. Silinghol, see No. 2526. Sin, see No. 5616. Sin-chang, see No. 2727. Sin-chau, see No. 2955. Sin-ching, see Nos. 2729, 2731.

Sin-fan, see No. 2749.

Sin-fung, see No. 2805. Sin-hiang, see No. 2759.

Sin-hing, see No. 2761. 
Sin-HO.

Sin-ho, see No. 2754.

Sin-lıwa, see No. 2755.

Sin-liwui, see No. 2757.

Sin-i, see No. 2812.

Sin-kan, see No. 2765.

Sin-kien, see No. 2738.

Sin-loh, see No. 2768.

Sin-min, see No. 2771.

Sin-ngan, see No. 2724.

Sin-ning, see No. 2774.

Sin-ping, see No. 2775.

Sin-tai, see No. 2779.

Sin-tien, see No. 2784.

Sin-tsai, see No. 2786.

Sin-tsin, see No. 2740.

Sin-tu, see No. 2789.

Sin-yang, see Nos. 2792, 2810.

Sin-yé, see No. 2793.

Sin-yu, see No. 2797.

Sindachu, see No. 2930.

Sindafu, see No. 511.

Sing-tsz', see No. 2855.

Sinigul, see No. 2895.

Sinju, see Nos. 2483, 7468.

Sinjumatu, see No. 602 .

Siu-jin, see No. 2870.

Siti-shan, see No. 2865.

Siu-shwui, see No. 2866.

Siu-wăn, see No. 2873.

Sil1-wli, see No. 2874.

Siu-yen, see No. 2867.

Siuen-ching, see No. 2924.

Siuen-chuen, see No. 2925.

Sțuen-hwa, see No. 2930.

Sinen-ngăn, see No. 2926.

Siuen-ping, see No. 2932.

Sinen-wei, see No. 2934.
岪

So-LU.

Siun-hwa, see No. 2962.

Siun-tien, see No. 2949.

Siun-yang, see No. 2966.

Siün, see No. 2957.

\section{SO}

\section{莎}

5835 So-ch'ê染車, C.I.C. in Kashgar 'circ., New Dominion.

\section{菒}

5836 So 萎, see No. 4216.

5837 So-ch ‘ù | 渠, (obs.) in Ssŭch'uan; Trang, C. in Lung-yu 隴右 circ.

5838 So-k'ê | 克, T. S. in Kokonor.

5839 So-ku-mu | 古木, Sougom, town in Yarkand; Lat. $39^{\circ} 45^{\prime}$, Long. $69^{\circ}$ $45^{\prime}$.

5840 So-k110-k'ê | 郭克 Sugukur, town in Cobdo; Lat. $47^{\circ} 40^{\prime}$, Long. $87^{\circ} 30^{\prime}$.

5841 So-1u | 盧.

$1^{\circ}$ (obs.) Liang, H., S. of Hsin-hsing H., Kuangtung.

$2^{\circ}$ (obs.) N.Wei; H., 15 li E. of Tsao-ch 'iang $\mathrm{H}$., Chihli.

$3^{\circ}$ (obs.) S. Sung and $N$. Wei, H. near Chi-nan $\mathrm{Fu}$, Shantung. 
素 SO-T'ING.

5842 So-t'ing | 总, Y. near Jung-yang H., Honan.

\section{朔}

5843 So 䍭 Solr.

$1^{\circ} \mathrm{C}$. in So-p ing F 11 , Shan[376] si ; Lat. $39^{\circ} 25^{\prime}$, Isong. $112^{\circ} 27^{\prime} ;$ Han, Ma-yi 馬邑; Wei, Hsin-hsing 新 與; Tsin, Hsincli' ang 新昌; P. Chou, So $\mathrm{C}$.

$2^{\circ}$ See No. 1191.

5844 So-chou | 州, Fu, in P'ingan 补努 circ, Corea.

5845 So-fang | 方.

$1^{\circ}$ (obs.) W. Han, chou, comprising part of Kansulı; Tsin, chïn.

$2^{\circ}$ See Nos. 2266, 4687.

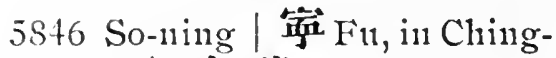
ch'i 京畿 circ., Corea.

5847 So-p “ing | 平 Soll-ping, [516] F'n in the Yen-Ping-'Ta-So-

Ning-Hsin-Tai - Pao circ., Shansi; forming also the district city of Yu-y ï ; Lat. $40^{\circ} 10^{\prime}$, Long. $112^{\circ} 13^{\prime}$. 桨

5848 So-li 桨里, see No. 5340. 恕

5849 So-t'i 䓡題, (obs.) S. of Tsao-ch'iang H., Clihli ; W. Han, H. in Ch'ing-ho 清河 chiin.
Ssŭ.

\section{梭}

5850 So-1110 梭磨, 'T.S., N.W. of Li-fan 'T., Ssŭch'uan. Thibetan name Sumung [Baber].

\section{䔖}

5851 So-jên 葆 人, (obs.) S. of Fan-chih H., Shansi; $W$. Han, H. in 'T'ai-yüan 太 原 chün； Tsin, H. in Ying-mên 磼閒 chïn.

Sodania, see No. 5967. Soh: see No. 5843. Soh-ping, see No. 5847.

\section{SOU}

\section{擢}

5852 Sou-chi 拽集, post-town near Wan-ch'ïan $H$, Chihli.

Sougom, see No. 5839.

Söul, see No. 363.

\section{ssü}

思

5853 Ssŭ 思 Sz'。

$1^{\circ}$ T. C. in 'T'ai-p'ing Fu,

[309] Kuangsi; Lat. $23^{\circ} 20^{\prime}$, Lonn. $106^{\circ} 45^{\prime} ;$ S. Sung, Ssŭ |C.

$2^{\circ}$ See No. 5858. 
思

Ssü-AN.

5854 Ssü-an | 努, S. Ts'i chïn in Ssŭch' uan.

5855 Ssŭ-chi | 吉, chên near Shang-lin H., Kuangsi.

5856 Ssŭ-chin | 勤,(obs.) N.E. of Cliao-p ing H., Knangsi ; r ang, H. in Fu 富 C.

5857 Ssŭ-chiung | IS , (obs.) $90 l i \mathrm{~S} . \mathrm{E}$. of Chên-yüan H., Kueichon; T"ang, H. in Ssŭ |C.

5858 Ssǔ-chou | 州 Sz-clıau, [410] Fu in the Kuei-tung circ., Kneichou; Lat. $27^{\circ} 11^{\prime}$, I ong. $108^{\circ} 35^{\prime}$; Ts'in, in Ch'ien-chung 默 中 chün; Han, in Wu-ling 武陵 chün; T⿱ang, Ssŭ $\mid$ C. and Ning-yi 漂 夷; Ming, Ssŭ-chou | 州 Fu.

5859 Ssŭ-ên | 恩 Sz-ngăn.

$1^{\circ} \mathrm{Fu}$ in the "I"ai-P'ing [416] Ssı̆-Shun circ., Knangsi ; Lat. $23^{\circ} 25^{\prime}$, Long. $107^{\circ} 54^{\prime}$; Han, in Chiaochil 交趾, chïn; T゙ang and Ming, Ssŭ-ên | 恩 Fi1.

$2^{\circ}$ H. in Ch 'ing-yüan F'u, [274] Knangsi ; Lat. $24^{\circ} 48^{\prime}$, Long. $107^{\circ} 58^{\prime}$; Ming, H. in Ho-ch' ih 河池 C. $3^{\circ}$ (obs.) S. of Ssŭ-ên H.,

- Kuangsi : Tang, H. in Huan 瞢 C.; Sung, H. in Ch'ing-yiian 麀遠 Fir.
思 SSU-NAN.

$4^{\circ}$ (obs.) 150 li N. W. of Ssü-ên Fı, Knangsi; T'ang, C. in I,ing-nan 領南 circ。

$5^{\circ}$ (obs.) near Nan1-111ng Fu, Kuangsi ; T"ang, H. in Wei 椳 C.

5860 Ssŭ-knan | 管, chên in Yung H., Kuangsi.

5861 Ssŭ-knei | 踝, (obs.) near Hsiang-yang Fir, Hupeh ; S. Ts $i, \mathrm{H}$. in An-ting 桇 定 chïn.

5862 Ssŭ-ling | 陵 Sz'-ling, 'T. C. in T'ai-p'ing Fu, Kuangsi ; I at. $21^{\circ} 56^{\prime}$, Long. $106^{\circ} 50^{\prime} ;$ Sung, Ssŭ-ling | 陵 C.

5863 Ssŭ-1u11g | 龍, (obs.) $T^{6}$ ang, H. near Hsincl $^{\prime}$ êng 'T.H., Knangsi.

5864 Ssŭ-lung-yi | 龍馹, ss̆̌ near Pin C., Knangsi.

5865 Ssŭ-lu11g | 部, (obs.) S. of Itrugan H., Kuangsi; T*ang, H. in Yung 崖 C.

5866 Ssŭ-mao | 函 Sz'-11nan, 'T. [429] in P'n-êrlı I'u, Yünnan; Iat. $23^{\circ} 30^{\prime}$, I.ong. $101^{\circ}$ $40^{\prime}$; called locally Esmok and, by the Burmese, Muang-la.

5867 Ssŭ-111ng | 明; T゙ ang, C. in Kuangsi.

5868 Ssŭ-11an | 南 Sz'-11an, Fiu [513] in the Knei-tung circ. Kneichon; forming also 
思

SsŬ-NAN .

the district city of An-hua ; Lat. $27^{\circ} 56^{\prime}$, Long. $108^{\circ}$ $26^{\prime}$; S. A., and C. Wars, in $\mathrm{K}$. Cl' $\mathrm{n}$ 楚.

5869 Ssŭ-11ung | 震, (obs.) in Annam; T Tang, C. and H. in Ling-nan 領南 circ. 5870 Ssŭ-shun | 順, (obs.) $T^{6}$ ang, C.near Hsin-ch' êng H., Kuangsi.

5871 Ssư-t'ung|同, (obs.) 15 li S.W. of Yung-k'ang C., Kunangs; T'ang and Sung, Ssǔtt ung | 同 C.

5872 Ssŭ-wêng | 幾, (obs.) in Ssŭch 'nan; T'ang, H. in Hung 鴻 C.

\section{徙}

5873 Ssŭ 徙, (obs.) E. of 'T'iench'üan C., Ssùclı' uan; Han, H. in Shu蜀 chïn.

5874 Ssŭ-yang | 陽, (obs.) Han and $T \sin , \mathrm{H}$., S. of Yaclon Fru, Ssŭch'uan.

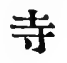

5875 Ssŭ-yai 寺崖, chên near Ning-hsia Fu, Kansuh.

\section{司}

5876 Ssŭ 司, see No. 2079.

5877 Ssŭ-na | 馬, chên near Huai-jên H., Shansi.

5878 Ssŭ-nung | 震, (obs.) near Chin-chou $\mathrm{F}_{\text {in }}$, Shing-king; Liao, H. in Chien 乾 C. 5879 Ssŭ-wu | 颌.
近 Ssü-CH'UAN.

$1^{\circ}$ (obs.) $60 l i$ N. of Such'ien H., Kiangsu; $W$. Han. H. in Tung-hai 東海 chün; E. Han, Marq. in Hsia-p'ei 下 阣 K.; Tsin, H. in Linluai 臨 淮 chiin.

$2^{\circ}$ (obs.) in Kiangsu; $S$. Sung and $S . T s^{\prime} i, \mathrm{H}$. in Huai-ling 淮陵 chün.

\section{峒]}

5880 Ssŭ-wu 㟃 峿, ssŭ near Shut-yang H., Kiangsu.

\section{汜}

5881 Ssŭ-shui 汇水 Sz'-shwui, [148] H. in K' ai-fêng Fu, Honan; Lat. $34^{\circ} 55^{\prime}$, Long. $113^{\circ} 20^{\prime}$; orig., Tung-kuo 東鼡; Han, Ch'êng-kao 成鼠; Sui, Ssı̌r-shui | 水.

\section{肆}

5882 Ssŭ 肄, see No. 2814.

5883 Ssŭ-lu | 慮, (obs.) W. of Hsin C., Shansi ; N. Wei, H. in Hsiu-jung 秀容 chïn.

\section{絲}

5884 Ssư 絲, (obs.) in Ssüch'nan; Sung, C. in Shaoch'ing 紹度 In.

约

5885 Ssŭ-ch'nan 四 川 Sz'chuen, a province of China ; 
四 SSÜ-CH'UAN.

literary nanes Chien-11an 劍南, Hsi-ch' 'ua! 西川 and Shu蜀; capital,Clı 'êng tu $\mathrm{Fu}$; it contains 5 circ., $12 \mathrm{Fu}, 7$ C. L. T., 8 T., 8 C. L. C., $11 \mathrm{C}$, and $113 \mathrm{H}$. 5886 Ssŭ-1ıui | 會 Sz'-hwui, H. [416] in Chao-ch 'ing Fu, Kuangtung; Lat. $23^{\circ} 22^{\prime}$, Long. $112^{\circ} 30^{\prime} ; T s^{\prime}$ in, Ssŭ-hui | 會; T'angr, Chêng 湞 C. 5887 Ssŭ-jih-shih | 日市, Yokkaichi; chief town of Miyé ken, Japan.

5888 Ssŭ-liu-shu| 柳 樹, chên near Ku-ch' êng H., Chihli. 5889 Ssŭ-111ing | 明, chên near Shang-ÿ̈ H., Chehliang. $1^{\circ}$ chên near Shang-yü $H$., Chehkiang.

$2^{\circ}$ See No. 4702.

5890 Ssü-p'ing-cliiel | 平街, ssŭ in Huai-jêı H., Shingking.

5891 Ssŭ-t' "nan | 漙, chên in Fêng-hsien H., Kiangsu. 5892 Ssŭ-tzŭ-pu-lao |子部落 Durban Kenked, Mongol Tribe.

\section{泗}

$5893 \mathrm{Ssu}$ 泗 Sz'.

$1^{\circ}$ C. L. C. in the Fêng[465] Yingcirc., Anhui ; Lat. $-33^{\circ} 08^{\prime}$, I ong. $118^{\circ}$ $20^{\prime}$; S.A., K. Hsii 徐; Han, Ch'in-yu 厹猴; Tsin, Sil-yï 宿唤;
Sis.

Wei, 'Tung-ch'u東楚;

Sui, An 㚣 C.; P. Chou, Ssü |C.

$2^{\circ} \mathrm{C}$. in Corea ; Lat. $34^{\circ}$ $43^{\prime}$, Isong. $127^{\circ} 27^{\prime}$.

$3^{\circ}$ See Nos. 1245, 7113.

5894 Ssŭ-ch 'êng | 城 Sz'-ching, [411] Fu in the Tso-chiang circ. Kuangsi ; Lat. $24^{\circ} 20^{\prime}$, Long. $106^{\circ} 16$; orig., land of N. Yüeh 鲴 tribes; Han, in Chiao-cliih 交 趾: chün; Sung, Ssŭ-ch'êng 1 城.

5895 Ssŭ-ching | 添, chên near Sung-chiang $F_{11}$, Kiangsu.

5896 Ssù-choul-chai | 州砦, ssŭ near Cl'ang-ning H., Hunan.

5897 Ssŭ-clı' ${ }^{\prime}$ an | 川, H. in Cl'ing-shang 屋份 circ., Corea.

5898 Ssü-shui | 水 $\mathrm{Sz}_{2}$-shwui, [412] H. in Yen-chou Fu, Shantung; Lat. $35^{\circ} 48^{\prime}$. Long. $117^{\circ} 30^{\prime} ;$ S. A., Hsia-yi 下邑; Ts'in and Han, Ssù-shui | 水 chün; Sui, Ssŭ-shui | 水 $\mathrm{H}$.

\section{SU}

蘇

5899 Su 雀䒩.

$1^{\circ}$ (obs.) Liao, C. in Tungching 東京 circ., Shingking.

$2^{\circ}$ See Nos. 5904, 7130 . 
蕉䒩 SU-ChANG.

5900 Su-cliang | 章.

$1^{\circ}$ Chên near Kai-hua H., Chehkiang.

$2^{\circ} \mathrm{Y}$. near Huo-chia H., Honan.

5901 Su-ch'i | 邽, (obs.) Han and $S$. Sung, H. 80 li N. of Hsi-ch'ang 西昌 H., Ssŭch'ıan; Sui, Su-chilı | 祀 $\mathrm{H}$.

5902 Su-chih | 祇, see the preceding.

5903 Su-cli'iao | 橋.

$1^{\circ}$ Ss ̌̆ near Yung-fu H., Kuangsi.

$2^{\circ}$ Chên near Pa C., Chihli.

$590+$ Su-chou | 州 Su-chan.

$1^{\circ} \mathrm{Fu}$ in the Su-SungT'ai-ts'ang circ, Kiangsu; forming the three aistrict cities of $\mathrm{Wu}$, Yüan-11o and Ch'angchoul I Iat. $31^{\circ} 28^{\prime}$, Long. $120^{\circ} 44^{\prime}$; orig., $\mathrm{K} . \mathrm{Wu}$ 吳; C. Wars, in, 1st, K. Yüieh 越, $2 n d, \mathrm{~K} . \mathrm{Ch}$ ' 1 楚; $T s^{\prime} i n$, in Kuei-clni 會稭 chün and 'Tung-liai 東 海 chïn; Three Ks., Tsin, 1st Sung, $T s^{\prime} i$ and Liang, $\mathrm{Wn}$ 吳 chün; Sui, Su | C.; T'ang, Ch'ang-cliou 長 洲; Sung, P'ing-chiang 平 江; Ming, Su-clion |州 Fi1. Suiu (Polo).
Su.

$2^{\circ}$ Ss̆̌ in Po-lo H., Knangtung.

5905 Su-êrl1-mang | 尔养, T. S. in Kokonor.

5906 Su-fang | 坊, chên near $P^{i}$ 11-ch êñg H., Sliensi.

5907 Sullu-k'ê | 角 克, T. S. in Kokonor.

5908 Su-mên | P月, see No. 2373. 5909 Su-min | 民, see No. 2751.

5910 Su-mu-kê-êrl | 穆唠尔 Sungarku, town in the New Dominion; Lat. $42^{\circ}$ $48^{\prime}$, Long. $93^{\circ} 10^{\prime}$.

5911 Su-ni-t' ê | 尼特 Sunid, Mongol Tribe.

5912 Su-Sung-T'ai-ts'ang | 松 太蒼, circ. in Kiangsu comprising Su-chou Fu, Sung-chiang Fin and T'aits'ang C.

5913 St1-wut-ch'êng | 武 城, (obs.) formerly a fort W. of Ta-t'ung Fu, Shansi.

\section{宿}

5914 Su 宿 Sul1.

$1^{\circ} \mathrm{C}$. in Fêng-yang Fin, [486] Anluni; I.at. $33^{\circ} 43^{\prime}$, Long. $117^{\circ} 07^{\prime}$; orig., in K.Su $\mid$; Han, Clih 銍 H.; Liang, Sui 睢 C.; T'ang; Su | C.; Sung, Ful-li 符 離.

$2^{\circ}$ C. in Corea; Lat. $40^{\circ}$ $15^{\prime}$, Long. $124^{\circ} 55^{\prime}$. $3^{\circ}$ (obs.) S. A., K. in Anhui. 


\section{宿 SU-CH'IEN.}

5915 Su-ch ien | 要 Suh-tsien, [322] $\mathrm{H}$. in Hsù-chou $\mathrm{Fu}_{\text {, }}$ Kiangsu ; Lat. $33^{\circ} 55^{\prime}$, Long. $118^{\circ} 44^{\prime}$; orig., $\mathrm{K}$. Chung-wu 鍾吾; $T s^{\prime}$ in, Hsia-hsiang 下相; Han, Cl'iu-yu 厹唒; Tsin, Suyü|豫; T゙ang, Su-ch'ien 遷. Siju (Polo).

5916 Su-sung|松 Sull-sung, H. [120] in An-ch'ing $\mathrm{Fu}$, Anhui; Lat. $30^{\circ} 16^{\prime}$, Long. $117^{\circ}$ $10^{\prime}$; Han, Sung-tzŭ | 滋; $T \sin$, Su - sung ! 松; Liang, Kao-t'ang 高塘.

5917 Su-yï | 楙, see Nos. 5893, 5915.

\section{粟}

5918 Su-ch'êng 粟城, chên near Liao C., Shansi.

5919 St1-yi | 邑, see No. 4858.

\section{費}

$5920 \mathrm{Su}$ 䶤 St1h, C.L.C. in the [467]An-Su circ., Kansulı; Lat. $39^{\circ} 46^{\prime}$, Long. $99^{\circ} 07^{\prime}$; Han, Chiu-ch üan 酒泉; Sui, Su-chou I 州 military district; Ming, Su-chou | 州 Fu.

$5920^{\wedge} \mathrm{Su}-\mathrm{ch}^{\prime}$ uan | 川 $\mathrm{Fu}$ in

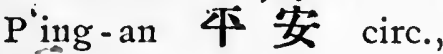
Corea.

5921 Su-chou | 州, see No. 5920.

\section{蒜 SUAN-L,ING。}

5922 Su-ning | 傢 Suh-ning, H. in Ho-chien Fu, Chihli; Lat. $38^{\circ} 30^{\prime}$, Long. $115^{\circ}$ 54'; T'ang, Ho-chien 河 間; Sung, Su-ning | 富.

\section{疏}

5923 Su-fu 疏附 H. in Su-lê $\mathrm{Fu}$, New Dominion.

5924 Su-1ê | 勒.

$1^{\circ} \mathrm{Fu}$, in Kasligar circ., New Dominion; $T^{6}$ ang, Su-lê | 勒 Fu.

$2^{\circ}$ See No. 3224.

\section{束}

5925 Su-fêng 束 鋒, (obs.) in Ya-chou Fu, Ssǔch'uan; T'ang, C. in Chien-11an 劍 南 circ.

5926 Su-1u | 鹿, see No. 5767.

Sil-cliau, see No. 5904. Sü-chau, see Nos. 2895, 2902.

Sü-kau, see No. 2905.

Sü-pu, see No. 2897.

Sï-wăn, see No. 2908. Sï-yung, see No. 2896.

\section{SUAN}

\section{就:}

5927 Suan-ling 祘 嶺, posttown near Fu-ch'ing $\mathrm{H}$., Fuhkien. 


\section{酸}

5928 Suan-tsao 酸 紫, see No. 7362 .

Sugukur, see No. 5840. Suh, see Nos. 5914, 5920. Suh-ning, see No. 5922. Suh-sung, see No. 5916. Suh-tsien, see No. 5915.

\section{SUI}

\section{妨}

5929 Sui 総, see No. 5942.

5930 Sui-an | 安.

$1^{\circ}$ (obs.) $T \sin$, town 100 li S. W. of Chang-p' $\mathrm{s}$ H., Fuhkien.

$2^{\circ}$ See No. 3417.

5931 Sui-ch' êng | 城, see Nos. 859, 5569, 6181.

5932 Sui-chien | 建, see No. 335.

5933 Sui-ching | 靖 Sui-tsing, or Ch'u-hu-ch'u 楚呼楚 Tuguchuk, Fu in Yi-T'a 伊塔 circ.; capital of Tarbagatai; Lat. $46^{\circ}$, Long. $83^{\circ}$. See No. 6140. 5934 Sui-ch'ing | 度 Kurkurausu, see No. 3329.

$5934^{\wedge}$ Sui-fên | 芬 T. in Wuch'ing C.L.'T., Kirin.

5935 Sui-hua | 化, Fu in SuiLan-Hai circ., Kirin.
效 5936 Sui-jung | 戎, (obs.) on
the frontier of Kansuh; Sung, pao in Hsi-an 西 安 C., Shensi.

5937 Sui-kuei | 蹛, (obs.) in Ssŭch'uan; S. Sung and S. $T s^{6} i$, Sui-kuei | 鯊 H.

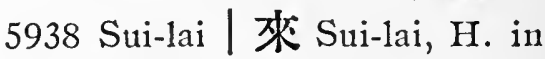
Ti-hua C., Kansuh; Lat. $44^{\circ} 10^{\prime}$, Long. $86^{\circ} 40^{\prime}$.

5939 Sui-Lan-Hai, 綏 蘭海, circ., in Kirin, comprising Sui-hua Fu, Hu-Lan Fu and Hai-lun C.L.'T.

5940 Sui-ning | 察 Sui-ning.

$1^{\circ}$ Town in the New Dominion; also called Ma-nassŭ 瑪納斯 Manas; Lat. $44^{\circ}$, Long. $87^{\circ}$. The town of Manas is situated a short distance from Sui-ning.

$2^{\circ} \mathrm{H}$. in Ching C., Hunan ; [169] Lat. $26^{\circ} 25^{\prime}$, Long. $109^{\circ} 49^{\prime}$; Sung, Suining | 富 $\mathrm{H}$.

$3^{\circ}$ (obs.) 1st Sing, $\mathrm{H}$. comprising parts of Nan-hai H. and P'anyü H., Kuangtung.

5941 Sui-shan | 山, (obs.) Tang, H., W. of O-mei H., Ssưch' uan.

5942 Sui-tê | 德 Sui-teh, C. L. [83] C. in the Yen-Yü-Sui circ., Shensi ; Lat. $37^{\circ} 38^{\prime}$, Long. 
総

$110^{\circ} 03^{\prime} ; T s^{\prime}$ in, Yang-chou

陽周; Han, Tiao-yin 雕

陰; 2nd Wei, Shang 上 chïn; Trang, Sui | C.;

Sung, Sui-tê | 德.

5943 Sui-ting | 定 Sui-ting.

$1^{\circ} \mathrm{Fu}$ in the $\mathrm{Ch}^{\prime}$ uan-tung [111] circ., Ssŭch'uan; also forming the district city of Ta; Lat. $31^{\circ} 18^{\prime}$, Long. $107^{\circ} 37^{\prime}$.

$2^{\circ} \mathrm{H}$.; in Yi-li $\mathrm{Fu}$ Lat. $43^{\circ} 50^{\prime}$, Long. $82^{\circ} 40^{\prime}$.

5944 Sui-yang | 陽 Sui-yang.

$1^{\circ} \mathrm{H}$. in Hsing-yi $\mathrm{Fu}$, [241] Kueichou; Lat. $27^{\circ} 55^{\prime}$, Long. $107^{\circ} 12^{\prime} ; T^{6}$ ang, Yi-ch'üan 義 泉 and Yi 夷 C.; Sung, Ch' êng 承 $\mathrm{C}$.

$2^{\circ}$ (obs.) Wei, H., W. of

Fang H., Hupeh.

5945 Sui-yüan | 遠 Sui-yuen, $T$. in So-p ing $F u$, Shansi ; Lat. $41^{\circ}$, Long. $112^{\circ} 05^{\prime}$; also known as Kukukoti.

5946 Sui-yüeh | 越, (obs.) Liang, H., S. of Ho H., Kuangsi.

5947 Sui-yün | 雲, (obs.) in Yünnan; $S$. Ts' $i, H$. in Chien-ning 建富 chïn.

\section{歲}

5948 Sui-fêng 歳豐, chên near Ho-shan H., Anhui.
隨

SuI.

\section{崔}

5949 Sui 舊, (obs.) Sui, C. near Li-chiang $\mathrm{Fu}$, Yünnan.

\section{穗}

5950 Sui-yüan 穗垣, see No. 3393.

\section{睢}

5951 Sui 睢 Sui.

$1^{\circ} \mathrm{C}$. in Kuei-tê Fu, Ho. [4」 nan; Lat. $34^{\circ} 28^{\prime}$, Long. $115^{\circ} 13^{\prime}$; Ts in, Hsiangyi 裏邑; Kin, Sui | C. $2^{\circ}$ See No. 5914.

5952 Sui-ling | 陵, see No. 5953. 5953 Sui-ning | 莩 Sui-ning, $\mathrm{H}$. in Hsü-chou $\mathrm{Fu}$, Kiangsu; Lat. $33^{\circ} 50^{\prime}$, Long. $118^{\circ} 15^{\prime} ;$ E. Han, Sui-ling | 陵; T'ang, Sui-ning | 恧。

5945 Sui-yang | 陽.

$1^{\circ}$ Post-town near $\mathrm{P}^{\prime}$ ei C. Kiangsu.

$2^{\circ}$ See Nos. 3464, 5552.

\section{隨}

5955 Sui 隨 Sui.

$1^{\circ}$ C. in Tê-an Fu, Hupeh; [568] Lat. $31^{\circ} 47^{\prime}$, Long. $113^{\circ} 16^{\prime}$; orig., K. Sui | ; W. Wei, Str- | C. Sui, Han-tung 漢 東; S. Sung, Sui |C. $2^{\circ}$ (obs.) formerly $\mathrm{K}$. in Hupelr. 
隨

5956 Sui-an | 怒, (obs.) T'ang, H., E. of 'T'êng H., Kuangsi.

\section{遂}

5957 Sui 遂, see No. 5963.

5958 Sui-an | 安 Sui-ngan.

$1^{\circ} \mathrm{H}$. in Yen-chou $\mathrm{Fu}$, [505] Chehkiang; Lat. $29^{\circ} 26^{\prime}$, Long. $118^{\circ} 50^{\prime} ; W u$, Hsin-ning 新察; Tsin, Sui-an | 安.

$2^{\circ}$ Chïn in Huang-hai 黃 海 circ., Corea.

5959 Sui-ch'ang | 昌 Sui-chang, [330] H. in Ch" ${ }^{\prime}$-chou Fu, Chehkiang; Lat. $28^{\circ} 35^{\prime}$, Long. $119^{\circ} 18^{\prime} ; W_{\imath l}, \mathrm{P}^{\prime}$ ing-ch' ang 平昌; T $\sin$, Sui-ch'ang

5960 Sui-ch'êng | 城, see No. 99.

5961 Sui-ch'i | 溪 Sui-ki.

$1^{\circ} \mathrm{H}$. in Lei-chou $\mathrm{Fu}$, [93] Knnangtung; Lat. $21^{\circ}$ $19^{\prime}$, Long. $109^{\circ} 46^{\prime}$; Han, Hsï-wên 徐聞; Sui, Shên-ch'uan 椹川; Sung, Yiüan and Ming, Sui-ch'i | 深 $\mathrm{H}$.

$2^{\circ}$ (obs.) $70 l i$ S. E. of the above; Tang, H. in Lei 霓 C.

5962 Sui-hsing | 與, see No. 4181.
隨

Sumung.

5963 Sui-ning | 晸 Sui-ning.

$1^{\circ} \mathrm{H}$. in ' $\mathrm{T}^{\prime}$ ung-ch' uan $\mathrm{Fu}$, [125] Ssŭch 'uan; Lat. $30^{\circ} 32^{\prime}$, Long. $105^{\circ} 36^{\prime}$; Han, Tê-yang 德陽; E. Tsin, Sui-ning | 察; P.Chou, Sui | C.; Sung and Yüan, Hsiao-ch' $\stackrel{\mathrm{i}}{\text { 小溪 }}$ H.; S. Sung, Sui-ning | 察 $\mathrm{Fu}$.

$2^{\circ}$ See No. 5964.

5964 Sui-p'ing | 平 Sui-ping, [201] H. in Ju-ning Fin. Honan; Lat. $33^{\circ} 08^{\prime}$, Long. $114^{\circ} 02^{\prime}$; S.A., K. Fang-tzŭ 房子; Han, Wu-fang 吳 房; 2nd Wei, Sui-ning | 穿; T'ang, Sui-p ing 平。

Sui-chang, see Nos. 3025, 5959.

Sui-chan, see No. 3027.

Sui-hing, see No. 3028 .

Sui-ki, see No. 5961.

Sui-kin, see No. 3026.

Sui-ngan, see Nos. 3024, 5958.

Sui-ping, see No. 5964. Sui-teh, see No. 5942.

Sui-tsing, see No. 5933. Sui-yuen, see No. 5945. Suju, see No. 5904. Sultania, see No. 5967. Sumung, see No. 5850. 
孫

Sun-HUANG.

\section{SUN}

\section{係}

5965 Sun-huang 係黃, posttown near Kung-an $\mathrm{H}$., Hupeh.

5966 Sun-nan | 南, chên near Yü-hsiang H., Shansi.

5967 Sun-tan-ni-ya |丹尼牙 Sultania, or Sodania, town in Persia, province Irakajemi.

\section{SUNG}

\section{松}

5968 Sung 松.

$1^{\circ}$ (obs.) Sui, C. near Yitu H., Hupel.

$2^{\circ}$ (obs.) Yüan, C., N. of Ch'êng-tê $\mathrm{Fu}$, Chihli.

$3^{\circ}$ See No. 5977.

5969 Sung-ch' $\mathrm{i}$ | 溪 Sung-ki, [420] $\mathrm{H}$. in Chien-ning $\mathrm{Fu}$, Fuhkien; Lat. $27^{\circ} 36^{\prime}$, Long. $118^{\circ} 46^{\prime}$; S. T'ang, Sung-yüan | 源; Sung, Sung-ch'i | 溪.

5970 Sung-chiang | 江. Sungkiang.

$1^{\circ} \mathrm{Fu}$ in the Su-Sung-T'aits'ang circ., Kiangsu; forming also the district cities of Lou and Huat'ing. Literary name, Jung-ch' êng 苜:城; Lat. $31^{\circ} 03^{\prime}$, Long. $121^{\circ}$
松 SUNG-LING.

$15^{\prime}$; orig., in $\mathrm{K}$. Wu 㕦, K. Yüeh 越 and K. Ch'u楚; Ts ' in, Lou 婁 H. in Kuei-chi 會 稭 chiin; E. Han, Wei and $T \sin$, in $\mathrm{Wu}$ 哭 chiin, T'ang, Hua-t'ing 華亭; Five dyn., in Hsin 秀 C.; Yüan and Ming, Sung-chiang | 江 Fu.

$2^{\circ}$ (obs.) in N. Chilhli; Liao, C. and H. in Tating 大定 $\mathrm{Fu}$.

$3^{\circ}$ Matsuyé, chief town of Shimané Ken, Japan.

5971 Sung-ch' uan | 川, see No. 5983.

$5971^{\mathrm{A}}$ Sung-110 | 禾 H. in Huanghai 黃海 circ., Corea.

5972 Sung-hsiang | 相, sš̌ in K'ai-p'ing H., Kuangtung.

5973 Sung-kang | 阔'T.S. north of Li-fan T., Ssŭch' 'uan. Thibetan name $Z$ unga [Baber].

5974 Sung-lin | 林.

$1^{\circ}$ Post-town near Litu-pa T., Shensi.

$2^{\circ}$ Post-town near $\mathrm{P}^{\prime}$ ing-yi H., Yünnan.

5974 ${ }^{\mathrm{A}}$ Sung-lin-ti | 林地, T.S. near Ch' 'ing-ch'i $\mathrm{H}$., Ssŭch'van.

5975 Sung-ling | 陵, see No. 7134. 
松

SUNG-MÊN.

5976 Sung-mên | 閒, sš̆ in T'ai-p'ing H., Chehkiang. 5977 Sung-p'an |潘 Sung-pwan, [307] C. L. T. in Ch 'êng-LungT'ung - Mien - Mou circ., Ssŭch 'uan; Lat. $32^{\circ} 38^{\prime}$, Long. $103^{\circ} 36^{\prime}$; P. Chou, I,ung-ho 龍涸 and Chiach'êng 嘉城 H.; T'ang, Sung |C. Terr. jur. 5978 Sung-p'ing | 坪.

$1^{\circ}$ T.S. near the preceding. $2^{\circ}$ T.S. near $\mathrm{Ch}^{\prime}$ ing-ch' $\mathrm{i}$ H., Ssŭch' uan.

5979 Sung-p u | 浦, sš̆ in Tzüch'i $\mathrm{H}$., Chehkiang.

5980 Sung-shan | 山.

$1^{\circ}$ Post-town near $\mathrm{P}^{6}$ ingfan H., Kansuh.

$2^{\circ}$ Matsuyama, chief town of Ehimé Ken, Japan.

5981 Sung-t ao | 桃 Sung-tau, [21] C. L. T. in Kuei-tung circ., Kueichou; Lat. $27^{\circ} 52^{\prime}$, Long. $109^{\circ} 10^{\prime}$.

5982 Sung-tzŭ | 滋 Sung-tsz'.

$1^{\circ} \mathrm{H}$. in Ching-chou $\mathrm{Fu}$, [528] Hupeh; Lat. $30^{\circ} 26^{\prime}$, Long. $111^{\circ} 34^{\prime}$; Han, $\mathrm{Kao}-\mathrm{ch}^{6}$ êng 高 城; Sung, Sung-tzŭ | 滋.

$2^{\circ}$ (obs.) 50 li N. of Susung $\mathrm{H}$., Anhui; $W$. Han, Marq. in Lüchiang 盧江 chïn.
松 SUNG-YÜAN.

$3^{\circ}$ (obs.) 15 li E. of Hoch'iu H., Anhui; Tsin, S. Sung, S. Ts' $i$ and $N$. Wei, H. in An-fêng 安䁷 chiün.

$4^{\circ}$ (obs.) W. of Sung-tzŭ H., Hupeh ; Tsin, H. in Nan 南 chïn; S. Sung and $S . T s^{\prime} i, \mathrm{H}$. in Hotung 河 東 chiin; Sui, H. in Nan 南 chün; Tang, $\mathrm{H}$. in Chiangling 江 陵 Fu.

$5^{\circ}$ (obs.) E. of Tê-hua H., Kiangsi ; S. Sung, H. in Hsün-yang 骂 陽 chïn.

$6^{\circ}$ See No. 5916.

5983 Sung-yang |陽 Sung-yang, [330] H. in Ch' 'u-chou $\mathrm{Fu}$, Chehkiang; Lat. $28^{\circ} 27^{\prime}$, Long. $119^{\circ} 27^{\prime}$; Han, Sung-yang 陽; T'ang, Sung-ch 'uan 川 Five dyn., Pai-lung 白龍.

5984 Sung-yen | 烟, chên near Ho-shun H., Shansi.

5985 Sung-yin | 隐, chên near Chin-shan H., Kiangsu.

5986 Sung-yüan | 源, see No. 5969.

5987 Sung-yüan | 原, (obs.) in Annam; Tsin, S. Sung and S. Ts $i, \mathrm{H}$. in Chiuchên 九 県 chïn. 
松 SUNG-CHOU-CH' ÊNG.

\section{湈}

5988 Sung-choul-ch'êng 崧 州 城, (obs.) formerly a fort N. W. of Tung-an H., Chilili.

\section{菘}

5989 Sung 菘, chên near Yü-yao H., Chehkiang.

$$
\text { 焗 }
$$

5990 Sung 荁 Sung, H. in Ho[413] nan Fu, Honan; Lat. $34^{\circ}$ $10^{\prime}$, Long. $112^{\circ} 08^{\prime}$; orig., Yi-ch'üeh 伊 閶; Han, Ln1-hun陸 渾; Sung, Yi 伊 H. and Sung | H. 5991 Sung-ming | 明 Sung[29] ming, C. in Yün-nan Fu, Yünnan; Lat. $25^{\circ} 23^{\prime}$, Long. $103^{\circ} 08^{\prime}$; orig., in $\mathrm{K}$. Tien 泪 ; Han, Sungming | 明.

5992 Sung-yang | 陽, see No. 6331.

\section{柇}

5993 Sung 宋.

$1^{\circ}$ (obs.) S. A., K. comprising parts of Honan and Kiangsu.

$2^{\circ}$ See Nos. 2902, 3464.

5994 Sung-ch'i | 溪, (obs.) formerly town near Ching $\mathrm{C}$., Hunan.

5995 Sưng-fêng | 豈, (obs.) in Lien-chou $\mathrm{Fu}$, Kuangtung; S. $T s^{6} i, \mathrm{H}$. in Ho-p' $\mathrm{u}$ 合 浦 chïn.
柇 SUNGARKU。

5996 Sung-ho | 河, ssŭ near Ching-shan H., Hupeh.

5997 Sung-hsi | 熙.

$1^{\circ}$ (obs.) near Pao-ning Fu, Ssüch' uan; S. Sung, $S$. $T s^{\prime} i$ and $N$. Wei, Sung-hsi | 熙 chïn. $2^{\circ}$ (obs.) $30 l i \mathrm{~W}$. of Kaoming $\mathrm{H}$., Kuangtung; S. Sung, chïn in Kuang 笛 chou.

$3^{\circ}$ (obs.) in Nan-chêng H., Shensi; S. Sung and $S . T s^{\prime} i, H$. in Shihp'ing 始本 chün。

5998 Sung-lung | 隆, (obs.) 30 li W. of Kao-ming H., Kuangtung; S. Sung, chün in Kuang 廣 chou.

5999 Sung-yi | 邑, see No. 1025. 6000 Sung-yüan | $\overrightarrow{\text { 兀゙. }}$

$1^{\circ}$ (obs.) near Hsin-hui H., Kuangtung; $S$. Sung, H. in Hsin-hui 新 會 chiün.

$2^{\circ}$ (obs.) in Ssŭ-hui H., Kuangtung; S. Sung and $S . T s^{\prime} i, \mathrm{H}$. in Lêch'ang 樂昌chün.

Sung-ki, see No. 5969. Sung-kiang, see No. 5970. Sung-pwan, see No. 5977. Sung-tau, see No. 5981. Sung-tsz, see No. 5982. Singarku, see No. 5910. 
Swatow.

Swatow, see No. 5463.

Sz', see Nos. 5854, 5893.

Sz'-chau, see No. 5858.

Sz'-ching, see No. 5894.

Sz'-chuen, see No. 5885.

Sz'-hwui, see No. 5886.

Sz'-ling, see No. 5862.

Sz'-mau, see No. 5866.

Sz'-nan, see No. 5868.

Sz'-ngăn, see No. 5859.

Sz'-shwui, see Nos. 5881, 5898.

Sz'-tsung, see No. 5663.

TA

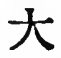

6001 Ta 大, Y. near Fan-chih H., Shansi.

6012 Ta-an | 安.

$1^{\circ}$ Post-town near Mien H., Shensi.

$2^{\circ}$ (obs.) S.Sung, military district in Li-chou 利 州 circ.

6003 Ta-ch'an | 座, ssü near Ta-pu H., Kuangtung.

6004 Ta-ch'ang | 昌.

$1^{\circ}$ Chên near Ning C., Kansuh.

$2^{\circ}$ See No. 6069.

6005 Ta-ch'ang | 常, chên near Ning C., Kansuh.

6006 'Ta-ch' êng | 城 'Ta-ching, $\mathrm{H}$. in Shun-t'ien $\mathrm{Fu}$, Chihli ; Lat. $38^{\circ} 50^{\prime}$, Long.
大

TA-CH'10.

$116^{\circ} 30^{\prime} ;$ Han, Tung $\mathrm{P}^{\prime}$ ing-shu 東平舒; Ts'in, Chang-wu 彰武; $N$. Wei, Sui and Tang, P'ing-shu 平舒 H.; Five dyn., Tach'êng | 城 H.

6007 Ta-chi | 紀, chen n near Fup'ing H., Shensi.

$6008 \mathrm{Ta}-\mathrm{ch}^{6} \mathrm{i}$ | 溪, ssŭ near Yung-ting H., Fuhkien.

6009 Ta-chia | 甲, ssü near Tamsui, Formosa.

6010 Ta-chiang | 港, chên near Tan-yang H., Kiangsu.

6011 Ta-chiao / 艾, chên near Yi-ch'êng H., Shansi.

6012 Ta-ch'iao / 橋, chên near Sui-ning H., Kiangsu.

6013 Ta-ch'ih-ch' ${ }^{\prime} \ddot{u}$ | 池埧, post-town near Ting-yüan T., Shensi.

6014 Ta-ch'in | 秦, The Roman Empire in Asia [Hirth].

6015 Ta-ching | 津 ôtsu, chief town of Shiga Ken, Japan.

6016 Ta-ching | 荆.

$1^{\circ}$ Chên in Hsiang-yin H., Hunan.

$2^{\circ}$ Y. near T'ai-p'ing H., Chehkiang.

6017 Ta-ching-pao | 靖堡, ssŭ near $P^{\prime}$ ing-fan H., Kansuh.

$6018 \mathrm{Ta}-\mathrm{ch}^{\prime} \mathrm{iu}$ / 邱, post-town near Yung-ch'êng H., Honan. 
大

TA-CHOU.

$6019 \mathrm{Ta}$-chou | 州, Y. near $\mathrm{K}^{\prime}$ ai-chien $\mathrm{H}$., Kuangtung. 6020 Ta-chu | 竹 Ta-chuh, H. [76] in Sui-ting Fu, Ssuch 'uan; Lat. $30^{\circ} 48^{\prime}$, Long. $107^{\circ}$ $08^{\prime} ;$ Han, in Tang-ch' ${ }^{\circ}$ 宕渠 chïn; T'ang, Taclut | 竹 $\mathrm{H}$.

602. Ta-pa |埧, Y. near Hsüyung T., Ssŭch' uan.

6022 Ta-fan | 坂, Ozaka (or Osaka) Fu and chief town, Japan.

6023 Ta-fên | 分 Oita, Ken in Japan; chief town Funai. 6024 Ta-fêngl封, (obs.) in Mou C., Ssưch'uan; T'ang, $\mathrm{H}$. in Yen 笑 $\mathrm{C}$.

6025 Ta-fêngl 豐, (obs.) Liang, town N.E. of 'T'ung-jên Fu, Kueichou.

6026 Ta-fu | 端, ssŭ near Shaochou $\mathrm{Fu}$, Kuangtung.

6027 Ta-hêng | 橫, post-town near Yent-p'ing Fu, Fuhkien.

6028 Ta-ho | 河.

$1^{\circ} \mathrm{Y}$. near Liang-chou $\mathrm{Fu}$, Kansuh.

$2^{\circ}$ See No. 6745.

6029 Ta-ho | 和 Ta-ho, T. in [125]T'ung - ch'uan Fu, Ssüch'uan; Lat. $31^{\circ}$, Long. $105^{\circ} 24^{\prime}$.

6030 Ta-hou | 候, (obs.) Ming, C. between Shun-ning Fu
大

TA-KU.

and Ching-tung T.; Yüan, Lu-ch' uan 麓川 circ.

$6031 \mathrm{Ta}-\mathrm{hua}$ | 華, ssŭ near Ch'ang-chai T., Kueichou.

6032 Ta-huai | 雨, T.S. near Mien-ning H., Ssŭch'uan.

6033 Ta-huang-chiang | 毌江, ssǚ near $\mathrm{P}^{\prime}$ ing-nan $\mathrm{H}$., Kuangsi.

6034 Ta-hung | 洪, ssŭ near Yi H., An-hui.

6035 Ta-hsia | 夏.

$1^{\circ}$ Bactria.

$2^{\circ}$ See No. 2062.

6036 Ta-hsin | 信, ssŭ in Tantı H., Anhui.

6037 Ta-hsing | 與 Ta-hing.

$1^{\circ} \mathrm{H}$. forming with Wanp'ing $H$. the prefectural city of Shun-t'ien, Chihli; Lat. $39^{\circ} 57^{\prime}$, Long. $116^{\circ} 29^{\prime} ;$ Ts $^{\prime}$ in, Chi 魝; Liao, Hsiching 析津; Kin, Tahsing | 與.

$2^{\circ}$ Chên near Yi-chou $\mathrm{Fu}$, Shantung.

$3^{\circ}$ See No. 2691.

6038 Ta-hsing | 姓, T.S. near Sung-p'an 'T., Ssŭch'uan.

6039 Ta-jun | 閣, chên near Wan-ch üan H., Shansi.

6040 Ta-ku | 姑, ssŭ in Tê-hua H., Kiangsi. 
TA-KU.

6041 Ta-ku | 活 Ta-ku, Y. at the entrance of the Peiho River; Lat. 39', Isong. $117^{\circ} 41^{\prime}$; here were situated the well-known Ta-ku Forts.

6042 Ta-ku | 谷, see No. 6166. 6043 Ta-kuan | 關 Ta-kuan, T. [214] in Chao-t ung Fu, Yünnan; Lat. $28^{\circ}$, Long. $104^{\circ}$.

6044 Ta-kuei / 桂, chên near San-yüan H., Shensi.

6045 Ta-kuei | 騩, (obs.) S. E. of Mi H., Honan; Kin, chên in Chêng 真陪 $\mathrm{C}$.

6046 Ta-lê | 樂, ssŭ near P'ingnan $\mathrm{H}$., Kuangsi.

6047 Ta-lei-ch'ih | 鼠池, see No. 6941.

6048 Ta-li | 里, town in the Liu-ch iu Islands.

6049 Ta-li | 理 Ta-li, Fu in the [121]Yi-hsi circ., Yünnan; forming also the district city of 'T'ai-ho; Lat. $25^{\circ} 42^{\prime}$, Long. $100^{\circ} 22^{\prime}$; Hsia, Yin and Chou, K' un-mi 昆彌; Han, in Yi 监 C.; Minor Han, Yeh-yü 葉 榆; T'ang, 1st, Yao 姚 C., 2nd, Nar-chao 南詔; Five dyn., and Yüen, Ta-li | 里; Ming, Ta-1i | 里 Fu. Carajan [Polo].

6050 个a-li | 利, see No. 2232.
大

TA-LU.

6051 Ta-li | 荔 Ta-li, H. forming the prefectural city of T'ung-cliou, Shensi; Lat. $34^{\circ} 50^{\prime}$, Long. $109^{\circ} 51^{\prime}$.

6052 'Ta-li | 歴.

$1^{\circ}$ Ssŭ near Yen-p'ing Fu, Fuhkien.

$2^{\circ}$ (obs.) $T^{6}$ ang, H., N. of Ning-yüan $\mathrm{H}$., Hunan.

6053 Ta-liang | 梁.

$1^{\circ} \mathrm{Y}$. near $\mathrm{K}^{\prime}$ ai-fêng $\mathrm{Fu}$,

Honan.

$2^{\circ}$ See Nos. 3256, 3417.

6054 Ta-lien Wan | 連灣, a bay on the east side of the Manclurian peninsula, once leased by Russia from China on the same terms as Port Arthur [No. 4132]. Russia here founded the town Dalny, the Far-Eastern terminus of the Siberian Railway. Lat. $39^{\circ} 05^{\prime}$, Isong. $121^{\circ} 15^{\prime}$.

6055 Ta-ling | 陵, see No. 7013. 6056 Ta-lin | 柳, post-town near Lai-an H., Anhui.

6057 Ta-liu-huang | 骝隍, ssü near $\mathrm{Ch}$ ao-chou $\mathrm{F} n$, Kuangtung.

6058 Ta-lo | 羅, Y. near Ying C., Shansi.

6059 Ta-1u | 爈, Y. near Kuei H., Kuangsi.

6060 Ta-1u | 路, chên near Lojung H., Kuangsi. 
大

TA-LUN.

6061 Ta-lun | 輪, post-town near $T^{\prime}$ ung-an H., Fuhkien.

6062 Ta-lung | 龍.

$1^{\circ}$ Sš̆ in Wu-ling H., Hunan.

$2^{\circ}$ Post-town near lingch'uan H., Kuangsi.

6063 Ta-ma | 馬.

$1^{\circ} \mathrm{Y}$. near Yung-ch'ang H., Kansul.

$2^{\circ} \mathrm{Y}$. near Chiang-hua H., Hupeh.

6064 Ta-mên | P月, chên near $\mathrm{Ch}^{\prime}$ in C., Kansuh.

6065 Ta-ming | 名 Ta-ming.

$1^{\circ} \mathrm{Fu}$ in the Ta-Shun-

[496] Kuang circ., Chihli; forming also the district cities of Ta-ming and Yüan-ch' êng; Lat. $36^{\circ}$ $21^{\prime}$, Long. $115^{\circ} 22^{\prime}$; Chou, feudal state of Wukêng 武庚; $S . A$., in $\mathrm{K}$. Chin 留; C. Wars, frontiers of K. Wei 衛 and K. Wei 魏; Han, Wei 魏 chiin; Wei, Yang-p'ing 陽平; Sui, Wu-yang 武陽; T'ang, T'ien-hsiung 天雄; Sung, Ta-ming | 名.

$2^{\circ} \mathrm{H}$. forming with Yüanch' êng H. the prefectural city of Ta-ming, Chibli ; Lat. $36^{\circ} 21^{\prime}$, Long. $115^{\circ}$ $22^{\prime} ;$ P. Chou, Wei 魏
太
TA-PIN.

C.; Sui, Wu-yang 武 陽; Five dyn., Ta-ming |各。

6066 Ta-mul-ch'ang | 木廠, sš̆ near Fang H., Hupeh.

6067 Ta-mu-ts'un | 木村, posttown near Chao-hua H., Ssŭch' 'uan.

6068 Ta-nien | 年, see No. 2691.

6069 Ta-ning | 篮 Ta-ning.

$1^{\circ} \mathrm{H}$. in Hsi C., Shansi ;

[174] Lat. $36^{\circ} 30^{\prime}$, Long. $110^{\circ}$ $43^{\prime}$; Han, Pei-ch 'ii 北 屈; 2 nd $W e i, W u-c h$ 'êng 任城; P. Chou，Taning | 耍.

$2^{\circ} \mathrm{H}$. in $\mathrm{K}^{\prime}$ uei-chou $\mathrm{Fu}$,

[12] Ssǔch 'uan; Lat. $31^{\circ} 37^{\prime}$, Long. $109^{\circ} 38^{\prime} ; T^{\prime}$ in, Wu 功 H.; Tsin, Chiench' ang 建昌 and $\mathrm{Ch}^{\text {' in- }}$ clíang 秦昌; P. Chou, Ta-ch'ang 昌; Sung, Ta-ning | 察 military post.

6070 Ta-pa-kuan | 巴關, ss near Ting-yüan T., Shensi. 6071 Ta-pai | 白.

$1^{\circ}$ Ssŭ near Wu-yuaan $\mathrm{H}$, Anhui.

$2^{\circ}$ Post-town near Lingch'iu H., Shansi.

6072 Ta-pin | 賓, (obs.) S. E. of Kuei-p ing H., Kuangsi ; Sui, H. in Yung-p'ing 永 平 chiun; T'ang, H. in $\mathrm{H}$ sün 浔 $\mathrm{C}$. 
6073 Ta-pin | 斌, (obs.) 120 li N. W. of Sui-tê C., Shensi ; Sui, H. in Tiao-yin 雕 陰 chïn; T'ang, H. in Sui 綏 C.

6074 Ta-p'ing | 平.

$1^{\circ}$ Ss̆̌̆ near Yung-ting H.,

Fuhkien.

$2^{\circ}$ Y. near Sung-t'ao T., Kneichou.

6075 Ta-p'o | 坡, Y. near Lingshui H., Kuangtung.

6076 Ta-pu | 埔 Ta-pu, H. in Chao-chou $\mathrm{Fu}$, Kuangtung; Lat. $24^{\circ} 40^{\prime}$, Long. $116^{\circ} 43$.

6077 Ta-shang-yüan | 商 原, chên near Pao-ch 'êng H., Shensi.

6078 Ta-shê | 凋, chên near Chi-shan H., Shansi.

6079 Ta-shê-t'ou | 蛇頭, chên near Lan H., Shansi.

6080 Ta-shil | 世, ssü in Ch'imên H., Anhui.

6081 'Ta-shih-k'ou | 石口, ssü near Yi-ning $C_{\text {., }}$ Kiangsi.

6082 Ta-shu-pao | 樹 堡, post-

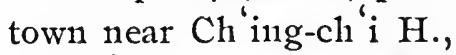
Ssŭch' uan.

6083 Ta-shni | 水, (obs.) S. Sung, military district in Li-chou 利州 circ.

6084 Ta-shin | 順, Kin walled town in Kansull.
天

TA-TSU.

6085 Ta-Shun-Kuang | 順㡺, circ. in Chihli, comprising Ta-ming Fu, Shun-tê Fu and Kuang-p ing Fu.

6086 Ta-sung | 营, military post near Hai-fêng H., Shantung.

6087 Ta-t'an | 潭, (obs.) Han, H. near Hsi-ho H., Kansuh. 6088 Ta-tang | 當, chên near Liu-chou $\mathrm{Fu}$, Kuangsi.

6089 Ta-t'ao-ch'êng | 葐埕 Twatutia, foreign settlement at Tamsui, twelve miles from Hobe.

6090 Ta-t'ien | 田 Ta-tien.

$1^{\circ} \mathrm{H}$. in Yung-ch'un C., [438] Fuhkien; Lat. $25^{\circ} 40^{\prime}$, Long. $118^{\circ} 08^{\prime}$.

$2^{\circ}$ Post-town near Fuch'ing H., Fuhkien.

$3^{\circ}$ T.S. near $\mathrm{Ch}^{\prime}$ ing-ch ${ }^{\prime} \mathrm{i}$ H., Ssǔch'uan.

6091 Ta-ting | 定 Ta-ting.

[238] $1^{\circ} \mathrm{Fu}$ in the Kuei-hsi circ., Kueichou; Lat. $27^{\circ} 05^{\prime}$, Long. $105^{\circ} 33^{\prime}$. $z^{\circ}$ (obs.) Kin, $\mathrm{Fu}$ in $\mathrm{N}$. Chihli, comprising $\mathrm{E}$. part of Inner Mongolia.

6092 Ta-ting-sha-pa | 定沙 垻, T.S. near Mou C., Ssŭch' wan.

6093 Ta-tsu | 足 Ta-tsuh, H. [563] in Chung-ch' 'ing Fu, Ssücli'uan; Lat. $29^{\circ} 50^{\prime}$, 
大

TA-TSU.

Long. $105^{\circ} 51^{\prime}$; orig., $\mathrm{Pa}$

巴C.; T'ang, Ta-tsu| 足. 6094 Ta-ts'un | 村, Y. near Mien-ning H., Ssŭch'uan. 6095 Ta-tu | 都, see No. 5822. 6096 Ta-tu | 肚, shê near Chang-hua H., Fuhkien. $6097 \mathrm{Ta}-\mathrm{t}^{\prime} \mathrm{un}$ | 屯, shê near Tamsui, Formosa.

6098 Ta-tung-ch 'ü-t’ai | 東渠 台, post-town near Yümên H., Kansuh.

6099 Ta-tung | 洞.

$1^{\circ}$ Ss ̌̌ near Hsing-an H., Kuangsi .

$2^{\circ}$ Ssŭ near Ts'ên-ch'i H., Kuangsi.

6100 Ta-t'ung | 通 Ta-tung.

[357] $1^{\circ} \mathrm{H}$. in Hsi-ning $\mathrm{Fu}$, Kansuh; Lat. $36^{\circ} 50^{\prime}$, Long. $102^{\circ} 50^{\prime}$,

$2^{\circ} \mathrm{H}$. in Yi-lan $\mathrm{Fu}, \mathrm{Kirin}$.

$3^{\circ}$ Ssü near T'ung-ling H., Anhui. Port of call. $4^{\circ}$ (obs.) Sung, post near T'ai-yüan $\mathrm{Fu}$, Shansi. $5^{\circ}$ (obs.) Sung and Kin, a fort W. of Ho C., Kansuh.

$6^{\circ}$ (obs.) Sung, a fort near Hsi-ning Fu,

- Kansuh.

6101 Ta-t'ung-ch'uan ! 通 川, T.S. near Ta-t ung $H$., Kansuh.
大 TA-wU-HsUt.

6102 Ta-t'ung | 同 Ta-tung.

[559] $1^{\circ} \mathrm{Fu}$ in the Yen-P'ingTa - So-Ning-Hsin - TaiPao circ., Shansi ; forming also the district city of the same name; Iat. $40^{\circ} 06^{\prime}$, Long. $113^{\circ} 13^{\prime} ;$ S.A., land of N. barbarians ; $C$. Wars, in K. Chao 趋; Ts'in, Yün-chn1ng 雲中, Yenmên 䳽閒 and Tai 代 chiin; 2nd Wei, Hêng 恒 C.; T'ang, Yün 堅 C.; Five dyn., Ta-t' ung 通.

$2^{\circ} \mathrm{H}$. forming the prefectural city of the same name, Shansi; Lat. $40^{\circ} 06^{\prime}$, Long. $113^{\circ} 13^{\prime}$; Chou, Tat'ung-ch'uan | 同 川;

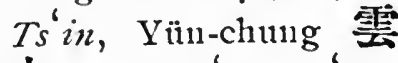

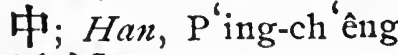
平城; Sui, Yün-nei 㙯闪; Kin, Ta-t'ung |通; Ming, Shan-hsi 山西 ssǔ。

$3^{\circ}$ Chên in $\mathrm{Ch}^{\prime} \mathrm{i} \mathrm{C}$., Hupeh.

6103 Ta-wan | 宛 Ferghana, or Kokand, on the upper course of the Sir Daria.

6104 Ta-wang | 王, chên near $\mathrm{Hu}$ H., Shensi.

6105 Ta-wu-hsï|鳥墟, sš̆ near T'êng H., Kuangsi. 
犬 TA-WU-LUNG.

6106 Ta-wu-lung | 武壠, ssï near T'ai-nan Fu, Formosa.

6107 Ta-yang | 陽, chên near Ch'ang-hua H., Chehkiang.

6108 Ta-yang | 洋, ssŭ near Hsing-hua Fu, Fuhkien.

6109 Ta-yao | 姚 Ta-yau, H. [435] in Ch' $u$-hsiung Fu, Yünnan; Lat. $25^{\circ} 46^{\prime}$, Long. $101^{\circ} 30^{\prime} ;$ Han, Ch 'ing-ling 蜻 (or 靑) 蛉; Yï̈an, Ta-yao | 姚.

6110 Ta-yeh | 野, see No. 1415. 6111 Ta-yeh | 治 Ta-yé, H. in [53] Wu-ch'ang Fu, Hupeh; Lat. $30^{\circ} 06^{\prime}$, Long. $114^{\circ}$ $57^{\prime} ;$ Sui, Wu-ch'ang 武 昌; T'ang, Ta-yeh | 诒.

6112 Ta-yen-ching | 監井, posttown near Yüelı-sui T., Ssuch' 'tan.

6113 Ta-yen-ling | 痛領, posttown near Chien-shih $\mathrm{H}$., Hupeh.

6114 Ta-yi | 義 Ta-i, T. in Luan $\mathrm{Fu}$, Shansi.

6115 Ta-yi | 儀, chên near Yichêng, H., Kiangsu.

6116 Ta-yi-wei | 宜 昧, town in the Liuch'iu Islands.

6117 'Ta-yi | 伊, chên near Hai C., Kiangsu.

6118 Ta-yi | 邑 Jo-yih, H. in [307] Chiung C., Ssưch'uan;
打 TA-KOU.

Lat. $33^{\circ} 37^{\prime}$, Long. $103^{\circ}$ $33^{\prime}$; Han, Chiang-yïan 江 原; $P$. Chou, Chin-yüan 琶原; T'ang, Ta-yi.

6119 Ta-yin | 寅, (obs.) 60 li S. E. of Yi-lung H., Ssŭch' 'uan; Sui, H. in Pa-hsi 巴西 chïn.

6120 Ta-ying-wan | 營灣, T.S. near Ku-lang H., Kansuh.

6121 Ta-yu | 有, (obs.) Yï̈an, H., S. of Chin-ning C., Yünnan.

6122 Ta-ỹü|英 Ta-yu, H. forming the prefectural city of Nan-an, Kiangsi; Lat. $25^{\circ} 42^{\prime}$, Long. $114^{\circ} 18^{\prime}$; Han, Ta-yü | 属 $\mathrm{H}$.

6123 Ta-yü | 峪, chên near Sanshui H., Shensi.

6124 Ta-yung | 鱸, ssü near Wu-yüan H., Anhui.

\section{打}

6125 Ta-chien-lu 打箭爐 Ta[268] tsien-1u, T. in Ya-chou Fu, Ssŭch 'uan; Lat. $30^{\circ} 15^{\prime}$, Long. $102^{\circ} 10^{\prime}$.

"The name Ta-chien-lu is a corruption of the Thibetan Tar-tsendo, i.e. the confluence of the Rivers Tar and T'sen." [Baber.]

6126 Ta-kou | 狗 Takow, a village near T'ai-nan $F u$, Formosa; Lat. $22^{\circ} 35^{\prime}$, Long. $120^{\circ} 15^{\prime}$; one of the ports in Formosa opened to foreign trade. The name 
打

TA-KOU.

was originally Ta-ku | 鼓, an allusion to the sound of the waves "drumming" on the rocky coast. Called locally Kiow 旗後.

\section{達}

$6127 \mathrm{Ta}$ 達 Tah, H. forming the prefectural city of Suiting, Ssŭch' ${ }^{\prime}$ tan; Lat. $31^{\circ}$ $18^{\prime}$, Long. $107^{\circ} 38^{\prime}$; Han, Hsüan-han 宣漢; Liang, Tung-kuan 東關; W. Wei and $T^{6}$ ang, T'ung 通 C.; Sui, 'T'ung-ch'uan 通川; Sung, Ta | C.; S. Sung and $S . T s^{\prime} i, \mathrm{~Pa}-\mathrm{ch}^{\prime} \mathrm{u} \mathrm{u} 巴$ 渠 chïn。

6128 Ta-ch'êng | 城, chên near Shê H., Honan.

6129 Ta-êrh-tsung | 尔登 Tardzung, town in Thibet; Lat. $29^{\circ} 40^{\prime}$, Long. $95^{\circ} 30^{\prime}$.

6130 Ta-hao | 豪, Y. nearClı'aoyang H., Kuangtung.

6131 Ta-lung | 隆, (obs.) 155 li N. W. of Fu-ch'iang H., Kansuh; Sung, pao in $\mathrm{Cl}^{\prime}$ in 泰 C.

6132 Ta-lung | 弄, T. S. near Sung-p' an T., Ssŭch' '1an.

6133 Ta-shili-kan |失干, see No. 154.

6134 Ta-wei | 違, (obs.) in Ssŭch'uan; T'ang, C. in Lung-yu階右 circuit.
他 TA-LANG.

Ta-ching, see No. 6006. Ta-chuh, see No. 6020. Ta-hing, see No. 6037. Ta-i, see No. 6114. Takow, see No. 6126. Ta-kwan, see No. 6043. Ta-lang, see No. 6137. Ta-pu, see No. 6076. Ta-tien, see No. 6090. Ta-tsien-lu, see No. 6125. Ta-tsuh, see No. 6093. Ta-tung, see Nos. 6100, 6102.

Ta-yau, see No. 6109.

Ta-yé, see No. 6111.

Ta-yih, see No. 6118.

Ta-yu, see No. 6122.

\section{T'A}

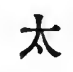

6135 个'a-mo 太末, see Nos. 1445, 2438, 4220.

\section{他}

6136 T'a-k'ê-hsin 他克新, or T'o-ku-sun 托古孫, Toksun, a city W. of Turfan. 6137 T'a-lang | 郳 Ta-lung, T. [367]in $\mathrm{P}^{\prime} \mathrm{u}$-êrh $\mathrm{Fu}$, Yünnan; Lat. $24^{\circ} 12^{\prime}$, Isong. $101^{\circ}$ $56^{\prime}$; orig., Ma-lung 馬龍 and 'T'a-lang | 郎; Yüan, Nei-fu 內附; Ming, Hsinhua 新化 $\mathrm{c}$. 
T'A-YAng.

\section{漯}

6138 T'a-yang 漯陽, (obs.) formerly a town $\mathrm{N}$. W. of Ch'ing-ch' êng H., Shantung.

\section{塔}

6139 T'a-ch'êng 塔城 C. L. T. in Yi-T'a circuit, New Dominion.

6140 T'a-êrh-pa-ha-t'ai | 尔巴 哈台 Tarbagatai.

$1^{\circ}$ Province in New Dominion bounded on the $\mathrm{N}$. by Siberia and Cobdo; on the E. by Cobdo ; on the S. by Ili and Outer Kanstih ; and on the W. by Independent T'artary. $2^{\circ}$ Capital of province. See No. 5933.

$6141 \mathrm{~T}^{\prime} \mathrm{a}-\mathrm{k}^{\prime}$ ê | 克 Tak, a town in Yarkand; I at. $36^{\circ} 20^{\prime}$, Long. $82^{\circ} 35^{\prime}$.

$6142 \mathrm{~T}^{6}$ a-kêng | 坑, chên near Hsin-ning H., Anhui.

$6143 \mathrm{~T}^{\prime}$ a-la-ssŭ $\mid$ 刺寺, or | 喇 思, Talas, a city near Sairam.

$6144 \mathrm{~T}^{\prime}$ a-la-ssŭ | 喇思, see the preceding.

6145 T'a-la-wu-su | 拉鳥蘇, Tarausu, a town in the New Dominion; Lat. $41^{\circ}$ $50^{\prime}$, Long. $94^{\circ} 55^{\prime}$.

6146 'T'a-lê-ch'i | 勒奇 Talki; a town in Ili; Lat. $43^{\circ}$ $50^{\prime}$, Long. $82^{\circ} 25^{\prime}$.
塔 $\mathrm{T}_{\mathrm{AH}}$.

6147 T'a-li-han | 里寒, see No. 6148.

$6148 \mathrm{~T}^{\mathrm{6}}$ a-li-kan $\mid$ 里干 (or, T'a-li-han 里 寒, or, Tan-la-chien 呾喇健,) Talecan, a city near the source of the Oxus. Tailan [Polo].

6149 T'a-mi-shê | 米設 Damegan, town in Persia, province of Khorassan.

6150 T'a-pa-hsin|八畒, Thabas Sistan, a town in Persia.

6151 T'a-shen | 山, ssŭ in Fênghia H., Chehkiang.

6152 个'a-shih-pa-1i | 失八里 Tashbalik, a town E. of Hami; also called Hsiaoshih-chou 小石州; for modern town see No. 6155 .

6153 T'a-shih-yüan | 失元, see No. 154.

6154 '`a-shih-kan | 什干, see No. 154.

6155 T'a-shih-mi-1i-k'ê | 什密 里克 Tashbalig, a town in Yarkand; Lat. $39^{\circ}$, Long. $68^{\circ}$; see No. 6152 .

6156 T'a-yai | 崖, a post-town near Kuang-cl'ang $H$, Chihli.

Tabriz, see No. 6303. Tadinfu, see No. 7373. Tah, see No. 6127. 
代

'TAI.

TAI

代

6157 'ai 代 'ai.

$1^{\circ}$ C. I. C. in the Yen[143] P'ing-Ta-So-Ning-HsinTai-Pao circ., Shansi; I at. $39^{\circ} 06^{\prime}$, Isong. $112^{\circ}$ $58^{\prime} ; T s^{\prime}$ in, Yen1-11ên 鵆

門; Wei, Kuang-wu鹰 武; Sui, Tai | C.; $N$. Wei, Ying-mên 赝閒 chiin.

$2^{\circ}$ (obs.) Ts ${ }^{i} \mathrm{in}$, chiun in Shansi.

$3^{\circ}$ See Nos. 6102, 7738.

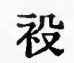

6158 ' 'ai-yü 袂初, see Nos. 6806, 7322, 7424.

\section{埭}

6159 'Tai-ch'i 埭潆, chên in Knei-an H., Chelıkiang.

\section{瑇}

6160 Tai-mei 瑇 琑, see No. 1322.

\section{戴}

6161 Tai 戴, see No. 521.

Tai, see No. 6175.

Tai-chau, see No. 6185.

Tai-hing, see No. 6180 .

Tai-ho, see Nos. 6163, 6179.

Tai-hu, see No. 6164.

Tai-kang, see No. 6165.
太

'I"AI-HU.

'Tai-kah, see No. 6166. 'lai-kung, see No. 6186. Tai-ngan, see No. 6176. Tai-ning, see No. 6181. 'Tai-peh, see No. 6192. Tai-ping, see No. 6169. 'Tai-shun, see No. 6182. 'Tai-tsang, see No. 6170. 'Tai-wan, see No. 6194. 'Tai-ynen, see No. 6174. 'Taianfu, see No. 6174. 'Taican, see No. 6148. 'Tairen, see No. 6054.

\section{T'AI}

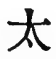

6162 T'ai-ch'iu 太邱, see Nos. 2829, 7838.

6163 'T'ai-ho | 和 Tai-ho。

$1^{\circ} \mathrm{H}$. in Ying-chou $\mathrm{Fu}$, [550] Anhui ; Iat. $33^{\circ} 10^{\prime}$, I.ong. $115^{\circ} 43^{\prime}$; Han, Hsi-yang 細陽; Sung, T'ai-ho | 和.

$2^{\circ} \mathrm{H}$. forming the prefectural city of Ta-li, Yünnan; Lat. $25^{\circ} 44^{\prime}$, Long. $100^{\circ} 22^{\prime}$; Han, Yeh-yii 葉榆; T'ang, T'ai-ho | 和.

$3^{\circ}$ See No. 3837.

$6164 \mathrm{~T}^{6}$ ai-hu | 湖 Tai-hu.

$1^{\circ}$ T. in Su-chou $\mathrm{Fu}$, Kiangsu; Lat. $31^{\circ} 10^{\prime}$, Long. $120^{\circ} 40^{\prime}$. Terr. jur. 
太

$[260] 2^{\circ}$ H. in An-ch'ing Fu, Anlu1ii; I at. $30^{\circ} 30^{\prime}$, I.ong. $116^{\circ} 20^{\prime}$; Han, Wan 皖 H.; 1st Sung, 'T"ai-hu1 和 $\mathrm{H}$.

6165 '个'ai-k'ang | 康 Tai-kang, [213] H. in Ch' ên-chon Fin, Honan ; Lat. $34^{\circ} 07^{\prime}$, Long. $114^{\circ} 54^{\prime} ; H$ sia, fendal state of T'ai-k'ang | 康; Han, Yang-chia 陽 夏; Sui, $\mathrm{K}^{\prime}$ 'trang-ch' êng 匡城.

6166 T'ai-ku | 谷 T'ai-kuh, H. in T'ai-y ïian Fu, Shansi; Lat. $37^{\circ} 25^{\prime}$, Long. $112^{\circ}$ $33^{\prime}$; Han, Yang-yi 陽邑; Sui, T'ai-ku | 谷.

6167 个'ai-lao | 年, see No. 3043. 6168 T'ai-ning | 第, Y. near

Ch'ing-ch' i H., Ssŭch' 'uan. 6169 T'ai-p“ing | 平 Tai-ping. [528] $1^{\circ} \mathrm{Fu}$ in the Hui-NingCh'ih - T'ai - Kuang-têchon circ., Anluni ; forming also the district city of Tinng-tur I Iat. $31^{\circ} 33^{\prime}$, Long. $118^{\circ} 30^{\prime}$; S. $A$. in $1 s t, \mathrm{~K}$. W 1 吳, $2 n d, \mathrm{~K}$. Yüeh 越; $C$. Wars, in K. Ch" 1 楚; $T s^{\prime} i n$, in Chang 鄣 chïn; Han, in Tan-yang 开陽 chiun; Tsin, 'Tang-tu 當塗; 1 st Sung, K11-shu1 姑孰; Tang, Nan-yü 南豫 C.; Sung, P'ing-11an 平 南 and 'T'ai-p'ing | 平.
太 バ'AI-P'ING.

[198] $2^{\circ} \mathrm{Fu}$ in the 'T'ai-p 'ingSsŭ-Shun circ., Knangsi ; forming also the district city of Cl' 'ningslian ; Lat $22^{\circ} 25^{\prime}$, Long. $107^{\circ} 07^{\prime}$; orig., land of the S. Yüeh 类 tribes; Han, L,i-chiang 麗 江 in Chiao-chil 交 趾

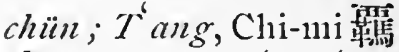
縻 C.; Sung; 'T'ai-p'ing 本。

[225] $3^{\circ}$ T.C. in 'T'ai-p ing Fu, Kuangsi ; Lat. $22^{\circ} 41^{\prime}$, Long. $106^{\circ} 55^{\prime}$; Sung, '“'ai-p'ing | 平 C.

$4^{\circ} \mathrm{H}$. in P'ing-yang $\mathrm{Fu}$, Shansi; Lat. $35^{\circ} 46^{\prime}$, Long. $111^{\circ} 18^{\prime}$.

[182] $5^{\circ} \mathrm{H}$. in Ning-kno Fit, Anlu1i ; Lat. $30^{\circ} 25^{\prime}$, Long. $118^{\circ} 08^{\prime}$; Han, Cling 湮 H.; T'ang, T’ai-p'ing|平.

$6^{\circ} \mathrm{H}$. in $\mathrm{T}^{\prime}$ ai-chon $\mathrm{F} 11$, Chellkiang: Lat. $28^{\circ}$ $26^{\prime}$, Long. $120^{\circ} 42^{\prime}$.

$[140] 7^{\circ} \mathrm{H}$. in Sui-ting Fu, Ssŭch'nan; Lat. $32^{\circ} 08^{\prime}$, Long. $108^{\circ} 14^{\prime}$.

$8^{\circ}$ Chên near Yang-wu H., Honan.

$9^{\circ}$ Chên near Ying-shan H., Hupeh.

$10^{\circ}$ Ss̆̌ near Lin-cliang $\mathrm{F} \mathfrak{u}$, Kiaingsi. 
太

$11^{\circ}$ Ss sú in Yrung-ting H., Fullkien.

$12^{\circ}$.5s $5 \check{t}$ in Yang-chiang '., Knangtung.

$13^{\circ}$ S.̌̆ in Ting-an H.,

Kuangtung.

$14^{\circ}$ See No. 381.5.

6170 'l'ai-p'ing-Ssŭ-Shun | 平 思㮌, circuit in Knangsi, comprising 'l'ai-p'ing Fun, Ssŭ-ên Fin and Kueishun C.L.C.

6171 T'ai-ts' ang | 倉 I'ai-tsang, C. I. C. in the Su-SungT'ai-ts' ang circ., Kiangsu; forming also the cistrict city of Chên-yang; Lat. $31^{\circ} 30^{\prime}$, Long. $120^{\circ} 52^{\prime}$.

6172 ' $\mathrm{T}^{6}$ ai-tu | 都, (obs.) Ÿ̈an, a town, built by Kublai Khan, 3 li from Peking. 6173 T'ai-yeh | 掖, see No. 5822.

6174 T'ai-yüan | 原 Tai-yuen. [123] $1^{\circ}$ Fu in the Clii-ning circ., Shansi; forming also the district city of Yang$\operatorname{ch}^{\prime}$ ï ; Irat. $37^{\circ} 54^{\prime}$, Long. $112^{\circ} 31^{\prime}$; capital of the province; Chou, K. T'ang 虍; S.A., in K. Chin 登: C. Wars, in K. Chao 趙; Ts'in, Chin-yang 嶳 陽 and 'P'ai-yüan | 原, chün; T'ang, Pei-ching 北京 and T'ai-yüan | 原 Fu; P. T ang, Hsi-ching 西
T'AI.

京; Sung, Ho-tung 河 東 circ. and 'T"ai-yüian 原 Fin； Kin and Ming, 'T'ai-yiian Fin. Taianfu (Polo).

[123] $2^{\circ}$ H. in T'ai-yüan Fin, Shansi; I at. $37^{\circ} 45^{\prime}$, Isong. $112^{\circ} 20^{\prime}$; S.A., Chin-yang 至晹; Han, 'T'ai-ỹüan | 原 chün; Tsin, K. 'T'ai-yüan |

原in Ping 扭 CHOv; T*ang, T'ai-yüan | 原 Fin.

$3^{\circ}$ (obs.) N.E. of Ch'angcling H., Shantung; S. Sung, chïn and H. in Ch'ing 青 cHOU; $N$. IVei, chïn and $\mathrm{H}$. in $\mathrm{Cl}_{1} \mathrm{i}$ 驾率 chou.

$4^{\circ}$ (obs.) near Ying-chou Fin, Anhui; N. Wei, H.in Jn-nan 汝南 chiön. $5^{\circ}$ (obs.) Sui, T'ang and Ming, H., N. E. of T'aiyüan Fu, Shansi.

\section{奉}

6175 T'ai 秦 Tai.

$1^{\circ} \mathrm{C}$. in Yang-chou $\mathrm{Fu}$, Kiangsu ; I at. $32^{\circ} 24^{\prime}$, Long. $119^{\circ} 55^{\prime}$; Han, Hai-ling 海陵; Tsin, Hai-yang 海陽; T'ang, $W u-l i n g$ 吳 陵; $S$. Sung, 'T'ai | C. Tiju (Polo).

$2^{\circ}$ See No, 6176. 
春

T'AI-AN.

6176 T'ai-an | 安 Tai-ngan.

[502]1 $1^{\circ}$ Fu in the Chi-Tung'T'ai - Wu - Iin circ., Shantung; forming also the district city of the same name; Lat. $36^{\circ}$ $10^{\prime}$, Long. $117^{\circ} 15^{\prime}$; S.A., Po-yi 博邑; Sui, Wên-yang 洨陽 and Po-cli'êng 博城; T'ang, 'T'ai C.; Sung, Fêng$\mathrm{f} n$ 奉符; Kin, T'ai-an 安.

$2^{\circ} \mathrm{H}$. forming the prefectural city of the same name, Shantung; Lat. $36^{\circ} 10^{\prime}$, Long. $117^{\circ} 15^{\prime}$.

6177 T'ai-ch'iao / 欈, chên near Frn-p'ing H., Shensi.

6178 T'ai-ch'nan| 川, ssü near P'ing-11an H., Kuangsi. • 6179 I'ai-ho | 利 Tai-ho, H. [208] in Chi-an Fin, Kiangsi; Lat. $26^{\circ} 52^{\prime}$, Long. $114^{\circ}$ $56^{\prime}$; Han, I, ï-ling 盧陵; E. Han, Hsi-cli'ang 西 泉; Sui, An-fêng 妾豐 and 'T'ai-ho / 和.

6180 T'ai-hsing | 與 Tai-hing, [528]H. in T'ung C., Kiangsu; I at. $32^{\circ} 03^{\prime}$, Long. $120^{\circ}$ $10^{\prime}$; Sui, Hai-ling 海陵; S. T'ang, T'ai-hsing |

興1. Port of call.

6180" 'T'ai-jên | 任, H. in Cl’ïan-1o 全羅 circ., Corea,
台

T'AI-KUNG.

6181 'T'ai-11ing | 家 'Tai-11ing. [298] $1^{\circ} \mathrm{H}$. in Shao-wu Fu, Fulukien; Lat. $26^{\circ} 55^{\prime}$, Lonig. $117^{\circ} 18^{\prime}$; $T \sin$, Sui - ch'êng 総 城; Tang, Knei-hua 歸 化; Sung, T'ai-ning | 富.

$2^{\circ}$ See No. 7373.

6182 'T'ai-shum | 順 Tai-shu11, [33] H. in IVêl1-cliou Fur, Chelıkiang; Lat. $27^{\circ} 35^{\prime}$, Long. $119^{\circ} 50^{\prime}$; orig., $\mathrm{P}^{\prime}$ ing-yang 平晹.

6183 T'ai-tai | 岱, see No. $5462^{\circ}$.

\section{台}

6184 T'ai 台, see No. 6185.

6185 T'ai-chon | 州 Tai-chan, [252] Fu in the Ning-Shao- $T$ ' ai circ., Chehkiang; forming also the district city of Lin-hai ; Lat. $28^{\circ} 54^{\prime}$, I.ong. $121^{\circ} 06^{\prime} ; S . A$. and $C$. Wars, land of the Yüeh 臱 tribes; $T s^{\prime}$ in, in Min-chung 閩中 chïn; Han, Tung-oul 東區; E. Han, Cliang-an 辛安; Liang, Ch'ih-ch'êng 赫 城; Tang, Hai 海 C. and T'ai | C.; Ming, T'aichou | 州 Fu.

6186 T'ai-kung | 拱 Tai-kung, [81] T. in - Clhên - yïan Fin, Kueichon; Lat. $26^{\circ} 30^{\prime}$, I.ong. $10835^{\prime}$. 
台

$T^{6}$ a $-T^{6}$ OU.

6187 T'ai-t'ou | 頭, chên near Hua-yin H., Sliensi.

\section{囊}

6188 T'ai 喜.

$1^{\circ}$ (obs.) 30 li N. E. of Li-ch'êng H., Shantung; Han, H. in Chinan 沼南 chion.

$2^{\circ}$ See No. 7121.

$6189 \mathrm{~T}^{6}$ ai-chung | 中, Fu in T'ai - nan - P'êng-hu circ., Formosa, forming also the district city of 'T'ai-wan. Not far from Chung-hua $\mathrm{H}$.

6190 T'ai-huai | 懷, chên in Wu-t'ai H., Shansi.

6191 T'ai-11an | 献, Fu in T'ai -nan-P'êng-hu circ., Formosa ; forming also the district city of the same name; Lat. $22^{\circ} 58^{\prime}$, Long. $120^{\circ} 14^{\prime}$. Previous to 1888 called 'T'ai-wan |

瀿. Capital of the Island of Formosa; also called T“ai-yang | 陽. Yüan, land of the Tung-fan 東 番, Eastern barbarians; Ming, 1st, land of the Hung-11nao-fan 紅毛番, red haired barbarians (i.e. the Ditch), 2nd, Cli êng$\mathrm{t}$ ien 丞天.

$2^{\circ} \mathrm{H}$. forming the prefectural city of the same
罂 TAKH'T SOLEIMAN.

name, Formosa ; I at. $22^{\circ} 58^{\prime}$, Loung. $120^{\circ} 14^{\prime}$. 6191' T'ai-11an-P'êng-hu|南澎 湖, circuit in Formosa, comprising T'ai-pei Fur, 'T'ai-nan Fu, 'T'ai-chung Fu and 'T'ai-tung C.I.C. Before 1888 called ' $\mathrm{T}$ 'aiwan-P'êng-lın circuit.

6192 T'ai-pei | 北 'Tai-peh, Fu in the ' $C$ 'ai-wan circ., Formosa ; forming also the district city of Tan-shu1; Lat. $25^{\circ} 07^{\prime}$, Isong. $121^{\circ}$ $32^{\prime}$.

6193 T'ai-tnng | 東, C.I.C. in the 'T'ai - wan - P'êng-hn circuit, Formosa. Formerly $\mathrm{P}^{6} \mathrm{i}$-11an $\mathrm{T}$.

6194 T'ai-wan | 漠 Tai-wan, see Nos. 6189, 6191.

6195 T'ai - wan - P'êng - hı, see No. $6191^{\wedge}$.

6196 'T'ai-yang | 風, see the preceding.

\section{䔩}

6197 T'ai 楚, (obs.) S.IV. of Wr-kung H., Shensi; Han, H. in Yn-fu-fêng 右扶陽 chiin.

Tak, see No. 6141.

Takasaki, sce No. 3130.

Takelik, see No. 6323.

Takhti Soleiman, see No. 5338. 
丹

Talas, see No. 6143.

Talecan, see No. 6148.

Talki, see No. 6146.

Tamba, see No. 6240 .

Tamsui, see No. 6217.

\section{TAN}

\section{丹}

6198 Tan f, see No. 7422 .

6199 Tan-cl' êng | 城, H. in

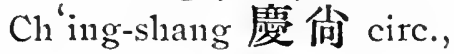
Corea.

6200 Tan-chi | 吉, Han, H. in Chihli.

6201 Tan-chia | 家, T'ang, C. in Ssŭch' 'uan.

6202 Tan-chiang | 汇 Tan[94] kiang, 'T. in 'Tulyün Fin, Kueichou ; Lat. $26^{\circ}, 10^{\prime}$, Long. $108^{\circ} 10^{\prime}$.

6203 Tan-chu-ch'êng | 朱城, see Nos. 1661, 1794.

6204 Tan-lisia | 霞, a posttown near Chang-chou Fu, Fulukien.

6205 Tan-kê-êrh | 噶尔 Tanko-'rh, T. in Hsi-ning $\mathrm{Fu}$, Kansuh ; Lat. $36^{\circ} 40^{\prime}$, Long. $101^{\circ} 30^{\prime}$.

6206 Tan-lêng | 稜 Tan-lăng, [414] H. in Mei C., Ssuch'uan; Lat. $30^{\circ}\left(05^{\prime}\right.$; Long. $103^{\circ}$ 35'; Han, Nan-an 南妿; P. Chou, Ch'i-lê 唯樂; Sui, Tan-lêng | 校.

6207 Tan-pi | 陛, see No. 5822.
丹 TAN-YANG-CH' $\hat{\text { FNG }}$.

6208 Tan-p'ing | 平, ssŭ near Ch'ang-chai T., Kueichou.

6209 'Tan-t' ${ }^{\prime}$ | 徒 Tan-tu.

$1^{\circ} \mathrm{H}$. forming the prefectural city of Chên-chiang, Kiangsu; Lat. $32^{\circ} 10^{\prime}$, I.ong. $119^{\circ} 29^{\prime}$; orig., Kn-yang 谷陽; $T^{6}$ ang, Tan1-t"n | 徒 H.; Sung, Nan-hsui 南徐; Sui, Yen-ling 延 陵.

$2^{\circ}$ (obs.) $18 l i$ S.E. of the above; Han, Tsin and S. Sung, Tan-t' $\mathrm{u} \mid$ 徒 $\mathrm{H}$.

6210 Tan-ya | 牙 Damietta, in Egypt.

6211 Tan-yang | 陽 Tan-yang.

$1^{\circ} \mathrm{H}$. in Chên-chiang Fun, Kiangsu; Lat. $32^{\circ} 04^{\prime}$, rong. $119^{\circ} 32^{\prime} ; T^{\prime}{ }^{\prime}$ in, Yün-yang 雲陽 and Ch'î-o 曲阿; Han, Tan-yang | chïn.

$2^{\circ}$ Chên near T'ai-p'ing H., Anluni.

$3^{\circ}$ (obs.) Han and $T^{6}$ ang, chün comprising parts of Kiangsin and Anhui. $4^{\circ}$ chiin in Cinung-ch'ing 忠 清 circ., Corea.

$5^{\circ}$ See No. 7422 .

6212 Tan-yang-ch'êng | 陽城, (obs.) formerly a town $\mathrm{E}$. of T'ai-p'ing Fu, Anhui. 
但

TAN-TIEN.

但

6213 'Tan-tien 但 店, ssü in Huang-kang H., Hupeh.

\section{㙂}

6214 Tan-1o 㙂 篗 Quelpaert Island, Corea. See No. 600.

\section{儋}

6215 Tan 㑾Tan, C. in Ch'iungcliou $\mathrm{F}$ 11, Krangtung; Lat. $19^{\circ} 32^{\prime}$, Long. $109^{\circ} 20^{\prime}$; Han, Tan-êrh | 耳, Sui, Chu-yai 珠 崖 chïn and Yi-1un 義 倫 H.; T ang, Tan |C. and Yi-lun 戔 倫 $\mathrm{H}$.

6216 Tan-êrh | 耳, see the preceding.

\section{谈}

6217 Tan-shui 淡水 Tan-shwui or Tamsui.

$1^{\circ}$ (obs.) formerly $T$. in T'ai-pei Fu, Formosa; I at. $24^{\circ} 49^{\prime}$, Long. $121^{\circ} 15^{\prime}$; see No. 1380.

$2^{\circ} \mathrm{H}$. forming the prefectural city of ' ' ${ }^{i}$ ai-pei Fi1, Formosa; Lat. $25^{\circ}$ $07^{\prime}$, I ong. $121^{\circ} 32^{\prime}$; one of the ports in $\mathrm{N}$. Formosa open to foreign trade; the foreign settlement lies in Lat. $25^{\circ}$ -22', Long. $121^{\circ} 28^{\prime}$. Called locally Hu-wei 渥尾 (Hobé). See No. 6089.
潭 T'AN-CHUNG.

\section{明}

6218 Tan-la-chien 呾刺侹, see No. 6148.

\section{單.}

6219 Tan 單; Tan-fu-yi 父 邑, etc.; see No. 5467.

'Tan-ching, see No. 6228. 'Tan1-kiang, see No. 6200. 'Tan-ko-'rli, see No. 6205. Tan-lăng, see No. 6206. 'Tan-shwui, see No. 6217. Tan-tu, see No. 6209.

\section{T'AN}

\section{濉。}

6220 'T'an1-t'ou灘頭, ssü in Iang-clı 'ïan H., Kiangsi.

\section{潭}

6221 T’an 潭.

$1^{\circ}$ (obs.) S. Sung, C. in the Hu-nan 湖南 circnit.

$2^{\circ}$ See No. 267.

$6222 \mathrm{~T}^{6}$ an-ch'i | 溪.

$1^{\circ}$ T.S. near Hsia-chiang

T., Kueichon; Ming, ssŭ in Li-p'ing F $\mathrm{F}$, Kneichon.

$2^{\circ}$ See No. 3720.

6223 T'an-chou | 洲, see No. 2158.

6224 'T'an-chung | 中. 
潭

T'AN-ChUNG.

$1^{\circ}$ (obs.) S.E. of Ma-p' in H., Kuangsi ; Han, $\mathrm{H}$. in Yü-lin 䈭 林 chün; $T \sin , \mathrm{H}$. in Kuei-lin 柱林 chïn。

$2^{\circ}$ (obs.) in Ma-p'ing H., Kuangsi ; $S . T s^{\prime} i, \mathrm{H}$. in Knei-lin 桂林 chïn. $3^{\circ}$ See Nos. 4298, 7980.

6225 'T'an-k'ou | 口, ssй in Nan-k'ang H., Kiangsi.

$6226 \mathrm{~T}^{\prime}$ 'an-yang | 陽, (obs.) T'ang, a town W. of Yüanchou $F u$, Hunan.

\section{㸚}

6227 T'an 炏 (obs.) S.A., feudal state comprising parts of Shantung and Kiangsi ; see Nos. 1913, 6228.

6228 T'an-ch'êng 城, Tan[404] ching, H. in Yi-chon Fin, Shantung; Lat. $34^{\circ} 47^{\prime}$, Song. $118^{\circ} 43^{\prime}$; orig., K. I'an | Han, T'an |; T'ang, 'T'an-cln'êng | 城. 檀

6229 T'an 檀, see No. 4423.

\section{TANG}

\section{當}

6230 Tang-hu-shilı 當湘㶦, see No. 5156.

6231 Tang-jên | 仁, (obs.) near Ya-chou Fu, Ssŭch 'uan; T'ang, C. in Chien-nan 劍 南 circuit.
TANG-Yi.

6232 Tang-po | 波, (obs.) Han, a town near Jung-tsê $H$., Honan.

6233 'Tang-t'ing | 亭, see No. 1887.

6234 Tang-tu, or Tang-t' $u$ | 叙 Tang-tı.

$1^{\circ} \mathrm{H}$. forming the prefectural city of 'T'aip'ing, Anhui ; Lat. $31^{\circ}$ $33^{\prime}$, Long. $118^{\circ} 30^{\prime}$; Han, Tan-yang 丹陽; Sui, Tang-tu| 叙 $\mathrm{H}$. and K11-shn 姑 敦.

$2^{\circ}$ (obs.) S.E. of Huaiyüan H., Anhui ; $W$. Han, Marq.; E. Han, H. in Chiu-cliang 九 江 chiin.

$3^{\circ}$ (obs.) N. of Nan-ling H., Anhui; $T \sin , S$. Sung and $S . T s^{\prime} i, \mathrm{H}$. in Huai-nan 淮南chiün.

$4^{\circ}$ See No. 6167.

6235 'Tang-wei | 爲, (obs.) in Yün-lien H., Ssŭch'nan; $T^{\prime}$ ang, H. in Lien 連 C. 6236 Tang-yang | 陽 Tang[109] yang, H. in An-lu Fu, Hupeh; Lat. $30^{\circ} 45^{\prime}$, Long. $111^{\circ} 36^{\prime}$; Han, Tang-yang | 陽; P. Chou, Cliangchiang 漳 江; Sui, Yiu 玉 C.

6237 'Tang-yi | 夷, (obs.) W. of Min C.., Kansulu; Sui, H. in Lin-t'ao 臨洮 chün。 
黨

TANG.

\section{黨}

6238 Tang 嚜, (obs.) T ang, C., $\mathrm{N}$. of Ch'ing-yang $\mathrm{Ft}$, Anhui.

6239 Tang-ch' êng | 城, a posttown near An-hsi C., Kansul.

6240 Tang-pa | 埧, T. S. west of Li-fan T., Ssŭch' uan. Thibetan name Djamba (="Sacred") [ Baber].

\section{碭}

6241 Tang 碭.

$1^{\circ}$ (obs.) Ts in, chïn con1prising parts of Honan and Kiangsu.

$2^{\circ}$ See Nos. 2570, 4697, 6242.

6242 Tang-shan | II Tang[278| shan, H. in Hsii-chon Fin, Kiangsu ; Lat. $34^{\circ} 29^{\prime}$, Long. $116^{\circ} 41^{\prime}$; $T s^{\prime} i n$, in Tang | chün; Han, K. Liang 梁; 2nd $\mathrm{Wei}$, Anyang 安陽。

\section{宕}

6243 lang 炭, (obs.) T'ang, C. near Min C., Kansuh. 6244 'Tang-ch' ang | 昌, see No. 7.980.

6245 'Tang-ch' ii | 渠.

$1^{\circ}$ (obs.) N.E. of $\mathrm{Cl}_{1}$ ï $\mathrm{H}$., Ssuch' '1an; Han, H. in -Pa巴chïn; Tsin, H. in Pa-hsi 巴间 chün; S. Sung and $S . T s^{\prime} i, \mathrm{H}$. in Tang-ch'ii | 渠chïn.
唐 T'ANG.

$2^{\circ}$ (obs.) near Pao-ning Fu, Ssŭch" uan; S. Sung and $S . T s^{6} i, H$. in Huayang 華陽 chïn.

$3^{\circ}$ (obs.) in Ssŭcl' ${ }^{\prime}$ tan; $S$. Sung, H. in Nan 'yangcls'ü 南 | 渠 chïn; S. $T s^{\prime} i$, H. in 'Tung 'Tangch'ï 少 | 渠 chïn.

$4^{\circ}$ (obs.) N. IV. of Yellt'ing H., Ssŭch'nan; S. Ts' $i, H$. in 'Tang-ch' ï | 渠 chïn.

$5^{\circ}$ N. E. of Ying-shan H., Sstuch '11an1; Simi, H. in Tang-ch' î | 渠 chïn; T'ang, H. in P'êng 逢 C.

$6^{\circ}$ (obs.) Sui, chïn in Iiang 梁 CHOU; see Nos. 1437, 2774, 3388, $3923,4568^{\mathrm{s}}, 5815,6020$, 6524, 6772, 7593, 7614.

Tang, see No. 6246.

Tang-ki, see No. 6262.

Tang-slian, see No. 6253.

Tang-yili, see No. 6271.

Tang-yin, see No. 6265.

\section{T'ANG}

\section{唐}

6246 T'ang 磨 Tang.

[122] $1^{\circ}$ H. in Shun-t'ien Fu, Chihli; Lat. $38^{\circ} 44^{\prime}$, Long. $115^{\circ}$; orig. , feudal 
唐

$T^{6}$ ANG.

state 'T'ang |; S. A., Hsien-yu 鮮虞; Han, T'ang | H.; Five dyn., Po-ling 博 陵.

[447] $2^{\circ}$ H.. in Nan-yang Fu, Honan; Lat. $32^{\circ} 47^{\prime}$, Long. $112^{\circ}$ 53'; Han, Pei-yang 北陽; 2nd Vei, Tung-ching 東 判; $W$. Wei, Hnai 淮 C.; Sui, Hsien 晶 C.; T'ang, $\mathrm{Pi}$ 泌 $\mathrm{C}$. and 'T'ang $\mathrm{C}$.

$3^{\circ}$ (obs.) Sui, feudal state - comprising parts of Chihli and Honan.

$4^{\circ}$ (obs.) T'ang, C. near $\mathrm{P}$ 'ing-nan $\mathrm{H}$., Kuangsi. $5^{\circ}$ See Nos. 5200, 6248.

6247 'T'ang-clt'ang | 昌, see No. 1594.

6248 T'ang-cl' êng | 㧞; (obs.) Sui, H. near Sui C., Ho11an ; this city was the capital of the feudal state $T^{\prime}$ ang 1 .

6249 T'ang-chia | 家.

$1^{\circ} \mathrm{Y}$. near Clı êng-ku H., Shensi.

$2^{\circ}$ Y. near Yung-ming $H$, Huna11.

6250 'T'ang-ching | 津, H. in Chung-ch 'ing 忠清 circ., Corea.

6251 T'ang-hsien | 縣, chên near Sui C., Hupeh.
晹 T'ANG.

6252 T' ${ }^{*}$ ang-kou | 洀, chên near Wu-hu H., Anlui.

6253 T'ang-shan | II Tangshan.

[432] $1^{\circ}$ H. in Shun-tê Fu, Chihli; Lat. $37^{\circ} 22^{\prime}$, Long. $114^{\circ} 48^{\prime}$; orig., Pai -jên-yi 栢人邑; N.Wei, Pai-jên 柏人 H.; Sui, Pai-jên 柏人, or 柏仁, H.; T゙ang, Yao-shan 䔷 U; Kin, T'ang-shan | Ill.

$2^{\circ}$ See No. 291.

6254 'T'ang-tien | 苫, chên near Têng-fêng H., Honan.

6255 T'ang-tui | 推, (obs.) near Yen-an Fu, Shensi; Sung, pao in Sui-tê 綏德 military district.

6256 'T'ang-yai | 崖,(obs.) near Hsien-fêng H., Hupeh ; Ming, ssü in Shih-chon 施州 military district.

\section{䛸}

6257 '“'ang-chi 䛸棲, chên near Tê-ch'ing H., Chehkiang.

6258 T'ang-chia | 家, ssŭ near Hsing-an H., Kuangsi.

6259 'T'ang-kê-êrh-chung | 噶 尔鍾, T.S. near Taclien-lu T., Ssŭch'nan.

\section{湯}

6260 T'ang 湯, Y. near Stung H., Honan. 
湯 T'ANG-CH'ÊNG.

6261 T'ang-ch'êng | 城, a posttown near Fêng-t'ien Fu, Shingking.

6262 T'ang-ch'i | 溪 'Tang-ki, H. in Chin-hua Fin, Chehkiang; I at. $29^{\circ} 05^{\prime}$, Long. $119^{\circ} 32^{\prime}$; Ming, 'T'ang$\mathrm{ch}^{6} \mathrm{i}$ | 潆 $\mathrm{H}$.

6263 T'ang-ch'ih | 池, (obs.) 60 li N.E. of Kai-p'ing H., Shingking; Liao, T'iell 鐵 C.; Kin, H. in Kai 蓋 C.

6264 T'ang-shan | IL; (obs.)

Liang and Sui, H., S. of Ho H., Kuangsi.

6265 T'ang-yin | 陰 Tang-yin, [446] H. in Clıang-tê Fu, Honan; I at. $35^{\circ} 56^{\prime}$, Long. $114^{\circ}$ $33^{\prime}$; orig., Y'ulli 美里; Han, T'any-yin 湯陵; Sui, T'ang-yin | 擦.

$6265^{\wedge} \mathrm{T}^{\prime}$ 'ang-yoian | 源 H. in Yi-lan Fn, Kirin.

\section{萿}

6266 'T'ang-ch'ang 蕩昌, Tsin, $H$. in Kuangsi.

6267 T'ang-yin | 陰, see Nos. 107,6265 .

\section{沿}

6268 'T'ang-t'ang 华䛸, a posttown near Hsüan-wei $\mathrm{C}$., Yünnan.

\section{儻}

6269 'I'ang-chêng 㙁城, see No. 7234 .
道 TAO.

\section{衰}

6270 T'ang-yang 堂晹, see Nos. 2754, 3419.

6271 T'ang-yi | 邑 Tang-yih, H. in Tung-ch'ang Fu, Shantung; Lat. $36^{\circ} 35^{\prime}$, I ong. $115^{\circ} 58^{\prime} ;$ Sui,

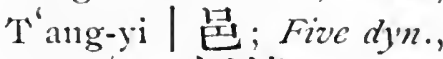
Ho-cling 河清.

\section{螳}

6272 T'ang-lang-cl' 11an 螳蛝 川, see No. 89.

\section{学}

6273 T'ang-yi 棠邑, see Nos. 753, 4007.

Tăng, see Nos. 6333, 6336, 6340.

Tăng-chan, see No. 6330 . Tăng-chuen, see No. 6334 . T'ăng-fung, see No. 6331. Tăng-yueh, see No. 6339. 'Tanpiju, see No. 5574.

\section{TAO}

演

6274 'Tao-kên 鼻 根 Shimané, Ken in Japan. Chief town Matsuyé.

\section{道}

6275 '个ao 道 'Tau, C. in Yung[180] chou Fu, Hunan ; Lat. $25^{\circ} 33^{\prime}$, Long. $111^{\circ} 28^{\prime}$; 
道

TAO.

Wu，Ying-yang 營陽; T'ang, Tao | C. and Chiang-hua 江華; S.Sung, Tao | C.

6276 Tao-ching | 米, a posttown near Hsin-yen $T$., Shingking.

6277 Tao-hsien | 賢, chên near P'u-ch' êng H., Shensi.

6278 Tao-jên |人, (obs.) S.E. of Yang-kao H., Shansi; Han, H. in Tai 代 chïn.

6279 'Tao-k'ou | 口, a post-town near Hua H., Honan.

6280 'Tao-t'ou | 預, ssŭ in Tinghai 'T., Chelıkiang.

\section{導}

6281 Tao-chiang 導江.

$1^{\circ}$ (obs.) 20 li E. of Kuan H., Ssurch' 'nan; T'ang, H. in P'êng 彭 C.; Sung, H. in Yung-k'ang 永庭 military district. $2^{\circ}$ See No. 3359.

\section{倒}

6282 Tao-111a-kuan 倒 馬 關, ssü in 'T'ang H., Chilhli.

\section{T'AO}

\section{桃}

6283 个'ao 桃, see Nos. 2033, 3417.

6284 'T'ao-ch'êng | 城, chên near Ying-shan H., Anhui.
洮 T'AO.

6285 'T'ao-h11a-p'ing | 花坪, a post-town near Hsin-luna H., Hunan.

6286 T'ao-hua-tien | 花店, chên near Huang-an $\mathrm{H}$., Hupeh. 6287 'T'ao-hui | 槐, (obs.) near Turfan; Tang, C. in Yüehchin 月支 Fu.

6288 'T'ao-lin 林.

$1^{\circ}$ (obs.) chou, H. in W. of Honan.

$2^{\circ}$ See No. 3965.

6289 'T'ao-shan | U, a posttow1 near Hsü-chou $\mathrm{Fu}$, Kiangsu.

6290 'T'ao-yüan | 園, see No. 6291.

6291 T'ao-yüan | 源 Tan-yuen. [148] $1^{\circ} \mathrm{H}$. in Huai-an $\mathrm{Fu}$, Kiangsu ; Lat. $33^{\circ} 40^{\prime}$; Long. $118^{\circ} 46^{\prime}$; Sui, Huai-pin 淮演; Yïan, T'ao-yüan | 園 H.; Ming, 'T'ao-yiuan | 源 $\mathrm{H}$.

$[561] 2^{\circ}$ H. in $\mathrm{Ch}^{\prime}$ ang-tê $\mathrm{F} 11$, Hunan ; Lat. $28^{\circ} 52^{\prime}$, I ong. $111^{\circ} 11^{\prime}$; Han, Yïan-11an 沅南; $T^{6}$ ang， '”ao-yüan|源 $\mathrm{H}$. $3^{\circ}$ Ss ̌̆ near 'Ta-t'ien $\mathrm{H}$., Fullkieu.

$4^{\circ}$ See No. 7840 .

\section{洮}

6292 'T'ao 洮. 
洮 T'AO.

$1^{\circ}$ Chên near Shun-hua H., Sliensi.

$2^{\circ}$ See No. 6293.

6293 'T'ao-chou | 州 Tau-chau, [455] T. in Kung-ch'ang Fu,

Kansulı; I at. $43^{\circ} 35^{\prime}$, Long. $103^{\circ} 31^{\prime} ; T s^{\prime} i n$ and Han, land of the Jung 我 tribes; $T \sin$, occupied by the Chinese; P. Chou, T'ao-yang | 晹; Sui, Lint'ao 臨|; T⿱ang and Sung, T' ao |C. Terr. jur. 6294 'T'ao-p'ing | 坪, chên near Li H., Kansul.

6295 T'ao-yang | 陽.

$1^{\circ}$ (obs.) Han, H., 35 li N.E. of Cli iian C., Kuangsi.

$2^{\circ}$ See No. 6293.

$6296 \mathrm{~T}^{\prime}$ 'ao-yüan | 源, (obs.)

S.W. of 'T'ao-chou T., Kansuh ; Sui, H. in Lint’ao 臨|chïn.

\section{陶}

6297 T'ao 陶, Y. near Hoch'ing C.. Yïnnan.

6298 T'ao-chia | 第, Y. near Nan-cl1' nan H., Ssŭclı' 'ıan; also, 'T.S. near Yüeh-sui 'T., Ssŭch' 'nan.

6299 'T'ao-ch'in | 邱, see No. 64.58.

6300 T'ao-ch'ung | 冲, a posttown near 'T'ung-ch' êng H., Anhui.
'’̂.

6301 'T'ao-hsü | 㙈, Y. near Pin C., Kuangsi.

6302 T'ao-t'ang | 府, (obs.) the princedon of Yao 堯, near Clivao-clı'êng H., Shansi.

\section{討}

6303 ' ${ }^{6}$ 'ao-lai-ssŭ 討來思 (or, T'ao - lao-ssu | 落 思) Tabriz (or, Tauris, the Chinese nanle being evidently adapted to the latter), a town in Persia; Lat. $38^{\circ} 02^{\prime}$, Long. $46^{\circ} 12^{\prime}$. 630+ 'T'ao-lao-ssin | 落思, see the preceding.

Tapatakze, see No. 6352. Tara-usu, see No. 6145. Tarbagatai, see No. 6140. Tardzung, see No. 6129. Tar-tsen-do, see No. 6125. Tashbalig, or 'Tashbalik, see Nos. 6952, 6152, 6155.

Tashkend, see No. 154. Tau, see No. 6275.

Tat1-chat1, see No. 6293.

Tauris, see No. 6303.

Tan-yuen, see No. 6291.

'Tchrango, see No. 2102.

TE

德

6305 'Tê 德 'Teh. 
德

[322] $1^{\circ}$ C. in Chi-nan Fu, Shantung; Lat. $37^{\circ} 27^{\prime}$, Long. $116^{\circ} 23^{\prime}$; Han, $P^{6}$ ing-yiuan 平原; 2nd IVci, An-tê 安|; Sui, Tê |C.

$2^{\circ}$ C. in Corea; Iat. $40^{\circ}$, I,ong. $127^{\circ} 10^{\prime}$.

$3^{\circ}$ See No. 6319.

6306 'Tê-an | 安 Teln-ngan.

$[568] 1^{\circ} \mathrm{Fu}$ in the Han-HuangTê circ., Hupeh ; forming also the district city of An-lu; Lat. $31^{\circ} 20^{\prime}$, Long. $113^{\circ} 37^{\prime}$; orig., Y ïn-11êng 雲萝; S.A., in K. Yiun 郥; $7 s^{\prime} \mathrm{in}$, in Nan 南 chün; Han, in Chiang-lisia 江夏 chiun; 1st Sung, Anling 学陵; W. Wei, An 岁 C.; P. Chou, Yün 鄖 C.; T Tang， An-huang 究頡; Five dyn., Hsïan-wei 宣威 and An-yüan 妾遠; $S$. Sung, Tê-an 安 Fin.

$2^{\circ} \mathrm{H}$. in Chin-chiang $\mathrm{F}_{11}$, Kiangsi ; Lat. $29^{\circ} 19^{\prime}$, Long. $115^{\circ} 43^{\prime}$; Han, Li-ling 歴陵; Wangmang (usurper), P'nt'ing 蒲亭; Five dyn., Tê-an 妏:

6307 'Tê-ch' ang-so | 莦所, ssü S. of Hsi-ch'ang $\mathrm{H}$., Ssŭch'uan.
德

'T̂E-HUA.

6308 Tê-ch'in | 沈 Detsin, a town in Thibet; Iat. $29^{\circ}$ $40^{\prime}$, Long. $92^{\circ} 30^{\prime}$.

6309 Tê-ch'ing | 慶 Teln-king, L165] C. in Chao-ch'ing Fu, Kuangtung ; Lat. $23^{\circ} 14^{\prime}$, Long. $111^{\circ} 14^{\prime}$; Han, Tsin, S. Sung, S. Ts' $i$ and Sui,

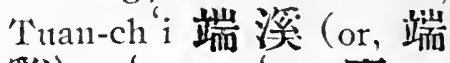
彩谷); $T^{\prime}$ ang, $\mathrm{K}$ ' ang 康 $\mathrm{C}$.; Sung, Tê-ch'ing | 慶 Fu.

6310 Tê-ch'ing | 清 Tell-tsing, $\mathrm{H}$. in Hu-chou Fu, Chekiang; Lat. $30^{\circ} 33^{\prime}$, Long. $120^{\circ} 06^{\prime} ; T^{\prime}$ ang ; Wu-yüan 武原, Lin-ch“i 臨溪 and Tê-cling | 漹.

$6310^{3}$ Tê-ch" ${ }^{6}$ 11n11 | 川, chün in P'ing-an本等 circ, Corea.

6311 'Tê-êrh-kê-t'ê | 尔格武, T.S. near Sung-p an T., Ssìch' nan.

6312 Tê-hua | 化 Teh-hwa.

$[517] 1^{\circ} \mathrm{H}$. forming the prefectnral city of Chin-chiang, Kiangsi ; Lat. $29^{\circ} 28^{\prime}$, I.ong. $116^{\circ} 08^{\prime}$; Han, Hsün-yang 浽陽; Tsin, $\mathrm{Ch}^{\prime}$ ai-sang 柴桑; Sui, P'ên-ch'êng 湓 城; T'ang, Sung and Yïan, Chiang 江. C.

$2^{\circ} \mathrm{H}$. in Yung-ch' $11 n \mathrm{C}$., Fuhkien ; Lat. $25^{\circ} 26^{\prime}$, Long. $118^{\circ} 27^{\prime}$; orig., Yung-fu 永酔; Five, dyn., Tê-luua | 化. 
德

'I'Ê-HSING.

6313 Tê-lısing | 與 Tell-hing.

$1^{\circ} \mathrm{H}$. in Jao-chon $\mathrm{F}_{11}$, [240] Kiangsi; I,at. $28^{\circ} 55^{\prime}$, I.ong. $117^{\circ} 42^{\prime}$; orig., L.ê-p ing 樂 本; $S$. T゙ang, Tê-hsing | 與. $2^{\circ}$ See No. 4947 .

6314 Tê-p'ing | 4 'Telı-ping, [284] H. in Clii-nan Fu, Shantung; Lat. $37^{\circ} 34^{\prime}$, Long. $117^{\circ} 04^{\prime}$; Han, P'ingch'ang 平昌; E. Han, Hsi-p ing-ch'ang 西平昌; Five dyn., Tê-p ing 1 平. 6315 Tê-shan | IL, H. in Chungch'ing 忠清 circ., Corea. 6316 Tê-shêng |勝 (or. 得勝) Teh-shing, T. in Clingyüan $F u$, Kuangsi; Iat. $21^{\circ} 42^{\prime}$, Long. $107^{\circ} 59^{\prime}$. 6317 Tê-tao | 島, Ken in Japan. 6318 Tê-yang | 韵 Teh-yang.

$1^{\circ} \mathrm{H}$. in Mien C., Ssŭ[305] ch'uan; I.at. $31^{\circ} 10^{\prime}$, I.ong. $104^{\circ} 22^{\prime}$; Han, Mien-chu 綿 竹; $E$. Han, Tê-yang | 晹; Yïan, 'Tê |C.

$2^{\circ}$ See No. 5963.

6319 Tê-yüan | 源, Fu in Hsienching 咸鏡 circ., Corea. 得

6320 Tê-shêng 得勝, see No. 6316.

6321 Tê-shêng-hsün | 勝 汛, ssŭ near Wei-ning C'., Kneichou.
武 'Ten-Shing.

6322 'lê-shêng | 滕, Y. near

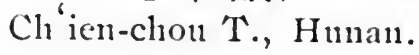

\section{T‘र्E}

特

6323 'T'ê-k'ê-li-k'ê特克里克 Takelik, a town in The New Dominion; Lat. $40^{\circ}$ $50^{\prime}$, Long. $96^{\circ}$.

$6324 T^{\prime}$ ê-k'ê-ssŭ| 总斯 Tekes, a town in Ili; Iat. $42^{\circ}$ $30^{\prime}$, I,ong. $82^{\circ} 05^{\prime}$.

6325 'T'ê-liang | 莌, (obs.) T'ang, H., N.W. of Hsin-yi H., Kuangtung.

6326 T'ê-mo | 磨, see No. 3410. 6327 T'ê-mu-êrh-li-k'ê | 穆尔 哩鼔 Timurlik, a town in Ili ; Lat. $43^{\circ} 15^{\prime}$, Long. $80^{\circ}+5^{\prime}$.

\section{式}

6328 ' '’ê-êrh-mi 武耳迷（or Tieh-li-mi 迭里密, or, T'ieh-li-ma 帖里派)。

Termed, a town on the Oxus, 30 miles $\mathrm{N}$. of Balkh.

Telr, see No. 6.305.

'Teh-hing, see No. 6313.

'Teh-hwa, see No. 6312.

Teh-king, see No. 6309.

Telı-ngan, see No. 6306.

Teh-ping, see No. 6314.

Teh-shing, see No. 6316, 
武

TEh-'TSing.

Teh-tsing, see No. 6310. Tein-yang, see No. 6318. Tekes, see No. 6324.

Tenduc, see No. 6423.

\section{TÊNG}

\section{登}

6329 T'èng 登, see No. 6330.

6330 Têng-chon|州 Tăng-clıau, Fil in the T'êng-Lai-Clı'ingChiao circ., Shantung; forming also the district city of $\mathrm{P}^{\prime}$ êng-lai; Lat. $37^{\circ}$ $45^{\prime}$, Long. $120^{\circ} 42^{\prime}$; orig., land of the Yü 嵎 tribes; S.A., K. Mou 牟; $C$. Wars, in K. Chri 蕉; Han, in Tung-lai 東萊 chïn; 2nd Wei, Trnng-mon 東牵; Sui, Mou 牟 C.; $T^{6}$ ang, Têng |C.

6331 'Têng-fêng | 封 Tăng[550]fung, H. in Ho-nan Fu, Honan; I at. $34^{\circ} 30^{\prime}$, I tong. $114^{\circ} 23^{\prime}$; W. Wei, Stung-yang 昜陽; T⿱㇒ang, Têng-fêng | 封 $\mathrm{H}$.

6332 Têng-Lai-Ch' ing-Clriao | 萊青疹, circ. in Shantung, comprising Têngchou Fu, Iai-chou Fu,

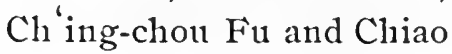
C.

鄧

6333 Têng 鄧 Tăng.
榉

T'ÂNG-YANG.

$[477] 1^{\circ}$ C. in Nan-yang $\mathrm{Fu}$, Honan; Lat. $32^{\circ} 46^{\prime}$, Long. $112^{\circ} 08^{\prime} ; 2^{\prime}$ in, $^{\circ}$, Jang-yi 程邑; 2nd Wei, Ching 荆 C.; Sui, Têng | C.

$2^{\circ}$ (obs.) formerly a town N.E. of Hsiang-yang Fu, Hupel.

$3^{\circ}$ See No. 4641).

6334 'Têng - ch' 'uan | 川 Tăng[303] clunen, C: in Ta-li Fu, Yünnan; ; Lat. $26^{\circ} 02^{\prime}$, I.ong. $100^{\circ} 15^{\prime} ;$ Han, Yeli-yü 葉榆; under the Mêng 蒙 tribes, Têngch' 1 tan | 川.

6335 Têng-pu|垚, chên near Kao-shun H., Kiangsu.

\section{T'Ê N}

滕

6336 'T'êng 滕 Tăng.

[68] $1^{\circ} \mathrm{H}$. in Yen-clion Fu, Shantung; Lat. $35^{\circ} 23^{\prime}$, Long. $117^{\circ} 24^{\prime}$; orig., K. Hsiao-chu 小制; Han, P'i 蕃 H., Sui, 'T'êng | $\mathrm{H}$.

$2^{\circ}$ (obs.) formerly a town

$\mathrm{S}$. of Hsü-chou $\mathrm{Fu}$, Kiangsu.

6337 Têng-chia | 家, Y. near $\mathrm{Ch}^{\prime}$ ên-chou Fu, Honan.

6338 T'êng-yang | 陽, a posttown near 'T'êng $H$., Shantung. 


\section{滕}

T'ÊNG-YƯ๋H.

6.339 T'êng-ÿïh | 越, see No. 6344.

\section{藤}

6340 T'êng 藤 Tăng, H. in [416] Wu-chou Fu, Kuangsi; Lat. $23^{\circ} 26^{\prime}$, L,ong. $110^{\circ}$ 31'; Han, Mêng-ling 猛 陵; Sui and S. Sung, T'êng $\mid C$.

6341 T'êng-ch' 'iao | 橋, ssünear Ling-shui H., Kuangtung.

\section{騰}

$6342 \mathrm{~T}^{6}$ êng-ch' $\mathrm{i}$ 䲲溪, (obs.)

S. Tsi, H. in Knangsi.

6343 'T'êng-chung / 衝, see No. 6344.

6344 T'êng-yueh | 越 (or, 滕

[568] 越) Tăng-yueh (by the Burmese called Momein), $T$. in Yung-ch' ang $F u$, Yünnan; Lat. $25^{\circ} 02^{\prime}$, Long. $98^{\circ} 45^{\prime}$; orig., Yüel1t'an 越賧; Sung, Yuiehchung 越衝; Yüan, 'T'êngchung $\mid$ 衝 Fu; Ming, T'êng-yüel. | 越 C.

Termed, see No. 6328.

Teshigang, see No. 136.

Teshu-11'lumbu, see No. 133.

Thabas-Sistan, see No. 6150.

Thribet, see Nos. 2502, 7167.

Thus, see No. 6672.

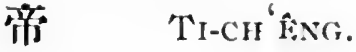

\section{TI}

遞

6345 Tii-p'u遞鋪, chênn near An-chi H., Chehkiang.

6346 Ti-t 'ing | 亭, a post-town near Hsiang-yüian H., Shansi.

\section{棣}

6347 Ti 棣, see Nos. 6521, 7097.

\section{氏}

6348 Ti-ch'iang 氏美, see Nos. 761, 4169.

\section{低}

6349 Ti-lien 低䈒 Dilem, a district of Persia, near the Caspian Sea.

\section{底}

6350 Ti-chao 底照, chên near Li-ch' üan H., Shensi.

6351 Ti-miao | 廟, chên near San-shui H., Shensi.

6352 'Ti-pa-ta-k'ê-sa / 巴達克 薩 Tapatakze, town in Thibet; Lat. $50^{\circ}$, Long. $92^{\circ} 30^{\prime}$.

6353 Ti-tien | 店, chên near Paochi H., Shensi.

\section{邸}

6354 Ti-chang 邸張, a post-town near $I, i-c h$ 'tüan $H$., Shensi.

\section{帝}

6355 Ti-ch'êng 帝城, see No. 5822. 
帝

TI-ChIng.

$6355^{\mathrm{A}}$ Ti-ching | 京, see No. 5822.

$6355^{\mathrm{B}}$ Ti-hu1 | 䦵, see No.5822. 迪

6356 Ti-hua 迪化 Tih-liwa.

$1^{\circ} \mathrm{Fu}$ in the Chên-'Ti circ., New Dominion, of which it is the capital; forming also the District city of the same name; Lat. $43^{\circ} 45^{\prime}$, Long. $89^{\circ}$; also known as Kungning 垡夏, or W11-lumu-ch'i 島魯木呍 Ourumtsi. The city of Ourumtsi is close to, but distinct from, that of Ti-hua.

$2^{\circ} \mathrm{H}$. forming the prefectural city of the same name.

\section{狄}

6357 Ti-han 狄 寒, chên near Lin-tung H., Shensi.

6358 Ti-tao | 道 Yin-tau, C. in [455] Lan-chou Fu, Kansulı; Lat. $35^{\circ} 22^{\prime}$, Long. $103^{\circ}$ $59^{\prime} ; C$. Wars, land of the W. Ch'iang 羌 tribes; $T s^{\prime}$ in and Han, Lung-hsi 隴西 chïn; Tsin, Ti-tao |道; Sung, Wu-shih 武 始; Sui, Chin-ch'êng 金 城; T'ang, Lit1-t'ao 臨 洮; S. Sung; Hsi 熙 C.; Ming, Lin-t ao 臨洮 Fu.

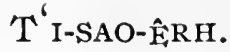

\section{荻}

6359 Ti-chiang 荻漠, chên near Fan-ch'ang H., Anhui.

翟

$6360 \mathrm{Ti}$ 翟, (obs.) Han, K. in Shensi; see No. 7358.

$6361 \mathrm{~T} i$ - ch'êng | 城, a posttown near Wang-tu $\mathrm{H}$,, Chihli.

6362 Ti-tao | 道.

$1^{\circ}$ Chên near Chung-pu

H., Shensi.

$2^{\circ}$ See No. 1547.

的

6363 Ti-an-li 的安里, see No. 6365.

6364 Tì-hsiang | 相, chên near Hsia H., Shansi.

6365 Ti-li-an | 里安 (also found written Ti-an-li | 安里) Daran, a town in Independent Turkestan, between Bokhara and Kath. 6366 Ti-mi-shih-chi | 迷失吉 Damascus, in Syria.

\section{堤}

6367 Ti-ch' uan 堤川, H. in Chuang-ch'ing 忠清 circ., Corea.

\section{T'I \\ 滞}

6368 T'i-sao-êrh 㱬埽尔, Sebzivar, a town in Persia, province Khorassan, W. of Nishapur. 
提 T'I-CH'I.

提

$6369 \mathrm{~T}^{\mathrm{T}} \mathrm{i}$-ch' $\mathrm{i}$ 提 溪, T.S. near T'ung-jên Fun, Kueichou； Ming, 'T'i-cli ${ }^{\prime} \mathrm{i} \mid$ 溪 ssü.

$6370 \mathrm{~T}^{\mathrm{T}} \mathrm{i}$-hsi | 突, (obs.) in Corea; Han, H. in Lềlang 樂泿 chiin; Tsin, H. in Tai-fang 帶方 chiin.

\section{屏}

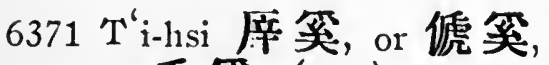
or 虎突, (obs.) N.E. of Mi-yïn H., Chihli; Han, H. in Y $\ddot{i}$-yang 漁陽 chiin.

\section{虎}

6372 T'i-hsi 㻺堂, see No. 6371.

\section{偱}

$6373 \mathrm{~T}^{\mathrm{i}} \mathrm{i}$-h hi 傆堂, see No. 6371.

\section{髦}

6374 T'i 囑, (obs.) T'ang, C. near Ta-yao H., Yünnan.

\section{TIAO}

\section{雕}

6375 Tiao-yin 雕陸, see Nos. 3080, 5942.

\section{沜}

6376 Tiao-o-pao 棩鶚堡, a post-town near Lung-miên H., Chihli.

熱 Tifh-chiang.

オ

6377 Tiao-yü 刀魚, (obs.) 50 li E. of Pa C., Clihli; Sung, a military post in Hsin-an 信安 military district.

\section{釣}

6378 Tiao-t' ai 釣臺, (obs.) 60 li N. of Ching-hai H., Chilli; Sung, a military post in $\mathrm{Cl}^{\text {'ing }}$ 清 $\mathrm{C}$.

\section{T'IAO}

條

6379 T'iao-chih 條支, (obs.) near Turfan; T'ang, Fu in Lung-yul 端右 circuit.

\section{調}

6380 T'iao-lisien 調絃, sš̈ near Shill-shorl H., Hupeh.

\section{TIEH}

\section{熱}

6381 Tieh-chiang 熱江 Tiel1kiang.

[210] $1^{\circ}$ H. in Chung C., Ssŭch' van; I Lat. $30^{\circ} 30^{\prime}$, Long. $107^{\circ} 24^{\prime}$; Han, Lin-chiang 臨江; $W$. Wei, Tieh-chiang 江; P. Chou, Wei-anl 魏安. $2^{\circ}$ (obs.) near Ch' êng-tı $F u$, Ssŭch' 'uan ; $S . T s^{\prime} i$, H. in Ning-shu察蜀 chiin.

$3^{\circ}$ See No. 2120. 
罟:

TIFH.

\section{鼠:}

6382 Tiel 嘼, (obs.) T'ang, C., $180 l i \mathrm{~S}$. of 'T'ao-chou T., Kansuln; Lat. $34^{\circ} 10^{\prime}$, Long. $103^{\circ} 30^{\prime}$.

6383 Tieh-ch' ${ }^{\mathrm{i}} \mid$ 溪, Y. near Mou C., Ssŭch' 'uan ; Ming, Tieh$\mathrm{clh}^{6} \mathrm{i} \mid$ 深 ss $\breve{u}$.

\section{迭}

6384 Tiell-li-1ni 迭里密, see No. 6328.

\section{牌}

6385 Tieh-chên 牌 珍, (obs.) in Ya-chou Fu, Ssuch' uan; $T^{6}$ ang, C. in Chien-nan 劍 南 circuit.

6386 Tieh-ts'ung | 琮, (obs.) near Ya-chou Fu, Ssŭch'uan; Sung, C.

Tieh, see No. 6387.

Tieh-kiang, see No. 6381. Tieh-ling, see No. 6390. 'Tieh-yıen, see No. 6394.

\section{T'IEH}

\section{鐵}

6387 ' $\Gamma^{\prime}$ ieh 鐵 Tieh.

$1^{\circ}$ C. in Corea ; Lat. $39^{\circ}$ $51^{\prime}$, Long. $124^{\circ} 50^{\prime}$.

$2^{\circ}$ Chên near Chên-ning C., Kueichou.

6388 'T'ieh-chên | 鎭, X. near Yung-pei T., Уüınan.
電 TIFN-PAi.

6389 'T'ien-k'êng | 坑, Y. near Yang-shan H., Kuangtung. 6390 T'iel-ling | 嶺 Tieln-ling, $H$. in Fêng-t' ien Fu, Shingking; Lat. $42^{\circ} 25^{\prime}$, Long. $123^{\circ} 45^{\prime}$; Liao, Yen-ching 延津 $\mathrm{H}$.

$6391 T^{\prime}$ 'ieh-niu $\mid$ 牛, chên near Pai-shui H., Shensi.

6392 T'ielı-pa | 把, (obs.) Sui, H., S.E. of Sui-ch'i H., Kuangtung.

6393 'T'iel1-wêng-ch' êng| 梁城, (obs.) formerly a town on the site of Chinkiang.

6394 T'ieh-yüan | 原 Tiehyuen, Fu in Chiang-yuian 江 原 circ., Corea; Lat. $38^{\circ} 15^{\prime}$, Long. $128^{\circ}$.

\section{帖}

6395 T'ieh-li-ma 帖里昨, see No. 6328.

6396 'T'ieh-yi | 夷, (obs.) W. of Wên H., Kansuh ; Suzi, H. in ' T'ung-ch' ang 闰昌 chiün; Tang, $\mathrm{H}$. in $\mathrm{Fu}$ 扶 $\mathrm{c}$.

\section{TIEN}

\section{電}

6397 Eien-pai 電白 Tien-peh, $\mathrm{H}$. in Kao-chou $\mathrm{Fu}$, Kuangtung; Lat. $21^{\circ} 30^{\prime}$, Long. $111^{\circ} 22^{\prime}$; Han, Kao-liang 高凉; Liang, Tien-pai |白. 
店

TIEN-CHANG.

\section{店}

6398 Tienl-chang 店張, chên in Hsing-p' ing H., Shensi.

6399 Tien-pu | 阜, a post-town near Lu-chon $\mathrm{Fu}$, Anhni. 6400 Tien-wei | 尾, chên near Fên-hsi H., Shansi.

\section{沾}

6401 Tien 沾, see No. 3656.

\section{淔}

6402 Tien 滇.

$1^{\circ}$ (obs.) C. Wars, K. in Yünnan.

$2^{\circ}$ See Nos. 544, 1404, 2761, 3398, 5991, 7813.

6403 Tien-ch'ih | 池.

$1^{\circ}$ (obs.) E. of Chin-ning C., Yünnan; Han, $\mathrm{H}$. in Yi-chou 敛州 chïn.

$2^{\circ}$ See Nos. 1085, 7813.

6404 Tien-nan | 南, see No. 7813.

'Tien, see No. 6431.

Tien-cliang, see No. 6406. Tien-chin, see No. 6407. Tien-chu, see No. 6413. Tien-11o, see No. 6416. Tien-mun, see No. 6418. Tien-ngan, see No. 6405. Tien-pau, see No. 6419. Tien-peh, see No. 6397. Tien-tai, see No. 6421. Tien-tsin, see No. 6411. Tien-tsiuen, see Nò. 6414 .
天 T'TEN-CHNG.

\section{T'IEN}

\section{天}

6405 T'ien-ain 天岀 Tien-11gan, chiinn in Chung-ch 'ing 忠 清 circ., Corea; Lat. $37^{\circ}$ $10^{\prime}$, Long. $127^{\circ} 10^{\prime}$.

6406 'T'ien-ch'ang | 長 Tien[399] chang, H. in Ssŭ C., Anluti ; Lat $32^{\circ} 41^{\prime}$, Long. $118^{\circ} 55^{\prime}$; Han, in Kuangling 廣陵 chiin; Liang, Ching 涇 C.; P. Chou, Shih-liang 石粱; T'ang, T'ien-ch' ang | 長.

6407 'T'ien1-chên | 鎮 Tien-chin, H. in Ta-t ${ }^{6}$ nng Fu, Shansi ; Lat. $40^{\circ} 27^{\prime}$, Long. $113^{\circ}$ $10^{\prime}$.

$6408 T^{\prime}$ ien-clia | 嘉, see No. 3729.

6409 T'ien1-ch'iao-ch'ang | 橋 獙, sš̆ in Chin H., Shingking.

6410 '八'ien-ch'ih | 池.

$1^{\circ}$ (obs.) in Ching-lê $\mathrm{H}$, Shansi ; $T^{\prime}$ ang, H. in Hsien 䶊 $\mathrm{C}$.

$2^{\circ}$ (obs.) near $\mathrm{Ch}^{\prime} \mathrm{u}$-hsiung Fu, Yünnan; $T^{6}$ ang, $\mathrm{H}$. in $Y$ in $尹 \mathrm{C}$.

$3^{\circ}$ (obs.) near $\mathrm{H}$ sü-chon Fu, Ssŭch' 11an; T'ang, H. in Hsün 馿 C.

6+11 'T'ien-cling | 津'l'ien-tsin. 
天 T'IEN-CHING.

[360] $1^{\circ} \mathrm{Fu}$ in the $\mathrm{T}^{6}$ ien-chingHo-chien circ., Chihli; forming also the district city of the same name; Lat. $39^{\circ} 07^{\prime}$, Long. $117^{\circ} 11^{\prime}$; one of the ports open to foreign trade; Ming, T'ienching | 津 military district.

$2^{\circ} \mathrm{H}$. forming the prefectural city of the same name, Clihili ; Lat. $39^{\circ}$ $07^{\prime}$, Long. $117^{\circ} 11^{\prime}$.

6412 T'ien-ching-Ho-chien | 津 河間, circ. in Chillli comprising $T^{\prime}$ 'ien-cling $F u$ and Ho-chien Fu.

$6413 \mathrm{~T}^{6}$ ien-chn | 柱 Tien-chu, [456] H. in Chên-yüan Fu, Kueichon; Lat. $26^{\circ} 45^{\prime}$, Long. $108^{\circ} 58^{\prime}$.

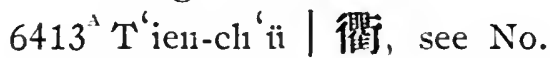
5822.

$6414 T^{\prime}$ 'ien-ch'ïan | 全 Tien-

[72] tsinen, C. in Ya-chou Fu, Sstuch 'nan; Lat. $30^{\circ}$, Long. $102^{\circ} 20^{\prime}$.

$6414^{\mathrm{A}} \mathrm{T}^{\mathrm{t}}$ ien-fu | 府, see No. 5822.

6415 T'ien-hêng | 衡, chên near Kung-ch' ang Fu, Kansulı. $6416 \mathrm{~T}^{6}$ ien-ho | 河 Tien-ho, $[481] \mathrm{H}$. in $\mathrm{Cl}_{1}$ 'ing-yüan $\mathrm{Fu}$, Kuangsi ; Lat. $24^{\circ} 48^{\prime}$, Long. $108^{\circ} 20^{\prime} ; T^{6}$ ang, T'ien-hol河, in Yi 宜 $\mathrm{C}$.
天 T'IEN-TÊ.

6417 T'ien-hsiung | 雄, see No. 6065.

6418 T'ien-mên | 門 Tien-mun. [525] $1^{\circ} \mathrm{H}$. in An-lu Fu, Hupeh; Lat. $30^{\circ} 40^{\prime}$, Long. $113^{\circ}() 5^{\prime}$.

$2^{\circ}$ Chên near Ning-yüan H., Kansul.

$3^{\circ}$ See No. 5721.

6419 T'ien-pao | 保 Tien - pau, $H$. forming the prefectural city of Chên-an, Kuangsi ; Lat. $23^{\circ} 20^{\prime}$, Long. $106^{\circ}$ $19^{\prime}$.

$6419^{A} T^{\prime}$ ien-pi | 毞, see No. 5822.

6420 T'ien-shui | 水.

$1^{\circ}$ Chên near $\mathrm{Cl}^{\text {'in }} \mathrm{C}$., Kansuli.

$2^{\circ}$ (obs.) formerly a town $50 l i \mathrm{E}$. of $\mathrm{P}^{\prime}$ êng-tsê H., Kiangsi.

$3^{\circ}$ See Nos. 324, 1105, 3521.

$6421 \mathrm{~T}^{\prime}$ ien-t'ai | 台 Tien - tai, [425] H. in T'ai-chou Fu, Chellkiang; Lat. $29^{\circ} 10^{\prime}$, Long. $121^{\circ} 03^{\prime}$; Han, Chang-an 章发; Tsin, Shih-fêng 始豐; Liang, T'ien-t'ai 台.

6422 'T'ien-t'ang | 堂, chên near Ling-t'ai H., Kansuh.

6423 T'ien-tê | 德, Liao, mil. district north of Oirad territory. Tenduc (Polo). 
天 T'IEN-TS'ANG.

6424 'T'ien-ts'ang | 藏, a town in Inner Mongolia; Lat. $41^{\circ} 54^{\prime}$, Long. $99^{\circ} 36^{\prime}$.

6425 T'ien-tu| 都, (obs.) Sung, a mịilitary post $170 l i$ N. W. of Ku-yüan C., Kansuh.

6426 T'ien-wei | 威, (obs.) see No. 1179.

$6427 \mathrm{~T}^{\prime}$ ien-yi | 夷, sonnetimes written wrongly for No. 1812.

$6428 \mathrm{~T}^{\prime}$ ien-yi-| 防, (obs.) in Kansulı ; W. Han, H. in Chiu-ch'üan 酒㫤 chiun.

\section{蒸}

6429 T'ien-mên菼門, chên near Ch'ing-shui H., Kansuh.

\section{甜}

6430 T'ien-shui 甜水, a posttown near Fêng-t ien $F u$, Shingking.

\section{田}

6431 T'ien 田 see No. 1613.

6432 T'ien-chia | 家, chên near $\mathrm{Ch}^{\prime} \mathrm{i}$ C., Hupeh.

6433 T'ien-chou | 州, see No. 1613.

6434 T'ien-êrh-pao | 兒保, a post-town near ' $T$ 'ien-mên H., Hupeli.

6435 T'ien-hsien | 縣, chên near Mêng-ching H., Honan.
定 TING-AN.

6436 T'ien-shih | 市, chên near Fu-p'ing H., Shensi.

6437 T'ien-t'ou | 頡, chên near Hsien-chü H., Chehkiang.

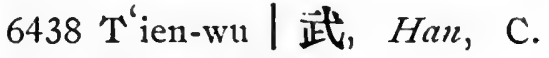
in Fuhkien.

6439 T'ien-yiuan | 園, $T \sin , \mathrm{H}$. in Kuaugtung.

Tih-hwa, see No. 6356.

Tiju, see No. 6175 .

Timurlik, see No. 6327.

\section{TING}

\section{定}

6440 Ting 定 Ting.

[448] $1^{\circ}$ C.I.C. in the Clingho circ., Chillit; Lat. $38^{\circ} 33^{\prime}$, Long. $115^{\circ}$ 09'; Han, Chung-shan 中 山 $\mathrm{K}$. and I, $\mathrm{nl}-\mathrm{nu}$ 盧奴 H.; N. Wei, Chnng-shan 中旧 chïn and I,u-nu 慮奴 H.; Sui, Po-ling 博陵 chiin and Hsie11-yü 鮮虞 H.; Trang, Ting $\mid \mathrm{C}$.

$2^{\circ}$ C. in P'ing-an 本发 circ., Corea; Lat. $39^{\circ}$ $40^{\prime}$, Long. $125^{\circ} 25^{\prime}$.

$3^{\circ}$ See Nos. 3473, 4251.

6441 Ting-an | 岀 Ting-11gan. $1^{\circ} \mathrm{H}$. in $\mathrm{Ch}$ 'iung-chon Fu, Kuangtung; Lat. $19^{\circ} 42^{\prime}$, Long. $110^{\circ}$ $18^{\prime} ; T^{\prime}$ ang and Sung, 
Ch'iung-shan 琽山; Ming, Ting-an 岁 $\mathrm{H}$.

$2^{\circ}$ Chên near 'Tung-yang H., Cliehkiang.

$3^{\circ}$ (obs.) in Annan; E.

Han and S. Sung, H. in Chiao-chil 交 趾: chiun.

$4^{\circ}$ (obs.) Liao, Kin and Yüan, H., 60 li N.E. of Yü C., Chihlil.

$5^{\circ}$ (obs.) Kin, in Fan H., Shantung.

$6^{\circ}$ (obs.) Yïan, H., S.E. of Ting-an H., Kuangtunig.

$7^{\circ}$ See No. 4672.

6442 Ting-cli' ang | 昌, see No. 5200 .

6443 'Ting-chiang | 江, see No. 1281.

6444 'Ting-ch'iang | 美.

$1^{\circ} \mathrm{A}$ post-town near Titao C., Kansull.

$2^{\circ}$ A post-town nearlungcli ang H., Kansul.

6445 'Ting-ch' wan| 川.

$1^{\circ}$ (obs.) Sui, H., E. of Yii-lin C., Knangsi.

$2^{\circ}$ See Nos. 3856, 7832.

6446 'Ting-fan | 番 'Ting-fan, C. in Kuei-yang Fu, Kneichour ; Lat. $26^{\circ} 06^{\prime}$, I.ong, $106^{\circ} 32^{\prime} ;$ Wing, 'Ting-fan 番 C.
6447 Ting-hai | 海 Ting-hai, C.L.'T. in Chehkiang, chief town of the Island Chonshan 舟IL Clinsan; Iat. $30^{\circ} 01^{\prime}$, Long. $122^{\circ} 14^{\prime}$; $T s^{\text {in }}$, Koll-cliang 句章; Five dyn., Wang-1nai 望海 and Ting-hai | 海 $\mathrm{H}$.

6448 Ting-lu | 湖, (obs.) 120 li W. of Yung-ning C., Shansi ; Sui, H. in Li-shih 離石 C.; Trang, H. in Shih 石 C.; Sung, H. in Chin-11ing 晉 察 nilitary district.

6449 'Ting-lisi | 西.

$1^{\circ}$ (obs.) Sung, a military post $45 \mathrm{li}$ N.W. of $\mathrm{Cl}_{1}^{6} \mathrm{in}$ C., Kansulı.

$2^{\circ}$ (obs.) Sung, fort $27 \mathrm{li}$ S. of An-ting H., Kansulı; Kin, Ting-hsi | 西 $\mathrm{H}$.

$3^{\circ}$ (obs.) Sung, fort near Ian-clon Fu, Kansul. $4^{\circ}$ See No. 101.

6450 Ting-lisi-ling | 唒領, T. S. near Chao C., Yünıan. 6451 'Ting-hssiang | 衰 Ting[143] siang, H. in Hsin C., Shansi ; Iat. $38^{\circ} 32^{\prime}$, I tong. $112^{\circ} 59^{\prime} ;$ Han, Yang-ch ii 陽曲; $W$. Han, Tinghsiang | 蓓; Tsin, Chincli'ang 좁 旨; Sui, Hsinjung 秀容. 
定

TING-HSING.

6452 'Ting-lnsing | 與 'Tingling; H. in Pao-ting $\mathrm{F}_{11}$, Chilili ; I at. $39^{\circ} 17^{\prime}$, I.ong. $115^{\circ} 56^{\prime}$; Sui, Fan-yang 范陽； Kin，Ting-hsing 與.

6453 Ting-jung | 我.

$1^{\circ}$ (obs.) N. Wei, chïn near Hsiao-yi H., Shansi.

$2^{\circ}$ (obs.) Sung, pao in Huan H., Kansul.

$3^{\circ}$ (obs.) Sung, pao on borders of $\mathrm{P}$ ing-liang Fu, Kansul.

$4^{\circ}$ (obs.) Kin, chên in

Fêng-lisiang Fu, Shansi.

6454 'Ting-lo | 羅, T.S. near Ssŭ-ên Fu, Kuangsi.

6455 Ting-11an | 南 Ting-11an, [478] T. in Kan-chon Fin, Kiangsi ; Iat. $24^{\circ} 45^{\prime}$, Loulg. $114^{\circ} 45^{\prime}$. Terr. jur.

6456 Ting-pien | 邊 Ting-pien.

$1^{\circ} \mathrm{H}$. in Yen-an $\mathrm{F} n$, Shensi ; Lat. $37^{\circ} 40^{\prime}$, Long. $107^{\circ} 30^{\prime}$.

$2^{\circ}$ (obs.) Yüan, H. near Mêng-hna 'I', Y'ünnan; Tang, Nan-chicn 湳 澗 H.; now Nan-chien 南簡 ssĭ.

$3^{\circ}$ (obs.) Sung, a town

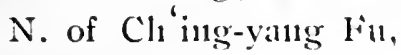
Kansul.
定 TING-Yن்.

$6456^{\circ}$ Ting-p'ing | 平, Fu in Hsien-ching 成鏡 circ., Corea.

6457 Ting-pu|步, chên near Chien-p ing H., Anhui.

6458 Ting-shan | 山, H. in Chung-ch'ing 忠声 circ., Corea.

-6459 Ting-t'ao | 陴 'Ting-tau, $[370] \mathrm{H}$. in Ts'ao-chon Fu, Shantung; Lat. $35^{\circ} 11^{\prime}$, Long. $115^{\circ} 45^{\prime}$; orig., 1st, San1-tsung 三 䰚, 2nd, T'ao-ch'in 陶邱; Chou, centre of feudal state 'T's'ao 曹; Ts'in, Ting-t'ao | 陶. 6460 Ting-tso | 葙, see No. 3737.

6461 'Ting-yang | 陽, see Nos. 319, 583.

6462 'Ting-yüian |邀 Ting-y"nen. [512] 1 ${ }^{\circ}$ '. in Han-chnng Fin, Shensi; Lat. $33^{\circ} 20^{\prime}$, I tong. $108^{\circ} 15^{\prime}$. 'Terr. jur.

$\left[50^{\mathrm{A}}\right] 2^{\circ}$ H. in Fêng-yang Fu, Anhui; Lat. $32^{\circ} 33^{\prime}$, I.ong. $117^{\circ} 33^{\prime} ; T^{\prime} i n$, Cl'ii-yang 曲陽; Han, 'Tung-ch'êng 東 城;

Liang, 'Ting-yüan/䔔;

Sui, I,in-11ao 臨湆.

[30] $3^{\circ}$ H. in Clunng-ch ing In, Ssŭch'nan; I,at. $30^{\circ} 25^{\prime}$, I.0119. $106^{\circ}$ $20^{\prime}$; orig., Ho 合 C.;

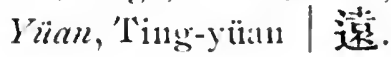


定

[277] $4^{\circ} \mathrm{H}$. in $\mathrm{Cl}^{6} \mathrm{u}-\mathrm{h}$ siung $\mathrm{Fu}$, Yünnan; Lat. 25。 22', Long. $101^{\circ} 44^{\prime}$; Han, Yüeh-sui 越䳡; T'ang, Mon 留 C.; Yïan, Ting-yüan | 䢬.

$5^{\circ}$ Post-town near Lancliou fin, Kansuh.

$6^{\circ}$ (obs.) Yüan, H., 200 li S. of Kuei-yang Fu, Kueichou.

$7^{\circ}$ (obs.) Han, a town near Hsi-lisiang $H$., Shensi.

丁

6463 Ting-pi 丁壁, chên near Kao-p ing H., Shansi.

6464 Ting-yen | 医, chên near Ju-kao H., Kiangsu.

\section{媔}

6465 Ting-ying 頂營, ssŭ near Chên-11ing C., Kueichou.

\section{鼎}

6466 Ting 鼎.

$1^{\circ}$ (obs.) Tang, C., W. of Ching-yang H., Shensi. $2^{\circ}$ See Nos. 321, 1114, 7033.

6467 Ting-ln | 湖, a post-town near Wên-hrsiang $H$., Honan.

Ting-chan, see No. 6470. Ting-hing, see No. 6452. Ting-11gan, see No. 6441. Ting-siang, see No. 6451.
丢

TIU-KU.

Ting-tau, see No. 6459.

Ting-yıen, see No. 6462.

\section{T'NG}

\section{汀}

6468 T'ing 汀, see No. 6470. 6469 T'ing-Chang-IAnng | 漳 龍, circ. in Fuhkien conprising 'T'ing-chon Fu, Chang-chou $F u$ and Lningyen $\mathrm{C}$.

6470 T'ing-chou | 州 Tingchan, Fu in the T'ingChang-Linng circ., Fullkien ; forming also the dis-

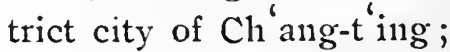
Lat. $25^{\circ} 45^{\prime}$, Long. $116^{\circ}$ 30'; Chou, Ch'i-min t 閩; Han, in Knei-chi 會 嵇 $\operatorname{chiin} n ; T \sin , \mathrm{H} \sin$-lo 新 羅; T'ang, T'ing | C. and Lin-t'ing 臨 |; Ming, T'ing-chou| 州 Fu.

\section{党}

6471 T'ing 亨, see Nos. 4257, 5673.

6472 T'ing-clı'ien | 前, ssü near Huang-mei H., Hupeh.

Tinju, see No. 6768.

\section{TIU}

\section{丢}

6473 'Tiu-ku丢 骨, T.S. near Sung-p'an T., Ssŭch' nan. 
單了

To.

TO

㠆

6474 To 鄲, see No. 4124.

\section{条}

6475 To-lun-no-êrh | 倫諾尔 Dolonnor, $T$. in Hsüanluna $F u$, and also included in the $\mathrm{K}^{\prime}$ ou-pei circ., Chilili ; Lat. $42^{\circ} 15^{\prime}$, I ong. $116^{\circ} 10^{\prime}$.

6476 To-mei | 梅, (obs.) T'ang, H., W. of Yi-shan H., Kuangsi.

6477 To-p'êng蓬, (obs.) T'ang, H. nearSsŭ-ên H., Kuangsi.

6478 To-tsung | 總 Tudzung, a town in Thibet; I.at. $28^{\circ}$ $20^{\prime}$, Long. $90^{\circ} 40^{\prime}$.

6479 To-tu11. | 冬 Dodon, a town in Thibet; Lat. $30^{\circ}$ $50^{\prime}$, Long. $90^{\circ} 50^{\prime}$.

6480 To-yün | 雲.

$1^{\circ}$ Chên in Lo-t'ien H., Hupeh.

$2^{\circ}$ (obs.) Tang, H. near Hsin-cli êng T. H., Kuangsi.

\section{染}

6481 To-tien 慜 殿, see No. 5822.

6482 To-yen | 顏 (obs.) Ming, a military district in Mongolia.

\section{垛}

6483 To-chuang 水获, ssŭ near Mêng-yin H., Shantung.
莠

T'O-KU.

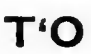

拕

6484 'T'o-lê 拕樂, T.S. near Sung-p'an 'I', Ssŭch'nan.

\section{鮀}

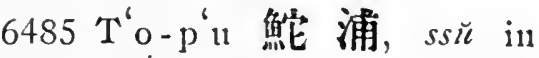
Ch' êng-hai H., Knangtung, in which Swatow is situated.

\section{歌}

6486 T'o-1u-t'ang 䭾虜热, sš̈ near 'Tso C., Kuangsi.

\section{托}

6487 T $^{6}$ o-ho-ch ${ }^{6} \mathrm{i}$ 托和唯 Tokotsi, a town in the New Dominion; Lat. $42^{\circ} 52^{\prime}$, Long. $92^{\circ} 50^{\prime}$.

6488 'T'o-ho-nai | 和 奈 Tokhonai, a town in Yarkand; Iat. $41^{\circ} 15^{\prime}$, I ong. $83^{\circ} 10^{\prime}$. 6489 T'o-k'ê-t'o|傥托 Tuktu, [475]'T. in So-p "ing Fu, Shansi ; Lat. $40^{\circ} 40^{\prime}$, Long. $112^{\circ}$ $05^{\prime}$.

6490 T'o-ku-sun | 古係, see No. 6136.

6491 T'o-li | 里 Toli, a town ii1 Ili; Lat. $43^{\circ} 30^{\prime}$, Long. $81^{\circ} 45^{\prime}$.

\section{索}

6492 T'o-ku 索臬, (obs.) N.W. of Ch ao H., Auhui; $W$. Han, H. in Chin-chiang 九江 chïn. 
绝 T'O-I.UNG.

陁

6493 'T'o-lung 阤隆, (obs.) T'ang, H. near Ssŭ-nan Fin, Kueichou.

Tochigi, see No. 2605. Tokhonai, see No. 6488. 'Iokio, see No. 6707. Toksı11, see No. 6136. Toli, see No. 6491.

\section{TOU}

\section{斗}

6494 Tou-lin-mêne斗六阴, a post-town in Chia-yi H., Formosa.

6495 Ton-11êen|門, chên near Hsi-an Fu, Shensi.

\section{竇}

6496 'Toul-chia 䆟家, ssŭ near Jung H., Knangsi.

6497 'Tont-ts' 1n | 村, chên near Wu1-t'ai H., Shansi.

陡

6498 'I'on-chiielı 陡覺, chênnear Shao-hsing $F u$, Chehkiang. 6499 'Ton-mên | F, Y. Yang-chiang 'T., Kuangtung.

\section{T'OU}

頭

6500 '个'ou1-111a 頭馬, Y. near Ning-wu Fu, Shansi.

'Tou11, see No. 6685 .
蔡

TS AI-KUAN.

\section{TSA}

咱

6501 Tsa 㕷, T.S. near Tachien-lı T., Ssŭch' 'uan.

\section{雜}

6502 'Tsa-ku 権谷 Tsall-kul, see No. 3691.

\section{擦}

6503 'Tsa-ch'a-êrl1-kang 擦作尔 岡 Dzacharkang, a town in 'Thibet; Lat. $29^{\circ}$, Long. $97^{\circ} 50^{\prime}$.

'Tsah-kulh, see Nos. 3691, 6502.

\section{TSAI}

載

6504 'Tsai-ning 載察, chün in Huang-lai 黄海 circ., Corea.

\section{TS'Al}

\section{蔡}

6505 'Ts'ai 蔡.

$1^{\circ}$ (obs.) S.A., K. in

\section{Honan.}

$2^{\circ}$ See Nos. 2786, 3010.

6506 Ts'ai-chia | 家.

$1^{\circ}$ Chền near Mei H., Shensi.

$2^{\circ}$ Y. near Hsiang-ch'êng H., Honan. [2596.]

6507 'T's'il-kuan | 官, Y. near Pin-clı' naı C., Yünnıаı. 


\section{蔡 TS'AI-T'ANG.}

6508 'T's'ai-t'ang | 售, chên in Han-yang H.. Hupel.

6509 Ts'ai-tien | 贴, sš̆ near Han-yang Fu, Hupel. 6510 Ts'ai-yang | 陽, see No. 6531.

\section{杂}

6511 Ts'ai-shih 采石, ss̆̌ near T'ai-p'ing H., Anhui.

6512 Ts'ai-yü | 肓, Y. near Liang-lisiang $\mathrm{H}$., Chihli.

\section{TSAN}

\section{贊}

6513 Tsan-chang 贊章 Zendjan, a town in Persia, province Irakajemi.

6514 Tsan-huang | 皇 Tsan[145] hwang, H. in Chêng-ting Fu, Chillil; Lat. $37^{\circ} 43^{\prime}$, Long. $114^{\circ} 32^{\prime}$; Han, Kaoyi 高邑; Sui, Tsan-liuany | 皇.

'Tsan-hwang, see No. 6514.

\section{TS'AN}

\section{然}

6515 Ts'an-chün 參軍, (obs.) Kin, chên in Chiao-ho H., Cliihli.

6516 Ts'an-fêng | 封, (obs.) $W$. Han, Marq. near Clingchou Fu, Shantung.

\section{䖯}

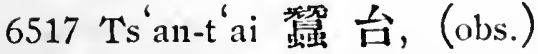
$W$. Han, H. in Corea.
湆 TS'ANG-HSIA.

\section{TSANG}

\section{非}

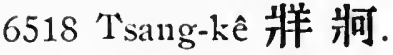

$1^{\circ}$ (obs.) Han and $T^{\prime}$ ang, chiun comprising parts of Ssŭch uan, Hunan, Kueichon and Kuangsi. $2^{\circ}$ See Nos. 1157, 2242, $3485,3838,4283$.

Tsang, see No. 6521. Tsang-ki, see No. 6524. 'Tsang-wu, see No. 6526.

\section{TS'ANG}

\section{倉}

6519 Ts'ang-tzü-pu 倉子垆, ss̆̌ near Huang-p'o H., Hupeh.

\section{峆}

6520 Ts'ang-yao 峆㯖, ssŭ near Ku-t'ien H., Fullkien.

\section{滄}

6521 Ts'ang 嵢 Tsang, C. in [322] ' $T$ 'ien-clning Fu, Chihli ; I at. $38^{\circ} 22^{\prime}$, Long. $116^{\circ}$ $47^{\prime}$; Han, Po-hai 渤 海; $W e i$, Ts'ang | C.; Sui, Ti 㴍 C.; T $T^{6}$ ang, Clı' ang-lı 長藘 H.; Kin, $\mathrm{Cl}^{\text {'ang-lu }}$ 長蘆 chên.

6522 Ts'ang-ch'i | 海, chền near Wu-hu H., Anluni.

6523 Ts'ang-lisia | 陝, ss̆̌t in Nan-p ing H., Fuhkien. 
蒼 TS'ANG-Ch' I.

\section{蒼}

6524 Ts'ang-ch'i 蒼溪 Tsang-ki [30] H. in Pao-ning Fu, Ssŭch 'uan; I Lat. $31^{\circ} 40^{\prime}$, Long. $105^{\circ} 56^{\prime} ; T^{\prime} \mathrm{in}$, in Tangch'ii 宕渠 cliün; Han, Han-chung 漢中; $T s^{\prime}$ in, Ts'ang-ch'i 溪.

6525 Ts'ang-sung 松 (obs.) Han and 7 sin, H., W. of Ku-lang H., Kansuh.

6526 Ts'ang-wu | 梧'Tsang-wu. $1^{\circ} \mathrm{H}$. forming the prefectural city of Wu-chou, Knangsi ; Lat. $23^{\circ} 29^{\prime}$, Lorig. $110^{\circ} 51^{\prime} ; W$. Han, Kuang-hsin 栱 信; E. Han, Tsin, S. Sung and $S$. $T s^{\prime} i$, Ts'ang-wu | 梧 chïn; Sui, Ts'ang-wu | 梧. $2^{\circ}$ See Nos. 335, 1701, 2812, 3515, 5169 .

Tsŭng-ching, see No, 6552 .

\section{TSAO}

\section{早}

6527 Tsao-shê 早社, chên near Ning C., Kansuh.

\section{是}

6528 'T'sao-k'oul 是口, ss̆̌ in Wan-an H., Kiangsi.

6529 Tsao-lin | 林, a post-town near. Shih-mên H.: Chel1kiang.
曹 Ts'Ao.

\section{事}

6530 Tsao-ch'iang 事强, Tsaushan H. in Chi C.L.C., Clihli; Lat. $37^{\circ} 40^{\prime}$, Long. $115^{\circ} 43^{\prime}$; Han, Tsaochiang | 彊; Tsin, Kuangch'uan 廣川.

6531 Tsao-yang | 陽 Tsaut-yang, $\mathrm{H}$. in Hsiang-yang $\mathrm{Fu}$, Hupel1 ; Lat. $32^{\circ} 10^{\prime}$, Long. $112^{\circ} 41^{\prime}$; Han, Ts'aiyang 蔡陽; $W$. Wei, Ch' ang 昌 C.; Sui, Clunanghing 春陵; S. Sung, Tsaoyang | 陽 military district.

\section{TS'AO}

\section{皆}

6532 'Ts'ao-ch'i 草溪, H. in Ch'ing-chang 慶分 circ., Corea.

6533 'Ts'ao-ting | 津 Kusatsa, Mineral Springs, Japan.

6534 'Ts'ao-kang | [開, ssü in Fu-ning H., Kiangsu.

6535 Ts'ao-p'ing| 平, chên near

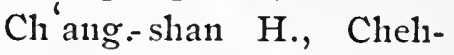
kiang.

6536 T's'ao-shil | 市, ssü in Hêng-shan H., Hunan.

6537 Ts'ao-t'ang | 塘, ssŭ near Wêng-an H., Kueichou.

\section{曹}

6538 Ts'ao 曹 Tsau。 
曹

Ts'Ao.

$1^{\circ} \mathrm{H}$. in Ts'ao-chou $\mathrm{Fu}$, Shantung; Lat. $34^{\circ}$ 56', Long. $115^{\circ} 38^{\prime}$; orig., K. Ts'ao | ; Sui, Chi-yin 沜陰; T'ang, Mêng-tsê 蒙澤.

$2^{\circ}$ (obs.) formerly K. in Shantung.

$3^{\circ}$ See Nos. $3574,6542$.

65.39 Ts'ao-chang | 張, chên near Wan-ch' ïan H., Shansi.

6540 'T's'ao-chia-chai | 家砦, Y. near Mi-yün H., Clihlil. 6541 Ts'ao-chia-ho | 家河, chên near Kuang-clii H., Hupel. 6542 Ts'ao-chou | 州 Tsan[15] clian, Fu in the Yen-Yi'Ts'ao-Chi circ., Shantung; forming also the district city of Kê-tsê; Lat. $35^{\circ}$ $20^{\prime}$, Long. $115^{\circ} 35^{\prime \circ}$; orig. , K. Ts'ao ; Tsin, Chiyang 濟陽; Sung, Hsingjên 興 仁; P. Chou, 'Ts'ao | C.; Sui, Chi-ying 墚陰. 6543 'Ts'ao-ti | 滴, ssŭ near Lip'ing Fu, Kueichou.

6544 Ts'ao-wu|務, chên near Lung-tê H., Kansuh.

Tsaprung, see No. 6547. Tsau, see Nos. 374, 6538, 6576.

Tsall-chan, see No. 6542 . Tsatu-ping, see No. 6578. Tsall-shan, see 6130 . Tsalt-yang, see 6131.
突 TS ${ }^{\prime} \hat{\mathrm{F}} \mathrm{N}-\mathrm{CH}{ }^{\prime} \mathrm{I}$.

\section{TSE}

\section{羉}

6545 Tsê 澤, see No. 6546. 6546 Tsê-chon | 州 Tseh-chan, [369] Fu in the Chi-11ing circ., Shansi; forming also the district city of Fêng-t'ai ; Lat. $35^{\circ} 30^{\prime}$, Long. $112^{\circ}$ $50^{\prime}$; orig., 1st in K. Chin 西宣, 2nd, in K. Han 韓 and $\mathrm{K}$. Chao 趋; $T s^{\prime}$ in, in Shang-tang 上黨 chün and Kao-tu高都 chïn; Han, Ho-tung 河 東; 2 nd Wei, Chien 建 C.; P. Chou, Kao-p'ing 高平; Sui, Tsê C. and Ch' ang-p'ing 長 本; T'ang, Kai 蓋 $\mathrm{C}$.; Ming, Tsê | C.

6547 Tsê-pu-lung | 布 隴 Tsaprung, a town in Thibet; Lat. $31^{\circ} 15^{\prime}$, Long. $79^{\circ}$ $20^{\prime}$.

\section{宅}

6548 Tsê-yang 宅 陽, (obs.) formerly a town $\mathrm{E}$. of $\mathrm{K}$ 'aifêng $F u$, Honan.

Tseh-chau, see No. 6546.

\section{TS'ÊN}

\section{宩}

6549 Ts'ên-ch'i 岑溪 Tsin-ki. 
岑 TS' $\mathrm{F}^{\circ} \mathrm{N}-\mathrm{CH}{ }^{6} \mathrm{I}$.

[457] $1^{\circ}$ H. in IV11-chon Fin, Knangsi ; Lat. $23^{\circ}$, I ong. $110^{\circ} 27^{\prime}$; Han, in T's'ang-wu荅梧 chün; Sung, Ts' ên-clı' $\mathrm{i} \mid$ 溪. $2^{\circ}$ (obs.) T'ang, H., 20 li $\mathrm{E}$. of the above.

6550 Ts'ên-wang | 王, ssŭ near Tz '̆

\section{TSÊNG}

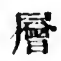

6551 Tsêng-shan 穈山, ssŭ in Yung-fêng H., Kiangsi.

\section{增}

6552 'Tseng-ch 'êng增城 Tsăng[472] ching, H. in Knang-chon Fu, Knangtung, Lat. $23^{\circ}$ $05^{\prime}$, Long. $113^{\circ} 58^{\prime}$; Han, Tsêng-cli'êng | the

\section{矰}

6553 Tsêng-shan 䙼 山.

$1^{\circ}$ H. in $\mathrm{P}^{\prime}$ ing-an 平努 circ., Corea.

$2^{\circ}$ See No. 1949.

\section{邵}

6554 Tsêng 鄫, (obs.) S.A., K. in Shantung; see No. 7518.

\section{TS'ÊNG}

筲

6555 T' êng 算, (obs.) T'ang, C. near Têng-ch' nan C., Yün111an.

6556 'Ts 'êng-k'ou | 口, (obs.) Tang, a town $70 l i \mathrm{~S}$. of
袋

Tsing.

Ch'êng-nnai H., Knangtung.

6557 'Ts' êng-ts'un | 村, chên near Fu-p'ing H., Shensi.

Tsetsell, see No. 397.

Tsi, see No. 600.

Tsi-hia, see No. 659 .

Tsi-1ıo, see No. 679.

Tsi-nan, see No. 601.

'Tsi-ning, see No. 602.

Tsi-tung, see No. 685.

'Tsi-yang, see No. 606.

Tsi-yuen, see No. 608.

Tsiang-lol, see No. 772.

Tsien-kiang, see Nos. 912, 932, 957.

'Tsien-ngan, see No. 911.

Tsien-slıan, see No. 933.

Tsien-shan-chai, see No. 938.

Tsient-tang, see No. 936. Tsilı-ki, see No. 598.

Tsil1-1nol, see No. 611. 'Tsil-shan, see No. 550 . Tsin, see Nos. 1073, 1098, 1105.

'Tsin-hien, see No. 1072.

Tsin-huang Tao, see No. 1108.

Tsin-ki, see No. 6549.

Tsin-kiang, see No. 1077. T'sin-11gan, see No. 1106. 'Tsin-ning, see No. 1085. 'Tsin-shwui, see No. 1100. Tsin-yuen, see No. 1103. Tsin-y'un, see No. 1090. 'Tsing, see Nos. 1157, 1197. 
T'sing-chau, see No. 1204.

'Tsing-ching, see No. 1199.

'sing-fung, see Nos. 1242 , 1243.

l'sing-hai, see Nos. 1169. 1206.

'lsing-ling, see No. 1179. 'Tsing-ho, see No. 1245. 'lsing-ki, see No. 1235.

'r'sing-kiang, see Nos. 1159 , 1236.

'Tsing-kien, see No. 1237. Tsing-liu, see No. 1249.

'Tsing-lol, see No. 1172.

'T'sing-ngan, see No. 1158.

'Tsing-ning, see No. 1173. 'Tsing-pien, see No. 1161. 'l'sing-ping, see No. 1250. 'T'sing-pu, see No. 1217.

'Ising-shin, see No. 1220. 'Tsing-shwui, see No. 1252. 'rsing-shwui-ho, see No. 1253.

'I'sing-tau, see No. 1256. 'l'sing-teh, see No. 1176. Tsing-tien, see No. 1224. 'Tsing-tsinen, see No. 1240. 'Tsing-yang, see No. 1227. 'Psing-yen, see No. 1181. 'I'sing-ynen, see Nos. 1162 , $1261,1265$.

'Tsitsihar, see Nos. 677, 2007.

Tsitsirkana, see No. 676. 'Tsiuen, see No. 1483. Tsiuen-chan, see No. 1491. 'Tsiuen-tsiau, see No. 1484.

\section{TSO}

tr.

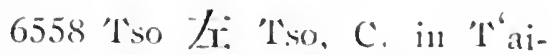
p’ing Fin, Kuangsi; I at. $22^{\circ}+z^{\prime}$, I.Ong. $107^{\circ} 11^{\prime}$; sung, 'liso |c.

6559 'To-an | 岁, ssü in I unnch"ïan H., Kiangsi.

6.500 'l'so-ch'i | 旗, ssü near Kuang-hua H., Hupelı.

6561 'rso-chiang | 江, circ., in Kuangsi comprising Ss:ucliêng Fin, Nan111ing Fun anci 'T"ai-p'ing Fin.

6562 'Tso-fêng | 封, (obs.) Sui and Tang, H. $220 \mathrm{li} \mathrm{W}$. of 'Tieh-ch'i Y., Mon C., Ssŭch' 'ran.

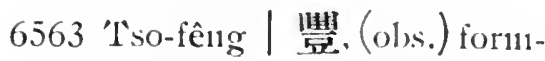
erly al town near Hai-fêng H., Kinangtning.

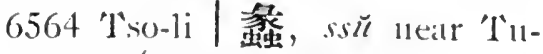
ch'ang H., Kiangsi.

6565 'Tso-lu! | 路, chên ne:ırSuining H., Kiulugsu.

6566 'T'so-so | 所, 'I'.S. near Yen-yüian H., Ssĭch' nan. 6567 'Tso-yi | 邑, see No. 7036”. 6568 T'so-yün 雲 T'so-yn11, H. in So-p ing Fu, Shansi; Lat. $39^{\circ} 55^{\prime}$, I ong. $112^{\circ}$ $23^{\prime}$.

\section{佐}

6569 Tso-fu 恢斩笅, a town in the Lin1-ch'in Islands. 6570 'Tso-ho | 頨, ken in Japan. 
贊 Tso.

\section{贊}

6571 Tso 赞.

$1^{\circ}$ (obs.) Han, H. near P'ei H., Kiangsu.

$2^{\circ}$ See Nos. $3317,3432$.

\section{作}

6572 Tso-lu 作路, T. S. near Sung-p an T., Ssŭch' 'uan.

\section{花}

6573 ' 'so - clh' in 萑秦, (obs.) Han, H. near Ning-yüan Fu, Ssŭch' uan.

Tso-yur, see No. 6568.

\section{TS'O}

\section{厝}

6574 Ts'o 厝, W. Han, H. South of Ch ing-p ing $\mathrm{H}$., Shantung.

\section{TSOU}

\section{走}

6575 Tsoul-ma 走馬, Y. near Lin-wu H., Hunan.

\section{邭}

6576 Tsou 雏 Tsau.

[343] $1^{\circ} \mathrm{H}$. in Yen-chou $\mathrm{Fu}$, Shantung; Lat. $35^{\circ} 30^{\prime}$, Long. $117^{\circ} 10^{\prime}$; Chou, K. Chu 制; Han, Tsou 㴔 H.; T'ang, Tsoul $\mathrm{H}$.

$2^{\circ}$ See No, 6578.
胙 TsU-CH ${ }^{6} \hat{\mathrm{ENG}}$.

6577 Tsou-lı | 盧, (obs.) W. Han, H. near Têng-chou Fu, Shantung.

6578 Tsout-p'ing $\mid$ 雨 Tsan-ping, [183] H. in Chi-nan Fu, Shantung; Lat. $35^{\circ} 56^{\prime}$, Long. $117^{\circ} 50^{\prime}$; orig., feudal state Tsou |; Han, Tsoup'ing | 本; N.Wei, Tung P'ing-yüan 東斥原 chün; T'ang, Tson | H.; see No. 685.

\section{龅}

6579 'Tson 䑐, see No. 6576.

6580 Tsoul-yü|虞, (obs.) $W$. Han, H. in Shansi.

\section{奏}

6581 'Tsou-lung 弮龍, (obs.) near $\mathrm{Ch}^{6}$ "1-hsiung $\mathrm{Fu}$, Yünnan; T'ang, C. in Chiennan 劍南 circ.

\section{TSU}

胙

6582 Tsu 胙.

$1^{\circ}$ (obs.) formerly K. in Honan.

$2^{\circ}$ See No. 6183.

6583 'Tsu-ch' êng | 城, (obs.) Sui, H. near Wei-hui Fu, Honan; Lat. $35^{\circ} 20^{\prime}$, Long. $114^{\circ} 22^{\prime}$; orig., K. Tsu |; $S$. A., Nanl-yen 南燕; Tsin, Tung-yen 東燕。 
祖

TSU-AN.

\section{祖}

6584 Tsu-an 瓶l 蒦, chên in Chou-chih H., Shensi.

6585 Tsus-chü | 居, (obs.) N. $W e i, \mathrm{H}$. near $\mathrm{P}^{\prime}$ ing-liang Fu, Kansulı.

6586 Tsu-yi-tu $\mid 乙$ 都, (obs.) formerly town on the site of Shun-tê Fu, Chilnli.

Tsu-hiung, see No. 1404.

\section{TS'UAN}

攢

6587 Ts' uaul-tu-kou 攢都㲘, T. S. near Wei-yüan H., Kansull.

\section{TSUI}

\section{槜}

6588 Tsui-li 槜李, see No. 699.

\section{TS'UI}

\section{崔}

$6589 \mathrm{Ts}^{\prime}$ 'ui 崔, chên near T'aoyüan $\mathrm{H}$., Kiangsu.

6590 Ts'ui-chia | 家, a posttown near T'ai-an Fu, Shantung.

\section{催}

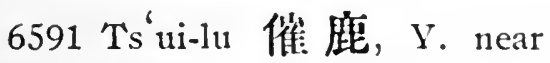
Tan C., Kuangtung.

\section{TSUN}

邅

6592 Tsun-hua 遵化 Tsun-hwa.
宗 Tsurg.

[380] $1^{\circ}$ C. L.C. in the Tingchou-Yün-ho circ., Chilhli; Lat. $40^{\circ} 11^{\prime}$, Long. $117^{\circ} 53^{\prime}$; orig., K. Wu-chung 無終; Han, Yu-pei-p'ing 右

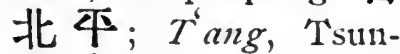
lina $\mid$ 化.

$2^{\circ}$ (obs.) Sui, H., S. of Ling-shan H., Kuangtung.

6593 Tsun1-yi | 義 Tsun-i.

[178] $1^{\circ} \mathrm{Fu}$ in the Kuei-hsi circ., Kueichou ; forming also the district city of the same name; Lat. $27^{\circ}$ $38^{\prime}$, I,ong. $106^{\circ} 58^{\prime}$; $T^{6}$ ang, Lang 郎 C. and Po 潘 C.

$2^{\circ} \mathrm{H}$. forming the prefectural city of the same name, Kueichou; Lat. $27^{\circ} 38^{\prime}$, Long. $106^{\circ} 58^{\prime}$; orig., K. Iang 郎.

\section{巂}

6594 Tsun 巂, see No. 1598.

Tsun-liwa, see No. 6592.

Tsun-i, see No. 6593.

\section{TSUNG}

\section{宗}

6595 Tsung 宗.

$1^{\circ}$ (obs.) Liao, C. in Corea.

$2^{\circ}$ See Nos. 431, 2073 , 3419. 
宗 TSUNG-CH'ÂNG.

6596 'Tsung-ch'êng | 城, see No. 3419.

6597 T'sung-chia | 家, Y. near Pao-ch' êng H., Shensi.

6598 Tsung-chü | 居, (obs.) Tang, H. near $\mathrm{Cl}^{\prime}{ }^{\prime} 1$ lısinng Fı, Yünnaı.

'Tsung-liwa, see No. $6601^{\mathrm{A}}$. Tsung-i, see No. 1599.

Tsung-jin, see No. 1591.

Tsung-king, see No. 1589.

Tsung-ming, see No. 1593.

Tsung-ngan, see No. 1586.

Tsung-ning, see No. 1594.

Tsung-shen, see No. 1595. Tsung-sin, see No. 1590. Tsung-yang, see No. 1598.

\section{TS'UNG}

\section{崖}

6599 Ts'ung 丵, (obs.) T'ang, C. in Ssŭch 'uan.

6600 Ts'ung-lo-yii | 羅峪, chên near Yung-ning C., Shansi.

\section{從}

6601 Ts'ung 從.

$1^{\circ}$ (obs.) T'ang and Sung,

C. near Hsü-chon Fu, Ssüch 'uan.

$2^{\circ}$ (obs.) Sung, C., IV. of Yung-ts ung H., Kueichon.

6601'Ts'ung-hua | 化 'Tsung$[527]$ hwa, H. in Kuang-chou Fu, Kuangtung; Lat. $23^{\circ}$
都 TU-AN.

$33^{\prime}$, Long. $113^{\circ} 27^{\prime} ; s^{\prime}$ in, P'all-yï 番禺; Tsin, 'Ts'ung-hua | 化.

\section{榄}

6602 T's'ung-yaung 杪陽.

$1^{\circ}$ Chên near An-ch 'ing F11, Anliui.

$2^{\circ}$ See No. 6808 .

\section{婘}

6603 'T's'11ng 琮, (obs.) T'ang, C. near $\mathrm{Cl}_{1}$ ing-yang $\mathrm{F} u$, Kansulı.

\section{賽}

6604 'Ts'ing 藚, (obs.) 12 li N. of Kuang-an C., Ssŭch'tuan; Sui, H. in Tangch'ii 宕渠 chiin.

'Tsushoul, see No. 1336. 'Tsz', see Nos. 6840, 6868. 'Tsz'-chnen, see No. 6834. 'Tsz'-ki, see No. 6863. 'Tsz'-1i, see No. 6865. 'Tsz'-shan, see No. 6867. Tsz'-tung, see No. 6853. 'Tsz'-yang, see Nos. 6837, $6843,6849$.

\section{TU}

\section{都}

6605 Tu 都, (obs.) T'ang, C. in Ssŭch' uan.

6606 Tu-an | 岁, see No. 3359. 
都

TU-CH'ANG.

6607 Tit-ch'ang | 昌 Tu-chang. [366] $1^{\circ} \mathrm{H}$. in Nan-k'ang Fin, Kiangsi ; Lat. $29^{\circ} 12^{\prime}$, Long. $116^{\circ} 25^{\prime}$; $\mathrm{Han}$, P'êng-tsê 彭澤; T⿱ang, 'lu-ch'ang| 昌. $2^{\circ}$ See No. 306.

6608 'Tu-ch' êng | 城, sš̆ near Hsi-ning H., Knangtung. 6609 Tu-chiang | 江. Tn-kiang, [473]T. in Tu-yün Fu, Kueichour; Lat. $25^{\circ} 45^{\prime}$, I,ong. $108^{\circ} 10^{\prime}$.

6610 Tu-chi | 嫘, (obs.) T'ang, circ.

6611 Tu-chieh | 結 Tu-kié, T. C. in T'ai-p'ing Fu, Knangsi ; Lat. $23^{\circ} 10^{\prime}$, Long. $107^{\circ} 08^{\prime}$; Yüan, Tu-chieh | 城 C.

6612 Tu-êrh-pên | 耳本 Derbend, a town in Russian Cancasus, on W. shore of the Caspian Sea.

6613 'in-êrh-kên沵根 Durgen, a town in Dsassaktu; Lat. $47^{\circ} 10^{\prime}$, I ong. $91^{\circ} 40^{\prime}$.

6614 Tu-hsia|下, see No. 5822. 6615 'Tu-ju | 濡, (obs.) T'ang, H: 200 li S. of P'êng-shui H., Ssŭch' inau.

6616 'Tu-k'ang | 康 Tu-kang, [577]T. C. in Chên-an F 11 , Kuangsi ; Iat. $23^{\circ} 04^{\prime}$, Long. $106^{\circ} 45^{\prime}$; Han, in Chiao-chih 交趾 chïn; Sung, Tu-k'ang | 㞗 C.
都 TU-ris.

6617 Tu-liang | 梁, (obs.) Han, a town E. of Wn-kang, C., Hunan.

6618 Tu-lı | 慮, (obs.) Tsin, H. in Kansun.

6619 Tu-lung / 龍, (obs.) T'ang, H. in Annam.

6620 Tu1-mên! !师, see No. 5822.

6621 Tu-ning | 䇛, ssŭ in Shuntê Fu, Kuangtung.

6622 Tu-pang | 邦, (obs.) T'ang, H., E. of Hsinch'êng T.H., Kuangsi.

6623 Tu-p ang | 龐, (obs.) $W$. Han and S. Sung, H. in Annam.

6624 Tutp'ing | 平, T. S. in Ssŭ-chou Fu, Kueichou.

6625 Tu-p'ing-o-yi-ch'i | 坪峩 暴溪, (obs.) Ming, ssü near Ssŭ-chou Fu, Kueichou.

6626 Tu-sı | 素, T.S. near Yüp'ing H., Kueichou. 6627 Tu-yang | 陽.

$1^{\circ}$ Ssü near Tung-lan C., Kuangsi.

$2^{\circ}$ T.S. near Lung-an H., Knangsi.

$3^{\circ}$ (obs.) Han, a town near Yü C., Honan (No. 7698).

$4^{\circ}$ (obs.) Tang, a town IV. of Yün-yang Fu, Hupeh.

6628 ' 'u-yi | 伊, (obs.) T'ang, H., S. of Yi-shan H., Kuangsi. 
都

TU-YÜ-CH' ${ }^{\text {ENNG. }}$

6629 Tu-yiu-ch'êng | 尉 城, (obs.) Han, a town E. of Lin-chiang $\mathrm{Fu}$, Kiangsi.

6630 Tul-yün | 雲, see No. 6631. $6631 \mathrm{Tu-yün}$ 匀 Tu-yun.

[288] $1^{\circ} \mathrm{Fu}$ in the Kuei-tung circ., Kueichou; forming also the district city of the same name; Lat. $26^{\circ}$ $12^{\prime}$, Long. $107^{\circ} 22^{\prime}$; Yüan, Tu-yïn | 雲; Ming, Tu-yün|匀.

$2^{\circ} \mathrm{H}$. forming the prefectural city of the same name, Kneichou ; Lat. $26^{\circ} 12^{\prime}$, Long. $107^{\circ} 92^{\prime}$. $3^{\circ}$ T.S. near the above.

\section{度}

6632 Tu-shih 侤市, chên near Shu11-an H., Chehkiang.

渡

6633 'Tu-k'ou 渡 $\square$, post-town near Wu-ch'êng H., Shantung.

6634 Tu-t'ou | 頙, ss ${ }^{\breve{u}}$ near Hsing-ning $\mathrm{H}$., Hunan.

\section{兔}

6635 Tu-shan 亮 仙, H. in Huang-hai 黃海 circ., Corea.

\section{塗}

6636 Tu-po-t ê 塗孛特, see No. 2502.

6637 Tu-shan | IU, see No. 1731
讀

TU-KU-Shan.

\section{杜}

6638 Tu-chia 杜家, chên near Lai-an H., Anhui.

6639 'Tu-chia-t'ai | 家台, a post-town neat Knang-ning H., Shingking.

6640 Tu-ch' ii | 曲, chên near Hsi-an Fu, Shensi.

6641 Tu-êrh-po-t tê | 爾伯特 Turbet, Mongol Tribe.

$6642 \mathrm{Tu}-1 \mathrm{in} \mid$ 林.

$1^{\circ}$ Chên near $\mathrm{Ch}^{\prime}$ ing $\mathrm{H}$., Chihli.

$2^{\circ}$ Chên near Chieh C., Shansi.

6643 Tu-ling | 陵.

$1^{\circ}$ (obs.) Liang, H., W. of Yang-chiang $T$., Knangtung.

$2^{\circ}$ (obs.) Han, H., E. of Hsi-an $\mathrm{Fu}$, Shensi.

$3^{\circ}$ See No. 2691.

6644 Tut-shêng | 勝, Y. near Ts'ao H., Shantung.

6645 Tu-t'ung | 同, (obs.) S. $T s^{t} i, \mathrm{H}$. on the W. frontiers of Kuangtung.

6646 Tu-yang | 踼, see No. 3920. 6647 Tu-yüan | 原, (obs.) Sui, H., $120 l i \mathrm{~W}$. of Yangchiang $T$., Kuangtung.

\section{讀}

6648 Tu-ku-shan 讀谷山, a town in the Lin-ch'in Islands. 
獨 TU-LIU.

\section{獨}

6649 Tu-liu 獨流, chên in Ching-hai H., Chihli.

6650 Tu-shan | II Tuh-shan, [474]C. in Tu-yün Fu, Kueichon; Lat. $25^{\circ} 45^{\prime}$, L,ong. $107^{\circ} 20^{\prime}$.

6651 Tu-shih-k'ou | 石口Tıhshih-kau, T. in Hsüan-hua $\mathrm{Fu}$, also included in the K'ou-pei circ., Chihli ; I at. $41^{\circ}$, I,ong. $11^{\circ} 40^{\prime}$.

\section{秃}

6652 Tu-ma-wên 秃馬溫 Demavend, a town in Persia, at the foot of the Elburz Mountains.

Tu-chang, see No. 6607 . Tu-kang, see No. 6616. Tu-kiang, see No. 6609. Tt1-kié, see No. 6611. Ti1-shan, see No. 6666. Tr1-yu1, see No. 6631.

\section{T'U}

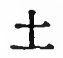

6653 T'u-clı'iao 士 橋, posttown near Wu-ch ang $\mathrm{H}$., Hupeh.

6654 T'u-ch'iao-p' $"$ | 橋 舖, ssǚ near T'ung-ling $\mathrm{H}$., Anliui.

6655 T'”.fan| 番, see No. 2502. 6656 T'u-liu-t'ê | 扈特Turgut, Mongol Tribe.
土 T'U-YIN.

6657 T'u-hsieh-t'u | 謝圖 Tuchetı, Khanate in Mongolia; bounded on the $\mathrm{N}$. by Siberia; on the E. by 'Tsetsen; on the S. by Inner Mongolia; and on the W. by Uliasutai and Sainnoin.

6658 T'u-lung | 龍, ssŭ near $\mathrm{P}^{\text {'ing-lê } F u, ~ K u a n g s i . ~}$

6659 T'u-1nêtı | P月.

$1^{\circ}$ Chên near Ning-lisiang H., Shansi.

$2^{\circ}$ Ss̆̈ near Lin-hsiang H., Hunan.

$3^{\circ}$ (obs.) N. Wci, H. 70 li N.W. of Futping H., Shensi.

6660 T'11-mêng | 孟, Kin, H. in Chihli.

6661 T'u-mo-t'ê默特, Tumed, Nongol Tribe.

6662 T'u-mu-pao | 木堡, posttown near Huai-lai H., Chilili.

6663 'I" $11-$ pa | 巴, Y. near Suiting Fu, Ssŭch' 'uan.

$6064 \mathrm{~T}^{\prime}$ "11-po | 波, see No. 2502. 6665 T'u-po-t'ê | 伯特, see No. 2502.

6666 ' $T$ '11-shan | U Tu-shan, H. in Corea; Lat. $38^{\circ}$ $22^{\prime}$, Isong. $127^{\circ} 05^{\prime}$.

6667 T'u-yin | 垠 (for which Shang-yin 上垠 is sometimes written by mistake). 
土

T"T"YIN.

$1^{\circ}$ (obs.) Hon, Tsin and N. Wir, H. 10 li E. of Fêng-jun H., Chihli. $2^{\circ}$ (obs.) N. Wei, H. 180 li N.Li. of Mi-yiun H., Chihli.

昩:

6668 '”11-ching 叶宗, see No. $571 s$.

6669 'l'11-fan|蕃, sce No. 2502.

6670 '"11-111-fan| 急番 Turfan (also called Knaing-an

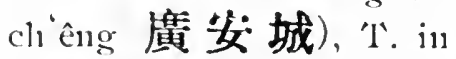
Chên-lısi C.I..'T., Kansul1; I.at. $42^{\circ} 40^{\prime}$, I.omg. $90^{\circ}$ $48^{\prime}$.

\section{徒}

6671 T'n-hsi 徒西, (obs.) T'ang, H., S. of Kao H., Ssücli nan.

6672 'T"u-ssin| 思 (or, 途思)

'Thus, the ancient capital of Khorassan, Persia.

\section{途}

6673 'T'11-lu-chi 途魯吉, nane for Turkestan.

6674 '个"11-ssul| 思, seeNo.6672. 副

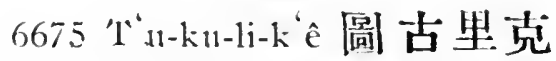
'Tugurik, a town in Cobdo; Iat. $47^{\circ} 05^{\prime}$, I.ong. $90^{\circ}$.

\section{TUAN E段}

6676 'Tuan-chia-chi段家集, ssü near Ku-shih H., Honan.
TUMED.

\section{端}

6677 Tuan 端, sec Nos. 335, 3169.

6678 Tr1an-ch'i | 溪, see Nos. 3967, 6309.

6679 Tuan-ch 'il給, another way of writing the preceding.

6680 T'uan-ch uan M, Fu in Hsien-ching 鏡 circ., Corea; Lat. $40^{\circ} 30^{\prime}$, I,ong. $129^{\circ}$.

6681 'ruan-shih | 氏, chên near Kao-p'ing H., Shansi.

6682 Tuan-yüan | 源, (obs.) T゙rng, H. in Mon C., Ssŭch แan.

\section{T'UAN}

\section{湍}

6683 ' $T^{6}$ uan-yang 湍陽, a posttown near Hsin-yeli H., Honan.

\section{團}

668+ 'l'nan-fêng 湽風, ssü in Huang-kang H., Hupeh. 6685 '个'uan-pa-la|八刺 Toun, a town in Persia, province lezd.

'luchetu, see No. 6657. Tudzung, see No. 6478. 'Tuguchuk, see No. 5933. Tugurik, see No. 6675. 'T'uh-shan, see No. 6650. Tuh-shih-kau, seeNo. 6651 . 'Tuktu, see No. 6489. 'Tumed, see No. 6061. 
頓

TUN-CH IU.

\section{TUN}

頓

6686 'Tu11-ch'in頓邱, see No. 1243.

\section{敦}

6687 Tun 敦.

$$
\begin{aligned}
& 1^{\circ} \text { (obs.) T'ang, C. in Ssŭ- } \\
& \text { chinan. } \\
& 2^{\circ} \text { See No. } 480 \text {. }
\end{aligned}
$$

6688 'Tun-hua | 化, H. in Wuch'ang C.L.'T., Kirin.

\section{墩}

6689 'Tnn-huang 墩煌, posttown near An-hisi C., Kansuh.

'Tun-liwang, see No. 6690. 'Tun-liu, see No. 6692.

\section{T'UN}

影

6690 T'un-luang 敦煌 'T’111hwang, H. in An-hsi C., Kansuh ; Iat. $39^{\circ} 40^{\prime}$. I.ong. $95^{\circ} 05^{\prime}$.

\section{屯}

6691 'T"un-ch'i 屯溪, chên near Hui-chon Fu, Anluui.

6692 T'un-liu | 猊 Tun-liu, H. in I,llan $\mathrm{F}_{11}$, Shansi; Lat. $36^{\circ} 15^{\prime}$, Long. $112^{\circ} 46^{\prime}$; . 5.-A., Yü-wu 余吾 in $\mathrm{K}$. Chin 晁; Han, 1st T'unlin 純留, 2nd, T“"n1-lin 留。
東 Tung-an.

6693 T'nn-mo | 磨, chên near Lo-ch'uan H., Shensi.

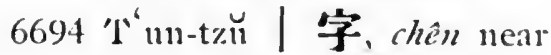
P'ing-liang Fu, Kansul.

純

6695 T'u11-liu 純留, see No. 6692.

\section{吞}

6696 T'un-shan 呑暗, shê nen Tan-shui H., Formosa.

\section{TUNG}

\section{東}

6697 Tung 東 Tung.

$1^{\circ}$ T. C. in 'T'ai-p ing Fu, Knangsi, near Tso $C$. $2^{\circ}$ (obs.) S. of $\mathrm{K}^{\prime} \mathrm{ai} \mathrm{C}$., Chihli ; Han, shïn in Yen 党 CHOv.

$3^{\circ}$ (obs.) in Ch' ên-chon Fin, Honan; S. Sung, chïn in S. Yï 豫 crov. $t^{\circ}$ (obs.) N.E. of Hua H., Honan; $N$. Wei and Sui, chïn in Yen 毫 chov. $5^{\circ}$ (obs.) near Fêng-t ien F*11, Shingking; Lino, C. in Tung-ching | 京 circuit.

$6^{\circ}$ (obs.) in Wên-hsi H., Shansi; Kin, chên in Chieh 解 $\mathrm{C}$.

$7^{\circ}$ See Nos. 2223, 5462". 6701.

6698 'Tung-an | 娄 Tung-ngan. 
東

$$
\text { TUNG-AN. }
$$

$1^{\circ} \mathrm{H}$. in Shun-t'ien $\mathrm{Fu}$, Clinhli; Lat. $39^{\circ} 25^{\prime}$, I,ong. $116^{\circ} 46^{\prime}$; Han, An-tz" 管次; $M i n g$, Tung-an|庭 H.

$2^{\circ} \mathrm{H}$. in Yung-chou $\mathrm{Fu}$, Hunan; Lat. $26^{\circ} 13^{\prime}$, Long. $111^{\circ} 14^{\prime}$; Han, Ling-ling 零陵; Sung, Tung-an | 安 $\mathrm{H}$.

[440] $3^{\circ}$ H.in Lo-ting C., Knangtung; Lat. $23^{\circ} 03^{\prime}$, Long. $111^{\circ} 44^{\prime}$; Ming, Tung-an | 发 $\mathrm{H}$.

$4^{\circ}$ Post-town near Hsüchou Fu, Kiangsu.

$5^{\circ} S s \breve{u}$ in 'T's'ang-wu H., Kuangsi.

$6^{\circ}$ (obs.) $W$. Han, Marq. W. of Hai C., Kiangsu. $7^{\circ}$ (obs.) Han and Tsin, H. 30 li S. of Yi-shni H., Shantung.

$8^{\circ}$ (obs.) $80 \mathrm{li}$ N. W. of Yishui H., Sliantung; $S$. Sung, chün in Hsï 徐 chov; N. Wei, chiü in Nan-ch'ing 南青 CHOU ; Sui, H. in Langyeh 䐚躯 chïn.

$9^{\circ}$ (obs.) S. Sung and $S$. $T s^{\prime} i$, H. near Huangchou $\mathrm{Fu}, \mathrm{Hupeh}$.

$10^{\circ}$ (obs.) T'ang, H., S.W. of Kung H., Ssŭch ' 1 an. $11^{\circ}$ (obs.) Yüan, C. $45 l i$ N. W. of Tung-an H., Chihli.
東 TUNG-CHIH.

$12^{\circ}$ See Nos. 1862, 4558. 6699 Tung-an | 岸, chên near Ning-kuo Fu, Anhui.

6700 'Tung-ch' 'ang | 長, chên near Fu-shan H., Shansi.

6701 Tung-ch' ang | 昌 Tungchang.

[322] $1^{\circ} \mathrm{Fu}$ in the Chi-Tung-T'aiWu-Lin circ., Shantung; forming also the district city of Liaoch'êng; Lat. $36^{\circ} 37^{\prime}$, Long. $116^{\circ} 12^{\prime} ; S . A$., W. part of K. Cl' ' $\mathrm{i}$ 唯; C. Wars, in K. Wei 衞, K. $\mathrm{Cl}^{\prime} \mathrm{i}$ 贸 and $\mathrm{K}$. Chao 趋; $T s^{6}$ in, in Tung | chün and Chü1 釬. 鹿 chün; Han, Chi-yin 派陵 and Ch'ing-loo 声河; Wei, Yang-ping 陽 平; Sung, Pu 沙 C.; Yüan, Tung-ch'ang | 昌.

$2^{\circ}$ (obs.) $W u$, a town $\mathrm{S}$. of Chi-an Fu, Kiangsi.

6702 'Tung-ch' ang | 鹪, ssü near Hsii-wên H., Kuangtung.

6703 'Tung-ch' êng | 城, see No. 6462.

6704 'Tung-chia | 嘉, see No. 7092.

6705 'Tung-chiang-k'ou | 江口， ssü near Hsiao-yi $\mathrm{T}$., Shensi.

6706 Tung-chih | 治 Tung-chi. 
東

TUNG-снін.

$1^{\circ} \mathrm{T}$. in Tsê-chon $\mathrm{Fu}$, Shansi.

$2^{\circ}$ Chên near Wu-t'ai H., Shansi.

6707 Tung-ching | 京.

$1^{\circ}$ Tokio, chief town of Tokio Fu, capital of Japan. Old name 江 戸 Yedo.

$2^{\circ}$ See Nos. 2079, 3061, 3805.

6708 T'ung - ching - ch'êng $\mid$ 京 城, a post-town near Fêng-t 'ien Fu, Shingking.

6709 Tung-ching / 婣, see No. 6246.

6710 Tung-chtt-shih | 朱提, (obs.) S. Ts'i, chiün near Hsü-chou Fu, Ssŭch' "uan.

6711 Tung-ch' । / 楚, see No. 5893.

6712 'Tung-ch' $\ddot{\text { in }} \mid$ 區.

$1^{\circ}$ (obs.) Tang, H., S.E. of Yung-an C., Kuangsi. $2^{\circ}$ (obs.) T"ang, H., E. of Hsin-ch êng T. H., Kuangsi.

6713 Tung-ch' inan | 川 Tungchuen.

$[547] 1^{\circ} \quad F u$ in the Yi-tung circ., Yünnan ; forming also the district city of Hui-tsê ; Lat. $26^{\circ} 25^{\prime}$, Long. $103^{\circ} 26^{\prime}$; orig., S. part of Tung-cl tuan 川 chïn.
東

Tung-hsiang.

$2^{\circ}$ (obs.) T'ang to Yiün, chiü comprising parts of Ssŭcl 'van and Yünnan. $3^{\circ}$ See Nos. 5815, 6795.

6714 Tung-ch' inan | 泉, chên nin Lin-ch' êng H., Kunangsi.

6715 Tung - fan | 番, see No. 6194.

6716 Tung-fêng-p'ing | 風本 a town in the Linch in Islands.

6717 Tung-hai | 海, see Nos. 1913, 3100.

6718 Tumg-hêng-nung | 恒脿. $1^{\circ}$ (obs.) N. Wei, chün in Anhui.

$2^{\circ}$ (obs.) N. Wei, chïn in Nei-hsiang H., Honan.

6719 'Tung-hu｜湖 Tung-hu, $\mathrm{H}$. forming the prefectural city of Yi-ch'ang, Hupeli; Lat. $30^{\circ} 49^{\prime}$, Long. $111^{\circ}$ $10^{\prime}$.

6720 Tung-hun | 昌.

$1^{\circ}$ (obs.) Han, H. $20 l i$ N.E. of Lan-yi H., Honan.

$2^{\circ}$ See Nos. 3594, 6740.

6721 Tung-hsia | 夏, see No. 7358.

6722 'Tung-hsiang | 鄉 Tunghiang.

$[375] 1^{\circ} \mathrm{H}$. in Fu-clion $\mathrm{Fu}$, Kiangsi ; Lat. $28^{\circ} 15^{\prime}$, L.ong. $116^{\circ} 35^{\prime}$. 
東

$\lceil 111] 2^{\circ} \mathrm{H}$. in Sni-ting Fu, Ssŭch'nan; Lat. $31^{\circ}$ $27^{\prime}$, Long. $107^{\circ} 51^{\prime}$; Han, Hsin-an 新发; Wei, Tung-hsiang / 拫.

$3^{\circ}$ Chên nen Writho H., Anhui.

6723 'lung-kê | 莺, a post-town near Chiang-p'n H., Kiangsu.

6724 Tung-k'e | 科, '.S. near 'Ta-chien-1n 'T', Ssŭch'nan.

6725 'lung-knan | 筞 'Tungkwall.

$[478] 1^{\circ}$ H. in Knang-chon Fu, Knangtung; Isat. $22^{\circ}$ $50^{\circ}$, I ong. $113^{\circ} 50^{\prime}$; Tsin, Pao-an 䫧安; T'ang, 'Tung - knan | 莞.

$2^{\circ}$ (obs.) Chou, fendal state in Shantung.

$3^{\circ}$ See No. 7482 .

6726 Ting-kuan | 關.

$1^{\circ}$ Chên near Shao-hsing Fin, Chehkiang. $2^{\circ}$ See Nos. 6127, 7354.

6727 Tung-kuang | 光 Tungkwang, $H$. in Ho-chien Fu, Chihli; Iat. $38^{\circ} 03^{\prime}$, I,ong. $116^{\circ} 36^{\prime}$; . Han, Tung-kuang | 光; Sui, Kuan 觀 C.; T $T^{*}$ an $g$, Anling 安陵.

6728 Tung-kung | 公. (obs.) T ang, H., N.W. of Yipin H., Ssŭch uan.
東

TUN-1, ̈̈.

6729 'Tung-kuo | 稂, see Nos. $3051,3536,5881$.

6730 'Tung-lai | 萊 Tung-lai.

$1^{\circ} \mathrm{H}$. in Ch'ing-chang 慶 们 circ., Corea; Lat. $34^{\circ} 48^{\prime}$, I ollg. $129^{\circ} 35^{\prime}$. $2^{\circ}$ (obs,) S. Sung, chïn 60 li N.E. of Yeh H., Shantung.

$3^{\circ}$ Sce Nos. 3570, 5062, 6330, 7326.

6731 'Tung-lan | 蘭 Tung-lan. [518] $1^{\circ} \mathrm{C}$. in $\mathrm{Cl}^{\prime}$ ing-yüan $\mathrm{Fu}$, Knangsi; I,at. $24^{\circ} 28^{\prime}$, I.ong. $106^{\circ} 45^{\prime}$; Sung, Lan 荿 C., Yïan, Tunglan |成 $\mathrm{C}$.

$2^{\circ}$ T.C. near the above.

6732 'nung-lê | 櫟, a post-town near Shan-tan H., Kansul.

6733 Tung-li | 黎, (obs.) $N$. $\mathrm{We} i, \mathrm{H} .60 \mathrm{li} \mathrm{W}$. of $\mathrm{K}^{6}$ ai C., Chihli.

6734 'lung-liang-shan | 梁山, ssü near 'T"ai-p'ing $H$., Anhni.

6735 Tung-liang | 凉, chên near Ning-hsia Fu, Kansul.

6736 Tung-ling | 陵, see No. 4773.

6737 Tung-lin | 流 Tung-lin, [528] H. in Ch ih-chon Fu, Anhui; I at. $30^{\circ} 13^{\prime}$, Long. $117^{\circ} 01^{\prime} ; H a n, P^{\prime}$ êng-tsê 彭澤; S. T'ang, Tunglin | 流。

6738 Trung-lï | 閭, chên near Ch' ${ }^{\prime} i$-wu H. Shansi. 
東

TUNG-Min.

6739 Tung-min | 緍.

$1^{\circ}$ (obs.) Han, H. 20 li

N.E. of Clin-lısiang $\mathrm{H}$,

Shantung.

$2^{\circ}$ See No. 1025.

6740 Tung-ming | 明 'Tungming.

[148] $1^{\circ} \mathrm{H}$. in la-ming Fu, Cliihli: Lat. $35^{\circ} 29^{\prime}$, Long. $115^{\circ} 18^{\prime} ; \mathrm{Han}$, 'Tung-hun | 苌; Wangmang (usurper) 'lungming | 明.

$2^{\circ}$ See No. 3594.

6741 Tung-1110n | 牟, see No. 6330.

6742 Tung-o | 阿 Tung-o, H. [148] in 'T'ai-an Fil, Shantung ; Lat. $36^{\circ} 23^{\prime}$, I,ong. $116^{\circ}$ $22^{\prime}$; orig., Pei-lising 北 杏; S.A., A-yi 阿 邑; Han, 'Tung-a |阿.

6743 Tung-on | 匰, (obs.) Han, K. in Chehkiang; see Nos. 1396, 6185, 7022, 7840.

6744 Tung-pien | 邊, Cirenit in Slungking, comprising Ch'ang-tu Fu, Fêng-luiang ' $\mathrm{C}$. and Hsing-ching ' $\mathrm{T}$.

6745 'lung-p'ing | 平 lungping.

$1^{\circ}$ C. in 'T"ai-anl I'il, Shantung; Iat. $36^{\circ}$ 15', I,ong. 116 27'; S.A., Hsï-cliü 須们 in K. Ln 惫; Han, Tung-p'ingl平 and Ta-
東 'TuNg-Yang.

ho 大河; P. Chou, Ln 魯 C.; Sui, Yün 鄆 C. $2^{\circ} \mathrm{H}$. in Hai-lung $\mathrm{F}_{11}$, Shingking.

6746 'Tung-p'ing-shu| 平舒, see No. 6006.

6747 'Tung-p'ing-yang | 平陽, see No. 2779.

6748 'Tung-p ing-yüan | 本原, see No. 6578.

6749 'lung-shan | IIf, 'T.C. near Ho-hsi H., Yümman.

6750 ' ' ung-shou-chiang 受降 see No. 3456.

6751 'rung-shui| 水, Y. near Chiang-lna H., Hunan.

6752 'lung-tai | 代, (obs.) $7 s^{\prime}$ in and Han, chïn.

6753 'lung-tai-pao | 柋堡, ssu near Lien-chiang $H$. , Fuhkien.

6754 'Tung-t ai | 臺 Tumg-tai, H. in Vang-chon lin, Kiangsu; I att. $32^{\circ} 35^{\prime}$, I.ong. $120^{\circ} 25^{\prime}$.

6755 'ling-tang-cl' ii | 告渠.

$1^{\circ}$ (obs.) s. Ts'i, chün in Ssuchinian.

$2^{\circ}$ See No. 2120.

6756 'lung-ts'un | 村, chênnear Ian H., Slanni.

6757 'Nung-tu | 都, see Nos. $2079,7308$.

6758 'lung-wu|武, S. Sung, H. in Kiangsu.

6759 Tung-yang|陽 Tung-yang. 
東

Tung-Yang.

[520] $1^{\circ}$ H. in 'T'ai-chon Fu, Chehkiang; Lat. $29^{\circ}$ $17^{\prime}$, Long. $120^{\circ}$; Han, Wu-shang 烏傷; T'ang, Tung-yang | 陽.

$2^{\circ}$ Sš̆ near Knang-tê C., Anlini.

$3^{\circ}$ See Nos. 225, 576, 2064, 5574.

6760 Tung-yel | 治, see No. 4448.

6761 Tung-yen | 蕧, see No. 6583.

6762 'Tung-yi | 暆, (obs.) S.IV. of the Capital of Corea; $W$. Han, H. in L,ê-lang 樂泿 chiin.

6763 Tung-yi | 篮, see No. 5046.

6764 'Tung-yïan | 垣.

$1^{\circ}$ (obs.) $N . W e i, \mathrm{H} ., \mathrm{E}$. of Hsin-an H., Honan. $2^{\circ}$ See Nos. 487, 574, 2724.

6765 Tung-yung | 䔨, see No. 769.

\section{涷}

6766 'Tung-ch' uan 涷川, a posttown near Wên-hsi H., Shansi.

\section{悯}

6767 Tung-pa 润 巴, T. S. in Kokonor.

Tung, see No. 6768.

Tung-chang, see No. 6701. Tung-chan, see No. 6802. Tung-chi, see No. 6706. Tung-ching, see Nos. 6771 , 6808, 7803.
T'UNG.

Tung-chuen, see Nos. 6713, $6777,6795$.

'Tung-hai, see No. 6779.

Tung-hiang, see Nos. 6722, 6811, 7806.

T'ung-hü, see No. 6782 .

Tung-jin, see No. 6822.

Tung-kiang, see No. 6772.

Tung-ku, see No. 6813.

Tung-kwan, see Nos. 6725 , 6796, 6806, 7792.

'Tung-kwang, see No. 6727 . Tung-liang, see No. 6824. Tung-ling, see No. 6825. 'Tung-lii, see No. 6815. Tung-1ngan, see Nos. 6698 , 6799.

Tung-o, see No. 6742.

Tung-pel, see No. 6817.

Tung-ping, see No. 6745. 'Tung-shan, see Nos. 6786, 6827.

Tung-tai, see No. 6754.

Tung-tau, see No. 6787.

'Tu11g-tsz', see No. 6818.

Tung-wei, see No 6789 .

\section{T'UNG}

\section{通}

6768 T'ung 通 'Tung.

[360] $1^{\circ} \mathrm{C}$. in Shun-t'ien Fu, and also included in the T'ung-chou circ., Chihli ; Lat. $39^{\circ} 54^{\prime}$, Long. $116^{\circ} 41^{\prime}$; Han, I, u 㴗 H.; T'ang, Hsüan 立 C.; Kin, Yïan 
通

T'uxg.

and Ming, 'T'ung |C. Called Pei-T'nug-Chou 北 | 州 to distinguish it from the city of the same name in Kiangsu. [528] $2^{\circ}$ C.L.C. in the Ch' ${ }^{\prime}$ angChên-T'nung-Hai circ., Kiangsı ; Lat. $32^{\circ}$, Long. $120^{\circ} 55^{\prime}$; Han, Hai-ling 海 陵; Five dyn., Sung, Yüan and Ming, 'T'ungC. Port of call. Tinju or Chinju (Polo).

$3^{\circ}$ (obs.) in $\mathrm{K}^{6}$ ai-yüan $\mathrm{H}$., Shingking; Liao, C. in Tung-ching 東京 circuit.

$4^{\circ}$ See No. 6127.

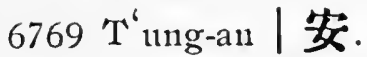

$1^{\circ} \mathrm{A}$ post-town near Anting H., Kansul.

$2^{\circ}$ See No. 3737.

6770 T'ung-an-choul学州 T.S. near Hui-li C., Ssüch' 'uau. 6771 'T'ung-ch'êng | 旅 Tung[.270] ching, H. in Wu-ch' ang Fu, Hupelı; Lat. $29^{\circ} 16^{\prime}$, Long. $113^{\circ} 47^{\prime}$; Han, Hsiatsun 下隽; $T^{6}$ ang, ' 'tungch'êng | 城.

6772 T'ung-chiang | 江 'lung[332] kiang, H. in Pao-11ing Fu, Ssưch 'uan; Lat. $31^{\circ} 55^{\prime}$, Lolig. $107^{\circ} 16^{\prime} ;$ Han, Tang-ch' $\mathfrak{u}$ 宕渠; 2nd Wei, No-shui 諾 水; T $T^{6}$ ang, T'ung-chiang | 江 $\mathrm{H}$.
通 T'UNG-HUA.

6773 'T'ung-ch'in | 秦, (obs.) Sung, military post N.W. of Chia C., Shensi.

$677+$ 'T'ung-ching | 津.

$1^{\circ}$ (obs.) Sung and Kin pao 160 li W. of Ho C., Kansul.

$2^{\circ}$ (obs.) Sung, pao near Mien C., Ssŭch' nan.

$3^{\circ}$ (obs.) Kin, pao near Shên-nu H., Sheusi.

$4^{\circ} \mathrm{Fu}$ in Cling-ch'i 京㽣 circ., Corea.

6775 'T'ung-chou | 州, circ. in Chiluli, comprising ' $T$ 'ung C., Tsun-hua C. and Yungp'ing Fu.

6776 T'ung-ch'ï-chieh | 浟街, ssü in Lung-ch ualu $\mathrm{H}$., Kuangtung.

6777 T'ung-ch' uan | 川 Tungchuen.

$1^{\circ}$ Chün in Chiang-yüen 江 原 circ., Corea; Lat. $38^{\circ}$ $06^{\prime}$, Long. $128^{\circ} 52^{\prime}$.

$2^{\circ}$ See No. 6127.

6778 'r'ung-ch'üan | 泉, see No. 5585.

6779 'T'ung-hai | 海 Tung-hai. [26] $1^{\circ} \mathrm{H}$. in Lin-an Fu, Yünnan; Lat. $24^{\circ} 12^{\prime}$, I Iong. $102^{\circ} 56^{\prime}$; Yizan, 'T'unghai $\mid$ 海 $\mathrm{H}$.

$2^{\circ}$ (obs.) Trang, chün in Yünnan.

$3^{\circ}$ See No. 3838.

6780 T'ung-hua | 化. 
通

$1^{\circ} \mathrm{H}$. in Hsing-ching ' $\mathrm{I}$., Shingking.

$2^{\circ}$ (obs.) Sui, H. east of Min-cli uan H., Ssŭch'uan.

6781 'l"ung-hsi | 西.

$1^{\circ}$ (obs.) 40 li S. of Anting H., Kansul ; Sung, a military post in Kung 翠 C.; Kin, H. in Kung 垍 C.

$2^{\circ}$ (obs.) Yüan, Fu in Yïnnan.

6782 'lung-hsï| 許 'Tung-hü, $\mathrm{H}$. in $\mathrm{K}$ ' ai-fêng $\mathrm{Fu}$, Honan; I.at. $34^{\circ} 35^{\prime}$, Long. $114^{\circ}$ $35^{\prime} ; \mathrm{Han}, \mathrm{Cl}^{\prime}$ ên-liu 陳留; Sung, Hsien-p ing 咸平。 6783 '’”ung-kouk'ou | 溝山, ss̆̌ in Huai-jên H., Shingking.

6784 'T'ung-knei | 螮, (obs.) Sung, pro $70 l i$ N.E. of Huan H., Kansul.

6785 'T"ung-min | 㞾, (obs.)

Sung, a military post near

Kung-ch' ang Fu, Kansub.

6786 T'ung-shan | II Tung[532] shan, H. in Wu-ch'ang Fu, Hupeh ; Lat. $29^{\circ} 40^{\prime}$, Long. $114^{\circ} 22^{\prime}$; Five dy'n., Yang-shan 羊龱 chên; $S$. T゙ang, Tung-shan | II.

6787 T"ung-tao | 道 Tung-tau. [110] $1^{\circ} \mathrm{H}$. in Ching C., Hunan; Lat. $26^{\circ} 17^{\prime}$, Long. $109^{\circ}$ 29'; T’ang, Kung-shui
潼 T'UNG-CH'UAN.

葆水; Sung, T'ungtao | 道.

$2^{\circ}$ Chên near 'l' ien-ho H., Kuangsi.

6 788 'l"ung-tsê | 涬, see No. 7842 .

6789 '个'ung-wei | 消 Twung-wei, $\mathrm{H}$. in Kung-ch 'ang $\mathrm{Fu}$, Kansuls ; Lat. $35^{\circ} 06^{\prime}$. I.ong. $105^{\circ} 13^{\prime} ; T^{\prime}$ ang, Lung-hsi 陪西; Sung, 'I"ming-wei | 渭.

6790 'Tung-yüan | 遠.

$1^{\circ}$ A post-town near Kungch'ang Fu, Kansuh.

$2^{\circ} \mathrm{A}$ post-town near $\mathrm{Ku}$ lang H., Kansuh.

$3^{\circ}$ See No. 2266.

6791 T'ung-yüan-pao | 遠 堡, a post-town near Fêng-t ien Fu, Shingking.

\section{堁}

6792 'T'ung-ti 㰣地, see No. 5822.

6792`'T'ung-t'ing | 廷, see No. 5822.

\section{潼}

6793 T'ung 潼, see Nos. 4433, 6795.

6794 'I'ung-ching | 津, see No. 2220.

6795 'T'ung-ch'nan | 川 Tung[125] chuen, $\mathrm{Fu}$ in the $\mathrm{Ch}^{\prime}$ uanpei circ., Ssŭch' 11an ; forming also the district city of Sant-t'ai ; Lat. $31^{\circ} 09^{\prime}$. 
long. $105^{\circ} 11^{\prime} ;$ S. A . and C. Hars, Shu 溞：Han，

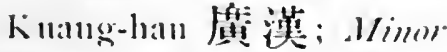

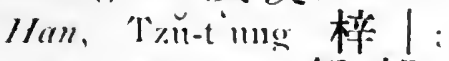
Sin, Hsin-tu 新都: liang, 'I"mu| C.; $\Pi$. Hei, chang-ch'êng 昌城。

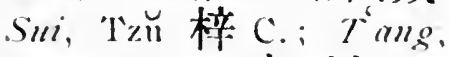
Trung-ch nan 東 川: 5 . Sung, T"mo-ch" wan| 川 Fil.

6796 T'ung-kuan | 關 'lung[148] kwan, T. in 'I"mw-clon Fu, and also in the 'lungShang circ., Shensi: Iatt. $31^{\circ} 09^{\prime}$, Long. $105^{\circ} 11^{\prime}$. 6797 ' $\mathrm{T}^{\prime}$ ung-slang | 商, circ. in Shensi, comprising 'T"muchon Fu, 'T’mu-knan 'l. and shang $C$.

\section{同}

6798 T'mug 同.

$1^{\circ}$ (obs.) Limo, C. $30 / i s$.

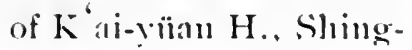
king.

$2^{\circ}$ See No. 6802 .

6799 个“ung-an| 娄Tung-ngan.

$1^{\circ}$ H. in Cli üan-chon Fn, Fuhkien: Iat. $2 t^{\circ}+t^{\prime}$. J.ong. $118^{\circ} 29^{\prime}$; Ham. Hon-knan 侯官 : Tsm, r”ung-an 妍.

$2^{\circ}$.Ss̆̈ in Hsin-ch êng H. Kiangsi.

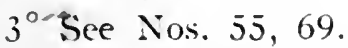

6800 'T"ung-chêng | IE. (ohs.) T"ang, C. near Vang-lic.. Kuangsi.
6801 'I"ung-ch'ing | 度、(obs.) S. Sung, Fun in Kimsul.

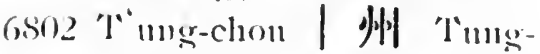
[264] cham, Fin in the "T"moShang circ. Shensi ; forming also the district city of Ta-li; I.a1t. $34^{\circ} .30^{\prime}$, I, ong. $109^{\circ} 51^{\prime} ; \%{ }^{\prime}{ }^{\circ}$ ill, Nei-shih 內史: Han, In-fêng-ıi 右蕉䄰. Ind Hei, Hsihua 西蕉; ゲ Kin, Yü̈rn anci lVing, 'I" 1111 | C.

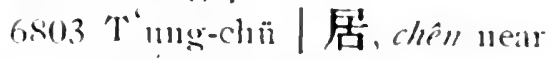
Ching H., Chiluli.

$6803^{\prime} \mathrm{T}^{\prime} \mathrm{mug}-\mathrm{fu}$ | 褔. H. in Chinall-10全羅circ., Corea. rsot 'I'mug-kê | 戈. a post-town near Hsï-kon H., Shamsi. $6805 T^{\circ}$ ing-kn 谷. see No. 503 . 6806 T'mug-kuan | 官 Tmu[397] bwan, H. in Hsi-an Fu, Shensi; I at . $35^{\circ} 06^{\prime}$, I.ong. $109^{\circ} 03^{\prime} ;$ Han, 'Tai-rï 卌 新; Tin, P’in-yang 频! 陽 ; 2nd Hici, 'T" mug-kman

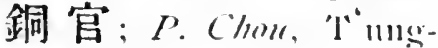
kuan | 官.

\section{样}

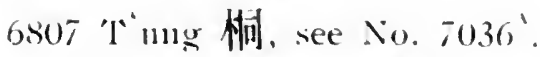
bogs T'ung-ch'êng | 城 Tum ching.

[451] $1^{\circ} \mathrm{H}$, in An-cling Fu, Anhui; Jat. $31^{\circ} 07^{\prime}$. I.ong. $116^{\circ} 56^{\prime}$; $\mathrm{Han}$, 个img - yang 佂晹: Tsin, L, ring-shu龍舒: 
相 T'UNG-CH' I.

T'ang, Tung-ch'êng | 城.

$2^{\circ}$ A post-town near $T$ ' 1 ungch'ang Fu, Shantung.

6809 T'ung-ch'i | 溪, see No. 6815.

6810 T'ung-ch'iao | 橋, chên near Fulp'ing H., Shensi.

6811 'T'ung-hsiang | 拫 Tunghiang, $H$. in Chia-hsing F11, Chehkiang; Lat. $30^{\circ}$ $45^{\prime}$, Long. $120^{\circ} 23^{\prime}$.

6812 'T'ung-hsü|墟, (obs.) Kin, chên S.W. of Su C., Anhui. 6813 T'ung-jui涡, |see No. 3417. 6814 T'ung-lou | 樓, Y. near Ling-shui $\mathrm{H}$., Kuangtung. 6815 'T'ung-lü | 盧 Tung-lü.

[231] $1^{\circ} \mathrm{H}$. in Yen-chou Fu, Chehkiang; Lat. $29^{\circ}$ $46^{\prime}$, Long. $119^{\circ} 40^{\prime}$; Han, T'ang-ch'i | 溪; T'ang, Sung, Yiüan, and Ming, T'ung-lï | 盧 $\mathrm{H}$.

$2^{\circ}$ (obs.) W. of the above; Tsin, S. Sung, S. Ts' i and Sui, T'ung-lü | 盧 $\mathrm{H}$.

6816 T'ung-mu | 木, chên near Yung-ning C., Kuangsi. 6817 ' T'ung-pai | 㮌 'Tung-peh, [144] H. in Nan-yang Fu, Honan; Lat. $32^{\circ} 20^{\prime}$, Long. $113^{\circ}$ $18^{\prime} ;$ Han, Fut-yang, 復陽; Sui, T'ung-pai | 栢. 6818 'T'11ng-tzŭ |梓 'Tung-tsz', [66] H. in Tsun-yi Fu, Kuei-
銅 T'UNG-LIANG.

chou; Lat. $28^{\circ}$, Long. $106^{\circ} 45^{\prime}$.

6819 T'ung - yang | 陽, chên near Ying-shan H., Anhui.

\section{銅}

$6820 T^{\prime}$ tung 銅.

$1^{\circ}$ (obs.) T'ang, C. near Pei-liu H., Kuangsi.

$2^{\circ}$ (obs.) Liao, C. 40 li E. of Hai-ch' êng H., Shingking.

6821 T'ung-ch'êng | 城.

$1^{\circ}$ Chên near Ch ung-hsin H., Kansuh.

$2^{\circ}$ Chên near 'T'ien-ch'ang H., Anhui.

6822 T'ung-jên | 仁 Tung-jin. [283] $1^{\circ} \mathrm{Fu}$ in the Kuei-tung circ., Kueichon ; forming also the district city of the same name; Lat. $27^{\circ} 38^{\prime}$, Long. $109^{\circ}$; Ming, T'ung-jên |仁 $\mathrm{Fu}$.

$2^{\circ} \mathrm{H}$. forming the prefectural city of the same name, Kuei-chon; $\mathrm{L}_{1}$ at. $27^{\circ} 38^{\prime}$, Long. $109^{\circ}$.

$6823 \mathrm{~T}^{\prime}$ ung-ku | 鼓 'Tung-ku, [193] T. in Jui-chou Fu, Kiangsi ; Lat. $28^{\circ} 30^{\prime}$, Long. $114^{\circ}$ $06^{\prime}$.

6824 ' T'ung-liang | 梁 Tung[538] liang, H. in Chung-ch'ing Fu, Ssuchl' 'uan; Lat. $29^{\circ}$ 59', Long. $106^{\circ} 11^{\prime}$; orig., in $\mathrm{Pa} 巴$ chïn; T'ang, T'ung-liang | 梁. 
语门

T'UNG-LING.

$6825 \mathrm{~T}^{6}$ 'ung-ling | 陵'Tung-ling. [528] $1^{\circ} \mathrm{H}$. in $\mathrm{Cl}_{1}^{\prime}$ ih-chion $\mathrm{Fu}$, Anhui ; Lat. $31^{\circ} 04^{\prime}$, Long. $117^{\circ} 50^{\prime}$; Liang, Nan-ling 南陵; $T$ 'ang， Yi-an 峩安; $S$. T'ang, T'ung-ling | 陵.

$2^{\circ}$ Liang, H., N. of Yangch un H., Knangtung.

6826 'T'ung-p'u|鋪, chên near Ch'ing-yang H., Anhui.

6827 T'ung-shan| IL T'ung-shan.

$1^{\circ} \mathrm{H}$. forming the prefectural city of Hsï-chon, Kiangsu; I at. $34^{\circ} 11^{\prime}$, Long. $117^{\circ} 32^{\prime}$.

$2^{\circ}$ (obs.) T'ang, H., S.E. of Chung-chiang $\mathrm{H}$., Ssüch' 'uan.

$3^{\circ}$ Chên in Chang-p'a H., Fuhkien.

6828 'T'ung-ti | 鞮.

$1^{\circ}$ (obs.) Han, $T \sin , N$. Wei and Sui, H. 40 li s. W. of Ch'in C., Shansi.

$2^{\circ}$ See No. 1098.

6829 'I'ung-yang | 楊, chênn near T'ai-ho H., Anhui.

6830 T'ung-yu | 峪, chên near Wu-hsiang H., Shansi.

\section{峒}

6831 'T'ung-wu詷捂, ssŭ in Su-ch.ien H., Kiangsu.

Turbet, see No. 6641.

Turfan, see No. 6670 .
骖 TZŬ-YANG.

'Turgut, see No. 6655.

Turkesta11, see No. 6673.

'Twan-chau, see No. 6680.

'Twatutia, see No. 6089.

\section{TZU}

\section{甾}

6832 Tzŭ 甾, (obs.) W. Han, H., S.E. of K'ao-ch' êng H., Honan .

\section{淄}

6833 ' $z$ ŭ 畄, see No. 6834.

6834 'Tzй-ch' [186] H. in Chi-nan Fu, Shan-

tung; I at. $36^{\circ} 43^{\prime}$, Long. $118^{\circ} 12^{\prime}$; Han, Pan-yang 般陽; 1st Sung, Pei-ch“iu 具邱; Sui, 'Tzŭ-cli'uan | 川; T'ang, Тzй | C.

\section{滋}

6835 Trü 滋, (obs.) Sung, C. S.E. of I,ï C., Sstuch "uan. 6836 'Гz̆̆-ho | 賀 Shiga, Ken in Japan ; chief town Ôtsu. 6837 'Tž̆-yang | 陽 'Tsz'-yang, H. forming the prefectural city of Yen-chon, Shantung; Lat. $35^{\circ} 47^{\prime}$, I,olig. $116^{\circ} 59^{\prime} ;$ Han, Hsia-ch in 层邱; Sung, Tzй-yang 厥陑。

\section{嵫}

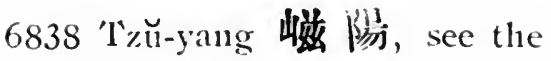
preceding. 


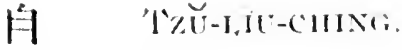

\section{白}

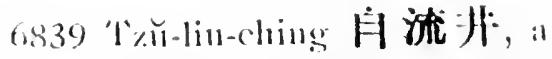
pent-town near shê-humg H., Ssĭch' n:m.

\section{犋}

6840 Tะŭ 筫 T\%u1。

[265] $1^{\circ}$ C.I.C. in the Ch nannan-Yung-ning circ., Ssŭch nan; Lat. $29^{\circ}$ $52^{\prime}$, l.ong. $104^{\circ} 52^{\prime}$; Ming, Tzŭ| H.; Han, 1\%и-chung | 中p; $P$. Chou, P'an-shil 盤不. $2^{\circ}$ (ols.) T"ang and Sung, C. 3 li $\mathrm{N}$. of the above.

ostl T\%ü-chung | 中, see Nos. 1650,0840 .

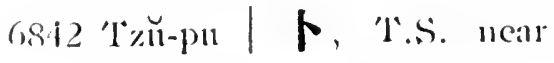
Io C., Kansul.

6843 Tž̆-yang | 陽 'T'sz'-yang,

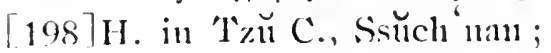
I,att. $30^{\circ} 15^{\prime}$, I,omg. $104^{\circ}$ $11^{\prime}$.

\section{紫}

6844 Tz̆̆-chên 紫宸, see No. 5822.

6845 T\%й-ch'i | 溪, see No. 291. $68+5{ }^{A}$ [\%苂-chin | 禁, see No. 5822 .

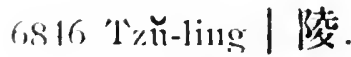

$1^{\circ}$ Chîn near Huai-ch ing. Iill, Honlan.

$2^{\circ}$ (obs.) P. Chou, a town S.IV. of ching-chon Fin, Hupeh.

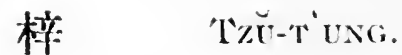

6S47 T\%u-mêng | 蒙, (ols.) J.igo, II., Fi. of I,i:1o-yang II., Shingking.

6848 'Trii-ni | 泷, ssŭ in Sllun1tê H., Kuangtung.

6849 'Tž̆-yang | 陽, 'Tsz'-yang, [133]H. in Hsing-an Fn, Shensi ; Lat. $32^{\circ} 27^{\prime}$, Long. $108^{\circ} 46^{\prime} ; H a n$, Hsüırang 洵陽； T ing, Hanvin 漢陰.

\section{䄰}

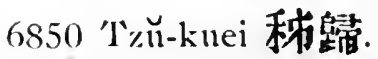

$1^{\circ}$ (obs.) Sung and Yürm, H., S. of Knei C., Hupeli. $2^{\circ}$ See No. 3454.

\section{子}

6851 Thŭ-hung 子洪, chên ncar ch'i H., Shansi.

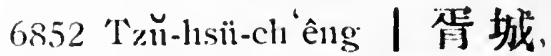
(obs.) formerly a town near Hsing-kno C., Hupel.

\section{样}

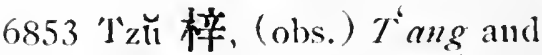
Sung, C. near 'T"m!ngch' "uan Fu, Ssŭch' uan.

6854 'T\%̆̆-t'ung | 潼, 'Tsz'-tıng. $[.192] 1^{\circ}$ H. in Mien C., Ssı̆ch nan; Lat. $31^{\circ} 37^{\prime}$, Jong. $105^{\circ} 16^{\prime} ; \mathrm{Han}$, H. in Kuang-han 㢈 漢 chiin; Tsin, chiun and H. I,iang 梁 cuov; S. Sung and $S$. Ts'i, 
梓

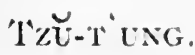

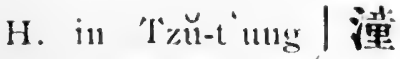
chiin, Sui, H. in I'"11-an

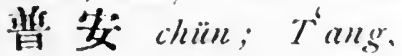
Yüan and Ming, H. in Chien 钢 $\mathrm{c}$.

$2^{\circ}$ See Nos. 850, 4433, 6795.

\section{萑}

6855 '\%崔, (obs.) Han, H. 40 li N.L. of Cli angch'ing H., Sliantung. See No. 5680 .

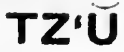

束

6856 1\%”萑-chon 束州、(obs.) I/an, a town near Hochien fiu, Chilsli.

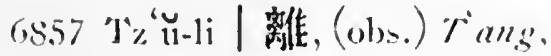
H. near 'Turfan.

6858 'T\%“̆̆-lin| 林, chên nean Mei H., Sliensi.

\section{鄞}

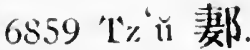

$1^{\circ}$ (obs.) S. of San-t ai H., Ssüch'uan; Han, S. Sung and $S . T s^{i} i, \mathrm{H}$. in Kuang-lian 庵漢 chiin.

$2^{\circ}$ See Nus. 5393, 5585.

$$
\text { 淡 }
$$

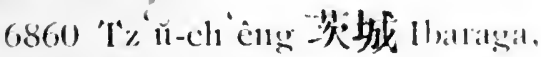
Ken in Japan! : chict town Mito.
碳

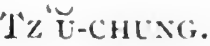

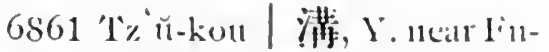
p’ing H., Clinhli.

\section{慈}

6862 '1\%“㴽, sec No. 583.

6863 'T\%“ “u-ch'i | 笑谷 'T's\%'-ki, II. in Ning-po Fin, Cleclikiang; Lat. $30^{\circ} 01^{\prime}$, Long. $121^{\circ}$ $17^{\prime}$; Han, Kott-chang 句 触; T゙ang, Sung, Y'üan

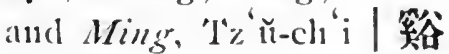
$\mathrm{H}$.

6864 'T\%“̆̆-jên | 仁,(obs.) I.iro, H. in the county of the Ongniods, N. Clinhli.

6865 'Tz ù-li | 利 'l's\%-li, H. in I, C., Human; I at. $29^{\circ}$ $22^{\prime}$, Long. $111^{\circ} 05^{\prime} ; \mathrm{W}^{\prime \prime}$, I,on-chung 嘍中; Tsin.

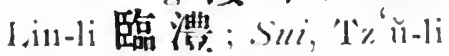
| 利。

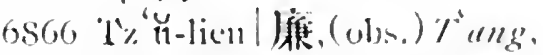
H., IV. of Hna C., Kinnugtu11y.

6867 'T\%' chün in P’ing-inl 本努 cire., Corea ; Iat. $39^{\circ} 34^{\prime}$. I.ong. $126^{\circ} 30^{\prime}$.

\section{碳}

6868 '个\% ‘̆ 磁'Tsz', C. in Kuangp'ing Fu, Chihli; Lat. $36^{\circ}$ $25^{\prime}$, I,ong. $114^{\circ} 33^{\prime} ;$ Ts in, Han-tan 仍鄲; 2nd Wei, I,in-shui 監水; $P$. Chou,

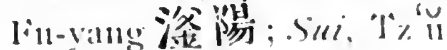
$1 \mathrm{c}$.

$68699 \%$ ' near Slian C.. Honan. 
Uchu1nuchin, see No. 7172 .

Ujukling, see No. 7196.

Ukol, see No. 7176.

Ulan Chap, see No. 7177.

Ulianglai. see No. 7182.

Uliasutai, see No. 7180 .

Uliasuto, see No. 7181.

Ung-11ga1, see No. 7044.

Ung-yuen, see No. 7041.

Unken, see No. 4451.

Urad, see No. 7178.

Urawa, see No. 5305.

Urga, see No. 3332.

Urgendj, see No. 7117.

Urustun11dan, see No. 7185.

Urusutai, see No. 4765.

Ush, see No. 7192.

Usliaktar, see No. 7190.

Ushi, see No. 7196.

Uzgend, see No. 1605.

Vladivostock, see No. 2376 .

Vocha11, see No. 7836.

Vuglin, see No. 7134.

Vuju, see Nos. 1023, 2154.

\section{WA}

\section{互}

6870 Wa-hsi-ti 玞多的 Wassit, a town situated between the Euphrates and Tigris, S. of Lat. $32^{\circ}$.

6871 Wa-lu-chih | 舊之, (obs.) Ming, ssü $280 l i \mathrm{~N}$. of Yung-ning T.F., Yünnan.

6872 Wa-shu-cl 'ï-têng | 述 曲 登, T.S. near Mien-ning H., Ssŭch' 'uan
6873 Wa-shn1-clı' ung-hisi | 述 桨喜, 'T.S.

6874 Wa-sln1-kêng-p'ing | 述 更本, 个.S.

6875 Wa-slu1 - kuo-lung | 述 嘓嚨, ז.s.

6876 Wa-sh11-1na-1i | 述 麻 里, '.S.

6877 Wa-slu1 - mao-liao | 述 毛了, T.S.

6878 Wa-sli11-sê-t'a | 速色 他, 'T.S.

6879 Wa-sluu-yï-k'ê | 述餘 科, T.S.

The above seven 'T.S. are all near Ta-chien-1u, Ssŭcli'nan.

6880 Wa-t'ing | 亨, a post-town near Lung-tê H., Kansulı.

6881 Wa-tı | 都, T.S. near Mien-ning H., Ssŭch'nan.

6882 Wa-tzŭ-yao |子窟; ss̆̆ near Chien-li H., Hupeh.

6883 Wa-wei | 尾, 'T.S. near Mien-ning H., Ssŭch' 'ran.

6884 Wa-yün | 雲, a post-town near Ching C., Kansuls.

\section{WAI}

外

6885 Wai-chiang 外江, see No. 7436.

6886 Wai-hwang | 黃, (obs.) formerly a town near Clı ênlin H., Honan. 
外

WAI-KAN-SU.

6887 Wai Kan-su | 甘藏 Outer Kansuh; that part of the province of Kansuh lying beyond the Great Wall, and extending from the Chia-yü pass to Kurkurausu. Now formed into a separate province under the name of the "New Dominion." See No. 2736.

6888 Wai-kang | 岡, chên near Chia-ting H., Kiangsu.

Wakayama, see No. 2048.

\section{WAN}

\section{萬}

6889 Wan 萬 Wan.

$1^{\circ} \mathrm{C}$. in $\mathrm{Ch}^{\prime}$ iung-chou $\mathrm{F} u$,

Kuangtung; Lat. $18^{\circ}$ $49^{\prime}$, Long. $112^{\circ} 20^{\prime}$; Han, Chu-yai 殊崖; Sui, Wan-an 发.

[528] $2^{\circ}$ H. in $K^{\prime}$ uei-chou $F u$, Ssŭch 'uan; Lat. $30^{\circ} 57^{\prime}$, Long. $108^{\circ} 32^{\prime}$; Minor Han, Wan-p'u | 浦; P. Chou, An-hsiang 安 拫; T'ang, and $S$. Sung, Wan | C.

6890 Wan-an | 㚣 Wan-ngan.

[208] $1^{\circ} \mathrm{H}$. in Chi-an Fu, Kiangsi ; Lat. $26^{\circ} 38^{\prime}$, Long. $114^{\circ} 54^{\prime}$; $W u$, Hsinhsing 新興; S. T ang, Wan-an 安.
萬

WAN-NIEN.

$2^{\circ}$ Chên near Hung-tung H., Shansi.

$3^{\circ}$ Chên, near Hui-clıou Fin, Anluvi.

$4^{\circ}$ Chên Chiang-lê H., Fulkien.

$5^{\circ}$ (obs.) S. Sung, military district in Kuang-hssi 廣 䲨 circ.

$6^{\circ}$ See Nos. 1870, 4014, 6889, 7829.

6891 Wan-ch'êng | 搏 Wanching, T.C. in T'ai-p'ing Fu. Kuangsi ; Lat. $23^{\circ} 02^{\prime}$, Long. $107^{\circ} 08^{\prime} ; T^{\prime}$ ang, Wan-ch'êng | 承 C.

6892 Wan-ch'ing | 頃, H. in $\mathrm{Ch}^{\prime}$ 'üan-1o 全羅circ., Corea.

6893 Wan-ch'üan | 全 Wantsiuen, H. in Hsüan-hua Fu, Chilili; Lat. $40^{\circ} 45^{\prime}$, Long. $114^{\circ} 38^{\prime}$.

6894 Wan-ch'üan | 泉 Wantsiuen, H. in $\mathrm{P}^{6} \mathrm{u}$-chon $\mathrm{F} u$, Shansi ; Lat. $35^{\circ} 25^{\prime}$, I fong. $110^{\circ} 46^{\prime} ;$ Han, Fên-yin 汾 陰; T'ang, Wan-chüin 家.

6895 Wan-ch'un | 春, see No. 7020 .

6896 Wan-li | 里, a post-town near Ch'êng-tı Fu, Ssŭch'uan.

6897 Wan-liu | 流, a post-town near Kuei C., Hupeh.

6898 Wan-nien | 年 Wan-11ien. 
雱 WAN-NIEA

$[103] 3^{\circ} \mathrm{H}$. in Jao-chon Fu, Kiangsi: Lat. $28^{\circ} 40^{\prime}$. I.ong. $116^{\circ} 51^{\prime}$.

$2^{\circ}$ See No. 2691.

6899 Wan-pei 早, (obs.) \% ang, C. in Ssurch 'uan.

6900 Wan-p " 1 溥, see No. 6890.

6901 Wan-shan | 望, chên near Huai-ch'ing Fu, Honan.

6902) Wall-shon | 壹.

$1^{\circ}$ Chên in Chiang-tu H., Kiangsu.

$2^{\circ}$ (obs.) T゙ang, H., S.ll. of Clinng-cling Fin, Ssüchinan.

, ,903 Winl1-t a| 塔, ihên near Cli'ang-chon Fu, Kiangsu. ront Wan-tan | 丹. (obs.) r゙ang, C. near Shun-t ien Iri, Chilhli.

6005 Wan-tsai | 載 Wan-tsai, [524]H. in Yüan-chon Fu, Kiangsi: Latt. $28^{\circ} 05^{\prime}$, Long. $114^{\circ} 20^{\prime}$ : " $" \prime \prime$, Vang-lê 陽樂; Tsin,

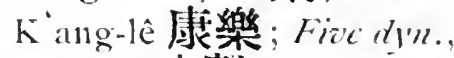
Wan-tsai | 䑤化.

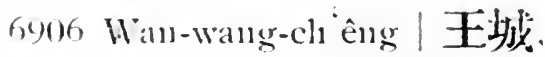
(obs.) formerly a town near Knei-tung H., Human.

6907 Nan-yai | 崖, (obs.).Sung, C. near 'T"ai-p'ing Fu, Knangsi.

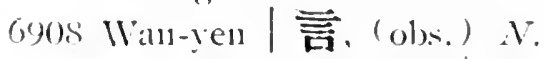
Hei, H. S.ll. of Cli angp’ing C., Chilili.
潾

IVAX-CH'

\section{完}

6909 IIan 完 Wan, H. in Pan[122] ting Fu, Clihli; Iat. $38^{\circ}$ $50^{\prime}$, L ollg. $115^{\circ} 13^{\prime} ; T^{\circ} \mathrm{in}$. Clioir-ni 曲遡; Han, P'ejping 北平; Kin, Wan | c.

\section{皖}

6910 Wan 皖， see Nos. 55,69. 2237.

6911 Wan-ch"êng | 城, see No. $223 \%$.

6912 Wan-chiang | 汭, see No. 69.

\section{多}

6913 Wan 宛, see No. 4640.

6914 Wan-cli'êng | 城, a posttown near Nin1-yang I'u, Honan.

6915 Wan-chin | 邱, see No. 467.

6916 Wanthon | 句, see No. TiT2.

6917 Wan-ling | 陵, see Xos. 2924, 4696.

6918 Wan-p ing | 平 Wan-ping, or Ynen-ping, H., forming with Tallising $H$, the prefectural city of Slnun-t ien. Chiluli; Lat. $39^{\circ} .57^{\prime}$, I, tong. $116^{\circ} 29^{\prime}$; orig., Y'11-t11 幽都; Licro, Wan-p ing.

6919 Wan-wên | 溫. see No. 7524.

\section{灣}

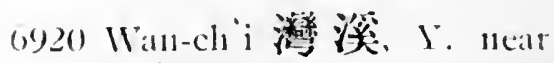
Ch'ien-chon ' 1 '., Hunan. 
6921 Wan-chih | 竦, chên near Ning-kuo H., Anluni.

6922 Wan-tien | 解, W.c. near [171].Mien-ning 'T. \'ünnan: I at. $24^{\circ} 28^{\prime}$, I,ong. $99^{\circ}$ $33^{\prime}$

6923 Wan-t on | 領, chenn near Yang-chou Fu, Kiangsu.

Wall-ching, see No. 6891. Wan-ping, see No. 6918. Wall-ngall, see No. 6890. Wan-tsinen, see Nos. 6893 , 6894.

Wăı, see Nos. 7007, 7018. Wăn-chang, see No. 7009 . Wăn-chau, see No. 7022. Wăı-chuen, see No. $+455^{\text {". }}$ Wăı-hi, see No. 7036.

Wăn-hiang, see No. 7033 . Wăn-kiang, see No. 7020 . Wănllngan, see No. Joos. Wăı-shan, see No. 7012. Wăn-shlang, see No. 7016. WV̆ll-shwui, see సo. 7013. Wăn-tăng, see No. 7015. Wăn-yang, see No. 7031.

\section{WANG}

\section{王}

6924 Wang-ch'êng 王城. (oł心) Han, a town W. of Honan fin, Honan.

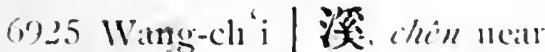
'I"uncr-lisiangr II., Chel1kiang.

6926 Willm-chia | 关.

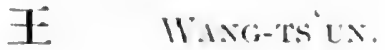

$1^{\circ}$ Y. near Huaj-in l:u Kiangsu.

$2^{\circ} \mathrm{Y}$. near Vien-11ing H. sintich nan.

6927 Wang-chial-pao | 莨 堡。 'I.S. near Ta-t ung H., Kallsul.

6928 IVang-chiang-ching | 江. 渓, chên near ch"ing-t 'ien H., Chehriang.

6929 Wang-chi iao / 欈, chên near Ching-yang H., Shensi.

6930 Wang-ch ii | 曲, chên near I, 11-ch'êng H., Shansi.

6931 Wang-chning | 生, ihen near Ch'êng-ch'êng H., Shensi.

6932 Wang-kan | F., ssu near Chi-ch'i H., Inhui.

6933 Wang-k nai 快, chénn nua Fin-p ing H. Chihli.

$693+$ Mang-kull1g | 光. Y. near Ch'in C.. Kuangtumg.

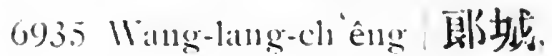
(obs.) formerly a town near Han-tan H., Chiluli.

6936 Wann-shan | 山. (obs.) Lians, al town N. of Hsiangvin H., Hunan.

6937 Wang-t ai | 台, a posttown near Yen-p ing $F u$, Fullien.

6)3s Milly-ti | 第, hinn near Yüiı1-ch ü H., Shlalus.

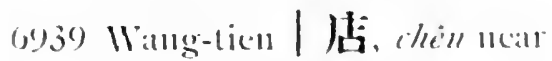
Hati-yen H., Chelskiang.

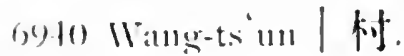


王 Wang-ts'un.

$1^{\circ}$ Chên near Ching C., Kansuh.

$2^{\circ}$ Ss ̌̆ near Yung-shun Fit, Hunan.

\section{望}

6941 Wang-cliang 望江, Wangkiang.

[528] $1^{\circ} \mathrm{H}$. in An-ch'ing Fit, Anhui; Lat. $30^{\circ} 15^{\prime}$, Long. $116^{\circ} 40^{\prime}$; orig., Ta-lei-ch'ih 大霄池; $T \sin , \mathrm{H}$ sin-yell 新结; Sui, Wang-cliang | 汀., T'ang, Cliih 智 $\mathrm{C}$.

$2^{\circ}$ (obs.) Tang, H. in

Kueicliou.

6942 Wang-hai | 海, see No. 6447.

6943 Wang-kao | 高, ss̆̌ near Yang-chiang T., Kuangtung.

6944 Wang-sung | 松, (obs.) $W$. Han, H. near Yen-an Fin, Shensi.

6945 Wang-t'ing | 亭, ssü in Chin-knei H., Kiangsu.

6946 Wang-ts'ai | 蔡, see No. 5512.

6947 Wang-tu | 都 Wang-tı.

[57] $1^{\circ} \mathrm{H}$. in Pao-ting $\mathrm{Fu}$, Chihli; Lat. $38^{\circ} 45^{\prime}$, Long. $115^{\circ} 14^{\prime} ; T^{\prime}$ ang, Wang-tu 都; Kin, Yïan and $M i n g$, $\mathrm{Ch}^{\text {'ing- }}$ tu产都。

$2^{\circ}$ (obs.) Han, $T \sin$ and W. Wei, H. $30 l i$ N.W. of the above.
威

WEI.

$3^{\circ}$ (obs.) Liao, H., S. of Lu-lung H., Chihli.

6948 Wang-yüan1 | 垣.

$1^{\circ}$ (obs.) Han, H., N.W. of $\mathrm{Ch}^{\prime}$ in C., Kansuh.

$2^{\circ}$ (obs.) formerly a town near Yüan-ch' ii $\mathrm{H}$., Shansi.

6949 Wang-yün | 雲.

$1^{\circ}$ (obs.) Liao and Kin, H., N. of Ch'ih-ch' êtug H., Chihli.

$2^{\circ}$ See No. 4197.

Wang-kiang, see No. 6941. Wassit, see No. 6870.

\section{WEI}

\section{威}

6950 Wei 威 Wei.

[10] $1^{\circ} \mathrm{H}$. in Kuang-p'ing $\mathrm{Fu}$, Chihli; Lat. $37^{\circ} 05^{\prime}$, Long. $115^{\circ} 05^{\prime}$; Han, Ch' ih-chang 哌章; Sui, Ming-shui 洛水; Sung, Wei $\mid \mathrm{C}$.

$2^{\circ}$ Chên near P`ing-lo H., Kansuh.

$3^{\circ}$ (obs.) Sung, C. in Ssŭch'uan; Lat. $31^{\circ} 25^{\prime}$, Long. $103^{\circ} 41^{\prime}$; T' Tang, $^{\prime}$ Wei 維 C.; now absorbed into Mou C.

$4^{\circ}$ See Nos. 2227, 2266, 6961. 
威 WEI-CH'U.

6951 Wei-ch'ul楚, see No. 1404.

6952 Wei-hai-wei | 海衛, a posttown near Wên-têng $H$., Shantung; I at. $37^{\circ} 30^{\prime}$, Long. $122^{\circ} 10^{\prime}$. 'I'he port. and a portion of the adjoining territory were leased from China by Great Britain, the duration of the lease being dependent on the tenure of Port Arthur by Russia.

6953 Wei-hrsin | 信, a post-town near Chên-hsiung C., Vünnan.

6954 Wei-jung | 犮.

1。 (obs.) Sung, fort N.E.: of An-ting H., Shensi. $2^{\circ}$ (obs.) 40 li S. of Chingning C., Kansuh ; Sung, pao in Tê-shun 德順 military district; Kin, H. in Tê-shun德順 C.

6955 Wei-lung | 龍, (obs.) 410 li S.E. of Hsi-ch'ang H., Ssŭch'uan; Yüan, C. in Tê - ch'ang 德昌, circ.; Ming, ssŭ in Ssŭch' uan.

6956 Wei-lung-chou | 龍 州, T. S. near Hui-li C., Ssŭch 'uan.

6957 Wei-ning | 窗 Wei-ning, [258] C. in Ta-ting Fu, Kueichou; Lat. $26^{\circ} 43^{\prime}$, Long. $104^{\circ} 15^{\prime}$.

6958 Wei-p'ing | 平, chên near Sui-ch' iang H., Chehkiang.
葦 Wlí-CH'III.

6959 Wei-shêng | 勝

$1^{\circ} \mathrm{A}$ post-town near $\mathrm{Cl}^{\prime}$ ien C., Shensi.

$2^{\circ}$ See No. 1098.

6960 Wei-wu | 武, see No.1871. 6961 Wei-yüan | 㖣 Wei-yuen. [362] $1^{\circ}$ 'T'. in $P^{\prime}$ u-êrlı F'u, Y'ünnan ; I at. $23^{\circ} 29^{\prime}$, I long. $100^{\circ} 55^{\prime}$; T'ang, Yinslêng 銀生Fu； Yüan, Wei-yüan厂遠 C.

[495] $2^{\circ}$ H. in Chia-ting Fu, Ssǔch 'uan; I at. $29^{\circ} 39^{\prime}$, I.ong. $104^{\circ} 40^{\prime}$; Sui, Wei $\mid C$.

$3^{\circ}$ Ss š̆ in Y 1 -yü H., Shansi.

尉 and 蔚

[See under the syllable vï.]

\section{常}

6962 Wei-chou 荤 州, (obs.) Ming, so $220 l i \mathrm{~S}$. E. of Ling C., Kansul.

6963 Wei-chuang | 左, chên near Chao-yi H., Shensi.

6964 Wei-fan | 番, T. S. near Kuei-yang Fu, Kueichou; Ming, Wei-fan | 番 ssü.

\section{漟}

6965 Wei-ch' uan 湋川, see No. 1817.

\section{蔁}

6966 Wei-cl'ill蕫池, (obs.) N. Wei, H., N. W. of P'ingyao H., Shansi. 


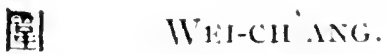

漶

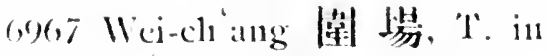
Ch' êng-tê I'n, Chihli.

\section{得}

6968 11 ci 衛.

$1^{\circ}$ (obs.) $C h_{0} l l$, fendal state E. of Wei-hui Fu, Honan.

$2^{\circ}$ (obs.) E. Han, feudal state in Shantung.

$3^{\circ}$ See No. 6971.

6969 Wei-chên | 德. (obs.) Sung and Kin, H. $10 \mathrm{li}$ E. of Lu-yi H., Honan.

6970 Wei-ch'êng | 城.

$1^{\circ}$ Chền in Chin-shlan H.. Kiangsin.

$2^{\circ}$ See No. 5266.

6971 Wei-hui | 輝 Wei-lowil, [496] Fu in the Ho-pei circ., Honan; forming also the district city of Chi ; Iat. $35^{\circ} 28^{\prime}$, Long. $114^{\circ} 16^{\prime}$; Chou, eapital of $\mathrm{K}$. Wei I: C. Wars, in K. Wei 魏: $7 s^{\circ}$ in, in Ho-tung 河 東 chiun; Han, in Ho-nei 河內 chün; Hei, Chao-kê 朝歌; Tsin, Chi 没 $2 n d$ Hei, Yi 載 C.; T"ang and Sung. Wei | C.; Ming, Wci-hui | 狏 Iin.

6972 Wiej-kunger 公. Y. in Inner Nongoiia; Lat. $41^{\circ}$ $30^{\prime}$, I.ong. $99^{\circ}+0^{\prime}$.
洧

IVEI-CH'U.IN.

6973 Wei-lu | 搵, a town in Inner Nongolia; Lat. $41^{\circ}$ $30^{\prime}$, I, ong. $99^{\circ} 2^{\prime}$.

097t Wei-tzŭ|子, chên neạr Ch'in C., Kansul.

6975 Wei-vi| 邑, see Nos. 2957, 7470.

6976 Wei-yüan| 原 [Chi-chwang in Williams's Map], a town in Inner Mongolia; I att. $42^{\circ}$, I tong. $100^{\circ}$.

6977 Wei-yỉan | 源, a posttown near Wei-hui fin, Honan.

\section{位}

6978 Wei-fêngr 位慧. (obs.) t’ang. H. near Ch'ingryang fill, Kansuh.

\section{微}

6979 Vei-tzun 微子、 chên nean I,n-ch'êngr H., Shansi.

6980 Wei-tzŭ-ch'êng $\mid$ 子城. (obs.) Shang, a town N.E. of 'l'ung-ch'ang Fin. Shantung.

6981 Wei-yang | 晹, (obs.) Three Ks., a town W. of Cliu-shan H., Hupeh.

\section{洧}

6982 Wei 洧, see No. 6983.

oys3 Wei-ch"nan | 川, Wei[405]chnen, H. in $\mathrm{K}^{\prime}$ ait-fên Fin, Honan; Ialt. $34^{\circ} 18^{\prime}$. I.o11g. $11 t^{\circ} 05^{\prime} ; .5 .1$. 
Ch' ${ }^{\circ i}$-wei 曲 |: /lan, Hsin_chi 新没; ‘゙an!

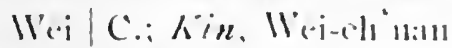
| 川.

\section{沙}

6984 Wei 渭, see No. 3521 .

698.5 Wei-ch'êng | 城, see No. 2695.

6986 Wei-chi | 溪, (obs.) Tang, H., 120 li 11 . of Chih-chiang $\mathrm{H}$., Hunan. 6985 Wei-nan | 南 Wei-nan, [498]H. in Hsi-an Fin, Shensi; Lat. $34^{\circ} 29^{\prime}$, I.ong. $109^{\circ}$ $27^{\prime} ;$ Han, Hsia-kne! 下 卦 and Hsin-fêng 新豐.

6988 Wei-yüan | 源 Wei-ynen, [498]H. in Lan-chon Fin, Kansuls; Lat. $35^{\circ} 08^{\prime}$, Lony. $104^{\circ} 16^{\prime} ;$ Han, Shon-yang 首陽：Sui, Wei-rian 源 $\mathrm{H}$.

6989 Vei-vian | 原 chïn in p'ing-an 平桨 circ. corea.

\section{魏}

6990 IVei 魏.

1 (obs.) C. Wars, K. comprising lower Shansi and part of Honan.

$2^{2}$ (obs.) Han, chïn comprising partis of Cliihli and Honan.

$3^{\circ}$ (obs.) Han, II. in Honan : Lat. $36^{\circ} 25^{\prime}, 1,011 \mathrm{~g}$. $115^{\circ} 06^{\prime} ;$ mris., Yiinan-

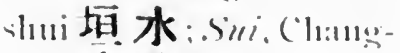
产漳陵。

to see Nos. 225, (0)6.5.

6991 Wei-an | 安, see No.6381.

6992 IVei-ch'êng | 娍.

$1^{\circ}$ Chển near Mn-hsimg H., Shansi.

$2^{\circ}$ A post-town near Changming H., Ssinch "иan.

6993 Wei-ch $\mathrm{i} /$ 其, (obs.) 11. Han, Marq. S. of I.an-shan H., Shantmug.

6994 IVei-chia-chai|家寨, chîn near I,int 'ung H., Shensi.

6995 Wei-chia-wan | 家灣、si near I.jn-ch ing C C.. Sthantming.

5996 IV ei-kung | 公, then near $\mathrm{Ch}^{i}$ ien C., Shensi.

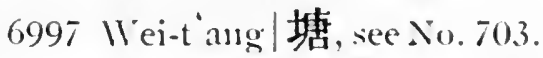
6998 llei-yeh | 娜, see No.225.

隗

6999 IVei-ts'un 隗村, Y'. near Huo-chia H., Honan.

唯

200011 ei-t ing | 亭, thin near su-chon lin, Kiangutr.

\section{維}

$700111 \times$ ei 維.

$1^{\circ}$ (obs.) $7^{\prime} a \mu$, , ( . . . . II. of Min-cli unan H., șinch nan. 
維

$2^{\circ}$ (obs.) Five dyn., C., W. of Wên-ch" uan H., Ssı̆ch'uan.

$3^{\circ}$ (obs.) Liao, C. in Shingking.

$4^{\circ}$ See No. 6950.

7002 Wei-hsi | 西 Wei-si, 'T. in [233] Li-chiang Fu, Xünnan ; Lat. $27^{\circ} 30^{\prime}$, Long. $100^{\circ}$ $05^{\prime} ; T^{6}$ ang, in Nan-chao 南詔 chïn; Sung, land of the Chia 菲氏 tribes; Yïan, Yung-ning 永耍; Ming, Yung-11ing 永察 Fu.

\section{濰}

7003 Wei 濰 Wei, H. in Lai[342] chon Fin, Shantung; Iat. $36^{\circ} 46^{\prime}$, Long. $119^{\circ} 20^{\prime}$; Han, Chiao-tung 晊東; Sui, Pei-hai 北海; Sung, Kin and Yüan, Wei C.; Ming, Wei | $\mathrm{H}$.

\section{䍃}

7004 Wei 瓷.

$1^{\circ}$ (obs.) T'ang, C'. near Hsï-chon Fu, Ssŭch'uan; Sung, Wei | H. $2^{\circ}$ (obs.) Tang, C. in Annain.

\section{畏}

7005 Wei-wu-êrh 異匹兒 (or, Hui-ho 可 紀: or, Hui-hu 间鶻，or，Wu-hu 烏護，
交

WÊN-AN.

or, Yüan-ho 袁紀) Ouigour, a tribe of Mongols ; their county was also called Kao-ch'ê 高車.

\section{椳}

7006 Wei 椳, (obs.) T Tang, C. near Nan-ning Fu, Kuangsi.

Wei-chin, see No. 7739. Vei-chuen, see No. 6983. Wei-hwui, see No. 6971. Wei-shi, see No. 7737. Wei-si, see No. 7002. Wei-yuen, see Nos. 6961, 6988.

\section{$W \hat{E} N$}

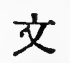

7007 Wên 文 Wăn.

[71] $1^{\circ} \mathrm{H}$. in Chieh C., Kansulh ; I at. $35^{\circ} 55^{\prime}$, Long. $105^{\circ} 18^{\prime} ; T^{\prime}$ ang, Ch ${ }^{\prime} \ddot{\mathrm{i}}-$ shni 曲水; Han, Yinp'ing-tao 陰平道; T'ang and Sung, Wên | C.; Ming, Wên | H. $2^{\circ}$ (obs.) $W$. Han, H. near Fêng-t'ien Fu, Shingking.

$3^{\circ}$ (obs.) S. W. of Tu11glan C., Kuangsi ; Sung, 1st 'T.C. in Ch'ing-ÿüan 慶樾 $\mathrm{F} u ; 2 n d, \mathrm{C}$. in Knang-1ısi 廣西 circuit. 7008 Wên-an | 安 Wăn-ngan. 
文

$1^{\circ} \mathrm{H}$. in Shun-t'ien Fu, Chihli; I at. $38^{\circ} 53^{\prime}$, Long. $116^{\circ} 34^{\prime}$; Han, Wên-an | 发 $\mathrm{H}$.

$2^{\circ} \mathrm{A}$ post-town near Yench' '1an H., Shensi.

7009 Wên -ch'ang | 昌 Wănchang, H. in Ch' iung-chou F.u, Kuangtung; Lat. $19^{\circ}$ $36^{\prime}$, Long. $110^{\circ} 43^{\prime}$; Han, Tzŭ-pei 紫 具; T'ang, W ên-ch' ang 昌.

7010 Wên-ch'nan | M chïn in Hsien-ching 咸鏡 circ., Corea.

7011 Wên-hıi |" 曾, ssŭ near Ling-shan $\mathrm{H}$., Kuangtung. 7012 Wên-shan | U IVăn-shan.

$1^{\circ} \mathrm{H}$. forming the prefectural city of $\mathrm{K}^{\prime a i}-\mathrm{hu}$, Yünnan; Lat. $23^{\circ} 10^{\prime}$, Long. $104^{\circ} 50^{\prime}$.

$2^{\circ}$ See No. 4498.

7013 Wên-shui| 水Wăn-shwui, [501] H. in T'ai-yüan Fu, Shansi ; Lat. $37^{\circ} 29^{\prime}$, I, song. $111^{\circ} 58^{\prime} ; T s^{\circ}$ in, 'Ta-ling 大陵; Sui, wên-shui | 水; T'ang, Wu-hsing 武興.

7014 Wên-tê | 德, ssünear K'aichien $\mathrm{H}$., Kuangtung.

7015 Wên-têng | 登 WWăn-tăng. $1^{\circ} \mathrm{H}$. in Têng-chou Fu, Shantung; I at. $37^{\circ} 12^{\prime}$, T.ong. $122^{\circ} 30^{\prime}$; orig., Pu-yeh 不佼; N.Ts' $i$, Wên-têng | 登.

$2^{\circ} \mathrm{Y}$. near the above.
温 WÊN-CHIANG.

$7015^{A}$ IVên-yi | 義 H. in Chungch'ing 忠清 circ., Corea. 汶

7016 Wên-shang | 上 Wăn[502] shang, H. in Yen-chou Fin, Shantu1ng; Lat. $35^{\circ} 50^{\prime}$, Long. $116^{\circ} 40^{\prime}$; S. A., centre of K. Lu魯; Han, $\mathrm{P}^{\prime}$ ing-lu 平陸; $N$. Ts $\mathrm{s}^{6} i$, Lê-p 'ing 樂平; T'ang and Sung, Chung-tu 中 都 H.; Kin, IVên-shang $\mid$ 上 $\mathrm{H}$. 7017 Wên-yang | 晹, see Nos. $1454,6176$.

\section{溫}

7018 IVên 溫 IVăn.

[148] $1^{\circ}$ H. in Huai-ch'ing Fu, Honan ; Lat. $35^{\circ} 06^{\prime}$, I ong. $113^{\circ} 13^{\prime}$; Chou, Inperial domain; T⿱ang, P'ing-ch'van 本 川; Sui, Tang, Sung, Kin, Yüan and Ming, IVên $2^{\circ}$ (obs.) Han, Tsin and N. Wei, H. 30 li S. IV. of the above.

$3^{\circ}$ Sse Nos. 83, 1125, 5428, 7022 .

7019 Wêi-chia | 管, chên in I i11ch' nan H., Kiangsi.

7020 Wên-clıiang | 江. Wănkiang.

[307] $1^{\circ} \mathrm{H}$. in Ch'êntg-tu F'u, Ssŭcli' 'ıan; I at. $30^{\circ} 40^{\prime}$, Long. $103^{\circ} 55^{\prime}$; Han, 
㴵 WĤN-CHIANG.

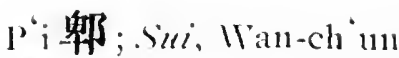
萬春; T゙ang, Sumg, lïrn and Ming. WV̂ेchianer | 江 $\mathrm{H}$.

$2^{c}$ (obs.) S. Sung, H. near

Cli ii-ching Fu, Yännan.

7021 Wèn-clíin | 池, (obs.) Tang, H., S. E. of I,ing C., Kansul.

7022 Wên-clou- | 州Wăn-chan, [330] Fin in the Wên-Ch' 11 circ., Chehkiang; forming also the district city of Yungchia: Lat. $28^{\circ} 01^{\prime}$, I.ong. $120^{\circ} 31^{\prime}$; one of the ports open to foreign trade; S.A. and C. IV Trs, in Yüeh 越; $T s^{\circ} \mathrm{in}$, in Min-chnng 閩中 chiin; Han, K. Tung-on 東陨； Tsin， lung-chia

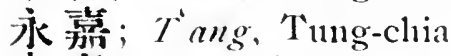
束嘉 and IVên | C..; S Sung. Jui-an 瑞安: lring, Wênchon | 州 Fu.

7) 2.3 Wên-Ch"n | 處, circ. in Chehkiang comprising Wên-chon Fu and Ch' ${ }^{\prime}$ chon Fu.

7024 IVên-ch'ïan| 泉, chênnear Ning C., Kansul1.

7025 W ên-hisiu| 秀, seeNo.3734. 7026 Wên-ling | 䫈, see No. 1586.

7027 Wên-ling | 陵, see No. 1491.

7028 Wên-ma|麻, see Nos. 1876 . 3822 .

7029 Wên-shui | 水, ss̆̌ near I.u-ch nan H., Kuangsi.
荓 ŴAN-HSI.

7030) Wên-sn | 䈹 C. I. C. in Aksu circ., New Dominion. ['The city of Aksu].

7031 Wên-yang | 陽Wăn-yans, chiün in Clunng-ch'ing 出 清 circ., Corea; Lat. $37^{\circ}$ $10^{\prime}$, I.ong. $126^{\circ} 41^{\prime}$.

\section{媪}

7032 Wên-wei or Ao-wei 媪嫴、 (obs.) Han, H., N.F. of Kan-lan H., Kansuh.

\section{閭}

7033 Wên-hsiang 䦔䐚 Wăn[148] hiang, H. in Shan C.. Honan; I at. $34^{\circ} 38^{\prime}$, I,ong. $110^{\circ} 30^{\prime} ; \mathrm{Han}, \mathrm{H}_{\mathrm{u}}$ 湖 $\mathrm{H}$; P. Chon, |Vên-hsiang | 拫; でang, Ting 㻗C.

䦡

7034 Wên-ching 䦓津, a posttown near Kuang-ÿian $H$., Ssurch' nan.

7035 Wên-shui | 水, chên in Ch'ang-chih H., Shansi.

聞

7036 Wvên-cl'ing 聞㢇, H. in Ching-shang 磨㛠 circ. corea.

7036 "Iên-hsi | 嫦 Wăn-hi, H. [415] in Chiang C., Shansi; I.at. $35^{\circ} 25^{\prime}$. I,ong. $111^{\circ} 03^{\prime}$ : Shang, T'ung 桐 town; Ts"in, 'Tso-vi 庄邑; Han. lst, Wên-hsi | 喜; 2nd, Wêu-p $\therefore$ | 破. 
聞

WÊN-P'O.

7037 Wên-p'o | 破, see No. $7036^{*}$.

\section{棌}

7037"Wên-cll' êtny 㮽"城, Fu in Hsien-ching 战鏡 circ., Corea.

\section{WÊNG}

\section{第}

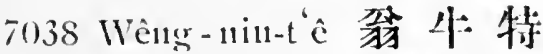
Ongniod, a tribe of Mongols to the N. of Jehol.

7039 Wêng-shan | |l, (obs.) Tang, H., N. of 'Ting-hai 'T., Chehkiang.

7 (1) ivêng-tang | 旉, ss̆̌ near Ch' ing-p "ing H., Kneichou.

7041 Wêng-yïan /源Ung-yuen, [504] H. in Shao-chou F Fil, Kuangtung; Lat. $24^{\circ} 25^{\prime}$, I,ong. $113^{\circ} 20^{\prime}$; Han, Chêng-yang 湞陽; Liang, Wêng-yüan | 源.

\section{槩}

7042 Wêng-ch'a 余盆, chên near Hua-yin H., Shensi.

7043 Wêng-cl' êng | 城, a posttown near Hun-yiian C., Sliansi.

7043 Wêrüg-ching | 津 chïn in Huang-hai 幊海 circ., Corea.
耻

WU-AN.

\section{鄢}

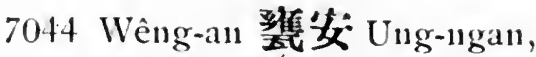
[44] H. in P'ing-yiueh C., Kueichou ; Iat. $27^{\circ}$, I,ong. $107^{\circ} 18^{\prime}$.

Whanpoa, see No. 1309.

IVladiwostock, see No. 2376.

\section{WO}

卧

7045 Wo-lung-fan 跃麦番, 'T.S. near Kuei-yang lin, Kueichon; Ming, sš̆.

\section{简}

7046 Wo-pu 焸卜, T.S. near Mien-ning H., Ssŭch' nan.

\section{倭}

7047 Wo-ch'ih 倭赤, see No. 7192 .

斡

7048 Wo-t'o-lo-êrly 翰脱羅兒, see No. 1604.

7049 Wo-tuan | 端, see No. 2057.

\section{WU}

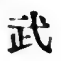

7050 W 11 武, see Nos. 321, 832, 2930, 5171, 7080. 
武

$7051 \mathrm{~W}_{11-a n}$ | 㚣 Wn-ngan.

$[308] 1^{\circ} \mathrm{H}$. in Chang-tê $\mathrm{F} n$,

Honan; Lat. $36^{\circ} 46^{\prime}$.

Long. $114^{\circ} 24^{\prime}$; Han,

IVu-an | 努 $\mathrm{H}$.

$2^{\circ}$ Chên near Kao-p'ing

H., Shansi.

$3^{\circ}$ (obs.) S. Ts' $i$, H. in

Hupeh.

$4^{\circ}$ (obs.) N. Wei, chün

S.IV. of Chên-fan H.,

Kansult.

$5^{\circ}$ (obs.) N. Wei, H. near Han-chung Fu, Shensi.

$6^{\circ}$ (obs.) T'ang, C. and

H. in Annaull.

$7^{\circ}$ (obs.) T'ang, H. near

Hsï-chou Fin, Ssĭch'nan.

$8^{\circ}$ (obs.) Lino C. in N. of Chihli.

$9^{\circ}$ See Nos. 267, 3413, 5704.

7052 Wu-clíangl昌 Wu-chang.

$1^{\circ}$ Circ. in Hupeh, con1prising Wu-ch' ang Fu.

[528]2 $2^{\circ}$ Ft in the Wu-ch' ang circ., Hupeh ; forming also the district city of Chiang-hsia ; Lat. $30^{\circ}$ $33^{\prime}$, Itong. $114^{\circ} 27^{\prime}$; orig., in $\mathrm{K}$. W11 哭; S.A., Hsia-jui 夏汭; Ts'in, in Nan 南 chïn; Han, Chiang-hsia 江. 夏; Wu Wu-ch' $W^{6}$ ang
政

WU-CHIEH.

昌; 1st Sung, Ying 趴 C.; Sui and T'ang, $\mathrm{O}$ 鄂 $\mathrm{C}$.; Five dyn., $\mathrm{V}_{11-\mathrm{cl}}$ 'ing | 清; Ming, Wu-clíang

[528] $3^{\circ}$ H. in Wu-ch' ang Fin, Hupel1; I at. $30^{\circ} 22^{\prime}$, Long. $114^{\circ} 48^{\prime} ; T s^{\prime}$ in, $\mathrm{O}$ 鄲 $\mathrm{C} . ; \mathrm{Wu}, \mathrm{Wu}$ ch'ang | 昌.

7053 Wu-ch'ê|果, (obs.) W. Han, H. in Shensi.

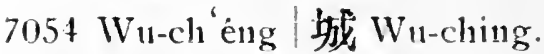
[322] $1^{\circ} \mathrm{H}$. in Liul-ch 'ing C., Shantung; Lat. $37^{\circ}$ $25^{\prime}$, Long. $116^{\circ}$; orig., fendal state $\mathrm{P}^{\text {'ing-yüan }}$ 平原; E. Han, Wnch'êng | 城; Sui, Ch'ing-lio 清河.

$2^{\circ}$ See Nos. 5178, 7102 .

7055 Wu-chiang | 江.

$1^{\circ}$ (obs.) S. Ts' $i, H$. in Ssŭch 'nan.

$2^{\circ}$ (obs.) T'ang, H. in Kuangsi.

7056 Wu-ch' iang/ 强 Wu-kiang.

$1^{\circ}$ H. in Shên C., Chihli ; Lat. $38^{\circ} 03^{\prime}$, Long. $115^{\circ}$ $56^{\prime}$; IIan, W11-sui | 隧; $7 \mathrm{sin}, \mathrm{Wu}$-ch iang $\mid$ 强.

$2^{\circ}$ See No. 268.

7057 Wu-chieh | 階.

$1^{\circ}$ (obs.) N. Wei, chün in Chieh C., Kansul.

$2^{\circ}$ See No. 832, 
武

WV-CHIH.

7058 Wu-chih | 陟 Wu-chih, [148] H. in Huai-ch ing Jiu. Honan ; Lat. $35^{\circ} 07^{\prime}$, Longr. $113^{\circ} 31^{\prime} ; 7 \sin$, Huai-yi 懷邑; Sui, Wu-chih | 䏚; Tang, Chih 揹 C.

7059 Wn-chin|勤, (obs.) T'ang. H. in Kuangsi.

7060 Wn-chin | 進 Wn-tsin, H. forming with Yang-lun $\mathrm{H}$. the prefectural city of Ch'ang-chou, Kiangsu : Lat. $3 !^{\circ} 47^{\prime}$, I.ong. $119^{\circ}$ 56'; Han, 'Tan-t'u丹徒 and $\mathrm{Ch}^{\prime}$ î.a 曲阿; Hei, Wtt-chin | 進; Liang, Lanling 阔陵。

7061 IVu-ching | 津.

$1^{\circ}$ (obs.) S. Sung, S. T'si and $N$. Wei, H., E. of Shang-ts' ai H., Honan.

$2^{\circ}$ See No. 5534.

7062 Wu-ch'ing | 声 Wu-tsing. $1^{\circ} \mathrm{H}$. in Shun-t ien Fu, Chihli; Iat. $39^{\circ} 33^{\prime}$, I.ong. $116^{\circ} 5.5^{\prime}$; Han, Yung-11! 辝奴; T゙ang， Wu-ching $\mid$ 清.

$2^{\circ}$ See Nos. 2590, 7052 .

7063 Wu-fêng | 豐.

$1^{\circ}$ (obs.) $T s i n$ and $.5 . T s^{i} i$, H. in Knangsi.

$2^{\circ}$ See No. 5059.

7064 Wu-hua| 化, (obs.) Liang, $H$., $S$. of Lien-shan $\mathrm{T}$., Kuangtung.
武 WU-KANG.

7065 Withsi | 慗, (obs.) Tsin, S. Sung and S. Ts' $i, H$. in Hsiang C., Kuangsi.

7066 Wn-hsiang | 狠 W11-hians. [31] $1^{\circ}$ H. in Cli in C., Shansi; Iat. $36^{\circ} 50^{\prime}$, I,oung. $112^{\circ} 50^{\prime} ; / 1 a n$, Nieh 涅; Tsin, Wu-lusiang I郷.

$2^{\circ}$ See No. 7669.

7067 Wu-hsicn | 仙, see No. 7069.

$7068 \mathrm{Wn}$-h1sing $\mid$ 舆, see Nos. $45,7013$.

7069 Wu-hoüan | 官. Wu-sinen, [416] H. in Hsïll-chou Fin, Knangsi ; Lat. $23^{\circ} 42^{\prime}$. I.ong. $109^{\circ} 21^{\prime} ; T^{\circ}$ ang, Wu-lisien | 们.

7070 Wu-hsieh $\mid$ 公 Village in [528] Wu-kang T., Hupch. Port of call.

7071 Wu-jung $\mid$ 几 , a post-town near Hsing-kuo C., Hupel.

2072 Wu-kang | 風 Wu-kang. [490] $1^{\circ} \mathrm{C}$. in Pao-ch'ing Fin, Hunan; Lat. $26^{\circ} 34^{\prime}$, Long. $110^{\circ} 30^{\prime}$; orig., in $\mathrm{Ch}^{\prime}$ ien-chung 點 chiun; Han, Tu-liang 都梁: S. Sung, Wukang ! 岡 military district.

$2^{\circ} \mathrm{T}$. in Huang-chon $\mathrm{Fu}$, Hupeh. The Sub-prefect resides at $\mathrm{Wu}_{\mathrm{u}}$-hsüeh [No. 7070]. 


\section{政}

7073 Wu-k'ang | 康 Win-kang, H. in Hu-chon Fu, Chel1kiang; Lat. $30^{\circ} 33$, Long. $119^{\circ} 53^{\prime}$; orig., feudal state Fang-fêng-sliil 於風氏; Han, Yü-pu-hsiang 餘不 拫; Tsin, Wu1-k'ang | 康. 7074 Wu-kêng | 庚.

$1^{\circ}$ (obs.) Chou, fendal state in Chilnii.

$2^{\circ}$ See No. 6065 .

7075 Wu-kuan | 關, a post-town near I.in-pa 'T'., Shensi.

7076 Wu-kung | 功 Wu-kung. [497] $1^{\circ}$ H. in Ch 'ien C., Shensi ; Lat. $34^{\circ} 20^{\prime}$, Long. $108^{\circ}$ $08^{\prime}$; orig., in K. 'T'ai 郃; Ts'in, Wu-kung 功; 2 nd $\mathrm{Wei}$, Mei-yang 美晹; r rang, Chi 穆 C.; Sung, Yüan and Ming, Wn-kwng|功 $\mathrm{H}$. $2^{\circ}$ (obs.) H. IIan, H. 40 li E. of Mei H., Shensi. 3" (obs.) S. W. of Wukung H., Shensi ; Han, Tsin, Sui and Tang, Wu-kung II.; N. Hei, Wu-kung | 功 chïn. $4^{\circ}$ (obs.) S. Sung and $S$. $T s^{\prime} i, H$. in Nan-clıêng H., Shensi.

7075 Wu-li|黎, see No. 2774. 7078 Wu-lien | 連, a post-town near Chien C., Ssŭch' uan (No. 880).

7079 Wu-lin | 林.
武 WU-LUNG.

$1^{\circ}$ A post-town near Hangchou Fu, Chehkiang.

$2^{\circ}$ (obs.) Yïan, H., N.E. of Hsiang-wu T. C., Knangsi.

70s0 Wu-ling | 陵 Wu-ling.

$1^{\circ} \mathrm{H}$. forming the prefectural city of Clıang-tê, Hunan; Lat. $29^{\circ} 01^{\prime}$, I.ong. $111^{\circ} 27^{\prime}$; Han, I ing-yiuan 陵沅; $E$. Han, | 陵 ; Liang, Wn I C.

$2^{\circ}$ (obs.) Ts' in and Hxn, chitn comprising parts of Kneichon and Hunan. $3^{\circ}$ (obs.) Han, H., W. of Chu-slian H., Hupe!. $4^{\circ}$ See Nos. 5858, 7764.

7081 Wu-lo | 羅, ssŭ near Pin C., Knangsi.

7082 Wu-lung | 龍.

$1^{\circ}$ (obs.) Trang, Sung and Yüan, H. 170 li S. E. of Foul C., Ssüch 'nan. $2^{\circ}$ (obs.) T'ang, H. 180 li W. of T'ien T.C. Knangsi.

7083 Wu-lung | 暗, (obs.) Sung, C. in Kuangsi.

7084 Wu-lung | 隆.

$1^{\circ}$ Sš̆̌ 107 li S.E. of Ifon C., Ssŭch 'nau; Lat. $29^{\circ}$ $20^{\prime}$, Long. $107^{\circ} 44^{\prime}$; Han, Font-ling 涪 陵; T'ang; Wu-lung | 隆; 
武

WU-I,UNG.

Sung,Chih, 枳H.; Ming, Wu-lung | 隆 H.

$2^{\circ}$ See No. 291.

7085 Wu-ning | 䡐 Wu-ning.

[193] $1^{\circ}$ H. in Nan-ch' ang Fu,

Kiangsi ; Lat. $29^{\circ} 16^{\prime}$, I,ong. $115^{\circ} 02^{\prime} ; H a n$, Hai-hun 海导; T'ang, Wu-ning | 富.

$2^{\circ}$ (obs.) Sung, H. near Chang-ning H., Ssŭclivan.

$3^{\circ}$ See No. 1145.

7086 Wu-pao | 堡 Wu-pau, H. [148]in Sui-tê C., Shensi; Iat. $37^{\circ} 35^{\prime}$, Long. $110^{\circ} 30^{\prime}$; T゙ang, Shih 石 C.; Sung, Wu-pao 员堡.

7087 Wu-p'ing | 本 Wu-ping, [134]H. in T'ing-chou Fu, Fuhkien; Iat. $25^{\circ} 05^{\prime}$, I,ong. $115^{\circ} 23^{\prime} ; T \sin , H \sin -10$ 新

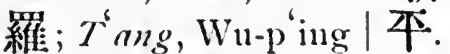
See also No. 4124.

7088 Wn-shan | I.L,(obs.)Liang, a town S. of Yi-ch' êng H., Hupeh.

7089 Wu-shêng $\mid$ 聖, see No. 362. 7090 Wu-shêng-pao | 勝堡, a post-town near $P$ 'ing-fan H., Kansul.

7091 Wu-shil ｜始, (obs.) formerly a town $W$. of Han-tan H., Chihli; see also No. 6358.

7092 Wu-so | 穒, ssŭ in Wanan H., Kiangsi.
武

WU-TU.

$7093 \mathrm{~W}_{11-\mathrm{sui}} \mid$ 隧, see No. 7056. 7094 Wu-t'ai | 泰, see No. 3051. 7095 Wuttang | 営, see No. 1521.

7090 Wu-tê | 德, chên near Huai-ch ing I'u, Honan.

7097 Wn-ting | 定 Wn-ting.

$1^{\circ}$ Fu in the Clit-T'nug'T’ai - Wu - Liu circuit, Shantung; forming also the district city of Huimin; Lat. $37^{\circ} 32^{\prime}$, Long. $117^{\circ} 41^{\prime}$; Han, P'ing-yiian 平原 and Po-hai 渤海; Wei, I,êling 㿟陵; Sui, 小i 棣 C.

[352] $2^{\circ}$ C.I.C. in the Yün-Wu circ., Yünnan ; Lat. $25^{\circ}$ $32^{\prime}$, L.ong. $102^{\circ} 33^{\prime}$; Y̌̈̈nn, Wn-ting | 定 C. 7098 Wu-tu | 都.

$1^{\circ}$ (obs.) so li W. of Ch'êng H., Kansulı; W. Han, chïn and $\mathrm{H}$.; Tsin, chïn and H. in $\mathrm{Ch}^{\prime}$ in 秦 cuot.

$2^{\circ} H$. Han, E. Han, H. in Wu-viian 五. 原 chion in the Oelot country, W. end of Inner Mongolia.

$3^{\circ}$ (obs.) E. Han, chïn $30 \mathrm{l} \mathrm{W}$. of $\mathrm{Cl}^{\prime} \mathrm{e}^{\mathrm{n}} \mathrm{g} \mathrm{H}$., Kansul.

$4^{\circ}$ (obs.) S. Sung and S. Ts'i, chïn in Nan-chêng H., Shensi. . 
证

$5^{\circ}$ (obs.) S. Sung, chïn and H., N.W. of Mienclı H., Ssŭch 'uan.

$6^{\circ}$ (obs.) N. Wei, chiin 50 li F. of Pao-chi H., Shensi.

$7^{\circ}$ (obs.) N. Wei and Sui, chiin N.W. of Chieh C., Kansul.

$\mathrm{S}^{\circ}$ (obs.) N. Hei, $\mathrm{H}$. near Han-chung Fu, Shensi.

$9^{\circ}$ (obs.) Sung, a fort near Lüi C., Ssŭ̌ch' nan. $10^{\circ}$ See Nos. 832, 3766, 4675.

7099 Wu-wei | 成 Wu-wei.

$1^{\circ} \mathrm{H}$. forming the prefectural city of Liangchou, Kansuh ; Lat. $37^{\circ}$ $59^{\prime}$, I,ong. $102^{\circ} 48^{\prime}$; $\mathrm{Han}, \mathrm{Tsin}, \mathrm{N}$. Wei and Sui, Wn-wei | 威 chiin.

$2^{\circ}$ (obs.) Man, H., N. of Chên-fan H., Kausull.

$3^{\circ}$ See Nos. 423, 7836.

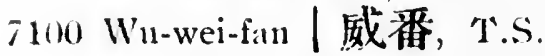
near Ku-lang H., Kansul.

7101 Win-yang | 隄.

$1^{\circ}$ Ssir in Ju-!iian H., Kuangtung.

$2^{\circ}$ (obs.) Liang, a town 50 li F. of Jao-chon Fu, Kiangsu.

$3^{\circ}$ See Nos. 871, 1181, 2740, 2992, 5049, 5616, 6065 .
五. WV-CHAI.

$7102 W_{11-y} \mid$ 军 Wn-i, H. in [297] Chin-hua Fu, Chehkiang; Lat. $28^{\circ} 53^{\prime}$, I,ong. $119^{\circ}$ $50^{\prime} ; \quad T^{6} a n g, \quad W_{11-c h}^{\prime} \hat{e ̂}_{1}$ |成 and $W_{11-y i} \mid$ 義.

7103 Wu-yi | 夷, (obs.) $7^{6} a n g$, H. in Annan.

7104 Wu-yi | 邑 Wu-yih, H. [143]in Chi C.I.C., Chihli; I at. $37^{\circ} 50^{\prime}$, I.ong. $116^{\circ}$; Han, Wu-yi| 邑.

7105 Wn-yiuan | 原.

$1^{\circ}$ (obs.) 80 li N.W. of P'ei C., Kiangsu; Han and Tsin, Wu-yüan | 原 H.; $N$. Wei, Wuyiian | 原 chïn and $\mathrm{H}$. $2^{\circ}$ (obs.) N. Sung and $N$. $T s^{6} i, H$. in Kiangsu.

$3^{\circ}$ See Nos. 1940, 6316.

7106 Wn-yüan | 垣.

$1^{\circ}$ (obs.) Han, Tsin and N.W., H. $35 l i \mathrm{~S} . W$. of Ho-chien Fu, Chilıli.

$2^{\circ}$ (olss.) S. Sung, H. in Shantung.

$3^{\circ}$ See No. 2064.

7107 Wu-yiian | 綵 Wu-ynen, H. in Sš̌-ên Fı, Kıangsi ; I.at. $23^{\circ} 07^{\prime}$, Long. $107^{\circ}$ $4.3^{\prime}$.

\section{五.}

7108 Wu-chai 五 棊 Wu-chai, [257]H. in Ning-wu Fu, Shansi ; Lat. $39^{\circ} 08^{\prime}$, Long. $111^{\circ}$ $40^{\prime}$. 
五

WU-CH'ANG.

7109 Wu-ch'ang | 常 C. I. T., in Clii-lin circ, Kirin.

7110 Wn-ch'êng | 城.

$1^{\circ}$ Chên near Hsiu-ning H., Anhui.

$2^{\circ}$ The Chinese translation of the word Bishbalik i.e. five cities. See No. 5099.

7111 Wu-chieh | 街, (obs.) $W^{\prime}$. Han, H. in Kansulı.

7112 Wu-fêng| 篻.

$1^{\circ}$ Ssünear Ho-fêng C.I. 'T. Hupel.

$2^{\circ}$ A post-town near Yiunyang H., Ssinch' $11: 11$.

7113 Wu-ho / 河 Wu-ho, H. in [162] Ssŭ C., Auhui; Iat. $33^{\circ}$ $12^{\prime}$, l,ong. $-117^{\circ} 43^{\prime}$, orig., Sss̆̌ 泪 C.; Sung, Wu-ho 河.

7114 Wn-hui| 同, (obs.) $T^{*} a n g$, H. $100 \mathrm{li} \mathrm{W}$. of $\mathrm{Yi} \mathrm{C}$., Chillit.

7115 Wu-hsiao / 㴾, chen near Ch'ung-ming H., Ki:mgsin.

$7116 \mathrm{Wu-li}$ | 生, chin near Ch"i H., Shansi.

7117 Wu-li-chien| 里特 (or Yii-lung-chieh-ch'ih 王能 傑赤) Urgendj, a town in The New Dominion, IV. of Bokluara.

7118 Wu-li-pa |星埧, a posttown near 'Ting-yüan $T$., Shensi.
五. WU-YUAN

7119 Wr1-lung | 龍, see No. 3389.

7120 Wn-shan | IU, chên near

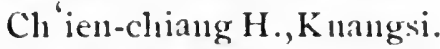

7121 Wu-tai | 臺 Wuttai, H. [91] in Tai C., Shansi; Lat. $38^{\circ}$ $46^{\prime}$, I,ong. $113^{\circ} 24^{\prime} ; N$. W'ii, I,ï-yi 艫挗 H., Sui, Tang, Sung, Kin, and Ming, Wn-tai | 擡 H.; Yäan, 'T'ai 臺 C.; Han, I ïi-ssŭ 虑 虎。

7122 Wn-ton| 斗, ssü in Nanhai $H .$, Knangtung.

7123 W11-ts'ao-han | 曹 |l, a post-town near Ch'in-pei H., Yïnnan.

7124 Wu-tuan / 端, see No. 2057.

7125 Wи-уӥ:11 | 原.

$1^{\circ}$ (obs.) Hinn, chiin in the Oelot conntry, W. end of Inner Mongolia.

$2^{\circ}$ (ols.) Han, H. in $\mathrm{W}_{11-}$ yian | 原 chizl.

$3^{\circ}$ (obs.) N. H'i, H. in Chin C., Shansi.

$4^{\circ}$ (obs.) N. Wei, H., N. of Shon-yang H., Shansi.

$5^{\circ}$ (obs.) Siui, chïn $100 \mathrm{li}$ IV. of the Ordos right wing (N. of Shen ii).

$6^{\circ}$ (ols.) Sui and $T^{\prime}$ ang, H., S.E. of ling C., Kansul.

$7^{\circ}$ See Nos. 5610, 7666. 
伍 WU-CH ${ }^{\circ} \hat{\mathrm{ENG}}$.

\section{伍}

7126 Wu-ch'êng 伍城, see No. 1532.

7127 Wu-chi | 没, chên near Ning-wı Fu, Slınısi.

\section{禁}

$7128 \mathrm{Wu}$ 梧.

$1^{\circ}$ (obs.) Han and Tsin, H. near Hsï-chon Fu, Kiangsu.

$2^{\circ}$ See Nos. 6526, 7129.

7129 Wu-chon | 州 Wu-chatu, [416] Fu in the Knei-P'ing-WuVï circ., Knangsi ; forming also the district city of 'T's'ang-wu; Lat. 230 $29^{\prime}$, I,ong. $110^{\circ} 51^{\prime} ;$ Han, 'r's'ang-wn 蒼 | chïn and Chino 交 C.; T ang and Sung, Wn | C.; Yüan, Wncho1! 州 circ. in H11kuang province; Ming., W11-chon|州 Fi1, Kuangsi.

\section{吳}

\section{$7130 \mathrm{~W}_{11}$ 吳 $\mathrm{W}_{11}$.}

$1^{\circ} \mathrm{H}$. forming with $\mathrm{Cl}_{1}^{\prime}$ angchon H. and Yüan1-ho H. the prefectural city of St1-chou, Kiangsu; Isat. $31^{\circ} 28^{\prime}$, I,ong. $120^{\circ} 44^{\prime}$; orig., capital of K. WuI; Ts'in,

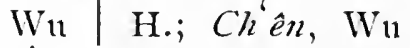
| C.; $W$. Han, Kueiclii 會稭 chiin ; T゙ang,
吳

WU-CH IAO.

Su 裮 C.; Sung, P'ingchiang 本. 江 Fu; S. Sung and $S$. Tsi, Wu chün and $\mathrm{H}$; Yïan, P'ing-chiang 平江. circuit.

$2^{\circ}$ Chên near Ch'ing-shlui H, Kansul.

$3^{\circ}$ (obs.) T'ang, C. near Ning-hısia Fu, Kansulh. $4^{\circ}$ (obs.) E. Han, chïn in Cinell-kiang.

$5^{\circ}$ (obs.) S. A., K. in Kiangsu.

$6^{\circ}$ See Nos. 755, 1993, 2974, 5904, 7134.

7131 W11-cli angl昌, see No.291.

7132 Wu-ch'êng | 城, chên in Chien-ch 'ang H., Kiangsi.

7133 Wn-chia | 家, ssü near Pachai 'T., Kueichon.

7134 Wn-cliang | 江. Wu-kiang, H. in Su-clion F'11, Kiangsu; this city serves also for Chên-tsê H.; I,at. $31^{\circ}$ $12^{\prime}$, I.ong. $120^{\circ} 20^{\prime}$; Han, \begin{tabular}{l|l}
$W_{11}$ & H.; Liang, Su1n-
\end{tabular} ling 忪陵; Yüan, W11cliiang | 江. C.; Ming, Wuchiang | 江. H. Vughin (Polo) .

7135 Wu-ch'ino | 筒 Wn-kian, H. in Ho-chien Fu, Cliilli ; Lat. $37^{\circ} 37^{\prime}$, Long. $116^{\prime \prime}$ $33^{\prime} ; T^{6}$ ang, Clinang-ling 将 陵; Sung, Wu-ch 'uan | 川; Kin, Wr1-clino | 橋. 
㕦

WU-CH'UAN.

71.36 Wu-ch'"111 | 川 Wn-chnen.

$1^{\circ}$ H. Kao-chon Fu, Kuangtung; I at. $21^{\circ} 19^{\prime}$, I.ong. $110^{\circ} 18^{\prime}$; Han, Kao-liang 高梁; Sui, Wu-ch'nan| 川.

$2^{\circ}$ See Nos. 7135 .

7137 Wn-fangl痛, see No. 5964.

7138 W11-lising | 興, see Nos. 2154, 5314, 7198 .

7139 Wu-kao ! 泉, see No. 1737.

7140 Wu-la-t' $\hat{\mathrm{e}}$ | 喇特, see No. 7178.

7141 Wnt-ling, | 陵, see No. 6175.

7142 IVu-11ing | 䆝, (obs.) IIan, a town 2.7 li Is. of 'Tungyang $\mathrm{H}$., Chehkiang.

7143 Wu-pao | 堡, see No.7086.

7144 Wn-p'ing | 平 (obs.) P. $H a n$, a town $100 \mathrm{li} \mathrm{F}$. of Hsin-ÿ̈ H., Kiangsi.

7145 Wu-shan | |山, see No.389. 7146 Wu-t'a | 塔, ss̆r in Ch'ingchon H., Kiangsı.

71.17 Wn-ts'un | 村, chên near P'u H., Shansi.

7148 Wu-wang-cliêng | 主城, (obs.) Han, a town near Wu-ch'ang H., Hupeh.

\section{巫.}

7149 W11 至.

$1^{a-(o b s .) ~ H a n, ~ H ., ~ E . ~ o f ~}$ Wu-shan H., Ssŭch 'uan. $2^{\circ}$ (obs.) formerly C. in Kneichon.
告类 WU'TI.

$3^{\circ}$ Sec Nos. 861, 4783, 6069,7150 .

7150 Wu-shan | I4 Wu-shan, [528]H. in K'nei-chou Fu, Ssŭclı' '1an; Lat. $31^{\circ} 09^{\prime}$, Long. $109^{\circ} 52^{\prime} ; \quad T 1^{\prime} i n$ and S. Sung, Wu $\mid \mathrm{H}$.; Tsin, Clicn-ping 建本; Sui, Wnu-shan | Ill.

\section{無}

7151 Wu-chi 無 極 Wu-ki, H. [491]in Chêng-ting Fu, Cliillli : Lat. $37^{\circ} 50^{\prime}$, Long. $116^{\circ}$; Sui, Wu-clii 册掼.

7152 Wn-clinng | 終.

$1^{\circ}$ (obs.) S.A., K. in Cliilili.

$2^{\circ}$ (obs.) Han, Tsin, $N$. Wei and Sui, H. in Chi C., Chihli (No. 580).

$3^{\circ}$ See Nos. 6592, 7732.

7153 Wu-fêngl 逢, (olss.) T゙ang, C. near Shnu-t'ien F'u, Chilili.

7 i54 Wn-l1si | 鍟 Wu-sih, H. in Ch'ang-chon Fn, Kiangsu; this city serves also for Clin-knei H.; Iat. $31^{\circ}$ $35^{\prime}$, Lonng. $120^{\circ} 20^{\prime}$; Han, Wu-lısi 錫; Sui, Chinling 睤陵.

7155 Wu-kung | 功,(obs.) Han, H. in Annam.

7156 Wu-lü | 虑, see No. 3412 .

7157 Wu-ti | 稢, see Nos. 1195 , 1918, 7352. 
笟

7158 Wu-wei | 䈠 Wu-wei, C. [150]in I,ü-chon Fu, Anhui; I,at. $31^{\circ} 23^{\prime}$, I,ong. $117^{\circ}$ $50^{\prime}$; orig., in $\mathrm{K}$. Ch' ao 巢; Sung, Wu-wei | 鼡 military district and $\mathrm{H}$.;

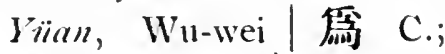
Ming, Wu-wei | 急 $\mathrm{H}$.

7159 Wu-yiu | 慮, (obs.) T ang, H., E. of Shang-lin H., Kuangsi.

\section{舞}

7160 Wu-yang 舞陽 Wu-yang. $1^{\circ} \mathrm{H}$. in Nan-yang $\mathrm{F} n$, Honall; I,at. $33^{\circ} 32^{\prime}$, Long. $113^{\circ} 38^{\prime}$. $2^{\circ}$ (obs.) $H a n$, H., N.W. of Clı '̂̂-ch'i H., Hunan.

7161 Wri-yin | 陵, Han, H. in Honan.

\section{重}

7162. Wu-ch'eng 带城, (obs.) formerly a town near Yang-chou Fu, Kiangsu.

7163 Wu-hu| 湖 Wu-hu.

[528] $1^{\circ}$ H. in T'ai-p’ing Fu, Anhni; Jat. $31^{\circ} 18^{\prime}$, Long. $118^{\circ} 21^{\prime}$; one of the ports on the Yangtsze open to foreign trade; Three Ks., Chin-

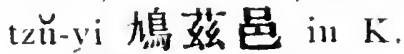
Wu 员; $T \sin , H$ siangyiian 賴坦; Sung, Yüan and Ming, W11lu $\mid$ 湖 $\mathrm{H}$.
岕 Wบ-СНIH.

$2^{\circ}$ (obs.) Han, H., E. of the above.

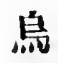

$7164 W_{11}$ 鳥.

$1^{\circ} C h \hat{e} n$ near $\mathrm{Cl}_{1}{ }^{6} \mathrm{ing}-\mathrm{t}^{6} \mathrm{ien}$ $\mathrm{H}$, Chehkiang.

$2^{\circ}$ (obs.) Tang, C. near Ch'ing-yang Fu, Kansuh.

$3^{\circ}$ (obs.) Liao, C. in Shingking.

$4^{\circ}$ See No. 7421.

7165 Wu-ch'a | 槎, ssŭ near Tapu H., Knangtung.

7166 Wu-ch'êng | 程, see No. 7198.

7167 Wn-chi | 雞, (obs.) formerly a town near T'ientai H., Chehkiang.

716S Wu-ch'i | 溪, chên near Wu-hu H., Anhui.

7169 Wu-chiang | 江., chên near Ho C., Anluni; $T \sin , S$. Sung, S. Ts'i, N. Wei, Sui, T゙ang, Sung and Yйan, Wu-chiang | 江 H. 7170 Wri-chils | 氏.

$1^{\circ}$ (obs.) $/ I a n$ and Tim, H., N.W. of P'ing-liang fill, Kansuh.

$2^{\circ}$ (obs.) N. Wei, H. in Ching C., Kansuh.

7171 Wn-chilı | 枝, (obs.)Han, H., N. W. of P'ing-liang Fu, Kansuh. 
鳥 WU-CHU-MU-CH'

7172 W11-chu1mu-clin | 珠穆 汇 Uchunnuchin, Mongol 'rribe.

7173 Wu-fei $\mid$ 瓜, (obs.) $7^{*} a n g$, H. near Turfan.

7174 Wu-hu| 護, see No.7005. 7175 Wu-jên | 仁, (obs.) Kin, a pass 80 li N.I. of Y $\mathrm{i}$ ch nan H., Sliensi.

7176 Wu-k'ê 克Ukol. a tow'1 in The New Dominion; I at . $40^{\circ} 10^{\prime}$, I,ong. $91^{\circ} 50^{\prime}$.

7177 Wu-k'ê-êrll 克尔, Onigour, a town in Cobdo; I, at. $49^{\circ}$, I,ong. $86^{\circ} 30^{\prime}$.

7178 W11-latt'ê | 喇特 (or 员 喇特)Urad, Mongol Tribe.

7179 Wu-lan-ch'a-pu | 菲察 布 Ulan Chap, Mongol Tribe.

7180 Wn-li-ya-su-t'ai | 里雅 蘇台 Uliasntái.

$1^{\circ}$ A region in Mongolia comprising Cobdo and Ulianghai.

$2^{\circ}$ A town in Saimnoin; I at. $47^{\circ} 30^{\prime}$, Long. $96^{\circ}$.

7181 Wu-li-ya-su-t $n$ | 里雅蕉 圆 Uliasuto, a town in 'Tarbagatai; Lat. $46^{\circ} 15^{\prime}$, Long. $82^{\circ} 30^{\prime}$.

7182 Wn-liang-hai医海Ulianghai, a district in Mongolia ; bonnded on the $N$. by Siberia; on the F. by 'Tuchetu; on the S. by Dsassaktu and Cobdo; and
鼠 IVU-SHIH.

on the $\mathrm{W}$. by Cobdo and Siberia

7183 Wu-lo | 薙, T.S. near Yin-chiangr H., Kueichon.

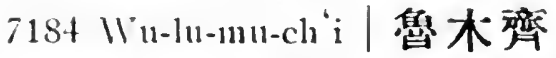
Ouruntsi, alsocalled Kungning, 腪富 Chü-shih 車 師, and anciently Bisilbalik; see Nos. 5099, 6356.

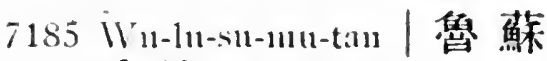
木丹 Urnsmundan, a town in Helıhnngkiang; Lat. $51^{\circ}$ $30^{\prime}$, Long. $126^{\circ} 30^{\prime}$.

$7186 W^{\prime} n$-lung | 龍,(obs.).sung and $K \mathrm{in}$, a military post 50 li S. II. of Chia C., Shensi.

$7187 W^{\gamma} n-l u n g \mid$ 籠, (obs.) T゙ang, C. near Ning-hsia Fin, Kansuh.

7188 Wu-mêng | 蒙, (obs.) Ming, Fu 20 li E. of Chaot’ung Fu, Yümman.

7189 Wu-mo | 麼, see No. 5730. 7190 Wu-sha-k'ê-t'a-êrh| 敞克 塔尔 Ushaktar, a town in Harashar; Lat. $42^{\circ}$, I.ong. $88^{\circ} 25^{\prime}$.

7191 Wu-shang | 稘, see Nos. 6759, 7463.

2192 Wu-shil | f (or, Noch'ih 倭赤,) Ushi or Ush ; C.I.T. in Aksu circ, New Donninion; Lat. $41^{\circ} 35^{\prime}$, I.ong. $77^{\circ} 50^{\prime}$; also called Yung-ning $\mathrm{Ch}^{\prime}$ êng 永察 城. 
晑

WU-TAN.

7193 Wu-tan | 月, (olss.) T'ang, C. near Turfan.

719t Wu-tu | 乫, see No. 3837. 7195 Wu-t"u-ssŭ|圖斯 Otus, a town in Yarkand; Lat. $40^{\circ}$, I,oung. $74^{\circ} 10^{\prime}$.

7196 Wn-yü-k'ê-lin-ina | 裕克 林哈 Ujukling; a town in 'Thibet; Iat. $29^{\circ} 25^{\prime}$, I.ong. $90^{\circ} 40^{\prime}$.

\section{郳}

$7197 W_{11}$ 䣕, (obs.) S.A. and $H a n$, a town near Chiehhsiu H., Shansi.

7198 Wu-cl ền | 程 (or, 烏 程) Wu-ching.

$1^{\circ} \mathrm{H}$. forming with Kueian $H$. the prefectural city of Hu-clion, Cliehkiang; Lat. $30^{\circ} 48^{\prime}$, I ong. $120^{\circ} 03^{\prime}$; Harn, $111-11 \operatorname{sing}$ 吴與.

$2^{\circ}$ See No. 2151.

\section{个}

7199 Wu-ching 午井, chên near Mei H. Shensi.

\section{仵}

7200 Wn-cl'êng 件城, see No. 6069.

\section{移}

7201 W11-an 務茹, H., in Ch' 'ian-lo 全 羅 circ., Corea.

7202 Wu-ch' 'uan | 川, see No. 5868.
沃 W'T-KAN.

\section{$7203 W_{u}$ 秋.}

$1^{\circ}$ (obs.) S. Sung, C. in the Chê-tunng 浙 東 circ.

$2^{\circ}$ See No. 1023.

720+ Wu-ch'nand 川 Wu-cluen, [163]H. in Ssin-nan Fu, Kueichon! I Iat, $28^{\circ} 24^{\prime}$, I, tong. $108^{\circ} 11^{\prime}$.

7205 Wn-yüan | 源 Wn-ynen, [519]H. in Hui-chon Fin, Anluii ; Lat. $29^{\circ} 18^{\prime}$, Long. $117^{\circ} 59^{\prime} ;$ Sui, Hsin-1ing 休察; T'ang; W11-ÿïan | 源 $\mathrm{H}$.

\section{$\pi$}

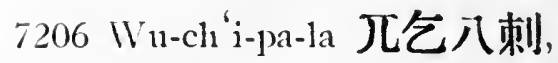
Okbara, a city S.E. of Moussoul, Asiatic 'Turkey.

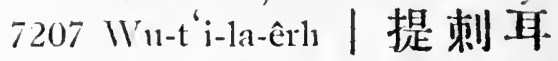
Otrar; sce No. 1604.

\section{册}

7208 W11-chi 册極, see No. 7151.

\section{物}

7209 Wu-tsang 物藏, T.S. near Sung-p' an T., Ssŭch' "uan.

\section{沃}

7210 Wu-ch'van 沃川 chïn in Chung-ch'ing 忠清 circ, Corea.

$7210^{\wedge} W_{11-k o n}$ 檴 H. in Ch ${ }^{6}$ ïan1o 全羅 circ., Corea. 
Wu-cliang, see No. 7052. IVu-chau, see No. 7129.

IV11-ching, see Nos. 7054, 7198.

Wu-chuen, see Nos. 7136, 7204.

Wu-hiang, see No. 7066.

W11-i, see No. 7102.

W11-kang, see No. 7073 .

IVu-ki, see No. 7151.

Wu-kiang, see Nos. 7056, 7134.

W11-kial1, see No. 7135.

W11-ngal1, see No. 7051.

W11-pall, see No. 7086.

Wu-ping, see No: 7087.

W11-sil, see No. 7154.

W11-sine11, see No. 7069.

IV11-tai, sce No. 7121.

Wu-tsin, see No. 7060.

IV1-tsing, see No. 7062.

W11-yil, see No. 7104.

I'n-yuen, see Nos. 7107, 7205 .

Xanadu, see No. 5535.

\section{YA}

\section{雅}

7211 Ya 雅.

$1^{\circ}$ (obs.) 5. Sung, C. in the Ch'êngr-tu 成都circ. $2^{\circ}$ See No. 7213.

7212 Va-an| 委 Sa-11gan, H. formingthe prefectural cit! of Ya-chon, Ssŭch' naw; Lat. $30^{\circ} 04^{\prime}$, I ong. $103^{\circ} 04^{\prime}$.
¡213 Ya-chon | 州 Ya-chan, Fin

[72] in the Chien-ch' ang-shangnan circ., Ssŭch' "1an; forming also the aistrict city of Ya-an; Lat. $30^{\circ} 04^{\prime}$, I,ong. $103^{\circ} 04^{\prime}$; Chou, in Yung 㠍 CHOU; Ts'in and Han, Yen-tao 䈑道 in $\mathrm{K}$. Ch'u楚; $W$. Wei, Mêngshan 蒙 III; Sui, I,inchinung 臨功; Tiang and sung Ya|C.

7214 Ya-k'ê-sa| 筧藷 Yaksa, a town in Hehlungkiang; Jat. $53^{\circ}$, Iang. $122^{\circ} 30^{\prime}$.

i215 Ya-lung | 龍, (obs.) S. $T s^{\prime} i, H$. near Ta-li fin, Y'ïn11.11.

7216 Ya-111i-t 1 | 馬间 Yanmato, a town in Ili; Iat. $44^{\circ}$ $20^{\prime}$, Long. $83^{\circ}$.

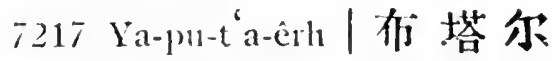
Yartobo, a town in 'Thibet; I.it. $56^{\circ}$, Jong. $78^{\circ} 30$.

\section{芽}

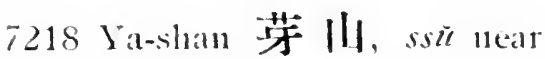
$\mathrm{Cl}_{1}$ i-shui H., Hupeh.

\section{位}

7219 Va-fu-ch'êng 亞i 父城. (obs.) formerly fort near Ho C., Anluni.

\section{叫?}

7220 Va-pai 唯星, chèn near Chon-chih H., Shensi. 
鼣

YA-LAN.

㴧

r221 Ya-lan 鹤欄, ssü near I.in-hsiang H., Hunan.

押

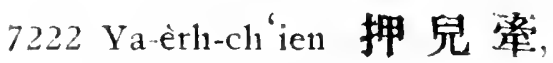
see No. 7337.

7223 Ya-t'un| 屯 T.S. near Sung-p'an T., Ssŭch'uan.

Ya-chan, see No. 7213.

Ya-ngan, see No. 7212.

\section{YAI}

崖

7224 Yai 崖 Yai.

$1^{n}$ C.L.C. in Ch'inng-Yin circ., Kuangtung; Lat. $18^{\circ} 22^{\prime}$, Long. $108^{\circ} 48^{\prime}$; Ilan, Chn1-yai 珠 |; Sui, Ning-yüan 要遠; r'ang, Clien 振 C.; Sung, Yai | C. and Chiyang 者陽; Ming, Yai |C.

$2^{\circ}$ (obs.) T'ang, C. $40 l i$ S. E. of Clining-shan H., Kuangtung.

$3^{\circ}$ See No. 1321.

Yaksa, see No. 7214.

Yamagata, see No. 5462."

Yamagnchi, see No. 5462."

Yamanashi, see No. 5462.

Yamato, see No. 7216.
洋

YANG.

\section{YANG}

\section{樣}

7225 Yang-p’ i 浗濞ssŭ in Mênghua 'T., Yüun11an.

\section{羊}

7226 Yang-ch'èng 羊城, see No. 3393.

722 ' Yang-ch'iian-tzu|圈子, a post-town near $\mathrm{Yi} \mathrm{C}$, Shingking.

7228 Yang-lin-tien | 流 店, a post-town near T'ai-an Fu, Shantung.

7229 Yang-shan | II. see No. 6786.

7230 Yang-t ung ! 峝, '1.S. near Sung-p'an T., Ssŭch' nan."

7231 Yang-yang | 陽, T.S. near Inng-li H., Kneichon.

7232 Yang-yu-ch'êng | 耎 城, (obs.) formerly fort near 'I"ai-an Fin, Shantung.

7233 Yang-yüan | 源, a posttown near Ch nng-an H., finhkien.

\section{洋}

7234 Yang 泽 Yang.

[133] $1^{\circ}$ H. in Han-chung F 11 , Shensi ; Lat. $33^{\circ} 05^{\prime}$, I.ong. $107^{\circ} 33^{\prime} ; T^{\prime}$ in, Ch'êng-kn 城固; 2 nd Wei, 'T'ang-ch' êng 僖 域; Sui, Yang |C. 


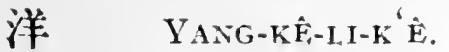

7235 Yang-kê-li-k'é | 柯里克 Inge-arik, a town in Yarkand ; Lat. $40^{\circ}+5^{\prime}$, Long. $79^{\circ} 30^{\prime}$.

7236 Yang-k'on | II, ssü near Kuang-fêng H., Kiangsi.

7237 Yang-po | 波, Y. near Shang-lin H., Kuangsi.

7238 Yang-sa-êrh | 薩尔 Yanghi; a town in Harashar; Lat. $41^{\circ} 30^{\prime}$, I , ong. $84^{\circ} 40^{\prime}$.

7239 Yang-yiian | 源, (obs.) T'ang, H., S. of Hsihsiang H., Shensi.

\section{養}

7240 Yang-chi-kan 養吉正, Yenguikend; a town in Independent 'I'urkestan, on R. Sillon; see No. 7243.

7241 Yang-li. 利 Yang-li, C. [567] in 'T'ai-p'ing Fin, Kuangsi ; I jat. $22^{\circ} 54^{\prime}$, L,ong. $106^{\circ} 56^{\prime}$; T'ang, Yang-li 利 $\mathrm{C}$.

7242 Yang-lung | 竜, T.S. near Kuei-yang Fu, Kueichon.

7243 Yang-yi | 资 Yangnikand, or, Yenguikend, or Yangki; a town in 'Turkestan, also called Otrar; sec Nos. 1604, 7240 .

\section{仰}

7244 Yang-chi-pa-li 仰吉八坐, Yạıkibalik, or Yang balgasun, a town in the New Dominion, E. of Manas.
隄 YAING-CHIA.

7245 Yang-chiang | 江 (or Yangknang | 炎) katngoon.

陽

7246 Yang 陽, see No. 2407.

7247 Yang-an | 女:

$1^{\circ}$ A post-town near Chien C., Ssüch' nan (No.904); Sui, T'ang and Sung, Yang-an | 岁 H.

$2^{\circ}$ (obs.) N.E. of Ch iiehshan H., Honan; Han and $T \sin$, Yang-an | 努 H., N.Wei, Yangan | 安 chiun and $\mathrm{H}$. $3^{\circ}$ (obs.) S. Ts' $i, \mathrm{H}$. near Pao-ning Fu, Ssuch ' nan. $4^{\circ}$ See No. 874.

7248 Vang-chai | 猝, see No. 7687.

7249 Yang-chên | 真, see No. 7255 .

7250 Yang-ch'èng | 城 Yangching.

[64] $1^{\circ}$ H. in 'Tsê-choul Fil, Shansi ; Lat. $35^{\circ} 26^{\prime}$, I.ong. $112^{\circ} 36^{\prime}$; $\mathrm{Han}$,

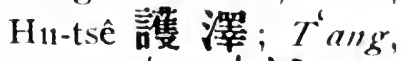
Yang-ch' êng | 城.

$2^{\circ}$ H. in Ching-chi $i$ 京 嫘 circ., Corea.

$3^{\circ}$ (obs.) Ts'in, a town near Su C., Anluui.

$4^{\circ}$ See Nos. 1098, 4257, 7847.

7251 Vang-chia | 夏, see No. 6165. 
7252 Yang-chiang | 江 Yang[529]kiang, C.L.'T. in Kao-LeiYang circ. Kuangtung; Lat. $21^{\circ} 50^{\prime}$, Long. $112^{\circ} 04^{\prime}$; $W u$, Hai-an 海父, and Hsip'ing 西本; Tsin, Chi-an 齊安; T⿱ang, An-p'ing 岀本; Sung and Yiran, Nan-ên 南恩 C.; Ming, Yang-chiang | ir $\mathrm{H}$. 2252 Yang-chil | 智, H. in Cling-ch ${ }^{\circ} \mathrm{i}$ 京畿, circ., Corea.

7253 Yang-ch'in | 邱, see No. 204.

7254 Yany-chon| 凬, see No. 5942.

7255 Yang-ch' iì | 曲 Yang-kiulh, $\mathrm{H}$. forming the prefectural city of 'T"ai-yüan, Shansi ; Lat. $37^{\circ} 54^{\prime}$, Long. $112^{\circ}$ 33'; $7 s^{\prime}$ in, Lang-111êng 狼 孟; Han, Yang-ch ii | 曲; Sui, Yang-chên | 真 and Fên 汾 C.

7256 Yang-ch'uan | II, H. in Ching-ch' i 京畿 circ., Corea.

7257 Yanng-ch'un | 春 Yang[529]chun, H. in Chao-ch'ing Fu, Knangtung; Lat. $22^{\circ}$ $08^{\prime}$, Long. $111^{\circ} 33^{\prime}$; Han, Kao-lianng 高 凉; liang, Yanir-ch' "un | 春; T゙ang, $\mathrm{Ch}^{4} \mathrm{t}+1$ 春 $\mathrm{C}$ :

7258 Yang-fêng|豐.
陽 Y৯NG-KUA.

$1^{\circ}$ (obs.) Tsin, S. Sung, and $S . T s^{\prime} i$, H. $60 \mathrm{li}$ IV. of Yung-fêng H., Kiangsi.

$2^{\circ}$ See No. 7847.

7259 Yang-lian | 漢, chên near $\mathrm{Cl}^{\prime}$ ien C., Shensi.

7260 Yang-ho | 和, a post-town near Yang-kao H., Shansi.

7261 Yang-hu | 湖 Yang-hu, H. forming with Wu-chin $\mathrm{H}$. the prefectural city of Ch'ang-chon, Kiangsu ; Lat. $31^{\circ} 47^{\prime}$, Isong. $119^{\circ}$ $56^{\prime}$.

7262 Yang-lisi | 西, chên, near Hsin C., Shansi.

7263 Yang-hsin | 信 Yang-sin. [284] $1^{\circ} \mathrm{H}$. in WVu-ting Fu, Shantung; Lat. $37^{\circ} 45^{\prime}$, Isong. $117^{\circ} 38^{\prime}$.

$2^{\circ}$ See Nos. 1195, 1918, 4677.

7264 Yang-lısün | 泌, ss̆̈ near Ch'ih-chou Fu, Anluui.

T265 Yang-kao | 高 Yang-kau, H. in 'Ta-t' 'ung Fin, Shansi; I at. $40^{\circ} 27^{\prime}$, Long. $113^{\circ}$ $04^{\prime}$.

7266 Yang-ku | 紫 Yang-ku, H. in Yen-chon Fu, Shantung; Lat. $36^{\circ} 09^{\prime}$, Long. $115^{\circ} 59^{\prime}$; orig., in $\mathrm{K}$. Ch'i 䂟; Sui, Yang-ku | 㥅。

7267 lang-kua | 瓜, see No. 4375. 
陽 Yang-kUo.

7268 Yang-kuo | 郭, chên near IVei-yïan H., Kansuh.

7269 Yang-lê | 葲, see No. 6905.

7270 Yang-ling | 陵, chên near Wu-kung H., Shensi.

7271 Yang-lo | 羅, ssŭ near Huang-p'o H., Hupel.

7272 Yang-mên | 閨, (obs.) Kin, chên E. of 'T' ien-chên H., Shansi.

7273 Yang-p'ing | 平。

$1^{\circ}$.Chên near Pao'chi H., Sliensi.

$2^{\circ}$ See Nos. 4945, 5616, 6701, 7062.

7274 Yang-pı | 彗, chên near Hsin-ts' ai H., Honan.

7275 Yang-shan | I. Y Yang-shan, [156]H. in Lien C., Kuangtunng; Lat. $24^{\circ} 30^{\prime}$, Long. $112^{\circ} 25^{\prime} ; H(m)$, Yang-shan | Ili.

7276 Yang-shil | 師, (obs.) Tang, H. in Fang-shan H., Chihli.

7277 Yang-so | 朔 Yang-soh, H. [224] in Kuei-lin Fu, Kuangsi; Lat. $24^{\circ} 28^{\prime}$, Long. $110^{\circ}$; Sui, Yang-so | 剙; $T^{*}$ ang, Knei-yi 踏尧.

7278 Yang-t'ai | 臺, (obs.) $W$. Han, Marq. in Chilnli.

7279 Yang-tê | 德 Yang-tel, , H. in P'ing-an 平努 circ., Corea; Lat. $39^{\circ} 46^{\prime}$, Long. $127^{\circ} 37^{\prime}$.
楊 YANG.

7280 Yang-ti-ai|地隠, T.S.near Lung-an Fu, Ssizch' "uan.

7281 Yang-tsung | 宗, (obs.) Yïan and Ming, H., 40 li N. E. of Ho-yang H., Yünnan.

7282 Yang-tı| 都, (obs.) Han and $T \sin , \mathrm{H}$., S. of $\mathrm{Yi}$ shui H., Shantung.

7283 Yang-wan | 萬, T.C. near Fêng-yi C., Kuangsi.

7284 Yang-wu | 武 Yang-wu, [148] H. in Huai-ch'ing Fu, Honan; Lat. $35^{\circ} 05^{\prime}$, Long. $114^{\circ} 08^{\prime}$; Ts' in, Po-langslia 博浪沙; Han, Yangwu | 武.

7285 Yang-yi | 我, T.S. near Ma-ha C., Kneichon.

7286 Yang-yi | 邑, see No. 6166. 7287 Yang-yi | 羡, see No.7429. 7288 Yang-yu-ch' ‘i | 右曲, chên near Fu-p'ing H., Shensi.

7289 Vang-yüan | 原, (obs.) $W$. Han, H. 10 li S. of Hsining H., Chihli.

\section{楊}

7290 Yang 楊 Yang.

$1^{\circ}$ C. in Ching-ch'i 京畿 circ., Corea; Iat. $38^{\circ}$, I rong. $127^{\circ} 20^{\prime}$.

$2^{\circ}$ (obs.) S. A., K. in Shansi.

$3^{\circ}$ See No. 2407. 
模

7291 Yang-clıạ| 猚, ssü in Huang-kang H., Hupeh.

7292 Yang-cli'i | 溪, ssüin Hsinfêng H., Kiangsi.

7293 Yang-chia-ho | 家 河, chên near $\mathrm{Cl}^{\prime}$ 'nug-ming $\mathrm{H}$., Kiangsu.

$7293^{\star}$ Yang-cliang | 省, chên in Yi-hsing H., Kiangsu.

7294 Yang-ch'ing | 葛, a posttown near ' $\mathrm{F}$ 'ien-ching Fu, Cliilili.

7295 Yang-ch'ï | H⿻, chên near Hung-tung H., Shansi.

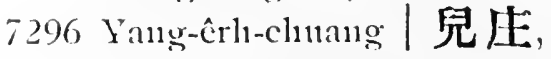
ssŭ near Y'en-slian H., Chilili; Isat. $38^{\circ} 20^{\prime}$, Isong. $117^{\circ} 36^{\prime}$.

7297 Yang-fang | j, Y. 11ear Ning-wu Fu, Shansi.

7298 Jang-kên | 根 Yang-kăn, chün in Ching-ch' $\mathrm{i}$ 玺 䍃 circ., Corea ; Lat. $38^{\circ} 53^{\prime}$, Jong. $127^{\circ} 23^{\prime}$.

$7298^{\circ}$ Yang-k' on | D, H. in Chiang-yian 江 原 circ., Corca.

7299 Yang-lao | 老, a post-town near Cl' ing-p'ing H., Kucichons.

7300 Yang-lin | 林, a post-town near Yi-liang H., Yünnan.

7301 Yang-liu | 柳, Y. near Slih-ping H., Kueichon.

7302 Yang-mei | 梅, Y. near Yuiell-sui 'T., Ssŭch' tuan.

7303 Yang-shê | 舍, chên near Chiang-yin H., Kiangsu.
揚 SANG-CHOL。

7304 Yang-ticn | 点, a post-town near Tê-an Fin, Hupelı.

7305 Yaug-wu-pa | 武 埧, ssĭ near Yiuan-chiang C., Yüınan.

7306 Yang-yai | 崖, chên near Clin'in-an H., Kansuh.

$7306^{\wedge}$ Yang-yi | 義, T.S. i11 P'ing-yiieh C., Kueichoı.

\section{掦}

7307 Yang 揚.

$1^{\circ}$ (obs.) one of the nine CHOL, or great divisions of the Eirupire established by Yii 重 ; comprising Chehkiang, Fuhkien, Kiangsi and the Cli aochow Prefecture in Kuangtung.

$2^{\circ}$ See No. 730 s.

7308 Yang-chon | 州 Yang-chan, [322] Fu in the Huai-yang circ., Kiangsu; forming the two district cities of Clniang-tı and Kan-ch' iian; Lat. $32^{\circ}$ $21^{\prime}$, I,ong. $119^{\circ} 15^{\prime} ;$ S.A., $1 s t$, in $\mathrm{K}$. W'n 只, $2 n d$, in K. Yiieh 越; C. Wars, in K. $C h^{\prime} n$ 楚; $7 s^{\prime} \mathrm{m}$, in Chin-chiang 九 论 chün; Han, Chiang-tı 讧者 Knang-ling 愿陵; Sui,

- Yang $\mid$ C.; $T^{6}$ ang, Kan FB C.; S. T゙ang, Tung-tu 東 都; Ming, Yang-chou 州 Fu. Yanjı: (Polo). 
揚 YANG-HSIN.

7309 Yang-lısin|新, (obs.) $T$ sin, $S$. Sung and S. T si, H. 60 li S.IV of Hsing-kno C., Hupel.

Vang-chan, see No. 7308. lang-ching, see No. 7250 . Yang-chu11, see No. 7257. Yang-hi, see No. 7238. Yang-kăn, see No. 7298. laug-kau, see No. 726.5. Yang-kiang, see No. 7252. Yang-kiuh, see No. 7255. Yang-sin, see No. 7263. lang-sol, see No. 7277. lang-tel, see No. 7279. Yangbalsagin, see $\mathrm{N}$. 7244.

Yangki, see No. 7243.

Yanguikand, see No. 7243. Yangju, see No. 7308.

lankibalik, see No. 7244.

\section{YAO}

\section{琶}

7310 Yao-ch'êng 堯 城.

$1^{\circ}$ Chên near Clıien-tê H., Anluui.

$2^{\circ}$ (obs.) formerly a fort near Wan H., Chihli.

7311 Y'ao-shan| IL, see No. 625.3. 姚

7312 Yao 姚 Yaut.

[435] $1^{\circ} \mathrm{C}$. in $\mathrm{Cl}_{1}^{\prime} n$-hsiung Fu, Y'ïnuan; I,at. 25 33', Long. $101^{\circ} 23^{\prime}$.

\section{耀} Yao.

$2^{\circ}$ See Nos. 6049, 7651.

7313 Yao-ch' $i$ 雄, Y. near Y'ỉan-wu H., Honan.

\section{窟}

7314 Yao-ao 䆗奥 a post-town near Y'ï-huan 'T', Chelıkiang.

7315 Yao-chê | 折, ssü in Chüauli H., Hupeh.

7316 Vao-tien | 噟, chên near Ch'ien-yang H., Shensi.

7317 Yao-t'on | 䫒, chên near Ch ung-hsin H., Kansulı.

\section{璠}

$7317^{1}$ Yao-chi'in 璠池, see No. 5822.

$7317^{\prime \prime}$ Yao-pi | 階, see No. 5822. $7317^{\mathrm{C}}$ Y ao-t'ai | 臺, see No. 5822.

\section{哣}

7318 Yao 要, chên near I.o-11an H., Shensi.

7319 Vao-ching | 津, see No. 2561 .

7320 Yao-clian 腰站, a posttown near Chi-shui H., Kiangsi.

\section{藥}

7321 Vao-ching 藥 徑, ssü in Ho-shan H., Kulugtung.

\section{耀}

7322 Yao 耀 Yau, C. in Hsi-an [397] Fu, Shensi; Lat. $34^{\circ} 56^{\prime}$, Long. $108^{\circ} 53^{\prime}$; Han, Tai- 
耀

YAO.

yii 机祅, 2nd Wei, Peiyung 北䔨; $W . W e i, \mathrm{Yi}$ 宜 C.; Sui, Hua-yüan 華 原 $\mathrm{H}$.

7323 Yao-chou | 州, post-town near Hai-ch 'êng H., Shingking.

Yarcan, see No. 7337.

Yarkand, see No. 7337.

Yartobo, see No. 7217.

Yau, see No. 7312, 7322.

Yê, see No. 7326.

Yedo, see No. 738.

\section{YEH}

\section{夜}

7324 Yeh-hu 夜獲, ssü in Hsining $\mathrm{H}$., Kuangtung.

7325 Yeh-1ang | 郎, see Nos. 1452, 2836, 3123, 5291, 5673 .

\section{掖}

7326 Yeh 掖 Yé.

$1^{\circ} \mathrm{H}$. forming the prefectural cit of Lai-chou, Shantung ; Lat. $37^{\circ}$ $10^{\prime}$, Long. $120^{\circ} 10^{\prime}$; W. Han, Tung-lai 東 萊 chïn；Tsin, Tunglai 東萊 K.; N.Wei, and Sui, Tung-lai 東 萊 chion.

$2^{\circ}$ See No. 360.

$7326^{\mathrm{A}}$ Yeli-cll $^{\prime} \mathrm{il}$ | 池, see No. 5822.
葉 YEH-ÂRH-CH IANG.

\section{野}

7327 Yeh-1i 野利, (obs.) formerly H., N. of Ch'ingyang Fu, Kansuh.

7328 Yeh-san-kuan |三關, ssü near Chien-shih H., Hntpeh.

7329 Yeh-wang | 王, see No. 2082.

\section{治}

7330 Yel1-ch'êng 治城。

$1^{\circ}$ (obs.) Han, H. near Fu-chou Fu, Fullkien. $z^{\circ}$ (obs.) formerly $H$. near Chiang-ning $\mathrm{Fu}$, Kiangsu.

7331 Yeh-shu | 戌, chên near Mêng H., Honan.

\section{览}

7332 Yeh 業, see No. 861.

7333 Yeh-111an | 淽, see No. 7339.

7334 Yeh-tu | 都, see No. 225.

\section{鄴}

7.335 Yelı 鄴, see Nos. 225， 3839.

\section{葉}

7336 Yeh-ch'êng 葉城, H. in So-ch'ê C. L. C., New Dominion.

7337 Yeh-êrlı-ch'iang | 尔耂 Yarkand. 
葉

YEH-ÊRH-CHIANG.

$1^{\circ}$ A province in The New Dominion, bounded on the N. by Ili and Russian Turkestan ; on the E. by Harashar; on the S. by Thibet; and on the W. by Russian Turkestan.

$2^{\circ}$ Capital of the above province; Lat. $38^{\circ} 19^{\prime}$, Long. $77^{\circ} 30^{\prime}$; the name is also written Ya-êrhch'ien 押兒萃. Yarcan (Polo).

7338 Yelı-fang | 坊, ssü near Chien-ning H., Fullkien.

7339 Yeh-mi-li | 密店 (or, Yehmi-shih 也迷失, or, Yel1man 業满) Enil or In11, a town N.E. of Ili city.

7340 Yel1-yü | 榆.

$1^{\circ}$ (obs.) W. Han, H., N.E. of Ta-li Fu, Yünnall.

$2^{\circ}$ See Nos. 6049, 6163.

\section{謁}

7341 Yell-cl' êng 謁 城, (obs.) Han, a town near Jênch'iu H., Chihli.

\section{也}

7342 Yeh-li 也里, see No. 1906.

7343 Yehi-mi-shih | 迷失, see No. 8398.

7344 Yeli-yün-ch'ih | 云赤, a town W. of Ili.
覧 YEN-SHAN.

\section{菜}

$7344^{A}$ Yeh-linis 茶陵 see No. 147.

\section{YEN}

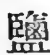

7345 Yell-ch'a 監朴 see No. $1915,1^{\circ}$.

7346 Yen-ch'ang | 㙫, Y. near Ta-tsu H., Ssuch' 'uan.

7347 Yen-cli'êng | 城 Yencling, H. in Huai-an Fu, Kiangsu ; Lat. $33^{\circ} 22^{\prime}$, Long. $120^{\circ}$; Han, Yen-tu 瀆; $C h^{\prime} e ̂ n$. Yen-ch'êng 城; Sui, Shê 射 C.

7348 Yen-ch'ih | 池, a posttown near Kao-t'ai H., Kansulh.

7349 Yen-ch'ïan | 泉, (obs.) Three Ks., H. near Mienchu H., Ssŭch' 'uan.

7350 Yen-k'ê | 課, ssŭ near Chuan-sha T., Kiangsu.

7351 Yen-kuan | 官.

$1^{\circ}$ Chên near Li H., Kansuh.

$2^{\circ}$ (obs.) formerly fort near Hsi-1ı H., Kansuli.

$3^{\circ}$ See Nos. 1934, 2988.

7352 Yen-shan | II Yen-shan, [196] J. in T'ien-ching Fैu, Chihli ; Lat. $38^{\circ} 07^{\prime}$, Long. $117^{\circ} 12^{\prime}$; orig., Wu-ti-yi 無棣邑; Han, Kaoch'êng 高城; Sui, Finsluii 浮水; Kin, I,i-fèng 
擥

YEN-SHAN.

利豐 chên; T Tang, Yenshan | I and Kê-ching 㻤津。

7353 len-shui | 水, (obs.) $P$. Chou, H., H. of Shih-nan Fin, Hupeh.

7354 Yen-t'ing | 营 Venting, [184] H. in 'T'mng-ch' nan Fu, Sstuch 'uan: I at. $31^{\circ} 14^{\prime}$, I.ong. $105^{\circ} 26^{\prime}$; Han, Knang-han 奮漢; Liang, Yen-t ing | 亭; T T ang, Tung-kuan 東關。

7355 Yen-tı| 瀆, see No. 7347. 7356 Yen-yüan | 源 Ven-yuen, [534]H. in Ning-yüan Fu, Ssüch'tran; Lat. $27^{\circ} 20^{\prime}$, I ong. $101^{\circ} 32^{\prime}$.

\section{还}

7357 len 延, see No. 7357.

7358 Yen-an | 努 Yen-1ngan.

$1^{\circ}$ I'n in the Ven-Yü-Sui [536] circ., Shensi; forming also the district city of Fu-shih; Lat. $36^{\circ} 42^{\prime}$, Long. $109^{\circ} 2 S^{\prime}$; S.A., land of the Pai-ti白萑 tribes; $T s^{\prime} \mathrm{in}$, Shang $上$. chion; Han, K. Ti 翟; 2nd Hei, Clnin-ming 金 明 and Tung-hsia 東 夏; $H^{\prime} . \mathrm{Hei}$, Yen C.: Sui, and $T^{*} a n g$, len-an | 发; Sung, Chang-wu彰武; Ming, Jen-an | 安 Fu.
遈 IEN-CH ING.

$2^{\circ}$ Fu in Huang-lnai 黃海 circ., Corea.

$3^{\circ}$ See No. 7359.

7359 Ven-ch'ang | 長 Yen[538]chang, H. in len-an Fu, Shensi ; I at. $36^{\circ} 37^{\prime}$, I,ong. $109^{\circ} 58^{\prime}$; 2nd Wei, Kuangan 笛缺; Sui, Ien-an 类; Thong, len-ch'ang

7360 Yen-ch'ang | 昌, see No. 4714.

$7360^{\text {A }}$ Yen-chi | 吉 T. in Wucha'ng C.I.'T', Kirin.

7361 Yen-Chien-Shao | 建 邵, circ. in Fuhkien comprising Yen-p ing Fn, Chienning $F u$ and Shao-wu Fu.

7362 len-ching | 津 Yen-tsin. $1^{\circ}$ H. in Wei-hui Fu, Honan ; Lat. $35^{\circ} 10^{\prime}$, Long. $114^{\circ} 20^{\prime}$; S.A., lingching 嘼 津; $7 s^{\prime} \mathrm{in}$, Suan-tsao 酸事; Sung, Ven-ching $\mid$ 津 $\mathrm{H}$.

$2^{\circ}$ (obs.) T゙ang, C. in T'ieln-ling H. Shingking.

7363 Yen-ch'ing | 慶 Ven-king [227] C. in Hsïan-hua Fn, Chilhi; Lat. $40^{\circ} 29^{\prime}$, Long. $116^{\circ} 03^{\prime}$; Han, Kuangning 廣富 in Shang-ku 上. 谷 chün; 2nd Hci, Pei-yen 北 䋳; Tang, Knei 娞 C. and Ju 儒 C.. 
Ming, 1st, Lunng-ch'ing 隆㡸, 2nd Yen-ch'ing | 愛.

7364 Yen-ch'uan| 玔 Yen[78] chnen, H. in len-an Fu, Shensi; Lat. $36^{\circ} 57^{\prime}$, Isong. $110^{\circ} 08^{\prime} ; H a n$, Lin - ho 臨河； 2nd Wei, Wên-an 文安; Sui, Yen-ch'nan | 川I.

7365 Yen-li | 㻃 H. in Chungcln'ing 忠清 circ., Corea.

7366 Ven-ling | 陵, see Nos. $312,3407,6209$.

7367 Yen-min | 民, (obs.) N. $\mathrm{Wei}, \mathrm{H} ., \mathrm{E}$. of $\mathrm{Cl}_{1}{ }^{\mathrm{i}} \mathrm{H}$., Sliansi.

7368 Yen-p ing | 平 Yen-ping, [306] $F u$ in the Jen-Chien-Shao circ., Fulukien ; forming also the district city of Nanp'ing; Lat. $26^{\circ} 38^{\prime}$, Lonng. $118^{\circ} 18^{\prime} ;$ Chou, in Min 閩 and Yüeh 越; Han, in Knei-chi 會稭 chiun; Tsin, and T'ang, Yen - p'ing | 平; Five dy'n., Hsiun 䤾 C. ; S. T'ang, Chien 劍 C.; Sung Nan-chien 南劍; Ming, Yen-p'ing | 本Fu.

7369 Yen-shui-ch'êng | 水城, (obs.) T'ang, a town E. of-Ỵen-an Fu, Shensi.

7370 Yen-tê | 德, (obs.) Sui, a towu W. of Yai C., Knangtumg.
7371 Yen-Yüi-Sui | 榆数, circ. in Shensi comprising Yenan Fu, Yii-lin Fu and Suitê $C$.

\section{兗}

7372 Yen 兗.

$1^{\circ}$ one of the nine criou, or great divisions of the Empire establislied by I ï 禹; comprising parts of Sliantung and Clilili. $2^{\circ}$ See No. 7373.

i373 Yen-clion | 川 Yen-clian, [130] Fu in the Yen-Yi-Ts'aoChi circ., Shantung; forming also the district city of Tžn-yang; Lat. $35^{\circ} 47^{\prime}$; Long. $116^{\circ} 59^{\prime} ;$ S.A., in K. In 魯; C. Wars, in K. Ch'u楚; Ts'in, in Hsiieh 蒒 chïn; Han, K. In 鼻 and Shan-yang I 陽 ; E. Han, Jên - ch' êng 任城; $T \sin$, in I, 㫘 chiin; Sui and T'ang, Yen|C.; Sung, T'ai-ning 泰察 and L, tung-ch'ing 櫳 度; Wing, Yen-chon| 州 Fin. Ta-din-fu (Polo).

7374 Yen-Yi-Ts'ao-Chi | 沂曹 沿, circ. in Slantung comprising Yen-chou $F u$, Yi-chon Fu, T's'ao-chou, Fil and Chi-ning $C$.

\section{晏}

7375 Yen 监。 
是

$1^{\circ}$ (obs.) T'ang, C. comprising part of the $\mathrm{N}$. of Knangsi.

$2^{\circ}$ See Nos. 2836, 2870.

7376 Yen - cl'êng | 城, a posttown nearCh'i-ho H., Shantung.

\section{宴}

7377 Yen-an 意数; by mistake for Ning-an 冥 发; see No. 4480.

\section{偃}

7.378 Yen-shih 遈師 Yen-sz, [263]H. in Ho-11an Fu, Honan; Lat. $34^{\circ} 45^{\prime}$, Long. $112^{\circ}$ $43^{\prime} ;$ Han, Suti, T'ang, Sung, Kin, Yüan and Ming, Yen-shih | 師 H.

\section{鄎}

7379 Yen 敫.

$1^{\circ}$ (obs.) formerly $\mathrm{K}$. in Honan.

$2^{\circ}$ See No. 8439.

7380 Yen-ch'êng | 班 Yen[384]ching, H.in Hsï C. . Honan; Lat. $33^{\circ} 38^{\prime}$. Long. $114^{\circ}$ $05^{\prime}$; orig., K. Yen |; Han, Yen-ch"êng | 城.

\section{璌}

7381 Yen-wu 演武, so near Hsin-yang C., Honan.

\section{鷹 or 倠}

7382 Yen-clı'a 䳆汊, chên near Tung-liu H., Anhui.
獡 YEN-LING.

7383 Ven-mên | 門 or 赝門. $1^{\circ} \mathrm{A}$ post-town near Tai C., Shansi ; also called Yen-mên Kuan | 师聯. $2^{\circ}$ (obs.) W. Han, chün, S. of Yu-yü H., Shansi. $3^{\circ}$ (obs.) H. Han, chün, $40 l i \mathrm{~N} . \mathrm{W}$. of Tai C., Snansi.

$4^{\circ}$ (obs.) Tsin, chïn W. of Tai C., Shansi.

$5^{\circ}$ (obs.) Kin, chên in Tai C., Shansi.

$6^{\circ}$ See Nos. 2378, 6102, 6157.

7384 Yen - P'ing - Ta - So - NingHsin-Tai-Pao 平大朔 管忻代保, circ. in Shansi comprising Yen-mên Kuan, P'ing-yang Fu, Tat'ung Fu, So-p'ing Fu, Ning-wu Fin, Hsin C., Tai C. and Pao-tê C.

翞

7385 Yen-ch'i 焉惹, Fu in the New Dominion. Formerly Harashar C.I.'T. See No. 1904, 2.

\section{鄢}

7.386 Jen-ch'êng 鄢城, a posttown near Yi-ch êng H., Hupel.

7387 Yen-ling | 陵 Yen-ling,

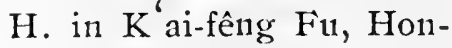
an ; Lat. $34^{\circ} 10^{\prime}$, Long. $114^{\circ} 21^{\prime} ;$ S.A., Chêng-yi 
漂

YEN-IING.

鄭邑; C. Wars, An-ling 努陵; Han, Yen-ling | 陵.

\section{沿}

7388 Yen-hai 沿海, ssŭ in Ch'in C., Kuangtung.

7389 Yen-ho | 河, T.S. in Ssŭnan Fu, Kneichou.

7390 Yen-ho-yu-ch'i | 河䂜溪, (obs.) Ming, ssü near Yin-chiang H., Kueichou.

\section{英}

7391 Yen-li 琶里, (obs.) formerly a town on the site of Ch' ï-fu H., Shantung.

\section{崦}

7392 Yen-tzŭ 崦 嵫, (obs.) T'ang, C. near Turfan.

\section{嚴}

7393 Yen 嚴.

$1^{\circ}$ (obs.) T'ang, C. comprising Lai-pin $\mathrm{H}$. and part of Ma-p'ing H., Kunangsi.

$2^{\circ}$ See No. 7394.

7394 Yen-chou | 州 Yen-chau, [231]Fu in the Chin-Cl' ii- Yen circ., Chehkiang; forming also the district city of Chien-tê ; Iat. $29^{\circ} 27^{\prime}$, Long. $119^{\circ} 35^{\prime}$; S.A. 1st, in $\mathrm{K}$. Wu 吳 $2 n d$, in $\mathrm{K}$. $\mathrm{Cl}^{\prime}$ n楚; $T s^{\prime}$ in in Knei-chi 會嵇 chïn and Tan-yang
荻 YHN-SUNG。

丹陽 chün; $W u$, Hsin-tu 新都; Sui and $I^{\prime} \mathrm{ang}$ ， Mu 㓐 C.; Sung, Yen| C. and Chien-tê 建德 H.; Ming, Yen-chor | 州 Fu. 7395 Yell-tao | 道, see Nos. $4568^{\mathrm{s}}, 7213$.

\section{岩}

7396 Yen-mên 岩門, T.S. near Huang-p'ing C., Kueichon.

7397 Yen-shou | 手 Iwate, ken in Japan; chief town Morioka.

7398 Yent-t'on | 頉, ssŭ near $\mathrm{Ch}^{\prime}$ ing-p'ing $\mathrm{H}$., Kneichon.

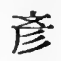

7399 Yen H., Honan.

7400 Yen-yang | 陽, H. in Ch'ing-shang 慶份 circ., Corea.

\section{顏}

7401 Yen-1o 顏羅, (obs.) Sui, $\mathrm{H}$. near $\mathrm{Ch}$ inng-chon $\mathrm{Fu}$, Kuangtung.

7402 Yen-shên | 触, chên near Po-shan H., Shantung.

\section{乲}

7403 len 炎, (obs.) T'ang, C. near Cliang-ming H., Ssŭch' lian.

7404 Yen-fang | 方, a post-town near P'ing-yi H., Yünnan.

7405 Yen-sung| 松, ssŭ in Chanyi C., Yünnan. 
㸚

YEN.

\section{䟝}

7406 Yen 剠, '(obs.) Han, H., N.E. of P'ei C., Kiangsu.

\section{燕}

7407 Yen 燕.

$1^{\circ}$ (obs.) K. in N. Cliihli.

$2^{\circ}$ See Nos. 1817. 3969, 5822 .

7408 Yell-ch'êng | 城, (obs.) Kim, capital of the Empire, lying S.W. of Peking.

7409 Yen-ch'i | 岐 Yen-ki, H. in Chung-ch'ing 忠清 circ., Corea ; Lat. $37^{\circ} 08^{\prime}$, I,ong. $126^{\circ} 50^{\prime}$.

7410 Yen-ching | 京, see No. 5822 .

7411 len-p ‘ingl平, see No. 299. 7412 Yen-shan|!l, see No.5822. $7412^{\mathrm{A}}$ Yeulshih | ifj, see No.5822. 7413 Ven-ruin | see No.950. 烟

7414 Iell-t'ai 烟喜, see No.977.

Ien-cha, see No. 7345.

Yen-chang, see No. 7359.

Yen-chan, see Nos. 7373, 7394.

Yen-ching, see Nos. 7347, 7381 .

len-chuen, see No. 7364.

Yen-ki, see No. 7409.

Yen-king, see No. 7363.

Ien-ngan, see No. 7358 .

len-ping, see No. 7368.
宜 YI.

Yel1-sz', see No. 7379.

Yent-ting, see No. 7354.

Yen-tsin, see No. 7362 .

Yel1-yuen, see No. 7356.

Yenguikend, see No. 7240.

lenkoji, see No. 5476.

Yerendeh: see No. 7487.

\section{YI}

\section{迎}

7415 Yi-hsi 迎 西, circ. in Yünnan, comprising 'Ta-li Fu, Cl1' 11-hisiung Fu, Yungch' 'ang Fu, Yung-pei T., Li-cliiang Fu and Mênghuta 'T.

7416 Yi-nan| 南, circ. in Yünnan, connprising $P^{\prime} 11$-êrh Fu, Shun1-ning Fu, Chênyüan $T$., Yüan-chiang 'T., Cling-tung ' $T$. and Lin-an Fit.

7+17 Yi-tı11g|東, circ. in Y'ünnan, comprising $\mathrm{Cl}^{4}$ ì-ching Fu, Tung-ch' wan Fu, Chaot'ung Fil and Ch'êngchiang Fu.

\section{宜}

$7418 \mathrm{Yi}$ 宜

$1^{\circ}$ (obs.) S. Sung, C. in Kuang-hsi 廣西 circ. $2^{\circ}$ (obs.) Liang, C. near Yi-ch'ang Fu, Hupeh.

$3^{\circ}$ See Nos. 1192, 6416. $7322,7+37,7443$. 
宜

Yi-chang.

7419 Yi-chang | 章 I-chang, $\mathrm{H}$.

[507]in Clı'ên C. Hunan; Iat. $25^{\circ} 47^{\prime}$, Long. $112^{\circ} 38^{\prime}$; Han, Ch'ên 森 H., Sui, Yi-cliang 義 $\mid$; Tang, Yi-chang | 章

$7420 \mathrm{Yi}$-cl1'ang | 昌 I-chang.

[528] $1^{\circ}$ Fin in the Sluang-chingnan circ., Hupeh; forming also the district city of 'Tung-lin; Lat. $30^{\circ}$ $49^{\prime}$, Lorig. $111^{\circ} 10^{\prime}$; one of the ports on the langtsze open to foreign trade; orig., in K. Ch' 1 楚, with capital Yi-ling 夷陵; Han, Yi-ling 茀 陵; Minor Han, Hsiling 西 陵; P. Chou, Hsia 恹 C.

$2^{\circ}$ (obs.) Tsin, a town near Y i-tu H., Hupelı.

7421 Vi-cli'êng | 虚 I-ching, H. in Hsiang-yang Fin, Hupeh; Lat. $31^{\circ} 40^{\prime}$, Long. $112^{\circ}$ $0 \mathrm{~S}^{\prime} ;$ C: Wars, Wu 鳥 $\mathrm{H}$.; Han, Yi-ch'êng | 城; Liang, Shuai-tao 本道.

7422 Yi-cli uan | 川 I-chnen.

$1^{\circ}$ H.in Yen-an Fu, Shensi ; Lat. $36^{\circ} 08^{\prime}$, J.ong. $110^{\circ} ;$ Tsin, in Shang $^{\circ}$ .上 chiun; P. Wei, Yich'nan 毒川: P. Chou, 'Tan-yang 乎陽; T" ang $^{\circ}$ Tan 丹 C., Sumg, Yichinan 川.
宜 YI-HUANG.

$2^{\circ}$ See No. 101.

7423 Yi-ch'un | 奉 I-chu11。

$1^{\circ} \mathrm{H}$. forming the prefectural city of Yüan-chon, Kiangsi ; Lat. $27^{\circ} 52^{\prime}$, Long. $114^{\circ} 22^{\prime}$; IIan, Yi-ch' 11 | 春 H.; Tsin, li-yang | 晹.

$2^{\circ}$ (obs.) $W$. Han, Marq. 60 li S.W. of Ju-yang H., Honan.

$3^{\circ}$ (obs.) Kin, H., N.I. of K'ai-yïan H., Shingking.

$4^{\circ}$ see Nos. 2797, 7743.

7424 Yi-cliün | 君 l-kiun, H. in Fu C., Shensi ; Iat. $35^{\circ}$ $28^{\prime}$, Long. $109^{\circ} 02^{\prime} ; T^{*}$ ang, Tai-yii 股秋; Ind Wei, Yi-chün | 虎.

742.5 Yi-fêng | 風, (obs.) $T^{6} a n g$ H., S. of ling-fu H., Kuangsi.

7426 Yi-fêng | 幽.

$1^{\circ}$ (obs.) $T \sin$, H. $30 l i \mathrm{~N}$. of Hsin-ch'ang H., Kiangsi.

$2^{\circ}$ (obs.) Lino and Kin, H. 100 li S.W. of Lianoyang $C$. , Slingking. $3^{\circ}$ See Nos. $2727,2738$.

7427 Yi-lio | 不 I-lio, H. in Chên-hssi T., Kansulı; Lat. $43^{\circ} 40^{\prime}$, I,ong. $94^{\circ}$.

7.28 Yi-hnang | 黄 I-liwang, $H$. [5+1]in lin-chon lin, Kiangsi; 
宜

Lat. $27^{\circ} 32^{\prime}$, Long. $116^{\circ}$ $12^{\prime}$; E. Han, Lin-ju臨汝; Wu, Yi-lnnang |黃.

7429 Yi-hsing | 與 I-hing, H. in Ch'ang-chou Fu, KiangS11; this city serves also for Ching-ch i $\mathrm{i}$.; L.at. $31^{\circ}$ $28^{\prime}$, Long. $119^{\circ} 49^{\prime}$; orig., Ching-ch' $\mathrm{i}$ 荆 溪; $T \sin$, Yang-yi 陽美; Tsin, Yihasing 義興; Sung, Yihsing | 興.

7430 Yi-lan | 菊 I-lan, H. in $T^{\prime}$ 'ai-pei $\mathrm{Fu}, \mathrm{N}$. Formosa.

7431 Yi-liang | 辰 I-liang, H. [335] in Yün-11an $\mathrm{Fu}, \mathrm{Yünnan;}$ Lat. $24^{\circ} 58^{\prime}$, Long. $103^{\circ}$ $14^{\prime} ; T^{6}$ ang, $\mathrm{K}^{\prime}$ 'nang 匡 C.; Yüan, Yi-liang | 良.

7432 Yi-ling | 陵, chên near T'ai C., Kiangsu.

7433 Yi-1n | 㵀, a post-town near $\mathrm{Cl}^{\text {' }}$ ang-wu $\mathrm{H}$., Shensi.

7434 Yi-1un | 倫.

$1^{\circ}$ (obs.) Liang, H. near Tan C., Knangtung.

7435 Yi-ning | 䆝 $\mathrm{H}$. in Ch'ingshang 慶何 circ., Corea.

7436 Yi-pin | 宾 I-pin, H. forming the prefectural city of Hsü-chou, Ssŭch'uan ; Lat. $28^{\circ} 38^{\prime}$, Long. $104^{\circ}$ $46^{\prime}$; Han, $\mathrm{P}^{\prime}$ o-tao 整道; $P$. Chou, Wai-chiang 外

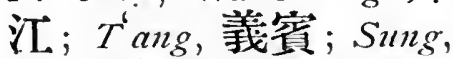
Yi-pin | 筫.
宜 YI-YANG。

7437 Yi-shan | III I-shan, H. forming the prefectural city of Cl' ing-yiian, Knangsi; Lat. $24^{\circ} 26^{\prime}$, Long. $108^{\circ}$ $25^{\prime} ; T^{6}$ ang, Yi | C. and Lung-shni 龍 水; Sung, Yi-shan | !l.

7438 Yi-shan | 善, ss̆̌ in I,ienshan T., Kuangtung.

7439 Yi-shon | 铝, see No. 1346. 7440 Yi-tı | 都 I-tu.

[528] $1^{\circ} \mathrm{H}$. in Ching-choir $\mathrm{Fu}$, Hupeh; Lat. $30^{\circ} 28^{\prime}$, Long. $111^{\circ} 19^{\prime}$; Han, Yi-tao 荑道; T'ang, Chiang-ch wan 汇. 川; Tang, and Sung, Yitu| 都 $\mathrm{H}$.

$2^{\circ} \mathrm{Y}$. near Ma-yang $\mathrm{H}$. Hunan.

$3^{\circ}$ (obs.) $W . H a n, H$. in Shensi.

$4^{\circ}$ (obs.) Tsin, S. Sung and $S . T s^{\prime} i$, chïn N.W. of Yi-tu H., Hupeh.

7441 Yi-yang | 陽 I-yang.

[263] $1^{\circ} \mathrm{H}$. it. Ho-nan Fu, Honan; Lat. $34^{\circ} 31^{\prime}$, Long. $112^{\circ} 10^{\prime}$; Han, Yi-yang |晹; Wei, Kan-t'ang 甘棠; $W$. Wei, Yang 隌 C.

$2^{\circ}$ A post-town nearCh 'ingyüan $\mathrm{F} u$, Kuangsi.

$3^{\circ}$ See No. 7423. 
宜 YI-YFH-WAN。

7442 Yi-yeh-wan | 野澋, a town in the Lin-ch in Islands.

\section{義}

7443 Yi 義 I.

[430] $1^{\circ}$ C. in Chin-chou Fu, Shingking; Lat. $41^{\circ}$ $30^{\prime}$, Long. $121^{\circ} 20^{\prime}$; Liao, $\mathrm{Yi}$ 宜 $\mathrm{C}$.

$2^{\circ}$ (obs.) Tang, C. in Ssǔch uan.

$3^{\circ}$ See Nos. 2218, 4038. 6971.

7444 Yi-an | 努.

$1^{\circ}$ (obs.) $T s^{6} i$, a town W. of Hsiang-yang Fin, Hupeh.

$2^{\circ}$ See Nos. 369, 6825.

7445 Yi-chang | 章, see No. 7419.

7446 Yi-ch'ang | 昌, a post-town near Hsin-an H., Honan.

7447 Yi-chao | 招, (obs.) Tsin, H., S. of Ta-pu H., Kuangtung

7448 Yi-ch' êng | 城 I-ching. $1^{\circ} \mathrm{H}$. in $\mathrm{Cl}^{\prime}$ ing-shang 慶 们 circ., Corea ; I.at. $36^{\circ}$ $20^{\prime}$, Long. $128^{\circ} 40^{\prime}$.

$2^{\circ}$ Chên near Ho-shun H., Shansi.

$3^{\circ}$ Chên near Hsi C., Shansi. $4^{\circ}$ - (obs.) T"ang, H., E. of Chên-an Fu, Kuangsi. $5^{\circ}$ (obs.) formerly a town near Ssu C., Anhui.
義 YI-NING。

7449 Yi-chou|州, Fn in P'ingan 本安 circ., Corea; Lat. $39^{\circ} 58^{\prime}$, Long. $124^{\circ} 43^{\prime}$.

7450 Yi-ch' î | 渠.

$1^{\circ} W$. Han, circ. N.IV. of

Ning C., Kansuh.

$2^{\circ}$ See Nos. 1191, 4672.

7451 Yi-ch'nan | 川, see Nos. $408 t, 7422$.

7452 Yi-ch üan | 泉, see Nos. $5701,5944$.

7453 Yi-fêng | 䇺.

$1^{\circ}$ A post-town near Fêngju11 H., Chihli.

$2^{\circ}$ (obs.) Liao H. in the Ongniod county, N. of Jehol.

$3^{\circ}$ See Nos. 652, 4154.

7454 Yi-hsiang/拫, (obs.) Tsin, $\mathrm{H}$. near $\mathrm{Cl}_{1}$ 'ang-lnsing $\mathrm{H}$, Chehkiang.

7455 Yi-hsing | 與 I-hing.

. $1^{\circ} \mathrm{H}$. in $\mathrm{Cl}_{1}{ }^{\text {ing-shang 慶 }}$ 何 circ., Corea; Lat. $35^{\circ}$ $54^{\prime}$, Long. $128^{\circ} 45^{\prime}$.

$2^{\circ}$ (obs.) Tsin, H. near

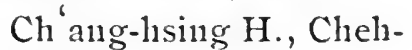
kiang.

7456 Yi-ling | 陵, see Nos. 321, 2897.

7457 Yi-lun | 倫, see No. 6215.

7458 Yi-ning | 窝 I-ning.

[193] $1^{\circ}$ C. in Nan-ch' ang Fu, Kiangsi ; I at. $29^{\circ} 01^{\prime}$, Long. $114^{\circ} 30^{\prime}$; Han, 
咅戈

VI-NING.

Ai 艾 H.; Tsin, Shnihısin 水修; Sung, Fênning 分富.

[542] $2^{\circ} \mathrm{H}$. in Kuei-lin Fu, Kuangsi ; Lat. $25^{\circ} 22^{\prime}$, I,ong. $110^{\circ}$; Tang,

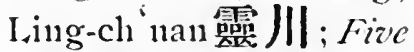
dyn., Yi-ning | 耍.

$3^{\circ}$ See No, 1098.

7459 Yi-pin | 顀.

$1^{\circ}$ (obs.) T'ang, H. 160 li N. IV. of Yi-pin H., Ssŭcli nan.

$2^{\circ}$ See No. 7436 .

7460 Yi-shan||l, Y. near Yungming H., Hnnan.

$7+61$ Yi-sui | 綏, (obs.) N.Wci, H., N. of Sui-ping H., Honal1.

7462 Yi-ts'un | 存, (obs.) $S$. Sung and $S . T s^{6} i, \mathrm{H}$. in Ssüch' nan.

7463 Yi-w1 | 烏 I-wu, H. in [520] Chin-hua $\mathrm{Fu}_{\mathrm{u}}$, Chehkiang; Lat. $29^{\circ} 20^{\prime}$, I.ong. $120^{\circ}$ $12^{\prime} ;$ Han, Wu-shang 鳥 穓; T"ang, Hua-cli uan 華川; and Yi-wu|島

7464 Yi-vang | 曜, see Nos. $2793,2810$.

\section{儀}

$7465 \mathrm{Yi}$ 儀, see No. 3801 .

7466 Yi-ch'ang|昌, seeNo. 3453. 7467 Yi-chên | 目, see No. 7468 .
YI-I,UNG.

7468 Yi-chêng | 徵 I-ching, H. [332] in Jang-chon Fu, Kiangs11; Lat. $32^{\circ} 18^{\prime}$, Long. $119^{\circ} 09^{\prime} ; H a n$, Chiang-tı 江. 都; T゙ang, Yang-tz̆ 楊子; Sung, Chên 眞 C.; Ming, Yi-chên $\mid$ 真 $\mathrm{H}$. Port of call. Sinju (Polo.)

7469 Yi-clı'êng | 城, (obs.) formerly a town near Lin H., Honan.

7470 Yi-fêng | 封 I-fung.

$1^{\circ}$ '. in K'ai-fêng Fu, Holla11; Iat. $34^{\circ} 55^{\prime}$, Long. $115^{\circ} 08^{\prime} ; .5 . A$., Wei-yi 衞邑; 2 nd $\mathrm{Wei}$, K'ao-rang 考陽; Ki川, Yi-fêng | 非 H..

$2^{\circ}$ Chên, near Wn-yang H., Honan ; Yüan, Y’ifêng | 封 $H$.

$3^{\circ}$ See No. 7471 .

7471 Yi-fêng-hsiang | 封拫, a post-town near Lan-yi H., Honan ; Ming, Yi-fêng | 封 $\mathrm{H}$.

7472 Yi-k un | 坤, (obs.) Lino, C. in the Ongniod conntry, N. of Jehol.

7473 Yi-lıng | 䈮 I-lu11g, H. in [253] Shı11-ch 'ing Fu, Ssŭch 'ıan; I at. $31^{\circ} 28^{\prime}$, Long. $106^{\circ}$ 25';Han, I,ang-chung 闃 中; Liang, Vi-lung | 隴; Tang, Fang 方 C. 
揖

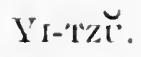

提

7474 Yi-tzŭ 揖次, (obs.) Tsin, H., N. of Kullang H., Kansul.

\section{易}

$7475 \mathrm{Yi}$ 易 Yih.

[545.1 $1^{\circ}$ C. L. C. in Ch'ing-ho circ., Chihli; Lat. $39^{\circ}$ $24^{\prime}$, I,ong. $115^{\circ} 35^{\prime}$; Ts'in, in Slang-kut. 谷 chïn; Kin, Yi |C. $2^{\circ}$ Sce No. 2877.

7476 Yi-cli'êng/城, see No.287\%. 7477 Yi-kn|古, sšr in Hsïntien C., Yünuan.

7478 Yi-lung | 籠, (obs.) Y'ïan, H. $180 l i$ N. of L,u-ch' iian H., Yünnan.

7479 Ji-n1ên | 門 Yilı-mun.

[544] $1^{\circ}$ H. in Yün-nan F ${ }^{\circ}$, Yünnan; Lat. $24^{\circ} 45^{\prime}$, Isong. $102^{\circ} 19^{\prime}$.

$2^{\circ}$ (obs.) Yïan and Ming, $\mathrm{H}$., $30 \mathrm{li} \mathrm{S}$. of the above.

\section{沂}

7480 Yi 励, see Nos. 358s, 7481. 7481 Yi-chon | 州 I-chan, Fu [546] in the Yen-Yi-Ts'so-Chi circ., Shantung; forming also the district city of Lan-shan ; I at. $35^{\circ} 15^{\prime}$, Long. $118^{\circ} \cdot 35^{\prime} ; T s^{\prime} \mathrm{in}$, I aurg-yeh 唧伢 chitn; 2nd $\mathrm{IVe}$, Pei-hsii 北徐 C., Sui, I,in-yi 臨 |; T゙ang,
伊

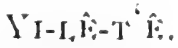

Yi | C. and Lang-yeh 瑯 躯 chiin.

7482 Yi-shui | 水 I-shwui, $H$. [546] in Yi-chou Fu, Shantung; Lat. $35^{\circ} 46^{\prime}$, Long. $119^{\circ}$; S.A., Yün-yi 鄆邑; Han, Tung-kuan 東莞; P. Chou, Hsin-t'ai 新泰; Sui, Yishui | 水.

$7483 \mathrm{Yi}$ 伊。

$1^{\circ}$ (obs.) T゙ang, C. 10 li S. of Hami, New Dominion. $2^{\circ}$ See No. 5990 .

7484 Yi-clion | 州 and Yi-wn-ln | 吾慮: names for Hani under the $T^{6}$ ang and $P$. Handynasties respectively. See No. 1907.

$7484^{4}$ Yi-ch'nan | 川, H. in Chiang-yüan 江. 愿 circ., Corea.

7485 Yi-ch ïieh | 閶, see Nos. 5990, 7495.

7486 Yi-êrh-h11-111u | 尔胡穆 Ilkoun, a town in Outer Kansulı ; Lat. $40^{\circ} 50^{\prime}$, I,ong. $95^{\circ} 25^{\prime}$.

$7486^{A}$ Yi-hsiang-pao | 香 保, Ikao, Mincral Springs, Japan.

$7486^{\mathrm{B}} \mathrm{Y} \mathrm{i}-\mathrm{k}$ è-chao | 克昭 Iklie Chao, Mongol League.

7487 Yi-lê-t' ê|勒特 Yerendell, a town in Yarkand; Iat. $39^{\circ} 45^{\prime}$, Long. $73^{\circ} 40^{\prime}$. 
伊 YI-LI-CH'

7488 Yi-li-ch'i | 里啒 Ilchi, or Khoten; see No. 2057.

7489 Yi-li | 物 Ili.

$1^{\circ}$ Province in The New Dominion bounded on the N. by Tarbagatai and Russian Turkestan; on the E. by Harashar; on the S. by Yarkand and Harashar; and on the IV. by Russian Turkestan.

$2^{\circ}$ Prefecture in Yi-T'a circ., Ili ; capital of the province; also called Kuldsha, and Hui-yïan 惠遠; in ancient times Yi-la-pa-li 亦刺八里 (or, Yi-li-pa-li 亦方 把里) Ilbalik; the ancient city of Almalik (see No. 16) has been identified with Ili. $T^{\prime}$ ang, Yüeh-p'an 悅 般. Lat. $43^{\circ} 46^{\prime}$, Long. $82^{\circ} 30^{\prime}$.

7490 Yi-ssŭ-pa-la-na | 思八刺 納, see No. 7522 .

7491 Yi-T'a | 塔, circ. in the New Dominion, comprising Ili and Tarbagatai

7492 Yi-t'ang | 通, C. in Chi-lin circ., Kirin.

7493 Yi-w11 | 吾.

$1^{\circ}$ (obs.) Tsin, H., N. of An-lisi C., Kansul.
资

YI-PIN.

$2^{\circ}$ (obs.) Tiang, H., 10 li S. of Hami, New Donninion.

7494 Yi-wu-1u| 吾盧, see No. 7484.

7495 Yi-yang | 陽 I-yang, H. [200] in Ju C., Honan; Lat. $34^{\circ}$ $12^{\prime}$, Long. $112^{\circ} 30^{\prime} ; \mathrm{Ts}^{\prime} \mathrm{in}$, Hsin-ch'êng 新城; Sui, $\mathrm{Yi}$-ch'üeh!閶; $T^{*}$ ang, Yi-yang | 陽.

$7496 \mathrm{Yi}$ 费.

\section{费}

$1^{\circ}$ (obs.) T'ang: C., N.W. of $\mathrm{L}$,ung-ch üan $\mathrm{H}$., Kueichon.

$2^{\circ}$ See Nos. 5701, 5944.

7497 Yi-an | 努。

$1^{\circ}$ (obs.) S. Ts $i$, H. in Nan-chêng H., Shensi. $2^{\circ}$ See No. 3151.

7498 Yi-ch'iian | 泉, see No. 5701.

7499 Yi-lin | 鄰, (obs.) $T^{6}$ ang, C., S. of Hsing-wên H., Ssǔch' van.

7500 Yi-ling | 陵, see No. 7420. 7501 Yi-1nên | H erly a town near Hsün H., Honan.

7502 Yi-1nêngl蒙,(obs.) T゙ang, H. in Yi-shan H., Kuangsi.

7503 Yi-pin | 䆩.

$1^{\circ}$ (obs.) $T^{6}$ aing, C., N.E. of Liang-lisiang $\mathrm{H}$, Chihli. 
策
$2^{\circ}$ (obs.) T"ang, H. near Hsï-cliou Fin, Ssŭ- cli nan.

7504 Yi-tao | 道, see No. 7440.

7505 Yi-yii | 舆, (obs.) $W$. Han, H., N.E. of Yench'ing C.. Chilili.

\section{移}

$7506 \mathrm{li}$ 移, (obs.) Tiang and Sung, C. in Hsii-chon $\mathrm{F}^{\mathrm{n}} \mathrm{n}$, Ssŭch' 'ıan.

$7507 Y$ Y i-fêng | 風, (obs.) Tsin, S. Sung, S. Ts'i and Sui H. in Annan.

\section{黟}

$7508 \mathrm{Yi}$ 黟 (or, 黝) I, H. in [314] Hui-chon Fu, Anhui ; Iat. $30^{\circ} 05^{\prime}$, Long. $117^{\circ} 58^{\prime}$; Ts'in, Han, Tsin, S. Sung, S. Ts'i, Sui, Tang, Sung, Yïan and Ming, Yi $\mid \mathrm{H}$.

\section{黝}

7509 Yi 黝奻, see No. 7038.

\section{猗}

7510 Yi-shih 猗氏 I-shi, H. in $\mathrm{P}^{\mathrm{i}}$-chon Fil, Shansi; Lat. $35^{\circ} 11^{\prime}$, Long. $110^{\circ} 43^{\prime}$; orig., feudal state Hsïn 的; $T s^{\prime} i n, Y i$-slih | 氏

\section{XII}

7511 Yi-ling X川陵, see No. 4099.

\section{翼}

7512 Yi-ch' êng 巽城, see No. 736.
嶧到。

\section{衣}

7513 Yi-chin 衣錦, ssü in Hsing-kno H., Kiangsi.

\section{墔}

7514 Yi-ch'êng 賞 城 I-cling, [498] H. in P'ing-yang Fin, Slansi ; Lat. $35^{\circ} 37^{\prime}$, I.ong. $111^{\circ} 47^{\prime}$; S.A., Chiang-ri 綘邑; Sui, Yi-ch'êng | 城; T"ang, Knei 墖 C.

\section{邑}

7515 Yi-shih 邑市, (obs.) formerly H., N.E., of Lt1-11an C., Jünnan.

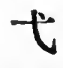

7516 Ii-chii 七居, (obs.) Han, H., S. of Ning C., Kansuh. 7517 Yi-yang | 陽 Yih-yang. [211] $1^{\circ} \mathrm{H}$. in Kuang-hsin Fu, Kiangsi ; Lat. $28^{\circ} 25^{\prime}$, Long. $117^{\circ} .33^{\prime}$; Han, Yü-lıan (or, Yü-kan) 餘汗; $W u$, Kê-yang 葛陽; Sui, Yi-yang | 陽.

$2^{\circ}$ See No. 3430 .

\section{啈}

7518 Yi 嶧 Yiln, H.in Yen-chon [106] Fu, Sliantung; Lat. $34^{\circ}$ $53^{\prime}$, Long. $117^{\circ} 51^{\prime}$; S.A., in K. Tsêng 鄫; Han, Lanl-ling 蘭陵 and Ch' êngch'êng 永城; Sui, Tsêng 鄭 C. ; T Tang, Ch' êng 永 $\mathrm{H}$; Kin, Yi $\mid \mathrm{H}$. 
ग्र

YI-I,A-P.A-L,I.

\section{施}

7519 Yi-la-pa-li 亦俥八里, see No. 7489.

7520 Yi-li-pa-li|少把里, see No. 7489.

7521 Yi-pi-êrl1-shih-pi-êrl | 必 兒头必自 Siberia。

7522 Yi-ssŭ-fa-liang | 思法椋 (or, Yi-ssü-fu-han | 思坓 突, or, Yi-ssü-pa-la-11a 伊 思八刺納) Ispahan in Persia (Persian nane also Isfahan); Lat. $32^{\circ} 45^{\prime}$, Long. $51^{\circ} 50^{\prime}$.

7523 Yi-ssü-fu-han| 思弗罕, see the preceding.

7524 Yi-tso | 佐, (obs.) Yïan, H. in Yünnan; Lat. $25^{\circ}$ $20^{\prime}$, Long. $104^{\circ} 46^{\prime}$; Han, Wan-wên 宛溫; T'ang, P'an 盤 C. in Ssuch' 'tant.

7525 Yi-tzŭ-k'ung | 筫 孔 , a post-town near $\mathrm{P}^{\prime}$ 11-an 'T., Kneiclou.

\section{尔}

7526 Yi-yi 奕费, T.S. near I,eipo T., Ssŭch' 'uaı.

\section{分}

$7527 \mathrm{Yi}$ 解.

$1^{\circ}$ (obs.) $W$. Hizn, сноU comprising parts of $Y$ ü11nan and Ssŭch' tran.

$2^{\circ}$ See Nos.511, 7535, 7813.

7528 Yi-clíang | 晶, see Nos. 340,7842 .
谷监 YIMANG。

7529 li-cliang | 将, ssŭ near Knei-yang H., Hunan.

$7530 \mathrm{Vi}$-ching | 津, see No.5881. 7531 li-li | 離, see No. 4792.

7532 Vi-nuên| 阿, chên near Pao-chi H., Shensi.

7533 Yi-p'ing | F , H. in Fêngt'ien Fin, Shingling.

$7534 \mathrm{Yi}-$ shan | |ll, chün in Ch'üan-lo 全 䉜 circ., Corea.

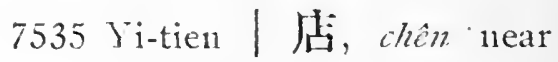
Fiu-fêng H., Shensi.

7536 Yi-tu | 都 Yilı-tu。

$1^{\circ} \mathrm{H}$. forming the prefectural city of $\mathrm{Cl}^{\text {'ing-chou, }}$ Sliantung; I at. $36^{\circ}$ 44', Long. $118^{\circ} 44^{\prime}$; Han, Yi | H.; Sui, T'ang and Sung, Yi-tu 都 H.; Kin, Yi-tu 都 Fn and H.; Yüan, Yi-tu | 都 circ. and H.; Ming, Yi-tu | 都 $\mathrm{H}$.

$2^{\circ}$ (obs.) S. Sung and $N$. Wei, H., S. of Shouknang H., Shantu1ng. $3^{\circ}$ (obs.) S. Ts'i, H. near Cliang-11ing Fin, Kiangsis.

$4^{\circ}$ See No. 1204.

7537 Yi-yang | 陽 Yih-yang. [490] $1^{\circ}$ H. in Ch'ang-sha Fu, Hunan; Lat. $28^{\circ} 35^{\prime}$, I.ong. $112^{\circ} 08^{\prime} ; T s^{\prime} \mathrm{in}$, Yi-yang | 榢 $\mathrm{H}$. 
䖝

$2^{\circ}$ See Nos. 2455, 4689, 7703.

$$
\text { 滃 }
$$

7538 Yi 溢, see No. 4457.

\section{郳}

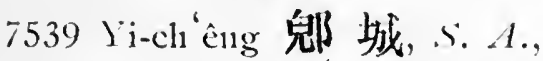
town near T'êng H., and T'son H., Shantung.

\section{遺}

7540 Yi-li 遺里, see No. 1906.

Yill, see Nos. 7475, 7518.

Yih-1111n, see No. 7479.

Yih-tu, see No. 7536.

Yih-yang, see Nos. 7517, 7537.

Yikar, see No. 129.

\section{YIN}

陵

7541 Yin 陰, (obs.) Han, a town E. of Kuang-hua H., Hupeli.

7542 Yin-an | 妿:

$1^{\circ}$ (obs.) W. Han, Tsin, N. Wei, H. $20 l i \mathrm{~S}$. of Ch'ing-fêng H., Chihli. $2^{\circ}$ (obs.) S. Smng and $S$. Ts'i, H. 180 li S.E. of 'T'ung-ch'êng H., An1hui.

$3^{\circ}$ (obs.) S. Sung, H. in Shantung.
因 YIN-I,

7543 Yin-cli êng | 城, see No. 34.32 .

7544 Yin-chin 丝, see No. 2220.

7545 Yin-chn | 竹, H. in Ching-

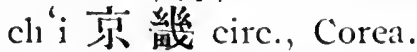

7546 Yin-kuan | 館, see Nos. $5462,{ }^{\mathrm{s}} 7568$.

7547 Yin-pingl本, see No. +169.

$75+8$ Yin-p'ing-tao | 平道, see No. 7007 .

7549 Yin-shan||l, (obs.) Han, a town N. W. of $\mathrm{Yu}$ H., Hinalu.

\section{落}

7550 Yin-cliêng 落娍, chên near Kao-p 'ing H., Slıansi.

\section{銀}

7551 Yin 銀.

(obs.) T"ang and Sung, C. 80 li N. W. of Michilı H., Sheusi.

7552 Yin-cl' êng | 旅, (obs.) chên, a town $120 l i \mathrm{E}$. of Tê-lısing H., Kiangsi.

7553 Yin - ch'uan| 川, a posttown near Hsün-hua 'T., Kansul.

7554 Yin-shan ||山, (obs.) Sui, H., S. E. of Tzŭ C., Ssŭch' nan.

7555 Yin-shêng | 生, see Nos. $442,1138,6961$.

\section{因}

7556 Yin-lü [太閫, (obs.) Tang, $\mathrm{H}$. near Hsï-chou Fu, Ssüch' uau. 
因

YIN-YIN.

7557 Yin-yin |., Y. near Yüanwu H., Honan.

7558 Yin-yüan | 遥, sš̆ in Yüan-chiang C., Yümnan.

\section{E[]}

7559 Yin-chiang 印 江 Yin-

[86] kiang, H. in Ssŭ-nan Fu, Kueichon ; Lat. $28^{\circ} 25^{\prime}$, Long. $108^{\circ} 30^{\prime} ; \mathrm{Ming}$, Yin-chiang | 江. $\mathrm{H}$.

\section{鄞}

7560 Yin 鄞 Yin.

$1^{\circ} \mathrm{H}$. forming the prefectural city of Ning-po, Chehkiang; I at. $29^{\circ}$ $49^{\prime}$, Long. $121^{\circ} 35^{\prime}$; Han, Koll-chang 句章; Tang, Yin | C.; Sung, Yïan and Ming, Yin | $\mathrm{H}$.

$2^{\circ}$ (obs.) Han, Tsin, S. Sung, and $S$. Ts' $i, \mathrm{H}$. $50 l i$ E. of Fêng-hura H., Chehkiang.

$3^{\circ}$ See No. 1766.

7561 Yin-chiang | 江, sš̆ in Yin H. Chehkiang.

\section{殷}

\section{Yin 殷.}

$1^{\circ}$ (obs.) T'ang, C. 293 li $\mathrm{N}$. W. of Yi-pin H., Ssŭch' nan.

$2^{\circ}$ Yïan, C. 100 li N.E. of $\mathrm{P}^{\mathrm{i}} \mathrm{ing}$-jang Fu, Corea.

$3^{\circ}$ See No. 2422.
YING.

7563 Yin-li | 栗, H. in Huanghai 黄海 circ., Corea.

$7563^{A}$ Yin-shan | ill, H. in P'ingan 本落 circ., Corea.

\section{洀}

7564 l'in-chiang 溵江, see No. 5559.

7565 Yin-shui | 水, see Nos. 3011, 5559.

\section{圄}

7566 Yin-yin 司 陉, see No. 715.

\section{引}

7567 Yin-chia-hui 引咓四, chên near Hsi-an Fu Shensi.

Yin-kiang, see No. 7759. Yin-tau, see No. 6358.

\section{YING}

\section{應}

7568 Ying 應 Ying.

[376] $1^{\circ}$ C. in 'Ta-t' $111 \mathrm{~g}$ Fin, Shansi ; Lat. $39^{\circ} 39^{\prime}$, Long. $113^{\circ} 14^{\prime} ; T s^{\prime}$ in, Yin-knan 院館; T'ang, Chin-ch'êng 金城; and ring $\mid \mathrm{C}$.

$2^{\circ}$ (obs.) Chou, fendal state in Honan.

$3^{\circ}$ (obs.) Yüan, C., N. of Ssŭ-nan F11, Kneichon. $4^{\circ}$ See Nos. 5582, 7573. 
應

YING-CH '̂ิTK.

7569 Ying-ch êng | 城 Yingching.

[170] $1^{\circ}$ H. in Tê-an Fu, Hupeh; Lat. $31^{\circ} 05^{\prime}$, Long. $113^{\circ}$ 27'; Han, An-ling 安 陵; 1.st Sung, Yingch'êng | 城.

$2^{\circ}$ Sce No. 7812 .

7570 Ying-chiang | 江, (obs.) T́ang, H. near Ssŭ-nan Fu, Kueichou.

7571 Ying-li | 里, see No. 3957. 7572 Ying-p'ing | 本, (obs.) Han, a town near ' $\mathrm{T}$ 'ungshan H., Hupeh.

7573 Ying-shan | |l| Ying-shan, [146] H. in Tê-an Fn, Hupeh; Lat. $31^{\circ} 40^{\prime}$, Long. $113^{\circ}$ $43^{\prime}$; Liang, Yung-yang 永 陽; Sui, Ying-shan|山: T゙ang, Ying |C.

7574 Ying-t'ien | 天, see Nos. $749,3464$.

7575 Ying-yang | 際, (obs.) Tsin, a town S. IV. of Yungchon Fin, Hunan.

\section{鷹}

7576 Ying-t'an 鷹潭, chên near Knei-ch'i H., Kiangsi.

\section{膺}

757 T Ying-ch'in 膺秋, Y. near I.ing-slui H., Kuangtung.

甸

7578 Ying-ti甸氏, (obs.) W. of Wên H., Kansulh; $W$. Han,
YING-YAO.

circ. in Kuang-han 廣漢 chiü, E. Han, circ. in K. Kuang-han 廣漢.

\section{頴}

7579 Ying 穎, see No. 7582.

7580 Ying-ch ang | 昌, see No. 2885.

7581 Ying-ch'iao | 㮕, chên near Yï C., Honan (No. 7687).

7582 Ying-chon 州 Ying-chan, [550]Fu in the Feng-ying circ. Anhui; forming also the district city of Fu-yang; Lat. $32^{\circ} 58^{\circ}$, Long. $115^{\circ}$ $57^{\prime} ; T s^{6} i u$, Ying-ch' inan | 川; Han, Ju-yin 汝 陰; Sui, Ying $\mid \mathrm{C}$.

7583 Ying-ch'nan | 川, (obs.) Ts'in and Han, chion in Anhui ; see Nos. 467, 3010, 7582 .

7584 Ying-shang | t Ying[550] shang, H. in Ying-chon Fin, Anhui; I at. $32^{\circ} 40^{\prime}$, Iong. $116^{\circ} 13^{\prime}$; Han, Shên 懴 H.; Liang, Hsia-ts'ai 下蔡; Sui, Ying-shang 上.

7585 Ying-yang | 陽, chên near Yi-yang H., Honan (No. 7495).

\section{癭}

7586 Ying-yao 皟遥, N. Wei, H. in Chihli. 
燍

Y'ING-T'Ao.

7587 Ying-t'ao|陶, see Nos. 4676, 5759 .

\section{然}

7588 Ying 營.

$1^{\circ}$ One of the twelve chOt, or great divisions of the Empire established by Shun 舜, comprising parts of Chihli and Shingking.

$2^{\circ}$ See Nos. 293, 3805.

7589 Ying-ch'in | 邱, see Nos. $292,3895$.

7590 Ying-k'on | 口, 'T. in Fêng-t'ien F'u, Shingking. See No. 4486.

7591 Ying-ling | 陵, see No. 292. 7592 Ying-p $n$ | 浦, see No.7860. 7593 Ying-shan | II Ying-shan, [232] H. in Shun-ch'ing Fu, Ssŭch' "tan; Lat. $31^{\circ} 08^{\prime}$, Long. $106^{\circ} 35^{\prime}$; Han, in 'Tang-ch'ï 宕渠 chün; Liang, Hsiang-ju相如; T'ang, Lang-ch ih 朗池; Sung, Ying-shan | II.

7594 Ying-t on | 頭, ssŭ near Chien-yang H., Fulıkien. 7595 Ying-tzŭ $\mid$ 子, see No. 4486. 7596 Ying-yang | 血, see Nos. $6275,7843$.

\section{迎}

$7596^{A}$ Ying-jih 迅 日, H. in Ch'ing-shang 慶分 circ., Corea.
英 YING-CH'AO.

\section{演}

7597 Ying 瀛.

$1^{\circ}$ (obs.) formerly C. near Tzŭ C., Ssŭch' 'uan. 20. See Nos. 369, 2064.

7598 Ying-an | 㚣, (obs.) formerly a town near Tzŭ C., Ssŭch' '1an.

7599 Ying-chou | 洲, see No. 5822.

7600 Ying-hai | 海, see No. 2064.

\section{盈}

$7600^{\wedge}$ ' ${ }^{\prime}$ ing-tê盈德, $\mathrm{H}$.in Ch' ingshang 慶佾 circ., Corea.

\section{趴}

7601 ling 趴.

$1^{\circ}$ (obs.) S. Sung, C. in Ching-hsi 京西 circ.

$2^{\circ}$ (obs.) formrely a town 20 li E. of Hsien-yang H., Shensi.

$3^{\circ}$ See Nos. 744, 2810, 7052 .

7602 Ying-chung $\mid$ 中, seeNo. 83.

7603 Ying-tung | 東, a posttown near An-lu H., Hupel.

\section{英}

7604 Ying 舢, see No. 7610 .

7605 Ying-ch' ao | 潮, Y. near Cli'ang-liua H., Kuangtung. 
笑 YING-CHI-SHA-ÊRH.

7605 Ying-chi-sha-êrlı | 吉沙 尔 Yingeshar, C.L.T. in Kasligar circuit, the New Dominion. Lat. $30^{\circ} 10^{\prime}$, Long. $70^{\circ}$.

7607 Ying-li | 利, a post-town near L,ei-chou Fu, Kuangtung.

7608 Ying-pu-ch' êng | 布 旅, (obs.) Han, a town N.W. of Jao-chou Fit, Kiangsi.

7609 Ying-shan | ILYing-shan, [215] H. in Lintan C., Anhui; Lat. $30^{\circ} 45^{\prime}$, Long. $115^{\circ}$ $45^{\prime}$; orig., L,o-t ien 羅田. 7610 Ying-tê | 德 Ying-ten, H. [22] in Shao-chon Fin, Kuangtung ; Lat. $24^{\circ} 12^{\prime}$, Long. $112^{\circ} 55^{\prime}$; Han, Chêng-yang 溑晹; T⿱anng, Ying |C.; Sung, Ying-tê 德 Fin.

Ying-chau, see No. 7582. Ying-ching, see No. 7569. Ying-teh, see No. 7610. lingeshar, see No. 7606.

\section{YO}

$T_{i}$

7611 Yo 孯, see No. 7615.

7612 Yo-a11 | 安, see No 2108 . 7613 Yo-Ch'ang-Li | 常澧circ. in ftuman comprising Yochou Fu, Chang-tê Fu and $\mathrm{Ii} \mathrm{C}$.

7614 Yo-ch'il | 池 Yolı-chi.
岳 Yo-YANG.

[535] $1^{\circ}$ H. in Shun-ch'ing Fu, Ssüch ' uan; Lat. $30^{\circ} 35^{\prime}$, Long. $106^{\circ} 26^{\prime} ; \mathrm{Han}$, in Tang-ch'ü 宕渠 chün; Ming, Yo-ch'ih | 池 $\mathrm{H}$. $2^{\circ}$ (obs.) T"ang, Sung. and Yiian, H., N.W. of the above.

7615 Yo-chon | 州 Yoh-chau, $\mathrm{Fu}$ in the Yo-Ch' ang-Li circ., Hunan ; forming also the district city of Pa-ling ; Lat. $29^{\circ} 18^{\prime}$, Long. $113^{\circ}$ $02^{\prime}$; orig. land of the Sanmiao 三苗 tribes; S.A., in $1 s t, \mathrm{~K} . \mathrm{Mi}$ 锈, $2 n d, \mathrm{~K}$. Lo 羅; C. Wars, in K. $\mathrm{Cl}_{1}{ }^{6} \mathrm{u}$ 楚; $T s^{\prime} \mathrm{in}$, in Ch'angsha 長 沙 chiin; $W u$, Chnng 重 chên; Tsin, Chien-ch"ang 建胃; 1 st Sung, Pa-ling 巴陵; Liang, Pa 巴 C.; Sui and Tang, Yol C.; Sung, Yo-yang | 殔; Ming, Yochon | 州 Fu.

7616 Yo-lisi | 希, T.S. near Mon C., Ssŭch' 'nan.

7617 Yo-shan | IL, ssüinMingkuo H., Auhui.

7618 Yo-yang | 陽 Yols-yang.

[43] $1^{\circ} \mathrm{H}$. in P'ing-yang Fu, Shansi; I,at. $36^{\circ} 15^{\prime}$, Long. $112^{\circ} 06^{\prime}$; Han, Ku-yüuln 䅥 遠; 2nd IVei, An-tsê 安澤; Sui, Yo-yang | 陽. 
岳

YU-CHIANG.

$2^{\circ}$ See Nos. 2066, 7615.

Ioh-chau, see No. 7615.

Yoh-chi, see No. 7614.

Yo-yang, see No. 7618.

Yokaichi, see No. 5887.

Yokohama, see No. 2026.

\section{YU}

\section{左}

7619 Yu-chiang 右江, circ. in Kuangsi comprising Ssŭ-ên Fin, Pai-sê T., Iin-chon Fu, Hsiun-chou Fiu, Ch' ingyüan $F u$ and Hsi-lung $C$. 7620 Yu-fêng-yi | 傌荡, see No. 6802.

7621 Yu-fu-fêng | 扶 風.

$1^{\circ}$ (obs.) $I V$. Han, chïu, F. of Hsicn-yang, H., Shensi.

$2^{\circ}$ (obs.) E. Han, chïn 10 li S. E. of Hsingp'ing H., Shensi.

$3^{\circ}$ See No. 1724.

7622 Yu-pei-p'ing | 北平, see Nos. 6592, 7965.

7623 Yu-so|所, T.S. near Yenyïan H., Ssŭch' nan.

7624 Yu-tien | 甸, a post-town near Shun1-ning Fu, Yüunnan.

7625 Y u-t'un-wei|屯衛, a posttown near $\mathrm{Yi} \mathrm{C}$., Shingking.
YU-LI.

7626 Yu-wu | 武, Y. near Kuyüan C., Kansuh.

7627 Yu-yü| $\mid$ 太 Yu-yul, H. forming the prefectural city of So-p'ing, Shansi; Lat. $40^{\circ} 10^{\prime}$, Long. $112^{\circ} 13^{\prime}$.

\section{伩}

7628 Yu 敒 Yu, H. in Ch'ang[302] sha Fu, Hunan; Lat. $27^{\circ}$ $08^{\prime}$, Long. $113^{\circ} 03^{\prime}$; Han, Yu| H. ; Chêen. Y'u-shini 水.

7629 Y 11 -shui | 水, see the preceding.

\section{有}

7630 Yu-shên 有等, see No. 469.

\section{宥}

7631 Yu 䈐, (obs.) T゙ang, C. near Yii-lin Fu, Shensi.

\section{由}

7632 Y11-ch' ïan 由拳, see Nos. 699, 1217.

\section{油}

7633 Yu-fang 油坊, chên in Ch'ing-ho H., Clihli.

\section{消存}

7634 Y I1-an 游发, (obs.) Liang, H., W. of Yang-shan H., Kuangtung.

\section{美}

7635 Y11-1i 美里, see No. 6265. 
幽

YU.

\section{幽}

7636 Yu幽.

$1^{\circ}$ Chou, CHOU comprising part of Chihli.

$2^{\circ}$ See No. 7965.

7637 Yu-tu| 都, see No. 6918.

7638 Yu-yen | 浾, see No.950.

\section{酉}

7639 Yu-chou西州 T.S. near Mien-ning H., Ssuch' 'uan. 7640 Yu-yang | 陽.

$1^{\circ}$ C.L.C. in the Ch' 'nantung circ., Ssưclı' nan; Lat. $28^{\circ} 45^{\prime}$, I.ong. $108^{\circ} 40^{\prime}$.

$2^{\circ}$ (obs.) Han, H. N.W. of Ch'ên-clıon Fu, Honan.

$3^{\circ}$ See No. 5051.

\section{韵}

7641 Y 11 猷.

$1^{\circ}$ (obs.) formerly C., F. of Shilı-tai H., Anhui. $2^{\circ}$ See No. 1112.

\section{尤}

7642 Yu-cli'i 尤 㳊 Yu-ki, H. [438] in Yen-p ing Fin, Fulhkien, Lat. $26^{\circ} 15^{\prime}$, Long. $118^{\circ} 10^{\prime}$; orig., Nan - p'ing; T'ang, Y11-ch'i | 溪.

Yï; see Nos. 7080, 7687, 7698.

Yu-ching; see Nos. 7653, 7688.
餘 $\mathrm{YU}$-CH'ING.

Yu-hang, see No. 7649.

Yu-liiang, see No. 7654.

Yu-kan, see No. 7650.

Yulki, see No. 7642.

Yu-king, see No. 7647.

Yu-lin, see No. 7666.

Yu-she, see No. 7669.

Yu-tai, see No. 7693.

Yu-tsi, see No. 7705.

Yu-tsz', see No. 7670.

Yu-tu, see No. 7656.

Yu-yau, see No. 9651.

Yu-yul, see No. 7627.

\section{Y}

余

7643 Yï-wu 余吾.

$1^{\circ}$ Chên near 'T' 'un-lin H., Shansi; $H$. Han, lu-

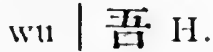

$2^{\circ}$ (obs.) $T^{\prime} a n g$, C. in the Oelot country, IV. end of Inner Mongolia.

7644 Yï-wu-ri|吾㫕, sce No. 6692.

\section{除}

7645 Yii 簿, (obs.) Trang, C. in Annan.

7646 Yïi-chi | 朢, see No. 2670. 7647 Yï-ch 'ing | 慶 Yu-king. [513] $1^{\circ} \mathrm{H}$. in $\mathrm{P}^{\prime}$ ing-y üeh C., Kuei-clon ; Lat. $27^{\circ} 06^{\prime}$, Long. $107^{\circ} 34^{\prime}$.

$2^{\circ}$. Ss̆̌ near the above. 
餘

7648 Yï-hang | 杭 Yu-hang, $\mathrm{H}$. in Hang-chon $\mathrm{Fu}$, Chehkiang; Lat. $30^{\circ} 17^{\prime}$, Long. $119^{\circ} 52^{\prime}$

7649 Yii-kan | 汗, see Nos. 3400, 3474, 7650.

7650 Yï-kan $\mid$ F Yn-kan.

[287] $1^{\circ} \mathrm{H}$. in Jao-chou $\mathrm{Fu}$, Kiangsi ; I at. $28^{\circ} 37^{\prime}$, Long. $116^{\circ} 43^{\prime}$; orig. in K. Yïel 越; Han, Yii-han (or, Yï-kan) 汗; Sui, Yï-kan |

$2^{\circ}$ See Nos. 75, 3656.

7651 Yii-yao | 姚 Yu-yan, H. in Shao-lising F'u, Cliehkiang; Lat. $30^{\circ} 07^{\prime}$, Long. $121^{\circ} 03^{\prime} ; \mathrm{Ts}^{\prime} \mathrm{in}$, Yii-yao | 姚; T"ang, Ya 姚 C.

\section{虞}

7652 Y ii 婜.

$1^{\circ}$ (obs.) Han and Tsin, H. 3 li S. W. of Yïclı êng H., Honan.

$2^{\circ}$ (obs.) S. Sung, and $S$. $T^{\prime} i$, H. near Huaiyiian H., Anhui.

$3^{\circ}$ (obs.) Chou, feudal state near An-ri H., Sliansi.

$4^{\circ}$ See Nos. 113, 5177, 7653.

7653 Yï-clı' êng | 城 J J1-ching. [161] $1^{\circ} \mathrm{H}$. in Kuei-tê Fu, Honan ; Lat. $34^{\circ} 38^{\prime}$, Long.
$Y \ddot{U}$.

$116^{\circ} 09^{\prime}$; Shang, feudal state Lun 綸 ; Han, Yii H.; Sui, Yüi-ch' êng 坊或.

$2^{\circ}$ (obs.) formerly a town nearHsiao-yi H., Shansi.

7654 Yï-hsiang | 鄉 Yu-hiang. [415] $1^{\circ} \mathrm{H}$. in $\mathrm{P}^{\prime}$ 11-chon $\mathrm{F} u$, Shansi ; Lat. $35^{\circ} 10^{\prime}$, Long. $110^{\circ} 35^{\prime}$.

$2^{\circ}$ See No. 829 .

\section{零}

$7655 \mathrm{Y} \mathrm{u}$ - $-\mathrm{ch}^{\prime} \mathrm{i}$ 零溪, chôn near Wan-an H., Kiangsi.

7656 Yii-tu | 都 Yu-tu (sometimes wrongly written Yïn$\mathrm{t} 11$ 雲都).

[293] $1^{\circ}$ H. Kan-chon Fu, Kiangsi ; Lat. $26^{\circ}$, Lonlg. $125^{\circ}$ 21'; $7^{6} a n g, \operatorname{Sung}$, Yüan and $\operatorname{Ving}$, Y Yï-tu | 都 $\mathrm{H}$.

$2^{\circ}$ (obs.) Han, Tsin, S. Sung, S. Ts'i and Sui, H., N.E. of the above. $3^{\circ}$ See Nos. 117, 3026.

\section{豫}

7657 Yii 豫.

$1^{\circ}$ one of the crrov, or great divisions of the Empire established by Yii 禹; comprising Honan, and parts of Hupeh, Chilili and Shantung. $2^{\circ}$ See Nos. 2079, 3010. 
瑔 YÜ-chaNG.

7658 Yü-clıang | 章.

$1^{\circ}$ (obs.) Han, chïn in Kiangsi.

$2^{\circ}$ See Nos. 740, 3027, $3847,4562$.

\section{猃}

7659 Yï-yüan 俞 元.

$1^{\circ}$ (obs.) Han, Ts' $i n$ and $S$. $T s^{i} i, \mathrm{H} .$, S. of Hoyang $H .$, Yünnan.

$2^{\circ}$ See No. 544.

\section{喻}

7660 Yü-mi 喻㖸, see No. 900.

\section{榆}

7661 Yii 榆.

$1^{\circ}$ (obs.) Liqo, C. in E. part of Inner Mongolia. $2^{\circ}$ See No. 7669 .

7662 Yii-ch'êng | 城, sec No. 2055.

7663 Yü-ch“i | 溪, (obs.) T゙ang, C., IV. of Yüi-lin H., Shensi.

7664 Yüi-chung | 中.

$1^{\circ}$ (obs.) Han, Tsin and N. Wei, H., N.W. of

Chin H., Kansuh.

$2^{\circ}$ (obs.) S. Sung and S. $7 s^{\prime} i, H$. in Nan-chêng H., Shensi.

7665 Yü-kuan | 關, a post-town near Lin-yü H., Chihli.

7666 Yï-lin 林 Yu-lin.
$\mathrm{Y} \ddot{\mathrm{E}}$

$[83] 1^{\circ} \mathrm{Fu}$ in the Yen-Yü-Sui circ., Shensi ; forming also the district city of the same name; Lat. $38^{\circ} 18^{\prime}$, Long. $109^{\circ} 33^{\prime}$; orig., land of the Jungti 戎狄 tribes; $T s^{\prime}$ in, in Yün - chung 雲 中 chïn ; Han, in Wu-yüan 五原 chïn; Sui and Tang, Shêng 幐 C. and Yï-lin | 林; Ming, Yii-lin | 林 military district.

$2^{\circ} \mathrm{H}$. forming the prefectural city of the same name, Shensi ; Lat. $38^{\circ}$ $18^{\prime}$, Long. $109^{\circ} 23^{\prime}$.

7667 Yï-lin-pas | 林堡, a posttown near Huai-lai H., Chihli.

7668 Yüi-shan| |山, see No. 5205. 7669 Yïi-slêe / 形 Yu-she, H.

[?] in L,iao C., Shansi; Iat. $37^{\circ} 04^{\prime}$, Long. $113^{\circ} 01^{\prime}$; $H a n$, Niel 涅 H.; $T^{6} \mathrm{in}$, Wu-hsiang 武郷; Sur, Yii-shê | 社; T Tang, Yii c.

7670 Y ̈̈̈-tž̆ | 次 Yu-tsz, H. in [485] T'ai-yiian Fn, Shansi; Lat. $37^{\circ} 42^{\prime}$, Long. $112^{\circ} 45^{\prime}$; S.A., Yiian-shui 原水; $H o n, Y$ Iï-tzü|次.

\section{瀑}

7671 Yii 渻, see Nos. $1583,4752$. 
隃

$$
\text { Y } \ddot{\mathrm{U}}-\mathrm{MI} \text {. }
$$$$
\text { 隃 }
$$

7672 Yü-mi 隃策, (obs.) 30 li E. of Ch'ien-yang H., Shensi; $W$. Han, H. in Y 11 -fu-fêng 右扶風 chün; E. Han, Marq. in Yu-fufêng 右扶風 chïn.

\section{于}

7673 Yü̈.

1. ${ }^{\circ}$ (obs,) Han, H. in Kiangsu.

$2^{\circ}$ (obs.) $T^{k} a n g$, C. in Ch'u-hssinng Fu, Yünnan.

7674 Yii-hu | 湖, (obs.) Tsin, S. Sung and $S . T s^{\prime} i, \mathrm{H}$. 38 li S. of Tang-tu H., Anl1ni.

7675 Y ï-1i | 離, (obs.) Han and Tsin, H. near Fênchon Fin, Shansi.

7676 Y'ii-mên | 阴, (obs.) $N$. Hei, H., N. W. of P'ingyao H., Shansi.

7677 Yiitt"ien| 闑.

$1^{\circ}$ H. in Ho-t'ien C.L.C., New Dominion.

$2^{\circ}$ See No. $205 \%$.

7678 Y ii-tum | 遁, see Nos. 2057.

$$
\text { 圢 }
$$

7679 Ÿï-t'ang 圩䛸, chên near Ching-chiang H., Kiangsu

$$
\text { 孟 }
$$

7680 Yü 孟 Yn. $1^{\circ} \mathrm{H}$. in P'ing-ting C., Shansi; Lat. $38^{\circ} 07^{\prime}$, Long. $113^{\circ} 22^{\prime}$; S.A., Ch'oul-yu 仇 猶; 2 d $W e i$, Shih-ai 石 艾; Sui, T'ang, Sung and Kin, Yü | H.; Yüan, Yï $\mid \mathrm{C}$.; Ming, Yü $\mid \mathrm{H}$. $2^{\circ}$ (obs.) Han and Tsin, H. $80 l i \mathrm{~N}$.E. of Yangeh ï H., Shanısi.

\section{糿}

7681 Yü 糿, (obs.) T'ang, C., E. of Hsin-ch'êng T.H., Knangsi.

\section{芋}

7682 Y ü-yüan 芋原, a post-town near Fin-chon Fin, Fulhkien.

\section{䄮}

7683 Yii 杼, (obs.) Chou, feudal state in Honan.

雨

7684 Yü-chin 丽金, chển near Kao-ling H., Shensi.

與

7685 Yiï-11a-ch' êng 與那戚, a town in the I,in-ch in Islands.

\section{牙}

7686 Yiu-ti 牙地, a town in the Lill-eh'in Islands. 
重

$\Upsilon \ddot{E}$.

\section{禹}

7687 Y ü 禹 Yn, C. in K'ai-fêng [550] Fu, Honan; Lat. $34^{\circ} 15$, I ong. $113^{\circ} 35^{\prime} ; \mathrm{Ts}^{\prime} \mathrm{in}$, Yang-chai 陽翟; Kin, Chün 鈞 C.

7688 Yü-clı'êng | 城 Yll-ching, [476] H. in Chi-nan Fu, Slinntung; Lat. $37^{\circ} 09^{\prime}$, I,ong. $116^{\circ} 47^{\prime}$; Chou, feudal state Chu 触; Han, Chun-a 预 阿; T'ang, Yü-ch'êng | 城.

7689 Yü-mên | 門 chên in Hoclining H., Shansi.

\section{魚}

7690 Yï-ch'in 魚邱, see No. 3160 .

7691 Yü-fu | 復, see No. 1763. 7692 Yü-mo | 汥, chên in Tingyüan T., Shensi.

7693 Yü-t'ai | 臺 Yu-tai, H. in [189] Chi-ning C., Shantung; I at. $35^{\circ} 07^{\prime}$, Long. $116^{\circ}$ $47^{\prime} ;$ S.A. Ch' ang-yi 常邑 in K. Lu魯; Han, Fangyii 方益; T'ang, Yü-t’ai 喜 $\mathrm{H}$.

\section{㴔}

7693^Y Yï-cl1'i 海溪, ssü in Shoulning H., Fulukien.

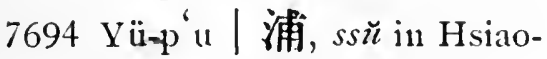
shan H., Chehkiang.

7695 Yïit'ing | 亭, chên near Hsiu-ning H., Anhui.
御

Y $\ddot{E}-Y \ddot{U} A x$.

7696 Yii-yang-knan | 洋關, a post-town near Ch'ang-tê H., Hupel.

7697 Yü-yang | 隄 Y11-yang.

$1^{\circ}$ A post-town near Chi C., Chilhli (No. 580).

[554] $2^{\circ}$ T. in Yi-ch 'ang Fin, Hupel1; Jait. $30^{\circ} 10^{\prime}$, Long. $111^{\circ} 03^{\prime}$, the name is also written | 洋。

$3^{\circ}$ (obs.) Ts' in and Han, chïn in Chilhli.

$t^{\circ}$ See Nos. 576, 4423, 5822 .

\section{裕}

7698 Y'ii 裕 Y'u, C. in Nan1-yang Fin, Honan; Lat. $33^{\circ} 23^{\prime}$, Long. $103^{\circ} 01^{\prime}$; orig., Fang-ch' êng 方城 in K. $\mathrm{Ch}^{\prime} \mathrm{u}$ 楚; Han, Tu-yang 都榢; E. Han, Shunyang 順陽; T゙ang, Lu 曽 C.; Kin, Xii |C.

7699 Yǜ-ch ‘ $\mathrm{i} \mid$ 溪, chên in Hanshan H., Anhui.

\section{峪}

7700 Yii-ho 峪河, chên in Huochia H., Honan.

\section{御}

7701 Yï-chieh 御 街, see No. 5822.

$7701^{A}$ Yü-ÿ̈̈an | 苑, see No. 5822. 
御

$$
Y \ddot{U}-Y \ddot{U} A N .
$$

$7701^{\prime \prime}$ Yü-yüiı | [ r see No. 5822.

$$
\text { 嵑 }
$$

7702 Yii 嵎, name for the Shantung promontory and its ancient inlabitants; see No. 6330 .

\section{推}

7ru3 Yü-êrh 集田, see No.699. 於

7704 Yüran 於岁, (obs.) formerly a town near No.7705, Chehkiang.

7705 I'ü-ch'ien | 海 In-tsien H. in Hang-chou Fin, Chel1kiang; Lat. $30^{\circ} 14^{\prime}$, Long. $119^{\circ} 23^{\prime}$; Han, Yï-ch' ien | 潛; T’ang, $\mathrm{Ch}^{\prime}$ 'ien 潛 C. 7706 Yü-ling | 陵, see No. 268. 7707 Yü-yi | 異, (obs.) T゙ang, C. near Yao C., Yünnan.

\section{淤}

7708 Yï-ch i 淤溪, chên near Hsing-hua H., Kiangsu.

\section{佛}

7709 Yii-chih 郁到, see No. 66 . 7710 Yüi-shan | III, chên near Ch'ien-chiang H., Ssŭch'uan.

7711 Yü-yi | 荑, (obs.) $W$. Han, H., $50 l i \mathrm{~W}$. of Lung C., Shensi.

整类

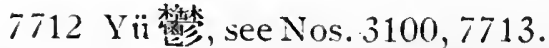

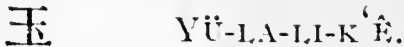

T13 Yï-lin | 林 Yuln-liu. [318] $1^{\circ}$ C. I. C. in the Tsochiang circ., Kuangsi; Lat. $22^{\circ} 43^{\prime}$, Long. $109^{\circ}$ $45^{\prime}$; Han, Yü-lin | 林; T゙ang, Y'̈̈ | C.; Sung, Nan-lin 南流; S. Sung; Yii-lin | 林 C.

$2^{\circ}$ Chên in Ta-yii H., Kiangsi.

$3^{\circ}$ See No. 2955.

7714 Yï-p'ingl平, see No.3473. 7ł15 yii-ho 峪河, chên near Huo-chia H., Honan.

$$
\text { 牙: }
$$

7716 Yii 王, see No. 6236.

7717 Yü-chang | 㲀, chên near IVei-yüan H., Kansuh.

7718 Yï-ch'êng | 城, a town in the Lin-ch'iu Islands.

7719 Yï-ching | 京, see No. 5822 .

7720 Vï-ching | 津, (obs.) T"ang, H. 90 li N. of Chienwei H., Ssüch'uan.

7721 Yü-ch'üan | 泉, Y. near Chum-wei H., Kansulı.

7722 Y:i-huan | 環 Yuh-hwan, C.L.T. in Chehkiang; Lat. $28^{\circ} 15^{\prime}$, Long. $122^{\circ} 05^{\prime}$.

7723 Yï-kê | 草, a post-town near Cling-liu H., Fulnkiell.

7724 Yü-la-li-k'ê / 喇里克Y ula-rik, a town in Yarkand; I at. $37^{\circ} 50^{\prime}$, Long. $75^{\circ} 30^{\prime}$. 
琵 Y

7725 Yïi-lu| 袮, Y. near Yüanwu H., Honan.

7726 Ÿ̈-lung-chieh-ch'ih | 龍 傑赤, see No. 7117.

7727 Y ̈ü-1ung-ha-shih | 隌哈什 V'urungkash, a town in Yarkand; Lat. $37^{\circ}$, Long. $81)^{\circ} 50^{\prime}$.

7728 Yii-111èn| 阴 Yulı-mun.

[368] $1^{c}$ H.in An-1ısiC., Kansulı; Lat. $39^{\circ} 40^{\prime}$, Isong. $97^{\circ} 05^{\prime}$.

$2^{\circ}$ (obs.) Han, Tsin, Sui and $T^{6}$ ang, H., E. of the above.

$7728^{\wedge}$ Y ü-pi | 陛, see No. 5822.

7729 Yü-p'ing | 居 Yuhl-ping, [561] H. in Ssŭ-chon Fu, Kueichou; Lat. $27^{\circ} 11^{\prime}$ Long. $108^{\circ} 40^{\prime}$.

7730 Yü-shan | U Yulı-shan, [211]H. in Kuang-hsin Fu, Kiangsi ; Lat. $28^{\circ} 46^{\prime}$, I.ong. $118^{\circ} 31^{\prime}$; Sui, Hsinan 新安; $T^{6}$ ang, Y Yï-shan | II.

7731 Ÿ̈̈-slu | 樹, T.S. in Kokonor.

7732 Yï-t'ien | 田 Yuh-tien, H. in Tsun-hua C., Chihli; Lat. $39^{\circ} 56^{\prime}$, I,ong. $117^{\circ}$ $40^{\prime}$; orig., K. Wu-chung 無終; T'ang, Yï-t'ien | 田:

7733 Yï-t'ing|亭, (obs.) T'ang, a town S.E. of Yii-kan H., Kiangsi.

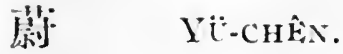

\section{摡}

$773+$ Yii-lu11 厩渾, (obs.) $W$. Han, H. in the Ordo. country, $\mathbf{N}$. of Shensi.

\section{充}

7535 Yii-li 青犁, U: Han, H. 120 li S.E. of P'èng-lai H., Shantung.

䒈

7736 Y ï 䦥, (obs.) Han and Tsin, . H. $45 \mathrm{li} \mathrm{W}$. of Cliang-t!ı H., Kiangsu.

\section{尉}

[This character may also be read WEI.] 7737 Yï-shil 叙氏 Wei-shi, H. [32] in K'ai-fêng Fu, Honan; Lat. $34^{\circ} 30^{\prime}$, Long. $114^{\circ}$ $23^{\prime}$; S.A., land of the $\mathrm{Y}$ ï tribes | 氏; $T s^{\prime}$ in, Yï-shih | 氏

\section{蔚}

[This character may also be read WEI.]

7738 Yii, 蔚 Yuh, or, Wei.

$1^{\circ}$ C. in "Hsüan-hua Fu, Chillli; I,at. $39^{\circ} 51^{\prime}$, Long. $114^{\circ} 36^{\prime} ; T s^{\prime} i n$, Tai 代 H., 2nd Wei, Ling - ch'in 靈 邱; $P$. Chou, Yï| C.

$2^{\circ}$ See No. 2134.

7739 Yii-chên | 珍Yuln-chin,or, Wei-chin, H. in Chiangyüan 江. 原 circ., Corea; Iat. $37^{\circ} 3.5^{\prime}$, Iong. $129^{\circ}$ $10^{\prime}$. 
尉

7740 Yï-fên | 汾, see No. 2814.

7741 Yii-shan | II Yuh-shan, or, Wei-shan, Fu in Ch' ingshang 慶佾 circ, Corea; Lat. $35^{\circ} 42^{\prime}$, Long. $129^{\circ}$ $35^{\prime}$.

\section{YÜAN}

\section{袁}

7742 Yiiral 袁, see No. 7743.

7743 Y üan-chou|州Y Yuen-chan, [552] Fu in the Jui-Yïant-Lin circ., Kiangsi ; forming also the district city of $\mathrm{Y}^{\mathrm{i}} \mathrm{-ch} \mathrm{ch}^{\prime} \mathrm{un}$; Lat. $27^{\circ} 52^{\prime}$, Long. $114^{\circ}$ $22^{\prime} ;$ S.A., in K. Wu 㕦; C. Wars, in $\mathrm{K} . \mathrm{Cl}_{1} \mathrm{n}$ 楚; Ts'in, in Chill-chiang 九 江 chïn; Han, Yi-ch' $u n$ 宜春; $W u$, in An-cll'êng 安城; T'ang and Sung， Y ̈̈an | C.

7744 Yïa11-110|紀, see No.7005.

\section{園}

7745 Y ïan-ch'ih 園池, (obs.) $N$. Wei, $\dot{\mathrm{H}}$. in Kausuh.

\section{遠}

7746 Y ïan-an避安 Yuen-1ngan; [109]H. in Ching-1nen C., Hupeh, I at. $31^{\circ} 10^{\prime}$, Long. $111^{\circ}$ $30^{\prime} ; \operatorname{Han}$, Lin-chï 臨沮; $T \sin , \mathrm{Kao}-\mathrm{an}$ 高努; $P$. Chou, Yüan-an| 安.

\section{原}

7747 Yiian 原 Ýnen.
源 Y门Ax.

$1^{\circ} \mathrm{C}$. in Chiang-yüan is: circ., Corea; Lat. $37^{\circ}$ $45^{\prime}$, I tong. $127^{\circ} 41^{\prime}$.

$2^{\circ}$ (obs.) Liao C., $300 l i$ N.E. of Knang-1ning $\mathrm{H}$., Shingking.

$3^{\circ}$ See Nos. 4+1, 3288.

7748 Y ïan-fêng | 崮.

$1^{\circ}$ (obs.) Tsin, S. Sung, and $S . T s^{\prime} i, H$. in Min H., Fuhkien.

$2^{\circ}$ See No. 4448.

7749 lïan-ho | 河, ssŭ near Yin-chiang H., Kueichou.

7750 Y an-ling | 陵, see 'No. 7754.

7751 Yïan1-p’ing | 平, a posttown near Kı1o H., Shansi.

7752 Yïan-shui | 水, see No. 7670 .

7753 Yüan1-tı | 都, (obs.) $W$. Han, H. in Kansuh.

7754 Yïan-w1 | 武 Ynen-wn, $H$. in Huai-cl 'ing $F u$, Honan; I at. $35^{\circ} 06^{\prime}$, Long. $113^{\circ} 06^{\prime}$; Han, Yüian-wu 武; $W$. Wei, Kuangwu 廣武; Sui, Y Yian-ling 陵。

\section{源}

7755 Yïan 源.

$1^{\circ}$ (obs.) Liao, C. in Shingking. 
元 YUंAN-CH ${ }^{\prime} \hat{\mathrm{E} N G}$.

\section{元}

7756 Yïan-clı' êng 元城 Yuenching, $H$. forming with 'Ta-1ning, $\mathrm{H}$. the prefectural city of Ta-ming. Chilli ; Lat. 36 21', Long. $115^{\circ}$ $22^{\prime}$; orig., Sha-lu沙簏; Han, Ÿ̈an-ch'êng | 城; Wei, Kuei-hsiang 貴鄉.

7757 Y ian-cl1'i | 笑谷, (obs.) Tsin, $S$. Sung and $S$. Ts' $i, \mathrm{H}$. 5 li E. of 'Tê-cl'ing C., Kuangtung.

7758 Yian-chiang | 江 Yuen[560] kiang, C. I. C. in Yi-nan circ., Yünnan; Iat. $23^{\circ}$ $36^{\prime}$, I on1g. $102^{\circ} 11^{\prime}$; Yïan, Yüan-chiang | 江 circ.; Ming, Yüan-chiang | 江 Fit.

7759 Yüan-ho | 和 Yuen-lio, H. forming with $\mathrm{W} u \mathrm{H}$. ano $\mathrm{Cl}^{\text {' }}$ ang-chon $\mathrm{H}$. the prefectural city of Su-chou, Kiangsu; Lat. $31^{\circ}$ Long. $120^{\circ} 44^{\prime}$.

7760 Yïan-1110u | 謀 Yuen-1nau, [277]H. in Wu-ting C., Yünnan;

- Lat. $25^{\circ} 38^{\prime}$, Lo11g. $102^{\circ}$ $03^{\prime}$; orig., Huan 㻴 C.; Yüan, Ÿ̈an-111011 | 謀.

$7760^{A}$ I ïan-shan | |ll Yensan, or Port I,azareff; port in Corea.

7761 Yüan-shih | 氏 Yuen-shi, [145] H. in Chêng-ting Fu, Chilhli; Lat. $37^{\circ} 48^{\prime}$, L,ong
淵 YÜAN-CH ${ }^{6} \mathrm{UAx}$.

$114^{\circ} 40^{\prime}$; orig., in K. Wei, 衛; Han, Yüan-shih | 氏.

\section{沅}

7762 Yiuan 沅, see Nos. 321, 7764.

7763 Yïan-chiang | 江 Yuen[490] kiang, H. in Clı' ang-tê Fu, Hunan; I at. $28^{\circ} 46^{\prime}$, Long. $112^{\circ} 14^{\prime}$; Han, Yiyang 益陽; Liang, Lêshan 樂 II; Sui, Yüanchiang | 汇. $\mathrm{H}$.

7764 Yïan-chon / 州 Yuen-chan, [561] Fu in the Ch'ên-Yïan Yung-Ching circ., Human ; forming also the district city of Chih-chiang; Lat. $27^{\circ} 41^{\prime}$, Lol1g. $109^{\circ} 25^{\prime}$; Han, Wn-ling 武 陵; Tang, Yuian | C. and $\mathrm{Cl}_{1}$ iao-chiang 橋 江 $\mathrm{H}$.

7765 Yüan-ling | 陵 Yuen-ling.

$1^{\circ} \mathrm{H}$. forming the prefectural city of Ch' ên-chou, Hunan; I Iat. $28^{\circ} 22^{\prime}$, Long. $110^{\circ} 09^{\prime}$; Han, Hsia-tsu11 下㩦；E. Han, Yïan1-ling | 陵. $2^{\circ}$ See No, 476.

7766 Yïan-11an | 献, see No. 6291.

\section{淵}

7767 Yüan-ch ïan 临泉, (obs.) Han, H. $160 l i$ E. of Anlısi C., Kausul. 
垣

Y ỸX.

\section{垣}

.7.768 Yiian 垣.

$1^{\circ}$ (obs.) Han and Tsin, H. 20 li W. of I üanch ii H., Shansi.

$2^{\circ}$ See No. 7769.

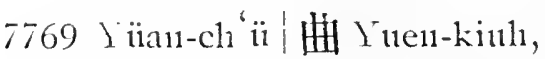
[148] H. in Chiang C., Sliansi; Lat. $35^{\circ} 05^{\prime}$, Long. $111^{\circ}$ $47^{\prime}$; orig., on the frontiers of K. Chon 周 and $\mathrm{K}$. Claao 趙; Han, Yüan ! H.; 2nd $\mathrm{W} e$ i, Pai-sluni 白 水; $P$. Chou, Po-ch' êng 堂城; でang, Shao 郡 C.; Sung, Yïan-cln ${ }^{6} \ddot{\text { i }}$ । 开.

7770 Yinan-slui | 水, see No. 6990.

\section{緣}

7771 Yüan 緣, see No. 2018.

\section{冕}

7772 Yiuan-kon | 䰟包 also written Wan-kon | 宛司. $1^{\circ}$ Han, Tsin, N. Woi, Sui and $T^{k} a n g$, H.S.W. of'Ts'ao-chon Fn, Shantung.

$2^{\circ}$ (obs.) S. Sung and S. $T s^{\prime} i, \mathrm{H}$. in Kiangsu.

\section{处}

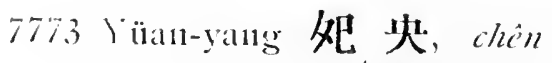
near Kung-ch':ang lin, K:a11:s11.
梡 I

\section{苑}

7374 Y ïan11-cl' êng 苑城, (obs.) formerly a town near Ch'ang-shan $\mathrm{H}$., Shantung.

7775 Yüian-cl' 'iang | 墙, see No. 3174.

7776 Yüan1-ch`nan | 川, see No. 4935.

7777 Yüian-wên | 溫, see No. 170.

\section{㳘}

7778 Yüan-11o 流河, chên near Iênng H., Honan.

\section{員}

7779 Y ü̉an-ch' ̈̈ 員曲, chển near Wei-yüan H., Kansull.

Y'uel1-sui, see No. 7791.

\section{YÜEH}

\section{月}

7780 Yüelı-chih 月 支, (obs.) T゙ ang, Fu near Turfan.

7781 Y'uiel1-p’i | 皮, a post-town near Chien-wei H., Ssŭcli' nan.

\section{梲}

7782 Yïeh 说，(obs.) T゙ang, C., S. of Clh ï-ching Fin, Yünnian.

Ti83 l'üen1-lê | 樂, chên near Ho-shuni H., Kallsull.

778+ Y'iell-p'an | 般, see No. 7489. 
越

$Y \mathrm{ẌHH}_{\text {. }}$

\section{越}

7785 Yüiels 越.

$1^{\circ}$ (obs.) C. llars, K. comprising parts of Kiangsi and Chelnkiang. $2^{\circ}$ (obs.) Yüan, C., S. of Clı ï-ching l'v, Y ümıаı. $3^{\circ}$ See Nos. 389, 3815 , $4702,5574,7650$.

7786 Yïel1-clinug | 中, (obs.) $S$. Ts' $i$, chün in Knangsi. 7787 Yiiel1-clı11ng | 衝, see No. 6344.

7788 Yïeh-lai | 來, a town in the I,iu-ch'in Isiancis.

7789 Yïeh-pin | 琵, (obs.) T゙ang, H. in I,ii C., Ssücli' nan.

7790 Yizeh-shang | 䄈, see No. 4504 .

7791 Yüeh-sui | 隽 lueh-sui.

[564] $1^{\circ}$ 'T. in Ning-yiian F ${ }^{\prime}$, Ssǔcl ' 11 an; I at $28^{\circ} 50^{\prime}$, Long. $102^{\circ} 45^{\prime}$. Terr. jur.

$2^{\circ}$ See No. 6162 .

7792 Yïeh-t'an | 賧, see No. 6364.

\section{㮐}

7793 Yïeh-yang 櫟踢, chên near Futp'ing H., Shensi.

\section{祭}

7594 Yiiel 残.

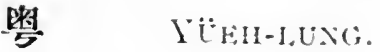

$1^{\circ}$ I iterary name for the Liang-Kuang provinces; Kuangsi being Y tiel1-l1si 话, and Kuangting liel1-tung | 東; see No. 3765.

$2^{\circ}$ Nanne of a tribe in S. China, occupying Knangtung and K nangsi during the 2 nd century I.C.

7795 Y ïeh-lung | 解, ss Wu-wei C., Anluni.

Yuen, see No. Ti4t.

Ynen-chan, see Nos. 7743, $7 \% 64$.

Yuen-ching, see No. $7 / 56$.

linen-ho, see No. 7759.

lne11-kiang, see Nos. 7758 , 7763.

luen-kinh, see No. 7769.

Yuen-ling, see No. 7765.

Ynen-111an, see No. 7760.

Y'uen-11gan, see No. 7746.

Y'nen-ping, see No. 6918.

Yuen-shan, see No. 930.

Ynen-slii, see No. T⿰61.

Yunen-w11, see No. 7754.

Jull, see No. 7738.

Yuli-hwan, see No. Tiz?.

Yul1-clin, see No. 7739.

Juhlin, see No. 7713.

Juh-mu1n, see No. 772s.

Yuh-ping, see No. 7729.

linh-shan. sec Nos. 7730, 7741. 
Yuh-tien, see No. 7732.

Yularik, see No. 7724.

Yu1, see Nos. 7797, 7820.

Yun-ching, see No. 7829.

Y'un-ho, see No. 7804.

Yun-lien, see No. 7832.

Yun-lung, see No. 7810.

Yun-mung, see No. 7812.

Yu11-nan, see No. 7813.

Y111-si, see No. 7821.

Yu11-siau, see No. 7805.

Yun-yang, see Nos. 7818,

7823.

\section{YÜN}

\section{云}

7796 Y ün-ting 云頂, ssü near $\mathrm{K} u-t$ 'ien H., Fuhkien.

\section{雲}

7797 Yiin 雲 Yun.

[408] $1^{\circ}$ C. in Shun-ning Fu, Y ̈̈nnan; Lat. $24^{\circ} 25^{\prime}$, Long. $100^{\circ} 20^{\prime}$.

$2^{\circ}$ (obs.) $W$. Han, marq. near Lai-chou Fu, Shantung.

$3^{\circ}$ (obs.) Yïan, H., N. of Ch 'ih-ch' êng H., Clihli.

$4^{\circ}$ (obs.) Yüan, C., N.E. of P'ing-hisiang Tro, Corea.

$5^{\circ}$ (obs.) Ming, pao $30 \%$ $\mathrm{N}$. of Ch'ili-ch'êng H., Chihli.

$6^{\circ}$ See No. 6102.
7798 Yün-an | 岀.

$1^{\circ} S$. Sung, military district in the $\mathrm{K}^{\prime}$ 'uei-chou 訾州 circ.

$2^{\circ}$ See Nos. 3491, 5963.

7799 Yün-ch'ang | 昌, T.S. near Sung-p'an T., Ssǔch 'uan.

7800 Yün-ch'i | 溪, a post-town near Lin-hsiang H., Hunan.

7801 Yün-chien | 間, see No. 2218.

7802 Yün-chon-pao | 州 堡, a post-town near $\mathrm{Ch}^{\text {ih }}$ clı' êng H., Chihli.

7803 Yün-chung | 中.

$1^{\circ} \mathrm{A}$ post-town near Tat'ungFu, Shansi; $T^{\prime}$ ang, Yün-chung | 中 $\mathrm{H}$.; Sung, Yün-chung | 中 Fu; Liqo and $K i n$, Yünchung 中 $\mathrm{H}$.

$2^{\circ}$ (obs.) Han, chïn and H.W. of Knei-hua T., Shansi.

$3^{\circ}$ (obs.) Tsin, H. $70 l i$ S.W. of K110 H., Shansi: $4^{\circ}$ (obs.) N. Wei, chïn E. of $\mathrm{Cl}^{\prime} \mathrm{i} \mathrm{H}$., Shansi.

$5^{\circ} T^{6}$ ang, Fu near Kueiha T., Shansi.

$6^{\circ}$ (obs.) $T s^{\prime} i n$, a town $N$. of 'Ta-t'ung Fin, Shansi.

$7^{\circ}$. See Nos. 2232, 6102, 7666. 
翣

Y ÜN.

$7803^{A}$ Yün-fêng | 攻, H. in Ch'iian-lo 全 篗 circ., Corea.

7804 Yïn-ho | 和 Yun-ho, H. [424] in Ch'u-chon $\mathrm{Fu}$, Chel1kiang; Lat. $28^{\circ} 07^{\prime}$, Long. $119^{\circ} 20^{\prime}$; orig., I,i-shui 曁水.

7805 Yun-lisiao | 霄 Yun-sian, $T$. in Chang-chou $F$, Fulkien.

7806 Yün-kên | 根, a post-town near Chung C., Ssŭclı' uan.

7807 Yün-Kuei | 貴, Yun-Kwei, collective name for the two provinces of Yümnan and Kueichou.

7808 Yün-lin | 林, H. in T'ainan Fu, Formosa.

7809 Yïn-ling | 陵.

$1^{\circ}$ (obs.) Han, a town 30 li N. of $\mathrm{Ch}^{\prime}$ üan C., Kuangsi.

$2^{\circ}$ See No. 5832 .

7810 Yün-lung | 龍 Yun-lung, [233] C. in Ta-li Fu, Yünnan; Lat. $25^{\circ} 54^{\prime}$, Long. $99^{\circ}$ $36^{\prime}$; orig., Lung-t'ien 龍 田; Ming, Yün-lung | 龍 C.

7811 Yün-lung-cling | 龍井, a post-town near the preceding.

7812 Yün=mêng | 曹 Y Yun-mung. [568] $1^{\circ} \mathrm{H}$. in Tê-an Fu, Hupeh; Lat. $31^{\circ} 05^{\prime}$, Long. $113^{\circ}$ $41^{\prime}$; Han, Hsi-ling 西陵;
䀜 YƯN-NAN.

$W$. Wei, Yün-mêng | 夢; T'ang, Ying-ch' êng 隹娍。

$2^{\circ}$ See No. 6306.

7813 Yün-nan | 南 Yun-nan.

$1^{\circ}$ Province of China; literary names Tien 滇, Tien-ch'ih 滇 池 and Tien-11an滇南; capital,

$Y$ ïn-nan Fu; it contains 4 circ., $14 \mathrm{Fu}, 4$ C.L.T., 13 T., 3 C.I.C., 27 C., 43 H., 3 T.F. and 6 T.C. Carajan (Polo).

[461] $2^{\circ} \mathrm{Fu}$ in the Yün-Wu circ., Yünnan; forming also the district city of K'un-ming; Lat. $25^{\circ}$ $03^{\prime}$, Long. $102^{\circ} 52^{\prime}$; the capital of the province; orig., land of the S. barbarians; $\mathrm{Han}$, $\mathrm{Yi}$ 瓮 chov: Minor Han, Chien-ning 建 家: Tsin, Ning 耍 C.; Sui, K'un 昆C.; T'ang, 1st, Nan-ning 南富, 2nd, country occupied by the Mêng 蒙 tribes; Yïan, Clnung-ch'ing ip 度; Ming, Ÿ̈̈n-nan 南 Fin. Yachi (Polo).

[70] $3^{\circ} \mathrm{H}$. in 'la-li Fu, Yün-nan; Lat. $25^{\circ} 32^{\prime}$, Long. $100^{\circ}$ $41^{\prime}$; Han, Yün-11an| 南; T"rng, $\mathrm{K}^{*}$ uaug $\mathrm{E} \mathrm{C}$. 
7814 Yün-11ei | 队文, sce No. 6102 . $7814^{\star}$ Yün-shan | IL, chïn in P'ing-an 平管 circ, Coren.

7815 Yün1-ts'u| 旗, chên near Y'ï-shê H., Shansi.

7816 Yün-tu｜都, see No. 7656. 7817 Yün-Wı | 武, circ. in Yünnlan connprising Yiünnan Fin and Wu-ting $C$.

7S18 Yün-yang | 陽 Yun-yang. [528] $1^{\circ} \mathrm{H}$. in $\mathrm{K}$ 'nei-chon Ft, Ssŭch' 'nan; I Iat. $31^{\circ} 05^{\prime}$, I,o11g. $109^{\circ} 06^{\prime} ;$ Han, Ch' ${ }^{\prime} 111-j e ̂ n$ 朐 䏰; $P$. Chon, Yün-an| 怒; lï̈rn, Yün-yang | 陽. $2^{\circ}$ (obs.) Han, H., II. of Ching-yang H., Shensi. $3^{\circ}$ Sce Nos. 5832, 6211.

フS19 Yün-yen| 岩, chên near Yen-ch'ang H., Shensi.

\section{鄖}

7820 Yün 鄖 Ýun。

$[133] 1^{\circ} \mathrm{H}$. forming the prefectural city of Yün-yang, Hupeh; Iat. $32^{\circ} 49^{\prime}$, I.ong. $110^{\circ} 42^{\prime}$; orig., fenclal state of $\mathrm{Mi}$ 籍; S.A., Hsi-lisiel 錫分; Sui, Yïn-l1siang | 県; Yüan, Yün | $\mathrm{H}$. $2^{\circ}$ (obs.) S.A., K.in Hupel. $3^{\circ}$ See Nos. 1967, 6306.

782i Yinn-lısi | 西, Yun-si, H. [3] in Yün-yang Fu, Hupelı; I at. $32^{\circ} 56^{\prime}$, I,ong. $110^{\circ}$ $53^{\prime}$.
7822 Yün-lisiang | 郷, sce Nos. 7820, 7823.

7823 Yïn-yang | 晹 Yun-yang, [133. F Fu in tle An-Hsiang YünChing circ., Hupeh; forming also the district city of Yïn; I at. $32^{\circ} 49^{\prime}$, I ong. $110^{\circ} 52^{\prime}$; S.A., Hsi-1nsüel 錫答, in K. Clin 楚; Han, Hsi 錫 H.; Sui, Yün-l1siang 拫; T $T^{6}$ ang, Nan-fêng 南㹃 and Hsi 淋 C.; Ming, Yüu-yang | 陽 Fi1.

\section{尤}

782.1 Yün 出, (obs.) Sung, C., N.W. of Huai-yiuan H., Knangsi.

7825 Yün-chielı | 街, Han and $T \sin$, H., S. of $\mathrm{P}^{i}$ ing-fan H. Kansulh.

\section{運}

7826 Yün-ch' êng 運城, a posttown near Clielı C., Shansi.

7827 Yün-chiang | 江, sš̆ near I,o-jung H., Kunangsi.

\section{鄆}

$7 \ddot{8} 28$ Y'ün 鄲, see Nos. 6745 , 7829.

7829 Y'ün-ch'êng | 城 Yun-

[15] ching, H. in Ch' ing-clon Fin, Shantung; Lat. $35^{\circ}$ $45^{\prime}$, Long. $116^{\circ} 14^{\prime}$; Han, I.ing-ch in 煘邱; P. Chon, 
䡉 Yiก-บ.

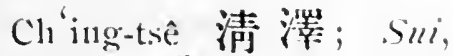
Wan-an 岱岁: and Yüncliêng | 城; T゙ang, YünC.

7830 Yïn-yi | 邑, seeNo.7482.

\section{鈞}

7831 Yün淕, seeNos.3027,7832. 7832 Yün-lien | 連 Yun-lien, [345] H. in Hsii-chou Fu, Ssüch 'nan; Lat. $28^{\circ} 06^{\prime}$, I, Ong. $104^{\circ} 40^{\prime} ;$ Han, Ting-

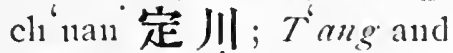
Sung, Yü̈|c.

7833 Yün-mên | 門, chên in Hui-ch ang H., Kiangsi.

\section{YUNG}

\section{永}

7834 Inug 永.

$1^{\circ}$ (obs.) Ming, C. near Nan-tan T.C., Knangsi. $2^{\circ}$ See No. 7843.

7835 Ying-an| 努Ying-ngan. [300] $1^{\circ}$ C. in P'ing-tê F 11 , Knangsi ; Lat. $24^{\circ} 01^{\prime}$, Long. $110^{\circ} 18^{\prime} ; T^{6} a n g$, Ii-shan 临 UL; Ming , Yung-an | 安 C.

[234] $2^{\circ}$ H. in Hui-chon Fin, Kuangtung; Lat. $23^{\circ}$ $42^{\prime}$, I,ong. $115^{\circ} 08^{\prime}$; $\operatorname{Ming}, J 111 \mathrm{~g}$-an $\mid$ 㚣 H. [38s] $3^{\circ}$ H. in lene ing I'n, Fullkien; I at. $25^{\circ} 54^{\prime}$, Long. $117^{\circ} 33^{\prime}$; orig, Y11-ch'i 尤㗛; Ming. ln11g-an | 管 $\mathrm{H}$.

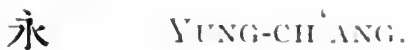

$4^{\circ}$ Chânn near Wnokn11g H., Sinensi.

$5^{\circ}$ Chên near Ning-yün H., Kansuly.

$6^{\circ}$ Chên near 'T’ $1111 \mathrm{C}$., Kiangsu.

$7^{\circ}$ Y. near Kni-lang H., Kansull.

$8^{\circ}$ A post-town near l $111 \mathrm{~g}$ slou H., Shensi.

$9^{\circ}$ (obs.) S. Ts $i$, H. in Hupeh.

$10^{\circ}$ (obs.) S. Sung and S. Tsi, H. in Sinng-t\%.̆ H., Hupel.

$11^{\circ}$ (obs.) S. Sung, and s. Ts'i, H. in Cl' êng-tu Fin, Sš̆ch nan.

$12^{\circ}$ (obs.) N. I'ci, chïn in Ying-slian H., Hupeh.

$13^{\circ}$ (obs.) N. Hé, chïn in Huang-kang H., Hupeh.

$14^{\circ}$ (obs.) N. Wri, chïn and H. in Honan.

$15^{\circ}$ (obs.) N. Hei, H. in Lin-fên H., Slan1si.

$16^{\circ}$ (obs.) N. Wei and Sui, H., S.E. of Hsiao-yi H., Shansi.

$17^{\circ}$ (obs.) N. I'ci, H., W. of An-su H., Clinlıli.

$1 S^{\circ}$ (ols.) N. Ilci, H. in Chilili.

$19^{\circ}$ (obs.) sui, chäm, 120 li N.IV. of Huang-kang H., Hupcls. 
永

YUNG-CH'ANG.

$20^{\circ}$ (obs.) Sung, H. $40 \mathrm{li}$ S. IV. of Kung H., Honan.

$21^{\circ}$ (obs.) Liao, H, in the Ongniod conntry, N. of Chillit.

$22^{\circ}$ (obs.) Kin, chên near Hsüan-hua Fu, Chillli.

$23^{\circ}$ (obs.) Kin, chên near $\mathrm{Cl}^{\prime}$ 'ên-chon $\mathrm{F} u$, Honan.

$24^{\circ}$ (obs.) Ming, ss̆̈ in Kuangsi.

$25^{\circ}$ See Nos. 119', 1800, 2095, 2282, 3752.

7836 Yung-cl1'ang | 昌 Yungcliang.

$[171] 1^{\circ} \mathrm{Fu}$ in the Yi-lisi circ., Yünnan; forming also the district city of Paoslian; Lat. $25^{\circ} 07^{\prime}$, Long. 99 26' ; Ming, Yung-ch'ang | 昌 Fu. Vochan (Polo).

$2^{\circ} \mathrm{H}$. in I,iang-chou $\mathrm{F} u$, Kansuh ; Lat. $38^{\circ} 20^{\prime}$, Long. $102^{\circ} 10^{\prime}$; Han, in Wu-wei 武威 chiun; Sung, Hsi-liang 西凉.

$3^{\circ}$ Chên near Wu-yüan H., Anhui.

$4^{\circ} Y$. near Cliang-hua H., Hunan.

$5^{2}$ (obs.) Wu, a town W. of Cli'i-yang H., Hunan. $6^{\circ}$ (obs.) $W u$, a town $\mathrm{V}$. of Hui-li C., Ssincli'nan. $7^{\circ}$ See Nos. 324, 521, 2845 j水 YUNG-CH'ING.

7837 Yung-chên | 貞, see No. 4047.

7838 Yung-ch'êng | 城 Yung[331] ching, H. in Kuei-tê Fu, Honan; Lat. $34^{\circ} 05^{\prime}$, I.ong. $116^{\circ} 24^{\prime}$; S. $A$., Mang-yi 芒邑; E. Han, 'T'ai-ch“iu 太邱 and Lin-sui 臨睢; Sui, Yung-ch'êng | 城.

7839 Yung-clii | 㳯 Yung-tsi, $\mathrm{H}$. forming the prefectural city of $\mathrm{P}^{6}$ 11-clion, Sliansi;

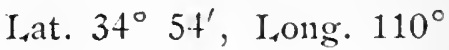
$15^{\prime}$.

7840 Yung-chia | 茄 Yung-kia.

$1^{\circ} \mathrm{H}$. forming the prefectural city of Wên-chou, Chelikiang; Lat. $28^{\circ} 01^{\prime}$, Long. $120^{\circ} 31^{\prime}$; Han, Tung-on 炛匰 and Yung-11ing | 蕣; Sung, Yung-chia | 鄯.

$2^{\circ}$ See Nos. 1396, 2326, 7022 .

7841 Yung-ch'ing | 度, ssŭ in Min H., Fulkien.

7842 Yung-cli'ing | 清 Yungtsing.

$1^{\circ} \mathrm{H}$. in Shun-t'ien Fu, Cliilili; Lat. $39^{\circ} 20^{\prime}$, Long. $116^{\circ} 35^{\prime}$; Han, Yi-ch'ang 谷易; Sui,

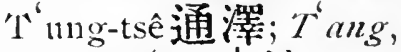
Yung-ch'ing | 清.

$2^{\circ}$ (obs.) $I / c i$, a town Is. of Fang H., Hupeh. 
永

YUNG-HOU.

$78+3$ Yung-chon | 州 Yung[177] chan, Fu in the HêngY'ung - Ch 'ên - Kuei circ., Hunan; forming also the district city of Iing-ling; I,at. $26^{\circ} 0 S^{\prime}$, Long. $111^{\circ}$ $35^{\prime}$; orig., S. frontier of K. Ch' ${ }^{\prime}$ 楚; Ts'in, in Clíang-sha 長沙 chün; Han, Ling-ling 零 陵; Tsin, Ying-yang 營陽; Sui, Trang and Sung, Yung | C.

7844 Yung-ch' nan | M Yungchuen.

$1^{\circ}$ chün in $\mathrm{Cl}^{6}$ ing-sheng 厝何 circ., Corea; Lat. $36^{\circ}$, Long. $129^{\circ} 05^{\prime}$.

[19] $2^{\circ}$ H. in Chung-ch 'ing Fu, Ssŭch 'uan; Lat. $29^{\circ} 30^{\prime}$, I.ong. $106^{\circ}$; orig., I'ï 滀 C.; T'ang, I'ungch'uan|川.

7845 Yung-ch'iian | 泉, a posttown near Yung-cli ang $\mathrm{H}$., Kansul.

7846 Yung-ch'm | 春 Yungchu11.

[45t] $1^{\circ}$ C.I.C. in the Hsing$\mathrm{Ch}^{\prime}$ ïan- Yung circ, Ful kien ; Lat. $25^{\circ} 18^{\prime}$, Long. $118^{\circ} 28^{\prime}$; Five dyn., T'ao-yüan 桃源; r. T'ang and Ming, lung-ch un | 春.

$2^{\circ}$ H. in Chung-ch ing 出 清 circ., Corea.
永 YUNG-FU.

7847 Yung-fêng| 배돌 Yung-fung. $1^{\circ} \mathrm{H}$. in Chi-an Fu, Kiangsi ; Lat. $27^{\circ} 25^{\prime}$, Lony. $115^{\circ} 20^{\prime}$; Han, Lï-ling 盧陵; $W u$, Yangcln'êng 陽城; Tsin, Yang-fêng 陽種; Sung, Yung-fêng | 豈 $\mathrm{H}$.

$2^{\circ}$ A post-town near Hsianghsiang H., Hunan.

$3^{\circ}$ (obs.) $T \sin , S$. Sung and $S . T s^{\prime} i$, H. 50 li N.IV. of Li-p $n$ H. Kuangsi.

$4^{\circ}$ S. Sung and S. T si, H. in Ssŭch "uan.

$5^{\circ}$ S. Sung and S. Ts $i$, H. in Yünnan.

$6^{\circ} N$. Wei, H. in An-sai H., Shens:

$7^{\circ}$ (obs.) Sui and $T^{*} a n g$, H. $260 \mathrm{li}, \mathrm{W}$. of the Ordos right wing, rear baumer, N. of Shensi.

$8^{\circ}$ (obs.) Sung, a military post 200 li S. of Chientê H., Anhui.

$9^{\circ}$ (obs.) Kin, chên E. of Chan-hua H., Shantung.

$10^{\circ}$ See No. 3395.

7848 l'ung-fu| 福 Y'ung-ful. [51 $] 1^{\circ}$ H. in Fin-chou Fu, Fuhkien; Lat. $25^{\circ} 4 \gamma^{\prime}$, I,ong. $119^{\circ} 02^{\prime} ; T^{\circ} \mathrm{ang}$, Yung-t'ai, 泰; Sung, lung-fu| 福. 
永

Yuxg-ho.

[570] $2^{\circ}$ H. in Knei-lin In, Knangsi ; Lat. $24^{\circ} 58^{\prime}$, Long. $109^{\circ} 51^{\prime} ; T^{6}$ ang, Yung-fu| 祸 $\mathrm{H}$.

$3^{\circ}$ See No. 6312.

7849 Yung-ho | 和 Yung-ho, [188] H. in Hsi C., Shansi ; Lat. $36^{\circ} 48^{\prime}$, I.ong. $110^{\circ}$ $36^{\prime} ;$ Han, Hu-chê 狐 讘; 2nd $\mathrm{H}^{\mathrm{C}} \mathrm{ci}$, Yung-ho 和.

'7850 lung-hsi | 熙.

$1^{\circ}$ (obs.) S. Ts $i$ and Sui, H., E. of Lo-ting C., Knangtung.

$2^{\circ}$ (obs.) Sui, chiin 100 li S. of Lo-ting C., Knangtung.

7S51 Vung-lisiang | 郎, chân near Lo-ch nan H. Shensi.

7852 Yung-hsin | 新 Yung-sin. [267] $1^{\circ}$ H. in Chi-an Fin, Kiangsi; Lat. $26^{\circ} 55^{\prime}$, I,ony. $114^{\circ} 07^{\prime} ; \mathrm{Han}$,

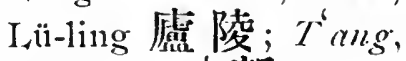
Yung-hisin | 新 $\mathrm{H}$.

$2^{\circ}$ A post-town near Hsinchêng H., Honan.

$3^{\circ}$ (obs.) Tsin, S. Sung and S. Ts' $i$, H. 35 li S. of Yung-hsin H., Kiangsi.

7853 Vuncr-lising | 藇 Junghing.

$1^{\circ}$ Fin in Hsien-ching 战 鏡 circ., Corea; Lat. $39^{\circ} 40^{\prime}$, Iollg. $128^{\circ} 48^{\prime}$.
永

YUNGi-I, î.

[23] $2^{\circ}$ H. in $\mathrm{Cl}^{\prime}$ ên C., Hunan; Lat. $26^{\circ} 05^{\prime}$, I long. $112^{\circ}$ $45^{\prime}$; Han, Pien 便 H., Targ, An-ling 威陵; Sung, Yung-hising | 興. $3^{\circ}$ (ols.) Trang, H. near Hsing-kuo C., Hupel. $4^{\circ}$ See Nos. 2438, 2670.

7854 Yung-jon | 柔 H.in P'ingan 平 娄 circ., Corea.

7855 Yung-k'ang | 康 Yungkang.

$1^{\circ}$ C. in T'ai-p'ing Fu, Knangsi ; Lat. $22^{\circ} 56^{\prime}$, Long. $10^{\circ} 36^{\prime}$.

[297] $2^{\circ}$ H. in Chin-huna Fu, Chellkiang; Iat. $28^{\circ}$ $58^{\prime}$, I.ong. $120^{\circ} 12^{\prime}$; Wu, Y'ung-k'ang | 庶; T'ang, I, 霹 C.

$3^{\circ}$ Chên near Sui-ning H., Kiangsu.

$4^{\circ}$ (obs.) S. Sung, military district in $\mathrm{Cl}_{1}{ }^{\prime} \mathrm{e} n \mathrm{n}-\mathrm{t} ı$ 成 者? circ.

$5^{\circ}$ See No. 4947.

7856 Yung-knei | 躈, (obs.) Sui and $T^{6}$ ang, H. 50 li S.E. of Chien C., Ssuch 'nan (No. 880).

7857 Yrung-lê | 樂 Yung-loh. $1^{\circ} \mathrm{T}$. in $\mathrm{P}^{\prime}$ 11-chon Fin, Sliansi; Iat. $34^{\circ} 40^{\prime}$, I.ong. $110^{\circ} 36^{\prime}$.

$2^{\circ}$ Chên near Ching-yang H., Shensi. 
永

Menci-iti.

7858 Yung-li | 豊, ssu in Nauching H., Fulıkien.

7859 Yung-ling | 陵, a posttown near F'êng-t' ien Fu, Shingking.

7860 Yung-1uing | 明 Yung[180] ming, H. in Yung-chou Fin, Hunan; I at. $25^{\circ} 20^{\prime}$, I,ong. $111^{\circ} 45^{\prime}$; Han, Yingp'u營浦; T゙ang, l lungining | 明.

7861 Vung-nien|年 Fung-nien, H. forming the prefectural city of Knang-p 'ing, Cliihli; I at. $36^{\circ} 46^{\prime}$, I.ong. $114^{\circ} 55^{\prime}$; Han, Ch' 'ii-liang. 曲梁; Sui, Wu-an 武笠 chïn and Yung-nien | 年. H.

7862 Jinng-11ing / 甯 Yung-111119. $[374] 1^{\circ}$ C. in Fiên-chou Fu, Shansi ; Iat. $37^{\circ} 34^{\prime}$, I.ong. $111^{\circ} 04^{\prime} ; C$. llors, Li-shili-yi 離不i 邑; Tsin, Hsi-ho 鸣 河; P. Tang, Yungning | 韜 $\mathrm{C}$.

[.358] $2^{\circ}$ C. in An1-slu11n Fu, Kueichou ; Lat. $27^{\circ} 53^{\prime}$, I.ong. $105^{\circ} 23^{\prime}$; orig., Huang-fu 紫服; Y ̈ӓаn, ling-11ing | 俩 $C$.

$3^{\circ} \mathrm{C}$. in Kuei-lin Fu, 'Knangsi ; Lat. $25^{\circ} 07^{\prime}$, Long. $109^{\circ} 36^{\prime}$; T’ang; Mu-hua 慕化; Sung, Yung-ning | 伃.
永

lowo-plit.

[263] $4^{\circ}$ II. in Ho-nan Fin, Honan; Lat. $34^{\circ} 22^{\prime}$, I.ong. $111^{\circ} 43^{\prime}$; Han, Mien-cl' ih 爿池; $\|$ ' II'ci, Hsinng-êrh 熊耳; Sur, I'mu-ning | 察.

[267] $5^{\circ} \mathrm{H}$. in Chi-an Fin, Kiangsi ; I at. $26^{\circ} 45^{\prime}$, I,ong. $114^{\circ} 02^{\prime} ; T^{\prime} a n g$, Clii 声 C.; Yiann, lungning | 俩.

[572] $6^{\circ} \mathrm{H}$. forming the subprefectural city of Hsiiynug, Ssŭcl' 'nan; Lat. $28^{\circ} 08^{\prime}$, I, ong. $105^{\circ} 18^{\prime}$. Formerly inclucled in Kneichon province.

$7^{\circ}$ 'I.F. North of Yungpei, 'T', Yünnan.

$S^{\circ} \Lambda$ post-town near Knyüan C., Kansul.

$9^{\circ}$ A post-town near $\mathrm{K}$ 'neichou Fu, Ssŭch "nan.

$10^{\circ}$ (obs.) Liang, H., IV. of Yang-chiang 'T'., Kuangtung.

$11^{\circ}$ (obs.) T"ang, H., N.E. of Li-p " 11 ., Knangsi. $12^{\circ}$ See Nos. 1583, 2326 , $3057,3491,3648,4609$, 7840 .

7963 Y'ung-ning-ch'êng|篗城。 see No. 7192.

7964 Iung-pei | 北 Yung-peh, [431] C.I.'T. in Jihsi circ., Yünnan ; I.at. $26^{\circ} 50^{\prime}$, I.ong. $101^{\circ} 15^{\prime}$; Choul, in 
永

YUNG-I'ING.

in K. Nan-chao 湳詔; Han, Chien-ch'iang 劍羌; Yïan, Pei-shêng 北 勝.

7965 Yung-p'ing | 平 Yungping.

[271] $1^{\circ}$ Fin in the $T^{\prime}$ ung-chon circ., Chilhli; forming also the district city of Lutlung; I at. $36^{\circ} 50^{\prime}$, Lolig. $118^{\circ} 50^{\prime}$; Clrou, Hu-chu 狐 竹 in In 幽 CHOU; Ts'in, Liaohsi 涾西 chïn and Inpei-ping在北本 chün; IVei，I,u-lung 盧 龍; 2nd Wci, Pei-p'ing 北 平; Sui and $T^{*}$ ang, P'ing 平 C. ; Sung, In 幽 C.; Hing, Yungp'ing | 尔 Fin.

[548] $2^{\circ} \mathrm{H}$. in Yung-ch'ang Fu, Yünnan; I at. $25^{\circ} 30^{\prime}$, Irong. $99^{\circ} 48^{\prime}$; Han, Po-nan 博献; Tsin, Yung-p'ing|平; T゙ang, Shêng-hsiang 勝 鄉.

$3^{\circ}$ H. in Ching-ch'i 京畿 circ., Corea.

$4^{\circ}$ Chên near Yen-ch" 'uan H., Shensi.

$5^{\circ}$ ChênnearLi H., Kansulul. $6^{\circ}$ Sis̆̈ in Ho-p 11 H., Kuangtung.

$7^{\circ}$ See No. 1157.

7966 Yung-p'ing-chai 本紫, ssü nea: Wu-p ing $\mathrm{H}$, Fuhkien.
永 YUNG-SHUN.

7967 Yung-shan | 善 Yungshen, H. in Chao-t 'ung F̀n, Yünnnan; Iat. $28^{\circ}$, I ong. $103^{\circ} 40^{\prime}$.

7968 Yung-shil | 世, see No. 3752 .

7969 Yung-shon | 䥾 Yung[312] slian, H. in Ch'ien C., Shensi ; I at. $34^{\circ} 48^{\prime}$, Long. $108^{\circ} 03^{\prime}$; orig., in K. Pin 奧; 2 nd Wei, Kuang-shou 廣壽; P. Chou, Yungshou| 菷.

7970 Y'ung-shnu | 順 Yrungshun.

[237] $1^{\circ} \mathrm{Fu}$ in the $\mathrm{Cl}{ }^{\prime}$ ên-YüanInng-Ching circ., Hunan; forming also the district city of the same name; Lat. $28^{\circ} 58^{\prime}$, I.ong. $109^{\circ} 51^{\prime}$; orig., land of the Man 纆 tribes and frontier of $\mathrm{K}$. Ch' $\mathrm{u}$ 楚; Ts'in, in Ch'iencliung 黔中 chün; Han, in Wu-ling 武陵 chün; T'ang, Ch'i 溪 $\mathrm{C}$. and I,ing-ch'i 靈渓; Sung and Hing, Yung-shun | 澒.

$2^{\circ} \mathrm{H}$. forming the perfectural city of the same name, Human; Lat. $28^{\circ} 58^{\prime}$, Isong. $109^{\circ}$ $51^{\prime}$.

$3^{\circ}$ 'T'.S. near Lo-ch êng H., Knangsi. 
永

Yung-shux.

$4^{\circ}$ (obs.) T"ang, "H. near Tung-an H., Knangtung.

7971 Yung-shun| 淐 Yung-shun. [198] $1^{\circ} \mathrm{H}$. in Nan-ning Fu, Kuangsi ; I.at. $22^{\circ} 41^{\prime}$, Long. $108^{\circ} 2^{\prime}$; Han,

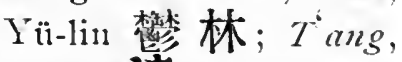
Shun 湆 C.; Sung, Yung-shun | 淐.

$2^{\circ}$ (obs.) Yüan, H., N. of the above.

7972 Yung-sui | 総 Yung-sui, [21] C. L. T. in Ch' ên-YüanYung-Ching circ., Hunan ; Lat. $28^{\circ} 30^{\prime}$, Long. $109^{\circ}$ $30^{\prime}$.

7973 Yung-t'ai 泰, see No.7848. 7974 Yung-ting | 定Yung-ting. [251] $1^{\circ}$ H. in I,i C., Hunan; Lat. $29^{\circ} 05^{\prime}$, Long. $110^{\circ} 15^{\prime}$.

$[575] 2^{\circ} \mathrm{H}$. in $\mathrm{T}^{\prime} \mathrm{ing}-\mathrm{ghou} \mathrm{Fu}$, Fulkielı; I at. $24^{\circ} 45^{\prime}$, J.ong. $116^{\circ} 53^{\prime}$; orig., Shang-hang 上杭.

$3^{\circ} Y$. near Ning-yiuan Fu, Ssŭcli tran.

$4^{\circ}$ T.S. near Ch'ing-yöan Ful, Kuangsi.

7975 Yung-ts'ung | 從 Inng[110] tsung, H. in I,i-p ing Fu,

Kueichou; Lat. $26^{\circ}$, 1.ong. $108^{\circ} 5 s^{\prime}$.

7976 Yung-t' ung | 同, H. in Clinng-ch'ing 忠清 circ., Corea,
多留 YUxa.

7977 lung-yang | 晹, see No. 7573.

7978 Y'ung-yang-p'u| 显鋪, sš̆ near T"ai-ho H., Kiangsi.

7979 Yung-yeh | 業, (obs.) Liang and Sui, H., N.E. of Ling-shan $\mathrm{H}_{\text {, , Knang- }}$ tung.

\section{融}

7980 Yung 融 Yung, H. in [255] Litt-chon Fit, Kuangsi; Lat. $24^{\circ} 59^{\prime}$, Long. $108^{\circ}$ $58^{\prime}$; Han, 'I'an-chung 漝 中: Sui, Iung | C.; Tong, Yung-shui | 水.

7981 '̌nung-chiang | 汇, (obs.) Sung, a military post near Jin-chou Fu, Knangsi.

7982 Yung-shui | 水, see No. 7980.

\section{庸}

7983 Yung 庸, (obs.) Sui, C. near Ch ien-chiang H., Ssŭch' nan.

\section{廑}

7984 Yung 确, (obs.) Chou, feudal state $S$. of IVei-hui Fin, Honan.

\section{銿}

7985 Y In1ng 錐, see No. 772.

\section{雃}

7986 Y $111 \mathrm{~g}$ 多隹. 


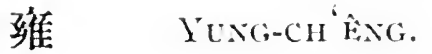

$1^{\circ}$ One of the nine chou, or great divisions of the Eumpire establisled by Jï 禹, comprising Shensi and Kansulu.

$2^{\circ}$ See Nos. 1724, 2438, 2616.

7987 Jung-cl'êng | 城, (obs.) formerly a town near $\mathrm{Hsh̆}$ C., Honan.

7988 Vinng-chi | 雞, (obs.) $H^{\prime}$. Han, H. in Clin'ung-shan H., Knangsi.

7989 Iung-chia | 蒙, chên near Wuhu H., Anluii.

7990 Vung-ch'in | 邱, see No. 623.

7991 linug-11u| 奴. $1^{\circ}$ (obs.) Han, Tsin, 1 . IVei and Sui, H. 8 li E. of Wu-cli'ing H., Chihli.

$2^{\circ}$ See No. 7062.

7992 lung-rï | 榆, (obs.) s.A. a town near In C., Honan.

\section{角}

7993 Yung 角, see No. 4702.

7994 Iung-tu1ng 策, see No. 4702.

\section{涌}

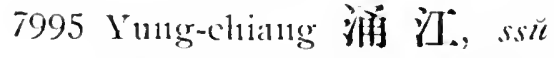
near Hsing-luta I'tu, liuhkien.
IUNG.

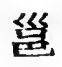

7996 Vu1ng 画, see Nos. 2930, 4609.

Yung, see Nos. 3036, 3043. Yung-cliang, see Nos. 3044, 7836.

Yung-chat1, see No. 7843.

Yung-ching, see Nos. 30.3\%, 3045, 7838.

Yung-clunen, see No. 7844.

Y'ung-cliun, see No. 7846.

Yung-ful, see No. 7848.

Yung-fung, see No. 7847 .

Yu11g-ling, see No. 7853.

Yung-ho, see No. 3048.

lung-kang, see No. 7855.

I'ung-kia, see No. 7840.

Yrung-king, see No. 3046 .

Yung-loh, see No. 7857.

Yung-ngan, see No. 7835.

Yung-pel, see No. 7964.

Yung-ping, see No. 7965.

Yung-slian, see No. 7969.

Yu11g-slien, see No. 7967.

Yung-sin, see No. 7852.

Yung-tseh, see No. 3050.

Yung-tsi, see No. 7839.

Yung-tsing, see No. 7842 .

Yung-tsung, see No. 7975.

Yung-yang, see No. 3051.

Iurungkash, see No. 7727 .

Zayfon, see Nos. 210, 1491.

Zendjan, see No. 6513.

Zhikatse, see No. 2997.

Zinieriek, see No. 597.

Zunga, see No. 5973. 


\section{APPENDICES}

1. Synoptical tables of Administrative Cities.

2. Towns in Thibet.

3. Towns in Corea.

4. Places mentioned by Marco Polo.

5. Distribution of Mongolian Tribes.

6. Towns in Japan.

7. The Principal Rivers and Lakes of China.

8. Radical Index. 



\section{ADMINISTRATIVE CITIES OF CHINA}

ARRANGED ALPHABETICALLYY

UNDER 'THEIR PROVINCES

\section{ANHUI}

\section{Circuits.}

I. An-Lü-Ch'u-Ho 安蒀滁和 A.C.E.I.

2. Hui-Ning-Ch'ih-'T'ai-Kuang-tê Chou 徽寉池太庶德州 B.F.G.J.L.

3. Fêng-Ying 鳳頴 D.H.K.M.

PREFECTURES AND INDEPF, NUENT DePAR'TMENTS.
A. An-CH'Ing Fu
安鹿
B. Ch'ih-chou Fu
池州
C. $\mathrm{Ch}^{\prime} \mathrm{u}$ C.L.C.
涂
E. Ho C.L.C.
利 J. Ning-kuo Fu
富國
F. Hui-chou Fu
徽州
G. Kuang-tê C.L.C. 廣德
K. Ssŭ C.L.C.
河
D. Fêng-yang Fu
H. Liu-an C.L.C.
六安
L. T'ai-p'ing $\mathrm{Fu}$
太平
I. Lü-chou Fu
M. Ying-chou $\mathrm{Fu}$ 颖州

SubrefFectures, Departments and Districts.

\begin{tabular}{|c|c|c|c|c|c|}
\hline Ch`ao 巢 & H. (i) & *Huai-ning 懷莩 & H. (a) & Su-sung 宿松 & H. (a) \\
\hline Chêng-yang Kuan & 正陽關 & Huai-yüan 懷遠 & H. $(d)$ & T'ai-ho 太和 & H. $(m)$ \\
\hline & T. $(d)$ & Hsiu-ning 休寀 & H. $(f)$ & T‘ai-hu 太湖 & Ii. $(a)$ \\
\hline Chi-ch`i 績哚 & H. $(f)$ & Hsü-yi 䀒眙 & H. $(k)$ & T'ai-p'ing 太本 & H. $(j)$ \\
\hline Ch'i-mên 䢼門 & H. $(f)$ & Hsüan-ch'êng 宣城 & U. $(j)$ & *Tang-tu 當鋈 & H. $(l)$ \\
\hline Chien-p'ing 建平 & H. $(g)$ & Kê-yang 渦陽 & H. $(m)$ & T'ien-ch'ang 天長 & H. $(k)$ \\
\hline Ch'ien-shan 潛山 & H. $(a)$ & *Kuei-ch'ih 貴池 & H. $(b)$ & Ting-yüan 定遣 & H. (d) \\
\hline Ching 泾 & H. $(j)$ & Lai-an 來安 & H. (c) & Tung-liu 東流 & H. $(b)$ \\
\hline Ching-tê 旅德 & H. $(j)$ & Ling-pi 靈幦 & H. $(d)$ & 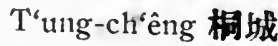 & H. (a) \\
\hline Ch'ing-yang 青陽 & H. (b) & Lü-chiang 盧江 & H. $(i)$ & $T^{\prime}$ 'ung-ling 銅陵 & H. (b) \\
\hline Ch'üan-chiao 全椒 & H. $(c)$ & Mêng-ch'êng 䔹城 & H. $(m)$ & hiang 哭江 & H. (a) \\
\hline Fan-ch'ang 繁昌 & H. (l) & Nan-ling 南陵 & H. $(j)$ & Wu-ho 五河 & H. $(k)$ \\
\hline *Fênlly-t'ai 臺鳳 & H. $(d)$ & Ning-kuo 豈國 & H. $(j)$ & 匴湖 & H. $(l)$ \\
\hline *Fêng-yang 鳳陽 & H. $(d)$ & Po 毫 & C. $(m)$ & Wu-wei 無第 & C. (i) \\
\hline *Fu-yang 阜陽 & H. $(m)$ & *Shê 歌 & H. $(f)$ & Wu-yüan 愁源 & H. $(f)$ \\
\hline Han-shan 含ll & H. (e) & Shih-tai 不埭 & H. $(b)$ & $\mathrm{Yi}$ 黝 & H. $(f)$ \\
\hline Ho-cli'iu 霍邱 & H. $(m)$ & Shou 寻 & C. $(d)$ & Ying-shan 英山 & H. $(h)$ \\
\hline *Ho-fei 分肥 & H. (i) & Shul-ch'êng 舒城 & H. (i) & Ying-shang 穎上 & H. $(m)$ \\
\hline Ho-shan 霍山 & H. (h) & $\mathrm{Su}$ 宿 & C. $(d)$ & & \\
\hline
\end{tabular}




\section{CHEHKIANG}

Cricuits:

I. Hang-Chia-Hu 杭嘉湖 A.E.F.

2. Ning-Shao-T'ai 豈紹太 G.H.I.J.

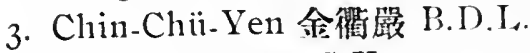

4. Wên-Ch'u 温處 C.K.

Prefecturfs and Inijepenient Subreitecture.

A. Chai-hsing Fu 嘉興| E. HanG-CHou Fu 杭州 1. T'ai-chou Fu 太必州

B. Chin-hua Fu

金華 F. Hu-chou Fu

湖州 J. Ting-hai C.I.T. 定海

C. Ch'u-chou $\mathrm{Fu}$

處州 G. Ning-po Fu

晸波 K. Wên-chou Fu 温州

D. Chü-chou Fu

臛州 H. Shao-hsing Fu

紹䀯 L. Yen-chon Fu嚴州

Subrefefectures. Departments and Districts.

\begin{tabular}{|c|c|c|c|c|c|}
\hline An-chi 安吉 & H. $(f)$ & Hsiao-shan 㶓! & H. (h) & n-an 渲妿 & H. (l) \\
\hline Ch'ang-hua 昌化 & H. (c) & Hsien-chü 仙居 & H. (i) & Sui-an 遂笠 & H. $(l)$ \\
\hline Ch'ang-hsing 長與 & H. $(f)$ & Hsin-ch'ang 新昌 & H. $(h)$ & Sui-ch'ang 遂昌 & H. $(c)$ \\
\hline 'ang-hsan 菏山 & $\mathrm{H}_{\mathbf{l}}(d)$ & Hsin-ch'eng 新城 & H. $(e)$ & Sung-yang 松湜 & H. (c) \\
\hline hai 鎛海 & H. $(g)$ & *Hsiu-shui 秀水 & H. $(a)$ & T'ai-p'ing 太本 & H. (i) \\
\hline 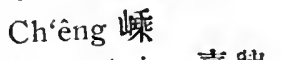 & H. (h) & p ‘ing 冝本 & H. $(c)$ & T'ai-shun 泰順 & H. $(k)$ \\
\hline hsing 嘉與 & H. (a) & 比利 & H. $(e)$ & T'ang-ch'i 晹溪 & H. (b) \\
\hline 嘉山 & H. (a) & Jui- & H. $(k)$ & ng 德清 & H. $(f)$ \\
\hline 江山 & H. $(d)$ & K'ai-h & H. $(d)$ & T'ien-t'ai 天台 & H. (i) \\
\hline 建德 & H. $(l)$ & * Kuei- & H. $(f)$ & Tung-yang 東陽 & H. $(b)$ \\
\hline * Ch'ien-t'ang 錢塘 & H. (e) & * Kue & H. $(h)$ & T'ung-hsiang 榈榔 & H. (a) \\
\hline ua 金華 & H. (b) & 阅窑 & H. (b) & T'ung & H. $(l)$ \\
\hline 緹雲 & H. $(c)$ & Lê-ch'ing 樂清 & H. $(k)$ & ; 慈閤 & H. $(g)$ \\
\hline g 崇蔫 & H. (c) & 雔水 & H. $(c)$ & *Wu-ch'êng 郳城 & H. $(f)$ \\
\hline $\mathrm{n}$ 青田 & H. $(c)$ & & H. (c) & Wu-k'ang 武康 & H. $(f)$ \\
\hline n 慶元 & H. $(c)$ & 魚海 & H. (i) & $\mathrm{Wu}$ & H. (b) \\
\hline 笪 & H. $(h)$ & 龍泉 & H. $(c)$ & $\mathrm{Yi}-$ & H. (b) \\
\hline 水 & H. $(l)$ & Lun & H. $(d)$ & $* \mathrm{Yi}$ & H. $(g)$ \\
\hline 泰化 & H. $(e)$ & i 䒠游 & H. (i) & Yü-ch'ien 於貲 & H. (e) \\
\hline Fu-y & H. $(e)$ & P'ing-he & H. $(a)$ & Yü-hang 俆杭 & H. (e) \\
\hline Hai-ning 海耍 & C. $(c)$ & $\mathrm{P}^{‘} \mathrm{u}$-chiang 浦江 & H. $(b)$ & Yü-huan 玉環 & T. $(k)$ \\
\hline Hai-yen 游監 & H. (a) & 的山陰 & H. $(h)$ & Yï-yao 俆姚 & H. (h) \\
\hline Huang-yen 誉㦑 & H. (i) & 上虞 & H. (h) & Yün-ho 雲利 & H. (c) \\
\hline & H. $(d)$ & Shih-mên 石門 & H. (a) & -chia 永嘉 & H. (b) \\
\hline & H. $(g)$ & Shih-p'u 石浦 & T. $(g)$ & Yung-k'an & H. (b) \\
\hline Isiao-fêng 孝贯 & H. $(f)$ & Shou-ch'ang 胥 & H. $(l)$ & & \\
\hline
\end{tabular}


Cincuits.

I. Ch'ing-lro 清河 A.C....I.O.Q.

2. K'oll-wai-Jê-l1o 口外熱河 B.L.

3. 'T'ung-chon-Yiin-ho 道州運河 P.R.

4. T'ien-ching-Ho-chien 天津河間 F.N.

5. Ta-Shun-Knang 大海塂 H.K.AI,

6. K'ou-pei 口北 $\mathrm{G}$.

PREFECTURES IND INUEPENIENT DEPARTMENTS
A. Chao C.L.C.
趙
G. Hsüan-lua $F_{1}$
宣化|M. Ta-ming F*u
大名
B. Ch'ao-yang Fu
H. Kuang-p'ing Fn 度本
N.'T"icu-ching Fu
天津
C. Chêng-ting $F_{u}$
D. Ch"èng-tè Fu
1. PaO-TING Fi
保定 O. Ting C.L.C.
E. Chi C.L.C.
J. Shén C.J.C.
深 P. Tsum-hua C.L.C. 燃化
F. Ho-chien $\mathrm{F}_{\mathrm{i}}$
冀 K. Shun1-tè Fu
順德 Q. vi C.L.C.
河閒 L. Shun-t'ien Fu
順天 R. I'ung-p'ing l'u
易

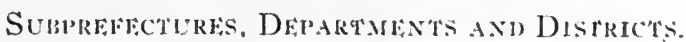

\begin{tabular}{|c|c|c|c|c|c|}
\hline An茹 & C. (i) & Ch'ing-fèng 清豐 & H. (ii) & Hsiang-ho 香河 & H. $(l)$ \\
\hline An-p’ing 安本 & H. $(j)$ & Cl'ing-110 清河 & II. $(h)$ & Hsien 龊 & 11. $(f)$ \\
\hline An-s1n 安徳 & H. (i) & *Ch'ing-yüan 清苑 & H. (i) & H Isin-ch"èng 新城 & H. (i) \\
\hline chang-chia K'ou & 張笑口 & Chring-yün 慶雲 & H. (II) & Hsin-ho 新河 & II. (c) \\
\hline & 'I. $(g)$ & Cho 㴍 & C. (l) & Hsin-lê 新樂 & H. (c) \\
\hline Ch'ang-li 昌黎 & If. $(r)$ & Chü-lu 鈿鹿 & II. $(k)$ & *Hsing-t'ai 形茥 & H. (k) \\
\hline Ch'ang-p'ing 昌平 & C. $(l)$ & Cli'ü-chou曲周 & H. $(/ h)$ & Hsing-tang 行噟 & H. (c) \\
\hline Ch'angr-yüan 長垣 & H. (m) & Chrï-yang 曲隄 & H. $(n i)$ & Hsiung 雉 & H. (i) \\
\hline *Chêng-ting 正定 & H. (c) & Fang-shan 房山 & 11. (l) & *HIüan-hua 宣化 & H. $(g)$ \\
\hline Ch’êng-an 成安 & H. $(h)$ & Fei-hsiang 肥鄉 & 11. $(h)$ & Jao-yang 饒隊 & H. $(j)$ \\
\hline Chi 魝 & C. (l) & Fèng-jun 罟潤 & II. $(p)$ & Jèn 任 & H. (k) \\
\hline Chi-tsê 矮紧 & H. $(h)$ & 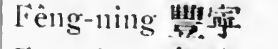 & H. $(d)$ & len-ch'in 任坵 & H. $(f)$ \\
\hline Ch'i 䃼 & C. (i) & 卓城 & H. $(f)$ & chèng 飸城 & H. (c) \\
\hline Chiao-110 交河 & H. $(f)$ & $\mathrm{I}^{*} 11-\mathrm{h}_{3}$ & H1. (b) & K’ai 開 & $C .(m)$ \\
\hline Chien-chang 建昌 & H. (b) & F'u-ning 撫偁 & 11. $(r)$ & Kao-cl'èng 萦城 & H. (c) \\
\hline Chien-p'ing 建本 & H. $(b)$ & Fu-p ing 皁本 & H. (c) & Kao-yang 高隊 & H. (i) \\
\hline Clïien-an 遷得 & H. $(r)$ & Han-tan 邯鄲 & H. (h) & Kao-yi 高出 & H. (a) \\
\hline Ch'ih-cl êtrg 浾城 & H. $(g)$ & Heng-shui 衡水 & H. (c) & Ku-an 固安 & H. (l) \\
\hline Clı̈in-fêng 洂峰 & H. (d) & *Io-chien 河間 & H. $(f)$ & Ku1-ch'êng 故城 & H. $(f)$ \\
\hline Chin 丝 & C. $(c)$ & U luai-an 裹桨 & H. $(x)$ & Kunang-cl'ang磨昌 & HI. $(r)$ \\
\hline Ching 景 & C. $(f)$ & IInai-jou 裹乘 & H. $(l)$ & Kuang-p 'ing 戌平 & H. (li) \\
\hline Ching-lai 静游 & H. (n) & Huai-lai 懷本 & H. $(g)$ & Kuang-tsung 篔宗 & II. $(k)$ \\
\hline Ching-hsing 非陘 & H. (c) & Huo-lu 獲鹿 & H. (c) & Lai-shui 淶水 & H. $(q)$ \\
\hline Cli'ing 青 & H. (n) & Hsi-ning 西莩 & H. $(g)$ & Lè-1'ing 樂亭 & H. $(r)$ \\
\hline
\end{tabular}




\section{CHIHLI Continued.}

$1, \mathrm{i}$ 䖝

11. (i) Pao-ting 保分

H. (l) ' Tung-an 東安

H. (l)

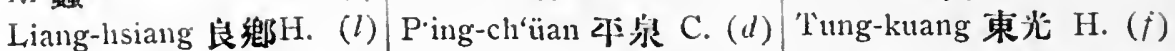

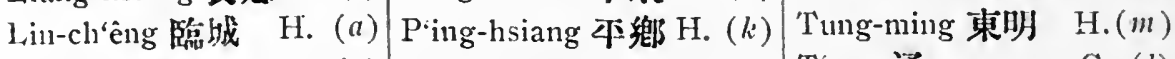

Lin-yii 隐榆

H. (r) P'ing-ku 不谷

H. (l) T'ung 通

C. $(l)$

Ling-shou 䍔语

H. (c) P'ing-shan 平小

H. (c) 'Tz'ù 磁

C. (h)

*L,u-lung 盧龍

II. (r) Po-yeh tijige

Luan 棌

C. $(r)$ San-ho 三汀

H. (i) Wan 完

H. (i)

Luan-ch'èng 戀城 H. (c) Sha-ho 沙讨

H. (l) Wan-chiian 茧全 H. $(g)$

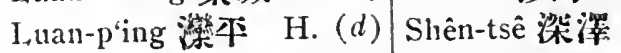

H. (k) *Wan-p'ing 宛平 H. (l)

Lung-hua 隆化

H. (d) Shun-yi 順妾

H. (i) Wang-tu 㕵都

H. (i)

Lumg-mèn 龍門

H. (s) Su-lu 束鹿

H. (l) Wei 成

H. (h)

H. (i) Wei-ch'ang 圈場 T. (d)

Lung-p'ing 隆近

H. (a) Su-ning 茛察

H. $(f)$ Wèn-an 文安

H. (l)

Ian-ch'êng 㴖城

H. (i) Ta-ch'èng 大岾

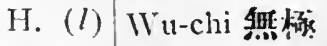

H. (c)

Mi-yün 惩雲

Nan-110 南利

H. (l) *Ta-hsing 大留

H. (l) Wu-ch'iang 试强 H. (II)

H. (k) *'Ta-ming 大名

H. (m) W Wu-ch'iao 吳橋

H. $(f)$

Nan-kung 哃宮

H. (c) 'T'ang 磨

H. (i) Wn-ch'ing 就涯

H. (l)

Nan-lê 南樂

H. (m) T'ang-shan 虎l

H. (k) Wu-yi 试弁

H. (e)

Nan-p'i 南皮

Nei-ch'iu 队形

H. (n) *T"ien-ching.天率

H. (n) Yen-ch'ing 延参

C. $(g)$

H. (k) l'ing-hsing 定罗

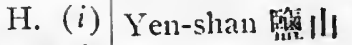

H. (")

Ning-chin 票蓝

Ning-ching 篮渄

H. (a) 'To-lun-1no-êrlı 多

倫諾尔 Y ü 薜

C. $(g)$

H. $(f)$

'l. (g) Yü̈-t'ien 王[I]

H. $(p)$

Ning-ho 话流

H. (l) Tsan-huang 贊皇

H. (c) *Yüan-ch'êng 元城 H. $(m)$

$\mathrm{Pa}$ 霖

C. (l) 'T's'ang 涗

C. (n) Yüan-shih 元比 H. (c)

Pai-hsiang 柏瑯

Pao-an 保安

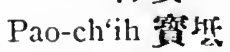

H. (a) Tsao-ch'iang 装强

H. (e) Yung-ch'ing 永沙

H. (l)

C. (g) Tu - shih - kou 獨石 I $*$ Y ung-nien 永年 H. $(h)$

iH. $(l)$

T. $(g)$

FORMOSA (Under Chinese Rule)

Circuit.

'小'ai-wan-P'êng-hu 臺潄澎湖 A.B.C.D.

PRFFECTIRES AND INDFPFAOFNT DEPARTMENT.
A. 'T'ai-clung
喜中
C. $12 \mathrm{~N}-\mathrm{PFI} \mathrm{Fu}$
B. 'T'ai-nan Fu
臺南
D. 'T'ai-tu1ng C.L.C. 辜東

SUBPREFECTURES AND DisTRIC'S.
An-p'ing妿不
H. (b) Fêng-shan 鳳山
H. (b) *T'ai-wan 臺灣
H. (a)
Chang-hua 彰化
Chi-lung 基隆.
H. (a) Hêng-ch' un 恒春
H. (b) *Tan-shui 淡水
H. (c)
T. (c) Hsin-cho 新竹
H. (c) Yi-lan 宜關
H. (c)
Chia-yi 嘉義
H. (b) $\begin{aligned} & \text { P'êng-hu 澎湖 } \\ & \text { Nan-li 南栗 }\end{aligned}$
T. (b) Yün-lin 雲林
H. (b)
* Head District of a Prefecture. 


\section{FUHKIEN}

Cincites.

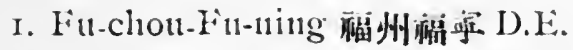

2. Hsing-Ch'üan-Yung 舆泉永 C.F.K.

3. Yen-Chien-Shao 延建郡 B.H.J.

4. 'T'ing-Chang-Lung 汀漳龍, A.G.I.

PREFECTURES AND INDEPENDENT DFPARTMENTS.
A. Chang-chou lin 漳州 E. Fin-ning Fu
福曾 I. 'T'ing-chon Fin 汀州
B. Chien-ning Fu 建管 F. Hsing-hua I'u 與化 J. Yen-p ing Fu 延平
C. Ch'iian-chou I'u 泉州 G. Lung-yeh C.I.C. 龍墏 K. Linng-ch'un C.L.C.
D Fu-criot: Fu 稫州 H. Shao-wuru 郡武
永春

\section{SUBPREFFCTURES .IND DISTRICTS.}

\begin{tabular}{|c|c|c|c|c|c|}
\hline An-chii 茹溪 & H. $(c)$ & \multicolumn{2}{|c|}{ Hung-chiang 虹江 T. (c) } & P'ing-ho 平利 & 11. $(a)$ \\
\hline Chang-p'ing 漳本 & H. $(s)$ & Hsia-mên 厦門 & 'T. (a) & P'ing-nan 屏南 & H. $(d)$ \\
\hline Chang-p'u 澶浦 & H. (a) & *Hsia-p’u霞浦 & H. $(\mathfrak{c})$ & P'ing-t'an 本增 & T. $(d)$ \\
\hline Ch'ang-lè 長樂 & H. $(d)$ & Hsien-yu 仙遊 & H. $(f)$ & P'u-ch'êng 浦贱 & H. (b) \\
\hline Ch'ang-t'ai 長泰 & H. $(a)$ & Ku-t'ien 古田 & H. $(d)$ & *P'u-t’ien 甫田 & H. $(f)$ \\
\hline *Cl1'ang-t'ing長汀 & H. $(i)$ & Kuang-tsè 光澤 & H. $(h) !$ & Sha 沙 & H. $(j)$ \\
\hline Chao-an 詔安 & H. $(a)$ & Kuci-luna 蹄化 & H. (i) & Shang-hang 上杭 & H. (i) \\
\hline Chêng-ho 政利 & H. (b) & I ien-ch'êng 連城 & H. (i) & *Shao-wu 郡武 & H. $(h)$ \\
\hline Chiang-lê 將樂 & II. $(j)$ & Lien-chiang 浬证 & H. $(d)$ & 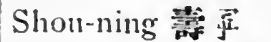 & H. (c) \\
\hline *Chien-an 建妿 & H. $(b)$ & Lo-yüan 囉源 & II. $(d)$ & Shun-ch'ang 順旨 & H. $(j)$ \\
\hline Chien-ning 建篎: & H. $(h)$ & *Lung-ch'i 龍溪 & H. $(a)$ & i 松溪 & II. (b) \\
\hline Chien-yang 建陽 & II. $(b)$ & Ma-chia-hsiang 黑 & 家萣 & 大田 & H. $(k)$ \\
\hline *Chin. chiang晋江 & -H. (c) & & T. (c) & 'T'ai-ning 泰富 & H. (h) \\
\hline Ch'ing-liu 清流 & H. (i) & *Min 閩 & H. $(d)$ & Tè-hua 德化 & H. $(k)$ \\
\hline Ch'ung-an 岁宗 & H. $(b)$ & Min-ch'ing 閔清 & H. $(d)$ & $\mathrm{T}^{c}$ ung-an 同安 & H. $(c)$ \\
\hline Fu-an 福奖 & H. (c) & Nan-an 南姦 & H. (c) & Wn-p'ing 武平 & H. (i) \\
\hline F'u-ch'ing 福清 & H. $(d)$ & Nan-ching 南靖 & II. $(a)$ & Yu-ch'i 尤渗 & H. $(j)$ \\
\hline lu-ting 福鼎 & II. (c) & *Yan-p 'ing 南本 & H. $(j)$ & Yün-hsiao 雲霄 & T. (a) \\
\hline Hai-ch'êng 海澄 & H. (a) & Ning-hula 跕化 & H. (i) & lung-an 永安 & H. (J) \\
\hline *Hon-kuan 侯官 & H. $(d)$ & Ning-te 苸德 & H. $(c)$ & Yung-iu 永蔽 & II. $(d)$ \\
\hline Hui-an 基妏 & H. $(c)$ & Ning-yang 偁隄 & H. $(g)$ & loung-ting 永定 & II. (i) \\
\hline & & & & & \\
\hline
\end{tabular}

* Head Distrid of a Prefecture. 


\section{HONAN}

\section{Cikctirs.}

1. K'ai-Knei-Clı'ên-Hsii 開菷淒許 B.C.J.I.

2. Ho-pei 河北 A.H.O.

3. Ho-nan-Slian-Ju 沫献傸汝 I).H.X.

4. Nai1-Ju-Kuang 南汝光 I. F . K.M.

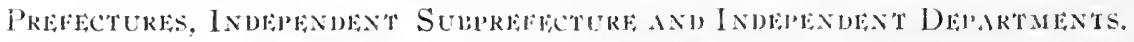
A. Chanlgy-tè Fơ
彰德 $F$
B. Clien-chon Fu 郪州 G. Hsü C.J.C.
許
K. Kuang C'L.C.C.
光
C. Chểng C.L.C.
鄭
H. In C.I.C:
D. Hu-nan lin
洏南 I. Ju-ning Fu
汝
L. Kuei-tê Fu
汝莠 N. Shan C.L.C.

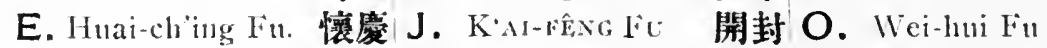
踁德
南陽
陝
街輝

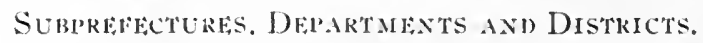

\section{Ari-yang 安陽}

Ch'ang-ké 長蒱

Chè-clièng 杯城

Chên-p'ing 鐭平

Clìèn-lin 陳馏

Cheneng-yang 正㧛

*Chi 汲

Chi-yiian 㴒源

Cl1 $\mathrm{i}$ 淇

* Chri 杷

Chia 邩

Chrieh-shan 確山

F'èng-ch'iu 封邱

liu-kou 扶满

*Ho-nei 咑內

Hua 滑

* Huai-ning 悴寀

Huang-ch'in 寅脑

Hui 輝

Iluo-chia 獲嘉

Hsi 息

I1si-hua 西華

Hsi-ping 西本

Hsia-yi 夏远

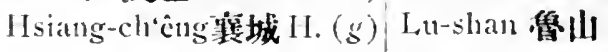

H. (l) Hsin-all 新姿

H. (c) Ku-shih 固始

H.(b) Kung 㳯

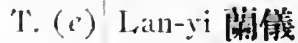

H. (o) Lin 林

H. (k) Lin-ying 隐疑

H. (b) Ling-pao 露琵

H. (i) Lo-shan 羅山
H. (a) Hsiang-ch'eng項城H. (b)|Lut-shih 盧氐

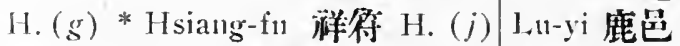

H. (d) Mêng 孟

H. (m) Hsin-chêng 新嘼

1. (j) Hsin-lhsiang 新塊

H. (j) Nêng-ching 系渄

H. (o) Mi 密:

H. (i) Nien-ch'ih 澠池

H. (i) Nat1-chao 南召

IH. (II) * Nan1-yang 南隊

H. (e) Nei-huang 內橫

H. (o) Nei-hsiang 內挀

H. (i) Ning-ling 富陵

H. (c) Pao-fêng 實豐.

H. (c) Pi-yang 泌陽

H. (o) Jung-yang 㷊陽

H. (b) K'ao-cli'êng 考城

H. (o) Kuang-shan 光山

H. (o) Lin-chang 臨游

H. (l) * lo-yang 洛䧄

H. (l) Shang-ch'èng 商城 $\mathrm{H} .(k)$

H. $(k)$ *Shang-cl' 'iu 商邱 H. (l)

H. (k) Shang-shni 商水 H. (b)

H. (d) Shang-ts'ai 上繁 H. (i)

H. (j) Shê 涉 H. (a)

H. (a) Shê葉 H.(m)

H. (a) Shèn-cb'in 沈邱 H. (b)

H. (g) Ssŭ-shui 氾水 H. (c)

H. (n) Sui 㫿

H. (i) Sui-p ing 遂平

C. $($ l)

H. (d) Sung 管

H. (i)

H. (h) T'ai-k'ang 太归 H. (b) 
HONAN Continued.

'T'ang 應

$\mathrm{T}$ 'ang-yin 湯陰

H. (m) Wu-an 武管

H. (a) Y Yi-yang 寉隄

H. (d)

Têng 鄧

H. (a) Wu-chih 武陟

H. (c) Yi-yang 伊陽

H. (h)

Tèng-fêng 登封

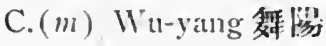

II. ( $m$ ) Yü 禹

C. $(j)$

H. (d) lang-wu隄武

H. (c) Y Yii 被

C. $(m)$

'T'ung-hsii 通許

H. (j) Yen-chèng 貙城

11. $(g)$ Y Yii-clı èng 篹城

11. (!)

'I’ung-pai 桐柏

If. (m) Yen-ching 延津

H. (o) Yii-shih 尉正

11. (j)

Wei-ch'van 洧川

Wên 溰

H. ( $j$ ) len-ling 酸陵

H. ( $j$ ) Yüan-W"n原武

II. ( ( $\left.{ }^{\circ}\right)$

H. (c) Y'cn-shih 㑑倧

II. (c) lung-ch'êng 永城 II. (l)

Wên-hsiang 䦣挀

H. (n)! \i-rèng 儀封

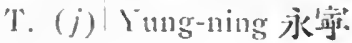

H. (d)

\section{HUNAN}

Cikceits.

I. Ch'ang-Pao 長窟 A.I.

2. Yo-Ch'ang-Li 岳常澧 B.M.K.

3. Hêng-Y'rung-Ch'ên-Kuei 衡永悚柱 C.H.J.O.

4. Ch'ên-Yüan-Yung-C'hing 辰沅永垟 D.E.F.G.I.N.P.Q.

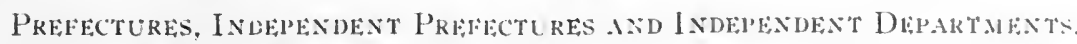
A. Cir'axg-sen-Fu
長沙 G. Fêng-huang
L. Pao-ch "ing lin
實度
B. Ch'ang-te I’u 常德
C.L.'I'. 鳳凰 M. lo-chou Fu
活州
C. Cliên Cil.c.
籿 H. I Iêny-cinon lin
衡洲 N. Iü:แ1-chun l':1
沅州
D. Ch"èn-chou Fu
定州 I. Huang-chon
O. linng-chun I:" 永州
E. Ch"ien-chun
C.1.'T. 光州
C.L.'I. 乾州 J. Kuci-yang C.L.C.样隄
F. Ching C.L.C. 锖 K. Ki C.L.C. 謴
P. Ving-slum $F$ แ 永脽

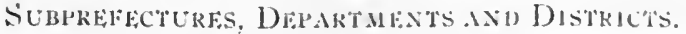

An-fu安福

An-hua 安化

An1-hsiang 妥鄉

H. (k) $\left.\right|^{*}$ Chilh-chiang 鿊江H. (n)' Ku-chang-p' ${ }^{\prime}$ ing

H. (a) *Ch'ing-ch'üan清泉H. (n)

古丈坪 I'（中）

H. (b) Hèng-shan 衡山 H. (h) Kuci-tung 桂東

H. (c)

An-jên 安仁

Ch'a-ling 茶陵

H. (h) *Hêng-yang 衡䍀 H. (h) Kuei-yang 棋隄

H. (c)

C. . (a) Hua-jung 華突

H. (m) Lan-shan 藍山

H. (j)

Ch'ang-an 長妿

H. (l) Hui-t'ung 會同

H. $(f)$ Lei-yang 来湜

H. $(n)$

Ch'ang-ning 常寀

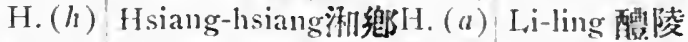

H. (a)

* Ch'ang-sha 長沙

Ch'ên-ch'i 辰溪

Ch'êng-pu 城步

Ch'i-yang 新陽

Chia-ho 嘉禾

H. (a) Hsiang-t'an 洲漂

H. (a) L,in-hsiang 绝湘

H. (mi)

H. (d) Hsiang-y in 淋䧔

H. (a) Lin-Wu 䜿试

H. (j)

H. (l) Hsin-hua 新化

H. (l) Ling 塥

H. (h)

H. (o) Hsin-ning 新寀

H. (c)* Ling-ling 露陵

H. (a)

Chiang-hua 汇華

Chiang-lan 江留

H. (j)

Liu-yang 硂陵

H. (a)

H. (o) Hsin-t'ien 新田

H. (o) Lï-ch'i 瀘溪

H. ( )

'T. (o) Hsing-ning 刞浮

I. (c) Luns-shan 龍山

H. $(\rho)$

Ch'ien-yang 駖㔭

H. (n) Hsü-p u洨浦

H. (d) Lung-yang 能隊

H. (b)

* Ilead Distriet of a l'beriedure. 


\begin{tabular}{|c|c|c|c|c|}
\hline 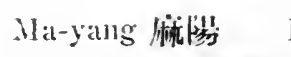 & H. (n) & *Shao-yang 例淓 & H. (l) & li-chang 宜章 \\
\hline Ning-hsiang 摹纱 & H. (a) & Shih-mên 不邽 & H. $(k)$ & Yi-yang 昷防 \\
\hline Ning-yüan 䆶邀 & H. (o) & Sni-ning 緩接 & H. $(f)$ & 广做 \\
\hline 巴陵 & H. $(m)$ & Tao 道 & $(o)$ & 119 沅江H \\
\hline Pao-ching 保站 & $\mathrm{H} .(p)$ & 'T'ao-ÿian 桃源 & H. $(b)$ & *Yüan-1 \\
\hline ang 本江 & H. (m) & -an 東安 & H. $(o)$ & 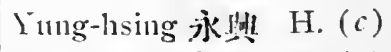 \\
\hline '11-shih & T. (d) & T’ung-tao 通篮。 & H. $(f)$ & ling-ming jikl1) H. $(o)$ \\
\hline al1g- & H. $(p)$ & 'Tz'ĭ-li 慈利 & H. (k) & *Yung-shun 永顺 H. $(r)$ \\
\hline & H. (a) & $\begin{array}{l}\text { Wu-kang 武雕 } \\
* \| \text {-ling 武洼 }\end{array}$ & $\begin{array}{l}\text { C. }(l) \\
\text { H. }(b)\end{array}$ & Vung-ting 永少 H. \\
\hline
\end{tabular}

\section{HUPEH}

Circeits.

1. Win-chang 武昌 J.

2. Han-Huang-'I'ê 漭黄德 D.IF.I.

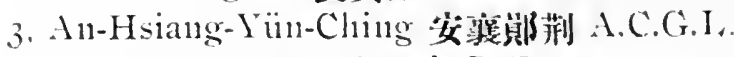

4. Shang-Ching-11an 上荆南 B.K.

5. Shil1-Ho 施噍 H.k.

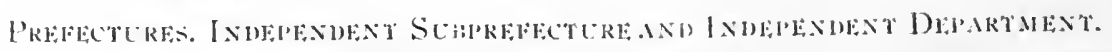
A. An-hu Fin
安陸
E. Ilo-fèng C.L.'T'
鹤篷 I. 'Tè-an I’u
德该
B. Ching-chon In 喑州
F. IInang-chon Fu 渭州 J.

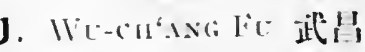
C. Ching-nnênC.L.C. 別例
G. Hsiang-yang Fu ther K. Yi-ch'ang Fu
亩: 3
D. 1lan-yang din
漢陵 H. Shih-nan Fin
施觕
椇陽

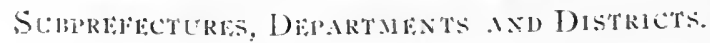

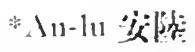
II. (c) Chu-shan 竹仙

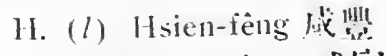
C. () Hsicn-11ing 咸蒋
H. (h)

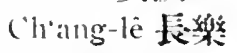
II. (k) Chün 均
Chang-yang 長隊 11. (k) *Chmug-hsiang 鍾游 Hsing-kuo 舆㨁
H. $(j)$
Chri 部
c. (i)
11. (a) Hsing-shan 興山
C. $(j)$
Chri-shni 散水
H. $(f)$ Chrung-yang 装隊 H. (i) Hsïan-ên 宣恩
H. (j)

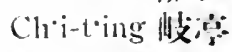
T. $(f) *$ En-shih 恩施
H. (h) K Ku-cll'êng 影城
H. $(h)$
Chia-yii 誉焦
H. (j) liang 房
H. (l) Kurung-chi 廣湾
H. $(g)$
*Chiang-hsia 汇军H. (j) Han-ch'tan 淡川 H. (d) Kuang-hna 光化
H. $(f)$

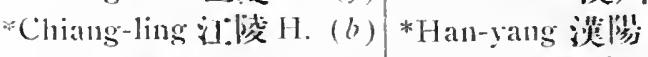
H. (d) Kuei 鼭
H. (s)
Chicn-li 篮利
H. (i) Hnang-all 监该
H. (f) Kung-an 公该
c. $(k)$
Chen-shih 建始
H. (h) *Hnang-kang 黄岡 H. (f) : Laii-fèng 来瓷
H. (b)
Chrien-chiang 整汇:H. ( a ) Huang-mei 黄柏 H. (f) Li-ch'uan 利川
H. $(h)$
Chih-chialng 枝:
II. (b) IInang-1)'o 迕波
H. (d) L(t)-t'ien 唄红
H. $(h)$
Cling-shan 影

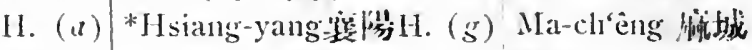
H. $(f)$
Chu-chri 竹繁
II. (l) Hsiao-kan 涍感
H. (d) Mien-yang 滥隄
H. (f)
C. (d)

* Mead Dislriet of a l'rejedure. 
HUPEH continuct.

Nan-chang 㣮滛

Pa-tung 巴束

Pao-k'ang 保䐂

P'u-cll'i 浦圻

Sha-han 沙谈

Shih-shou 不首

Sui 隨

Sunny-tzŭ 松涪
H. (g) Ta-yeh 大治

H. (k) Tang-yang 賞隊

H. (l) T'ien-mèn 天附

H. (j) T'sao-yang 将隊

T. (a) *Tung-hu 東湖

H. (b) T'ung-chêng 通城

c. (i) T'ung-shan 通ll

11. (b) Wu-ch'ang 试星

Vi-ch’èng 宜城
H. (j) Y i-tul 宜都

H. (c) Ying-ch'êng倠城 H. (i)

I1. (a) Ying-shan 確山 H. (i)

H. $(g)$ Y iu-yang 漁院

H. (k) Yüan-an 㒉安

T. $(k)$

H. (c)

H. (l)

H. (l)

II. (i)

\section{KANSUH}

I. Iarti-chon 藏州 K.

Circurts.

2. P'ing-Ching-Clining 本鹿汪 D.E.F.I.N.

3. Kung-Cl1'in-Cliieh 墅秦階 B.C.J.

4. Ning-hsia 窂夏 $\mathrm{M}$.

5. Hsi-ning 阮察 $\mathrm{G}$.

6. Kan-Liang 甘凉 H. I.

7. An-Su㒸肃 A.O.

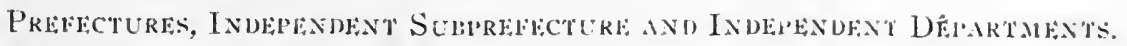
A. An-hsi C.L.C. 安西|F.Hua-p'ing-ch'nan
K. Lax-chou lie
B. Chich C.L.C.
C.I.'T'化不川
G. Clin C.L.C:
湝
G. Hsi-ning Fu
西桨
L. Liang-chon lin
米州
D. Clling C.L.C. 汪
H. Kan-chou In
甘州
M. Ningr-hsia I"n
猔州
E. Chring-yang Fu
I. Ku-yüan C.L.C. 固原 O. Su C.I.C.
J. Kung-chrang Fu瑟昌
繁夏
本谅
肃

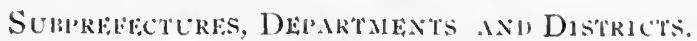

\begin{tabular}{|c|c|c|c|c|c|}
\hline An-1ua 安化 & H. (c) & uang-lang 诽浪 & 'T. (c) & Hsi-ho 西利 & H. $(j)$ \\
\hline An-ting 该徙 & H. $(j)$ & Chung-wei 中衛 & H. (m) & *Hsi-ning 西筫 & H. $(s)$ \\
\hline "Chang-yeh 張掖 & H. $(h)$ & Ch'tung-hsin 崣信 & H. $(d)$ & Hsiin-huna 循化 & T. $(g)$ \\
\hline ('hen-fan 鎮番 & H. (l) & nng 伏蒐 & H. (j) & *Kao-lan 叟菻 & H. $(k)$ \\
\hline Chèn-yüian 鑥原 & H. $(d)$ & I'u-y' 撫矮 & 'r. $(h)$ & Kao-t'ai 高台 & H. $(o)$ \\
\hline Chêng-ning 正茑 & H. (i) & Hai-ch'êng 游城 & H. (i) & Ku-lang 古浪 & H. (l) \\
\hline Clièng 成 & H. $(b)$ & Ho 河 & C. $(k)$ & Kuei-tè 貴德 & I. $(s)$ \\
\hline Chin 金 & H. $(k)$ & uii 合水 & H. (c) & $\mathrm{Li}$ 视㤟 & H. $(c)$ \\
\hline Chrin & H. (c) & Hua-t'ing 宑亭 & H. (n) & Liang-chuaing 凉邪 & I'T. (l) \\
\hline Ching-ning 郬售 & C. (u) & Huan 環 & 11. (c) & Liany-tang 兩當 & H. (c \\
\hline Cling-y tiatr 靖遠 & H. (k) & Hui 徽 & H. $(c)$ & ling & C. $(n$ \\
\hline Ch'ing-shui & H. (c) & Hui-niug 會媱 & H. $(j)$ & Ling-t’ai & 11. $(4)$ \\
\hline
\end{tabular}


*Lung-hsi 㜔西 H. (j) Pa-yen-jung-kê 巴燕戎格 T'ao-chou 洮州 T. ( $j$ ) Lung-té虐德 H. (n) T. (g) Ti-tao 狄道 C. $(k)$ Min 峎 C. ( $j)$ P.ing-fan 平番 H. (l) Tun-huang 敦煌 H. (a) Nien po 碾伯 H. (g) * P·ing-liang 本涥H. (n) T'ung-wei 通渭 H. ( $j$ )

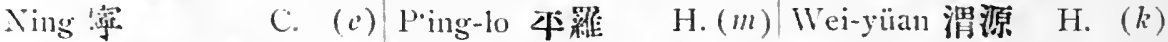

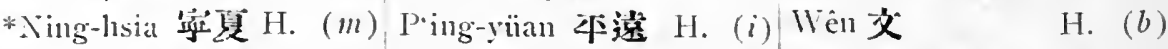
Ning-ling 综鼢 T. $(m)$ Shan-tan 山川. II. (h) Wu-wei 武威 H. (l)

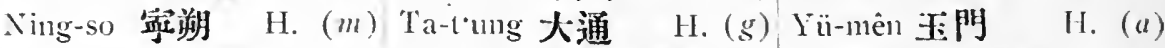

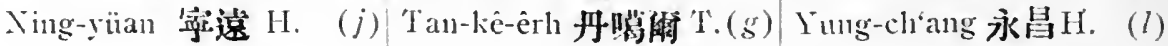

\section{KIANGSI}

Circuirs.

I. Nan-Fu-Chien 湳撫建 B.D.K.

2. Kuang-Jao-Chin-Nan 錯餚九南 C.E.H.L.

3. Chi-Nan-Kan-Ning 吉南䫓蒋 A.G.J.M.

4. Jui-Yüan-Lin 瑞㳖䰻 F.I.N.

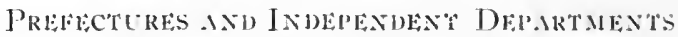
A. Cin-an lin
㨁安 F. J ui-chou liu

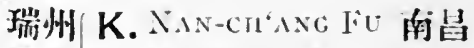
B. Chicn-chang Fu 建昌 G. Kan-chon I'u
贑州 L. Nan-h”ang Fu防接
C. Chiu-chiang F'u
九江 H.Kuang-hsin Fu 䍃信 M. Ning-tu C.L.C. 琈都
D. Fin-chon Iir
挴州 1. Lin-chiang I’u
E. Jao-chou I'u
䍌州J. Nan-an Fu
臨江 N. Yüan-chou Fu
装州

SURPREFECTRES, DEPARTALNT AND DISTRICTS.
An-fu 㚣福
H. (a) Ch'nug-yi 酱議
H. (j) Hsin-kan 新崄
H. (i)
An-jên 安仁
H. (c) Fên-yi 分管.
H. (n) Hsin-y ü 新喻
H. (i)
An-yi 妴義
H. (l) Fêng-ch'êng 5 In 城 H. (k) Hsing-an 舆范
H. (h)
An-yiian 安袁
II. (s) Fêng-hsin 奉新
H. (k) Hsing-kuo 與國 H. $(g)$
Ch'ang-ning 長预H. (s) Fu-liang 孚梁
H. (c) *Hsing-tzu星于 H. (l)
Chi-shui 吉水
H. (a) Hu-k'ou 湖口
H. (c) Jui-ch'ang 瑞昌
H. (c)
Chien-ch'ang 建昌H. (l) Hui-ch'ang 會昌 H. (f) Jui-chin瑞金
H. (m)
Chien-shan 銛山H. (h) Hsia-chiang 跌江: H.
Chin-ch"i 金鄭
II. (d) H sin-ch'ang 澵星
Chin-hsien 進筫
H. (k)
(i) * Kan 韸
H. $(g)$

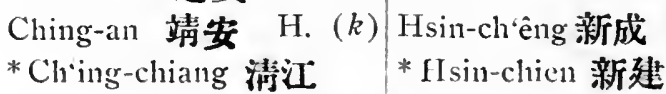
\begin{tabular}{l|l} 
Ching-an 靖安 H. $(k)$ & $\begin{array}{l}\text { Hsin-ch'êng 䉼成 } \\
\text { *Ch'ing-chiang 清江 }\end{array}$ \\
$*$ IIsin-chien 䉼建
\end{tabular}
* Kao-an 高安
H. (f)
H. $(f)$ Kualug-ch'ang 是昌
*Ching-chiang 清江 *IIsin-chien 激建

$$
\text { H. (i) }
$$
H. (k) Kuei-ch'i 贵溪
H. (b)
H. (b)

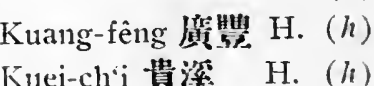

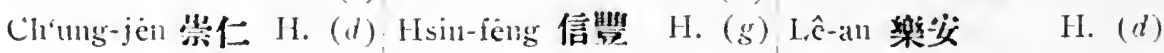


I.è-ping 樂平

H. (c) * Shang-jato 上镜

H. (1) Wan-nien 营年

H. $(i)$

Lien-hua 連化

1\%. (a) Shang-kao 上高

H. $(f)$ Wan-tsai 蕰載

H. (il)

* Lin-cli uan (if

H. (d) Shang-y 上俩

H. (j) W11-11ing in 宊

II. $\left(k^{\prime}\right)$

Lii-cli’i 湑深

H. (b) Shih-clıèng 不城 H. ( /ii) * Ji-cln'un 宜寿

H. (ii)

*Lü-ling 值陵

H. (a) * Ta-yii 大鵕

I1. (j) Ji-luang 苗黄

H. (d)

Lung-cl'tian 乳影11. ( a ) T:ai-bo 泰河

11. (a) Ji-ning 義漹

c. (k)

Lung-11au龍南

li. ( s) lè-an 德往

II. (c) lii-yang 过綮

H. (h)

* Nan-ch'ang 㑲昌 H. (k) Tê-hna 德化

11. (i) Yii-kan 集干

ii. (i)

* Nan-ch'êng 南城H. (b) * Tè-hsing 德县

I1. (c) Yu-sian 主山

H. (h)

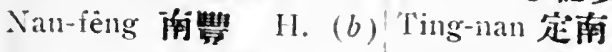

H. (g) \ï-tu雾都

11. $(g)$

Nan-k'ang 南康 H. ( j) 'lu-clrang 都昌

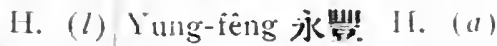

P'èng-tsê 彭澤

H. (c) Tung-lisiang 東獀 H

H. (d) Yung-hsin 永激

H. (a)

Ping-hsiang 萍鄉 H. ( n ) T'ung-kul 銅鼓

* P'o-yang 翻懏

H. (c) | Van-an 营安

T. (f) Yung-ning j每学 H. (a)

H. (a)

\section{KIANGSU}

Circuits.

I. Chiang-ning 江察 C.

2. Huli-Yang 淮提 F.I.

3. Hsti-Hai 徐游 D.G.

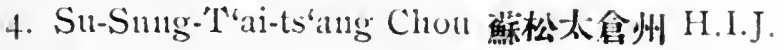

5. Ch'ang-Chên-'T'ung-Hai常鎮通海 A.B.E.K.

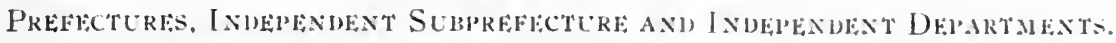
A. Ch'ang-chou Fiu 常州( E.
E. Hai-mên C.L.'T'.
海門 1. Sung-chiang Fin 松江
B. Chèn-cluiang Fu 錤江 F. Huai-an Fu

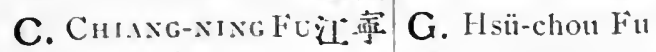
淮岀 J. T'ai-ts'ang C.L.C.太倉
D. Hai C.L.C.
海 H. Sn-chou liu
徐州 K. T'ung C.L.C.
䔳州 L. Jang-chon I'u
通
揚州

SUBPREFECHURES. DENARTMENTS ANU DISTRICTS.

An-tung 始東 H. (f) Chiang-p u 江浦 H. (c) Chii-jung 句容 H. (c) *Ch'ang-choi 長洲 *Chiang-tu 江都 H. (l) Ch'uan-sha 川沙 T. (i)

H. (h) Chiang-yin 江陰 H. (a) Ch'nng-ning 峉明 H. (j)

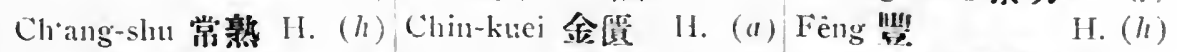

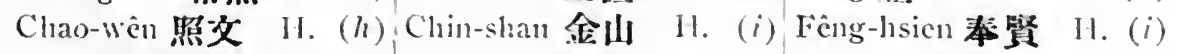

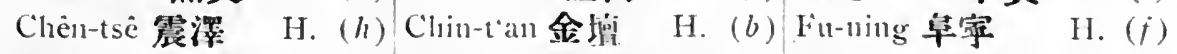

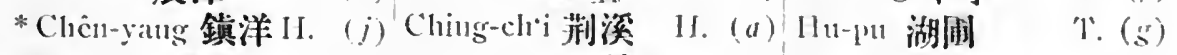

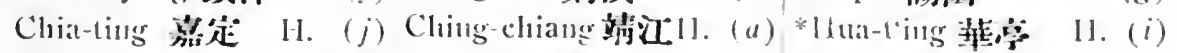

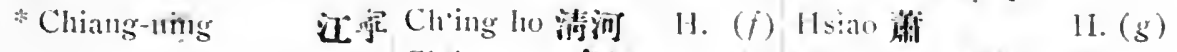

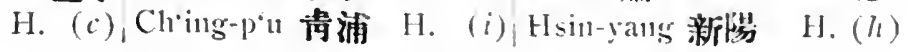

* Head Distrid of a Preficture. 


\section{KIANGSU Continued.}

\begin{tabular}{|c|c|c|c|c|c|}
\hline Hsing-hua 興化 & H. (l) & Paro-ying 钼應 & H. (l) & 'Tang-shan 舓山 & H. $(s)$ \\
\hline J11-kao 邚泉 & H. $(k)$ & P'ei 邳 & C. $(g)$ & 'T‘an-yiian 桃源 & H. $(f)$ \\
\hline *Kan-ch'iian 甘景 & H. (l) & $\mathrm{P}^{\prime} \mathrm{ei}$ 浦 & H. $(g)$ & 'Tung-t’ai 東辜 & H. $(l)$ \\
\hline Kan-yii 贑榆 & H. $(d)$ & *Shan-yang 山晹 & H. $(f)$ & *'T'u111g-shan 銅山l & H. $(g)$ \\
\hline Kao-shum 高湸 & 11. $(c)$ & Shang-hai 上䌕: & H. (i) & *Wu 䞨 & H. $(h)$ \\
\hline Kao-yı 高秘 & C. $(l)$ & $*$ Shang-yüan上元 & H. (c) & Wu-chiang 只江 & H. $(h)$ \\
\hline K'un-shan 崑山 & H. $(h)$ & Shu-yang 沭茜 & H. (d) & *Wu-chin 武進 & H. (a) \\
\hline Li-shui 溧水 & H. (c) & Su-ch'ien 㠅遷 & H. $(g)$ & IVt1-hsi 热鍚 & H. $(a)$ \\
\hline L,i-yang 溧隄 & H. $(b)$ & Sui-ning 睢密 & H. $(g)$ & *Yang-hu陽湖 & H. (a) \\
\hline Lit1-ho 宍合 & H. (c) & T'ai 泰 & c. $(l)$ & 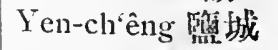 & H. $(f)$ \\
\hline *Lon 婁 & H. (i) & T’ai-hu 太湖 & I. (h) & Yi-ch'êng 猨徽 & H. (l) \\
\hline Nan-hui 南漼 & H. (i) & 'T'ai-l ${ }_{2} \operatorname{sing}$ 泰興 & H. $(k)$ & Yi-hsing 宜興 & H. (a) \\
\hline 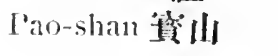 & H. $(j)$ & *'Tan-t'u 丹徒 & II. $(b)$ & *Yüian-ho 元利 & H. (h) \\
\hline & & Tan-yang 丹降 & H. $(b)$ & & \\
\hline
\end{tabular}

\section{KIRIN}

\section{Ha-êrh-pin 哈楼瓷 \\ Circuits.}

PRFFECTURES ANU JNPEPENUENT SUBPREFECTURES.

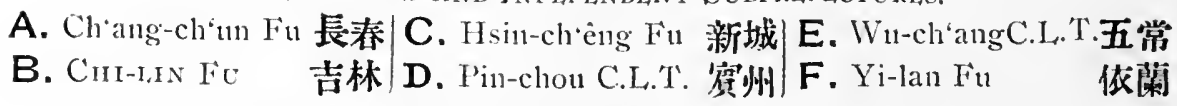
Subreirfictures. Depalitments and Districts.

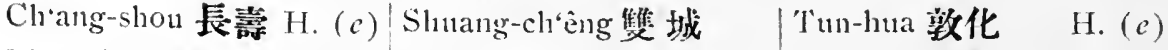

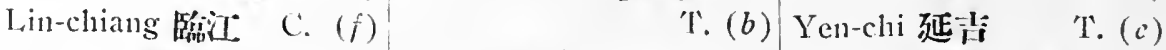

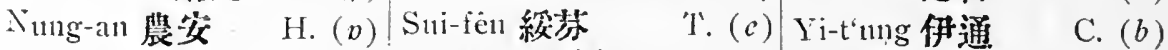
p'an-shih 磐不 H. (c)! Ta-t'ung 大通 H. $(f)$ Yü-shul 榆樹 H. (c) T'ang-yüaul 湯源 H. $(f)$.

\section{KUANGSI}

Circuits.

I. Kuei-Ping-Wu 桂本标 E.J.N.

2. Yu-kiang 右江 B.D.G.I.

3. 'Tso-kiang trit. A.C.H.K.O.

4. 'T'ai-p'ing-Sü-Shun 太本思顒 M.F.I.

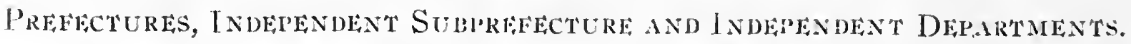
A. Chên-an Fì
鈛安
F. Kuei-shun Fu
蹛順|K. Ssŭ-ch'êng Fu
泪城
B. Ch'ing-yüian Fu 慶遠 G. Litu-chon I'u
柳州 L. Ssŭıên Fı̀
思恩
C. Hsi-lung C.I.C. 西隆
H. Nan-ning $\mathrm{Fu}$
南管 M. T'ai-p'ing F'u
太本
D. IIsün-chon F'u
浔州 I. Pai-sê C.L.'I.
百色 N.W11-chon Fu
吘州
E. KULT-I,IN I:
佧林 J. P'ingrg-lè F'n 本樂 O. Yü-lin C.I.C.
繁林 


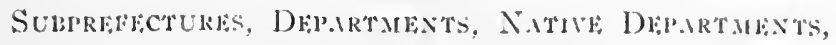

Districts and Native Districts.

An-p'ing 安本 T.C.(m) Kuan-yang 灌陽 H. (c)|Shang-lin 上林 H. (l)

Chao-p'ing 昭本 H. ( $j$ ) Kuei 贵 H. (d) Shang-lin 上林 'T.H. (l)

Chên-pien 鎮後 H. $(f)$ *Kuci-p ing 桂平 H. (d) Shang-lung 上龍

Chên-yiian 鎮邀 T.C. $(m)$ Kuei-tê 歸德 T.C. $(h)$ T.C. $(m)$

Chi-lun 佶偷 'T.C. ( $m$ ) Kung-ch'êng 港城 Sliang-ssŭ 上思 C. (h)

Chiang 江 T.C. $(m)$

H. (j) Sluang-ying 上聅

Chieh-an 結安 T.C. (m) Kuo-lıua 果化 T.C. (h)

Ch'ien-chiang 邉江 Lai-pin 來窟 H. $(g)$

Cli'iian 全

H. (l) Li-p'u素浦

Chung 忠

C. (c) *Lin-kuei 臨桂

H. (j) Ssŭ-ên 思恩

'T.C. (m)

Chung-shan 釷山 T. $(j)$

*C'l'tung-shan 詝山

En-lung 恩绦

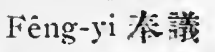

H. (m)

Ling-ch'van 靈川 H. (c)

I.C. $(m)$

H. (c) Ssŭ-ling 䍐陵 \%. C. ( (m)

Fu-ch'uan 急川

Hêng 模

Ho 賀

Ho-ch'ih 河池

Huai-chi 懹集

Huai-y ïan 懷遠

Hsi-lin 西林

H. (l)

Ling-yün 凌墨

Liu-cl'êng 柳城

H. $(k)$

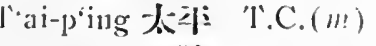

Tè-shèng 得勝

'T. (b)

H. (n)

Lo-cl'êng 羅城

H. $(g)$ T"êng 萨

'. C. (l)

T'ien 田

H. (b)

C. (a)

Lo-jung 雒容

H. $(g)$

T'ien-ho 天河

H. (a)

H. (j) Lo-pai 羅白

H. $(g)$

*'丁'ien-pao 天保

*'T's'ang-wu 萑梧 II. ( $(n)$

C. $(h)$

Lo-yang 羅陽

H. $(j)$

L-11-ch'nan 陸川

C. (b) Linng-an 隆安

T.H. (m)

\begin{tabular}{l|ll} 
H. (a) Ts'ên-clı’ 答溪 & II. $(n)$ \\
H. (h) Tso \% & C. $(m)$
\end{tabular}

H. (n) Lung-chou 龍州

Tu-chieh 都結 'T.C. (m)

$\mathrm{T} .(m)$

H. (g) Lung-sheng 㳶勝 T. (e)

I'u-kang 都䐂 T.C. (a)

H. ( $k$ Lung-ying 龍英

Hsia-lei F雷 T.C. (a)

Hsia-shih-hsi 下石西

T.C. $(m)$

*Ma-ping 馹本 H. $(\mathrm{g})$

Tung 束

I.C. (in)

Tung-lan 東阑 C. (b)

Wan-ch'êng 萬城

T.C. (III)

C. $(g)$

Hsiang 象

Ning-chiang 明江 T. (m) Wu-lssïan 武笪.

H. $(d)$

Ming-ying 薄盈 I.C. ( m $)$ Wu-yüan 武緣

H. (l)

Na-ti 那地 I.C. (b) Yang-li 澺利 C. (m)

IIsiang-Wu问武 T.C. (a)

Hsin-cl'êng 析城 H. (b)

Hsin-ch'êng忻城'l'H. (b)

Van-tan 解丹 I.C. (b) Yang-so 晹溯

H. $(f)$

Hsin-lun 信偷 T.C. ( $m$ )

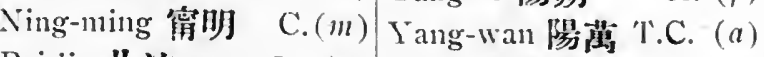

Pei-liu 北流

Hsin-ning 䉼窝

C. $(h)$ Pin 旗

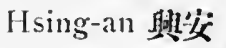

H. (e) P'ing-hsiang 悲皏

H. (d) Yi-ning 義蒋

Fl. (b)

Hsing-yen 興淡

H. (o)

Hsin-iên 修仁

*Hsüan-his itist H. (h)

Jung 谷

Ku-shang-shih

H. (n)

*P'ing-lé 平樂

P'ing-nan 平南

C. (l) *Yi-shan 宜山

H. $(b)$

T.C. $(m)$ Yung-an 永管

H. $(g)$

C. $(j)$

H. ( $j$ ) Yung-fu 永福 H. $(f)$

H. (d) Yung-kang 永康 C. $(m)$

Po-pai 博白

Shang-hsia-tung

H. (o) Yung-ning 永踪

Y ung-shun 永順 H. (h) 


\section{KUANGTUNG}

Circuits.

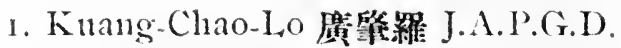

2. Hui-Ch'ao-Chia 善潮嘉 P.C.H.

3. Kao-Lei-Yang 高雷湿 I.K.S.

4. Lien-Ch'in 廉䣄 N.F.

5. Shao-Nan-Lien 韶南連 L.MI.Q.R.O.

6. Ch'iung-Yai 㻅崖 F.'T.

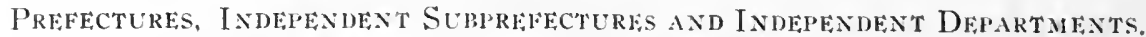
A. Chao-ch'ing Fu 㢣愿 $\mathrm{H}$. Hui-chou $F_{u}$
惠州 P. Lo-ting C.L.C. 䍜定
B. Chao-chou Fu 潮州 1. Kao-chou Fu
高州 Q. Nan-hsiung C.L.C.

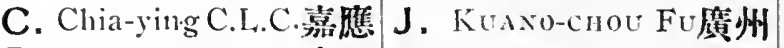
D. Chiih-ch·i C.L.T.赫溪 K. Lei-chou Fu
雷州
E. Chiih C.I.C. 銫 L. I.i-yan C.L.'T.
理滛
R. Shao-chou $\mathrm{F}_{\mathrm{u}}$
南堆
F. Chiinng-chou Fu 隻州
M. Lien C.L.C.
連
G. Fo-kang C.L.T.
N. Lien-chon $F_{u}$
筆州 T. Yai C.L.C.
O. Lien-slian C.1.'个.速山
槊州
S. Yang-chiang C.L.'T.
晹江
最

SLBPRFFECTERFS. DFPARTMFATS INI DISTKICTS.
Ch'ang-hua 昌化
H. (t) *Ho-p
H. ( $u$ ) Kan-ên 贴
H. $(t)$
Clrang-Lè 辰樂
H. (c) Ho-shan 猚山
H. (a) Kao-ming 高明
H. $(a)$
Ch'ang-11ing 長参
H. (h) Ho-yüan 河源
H. (h) *Kao-yao 高要
H. (a)
Ch'ao-yang 激陵
H. (b) Hu-nên 虎嬥
T. (j) Kuang-ning 鹿孚
H. (a)
Chên-p'ing 鋋雨
H. (c) Hua 化
H. (j) *Kuei-shan 歸善
H. $(h)$
Chèng-hai 澄游
i. (b) Hua 化
C. (i) Lè-ch'ang 樂昌
H. $(q)$
Ch‘êng-mai 澄萬
H. (f) Huang-kang 击间
'. (b) Lè-hui 樂鿖
H. $(f)$
Chich-shih 礍不i
T. (h) Hui-lai 惖爽
H. (b) Lien-p'ing 监平
C. $(h)$
Chich-yang 揭陽
H.(b) Hui-t'ung 會同]
H. (i) Lin-kao 路高
H. $(f)$
Cllien-shan-chai
前山寨 Hsi-ning 西要
H. (o) Ling-shan 霓 II
H. (h)
T. ( $j$ ) Hsiang-shan 香山
Ching-yïan 爱逗 H. ( j ) Hsin-an 新安
H. (j) Ling-shui 陵水
H. $(t)$
*Ch'iung-shanf(ill H. (f) Hsin-hui 新會
H. (j) L.u-fêng 陸糬.
H. (h)
H. ( $j$ ) Lung-ch'uan 龍川 H. (h)
*Ch'ï-chiang Hit 证 H. (q) Hsin-hsing 新與
II. (a) Lung-mên 龍門
H. (j)
ín-p'ing 恩本
H. (a) Hsin-yi 信宜
II. (i) *Mon-ming 茂名
H. (i)
Fêng-ch'uan 封川

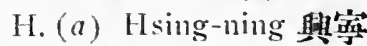
H. (c) Nan-ao 南澳
T. (b)
Fèng-shun 曋顼
H. (b) Hsï-Wên 徐聞
Eo-sluan 佛山

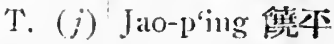
H. (k) *Nan-hai 南游
H. $(j)$
Hai-fêng 旅絭
H. (in) Jên-hua 仁化
H. (b) *P'an-y ï 番禺
H. $(j)$
*Hai-k'ang 游康
H. (k) Ju-yüan 孚源
H. (q) P'ing-ÿian 本裳
H. (c)
*Hai-yang 海晹
H. (b) Kai-chicn 開建
H. (q) P'n-ning 普䟢
H. (b)
Ho-p'ing 利本
H. (i) K'ai-p’ing 開本
H. (a) San-shui 三水
H. $(j)$
H. (a) shih-ch'êng 不城 H. (i)
* Head Dislriat of a Prefecture. 


\section{KUANGTUNG Continued.}

Slum11-tê 順鿉

Ssŭ-hui 四會

Sui-ch'i 遂溪

la-pu 大找

'an 霄

'l'ê-ch'ing 德重

Tien-pai 電白
Hi. (j) 'Ting-an 定妾

H. $(f) \mid$ Wêng-yitan 㕮源

H. (a) 'l'sêng-ch'êng 㙁城 H. (j) Wu-ch'nan 号川

H. (k) 'Ts'ung-hua 從化 H. (j) Yang-ch'un 隄涪

H. (b) 'Tung-an 乘安

C. $(f)$ Tung-kuan 東莞

H. (o) Vang-shan 修 IJ

H. $(j)$ ling-tê 热德

C. (a) Wan 萬

H. (i) Wên-ch’ang 交淐
II. (q)

II. (i)

H. (a)

I. (m)

H. $(q)$

II. (h)

\section{KUEICHOU}

Circurs.

I. Kuei-P‘ing-Shih 貴平存 D.E.G.I.

2. Kuei-hsi 貴西 A.C.H.M.N.

3. Kuei-tung 贵東 B.F.J.K.L.O.P.

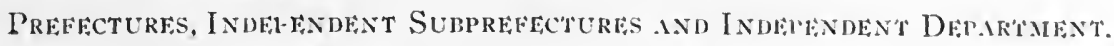
A. An-shum Fu
安倠 F. Li-p'ing Fin
黎平 L. Sung-t’ao C.L.'T. 松桃
B. Chen-ÿian Fu
鎮遠
C. Hsing-yi Iin
舁着
G. P.ing-yüch C.1.C.相越 M. Ta-ting Fu
大定
D. Jên-huai C.I..'l'.
仁懷
H. P'u-an C.L.'I'.
㘳发 N. 'l'sun-yi F"
漼䣡
E. Kunt-y.ne I'u
貴隄
I. Shill-ch.ien Fin

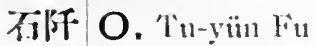
都勾
J. Ssŭ-chon Fin
K. Ssŭ-nan F'u
思州 P. 'T'nng-jên F'u
銅仁

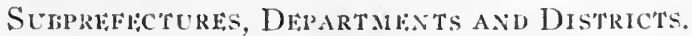

*An-hua 荌化

An-11an 安諵

An-p “ing 安本

Ch'ang-chai 長寨 'T

Chên-fêng 直啨

Chên-ning 敛罩

*Chên-yiian 鎮部

Chêng-an 正妿

H. $(k) \mid * K^{\prime}$ ai-t'ai 開泰

H. $(f) \mid$ Sui-Y'ang 綏陽

T. $(f)$ T”ai-kung 台拱

H. (c) Ku-chou 古州

H. (

C. (c) Tan-cliang 丹江

C. (c) Kuei-hua 部化

H. (c) T'ien-chn 天柱

C. (i) Knei-ting 貴定

T. (a) Ting-fan 定番

H. (b) Lang-tai 郎岱

H. (c) *'l'sun-yi 遥義

尔（i) Tu-chiang 都江

C. (ni) Li-po 斾波

H. (o) Tw-shan 獨山

H. (n)

个. (b)

T. (o)

H. (b)

C. $(c)$

H. $(n)$

T. (o)

C. $(o)$

Ch'ien-hsi 黑西

H. (o)

Ch'ing-chên 清鎮 H. (i) Lung-li 龍里

H. (i) *1'n-yün 都匀

H. $(p)$

Ch“ing-ch'i 清矤

H. (j) Ma-ha 麇哈

H. (c) *T"ung-jên 銅仁

H. (II)

Ch'ing-chiang 消江T. (b) Mei-t’an 渭漂

C. (o) T"ung-tzŭ 桐榜

C. $(m)$

Ch'ing-p'ing 清年 II. (o) Pa-chai 八寨

H. (g) Wci-ning 威涪

H. $(g)$

Huang-p ing 䜤本 C. (b) Pi-chich 里節

T. (o) IIèng-an 留安

H. $(k)$

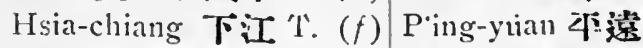

*H Ising-yi 舆我

H. (c) P'

Usin-wên 修文

Jên-huai 仁懷

H. (m) Wo-ch'uan 嫯川

H. (k)

C. (iii) Yin-chiang 印江

H. $(g)$

H. (c) Y'ï-ch'ing 馀栯

H. $(j)$

H. (c) *P'ut-ting 普定

H. (a) Yüi-pi'ng 还屏

C. (a)

K'ai 開

H. ( i ) Shih-ping 施来

H. (b) Yung-ning 永踏

H. $(f)$

* Head District of a Prefecture. 


\section{NEW DOMINION}

C'IrCUI'ts.

I. Ak-su 阿克蕉 D.E.K.L.M.

2. Chên-ti 鎮延 A.J.

3. Kashgar 喀什噶尔 C.F.(B.H.().

4. Y'i-T'a 例塔 B.N.I.

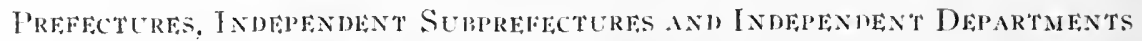
A. Chên-hsi C.L.'T. 鎮西 |F. Lurkurausu C.L.T.
B. Ching-ho C.L.'I. 精沙
央尔喀拉鳥蘇
K. Wên-su C.I.C.
温寉
C. Ho-tien C.L.C. 利閪
G. So-ch ê C.L.C. 莎車
L. Ush C.L.'T'.
鳥什
D. K’u-ch'ê C.L.'T'. 庫車
H. Su-lê C.L.C.
疏勒
M. Yen-ch"i Fu
延老
E. Marapash C.L.T.
I. 'T’a-ch'èng Fin
N. Ili Fu
伊犁
瑪喇巴什 J. 'Tr-11CA Fi 廸化
英诘沙你

SUBPREFECTURES AND DISTRICTS.

\begin{tabular}{|c|c|c|c|c|c|}
\hline Ch'ang-chi 昌吉 & H. $(j)$ & Pai-ch'êng 拜城 & H. $(k)$ & leh-ch êng 苝城 & H. $(g)$ \\
\hline Ch“i-t’ai 奇台 & II. (a) & $S u-f u$ 疏附 & H. $(h)$ & Yü-tien 于閐 & H. $(c)$ \\
\hline l'u-k’ang 皁層 & H. $(j)$ & Sui-lai 総棑 & H. $(j)$ & Erh-ch iang 婼㴽 & H. $(m)$ \\
\hline Ha-11i 昐密 & '1. $(a)$ & Sui-ting 綏定 & H. $(n)$ & Hsin-p ing 新尔 & H. $(m)$ \\
\hline 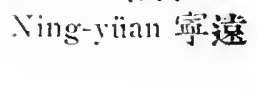 & H. $(n)$ & 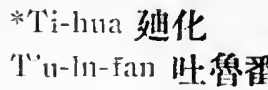 & $\begin{array}{l}\text { H. (j) } \\
\text { 'T. (a) }\end{array}$ & Lun-t'ai 輸台 & H. $(m)$ \\
\hline
\end{tabular}

\section{SHANSI}

Circeits

I. Chi-Ning 冀察 C.D.H.I.L.R.S.

2. Ho-tung 河東 A.B.E.F.MI.N.

3. Yen-P'ing-Ta-So-Ning-Hsin-Tai-Pao解平大朔学析代保G.J.K.O.P.Q. 4. Kuei-Sui 歸緩 a.b.c.d.e.f.g.

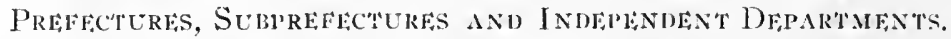
A. Chiang C.L.C.
B. Chieh C.L.C.
C. Ch'in C.L.C.
D. Fë1i-chon Fu
E. Ho C.L.C.
F. Hsi C.L.C.

綘 $\mid$ G. H sin

解 H. L iao C.L.C.

沁 1. Lu-an Fu

汾州

J. Ning-wo $\mathrm{Fu}$

K. Pao-tê C.L.C.

岓
遼
潞安
窑武
保德
C.体定

岓 N. P'u-chou Fu

浦州

O. So-p'ing $F u$

湖平

P. ' $1 \mathrm{a}-t^{\prime} \mathrm{ung} \mathrm{F} u$

大闹

霍

L. P'ing-ting C.L.C.平定 Q. Tai C.L.C.

M. P'ing-yang Fu 平晹

R. 'T"AI-YÜAN Fu 太原

S. Tsê-chou Fin 澤州

c. Knei-hua T. 蹄化 f. Sui-yüan 'T.

綏遠

a. Ch'ing-shui-ho T.

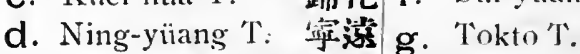

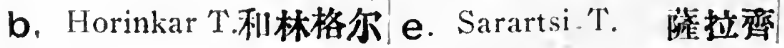

托克拒 
SHANSI Continucd.

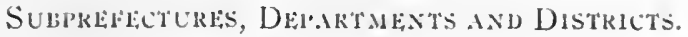

\begin{tabular}{|c|c|c|c|c|c|}
\hline A11-yi 芜出 & H. (b) & Hsing 骵 & H. (r) & T”ai-ku 太谷 & $H(r)$ \\
\hline *Ch'ang-chih 長治 & 台H. (i) & Hsï-kou 徐蓝 & H. $(r)$ & 'T’ai-p'ing 太45 & H. (ni) \\
\hline Ch'ang-tzŭ 長子 & H. (i) & Jui-ch'êng 芳城 & H. (b) & 'T'ai-yüan 太原 & H. $(r)$ \\
\hline Chao-ch'êng 趙城 & H. (c) & Jung-ho 策 & H. $(n)$ & T'ien-chenn 天鎮 & H. $(p)$ \\
\hline Chi 年 & C. $(m)$ & Kao-p'ing & H. (s) & Ting-hsiang 定高要 & H. $(g)$ \\
\hline Chi-shan 稜山 & H. (a) & $K^{\prime} \hat{e}-l a 11 \dot{c}$ & C. $(r)$ & Tso- & H. (u) \\
\hline Ch'i 征 & H. (r) & Kuang-1 & H. $(p)$ & T‘u & H. (i) \\
\hline hiang 释 & H. (it) & 110 崞 & H. $(q)$ & T'un & \\
\hline Chiao-ch & 戗H. $(r)$ & Lan 嵐 & H. $(r)$ & 5 & 'l. $(s)$ \\
\hline 介休 & H. $(d)$ & Li-cl'êng 黎城 & H. (i) & 总察 & $\mathrm{H}$. \\
\hline 沁水 & H. $(s)$ & 器 & H. $(d)$ & IVên & H. (a) \\
\hline 沁源 & H. $(c)$ & -chin & H. (u) & 水 & H. $(r)$ \\
\hline 静樂 & H. $(g)$ & ${ }^{*} \operatorname{Lin}-\hat{\mathrm{f}} \hat{\mathrm{e}}$ & H. (m) & I & H. $(j)$ \\
\hline IVE & H. $(m)$ & l.ing-c & HI. $(p)$ & 鄢缴 & H. (c) \\
\hline 㢣峙 & H. $(q)$ & 陵川 & H. $(s)$ & & H. $(q)$ \\
\hline 西 & H. $(m)$ & Ling-s & H. (c) & 陽城 & H. $(s)$ \\
\hline 济隄 & H. $(d)$ & Lit-c & H. (i) & 晹曲 & H. $(r)$ \\
\hline 紫鎮 & H. $(p)$ & Ning- & H. (d) & Yan & H. $(p)$ \\
\hline i 配唯 & H. $(s)$ & & H. $(j)$ & Yi-cl & H. $(n)$ \\
\hline 孚山 & H. $(m)$ & 偏關 & H. (j) & Yi-shil & H. (n) \\
\hline J津 & H. (a) & P'ing & H. $(b)$ & Ying 㗹 & C. $(p)$ \\
\hline Ho-ch'ii & H. $(k)$ & P'in & H. $(o)$ & Yo-yang & H. (m) \\
\hline Ho-shum & H. $(h)$ & P'ing-yao 乐粕 & H. (d) & $10-y$ & H. $(o)$ \\
\hline Hu-kuan 旡關 & H. (i) & P'u illis & H. $(\dot{f})$ & 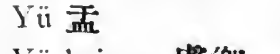 & H. (o) \\
\hline Huai-jên 懷仁 & H. $(p)$ & Shan-yin & H. $(p)$ & Yï-hsiang 虞榔 & H. (n) \\
\hline Hun-yüan 渾源 & C. $(p)$ & ih 神池 & H. $(j)$ & 11-5ne & H. $(h)$ \\
\hline Hung-tung 洪润 & H. (m) & Shih-lo & H. $(d)$ & 1 सारा & H. $(r)$ \\
\hline Isia 夏 & H. (b) & Shon-yang & H. $(l)$ & 11-ch'̈̈ 坦典 & H. (a) \\
\hline Hsiang-ling 垔陵 & H. (m) & So 湖 & C. $(o)$ & *Yung-chi j泳 & H. $(n)$ \\
\hline Hsiang-ni & H. (m) & 'l’a-ning 大㜔 & 11. $(p)$ & Yuug-ho 永利 & H. $(f)$ \\
\hline Hsiany-yi & & *Ta-tung 大同 & H. $(p)$ & Yung-lè 永樂 & Т. (n) \\
\hline Isiato-yi & H. $(d)$ & |'la-yi 大我 & '个. (i) & Yung-ning 永孚 & C. $(d)$ \\
\hline
\end{tabular}

\section{SHANTUNG}

CiRcLits.

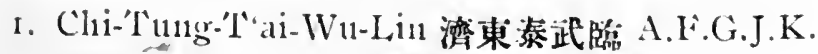

2. Yent-Yi-'T's'ao-Chi 交沂曹㴒 B.I.I...I.

3. 'Tềng-Lai-Ch'ing-Chiao 登萊青膠

* Head District of a Prefecture. 


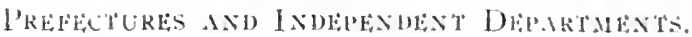
A. Cu-NAx liv 濟南 E. lai-chou Fu
萊州
B. Chi-ning C.L.C. 看軍
F. Lin-ch'ing C.L.C. 䠛奉
C. Chrao C.L.C.

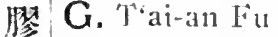
泰安
J. T'ung-chrang lin 東昌
D. Ch'ing-chou Fin 青州
K. W'tu-ting I'u
武定
L. Yen-chon
H. 'Tenng-chou F'u 登州 M. Ti-chon I'u
宽州
I. 'Ts'ro-chou Fin 曹州
沂州

DEPARTMFTS AND JISTRICTS.

\begin{tabular}{|c|c|c|c|c|c|}
\hline l11-cl1·iu 㒸邱 & H. $(d)$ & in-t'ai 新臺 & H. $(g) \mid$ & & H. (i) \\
\hline Chan-hura 霑化 & H. $(k)$ & Jih-chao 日照 & H. $(m)$ & Shang-ho 㫟润 & H. $(k)$ \\
\hline Chang-ch'in 章邱 & H. $(a)$ & Jung-ch'êng 榮成 & H. (h) & Shên 装: & H. $(j)$ \\
\hline 1'ang-ch'ing 長济 & H. (a) & Kao-nii 高密 & H. (c) & Shih-p'ing 往性 & II. $(j)$ \\
\hline g-lê 昌樂 & H. $(d)$ & Kao-t’ang 高焦 & C. $(j)$ & Shon-chang 穜張 & H. (l) \\
\hline h'any-shan 長山 & H. (a) & Kao-yüan 高苑 & H. $(d)$ & Shou-kuang 关光 & HI. $(d)$ \\
\hline 昌色 & H. (e) & *Kê-1sê 嗬澤 & H. (i) & Ss̆̌-shui 泪水 & H. $(I)$ \\
\hline ng 朝城 & H. (i) & Kuan 管 & H. $(j)$ & *'T’ai-an 泰奖 & H. $(f)$ \\
\hline 13 招遌 & H. $(h)$ & Knan-clıềng 敬城 & H. (i) & T'an-ch'êng 矨城 & H. $(m)$ \\
\hline 城武 & H. (i) & Kuan-t’ao 銠隐 & H. (j) & ng-yi 堂色 & H. $(j)$ \\
\hline & H. $(c)$ & Lai-wn 萊苝 & H. $(\xi)$ & 'T'ê 德 & C. $(a)$ \\
\hline 濟湜 & H. (a) & ig 桻隄 & H. $(h)$ & p’ing 德头 & H. $(a)$ \\
\hline 育河 & H. (a) & $n$ 闌山 & H. (m) & 'T’êng 滕 & H. $(l)$ \\
\hline 棲霞 & H. (h) & 彩装 & H. $(d)$ & g-t'ao 徙湖 & H. (i) \\
\hline 䨗東 & H. (a) & Lêe-ling 樂陵 & H. $(k)$ & Ts'ao 曹 & H. (i) \\
\hline g䪯群 & H. $(b)$ & *Li-ch'êng 糜:城 & H. (a) & T'sou 鄒 & H. $(l)$ \\
\hline 金郷 & H. (b) & Li-ching 利津: & H. $(k)$ & 'Tsou-p'ing 鄒平 & H. (a) \\
\hline $\mathrm{g}$ 青城 & 棧H. $(k)$ & 'êng 聊城 & H. $(j)$ & Tung-o 東阿 & H. $(g)$ \\
\hline $\mathrm{Ch}^{\mathrm{i}}$ & H. $(j)$ & 臨朐 & H. $(d)$ & 'Tung-p'ing 東本 & C. $(g)$ \\
\hline $\mathrm{Ch}^{\circ} \mathrm{i}$ & H. $(f)$ & 筑液 & H. $(d)$ & Tzŭ-ch'tuan 淄川 & H. $(a)$ \\
\hline Chu-ch'êng 諸城 & H. $(d)$ & 足 & & *Tz'ŭ-yang 滋隄 & H. (l) \\
\hline & C. $(m)$ & & H. (a) & Wei 篧 & H. $(c)$ \\
\hline 11 鈀影 & H. (i) & in 㭬陰 & H. $(m)$ & Wên-shang 汶上 & H. (I) \\
\hline & H. (l) & i 窗海 & C. (h) & têng 文登 & H. $(h)$ \\
\hline En 恩 & H. $(j)$ & 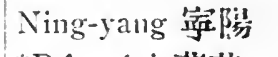 & H. (l) & 武城 & H. $(f)$ \\
\hline Fan 范 & H. (i) & i嚾莱 & H. (h) & 隄信 & H. $(k)$ \\
\hline Fei-ch'êng 肥城 & H. $(g)$ & $\mathrm{Pi}$ & H. $(m)$ & Yas & H. (l) \\
\hline 福川 & H. $(h)$ & 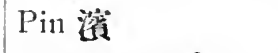 & C. (k) & *Yeh 掖 & H. (c) \\
\hline 游整 & H. (k) & 11 本度 & C. $(e)$ & $\mathrm{Yi}$ 嚗 & H. $(l)$ \\
\hline 游阮 & H. $(h)$ & yüan 稩原 & H. (a) & Yi-shui 沂水 & $\mathrm{H}(m)$ \\
\hline & H. $(h)$ & Po-hsing 博興 & H. $(d)$ & *Ti-tu 徐都 & H. $(d)$ \\
\hline in 㭧民 & H. $(k)$ & 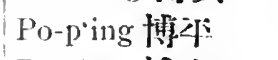 & H. $(j)$ & Yü-ch'èng 禹城 & H. (a) \\
\hline ing 夏津 & H. $(p)$ & Po-shan $f$ & H. $(d)$ & & H. $(b)$ \\
\hline Hsin-cl'êng 新城 & H. (a) & & & Yinn-cli: & H. (i) \\
\hline & & & & & \\
\hline
\end{tabular}




\section{SHENSI}

Circuits.

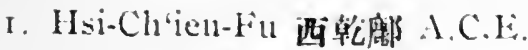

2. Fèny-l'in 鳳邠 B.G.

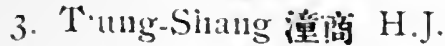

4. Shan-An 傸姿 D.F.

5. Yen-Yii-Sui 延榆䍃 I.K.I。

PREFFCTLRES ANI INUEHENUENT DHRAKTMLNTS.
A. Ch'ien C.L.C.
乾 E. Hsi-an Fu
B. Fèng-lisiang Fu 鳳叛 F. Hsing-an Fu
C. Fu C.L.C.
鄜 G. Pin C.1..C.

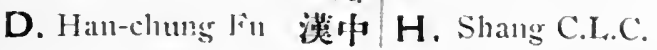
晒睃 I. Suite C.L.C.
綏德
舆安 J. 'l'ung-chou Fu
闹州
檚 K. Yen-an Fin
商 L. Iii-lin Fin
趜桨:
榆林

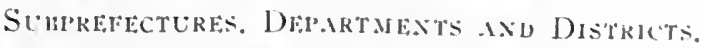

\begin{tabular}{|c|c|c|c|c|c|}
\hline An-k'ang 妾康 & H. $(f)$ & & c. $(j)$ & Eng 集城 & 11. (a) \\
\hline An-sai 妥塞 & H. (k) & liua-yin 萑陰 & H. $(j)$ & 筫雞 & H. (b) \\
\hline An-pien 安宾 & T. $(k)$ & Huai-yüan 懹遠 & H. (l) & 怢利 & H. $(f)$ \\
\hline tn-ting 岁定 & H. $(k)$ & Hsi-hsiang 西啲 & H. $(d)$ & ing 蒲城 & H. $(j)$ \\
\hline y-an 長泣 & H. (i) & Hsiao-yi 孝義 & '. (c) & San-shui 三水 & H. 18 \\
\hline w長武 & H. $(g)$ & *Hsien-ning 威晸 & H. (c) & 监三原 & H. $(e$ \\
\hline 朝岜 & H. $(j)$ & Hsien-yang 咸茄 & H. $\left(c^{\circ}\right)$ & mg 山昜 & H. (h) \\
\hline Chèn-an 鐄安 & H. $(h)$ & Hsing-p'ing 期斥 & H. $(c)$ & 就商南 & H. $(h$ \\
\hline Ch'eng-ch"èng 澄城 & 过 & Hsün-yang 洵陽 & H. $(f)$ & 神木 & H. (l) \\
\hline Ch'èng-ku 城固 & H. (d) & Kan-chiian 甘留 & H. $(k)$ & 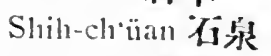 & H. $(f$ \\
\hline 岐山 & 11. $(b)$ & Kan-ling & H. $\left(e^{\prime}\right)$ & 洷化 & H. $(g$ \\
\hline Chia & C. (l) & ien 藍田 & H. (c) & $* \mathrm{~T}$ & H. (j) \\
\hline yang 派隄 & H. (b) & 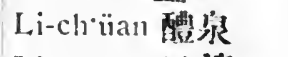 & H. (i) & 定邀 & H. $(k)$ \\
\hline 站遑 & H. $(k)$ & 隔境 & H. $(e)$ & 定嗃 & 1. (d) \\
\hline 涇陽 & H. $\left(e^{3}\right)$ & 粼遊 & H. (b) & 响闹官 & H. $(e)$ \\
\hline 清澗 & 11. (i) & 燿壩 & 'T. $(d)$ & 潼關 & T. $(j$ \\
\hline 盆屋 & H. $(e)$ & uan 洛川 & H. (c) & 紫隄 & H. $(i)$ \\
\hline pu中部 & H. (c) & yang 略隄 & H. $(d)$ & 渭觕 & (e) \\
\hline èng 鳳 & H. (d) & 雉南 & H. (h) & 武功 & H. (a) \\
\hline *Fèng- & H. $(b)$ & Lum & C. $(b)$ & 武堡 & H. (i) \\
\hline 佛文 & 'I. (d) & Mei 刷 & H. (b) & & H. $(d)$ \\
\hline 扶国 & H. $(b)$ & Mi-chih 米脂 & H. (i) & lao & C. (c) \\
\hline 付谷 & H. (l) & Mien ir & H. $(d)$ & 1'ang 延長 & H. (k) \\
\hline u-p'ing 富平 & H. $(c)$ & *Nan-chengy 南䂓 & H. (d) & 延川 & H. $(k)$ \\
\hline Fu-shih 凮施 & HI. $(k)$ & Ning-cin'iang 莩箅 & $C \cdot(d)$ & 管川 & H. $(k)$ \\
\hline “èng 韓城 & H. $(j)$ & Ning-shan 晏傸 & T. $(e)$ & 1 宜君 & H. (c) \\
\hline 漢陰 & T. $(i)$ & & & *Yï-lin 榆林 & H. (l) \\
\hline o-yang & H. (j) & & H. $(j)$ & Yung-shon 永琵 & H. (a) \\
\hline 1 部 & & & & & \\
\hline
\end{tabular}




\section{SHINGKING}

\section{Circeitss.}

I. Feng-Clnin-Shan-hiai 举䤼山游 B.D.E.

2. Tinng-pien 東邊 A.C.I.

PreFeCtURES AND INDEPENDFN SUbPRFECTURE.
A. Ch'ang-t'u Fiu 昌圖
C. Feng-huang C.L.T.
E. Hai-lung Fu
B. Chin-chou Fu 錦州
腺凰 F. Hsing-ching C.L.'T.

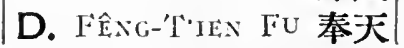
舆京

SUmprefectures, Departintes and Districtis.

\begin{tabular}{|c|c|c|c|c|c|}
\hline 安東 & H. $(c)$ & Hsi-an 西安 & H. $(e)$ & Liao-yang 遼陽 & C. $(d)$ \\
\hline *Ch'êng-tê 承德 & H. (d) & 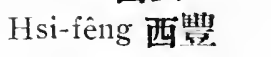 & H. $(\mathfrak{c})$ & Liu-hu 柳河 & H. $\left(c^{\circ}\right)$ \\
\hline Chin 錦 & H. (b) & in 新民 & T. $(d)$ & Ning-yüian 跕䔔 & C. $(b)$ \\
\hline $10 u$ 金州 & 'T. $(d)$ & 蓋本 & H. $(d)$ & 鐵嶺 & H. (d) \\
\hline a 奉化 & H. (a) & n 開原 & H. $(d)$ & $\lg$ 東头 & H. $(e)$ \\
\hline ing 海城 & H. $(d)$ & ing 康本 & H. (a) & $T^{\prime} \mathrm{w}$ & H. ( \\
\hline & H. $(f)$ & & H. $(c)$ & Ying-k'o & T. 6 \\
\hline Huai-tê 懵 & H. $(a)$ & Kuang-ning 席窝 & H. (b) & Yi 義 & C. $(b)$ \\
\hline
\end{tabular}

\section{SSÜCH'UAN}

Cincurts.

I. Ch'êng-Lung-T'ung-Mien-MIon 成龍港綿茂 B.K.M.O.P.Q.W.X.

2. Chien-ch'ang-Shang-11an 建昌上南 C.D.N.R.Z.

3. Ch'uan-pei 川北 S.U.

4. Ch'uan-nan-Yung-ning 川南永䗓 G.H.J.L.Y.

5. Ch'uan-tung 川東 A.E.F.I.T.V.Aa.

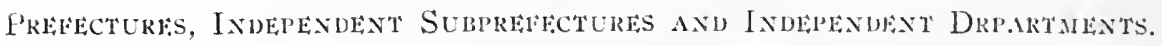
A. Ch'êng-k'ou C.L.'T.
1. K'uei-chou Fu
薄州|S. Pao-ning

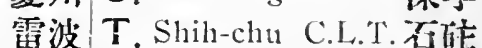 城口 J. Lei-po C.I..T.
B. CH. ÊNG-TU FU
成都 K. Li-fan C.L.'T.
C. Chia-ting Fu
琹定 L. Lü C.L.C.
D. Chiung C.L.C. · I I M. Lang-an F'n
㻎番 U. Shun-ch'ing Fu 順慶
憈 V. Sui-ting Fu
綏定
E. Chung C.I..C.
忠 N. Mei C.L.C.
F. Ch'ung-ch'ing Fu 管重 O. Mien C.L.C.
龍安 W.Sung-p’an C.L.'T.松潘
眉 X. T'tu1g-ch'uan Fu潼川!
G. Hsü-chou Fu 叙州 P. Mou C.L.C.
綿 Y. Tzú C.L.C.
茂 Z. Ya-chou Fu 雅州
H. Hsü-yung C.L.'T. 叙永 Q. Nou-kung C.L.'T.梦功 Aa. Yu-yang C.L.C. 西陽
R. Ning-yüan Fu 酒遠
* Head District of a Prefecture. 


\section{SSÜCH'UAN Continued.}

Surprefectures, Dfipartanests and Districtrs.

An 安

An-yo 安岳

Chang-ming 彰明 H. (m) Hsin-ching 新浙

\begin{tabular}{l|l} 
H. (d) & $*$ Hsi-ch'ang 酉 \\
H. (x) & Hsi-ch'ung 西古 \\
H. $(m)$ & Hsin-ching 新㜊 \\
H. $(g)$ & Hsin-fan 新繁
\end{tabular}

Chiang-shou 長㗲 H. $(f)$ Hsin-ning 新掌

Chao-hua 昭化

H. (s) Hsin-tu 新都

*Ch'êng-tu 成都

H. (b) Hsing-wên 舆交

$\mathrm{Cl}^{\prime}$ ' -chiang 基江

H. $(f)$ Hsill-shan 秀山

Chia-chiang 夾江

Chiang-an 江安

H. (c) Jên-shou 仁兽

Chiang-ching 江津: H. $(f)$ Jung-ch'ang 㮡昌

Chiang-pei 江北

Chiang-yu 江沙

T. $(f)$ Jung-ching 策經

H. (m) K'ai 開

Chien 簡

Chien 劍

C. (b) Kao 高

C. (s) Kuan 灌

Chien-wei 徒第

$\mathrm{Ch}^{\prime}$ ien-chiang 默江.

\section{H. (c) Kuang-an 廣安}

Kuang-ÿian 原元
H. ( $r) \mid P a 巴$

H. (u) * $\mathrm{Pa} 巴$

H. (b) P'êng 彭.

H. (b) P'êng 逢.

H. ( $\left.a^{\prime}\right)$ P'êng-ch'i 蓬溪

H. (b) P'êng-shan 彭山

H. $(g)$ P'êng-shui 彭水 H. $(A a)$

H. $(A a)$ एi-shan 壁山

H. (y) P'i 郫

H. (c) P'ing-shan 屏山

H. $(f) *$ P'ing-wu 平武

H. (z) P'w-chiang 蒲江

H. (i ) * San-t'ai 三臺

H. $(g)$ Shê-hung 射洪

H. (b) Shih-ch'üan 石泉

C. (u) Shih-fang 什邡

H. (Aa) Kung 珙

H. (s) Shuang-liu 隻流

C. $(s)$

H. $(f)$

H. (b)

C. (ii)

IH. $(x)$

H. (n)

H. $(f)$

H. $(b)$

H. $(g)$

H. (m)

H. $(d)$

H. $(x)$

H. $(x)$

H. $(m)$

H. $(b)$

H. (b)

H. $(g)$ Stui-ning 遂察

H. $(x)$

H. (i')

Chin-t'ang 金堂 H. $(b) *$ Lang-chung 間中 II. $(s) *$ Ta 達

H. (r) Ta-chien-lu打箭堵H. (z)

Ching-hsi Kuan 靖西關 Lê-chih 樂至

Ching-yen 非䂙

T. $(z) *$ Lê-shan 樂山

H. (c) Ta-chu 大竹

H. $(v)$

H. (y') Liang-shan 梁山

$\mathrm{Ch}^{\prime}$ ing-cl'i 清溪

H. $(z)$ Lin-shui 鄰水

$\mathrm{Ch}$ 'ing-ful 慶符

H. (g) Lo-chiang 羅江:

II. (c) Ta-bo 大和

T. $(x)$

H. (ii) Ta-ning大脢

H. $(i)$

H. (o) Ta-tsu大足

H. $(f)$

Ch'ing-shên 青种

H. (n) L,u1-shan 渵山

H. (z) Ta-yi 大邑

H. $(d)$

$\mathrm{Cl}^{\prime}$ ii 渠

H. (i) Lung-ch'ang 隆昌

H. $(g)$ 'T'ai-p'ing 太平

H. $\left(a^{\prime}\right)$

Chung-chiang中江 H. ( $x$ ) Ma-pien 喣邊

T. (g) Tan-lêng 丹稜

H. (n)

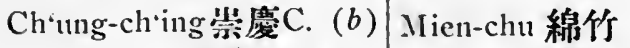

Ch'sung-ning 岁察 H. (b) Nien-ning 痏寀

H. (o) Tê-yang 德惕

H. $(o)$

H. $(r)$ Tien-chiang 熱江 H. $(e)$

*Fèng-chieh 奉節 H. (i) Min-ch'uan 汶川

Fêng-tu 㔂都

Fon 谣

H. (c) Ming-shan 名山

H. (p) T'ien-ch'iian 天全

H. (z) Ting-yüan 定薏

H. $(f)$

H. (l) T's'ang-ch'i 荅溪 H. $(s)$

Fu-shun 富顼

Han 淡

C. (f) Na-ch'i 納絺

H. $(g)$ Tung-hsiang 東榔 H. ( ( $\left.{ }^{\prime}\right)$

Ho 合

H. (g) Nan-chi i 南溪

C. (b) Nan-chiang 南江

H. $(s)$ 'T'ung-chiang 通 江H. $(s)$

C. $(f)$ Nan-ch'uan 南川 H. $(f)$ T'ung-liang 銅梁 H. $(f)$

Ho-chiang 合江

H. (l) *Nan-ch'ung 南充

H. $\left(v^{\prime}\right)$ ' 'zŭ-t'tung 样潼

H. (o)

*Hna-yang 華隄

H. (b) Nan-pu 㑲部

H. $(s)$ Tzŭ-yang 資隄

H. $(y)$

Huang-chiao 黄得 T. (c) Nei-chiang 內江

Hui-li 會理

C. $(r)$ O-mei 峨居

Hung-yai 谐雅

II. (c) O-pien 峨邀

H. (y) Wan 萬

II. (c) Wei-yüan 威遠

H. (i)

T. (c) Wên-chiang 溜江 H. $(b)$

* Head Distrial of a Piefecture. 
$x \times i i$

\section{SSŬCHUAN Continued.}

Wu-shan 巫川

11. ( $i$ ) Yi-lung 儀陪

H. (n) Yün-lien 均遇

H. $(g)$

*Ya-an 雅:妾

Yen-t’ing 㽭亭

H. (z)*Yi-pin 宜筫

H. (g) Yün-yang 雲隄

H. ( $i)$

Yen-ÿ̈an 㲯源

H. (x) Jing-shan 營川

H. (i) Yung-ch'uan 永川 H. $(f)$

H. $(r)$ Yo-chih 零池

H. $(u)$ *Yung-ning 永学 H. $(h)$

Yüel1-sui 越售

T. $(r)$

\section{TSITSIHAR}

CiRCUTS.

1. Hei-shni-Ta 黑水大 C.E.

2. Sui-Lan-Hai 緮藏游 B.D.A.

PREFFCTURES AND INUFPENDEN' SUBPRFFCTURES.
A. Hai-lun C.L.T. 海倫
B. Hutan Iin
C.Hei-shuiC.L.T.
黑水 E. Ta-pin C.I..'T.
綏化

大頞

DEPARTMFAT AND DISTRICTS.
Chring-kang 毒周 H. (a) Mu-lan 木藏
Lan-hsi 苗西
H. (b) Pa-yen U旁

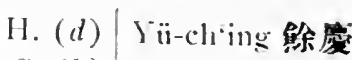
H. (d)
C. (b)

\section{YÜNNAN}

Cikcursts.

I. Y'ï1-IV'u 雲武 R.'T.

2. Yi-tung 沲束 A.D.G.I.

3. Lin-K'ai-Kuang 臨開廣 H.J.I.

4. Yi-hsi 迤西 F.K.M.P.U.V.

5. Yi-nan1 沲㑲 B.C.E.N.O.S.

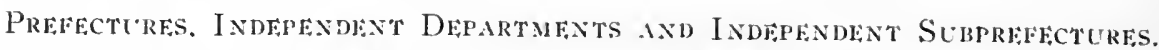
A. Chao-t'ung Fin 昭通
B. Chên-pienC.L.'T.鍞櫋
I. Kuang-hsi C.L.C. 鹰西
Q. Tung-clinuan Iin 東川
C. Chên-yuanC.J.T.鎮沅 K. Li-chiang Fu
廣防
R. Wu-ting C.L.C. 武定
D. Chiêng-chiangFu溦䜣 L. Lin-an
麗江 S. Yüan-chiang C.L.C.
E.Ching-tungC.J.T.崇東
沅证
F. Ch'u-hsiung Fu 楚雄
M. Mêng-hua C.L.T.蒙化 T. Yix-NAN Fu
雲南
G. Ch'ii-ching Fin 䙵靖
N. P'u-êth F’u
埥洱 U. Yung-ch'ang $F_{u}$ 永昌
H. K'ai-hua Fu
開化 P. Ta-li F.1
順寀 V. Iung-pei C.I..T. 永地 大理 


\section{YÜNNAN Continued.}

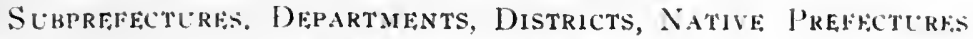
and Nathe Dipartinents.

\begin{tabular}{|c|c|c|c|}
\hline -mi 阢墚 & $\cdot(l)$ & $(g)$ & 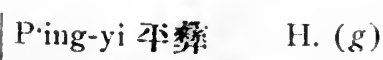 \\
\hline n-ning 妾宰 & C. $(t)$ & Cuang-t'ung 盛通 H. $(f)$ & Shih-p'ing 不屏 \\
\hline$n$-p'ing 安平 & T. $(h)$ & H. $(t)$ & 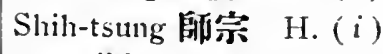 \\
\hline han-yi 鼠谷 & C. $(g)$ & C. $(t)$ & Shun 順 \\
\hline hao 趙 & C. $(p)$ & 主穹H. $(p)$ & * Shun-ning \\
\hline -hsiung 鐄堆 & C. $(a)$ & U. H. $(j)$ & T. $(n)$ \\
\hline 鏑康 & 'Т.C. (o) & C. $(g)$ & C. $(t)$ \\
\hline 消 & C. $(j)$ & H. $(t)$ & T. (a) \\
\hline 星耍 & H. $(t)$ & $(r)$ & $(f)$ \\
\hline & & H. $(t)$ & T. $(n)$ \\
\hline & & C. $(f)$ & H. $(p)$ \\
\hline & '1. $(q)$ & c. $(d)$ & 㔁川 C. $(p)$ \\
\hline his & & (a) & 戰 T. (u) \\
\hline$v_{1}$ & (l) & & H. $(i)$ \\
\hline 富 & C. $(t)$ & C. $(g)$ & $(l)$ \\
\hline 東 & I.F. $(c)$ & 偭 T. $(o)$ & H. (l) \\
\hline 北 & H. $(i)$ & T.F. (c) & T.C. $(o)$ \\
\hline 楚梦 & H. $(f)$ & H. (l) & T. $(k)$ \\
\hline 伯; & T. (k) & H. (i) & T. $(n)$ \\
\hline$E_{1}$ & (a) & 'T. $(o)$ & H. $(h)$ \\
\hline$a^{1-1}$ & H. $(1)$ & C. $(f)$ & C. $(i)$ \\
\hline 猚鹿 & C. $(k)$ & en 南潤 T.C. ( & I. $(t)$ \\
\hline $0-$ & H. (l) & $g$ 㑲豈 $H .(g)$ & H. $(t)$ \\
\hline $\mathrm{H}$ & H. $(d)$ & C. (l) & $\mathrm{H} \cdot$ \\
\hline 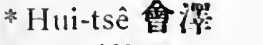 & H. (q) & (n) & C. $(o)$ \\
\hline si & $(l)$ & H. (j) & C. $(p)$ \\
\hline si & & han 保山 H. (\#) & H. $(p)$ \\
\hline & & ng 北腃 T.C. ( & H. $(u)$ \\
\hline O. & $C \cdot(s)$ & Pin-climan 筫川 C. $(p)$ & Vung-shan \\
\hline
\end{tabular}

\section{2.-TOWNS IN THIBET}

Chando

Detsin

Djaya

Dodon

Dzacharkang

Dzangamrin

Ghiamclat

Gumboshotia

Gyangtse

H'lari

H'lassa

\begin{tabular}{|c|c|}
\hline 158 & Il'lunchuls \\
\hline (i:30s & H'lurıujung \\
\hline $1: 35$ & Jansujung \\
\hline 6479 & Medjugung \\
\hline fi:o:):3 & Niuloordzung \\
\hline 5650 & Painamjung \\
\hline $1: 32$ & Paiti \\
\hline 350 & Pitala \\
\hline $37 \%$ & Sangri \\
\hline 3558 & Shobando \\
\hline 83.55 & 'l'apatakse \\
\hline
\end{tabular}

\begin{tabular}{|c|c|}
\hline+15 ? & I'ardzung \\
\hline $402 i$ & 'leshigang \\
\hline ir & Teshin'lumbu \\
\hline $4+5 ?$ & Tsaprung \\
\hline 4i2: & Tsushoul \\
\hline $4 \$ 1 !$ & ludizung \\
\hline 4520 & Ujukling \\
\hline 8.5 .5$. & Sartobo \\
\hline $5+11$ & Yikar \\
\hline & Zhikatse \\
\hline
\end{tabular}

619?

$1: 31 ;$

$1: 3$

(i.) $4 i$

1:3:36

(itis

;196

凊1\%

129

$1: 38$ 
xxiv

\section{3.-TOWNS IN COREA.}

I. Chiang-yuan 2. Ching-ch'i 3. Ch'ing-shang 4. Ch'iian-lo

5. Chung-ch'ing 6. Huang-Hai 7. Hsien-ching 8. P'ing-an

\begin{tabular}{|c|c|c|c|c|c|c|c|c|}
\hline $\operatorname{lng}$ & Fu. & (2) & Chien-ch'nan & Chum. & (s) & Hêng-clı'êng & H. & (1) \\
\hline chou & Fu. & (8) & Chih-li & $\mathrm{H}$. & (3) & Ho-shun & $\mathrm{H}$. & (4) \\
\hline hsia & H. & (1) & $\mathrm{Cl}_{1} \mathrm{inh}$ & C. & (2) & tung & $\mathrm{H}$. & (3) \\
\hline pien & Fu. & $(i)$ & Clun-ch'êng & $\mathrm{H}$. & (1) & yang & H. & (3) \\
\hline & Chïn. & (2) & ch'tan & Chïn. & (6) & Hou-chou & Fin. & $(\tilde{z})$ \\
\hline & $\mathrm{Fu}$. & (3) & & H. & (2) & Huai-shan & Chün. & (5) \\
\hline$y \mathrm{i}$ & $\mathrm{H}$. & (3) & hai & l*u. & (3) & tè & $\mathrm{H}$. & (5) \\
\hline yo & Chün. & (6) & luta & $\mathrm{Il}$ & (1) & yang & Fin. & (1) \\
\hline hang-cheng & Ful. & (8) & ko & $\mathrm{H}$. & (4) & Huang-chien & H. & (5) \\
\hline & $\mathrm{H}$. & (3) & $p^{6} 11$ & H. & (1) & & Fu. & (6) \\
\hline & Fur. & (7) & sh & Chïn. & (3) & & Fut. & $(4)$ \\
\hline & Fu. & (2) & shat & Chïn. & (4) & Hui-nis & Fu. & (7) \\
\hline hsiao & H. & (6) & $t^{*} \mathrm{i}$ & Chün. & (4) & Hung & C. & (5) \\
\hline lie & H. & (6) & Ching-ch'èng & Fu. & (i) & $\operatorname{ch}^{\prime}$ & $\mathrm{H}$. & (1) \\
\hline & H. & (3) & shan & Chün. & (1) & & H. & (5) \\
\hline & H. & (4) & Ch'ing & C. & 5) & & $\mathrm{H}$. & $(8)$ \\
\hline & H. & (4) & & H. & (5) & Hsi-ch & Chün. & (8) \\
\hline & Ful. & (3) & & H. & (5) & $\mathrm{Hs}^{6}$ & Chün. & ( \\
\hline hên-an & H. & $(4)$ & & Fu. & $(\tilde{y})$ & Hsial & Chün. & (3) \\
\hline & H. & $(5)$ & & H. & (3) & & Chïn. & (8) \\
\hline he & $\mathrm{H}$. & (3) & & $F_{\text {n1. }}$ & (3) & Hsien & Chün. & (3) \\
\hline & $\mathrm{H}$. & (3) & & Chün. & (3) & & $\mathrm{H}$. & (3) \\
\hline & Chiin. & (4) & & $\mathrm{H}$. & (5) & & $F_{\mathrm{u}}$ & $(7)$ \\
\hline & Chiin. & 4) & & Fit. & (7) & & H. & (4) \\
\hline & $\mathrm{H}$. & (5) & $\mathrm{Ch}^{6} \mathrm{u1}-\mathrm{s}$ & Fu. & (2) & & Chün. & (3) \\
\hline & H. & (2) & Chü-c & Fu. & (3) & & $\mathrm{H}$. & (4) \\
\hline & $\mathrm{H}$. & $(\tilde{c})$ & & H. & (3) & $H \sin -$ & H. & (5) \\
\hline Ch'êng-ch'uan & Fu. & (8) & Ch'ul-shan & Fin. & (8) & & H. & (6) \\
\hline & C. & (i) & $\mathrm{Ch}^{`}$ üan & c. & (4) & & Chün. & (6) \\
\hline & C. & & $y$ & $\mathrm{H}$. & (5) & & H. & (3) \\
\hline Chi-ch & H. & $(3)$ & Chung-ch'êng & Fu. & (\%) & Hsing-hai & H. & (3) \\
\hline ch'êng & H. & & & Fut. & (8) & tê & H. & (4) \\
\hline ku & Fur. & (3) & En-ching & $\mathrm{H}$. & (5) & yang & H. & $(1)$ \\
\hline & $\mathrm{H}$. & $(5)$ & Fêng- & $F_{11}$. & (6) & Hsiu & $\mathrm{H}$. & (3) \\
\hline Chri-ping & Chïn. & (2) & & H. & (3) & Hsiia & Fu. & (s) \\
\hline Chia-p' & Fil. & (2) & shan & Chïn. & (6) & & H. & (3) \\
\hline & Fu. & (अ) & Fin-an & $\mathrm{H}$. & (4) & Jên-ch'uan & Fu. & $(2)$ \\
\hline shan & Chün. & (8) & & Fin. & $(7)$ & & $\mathrm{H}$. & (4) \\
\hline Chiang-chieh & Fu. & & $p^{\prime}$ & Fin. & (2) & & Fu. & $(\delta)$ \\
\hline & Fin. & $(2)$ & ÿü & H. & (5) & Jui-hsing & Fu. & $(6)$ \\
\hline & Fu. & (1) & Hai & C. & (6) & Jung-ch'tuan & Chün. & (3) \\
\hline & H. & (8) & mei & $\mathrm{H}$. & (5) & K’ai-clı'êng & Fu. & $(2)$ \\
\hline & $\mathrm{H}$. & & nan & H. & (4) & & $\mathrm{H}$. & (3) \\
\hline & Fu. & (2) & Han-ch' & Fun. & (2) & Kan-c & Chiin. & (1) \\
\hline Cliciaot'ung & H. & (2) & shan & Chiin. & (5) & | K'ang-ching & H. & (4) \\
\hline
\end{tabular}




\begin{tabular}{|c|c|c|c|c|c|c|c|c|}
\hline sung & $\mathrm{H}$. & (6) & Mon-ch'ang & H. & (t) & 'T'ai-an & Chïn. & (3) \\
\hline Kalo-ch'êng & Chü!ı. & (1) & $\operatorname{ch}^{\circ} \mathrm{i}$ & H. & (3) & ch'llan & H. & (s) \\
\hline ling & H. & (3) & chu & H. & (4) & jên & H. & (4) \\
\hline pi & H. & (4) & shan & Fu. & $(\tau)$ & 'Tan-ch'êng & H. & (3) \\
\hline shan & H. & (4) & Mu-ch'uan & H. & $(5)$ & yang & Chïi. & \\
\hline yang & H. & (1) & Xan-hai & H. & $(3)$ & 'T'an-yang & $F_{u}$. & \\
\hline ÿ̈аn & Chün. & $(i)$ & p'ing & $\mathrm{H}$. & (4) & T'ang-ching & $\mathrm{H}$. & \\
\hline in-an & $\mathrm{H}$. & (B) & yang & I'u. & (2) & Tê-chuan & Chün. & \\
\hline ch'êngr & H. & (4) & yüan & Fu. & (4) & shan & H. & \\
\hline fou & Chün. & (4) & $\mathrm{Ni}$-shan & $\mathrm{H}$. & $(5)$ & ÿ̈аn & livu. & \\
\hline slan & Fu. & (6) & Ning-hai & Fin. & (3) & 'T'i-ch'uan & $\mathrm{H}$. & \\
\hline Kuang & C. & (t) & pien & $F u$. & (8) & T'ien-shan & Fu. & \\
\hline choil & F'u. & $(2)$ & yïan & Chüin. & (8) & ÿ̈̈an & Fin. & \\
\hline yang & H. & (4) & ÿ̈еn & Chäin. & (1) & Ticn-an & Chüu. & \\
\hline Cuei-ch'êng & Fur. & (8) & Pai-ch'uan & Chün. & 6) & Ting-p'ing & Fu. & \\
\hline হ'un-yang & Chün. & (i) & Pao-ch'êng & Chüı. & (4) & shan & $\mathrm{H}$. & \\
\hline Fung & C. & (5) & ên & iI. & (5) & 'Tuan-ch'uan & Fu. & \\
\hline Kuo-ch'uan & H. & (2) & ning & $\mathrm{H}$ & (5) & shan & Chiin. & \\
\hline shan & Chïn. & (8) & Pei-ch'ing & Fu. & y) & 'Tung-lai & Fu. & \\
\hline Lan-p'u & H. & (5) & Pi-t'ung & Chïn. & (8) & 'T'ung-ching & Fu. & \\
\hline Lang-cli'uan & H. & $(\gamma)$ & $P^{\prime}$ ing-ch'ang & Chün. & (1) & lai & Fu. & \\
\hline Li-an & H. & (3) & chih & H. & (i) & 'l'sai-Ning & Chün. & \\
\hline chi & Chiii. & (3) & ha & Chüю. & 1) & Ts'ao- & H. & \\
\hline ch'uan & $F u$. & (2) & jang & F & 3) & 'T sèılg-shan & $\mathrm{H}$. & \\
\hline ch'rian & Chün. & (3) & $k^{*}$ ang & H. & 1) & '̂zŭ-jêu & H. & \\
\hline shan & H. & (5) & Po-ch'uau & Cḧ้̈1. & (8) & shan & Chün. & 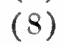 \\
\hline tê & Fu. & (2) & $\mathrm{P} \cdot \mathrm{O}$ & C. & (z) & Wan-ching & H. & \\
\hline yïan & $\mathrm{H}$. & $(7)$ & Saln-chia & IH. & (3) & Wvei-yüan & Chïn. & $12+3$ \\
\hline Liang-shan & Chün. & (3) & & Fu. & & Wên-ch'êng & Fru. & \\
\hline Lien-ch'uan & H. & (2) & Cluper & Fu. & $(\gamma$ & ch'ing & H. & \\
\hline shan & H. & (5) & Shan- & Fu. & & tan & Chün. & \\
\hline Lin-ch'uan & Chün. & (5) & Shang & c. & & & & \\
\hline po & H. & (4) & Shê-ku & $\mathrm{H}$. & (1) & llêng-ching & Chün. & \\
\hline ti & H. & (1) & Shu-ch'uan & Chün. & (5) & Wu-an & H. & $(4$ \\
\hline Ling & C. & & Shui-ÿïan & Fin. & (2) & ch'uan & Chiin. & \\
\hline & $\begin{array}{r}\text { Chün. } \\
\text { H. }\end{array}$ & $(4$ & Shun-an & $\mathrm{H}$. & (8) & Wu-kou & $\mathrm{H}$. & \\
\hline Lin-Yen & bïn & & ch'ang & Chiin. & (4) & lang & C. & \\
\hline $1,1 n-1 \mathrm{en}$ & & & ch'uan & Chün. & (s & ch'êng & $\mathrm{H}$. & \\
\hline I & C. & & Slum-lising & H. & $(3)$ & ch'uan & I. & (: \\
\hline I,lung-ch't1an & I*u. & (8) & t'ien & Fiu. & (4) & kên & Chün. & \\
\hline jêı & $\mathrm{H}$. & & Sü-chou & Fin. & (s & kour & H. & \\
\hline kang & $\mathrm{H}$. & (8) & ning & Fin. & (2) & $t \hat{\mathrm{e}}$ & H. & \\
\hline kung & Chiin. & (3) & Ssŭ-ch'uan & H. & (3) & Ien-an & $F_{u}$. & ) \\
\hline Ma-t'ien-4 & C'hün. & $(2)$ & Su-ch'uan & $F$. & 10 & $\mathrm{ch} \cdot \mathrm{i}$ & $\mathrm{H}$. & \\
\hline Mièng-shan & $\mathrm{H}$. & (S) & Sui-an & Cliiin. & (6 & li & H. & \\
\hline Mi-yang & Fu. & (3) & Sung-ho & & & & $\mathrm{H}$. & \\
\hline Micu-ch'uan & Chiin. & (5) & 'Ta-ch‘iu & Fu. & (3) & Yi-ch'êng & $\mathrm{H}$ & \\
\hline Ming-ch'van & Fi1. & $(7)$ & hsing & II. & 5) & Yi-chou & Fu. & \\
\hline
\end{tabular}


TOWNS IN COREA Contimucd.

\begin{tabular}{|c|c|c|c|c|c|c|}
\hline ch'uan & H. (1) & jih & H. & (i) & shan & Chïn. \\
\hline hsing & $(3)$ & $t \hat{e}$ & H. & (3) & wei & H. \\
\hline ning & (3) & Yü-chên & $\mathrm{H}$. & (1) & Jung-ch'uan & Chün. \\
\hline Vin-chu & (2) & ch‘üan & H. & (4) & ch't111 & H. \\
\hline li & (i) & Yü-shan & $F_{u l}$. & (3) & jout & H. \\
\hline shan & (8) & Yün-ch'nan & Chün. & (3) & p'ing & $\mathrm{H}$. \\
\hline 'ing-ch'êng & $(5)$ & fêng & H. & $(4)$ & . t'ung & H. \\
\hline
\end{tabular}

\section{4.-PLACES MENTIONED BY MARCO POLO}

Acbaluc
Anin
Cacaufu
Cachanfu
Caichı
Caiju
Caindı
Canbaluc
Campichı
Caracoran
Carajan
Cascar
Caugigu
Cauly
Cayu
Chandu
Changlu
Chanshan
Charchan
Chinangli
Chinghianfu
Chinginju

\begin{tabular}{r|l}
487 & Chinju \\
20 & Ciangan \\
2064 & Coiganju \\
5318 & Cotan \\
829 & Cuiju \\
3834 & Cuncun \\
171 & Fuju \\
5522 & Fungul \\
$30 \% 9$ & Ganfu \\
1901 & Ghiuju \\
6049.7813 & Juju \\
3224 & Kenjanfu \\
786 & Kinsay \\
363 & Lingin \\
$31 \% 1$ & Nanglin \\
5535 & Paukin \\
6.521 & Pianfu \\
$: 319$ & Poju \\
401 & Quelinfu \\
601 & Quinsay \\
418 & Sachiu \\
$: 12$ & Saianfu \\
& Siju
\end{tabular}

\begin{tabular}{|c|c|}
\hline $6 \tilde{7} 6$ & Sindachu \\
\hline 69 & Sindafu \\
\hline 224 & Sinigul \\
\hline 205 & $\operatorname{Sinju}$ \\
\hline 847 & Sinjumatu \\
\hline 1950 & Suju \\
\hline $18 \% 1$ & Taianfu \\
\hline 2895 & Taican \\
\hline 3101 & Tadinfu \\
\hline 1445 & 'Tanpiju \\
\hline 1324 & Tenduc \\
\hline 2435 & Tiju \\
\hline 1998 & Tinju \\
\hline$: 345$ & Unken \\
\hline & Vochan \\
\hline $494:$ & Vughin \\
\hline .200 & Vuju \\
\hline $50: 3$ & Xanarlu \\
\hline $85 !$ & Yachi \\
\hline $199:$ & Yanju \\
\hline .42 & Yarcan \\
\hline & Zayton \\
\hline
\end{tabular}




\section{5.-DISTRIBUTION OF MONGOLIAN TRIBES.}

\begin{tabular}{|c|c|c|}
\hline DIvisions OF MONGOLAA & LFAGLES & 'TKIBES \\
\hline \multirow{6}{*}{$\begin{array}{c}\bar{\Xi} \\
\vdots \\
\vdots \\
0 \\
0 \\
0 \\
0 \\
0\end{array}$} & Cherin & $\begin{array}{l}\text { Khorchin } \\
\text { Djalait } \\
\text { Turbet } \\
\text { Ghorlos }\end{array}$ \\
\hline & Chosotu & $\begin{array}{l}\text { Kharachin } \\
\text { Tumed }\end{array}$ \\
\hline & Chao Lda & $\begin{array}{l}\text { Aokhan } \\
\text { Naiman } \\
\text { Barin } \\
\text { Djarid } \\
\text { Aru Khorchin } \\
\text { Ongniod } \\
\text { Keshikteng } \\
\text { Khalka (left wing) }\end{array}$ \\
\hline & Silinghol & $\begin{array}{l}\text { Lchumuchin } \\
\text { Khaochid } \\
\text { Sunicl } \\
\text { Abaga } \\
\text { Abaganar }\end{array}$ \\
\hline & Ulan Chap & $\begin{array}{l}\text { Durban Kenked } \\
\text { Mow Mingan } \\
\text { Urad } \\
\text { Khalka (right wing) }\end{array}$ \\
\hline & Ikh Chao & Ordos \\
\hline Outer Mongolia & Kalmuks & $\begin{array}{l}\text { Oelots, (Elenths) } \\
\text { Turgut }\end{array}$ \\
\hline Koknonor & () & $\begin{array}{l}\text { Khoshoit } \\
\text { Choros } \\
\text { Khoit }\end{array}$ \\
\hline Uliasutai & \{ & $\begin{array}{l}\text { Mingad } \\
\text { Djakchin }\end{array}$ \\
\hline
\end{tabular}




\section{6. - TOWNS IN JAPAN}

\begin{tabular}{lr|l} 
Aichi & 41 & Kioto \\
Akita & $130 \%$ & Kobé \\
Awomori & 1218 & Kóchi \\
Chiba & 945 & Kofu \\
Ehimé & 43 & Kunamoto \\
Fukuoka & 1868 & Kusatsu \\
Fukushima & $18 \% 9$ & Matsuyama \\
Funai & 1867 & Matsuyé \\
Gifu & 640 & Mito \\
Gumma & 1524 & Miyagi \\
Hakodate & 2626 & Morioka \\
Hakone & 2624 & Nagano \\
Hiogo & 5138 & Nagasaki \\
Hiroshima & $3416 a$ & Nagoya \\
Ibaraga & 6860 & Nemuro \\
Ikao & $8466 a$ & Niigata \\
Ishikawa & $5 \% 05$ & Nikko \\
Iwate & $\gamma 396$ & Oita \\
Kagoshima & 4059 & Okayama \\
Kamazawa & 1049 & Osaka
\end{tabular}

\begin{tabular}{|c|c|c|}
\hline $112 \gamma^{\prime}$ & Otsu & 6015 \\
\hline $.561 \gamma_{a}$ & Saitama & 63S \\
\hline $3134 a$ & Sakai & $\$ 2 S$ \\
\hline$r 18$ & Sapporo & $13 \gamma$ \\
\hline 2883 & Sendai & $2 \gamma 11$ \\
\hline 6532 & Shidzlioka & $11 \% 1$ \\
\hline 5980 & Shiga & 6816 \\
\hline 5980 & Shimané & $62 \gamma 4$ \\
\hline $5 \% 98$ & 'Takasaki & 3130 \\
\hline 3511 & Tochigi & 2605 \\
\hline 5645 & 'l'okio & $6 \% 07$ \\
\hline 281 & Urawa & 5305 \\
\hline 234 & Wakayama & 2048 \\
\hline 4461 & Yamagata & $5462 \mathrm{c}$ \\
\hline 3240 & Yamaguchi & $5462 a$ \\
\hline $2 \% 60$ & Yamanashi & $5462 \mathrm{~b}$ \\
\hline 2998 & Yedo & 738 \\
\hline 6023 & Yenkoji & $54 \% 6$ \\
\hline 3112 & Iokkaichi & 5887 \\
\hline 6022 & Yokohama & 2026 \\
\hline
\end{tabular}




\section{7.- THE PRINCIPAL RIVERS AND LAKES OF CHINA.}

\section{CONTRACTONS IND DH:RNTRONS.}

$h=$ 河; ch. = 江; $\quad$ ch' $\mathrm{i}=$ 溪; trib. = tributary $; \quad$ affl. = affluent. A tributary is a strean entering a river which flows directly into the sea An affluent is a stream which enters a tributary.

\begin{tabular}{|c|c|c|c|c|c|}
\hline & & & & & \\
\hline 1 & A-chiao-ho & |阿艾合 & $\operatorname{ch}{ }^{\mathrm{i}}$ & trib. of $52 \mathrm{~s}$ (Yümnan) & \\
\hline 2 & & 艾 & $\operatorname{cin}^{\prime} \mathrm{i}$ & affl. of $3 \% \%$ & \\
\hline 3 & An-clial & 晏冢 & h. & affl. of $13:$ & \\
\hline$t$ & $\eta \quad$, & 安家 & h. & affl. of $15 \%$ & \\
\hline 5 & , ning & 妥富 & h. & trib. of 5:s (Ssŭch'uan) & \\
\hline (j) & , , , & 要垩 & h. & afifl. of 436. & \\
\hline 8 & Ch'a-ch'uan & 爸川 & li. & affl. of 31 & \\
\hline S & , & 祭 & $\operatorname{ch} \mathrm{i}$ & enters $: 360$ & \\
\hline 9 & , p.ing & 茶坷 & h. & affl. of 125. & \\
\hline 10) & Chang & & h. & trib. of 495 . & \\
\hline 11 & $\begin{array}{r}\text { Ch'ang-ch'iao- } \\
\text { hai }\end{array}$ & 長橋游 & Lake & in Yünnan. & \\
\hline 12 & , t'an & 長㵀 & h. & trib. of 528 (Ssŭch'uan) & \\
\hline 13 & , t'èng & 唇滕 & i.. & affl. of $40: 3$ & \\
\hline 14 & , & 昌 & ch. & enters $: 60$. & \\
\hline 15 & Chao-wang & 趙王 & h. & enters 5it. & \\
\hline 16 & Ch'ao & 潮 & h. & affl. of $3: 3 \gamma$ & \\
\hline $1 \%$ & Chè-111u & 杯术 & ch. & affl. of $1 \% \%$ & \\
\hline 15 & Ch'ê & 車 & h. & affl. of $16 \%$ & \\
\hline 19 & ,, tui & 車對 & h. & trib. of 528 (Ssŭch uan) & \\
\hline 20 & Cliên-yang & 鍾陟 & cls. & affl. of 561 . & \\
\hline 21 & Cln'ên1 & 辰 & h. & affil. of 561 & \\
\hline $2: 2$ & Chêng & 槕 & ch. & enters sea near Canton. & North River \\
\hline 23 & Ch'ềng: & 程 & ch. & name of part of $1 \%$ & \\
\hline 21 & Chi-kung & 彩公 & li. & affl. of $51: 3$ & \\
\hline 25 & ," chiang & 基江 & h. & trib. of $52 \mathrm{~S}$ (Ssŭcli'uan) & \\
\hline 26 & , li & 枪麗 & Lake & in Yünnan & \\
\hline 28 & Ch'i-ting & 岐管 & h. & trib. of 5:2 (Hupels) & \\
\hline 28 & , ts tun & 萦朴 & li. & enters $16 \%$ & \\
\hline 光!) & Chia-li & 滰利 & Lake & in Yünnan. & \\
\hline 30 & :, ling & 落陵 & ch. & trib. of 52s (Ssǔch'uan) & Kia-ling \\
\hline :11 & , shui & 琞水 & h. & affl. of 104 . & \\
\hline $3: 1$ &,, 11 & 賈㶵 & 1. & affl. of 550 & \\
\hline 8:3 & Chiaro & 交 & clïi & cuters sea in liulkien. & \\
\hline 31 & , & 洨 & li. .. & cuters $3: 5$ & \\
\hline :3ü & .. & & chi & afil. of $: 3: 30$ & \\
\hline$: 36$ & Chino-wan & 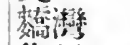 & h. & trib. of 5.s. (Anhui) & \\
\hline $3 \%$ & Chich-tzŭ & 街子 & h. & affl. of 264 & \\
\hline 83 & Chien & 建 & l. & affl. of 408 & \\
\hline ;9 & , yang & 建隄 & li. & trib. of $5 \pm 3$. (Hupeh) & \\
\hline 10 &. & 劍 & ch. & affl. of $40 \mathrm{~s}$ & \\
\hline 11 & ,. & 锿!! & ch. & affl. of :agt. & \\
\hline 42 & , & 劍 & Lake & in Yïnnan. & \\
\hline 43 & , & 澗 & h. & affl. of 120 & \\
\hline
\end{tabular}




\section{THE PRINCIPAL RIVERS AND LAKES OF CHINA Continucd.}

\begin{tabular}{|c|c|c|c|c|c|}
\hline$\$ t$ & Clïien & $v_{c}^{\prime}$ & $\operatorname{ch} \ddot{i}$ & affl. of $51:$. & $a m s m$ \\
\hline 45 &,$\quad k u$ & 乾枯 & h. & atfl. of $13: 3$ & \\
\hline 46 & .. ta & 乾大 & 1. & enters lake in tnhui. & \\
\hline $4 \gamma$ &. & 铪 & cli, & name of part of 416 . & \\
\hline is & . sluui & 活水 & h. & affl. of 498 & \\
\hline 49 & ... tang & 鈛堵 & ch. & enters sea near Hangchon. & T'sientang \\
\hline 50 & Chilı & 氾 & ch. & affl. of 208 & \\
\hline $\begin{array}{l}50 \mathrm{~A} \\
.11\end{array}$ & .. & 染 & h. & enters 162 & \\
\hline $\begin{array}{l}-1 \\
52 \\
52\end{array}$ & $\begin{array}{cc}y & \text { shti } \\
. . & .,\end{array}$ & $\begin{array}{l}\text { 亦水 } \\
\text { 淥水 }\end{array}$ & $\begin{array}{l}\text { h. } \\
\text { h. }\end{array}$ & trib of 528 (Ssŭch nan) & Chihshwui \\
\hline $5: 3$ & Chin & 俭 & h. & $\begin{array}{l}\text { aftl. of } 509 \text {. } \\
\text { trib. of } 528 \text {. (Hupeh) }\end{array}$ & \\
\hline .54 &.. & 金 & b. & trib. of 148 (Shensi) & \\
\hline is & " & 金 & $\operatorname{cln} i$ & affl. of 306 & \\
\hline .66 & $\because \quad$. & 金 & $\operatorname{ch} \cdot \mathrm{i}$ & offl. of 112 & \\
\hline $5 \%$ & . hsien. & 金楾 & 1.. & affl. of 101 & \\
\hline 58 & $\because$ & 敘 & ch. & enters 360 & \\
\hline 59 & .. & 䤼 & ch. & affl. of 116 . & \\
\hline 60 & " & 錦 & ch. & aftl. of 22 & \\
\hline 61 & 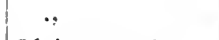 & 筧 & ch. & enters sea in Fuhkien. & \\
\hline 62 & Ch'in-shui & 琴水 & ch. & afil. of 293 & \\
\hline 63 & , & 欽 & ch. & enters sea in Kuangtung. & \\
\hline 64 & .. & 沁 & li. & trib. of 148 . (Honan) & $\Gamma \sin$ \\
\hline $6 j$ & .. huai & 秦淮 & h. & trib. of 528. (Kiangsu) & \\
\hline 66 & , & 溱 & $\operatorname{ch} \ddot{i}$ & affl. of 51. & \\
\hline $6 \%$ & Ching & 浛 & h. & affl. of $49 \%$. & \\
\hline 65 & , kou & 荆满 & h. & enters 822 & \\
\hline 69 & , & 靖 & ch. & aftil. of $1 \gamma \gamma$. & \\
\hline 80 & Ch'ing-lung-liai & 青龍海 & Lake & in Yünnan. & \\
\hline$\gamma 1$ & , shui & 离水 & ch. & affl. of $3+\%$. & \\
\hline 28 & $=\quad y i$ & 青夌 & ch. & affl. of $30 \%$ & \\
\hline 83 & $. . \quad .$. & 羍七 & ch. & trib. of 528 (Anhui) & $T \operatorname{sing}$ \\
\hline 84 &.. & 港 & ch. & trib. of 528. (Hupeh) & \\
\hline 85 & .. chang & 溻漳 & h. & affl. of 10 . & \\
\hline 86 & .. ch'i & 清溪 & 1.. & affl of 111 & \\
\hline$\gamma$ & $\because \quad$ chiang & 筇江 & $\operatorname{ch}{ }^{\circ}$ & affl. of 239 & \\
\hline is &.. chien & 䧼澗 & h. & trib. of 148 . (Shensi) & \\
\hline '9 & .. ku & 活谷 & h. & affl. of 498 & \\
\hline 80 &. liu. & 涪流 & h. & affil. of 265 & \\
\hline 81 & .. shui & 清水 & ch. & affl. of $56 i$ & \\
\hline 82 & $. . \quad .$. & 㵋水 & h. & trib. of 148. (Shansi) & \\
\hline 83 & $\because$ & 声水 & h. & trib. of 148 . (Shensi) & \\
\hline 84 &.. & 声水 & h. & trib. of 148 . (Kansuh) & \\
\hline 85 &.$\cdot$ & 湢水 & ch. & affl. of 513 & \\
\hline 86 & .. & 清水 & h. & affl. of 513 & \\
\hline $3 \%$ &.. & 清水 & h. & trib. of 528 (Ssüchuan) & \\
\hline 88 & , & 清水 & h. & affl. of $30 \%$ & \\
\hline 89 & ., & 声水 & h. & affil. of 96 & \\
\hline 90 &.$\cdot$ & 清水 & h. & trib. of 560 . & \\
\hline 91 & $\cdot$. & 洋水 & h. & affle of 143 & \\
\hline 92 & . $\quad$ lall & 清苑 & h. & aftil. of 101 . & \\
\hline
\end{tabular}




\section{THE PRINCIPAL RIVERS AND LAKES OF CHINA Continued.}

William's map.

\begin{tabular}{|c|c|c|c|c|c|}
\hline !:? & Clitu-chuu & 九州 & cli. & cutcrs scal in kuanglung. & \\
\hline y4 & ., kill & 九股 & li. & afil. of st. & \\
\hline 15 & . lung & 九龍 & ch. & enters seit neal \moy. & \\
\hline 96 & ., lungr & 九龍 & ch. & afill of j1s. & \\
\hline$! i$ & .. p'êng & 九鹏 & chi. & affl. of 95 & \\
\hline 98 & Chöis & 湫 & li. & trib. of 11s. (Shensi) & \\
\hline 99 & Chon-shui & 凬水 & h. & affll oi 20 is. & \\
\hline 100 & Chu & & in. & afril. oi .5.50. & \\
\hline $\begin{array}{l}101 \\
102\end{array}$ & .. lumy & 猪能 & h. & trib. of 360. & C'hu-lumy \\
\hline $\begin{array}{l}102 \\
103\end{array}$ & $\begin{array}{l}\text { sluii } \\
. . \\
\text { t'un }\end{array}$ & 浽水 & h. & enlers bit. & \\
\hline 104 & .. changr & 湿漳 & $\begin{array}{l}\text { hi. } \\
\text { h. }\end{array}$ & enters :30s. & \\
\hline 105 & Ch'u & 淔余 & h. & "pper waters of 10 . & \\
\hline $\begin{array}{l}106 \\
19 \%\end{array}$ & Chail-lungy & 巨除 & h. & $\begin{array}{l}\text { trib. of i2s. ( Kiangsu) } \\
\text { culters } 822 . \text {. }\end{array}$ & \\
\hline $\begin{array}{l}10 \% \\
108\end{array}$ & 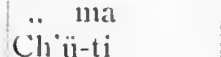 & 拒惩 & h. & aifl. of $3 \%: \%$ & \\
\hline $\begin{array}{l}108 \\
109\end{array}$ & Cl1 $11-\mathrm{t} 1$ & 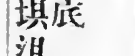 & hi. & affil. of 125 & \\
\hline 110 & $\begin{array}{l}\ddot{\prime} \\
. \cdot\end{array}$ & 㴪 & h. & trib. of 528 . (Hupcls) & \\
\hline 111 & .. & 梁 & h. & affi. of $\mathrm{s} 1$. & Kii \\
\hline $\begin{array}{l}112 \\
113\end{array}$ & .. & 雚 & h. & affl. of 30 & \\
\hline $\begin{array}{l}113 \\
114\end{array}$ & , & 此 & cli. & affl. of $2: 31$. & \\
\hline $\begin{array}{l}114 \\
115\end{array}$ & & & li. & affl. of 335 . & 1 \\
\hline $\begin{array}{l}115 \\
116\end{array}$ & .. ÿ̈̈m shui & 曲源水 & & affll. of $14 t$ & \\
\hline 116 & , ch'an & 崫產 & h. & trib. of 14S. (Shensi) & \\
\hline $\begin{array}{l}11 \gamma \\
118\end{array}$ & Chuang-lang & 誑浪 & h. & trib. of 148 . (Shansi) & \\
\hline $\begin{array}{l}118 \\
119\end{array}$ & Chung & 中 & h. & trib. of 1ts. (Kansuh) & \\
\hline 119 & ., tæing & 中宗 & h. & affl. of 111 & \\
\hline $1 \geq 0$ & Errh-lang & 二郎 & h. & affl. of 360 & \\
\hline $\begin{array}{l}121 \\
122\end{array}$ & .. hai & 泟海 & Lake & trib. of $52 \mathrm{~s}$. (Anluui) & Urh-hai \\
\hline $\begin{array}{l}122 \\
123\end{array}$ & Fang-shun & 方順 & & in Yünnan. & \\
\hline 123 & Fên & 染 & h. & affl. of 101 & Făı \\
\hline $\begin{array}{l}124 \\
125\end{array}$ & Fêng-shini & 燞水 & h. & trib. of $14 \mathrm{~s}$ (Shansi) & \\
\hline $\begin{array}{l}125 \\
126\end{array}$ & Fou & 涪 & $\mathrm{ch}$. & enters 860. & Pei \\
\hline $\begin{array}{l}126 \\
128\end{array}$ & $F_{t 1-t} u n g$ & 傅疃 & li. & affl. of 30 & \\
\hline $\begin{array}{l}1.28 \\
1.28\end{array}$ & . cli $i$ & 棈深 & h. & enters sea in Shantung. & \\
\hline $\begin{array}{l}128 \\
129\end{array}$ & ,. yang & 淦芬 & & aifl. of 400 & \\
\hline $\begin{array}{l}129 \\
1: 30\end{array}$ & $\therefore \quad$ toll & 究㖽 & Lake & enters 4:32. & \\
\hline $\begin{array}{l}1: 30 \\
1: 1\end{array}$ & $F_{11}$ & 府 & h. & in Hupeh & \\
\hline $\begin{array}{l}1: 3 \\
1: 1 A\end{array}$ & , t'un. & 菑电 & cli $\mathrm{i}$ & enters 322 & \\
\hline $1: 31_{A}$ & , yi shui & 夫浔水 & b. & afll. of 300. & \\
\hline $\begin{array}{l}1: 32 \\
133\end{array}$ & $\because$ shnii & 洑水 & h. & :iffl. of 490. & \\
\hline $\begin{array}{l}133 \\
1: 34\end{array}$ & Han & 谟 & ch. & afil. of 1810. & Han \\
\hline $\begin{array}{l}134 \\
135\end{array}$ & 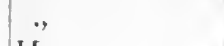 & 暲 & ch. & $\begin{array}{l}\text { trib of } 528 . \text { (Hupeh) } \\
\text { enters sca in Kuangtung }\end{array}$ & \\
\hline $\begin{array}{l}135 \\
1: 30\end{array}$ & Häo & 好 & $\operatorname{ch} i$ & $\begin{array}{l}\text { enters sca in Kilangtung } \\
\text { trib, of :3:30. }\end{array}$ & \\
\hline $\begin{array}{l}1: 30 \\
18 \%\end{array}$ & Hei & 器: & $\operatorname{ch} \ddot{i}$ & trib. of :3:30. & \\
\hline $\begin{array}{l}13 i \\
138\end{array}$ & Hêng & 櫗 & l.. & $\begin{array}{l}\text { aftl. of } 2 \pi s \text {. } \\
\text { alth. of } 26 \text { (6). }\end{array}$ & \\
\hline $\begin{array}{l}138 \\
1: 39\end{array}$ & ,. slitn & 槙水 & li. & $\begin{array}{l}\text { aift of } 26 i 1 . \\
\text { alti. of } 1 \text {. }\end{array}$ & \\
\hline $\begin{array}{l}1: 39 \\
1+0\end{array}$ & Ho-shui & 賀水 & h. & $\begin{array}{l}\text { atil. of 1:ii. } \\
\text { atil. of 1:3. }\end{array}$ & \\
\hline $\begin{array}{l}1+0 \\
1+1\end{array}$ & Hon & & h. & 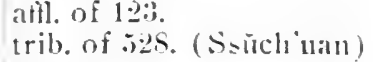 & \\
\hline $\begin{array}{l}141 \\
142\end{array}$ & & 嵝。 & h. & affl. of 111 . & \\
\hline 142 & | I Hu-tu & 虎渡 & h. & trib, of izs (Hupeh) & \\
\hline
\end{tabular}


THE PRINCIPAL RIVERS AND LAKES OF CHINA Continucd.

\begin{tabular}{|c|c|c|c|c|c|}
\hline 143 & Hu-t’o & 滹沱 & h. & enters 325. & Hu-to \\
\hline 144 & Huai & 淮 & h. & enters $16 \%$ & Hwai \\
\hline 145 & , & 槐 & h. & cnters 825. & \\
\hline 146 & Huan & 潧 & h. & affl. of $13: 3$. & \\
\hline $14 \%$ & & 環 & h. & affl. of 285 & \\
\hline 148 & Huang & 黄 & h. & enters Gulf of Pechihli. & Y'cllow River. \\
\hline 49 & ," kang & 黄夙 & h. & enters sea in Kuangtung. & \\
\hline 50 &,$\quad 1$ ao & 黄落 & h. & trib. of 528. (Anhui) & \\
\hline 151 & " lung & 黄龍 & ch. & affl. of 416 & \\
\hline 152 & " p'u & 黄浦 & ch. & enters 528 near Shangliai & Hwangpoo. \\
\hline 53 & , shih & 黄石 & h. & affl. of 561 & \\
\hline 54 & , & 㣴 & h. & affl. of $14 t$ & \\
\hline 155 & "shui & 洤水 & h. & affl. of 148. (Kansuls) & Si-ning \\
\hline 156 & , , , & 隍水 & h. & affl. of 22 . & \\
\hline $15 \%$ & Hui-chi & 惫灌 & h. & affl. of 213 & \\
\hline 158 & Hu11 & 渾 & h. & affl. of 240. & \\
\hline 159 & , & 渾 & h. & trib. of 360 . & \\
\hline 160 & & 渾 & li. & affl. of $3 \gamma 6$ & \\
\hline 161 & Hung-kou & 㴀㸺 & h. & affl. of $2 \% 8$. & \\
\hline 162 &,$\quad$ tsê & 洪漯 & Lake & in Kiangsu. & \\
\hline 163 &,$\quad$ tu & 洪渡 & h. & aff. of 513 . & Hungtsilu \\
\hline 164 & , yen & 浓㴔 & ch. & affl. of 416 . & \\
\hline 165 & Hsi & 西 & ch. & enters sea near Cauton & West River \\
\hline 166 &, & 西 & h. & trib. of 148 . (Kansuh) & \\
\hline 167 & $\because$, & 西 & 11. & trib. of 528. (Anhui) & \\
\hline 168 & $"$ & 西 & h. & affl. of 30. & \\
\hline 169 & , & $\sqrt{4}$ & 11. & affl. of 561 . & \\
\hline $1 \% 0$ & $"$ & 西 & h. & affl. of 133 & \\
\hline $1 \% 1$ & & 西 & $\operatorname{ch}$ 'i & enters Burmah from Ÿ̈nnan & \\
\hline $1 \% 2$ &, fei & 西佩 & h. & affl. of 115 . & \\
\hline 173 &, p’o & 西坡 & h. & affl. of 358. & \\
\hline 174 & & 隰 & h. & affl. of 1 te (Shansi) & \\
\hline 175 &, niu & 雨年 & ch. & affl. of $: 30$. & \\
\hline 176 & Hsia-11111y & 下照 & ch. & affl. of 315 . & Si. \\
\hline $17 \%$ & Hsiang & & ch. & enters $\$ 81$. & Siang \\
\hline $1 \% 8$ & , & & ch. & affl. of 513 . & \\
\hline 179 &,$y$ tung & 湘果 & ch. & affl. of $1 \% \%$. & \\
\hline 180 & Hsiao & & ch. & affl. of $1 \gamma \%$. & \\
\hline 181 & , & & h. & afil. of 84. & \\
\hline 182 & $\Rightarrow \quad$ ch'i & 小溪 & h. & affl. of 73 & \\
\hline 183 & , ch'ing & 小清 & h. & enters sea in. Shantung. & \\
\hline 181 & "sha & 小沙 & h. & affl. of 125 & \\
\hline 185 &, wu & 小照 & ch. & affl. of $\breve{1}: 3$ & \\
\hline 186 &,$y \mathrm{fu}$ & 孝璔 & h. & trib. of $18: 3$. & \\
\hline 187 & Hsien-chiang & 獎系港 & h. & affl. of $: 3 \% \%$ & \\
\hline 188 &, chih & 似筲 & h. & trib. of 148. (Shansi) & \\
\hline 189 & Hsin-k'ai & 新開 & h. & enters $5 \% 4$ & \\
\hline 190 & Hsing-yïn & 星焉 & Lake & in Yünnan. & \\
\hline 191 & Hsiut-chên & & h. & affl. of 360 . & \\
\hline 109 & , & & & affl. of 224 . & Siu \\
\hline
\end{tabular}




\begin{tabular}{|c|c|c|c|c|c|}
\hline & & & & & William's mo \\
\hline 193 & Hsiu-chên & 修 & h. & enters 366 & \\
\hline 194 & Hsü . & 㱏 & h. & enters Lake in Kiangsu. & \\
\hline 195 & ,, shui & 溆水 & h. & affl. of 561 & \\
\hline 196 & Hsüan-hui & 宣忽 & i.. & lenters sea in Chihli. & \\
\hline 197 & Hsün-wu & 䣕 & cls. & affl. of 428 . & \\
\hline 198 & , & 潯 & ch. & affl. of 416 . & \\
\hline 199 & Jê & 教 & h. & affl. of 271 & \\
\hline 200 & $J u$ & 洝. & h. & aff. of $14 t$. & $\mathrm{Ju}$ \\
\hline 201 & , & 汝 & h. & affl. of 384 & \\
\hline 202 & Jui-shui & 脑水 & h. & aff. of $6 \%$ & \\
\hline 203 & Jung & 溶 & ch. & affl. of 208 & \\
\hline 204 & , & 策 & ch'i & affl. of 528. (Ssŭch'ıan) & \\
\hline 205 & $K^{\prime} a i-t u$ & 開都 & h. & enters 365 & \\
\hline 206 & Kan & t5 & h. & affl. of $6 \%$. & \\
\hline $20 \%$ & , ch’üaı & 甘家 & h. & aff. of 261. & \\
\hline 208 & & 韸 & cls. & enters 366 . & $K_{i l u}$ \\
\hline 209 & , ch $\mathrm{i}$ & 乾溪 & h. & aftl. of 513. & \\
\hline 210 & Kao-t'an & 高灘 & li. & aff. of 528. (Ssŭch iuan) & \\
\hline 211 & Kê & 齿 & ch $\because \mathrm{i}$ & aff. of 58 . & \\
\hline 212 & , & 滑 & h. & affl. of 550 . & \\
\hline 213 & & 滑 & 11. & atrt. of 144 . & Ko \\
\hline 214 & K'ê-l'uei & 戈魁 & h. & affl. of $38 \%$ & \\
\hline 215 & & 古 & h. & trib. of 528 . (Hupeh) & \\
\hline 216 & , lang & 古浪 & h. & enters lake in Hupeh. & \\
\hline $21 \%$ & & & h. & trib. of 360 . & \\
\hline 218 & K'u-chu Sluni & 苦竹水 & h. & aft. of 509 . & \\
\hline 219 & ,y sluti & 苦水 & h. & aftl. of 498 . & \\
\hline 220 & K'uai-tsê & 塊虂 & h. & afil. of 96 & \\
\hline 221 & Kuan-ch'uan & 關川 & h. & affl. of 148. (Kansul1) & \\
\hline 222 & ,y yin & 観音 & h. & affl. of 133. & \\
\hline 223 & , & 滺 & ch. & affi. of $1 \% \%$ & \\
\hline 224 & Kuei & 桂 & ch. & affl. of +16 . & \\
\hline 225 & Kuei-shun & 垖素順 & h. & afit. of 198 . & \\
\hline 226 & , shu & 鵝樞 & h. & affl. of 560 & f \\
\hline 227 & , & 㖹 & h. & affl. of $5 \% 3$. & \\
\hline 228 & Kung & 13 & ch. & affl. of 208 . & \\
\hline 229 & , & 珙 & ch'i & affl. of 5. & \\
\hline 230 & Kuo & 单了 & h. & enters Lake in Kanswh. & Sha \\
\hline 231 & Lan & 蘭 & $\mathrm{ch}^{\prime} \mathrm{i}$ & affl. of $4 ?$ & \\
\hline 232 & ,y ch'i & 桠溪 & h. & affl. of $25 \%$ & \\
\hline 233 & , ts'ang & 瀾渝 & ch. & enters Annam from Y'ünnan. & Lantsan or \\
\hline 234 & ., & 摬 & ch'i & affl. of $4 \% 8$. & \\
\hline 235 & ,, shui & 藍水 & h. & aff. of 498 . & \\
\hline 236 & ., ch’i & 咸渵 & h. & trib. of 148. (Shansi) & \\
\hline 237 & Lang & 榔 & $\operatorname{ch} i$ & aftl. of 21. & \\
\hline $23 \varsigma$ & Lao-chüeh & 落脚 & h. & aff. of 2.58 . & \\
\hline 239 & , ha & 老哈 & h. & affl. of 246 . & Loha \\
\hline 240 & Lề-an & 樂安 & i. & enters 360 & \\
\hline 241 & , , shui & 樂安水 & h. & affl. of 178 . & \\
\hline 242 & & 墭 & ch. & affl. of 224. & \\
\hline
\end{tabular}




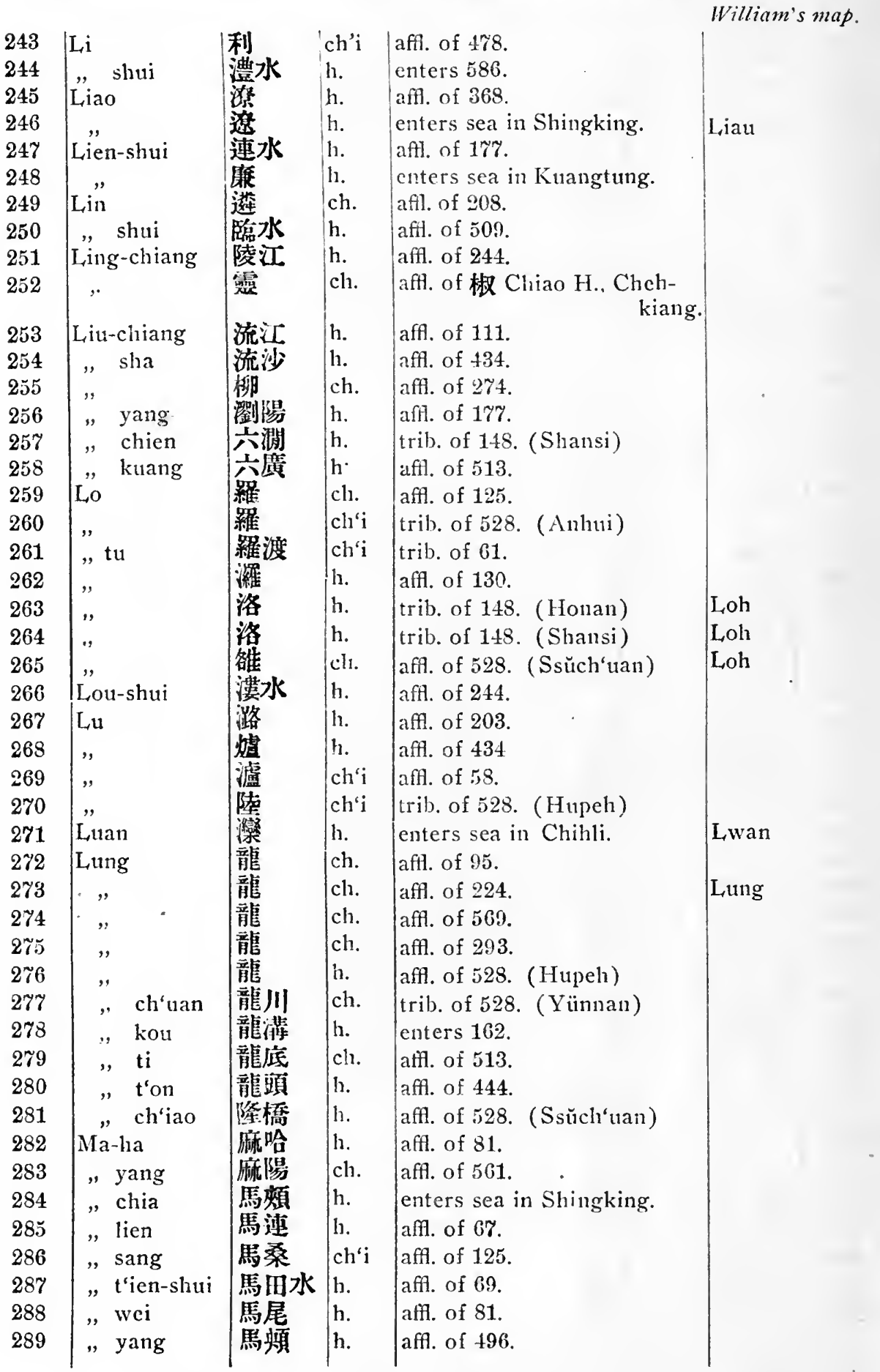


THE PRINCIPAL RIVERS AND LAKES OF CHINA Continued.

\begin{tabular}{|c|c|c|c|c|c|}
\hline 290 & Man & 䌕 & h. & |aff. of 133 . & $\operatorname{zam}^{\prime} s$ \\
\hline 291 & , tzŭ & 篗子 & h. & arf. of 30. & \\
\hline 292 & , chin & 涭金 & h. & aff. of $\Sigma 64$. & \\
\hline 293 & Mei & 梅 & ch. & affl. of 228 . & \\
\hline 294 & & 检 & h. & aff. of 64 & \\
\hline 295 & " $\operatorname{ch}^{\prime} \mathrm{i}$ & 梅溪 & h. & trib. of 528 . (Ssŭch'uan) & \\
\hline 296 & $"$, & 栍哚 & h. & affl. of 98. & \\
\hline 297 & $"$, & 梅深 & h. & affl. of 231 . & \\
\hline 298 & , , & 梅深 & h. & aff. of 55 & \\
\hline 299 & $"$ lin & 梅林 & ch. & affl. of 228 . & \\
\hline 300 & Mêng & 蒙 & ch. & aftl. of 416 . & \\
\hline 301 & $\mathrm{Mi}$ & & ch. & enters 586. & \\
\hline 302 & & * & ch. & affl. of $17 \%$. & \\
\hline 303 & , ton & 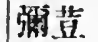 & h. & enters 121 & \\
\hline 304 & & 涨 & h. & enters sea in Shantung. & \\
\hline 305 & Mein-yang & 線陽 & h. & affl. of 265 & \\
\hline 306 & Min & 閩 & ch. & enters sea near Foochow. & Min \\
\hline 307 & & 峎。 & ch. & trib. of 528. (Ssŭclı'tıan) & Min \\
\hline 308 & Ming & 洛 & h. & enters 432 & \\
\hline 309 & , & 明 & ch. & affl. of 198. & \\
\hline 310 & $\mathrm{Mu}$ & 莫 & $\operatorname{ch}^{\circ} \mathrm{i}$ & affl. of 22 & \\
\hline 311 & & 淋 & h. & affl. of 478 . & \\
\hline 312 & ", yü & 泍浴 & h. & aff. of 508 . & \\
\hline 313 & Nan & 南 & h. & affl. of 133 . & \\
\hline 314 & & 㨁 & h. & aft. of 14. & \\
\hline 315 & " ch'in & 南琴 & ch. & enters sea in Shantung. & \\
\hline 316 & " ch'uan & 消川 & h. & affl. of 374 & \\
\hline 317 &., fei & 南淝 & h. & affl. of 144 . & \\
\hline 318 &, liu & 南流 & ch. & affl. of 248 . & \\
\hline 319 & $\Rightarrow p^{i} u$ & 南浦 & $\operatorname{ch}^{\prime} \mathrm{i}$ & affl. of 306 . & \\
\hline 320 & "sha & 南沙 & h. & aftl. of 359 & \\
\hline 321 & " t'ing & 陑汀 & h. & enters Annam from Yünnan. & Nan-ting. \\
\hline 322 & " yün & 觕軍 & h. & $\begin{array}{r}\text { Grand Canal between 'Tien- } \\
\text { tsin and Chinkiang. }\end{array}$ & \\
\hline 323 & $\mathrm{Ni}-\mathrm{ch}^{\prime} \mathrm{i}$ & 泥哚 & h. & affl. of $30 \%$ & \\
\hline 324 & Ning & 窖 & $\operatorname{ch}^{\prime} \mathrm{i}$ & affl. of 55 . & \\
\hline 325 & , chin & 㧘晉 & Lake & in Chihli. & Peh-hus \\
\hline 326 & $\because \mathrm{ku}$ & 蔫谷 & h. & affl. of $35 \mathrm{~s}$. & \\
\hline 327 & Niu-lu & 牛路 & h. & affl. of 8.5 & \\
\hline 328 & , t'on & 牛䫓 & h. & affl. of 498 . & \\
\hline 329 & , wei & 牛尾 & h. & enters 432 & \\
\hline 330 & On & 斯 & ch. & enters sea near Wenchow. & \\
\hline 331 & Pa-kon & 巴悲 & h. & enters 162 & \\
\hline 332 & "shni & 巴水 & h. & aff. of 36. & \\
\hline 333 & ,, pien & 把莩 & ch. & affl. of 560 & \\
\hline 334 & , t'ung & 筜通 & h. & 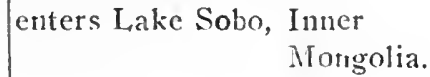 & \\
\hline
\end{tabular}


THE PRINCIPAL RIVERS AND LAKES OF CHINA Continued.

William's map.

\begin{tabular}{|c|c|c|c|c|c|}
\hline 335 & $\mathrm{~Pa}-\mathrm{ta}$ & 八達 & h. & affl. of 96 . & \\
\hline 336 &, tu & 八渡 & h. & affl. of 30. & \\
\hline 337 & Pai & 白 & h. & affl. of 360 . & \\
\hline 338 & ," & 白 & h. & affl. of 133 . & \\
\hline 339 & , & 白 & h. & affl. of 443 . & \\
\hline 340 & $\because$ hua & 白花 & hi. & affl. of 189 . & \\
\hline 341 & ", kou & 白輫 & h. & affl. of 360 . & \\
\hline 342 & , lang & 白狠 & h. & enters sea in Shantung & \\
\hline 343 & , ma & 白熙 & h. & affl. of 412 & \\
\hline 344 &,$\quad \mathrm{ni}$ & 白泥 & Lake & in Hupeh. & \\
\hline 345 & , shui & 白水 & ch. & trib. of 528 . (Ssŭch'uan) & \\
\hline 346 &,$\quad$, & 白水 & h. & affl. of 358. & \\
\hline 347 &, & 白水 & ch. & affl. of 30. & \\
\hline 348 & $\begin{array}{l}\prime \prime \\
, \quad, \quad r\end{array}$ & 白水 & h. & affl. of 264 . & \\
\hline $348 \mathrm{~A}$ & t'a & 白塔 & & affl. of $2 \%$ & \\
\hline 349 &, mo & 百脈 & Lake & in Shantung. & Peh-11uh \\
\hline 350 & $P^{\prime} a i$ & 派 & $\operatorname{ch}^{\circ} \mathrm{i}$ & aftl. of 112 & \\
\hline 351 & P'an-lung & 盤龍 & h. & enters Annam from Yünnan. & Pwan-iun \\
\hline 352 & $\because, \quad$, & 盤龍 & h. & aff. of 371. & \\
\hline 353 & Pang & 袄 & h. & affl. of 546. & \\
\hline 354 & $\mathrm{Pao}$ & 瀑 & h. & trib. of $2 \% 1$. & \\
\hline 355 &, pu & 瀑布 & h. & affl. of 335 & \\
\hline 356 & & 䨌 & h. & affl. of 101. & \\
\hline $35 \%$ & Pei-ch'uan & 兆川 & h. & affl. of 155 . & \\
\hline 358 & , p'an & 北盤 & ch. & affl. of 96. & \\
\hline 859 & , sha & 北沙 & h. & affi. of 144 & \\
\hline 360 & Pei-yün & 北運 & h. & enters sea at Taku & Peiho \\
\hline 361 & & 渒 & h. & affl. of 144 & \\
\hline 362 & Pin & 涁 & ch’i & affl. of 233. & \\
\hline 363 & P'ing-tien & 降何 & h. & affl. of 226 . & \\
\hline 364 & - „, ch'ü & 呠坦 & h. & affl. of 125 & \\
\hline$\$ 65$ & Po-ssŭ-t'êng & 博斯騰 & Lake & in Harashar. & Bosteng \\
\hline 366 & Po-yang & 鄱婸 & Lake & in Kiangsi. & Poyang \\
\hline 367 & $\mathrm{Pu}-\mathrm{ku}$ & 布固 & ch. & affi. of 560 & \\
\hline 368 & Pu-lung-chi & 布隆吉 & il. & enters Lake Kara, Kansulı & Purunki \\
\hline 369 & P'u-yang & 尃陽 & h. & trib. of 49 & \\
\hline $3 \% 0$ & , êrh & 普洱 & h. & aff. of 233 & \\
\hline 371 &, tı & 普渡 & h. & affl. of 528. (Yünman) & Puto \\
\hline 372 & San & $\equiv$ & h. & trib. of 191 & \\
\hline 373 & ,, ch'a & 三永 & h. & affl. of 513 & \\
\hline 374 & , ch'uan & 三川 & li. & trib. of 148. (Shansi) & \\
\hline $3 \% 5$ & , chiang-shui & 三港水 & h. & affl. of 509 & \\
\hline $3 \%$ & Sang-kan & 杀草i & h. & affl. of $5 \% 3$. & Sangkan \\
\hline $37 \%$ &,$\quad l i$ & 桑里 & Lake & in Central Thibet. & \\
\hline 378 & Sè & 色 & h. & affl. of 133 & \\
\hline 379 & Sha & 沙 & h. & affl. of 151 . & \\
\hline 380 & ", & 沙 & h. & affl. of $21 \%$ & \\
\hline
\end{tabular}


xxxvii

THE PRINCIPAL RIVERS AND LAKES OF CHINA Continued.

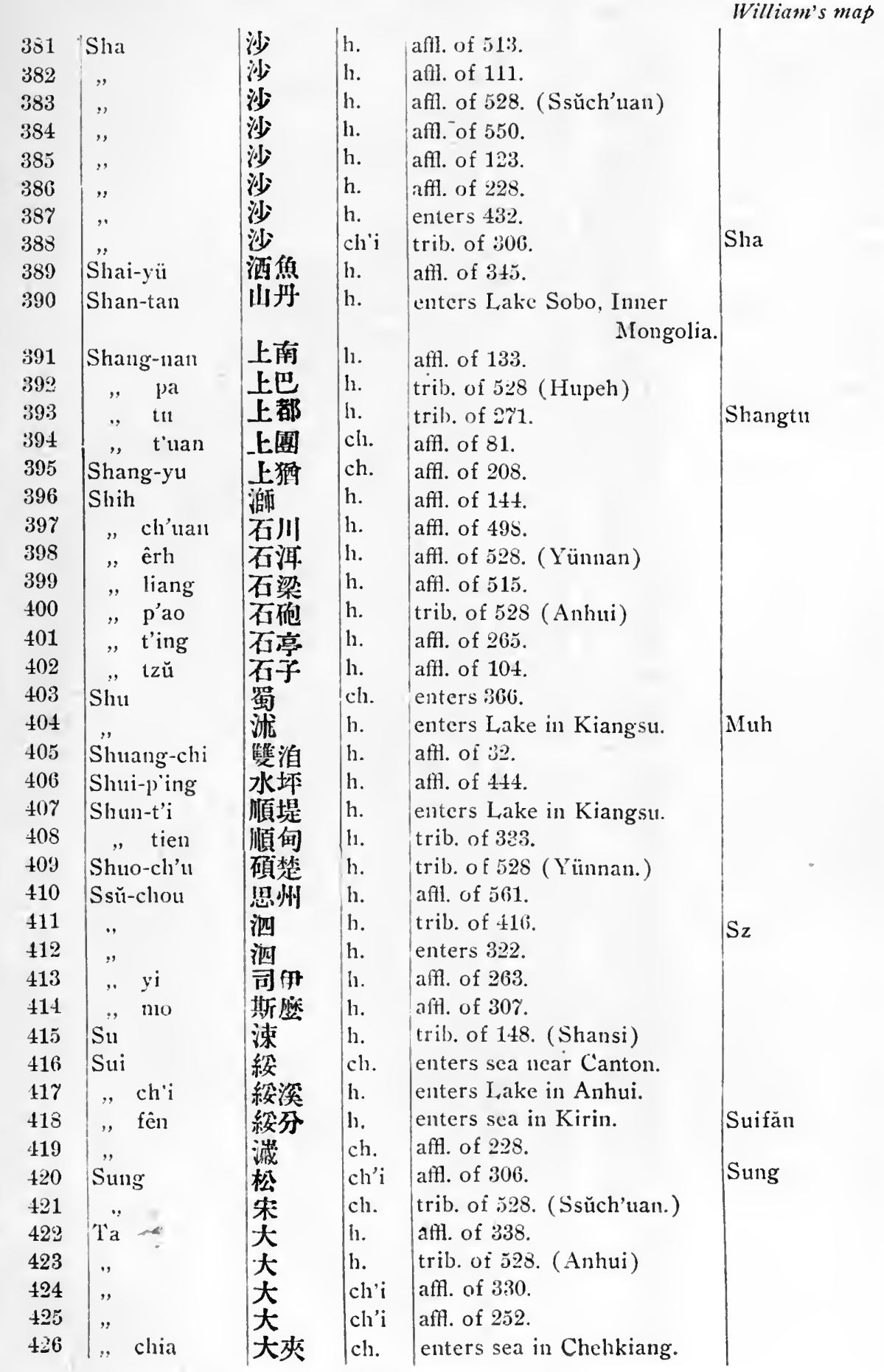




\begin{tabular}{|c|c|c|c|c|c|}
\hline $42 \%$ & Ta-chu & 大竹 & h. & affl. of 133 . & \\
\hline 428 & ,hsia & 大夏 & h. & trib. of 148 . (Kansuh) & \\
\hline 429 & , k'ai & 大開 & h. & trib. of 233 & \\
\hline 430 & , ling & 大凌 & h. & enters sea in Shingking. & 'Taling \\
\hline 431 & , 10 & 大羅 & h. & trib. of 528. (Yünnan) & \\
\hline 432 & ,lu-tsê & 大陸澤 & Lake & in Chihli. & \\
\hline 433 & „, chien-kou & 大澗挐 & h. & affl. of 133 . & \\
\hline 434 &, tu & 大渡 & h. & affl. of $30 \%$ & 'Tatu \\
\hline 435 & " уао & 大姚 & h. & trib. of 528. (Ssŭch'uan) & \\
\hline 436 & , ch'ang & 打计 & h. & trib. of 528 (Ssŭch'uan) & \\
\hline 457 &,, $\tan$ & 答旦 & h. & trib. of 528. (Yünnan) & \\
\hline 438 & T'a-ton & 塔嵬 & ch'i & trib. of 306. & \\
\hline 439 & 'T'ai-tzŭ & 太子 & h. & affl. of 158 . & \\
\hline 440 & Tan-t'ung shui & 射筒水 & h. & affl. of 165 . & \\
\hline 441 & Tan & 丹 & ch. & affl. of 133 & Tan \\
\hline 442 & , & 舟 & h. & trib. of 148 . (Honan) & \\
\hline 443 &. & 舟 & h. & ipper waters of 496 & \\
\hline 444 & T’a1ı & 潭 & ch. & enters sea near Canton. & \\
\hline 445 & , zang & 潭陽 & Lake & in Kiangsu. & \\
\hline 446 & T'ang & 港 & h. & affl. of 496 . & \\
\hline 447 & $"$ & 唐 & h. & affl. of 338 . & 'Tang \\
\hline 448 & & 唐 & h. & affl. of 101. & \\
\hline 449 & ", yai & 虍喾 & h. & affl. of 513 . & \\
\hline 450 & Tao & 道 & $\operatorname{ch}^{*} \mathrm{i}$ & affl. of 244 & \\
\hline 451 & , liu & 倒流 & h. & enters Lake in Anhui. & \\
\hline 452 & T"ao & 桃 & ch. & affl. of 208 & \\
\hline 453 & & 桃 & h. & affl. of 143 . & \\
\hline 454 &, $\operatorname{lin}$ & 䄻林 & $\operatorname{cln} \mathrm{i}$ & trib. of 61 & \\
\hline 155 & $"$ & 洮 & h. & trib. of 148. (Kansuh) & Tau \\
\hline 456 & Têng & 等 & ch'i & affl. of 416 & \\
\hline 457 & T'êng & 藤 & ch. & affl. of 416 . & \\
\hline 458 & Ti-shui & 滴水 & h. & affl. of 123 . & \\
\hline 459 & $\mathrm{Ti}$ & 㭽 & h. & enters 325. & \\
\hline 460 & Tien-shan & 店山 & h. & enters 366 . & \\
\hline 461 & & 溴 & Lake & in Yünnan. & \\
\hline $46 ?$ & Tien-shui & 甜水 & h. & affl. of 219 . & \\
\hline 463 & 'Ting-k'uei & 丁癸 & ch'i & affl. of 560 . & \\
\hline 461 & T’ing & 汀 & ch. & affl. of 134 & \\
\hline 465 & T’o & 沱 & h. & affl. of 144 & \\
\hline 466 & $"$ & 托 & ch'i. & trib. of 33 & \\
\hline $46 \%$ & , lai & 托賴 & h. & $\begin{array}{r}\text { enters Lake Sobo, Inner } \\
\text { Mongolia. }\end{array}$ & \\
\hline 468 & 'Tou & 䝶 & ch. & enters sea in Kuangtung. & \\
\hline 469 & 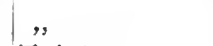 & 陡 & h. & affl. of 133 & \\
\hline 470 & Ts'ai & 唀 & h. & affl. of 550 . & \\
\hline $4 \% 1$ & Tsê-ti & 則底 & h. & affl. of 258 . & \\
\hline $4 \% 2$ & Tsêng & 䏆 & h. & aff. of 133 . & \\
\hline
\end{tabular}


THE PRINCIPAL RIVERS AND LAKES OF CHINA Continued.

\begin{tabular}{|c|c|c|c|c|c|}
\hline 473 & $\mathrm{Tu}$ & 都 & ch. & affl. of $1 \% \%$. & \\
\hline 474 & , shan & 㛚山 & h. & affl. of 4 \% 3 & \\
\hline 475 & I'u-êrh-kên & 㽞閶根 & h. & trib. of 148. (Shansi) & \\
\hline 476 & , hai & 徒駭 & h. & enters sea in Shantung. & \\
\hline $47 y$ & Tuan & 满 & h. & aff. of 338. & \\
\hline 478 & Tung & 東 & ch. & enters sea near Canton. & Tung \\
\hline 479 & " an & 東安 & ch. & trib. of 528. (Ssŭch'uan) & \\
\hline 480 & ", chieh & 東界 & h. & aff. of 49 & \\
\hline 481 & ,h hsiao & 東小 & ch. & affl. of 274 . & \\
\hline 482 &, liao & 東遼 & h. & affl. of 246 . & \\
\hline 483 & : tsung & 東㥎 & h. & affl. of 408 . & \\
\hline 484 & " wên & 東洨 & h. & affl. of 546 . & \\
\hline 485 & ,, ko shui & 洞娍水 & h. & affl. of 123. & . \\
\hline 436 & T'ung & 淕 & h. & aff. of 144 . & \\
\hline 487 & " ch'i shui & 桐溪水 & h. & trib. of 49 & \\
\hline 483 & , shan & 桐山 & $\operatorname{ch}^{\prime} \mathrm{i}$ & enters sea in Chehkiang. & \\
\hline 489 & Tž̆ & 浛 & h. & enters sea in Shantung. & \\
\hline 490 & , & 筫 & ch. & enters 556 & Tsz'kiang \\
\hline 491 &, & 㬎。 & h. & affl. of 101 . & \\
\hline 492 & , t'ung & 梓潼 & h. & affi. of 125 . & \\
\hline 493 & Wải-hsieh & 外卸 & $\operatorname{ch}^{\prime} \mathrm{i}$ & trib. of 330 . & \\
\hline 494 & Wang-lu & 絧鹿 & ch'i & trib. of 528. (Ssŭch'uan) & \\
\hline 495 & Wei-yüan & 威遠 & h. & affl. of 204 & \\
\hline 496 & & 徫 & h. & enters 322 & \\
\hline 497 & Wei-shui & 湋水 & h. & aff. of 508 & \\
\hline 498 & Wei & 渭 & h. & trib. of 148. (Shensi) & Wei \\
\hline $498 \mathrm{~A}$ &, & 溜 & h. & aff. of 123 . & \\
\hline 499 & $"$ & 魏 & h. & trib. of 148. (Shantung) & \\
\hline 500 & & 䧽 & h. & enters sea in Shantung. & \\
\hline 501 & Wên-shui & 交水 & h. & affl. of 123. & \\
\hline 502 &, & 汶 & h. & enters 322. & \\
\hline 503 &, & 汶 & h. & affl. of 500 . & \\
\hline 504 & iVêng & 复 & ch. & affl. of 22 & \\
\hline 505 & Wu-ch'iang & 武强 & ch'i & affl. of 49 . & \\
\hline 506 & Wu-hu-shui & 耻湖水 & h. & atf. of 133. & \\
\hline 507 & , shui & 武水 & h. & affl. of 22 . & \\
\hline 508 &, t'ing & 武亭 & h. & affl. of 498 . & \\
\hline 509 & "yang shui & 武降水 & li. & enters 366 & \\
\hline 510 & $"$ ch’i & 五溪 & h. & trib. of 528 . (Antui) & \\
\hline 511 & , lang & 五郎 & h. & trib. of 528 . (Yünnan) & Wulang \\
\hline 512 & " tao ch'uan & 五道川 & h. & affl. of 133 & \\
\hline 513 & & 烏 & ch. & affl. of 523. (Kueichou) & - \\
\hline 514 &, chao & 舄集 & ch. & affl. of 561 & \\
\hline 515 & "hsia & 鳥下 & ch'i & affl. of 416 & \\
\hline 516 & "lan-mu-lun & 泉䒽标 & h. & trii. of 148 . (Shansi) & \\
\hline 517 & , lung & 奥龍 & ch. & enters sea in Fuhkien. & \\
\hline
\end{tabular}

William's map. 
THE PRINCIPAL RIVERS AND LAKES OF CHINA Continucd.

\begin{tabular}{|c|c|c|c|c|c|}
\hline & & & & & 'illiam's \\
\hline 518 & Wu-ni & 鳥泥 & ch. & affl. of 416 . & \\
\hline 519 &,$\quad \ldots$ & 媝 & ch. & enters 366. & \\
\hline 520 & , chiang & 嬥港 & h. & affl. of 231. & \\
\hline 521 & Yang-ch'ang & 羊腸 & h. & affl. of 24 & \\
\hline 522 & & 洋 & h. & affl. of 573 . & \\
\hline 523 & "chin-sliui & 楊晉水 & h. & affl. of $6 \%$ & \\
\hline 524 &, lin & 楊林 & Lake & in Kiangsi. & \\
\hline 525 & , tang & 楊桑 & Lake & in Hupeh. & \\
\hline 526 & , shui & 楊水 & ch. & affl. of 13 . & \\
\hline $32 \gamma$ &, ts'un & 楊村 & ch. & enters sea near Canton & \\
\hline 528 &,, tzŭ & 楊子 & ch. & enters sea near Shanghai. & Yangtsze \\
\hline 529 & ,' & 陽 & ch. & enters sea in Kuangtung. & \\
\hline 530 & Yang & 隄 & ch. & affl. of 560. & \\
\hline 531 & , ch'i & 隄溪 & h. & affl. of 73 & \\
\hline 532 & ,hsin & 隄辛 & h. & trib. of 528 . (Hupeh) & \\
\hline 538 & Yao-hsien & 姚遚 & 1 . & affl. of 415 . & \\
\hline 534 & Yen-ching & 鹽井 & h. & affl. of 436 . & \\
\hline 535 &, t'an & 監潭 & ch'i & affl. of 30. & \\
\hline ذ36 &, , slius & 延水 & h. & trib. of $1 \pm 8$ (Shansi) & \\
\hline 537 & , shout & 延䩧 & ch'i & enters sea in Fuhkien. & \\
\hline 538 &, po & 晏波 & h. & affl. of 30. & \\
\hline 539 & & 剡 & $\operatorname{ch}^{\prime} \mathrm{i}$ & enters sea near Ningpo. & \\
\hline 540 & Yi-mei & 臬梅 & h. & affl. of 21 . & \\
\hline 541 & ., huang & 冝黄 & h. & affl. of 509 . & \\
\hline 542 & , & 茙 & ch. & affl. of $1 \% \%$ & \\
\hline 543 & , & 義 & h. & aff. of 123 . & \\
\hline 544 &, & 易 & ch. & affl. of 463 . & \\
\hline 545 & , shui & 易水 & h. & trib. of 360. & \\
\hline 546 & , & 沂 & h. & enters Lake in Kiangsu. & I-ho \\
\hline 547 & ,, kuan & 以灌 & h. & trib. of 528. (Yünnan) & . \\
\hline 548 & Yin-lung & 銀龍 & h. & affl. of 233 . & \\
\hline 549 & 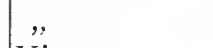 & 引 & h. & aff. of 360 . & \\
\hline 550 & Ying & 穎 & h. & affi. of 144 . & \\
\hline 551 & Yu & 游 & $\operatorname{ch}^{\prime} i$ & trib. of 528. (Ssŭch'uan) & \\
\hline 552 & $\mathrm{Y} \ddot{\mathrm{u}}$ & 箣 & h. & affl. of 208 . & \\
\hline 553 & , hai & 魚旅 & h. & affl. of 125 . & \\
\hline 554 & , yang & 漁洋 & h. & affl. of 74 & \\
\hline 555 & , fên-shui & 蔚沙水 & h. & trib. of 148 . (Shansi) & \\
\hline 556 & , shui & 越水 & h. & enters sea in Kuangtung. & \\
\hline 557 & , & 王 & h. & aifl. of 528. (Y ünnan) & \\
\hline こૅ8 & , tai & 玉愽 & h. & affl. of 360. & \\
\hline 559 &, & 御 & h. & affl. of 376. & \\
\hline 560 & Yüan & 元 & ch. & enters Annanı from Yünnan & Songka \\
\hline 561 &, & 沅 & ch. & enters 586 & Ytuen \\
\hline 562 & , & 洹 & h. & affl. of 496 & \\
\hline 563 & , shui & 洹水 & h. & affl. of 528. (Ssŭch'uan) & \\
\hline 564 & Yüeh-sui & 越售 & h. & affl. of 434 & \\
\hline 565 & Yün-chung & 紫中 & h. & affl. of 143. & \\
\hline
\end{tabular}


THE PRINCIPAL RIVERS AND LAKES OF CHINA Continucd.

William's map.

\begin{tabular}{|c|c|}
\hline 566 & Yün-lung \\
\hline 567 & , mèn \\
\hline 568 & ", \\
\hline 569 & $\therefore$ \\
\hline 570 & Yung-fu \\
\hline 571 & ,, ning \\
\hline 572 & ", \\
\hline ة\%3 & , ting \\
\hline
\end{tabular}

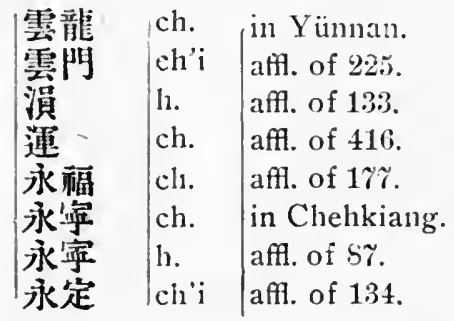

$\mid$

Other Notabe Rivers and Lakes in China, on which none of the towns enumerated in this volume is Situated.

\begin{tabular}{|c|c|c|c|c|c|}
\hline 574 & Chin-yün & 舊運 & h. & $\begin{array}{l}\text { Branch of Grand Canal } \\
\text { between Shantung and } \\
\text { Kiangsu. }\end{array}$ & $\begin{array}{l}\text { Old Grand } \\
\text { Canal }\end{array}$ \\
\hline 575 & Hei-lung & 黑龍 & ch. & in Manchuria & Amoor. \\
\hline $5 \% 6$ & Kan & 甘 & h. & ต1. of 581 . & $\mathrm{Ka}$ \\
\hline 577 & Kao-yı & 高郵 & Lake & in Kiangsu. & $1-y_{11}$ \\
\hline 578 & Li-hsien & 李仙 & ch. & affl. oi 560. & Li-sien-kiang \\
\hline 579 & Lung-ch'uan & 龍了 & h. & enters Anman from Yünnan. & $\left\{\begin{array}{l}\text { Lun } \\
\text { or } \mathrm{S}\end{array}\right.$ \\
\hline 80 & $\mathrm{Nu}$ & 㤂 & ch. & enters Burmah from Yünnan. & $N_{11}$ \\
\hline 81 & $\mathrm{Nu}$ & & h. & $\%$ & Nonin \\
\hline 82 & San-ch'a & 三登 & h. & enters Annam from Yünnan & Sanclia \\
\hline 83 & hua & 松花 & & trib. of $5 \% 5$ & $\mathrm{Su}$ \\
\hline 884 & T'ai & & Lake & in Kian & Ta \\
\hline 585 & T'u-mèn & 圆門 & ch. & $\begin{array}{l}\text { on frontiers of Shing king and } \\
\text { Corea. }\end{array}$ & Tumen \\
\hline 86 & Tung-t ing & & Lake & & \\
\hline 87 & & & Lake & in $\mathrm{Ki}$ & \\
\hline 88 & Wi1 & 烏蘇里 & ch. & tril). & \\
\hline 559 & Ya-lu-hang-pu & $\begin{array}{l}\text { 雅荷 } \\
\text { 藏在 }\end{array}$ & ch. & flows iinto India from Yünnan. & Brahmapootra \\
\hline 590 & ," & 鴨綠 & h. & $\begin{array}{l}\text { on fronticr of Shingking and } \\
\text { Coreat. }\end{array}$ & Yaluh \\
\hline 91 & Yung-ting & & h. & trib. of 360 . & Hun. \\
\hline
\end{tabular}


xlii

NAMES OF RIVERS AND LAKES AS ROMANISED IN WILLIAMS' MAP OF CHINA.

\begin{tabular}{lr|l} 
Amoor & 575 & Meikong \\
Bosteng & 365 & Min \\
Brahmapootra & 589 & Muh \\
Chih shwui & 51 & Nan ting \\
Chu lung & 101 & North River \\
Făn & 123 & Noun \\
Grand Canal & 322 & Nu \\
Han & 133 & Old Grand Canal \\
Hu to & 143 & Peh hu \\
Hung tsih & 591 & Peh muh \\
Hwai & 144 & Pei \\
Hwang poo & 152 & Peiho \\
I ho & 591 & Poyang \\
Ju & 200 & Purtungki \\
Kan & 208,576 & Puto \\
Kan yu & 577 & Pwanlung \\
Kia ling & 30 & Salwen \\
Ko & 213 & Sancha \\
Kü & 110 & Sangkan \\
Lantsan & 232 & Sha \\
Liau & 246 & Shangtu \\
Lisienkiang & 578 & Shweli \\
Loh & $263,261,265$ & Si-ning \\
Loha & 239 & Siang \\
Lung & 273 & Sin \\
Lung chuen & 579 & Songari \\
Lwan & 271 & Songka \\
& & \\
\hline & &
\end{tabular}

\begin{tabular}{|c|c|c|}
\hline 232 & Sui făn & 418 \\
\hline $306,30 \%$ & Sung & 420 \\
\hline 404 & $\mathrm{Sz}$ & 411 \\
\hline 321 & Ta liia & 428 \\
\hline 22 & T’a ling & 430 \\
\hline 581 & Ta tu & 434 \\
\hline 580 & Tai hı1 & 584 \\
\hline 574 & Tan & 441 \\
\hline 325 & Tang & 447 \\
\hline 349 & Tau & 455 \\
\hline 125 & Tsientang & 49 \\
\hline 360 & Tsin & 64 \\
\hline 366 & Tsing & 74 \\
\hline 368 & Tsing shwui & 83 \\
\hline 371 & Tumen & 585 \\
\hline 351 & Tung & 478 \\
\hline 580 & Tungting & 556 \\
\hline 582 & Tzkiang & 490 \\
\hline $376^{\circ}$ & Urh hai & 121 \\
\hline 230 & Usuri & 588 \\
\hline 393 & Wei & 498 \\
\hline 579 & West river & 165 \\
\hline 155 & Wishan & 587 \\
\hline $17 \%$ & Wulang & 511 \\
\hline 192 & Yaluh & 590 \\
\hline วั83 & Yangtsze & 528 \\
\hline 560 & & \\
\hline
\end{tabular}




\section{8.-RADICAL INDEX}

\section{$1 \longrightarrow Y I$}

- Yi 5688 .

1 J Ting 6463.

七 Ch'i 661,47\%8.

2 丈 Chang 22S, 3253,487\%.

下 Hsia Passim.

三 San 194, 3700, 5353, 7328.

上 Shang $\{848,2492,32 \gamma 8, " 443 \gamma$,

不 $\mathrm{Pu} \quad\left\{\begin{array}{l}3233,4539,4555, \\ 4665,5280 .\end{array}\right.$

4 且 Ch'ieh 842.

世 Shih 7968,6081.

5 丞 Ch'êng 532.

王 Tiu 6473 .

7 正 Ping 4246 .

\section{$2 \mid$ KUN}

3 中 Chung Passim

\section{$3 \cdot \mathrm{CHU}$}

3 丹 Tan $\left\{\begin{array}{l}1465,4626,5462 \mathrm{~J}, \\ 5464,5822,5967,6198, \\ 6904,7185,7193 .\end{array}\right.$

4 进 Chu 1379

\section{$4 \int \mathrm{P} \cdot \mathrm{IEH}$}

1 乃 Nai 4555.

2 人 Chiu 1275.

3 之 Chih $97 \%, 6811$.

4 乍 Ch'a 152,6503.

7 非 K'uai 3340 .

9 乘 Ch'êng 529, 733, 941, 1754.
$5 乙 Y I$

$\begin{array}{rll} & \text { 乙 Yi } & 6586 . \\ 1 \text { 兑 Chiu } & 12 \% 8 . \\ 2 & \text { 乞 Chi } & 656,7206 . \\ \text { 也 Yeh } & 7342 . \\ 7 \text { 乳 Ju } & 3020 . \\ 10 & \text { 乾 Chien } & 920,2446 .\end{array}$

\section{$6 \int \mathrm{CHÜEH}$}

1 了 Liao 687\%.

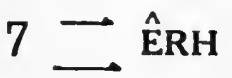

二 Erh 1619 .

1 于 Yü $2512,5469,5834,7673$.

2 云 $Y$ ün $3232,7344,7 \% 96$.

井 Ching Passim.

五. $\mathrm{Vu} 2508,7108$.

6 亞 $\mathrm{Ya} 7219$.

\section{8 ¿ T'OU}

2 䒕 K'ang $1711,4192$.

4 交 Chiao $783,2441,4055,535 \AA$.

亦 $\mathrm{Yi} \quad 4258,6011$.

6 亨 Hsiang 4506, 7521.

京 Ching Passim.

$\gamma$ 芫 Liang 3800,6325 .

苧 T'ing Passim.

8 毫 Po 5229.

18 舟 Mên 3182. 


\section{9 人 JÊN}

\begin{tabular}{|c|c|}
\hline 人 Jèn & $\left\{\begin{array}{l}1810,2981,3810,4692, \\
48 \% 1,4893,5833,5851, \\
62 \% \% .\end{array}\right.$ \\
\hline 仇 Ch'ou & $13+\%$ \\
\hline 二 Jên & Passim. \\
\hline 们 Chieh & 818. \\
\hline Chin & 1068. \\
\hline 付 Shih & Passim. \\
\hline 命 Ling & $3293,3926$. \\
\hline Liell & 3836. \\
\hline 仙 Hsien & $1354,2 \% 05, .5608,706 \%$. \\
\hline 他 ' I'a & $6136,68 \% 8$ \\
\hline 代 Tai & $615 \%, 6752$. \\
\hline 伐 Fa & 5162. \\
\hline 仰 Yang & $\% 244$ \\
\hline 伏 Fu & $1886,482 \%$. \\
\hline 休 Hsiu & $81 \%, 2856$. \\
\hline 伊 $\mathrm{Yi}$ & $611 \%, 6628, \gamma 483$. \\
\hline 仵 $\mathrm{Wu}$ & $\gamma 200$ \\
\hline 任 Jên & $2540,2982$. \\
\hline 价 Chieh & \$21. \\
\hline 伤 Wu & 8126. \\
\hline 住 Chu & 2744 \\
\hline 你 $\mathrm{Ni}$ & $591,4665$. \\
\hline 低 $\mathrm{Ti}$ & 6349 \\
\hline 余 Yü & $4046, \gamma 643$ \\
\hline 位 $\mathrm{Wei}$ & $69 \% 8$. \\
\hline 佛 Fo & $1 \%$ ชช, 5\%08. \\
\hline 何 $\mathrm{Ho}$ & 2094 \\
\hline 估 Ku & 4905 \\
\hline 伯 Po & $\left\{\begin{array}{l}252 \%, 2565,3189,3524, \\
46 \% 1,5235,5568,6665 .\end{array}\right.$ \\
\hline 但 'Tan & 6213 \\
\hline 佐 'Tso & $3122,6569.7524$ \\
\hline 作 'so & $65 \% 2$ \\
\hline 佶 Chi & 593. \\
\hline 侍 Shil & $568 t$. \\
\hline 佳 Chia & 2364 \\
\hline 秝 Lai & $\left\{\begin{array}{l}610,963,1819,2209, \\
2368,3556,4524,5938, \\
6303, \text { \% } 88 .\end{array}\right.$ \\
\hline 侯 Hou & 2139 \\
\hline 保 Pao & $\left\{\begin{array}{l}393,2094,290 \%, 4946, \\
6419 .\end{array}\right.$ \\
\hline 便 Pien & 5110. \\
\hline 信 Hsin & $\left\{\begin{array}{l}355,1510,1590,2800, \\
3400,5213,6036,6953, \\
7263 .\end{array}\right.$ \\
\hline
\end{tabular}

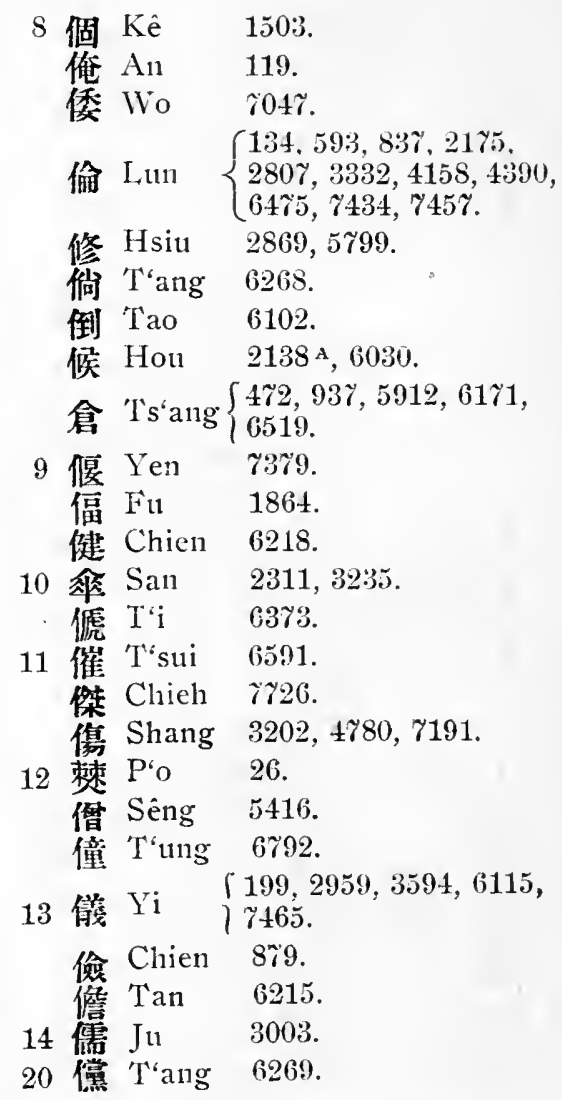

\section{0 儿ÊN}

1 兀 Wu $\{4263,466 \%, 5339.7005$,

2 元 Yüan Passim.

允 Yün 3906, 504t, 7824 .

3 充 Ch'ung 1588, 2453, 4568 s.

4 兆 Chao 1221.

光 Kuang $\left\{\begin{array}{l}2998,3430,3963, \\ 4588,5753,6727 \\ 6934,7245,7956 .\end{array}\right.$

先 Hsien 1456, 176\%.

5 克 $\mathrm{K}^{\prime} \mathrm{e}$ Passinn.

兔 $\mathrm{Tu} 4861,6635$.

6 兒 Erh Passim.

兵 Ping 5132.

$\gamma$ 交 Yen \%372. 


\section{1 入 ${ }^{\mathrm{ju}}$}

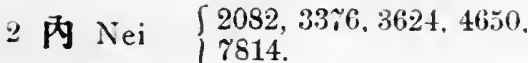

4 소 $\mathrm{Ch}^{\prime}$ üan $1483,6414,6893$.

6 角 Liang $3 \% 60$

$\gamma$ 角 $\mathrm{Y} \ddot{\mathrm{u}} \mathrm{i} 6.59$.

\section{2 八 $\mathrm{PA}$}

入 $\mathrm{Pa}\left\{\begin{array}{l}23,31,32,224,298 . \\ 4795,5099,6631 . \\ 7490,7519 .\end{array}\right.$

2 公 Kung Passim.

兵 Liu 4005, 6494 .

4 热 Kung 3509 .

6 其 $\mathrm{Ch}_{1} \mathrm{i} \quad\left\{\begin{array}{l}1506,1815,2334,5230, \\ 6993,\end{array}\right.$

"Chi 1391.

具 Chü 1419.

14 翼 Chi 5í6.

\section{3 「J chIUNG}

3 厄 Hui $\{2349,236 \%, 369 \%, 7114$,

6 周 Kang Passim.

9 笔 Mien $4+21$.

\section{$14 \mapsto \mathrm{MI}$}

2 圥 Jung ror1.

$\gamma$ 冠 Kuan 336\%.

8 宗 Ming 4480.

9 冢 Chung 5224.

\section{5 У PING}

3 冬 Tung 6479.

冲 Chiung 4552, 6300 .

5 汾 Lêng 1507, :3679. 治 Yeh $\left\{\begin{array}{l}2785,3271,6110,6 \% 60, \\ 7330 .\end{array}\right.$

8 凉 Liang $\int 24 \% 4,3085,3144,3796$.

(4131, 5171,6\%35

凌 Ling 2661, 3943.

谏 Tung 5.509.

\section{$1 6 \longdiv { \mathrm { CHI } }$}

1 凡 Fan $162 \%$.

原 Huang $1 \% 22$.

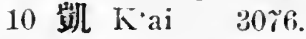

\section{7 | K·AN}

4 [II] $\mathrm{Wa} \quad \gamma 23,3210$.

6 函 Han 1988.

\section{TAO}

ᄀ 'Tiao 637\%.

2 分 Fên $168 \%$.

犾 $\mathrm{Yi} \quad 1511$.

4 刑 Hsing 5161 .

列 Lieh 1906, 3810.

$\therefore$ 利 $\mathrm{Li}$ Passim.

初 $\mathrm{Cl}^{\circ}$ ㄴ 851, 1399, 1948.

别 Pieh $39336,5099$.

删 Shan 5+64.

$\gamma$ 則 Tsê $2997,5 \% 90$.

制 Chih 9s2.

刺 La Passim.

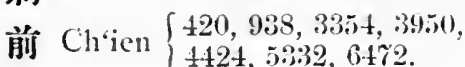

8 㓠 Yen itof.

"Shan 5tris.

峒 Kang 3112.

13 劍 Chien 880, 4568'.

劉 Liu $\quad 3994$.

剧 Chi 55 .

\section{9 力}

J $\mathrm{Li}\left\{\begin{array}{l}1 \%,+01,3 \%+9,4290, \\ \gamma 520 .\end{array}\right.$

3 加 Chia 690 .

功 Kung 3506, 4496, 8076, 6155 .

$\%$ 勇 Yung 1841, $28 \% 9$.

8 務 $\mathrm{Wu}_{\mathrm{u}} 65+4, \mathrm{r} 201$.

9 勒 Lê $\{15,2526,3330,3663$, 勒 Lè $\left\{\begin{array}{l}4406,4906,5924,6146, \\ 748 \% .\end{array}\right.$

10 等 Lan 5245, 54ri.

勝 Shêng Passim.

11 勤 Chin $10 \% 0,1609,5856.8059$.

14 顋 Hsïn 2943.

17 勸 Ch'iian 1482,411\%. 


\section{$20 / 7$ PAO}

2 匀 Yün 6631.

3 包 $\mathrm{Pao} 4965$.

\section{$21 \leftarrow$ PI}

2 化 Hua Passim.

3 北 Pei Passim.

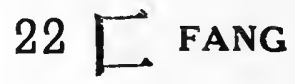
4 压 K'uang 3439.
厈 Chiang 118.
5 臧 Hsia $5 \% 10$.
12 匱 Knei 1030.

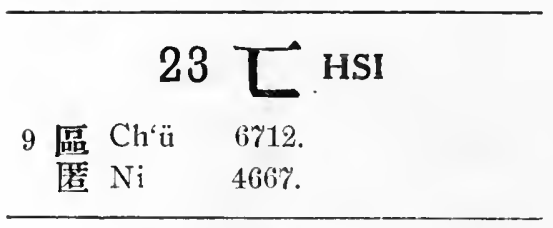

\section{$24-\mathrm{SHIH}$}

+ Shih 5391, 5686 .

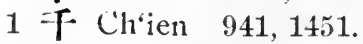

2 升 Shêng 5638 .

午 $\mathrm{IV}_{\mathrm{u}} \mathrm{7209}$.

6 卑 Pei 5028, 6899.

卓 Cho 1328, 1900.

協 Hsieh 2672 .

7 南 Nan Passim.

10 㙛 Po $1895,2550,4723,5238$.

\section{PU}

ト $\mathrm{Pu} \quad\left\{\begin{array}{l}2385,4336,4666.528 \%, \\ 6842, \gamma(046 .\end{array}\right.$ 3 占 Chan $166,2414$.

\section{6 П}
3 邱 Mao 4344 .
4 印 Yin 7559 .
5 却 $\mathrm{Ch}^{\prime}$ üeh 1433.
6 卷 Chïan 1480 .
7 即 Chi 601 .

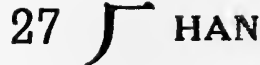

6 厔 Chih 1346.

7 厚 Hou 2142.

席 $\mathrm{i}$ “ $63 \% 1$.

8 底 Ts'o $65 \% 4$.

原 Yïan Passim.

厥 Chüeh 1501.

10 厦 Hsia $25 \%$.

12 哃 Ch'ang 6066, 6410.

17 痛 P'ang 589, 6623.

\section{$28 \perp$ MOU}

2 厹 Ch'iu 1299 .

9 幾 Ts'an 6565.

,, Shên $193 \%$.

\section{$29 \mathbb{X}$ YU}

1 叉 $\mathrm{Ch}^{\prime} \mathrm{a} 150,3320$.

2 叉 Chao 2638, 2802, 4560 .

6 受 Shou $\left\{\begin{array}{l}1549,1894,2494,5758 \text {, } \\ 6750 .\end{array}\right.$

双 Shu 2110 .

取 $\left.\begin{array}{l}\text { Ch'ü or } \\ \text { Chiu }\end{array}\right\} 1466,4668$.

8 無 $\begin{gathered}\text { Chia, } \\ \text { hsia }\end{gathered}$ \%14.

段 Tuan $66 \% 6$.

叙 Hsü 2894.

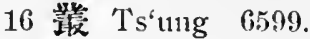

\section{0 П $\mathrm{k} \cdot \mathrm{OU}$}

口 K'on Passim.

2 石 Chao $336,4563$.

何 Chü 1408,3136, 313\%, 6916 .

,Ch'ii 1436, 2913.

" Kou 3246, 7r72.

區 $\mathrm{P}^{\prime} \mathrm{o} 5259$.

只 Chih 973,4881 .

史 Shil $465 \%$.

昛 Ch'ih 1006.

if $\mathrm{Ku}$ Passim.

可 $K \hat{e} \quad 3232$.

司 Ssir $\left\{\begin{array}{l}808,14 \% 3,2165,4622, \\ 58 \% 6 .\end{array}\right.$ 
台 T'ai Passim.

右 $\mathrm{Yu}_{\mathrm{ul}} \quad 4236,5462^{\mathrm{T}}, \mathrm{\gamma} 288, \tilde{\gamma} 619$.

3 向 Hsiang 2584.

合 Ho 2045, 2120,4007, 5155 .

者 Chi Passim.

名 Ming 4460, 4504, 6065 .

些. T'u 6068 .

同 T'ung $\left\{\begin{array}{l}2945,2493,3563,4253, \\ 58 \% 1,0102,6645,0 \% 98 .\end{array}\right.$

4 咥 Cheng 895,4568 A

吹 Ch'ui $150 \%$.

含 Han 1989, 2044 .

君 Chïn $404,7 \pm 24$.

魚 Lii 4144.

忝 T'ull 6696 .

五 $W^{551,1363,15 \% 5,3744, ~}$ 吾 $W_{11}\left\{\begin{array}{l}4085,5327,5879,7493, \\ 7494,\end{array}\right.$ 㟧 $\mathrm{Wu} \quad 106,2 \% 91,3640,7130$.

5 周 Chou 1339, 1458, 2449, 7254.

呼 $\mathrm{Hu} \quad 1403,1912,21 \%$.

呾 Tan 6218.

呚 Hsia 2584.

和 Ho Passim。

各 Ch'iu 1298.

命 Ming 651 .

味 Wei 4364,6116.

"Mei 4364.

6 威 Hsien 2686 .

哈 $\mathrm{Ha}$ Passin.

恦 Hsiang 4262 .

唃 T'sa J2116, 2592, 3209, 3238 ,

哇 $\mathrm{Wa} 29 \% 8$.

7 吹 Chia 729.

哩 $\mathrm{Li} 632 \%$.

哲 Chê 4743,5695 .

哥 Kê 3205,5226 .

揹 Shao 5580 .

唐 T'ang $\left\{\begin{array}{l}2845,3160,5121,6246, \\ 3302,6508,\end{array}\right.$

員 Y̌̈ian ry\%9.

8 商 Shang 4810,60\%\%。

開 Wên ros4.

唯 Wei 3564, 7000 .

㖶 $\mathrm{Ya} \quad 3551,7220$. 9 声 Hsi $\left\{\begin{array}{l}2429,4187,4516,4738 \text {, } \\ 68 \% 3, \gamma 036 .\end{array}\right.$

喬 Chíao 803.

喀 K’ê 15, 2056,3220,3329.
喇 La Passim. 普 Shan $\left\{\begin{array}{l}703,1475,1595,2404, \\ 3460,3791,5474,6901,\end{array}\right.$ ช $438,796 \%$.

單 Tan $4 \pi 06$.

Shan $546 \%$.

10 唯 Ma 4312.

愉 $\mathrm{Yü} \quad 2 \% 9 \%, 7660$.

11 嘉 Chia $\left\{\begin{array}{l}695,1946,1992,2363, \\ 2422,5356,6409,6704, \\ 7840 .\end{array}\right.$

咽 Kno $68 \% 5$.

13 荧 Kê Passim.

15 喚 Lu 19.

16 暗 Lung $68 \% 5$.

17 嚴 Yen 1019, 7393.

19 萿 Nang 4258, 4789.

\section{$31 \square$ WEI}

2 四 Sisŭ 5885.

3 回 Hui $\int 2349,2367,3637$,

因 Yin $3049,4806^{3}, 7556$.

4 园 Yün 4359.

5 固 $\mathrm{Ku} \quad\{79,51 \%, 1743,2469$,

7 周 Yin

闻 $\mathrm{Pu} 2061,2308,529 \%$ 。

8 圈 $\mathrm{Ch}^{\circ}$ üan $722 \%$.

國 Kuo 1\%i70,2824,3547,4696.

9 園 Wiei 4043, 7032.

10 園 Y ïan $\left\{149,1385,3793,7 \% 01^{B}\right.$,

11 㘣 'T'u Passim.

橉 T'uan $3110,5891,6084$.

\section{$32 \pm \mathbf{T}^{\mathrm{T} U}$}

土 T'u $\left\{\begin{array}{l}2323,35 \% 6,4860,5536, \\ 6653 .\end{array}\right.$

3 地 $\mathrm{Ti} \int 4319,4546,5013,5974$, 圩 $\mathrm{Yii}$ \%org.

4 坊 Fang $\{1656,2750,4242,4281$,

坎 K'an 138; ${ }^{\mathrm{A}}, 2717,2718$.

坆 Fên 1697 . 


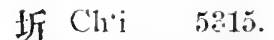

坂 Fan 5319 .

坑 K'êng $3245,6142,6389$.

5 坻 $\mathrm{Ch}^{6} \mathrm{ih} 4936$.

均 Chün 1521.

坐 'Tso 4965 .

胁 to 3722 .

坤 $\mathrm{K}$ 'un $4 \% \% 1, \% 4 \%$.

坡 Ping Passini.

$\{1216,1251,230 \%, 2490$. 坡 $\mathrm{P} \cdot \mathrm{O} \quad\{36 \% 2,5261,5462 \mathrm{~F}$.

6 㙅 To 6483.

捻 $\mathrm{Yin} 5544,666 \%$.

坦 Yuan Passim.

$\gamma$ t然 Ying 4358 .

垻 $\mathrm{Pa}$ Passim.

城 Chềng Passim.

垛 Ch'êng 6089.

埔 $\mathrm{Pu} \quad 2309,60 \% 6$.

8 基 Chi $49,560,675,1329.3 \% 24$ 。

壻 Sao 5415, 6368 .

焚 Yeh 4641 .

掔 Chien 891.

埧 $\mathrm{P} \cdot \mathrm{i} \quad 5285$

埠 $\mathrm{Pu}$ Passim.

埭 Tai 2168.5735.6159.

埼 $\mathrm{Ch}^{\circ} \mathrm{i} \quad 636$.

堂 T’ang $\left\{\begin{array}{l}1046,1550,4 \% 24,62 \% 0, \\ 6422 .\end{array}\right.$

堆 Tui 1051 .

倜 $\mathrm{Ku} \quad 5 \% 16$.

9 堪 K'an 3109 .

堡 Pao Passim.

報 Pao 4962 .

堤 $\mathrm{Ti} \backslash 104 \%, 1461,2 \% 80,4922$.

ก $5014,5 \% 40,6367$.

堯 Yao 8310 .

Y $198,202,765,164 \%$

垵 Yen $\left\{\begin{array}{l}2 \gamma 95,414 \%, 435 \%, 5 \% 46 . \\ 6464\end{array}\right.$

10 塚 Chung 3297.

塞 Sai $94,534 \%$ 。

塊 K’11ai 4286 .

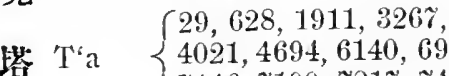

7146, 7190, 7217, 7491.

塔 'T'ang Passim.

棌 $\mathrm{T} \mathrm{i}, \mathrm{t}^{\mathrm{u}} \mathrm{u} \quad 6234,6635$.
11 㷪 Ch'ang $\left\{\begin{array}{l}7 \% 8,793,1365,3132, \\ 3247,3341,4170, \\ 53 \% 6,6702,7346 .\end{array}\right.$

麼 Ch'ên 3841,4108 .

墓 $\mathrm{Mu} 4 \% 1$.

矜 Sh11 1043.

熱 Tieh 6886 .

塹 Chan 1380 .

12 猺 Chui 2164.

塐 Fên 3007, 3859 . 墟 Hsü $\left\{\begin{array}{l}2 \% 62,3499,3911,6301, \\ 6812 .\end{array}\right.$

墨 Mo 611, 448\%

墩 Tun $173,2324,3103,6689$.

增 Tsêng 6152 .

13 鈿 Chiang \%r\%5.

壁 $\mathrm{Pi} \quad 410,3541,5412,6463$,

壇 T'an 1045, 5187.

15 壘 Lei 4526,5255 .

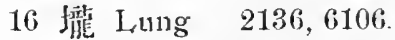

17 壈 Jang 2962.

21 墇 $\mathrm{Pa} 2300,3992$.

\section{3 工 $\mathrm{SHIH}$}

9 覃 $\mathrm{Hu} 21 \%$.

17 䃅 Shol $\left\{\begin{array}{l}2 \% 1,1963,2992, \\ 3414,3969,5749, \\ 6902 . \% 439,7969 .\end{array}\right.$

\section{5 攵 Tsur}

○夏 Hsia Passim.

„. Chia $\% 251$.

20 薆 $\mathrm{K}$ 'nei 3490.

\section{6 条 $\mathrm{HSI}$}

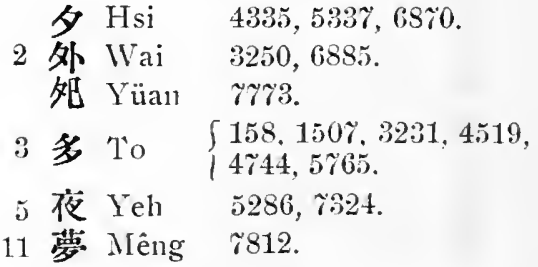




\section{$37-\mathrm{TA}$}

大 $\mathrm{Ta}\lceil 486,249 \%, 2498,4624$,

1 夫 $\mathrm{Fu} \quad 1810,4259$.

太 T'ai $\{604,2359,4625,5912$,

T'a 6135.

天 T.ien $\int 365,17 \% 3,5822,6405$,

2 夹 Yang 1 rivis.

失 Shih Passim.

3 灰 $\mathrm{Yi}$ Passim.

4 㚒 Chia $\% 24$.

5 奉 Fêng $1 \% 63$.

奇 $\mathrm{Ch}^{6} \mathrm{i} \quad 034,6146$.

奈 Nai 4556, 5011, 6488 .

花 Yen 7391 .

6 花 Pên 5042 .

奏 Tsou 5194, 6581.

奕 $\mathrm{Yi} 7526$.

7 奚 Hsi $\int 653,68 \% 0,63 \% 1,6872$,

9 花 She

10 鼠 Ao 121 .

11 栱 $\mathrm{S}$ 'o 5525.

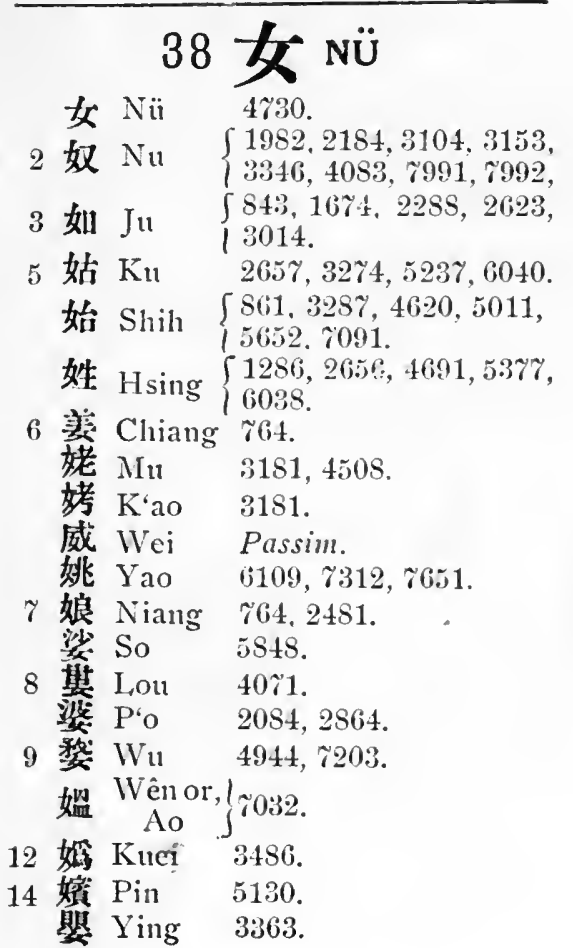

\section{9 子 TZŬ}

\section{子 Tzŭ Passim.}

1 孔 K'ung $2105,352 \%, 7525$.

3 骂 Тzŭ 565\%, 5690,6694.

存. Ts'un 7462 .

4 等 $\mathrm{F}_{13} 1791$.

㝵 Po 5226, 6036.

孜 Tzŭ 2104.

孝. Hsiao $804,1562,2046,2638$.

5 㧓 Ku $3298,582 \%$.

孟. Mêng 3621, 4345, 4385 .

7 孫 Sun 5965, 6490.

8 乾 Shu 3274 .

9 㨞 Ch'an 175.

\section{0 円 MIEN}

3 落 An Passim.

宅 Chai, tsê 159, 65t8.

4 芸 Hung 2389.

策 Sung 2496, 5993.

完 Wan 4535, 6909 .

$\left\{\begin{array}{l}10 \%, 282,5 \% 3,1150, \\ \text { 5 }\end{array}\right.$

5 宜 $\mathrm{Yi}\left\{\begin{array}{l}1686,2812,5025,5542, \\ 6116,5418,\end{array}\right.$ (6116, 7418 .

官 Kuan $\left\{\begin{array}{l}1064,1366,2139,3341, \\ 3503,3685,4889,650 \%, \\ 6806,7351 .\end{array}\right.$

䂞 Tang $\{2499,462 \%, 5012,6243$,

定 Ting Passim.

宗 Tsung Passim.

胣 Wan 5538,6103, 6913.

6

各 K'ê 4914, 5801 .

苗 Isüan 2923,7069 .

穿 Shih 3240.

穿 $\mathrm{Yu} 7631$.

$\%$ 家 Chia Passim.

笼 Ch'ên 5822,6844.

商 Jung Passim.

宮 Kung $\left\{\begin{array}{l}1542,3511,4194,4595, \\ 5822,5415 .\end{array}\right.$

寞 Yen 73\%\%.

8 汼: Mi | $190 \%, 3151,3219,4418$,

(4733, 6155, 6384, 7339.

î $\mathrm{ini1} 6119$.

寃 Yüan $7 \%$ \%.

寉 $\mathrm{Su} 526,1552,5914$. 
9 富 Fu 1844, 3345.

黄 Han 1986, 5104,614\%,635\%.

11 察 Ch'a $\left\{\begin{array}{l}154,15 \%, 4293,466 \% \\ 71 \% \% .\end{array}\right.$

宾 Ning Passim.

寨 Chai Passim.

䆓 Shih 1909, 2965, 5685.

寢 $\mathrm{Ch}^{\circ}$ in 1104.

12 筧 K'uan 3382.

13 壊 Huan 2263 .

17 顊 $\mathrm{Pao} \int 264,666,3066,3965$, 4933.

\section{1 才 TS'UN}

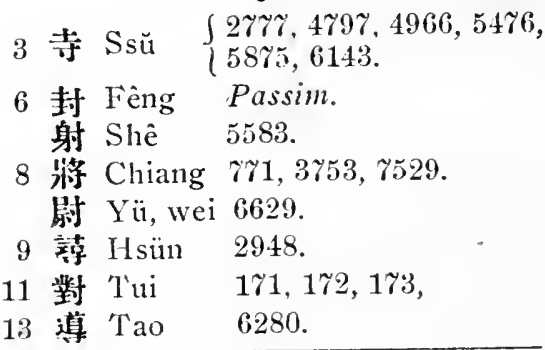

\section{J HSIAO}

$\begin{array}{lll} & \text { 小 Hsiao } & 2644 . \\ 1 & \text { 少 Shao } & 55 \% \% . \\ 2 & \text { 尔 Erh } & \text { Passim. } \\ 5 & \text { 傅 Shang } & 1189,2054,5562 .\end{array}$

1 尤 Yu 7642.

2 就 Chiu 1297.

\section{SHIH $_{\text {SH }}$}

1 尺 Chiih 4802.

2 尼 $\mathrm{Ni} \int 4312,4316,4483,4543$,

2 尼 $N i \quad 4660,5255,5911,596 \%$.

4 尾 Wei $\left\{\begin{array}{l}2180,4310,5808,6400, \\ 6882 .\end{array}\right.$

5 居 Chü Passim.

屈 Ch'ü 1465,4984 .

, Chü 1418.

6 屋 $\mathrm{Vu} \quad 2429,2567,3601,4461$.

7 展 Chan 5090 .

屏 Ping $1065,5215,5730,7 \% 29$.

9 屠 T'u 2859.

12 愿 'Ts'êng 5396, 6551.

\section{H $\mathrm{CH} \cdot \hat{\mathrm{E}}$}

1 屯 T'un Passim.

\section{SHAN}

山 Shan Passim.

4 徙 Ch'a 143, 1529, 5353, 7042.

岑 Ts'ên $43 \%, 6549$.

岐 Ch'i 639, 1816, 7409 。

5 岡 Kang Passim.

㟢 K'ê 3239 .

峎 Min $445 \%, 6 \% 85$.

坚 An 990,6699 .

佔 Hsin $286 \%$.

峒 Ssŭ 5880 .

貸 Tai $3610,6183,6753$.

岩 Yen $\int 1003,3974,4045,6113$,

质 Yo $\quad \int 116,200,2221,2884$,

岳 Yo 17611.

6 峝 T'11ng $\left\{\begin{array}{l}4,914,3015,5021, \\ 6831,7230 .\end{array}\right.$

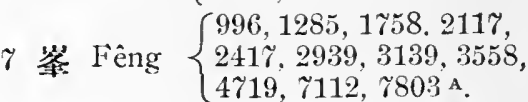

鳥 Tao $\left\{\begin{array}{l}448.1223,1879,3416 \text { ^ } \\ 5259,5 \% 39,5822,6274, \\ 631 \% .\end{array}\right.$

峿 Wu $\mathrm{r}$ i12.

姨 Hsia 426,994, 2464, 2578 .

峩 $\mathrm{O} \quad 2519,473 \%, 6625$.

峪 Yü Passim.

8 䕀 Ch'ung 1585, 6873.

崦 Yell 7392 .

崎 Ch'i $63 \%, 1391$.

淌 Kang 102\%, 1208.

崌 Chü 1430.

㵓 Kino 3538 .

崖 K'u11 3499, 38\%7.

㟯 $\mathrm{Ku} 3289$.

崆 K'1111g 5325 .

禁 Sung 5988 .

㕠 Ts'ui 6589.

崖 Yai Passim.

9 崶 Fèng 2213.

湈 K'an $4568^{\prime}$.

峲 Lan 3239,3602 .

楸 Cli'êng 531 . 
嵎 Ÿ̈ $7 \% 02$.

装 Sung 5990, 6085.

峤 Tzü

苵 Ch'in 1111.

峆 'T's'ang 6520.

11

嘴 Hsi 2519 .

12

嶓 1'o 5224 .

焦 Ch'iao 814.

叟 Ao 1314.

㼛 Yia 6520.

13 焦 Sui 5949, \%791。

嶧 $\mathrm{Yi} 7519$ 。

14 領 Ling Passim.

嗗 Hsü 1111, 2893.

18 魏 Wei 3639.

19 Luan 4151.

20 䈨 Yen 2326, 286\%, $421 \%$.

\section{7 《IK CH'UAN}

川 Ch'uan Passim.

3 州 Chou Passim.

4 巡 Hsïn 2963.

8 集 Ch'ao $3 \gamma_{4}, 792,1426$.

\section{I. KUNG}

\begin{tabular}{|c|c|c|}
\hline$T$ & Kung & $448 \%$. \\
\hline 2 巨 & Chüi & 1410. \\
\hline IJ & $\mathrm{Ch}^{6}$ iao & S11. \\
\hline Lr. & Tso & $1556,5462 \mathrm{x}, 655 \mathrm{~S}$. \\
\hline 4 쪼 & $\mathrm{W}_{\mathrm{t}}$ & 5023. \\
\hline
\end{tabular}

\section{E $\mathrm{CHI}$}
己 Chi 551
$1 \mathrm{~Pa}$ Passim.
6 巷 Hsiang $42 \% 6$.

\section{0 巾 CHIN}
2 布 $\mathrm{Pu}$ Passim.
市 Shih Passim.
4 希 Hsi- 7616 .
5 帖 T'ieh 6395 .
6 策 $\mathrm{Ti} \quad 4859,5822,6355$.

7 所 Shih ช $726,79 \% 9$.

8 艮 Chang $1 \% 5 \%$.

常: Ch'ang $309,6005, \% 109, \% 613$.

製 Tai 15\%4, 27\%8, 4\%82,4\%33.

11 花 Mu 5524 .

\section{F KAN}

F Kan Passim.

2 ये P'ing Passim.

3 年 Nien 2773,6068, 6595, rs61.

拃 Ping 5132 .

10 翰 Kan 1982, 3104。

\section{$52 \longleftarrow$ YAO}

6 Iu 7686.

\section{$53)^{x}$ YEN}

3 庒 Chuang Passim.

4 庇 $\mathrm{Pi} 5069$.

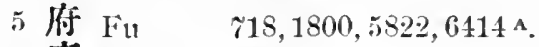

度 Kêng ro\%4.

底 $\mathrm{Ti}\left\{\begin{array}{l}3350,3535,4304,4782, \\ 4820,5075,0350 .\end{array}\right.$

店 Tien Passim.

6 度 T'u 400,4634, 5196, 6632.

7 㡹 K'u $3228,3328,4763,5133$.

庭 'T'ing 5616 .

8 捄 $\mathrm{K}$ a ang Passim.

底 $\mathrm{Ch}+\mathrm{i} 629$ 。

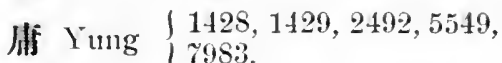

9 廂 Hsiang 2626

淀 $\mathrm{Yi}$ (6122.

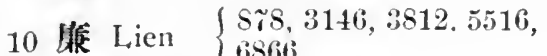

12 廒 Fei 1682.

蕰 Kuang $\left\{\begin{array}{l}2359,8386,8873,45.54, \\ 6054 .\end{array}\right.$

湖 Miao $\{1564,3665,4424,4855$,

13 㸃 Ling 3956.

16 监 Lï $84,1360,1545,4138$,

$1 \%$ 綮 Ying $\% 586$. 


\section{4 多 YIN}

4 廷 T'ing 5822,6792A.

5 延 Yen 5591, 735\%.

6 建 Chien $\left\{\begin{array}{l}845,1867,2738,3060, \\ 45 \% 2,5932,7361 .\end{array}\right.$

\section{HUNG}

4 彗 Lung 6132.

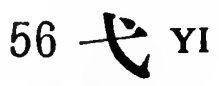

セ $\mathrm{Yi} 7516$.

\section{KUNG}

弓 Kung 3525 .

1 引 Yin 669, $756 \%$.

2 弘 Hung 1392, 4990.

弗 $\mathrm{F}_{\mathrm{u}} \mathrm{7523.}$

5 弦 Hsien 2690 .

8 張 Chang $\left\{\begin{array}{l}178,5 \% 3,5 \% 50,6354 \text {, } \\ 6539,7 \% 1 \% .\end{array}\right.$

9 强 Chiiang 6530, r056.

13 疆 Ch'iang 782, 2 \%36.

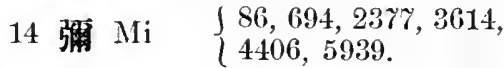

\section{8 工 $\mathrm{CHI}$}

$\begin{array}{rll}9 & \text { 㬎 Chih } & 955 . \\ 13 & \text { 絭 } \mathrm{Yi} & 1833,5202 .\end{array}$

\section{$59 \geqslant$ SHAN}

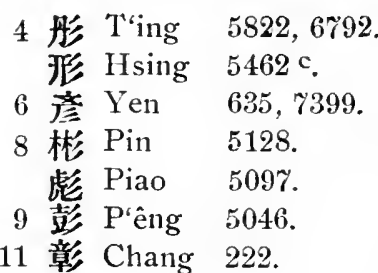

\section{0 个 $\mathrm{CH} \cdot \mathrm{IH}$}

4 役 $\mathrm{Yi} 4512$.

5 征 Chêng 493.

6 後 Hou 613,2136 .

徊 Hui 2358,5038 .

徒 'T'u 5122,6209, $66 \% 1$.

7 徐 Hsii $\int 555,2898,3870,4586$, $499 \%$.

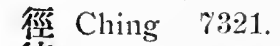

8 徠 Lai $356 \%$.

徘 $\mathrm{P}^{i} \mathrm{ei} 5038$.

徙 Ssŭ $58 \% 3$.

得 Tè $\left\{\begin{array}{l}30,11 \% 6,1565,5533, \\ 6320\end{array}\right.$

從 Ts'ung 2694,6601, 7975.

御 $\mathrm{Yü} 5822, \% \gamma 01$.

9 復 $\mathrm{Fu} \quad 1882,3292,4513, \gamma 691$.

循 Hsün 2961.

10 微 IVei 1053, 2663, 6979.

12 徽 Chêng 494,7468 .

德 Tê Passim.

1 徽 Hui $69,235 \%$.

\section{HSIN}

1

心 $\mathrm{H} \sin 4354$.

1 必 $\mathrm{Pi} 21 \% 6,5071,7521$.

3 志 Chih 406, 12\%6, 1419.

成 T'ê $\{12,31,5284,6311$,

4 虫 Chung $852,1560,2283$.

忽 $\mathrm{Hu} \quad 1982,2189,3104$.

㠼 Hsin $2 \% 21$.

快 K'uai 6933 .

念 Nien $9 \% 0$.

5 思 Ssŭ Passim.

怯 Ch'ieh 840 .

怀 $\mathrm{P}^{\prime} \mathrm{ei} 5036$.

6 恒 Hêng 2013, 245\%,6\%18.

悐 So 5849 .

恭 Kung Passin.

恰 Chia $71 \%$.

恩 En Passim.

息 Hsi $11,2522$.

7 悉 Hsi 2520.

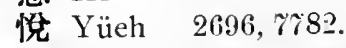

8 恵 Hui 1455, 2360, 5641. 


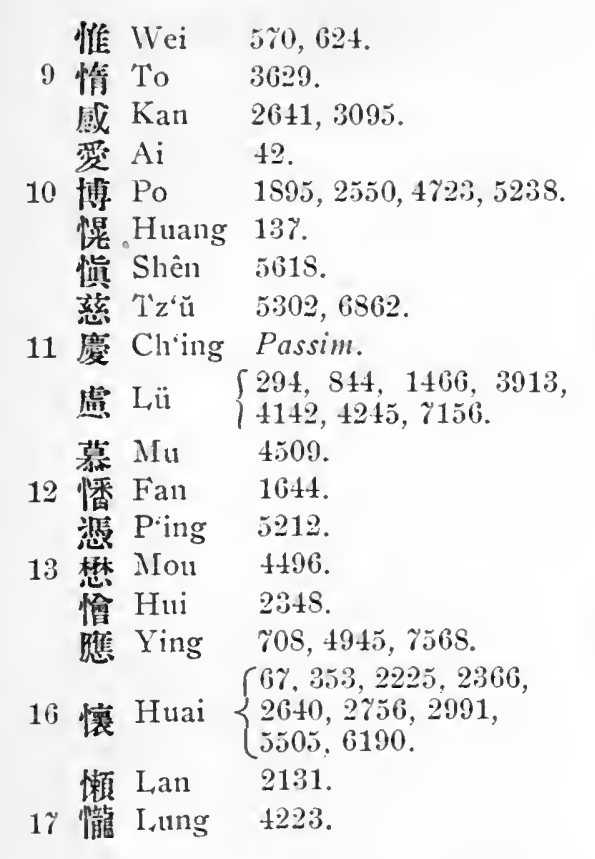

\section{2 戈 $\mathrm{KE}$}

戈 Kê 1209,6804.

2 我 Jung Passim.

戌 Shu r331.

3 成 Ch'èng $47,503,3645,4271$.

13 戯 Hsi $2+35$.

14 戴 Tai 6161.

\section{3 兵 HU}

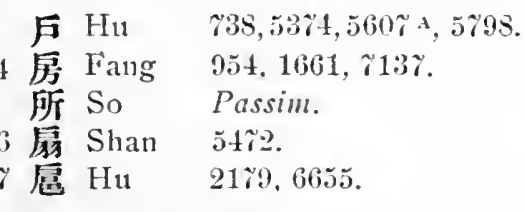

\section{4 手 SHOU}

乎 Shan $\% 389$.

才 'Ts'ai 635, 3164 .

1 扎 Cha- 129.

2 打 Ta $4760,4 \% 99,6125$.

3 托 T'o 156, 4495, 648\%.
4 承 Ch'êng 535, 6891.

折 Chè $3 \% 6,7315$.

把 $\mathrm{Pa} 7520$.

扶 $\mathrm{Fu} \int 1813,2456,453 \%$,

\498\%, 7621 .

扯 Chê 401 .

投 'T'ou 4631 .

5 招 Chao $352,2728,744 \%$.

极 $\mathrm{Pa} 4 \% 9 \%$.

拓 Chih 959 .

拒 Chï 1416 .

拉 La Passim.

拏 $\mathrm{Na} 454 \%$.

拜 Pai 4S1\%。

披 $\mathrm{P} i \mathrm{i} 5059$.

摍 $\mathrm{T}^{\prime} \mathrm{o} 6481$.

押 $\mathrm{Ya}$ \% 222 .

6 指 Chih $9 \% 2$.

拳 Ch“̈̈all $\gamma 632$.

拱 Kung 3512,6156 .

括 Kua 3338 .

7 振 Chên 150 .

捍 Han 1951.

捂 W'u 6S31.

捕 $\mathrm{Pu} 52 \% 8$.

$\mathrm{S}$ 排 P'ai 4594 .

捨 Shê 5590 .

推 Tui 6255 .

掖 Yeh, yi $197,305,5822,6173$,

9 捧 Fêng $1 \%$ r6.

掉 $\mathrm{Hui} 92 \mathrm{2}$.

揟 Hsü 2911.

偨 Shê 5601 .

挨 Huan 2269.

揾 $\mathrm{Yi}$ i 4 it.

㖞 Chieh 838.

提 Yang $830 \%$ 。

提 $\mathrm{T} \cdot \mathrm{i} \quad\left\{\begin{array}{l}2 \gamma 13,3203,3530 \\ 4568 \mathrm{P}, 6369, \gamma 20 \%\end{array}\right.$

., Shih $4913,5769,6710$.

10

接 Sou 155,5852 .

俟 $\mathrm{P}$ i 4722 .

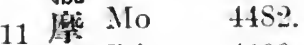

摽 P'iao 4199.

挴 $\mathrm{Fu} \quad 182 \%, 2753,292 \%, 45 \% 2$.

12 撚 $\mathrm{Po}$ 3694, 5225.

措 Sa I 1 $83,2106,3201,3528$, (358\%, $5333 \%$. 


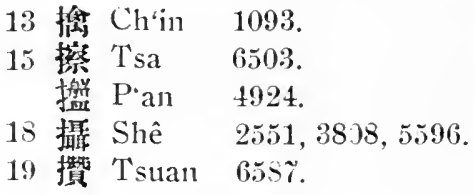

13 捻 Ch'in 1093.

15 櫒 Tsa 6503 . 瀊 P'an 4924 .

18 攝 Shê 2551,3838,5596.

19 摜 Tsuan 65s\%.

\section{5 支 $\mathrm{CHIH}$}

支 Chih $\{380,956,1283,3206$,

支 Chih $\{392 \%, 41 \% 4,63 \% 9, \% \gamma 80$.

" Ch'i 3926.

\section{6 × PU}

\section{2 收 Shou 2\%0, 5\%5\%.}

3 侦 $\mathrm{Yu} \quad 32 \mathrm{2}, 7628$.

教 Kai 5164 .

攻 Kung 3105 .

5 政 Chêng 490, 5814.

故 Ku1 3275, 3458。

7 教 Chiao 193.

敖 Min 4454 .

敖 Ao 124.

8 敨 Tun $2 \% \%, 91 \%, 665 \%$.

, T'un 6690 .

斐 San 323t.

11 数 Fu1 1836, 6569 。

15 策 $\mathrm{T}$ ' $619 \mathrm{y}$.

\section{7 妾 WÊN}

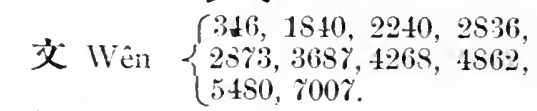

\%越 Pin 60\%3.

\section{8 if $\mathrm{TOU}$}

斗 Tou $1043,6494,7122$.

y 解 $\mathrm{Hu} 2182$.

斜 Hsieh $788,2769$.

9 諶 Chên 44.

10 倠 Wo rots.

\begin{tabular}{|c|c|c|}
\hline & $6 ?$ & F $C H I N$ \\
\hline 厂 & Chin & $556,995$. \\
\hline 1 穴 & Chih & $100 \mathrm{t}$ \\
\hline 8 斯 & Ssŭ & $\left\{\begin{array}{l}22,1 \gamma 09,4315,4 \gamma 63, \\
5223,5431,632+, \gamma 195 .\end{array}\right.$ \\
\hline 9 䉼 & Hsin & Passim. \\
\hline
\end{tabular}

\section{0 万 FANG}

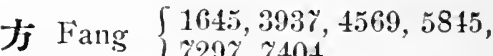

4 於 Yü 5 $560,8 \% 0 \%$.

5 施 Shih $\left\{\begin{array}{l}1616,18+3,4204,4331 \\ 5668\end{array}\right.$

6 旅 $\mathrm{Lii}$

$\gamma$ 旌 Ching $11 \% 6$. 10 旅 Ch'i $\left\{\begin{array}{l}613,2381,4823,5594, \\ 6560,7313 .\end{array}\right.$

\section{Jiн}

\section{日 Jih 2995 .}

1 旦 $\operatorname{Tan} 1443,2406,3313$.

2 旭 Hsï 2914.

早 Tsao 652\%。

4 昌 Ch'ang Passim,

易 $\mathrm{Yi} \quad 1259,346 \%, 4416,7475$.

昆 K'uil $891,3493,4414,5 \% \% 4$.

朋 Ming $\{223,262,296,342, \gamma 01$, $1036,1593$.

昔 Hsi 2531, 3015, 4419 .

昇 Shêng 5839 .

旺 IVang $1406,148 \%, 3686,4786$.

5 顾 Chao $33 \%$

唇 Fiun 1919, 6\%20.

春 Ch'un Passim.

星 Hsing 2852,4020 .

6 晃 Huang 2332.

時 Shih 5659 .

亚 Chin $\int 506,10 \% 3,3392,3848$,

百 Chin $\{4568 \mathrm{~J}, 46 \% 6,7544$.

晏 Yen 161\%, $85 \% 5$.

7 㦼 Shêng 5649

8 智 Chih 9\%1.

붕 Ching 1130, 5\%02.

䪧 $\mathrm{P} \cdot u \quad 3996,4809,5290$.

9 暯 Ying 5545 .

暗 Yang 1228.

暖 Nuan 4731.

蝶 Hui $2: 3 \% 2$.

昉留 $\mathrm{Yi} \quad 6 \% 62$.

12 㗨 Chi $5 \% 1,1375,7646$. 


\section{3 日 YÜЕH}

2 Ill Ch'ü Passim.

3 i⿱ 万) Kêng $14: 31$.

6 卷 Shu 2104, 3094, 8265.

$\gamma$ 曼 Man 4556 .

曹 Ts'ao 1554, 6538, 7123, 7374.

8 筧 'Ts'êng 6555.

9 会 Hui $\left\{\begin{array}{l}2072,2129,2335,275 \%, \\ 3649,4261,5886,2011 .\end{array}\right.$ " Kuei 3469 .

\section{4 月 YÜEH}

\begin{tabular}{|c|c|c|}
\hline 月 & Yüeh & $13 \gamma 9,8596$. \\
\hline 有 & Yu & $6121,7630$. \\
\hline 朋 & P'êng & 3826. \\
\hline 谢 & So & $4621,4 \gamma 05,5843$, ช2\%. \\
\hline 樶 & Lang & 3604,4280 \\
\hline 㕵 & Wang & $5198,5256,5400,6941$. \\
\hline 朝 & Chao & 362 \\
\hline & Chu & $138 \%$ \\
\hline 期 & Ch'i & $615,3782,5281$. \\
\hline
\end{tabular}

\section{5 木 $\mathrm{MU}$}

\begin{tabular}{|c|c|c|}
\hline 木 & $\mathrm{Mu}$ & Passim. \\
\hline & Mo & $5343,5346,6135$. \\
\hline 札 & Cha & 136. \\
\hline & Pên & $2853,5040,6612$. \\
\hline 成 & Shu & 4532. \\
\hline 来 & Chtt & $\left\{\begin{array}{l}\gamma \gamma 9,1349,4501,4563 \mathrm{~s} . \\
4 \gamma 61,4983,6203,6 \% 10 .\end{array}\right.$ \\
\hline & Shu & $5 ; 69$. \\
\hline 染 & To & $5822,6482$. \\
\hline 束 & 'Tz' $z^{6} \breve{u}$ & 6556 \\
\hline & $\mathrm{Ch}^{\prime} \mathrm{i}$ & 623. \\
\hline 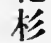 & Shan & 5465 \\
\hline 杆 & Kan & 3108. \\
\hline 藏 & Hsing & $284 \%, 4996,5822$. \\
\hline & $\mathrm{Li}$ & :36S5, 6588. \\
\hline & Su & 5925 \\
\hline 材 & Ts'ai & 2034. \\
\hline 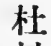 & $\mathrm{T} u=$ & $5018,66: 35$. \\
\hline P & Ts'un & Passim. \\
\hline & Chih & $95 \%, 7171$. \\
\hline
\end{tabular}

极 Chi 575 .

杭 Hang $\left\{\begin{array}{l}65 \%, 1022,1991,5504, \\ 7522,7648 .\end{array}\right.$

果 Kuo $210 \%, 3532,4840,5 \% 14$.

林 Lin Passim.

板 Pan 4899, 5386.

杷 $\mathrm{Pa} 6392$.

析 $\mathrm{Hsi} 2528$.

松 Sung $\left\{\begin{array}{l}273,1212,2010.3090, \\ 385 \%, 5912,5916,5968, \\ \gamma+05 .\end{array}\right.$

東 Tung Passim.

5 柳 Liu | $1358,39 \% 7,5888,6056$,

李 Ch'a 153, 2019.

某 Mou 4\%y.

柷 Chu 1394 .

楠 Shih $566 \%$.

柘 Chè $375,14 \%$.

枳 Chih 9rt.

样 Chu 6413.

卑 Jou 2233,3002,3404.

架 Chia 692 .

柯 Kê 319\%。汭35.

柏 Pai 22056,326t, $45 \% 0$. 6 根 Kên $\left\{\begin{array}{l}2624,321 \%, 3240,4488, \\ 62 \% 4,6613, \gamma 298, \gamma 80 \% .\end{array}\right.$

桂 Kuei 2036.3443, 3875, 6044.

桂 $\mathrm{Li}$. $3 \% 50,4598,4900,7563$.

㝝 $\mathrm{Pai} \quad 4891,681 \%$.

Sang 161,5405, 5462 G.

烑 T'ao $\int 19 s 8,2 \gamma 12,3889,4628$,

柴 Ch'ai 160, 3125.

格 Ke !2050,3214,4788,4938,

㭣 T'ung $505,680 \%$.

\% 模 Kan 898 .

榑 Kèng 3244 .

梨 $\mathrm{Li}$

泇 Liang

㭪 Fu

梅 Mci \{ $\begin{aligned} & 1508.2297,2381,434 \% \\ & 6476.7302 .\end{aligned}$ 2484, 3711, 5462 ${ }^{\text {} . ~}$

\section{Passim.}

1835.

榜 T往

條 'T'iao 4478,6379.

棇 So $\$ 850$.

楛 $\mathrm{Vu}-1155,3449,6526$, \% 128 .

8 棘 Chi 558 . 
椎 $\mathrm{Ch}^{\circ}{ }^{\circ 11} 1508$.

椑 $\mathrm{P} \cdot \mathrm{i} 5084$.

楼 Chi $659,1 \% 19,625 \%$.

植 Chih $540 \%$.

棋 Ch'i 614 .

森 Sên 1218.

栄: 'T'ang 150, 3091, 6273 .

㙌 $\mathrm{T} i$

苯 Tsao

$634 \%, 815 \%$.

椒 Chiao

9 楚 $\mathrm{Ch}^{6} \mathrm{u}$

冎 Huo

椷 Fêng

楖 Lang

椳 Wei

稆 Lêng

㛫 Yang

桫 Yeh $\left\{\begin{array}{l}108,868,2839,7332, \\ 7979 .\end{array}\right.$

霞 $\mathrm{Chi}$

棭 $\mathrm{Y} \ddot{\mathrm{ii}}$

椹 Shên

10 槐 Huai

, Hui

榜 Pang

榛 Chên

榴 Liu

籍 Jung

榕 Jung

5462 ×, 5928, 6530 .

$801,1484$.

( $15,1401,2450,4660$, $\{4 \% 62,6 \% 11,6951$.

2424.

$24 \%, 1 \% 12$.

5131 .

8006 .

3681.

2252,7290 ,

559,7208 .

Passim.

5632 .

2255 .

$3623,628 \%$.

$3655,4931$.

458.

$5 \% 16$.

3043.

3039 .

11 棓 Chang 218.

模 Mu $\left\{\begin{array}{l}12,21,2192,2481, \\ 3190\end{array}\right.$

㑧 Fan 1033.

模 Lou \{40\% $4,4553,4604,4856$,

樂 Lê Passim.

從 'T's'ung 6602.

12 蓝 Hêng 2018, 602\%

樿 Hsïn 2952.

溗 Ch·iao Passim.

機 Chi $5 \%$ \%

橡 Hsiang 260.

楼 $\mathrm{P} c \mathrm{u} \quad 5336$.

th Shu $\quad 28,220,1336,3264$.

S3302, 5888, 6082, \%731.

囊 $\mathrm{T}$ \% 6492.

樵 $\mathrm{Cl}_{1}$ iao 815.

族 'T'sui 6588.

椎 Kao 382 。
13 棆 Kuei 3470.

14 橧 Pin 5131.

檢 Chien 2963.

憻. T'an 6229 .

15 榴 Yüch r\%93.

17 鮎 Lung 4225 .

欄 Lan $35 \% 5,4288,7221$.

19 戀 Luan 4152.

21 欖 Lan 4i91.

\section{6 久 CH'IEN $^{2}$}

2 次 $\mathrm{Tz} \breve{\mathrm{u}} 105,4042,7474$.

, Тzŭ 2911, $76 \%$.

4 欣 Hsin $2 \% 1 \%$.

8 釷 Ch'in 1095.

9 歇 Hsieh $26 \gamma 1$.

10 歌 Kè $364,2048$.

11 践 $\mathrm{Ou} 4 \% 48$.

12 歌 Shê 5599.

18 歡 Huan 18.

\section{7 止}

1 正 Chêng $4482,158 \%, 2039,2440$,

1 II. Cheng / 4115, 44\%0, 6800 .

3 少 $\mathrm{Pu} 5 \% 5,4123,4808,645 \%$.

4 武 W Vu Passim.

9 藏 Sui 5948.

12 厢 $\mathrm{I} i \mathrm{i} 2545,3755,6052$.

14 靕 Kuei Passim.

\section{8 齐 $\mathrm{TAI}$}
6 架 Shu
$1981,5 \% \% 0$.
8 殖 Chih
951.

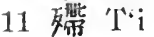
6.368.

\section{9 炎 SHU}
6 般 Yin
$5543,7562$.
$\gamma$ 殺 Sha
$\therefore 453$.
9 殿 Tien 5822, 6481. 


\section{0 曲: wu}

Hf $\mathrm{IVu}$ \% $\mathrm{vos}$.

显 $\mathrm{Ma} 543 \%$.

\section{1 比 PI}

$\begin{array}{lll} & \text { 比 } \mathrm{Pi} & 5066 . \\ 5 & \text { 蹗 } \mathrm{P} i & 5021 . \\ 7 & \text { 㡙 } \mathrm{P} \cdot \mathrm{i} & 5082 .\end{array}$

\section{2 毛 MAO}

毛 Mao 2118,499\%,68\%\%,

5 毡 Chan 168 .

13 㲘 Chan 2189.

\section{3 进 SHIH}

\section{It Shih Passinl.}

"Chih r1\%o.

1 IE $\mathrm{Ti} \mid 596,94 \%, 2459,3113$, $1 \%$ \%

"Chih $94 \%$.

民 Min Passin.

\section{5 入}

水 Shui Passim.

1 永 Yung Passim.

2 汀 'T'ing 215, 2\%6, 3s91, 6468.

求 $\mathrm{Ch}^{\circ}$ in 1304.

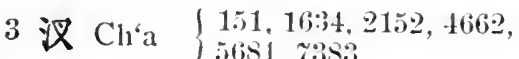

浙 Chilih Passim.

汻 Kan $\quad$ 64t?.

汝 Ju I $2081,3005,35 \% 1,4585$,

江 Chiang Passim.

汕 Shan 5463\%

沉 I Isün $(1002,1 \% 36,20 \% 5,83 \% 5$. ก 6321, 7132.7264.

汇 Ssŭ 5881.

4 流 Shến 5622.

泟 Chil 6921.

沖 Ch'ang 1600 .
汾 Fê11 168\%, 385ร, テชะ0.

注 Wang 5603 .

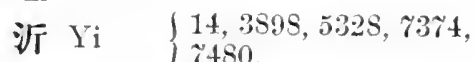

汭 Jui 2563, 6813.

泛 Chi $5 \%+, 2 \pi 30$ A, $712 \%$.

涩 Mien 4442 .

沒 No 44s6, 61692 .

沙 Sha Passim.

媇 Pien 5106.

胨 Mu 26\%?

沌 Ch'uan 1469, 4536.

汇 Chin $\{19,128,1095,2281$. $\{2382,3220,+10 \%, 6308$.

没 Wên \%o16.

"Nin 445.

治 Yüan $\%$ \% $\%$.

沃 $\mathrm{Wu} 1463,251 \%, \mathrm{2} 210$.

沅 Yüan Passim.

浦 $\mathrm{P}^{\prime} \mathrm{ei} 503 \%$.

5 沾 Tien 6401.

況 Huang 2:334.

治 Chih 283, 952,5\%95, 6706.

洺 Hsich 2685.

拈 Chu 2196.

河 Ho Passim.

泣 Hsüan 2920,

沶 Shih 5651.

洁 $\mathrm{Ku} \quad 3185,82 \% 9,4136,6041$.

沦 Ling 3930 .

涞 Mei $43 \% 1$.

泥 $\mathrm{Ni} \quad(1330,2295,2299,2548$,

泌 $\mathrm{Pi} 50 \%$ 注。

波 $\mathrm{Po}$ Passim.

泊 $\mathrm{Po} 52: 32$.

沭 Shu 5\%ช:

淌 $\mathrm{Ch}^{\circ}$ ii $5: 3 \%$.

河 Ssï 5893.

沱 T”。 2445.

泰 Tai | 2rt. 1254, 2\%ช9, 30\%0,

6175, $7094,79 \% 3$.

湛 Chii $38,5,41 \% 8$.

"Ch11 1348.

沿Yen ril

点 Choiian Passim.

沰. Hung 2:394.

油 Yu F61, 760333.

法 Fa 1621, 3324, 7522。 
6 洲 Chou $\{2+2,1254,1315,46+8$,

洽 Hsia 873.

洪 Hung Passim.

活 Ch'ien 899 .

洛 Lo ４051, 5175, 5518, 5554.

洛 Ming 4465 .

海 Erli 46\% 52996 .

泊 Hsün 2965 .

洮 T'ao 3890,6294 .

洞 'Tung $\{459,632,2604,4323$,

调 Tung $\{4344,4535,6099,6 \% 6 \%$.

" 'T'ung $240 \%$.

流 Liu $\left\{\begin{array}{l}1249,3998,4603,5001, \\ 5 \% 88,6 \gamma 3 \%, 6839,689 \% \\ \gamma 228 .\end{array}\right.$

洊 Chien $\$ 95$.

津 Ching Passim.

洧 Wei 1462,6983.

洋 Yang $\left\{\begin{array}{l}388,438,1799,4520, \\ 1866,5448,5540,6108, \\ \tau 234 .\end{array}\right.$

$\gamma$ 浙 Chê $389,3 \% 60$.

浮 Fu1 1892 .

游 Hai Passim.

浩 Hao 10r1, 2003.

„Kê 3182 .

浠 Hsi 2428.

浛 Ching $\left\{\begin{array}{l}946,1112,1 \gamma 13,2328, \\ 2 \gamma+2,5144,5895,6928 .\end{array}\right.$

浪 Lang $\left\{\begin{array}{l}1496,3149,3260,3261, \\ 3612,5168,5244\end{array}\right.$

沙 Hsiao 4202.

Nieh 4669 .

浦 $\mathrm{P}^{\prime} \mathrm{u}$ Passim.

涉 Shê $559 \%$.

淁 Hsïn 2958.

, Chün 1520.

涌 Yung $\% 995$.

源 Cho 1324.

䉼 Hsi 2427, 2530.

Hun $23 \% 9$.

涪 Fou 1\%80,4986.

涪 Lin $391 \%$ 。

淮 Huai $\int 119,249,2243,2462$,

准

淪 I,un 3930.

Kan $2 \% 65$.

涺 Ho 4183 .

淇 $\mathrm{Ch}^{\prime} \mathrm{i} \quad 616,3345$.
淶 Lai 3568 .

涼 Liang $\int 2474,3085,3144,3 \% 96$.

涼 Liang | 4131, 51\%1, 6735 .

媣 Shên 5628 .

淡 Tan $621 \%$.

添 $\mathrm{T}$ 'ien 2\%83, 5308.

浦 'Tung $6 \gamma 66$.

浿 Fei $16 \%$ \%

淛 Chê 396 .

啝 $\mathrm{Ho} 2060$.

潗 Chiing Passim.

淄 Tzŭ - 3895, 6833.

淤 $\mathrm{Yü}$ Gros.

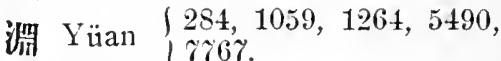

9 楨 Chêng 500 .

㵔 Chu | 188, 13\%1, 2852, 4351,

| $4743,5593$.

湔 Chien 896 .

湖 $\mathrm{Hu}$ Passin.

棌 Huan $22 \% 0$.

渴 Kê 3183 .

湏 Hui 2356 .

涵 Han $19 \% 1$.

泳 Lu 4116 .

湟 Huang 2330 .

搌 Hun $\mid 2375,4130,4605,4691$,

洋 Hun $5002, \% 734$.

湝 Chieh 831 .

港 Chiang Passim.

洋 Kê 3204 .

渠 Ch’ü Passim.

濯 Mei 4368 .

渤 Po 5228,

湘 Hsiang 1248, 262\%, 2S85, 3869 .

漠 Shun 2693, 3158, 5829, ช9ช1.

晹 T'ang 6260 .

(203, 333, 113\%, 1618,

渡 $\mathrm{Tu}\{2009,21 \% 1,2322,3353$,

橧 T'uan 6683.

,, Ch'uan 244.

湓 P'ên 2303, 5245.

㵋 IVên $\int 3660,6652,6919,7018$,

im tren fry\%.

漟 Wei 6965 .

满 Wei $4639,6 \gamma 89,6984$.

淌 Kê 3195 .

游 $\mathrm{Yu} 4220,7034$.

渝 $\mathrm{Y} \ddot{\mathrm{u}} \quad 3902, \% 6 \gamma 1$. 
10 溱 Chềil 455.

除 $\mathrm{Ch}^{\prime} \mathrm{u} \quad 81,139 \%, 3852$.

滈 $\mathrm{Hao} 1995$.

滑 Hua 2223.

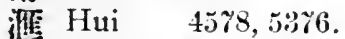

滧 Hsiao 4913, 7115.

浴签 1809.

灌 Kou Passim.

溪 Chithsi Pussim.

溧 $\mathrm{Li} \quad 8751$.

滕 'T'êng 6336.

湩 'T'ien 6402 .

洀 Yin 7564 .

渝 Ts'ang 6521.

浽 Tzй 1307, 5982, 6835 .

泎 Chnı 1509.

沽 Chung 1565 .

洤 $\mathrm{Yi}$ \%538.

源 Yüan Passim.

管 Jung 3050 .

溶 Jung 20\%6, 56\%1, 56\%2,

11 涪 Chang | 208, 1004, 22\%5, 3839,

| 4561, 6469.

渿 Yang 7221 .

洞 Shang 5561 .

湌 Ch'an 176.

涌 $\mathrm{Ti} 6543$.

漢 Han $\left\{\begin{array}{l}65.126,155,156,1942, \\ 2505,2928,3396,45 \% 4, \\ 542 \% .7259 .\end{array}\right.$

漏 Lou $40 \%$ \%

激 $\mathrm{H}_{11} \quad \% 3 \%, 2189,2286$.

萁 Lü 4150 .

激 Kuo 3545.

淧 $\mathrm{Chi} 659,4193$.

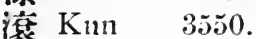

㴔 $\mathrm{Hu} 2180$.

满 Man 4061, 4325, 5102.

满 $\mathrm{Ti} \quad 6543$.

獎 Chiang irs.

漫 Man 62 $\gamma, 25 \gamma 5,4329$.

漯 'T'a 6138.

演 Yin r3s2.

漁 $\mathrm{Yii} \quad 769.5$.

12 潮 $\mathrm{Cl}^{6}$ ao $368,2363,7605$.

澄 $\mathrm{Ch}^{\prime} \hat{\mathrm{e}} \mathrm{ng}$ 538, 1916 .

溦 $\mathrm{Ch}^{\circ} \hat{\mathrm{e}} \mathrm{ng} 543$.

涺 Chitran 1478.

膶 Jun ${ }^{-1744,2155,3035,5890 . ~}$

润 Chien $\left\{\begin{array}{l}151,8 \% 2,1203,2279, \\ 4568 \text { F } 5421\end{array}\right.$
澇 Lao 3641 .

4600 .

$4928,59 \% \%$.

;101.

$505 \%$.

2953, 458\%.

Passim.

$409 \%$.

淀 Hsieh 2 \% 60.

潼 Tung $\{45 \%, 509,3894,463 \%$,

理 Tung | 5022, 5078, 6793, 685t

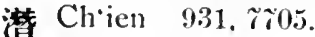

13

Knei 34 is.

澧 $\mathrm{Li} \quad 3 \% 2 \%, 35 \% 9,7613$.

畄 $\mathrm{P}^{\circ} \mathrm{i}$; 221 .

澤 Huan 2164.

澳 Ao $122,61 \%, 2396,4559$.

澛 Shan 5489.

漂 Tsê Passim.

湭 Mien $444 \%$.460\%, 5004. 14 演 Fin $\left\{\begin{array}{l}1935,2026,2034,2249, \\ 2632.3803,5125 .\end{array}\right.$ Hao $2001,3561$.

佂 $\mathrm{Pu} \quad 5266$.

灀 $\mathrm{Ju} \quad 3004,6615$.

影 Hsŭn $295 \%$

湿 Shih 694 .

Chi Passim.

济 Mêng 4381.

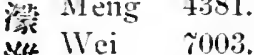

滩 Wei

15

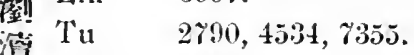

深

瀋

16

Shên 5620 .

濾 Lü $24 \% 9,4133$.

㳊 $\mathrm{Li} 20 \% 8$.

嘢

17 瀧 Shnang 4 220,

I.ung or)

ร59\%.

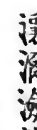

Jan 2968.

$\mathrm{Mi} 3615$.

Lien 3516.

18

Kuan 145, 3359.

$\mathrm{Ch}^{4} \mathrm{i} \quad 633,2833,5263$.

5610 .

19

Shê

濉 T'an 3is $19,62.20$.

20 唀 $\mathrm{Cl}$ (1) $91 \mathrm{~s}$

W $184,326,2299,2889$,

22 㩐 Wan $\left\{\begin{array}{l}3393^{\mathrm{A}}, 3535,4215.4427, \\ 5 \% 44,6054,6120,6194\end{array}\right.$

23 㮾 Luan 4154 . 


\section{HUo}

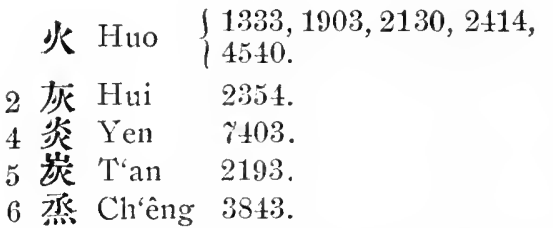

烏 Wu $\left\{\begin{array}{l}348,1225,1681,250 \%, \\ 5615,6105,6145,7164, \\ 7463 .\end{array}\right.$

烟 Yen 2510,5984, $7412^{\mathrm{A}}$.

7 烽 Fêng 2523.

琵 Yen 7385.

8 焦 Chiao 898.

焙 Pei $49 \% 2$.

然 Jan 3825.

每: W $W_{11}\{2346,2909,3470,5481$,

$\left\{\begin{array}{l}751 . \\ \text {. }\end{array}\right.$

焚 Fên 1695.

9 然 Chao 351, 2995, 4564, 6350 .

蜾 Lien 3818 .

煌 Huang 6689, 6690 .

煩 Fan 40\%3,40\%t, 4604 .

熙 $\mathrm{Hsi}$ Passim.

10 熊 Hsiung 2880.

11 蔱 Jê $297 \%, 3250$. 熟 Shu 320,2162.

12 倠 Shao $2419,2120$.

㴖 Yen $\{4642,4788,5822,6 \% 61$, 73\%6, 7638 .

13 營 Ying $\left\{\begin{array}{l}2511,3034,3986,6120, \\ 6465,7588 .\end{array}\right.$

熘 Chu 1388.

16 爐 Lu1 6061, 6125.

17 爛 Lan $35 \% 6$.

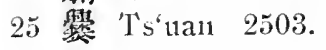

\section{7 М САO}

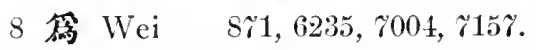

\section{FU}

丘 Fu 1702, 1807, 7219.

\section{9 爻 YAO}

爻 $\mathrm{Yao} 5462$ R.

10 爾 Erh See 尔.

[Under Radical 42].

\section{0 芀 CH'UANG}

5 垶 Kê 3206,6518 .

6 洋 Tsang 6518 .

\section{H. P'IEN}

8 牌 P'ai 5 \%25.

9 牒 Tieh 6385.

\section{2 牙 YA}

\section{牙 Ya $5342,596 \%, 6210$.}

\section{3 斗 NIU}

4. Niu $\left\{\begin{array}{l}103 \%, 1961,4122,440 \%, \\ 4716,5042,5 \% 24,6391, \\ 7038 .\end{array}\right.$

2 有 Mou 690, 1546, 4492, 6741 .

3 牢 Lao $616 \%$.

4 物 $\mathrm{Wu} 7209$.

特 T伦 $\{1912,4541,5269,52 \% 6$,

6 特 'T'ê $\left\{\begin{array}{l}6323,6636,6665,7038, \\ 748 \%\end{array}\right.$

$\gamma$ 等 $\mathrm{Ch}^{\prime}$ ien 2191.

居 Hsi 2533, 3225,5624 .

8 犁 $\mathrm{Li} 7489,7 \% 35$.

9 徒 Chien $870,711 \%$.

15 㹙 $\mathrm{Tu} .4479$. 


\section{4 СH“ÜAN}

\begin{tabular}{|c|c|c|}
\hline 4 狄 & $\mathrm{Ti}$ & $635 \%$ \\
\hline 狗 & Kou & $324 \gamma, 6126$ \\
\hline 5 狐 & $\mathrm{Hu}$ & $\left\{\begin{array}{l}983,1679,2184,3696, \\
3925 .\end{array}\right.$ \\
\hline 狠 & I.ang & 3615 \\
\hline 猛 & Mêng & $439 \%$. \\
\hline 猗 & $\mathrm{Yi}$ & 7510. \\
\hline 猫 & Mao & 4845 \\
\hline 猴 & Hou & 2141,4631 \\
\hline 解 & Yu & $\gamma 6 \cdot 11$ \\
\hline 眡 & Yu & $1299,134 \%, 5546$. \\
\hline 獅 & Shih & 5664 \\
\hline 㹲 & Yüan & 3566. \\
\hline 洺 & Yao & 3693,5021 . \\
\hline 獜 & Lin & 3925 \\
\hline 獨 & $\mathrm{T} u$ & 6650 \\
\hline 獲 & $\begin{array}{l}\text { Huo, } \\
\text { htiai }\end{array}$ & 2262,2422 \\
\hline 5 & Hsien & $269 \%$ \\
\hline
\end{tabular}

\section{HSÜAN}

方 Isüar, 2915 .

6 萑 Shuai 5\%\%, 7222.

\section{TE YÜ}

王 $Y_{\text {iิ }} \mid 3811,50 \% 9,5822,762 \%$,

¥: Wang Passim.

4 䚳 Chih 4290 .

5 㻇 Chên $446,350 \%, 6355$, 7 \%39.

6 珠 Chu $1365,4159,5408,7172$.

班 Pan 4904,5\%65.

珙 Kung 3513 .

㺲 Errh 1620 .

7 球 $\mathrm{Ch}^{\prime} \mathrm{iu} 4004$.

琅 Lang 3622 .

㻎 $\mathrm{Li} \quad 2341,3691,6049$.

珓 Liu 4004.

㑚 Yeh 3625 .

$S$ 琴 $\mathrm{Ch}^{\prime}$ in 5143.

琶 $\mathrm{P}$ 合 4816 .

琳 Lin 2051 .

娫 Y'en 4311, 431\%。
琮 'T's'ung 391\%, 69896, 6603.

9 层 Hsia $25 \% 2$.

瑞 Jui 3023 .

琩 Mei 6160 。

瑇 Tai 6160 .

理 Hun $23 \%$.

郎 Lang 3625.

10 瑪 Ma 3191, 4313.

瑰 Kuei 3623.

璘 $\mathrm{YaO} 731 \%$ A

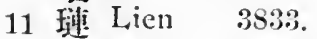

满 Man 3254 .

12 軖 Huang 2328.

畨 Fan $218 \%$.

13 環 Huan 2266, 7r22.

璧 $\mathrm{Pi} \quad 3960$.

15 舅 Ch'iung 1320, 366\%.

16 㠃 Kuei 4060.

\section{II KUA}

瓜 Kua 3393, 4523, 726\%。

\section{J WA}

王 Wa $\{2150,32 \% 0,3 \gamma 41,450 \%$,

4 咸 Wèng 7042.

6 瓶 Ping 521\%.

11 颚 Ou 4 \% $49.6 \% 23$.

12 毁 'Tsêng 6553.

13 䧳 Wèng 6393, \%044.

\section{9 甘 KAN}

甘 Kan $2104,30 \% 8,5488,6856$. 6 甜 $\mathrm{T}$-ien 6430 .

\section{0 告 SHÊNG}

生 Shêng 7555 .

6 道 $\mathrm{Ch}$,an 141s, 1759, 6003. 


\section{1 用 YUNG}

\author{
1 角 $\mathrm{Lu} 411 \%$ 。

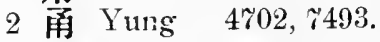 \\ 3 甫 $\mathrm{Fu} \quad 884,2284,4826$.
}

\section{2 田 T'IEN}

\section{田 T'ien Passim.}

1 甲 Chia $\left\{\begin{array}{l}719,22 \% \%, 5151,5494, \\ 6009\end{array}\right.$

申 Shên 5604 .

由 $\mathrm{Yu} \quad \mathrm{r} 632$.

2 町 T'ing 1436.

甸 Tien Passim.

"Ying r5\%s.

3 叟 Тzŭ 6832.

4 哭 Chieh $\mid 734,822,2445,3355$,

畏 Wei 5339, 7005.

5 留 Liu $\{469,398 \%, 4602,6692$,

$\{6695$.

㽣 Pan 4909 .

6 略 Lüeh 1116, 415\%。

哣 $\mathrm{Pi} 50 \% 4$.

7 潘 P'an Passim.

畕 Yi 6625, 7512, $7 \% 0 \%$

8 賞 Tang $\mid 29 \%, 3766,4303,608 \%$,

† 6230, 8095.

10 畿 $\mathrm{Ch}^{\prime} \mathrm{i} \quad 1122,5822,6610$.

17 覺 Tieh 4811, 6382.

\begin{tabular}{|c|c|}
\hline & $\vec{F}$ PI \\
\hline 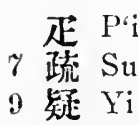 & $\begin{array}{l}5093 . \\
5933 . \\
1291 .\end{array}$ \\
\hline
\end{tabular}

\section{$104 y^{2} \mathrm{NI}$}

\section{5 疾 Chi 3205,3232 . \\ 17 疸 Ying $758 \%$}

\section{5 y}

$\gamma$ 登 Têng $\int_{(7015 .}^{195,5394,6329,68 \% 2,}$

\section{E PAI}

白 $\mathrm{Pai}\left\{\begin{array}{l}\% 80,3154,4029,4 \% 2 \%, \\ 4821,5252,60 \% 1,639 \%, \\ \gamma 220 .\end{array}\right.$

1 百 $\mathrm{Pai} 48 \%$.

2 皇 Tsao 6528.

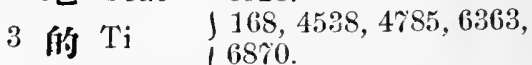

4 皇 Huang 2329, 5822, 6514.

5 卓 $\mathrm{K}$ 6492 .

„Kao $195 \%$.

7 皖 Wan 226\%,6910.

12 僠 $\mathrm{Pro} 5263$.

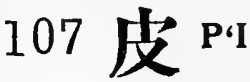

皮 $\mathrm{P}^{\prime} \mathrm{i}$

1892, 4612, 5088, ซร81.

\section{III MIN}

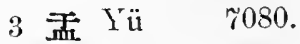

4 桑 Ying 4464.

盛 P'ên 5043 .

5 黄 $\mathrm{Yi} \quad 1 \% 0,4889,6 \% 63,7527$.

y 盛 Shêng 5614 .

8 蓝 Chan 165, 3321 .

9 監 Chien 892, 5363.

輫 Chin 5 \% 96.

10 盤 P'a1 399, 4485, 4911, 5526.

11 盧 Lu Passim.

12 贊 Chou 1346.

\section{E MU}

目 $\mathrm{Mu} 3 \% 08$.

3 㨁 Chih $950,2520,411 \%$.

时 Hsü 2892.

4 眉 $\mathrm{Mei}$ | 2424, 2425, 4365, 4737, 省 Shêng 5650

相 Ifsiang 1651, 2539, 2619, 6364.

真 Chên $\int 402,12 \%, 3840,4804$,

其 Chèn $159 \% 2,6970,7249,746 \%$.

眙 $\mathrm{Yi} 2892$.

6 兓 Yen 5402.

8 睦 M11 4514 .

㫿 Sui $3886,5951$.

9 鱣 Jui 3034.

12

$1376,1443$.

13 鲬 Chan 172.

薣 $\mathrm{K}_{11} 3297$. 


\section{0 牙 MOU}

4 秎 Ching 1139.

\section{1 矢 $\mathrm{SHIH}$}

3 知 Chih 969, $3134^{\mathrm{A}}, 4969,5219$.

\begin{tabular}{|c|c|c|c|}
\hline & & 12 & SHIH \\
\hline & $\sqrt{1}$ & Shih & Passim. \\
\hline 3 & 研 & Wu & $46 \Omega$ \\
\hline 4 & 唦 & Sha & 5452 \\
\hline 5 & 砦 & Chai & $\left\{\begin{array}{l}2155,2604,3361,4163, \\
4976,5996,65+0\end{array}\right.$ \\
\hline & 研 & $\mathrm{Ch}^{\circ} \mathrm{i}$ & 614 . \\
\hline & 砫 & Chut & $5 \% 98$ \\
\hline & 破 & $\mathrm{P}^{\prime} \mathrm{o}$ & $5262, \% 03 \%$ \\
\hline 6 & 斫 & Yen & 1181. \\
\hline & 砲 & Nao & 4648. \\
\hline 7 & 䂯 & Hsia & $25 \%$ \\
\hline 8 & 碉 & Tiao & 4801. \\
\hline & 确 & L,11 & 4112 \\
\hline & 矿低 & Chieh & 839,1509 . \\
\hline & 珀 & $\mathrm{Pi}$ & $50 \% 6$ \\
\hline & 磌 & Shuo & $14,2056,3505$. \\
\hline & 碭 & Tang & 6241 \\
\hline 10 & 確 & Ch'üch & 1504. \\
\hline & 礁 & $\mathrm{Ma}$ & $5 \approx 20$ \\
\hline & 能步 & $\mathrm{O}$ & $4 \gamma 41$ \\
\hline & 硙 & Nien & $46 \% 1$ \\
\hline & 磁 & $\mathrm{Tz}^{\prime} \breve{\imath}$ & 6868. \\
\hline 11 & 桃 & Mo & $4484,5850,6326,6693$. \\
\hline & 䃯 & Chuan & 1469 \\
\hline 12 & 磢卷 & Chi & $3 \% \mathrm{~s}, 1052$. \\
\hline & 硔 & Huang & $232 \%$ \\
\hline 15 & $\begin{array}{l}\text { 杽售 } \\
\end{array}$ & Fan & $3 \$ 19$. \\
\hline
\end{tabular}

\section{3 分 SHIH}

3 䄮 $\mathrm{Ch}^{\circ} \mathrm{i} \quad 652,4730,5901$.

就 Shê $\int 269,5032,5592,5690$,

4 沙 $\mathrm{Ch}^{\prime} \mathrm{i} \quad 650$.

祋 $\mathrm{Tai} 6158$.

旅 Chih 5902.

5 祝 Chu 1389.

神 Shên $\int 1220,3993,4855,5214$,

ना Tsu - $\begin{aligned} & 5606,5822, \% 40 \% . \\ & 430 \%, 5053,6585 .\end{aligned}$

就 'T'zŭ $108 \%$.
誵 Yu 927, 7232,7390 .

6 样 Hsiang $698,1570,260 \%, 5212$.

形 $\mathrm{Yü} \quad 6158$.

祭 $\mathrm{Chi} 612$.

8 政 L 11 (1852, 18\%5, 2662, 2940, 禁 Chin $4113,5520,7433,7$ \%25.

樉 Chin 5S22, 6845.

形 $\mathrm{Ch}^{\circ} \mathrm{i} 35 \% \mathrm{~s}$.

9 就î Chên 462 .

觘 Huo 2421.

解 Ful

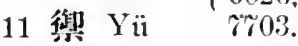

13 灌 Li $1304,3 \% 29$.

\section{4 五 Jou}

4 虽 Ÿ̈ rosi。

诎 $\mathrm{Yü} 492 \%, 520 \%$ 。

\section{5 주}

条 Ho $69 \%, 203 \gamma, 3 \% \gamma 3, \gamma+2 \%$.

3 秀 Hsiu 2860,4022, 7025.

秃 $\mathrm{T}_{\mathfrak{1}} 6652$.

3 奉 Ping $56 \%$.

4 科 $\mathrm{K} \cdot \hat{\mathrm{e}} \quad\{19,1892,1514,2103$, 科 $\mathrm{K} 231,6 \% 2 \%, 65 \% 9$

秋 Ch'in 18\%,1305, 75\%\%。

5 科 Ch'êng 524.

租 Tsu 1811 .

敉 Chih 960 .

秝 Mo 4489 .

秦 Ch'in $\{1105,2$ \% 39,3522 ,

1568 к, 65\%3, 6\%\%3.

稊 Tž̆ 6850 .

6 移 $\mathrm{Yi} \quad 4093,4643, \tau 506$.

$\gamma$ 秒 Shao 2203,5581 .

程 Ch'êng 52\%, 264t, \%166, r198.

8 稜 Lêng 6206 .

10 䖽 Chi $\{555,1512,1520,293 \%$, | 3469, 3636.

榣 Chi 549 .

稻 'T'ao 6059 .

踒 $\mathrm{K} 11 \quad 1515,3315,7266$ 。

皘 Chi 548 .

11 穆 Mu Passim.

穎 Ying 1260, 1732, 3900, 7579.

12 愁 Sui 5950 .

14 程 Wên rosy A.

1\%穬 Jang $29 \% 0$. 


\section{$116 \stackrel{\rightarrow}{\rightarrow}$ HSÜEH}

穴 Hsüeh 2525 .

3 穹 Ch'iung 1323, 3612. 空 K't1ng 541, 1473 .

4 穿 Ch'uan 545\%. 然 $\mathrm{Tu} \quad \mathrm{i} 194$.

9 䆟 Wo 3s31, r046.

10 篦 Yao 㝗 Yü 7 \% 734.

\section{LI \\ 立. $\mathrm{Li} 3754,7339$. \\ 5 站 Chan 2302, 5789,7320 . 童 Lung \121, 261, 1214, 4162, 站 Pan 4908 . \\ 6 音 Chang $\{201,135 \%, 2102,3246$, (7419, 7445, 765s. 竟 Ching 1118 . \\ 9 端 Tuan $\{34,3548,4 \% 46,66 \tilde{\tau}$, r049, 7124 .}

\section{8 解 $\mathrm{CHU}$}

竹 Chu Passim.

5 符 $\mathrm{F}_{11} \quad\left\{\begin{array}{l}405,925,1186,1765, \\ 1801,260 \%, 3258 .\end{array}\right.$ 笑 Pên 5041 .

第 Ti 6938 .

6 筑 Chu 1386, 34\%

筑 Chiung 1315 .

答 $\mathrm{Ta} 4810$.

7 篟 Yün 7831.

篗 'Tso 6460 .

8 簧 $\mathrm{Chi} 561$.

簓 Kê 3219 .

箱 Hsiang 2624.

管 Kuan 2\%67, 33506, 5860 .

9 節 Chieh 1763, 50\%4. 箭 Chien $89 \%, 6125$.

12 簡 Chien $8 \tilde{r} 4,4568 \mathrm{G}$,

13 䉀 Lien 6349 .

17 籠 Lung $\{565,4229,5865,7083$,

\section{9 米 MI}

米 $\mathrm{Mi}$

6 粟 Su

罾 Yüeh $3 \% 69,4890$, , 8994.

8 精 Ching 11\%t.

14 糆 No $4 \% 2 \%$.

\section{0 系 Ml}

3 紅. Hung 2380, 3022.

, Chiang $86 \%$.

総. Ho 2349, 4906, r744.

紀 Chi 505,552,600\%。

紟 Yü ros1.

4 綎 Chih 954 .

納 $\mathrm{Na}$ Passim.

純 Ch'un 1518.

.. T'un 6695.

索 So 1331, 5185, 5836 .

素 Su 6626.

5 絊 Ch'ou 4756.

累 Lei 1883.

", Lieh 1675.

終 Chung 157\%, r152.

絃. Hsien 6380.

紹 Shao 4\%04,55\%4.

細 Hsi 9, 2436 .

紫 Tzŭ $1149,5822,6845$.

6 桻 Chiang 869 .

參 Lei $36 \% 8$.

結 Chieh 835, 2215, 43\%6, 6611.

絲 Ssŭ 2315,5884 .

統 T'ung 4400 .

7 經 Ching 1116, 1\%10, 3046.

綘 Fêng 1\%5\%。

㛊 Sui $\quad(1086,5929,7371,7461$, 䌊 Stit $179 \% 2$.

8 䔦 Chou 1344.

基 $\mathrm{Ch}^{\circ} \mathrm{i} \quad 619$.

綽 Cho 1332.

縟 Lü 3935 .

綺 $\mathrm{Ch}^{\circ} \mathrm{i} 5048$.

緰 Lun 4160.

䄸 Mien 509, 4433 .

維 Wei 1654,7001.

9 觻 Mien 4439. 


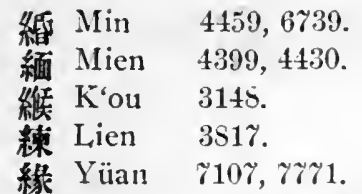

10 將 Hsien $\left\{\begin{array}{l}211,1268,2699,3141, \\ 4008,6251,6435 .\end{array}\right.$ 萦没 Ho $209 \%$.

結 Chin 1090.

11

慗 Fan $1625,2 \% 49$.

綪 Chi 598, 4273.

魔 $\mathrm{Mi} \quad 5 \pm \gamma, 4404$.

㣰 Tsung 1392, 6478 .

12

緄 Chih 966 .

紼 Hsiu 2868.

13 綆 Shêng 1600,5642.

14 算 'Tsuan 1342.

15 縓 Chan 2190.

果 Lieh $16 \%$.

\section{1 年}

4 鲉 $\mathrm{Ch}^{\circ}$ üeh 1502.

\section{2 奴 WANG}

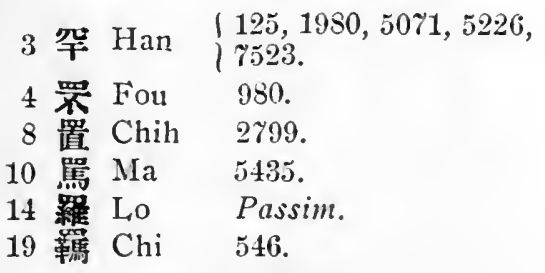

\section{3 单 YANG}

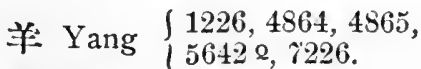

美 Mei 192\%, 4360 .

3 芙 $\mathrm{Yu} 7635$.

Ch' $419,780,852,188 \%$

4 虏 $\mathrm{Ch}^{\prime}$ iang $\left\{\begin{array}{l}419,780,852,188 \%, \\ 2443,4675,5262,6348, \\ 6444 .\end{array}\right.$

5 差 $\mathrm{Hsiu} 28 \% 6$.

渼 $\mathrm{Yi} \quad 5449,728 \%$.

7 義 $\mathrm{Yi}^{-\infty}$ Passim.

群 Chün 1524.

11 羲 $\mathrm{Hsi} 2430$.

\section{4 秽 Y}

物 Yü 1733, 7686.

4 翁 Wêng 5s\%2, 8038.

5 站 $\mathrm{Y}^{\mathrm{i}} 7620$.

齐 Hsi $2515,7249,7291$.

6 翔 Hsiang $1 \% 24,2606,45 \$ 0$ 。

8 膲 $\mathrm{Ti} 6330$.

"Chai rets.

9 蒋 Chien 1238.

12 翼 $\mathrm{Yi} 7514$.

14 耀 $\mathrm{Yao}$ \% 322 .

\section{5 艺 LAO}

$\begin{aligned} \text { 老 Lao } & 2864,3225,3632,7299 . \\ \text { 考 K'ao } & 3179 . \\ 4 \text { 著 Chê } & 393,1903,2130 . \\ \text { 惹 Ch'i } & 73,55 .\end{aligned}$

\section{6 面 $\hat{E R H}$}

而 Erh 52S2.

\section{7 本 LEI}

未 Lei $36 \%$ \%

4 耗 Hao 4322 .

\section{8 耳 $\mathrm{E}$ ERH}

耳 Erh Passim.

4 耿 Kêng 3242.

耿 Tan 6214.

5 聊 Liao $280 \%$.

$\gamma$ 留 Shêng $\left\{\begin{array}{l}1 \% \% 2,2831,5390,5822, \\ \text { ros9. }\end{array}\right.$

S 聞 IVên 2449, 2908, ro36.

聚 Chü 1434.

12 職 Chih $96 \%$.

\section{9 拝 YÜ}

8 萧 Su 99, 3089, 5920,6887.

肆 Ssŭ 5582 .

集 Chao 335. 


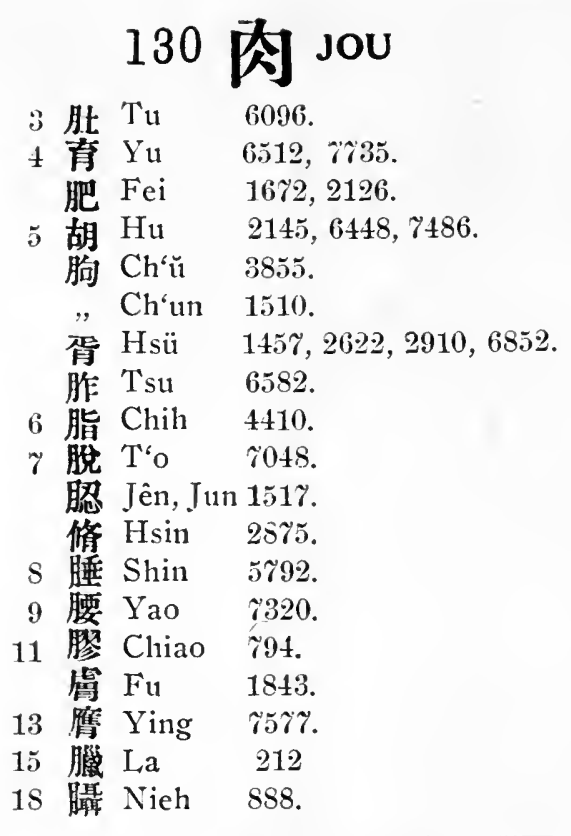

\section{E CH'ÊN $^{-}$}

战 Ch'èn $39 \%$.

2 卧 Wo 7045.

11 躁 Lin 604, 3031, 383\%.

\section{TZU \\ 自 Tž̆ 4380,6839 . \\ 5 臬 $\mathrm{Ku} 6492$. \\ 6 㫫 Kao $509,3016,31 \% 6,7139$.}

\section{3 茞}

$\begin{array}{rll} & \text { 䪞 Chih } & 963,3648 . \\ 8 & \text { 䵤 T'ai } & \text { Passim. } \\ 10 & \text { 瑧 Chên } & 459 .\end{array}$

\section{4 然 CHIU}

2 尖 $Y \ddot{u} 1470$.

5 春 $\mathrm{Ch}^{\prime}$ uang 1499.

8 與 $\mathrm{Y} \ddot{\mathrm{u}} \mathrm{Y} 685$.

9 鼠 Hsing Passim.

12 萑 Chiu 1266, 2305,

\section{5 卢 SHE}

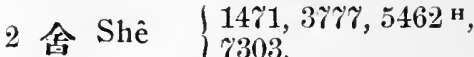

6 舒. Shu \3301, 4205, 5182, 5558. | $5 \% 61,6 \% 46$.

\section{6 多 CH'UAN}

8 舞. Wu 3897, 7161.

\section{7 年 $\mathrm{CHOU}$}

舟 Chou 4803 .

般 P'an 4910, 77S4.

5 舺 Chia 4402.

船 $\mathrm{Ch}^{\circ}$ uan $14 \% 1$.

8 艋 Mêng 4402.

11 艘 Lou 1696.

\section{8 层 KÊN}

1 通 Liang Passim.

\section{SE}

色 Sê $4545,4887,5413,68 \% 8$

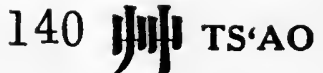

2 艾 $\mathrm{Ai} \quad 39,5693$.

3 巳 Mang 4330,4848 .

菜 $\mathrm{Yü} \quad$ i 682 .

4 奖 Chih $978,1132,2394$.

苏 Fề 313.

莎 Chih 961.

芭 $\mathrm{Pa} 4 \% 91$.

芳 Fang 1659.

花 $\mathrm{P}^{\prime} \mathrm{i} \quad 5092$.

芙 $\mathrm{Fu} 1825$.

留 $\mathrm{Ch}^{\circ} \mathrm{u} 4482$.

花 Hua I $2205,2383,3834,4834$, 花 Hua $\quad 5283,5289,6285,6286$. 
苏 Jui 3032 .

芹 $\mathrm{Ch}^{\prime}$ in 1094.

莱 $\mathrm{Ya} 1260$.

5 范 Fan 1640.

苮 $\mathrm{PaO} 4611$.

荏 Shih 5680,6855.

若 Jo 3000 .

苦 $\mathrm{K}^{\prime} \mathrm{u} \quad 3320$.

茅. M.аo 2296, 4341,5866 .

茂 Mou 509,4498 。

苗 Miao 4428.

苴 Chï 1433, 2385.

菼 Ying 4219, r604.

获 Mao 4849 .

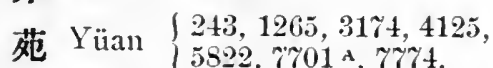

6 茶 Ch'a 146,7345.

茫 Mang 65\%, 4391, 480\%, 5383 .

荒 Huang 2333.

荅 Ta 591,4 \% 5 .

莎 $\mathrm{Ju} 1 \% 2,301 \%$.

荏 Jên 5462 ',

获 Chiao 4979 .

荆 Ching $\left\{\begin{array}{l}72,1140,5496,6016, \\ 6709\end{array}\right.$

6709

茬 Shih 5650,6855.

藮 Jung 3040 .

落 Tzŭ 1293.

修 $\mathrm{Li} \quad 1889,3745,6051$.

䓔 Ming 4464 .

淡 $\mathrm{Tz}^{\prime} \dot{\mathrm{u}} 668 \mathrm{s1}$

6 荀 Hsün 2964.

营 Ts'ao $2593,5806,6535$.

茜 Ch'ien 9.46.

$\gamma$ 莊 $\mathrm{Ch}$ uang $\left\{\begin{array}{l}59,1494,1847,2538, \\ 3118,3 \% 86,3 \% 98,5 \% 06, \\ 6453 .\end{array}\right.$

花 'I'so $65 \%$ \%

莎 So 5835 .

莎 Chü 1422.

荧 Kuan 6r25.

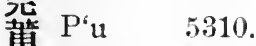

落 Shên $5616, \% 630$ 。

余 $\mathrm{T}$. 894 .

荻 $\mathrm{Ti} \quad 6359$.

8 鄯

華 Hua Passim.

萌 Mèng $\% 16$.

稂 $\mathrm{Ku}$ “ 3305 .

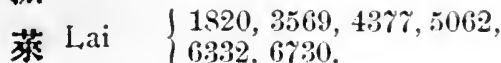

菟 T'u 2918.

菲 Chï 141\%.

䒭 Mang 2056,5905.

萑 T'ien 6429 .

萈 Huan $1 \% 41,22 \gamma 1$.

菜 P'ing 5211.

港 An 120,6834 .

茂 Ling 3948 .

蔁 $\mathrm{P}^{\prime} \mathrm{u} 5335$.

茹 Sung 5989 .

9 落 Kè | $253,3154,3 \% 13,43 \% 6$,

葵 K'uei 3488 .

䔖 So 5 S51.

落 Lao \{ $2294,2659,3636,5892$,

些 Wci 6966 .

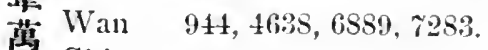

落 Chia 715 .

集 Yeh 4293, \%39\%.

"Shê 5582 .

10 薀 Kai 3058 .

,. $\mathrm{Ka} \quad 3052$.

莨 Lang $361 \%$ 。

䈬 $\mathrm{P}^{\prime} \mathrm{u} 5334$.

蒛 Mêng Passim.

㤅 Shih 5678 .

蒲 $\mathrm{P}^{\mathrm{i}} \mathrm{u} \quad 300,4852,5313$.

" Po $523 \%$.

蒜 Suan $592 \%$.

菨 Ts'ang 3339,6524 .

蓉 Jung 1925 .

11 暬 Liao 3809,4009 .

婵 Lien 3834,4842 .

途 P'èng Passim.

蔡 Ts'ai (2554, 28月6, 5534,65i)5,

繁 Ts'ai 16946.

辚 Chiang $7 \% 5$.

漖 $\mathrm{Ts}$ ' 7815 .

蔚 Yü. Wei rrüs.

陰 $\mathrm{Yin} \% 550$.

12 蕃 Fan 116\%, 1643, 6669 .

, P·i 5095 .

蓝 $\mathrm{Ch}$ ii $\$ 81 \%$.

染 Hsiao 2668.

灌 Tang 8040 .

,T'aing 6266.

䊉 $\mathrm{Pi} \quad 3155$.

蕉 Chiao $799,4 \% 90$. 


\begin{tabular}{|c|c|c|}
\hline 䔶 & $\mathrm{T}^{\prime} \mathrm{i}$ & 5335 \\
\hline 無 & $\mathrm{Wu}$ & 3573,7162 . \\
\hline 牌 & $P^{\prime}$ ai & 4898. \\
\hline 繌 & Chi & 580. \\
\hline 欂 & Pao & 4969. \\
\hline 限 & $\mathrm{Sa}$ & $\left\{\begin{array}{l}27,139,1443,3555 \\
5344,6352,7214,7238 .\end{array}\right.$ \\
\hline 葸 & Ts'ung & 3914. \\
\hline 薛 & Hsüeh & $2683,2935$. \\
\hline 蒡 & Kao & 3175 \\
\hline 監 & Lan & $743,3597,5347,5350$. \\
\hline 藏 & Ts'ang & 6424 \\
\hline$\therefore$ & Tsang & $940,2137,2502,7209$. \\
\hline 藕萬 & $\mathrm{Ou}$ & 4750 \\
\hline 藥 & Yao & 7321. \\
\hline 藤 & T'êng & 6340. \\
\hline 暲 & $\mathrm{Ch}^{\circ} \mathrm{i}$ & 645. \\
\hline & Chi & 545. \\
\hline 䦧 & Lin & 3262 \\
\hline 盛 & $\mathrm{Lu}$ & $260,408 \%$ \\
\hline 懵 & $\mathrm{Su}$ & Passim. \\
\hline & Lan & Passim. \\
\hline & Nieh & 3188. \\
\hline 总 & Lo & $41) 50$ \\
\hline
\end{tabular}

\section{HU}

2 虎 $\mathrm{Hu} \quad 2165,3620,4833,5453$.

4 虔 Ch'ien 908.

Ssŭ $, \int 2164,4142,4439,4441$.

虎 ${ }^{2} \mathrm{~T}$ ' $\mathrm{i}\{63 \% 2$.

5 處 $\mathrm{Ch}^{*} \mathrm{u} 1395$.

虚 Hsü 1355, 2890,4075, 6105,

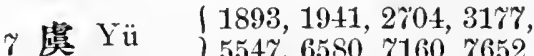

9 煞 Kuo 3536, $6 \% 29$.

\section{2 出 $\mathrm{CH} \cdot \mathrm{UNG}$}

3 虹 Hung 4018 .

, Chiang 768 .

5 蛇 Shê 6079 .

蛉 Ling 1156.

7 虽 Shu 4395, 57\%1.

蜋 Lang $62 \% 2$.

8 蜻 Ching, 1156.

9 蝢 Chieh 834.

蛼 $\mathrm{T}$ ' $\mathrm{i} 16 \%$.

蝍 Lang 2290 .

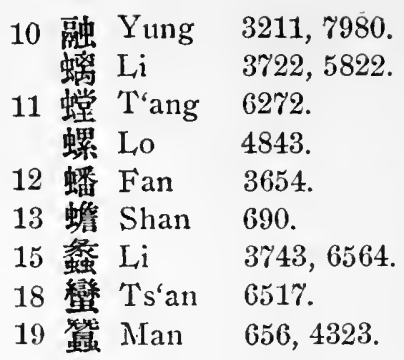

\section{3 向. HSIEH}

6 衆 Chung 60 .

\section{4 行 HSING}

行 Hsing $\{251,1495,2814,4453$.

6 街 Chieh Passim.

衙 Ch'ung 6343, ry8\%.

10 衡 Hêng 2030, 6415.

衛 Wei Passim.

16 聵 Ch'ü $\{1019,1444,5366,5822$, $\{6413 \mathrm{~A}, 67 \% 6$.

\section{5 衣 $\mathrm{YI}$}

农 $\mathrm{Yi} \quad 1230,4743,7513$.

3 表 Piao 5096 .

4 袁 Yüan 3031, 7742 .

7 裹 $\mathrm{Li} \quad 1498,2942,3694,4384$.

補 $\mathrm{Pu} \quad 47 \% 5$.

福 Shu $5 \% 68$

浴 $\mathrm{Yü} \quad 7196,7698$.

8 裳 Shang 7790.

装 $\mathrm{P}$ ei 5032.

Fei 609.

9 葆 $\mathrm{Pao} 4956$.

10 䇾 Huai 2224, 2990, 6032.

11 褒 Hsiang $\left\{\begin{array}{l}72,1353,2609,5162, \\ 6751 .\end{array}\right.$

Pao 4958

12 鼬 Tsa 4320.

\section{6 西 SHA}

西 Hsi Passim.

3 娶 $\mathrm{Yao} 3169,5401,7318$.

12 㩲 $\mathrm{Fu} 1885$. 


\section{7 見 CHIEN}

9 親 $\mathrm{Ch}^{6}$ in $2 \% 01$.

13 覺 Chüeh 1500, 6498.

18 観 Kuan 3364, 4909.

\section{8 争 CHIAO}

角 Chiao Passim.

5 觜 Tsui 568,756.

解 $\mathrm{Ku} 369 \%$ 。

6 解 Chieh 829.

\begin{tabular}{|c|c|c|}
\hline & 149 & 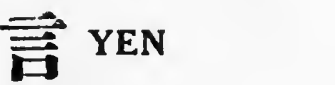 \\
\hline 言 & Yen & 6908. \\
\hline 2 計 & Chi & 556. \\
\hline 3 討 & T'ao & 6303. \\
\hline 4. 許 & Hsü & $2080,2885,6 \gamma 82$. \\
\hline 設 & Shê & 5602,6149 \\
\hline 䛴 & No & $4728,5403$. \\
\hline 5 娚 & Chao & 361.4565 \\
\hline 7 誠 & Ch'êng & 523. \\
\hline 8 樑 & $K^{\prime} \hat{\mathrm{e}}$ & 7350 \\
\hline 諭 & Lun & 356. \\
\hline 㵊 & T'iao & 6380. \\
\hline 9 謀 & Mou & $7 \% 60$ \\
\hline 榙 & Chu & 1373,4434 \\
\hline 諾 & No & $4729,6475$. \\
\hline 諭 & $Y \ddot{u}$ & $2 \% 98$ \\
\hline 謁 & Yeh & 7341 \\
\hline 10 謝 & Hsieh & $2673,665 \%$ \\
\hline 謴 & Ch'ien & $26 \% 3$ \\
\hline 11 謨 & $\mathrm{Mu}$ & 2794,4789 \\
\hline 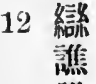 & $\begin{array}{l}\text { Luan } \\
\text { Ch'iao }\end{array}$ & $\begin{array}{l}59 \%, 456 \mathrm{~S} \text { Е, } 4980 . \\
812,4606 .\end{array}$ \\
\hline 13 議 & $\mathrm{Yi}$ & $17 \% 4$ \\
\hline 14 護 & $\mathrm{Hu}$ & $21 \mathrm{s6}, 4460 ; 7174,7324$ \\
\hline 15 詰賁 & $\mathrm{Tu}$ & $66+8$ \\
\hline 18 弾 & Chê & 2153. \\
\hline
\end{tabular}

\section{0 公 $\mathrm{KU}$}

谷 $\mathrm{Ku}$ Passim.

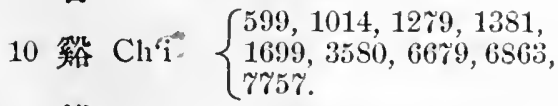
彭 Huo 2426, $52 \% 1$.

\section{1 昰 Tou}

6 豊 $\mathrm{Li} \quad 3725,5803,7365,7858$.

11 Fêng Passim.

\section{2 㚕 SHIH}

5 象 Hsiang $2599,3818$.

$\gamma$ 豪 Hao 1999,6130 .

9 豫 Yï $660,5917,765 \%$.

10 函 Pin 4614, 5126.

\section{3 若 CHAI}

3 豹 $\mathrm{Pao} 4959$.

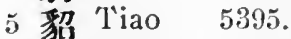

6 徝 Mo 4481, 5598.

11 婇 Hsiang 4481

\section{4 具 PEI}

具 Pei 2175, 4973.

2 貞 Chên $460,733 \%$.

3 顡 Kung $\int 206,525,24 \% 6,350 \%$, 然

IIt Ts'ai 1 r8s.

5 費 $\mathrm{Pi} 5064$.

Mai 4321.

貿 Kuan 944.

賀 Ho $2096,3563,6570$.

貫 Kuei $80,3473,780 \%$ 。

幕 Pan 2614 .

6 空 Chia, ku 711, 3290.

饯 Tsei $464 \%$.

資 Tzŭ $\int 1990,316 \%, 5590,6840$,

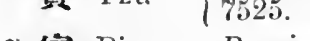

y 凟 Pin Passim.

鉴 Shê 5594 .

8 賧 T'an 4096, 7\%92.

賈 T's'ung 6604.

賴 Lai 130 .

賢 Hsien $\begin{aligned} & 354,596,1072,1768, \\ & 62 \% 6 .\end{aligned}$

質 Chih 983.

竞 Mai 4321.

10 蕉 Sai 5349.

滕 Shèng 6322 .

11 雔 Chui 1506.

12 積 Tsau 6513.

$1 \%$ 竷 Kan 588, 3099 . 


\section{5 羿 $\mathrm{CH}$ ‘IH}

\begin{tabular}{|c|c|c|}
\hline 赤 & Ch'ih & $\left\{\begin{array}{l}154,990,4759,7047, \\
7344,7726 .\end{array}\right.$ \\
\hline 5 赫 & Nan & $464 \%$ \\
\hline 赫 & Ho & 4728. \\
\hline 籍 & Chê & 395. \\
\hline
\end{tabular}

\section{6 走 TSOU}

起 Tsou 3531, 6575 .

3 起 $\mathrm{Ch}^{\prime} \mathrm{i} 626$.

5 越 Yüeh | $4715,5210,5946,6339$,

$\gamma$ 趙 Chao $324,2099,3184$.

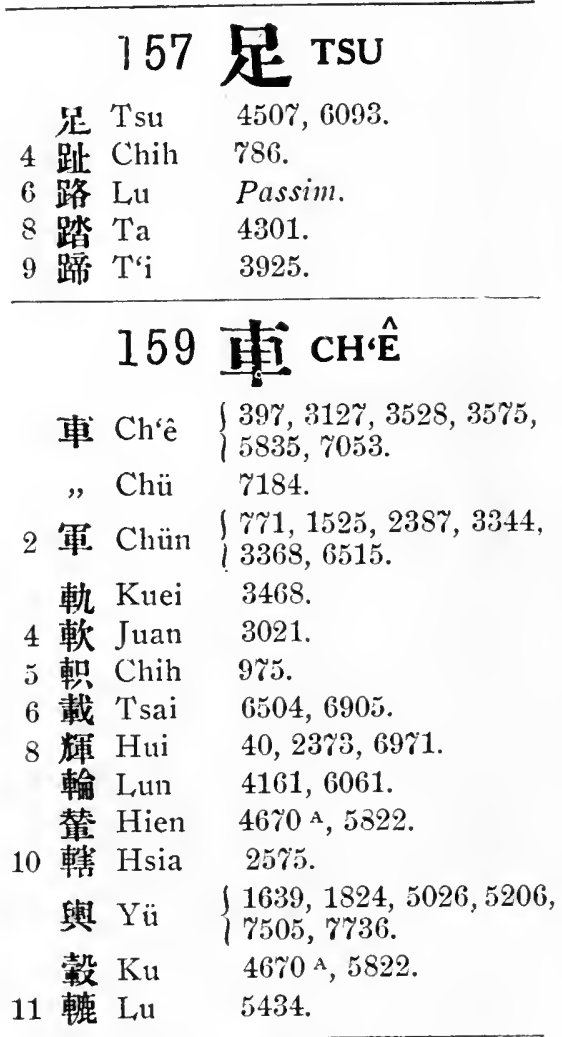

\section{0 斗 HSIN}

辛 $\mathrm{H} \sin 2799,6150$ 。

9 辦 $\mathrm{Pan} 4903$.

辨 Pien 4031.

14 辯 Pien 5105 .

\section{1 展 $\mathrm{CH} \cdot \hat{\mathrm{EN}}$}

辰 Ch'ên $474,3512$.

6 辳 Nung Passim.

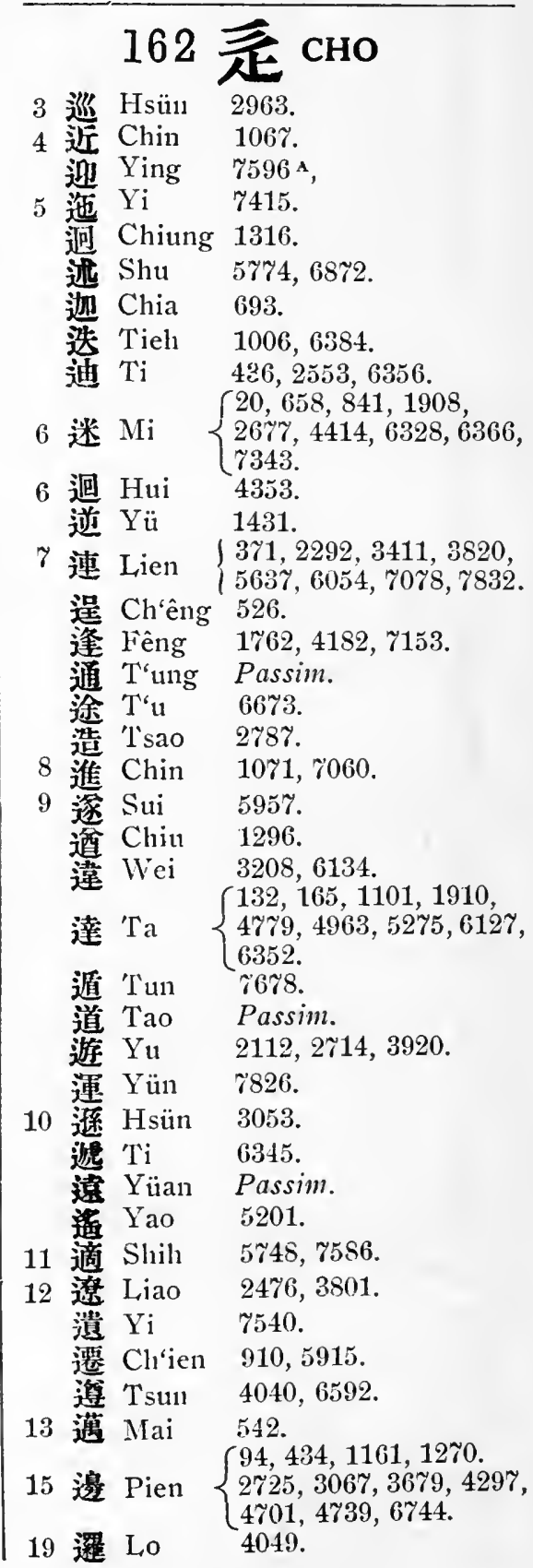




\section{3 邑 YI}

\begin{tabular}{|c|c|c|}
\hline 芭 & $\mathrm{Yi}$ & Passim. \\
\hline 郎 & Ch'i & 625 \\
\hline \#B & Kan & $3105,4759$. \\
\hline 我 & $Y \ddot{\mathrm{u}}$ & 7683. \\
\hline IS & Chiung & g 1309, 21s\%, 3S51, 585\%. \\
\hline 毕 & Yung & 7996 \\
\hline 邡 & Fang & $\check{5} 6 \varsigma 9$. \\
\hline 邞 & $\mathrm{Fu}$ & 1826. \\
\hline 形 & Hsing & 2849 . \\
\hline ", & Kêng & 3241 \\
\hline 那 & $\mathrm{Na}$ & $\begin{array}{l}\text { 750, 138\%, 1443, 2270, } \\
\text { | 4537, 7685. }\end{array}$ \\
\hline 邦 & Pang & $452 \%, 4932,6622$ \\
\hline 分 & Pin & $1 \% 27,512 \%$ \\
\hline 邪 & Hsieh & $26 \% 6$ \\
\hline & Yeh & 3625. \\
\hline 邯 & Han & 1978. \\
\hline 邱 & $\mathrm{Ch}^{\prime} \mathrm{in}$ & Passim. \\
\hline 丕 & P'ei & $2547,4993,5035$. \\
\hline 猅 & Pei & $502 \%$ \\
\hline 郡 & Shao & 5565, ช361. \\
\hline 邸 & $\mathrm{Ti}$ & 6354 \\
\hline 制 & Chu & $1369,2536,2652,5343$. \\
\hline 到 & Chib & รั\%9. \\
\hline 郝 & Shih & 5683. \\
\hline 郃 & Ho & 2135 \\
\hline 邽 & Kuei & $2543,5514$. \\
\hline 郁 & $Y \ddot{i}$ & \% $\% 09$ \\
\hline 的 & Hsün & 2966 A. \\
\hline 灶 & Cliia & $72 \%$ \\
\hline 郎 & Lang & $\left\{\begin{array}{l}2165,2978,3504,362 \%, \\
538 \%, 5681,6137,6935, \\
7325 .\end{array}\right.$ \\
\hline 虽 & Ying & $\tau 601$ \\
\hline 郜 & Kao & $31 \% 5$. \\
\hline 郡 & Chün & 1325 \\
\hline 林 & Ch'ên & 450,2036 \\
\hline 部 & Kuo & $\left\{\begin{array}{l}1317,2526,3539,5840, \\
7268 .\end{array}\right.$ \\
\hline 鄅 & $P \cdot i$ & 5083. \\
\hline 炏 & 'T'an & $622 \%$ \\
\hline 兒 & $\mathrm{Yi}$ & 7539 \\
\hline 部 & $\mathrm{Pu}$ & $\left\{\begin{array}{l}1312,154 \%, 213 \%, 461 \%, \\
5040,5892 .\end{array}\right.$ \\
\hline 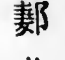 & $\mathrm{Ch}^{\circ} \mathrm{i}^{-}$ & 2734 \\
\hline & 'Tz' $\breve{u}$ & 6859. \\
\hline
\end{tabular}

郵 $Y$ แ1 $1110,31 \% 1$.

9 鄀 Jo 3001.

邻 Shu $5 \% 66$.

鄄 Chïan 1451.

鄂 Nei 4369 .

啊 $\mathrm{O} 4 \% 42,4 \% 65$.

㖪 Yün \% \%28.

都 Tu Passim。

㯦 Yen 1380.

10

䣓 Hao 1996 .

卿 Hsiang Passim.

駺 T'sou $65 \% 6$.

坬 $\mathrm{W}_{11} \% 19 \%$.

鄖 $\mathrm{Y}$ ïn 72,7820 .

鄣 Chang 22\%, 3276 .

11 麇 $\mathrm{Fu}$ 1805, 2446. 鄚 Mo 4491 .

梆 $\mathrm{Hu} 2178$.

滒 Yen $838 \%$.

鄞 Yin 7560 。

Yung 7984.

12 鄚 Chêng 495, 2ช29, 456\%.

鄰 $\operatorname{Lin}\{1031,1924,2521,3921$,

$\left\{\begin{array}{l}1999 . \\ 5904 .\end{array}\right.$

翻 $\mathrm{P}$ 'o 5264 .

都 Hsiin 444.

郘 Tan $19 \% 9$.

To 6474.

站 Têng $633 \%$.

暗 Tsêng 6554.

13 险 Kuei $34 \% 2$.

鄴 Yeh 6998, 7335 .

鄔 Mêng 4403 .

17 糙 Ling $39 \% 6$.

15 数 Fèng 1753 .

19 貲 'Tso $65 \% 1$.

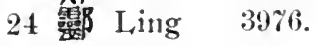

\section{4 开 YU}

$\begin{array}{rlr} & \text { 西 Yu } & \text { \% } 639 . \\ 3 \text { 酒 Chiu } & 1294 . \\ 7 \text { 酸 Suan } & 5928 . \\ 13 & \text { 醴 Li } & 3734 .\end{array}$

\section{5 尔 TSAAl}

采 Ts'ai 6511 . 


\section{6 里 LI}

\section{里 Li Passim.}

2 重 Chung 1566, 5369.

„Ch'ung 1582.

4 野 Yeh $\left\{\begin{array}{l}281,1415,2347,2793, \\ 3331,5256,6110,7327, \\ 7442 .\end{array}\right.$ 11 䃏 $\mathrm{Li} 3695$.

\section{7 金 CHIN}

\begin{tabular}{|c|c|c|}
\hline 金 & Chin & $\left\{\begin{array}{l}186,755,993,994, \\
1007,2649,3026,3663 \\
7684 .\end{array}\right.$ \\
\hline 2 針 & Chên & 443 \\
\hline 3 釷 & $\mathrm{Ch}^{\prime} \mathrm{ai}$ & 1008. \\
\hline 鈞 & Tiao & 6378. \\
\hline 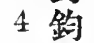 & Chün & 1523. \\
\hline 釜 & $F u$ & 1808. \\
\hline 5 鉅 & Chï & 1413. \\
\hline & Ch'ien & 916. \\
\hline & Po & 4531. \\
\hline & Kou & 524. \\
\hline 鉛 & $\begin{array}{l}\text { Ch'ien }^{6} \\
\mathrm{~T}^{\prime} \text { 'ung }\end{array}$ & $\begin{array}{l}930 . \\
6820 .\end{array}$ \\
\hline 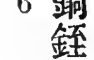 & Chih & 965 \\
\hline 銀 & Yin & $115,8565,7551$. \\
\hline 鋪 & $P^{\prime} u$ & Passim. \\
\hline 鋒 & Fêng & 5925. \\
\hline 錦 & Chin & 1060,1764 \\
\hline 錫 & $\mathrm{Hsi}$ & $136,2524,7154$ \\
\hline 錢 & Ch'ien & 934,951 \\
\hline 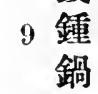 & $\begin{array}{l}\text { Chung } \\
\text { Kuo }\end{array}$ & $\begin{array}{l}381,1569,4313,6259 \text {. } \\
3535 \text {. }\end{array}$ \\
\hline 鋤 & Chên & $\left\{\begin{array}{l}311,415,1566,1198, \\
1234,1 \% 36,2 \% 06,3282, \\
6388,640 \%\end{array}\right.$ \\
\hline & Hao & 1998. \\
\hline & $\begin{array}{l}\text { Ching } \\
\text { Yung }\end{array}$ & $\begin{array}{l}1119,2688,4015 . \\
7985 .\end{array}$ \\
\hline 12 鐔 & Hsün & 2944. \\
\hline & Chung & $1581,3525,6869$. \\
\hline 5 & T'ieh & $638 \%$ \\
\hline 9 鑽 & Tsuan & 2421. \\
\hline
\end{tabular}

\section{8 長 CH·ANG}

長 $\mathrm{Ch}^{\circ} \quad\{229,432,881,1275$,

長 ang $4499,6406,6700,7359$.

"Chang 1rir.

\section{9 門 MÊN}

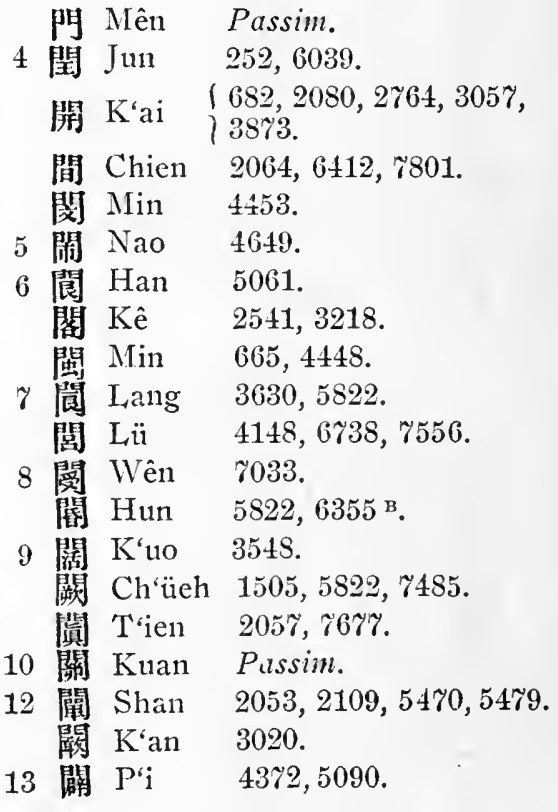

\section{FU}

皁 Fu $\left\{\begin{array}{l}63,640,1454,1 \% 40, \\ 1783,1952,325 \%\end{array}\right.$

3 泝 Ch'ien $5 \% 01$.

4 阯 Chih 786.

防 Fang 1669.

5阿 $\mathrm{A}\{1,1390,1447,338 \%$ ，

5 阿 A $\{4255,4760$.

, O 6742 .

附 $\mathrm{Fu}_{1} \quad 1803,4652$.

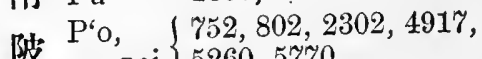

陂

陁 T'o 6493.

陀 'T'0 5255, 5325 .

6 陌 Mo 1113, 4608, 5523.

䧇 $\mathrm{Yi} 6428$. 
降 Chiang 1549, 2494, 6750 .

7 陟 Chih 952, 7058.

除 $\mathrm{Ch}^{\prime} \mathrm{\prime} 2069$.

陘 Hsing $11 \% 9,2842$.

陜 Hsia 97, 2576, 6523.

陛 $\mathrm{Pi} 5822,6419 \mathrm{~A}, 7317^{\mathrm{B}}$.

Shan 4703,5483.

陡 Tou 6498 .

䏚 Ch'iao 816 .

8 陳 Ch'ên $\left\{\begin{array}{l}463,1310,2080,4006, \\ 4103,4566,497 \%, 5492\end{array}\right.$

陪 P'ei 5039 .

㵵 Tsou 906.

陲 $\mathrm{Ch}^{\mathrm{r}}{ }^{n i} 4568^{\mathrm{R}}$.

陵 Ling Passim.

阹 $\mathrm{L}_{11}\{83,498,1891,412 \%$,

塺 Lil $\{4605,5002$.

陶 'T'ao $\int 3355,5189,6297,6459$,

陰 Yin Passim.

9 階 Chieh $\left\{\begin{array}{l}483,832,26 \% 9,3522, \\ 3926,705 \%\end{array}\right.$

隃 Yü $76 \gamma_{2}$.

隆. Linng Passim.

隄 $\mathrm{Ti} \quad 3162,4234,4923,5190$.

陽 Yang Passim. 隍 Huang 3988, 605\%.

10

隔 $\mathrm{Ai} 330 \%, 5462$ \&, 7280 .

聰 Liu $605 \%$.

隔 Kê 3995 .

隗 IVei 6999.

12

隣 Lin 3924

榢 Sui 8093.

13 隨 Sui 5955.

14 隰 Hsi 2811.

隐 Yin 5985 .

17 龖 Lung $\left\{\begin{array}{l}3150,4230,654 \%, 7473, \\ 7 \% 2 \%\end{array}\right.$

\section{1 承 TAI}

\section{8 隸 $\mathrm{Li} \quad 950,3374,3682$.}

\section{2 俸 CHUI}

2 寉 Ho. 2112.

3 雀 Chiao 800.

4 雄 Hsiung $\left\{\begin{array}{l}427,855,1153,1404, \\ 287 \%, 4023,4585,641 \%\end{array}\right.$
集 Chi Passin.

雅 $\mathrm{Ya} \mid 135,2408,322 \%, 5446$,

㕍 Yen 7384.

5 雉 Chih 948.

, Yi 2558.

售 T'sun 2555, 6594.

䔨 Yung $3273,6765,7956$.

6 倠 Lo $4066,5519,5555$.

8 雕 Tiao 6375.

10 雞 Chi $\quad$ (563, 782, 1011, 2621,

維 Chi 14935,7988 .

雙 Shuang 577\%。

獾 Tsa 6502 .

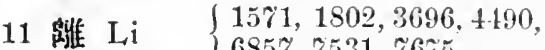

12 難 Nan $2 \% 72,4646$.

\section{3 雨 Yü}

雨 Yü 7084 .

3 雱 Hsüeh 2342.

零 Yü 7655 .

4 雲 Yün Passim.

5 雷 Lei $\quad\{1755,2544,2602,3664$

零 Ling 3263, 3933 .

電 Tien $639 \%$.

7 震 Chên 413.

零 Hsiao 780 .

8 霑 Chan 169 .

霍 Ho 19'2, 2098, 5255.

9 霞 Hsia $\quad 659,2574,4836,6204$.

11 霧 $\mathrm{Wu} \quad 2980,4346$.

13 霸 $\mathrm{Pa} \quad 4544,4$ \% 92.

16 諨 $\mathrm{Ai} 44$.

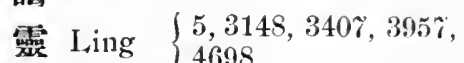

\section{4 春 CH'ING}

等 Ch'ing $\left\{\begin{array}{l}119 \% \\ 7294\end{array}\right.$

5 靖 Ching $\left\{\begin{array}{l}421,479,115 \%, 1452, \\ 4568^{\mathrm{M}}, 4949,5933, \\ 601 \% .\end{array}\right.$

8 静 Ching $1163,1999,4568 \mathrm{~N}$. 


\section{5 非 FEI}

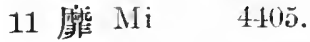

\section{6 值 MIEN}

面 Mien $\check{1} 435$.

\section{7 芦 $\mathrm{KE}$}

革 Kê 9, 3209, 4334, 7\%23.

3 靬 Chien 984.

6 瑟 Kung 664, 2472, 3520.

鞍 An 118 .

9 鞮 $\mathrm{Ti} 6828$.

11 韃 $\mathrm{Ta} 5346$.

\section{8 韋 WEI}

韋 Vei 4709, 5285, 6962.

8 韓 Han $\{4 \%$, 19\%2, 4282, 5108,

15371.

10 韜 T'ao 4959, 6696.

\begin{tabular}{|c|c|c|c|}
\hline 5 & $\begin{array}{l}\text { 音 } \\
\text { 韶 }\end{array}$ & $\begin{array}{l}180 \\
\text { Yin } \\
\text { Shao }\end{array}$ & $\begin{array}{l}\vec{E} \text { YIN } \\
3403 . \\
3411,5575 .\end{array}$ \\
\hline & & 81 & YEH \\
\hline 2 & 頂 & 'l'ing & $6465,7 \gamma 96$. \\
\hline & 頃 & Ch'ing & 6892 \\
\hline 3 & 項 & Hsiang & 2489 \\
\hline & 順 & Shun & Passim. \\
\hline & 須 & Hsü & $2912,4421$. \\
\hline 4 & 頓 & 'I'un & 6686. \\
\hline & 頌 & Sung & 3120. \\
\hline 5 & 領 & Ling & $393 \%$ \\
\hline 7 & 頸 & Kêng & 4284. \\
\hline & 頽 & T'ui & $5 \% \% 0$. \\
\hline & 頻 & $\mathrm{P}^{\prime}$ in & 5129. \\
\hline & 頭 & 'T'ou & Passim. \\
\hline 9 & 額 & E & $1601,4279$. \\
\hline & 蕷 & Chuan & $14 \% 0$ \\
\hline & 顏 & Yen & $4789.5403,64 S 2,7401$. \\
\hline 12 & 顧 & $\mathrm{Ku}$ & 3251 \\
\hline & 題 & $\mathrm{T} \cdot \mathrm{i}$ & 5849 \\
\hline 14 & 顯 & Hsien & 2700 \\
\hline
\end{tabular}

\section{2 風 FÊNG}

風 Fêng Passim.

\section{3 茼 FEI}

我 Fei 16\%8, 7173.

\section{4 食 SHIH}

6 峜 Yang $\pm 401,7240$.

7 餘 Yü 1823, 1881, 6879, 7645.

8 館 Kuan (1985, 2314, 2625,2669, $3354,7546$.

12 饒 Jao $2972,3402,5510$.

\section{5 首 SHOU}

首 Shou $826,4299,5734,5759$.

\section{6 自 HSIANG}

香 Hsiang $1714,2587$.

\section{7 影 MA}

望 Ma Passim.

2 䳐 Fêng 1\%54, 7620.

3 那 Hsün 2960.

4 䭾 'T'o 6486.

馹 $\mathrm{Yi} \cdot 5864$.

5 駕 Chia $756 \%$.

駒 Chü 4168 .

駝 $\mathrm{T}^{\circ} \mathrm{o} \quad 40 \%$ ().

䑐 P'ien $5 \% 15$.

6 駱 Lo $40 \% 0$.

8 騏 Ch'i 622.

駢 P'ien 5116.

10 滕 'T'êng $3229,4167,6342$.

騩 Kunei 6045.

騘 Tsou 6579.

11 驃 P'iao 5098.

16 驢 Lii 4143.

19 䱊 Li $313 \%, 3741$.

,Ch'ih 984. 


\section{8 䆒 $\mathrm{KU}$}

管 $\mathrm{Ku} 3306,6473$.

13 嘌 $\mathrm{T}$ ' $\mathrm{i} 32$.

\section{9 물 KAO}

高 Kao $\left\{\begin{array}{l}559,14 \% 1,3123,3875, \\ 5512,6943, \% 265 .\end{array}\right.$

\section{0 欫 PIAO}

5 䯮 Mou, $\{449 \%$.

\%敂 $\mathrm{Ti} 63 \% 4$.

\section{CH'ANG}

17 攀 143

\section{3 鬲 $\mathrm{KE}$}

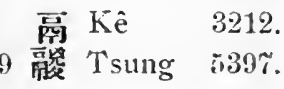

\section{4 男 KUEI}

4 魁 K'uei 554 .

8 魏 IVei 303, 6990 .

\section{5 魚YÜ}

份 Yü $\quad$ iu $\% 99,31 \% 2,46 \% 0,69 \%$,

4 售 Lu

\% 690 .

Passim.

$46 \% 0$.

$1 \% \approx 6$.

4968

6485.

6 鮮 Hsien 368,2704 .

11 鱗 Yung 612.4.

鳌 Ao $12 \%$.

\section{6 息 NIAO}

鼠 Niao 999, 1680, 4668 .

2 藏 Chiu 1293, $5 \% 63$.

毁 $\mathrm{Fu} 2 \% 09$.

3 唒 Fêng $\left\{1716,355 \tau^{\mathrm{A}}, 5785\right.$,

鳴 Ming 1581,4474

4 鴨 $\mathrm{Ya} 3635$.

鷹 Yen Fis3.

5 燋 $\mathrm{Ya}$ \% 221.

6 鸿 Hung 2410,3650,5768.

\% $\mathrm{O} 4$ 4 36 .

8 龍 Shun $582 \%$.

鵰 Tiao 6376.

鹊 Ch'üeh 1503.

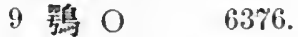

10 鶴 Ho $2113,44 \% 5,4830$,

鹤 $\mathrm{Hu} 2350$.

12 潐 Ying 5822, $75 \% 6$.

\section{7 或 LU}

13 監 I‘n $\left\{\begin{array}{l}16-5,1940,2011,3626, \\ 4867,5501,6112,7345\end{array}\right.$

\section{8 臂 LU}

Lu $\left\{\begin{array}{l}1326,1414,2262,2423 . \\ 4118,4294,4476,4845,\end{array}\right.$ $5 \% 6 \%, 6591$.

6 鹿 $\mathrm{Mi} \quad 441 \%, 7660,76 \% 2$.

魔 $\mathrm{Li} \quad 3136,3143,3736$.

麓 Lu 3229.

雁 $\mathrm{Ni} \quad 4126,5433$.

12 蹸 Lin 900,3918 .

\section{9 檴 MAI}

綮 Nai 4:18.

\section{0 庯 MA}

$\begin{aligned} \text { 魔 Ma } & \left\{\begin{array}{l}1 \%, 2104,3018,3094, \\ 4256,4391,5336,6395, \\ 6576,7028 .\end{array}\right. \\ 3 \text { 㦄 Mo } & 7189 .\end{aligned}$




\section{1 黃 HUANG}

黄 Huang $\left\{\begin{array}{l}68,1953,2188,2272, \\ 4653,5506,5965,6033, \\ 6886,7428 .\end{array}\right.$

\section{2 害 SHU} 3 黎 $\mathrm{L}_{\mathrm{i}} \mathrm{i} \quad\left\{\begin{array}{l}293.650,790,1441, \\ 2769,3 \% 13,5000,5093 . \\ 5625,6783,70 \% \%\end{array}\right.$

\section{3 매 $\mathrm{HEI}$}

黑 Hei $\left\{\begin{array}{l}2005,2652,4539,47 \% 0, \\ 4810\end{array}\right.$

4 黔 Chiien 902 .

默 Mo 6661.

5 㙻 $\mathrm{Yi} 7509$.

6 秝 $\mathrm{Yi} 7508$.

8 黨 Tang 5531, 6238.

\section{5 腱 MENG}

11

迎 Mien 4446 .

簿 $\mathrm{Pi} 50 \% 0$.

謷 Ao $122^{\mathrm{A}}, 5822$.

\section{6 舀 TING}

鼎. Ting $1850,2321,4942,6466$.

\section{7 鼓 $\mathrm{KU}$}

鼓 $\mathrm{Ku} \quad 3295,5 \% 13,6823$.

\section{0 峦新 $\mathrm{CH}$ 'I}

咱 Chi $\quad\left\{\begin{array}{l}6 \% 1,921,1911,2024, \\ 5344,648 \%, \gamma 184, \gamma 488 .\end{array}\right.$

\section{2 韵 LUNG}

\section{龍 Lung Passim.}

6 登辈 Kung $351 \%$.

\section{3 峩廷 KUEI}

帠 Ch'iu $130 \%$. 





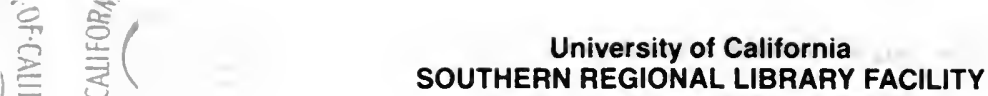 \\ Return this material to the library from which it was borrowed.}

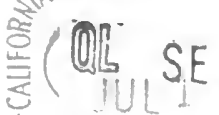
$\bar{t}_{1 / 5}$

焉
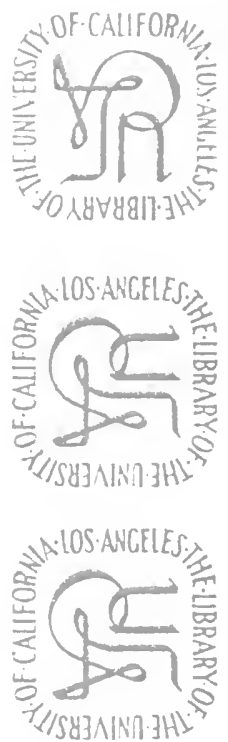

MEDIIBRARYCF.
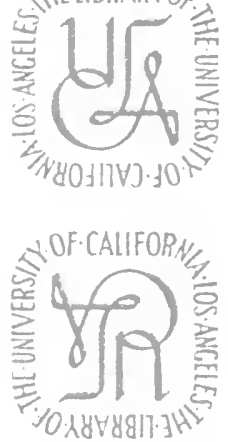

PC'O LDURL

H. APR 091990

FEB 0 , $\mathrm{kel}$

\section{SRLF}

QL

$$
\text { APR O } 91990
$$

REC:P LP- 4 RT:

SNN 201996
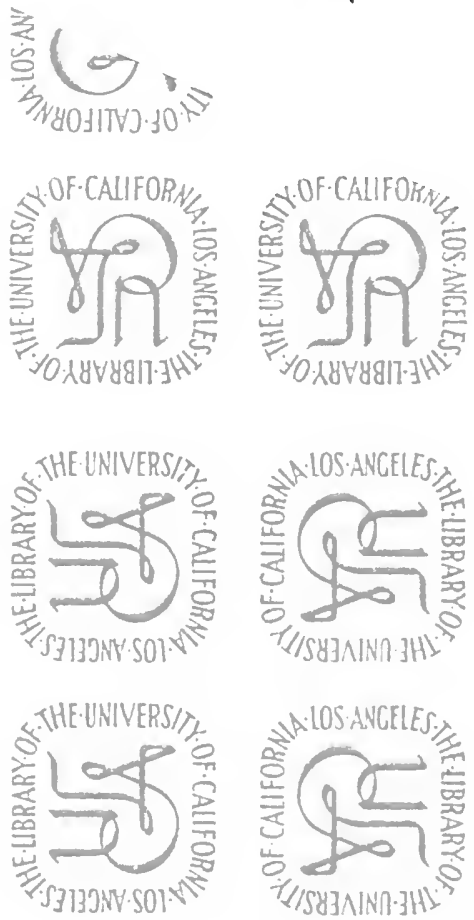

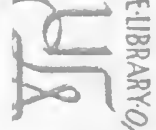

I3NING.3HI

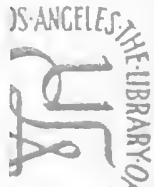

IJNNA.JHL
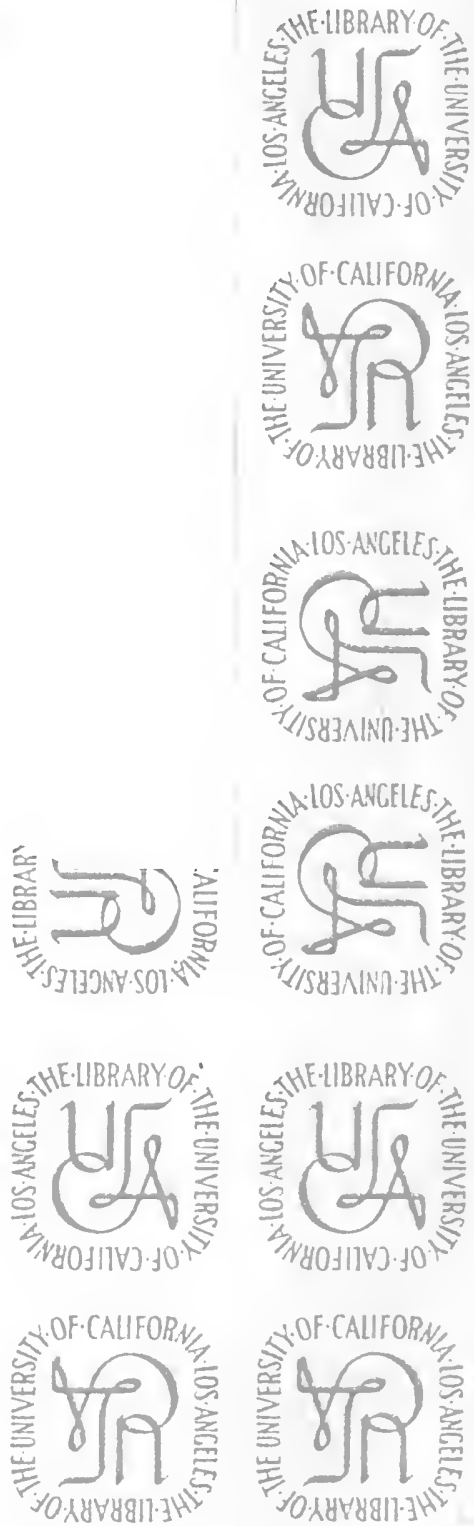
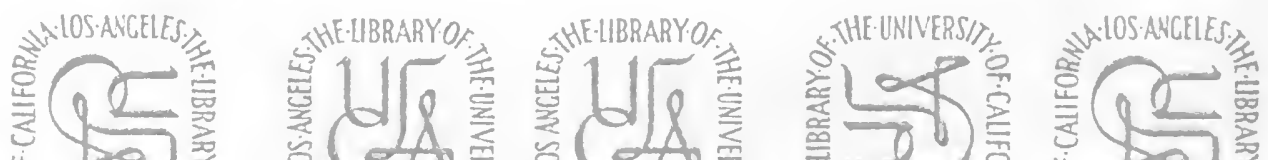


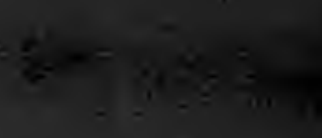

